H.

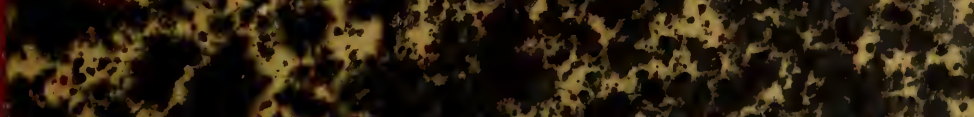

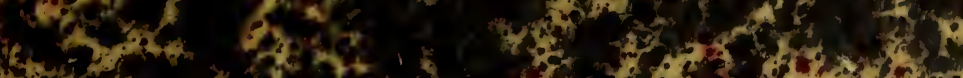

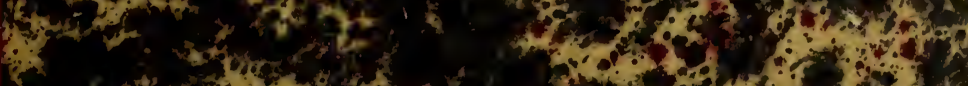

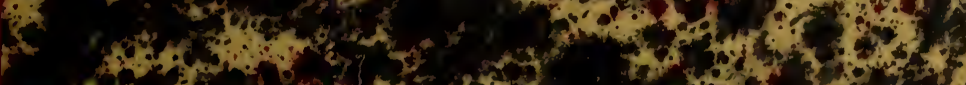

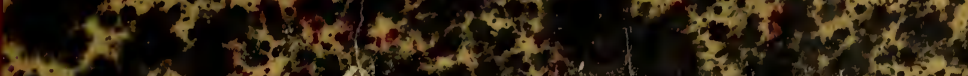

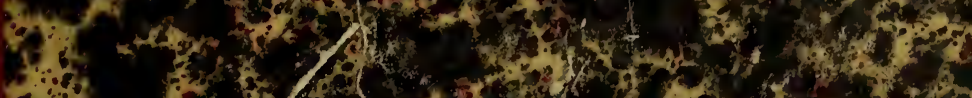

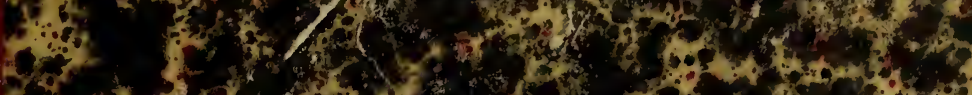

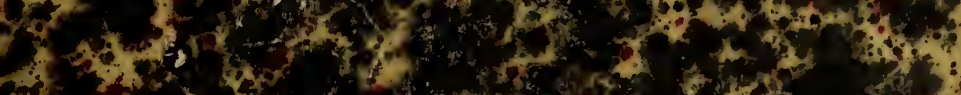

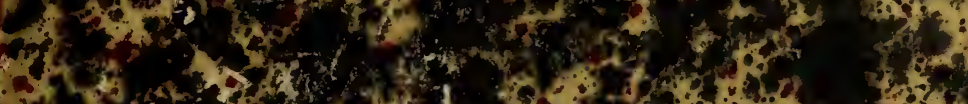

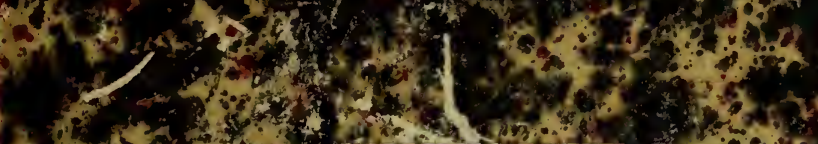

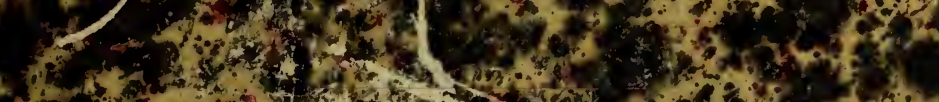
1.3.

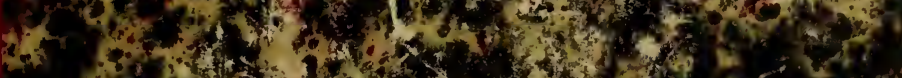

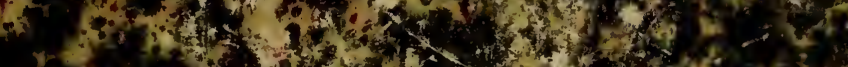

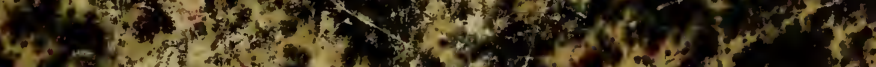

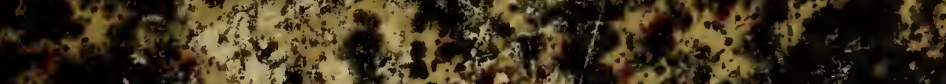

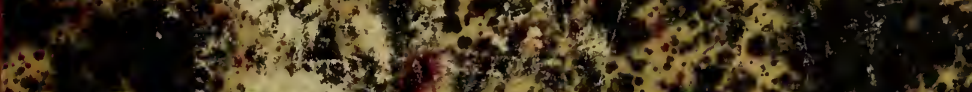

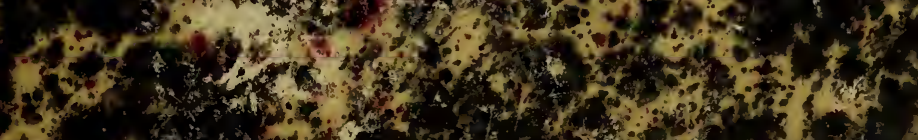

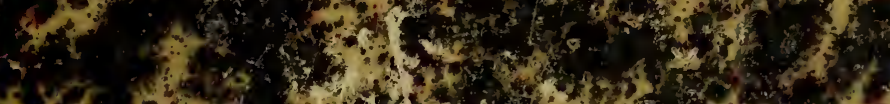

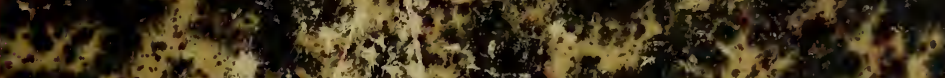

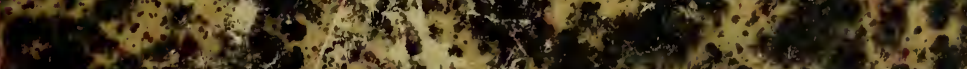

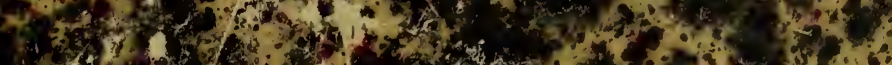

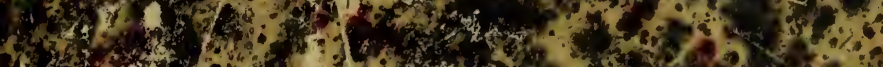

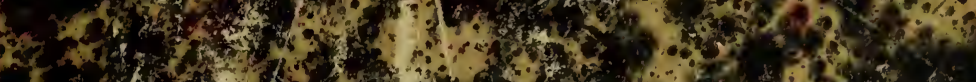

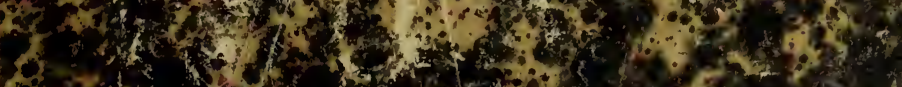

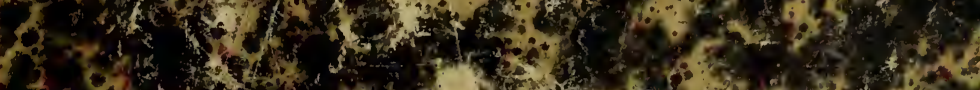

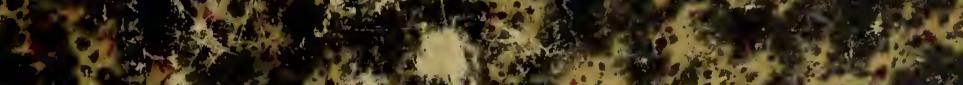

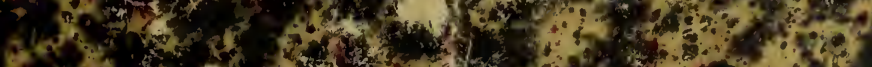

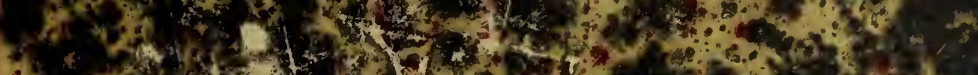

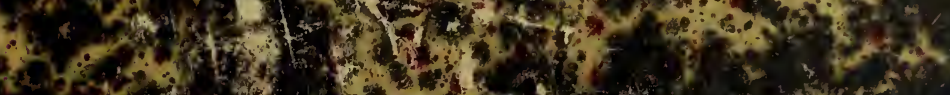

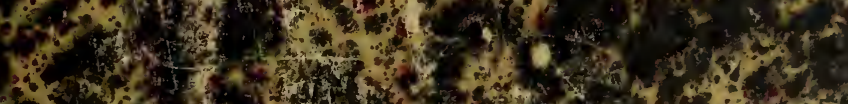

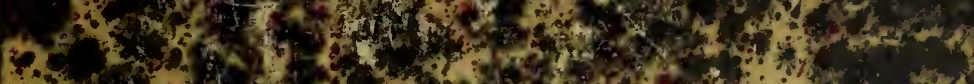

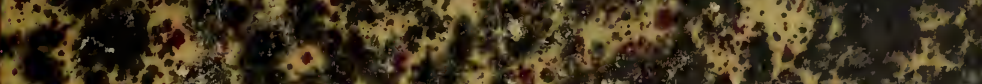

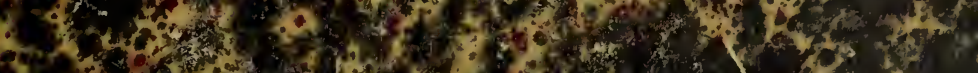

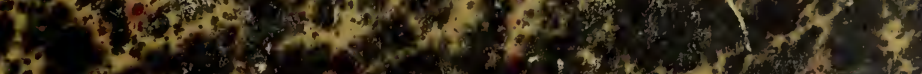

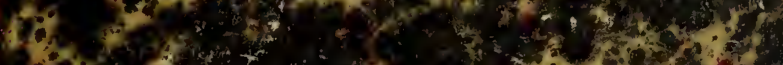

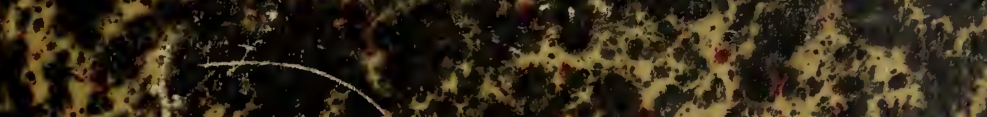

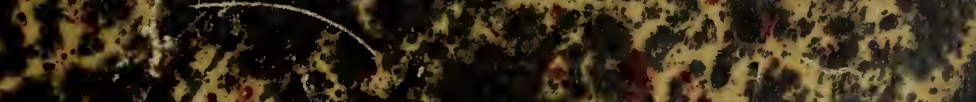

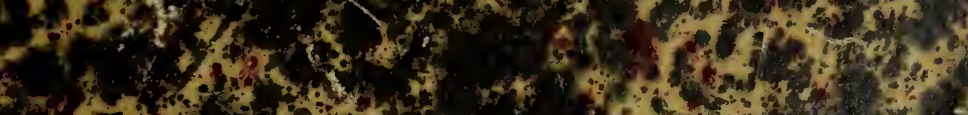

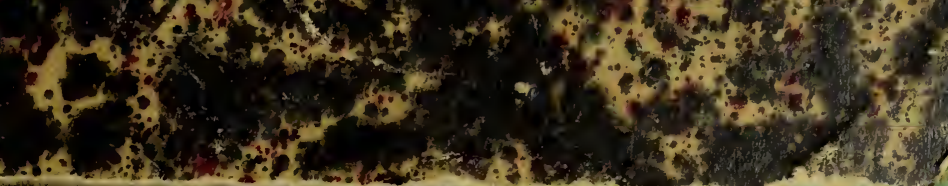




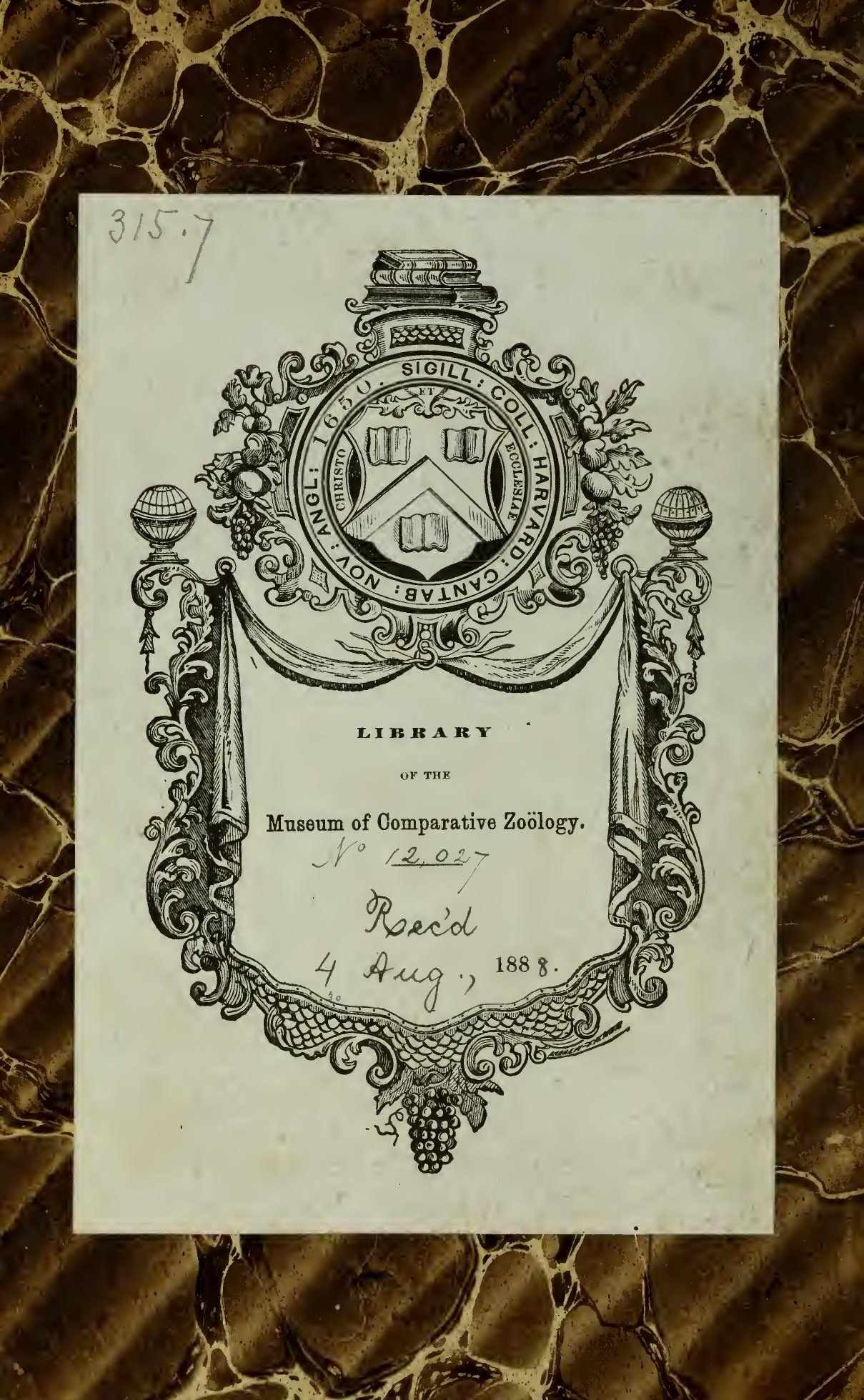




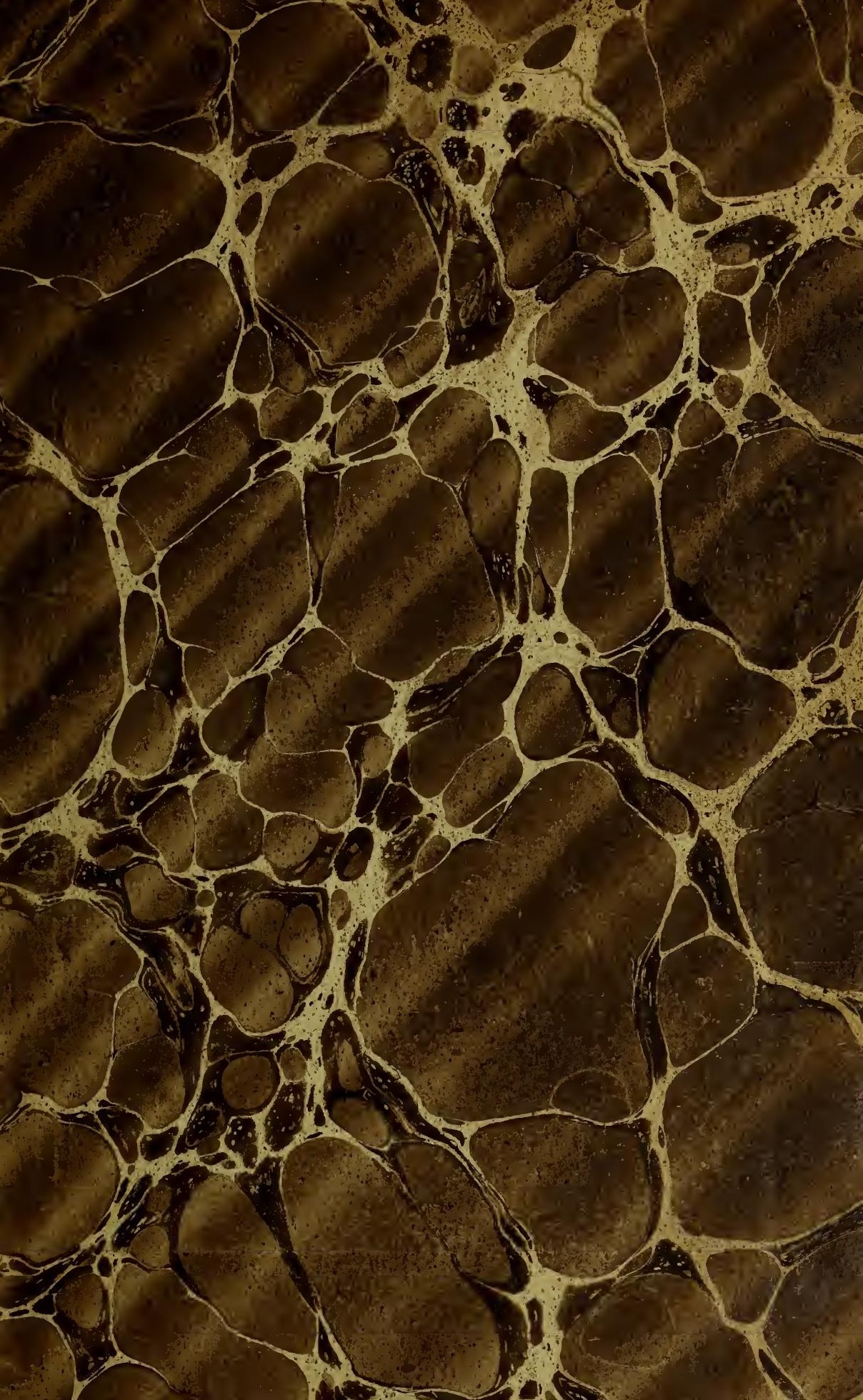







\section{MÉMOIRES}

D'ANTHROPOLOGIE 

0

\section{MÉMOIRES}

SUR LE

\section{CERVEAU DE LHOMME}

\section{ET DES PRIMATES}

PAR

\section{PAUL BROGA}

SEGRÉTAIRE GÉNÉRAL DF: LA SOGIÉTÉ D'ANTHROPOLOGIE DE PARIS PROFESSEUR A LA FAGULté DE MÉdeCiNe MEMBRE DE L'ACADÉMIE DE MÉDECINE

PUblís AVEC UNE INTRODUC'ION ET DES NOTES

PAR

Le docteur S. P0ZZI

PHÉSIDENT DE LA SOCIÉTÉ D'ANTHROPOLOGAE DE PARIS

AGRÉGÉ a LA FAGULTÉ DE MÉdECINE

SEGRÉTAIRE DE LA SOCIÉTÉ DE CHIRURGIE.

\section{SPARIS}

(i. REINWALD, ÉDITEUR

15, rue des SaINTS-PĖres, 15 


$$
\text { isylizil }
$$

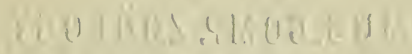

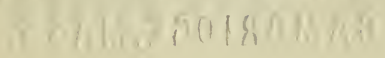




\section{INTRODUCTION}

Les dernières années de la vie de Broca ont été presque entièrement consacrées à l'étude du cerveau, et quand la mort est venue le frapper, il mettait la dernière main à une description du cerveau schématique, destinée à vulgariser le résultat de ses recherches.

On peut affirmer que cette étude l'a de tout temps attiré. Alors même qu'il semblait consacrer tous ses efforts au cràne, il pensait à son contenu, et ne manquait jamais de faire remarquer, dans un langage qui rappelait l'ancien candidat à l'École polytechnique, que le crâne est fonction $d u$ cerveau.

J'ai été chargé par la confiance de sa famille du pieux devoir de réunir et de compléter par quelques notes et additions discrètes les travaux de mon bien-aimé maître qui se rapportent au cerveau (1). Ils forment le plus étendu des volumes où, sous le titre de Mémoires d'anthropologie, il avait commencé à réunir toutes ses œuvres sur l'histoire naturelle de l'homme. J'ai cru pouvoir classer les nombreux articles qui forment ce tome $\mathrm{V}^{\mathrm{e}}$, en y établissant quelques divisions destinées surtout à favoriser les recherches. Les voici :

(1) Je ne saurais oublier de mentionner ici l'aide intelligente et dévouée que m'a prêlée dans ce travail l'ancien secrétaire de Broca, Charles Drouault ; une mort récente vient de l'enlever à l'estime et à l'affection de ses nombreux amis. 
I. Siège de la faculté du langage articulé, p. 1 à 162 ;

II. Anatomie pathologique du cerveau, p. 163 à 246 ;

III. Anatomie comparée du cerveau, p. 247 à 464;

IV. Morphologie comparée de l'homme et des primates, p. 465 ad finem.

Enfin, on trouvera en appendice quelques notes relatives aux procédés de conservation des cerveaux.

Pour la plupart des médecins et des savants, le nom de Broca se trouve lié à la détermination du siège précis de la faculté du langage articulé. Les Anglais, plus justes que ses compatriotes, désignent toujours la troisième circonvolution frontale gauche sous le nom de Broca's convolution. Certes, ce fut un grand mérite que de mettre hors de doute un fait aussi remarquable, et d'établir cette première localisation cérébrale, à peine entrevue par ses devanciers. Il ne faut pourtant pas que l'éclat et le retentissement d'une pareille découverte éclipsent et fassent méconnaître l'œuvre considérable de Broca dans l'étude de l'anatomie comparée et de la morphologie du cerveau de l'homme et des primates. Je n'hésite pas à proclamer, pour ma part, que la détermination du lobe limbique, de ses connexions et de sa signification a une portée qui dépasse de beaucoup celle des études sur le rôle de la troisième circonvolution frontale.

Le Mémoire sur la nomenclature cérébrale a fait faire à la science un pas plus grand encore et plus décisif que les travaux sur l'aphémie.

Mais tandis que ces derniers s'adressent à la généralité des médecins, et étaient par suite assurés d'emblée d'un retentissement immédiat et considérable, les recherches d'anatomie comparée ne sont accessibles qu'à des lecteurs peu nombreux. De plus, comme toutes les curres de science 
pure, étant sans application pratique, elles n'étaient pas faites pour émouvoir le grand public dès leur apparition. De là, sans doute, le peu de notoriété de ces travaux de premier ordre. Enfin, peut-être faut-il faire entrer pour quelque part dans cette injustice de la renommée, le mode de leur publication. Si Broca avait eu le temps de réunir en un ouvrage d'ensemble ces études partielles et éparses dans des revues périodiques, elles auraient sans doute forcé davantage l'attention du monde savant.

Pour se rendre un compte exact de leur haute valeur, il est nécessaire de se reporter à l'état de nos connaissances au moment où Broca, en 1877, publia successivement, dans les Bulletins de la Société d'anthropologie, de courts résumés des mémoires capitaux qui devaient paraître l'année suivante dans la Revue d'anthropologie.

La morphologie du cerveau de l'homme et des primates avait été presque définitivement établie en 1855 par l'immortel Mémoire sur les plis cérébraux de Gratiolet. Partant de cette parole profonde de Leuret : « le cerveau du singe peut, jusqu'à un certain point, être considéré comme une ébauche de celui de l'homme (1) ", Gratiolet avait débrouillé le chaos des circonvolutions du cerveau humain. Si quelques points de détails ont dû être corrigés et complétés dans son œuvre, elle n'en est pas moins restée la base de tous les travaux parus depuis en France ou à l'étranger. Elle a marqué véritablement une ère nouvelle dans nos connaissances.

Le chaos avait disparu pour l'homme et les singes, mais il continuait à exister au-dessous d'eùx. Des quatorze groupes que Leuret avait laborieusement établis dans les mammifères « d'après les caractères tirés des circonvo-

(1) Anatomie comparée du système nerveux, t. Ier, p. 399. Paris, 1839. 
lutions elles-mêmes ", treize demeuraient à l'état d'énigme, sans qu'aucun effort sérieux eût été tenté pour établir l'homologie de parties en apparence tout à fait incomparables. C'est que, pour s'orienter dans ce dédale, il fallait trouver un fil d'Ariane, un principe directeur jouant le rôle que le schéma simien avait eu pour Gratiolet. La conception de ce dernier, quoique entrevue par Leuret, avai été un trait de génie. On ne peut davantage refuser ce nom à la détermination, par Broca, de ce qu'il a appelé le grand lóbe limbique de l'hémisphère.

Considérons comparativement: $1^{\circ}$ le dessin d'un cerveau d'homme ramené à ses divisions élémenta

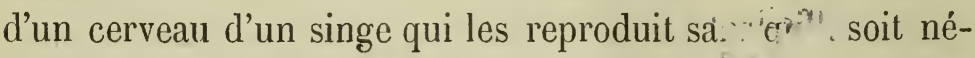
cessaire de le réduire à l'état de schéma; $\%$ des figures représentant des cerveaux de rongeurs, de pachydermes, de carnassiers.

Un fait frappe au premier coup d'œil : dans la première série manque un grand prolongement antérieur de la substance cérébrale (le lobe olfactif) qui, dans la seconde, s'avance au-dessous de la partie antérieure ou frontale de l'encéphale et d'où partent des filets nerveux qui traversent la lame criblée de l'ethmoïde pour al'er à la muqueuse olfactive. A sa place, chez l'homme et $\%$ singes, existe une dépression au fond de laquelle rampe un filet grêle, à peine un peu renflé à son extrémité, ayant plutôt l'aspect d'un nerf que d'une division du manteau. Aussi, l'anatomie n’ayant longtemps été que de l'anthropotomie, lui a-t-on appliqué la dénomination de nerf olfactif qu'il mérite si mal. L'étude minutieuse des connexions, où nous ne pouvons entrer ici, démontre péremptoirement que le nerf olfactif de l'homme et des singes n'est que le vestige atrophié du lobe olfactif des autres animaux. Celui-ci se continue avec le lobe, 
très agrandi, de l'hippocampe, qui se prolonge sans ligne de démarcation à la face interne de l'hémisphère par la prétendue circonvolution du corps calleux. Une même scissure cerne cet arc complet concentrique au limbe (1) de l'hémisphère, et la division qu'elle limite a par suite reçu de Broca le nom de grand lobe limbique, subdivisé en trois lobes (secondaires), celui du corps calleus, celui de l'hippocampe et enfin Ie lobe olfactif. Ce dernier, continu en arrière avec l'hippucampe, l'est, en haut, avec l'extrémité antérieure du lobe du corps calleux.

Un second fait frappe l'observateur. Ce lobe limbique, chez les gyrencénhales inférieurs, se distingue absolument du reste du intęau par son aspect lisse et sa simplicité. « Le contrasté cos ..., dit Broca, qu'il devient évident que ces deux parties de l'hémisphère, si différentes par leur structure, le sont aussi par la nature de leurs fonctions, et si l'on considère que l'une reste stationnaire et imperfectible pendant que l'autre se perfectionne et se développe, que celle-là perd de son importance à mesure que celle-ci fait des progrès, on est conduit à reconnaître que la première est le siège des facultés inférieures qui prédominent chez la brute, que la seconde est $\left.{ }^{l}\right\lrcorner$ siège des tacultés supérieures qui prédominent chez les : aimaux intelligents, et l'on peut exprimer cette opposition de leurs caractères respectifs en disant que le manteau de l'hémisphère se compose de deux parties : l'une brutale, représentée par le grand lobe limbique; l'autre, intellectuelle, représentée par le reste du manteau. »)

Ce sont les connexions du grand lobe limbique qui donnent la clef du plissement du reste du manteau.

Nous avons vu comment l'arc limbique était fermé de

(1) Sur la circonvolution limbique et la scissure limbique (Bulletins de la Société d'anthropologie, $2^{\circ}$ séric, t. XII, 187\%, p. 648). 
toutes parts par l'union antérieure de la prétendue circonvolution du corps calleux avec le lobe de l'hippocampe par l'intermédiaire du lobe olfactif. Ainsi bridé, pour ainsi dire, partout fixé du reste par le pourtour du grand lobe limbique, le manteau ne peut s'accroître en longueur et en largeur sans se plisser. "Le plissement dans le sens transversal produit les circonvolutions longitudinales; celles-ci étant devenues plus longues que le lobe limbique sur lequel leurs deux extrémités vont aboutir, décrivent des arcs dont ce lobe représente la corde. La plus élevée, celle qui longe la pente inter-hémisphérique et qu'on peut appeler circonvolution sagittale, forme un grand arc peu flexueux; mais la plus inférieure et la plus externe, celle qui longe l'arc inférieur de la scissure limbique, forme un arc plus court, mui se replie en s'adossant à lui-même et ce plissement donne naissance à la scissure de Sylvius. Lorsqu'il y a plus de deux circonvolutions longitudinales, les circonvolutions intermédiaires décrivent des arcs décroissants entre la sagittale et la sylvienne. )

Le plissement dans le sens longitudinal dont la scissure de Sylvius était déjà un indice, donne encore naissance, en avant, à la scissure de Rolando. Chez les gyrencéphales inférieurs aux primates, elle naît tout près de la scissure limbique (dont elle est le plus souvent séparée par un petit pli) très en avant de la scissure de Sylvius; on aurait peine, tout d'abord, à y reconnaître l'homologue de la scissure de Rolando de l'homme et des singes. Son origine a lieu près du point où le lobe olfactif commence à se réunir au reste de l'hémisphère. Elle se dirige très obliquement en avant, puis se recourbe en haut et en dehors, ne laissant au-devant d'elle qu'un très petit lobe frontal.

Le plissement transversal ne se produit pas en arrière 
(chez les mêmes animaux) de manière à limiter un lobe occipital. Il en résulte que tout ce qui est postérieur au lobe frontal forme ici un lobe pariéto-occipito-temporal, ou plus simplement pariétal. La circonvolution temporale de l'hippocampe des primates est ici, en effet, détachée du reste du manteau pour entrer dans le lobe limbique.

Telle est la constitution essentielle (chez les gyrencéphales inférieurs) de la face externe de l'hémisphère.

Quelques mots sur la face interne :

Là le lobe limbique s'étale dans toute sa longueur; audessus de lui, déborde la circonvolution sagittale, séparée du lobe limbiqne par une scissure qui a reçu de Broca le nom de scissure sous-pariétale; cette scissure, antérieurement, vient inciser, chez beaucoup d'animaux, la face externe de l'hémisphère en formant le sillon crucial de Leuret (1). En avant d'elle existe une large communication du lobe limbique avec le lobe frontal; cette fusion est empêchée à la partie antérieure par un sillon sous-frontal (Broca).

Si nous considérons maintenant le cerveau d'un carnassier, d'un rongeur, etc., suivant sa face ou norma inférieure, nous y voyons encore le grand lobe limbique représenté par deux saillies importantes, le lobe olfactif en avant et le lobe de l'hippocampe en arrière. Entre la base

(1) Pour le dire en passant, ce sillon n'est nullement l'homologue de la scissure de Rolando des primates et de l'homme, comme on l'enseigne généralement, mais bien de l'encoche qu'on observe sur ces derniers, au niveau du bord sagittal de l'hémisphère immédiatement en arrière du lobule paracentral ou ovalaire. Nous avons dit que la scissure de Rolando des mammifères en général et en particulier des carnassiers est située très en avant du sillon crucial, entre lui et la partie antérieure de la scissure limbique. On voit, d'après cela, que les recherches relatives aux localisations cérébrales faites en se basant sur cette détermination morphologique erronée du sillon crucial, péchaient par la base dans l'application qu'on en a faite au cerveau humain. 
de celui-ci et la partie antérieure de celui-là est une dépression transversale peu profonde qui établit une simple ligne de démarcation entre ces deux lobes sans interrompre en aucune façon leur continuité. C'est l'analogue de la partie interne de la scissure de Sylvius des primates. Cette partie interne, on le voit, est très différente par son origine de la partie externe. Tandis que cette dernière est le résultat du plissement de l'écorce, la première est due, chez l'homme et les primates, à une atrophie du grand lobe limbique qui a permis l'isolement de ses deux parties constituantes, olfactive et hippocampique. Broca a judicieusement consacré cette différence capitale en réservant à la partie interne de la scissure de Sylvius, chez l'homme et les primates, le nom de vallée de Sylvius (1).

Dans la base du lobe olfactif on distingue trois racines nerveuses : l'une externe, blanche, large bandelette qui longe la scissure limbique et se perd dans la partie externe de l'hippocampe; la seconde interne, grise, qui passe sur la face interne de l'hémisphère et se continue avec l'extrémité antérieure de la circonvolution du corps calleux; la troisième, intermédiaire, encore plus grise, qui se porte directement en arrière dans la partie interne de l'hippocampe; sa partie profonde est formée de fibres blanches qui vont se continuer avec les fibres inférieures du pédoncule cérébral. Ces trois racines sont continuées entre elles par leurs bords, et assez étroitement confondues à leur origine (2). La racine olfactive externe sépare par son relief la dépression, qui est l'analogue de la vallée de Sylvius, d'avec la scissure de Sylvius pro-

(1) Elle correspond sur le cerveau humain à ce qu'on appelle l'espace crible de Vicq d'Azyr.

(2) Broca a décrit une quatrième racine ou racine olfuctive supérieure. Recherches sur les centres olfuctifs (Revue d'anthrop., 1879, I. II, p. 396). Je ne fais que la mentionner pour mémoire. 
prement dite. Cette séparation rend très difficile au premier coup d'œil l'homologic entre la scissure de Sylvius des primates et celle des autres gyrencéphales.

Récapitulons maintenant les principaux caractères du cerveau des gyrencéphales autres que les primates, en opposition avec ceux du cerveau des primates et de l'homme. Ces caractères sont :

$1^{\circ}$ Existence d'un grand lobe limbique constitué par le développement et la fusion d'éléments qui demeurent plus ou moins distincts et en partie atrophiés chez les primates : le lobe du corps calleux, le lobe de l'hippocampe et le lobe olfactif. - C'est la présence de ce grand lobe à l'état de division maîtresse du manteau qui est la cause supérieure et déterminante de tous les autres caractères que je vais énumérer.

$2^{\circ}$ Réplétion de la vallée de Sylvius par un énorme lobe olfactif (si réduit dans le type primate qu'il a reçu l'humble nom de nerf). Séparation établie entre le vestige de cette vallée et la scissure de Sylvius sur la face externe par la grosse racine olfactive externe.

$3^{\circ}$ Exiguité extrême du lobe frontal réduit le plus souvent à une seule circonvolution (1). L'extrémité inférieure de la scissure de Rolando est toujours dirigée en arrière et tombe toujours dans la scissure limbique en avant de l'extrémité antérieure du lobe de l'hippocampe.

$4^{\circ}$ Absence de lobe occipital et de lobe temporal. Tout ce qui dans le manteau n'appartient pas au lobe frontal ou au lobe pariétal est absorbé par le grand lobe limbique. La première circonvolution temporale ou circonvolution de l'hippo-

(1) Sur les cerveaux les plus compliqués (cheval, chameau, bœuf, tapir), le lobe frontal est un peu plus grand et subdivisé par un ou deux sillons ; sur le cerveau de l'éléphant, cette complication est encore supérieure. 
campe des primates est représentée seule sur le cerveau des autres mammifères par la terminaison antérieure du grand lobe limbique ou lobe de l'hippocampe. Quant aux autres circonvolutions temporales et aux circonvolutions occipitales, elles n'existent pas ou, pour mieux dire, elles sont confondues en une seule masse avec les circonvolutions pariétales.

$5^{\circ} \mathrm{A}$ la face interne de l'hémisphère, situation du lobe du corps calleux placé presque tout entier au-dessous d'un lobe pariétal immense, tandis qu'une très petite portion seulement se trouve en contact et presque fusionnée avec un lobe frontal rudimentaire. La scissure sous-frontale est presque effacée et réduite à l'état de sillon, la scissure sous-pariétale au contraire est grande et accusée. C'est précisément l'inverse chez les primates et l'homme. Le lobe frontal étant devenu très grand et le pariétal très petit, le sillon sous-frontal devient une profonde scissure, la scissure sous-pariétale se dégrade en un simple sillon. De même que les rapports, les connexions sont renversées. Un simple pli de passage rétro-limbique est ici le vestige de la fusion interne du lobe pariétal avec le lobe du corps calleux au niveau du lobuls quadrilatère: inversement, pour le lobule frontal chez l'homme, un ou deux plis de passage allant au lobe du corps calleux remplacent les connexions étroites qu'on voit chez les gyrencéphales inférieurs.

Tel est, en résumé, le tableau des différences qui existent entre les deux types cérébraux que nous avons entrepris de comparer. Certes, elles sont nombreuses et profondes, mais cependant, partout il nous a été possible, après Broca, de retrouver les parties homologues, de reconnaître leurs connexions identiques, en un mot de comparer effectivement 
le cerveau des primates et des animaux placés au-dessous d'eux dans la série animale.

Ainsi que l'écrit le maître: «Toutes les parties du cerveau des primates ont leur analogie dans les autres cerveaux; vice versa, la détermination de ces analogies permettra de constater un type commun à tous les mammifères, type généd ral dont les divers types spéciaux ne sont que des dérivés (1).»

Avant d'aller plus loin dans l'interprétation des faits, il est nécessaire de nous arrêter un instant à l'étude faite par Broca d'un autre type cérébral qui se rapproche par des traits remarquables du type primate tout en demeurant profondément séparé. Je veux parler du cerveau des cétacés et des amphibies.

Le point commun à ces deux types est le peu de développement du grand lobe limbique corrélatif à l'atrophie de sa portion antérieure ou lobe olfactif.

Qu'on examine par exemple un cerveau de dauphin (cétacé). L'appareil olfactif a entièrement disparu, tant le lobe olfactif que ses filaments (si bien que la lame de l'ethmoïde n'est plus criblée); le lobe de l'hippocampe est encore plus réduit que chez les primates, et se fusionne antérieurement avec les circonvolutions adjacentes (2). La scissure de Rolando, détachée en avant de la scissure de Sylvius, limite un lobe frontal très petit, presque entièrement réduit à un étage orbitaire plissé antérieurement, mais tout à fait lisse en arrière. Il y a là, dit Broca, « une large surface dont la simplicité absolue contraste avec la grande complication de tout le reste du manteau, surface comparable à celle que forme, sur une

(1) P. Broca. Le grand lobe limbique, etc. (Revue d'anthrop., 1878, p. 465).

(2) Cette fusion est l'ébauche de la formation d'un lobe temporal, comme dans le type primate où l'hippocampe ne forme plus qu'une circonvolution de ce lobe. 
carte de géographie, un désert entouré de pays fertiles ». Broca lui a effectivement assigné le nom pittoresque de désert olfactif pour marquer à la fois son aspect et sa relation avec la disparition totale de l'appareil olfactif.L'absence du lobe olfactif et des racines qui en partent (en particulicr de la racine grise ou moyenne) amène la profonde dépression de la portion interne de la scissure de Sylvius en vallée de Sylvius, qui se continue sans démarcation aucune avec la portion externe de la scissure et l'apparition d'un espace quadrilatère ou espace perforé. On reconnaît là le type humain. Chez les amphibies (exemple, le phoque), où l'appareil olfactif n'est pas anéanti, mais seulement atrophié, on observe des caractères analogues, quoique moins marqués.

Chez les amphibies comme chez les cétacés, la présence de la vallée de Sylvius constitue une similitude d'autant plus frappante avec les primates que les types cérébraux sont du reste entièrement différents. En effet, l'atrophie de l'appareil olfactif coexiste chez les cétacés et amphibies avec un développement rudimentaire du lobe frontal; chez l'homme et les primates, au contraire, cette même atrophie coïncide avec l'exagération du lobe frontal.

Le développement du lobe olfactif étant toujours corrélatif à celui des autres portions du grand lobe limbique sur lesquelles s'établissent du reste les connexions de ses racines, il est légitime d'induire que toute cette portion du cerveau est subordonnée au sens de l'olfaction. Broca a consacré à l'établissement de cette proposition une argumentation remarquable et tout à fait probante (1). Il a de plus parfaitement mis en relief le rôle capital de l'odorat dans

(1) Revue d'anthrop., 1878, p. 392 et suiv. 
l'immense majorité des mammifères : «L'odorat joue chez eux un rôle souvent égal et même supérieur à celui de la vue. C'est lui qui lés guide dans le choix de la nourriture, dans la poursuite de la proie, dans la fuite du danger, dans la recherche de la femelle, dans le retour au gîte. L'exercice de ce sens est simple et n'exige qu'une faible opération intellectuelle. L'odorat perçoit une certaine odeur qui est propre à un certain corps; pour reconnaître ce corps, il suffit d'un peu d'expérience; pour en apprécier la distance, il suffit d'apprécier l'intensité de la sensation. L'animal qui fait le mieux ce diagnostic n'est pas le plus intelligent, c'est celui qui possède l'appareil olfactif le plus développé; et les quadrupèdes les plus stupides se trouvent souvent par là bien supérieurs à l'homme. Ce fait seul (ajoute Broca) suffit pour montrer toute l'infériorité du sens de l'odorat. On ne peut pas dire qu'il soit en raison inverse de l'intelligence, mais on peut dire du moins qu'il prédomine chez la brute, et on peut le qualifier de sens brutal, car il tire son importance du degré de perfection de l'appareil organique qui lui est attribué, bien plus que des actes intellectuels qu'il met en jeu dans l'ensemble du cerveau. »)

Broca a donné le nom de mammifères osmatiques (de o $\sigma \mu \dot{n}$, odorat) aux mammifères ordinaires chez lesquels la prédominance du sens de l'odorat est attestée par le grand développement de l'appareil olfactif; il a appelé anosmatiques ceux chez lesquels ce sens pour un motif quelconque a perdu sa suprématie et où on observe par suite la disparition partielle ou l'amoindrissement du grand lobe limbique. Ces anosmatiques, nous l'avons vu, forment deux catégories distinctes, placées à des degrés bicn éloignés de l'échelle zoologique: d'une part, l'homme et les primates, de l'autre, les cétacés et les amphibies. 
Pour ces derniers, la cause déterminante de l'atrophie de l'appareil olfactif et de ses dépendances est d'une grande simplicité. Elle est la conséquence de leur vie aquatique, rendant inutile ou à peu près l'exercice du sens de l'odorat (1). La fonction cessant, l'organe disparaît ou s'amoindrit.

Devons-nous rapporter aussi simplement à la déchéance du sens de l'olfaction, chez l'homme et les primates, l'extrême simplicité de l'appareil olfactif et de ses dépendances? Un observateur superficiel pourrait être tenté de le supposer. Il est certain, en effet, que l'homme, par exemple, est infiniment moins bien doué relativement au flair que le plus vulgaire carnassier. Mais un examen plus attentif du cerveau montre aussitôt que la raison de cette modification anatomique est plus haute que la simple diminution d'une fonction. L’immense développement du lobe frontal s'impose comme la cause déterminante de celte véritable révolution morphologique. Et qu'on ne vienne pas dire que ce développement frontal est l'effet et non la cause de l'atrophie olfactive. Le cerveau des cétacés et des amphibies est là pour attester le contraire: la dégradation du lobe limbique n'y est-elle pas restée sans influence sur les dimensions du lobe antérieur du cerveau? Tout nous l'indique, c'est bien au contraire ce développement insolite du lobe frontal, chez les primates, qui a été le facteur primordial et suffisant de la diminution du lobe olfactif en particulier, et du grand lobe limbique en général. Ce lobe frontal, siège privilégié de la pensée, arrivé à des proportions extraordi-

(1) Cela est si vrai que chez les carnassiers de la famille des martres comme la loutre, qui passent une partie de leur existence dans l'eau el l'autre sur terre, le grand lobe limbique, sans subir d'atrophie, offre une réduction sensible de volume, ce qui le rend très propre à la comparaison avec le cerveau des primates pour la recherche des parties homologues (Broca). 
naires chez l'homme, a exproprié pour ainsi dire et remplacé dans l'étage antérieur du crâne l'énorme masse sensorielle qui gênait son expansion. Désormais l'odorat ne sera plus qu'un sens secondaire et dont on pourra se passer au besoin. - Comparez l'homme qui en est serait dépourvu avec l'animal osmatique qui en serait privé. Le premier y perd : à peine quelques sensations; le second, désarmé dans la lutte pour l'existence, est presque fatalement destiné à périr.

L'animal était quadrupède, posture essentiellement favorable à l'exercice du flair. Le primate se relève, l'homme détache définitivement la tête du sol et dirige son regard parallèlement à l'horizon. L'hégémonie du sens de la vue se substitue à celle de l'odorat et la station bipède est le corollaire obligé de ce changement. Enfin, comme tout dans la nature s'enchaîne et se commande, les extrémités antérieures qui sont devenues libres se modifient, deviennent des mains, et le toucher prend place à côté de la vue, au premier rang parmi les sens.

Quelle révolution radicale! N'est-il pas curieux pour l'anatomiste de pouvoir la rattacher légitimement à ce premier fait si simple en apparence : le développement du lobe frontal?

Il ne l'est pas moins, au même point de vue, de retrouver dans le cerveau de l'homme les vestiges de cet organe détrôné, le lobe limbique. Au lieu de former un tout imposant, il est réduit à l'état de tronçons à peine reliés entre eux et, pour ainsi dire, de débris. !

En-ferons-nous l'énumération?

D'abord, à la face interne, la circonvolution crêtée ou circonvolution du corps calleux dont l'aspect lisse garde bien toujours, si je puis m'exprimer ainsi, quelque chose du type limbique, mais qui dégénère, surtout en avant, en un simple 
ruban, tandis que c'était, au contraire, cette partie, voisine du lobe olfactif, qui possédait chez les mammifères osmatiques la plus majestueuse ampleur.

Ensuite, à la face inférieure, la circonvolution de l'hippocampe, réduite aussi, et ne méritant plus le nom de lobe, simple appendice, maintenant, du lobe temporal dont elle forme la dernière circonvolution. Le lobule qu'elle présente chez l'homme et les primates paraît être tout ce qui reste du centre olfactif qui chez les osmatiques occupait la totalité du lobe de l'hippocampe (1). Au-dessous de ce lobule on voit nettement, chez les singes, un sillon (sillon limbique) qui, partant de la vallée de Sylvius, entaille plus ou moins profondément d'avant en arrière la pointe du lobe temporal. Chez l'homme de race blanche, ce sillon est le plus souvent effacé et réduit à une légère dépression longitudinale, plus large que profonde et sur laquelle la pie-mère s'applique sans former un repli. Broca a cru longtemps que l'absence du sillon limbique différenciait le cerveau humain de celui des singes. Mais il l'a retrouvé plus tard sur quatorze cerveaux de nègres, sur celui d'un Annamite, d'un Chinois, d'un noir de l'Hindoustan, d'un indigène du Pérou, en un mot sur tous les cerveaux non caucasiques qu'il a étudiés. Il en a conclu que la présence de ce sillon chez l'homme est un véritable caractère d'infériorité. Il est remarquable que l'évolution du sillon limbique soit l'inverse de celle des autres sillons. Ceux-ci se développent et se multiplient d'autant plus que le cerveau se perfectionne davantage, tandis que dans les mêmes conditions le sillon limbique tend au contraire à s'effacer. N'est-ce pas précisément parce qu'il est en rapport avec une cause spéciale et locale, la fonction olfactive, qui,

(1) Broca, Recherches sur les centres olfuctifs (Revue d'anthropologie, 1879). 
( portée au maximum dans la vie de nature, tombe au minimum chez les nations civilisées ? (1) ). Son absence presque constante chez le blanc n'est probablement pas un véritable caractère ethnique, mais s'explique par l'irnfluence de l'état social et l'action prolongée de la désuétude.

Le plus dégradé, si l'on peut ainsi dire, des vestiges du grand lobe limbique des osmatiques, chez l'homme et les primates, c'est sa partie antérieure; qui reconnaîtrait, dans ce mince tractus qui rampe humblement sous le lobule orbitaire, ce qui est une des plus importantes portions du manteau dans la grande majorité des mammifères? Le lobe olfactif de l'homme ressemble tant, par ses proportions réduites, aux nerfs qui naissent de l'encéphale, que les anatomistes n'ont pas hésité à le ranger parmi eux. Au point de vue de la structure et de la morphologie, c'est pourtant bien plutôt comme je l'ai déjà dit, une circonvolution qu'un nerf; les radicules qui partent de son renflement, pour passer dans les fosses nasales à travers la lame criblée de l'ethmoïde, méritent seules cette dénomination.

Mais le Primate a de quoi se consoler de cette atrophie de la partie brutale de son encéphale. Elle a pour magnifique compensation le développement imposant de la partie intellectuelle. Cette conquête par le lobe frontal de la surface du cerveau est une invasion véritable : en avant elle émousse et arrondit l'extrémité effilée ou bec de l'encéphale; sur toute la face externe, elle repousse loin en arrière sa ligne frontière, la scissure de Rolando, et, en refoulant ainsi tous les éléments voisins, elle les force, pour ainsi dire, à se resserrer et à se contourner en groupements nouveaux : le lobe occipital se sépare ainsi du lobe pariétal

(1) Broca, loc. cit. 
par une démarcation distincte, le lobe temporal prend naissance : le cerveau primate est constitué.

La détermination véritablement géniale de ces connexions et de ces transformations morphologiques est peut-être le plus grand titre de Broca à l'admiration de la postérité. 


\section{MÉMOIRES}

\section{D'ANTHROPOLOGIE}

S马LGE DE LA FACULTÉ DU LANGAGE ARTICULE

\section{PERTE DE LA PAROLE}

\section{RAMOLLISSEMENT CHRONIQUE ET DESTRUCTION PARTIELLE}

DU LOBE ANTÉRIEUR GAUCHE DU CERVEAU ${ }^{1}$

(Bulletins de la Société d'anthropologie, 1861, 18 avril, $1^{\text {re }}$ série, t. II, p. 235.)

M. Broca, à l'occasion du procès-verbal, présente le cerveau d'un homme de cinquante et un ans qui est mort dans son service à l'hôpital de Bicètre, et qui avait perdu depuis vingt et un ans l'usage de la parole. La pièce devant être déposée au Musée Dupuytren, et l'observation devant être publiée dans le Bulletin de la Société anatomique, nous nous bornerons à donner ici un court résumé de ce fait, qui est tout à fait semblable à quelques-uns de ceux dont M. Auburtin a parlé dans la dernière séance (2).

(1) Nous avons reproduit ici ce résumé de l'observation publiée in extenso quelques jours après à la Société anatomique, et qu'on trouvera ci-après, à cause de son importance au point de l'évolution des idées de Broca sur le siège de la faculté du langage.

(2) Séance du 4 avril 1861. - Comme Broca, Auburtin montre Gratiolet hésitant sur la question des localisations, et il soutient, malgré Gratiolet, qu'il faut admettre l'importance des faits pathologiques prouvant, comme l'a dit Bouillaud, que la faculté du langage siège dans les lobes antérieurs. Mais il ajoute (p. 219) que le point précis où réside cette faculté n'a pas encore été déterminé.

Périer, à son tour, défend le principe des localisations cérébrales.

C'est à la suite de cette discussion que le 18 avril, Broca publie à la Société d'anthropologie cette première autopsie d'aphémique. 
Lorsque le malade fut admis à Bicêtre, il y a vingt ans, il avait perdu, depuis peu de temps, l'usage de la parole; il ne pouvait plus prononcer qu'une seule syllabe, qu'il répétait ordinairement deux fois de suite; quelle que fût la question qu'on lui adressât, il répondait toujours: tan, tan, en y joignant des gestes expressifs très váriés. C'est pourquoi, dans tout l'hospice, il n'était connu que sous le nom de Tan.

A l'époque de son admission, Tan était parfaitement valide et intelligent. Au bout de dix ans, il commença à perdre le mouvement du bras droit, puis la paralysie gagna le membre inferieur du même côté, si bien que, depuis six à sept ans, il a continuellement gardé le lit. Depuis quelque temps, on s'est aperçu que sa vue s'affaiblissait. Enfin, ceux qui étaient en rapports particuliers avec lui avaient remarqué que son intelligence avait beaucoup baissé dans ces dernières années.

Le 12 avril 1861, il fut transporté dans le service de chirurgie de l'hospice pour un vaste phlegmon diffus gangreneux, qui occupait toute l'étendue du membre inférieur droit (du côté paralysé), depuis le cou-de-pied jusqu'à la fesse. Ce fut alors que M. Broca le vit pour la première fois. L'étude de ce malheureux, qui ne pouvait parler et qui, étant paralysé de la main droite, ne pouvait écrire, offrait bien quelque difficulté. On constata toutefois que la sensibilité générale était partout conservée; que le bras et la jambe gauches obéissaient à la volonté; que les muscles de la face et de la langue n'étaient point paralysés et que les mouvements de ce dernier organe étaient parfaitement libres.

L'état de l'intelligence n'a pu être exactement déterminé, mais on a eu la preuve que Tan comprenait presque tout ce qu'on lui disait. Ne pouvant manifester ses idées ou ses désirs que par les mouvements de sa main gauche, il faisait souvent des gestes incompréhensibles. Les réponses numériques étaient celles qu'il faisait le mieux, en ouvrant ou fermant les doigts. Il indiquait, sans se tromper, l'heure d'une montre à secondes. Il savait dire exactement depuis combien d'années il était à Bicêtre, etc. Toutefois, diverses questions, auxquelles un homme d'une intelligence ordinaire aurait trouvé le moyen de répondre par le geste, sont restées sans réponse intelligible; d'autres fois 
la réponse était claire, mais ne se rapportait pas à la question. Il n'est donc pas douteux que l'intelligence du malade avait subi une atteinte profonde, mais il en conservait certainement plus qu'il n'en faut pour parler.

Le malade est mort le 17 avril 1861. A l'autopsie, on a trouvé la dure-mère épaissie et vascularisée, tapissée, à sa face interne, d'une épaisse couche pseudo-membraneuse, la pie-mère épaissie, opaque et adhérente sur les lobes antérieurs, surtout sur le lobe gauche. Le lobe frontal de l'hémisphère gauche est ramolli dans la plus grande partie de son étendue; les circonvolutions du lobe orbitaire, quoique atrophiées, ont conservé leur forme; la plupart des autres circonvolutions frontales sont entièrement détruites. Il est résulté, de cette destruction de la substance cérébrale, une grande cavité, capable de loger un œuf de poule, et remplie de sérosité. Le ramollissement a gagné en arrière le pli ascendant du lobe pariétal; en bas, le pli marginal du lobe temporo-sphénoïdal; enfin, dans la profondeur, le lobule de l'insula et le noyau extraventriculaire du corps strié; c'est à la lésion de ce dernier organe qu'on doit attribuer la paralysie du mouvement des deux membres dı côté droit; mais il suffit de jeter un coup d'œil sur la pièce pour reconnaître que le foyer principal et le siège primitif dı ramollissement sont la partie moyenne du lobe frontal de l'hémisphère gauche; c'est là qu'on trouve les lésions les plus étendues, les plus avancées et les plus anciennes. Le ramollissement s'est ensuite propagé très lentement dans les parties environnantes; et l'on peut considérer comme certain qu’il y a eu une très longue période pendant laquelle le mal n’occupait que les circonvolutions du lobe frontal. Cette période correspond probablement aux onze années qui ont précédé la paralysie du bras droit et pendant lesquelies le malade, ayant conservé toute son intelligence, n'avait perdu que la parole.

Tout permet donc de croire que, dans le cas actuel, la lésion du lobe frontal a été la cause de la perte de la parole. 
REMARQUES SUR LE SIÈGE

DE LA

\title{
FAGULTÉ DU LANGAGE ARTIGULÉ
}

\author{
SUIVIES D'UNE OBSERVATION D'APHÉMIE ${ }^{1}$
}

(Bulletins de la Société analomique, 1861, $2^{\text {e }}$ série, t. VI, p. 330-357.)

La pièce et l'observation que je présente à la Société anatomique viennent à l'appui des idées que professe M. Bouillaud sur le siège de la faculté du langage. Cette question, à la fois physiologique et pathologique, mérite plus d'attention que la plupart des médecins ne lui en ont accordé jusqu'ici, et la matière est assez délicate, le sujet assez obscur et assez compliqué, pour qu'il me paraisse utile de faire précéder de quelques remarques la relation du fait que j'ai observé.

On sait que l'école phrénologique plaçait à la partie antérieure du cerveau, dans l'une des circonvolutions qui reposent sur la voûte orbitaire, le siège de la faculté du langage. Cette opinion, que l'on avait admise, comme tant d'autres, sans preuve suffisante et qui, d'ailleurs, ne reposait que sur une analyse très imparfaite des phénomènes du langage, aurait sans doute disparu avec le reste du système, si M. Bouillaud ne l'eût sauvée du naufrage en lui faisant subir d'importantes modifications et en l'entourant d'un cortège de preuves empruntées surtout à la pathologie. Sans considérer le langage comme une faculté simple dépendant d'un seul organe cérébral, et sans chercher à circonscrire dans une étendue de quelques millimètres la situation

(1) Voir la note 1, p. 1. 
de cet organe, comme l'avait fait l'école de Gall, ce professeur a été conduit par l'analyse d'un grand nombre de faits cliniques, suivis d'autopsies, à admettre que certaines lésions des hémisphères abolissent la parole sans détruire l'intelligence, et que ces lésions ont toujours leurs sièges dans les lobes antérieurs du cerveau. Il en a conclu qu'il y a quelque part, dans ces lobes, une ou plusieurs circonvolutions qui tiennent sous leur dépendance l'un des éléments essentiels du phénomène complexe de la parole, et c'est ainsi que, moins exclusif que l'école de Gall, il a placé dans les lobes antérieurs, sans spécifier davantage, le siège de la faculté du langage articulé, qu'il faut bien se garder de confondre avec la faculté générale du langage.

Il y a, en effet, plusieurs espèces de langages. Tout système de signes permettant d'exprimer les idées d'une manière plus ou moins intelligible, plus ou moins complète, plus ou moins rapide, est un langage dans le sens le plus général du mot: ainsi la parole, la dactylologie, l'écriture figurative, l'écriture phonétique, etc., sont autant d'espèces de langages.

Il y a une faculté générale du langage qui préside à tous ces modes d'expression de la pensée et qui peut être définie: la faculté d'établir une relation constante entre une idée et un signe, que ce signe soit un son, un geste, une figure ou un tracé quelconque. De plus, chaque espèce de langage nécessite le jeu de certains organes d'émission et de réception. Les organes de réception sont tantôt l'oreille, tantôt la vue, quelquefois même le toucher. Quant aux organes d'émission, ils sont mis en jeu par des muscles volontaires, tels que ceux du larynx, de la langue, du voile du palais, de la face, des membres supérieurs, etc. Tout langage particulier suppose donc l'intégrité: $1^{\circ}$ d'un certain nombre de muscles, des nerfs moteurs qui s'y rendent, et de la partie du système nerveux central d'où proviennent ces nerfs $; 2^{\circ} d^{\prime}$ un certain appareil sensorial externe du nerf sensitif qui en part, et de la partie du système nerveux central où ce nerf va aboutir; $3^{\circ}$ enfin de la partie du cerveau qui tient sous sa dépendance la faculté générale du langage telle que nous venons de la définir.

L'absence ou l'abolition de cette dernière faculté rend impossible toute espèce de langage. Les lésions congénitales ou acciden- 
telles des organes de réception et des organes d'émission peuvent nous priver de l'espèce particulière de langage qui met ces organes à contribution; mais, si la faculté générale du langage persiste en nous avec un degré suffisant d'intelligence, nous pouvons encore suppléer par une autre espèce de langage à celle que nous avons perdue.

Les causes pathologiques qui nous privent d'un moyen de communication ne nous en font ordinairement perdre que la moitié, parce qu'il est assez rare que les organes d'émission et les organes de réception soient affectés en même temps. Par exemple, l'adulte qui devient sourd continue à s'exprimer par la parole, mais pour lui transmettre une idée on se sert d'un langage différent, tel que le geste ou l'écriture. C'est l'inverse qui a lieu lorsqu'une paralysie frappe les muscles de la parole; le malade à qui nous nous adressons dans le langage articulé, nous répond alors dans un autre langage. C'est ainsi que les divers systèmes de communications peuvent se suppléer mutuellement.

Ceci n'est que de la physiologie élémentaire, mais la pathologie a permis de pousser plus loin l'analyse pour ce qui concerne le langage articulé, qui est le plus important et probablement le plus complexe de tous.

Il y a des cas où la faculté générale du langage persiste inaltérée, où l'appareil auditif est intact, où tous les muscles, sans en excepter ceux de la voix et ceux de l'articulation, obéissent à la volonté, et où pourtant une lésion cérébrale abolit le langage articulé. Cette abolition de la parole chez des individus qui ne sont ni paralysés, ni idiots, constitue un symptôme assez singulier pour qu'il me paraisse utile de la désigner sous un nom spécial. Je lui donnerai donc le nom d'aphémie (à privatif; qnul, je parle, je prononce); car ce qui manque à ces malades, c'est seulement la faculté d'articuler les mots. Ils entendent et comprennent tout ce qu'on leur dit; ils ont toute leur intelligence; ils émettent des sons vocaux avec facilité; ils exécutent avec leur langue et leurs lèvres des mouvements bien plus étendus et bien plus énergiques que ne l'exigerait l'articulation des sons, et pourtant la réponse parfaitement sensée qu'ils voudraient faire se réduit à un très petit nombre de sons articulés, 
toujours les mêmes et toujours disposés de la même manière; leur vocabulaire, si l'on peut dire ainsi, se compose d'une courte série de syllabes, quelquefois d'un monosyllabe qui exprime tout, ou plutôt qui n'exprime rien, car ce mot unique est le plus souvent étranger à tous les vocabulaires. Certains malades n'ont même pas ce vestige du langage articulé; ils font de vains efforts sans prononcer une seule syllabe. D'autres ont, en quelque sorte, deux degrés d'articulation. Dans les circonstances ordinaires, ils prononcent invariablement leur mot de prédilection; mais, lorsquïls éprouvent un mouvement de colère, ils deviennent capables d'articuler un second mot, le plus souvent un juron grossier, qui leur était familier probablement avant leur maladie, puis ils s'arrêtent après ce dernier effort. M. Auburtin a observé un malade qui vit encore et qui n'a besoin d'aucune excitation pour prononcer ce juron stéréotypé. Toutes ses réponses commencent par un mot bizarre de six syllabes et se terminent invariablement par cette invocation suprême : Sacré nom de D....

Ceux qui ont pour la première fois étudié ces faits étranges ont pu croire, faute d'une analyse suffisante, que la faculté du langage, en pareil cas, était abolie; mais elle persiste évidemment tout entière, puisque les malades comprennent parfaitement le langage articulé et le langage écrit; puisque ceux qui ne savent pas ou ne peuvent pas écrire ont assez d'intelligence (et il en faut beaucoup en pareil cas) pour trouver le moyen de communiquer leur pensée, et puisque enfin ceux qui sont lettrés, et qui ont le libre usage de leurs mains, mettent nettement leurs idées sur le papier. Ils connaissent donc le sens et la valeur des mots, sous la forme auditive comme sous la forme graphique. Le langage articulé qu'ils parlaient naguère leur est toujours familier, mais ils ne peuvent exécuter la série de mouvements méthodiques et coordonnés qui correspond à la syllabe cherchée. Ce qui a péri en eux, ce n'est donc pas la faculté du langage, ce n'est pas la mémoire des mots, ce n'est pas non plus l'action des nerfs et des muscles de la phonation et de l'articulation, c'est autre chose, c'est une faculté particulière considérée par M. Bouillaud comme la faculté de coordonner les nouvements propres au langage articulé ou plus simplement comme la 
faculté du langage articulé, puisque sans elle il n'y a pas d'articulation possible.

La nature de cette faculté et la place qu'il faut lui assigner dans la hiérarchie cérébrale peuvent donner lieu à quelque hésitation. N'est-ce pas une espèce de mémoire, et les individus qui l'ont perdue ont-ils perdu seulement non la mémoire des mots, mais le souvenir du procédé qu'il faut suivre pour articuler les mots? Sont-ils revenus par là à une condition comparable à celle du jeune enfant qui comprend déjà le langage de ses proches, qui est sensible au blâme et à la louange, qui montre du doigt tous les objets qu'on lui nomme, qui a acquis une foule d'idées simples et qui, pour les exprimer, ne sait encore balbutier qu'une seule syllabe? Peu à peu, après des efforts innombrables, il réussit à articuler quelques syllabes nouvelles. Pourtant il lui arrive encore souvent de se tromper, et de dire, par exemple, papa lorsqu'il voudrait dire mama, parce qu'au moment de prononcer ce dernier mot il ne se souvient plus de la position qu'il faudrait donner à sa langue et à ses lèvres. Bientôt il connaît assez bien le mécanisme de quelques syllabes simples et faciles pour les prononcer à tout coup sans erreur et sans hésitation; mais il hésite et se trompe encore sur les syllabes plus difficiles, et lorsque enfin il possède bien la pratique de plusieurs monosyllabes, il a besoin d'acquérir une nouvelle expérience pour apprendre à passer tout à coup d'une syllabe à une autre, et pour prononcer, à la place des monosyllabes redoublés qui constituaient son premier vocabulaire, des mots composés de deux ou trois syllabes différentes. Ces perfectionnements graduels du langage articulé chez les enfants sont dus au développement d'une espèce particulière de mémoire qui n'est pas la mémoire des mots, mais celle des mouvements nécessaires pour articuler les mots. Et celte mémoire particulière n'est nullement en rapport avec les autres mémoires ni avec le reste de l'intelligence. J'ai connu un enfant de trois ans qui avait une intelligence et une volonté au-dessus de son âge, qui avait la langue bien conformée, et qui ne savait pas encore parler. Je connais un autre enfant très intelligent qui, à l'âge de vingt et un mois, comprend parfaitement deux langues, qui, par conséquent, possède au plus haut degré la mémoire des mots, et qui jus- 
qu'ici n'a pu s'élever au-dessus de la prononciation des monosyllabes.

Si les adultes qui perdent la parole ont seulement oublié l'art de l'articulation, s'ils sont revenus simplement à la condition où ils étaient avant d'avoir appris à prononcer les mots, il faut ranger la faculté dont la maladie les a privés dans l'ordre des facultés intellectuelles. Gette hypothèse me paraît assez vraisemblable. Il serait possible; toutefois, qu'il en fût autrement, et que l'aphémie fût le résultat d'une ataxie locomotrice limitée à la partie de l'appareil nerveux central qui préside aux mouvements de l'articulation des sons. On objecte, il est vrai, que ces malades peuvent exécuter librement avec leur langue et leurs lèvres tous les mouvements autres que ceux de l'articulation; qu'ils peuvent porter immédiatement, lor'squ'on les en prie, la pointe de leur langue en haut, en bas, à droite et à gauche, etc.; mais ces mouvements, quelque précis qu'ils nous paraissent, le sont infiniment moins que les mouvements excessivement délicats qu'exige la parole. Dans l'ataxie locomotrice des membres, on observe que les malades exécutent à volonté tous les grands mouvements : si on leur dit de lever la main, de l'ouvrir, de la fermer, ils le font presque toujours sans hésitation; mais, quand ils veulent exécuter des mouvements plus précis, saisir par exemple, d'une certaine manière, un objet de peu de volume, ils vont au-delà ou restent en-deçà du but; ils ne savent pas coordonner la contraction de leurs muscles de manière à obtenir une résultante d'une valeur déterminée, et ils se trompent bien moins sur la direction de leurs mouvements que sur la quantité de force qu'il faudrait déployer et sur l'ordre de succession des mouvements partiels dont se compose la préhension des objets.

On peut donc se demander si l'aphémie ne serait pas une espèce d'ataxie locomotrice limitée aux muscles de l'articulation des sons, et, s'il en était ainsi, la faculté que les malades ont perdue ne serait pas une faculté intellectuelle, c'est-à-dire une faculté appartenant à la partie pensante du cervean, et ne serait qu'un cas particulier de la faculté générale de coordination des actions musculaires, faculté qui dépend de la partie motrice des centres nerveux. 
On peut donc faire au moins deux hypothèses sur la nature de la faculté spéciale du langage articulé. Dans la première hypothèse, ce serait une faculté supérieure, et l'aphémie serait un trouble intellectuel; dans la seconde hypothèse, ce serait une faculté d'un ordre beaucoup moins élevé, et l'aphémie ne serait plus qu'un trouble de la locomotion. Quoique cette dernière interprétation me paraisse beaucoup moins probable que l'autre, je n'oserais pourtant pas me prononcer d'une manière catégorique, si j'en étais réduit aux seules lumières de l'observation clinique.

Quoi qu'il en soit, sous le rapport de l'analyse fonctionnelle, l'existence de la faculté spéciale du langage articulé, telle que je l'ai définie, ne peut être révoquée en doute, car une faculté qui peut périr isolément, sans que celles qui l'avoisinent le plus soient altérées, est évidemment une faculté indépendante de toutes les autres, c'est-à-dire une faculté spéciale.

Si toutes les facultés cérébrales étaient aussi distinctes, aussi nettement circonscrites que celle-là, on aurait enfin un point de départ positif pour aborder la question si controversée des localisations cérébrales. Il n'en est malheureusement pas ainsi, et le plus grand obstacle au progrès de cette partie de la physiologie vient de l'insuffisance et de l'incertitude de l'analyse fonctionnelle qui doit nécessairement précéder la recherche des organes en rapport avec chaque fonction.

La science est si peu avancée sur ce point qu'elle n'a même pas encore trouvé sa base, et ce qui est en contestation aujourd'hui, ce n'est pas tel ou tel système phrénologique, mais le principe même des localisations, c'est-à-dire la question préalable de savoir si toutes les parties du cerveau qui sont affectées à la pensée ont des attributions identiques ou des attributions différentes.

Une communication de M. Gratiolet, relative au parallèle cérébral et intellectuel des races humaines, a mis, il y a quelque temps, la Société d'anthropologie de Paris en demeure d'examiner cette importante question, et M. Auburtin, partisan du principe des localisations, a pensé, avec juste raison, que la localisation d'une seule faculté suffirait pour établir la vérité de ce principe; il a donc cherché à démontrer, conformément à la 
doctrine de son maître, M. Bouillaud, que la faculté du langage articulé réside dans les lobes du cerveau.

Pour' cela, il a d'abord passé en revue une série de cas où une affection cérébrale spontanée avait aboli la faculté du langage articulé sans détruire les autres facultés cérébrales, et où l'on a trouvé à l'autopsie une lésion profonde des circonvolutions antérieures du cerveau. La nature spéciale du symptôme de l'aphémie ne dépendait pas de la nature de la maladie, mais seulement de son siège, puisque la lésion était tantôt un ramollissement, tantôt une apoplexie, tantôt un abcès ou une tumeur. Pour compléter sa démonstration, M. Auburtin a invoqué une autre série de cas où l'aphémie a été la conséquence d'une lésion traumatique des lobes antérieurs du cerveau; ces faits, suivant lui, équivalent à des vivisections, et il a terminé en disant qu'à sa connaissance on n'avait jamais trouvé les lobes antérieurs du cerveau dans un état d'intégrité complète, ni même dans un état d'intégrité relative, à l'autopsie des individus qui avaient perdu la faculté du langage articulé sans perdre le reste de leur intelligence.

On lui a opposé plusieurs faits remarquables relatifs à des individus qui avaient parlé jusqu'au dernier jour, et chez lesquels pourtant les lobes antérieurs du cerveau étaient le siège de profondes lésions spontanées ou traumatiques; mais il a répondu que cela ne prouvait rien, qu'une lésion, même étendue, des lobes antérieurs pouvait ne pas atteindre la partie de ces lobes où siège la faculté du langage articulé, que l'objection ne serait valable que si toutes les circonvolutions frontales avaient été détruites des deux côtés dans toute leur étendue, c'est-à-dire jusqu'au sillon de Rolando, et que, dans les cas qu'on lui opposait, la destruction de ces circonrolutions n’avait été que partielle. ll a donc reconnu qu'une lésion des lobes antérieurs n'entraîne pas nécessairement la perte de la parole, mais il a maintenu que celle-ci est l'indice certain de celle-là, qu'elle permet de la diagnostiquer, que ce diagnostic a été fait plusieurs fois pendant la vie, et n'a jamais été démenti par l'autopsie; enfin, après avoir cité l'observation d'un individu encore vivant qui présente depuis plusieurs années, de la manière la plus nette, les symptômes de l'aphémie, et qui est actuel- 
lement à l'hospice des Incurables, il a déclaré qu'il renoncerait sans retour à la doctrine de M. Bouillaud, si l'autopsie de ce malade ne confirmait pas le diagnostic d'une lésion cérébrale occupant exclusivement ou principalement les lobes antérieurs. (Voir Bulletins de la Société d'anthropologie, t. II, séance du 4 avril 1861.)

J'ai cru devoir résumer en quelques mots cette discussion pour faire ressortir l'intérêt et l'actualité de l'observation que je présente aujourd'hui à la Société anatomique. Sans doute, la valeur des faits n'est pas subordonnée aux circonstances au milieu desquelles on les observe; mais l'impression qu'ils font sur' nous en dépend en grande partie, et lorsque, peu de jours après avoir entendu l'argumentation de M. Auburtin, je trouvai un matin, dans mon service, un moribond qui, depuis vingt et un ans, avait perdu la faculté du langage articulé, je recueillis avec le plus grand soin cette observation, qui semblait venir tout exprès pour servir de pierre de touche à la théorie soutenue par mon collègue.

Jusqu'alors, sans repousser cette théorie et sans méconnaître en rien l'importance des faits qui 'lui sont favorables, j'avais éprouvé beaucoup d'hésitation en présence des faits contradictoires qui existent dans la science. Quoique partisan du principe des localisations, je me demandais, et je me demande encore, dans quelles limites ce principe est applicable. Il y a un point qui me paraît à peu près établi par l'anatomie comparée, par le parallèle anatomique et physiologique des races humaines, et, enfin, par la comparaison des variétés individuelles normales, anormales ou pathologiques des hommes de même race, savoir : que les facultés cérébrales les plus élevées, celles qui constituent l'entendement proprement dit, comme le jugement, la réflexion, les facultés de comparaison et d'abstraction, ont leur siège dans les ciconvolutions frontales, tandis que les circonvolutions des lobes temporaux, pariétaux et occipitaux sont affectées aux sentiments, aux penchants et aux passions. En d'autres termes, il y a dans l'esprit des groupes de facultés, et dans le cerveau, des groupes de circonvolutions; et les faits acquis jusqu'ici à la science permettent d'admettre, comme je l'ai dit ailleurs, que les grandes régions de l'esprit correspondent aux grandes régions 
du cerveau. C'est dans ce sens que le principe des localisations me paraît, sinon rigoureusement démontré, du moins extrêmement probable. Mais de savoir si chaque faculté particulière a son siège dans une circonvolution particulière, c'est une question qui me paraît tout à fait insoluble dans l'état actuel de la science.

L'étude des faits relatifs à la perte de la faculté du langage articulé est une de celles qui ont le plus de chance de nous conduire à une solution positive ou négative. L'indépendance de celte faculté est mise en évidence par l'observation pathologique, et, quoique l'on puisse élever quelques doutes sur sa nature, quoique l'on puisse se demander, comme on l'a vu plus haut, si elle fait partie des fonctions intellectuelles ou des fonctions cérébrales qui sont en rapport avec la musculation, il est permis de se placer, au moins provisoirement, au point de vue de la première hypothèse qui déjà, au premier coup d'œil, paraît la plus probable et en faveur de laquelle l'anatomie pathologique de l'aphémie établit de fortes présomptions. En effet, dans tous les cas où jusqu'ici l'autopsie a pu être pratiquée, on a trouvé la substance des circonvolutions profondément altérée dans une notable étendue; chez quelques sujets la lésion portait même exclusivement sur les circonvolutions : d'où il est permis de conclure que la faculté du langage articulé est une des fonctions de la masse circonvolutionnaire. Or, on admet généralement que toutes les facultés dites intellectuelles ont leur siège dans cette partie de l'encéphale, et il paraît dès lors fort probable que, réciproquement, toutes les facultés qui résident dans les circonvolutions cérébraless sont des facultés de l'ordre intellectuel.

En nous plaçant donc à ce point de vue, nous reconnaîtrons aisément que l'anatomie pathologique de l'aphémie peut donner quelque chose de plus que la solution d'une question particulière et qu'elle peut jeter beaucoup de jour sur la question générale des localisations cérébrales, en fournissant à la physiologie du cerveau un point de départ, ou plutôt un point de comparaison fort précieux. S'il était prouvé, par exemple, que l'aphémie peut être le résultat de lésions affectant indifféremment n'importe quelle circonvolution de n'importe quel lobe cérébral, on aurait 
le droit d'en conclure non seulement que la faculté du langage articulé n'est pas localisée, mais encore que très probablement les autres facultés de même ordre ne sont pas localisées non plus. S'il était démontré, au contraire, que les lésions qui abolissent la parole occupent constamment une circonvolution déterminée, on ne pourrait guère se dispenser d'admettre que cette circonvolution est le siège de la faculté du langage articulé, et, l'existence d'une première localisation une fois admise, le principe des localisations par circonvolutions serait établi. Enfin, entre ces deux alternatives extrêmes, il en est une troisième qui pourrait conduire à une doctrine mixte. Supposons, en effet, que les lésions de l'aphémie occupent constamment le même lobe cérébral, mais que, dans ce lobe, elles n'occupent pas constamment la même circonvolution, il en résulterait que la faculté du langage articulé aurait son siège dans une certàine région, dans un certain groupe de circonvolutions, mais non dans une circonvolution particulière, et il deviendrait très probable que les facultés cérébrales sont localisées par régions, et non par circonvolutions.

Il importe donc d'étudier avec le plus grand soin une question spéciale qui peut avoir des conséquences doctrinales si générales et si importantes. Il ne s'agit pas seulement de chercher dans quelles régions du cerveau siègent les lésions de l'aphémie; il faut, de plus, désigner par leur nom et par leur rang les circonvolutions malades et le degré d'altération de chacune d'elles. Ce n'est pas ainsi qu'on a procédé jusqu'ici. On s'est borné, dans les observations les plus complètes, à dire que la lésion commençait et finissait à tant de centimètres de l'extrémité antérieure de l'hémisphère, à tant de centimètres de la grande scissure médiane ou de la scissure de Sylvius. Mais cela est tout à fait insuffisant, parce que, avec ces indications, quelque minutieuses qu'elles soient, le lecteur ne peut pas deviner quelle est la circonvolution malade. Ainsi, il y a des cas où le mal est situé à la partie la plus antérieure de l'hémisphère, d'autres où il est situé à $\breve{g}$ et même à 8 centimètres en arrière de ce point, et il semble, d'après cela, que le siège de la lósion soit très variable; mais si l'on songe que les trois circonvolutions antéro-postérieures de la convexité du lobe frontal commencent au niveau 
de l'arcade sourcilière et cheminent côte à côte, d'avant en arrière, pour aller se jeter toutes les trois dans la circonvolution frontale transversale qui forme le bord antérieur du sillon de Rolando; si l'on songe que ce sillon est situé à 4 centimètres en arrière de la suture coronale (1), et que les trois circonvolutions antéro-postérieures occupent plus des deux cinquièmes de la longueur totale du cerveau, on comprendra que la même circonvolution puisse être atteinte par des lésions situées en des points très différents et très distants les uns des autres. Il est donc beaucoup moins important d'indiquer le niveau dı mal que de dire quelles sont les circonvolutions malades.

Ce genre de description est sans doute moins commode que l'autre, car les traités classiques d'anatomie n'ont pas vulgarisé jusqu'ici l'étude des circonvolutions cérébrales que les phrénologistes eux-mêmes ont eu le grand tort de négliger. On s'est laissé dominer par ce vieux préjugé que les circonvolutiuns céré-

(1) On croit généralement que le sillon de Rolando est silué directement audessous de la suture coronale, et M. Gratiolet, d'après cela, accorde une importance toute particulière à l'étude de cette suture, qui permettrait d'établir une relation bien précise entre la région frontale du crâne et les lobes antérieurs du cerveau. Ce serait une donnée infiniment précieuse dans le parallèle des races liumaines. Malheureusement, cette donnée est tout à fait inexacte; le cerveau, retiré du crâne et déposé sur une table, s'étale, se déforme, et si l'on mesure alors la longueur du lobe antérieur de l'hémisplière, on trouve qu'elle est à peu près égale à celle de l'os frontal. Mais, en examinant les organes en place, je suis arrivé à un résultat tout à fait différent. Voici comment je procède : après avoir enlevé les téguments et le péricrâne, j'enfonce des vrilles dans les divers points de la suture coronale, et je pousse à travers les trous de vrilles de petites clievilles de bois jusque dans la substance cérébrale. Le crâne est alors ouvert à la scie; le cerveau est enlevé et dépouillé de ses membranes, et j'étudie la situation des chevilles par rapport au sillon de Rolando. J'ai fait cette recherche sur onze sujets du sexe masculin, ayant atteint ou dépassé l'âge adulte, et j'ai trouvé que constamment le sillon de Rolando commence, sur la ligne médiane, à 4 centimètres au moins en arrière de la suture coronale (minimum, 40 millimètres; maximum, 63 millimètres). A sa partie externe, ce sillon qui est oblique et non transversal, se rapproclie de la suture coronale; à 4 centimètres de là ligne médiane, il n'est plus situé qu'à 2 centimètres au moins, 3 centimètres au plus, en arrière de cette suture. Le même procédé m'a permis de constater qu'il y a, au contraire, une relation assez constante entre la suture lambdoïde et le sillon occipital transverse qui sépare le lobe pariétal du lobe occipital de l'hémisphère. Les chevilles enfoncées dans la suture lambdoïde pénètrent ordinairement dans le sillon occipital ou tout près de là. Je ne les ai jamais trouvées à plus de 15 millimètres de ce sillon, et l'écart est rarement de plus de ŏ millimetres, 
brales n'ont rien de fixe, que ce sont de simples plis faits au hasard, comparables aux flexuosités désordonnées des anses intestinales, et ce qui a accrédité cette idée. c'est que les plis secondaires, qui dépendent du degré de développement des circonvolutions fondamentales, varient non seulement d'individus à individus, mais souvent même chez le même individu d'un hémisphère à l'autre. Il n'en est pas moins vrai que les circonvolutions fondamentales sont fixes et constantes chez tous les animaux de même espèce, et que, considérées dans la série animale, elles se comportent comme autant d'organes parfaitement distincts. La description et l'énumération de ces circonvolutions fondamentales, de leurs connexions et de leurs rapports, ne sauraient trouver place ici. On les trouvera dans les ouvrages spéciaux de MM. Gratiolet et Rodolphe Wagner (1).

Et puisque j'ai exprimé des regrets sur le peu de précision des descriptions relatives aux lésions des hémisphères cérébraux, je signalerai une confusion fâcheuse qui a induit en erreur plusieurs observateurs. Beaucoup de personnes, qui ont l'habitude d'étudier surtout le cerveau par sa face inférieure, s'imaginent que les lobes antérieurs comprennent seulement la partie des hémisphères qui est située en avant du chiasma des nerfs optiques et de l'extrémité antérieure du lobe temporo-sphénoïdal. G'est bien là, en effet, que s'arrête la face inférieure des lobes antérieur's; mais, du côté de la convexité des hémisphères, ces lobes ont une longueur au moins double de la précédente et se prolongent au-dessus de la scissure de Sylvius, dont ils forment le bord supérieur, jusqu'au sillon de Rolando, qui les sépare des lobes pariétaux. Quand on lit, dans certaines observations, que des malades dont les deux lobes antérieurs étaient entièrement détruits ont continué à parler jusqu'au moment de leur mort, il est permis de croire, en l'absence de toute autre indication, que l'auteur a voulu parler surtout de la partie de ces

(1) Gratiolet el Leuret, Anatomie compar'e du système nerveux, t. II, p.110. Paris, 1857, it1-8. Ce second volume est exclusivement l'œuvre de M. Gratiolet. - Gratiolet, Mémoire sur les plis cérébraux de l'homme et des primates, Paris, 1854 , in-4\%, avec allas in-folio. - Rodolphe Wagner, Abhandlung über die typischen Verschiedenheiten der Windunger der Hemispharen, etc., Göttingen, 1860 , in- $4^{\circ}$, avec atlas, p. 13 aे 25 . On trouvera plus loin, dans une autre note, une description abrégée des circonvolutions antérieures ou frontales. 
lobes qui recouvre la voûte orbitaire. Il est dit, par exemple, dans la plus célèbre de ces observations, qu'un homme, atteint au front par un éclat de mine, eut les deux lobes antérieurs entièrement écrasés et réduits en bouillie. Mais il est clair qu'aucune action traumatique ne peut broyer immédiatement, complètement et d'un seul coup, la totalité des deux lobes antérieurs sans écraser en même temps toute la moitié antérieure de l'encéphale, y compris les insula, les corps striés, le corps calleux, la voûte à trois piliers, etc., et une semblable lésion n'est pas admissible chez un homme qui avait pu marcher jusqu'à son lit, qui avait conservé toute son intelligence et qui survécut vingtquatre heures sans avoir présenté ni contracture, ni paralysie. De même, lorsque j'ai montré pour la première fois, dans une réunion de médecins, le cerveau de l'homme dont je publie aujourd'hui l'observation, plusieurs personnes se sont écriées que cette pièce était en contradiction avec les idées de M. Bouillaud, que les lobes antérieurs étaient à peu près sains, que la lésion était presque tout entière en arrière de ces lobes. On va voir pourtant que les circonvolutions frontales (ou antérieures) étaient détruites dans une étendue très considérable.

Mais j'ai à m'excuser d'avoir donné tant de développement à ces remarques préliminaires. Il est temps de passer à la relation de mon observation d'aphémie.

\section{II}

Aphémie datant de vingt et un ans, produite par le ramollissement chronique et progressif de la seconde et de la troisième circonvolution de l'étage supérieur' du lobe frontal gauche. Le 11 avril 1861, on transporta à l'infirmerie générale de Bicêtre, service de chirurgie, un homme de cinquante et un an, nommé Leborgne, atteint d'un phlegmon diffus gangreneux de tout le membre inférieur droit, depuis le cou-de-pied jusqu'à la fesse. Aux questions que je lui adressai le lendemain sur l'origine de son mal, il ne répondit que par le monosyllabe tan répété deux fois de suite et accompagné d'un geste de la main gauche. J'allai aux renseignements sur les antécédents de cet homme,

T. V. 
qui était à Bicêtre depuis vingt et un ans. On interrogea tour à tour ses surveillants, ses camarades de division et ceux de ses parents qui vinrent le voir, et voici quel fut le résultat de cette enquête :

Il était sujet, depuis sa jeunesse, à des attaques d'épilepsie; mais il avait pu prendre l'état de formier qu'il exerça jusqu'à l'âge de trente ans. A cette époque il perdit l'usage de la parole et ce fut pour ce motif qu'il fut admis comme infirme à l'hospice de Bicêtre. On n'a pu savoir si la perte de la parole était survenne lentement ou rapidement, ni si quelque autre symptôme avait accompagné le début de cette affection.

Lorsqu'il arriva à Bicêtre, il y avait déjà deux ou trois mois qu'il ne parlait plus. Il était alors parfaitement valide et intelligent, et ne différait d'un homme sain que par la perte du langage articulé. Il allait et venait dans l'hospice où il était connu sous le nom de Tan. Il comprenait tout ce qu'on lui disait; il avait même l'oreille très fine; mais, quelle que fût la question qu'on lui adressât, il répondait toujours : tan, tan, en y joignant des gestes très variés au moyen desquels il réussissait à exprimer la plupart de ses idées. Lorsque ses interlocuteurs ne comprenaient pas sa mimique, il se mettait aisément en colère, et ajoutait alors à son vocabulaire un gros juron, un seul, et le même précisément que j'ai indiqué plus haut, en parlant d'un malade observé par M. Auburtin. Tan passait pour égoïste, vindicatif, méchant, et ses camarades, qui le détestaient, l'accusaient même d'être voleur. Ces défauts pouvaient être dus en grande partie à la lésion cérébrale; toutefois ils n'étaient pas assez prononcés pour paraître pathologiques, et, quoique le malade fût à Bicêtre, on n'eut jamais la pensée de le faire passer' dans la division des aliénés. On le considérait au contraire comme un homme parfaitement responsable de ses actes.

Il y avait déjà dix ans qu'il avait perdu la parole lorsqu'un nouveau symptôme se manifesta: les muscles du bras droit s'affaiblirent graduellement, et finirent par être entièrement paralysés. Tan continuait à marcher sans difficulté, mais la paralysie du mouvement gagna peu à peu le membre inférieur droit, et, après avoir traîné la jambe pendant quelque temps, le malade dut se résigner à garder constamment le lit. Il s'était 
écoulé environ quatre ans depuis le début de la paralysie du bras jusqu'au moment où celle du membre abdominal avait été assez avancée pour rendre la station tout à fait impossible. Il y avait donc à peu près sept ans que Tan était alité lorsqu'il fut conduit à l'infirmerie. Cette dernière période de sa vie est celle sur laquelle nous avons le moins de renseignements. Comme il était devenu incapable de nuire, ses camarades ne s'occupaient plus de lui, si ce n'est pour s'amuser quelquefois à ses dépens (ce qui lui donnait de vifs accès de colère), et il avait perdu la petite célébrité que la singularité de sa maladie lui avait donnée autrefois dans l'hospice. On s'était aperçu que sa vue baissait notablement depuis environ deux ans. C'était la seule aggravation qu'on eût remarquée depuis qu'il gardait le lit. Du reste, il n'avait jamais été gâteux; on ne changeait ses draps qu'une fois par semaine, de telle sorte que le phlegmon diffus, pour lequel il fut transporté à l'infirmerie le 11 avril 1861, ne fut reconnu par les infirmiers que lorsqu'il eut fait des progrès considérables et envahi la totalité du membre abdominal droit, depuis le pied jusqu'à la fesse.

L'étude de ce malheureux, qui ne pouvait parler et qui, étant paralysé de la main droite, ne pouvait écrire, offrait bien quelque difficulté. Il était d'ailleurs dans un état général tellement grave, qu'il y aurait eu cruauté à le tourmenter par de trop longues investigations.

Je constatai toutefois que la sensibilité générale était partout conservée, quoiqu'elle le fût inégalement. La moitié droite du corps était moins sensible que l'autre, et cela avait contribué sans doute à atténuer la douleur du phlegmon diffus. Le malade n'en souffrait pas beaucoup lorsqu'on n'y touchait pas, mais la palpation était douloureuse, et quelques incisions, que je fus obligé de pratiquer, provoquèrent de l'agitation et des cris.

Les deux membres droits étaient complètement paralysés du mouvernent ; les deux autres membres obéissaient à la volonté, et, quoique affaiblis, pouvaient, sans aucune hésitation, exécuter tous les mouvements. L'émission des.urines et des matières fécales était naturelle, mais la déglutition se faisait avec quelque difficulté ; la mastication, au contraire, se fáisait très bien. lee visage n'était pas dévié; toutefois, dans l'action de souffler, la 
joue gauche paraissait un peu plus gonflée que la droite, ce qui indiquait que les muscles de ce côté de la face étaient un peu affaiblis. Il n'y avait aucune tendance au strabisme. La langue était parfaitement libre ; elle n'était nullement déviée; le malade pouvait la mouvoir en tous sens et la tirer hors de la bouche. Les deux moitiés de cet organe étaient d'une égale épaisseur. La difficulté de déglutition que je viens de signaler était due à la paralysie commençante du pharynx, et non à la paralysie de la langue, car c'était seulement le troisième temps de la déglutition qui était laborieux. Les muscles du larynx ne paraissaient nullement altérés, le timbre de la voix était naturel, et les sons que le malade rendait pour prononcer son monosyllabe étaient parfaitement purs.

L'ouïe avait gardé sa finesse; Tan entendait bien le bruit de la montre; mais sa vue était affaiblie; quand il voulait regarder l'heure, il était obligé de prendre la montre lui-même avec sa main gauche et de la placer dans une position particulière, à 20 centimètres environ de l'œil droit, qui paraissait meilleur que le gauche.

L'état ảe l'intelligence n'a pu êtrè exactement déterminé. Il est certain que Tan comprenait presque tout ce qu'on lui disait; mais, ne pouvant manifester ses idées ou ses désirs que par les mouvements de sa main gauche, notre moribond ne pouvait se faire comprendre aussi bien qu'il comprenait les autres. Les réponses numériques étaient celles qu'il faisait le mieux, en ouvrant ou fermant les doigts. Je lui demandai plusieurs fois depuis combien de jours il était malade, il répondit tantôt cinq jours, tantôt six jours. Depuis combien d'années il était à Bicêtre, il ouvrit la main quatre fois de suite, et fit l'appoint avec un seul doigt; cela faisait vingt et un ans, et l'on a vu plus haut que ce renseignement était parfaitement exact. Le lendemain, je répétai la même question, et j'obtins la même réponse ; mais lorsque je voulus y revenir une troisième fois, Tan comprit que je lui faisais faire un exercice; il se mit en colère, et articula le juron déjà nommé que je n'ai entendu de sa bouche qu'une seule fois. Je lui présentai ma montre deux jours de suite. L'aiguille des secondes ne marchait pas; il ne pouvait par conséquent distinguer les trois aiguilles qu'à leur forme ou à leur 
longueur ; néanmoins, après avoir examiné la montre quelques instants, il put indiquer chaque fois l'heure avec exactitude. Il est donc incontestable que cet homme était intelligent, qu'il pouvait réfléchir, et qu'il avait conservé, dans une certaine mesure, la mémoire des choses anciennes. Il pouvait même comprendre des idées assez compliquées; ainsi je lui demandai dans quel ordre ses paralysies s'étaient succédé; il fit d'abord avec l'index de la main gauche un petit geste horizontal qui voulait dire : compris! puis il me montra successivement sa langue, son bras et sa jambe droits. C'était parfaitement exact, à cela près qu'il attribuait la perte de la parole à la paralysie de la langue, ce qui était bien naturel.

Pourtant, diverses questions auxquelles un homme d'une intelligence ordinaire aurait trouvé le moyen de répondre par le geste, même avec une seule main, sont restées sans réponse. D'autres fois on n'a pu saisir le sens de certaines réponses, ce qui paraissait impatienter beaucoup le malade; d'autres fois, enfin, la réponse était claire, mais fausse: ainsi, quoiqu'il n'eût pas d'enfants, il prétendait en avoir. Il n'est donc pas douteux que l'intelligence de cet homme avait subi une atteinte profonde, soit sous l'influence de son affection cérébrale, soit sous l'influence de la fièvre qui le dévorait ; mais il était évidemment bien plus intelligent qu'il ne faut l'être pour parler.

Il résultait clairement des renseignements obtenus et de l'état présent du malade qu'il existait une lésion cérébrale progressive, qui, dans l'origine et pendant les dix premières années de la maladie, était restée limitée à une région assez circonscrite, et qui, dans cette première période, n'avait atteint ni les organes de motilité, ni les organes de sensibilité; qu'au bout de dix ans, la lésion s'était propagée à un ou plusieurs organes de motilité, en respectant encore les organes de sensibilité; et que, plus récemment enfin, la sensibilité générale s'était émoussée en même temps que la vision, surtout la vision de l'œil gauche. La paralysie complète du mouvement occupant les deux membres du côté droit, et la sensibilité de ces deux membres étant en outre un peu affaiblie, la lésion cérébrale principale devait occuper l'hémisphère gauche, et ce qui confirmait cette opinion, c'était la paralysie incomplète des muscles de la 
joue gauche et de la rétine du même côté, car il est inutile de rappeler que les paralysies de cause cérébrale sont croisées pour le tronc et les membres, et directes pour la face.

Il s'agissait maintenant de déterminer plus exactement, si c'était possible, le signe de la lésion primitive, et, quoique la dernière discussion de la Société d'anthropologie eût laissé planer quelque doute sur la doctrine de M. Bouillaud, je voulus, dans l'attente d'une autopsie prochaine, raisonner comme si cette doctrine était vraie; c'était le meilleur moyen de la mettre à l'épreuve. M. Auburtin ayant déclaré quelques jours auparavant qu'il y renoncerait, si on lui montrait un seul cas d'aphémie bien caractérisée sans lésion des lobes antérieurs, je l'invitai à venir voir mon malade pour savoir avant tout quel serait son diagnostic, et si cette observation était une de celles dont il accepterait le résultat comme concluant. Malgré les complications qui étaient survenues depuis onze ans, mon collègue trouva l'état actuel et les antécédents suffisamment clairs, pour affirmer sans hésitation que la lésion avait dû débuter dans l'un des lobes antérieurs.

Raisonnant d'après cette donnée pour compléter le diagnostic, je conșidérai que le corps strié était l'organe moteur le plus rapproché des lobes antérieurs; c'était sans doute en se propageant graduellement à cet organe que la lésion primitive avait produit l'hémiplégie. Le diagnostic probable était donc lésion primitive du lobe antérieur gauche, propagée au corps strié du même côté. Quant à la nature de cette lésion, tout indiquait qu'il s'agissait d'un ramollissement chronique à marche progessive, mais extrêmement lente, car l'absence de tout phénomène de compression excluait l'idée d'une tumeur intra-crânienne.

Le malade mourut le 17 avril, à onze heures du matin. L'autopsie fut pratiquée le plus tôt possible, c'est-à-dire au bout de vingt-quatre heures. La température était peu élevée. Le cadavre ne présentait aucun signe de putréfaction. Le cerveau fut montré quelques heures après à la Société d'anthropologie, puis plongé immédiatement dans l'alcoul. Cet organe était tellement altéré, qu'il a fallu de très grandes précautions pour le conserver. Ce n'est qu'au bout de deux mois et après plusieurs changements de liquide que, la pièce a commencé à se raffermir. 
Aujourd'hui elle est en parfait état, et elle est déposée dans le musée Dupuytren sous le numéro $5 ّ, a, d u$ système nerveux.

Je passe sous silence les détails relatifs au phlegmon diffus. Les muscles des deux membres droits étaient entièrement graisseux et réduits à un petit volume. Tous les viscères étaient sains, excepté l'encéphale.

Le crâne a été ouvert à la scie avec beaucoup de soin. Toutes les sutures sont soudées; l'épaisseur des os est un peu accrue; le diploé est remplacé par du tissu compact. La surface interne de la voûte crânienne présente dans toute son étendue une apparence de fine vermoulure, indice certain d'une ostéite chronique $\left(\mathrm{n}^{\circ} 55, b\right)$.

$\mathrm{La}$ face externe de la dure-mère est rouge et très vasculaire ; cette membrane est très épaisse, très vasculaire, comme charnue, et tapissée intérieurement d'une couche pseudo-membraneuse, infiltrée de sérosité, et d'apparence lardacée. La duremère et la fausse membrane réunies ont une épaisseur moyenne de 5 millimètres (minimum, 3 millimètres; maximum, 8); d'où il résulte nécessairement que l'encéphale a dû perdre une notable partie de son volume primitif.

La dure-mère enlevée, la pie-mère apparaît très injectée en certains points, épaissie partout, et par places, opaque, infiltrée d'une matière plastique jaunâtre qui a la couleur du pus, mais qui est solide et qui, examinée au microscope, ne renferme pas de globules purulents.

Sur la partie latérale de l'hémisphère gauche, au niveau de la scissure de Sylvius, la pie-mère est soulevée par une collection de sérosité transparente qui se loge dans une large et profonde dépression de la substance cérébrale. Ce liquide étant évacué par une ponction, la pie-mère s'affaisse, se déprime profondément, et il en résulte une cavité allongée d'une capacité équivalente au volume d'un œuf de poule, correspondant à la scissure de Sylvius, et séparant par conséquent le lobe frontal du lobe temporal. Elle se prolonge en arrière jusqu'au niveau du sillon de Rolando, qui sépare, comme on sait, les circonvolutions antérieures ou frontales des circonvolutions pariétales. La lésion est donc située tout entière en avant de ce sillon, et le lobe pariétal est sain, au moins d'une manière relative, car 
aucune partie des hémisphères n'est dans un état d'intégrité absolue.

En incisant et écartant la pie-mère au niveau de la cavité que je viens d'indiquer, on reconnaît au premier coup d'œil que celle-ci correspond non à une dépression, mais à une perte de substance de la masse cérébrale; le liquide qui la remplissait y a été exhalé consécutivement pour remplir le vide à mesure qu'il se formait, comme cela a lieu dans le ramollissement chronique des couches superficielles du cerveau ou du cervelet. L'étude des circonvolutions qui limitent la cavité montre effectivement qu'elles sont le siège d'un de ces ramollissements chroniques dont la marche est assez lente pour que les molécules cérébrales, dissociées en quelque sorte une à une, puissent se résorber et être remplacées par une exhalation de sérosité (1). Une partie notable de l'hémisphère gauche a été ainsi détruite graduellement ; mais le ramollissement s'étend bien au-delà des limites de la cavité ; celle-ci n'est nullement circonscrite, et ne peut sous aucun rapport être comparée à un kyste. Ses parois, presque partout irrégulières, anfractueuses, sont constituées par la substance cérébrale elle-même, qui est extrêmement ramollie à ce niveau, et dont la couche la plus interne, en contact direct avec la sérosité exhalée, était en voie de dissolution lente et graduelle lorsque le malade a succombé. Seule la paroi inférieure est lisse et offre une consistance àssez ferme.

Il est clair, par conséquent, que le foyer primitif du ramollissement existait là où se trouve aujourd'hui la perte de substance, que le mal s'est ensuite étendu de proche en proche par continuité de tissu, et que le point où il a débuté doit être cherché, non parmi les organes actuellement ramollis ou en voie de ramollissement, mais parmi ceux qui sont plus ou moins complètement détruits. Nous allons donc, d'après l'inspection des parties qui

(1) Ce n'est pas ainsi que les choses se passent dans le ramollissement qui débute dans la couche médullaire des circonvolutions : c'est seulement lorsque la lésion a son point de départ sous la pie-mère, e'est-ì-dire dans la couche corticale des circonvolutions, que la substance ramollie et lentement résorbée est remplacée par de la sérosité. J'ai observé les diverses phases de ce mécanisme sur le cervelet aussi bien que sur le cerveau. La première pièce que j'ai recueillie (et que j’ai présentée en 1861 à la Société anatomique) m’avait d'abord embarrassé; mais depuis lors plusieurs autres ont levé mes doutes. 
limitent la perte de substance, dresser la liste de celles qui ont disparu.

La cavité que nous allons décrire est située, comme on l'a déjà vu, au niveau de la scissure de Sylvius; elle est comprise, par conséquent, entre le lobe frontal et le lobe temporo-sphénoïdal, et si les organes qui l'entourent n'étaient que refoulés sans ètre détruits, on devrait trouver sur son bord inférieur ou temporal la circonvolution marginale inférieure (1) et, enfin, sur

(1) Il m'a paru nécessaire, pour l'intelligence de ce qui va suivre, de rappeler ici très succinctement la disposition et les rapports des organes cérébraux que je devrai mentionner.

l.e lobe antérieur du cerveau comprend toute la partie de l'hémisphère située au-dessus de la scissure de Sylvius, qui le sépare du lobe temporo-sphénoïdal, et en avant du sillon de Rolando, qui le sépare du Jobe pariétal. La situation de ce dernier sillon a été précisée dans une note précédente (p. 15). Sa direction est presque transversale; partant de la ligne médiane, il va presque en ligne droite, et en décrivant à peine de légères flexuosités, ahoutir en bas et en dehors à la scissure de Sylvius qu'il rencontre presque à angle droit, en arrière du bord postérieur du lobe de l'insula.

Le lobe antérieur du cerveau se compose de deux étages, l'un inférieur ou orbitaire, formé de plusieurs circonvolutions dites orbitaires, qui reposent sur la voûte de l'orbite, et dont je n’aurai pas à parler; l'autre supérieur, situé sous l'écaille de l'os frontal, et sous la partie la plus antérieure du pariétal.

Cet étage supérieur se compose de quatre circonvolutions fondamentales qu'on appelle les circonvolutions frontales proprement dites : l'une est postérieure, les autres sont antérieures. La postérieure, peu flexueuse, forme le bord anlérieur du sillon de Rolando; elle est donc presque transversale, et elle remonte de dehors en dedans, de la scissure de Sylvius à la grande scissure médiane qui reçoit la faux du cerveau; c'est pourquoi on la désigne indifféremment sous les noms de circonvolution fruntale postérieure, transversale ou ascendante. Les trois autres circonvolutions de l'étage sont très flexueuses, très compliquées, et il faut une certaine habitude pour les distinguer dans toute leur longueur, en évitant de confondre les sillons fondamentaux qui les séparent, avec les sillons secondaires qui séparent les plis de second ordre et qui varient, suivant les individus, d'après le degré de complication, c'est-à-dire d'après le degré de développement des circonvolutions fondamentaies. Ces trois circonvolutions antérieures sont antéro-postérieures, et, cheminant côte à côte, parcourent d'avant en arrière toute la longueur du lobe frontal. Elles commencent au niveau de l'arcade sourcilière, où elles se réfléchissent pour se continuer avec les circonvolutions de l'étage inférieur, et elles vont aboutir, en arrière, à la circonvolution frontale transversale, dans laquelle elles se jettent toutes trois. Elles portent les noms de première, deuxième et troisième circonvolution frontale. On peut encore les appeler interne, moyenne et externe; mais les noms ordinaux ont prévalu.

La première longe la grande scissure du cerveau; elle présente constamment dans l'espèce humaine un sillon antéro-postérieur plus ou moins complet, qui la 
sa paroi profonde, le lobe de l'insula. Or, il n'en est rien. $1^{\circ}$ Le bord inférieur de la cavité est limité par la seconde circonvolution temporo-sphénoïdale, qui est d'ailleurs bien entière et qui possède une consistance assez ferme. La circonvolution marginale inférieure a donc été détruite dans toute son épaisseur, c'est-àdire jusqu'à la scissure purallèle. $2^{\circ}$ La paroi profonde de la cavité ne présente plus de traces du lobe de l'insula; ce lobe est entièrement détruit, ainsi que la moitié interne du noyau extraventriculaire du corps strié; enfin, la perte de substance se prolonge de ce côté jusqu'à la partie antérieure du noyau ventriculaire du corps strié, de telle sorte que notre cavité communique, par une ouverture longue d'un demi-centimètre et à bords irréguliers, avec le ventricule latéral du cerveau. $3^{\circ}$ Enfin,

divise en deux plis de second ordre. On l'a donc subdivisé en deux circonvolutions; mais l'anatomie comparée montre que ces deux plis ne forment qu'une seule circonvolution fondamentale.

La seconde circonvolution frontale n'offre rien de particulier; il n'en est pas de même de la troisième qui est la plus externe. Celle-ci présente un bord supérieur ou interne continu avec le bord flexueux de la circonvolution moyenne, et un bord inférieur ou externe dont les rapports diffèrent suivant qu'on les examine en avant ou en arrière. Dans sa moitié antérieure, ce bord est en contact avec le bord externe de la circonvolution orbitaire la plus externe. Dans sa moitié postérieure, il est libre, au contraire, et séparé du lobe temporo-sphénoïdal par la scissure de Sylvius, dont il forme le bord supérieur. C'est à cause de ce dernier rapport que la troisième circonvolution frontale est quelquefois désignée sous le nom de circonvolution marginale supérieure. C'est un pli an téro-postérieur, mince et presque rectiligne, qui est séparé de la seconde circonvolution temporo-sphénoïdale par un sillon parallèle à la scissure de Sylvius. Ce sillon est désigné sous le nom de scissure parallèle (sous-entendu à la scissure de Sylvius).

Enfin, lorsqu'on écarte les deux circonvolutions marginales, supérieure et inférieure, de la scissure de Sylvius, on aperçoit une éminence large et peu saillante dont le sommet donne naissance à cinq petites circonvolutions simples, ou plutôt à cinq plis rectilignes radiés en éventail; c'est le lobe de l'insula qui recouvre le noyau extraventriculaire du corps strié, et qui, s'élevant du fond de la scissure de Sylvius, se trouve en continuité de substance, par sa couche corticale, avec la partie la plus enfoncée des deux circonvolutions marginales, par sa couche médullaire avec le noyau extraventriculaire du corps strié.

Il résulte de ce rapport qu'une lésion qui se propage, par voie de continuité, du lobe frontal au lobe temporo-sphénoïdal, ou réciproquement, passe presque nécessairement par le lobe de l'insula, et que de là elle a toute chance de se propager au noyau extraventriculaire du corps strié, attendu que la substance propre de l'insula qui sépare ce noyau de la surface du cerveau ne forme qu'une couche mince. 
le bord supérieur, ou plutôt la paroi supérieure de la cavité, empiète considérablement sur le lobe frontal, qui présente, à ce niveau, une échancrure large et profonde. La moitié postérieure de la troisième circonvolution frontale est complètement détruite dans toute son épaisseur; la seconde circonvolution frontale est un peu moins altérée. Ses deux tiers externes au moins ont disparu, et le tiers externe, qui se retrouve encore, est extrêmement ramolli. En arrière, le tiers inférieur de la circonvolution frontale transversale est détruit, dans toute son épaisseur, jusqu'au sillon de Rolando.

En résumé, par conséquent, les organes détruits. sont les suivants :

La petite circonvolution marginale inférieure (lobe temporosphénoïdal) ; les petites circonvolutions du lobe de l'insula, et la partie subjacente du corps strié; enfin, sur le lobe frontal, la partie inférieure de la circonvolution transversale, et la moitié postérieure des deux grandes circonvolutions désignées sous les noms de seconde et troisième circonvolution frontale. Des quatre circonvolutions qui forment l'étage supérieur du lobe frontal, une seule, la première et la plus interne, a conservé non son intégrité, car elle est ramollie et atrophiée, mais sa continuité; et si l'on rétablit par la pensée toutes les parties qui ont disparu, on trouve que les trois quarts au moins de la cavité ont été creusés aux dépens du lobe frontal.

Il s'agit maintenant de déterminer le point où la lésion a dû débuter. Or, l'examen de la cavité laissée par la perte de substance montre tout d'abord que le centre du foyer correspond au lobe frontal. Par conséquent, si le ramollissement s'était propagé uniformément en tous sens, ce serait bien ce lobe qui aurait été le point de départ du mal. Mais ce n'est pas seulernent l'étude de la cavité qui doit nous guider, nous devons tenir compte aussi de l'état des parties qui l'entourent. Ces parties sont très inégalement ramollies, elles le sont surtout dans une étendue très variable. Ainsi, la seconde circonvolution temporale, qui limite inférieurement le foyer, présente une surface lisse et une consistance assez ferme ; elle est ramollie, sans doute, mais elle ne l'est pas beaucoup, et elle'ne l'est que dans sa couche superficielle. Du còté opposé, sur le lobe frontal, le ramollissement est, au 
contraire, presque diffluent au voisinage du foyer; à mesure qu'on s'en éloigne, la substance cérébrale se raffermit graduellement, mais le ramollissement s'étend, en réalité, jusqu'à une distance considérable et atteint presque tout le lobe frontal. G'est, donc surtout dans ce lobe que le ramollissement s'est propagé, et il est à peu près certain que les autres parties n'ont été envahies que consécutivement.

Si l'on cherchait à préciser davantage, on remarquerait que la troisième circonvolution frontale est celle qui présente la perte de substance la plus étendue, qu'elle est non seulement coupée en travers au niveau de l'extrémité antérieure de la scissure de Sylvius, mais encore entièrement détruite dans toute sa moitié postérieure, qu'elle a subi, à elle seule, une perte de substance égale à la moitié environ de la perte de la substance totale; que la seconde circonvolution ou circonvolution moyenne, quoique très profondément entamée, conserve encore sa continuité à sa partie la plus interne et que, par conséquent, selon toutes probabilités, c'est dans la troisième circonvolution frontale que le mal a débuté.

Les autres parties des hémisphères sont relativement saines ; elles sont, il est vrai, un peu moins fermes que d'habitude, et on peut dire que toutes les parties extérieures de l'encéphale ont subi une atrophie notable, mais elles ont conservé leur forme, leur continuité, leur aspect normal. Quant aux parties profondes, j'ai renoncé à les étudier, afin de ne pas détruire la pièce qu'il me paraissait important de déposer dans le musée. Toutefois, l'ouverture qui faisait communiquer aver, l'extérieur la partie antérieure du ventricule latéral gauche s'étant agrandie malgré moi pendant la dissection de la pie-mère, j’ai pu examiner à demi la surface interne de ce ventricule et j'ai vu que tout le corps strié était plus ou moins ramolli, mais que la couche optique avait sa couleur, son volume et sa consistance normaux.

L'encéphale tout entier, pesé avec la pie-mère, après l'évacuation du liquide qui remplissait le foyer, ne dépasse pas le poids de 987 grammes. Il est donc inférieur de près de 400 grammes au poids moyen du cerveau chez les hommes de cinquante ans. Cette perte considérable porte presque entièrement sur les hémisphères cérébraux. On sait, en effet, que le reste de l'encéphale 
à l'état normal n'atteint jamais le poids de 200 grammes et reste presque constamment au-dessous de 180. Or, le cervelet, la protubérance et le bulbe, quoique peu volumineux sur notre sujet, ne sont certainenient pas beaucoup au-dessous de la moyenne, et en supposant, par impossible, qu'ils eussent perdu le quart de leur poids, cela ne rendrait compte que d'une partie minime de la déperdition totale.

La destruction des organes qui entourent la scissure de Sylvius de l'hémisphère gauche contribue, sans doute, beaucoup à diminuer le poids du cerveau; mais jai fait, dans un cerveau sain, une perte de substance de même étendue, et la masse que j’ai enlevée ainsi ne pesait pas tout à fait 50 grammes. Il est donc infiniment probable que les hémisphères cérébraux ont subi, dans toute leur étendue, une atrophie assez notable, et cette probabilité se change en certitude si l'on songe à l'épaisseur considérable des méninges et de la fausse membrane arachnoïdienne, épaisseur qui s'élève, en certains points, à 5 ou 6 millimètres.

Après avoir décrit les lésions et cherché à en déterminer la nature, le siège et la marche anatomique, il importe de comparer ces résultats avec ceux de l'observation clinique, afin d'établir, si c'est possible, un rapport entre les symptômes et les désordres matériels.

L’inspection anatomique montre que la lésion était encore en voie de propagation lorsque le malade a succombé. Gette lésion a donc été /progressive, mais elle a marché avec beaucoup de lenteur, puisqu'elle a mis vingt et un ans à détruire une partie assez limitée de la masse cérébrale. Il est permis de croire, par conséquent, qu'il y a eu au commencement une longue période pendant laquelle elle ne dépassait pas les limites de l'organe où elle avait débuté. Or, on a vu que le foyer principal du mal était situé dans le lobe frontal et très probablement dans la troisième circonvolution frontale. Gela nous conduit à admettre qu'au point de vue de l'anatomie pathologique il y a deux périodes, l'une, dans laquelle une seule circonvolution frontale (probablement la troisième) était altérée; l'autre, dans laquelle le mal s'est propagé de proche en prúche à d'autres circonvolutions, au lobe de l'insula et au noyau extra-ventriculaire du corps strié. 
Si maintenant nous examinons la succession des symptômes, nons trouvons également deux périodes : une première période qui a duré dix ans, pendant laquelle la faculté du langage était abolie et où toutes les autres fonctions de l'encéphale étaient intactes; et une seconde période de onze ans, pendant laquelle une paralysie du mouvement d'abord partielle, puis absolument complète, a envahi successivement le membre supérieur et le membre inférieur du côté droit.

Cela posé, il est impossible de méconnaître qu'il y a eu correspondance entre les deux périodes symptomatologiques. Nul n'ignore que les circonvolutions cérébrales ne sont pas des organes moteurs. Le corps strié de l'hémisphère gauche est donc de tous les organes lésés le seul où l'on puisse chercher la cause de la paralysie des deux membres droits, et la seconde période clinique, celle où la motilité a été altérée, correspond ainsi à la seconde période anatomique, c'est-à-dire à celle où le ramollissement, débordant les limites du lobe frontal, a gagné l'insula et le corps strié.

Dès lors, la première période de dix ans, caractérisée cliniquement par l'unique symptôme de l'aphémie, doit correspondre à l'époque où la lésion était encore limitée au lobe frontal.

Jusqu'ici, dans ce parallèle des lésions et des symptômes, je n'ai parlé ni des troubles de l'intelligence, ni de leur cause anatomique. On a vu que l'intelligence de notre malade, parfaitement conservée pendant longtemps, avait décliné notablement à partir d'une époque qui n'a pu être déterminée, et qu'elle était fort affaiblie lorsque nous l'avons vu pour la première fois.

Nous avons trouvé, à l'autopsie, des altérations plus que suffisantes pour expliquer cet état. Trois circonvolutions frontales sur quatre étaient profondément lésées dans une étendue considérable, presque tout le lobe frontal était plus ou moins ramolli; enfin toute la masse des circonvolutions des deux hémisphères était atrophiée, affaissée et sensiblement plus molle qu'à l'état normal. On a peine à comprendre que le malade ait pu conserver encore quelque intelligence, et il ne paraît pas probable qu'on puisse vivre bien longtemps avec un pareil cerveau. Je pense, pour ma part, que le ramollissement général du lohe 
frontal gauche, l'atrophie générale des deux hémisphères et la méningite chronique génórale, ne remontaient pas à une époque fort reculée; je suis disposé à croire que ses lésions sont survenues longtemps après le ramollissement du corps strié, de telle sorte qu'on pourrait subdiviser la seconde période en deux périodes secondaires et résumer ainsi l'histoire du malade:

Première période (dix ans) : $\begin{gathered}\text { Lésions. } \\ \text { Ramollissement d'une cir- } \\ \text { convolution frontale (pro- } \\ \text { bablement la troisième). Aphémie simple. }\end{gathered}$

Les faits qui, comme celui-ci, se rattachent à de grandes questions de doctrine, ne sauraient être exposés avec trop de détails, ni discutés avec trop de soin. J'ai besoin de cette excuse pour me faire pardonner l'aridité des descriptions et la longueur des discussions. Je n'ai plus maintenant que peu de mots à ajouter pour faire ressortir les conséquences de cette observation.

$1^{\circ}$ L'aphémie, c'est-à-dire la perte de la parole, avant tout autre trouble intellectuel et avant toute paralysie, a été la conséquence d'une lésion de l'un des lobes antérieurs du cerveau;

$2^{\circ}$ Notre observation vient donc confirmer l'opinion de M. Bouillaud, qui place dans ces lobes le siège de la faculté du langage articulé;

$3^{\circ}$ Les observations recueillies jusqu'ici, celles du moins qui sont accompagnées d'une description anatomique claire et précise, ne sont pas assez nombreuses pour qu'on puisse considérer cette localisation d'une faculté particulière dans un lobe déterminé comme définitivement démontrée, mais on peut la considérer du moins comme extrêmement probable;

$4^{\circ}$ C'est une question beaucoup plus douteuse de savoir si la faculté du langage articulé dépend du lobe antérieur considéré 
dans son ensemble, ou spécialement de l'une des circonvolutions de ce lobe; de savoir, en d'autres termes, si la localisation des facultés cérébrales a lieu par faculté et par circonvolution, ou seulement par groupes de facultés et par groupes de circonvolutions. Les observations ultérieures devront être recueillies dans le but de résoudre cette question. Il faut pour cela indiquer exactement le nom et le rang des circonvolutions malades, et, si la lésion est très étendue, chercher à déterminer, autant que possible, par l'examen anatomique, le point ou plutôt la circonvolution où le mal paraît avoir débuté ;

$5^{\circ}$ Chez notre malade, le siège primitif de la lésion était dans la seconde ou troisième circonvolution frontale, plus probablement dans cette dernière. Il est donc possible que la faculté du langage articulé siège dans l'une ou l'autre de ces deux circonvolutions; mais on ne peut le savoir encore, attendu que les observations antérieures sont muettes sur l'état de chaque circonvolution prise en particulier, et on ne peut même pas le pressentir, puisque le principe des localisations par circonvolution ne repose encore sur aucune base certaine;

$6^{\circ}$ En tous cas, il suffit de comparer notre observation avec celles qui l'ont précédée pour écarter aujourd'hui l'idée que la faculté du langage articulé réside dans un point fixe, circonscrit, et situé sous n'importe quelle bosse du crâne; les lésions de l'aphémie ont été trouvées le plus souvent dans la partie la plus antérieure du lobe frontal, non loin du sourcil, et au-dessus de la voûte orbitaire; tandis que, chez mon malade, elles existaient bien plus en arrière, et beaucoup plus près de la suture coronale que de l'arcade sourcilière. Cette différence de siège est incompatible avec le système des bosses; elle serait parfaitement conciliable, au contraire, a vec le système des localisations par circonvolutions, puisque chacune des trois grandes circonvolutions de l'étage supérieur du lobe frontal parcourt successivement, dans son trajet antéro-postérieur, toutes les régions où ont été trouvées jusqu'ici les lésions de l'aphémie. 


\section{NOUVELLE OBSERVATION D'APHÉMIE}

PRODUITE PAR UNE LÉSION DE LA TROISIÈME CIRCONVOLUTION FRONTALE

(Bulletins de la Société d'anatomie, 1861, 2e série, t. VI, p. 398-407.)

Dans la première observation d'aphémie que j'ai communiquée à la Société il y a quelques mois, j'ai décrit une lésion cérébrale assez compliquée, un ramollissement progressif qui avait détruit successivement trois circonvolutions frontales, le lobule de l'insula, le noyau extraventriculaire du corps strié, et enfin la circonvolution marginale supérieure du lobe temporo-sphénoïdal. Sachant qu'au début de la maladie, et pendant une longue période de dix ans, le malade avait perdu uniquement la faculté d'articuler les mots, en conservant tout à fait intactes toutes ses autres facultés intellectuelles, sensitives ou motrices, j'ai été conduit à penser que la perte de la parole avait été la conséquence d'une lésion primitivement assez circonscrite, et que l'organe central du langage articulé était probablement celui dans lequel cette lésion avait débuté ; pour découvrir cet organe, parmi tous ceux qui étaient lésés au moment de la mort, j'ai cherché quel était le point où l'altération paraissait la plus ancienne, et j'ai trouvé que, selon toute probabilité, la troisième circonvolution frontale, peut-être aussi la seconde, avaient dû être le point de départ du ramollissement.

Je re me dissimulais pas, toutefois, que l'ancienneté du mal et l'étendue de ses ravages rendaient cette détermination un peu incertaine. J'avais donc laissé planer quelques doutes sur mes conclusions. Le nouveau fait que je présente aujourd'hui à la Société me permettra d'être plus affirmatif. L'aphẻmie avait débuté brusquement un an et demi avant la mort; la lésion que j'ai trouvée à l'autopsie est parfaitement circonscrite et existe 
précisément, sans la moindre différence, dans le point où j'avais admis que la lésion avait dû débuter chez mon premier malade.

Observation. - Le nommé Lelong, âgé de quatre-vingtquatre ans, ancien terrassier, fut porté à l'infirmerie de l'hospice de Bicêtre, service de chirurgie, salle Saint-Prosper, le 27 octobre 1861, pour y être traité d'une fracture du col du fémur gauche.

Cet homme avait été admis dans l'hospice, huit ans auparavant, pour cause de débilité sénile. Il n'avait alors aucune paralysie, il avait conservé tous ses sens, toute son intelligence; mais ses membres, affaiblis par les progrès de l'âge, se refusaient au travail, et sa main, devenue tremblante, ne pouvait plus écrire; jamais d'ailleurs il n'avait su écrire couramment.

Au mois d'avril 1860, pendant les fêtes de Pâques, en descendant un escalier, il s'affaissa tout à coup sur lui-même. On le retint à temps pour l'empêcher de se blesser, mais il paraissait avoir perdu connaissance. Il fut transporté à l'infirmerie, service de médecine, et traité pour une apoplexie cérébrale.

Il fut sur pied en peu de jours. Il n'avait jamais présenté la moindre apparence de paralysie des membres; mais sa fille, de qui je tiens ces renseignements, pensa qu'il avait la langue paralysée. Le fait est que depuis le moment de son accident il avait perdu subitement et définitivement la faculté de parler; il ne prononçait plus que certains mots, articulés avec difficulté; sa démarche était un peu incertaine, mais il ne boitait pas; son intelligence n'avait subi aucune atteinte appréciable; il comprenait tout ce qu'on lui disait, et son court vocabulaire, accompagné d'une mimique expressive, lui permettait d'être compris, à son tour, par les personnes qui vivaient habituellement avec lui.

Cet état se maintint sans changement jusqu'au 27 octobre 1861. Ge jour-là, en montant au lit, il perdit l'équilibre, tomba sur la hanche gauche, et se brisa le col du fémur. Je passe sous silence tout cc qui est relatif à cette fracture.

La paralysie de la langue qu'on nous avait annoncée n'existait pas. Cet organe était bien mobile, il n'était nullement dévié, eí offrait la même épaisseur à droite et à gauche. La déglutition se faisait bien; la vue et l'oüe étaient conservées, les membres 
obéissaient à la volonté, à l'exception du membre fracturé, qui la veille encore était aussi fort que son congénère. L'émission des urines et des matières fécales était régulière; enfin la sensibilité persistait sans altération, et le malade souffrait beaucoup de sa fracture, qui était compliquée d'une assez forte contusion.

Aux questions qu'on lui adressait, cet homme ne répondait que par des signes, accompagnés d'une ou deux syllabes articulées brusquement avec un certain effort. Ces syllabes avaient un sens, c'étaient des mots français, savoir : oui, non, tois (pour trois) et toujours. Il y avait un cinquième mot qu'il prononçait quand on lui demandait son nom : il répondait alors Lelo pour Lelong, qui était son véritable nom.

Les trois premiers mots de son vocabulaire correspondaient chacun à une idée déterminée. Pour affirmer ou approuver, il disait oui. Pour exprimer l'idée opposée il disait non. Le mot trois exprimait tous les nombres, toutes les idées numériques. Enfin toutes les fois qu'aucun des trois mots précédents n'était applicable, Lelong se servait du mot toujours, qui, par conséquẹt, n'avait aucun sens déterminé.

Je lui demandai s'il savait écrire? Il répondit oui. - S'il pouvait ? - Non. - Essayez. - Il essaya, mais il ne put réussir à diriger la plume.

Les applications qu'il faisait du mot trois sont assez curieuses pour être indiquées avec quelques détails. Ce mot était toujours accompagné d'un signe fait avec les doigts, parce que notre malade, sachant que sa langue trahissait sa pensée, rectifiait par le geste cette erreur involontaire. On me permettra, pour plus de clarté, de rapporter ici quelques-unes de ses réponses.

- Depuis combien d'années êtes-vous à Bicêtre? - Trois, et il levait huit doigts.

- Avez-vous des enfants? - Oui.

- Combien? - Trois, et il levait quatre doigts.

- Combien de garçons? - Trois, et il levait deux doigts.

- Combien de filles? - Trois, et il levait encore deux doigts.

Tout cela était parfaitement exact.

- Savez-vous lire l'heure à une montre? - Oui.

- Quelle heure est-il? - Trois, et il levait dix doigts. (Il était dix heures.) 
- Quel âge avez-vous? Nous nous attendions à le voir ouvrir huit fois les deux mains, et faire ensuite un appoint de quatre doigts, car nous savions qu'il avait quatre-vingt-quatre ans. Au lieu de cela, il fit seulement deux gestes, en disant trois, et je crus un instant qu'il avait perdu la notion des nombres plus grands que dix. Mais l'interne du rang, M. Bernadet, fit une remarque qui nous révéla tout à coup que cet homme savait très bien son âge, et qu'il comptait parfaitement. Au premier geste il avait levé huit doigts; au second geste, il en avait levé quatre. Cela voulait dire sans doute huit dizaines quatre unités. La chose valait la peine d'être vérifiée; je répétai la question, et il reproduisit exactement les mêmes signes accompagnés du mot trois. Et quand il vit que nous avions compris cette fois son langage, il ajouta oui avec un signe de tête affirmatif.

Il avait des gestes fort expressifs qui lui permettaient de faire des réponses très intelligibles. Ainsi il m'a fait connaître sa profession avant qu'on m’eut renseigné sur ce point. "Quel état faisiez-vous avant d'entrer à Bicêtre? - Toujours. Et ce disant, il a fait aver ses deux mains le geste d'un homme qui prend une pelle, qui l'enfonce dans le sol, qui la soulève, et enfin jette une pelletée de terre. - Vous êtes donc terrassier? - Oui, avec un signe de tête affirmatif. » Il était effectivement terrassier.

Ses réponses ne nous ont trompés qu'une seule fois. Lorsque nous lui avons demandé depuis combien de temps il avait perdu la parole, il a répondu trois en levant huit doigts. Il confondait peut-être la date de son apoplexie avec celle de son entrée à Bicêtre, peut-être aussi voulait-il dire huit mois, ce qui était encore inexact. Nous pensions alors, d'après le rapport de la surveillante, que cet accident datait de trois ans. Ce fut seulement après sa mort que sa fille aînée, en nous donnant la confirmation de ses autres réponses, nous apprit qu'il avait perdu la parole en avril 1860 , ce qui faisait dix-huit mois révolus. Je me suis demandé depuis si le geste huit n'avait pas été précédé du geste un, qui aurait très bien pu passer inaperçu. Cette interprétation me paraît aujourd'hui très probable; mais alors même que cette seule et unique fois le malade se serait trompé ou n'aurait pas su s'exprimer, nous en savons assez pour pouvoir affirmer : $1^{\circ}$ qu'il comprenait tout ce qu'on lui disait; $2^{\circ}$ qu'il 
appliquait avec discernement les quatre mots de son vocabulaire; $3^{\circ}$ qu'il était sain d'esprit ; $4^{\circ}$ qu'il connaissait la numération écrite, et au moins la valeur des deux premiers ordres d'unités; 5゚ qu'il n'avait perdu ni la faculté générale du langage, ni la motilité volontaire des muscles de la phonation et de l'articulation, et qu'il n'avait perdu, par conséquent, que la faculté du langage articulé.

Il était donc atteint d'aphémie, mais cette aphémie différait, à plusieurs égards, de celle que j'avais étudiée chez mon premier malade. Celui-ci n'avait qu'une réponse invariable pour toutes les questions : c'était le monosyllabe tan, toujours répété deux fois, et ce mot n'était pas même un mot français; ce n'était pas le dernier débris de sa langue natale: c'était un son de hasard, entièrement dénué de sens. On peut dire au contraire que Lelong avait un vocabulaire; outre son nom de famille (Lelo), quatre mots, des mots français, avaient survécu au naufrage, et il en tirait tout le parti possible, en donnant à trois d'entre eux des acceptations déterminées. C'étaient donc deux variétés d'aphémie parfaitement distinctes l'une de l'autre. 0n pouvait supposer, il est vrai, qu'à la longue, par les progrès de son mal, Lelong serait descendu au niveau de l'autre malade. Il y avait, toutefois, une circonstance embarrassante, c'est que, chez ce dernier, l'aphémie avait été, dès le début, aussi complète qu'elle l'était vingt et un ans plus tard au moment de la mort. Le ramollissement cérébral, en se propageant, avait altéré ou aboli des fonctions et des organes divers; n'ayant détruit d'abord qu'une partie peu étendue des circonvolutions frontales, le mal avait fini par creuser dans le lobe antérieur une vaste perte de substance, et l'intelligence du malade avait subi en même temps une atteinte sensible; et pourtant, tandis que tous les autres troubles fonctionnels avaient été en croissant, le seul symptôme de l'aphémie était constamment resté le même. Il était donc permis de se demander si l'aphémie de Tan et celle de Lelong étaient de même nature; il y avait des raisons pour la négative et des raisons pour l'aftirmative. J'hésitai, par conséquent, à admettre que le siège de la lésion dût être le même dans le second cas que dans le premier, et j'attendis, sans me prononcer, les résultats de l'autopsie, qui devait être prochaine. Le 
malade, en effet, s'affaiblissait rapidement; il lui survint des eschares au sacrum, et il mourut le 8 novembre 1861, douze jours seulement après sa chute.

Autopsie. - Les viscères thoraciques et abdominaux ne présentaient rien de remarquable. Je n'ai pas à parler ici de la fracture du col du fémur; je ne parlerai que du cerveau.

Toutes les sutures sont ossifiées. Les parois du crâne sont un peu épaissies, mais ne sont pas plus dures que d'habitude; les os sont sains, ainsi que la dure-mère qui n'est pas épaissie. Il y a dans la cavité de l'arachnoïde une assez grande quantité de sérosité ; la pie-mère n'est ni épaissie ni congestionnée.

L'encéphale entier pèse, avec ses membranes, au sortir du crâne, 1136 grammes, chiffre très inférieur à la moyenne, et à peine égal au poids minimum de l'encéphale des individus adultes, du sexe masculin, et sains d'esprit. Dans les pesées connues jusqu'ici, ce minimum a été de 1133 grammes. Mais on sait que, chez les vieillards, le poids moyen đe l'encéphale diminue d'une manière notable. On verra, en outre, tout à l'heure que l'un des hémisphères avait subi une atrophie assez prononcée. Ces deux causes réunies ont contribué à faire descendre bien au-dessous de la moyenne le poids de l'encéphale de notre malade.

Le cerveau étant placé sur une table, on aperçoit au premier coup d'œil une lésion superficielle qui occıpe le lobe frontal gauche, immédiatement au-dessous de l'extrémité antérieure de la scissure de Sylvius. A ce niveau, la surface de l'hémisphère est sensiblement affaissée, et la pie-mère déprimée laisse apercevoir par transparence une collection de sérosité qui occupe en surface une étendue à peu près égale à celle d'une pièce d'un franc. Cette lésion est incomparablement plus circonscrite que celle qui existait sur le cerveau de Tan; mais, en comparant les deux pièces, on constate que le centre de la lésion est identiquement le même dans les deux cas.

Avant d'enlever la pie-mère, je sépare le cervelet, la protubérance et le bulbe, qui pèsent ensemble 142 grammes; puis je divise, sur la ligne médiane, le cerveau proprement dit et je pèse séparément les deux hémisphères. Le droit pèse 514 grammes; le gauche ne pèse que 480 grammes. Ce dernier, 
qui est le siège du mal, est donc inférieur de 34 grammes à l'hémisphère sain. La pesée comparative est répétée après l'ablation de la pie-mère et l'écoulement de la sérosité des ventricules latéraux. L'hémisphère droit pèse alors 487 grammes, le gauche 455 ; différence, 32 grammes au lieu de 34 . Cela indique que le foyer du lobe frontal gauche renfermait environ 2 grammes de sérosité. On voit que l'hémisphère gauche a subi une diminution de poids bien plus considérable qu'on aurait pu s'y attendre, d'après le peu d'étendue de la lésion du lobe frontal. Malgré cela, la consistance de la substance cérébrale est exactement la même des deux côtés; elle est très ferme et supérieure même à celle que présente ordinairement le cerveau des vieillards. La surface des circonvolutions offre, à droite et à gauche, la couleur la plus normale ; on n'a eu aucune difficulté à enlever la pie-mère.

L'hémisphère droit est parfaitement sain dans toutes ses parties, ainsi que le cervelet, le bulbe et la protubérance. Il n'y a de lésions appréciables que sur l'hémisphère gauche.

Dans cet hémisphère, la couche optique, la voûte, le corps calleux, le ténia, le corps strié, les lobes occipital et pariétal, le lobe de l'insula, et les circonvolutions orbitaires, qui forment l'étage inférieur du lobe frontal, sont à l'état normal; il m'a paru toutefois qu'à l'union de l'extrémité antérieure du noyau ventriculaire du corps strié avec la substance médullaire du lobe frontal la consistance du tissu cérébral était légèrement diminuée ; mais cette lésion, si c'en est une, est tout à fait indépendante de la lésion principale, dont elle est séparée par une épaisseur considérable de tissu sain. C'est cette dernière lésion que je vais maintenant décrire.

La collection de sérosité située sous la pie-mère, et dont le siège a été indiqué plus haut, occupait une cavité creusée dans la substance des circonvolutions. A ce niveau, la troisième circonvolution frontale, qui longe, comme on sait, le bord supérieur de la scissure de Sylvius, est complètement coupée en travers et a subi, dans toute son épaisseur, une perte de substance dont l'étendue paraît être d'environ 15 millimètres. Notre cavité est donc continue en dehors avec la scissure de Sylvius, au niveau du lobe de l'insula. En dedans, elle empiète 
sur la seconde circonvolution frontale, qui est très profondément échancrée, mais dont la couche la plus interne est respectée dans une épaisseur de 2 millimètres. G'est cette mince languette qui maintient seule la continuité de la deuxième circonvolution frontale. La première est parfaitement saine; la circonvolution frontale transversale ou supérieure, qui limite en avant le sillon de Rolando, est saine également; enfin; les deux circonvolutions malades, dans les deux tiers antérieurs, présentent une intégrité complète. On peut affirmer, par conséquent, que chez notre malade l'aphémie a été le résultat d'une lésion profunde, mais très nettement circonscrite, de la deuxième et de la troisième circonvolution frontale, dans une partie de leur tiers postérieur.

Il est certain que cette lésion n'est pas un ramollissement; le tissu cérébral est si loin d'être ramolli sur les parois du foyer, qu'aujourd'hui encore, qnoique la pièce ait été plusieurs fois examinée et maniée, la mince languette qui maintient la continuité de la deuxième circonvolution frontale a conservé sa solidité; cela me paraît même indiquer que le tissu cérébral, au voisinage immédiat, du foyer, est plus résistant qu'à l'état normal.

D'un autre côté, sur les parois du foyer on aperçoit quelques petites taches d'un jaune orangé qui paraissent d'origine hématique, et l'examen microscopique, fait par mon interne, M. Piedvache, a montré qu'il y avait à ce niveau des cristaux d'hématine. Il s'agit donc d'un ancien foyer apoplectique, et l'on n'a pas oublié que notre malade avait perdu la parole subitement dans une attaque d'apoplexie, dix-huit mois avant sa mort.

Cette observation présente des analogies très remarquables avec celle de mon premier malade et elle est beaucoup plus démonstrative, puisque le mal est ici parfaitement circonscrit. Dans le premier cas, il pouvaity avoir quelque doute sur le siège primitif de la lésion; ce n'était que par une analyse raisonnée des désordres anatomiques qu'on était conduit à reconnaître que l'aphémie avait été le résultat du ramollissement des deux circonvolutions frontales externes; chez mon second malade, au contraire, cette détermination est évidente. Il n'y a eu d'autre symptôme que l'aphémie, d'autre lésion que celle des deux cir- 
convolutions susdites, et il est incontestable que chez cet homme la maladie qui a frappé ces deux circonvolutions a été la cause directe de l'aphémie.

Dans les deux cas, la deuxième circonvolution frontale était beaucoup moins profondément altérée que la troisième; il est permis d'en conclure que celle-ci avait été, selon toute probabilité, le siège principal de la lésion primitive. Deux faits sont peu de chose, lorsqu'il s'agit de résoudre une des questions les plus obscures et les plus controversées de la physiologie cérébrale ; je ne puis toutefois m'empêcher de dire, jusqu'à plus ample informé, que l'intégrité de la troisième circonvolution frontale (et peut-être de la deuxième) paraît indispensable à l'exercice de la faculté du langage articulé.

Dans ma précédente note, j’avais émis cette pensée que, si la doctrine des localisations particulières était un jour reconnue exacte, ce ne serait probablement pas dans des points circonscrits, dans des districts plus ou moins nettement limités, correspondant à des points déterminés de la voûte du crâne, qu'on pourrait localiser les diverses facultés cérébrales; en d'autres termes, j'avais pensé que, s'il y avait une science phrénologique, ce serait la phrénologie des circonvolutions, et non la phrénologie des bosses. Je ne cacherai donc pas que j'ai éprouvé un étonnement. voisin de la stupéfaction, lorsque j’ai trouvé que, sur mon second malade, la lésion occupait rigoureusement le même siège que chez le premier; ce n'étaient pas seulement les mêmes circonvolutions qui étaient malades, elles l'étaient exactement dans le même point, immédiatement en arrière de leur tiers moyen, vis-à-vis du lobe de l'insula et précisément du même côté (côté gauche). Le siège primitif du mal, par conséquent, correspondait, dans les deux cas, au même point de la paroi du crâne. Je ne me dissimule pas que les partisans de la phrénologie des bosses pourront tirer des rapprochements de ces deux faits un argument favorableà leur système ; mais, sans oser me prononcer encore et sans attacher trop d'importance aux observations trop peu détaillées qui ont précédé les miennes, je ne puis oublier que, dans plusieurs des faits publiés, on a vu l'aphémie succéder à des lésions qui occupaient principalement (sinon exclusivement) la moitié antérieure des lobes frontaux. Ces faits, parfaitement 
compatibles avec l'hypothèse des localisations par circonvolutions, paraissent très difficiles à concilier avec le principe des localisations par districts, ou, si l'on veut, par compartiments correspondant à des points invariables de la boîte crânienne. Je suis donc disposé à attribuer à une pure coïncidence l'identité absolue du siège des lésions chez mes deux malades.

Quant à la différence bien manifeste qui existe entre les deux variétés d'aphémie que j'ai décrites dans mes deux observations, différence que j'étais tenté d'attribuer, avant la seconde autopsie, à une différence de siège, je suppose maintenant qu'elle dépendait de la nature des lésions. Chez le premier malade, il s'agissait d'un ramollissement progressif, lésion qui n'est jamais nettement circonscrite; le second n'avait qu'un foyer apoplectique très limité. Il est donc probable que, dans le premier cas, la circonvolution malade était, dès l'origine, altérée dans une étendue plus considérable. On peut comprendre ainsi, jusqu'à un certain point, pourquoi l'aphémie était plus complète et plus grave chez cet homme qu'elle ne l'était chez celui dont je viens de rapporter l'observation. 


\section{LOCALISATION DES FONCTIONS GÉREBRALES}

\section{SIÈGE DU LANGAGE ARTIGULÉ}

(Bulletins de la Société d'anthropologie, 1863, t. IV, p. 200-202 et 208.)

Déjà la Société s'est occupée longuement de la localisation des fonctions cérébrales, et les observations de lésions des lobes antérieurs ont surtout été produites dans la discussion. Ces observations étaient souvent contradictoires et prêtaient à des interprétations diverses, mais il faut penser que les lobes antérieurs représentent à peu près la moitié du cerveau, et que sans doute les fonctions peuvent avoir un siège infiniment plus restreint.

Des observations assez nombreuses me permettent de supposer avec quelque apparence de certitude que cela est vrai, au moins pour la fonction du langage articulé.

J'ai recueilli et communiqué à la Société anatomique une première observation prise chez un malale atteint de cette privation de la faculté de parler, à laquelle j’ai donné le nom d'aphémie. Il y avait une lésion de la troisième circonvolution frontale, et peut-être aussi de la seconde.

A quelque temps de là, je fis l'autopsie d'un malade, âgé de quatre-vingt-neuf ans, ayant eu dix-huit mois auparavant une hémorrhagie cérébrale. Il avait conservé son intelligence, mais avait perdu la faculté de parler. Il n'avait plus que cinq mots à sa disposition : oui, non, toi, toujours et Lelo. Nous trouvâmes un ancien foyer hémorrhagique à 2 centimètres de l'extrémité postérieure de la troisième circonvolution frontale, qui était coupée en travers.

Depuis ce temps, M. Charcot a trouvé trois malades aphémiques offrant des lésions cérébrales qui avaient exactement le 
même siège. M. Gubler a présenté un fait semblable à la Société de biologie.

Voici deux autres observations très importantes :

M. Charcot présente à la Société de biologie un cerveau d'aphémique où il constate que la lésion siège sur le lobe pariétal; c'était un ramollissement jaune avec atrophie. J'avoue que j'étais un peu décontenancé ; cependant je dissèque les membranes, et je trouve que le ramollissement file le long de la scissure de Sylvius et va jusqu'à la troisième sirconvolution frontale qui est détruite dans sa moitié profonde.

Un jour, M. Duchenne, de Boulogne, vient me dire qu'on a observé à l'Hôtel-Dieu, dans le service de M. Trousseau, un fait contraire aux idées que je professe sur le siège du langage articulé. Je me rends à l'hôpital et je constate, en effet, que le lobe pariétal était malade; mais m'appuyant sur les faits antérieurs, j'annonce, en enfonçant un scalpel dans l'épaisseur de la troisième circonvolution, que là doit se trouver une lésion. Effectivement la circonvolution était altérée dans ses 3 centimètres postérieurs.

Ainsi, voilà huit faits où la lésion a siégé dans le tiers postérieur de la troisième circonvolution frontale. Ce chiffre me paraît suffisant pour. donner de fortes présomptions. Et, chose bien remarquable, chez tous ces malades la lésion existait du cổté gauche. Je n'ose tirer de là une conclusion et j'attends de nouveaux faits.

MM. Marcé et Périer voient en ce moment des malades à la fois aphémiques et paralytiques; la paralysie est à droite, donc les lésions sont à gauche.

M. Charcot vient de recevoir dans son service une malade malheureusement démente, aphémique et paralytique. Peut-être chez elle la lésion sera-t-elle située à droite, la paralysie existant à gauche.

Tout ce que je viens de dire ne se rapporte qu'à une seule localisation, celle du langage articulé; chose bien définie et c'est déjà beaucoup, que d'être à peu près certain qu'une faculté au moins a un siège rigoureusement déterminé ; mais sera-t-il possible de fournir la même démonstration pour d'autres facultés moins circonscrites? Peut-être sera-ce bien difficile. 


\section{DISCUSSION}

M. Linas. Pendant mon internat à Charenton, j'ai eu souvent l'occasion de constater chez les malades atteints de paralysie générale et qui au début éprouvent un embarras de la parole, des adhérences de la pie-mère à toute la partie du lobe frontal qui borde la scissure de Sylvius, si bien que nous considérions ce point comme le siège d'élection des lésions qui causent la paralysie générale.

M. Broca. Bien que le fait de M. Linas soit intéressant à connaître, j'hésite à établir un rapprochement avec ceux que j'ai cités, car dans la paralysie générale ce n'est pas la faculté du langage, mais bien la possibilité d'articuler qui est perdue. Il y a donc une différence majeure.

M. Livas. M. Broca distinguerait donc la possibilité d'articuler les mots de la faculté du langage?

M. Broca. Celte distinction est évidente. Un des malades dont j'ai parlé avait conservé la possibilité de prononcer cinq mots. La plupart des aphémiques ont un vocabulaire restreint, mais dont ils usent de façon à prouver que l'articulation des mots reste possible, tandis que la faculté du langage est éteinte.

M. Gratiolet. Ces observations soulèvent une grande difficulté pliilosophique. Comment comprendre la conservation de l'intelligence coïncidant avec la perte du langage? Ce fait ne pourrait s'expliquer qu'en admettant certaines compensations et certaines substitutions de signes dont il serait curieux d'étudier le mécanisme.

M. Martin. Il y a eu à Montpellier un exemple célèbre de perte partielle de la faculté du langage. Broussunnet avait perdu la mémoire ou la possibilité de prononcer des substantifs: il n'en pouvait articuler aucun.

M. DE Jouvencel. Il me semble que la perte de la faculté du langage n'implique nulleınent la perte de l'intelligence comme tendraient à le faire croire les paroles de $\mathbf{1 1}$. Gratiolet. La pensée ne s'élabore pas sous forme de mots. C'eux-ci ne sont qu'une formule, une expression qui peut faire défaut pour une raison quelconque sans que l'intelligence ait perdu son activité.

M. Brocia. Il n'y a pas autant de différence qu'on pourrait le croire entre le cas de Broussonnet et ceux que j'ai cités. Un des malades que j'ai observés pouvait entretenir une sorte de conversation à l'aide d'une collection de petits papiers où étaient inscrites les réponses aux questions les plus usuelles qu'on lui 
adressait. Nous essayâmes de lui faire répéter quelques mots dont il avait conservé le souvenir, nous parvînmes même à lui apprendre quelques mots à la lecture, mais il nous fut impossible d'obtenir jamais un substantif.

(Séance suivante, p. 208.)

M. Broca. Depuis notre dernière séance, deux nouvelles observations d'aphémie avec autopsie ont été recueillies dans le service de M. Gharcot, à la Salpêtrière. La lésion siégeait encore du côté gauche et sur le tiers postérieur de la troisième circonvolution frontale. Je signale ces nouveaux faits à l'attention de la Société. 


\section{REMARQUES}

\section{SUR LE SIÈGE, LE DIAGNOSTIG ET LA NATURE}

\section{DE L'APHÉMIE}

(Bulletins de la Société anatomique, 1863, 2e série, t. VIII, p. 379-385 et 393-399.)

M. Lévy, interne de Parrot, présente une pièce sur laquelle on voit une atrophie complète du lobule de l'insula et de la troisième circonvolution du lobe frontal avec conservation de l'intelligence et de la faculté du langage.

La lésion siège à droite.

Pour Laborde, cette observation est une objection grave à la doctrine localisatrice du langage articulé telle qu'elle a été formulée dans ces derniers temps. Il y a une observation analogue de Charcot. Il est vrai que, dans le fait de Parrot, la lésion siège à droite et non à gauche, comme on l’a vu jusqu'à présent. " Il y a tout lieu de croire que cette différence de siège ne constitue pas une objection sérieuse à la signification de ce fait; il est difficile d'adnettre que deux portions si identiques d'un même organe ne présillent pas à la même fouction, en admettant même qu'elles y président réellement. En second lieu, on a tort de penser que chez les aphémiques l'intelligence est intacte. Presque tous écrivent plus ou moins mal, et c'est une preuve contre cette intégrité de l'intelligence.

Mais Axenfeld cite l'observation d'un homme intelligent qui au cours d'une congestion cérébrale fut frappé d'impossibilité de parler et d'écrire ; et après, le malade raconta toutes ses sensations. L'intelligence et la pensée étaient certainement restées intactes.

Laborde invoque alors des troubles de motilité de la langue et de la main.

M. Broca. Le fait de M. Parrot est remarquable à plus d'un titre, et mérite d'être pris en sérieuse considération. Le malade n'avait pas perdu la faculté du langage articulé, et cependant à 
l'autopsie le lobe frontal du côté droit a présenté une lésion semblable à celle que produit l'aphémie.

La Société n'a peut-être pas oublié que j'ai décrit il y a deux ans sous ce nom d'aphémie une affection caractérisée par l'impossibilité de parler, sans abolition de l'intelligence et sans paralysie des muscles de la phonation et de l'articuiation. Chez les deux malades dont je l'ai entretenue alors, j'avais constaté pendant la vie l'existence de l'aphémie, et j'ai trouvé à l'autopsie une lésion qui occupait dans les deux cas le tiers postérieur de la troisième circonvolution frontale de l'hémisphère gauche. J'avais été conduit à dire, d'après cela, que l'intégrité de cette circonvolution paraissait indispensable à l'exercice de la faculté du langage articulé ; mais je m'étais hâté d'ajouter que deux faits étaient peu de chose, lorsqu'il s'agissait d'une des questions les plus obscures et les plus controversées de la physiologie cérébrale; c'était aux faits ultérieurs à résoudre cette question, et à montrer si le rapport des symptômes et des lésions avait été chez mes deux malades le résultat d'une pure coïncidence, ou d'une relation de cause à effet.

Depuis lors, un assez grand nombre d'observations d'aphémie ont été recueillies, et complétées par l'autopsie, à laquelle j'ai le plus souvent assisté. Il y en a aujourd'hui une quinzaine. Dans tous ces cas, à l'exception d'un seul, sur lequel je vais revenir, et qui s'est présenté tout récemment à la Salpêtrière dans le service de M. Charcot, on a trouvé une lésion cérébrale plus ou moins étendue, mais atteignant toujours très profondément le tiers postérieur de la troisième circonvolution frontale visà-vis de l'insula. Cette série remarquable vient à l'appui de mon hypothèse sur le siège de la faculté du langage articulé. Mais ce qui m'a le plus frappé, c'est que, dans tous ces cas, la lésion occupait l'hémisphère gauche du cerveau. C'était aussi du côté gauche qu'existait la lésion dans le cas exceptionnel de M. Charcot. La troisième circonvolution frontale était à peu près saine ; mais le lobe pariétal gauche était profondément altéré, ainsi que le fond de la scissure de Sylvius du même côté. Enfin j'ai observé plusieurs aphémiques vivants, plusieurs confrères m'ont communiqué des observations analogues; la plupart de ces malades, dont l'autopsie n'a pas été faite, sont hémiplégiques, et 
ils le sont dı côté droit, d'où il est permis de conclure que leur lésion cérébrale existe du côté gauche. 'Toutes ces observations, avec ou sans autopsie, constituent au moins vingt-cinq faits d'aphémie coïncidant avec des lésions de l'hémisphère gauche du cerveau, sans que l'on ait pu trouver jusqu'ici un seul cas d'aphémie coïncidant avec une lésion de l'hémisphère droit.

C'est là ce qu'il y a de plus grave au point de vue physiologique. Que les diverses facultés cérébrales aient ou non des sièges distincts dans telle ou telle circonvolution, c'est une question extrêmement importante, sans aucun doute. Mais s'il était démontré qu'une faculté particulière, et parfaitement déterminée, comme la faculté du langage articulé, ne peut être altérée que par les lésions de l'hémisphère gauche, il faudrait en conclure que les deux moitiés de l'encéphale n'ont pas les mêmes attributions, et ce serait toute une révolution dans la physiologie des centres nerveux. J'avoue que je ne me résoudrai pas facilement à accepter une conséquence aussi subversive. J'ai donc cru devoir, dans une publication récente, poser des réserves expresses; j'ai demandé une contre-épreuve; après a voir signalé l'étrange prédilection des lésions de l'aphémie pour l'hémisphère gauche, j'ai ajouté " qu'avant d'accepter les conséquences qui pourraient en découler, il faudrait prouver, par des observations suivies d'autopsies, que les lésions du tiers postérieur de la troisième circonvolution frontale droite ne portent pas atteinte à la faculté du langage articulé ). (Exposédes titres et travaux scientifiques de 11 . Broca, Paris, avril 1862 , in $-4^{\circ}$, p. 67 , et dans ce volume, p. 61.)

L'observation de M. Parrot est un de ces faits que je demandais; mais elle n'est pas favorable à l'espérance que j'avais conçue. Je demandais un cas où l'aphémie fût la conséquence d'une lésion de la troisième circonvolution frontale droite. M. Parrot a trouvé cette lésion, qui, si elle eût été située du. côté gauche, aurait, selon toute probabilité, produit l'aphémie. Voilà donc le commencement de la contre-épreuve que j’attendais. Or le malade n'était pas aphémique. Ce fait ne prouve donc rien eu égard à la localisation de la faculté du langage dans la troisième circonvolution frontale gauche, et si les observations ultérieures continuaient à établir d'une part que certaines lé- 
sions de l'hémisphère gauche sont accompagnées d'aphémie, et que les mêmes lésions ne produisent pas l'aphémie lorsqu'elles sont situées à droite, il faudrait bien reconnaitre que la faculté du langage articulé est localisée dans l'hémisphère gauche.

Quoi qu'il en soit, le fait de M. Parrot ne pourra être mis en contradiction avec mon hypothèse, que lorsqu'il existera des observations d'aphémie produite par des lésions de l'hémisphère droit.

Jusqu'ici, à ma connaissance, cette hypothèse n'est en contradiction qu'avec un seul fait, celui de M. Charcot. Dans plusieurs autres cas, qui, au premier examen des pièces anatomiques, avaient paru contradictoires, une étude plus complète a démontré que la troisième circonvolution frontale, quoique ayant conservé un aspect à peu près normal, était profondément altérée dans son tissu. Mais dans la dernière observation de M. Charcot, cette circonvolution pouvait être considérée comme saine. Les minimes lésions microscopiques que M. Cornil y a trouvées, et que j'ai constatées après lui, ne m'ont pas paru de nature à en abolir les fonctions. J'accepte donc comme parfaitement exact, que la lésion ordinairè de l'aphémie n'existait pas dans ce cas. Si quelque doute pouvait être élevé, ce serait seulement sur le diagnostic de l'aphémie. Je n'ai pas vu la malade, je n'en puis parler par moi-même; je me hâte d'ajouter que M. Charcot est un des observateurs les plus compétents, puisqu'il a eu l'occasion d'étudier depuis un an un assez bon nombre de cas d'aphémie.

Mais je ne dois pas cacher que le diagnostic de cette affection est souvent entouré de beaucoup de difficultés. D'une part, en effet, des causes très diverses, et souvent réunies chez le même individu, peuvent par des mécanismes très différents abolir la parole. J'ai été souvent appelé cette année, soit par M. Charcot, soit par d'autres collègues, à donner mon avis sur des maladés qui ne parlaient pas ou qui ne marmottaient que quelques mots, et j'ai déclaré plusieurs fois que ces malades n'étaient pas aphémiques ou que le cas était trop complexe pour se prêter à un diagnostic certain. D'une autre part, une aphémie incomplète, existant chez un sujet dont l'intelligence est affaiblie ou pervertie, peut échapper à l'attention de l'observateur. Cela m'est 
arrivé il y a quelque temps dans un cas que je vous demande la permission de rapporter. Une vieille femme de quatre-vingt-un ans, nommée Anne Perchaud, gâteuse, paralytique et imbécile, fut portée à l'infirmerie de la Salpêtrière pour être traitée d'une fracture de l'extrémité inférieure du fémur, qu'elle s'était faite en tombant de son lit. Je voulus lui demander comment s'était produite la fracture, elle ne me répondit que par des cris, des gémissements, des mots confus et tout à fait inintelligibles. J'attribuai l'incohérence et l'insuffisance de ses réponses à la démence sénile, qui était d'ailleurs évidente. J'appliquais chaque matin un appareil qu'elle défaisait dans la journée; ses cris et ses plaintes troublaient toute la salle. J'ai continuellement sous les yeux des cas du même genre. Il ne me vint pas à l'idée que cette femme fût aphémique et je ne l'interrogeai pas en vue de ce diagnostic. Elle mourut au bout de sept ou huit jours. A l'autopsie, qui fut faite le 16 mai dernier, nous trouvâmes des lésions dans les deux hémisphères. Plusieurs petits foyers de ramollissement superficiels kystiforme existaient sur le lobe pariétal de l'hémis hère droit. Leurs deux lobes occipitaux étaient ramollis dans toute leur épaisseur. Enfin, les trois circonvolutions frontales de l'hémisphère gauche étaient détruites dans leurs deux cinquièmes postérieurs et remplacées par un kyste rempli de sérosité. Cette lésion était celle de l'aphémie, et mon interne, M. Dard, me rappela aussitôt que la malade n'avait pas répondu à nos questions. Le lendemain nous interrogeâmes les surveillantes et les gens de service. Puis nous allâmes faire une petite enquête dans la division des infirmes où notre vieille femme séjournait avant d'entrer à l'infirmerie. Voici ce que nous apprîmes. Elle ne parlait presque jamais d'elle-même, si ce n'était pour répéter de temps en temps un grand nombre de fois de suite: " J'aime mieux mourir ! j'aime mieux mourir! " Elle restait quelquefois plusieurs jours sans parler. Ses enfants, pleins d'attentions pour elle, venaient la voir très fréquemment. Elle les reconnaissait fort bien, mais ne leur répondait que des mots sans suite. Une chose qui avait frappé ses voisines, c'est qu'elle ne prononçait jamais les noms propres, et qu'elle ne pouvait pas même dire le nom de sa fille. Ces renseignements, qu'on me donna avant que je l'eusse demandé, me furent con- 
firmés par la surveillante et les infirmières. Cette vieille femme était donc aphémique, et c'est par la connaissance de la lésion que nous a vons pu remonter au diagnostic de la maladie. Mais elle n'était pas complètement aphémique, puisqu'elle balbutiait un assez grand nombre de mots, et cependant la troisième circonvolution frontale était entièrement détruite dans sa moitié postérieure. A cette occasion je ferai une remarque qui résulte de la comparaison de toutes mes observations d'aphémie, c'est qu'il n'y a pas un rapport constant entre l'intensité de l'aphémie et l'étendue de la lésion de la troisième circonvolution frontale. J'ai vu un cas d'aphémie presque complète, où le malade (nommé Lelong) ne prononçait plus que quatre mots, et où la troisième circonvolution cérébrale, détruite seulement dans une étendue de 15 millimètres, était saine partout ailleurs comme le reste du cerveau (voir Bulletin de la Société anatomique, 1862, p. 405 , et dans ce volume, p. 33). D'un autre côté, la vieille femme dont je viens de parler, était beaucoup moins aphémique, quoique la lésion chez elle occupât une étendue bien plus considérable.

Somme toute, en faisant seulement quelques réserves sur le diagnostic, j'accepte le dernier fait de M. Charcot comme étant en opposition avec mon hypothèse sur le siège de la faculté du langage articulé. Dans ce cas, la circonvolution pariétale inférieure ou externe, qui constitue le bord supérieur de la scissure de Sylvius, était entièrement désorganisée. J'ai pu me demander si le siège de la faculté du langage articulé, au lieu d'être localisé exclusivement dans la partie postérieure de la troisième circonvolution frontale, ne s'étendrait pas aussi à la circonvolution pariétale externe qui se continue directement avec elle. On sait que pour plusieurs anatomistes ces deux circonvolutions n'en font qu'une, désignée sous le nom de : circonvolution d'enceinte de la scissure de Sylvius. Et si cette manière de voir était exacte, on concevrait qu'une lésion de la partie postérieure de la circonvolution d'enceinte pût produire l'aphémie, alors même que la partie antérieure de cette circonvolution, celle qui fait partie du lobe frontal, serait à peu près intacte. Mais tout cela est encore trop hypothétique, et il faut attendre les faits ultérieurs. N'oublions pas, en effet, que, dans les quinze autres 
observations d'aphémie, la lésion a occupé constamment le même siège sur la troisième circonvolution frontale. Un fait négatif ne détruit pas cette série de faits positifs; en pathologie, et surtout en pathologie cérébrale, il n'y a guère de règle sans exception. La loi la plus générale de la pathologie cérébrale est certainement celle des paralysies croisées; elle souffre cependant quelques exceptions, qui ont été invoquées contre la théorie de l'entre-croisement, mais qui n'ont pas empêché cette théorie de se maintenir et de prévaloir dans la science. Si l'on me disait qu'à l'avenir les observatious d'aphémie fourniraient seulement un fait négatif pour douze ou quinze faits positifs, je n'en demanderais pas davantage pour considérer comme suffisamment démontrée mon hypothèse sur la localisation de la faculté du langage articulé.

Quant à ces faits négatifs, tant qu'ils resteront à l'état d'exception, on ne devra les accepter qu'après les avoir soumis à une critique sérieuse, et ils ne seront valables que s'ils sont accompagnés de détails précis, complets, propres à établir le diagnostic d'une manière rigoureuse. On a le droit de se montrer aujourd'hui plus exigeant pour ces faits négatifs que pour les faits positifs, parce que ceux-ci trouvent un appui dans les observations antérieures, tandis que les autres se trouvent au contraire en opposition arec elles. Ce n'est pas avoir deux poids et deux mesures, car c'est ainsi qu'on a procédé constamment dans l'histoire des sciences d'observations. Lorsqu'une idée nouvelle se manifeste, elle est presque toujours plus ou moins en contradiction avec des idées antérieures, et elle n'aurait droit à aucune attention si celui qui l'émet n'apportait que des faits incomplets et sommaires, sur lesquels le contrôle de la critique ne pourrait s'exercer, c'est ce qui vous explique la longueur des deux observations d'aphémie que je vous ai communiquées il y a deux ans. Telle idée succombe dans cette période initiale. Telle autre survit à ce premier contrôle : elle prend une consistance croissante à mesure que de nouveaux faits viennent la confirmer, et il arrive ainsi un moment où elle est entourée de grandes probabilités : mon hypothèse sur l'aphémie en est aujourd'hui là. Pour que cas probabilités se changent en certitude, il faudra sans doute qu'elles trouvent un nouvel appui dans 
les observations ultérieures; mais les premiers faits qu'on lui opposera, ayant contre eux les probabilités, devront, pour être valables, ne donner prise à aucune objection. Par exemple il ne suffira pas de dire que l'on a diagnostiqué une aphémie ; il faudra fournir à l'appui de ce diagnostic des preuves parfaitement claires. Il faudra établir que si le malade ne parlait pas, ce n'était ni faute d'intelligence, ni faute d'innervation musculaire. Il faudra dire de quelle manière on s'est assuré qu'il avait des idées à exprimer, qu'il avait conservé assez d'intelligence pour comprendre le langage d'autrui, et pour y répondre, tant bien que mal, par un procédé vocal, graphique ou mimique. Mais cette partie du diagnostic est quelquefois entourée des plus grandes difficultés; elle est quelquefois tout à fait impossible, et c'est pour cela qu'une observation d'aphémie, pour être concluante, doit être accompagnée de détails nombreux et précis. Je me suis imposé ces exigences lorsque j’ai apporté mes premières observations, et $j$ 'ai bien le droit de me montrer aussi difficile pour les faits contradictoires que l'on pourra m'opposer.

\section{DISCUSSION.}

M. LABorde. Il est spécieux de considérer le fait de Parrot comme confirmatif de la localisation. On ne saurait admettre la localisation à gauche seulement. Puis Laborde revient sur les troubles de l'écriture comme prouvant la participation de l'intelligence. Et souvent, quand l'intelligence est conservée, il faut tenir compte des troubles des mouvements de la langue.

M. Broca. - Je crois avoir répondu, dans mon premier mémoire sur le siège de la faculté du langage (Bull. de 1861, p. 330 , et dans ce volume, p. 4), à quelques-unes des questions que pose aujourd'hui M. Laborde.

Notre collègue donne trop d'extension au mot aphémie; aussi je tiens à bien établir le sens que j'attache à cette expression. J'entends par aphémie un état dans lequel le malade ne peut parler, bien qu'il ait plus d'intelligence qu'il n'en faut pour parler et bien que, d'autre part, les organes de la phonation et de l'articulation soient en état de fonctionner. On observe l'embarras, la diminution ou l'abolition de la parole 
chez des individus atteints d'idiotie, de démence, d'imbécillité, ou démence sénile, de ramollissement cérébral, de paralysie générale, d'apoplexie, etc. Ces individus ne sont pas aphémiques pour cela. Le langage articulé est soumis à trois conditions distinctes; il dépend de trois fonctions ou plutôt de trois groupes de fonctions inégales en dignité. Pour parler, il faut concevoir une idée, c'est la faculté d'ordre supérieur; il faut, en second lieu, établir un rapport entre ces idées et les signes de convention qui constituent les formes verbales du lanzage ; c'est une faculté fort élerée encore, puisqu'elle est particulière à l'homme, mais bien inférieure à la précédente, puisqu'on voit tous les jours des individus très peu intelligents qui parlent encore très aisément et très distinctement. Enfin, l'exercice de la parole met en jeu un troisième groupe de fonctions qui ne sont pas de l'ordre intellectuel : lorsque l'idée est conçue, lorsque la forme verbale est trourée, il faut que les muscles de la phonation et de l'articulation puissent obéir à la volonté. Le langage peut donc être altéré ou aboli par trois ordres de causes très différentes, portant atteinte soit à la pensée elle-même, soit à la faculté spécialẹ de coordination des mots, soit enfin à la mécanique de l'articulation des sons. L'individu qui, faute d'idées, ne parle pas ou ne prononce que des mots sans suite, n'est pas plus aphémique que celui qui a seulement la langue paralysée. Mais celui qui, n'ayant perdu ni l'intelligence ni les mouvements de la langue, des lèvres et du voile du palais, ne peut réussir à rendre ses idées par la parole, celui-là est privé d'une faculté particulière, qui, dans la hiérarchie fonctionnelle, est subordonnée à la pensée, tandis qu'elle tient sous sa dépendance la fonction mécanique de l'articulation des sons, et c'est la perte ou l'altération de cette faculté qui constitue l'aphémie.

En théorie, rien n'est plus net et plus simple que la distinction de l'aphémie, et, dans la pratique, on troure des cas où cette distinction est tout à fait évidente. Il y a des malades qui, n'ayant aucune paralysie, ayant conservé toute la plénitude de leur intelligence, peuvent exprimer par écrit, sans la moindre hésitation, les idées les plus compliquées et qui ont cependant perdu la faculté de s'exprimer par le langage articulé; ceux-là sont aphémiques, sans que la nature de leur affection puiše donner lieu à la 
moindre incertitude. Ces exemples prouvent qu'on peutêtre rendu aphémique par des lésions cérébrales peu profondes et relativement peu importantes. Mais une lésion plus grave, plus étendue, peut occuper un grand nombre de circonvolutions, envahir le corps strié, la couche optique, et porter atteinte à la fois à l'intelligence, à la faculté du langage et à l'articulation. Ces cas sont nombreux et donnent lieu à de grandes difficultés de diagnostic. On arrive le plus souvent, sans trop de peine, à s'assurer que l'altération de la parole n'est pas due à la paralysie des muscles de la phonation et de l'articulation. Mais, lor'squ'il s'agit de savoir si elle dépend ou non de l'altération de l'intelligence, la question se complique singulièrement. Le diagnostic de l'aphémie ne peut être établi que s'il est dûment constaté que le malade possède encore une quantité d'intelligence égale ou supérieure à celle qui est nécessaire pour parler. Mais l'intelligence ne se mesure pas et, s'il est déjà très difficile d'apprécier celle d'un homme qui parle, il l'est bien plus encore d'apprécier celle d'un homme qui n'a, pour exprimer ses idées, que des moyens tout à fait défectueux. On peut admettre qu'un individu privé de la parole est assez intelligent"pour parler, lorsqu'il comprend les questions qu'on lui adresse et qu'il trouve le moyen d'y répondre par des signes, des gestes, des attitudes, ou par l'emploi judicieux des quelques sons articulés qui constituent tout son vocabulaire. Lorsqu'on a acquis la preuve qu'il comprend le langage d'autrui, on peut en conclure qu'il est aphémique, c'est-à-dire que ce qui lui manque pour parler, c'est la faculté spéciale du langage articulé. Or, dans beaucoup de cas, on n'acquiert cette preuve qu'après de longs tâtonnements et, quelquefois, malgré toutes les investigations, on reste dans l'incertitude. Il n'en serait pas ainsi si le malade avait conservé toute son intelligence, tous ses sens, tous ses mouvements. Il trouverait toujours le moyen de manifester ses principales idées, soit par l'écriture, soit par la mimique. Mais nous considérons le cas où son intelligence est très affaiblie ; où l'une des moitiés du corps, et c'est toujours la moitié droite, est paralysée. C'est la main, et la main droite spécialement, qui est, après la parole, le principal moyen de communication. Un hémiplégique ordinaire, privé de l'usage de la main droite, s'exerce de la main gauche et 
finit par apprendre à s'en servir adroitement, même pour écrire. Mais il n'en est plus de même si la cause, qui a produit sa paralysie a en même temps dégradé son intelligence: les aphémiques dont nous parlons sont dans ce dernier cas.

Il y a autre chose encore de plus embarrassant. Le langage articulé n'est qu'une espèce de langage ; il y a plusieurs autres langages, les uns spontanés, les autres de convention. Les animaux, quoique privés de la parole, se transmettent leurs idées rudimentaires par des signes que nous ne comprenons pas, mais qui sont une espèce de langage. Tous les langages reposent sur un fonds commun, qui est la relation établie entre une idée et un signe verbal, vocal, graphique ou mimique. Qu'ils dépendent de plusieurs facultés distinctes, c'est ce que la pathologie démontre d'une manière irrécusable, puisque l'un d'eux, le langage articulé, peut périr isolément; mais que ces facultés soient très voisines les unes des autres, soit dans l'ordre psychique, soit dans l'ordre anatomique, c'est ce qui paraît infiniment probable ; et voilà pourquoi les lésions qui altèrent ou abolissent le langage articulé portent fréquemment atteinte aux autres langages. Ainsi beaucoup d'aphémiques, quoique n'étant ni aveugles ni paralysés, ne savent plus ni lire ni écrire. Quelques-uns peuvent encore lire, mais ne peuvent plus assembler les signes de l'écriture. $0 \mathrm{n}$ peut, avec de la persévérance, leur apprendre à lire un certain nombre de mots, comme à les écrire, et même à les prononcer. Mais le procédé ordinaire de la décomposition des mots en syllabes et en lettres n'est plus à leur portée. Ils apprennent à reconnaître un mot à sa forme, à sa physionomie, comme ils reconnaissent une montre, un visage, sans se rendre compte des éléments qui les composent. J'ai pu étudier ces curieux phénomènes sur un aphémique de Bicêtre, que j'ai gardé tout un an à l'infirmerie et dont nous avons tant bien que mal refait l'éducation. Or, les aphémiques, qui ont perdu à la fois le langage articulé et le langage écrit, peuvent perdre aussi le langage du geste, celui de l'expression, et ces complications peuvent les mettre hors d'état de se faire comprendre. On pourra croire qu'ils ne comprennent pas les questions, qu'ils n'ontippas d'idées à exprimer, et l'aphémie pourra être méconnue.

On voit combien de causes concourent à rendre difficile le 
diagnostic de l'aphémie. Il faut donc multiplier les questions, interroger le malade de mille manières en lui demandant d'abord les choses les plus simples, celles auxquelles il peut répondre intelligemment par les gestes de la langue universelle. Il faut insister tout particulièrement sur les notions qui le concernent directement et qu'il ne pourrait oublier sans oublier toutes les autres ; et, dans les cas les plus graves, il faut tourner les questions de manière qu'il puisse y répondre par oui ou par non, ou par les gestes équivalents. Enfin, je recommande surtout les questions auxquelles on peut répondre par un nombre. La notion des nombres survit quelquefois chez des aphémiques dont l'intelligence est profondément dégradée. J'en ai connu plusieur's qui pouvaient compter l'heure sur leurs doigts et qui ne se trompaient jamais sur les nombres plus petits que dix. J'en ai connu un autre - mais celui-là était très intelligent pour un aphémique - qui savait compter par unités du second et du premier ordre et qui, pour 84 , levait d'abord 8 doigts, puis 4 doigts.

On s'est demandé si les aphémiques avaient perdu une faculté spéciale, ou s'ils n'avaient pas simplement perdu la mémoire des mots. Cete opinion a été émise surtout par ceux qui, en admettant l'unité de l'intelligence, répugnent à accepter l'existence des facultés isolables et indépendantes les unes des autres. Mais je ferai remarquer d'abord que les aphémiques n'ont pas perdu la mémoire des mots, puisqu'ils comprennent ce qu'on leur dit. Beaucoup d'entre eux font de vains efforts pour répéter un simple monosyllabe qu'on leur demande avec insistance en le répétant soi-même un grand nombre de fois - tandis qu'ils retrouvent très bien chaque matin à leur réveil les quelques mots qui composent leur petit vocabulaire. Je ferai remarquer ensuite que, si la mémoire des mots pouvait périr isolément, ce serait une faculté parfaitement distincte de toutes les autres ; l'aphémie dès lors ne serait plus la perte de la mémoire en général, mais d'une mémoire particulière, indépendante des autres espèces de mémoires et des autres espèces de facultés; et ce point de vue se confondrait en une même doctrine avec celui que j'ai adopté.

Pour ma part, je ne considère pas la mémoire comme une faculté simple, ni même comme une faculté complexe, ınais 
comme un état, ou si l'on veut comme une qualité propre à chacune de nos facultés, et inégalement développée dans chacune d'elles. Ghaque faculté a sa mémoire, qui est plus ou moins parfaite et qui n'est nullement solidaire des autres mémoires. Je ne sais s'il y a des cerveaux assez bien équilibrés pour se sollvenir également bien de toutes choses. J'en doute fort, et je dois déclarer que je n'en connais pas. Tel qui, après avoir entendu une seule fois la partition d'un opéra, peut la chanter d'un bout à l'autre, sans se tromper d'une note, sera incapable d'apprendre par cœur dix lignes de prose. Tel autre, qui n'a jamais pu retenir une date, ni une formule, pourra réciter, mot par mot, un demi-volume après l'avoir lu une seule fois. Un peintre, doué de ce que, dans le langage ordinaire, on appelle la mémoire de l'œil, pourra faire de souvenir le portrait d'un personnage qui aura simplement causé avec lui pendant quelques heures, et qui, le rencontrant le lendemain, ne reconnaîtra même pas son interlocuteur de la veille. Lorsqu'on dit d'un homme qu'il a une bonne mémoire, on entend par là qu'il se souvient longtemps d'un grand nombre de faits. Mais la mémoire des faits peut être très développée chez des individus qui n'ont aucune aptitude à apprendre par cœur, et qui n'ont jamais pu réussir à réciter cinquante vers de suite. Par la rime et par la cadence, la poésie participe de la musique; aussi voit-on des personnes qui, étant douées de la mémoire musicale, apprennent très aisément les vers, et qui ne peuvent retenir la prose. Il y a une mémoire très distincte des autres, c'est celle des localités; on pourrait être tenté de la prendre pour un instinct spécial. Ceux qui la possèdent peuvent reconnâ̂tre au bout de trente ans un sentier qu'ils n'ont parcouru qu'une fois, une pierre sur laquelle ils se sont assis, des objets insignifiants auxquels ils n'ont accordé en passant aucune attention. Je connais un médecin qui a beaucoup cultivé la botanique, et qui oublie promptement les noms spécifiques, mais qui, voyant une plante dont le nom lui échappe, peut indiquer immédiatement tous les endroits où il l'a rencontrée; et c'est en se souvenant du lieu où il l'a vue pour la première fois qu'il arrive, après un moment de réflexion, à retrouver le nom de l'espèce. Notez qu'il connaît fort bien les caractères distinctifs des espèces. Ce sont 
les noms seuls qu'il oublie, tandis que d'autres botanistes, doués de la mémoire des noms, se souviennent immédiatement du nom d'une espèce quelconque, mais seraient souvent fort embarrassés de dire en quoi elle diffère des autres. Cette mémoire des noms est une des plus singulières, une des plus inégalement réparties; elle est compatible avec l'intelligence la plus obtuse, elle existe même à un degré remarquable chez quelques idiots, et elle fait lacune dans des esprits éminents. Il y a, par conséquent, beaucoup d'espèces de mémoires indépendantes les unes des autres, et les procédés miémoniques ont précisément pour but de suppléer aux mémoires dont on est plus ou moins dépourvu, à l'aide des mémoires dont on est plus heureusement doué.

Les phénomènes si variables que l'on observe chez les aphémiques pourraient donc à la rigueur être attribués à une altération de la mémoire des mots, accompagnée, en certains cas, d'une altération de la mémoire des signes de l'écriture ou d'une altération de la mémoire des faits. Pour expliquer comment un aphémique comprend le langage parlé, sans pouvoir cependant répéter les mots qu'il vient d'entendre, on pourrait dire qu'il a perdu, non la mémoire des mots, mais la mémoire des moyens de coordination que l'on emploie pour articuler les mots. Mais cette analyse serait un peu subtile. Il est bien plus simple de se horner à constater que la faculté du langage articulé est altérée, sans chercher si l'altération porte sur cette faculté tout entière, ou seulement sur l'espèce particulière de mémoire qui en fait partie intégrante. 


\section{RECHERCHES SUR LES FONCTIONS CÉRÉBRALES ${ }^{1}$}

(Exposé des titres et travaux scientifiques de M. Paul Broca, avril 1863.)

Sans oser encore affirmer qu'il a découvert le siège précis et circonscrit de la faculté du langage articulé, M. Broca pense du moins que la solution du problème des localisations cérébrales est bien avancée. Onze observations, recueillies coup sur coup et déposant toutes dans le même sens, laissent à peine entrevoir l'hypothèse que l'identité des résultats ait pu être due à de pures coïncidences. Si, comme tout permet de le prévoir, les observations ultérieures confirment ces résultats, la troisième circonvolution frontale, siège de la faculté la plus caractéristique de l'homme, pourra prendre le nom de circonvolution du langage, et la question, si longtemps douteuse, de la localisation des facultés sera définitivement résolue; car, dès le moment qu'il sera démontré sans réplique qu'une faculté intellectuelle réside dans un point déterminé des hémisphères, la doctrine de l'unité du centre nerveux intellectuel sera renversée, et il sera hautement probable, sinon tout à fait cerlain, que chaque circonvolution est affectée à des fonctions particulières.

L'auteur espère encore que d'autres, plus heureux que lui, trouveront enfin un exemple d'aphémie produite par une lésion de l'hémisphère droit. Jusqu'ici, c'est toujours la troisième circonvolution frontale gauche qui a été atteinte. S'il fallait admettre que les deux moitiés symétriques de l'encéphale ont des attributions différentes, ce serait une véritable subversion de nos connaissances en physiologie. L'auteur, qui a le premier signalé

(1) Ce résumé fait par Broca lui-même du résultat de ses rechershes à la date de 1863 , sera rapproché avec intérêt de celui qu'il a donné cinq ans plus tard (voir plus loin, année 1868). On peut y mesurer l'espace parcouru et se rendre compte de l'extrême prudence scientifique des affirmations. 
à la Société de biologie, dans la séance du 17 janvier 1863, l'étrange prédilection des lésions de l'aphémie pour l'hémisphère gauche, pense donc qu'avant d'accepter les conséquences qui pourraient en découler, il faudrait prouver, par des observations suivies d'autopsie, que les lésions du tiers postérieur de la troisième circonvolution frontale droite ne portent pas atteinte à la faculté du langage articulé. 


\title{
APHÉMIE, APHASIE ET APHRASIE
}

\author{
LETTRE A M. LE PROFESSEUR TROUSSEAU
}

(Gazette des hôpitaux, 23 janvier 1\$64.)

\section{Mon cher Maître,}

En apprenant, il y a quelques jours, que vous vous proposiez de faire à l'Hôtel-Dieu une série de leçons sur l'affection que j’ai désignée sous le nom d'aphémie, j'ai vivement regretté d'être privé du plaisir d'aller vous entendre. Si mon service d'hôpital ne m'en avait pas empêché, j'aurais été un de vos auditeurs les plus assidus. Je me dédommage de cette privation en lisant vos leçons dans la Gazette des hôpitaux, et j'ai trouvé dans votre première leçon une sorte de préambule philologique à l'occasion duquel je vous demande la permission de vous présenter quelques observations.

Lorsque j’ai éprouvé le besoin de chercher un nom particulier pour désigner l'une des affections qui privent l'homme de l'usage de la parole, j'ai dû me résigner à faire un mot nouveau, et, afin d'innover le moins possible, j'ai d'abord essayé de donner une terminaison française à l'un des substantifs que les Grecs employaient pour exprimer la perte ou l'absence de la parole. Mais ils n'ont pu me servir, parce qu'ils ont des significations précises dont on ne peut les détourner. Le mot aphonie est employé pour désigner la perte de la voix; il implique l'idée d'une affection des organes vocaux. Le mot alalie signitie mutisme, et plus spécialement surdi-mutité. Le mot aphasie, enfin, exprime l'état d'un individu qui s'exprime comme tout le monde, mais que la timidité ou la confusion empêchent momentanément de parler. Rien de tout cela ne pouvait me convenir. 
Il fallait donc faire un mot avec l' $\alpha$ privatif et l'un des trois

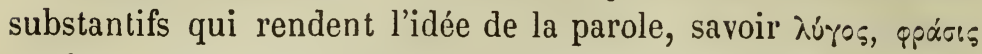

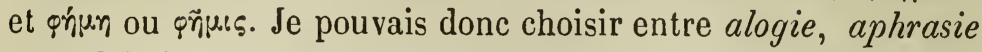
et aphémie. Pour me diriger dans mon choix, je me dis qu'il ne suffisait pas de mettre du grec dans un mot, qu'il fallait encore que ce mot fût immédiatement compréhensible, et que tout lecteur pût au premier coup d'œil, sans erreur et sans confusion possible, découvrir aussitôt le véritable sens. Certains mots grecs, en passant dans la langue française, ont reçu une acception particulière, désormais consacrée par un usage qui

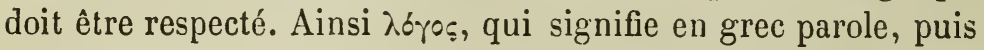
discours, raisonnement, etc., est employé dans les langues modernes pour rendre l'idée de logique ou l'idée de science. Le mot alogie aurait fait naître inévitablement l'idée d'absurdité; je ne m'y arrêterai pas.

Restaient deux autres mots, aphrasie et aphémie, tous deux également faits pour être compris immédiatement par tout le monde. Personne n'ignore en effet que ce n'est qu'une phrase, et il n'est pas un seul bachelier qui ne sache que qnui veut dire je parle.

Le mot aphrasie me séduisait d'autant plus qu'il caractérisait parfaitement, et qu'il caractérisait seul rigoureusement la maladie que je voulais désigner. Le verbe $q$ pár. ne veut pas dire seulement je parle, mais je parle clairement. De là est venu en grec, en latin, en français, et dans presque toutes les langues modernes, le mot phrase, qui signifie une série de mots formant un sens complet. Or, ce qui manque aux aphémiques (souffrez que provisoirement du moins je leur donne encore ce nom), ce n'est pas la faculté de prononcer un certain nombre de mots. Presque tous, vous le savez, ont un petit vocabulaire, mais ils ont perdu la faculté de combiner leurs mots pour construire de véritables phrases. Aphrasie me convenait donc mieux que tout autre terme, et je l'avais même adopté dans la rédaction de mon premier manuscrit. Ce qui m'y fit renoncer, au moment de l'impression, ce fut le désir de m'écarter le moins possible des mots usités dans la langue grecque - je parle du grec ancien et non du grec moderne, qui n'a pas voix au chapitre. - Le mot ä.pnp.os existe en grec. G'est un adjectif dont le masculin et 
le féminin sont semblables. Il a deux sens; il signifie : $1^{\circ}$ dont

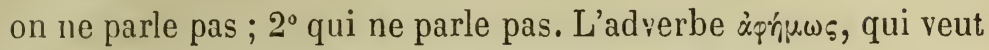
dire sans parler ou en se taisant, correspond exclusivement à ce second sens. Le mot ạ̀rn.os étant encore vierge d'application scientifique, il m'était permis de le prendre dans l'acception qu'il me convenait. Je le traduisis en français par l'adjectif aphémique, d'où le substantif aphémie se dégagea naturellement. Obligé de faire un néologisme, je l'avais fait aussi bien que possible. Je m'étais borné à donner une terminaison française à un mot de la langue grecque classique. J'ajoute que ce mot eut l'avantage d'être compris sans aucune hésitation, et la facilité avec laquelle il s'est répandu le prouve suffisamment.

Permettez-moi maintenant, cher et savant maître, d'examiner les arguments que vous invoquez contre ce nom d'aphémie. Un Grec moderne vous a dit qu'il avait été choqué de voir appliquer à d'honnêtes malades une expression déshonorante. Aphémie, pour lui, est synonyme d'infamie. Je ne le conteste pas; ce ne serait pas la première fois qu'un mot aurait changé de sens pendant l'évolution ascendante ou rétrograde d'une langue. "Acrinos voulait dire, entre autres choses, un individu dont on ne parle pas. Pour une femme, c'était sans doute un éloge; mais un homme aime qu'on parle de lui, et je conçois très bien qu'à la longue, dans un pays qui a gardé le souvenir d'hommes illustres, l'épithète àprnuos ait fini par être prise en mauvaise part. Si le mot aphémie survivait à votre critique, et s'il produisait chez les Athéniens modernes quelque étonnement, ils en seraient quittes pour faire un petit retour sur leurs étymologies, et ce ne serait certes pas un grand malheur.

Une autre objection a été dirigée contre le mot aphémie par un de vos auditeurs qui connaît parfaitement la règle de l'esprit rude, et qui, en appliquant cette règle à la dissection de ce mot,

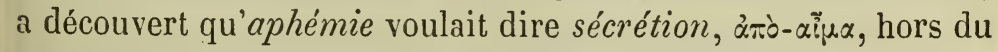
sang. Cela prouve que le grec, comme le français, peut prêter au calembour, pour peu qu'on ait non pas l'esprit rude, mais l'esprit gaulois. Je pourrais aisément soumettre à de semblables dissections et donner des interprétations multiples à un grand nombre de termes scientifiques dérivés du grec. Je m'en garderai bien; je me bornerai à vous rappeler qu'il est entré dans 
nos usages d'éliminer l'h de la terminaison hémie lorsque la lettre précédente est une consonne. Ainsi on peut dire leucohémie; mais lorsqu'on a voulu élider l'o pour abréger le mot, on a écrit leucémie et non leuchémie; de même on écrit hypohémie ou hypémie et non hyphémie; hydrohémie ou hydrémie et non hydrhémie; de sorte que si l'on éprouve jamais le besoin de désigner la sécrétion sous un nom grec, on pourra dire apohémie ou apémie, mais non aphémie.

Votre dernière objection me touche beaucoup plus, car elle émane d'un de nos hellénistes les plus éminents, de M. Littré, qui, comme vous le dites, "sait le grec aussi bien que le français », et j'ajoute que ce n'est pas peu dire. M. Littré se base sur une loi de dérivation en vertu de laquelle les mots composés qui renferment un verbe doivent être faits avec la racine de ce verbe et non avec la forme particulière au présent de l'indicatif. Aphémie, venant de a privatif et de çnú, je parle, serait donc un mauvais mot.

Ici, mon cher maître, je crains que M. Littré, consulté dans une simple conversation, n'ait pas eu le temps de rassembler tous ses souvenirs. S'il avait songé qu'il y a un substantif

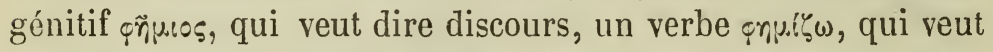

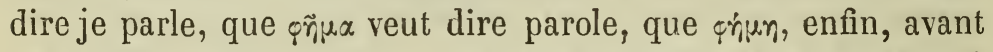
de signifier renommée ou oracle, signifiait mot et parole, il aurait, je n'en doute pas, reconnu que ces mots, qui expriment la même idée et qui commencent par ẹnu, pouvaient aussi bien que gnuí servir à composer le mot aphémie; si surtout il avait considéré que le mot àộn.os est un adjectif gr'ec, que l'adjeclif aşno.(u)v, synonyme du précédent, se trouve dans les anciens

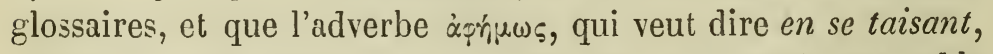
est usité par les poètes, il se serait gardé de déclarer inacceptable un mot qui n'est pas de mon cru, et qui a été composé par les vrais Grecs il y a plusieurs milliers d'années. J'ose donc, sur ce point, en appeler de M. Littré à M. Littré lui-même. Et ce qui me donne cette confiance, c'est que M. Renan, mon savant collègue de la Société d'anthropologie, et le collègue de M. Littré à l'Institut, a bien voulu me dire que le mot aphémie était à ses yeux parfaitement correct.

Ainsi, mon cher maître, nous pourons, sans craindre de passer 
pour des béotiens, désigner comme par le passé sous le nom d'aphémie la maladie qui est actuellement le sujet de vos intéressantes leçons. Nais nous restons libres de choisir un autre nom, si nous trouvons que cela soit réellement utile pour la science. Je vous ferai seulement remarquer que la langue scientifique est déjà bien assez chargée, et que, pour changer un nom qui est déjà en circulation, il faut invoquer des motifs tout à fait péremptoires. Si un mot, par exemple, rend d'une manière très inexacte l'idée qu'on veut exprimer, ou s'il est complice d'une théorie fausse, l'avantage qu'on trouve à le remplacer par un mot plus exact l'emporte sur l'inconvénient de compliquer la synonymie, et on se décide alors à faire un néologisme. Examinons donc à ce point de vue le nouveau nom d'aphasie, proposé par M. Littré.

Mais auparavant, permettez-moi de vous faire remarquer que les mots sont faits pour être compris, et qu'un mot nouveau doit être choisi de manière à ne faire naître aucune confusion dans l'esprit du lecteur. J'ai rejeté alogic parce que le mot $\lambda .6$ \%os, en fournissant aux langues modernes la terminaison logie, a pris une acception que tout le monde connaît, et que je ne devais pas changer. Aphasie, sous ce rapport, est tout aussi défectueux. Il ne dérive pas de pásı, qui ne se trouve pas dans les dictionnaires, mais de ọásıs, qui a un double sens, parce qu'il a une double racine. Lorsqu'il dérive du verbe poétique $\varphi^{\alpha} \omega$, je brille (racine de $\varphi \ddot{\omega} \varsigma$, lumière, et de $\alpha^{\prime}(v \omega$, je fais voir), il veut dire apparition, apparence, phase. Il signifie au contraire mot, ou parole, lorsqu'il dérive du verbe inusité $\varphi^{\alpha} \omega$, je parle, verbe fossile, qui disparut avant l'organisation de la langue grecque, mais dont les grammairiens ont retrouvé la trace dans certaines formes de la conjugaison du verbe opnuí. C'est ainsi que le substantif français son a deux acceptions différentes, suivant qu'il dérive de sonus, bruit, ou de summum, qui, dans la basse latinité, désignait l'épiderme du grain de blé. En grec, le sens le

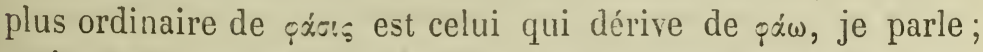
mais ce mot a passé dans le latin, et de là, sans exception, dans toutes les langues modernes de l'Europe, avec le sens dérivé de $̧ x_{x}(\omega$, je brille. Les astronomes s'en sont d'abord servis pour désigner les diverses apparences de la lune et de quelques autres 
astres ; puis les médecins, pour désigner les aspects successifs d'une maladie; puis on a dit: les phases du développement, les phases d'une discussion, les phases de l'histoire, etc.; si bien que ce mot, dans le langage ordinaire, a fini par devenir à peu près synonyme de changement. Interrogez qui vous voudrez, hors les hellénistes ; ouvrez n'importe quel vocabulaire moderne, français, allemand ou autre, et vous trouverez partout, à la définition du mot phase, l'idée de plusieurs choses diverses qui se succèdent. Les hellénistes peuvent protester; mais c'est là une situation toute faite devant laquelle il faut s'incliner. Dites à un médecin qu'il y a une chose qui s'appelle l'aphasie, il se creusera la tête sans pouvoir deviner qu'il s'agit d'une maladie, d'un symptôme, d'une lésion ou d'une fonction, et il y a bien quelque chance pour qu'il songe à l'arrêt de développement, qui est la suppression d'un certain nombre de phases embryonnaires, et qui, par parenthèse, n'a pas encore de nom grec. Certes, ce mot n'est pas fait pour embarrasser les membres de l'Académie des inscriptions et belles-lettres, qui tous probablement savent Platon par cœur; mais il sera fort obscur pour les simples mortels, habitués à donner 'au mot phase une certaine acception, qui n'a rien de commun avec l'idée de langage.

Maintenant, ce mot aphasie, qui pèche contre la clarté, caractérise-t-il du moins exactement la maladie qui nous occupe? Nullement. Vous l'avez dit vous-même, l'aphasie, àqaría, est l'état d'un homme à bout d'argument, et qui n'a rien à répondre. 0 n prétend que Platon aimait à mettre ses contradicteurs dans cet état; c'est même pour y avoir mis un jour Denys le Tyran, qu'il faillit être livré au bourreau, et qu'il fut vendu comme esclave. Par suite des progrès de la dialectique, l'aphasie est assez rare de nos jours, excepté pourtant aux examens. Ce qui manque à l'aphasique, ce n'est pas la parole, c'est l'idée. Il pourrait parler sans rien dire, il se tait; c'est une preuve du bon sens. Soufflez-lui l'idée qu'il cherche, il parlera aussitôt, et peutêtre fatiguera-t-il vos oreilles. L'aphémique, au contraire, a des idées à exprimer, mais la parole lui fait défaut. Vous savez comment l'ingénieux Desgenettes s'y prit, dans un examen, pour démontrer à ses collègues qu'un candidat aphasique n'était pas aphémique. Il lui demanda son âge, le lieu de sa naissance, la 
profession de son père; et, se retournant alors vers les deux autres juges: «Vous vous trompez, dit-il, il parle fort bien; mais comment voulez-vous qu'il vous réponde? Vous lui demandez des choses qu'il ne sait pas! "Je me trompe fort, mon cher maître, ou c'est de vous que je tiens cette histoire, et il est impossible d'établir plus nettement la distinction de l'aphasie et de l'aphémie. Vous voyez bien que le même nom ne peut s'appliquer à ces deux choses différentes; et comme il ne dépend plus de nous de changer l'acception du mot àoacía, consacré par Platon, nous devons chercher un autre mot pour désigner la perte de la faculté de la parole.

J'avais choisi aphémie; je crois avoir réfuté victorieusement les diverses objections auxquelles ce mot a donné lieu; mais il en est une que je lui fais moi-même et que je veux soumettre à votre appréciation. Pris dans un sens absolu, l'a privatif implique l'absence totale d'une chose. Le nom d'aphémie pourrait donc à la rigueur faire supposer que le malade ne prononce pas même un seul mot, et comme il y a effectivement un degré d'aphémie où le mutisme est absolu, on pourrait être tenté de croire que l'aphémie est le nom particulier de l'abolition complète de toute espèce de parole. L'usage permet, il est vrai, de ne donner à l'à privatif qu'une signification relative, de s'en servir pour exprimer simplement l'idée de diminution. Ainsi anémie ne veut pas dire qu'il ne reste plus une goutte de sang dans les vaisseaux; atrophie ne veut pas dire suppression de la nutrition; l'adynamie, l'ataxie, l'aphonie sont plus ou moins complètes, etc. Le nom d'aphémique peut donc s'appliquer de la même manière aux individus qui prononcent encore quelques mots ; mais j'ai déjà dit que le mot aphrasie, indiquant l'impossibilité de construire des phrases, caractériserait mieux que tout autre la maladie en question.

Il est aussi compréhensible, aussi clair qu'on puisse le désirer, car le radical phrase est devenu latin, français, anglais, italien, etc., en conservant partout et toujours une acception uniforme. Aphrasie est donc un mot excellent, et le petit motif pour lequel je l'ai écarté est d'un ordre tout à fait secondaire. Voyez maintenant, mon cher maître, s'il vaut la peine de le substituer au mot aphémie, qui n'est pas encore assez ancien 
pour être devenu respectable. Je m'en rapporterai pour ma part à votre décision. J'ai hésité entre ces deux mots ; ma balance a penché vers l'aphémie, mais il dépend de vous de faire descendre l'autre plateau. C'est à vous de mettre à votre tour en balance le tout petit avantage qu'il résulterait de ce changement de nom, avec la petite perturbation que cela apporterait dans le langage.

J'avais pris la plume pour ne vous écrire que quelques lignes, et voilà que $\mathrm{j}$ 'ai presque fait une dissertation. Vous me pardonnerez, je l'espère, mon cher maître, la longueur de cette lettre, et vous n'y verrez qu'une preuve de l'importance que j'attache à tout ce qui vient de vous.

Agréez, etc.

PAUL BROCiA.

18 janvier 1864. 


\section{DEUX CAS D'APHÉMIE TRAUMATIQUE}

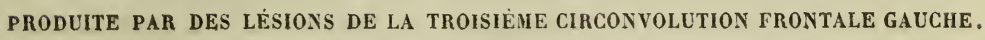

DIAGNOSTIC ChIRURGical.

(Bulletins de la Société de chirurgie, 1864, 2e série, t. V, p. 51-54.)

M. Broca. Notre collègue M. Ange Duval, professeur de clinique chirurgicale à l'Ecole de médecine navale de Brest et membre correspondant de la Société, m’a prié de vous présenter un mémoire dans lequel il a consigné deux observations de fracture du crâne compliquées de lésions cérébrales qui ont produit l'aphémie.

J'ai décrit sous ce nom d'aphémie, il y a près de trois ans, les altérations de la faculté du langage articulé, et j'ai été conduit par les résultats des autopsies à considérer la partie postérieure de la troisième circonvolution frontale comme étant le siège à peu près exclusif des lésions de l'aphémie.

Pour établir cette opinion, je n'ai pu me servir des faits antérieurs à mes recherches, parce que dans ces observations, précieuses sous le rapport des symptômes, le siège des lésions sur telle ou telle circonvolution n'était pas indiqué. Jusqu'à une époque toute récente, la plupart des anatomistes croyaient que les circonvolutions du cerveau étaient aussi désordonnées que celles de l'intestin. Cette opinion est encore assez généralement répandue, quoique des recherches déjà anciennes aient démontré la fixité de certains sillons et de certaines circonvolutions, et quoique des recherches plus récentes aient prouvé que toules les circonvolutions fondamentales sont parfaitement fixes quant à leur nombre, leurs connexions et leurs rapports. L'étage supérieur du lobe frontal, en particulier, se compose de quatre circonvolutions très distinctes, l'une postérieure et transversale, 
qui forme le bord antérieur du sillon de Rolando, les trois autres antéro-postérieures, qui commencent en avant derrière l'arcade orbitaire et qui, cheminant d'avant en arrière, vont se jeter isolément dans la circonvolution transversale. Ces trois circonvolutions antérieures sont désignées de dedans en dehors sous les noms de première, deuxième et troisième circonvolution frontale. La première longe la scissure médiane du cerveau; la troisième forme en arrière le bord supérieur de la scissure de Sylvius, et la couche de substance grise qui la tapisse, se réfléchissant dans le fond de cette scissure, se prolonge immédiatement sur le petit lobe central désigné sous le nom d'insula de Reil.

Ces rapports sont absolument fixes, mais ils sont encore peu connus, et ils étaient très généralement inconnus lorsque j'ai publié mes premières observations d'aphémie. On ne pouvait donc pas trouver, dans les faits antérieurs à cette époque, l'indication nominative des circonvolutions lésées. Les auteurs se bornent à dire que la lésion était située à la partie antérieure, moyenne ou postérieure de l'hémisphère, et, quoique leur's observations soient fort précieuses à consulter, la plupart d'entre elles ne peuvent servir à déterminer avec une précision suffisante le siège des lésions de l'aphémie.

M. Bouillaud, il est vrai, avait réuni une masse imposante de faits tendant à démontrer que la perte de la faculté du langage articulé dépendait des lésions des lobes antérieurs. Mais on lui avait opposé d'autres faits relatifs à de graves lésions de ces lobes sans perte de la parole. La lecture de ces faits prouve qu'on ne s'entendait même pas sur ce que c'est que les lobes antérieurs. La plupart des auteurs ne donnaient ce nom qu'à la partie du lobe frontal qui est située au-dessus de la voûte orbitaire et qui forme à peine la rnoitié de ce lobe. Or, c'est dans la moitié postérieure du lobe frontal, dans celle qui est au-dessus de la scissure de Sylvius, que sont situées les lésions de l'aphémie.

Il n'en faut pas davantage pour expliquer le nombre assez considérable des observations qui ont été invoquées contre les idées de M. Bouillaud. J'ajoute que la plupart de ces observations contradictoires sont relatives à des lésions du lobe frontal 
droit, tandis que les observations nombreuses recueillies depuis près de trois ans tendent à établir que les lésions de l'hémisphère gauche sont seules capables de produire l'aphémie. Cette proposition est étrange sans doute, mais, quelque embarrassante qu'elle soit pour la physiologie, il faudrait bien l'admettre, si les faits ultérieurs continuaient à déposer dans le même sens que le premier.

On a recueilli depuis environ trois ans plus de vingt observations d'aphémie suivies d'autopsies. Dans tous ces cas, sans exception, la lésion était située sur l'hémisphère gauche, et dans tous ces cas, à une seule exception près, le tiers postérieur de la troisième circonvolution frontale était profondément altéré. Ce fait exceptionnel, jusqu'ici unique, a été recueilli par II. Charcot. La troisième circonvolution frontale gauche était à peu près saine, mais la circonvolution pariétale externe, avec laquelle elle se continue sans interruption et qui forme la moitié postérieure du bord supérieur de la scissure de Sylvius, était détruite.

On sait que pour plusieurs anatomistes ces deux circonvolutions n'en font qu'une seule, désignée par eux sous le nom de circonvolution d'enceinte de la scissure de Sylvius, ou de circonvolution marginale supérieure. Si l'on interprétait dans ce sens le fait de M. Char'cot, on pourrait aisément le concilier avec les autres. Mais il faut attendre d'autres observations avant de se prononcer.

Les faits déjà nombreux sur lesquels reposait mon opinion étaient jusqu'ici exclusivement relatifs à des cas pathologiques, à des lésions spontanées, telles que l'apoplexie et les diverses espèces de ramollissement. Mais ces affections n'entraînent ordinairement la mort qu'après avoir produit dans le cerveau des dégâts assez étendus. On trouve presque toujours plusieurs circonvolutions malades. Le lobule de l'insula, si voisin de la troisième circonvolution frontale, cornme je l'ai dit, est presque toujours lésé en mème temps qu'elle; le noyau extraventriculaire du corps strié, immédiatement sous-jacent aux minces circonvolutions radiées de l'insula, est aussi atteint le plus souvent, ce qui donne lieu à une hémiplégie du côté droit. Parmi tant de lésions diverses, il est difficile de découvrir celle qui a 
produit l'aphémie. Toutefois, en comparant les autopsies, on est autorisé à considérer comme éventuelles les lésions qui ne sont pas constantes, et c'est ainsi que par voie d'élimination, j'ai été conduit à indiquer la troisième circonvolution frontale comme le siège des lésions de l'aphémie.

Mais cette voie est indirecte, et avant d'admettre comme démontré un fait physiologique, on attend ordinairement qu'il ait été confirmé par l'expérimentation directe. S'il s'agissait d'une tout autre faculté que de la faculté du langage, on pourrait songer peut-être à instituer des vivisections; mais l'homme seul possède cette faculté, et il ne reste d'autre ressource que d'étudier les effets des lésions traumatiques accidentelles du cerveau de l'homme. Il peut arriver que ces lésions soient assez circonscrites pour être équivalentes à des vivisections méthodiques. Il s'agit donc de constater le siège précis des plaies ou des contusions cérébrales chez les individus qui deviennent aphémiques à la suite d'une chute ou de tout autre accident. C'est une nouvelle série de recherches à entreprendre, et les deux observations que M. Ange Duval nous envoie aujourd'hui, quoique inégalement démonstratives, doivent être signalées comme les premières de ce genre qui aient été recueillies.

L'une de ces observations date déjà d'une quinzaine d'années, et le nom de la circonvolution lésée n'a pu être indiqué sur le procès-verbal de l'autopsie. Mais le siège de la lésion est si nettement spécifié, qu'il est impossible de méconnaître qu'elle occupait principalement, sinon exclusivement, la troisième circonvolution frontale.

L'autre observation a été recueillie tout récemment. Le sujet a été étudié attentivement, pendant la vie, au point de vue de l'aphémie, et il y a ceci de remarquable, que M. Ange Duval a pu, avant l'autopsie, diagnostiquer le siège et la nature de la lésion. La question de l'aphémie rentre par là dans la catégorie de celles qui intéressent directement les chirurgiens, puisqu'elle fournit un nouvel élément au diagnostic des lésions traumatiques du cerveau. Il faut maintenant attendre de nouveaux faits, et la communication faite à la Société de chirurgie par notre collègue de Brest contribuera certainement à attirer sur ce sujet l'attention des chirurgiens. 


\title{
SIÈGE
}

DE LA

\section{FAGULTÉ DU LANGAGE ARTIGULÉ}

\author{
DEUX CAS D'APHÉMIE TRAUMATIQUE
}

PRODUITE PAR DES LÉSIONS DE LA TROISIÈME GIRGONVOLUTION FRONTALE

GAUCHE

(Bulletins de la Société d'anthropologie, 1864, t. V, p. 213 à 217.)

RÉSUMÉ DES OBSERVATIONS DE M. ANGE DUVAL.

10 Homme de trente-quatre ans. Chute sur la partie postérieure et droite de la tête. Perte immédiate et complète de la parole. L'intelligence persiste néanmoins. Le blessé conserve une physionomie expressive, et se fait comprendre par signes. M. Ange Duval diagnostique une lésion du lobe frontal gauche. La mort survient le douzième jour sans que le blessé ait recouvré la parole. A l'autopsie, on troure une fracture transversale du rocher du même côté. Il y a dans l'extrémité antérieure du lobe frontal droit un tout petit foyer de contusion. Enfin, et surtout, il existe dans le lobe frontal gauche une contusion profonde et très étendue qui a entièrement désorganisé la moitié postérieure de la troisième circonvolution frontale. En dedans, la lésion empiète sur la deuxième circonvolution frontale qui n'est que partiellement divisée; en dehors, elle se prolonge quelque peu sur l'une des circonvolutions radiées de l'insula de Reil. Cette observation est toute récente, et le diagnostic porté pendant la vie lui donne une grande valeur.

20 Enfant de cing ans. Chute sur la tête. Fracture de la partie gauche de l'os frontal. Perte immédiate, complète et définitive de la parole. La guérison s'effectue sans accidents, mais l'enfant reste complètement aphémique en conservant son intelligence. Un an après, on le trouve noyé dans une petite flaque d'eau entourée de rochers. En jouant sur le bord de la mer, il était tombé dans cette flaque qui n’avait pas plus de 50 centimètres de profondeur. Après avoir fait de vains efforts pour en sortir, il s'y était noyé, faute de pouvoir appeler à son aide des ouvriers qui travaillaient près de là. A l'autopsie, on retrouve sur la moitié externe du lobe 
frontal gauche du cerveau, un kyste sphérique de 33 millimètres de diamètre, situé sur la pie-mère, un peu en arrière de la fracture, et logé dans une excavation profonde de la substance cérébrale. Ce kyste avait succédé à un foyer sanguin dû à une contusion de la partie correspondante du cerveau. L'observation a été recueillie à Toulon, en 1849, et à cette époque, il ne pouvait être question de désigner nominativement les circonvolutions lésées. Mais la situation dn kyste a été déterminée avec assez de précision pour qu'il soit impossible de mettre en doute la lésion de la troisième circonvolution frontale. Cette circonvolution, en effet, occupe les deux cinquièmes externes de la largeur totale du lobe frontal. Le procès-verbal de l'autopsie porte que la moitié interne de ce lobe était saine, que le kyste était contenu tout entier dans la moitié externe. Or, sur le cerveau d'un enfant de six ans, il est impossible de creuser, dans la moitié externe du lobe frontal, une cavité arrondie de 33 millimètres de diamètre, sans détruire la plus grande partie de la troisième circonvolution. Il est donc parfaitement certain que cette circonvolution a été très gravement lésée au moment de l'accident (1).

Après avoir communiqué au nom de l'auteur ces deux observations, M. Broca constate qu'elles font faire un grand pas à la question de l'aphémie et de la localisation des facultés cérébrales. Jusqu'ici, dit-il, tous les cas d'aphiémie, qui ont été étudiés dans le but de vérifier mon hypothèse sur le siège de la faculté du langage articulé, étaient relatifs à des lésions spontanées, presque toujours progressives qui, avant de déterminer la mort, avaient produit dans le cerveau des dégâts assez étendus. Ces faits sont assez nombreux aujourd'hui pour constituer une démonstration indirecte; mais, en physiologie, on donne avec raison la préférence aux démonstrations directes, fournies par l'expérimentation sur les animaux. Or, les animaux ne peuvent servir à l'étude d'une fonction dont ils sont privés. - Il faut donc attendre que les hasards du traumatisme produisent sur l'homme des lésions analogues à celles que l'on pourrait chercher à produire par des vivisections. Les deux observations de M. Duval, la première surtout, commencent la série des faits propres à établir cette démonstration directe. Dans ces deux cas, la troisième circonvolution frontale gauche a été subitement dés-

(1) Nous ne donnons ici qu'un extrait de ces deux observations, qui offrent un grand intérêt chirurgical, et qui seront publiées in extenso dans les Bulletins de la Société de chirurgie, mars 1864. 
organisée et une aphémie complète, immédiate et définitive en a été la conséquence.

Ces deux faits viennent à l'appui d'une remarque que j'ai faite depuis près de deux ans, et qui est tellement étrange, tellement subversive, qu'on ne saurait la présenter sans y mettre beaucoup de réserve. J'avais été frappé de ce fait que, chez mes premiers aphémiques, la lésion occupait toujours non seulement le même point du cerveau, mais encore le même côté, le côté gauche. Depuis lors, sur un nombre d'autopsies qui s'élève maintenant à plus de vingt, on a trouvé constamment que les lésions de l'aphémie étaient situées à gauche. On a observé, en outre, un très grand noímbre d'aphémiques vivants qui, pour la plupart, présentent une hémiplégie plus ou moins complète, et cette hémiplégie occupe les membres du côté droit, ce qui prouve que la lésion cérébrale existe du côté gauche. On a cité, il est vrai, un ou deux cas d'aphémie coïncidant avec une paralysie des membres gauches, c'est-à-dire avec une lésion de la moitié droite du cerveau. Mais il y a loin d'une observation recueillie sur le vivant à une autopsie; une lésion de l'hémisphère droit n'exclut pas l'existence d'une lésion de l'hémisphère gauche ; la chose reste douteuse jusqu'au jour de l'autopsie, et, je le répète, toutes les autopsies d'aphémiques ont révélé des lésions de l'hémisphère gauche. Et voici un autre fait bien remarquable: on a plusieurs fois trouvé, sur la troisième circonvolution frontale de l'hémisphère droit, des lésions qui n'avaient pas altéré la faculté du langage; ces lésions étaient pourtant exactement pareilles à celles qui, étant situées à gauche, produisent toujours l'aphémie, ou qui du moins l'ont toujours produite dans les cas connus jusqu'ici. Il semble résulter de là que la faculté du langage articulé est localisée dans l'hémisphère gauche du cerveau ou au moins qu'elle dépend principalement de cet hémisphère. J'ai cru pouvoir profiter de la communication de M. Ange Duval pour présenter quelques observations sur la question de l'aphémie, et la Société me le pardonnera peut-être, si elle veut bien se rappeler que cette question est née à la suite d'une discussion qui a rempli, il y a trois ans, plusieurs de nos séances. 


\title{
SUR LE SIÈGE
}

DE LA

\section{FACULTÉ DU LANGAGE ARTIGULÉ APHÉMIE TRAUMATIQUE}

\author{
LÉSION DE LA TROISIÈME CIRCONVOLUTION FRONTALE
}

(Bulletins de la Société d'anthropologie, 1864, t. V, p. 362-365.)

M. Broca présente, au nom de M. Perier, le cerveau d'un blessé qui a perdu la parole par suite d'une chute sur la tête et qui est mort au bout de dix jours.

Cet homme avait fait une chutė sur la partie latérale droite de la tête et s'était fait une fracture de la fosse temporale droite. Il resta d'abord sans connaissance; quelque temps après, lorsque M. Perier le vit pour la première fois, il présentait encore quelques symptômes de commotion; les membres étaient dans le relâchement. A toutes les questions qu'on lui faisait, il répondait uniquement: La tête, la tête. Pouls faible et lent, vomissement, ecchymose sous-orbitaire à droite, écoulement de sang par le nez.

Pendant les trois ou quatre jours suivants, le malade parut aller mieux. Il soutenait sa tête plus facilement. L'intelligence était revenue; il comprenait ce qu'on lui disait, et répondait par gestes, mais il ne pouvait prononcer que le monosyllabe oui.

Quoiqu'il fût évident que le blessé était tombé sur le côté droit de la tête, M. Perier, d'après le symptôme de l'aphémie, diagnostiqua une lésion par contre-coup de l'hémisphère gauche du cerveau et annonça que la troisième circonvolution frontale devait être au nombre des parties lésées.

Il n'y avait eu jusqu'alors ni convulsion ni paralysie. Mais le 
huitième jour survinrent des mouvements convulsifs, comme épileptiformes, occupant plus particulièrement les muscles de la moitié droite de la face et ceux des membres du côté droit. Cela confirmait l'idée d'une lésion de l'hémisphère gauche. En même temps, et tout en conservant d'ailleurs sa connaissance, le blessé cessa de prononcer son unique monosyllabe. - Les mouvements convulsifs se rapprochèrent de plus en plus, les muscles postérieurs du cou se raidirent; le malade mourut le dixième jour, sans avoir présenté aucun symptôme de paralysie.

A l'autopsie, qui a été pratiquée ce matin, on a trouvé d'abord une fracture s'étendant de la fosse temporale droite à la fente sphénoïdale du même côté. L'hémisphère droit du cerveau paraissait parfaitement sain ainsi que le bulbe, le cervelet et la protubérance. Une nappe de sang coagulé recouvrait l'hémisphère gauche. Ce sang a été enlevé, ınais on a laissé la pie-mère en place, et M. Broca, à qui M. Perier a eu l'obligeance d'envoyer la pièce, n'a pas voulu la disséquer avant de l'avoir montrée à la Société. On aperçoit, à la face externe de l'hémisphère gauche, trois petits foyers hémorrhagiques parfaitement distincts. Le premier, situé sur la partie moyenne de la deuxième circonvolution du lobe temporo-sphénoïdal, correspond à une contusion superficielle de la substance cérébrale ; la pie-mère est déchirée à ce niveau, et c'est par là sans doute que le sang s'est épanché dans l'arachnoïde. Ce premier foyer a environ 15 millimètres d'étendue. Le second ést situé sur la même circonvolution, à 2 centiinètres en arrière du précédent. Il a environ 1 centimètre de large. Enfin, le troisième foyer est situé sur le bord supérieur de la scissure de Sylvius, à 1 centimètre et demi en avant de l'extrémité externe du sillon de Rolando, et masque entièrement et exclusivement le méandre postérieur de la troisième circonvolution frontale. M. Broca n'hésite pas à considérer cette dernière lésion comme la cause de l'aphémie, car elle occupe strictement le point qu'il a indiqué comme étant le siège de la faculté du langage articulé.

M. Trélat procède alors avec le plus grand soin à la dissection de la pie-mère. En enlevant cette membrane, on constate d'abord qu'il existe deux caillots allongés, couchés respectivement dans les deux sillons qui limitent le méandre postérieur de la 
troisième circonvolution frontale. Cette partie de la troisième circonvolution, comprimée entre les deux caillots, est notablement amincie; en la comparant à la même partie de la troisième circonvolution de l'hémisphère droit, on trouve qu'elle a perdu au moins un tiers de son épaisseur. Elle présente, en outre, le ramollissement et la couleur rouge qui caractérisent l'encéphalite.

L'encéphalite n'est pas limitée à cette circonvolution. Les deux premières circonvolutions frontales et les trois cinquièmes antérieurs de la troisième sont parfaitement sains. Mais, en arrière, l'encéphalite s'est propagée à la partie inférieure de la circonvolution frontale transverse et de la circonvolution pariétale transverse. Les lésions de l'encéphalite sont manifestes également autour des deux foyers du lobe temporo-sphénoïdal ; mais une étude minutieuse montre qu'il n'y a aucune continuité entre l'inflammation de ce dernier lobe et celle qui a eu pour point de départ la lésion de la troișième circonvolution frontale. Le lobe de l'insula, le fond de la scissure de Sylvius et le bord inférieur de cette scissure sont parfaitement sains, et il est digne de remarque que le foyer sanguin de la troisième circonvolution ne s'est même pas prolongé par infiltratiơn dans l'intérieur de la scissure de Sylvius. 


\section{SUR L'APHÉMIE}

(Bulletins de la Société anatomique, 2e série, t. IX, juillet 1864, p. 293 et 296.

\section{Broca présente :}

A. Plusieurs cerveaux destinés au musée Dupuytren et qui présentent la lésion caractéristique de l'aphémie, à savoir une altération de la troisième circonvolution frontale gauche :

$1^{\circ}$ Femme complètement aphémique (service de M. Charcot) : l'observation a été publiée dans les Bulletins de notre Société, année 1863.

Autopsie. Ramollissement du lobe pariétal et occipital de l'hémisphère gauche.

La troisième circonvolution frontale est séparée des autres par une perte de substance dans laquelle on peut loger le doigt.

$2^{\circ}$ Fille épileptique depuis plusieurs années et en même temps aphémique (service de M. Moreau, de Tours).

Autopsie. Ramollissement de l'insula et de la troisième circonvolution frontale.

La substance grise de l'insula et le noyau extraventriculaire du corps strié ont complètement disparu.

Je n'ai pas vu l'hémisphère droit; mais on m'a dit qu'il ne présentait aucune altération.

$3^{\circ}$ Femme de quatre-vingts ans, entrée à l'infirmerie pour une fracture de l'extrémité inférieure du fémur.

Elle n'avait aucune intelligence et ne poussait que des cris inarticulés ; son mutisme était attribué à une altération sénile ; mais on apprit qu'elle avait une phrase, toujours la même : J'aime mieux mourir, et qu'elle reconnaissait sa fille.

Autopsie. Ramollissement de la troisième circonvolution frontale. 
$4^{\circ}$ La nommée Lagacke (femme Forget), soixante-douze ans, est morte le 27 juin 1864, à l'infirmerie de la Salpêtrière (service de M. Broca).

Elle a succombé aux suites d'un phlegmon diffus chronique avec abcès multiples dı membre inférieur droit, après un séjour de plus de deux mois à l'infirmerie. Elle venait de la division des aliénés (service de M. Falret).

Nous ne savions rien de ses antécédents, car elle ne répondait à aucune question. Elle n'a jamais parlé au moment de la visite, et elle ne comprenait certainement pas ce qu'on lui disait. Elle ne connaissait personne, n'appelait jamais ni ses voisines, ni les filles de service qui la soignaient. Sa fille n'est venue la voir que deux fois pendant son séjour à l'infirmerie ; la première fois elle a paru la reconnâttre, mais ne lui a rien dit ; la seconde fois elle ne l'a même pas reconnue.

Cette pauvre femme est restée continuellement au lit à cause de son phlegmon diffus. Elle gâtait, mais elle n'était nullement paralysée. Elle se retournait librement dans son lit, se servait de ses deux mains et remuait ses deux jambes; à plusieurs reprises j'ai dû lui faire des incisions sur la jambe et la cuisse droites; elle les a bien senties et a poussé quelques gémissements ; mais ne s'est pas agitée ; il pourrait se faire que la sensibilité fût un peu émoussée. Lorsqu'on préparait devant elle des instruments pour une nouvelle incision, elle regardait faire sans comprendre. Il était clair qu'elle ne se souvenait pas des incisions précédentes.

Elle n'a jamais répondu à une question; mais elle ne gardait cenendant pas toujours le silence. Elle disait souvent un grand nombre de fois de suite: "Je veux m'en aller », ou encore: "Qucl malheur! » ou enfin : “Mon Dicu, mon Dieu! » D'autres fois, elle marmottait pendant des heures entières des sons inarticulés accompagnés de gémissements.

Son intelligence était assez profondément altérée pour expliquer son mutisme; nous ne nous sommes pás demandé pendant la vie si ce mutisme dépendait d'une cause spéciale.

Autopsic. Enorme quantité de sérosité dans l'arachnoïde, dans la pie-mère et dans les ventricules. Léger ramollissement de la protubérance. Bulbe et cervelets sains. 
Poids de l'encéphale entier avec ses membranes, 1095 grammes. Les membranes enlevées, l'hémisphère droit pèse 455 grammes et le gauclie 417 seulement. Différence, 38 grammes.

L'hémisphère droit est sain, l'hémisphère gauche présente : $1^{\circ}$ un ramollissement diffus de la circonvolution marginale inférieure du lobe temporo-sphénoïdal ; $2^{\circ}$ une petite perte de substance au deuxième pli de l'insula, en forme d'abcès, peu profond, mais pénétrant cependant jusqu à la substance blanche ; $3^{\circ}$ une couleur brunâtre des circonvolutions groupées autour de la scissure de Sylvius, même sur celles d'entre elles qui ne présentent aucune autre lésion appréciable; $4^{\circ}$ enfin deux lésions de la troisième circonvolution frontale; l'une consiste en une atrophie avec un léger ramollissement diffus du méandre postérieur de cette circonvolution; l'autre est un foyer de ramollissement, jaune-brun, limité à la partie de cette circonvolution qui décrit une dernière courbe avant de quitter la scissure de Sylvius, pour se prolonger au-dessous du lobule orbitaire. Le reste de l'hémisphère gauche est un peu atrophié, mais ne paraît pas autrement malade.

L'artère sylvienne gauche présente des plaques et même des anneaux athéromateux, mais n'est ni ne peut être la cause du ramollissement; elle dépend sans doute de la même cause que le ramollissement. Le cœur et les gros vaisseaux sont parfaitement sains.

La lésion de l'hémisphère gauche est exactement celle de l'aphémie, et la malade ne parlait pas mieux que beaucoup d'aphémiques. Ce fait n'a pas beaucoup de valeur, puisque l'intelligence était à peu près abolie. Il est bon de noter cependant que la perte de la parole a coïncidé avec une lésion de la troisième circonvolution frontale gauche. 


\section{DU SIÈGE}

DE LA

\section{FAGULTÉ DU LANGAGE ARTIGULÉ}

(Bulletins de la Socièté d'anthropologie, 1865, t. VI, p. 377-393.)

La question de la localisation de la faculté du langage articulé, dont je vous demande la permission de vous entretenir pendant quelques instants, a donné lieu depuis plusieurs mois, à l'Académie de médecine et dans 'la presse médicale, à une longue discussion qui n'est pas encore terminée, et où j'ai été personnellement mis en cause. Absent de Paris pendant la plus grande partie de cette discussion, je n'ai pu la suivre que de loin et d'une manière très insuffisante. Il m'a paru que mes opinions n'avaient pas toujours été bien exactement rendues ; mais ces détails presque personnels n'auraient que peu d'intérêt pour vous. Aussi bien ne serait-il pas convenable de transporter ici un débat qui s'agite encore ailleurs. Je me bornerai donc à vous exposer, sans discussion, ma manière de voir sur l'un des éléments les plus curieux de cette question complexe.

Je vous ai communiqué dans les séances des 2 et 7 avril 1863 dix observations d'aphémie dans lesquelles on a trouvé à l'autopsie des lésions diverses de la troisième circonvolution frontale. Depuis lors, j'ai recueilli moi-même et plusieurs observateurs ont publié bon nombre d'autres faits semblables. On en a cité quelques-uns qui paraissent contradictoires. J'aurai peutêtre une autre fois à revenir sur ces derniers. Ceux qui jusqu'ici sont venus à ma connaissance sont loin, je dois le dire, d'être aussi décisifs qu'on l'a prétendu; tous, au contraire, m'ont paru sujets à controverse ; de sorte que je persiste à penser, jusqu'à 
plus ample informé, que l'aphémie véritable, c'est-à-dire la perte de la parole sans paralysie des organes de l'articulation et sans destruction de l'intelligence, est liée aux lésions de la troisième circonvolution frontale. Mais ce n'est pas de ce fait que je veux vous entretenir. Ma communication est relative à la singulière prédilection des lésions de l'aphémie pour l'hémisphère gauche du cerveau.

Vous vous souvenez sans doute que, dans tous les cas que je vous citai en 1863 , la lésion était située à gauche; je fis cette remarque sans en rien conclure (voir Bull., t. IV, p. 202, et dans ce volume, p. 43), et j'ajoutai qu'avant de chercher l'explication d'un fait aussi étrange, il fallait attendre de nouveaux faits.

Depuis lors les faits se sont présentés en grand nombre, et presque tous ont déposé dans le mêrne sens. Les cas où la lésion de l'aphémie a été trouvée à droite ne sont que des exceptions très rares. De plus, on a observé un grand nombre d'aphémiques vivants chez lesquels il existait, en outre, une paralysie du côté droit, ce qui était la preuve non équivoque de l'existence d'une lésion dans l'hémisphère gauche. (Car vous savez que l'action du cerveau est croisée.)

D'un autre côté, on a trouvé plusieurs fois dans l'hémisphère droit des lésions profondes de la troisième circonvolution frontale, à l'autopsie d'individus qui n'étaient nullement aphémiques. Quelques-unes de ces observations ont même été publiées dans le but de prouver que la troisième circonvolution n'est pas l'organe du langage ; or, elles ne faisaient que corroborer mon opinion, puisque c'est dans la troisième circonvolution frontale gauche, et non dans la droite, que j'avais localisé la faculté du langage articulé.

Depuis que j'ai signalé cette curieuse particularité, M. Dax fils a envoyé à l'Académie de médecine un mémoire d'où il résulte que son père avait depuis longtemps reconnu que les lésions qui détruisent la faculté du langage ont toujours leur siège dans l'hémisphère gauche du cerveau. M. Dax père aurait même consigné le résultat de ses observations sur ce sujet dans une note lue au Congrès méridional de Montpellier. Cette note manuscrite, retrouvée par M. Dax fils, a été reproduite par ce dernier dans son récent mémoire à l'Académie. 
Je n'aime pas les discussions de priorité, et j'aurais évité de faire remarquer que la découverte de Dax père, n'étant pas publiée, était comme non avenue au point de vue de l'histoire, si plusieurs personnes n'avaient donné à entendre que j'aurais bien dû citer l'opinion de Dax père, lorsque j'ai signalé à mon tour l'influence spéciale de l'hémisphère gauche du cerveau sur la faculté du langage. Je ne veux pas laisser croire plus longtemps que j’aie péché par ignorance ou par omission volontaire. L'existence 'du mémoire de Dax père, avant la mention qui en a été faite par son fils, était aussi inconnue à Montpellier qu'à Paris. Après avoir vainement sherché dans tous les journaux de 1836 une trace quelconque de ce mémoire, j'ai prié M. Gordon, bibliothécaire de la Faculté de Montpellier, de vouloir bien faire une petite enquête à ce sujet. M. Gordon n'a pas été plus heureux que moi. Le Congrès méridional a tenu sa troisième session à Montpellier du $1^{\text {er }}$ au 10 juillet 1836 . Il n'a pas publié de travaux, et il ne reste aucune trace de ses procèsverbaux. Il avait pour président le professeur Ribes et pour secrétaire le docteur Trinquier. La Revue de Montpellier (1836, t. II, p. 51 et 53) a donné un aperçu des sujets de philosophie médicale qui y furent discutés. La question du langage n'y est pas mentionnée. M. Gordon a interrogé personnellement vingt médecins qui étaient alors à Montpellier. Il n'est pas à leur connaissance que le mémoire en question ait été lu au Congrès ou publié quelque part. Tels sont les renseignements que j'ai pu obtenir. Je ne partirai pas de là pour contester l'authenticité de ce mémoire; il ne serait pas impossible que, préparé pour le Congrès, il n'y eût pas été lu. Mais ce que je veux faire constater, c'est que je ne pouvais vraiment pas deviner l'existence d'un manuscrit qui n'a été exhumé que deux ans après mes premières publications sur l'aphémie.

J'aborde maintenant les difficultés théoriques que soulève le fait de l'influence spéciale de l'hémisphère gauche sur le langage articulé, et probablement même sur le langage en général.

Des recherches statistiques très étendues faites par divers auteurs, et notamment par MM. Charcot et Vulpian, médecins de la Salpêtrière, ont établi que, d'une manière générale, les maladies de l'hémisphère droit sont aussi fréquentes que celles de 
l'hémisphère gauche ; et pourtant l'immense majorité des aphémiques, les dix-neuf vingtièmes peut-être, ont leur lésion dans l'hémisphère gauche.

Y a-t-il donc une différence fonctionnelle entre les deux moitiés de l'encéphale? Cette proposition, si elle était acceptée, bouleverserait toutes nos connaissances en physiologie. Il est bien certain, en effet, que les deux hémisphères du cerveau sont parfaitement semblables; si les circonvolutions cérébrales présentent des variations légères et accessoires d'individu à individu, il n'y en a pas du moins qui soient appréciables d'un côté ì l'autre de l'encéphale. Or, il y a une loi physiologique qui, partout ailleurs, est sans exception : c'est que deux organes pairs ou symétriques ont les mêmes attributions; et il serait tout à fait étrange que cette loi eût à subir ici une exception violente. Certes, l'observation est supérieure aux théories, et il faut savoir quelquefois s'incliner devant un fait, quelque inexplicable, quelque paradoxal qu'il puisse nous paraître. Mais avant de faire ce sacrifice, il faut chercher si ce fait ne serait pas susceptible d'être concilié aver les vérités générales qu'il semble contredire. C'est ce que je vais essayer de faire pour le cas particulier qui nous occupe.

Je procéderai pour cela du simple au composé, et je parlerai d'abord de l'action motrice de l'encéphale.

Un grand nombre d'actes mécaniques sont dirigés principalement, ou même exclusivement, par l'hémisphère gauche du cerveau. Il y a partout un certain nombre de gauchers; mais presque tous les hommes sont droitiers. D'où vient cette préférence accordée à la main droite? L'éducation et l'imitation y contribuent sans doute beaucoup ; et il est digne de remarque que, dans les actes compliqués et délicats qui exigent une éducation longue et toute spéciale, comme l'écriture, le dessin, le jeu de la plupart des instruments de musique, etc., la répartition du travail entre les deux mains est toujours la même, chez les gauchers comme chez les droitiers. Mais, dans cette répartition, le rôle le plus difficile, celui qui demande le plus d'habileté, de précision, d'expression ou de force, le rôle prépondérant, en un mot, est très généralement assigné à la main droite, et si nous pouvons dire que cet usage nous est venu de nos de- 
vanciers, il faut bien reconnaître que les premiers inventeurs ont dû être dirigés dans leur choix par des causes liées à l'organisation elle-même. Si, en effet, nous laissons de côté les actes qui exigent une éducation spéciale, si nous considérons seulement ceux que nous faisons spontanément, comme l'action de lancer une pierre, de frapper avec les poings, de manier un bâton, de soulever un fardeau, etc., nous trouvons, qu'à l'exception d'un très petit nombre d'individus, désignés pour cela sous le nom de gauchers, tout le monde se sert naturellement de la main droite, dont la main gauche n'est, en quelque sorte, que l'auxiliaire.

Dira-t-on que c'est un phénomène d'imitation? Mais alors comment se fait-il que tous les peuples soient droitiers, même ceux qui paraissent n'avoir jamais eu de communication avec les autres? Si c'était le hasard qui eût déterminé le choix de la main droite, on aurait trouvé certainement des peuples gauchers. Les polygénistes n'auront rien à répondre à ce raisonnement; et les monogénistes l'admettront également, puisqu'ils reconnaissent que la séparation de certains groupes humains remonte à une époque antérieure à l’invention des arts les plus rudimentaires. Il y a d'ailleurs une circonstance qui ne permet pas d'attribuer à l'imitation le choix de la main droite : c'est qu'il y a partout quelques individus qui, malgré tous leurs efforts, toute leur persévérance, restent gauchers. Pour ceux-là, on est bien obligé d'admettre l'existence d'une prédisposition organique inverse, contre laquelle l'imitation et même l'éducation ne peuvent prévaloir.

Cette prédisposition organique est accusée, en outre, par l'inégale force des deux mains. Les expériences dynamométriques montrent que la main droite, chez les droitiers, est beaucoup plus forte que la gauche. La différence est considérable; elle oscille ordinairement entre le quart et le tiers de la force de pression de la main droite. Si cette inégalité n'existait que chez les manouvriers, on pourrait supposer qu'elle est la conséquence de leur profession, et l'attribuer à ce fait bien connu que l'exercice développe la puissance musculaire ; mais elle est tout aussi prononcée chez leshommes vouésàdes professions intellectuelles.

Enfin, notre regrettable collègue Gratiolet a signalé un fait 
qui a été rappelé il y a quelques mois par M. Bertillon, et tout récemment par M. Baillarger dans son discours à l'Académie : c'est que, dans le développement du cerveau, les circonvolutions de l'hémisphère gauche sont en avance sur celles de l'hémisphère droit. Les premières sont déjà dessinées à un moment où les autres ne sont pas encore apparentes. L'hémisphère gauche, qui tient sous sa dépendance le mouvement des membres droits, est donc plus préeoce dans son développement que l'hémisphère opposé. (On comprend ainsi pourquoi, dès les premiers temps de la vie, le jeune enfant se sert de préférence des membres dont l'innervation est alors la plus parfaite, pourquoi, en d'autres termes, il devient droitier. Le membre supérieur droit, étant dès l'origine plus fort et plus adroit que le gauche, est appelé, par cela même, à fonctionner plus souvent; et il acquiert, dès lors, une supériorité de force et d'adresse qui ne fait que s'accroître avec l'âge.

Jusqu'ici, j’ai appelé droitiers ceux qui se servent de préférence de la main droite, et gauchers ceux qui se servent de préférence de la main gauche. Ces expressions sont tirées de la manifestation extérieure du phénomène ; mais si nous considérons le phénomène par rapport au cerveau, et non par rapport à ses agents mécaniques, nous dirons que la plupart des hommes sont naturellement gauchers du cerveau, et que, par exception, quelques-uns d'entre eux, ceux qu'on appelle gauchers, sont, au contraire, droitiers du cerveau.

Je passe maintenant aux phénomènes beaucoup plus complexes du langage articulé. Je laisse de côté tout ce qui concerne l'articulation elle-même, phénomène purement musculaire, et l'action motrice qui, partant des organes cérébraux affectés au mouvement, se transmet, par l'intermédiaire des nerfs moteurs, aux muscles de la langue, des lèvres, du voile du palais, etc. L'articulation dépend à un égal degré des deux hémisphères cérébraux, puisqu'elle est produite simultanément et uniformément par les muscles des deux côtés, associés dans leurs mouvements.

Mais ce n'est ni dans les muscles, ni dans les nerfs moteurs, ni dans les organes cérébraux moteurs tels que les couches optiques ou les corps striés, que gît le phénomène essentiel du 
langage articulé. Si l'on n'avait rien de plus que ces organes, on ne parlerait pas. Ils existent quelquefois, parfaitement sains et parfaitement conformés, chez des individus devenus complètement aphémiques, ou chez des idiots qui n'ont jamais pu ni apprendre ni comprendre aucun langage. Le langage articulé dépend donc de la partie de l'encéphale qui est affectée aux phénomènes intellectuels, et dont les organes cérébraux moteurs ne sont en quelque sorte que les ministres. Or, cette fonction de l'ordre intellectuel, qui domine la partie dynamique aussi bien que la partie mécanique de l'articulation, paraît être l'apanage à peu près constant des circonvolıtions de l'hémisphère gauche, puisque les lésions qui produisent l'aphémie occupent à peu près constamment cet hémisphère.

Cela revient à dire que, pour le langage, comme pour les actes beaucoup plus simples et beaucoup plus grossiers dont je parlais tout à l'heure, nous sommes gaucher's du cerveau. De même que nous dirigeons les mouvements de l'écriture, du dessin, de la broderie, etc., avec l'hémisphère gauche, de même nous parlons avec l'hémisphère gauche. G'est une habitude que nous prenons dès notre première enfance. De toutes les choses que nous sommes obligés d'apprendre, le langage articulé est peut-être la plus difficile. Nos autres facultés, nos autres actions existent au moins à l'état rudimentaire chez les animaux; mais, quoique ceux-ci aient certainement des idées, et quoiqu'ils sachent se les communiquer par un véritable langage, le langage articulé est au-dessus de leur portée. C'est cette chose complexe et difficile que l'enfant doit apprendre à l'âge le plus tendre, et il y parvient à la suite de longs tâtonnements et d'un travail cérébral de l'ordre le plus compliqué. Eh bien! ce travail cérébral, on le lui impose à une époque très rapprochée de ces périodes embryonnaires où le développement de l'hémisphère gauche est en avance sur celui de l'hémisphère droit. Dès lors, il ne répugne pas d'admettre que l'hémisphère cérébral le plus développé et le plus précoce soit, plutôt que l'autre, en état de diriger l'exécution et la coordination des actes à la fois intellectuels et musculaires qui constituent le langage articulé. Ainsi naît l'habitude de parler avec l'hémisphère gauche, et cette habitude finit par faire si bien partie de notre nature, que, lorsque nous sommes privés des 
fonctions de cet hémisphère, nous perdons la faculté de nous faire comprendre par la parole. Cela ne veut pas dire que l'hémisphère gauche soit le siège exclusif de la faculté générale du langage, qui consiste à établir une relation déterminée entre une idée et un signe, ni mêrne de la faculté spéciale du langage articulé, qui consiste à établir une relation déterminée entre une idée et un mot articulé; l'hémisphère droit n'est pas plus étranger que le gauche à cette faculté spéciale, et ce qui le prouve, c'est que l'individu, rendu aphémique par une lésion profonde et étendue de l'hémisphère gauche, n'est privé, en général, que de la faculté de reproduire lui-même les sons articulés du langage; il continue à comprendre ce qu'on lui dit, et, par coņséquent, il connaît parfaitement les rapports des idées avec les mots. Én d'autres termes, la faculté de concevoir ces rapports appartient à la fois aux deux hémisphères, qui peuvent, en cas de maladie, se suppléer réciproquement; mais la faculté de les exprimer par des mouvements coordonnés, dont la pratique ne s'acquiert qu'à la suite d'une très longue habitude, parait n'appartenir qu'à un seul hémisphère, qui est presque toujours l'hémisphère gauche.

Maintenant, de même qu'il y a des individus gauchers, chez lesquels la prééminence native des forces motrices de l'hémisphère droit donne une prééminence naturelle et incorrigible aux fonctions de la main gauche; de même, on conçoit qu'il puisse y avoir un certain nombre d'individus chez lesquels la prééminence native des circonvolutions de l'hémisphère droit renversera l'ordre des phénomènes que je viens d'indiquer, chez lesquels dès lors la faculté de coordonner les mouvements du langage articulé deviendra, par suite d'une habitude contractée dès la première enfance, l'apanage définitif de l'hémisphère droit.

Ces individus exceptionnels seront, par rapport au langage, comparables à ce que sont les gauchers par rapport aux fonctions de la main. Les uns et les autres seront droitiers du cerveau. Mais je n'en veux pas conclure qu'il doit y avoir coïncidence entre ces deux catégories d'exceptions ; car il ne me semble nullement nécessaire que la partie motrice et la partie intellectuelle de chaque hémisphère soient solidaires l'une de l'autre, eu égard 
à la précocité de leur développement respectif dans les deux hémisphères.

L'existence d'un petit nombre d'individus qui, par exception, parleraient avec l'hémisphère droit, expliquerait très bien les cas exceptionnels où l'aphémie est la conséquence d'une lésion de cet hémisphère.

Il suit de ce qui précède qu'un sujet chez lequel la troisième circonvolution frontale gauche, siège ordinaire du langage articulé, serait atrophiée depuis la naissance, apprendrait à parler et parlerait avec la troisième circonvolution frontale droite, comme l'enfant venu au monde sans la main droite devient aussi habile avec la main gauche qu'on l'est ordinairement avec l'autre main.

C'est ainsi, sans doute, qu'il faut expliquer un fait remarquable observé l'année dernière à la Salpêtrière, dans le service de M. Moreau (de Tours). A l'autopsie d'une malade de quarante-sept ans, épileptique depuis sa plus tendre enfance, on constata que la troisième circonvolution frontale gauche faisait défaut, ainsi que la circonvolution pariétale inférieure et la circonvolution temporo-sphénoïdale supérieure. En d'autres termes, on constata l'absence de toute la partie de l'hémisphère gauche qui borde la scissure de Sylvius et qui constitue, dans la nomenclature de M. Foville, la circonvolution d'enceinte de cette scissure. Or, cette malade n'était pas aphémique, et elle aurait dû l'être si la troisième circonvolution gauche était le siège exclusif et constant de la faculté de coordonner l'articulation des mots. Au reste, les parties qui manquaient.n'avaient pas été détruites par une maladie: il était aisé de reconnaître que ces parties ne s'étaient jamais développées; en effet, à la place de la circonvolution d'enceinte, on trouvait un petit pli sinueux, gros comme un boyau de rat, qui présentait exactement les connexions normales et les rapports de la circonvolution d'enceinte. Il s'agissait donc d'une atrophie congéniale, d'un véritable arrêt de développement, dû peut-être à l'absence congéniale de l'artère sylvienne gauche, artère dont nous ne pûmes retrouver la trace. Le reste de l'hémisphère gauche paraissait sain, mais était cependant loin d'être normal, car toutes sez̃ parties, les circonvolutions aussi bien que le corps strié, la couche optique et le pédoncule, étaient 
bien moins volumineuses que les parties correspondantes de l'hémisphère droit. Celui-ci pesait, sans les membranes, 540 grammes, tandis que l'autre pesait seulement 297 grammes. Cette différence de $\mathbf{2} 43$ grammes paraîtra d'autant plus considérable que l'encéphale entier, avec ses membranes, ne pesait que 1043 grammes. On ne s'étonnera pas, dès lors, que les fonctions de l'hémisphère gauche fussent imparfaites. Les deux membres du côté droit étaient très faibles et ne jouissaient que d'une sensibilité obtuse ; ils étaient, en outre, moins longs et moins volumineux que ceux du côté opposé. La main droite, presque inutile, était fléchie sur l'avant-bras, et la marche s'accompagnait d'une claudication manifeste. Cette inégalité anatomique et fonctionnelle des deux moitiés du corps était évidemment la conséquence de l'inégalité congéniale des deux moitiés du cerveau, et ce qui le prouve, c'est qu'à la face, dont les nerfs prennent naissance au-dessus de l'entre-croisement du bulbe et dont l'innervation, par conséquent, est directe et non pas croisée, l'atrophie des chairs et du squelette se montrait seulement du côté droit.

L'intelligence, sans aucun doute, devait se ressentir de l'état défectueux du cerveau. Mais la malade n'était nullement idiote; elle n'avait reçu qu'une instruction très rudimentaire; pourtant elle savait lire, elle s'occupait des travaux de son état, et, par parenthèse, elle cousait de la main gauche; enfin, elle parlait convenablement et elle exprimait ses idées sans difficulté.

Ce fait me donna à réfléchir. Les opinions que je vous soumets aujourd'hui s'étaient déjà présentées à mon esprit, mais ne s'y étaient pas encore fixées. Plusieurs fois déjà, en étudiant des cerveaux d'aphémiques, j'avais trouvé que la lésion de la troisième circonvolution frontale gauche n'était pas toujours en rapport direct d'intensité avec l'altération du langage; par exemple, j'avais vu la parole complètement anéantie par une lésion de huit à dix millimètres seulement d'étendue, tandis que, dans d'autres cas, des lésions dix fois plus considérables n'avaient altéré qu'en partie la faculté du langage articulé. J'en avais déjà conclu que, selon toutes probabilités, les deux hémisphères concouraient au langage et pouvaient se suppléer plus ou moins suivant les cas, quoique la troisième circonvolution frontale de l'hémisphère gauche fût toujours le siège principal de la fonction. 
Mais c'était la première fois que je voyais cette circonvolution du langage faire défaut dans toute son étendue chez un sujet non aphémique. Ici, il était parfaitement évident que la troisième circonvolution droite avait suppléé à l'absence de la gauche. Et je me demandai aussitôt comment il se faisait que cela n'eût pas lieu dans tous les cas d'aphémie.

Il semble, en effet, que, si les deux hémisphères concourent à la fonction du langage, la lésion d'un seul hémisphère ne devrait pas suffire pour produire l'aphémie. De même qu'on voit avec un seul œil, qu'on entend avec une seule oreille, on devrait pouvoir parler avec un seul hémisphère. En admettant même que l'hémisphère gauche joue dans le langage articulé un rôle prépondérant (et il est impossible de nier cette évidence), il semble que l'hémisphère droit resté sain devrait toujours pouvoir prendre la parole à la place de l'hémisphère gauche, rendu impuissant par une lésion. C'est ainsi, par exemple, que chez un amputé du bras droit, la main gauche acquiert une grande dextérité et finit même par écrire. Comment se fait-il donc que l'individu, rendu aphémique par une destruction partielle ou totale de la troisième circonvolution frontale gauche, $\mathrm{n}^{2}$ apprenne pas à parler avec l'hémisphère droit?

A cela, je répondrai d'abor'd que l'amputé conserve toute l'intégrité de son intelligence, tandis que, chez la plupart des aphémiques, il existe des lésions cérébrales plus ou moins étendues, qui, sans abolir l'intelligence, lui portent une atteinte notable. Lorsque la lésion est très circonscrite, il peut se fairc que le langage soit seul atteint, et que l'intelligence reste intacte, autant du moins que nous pouvons en juger; j'en ai vu un exemple tout à fait décisif. Ces faits prouvent bien manifestement que la faculté du langage est entièrement indépendante des autres facultés cérébrales; mais ils sont rares. Le plus souvent l'altération anatomique occupe une étendue assez considérable pour porter de graves atieintes à l'intelligence proprement dite. Il en résulte que la plupart des aphémiques ont l'esprit affaibli, et cette circonstance est bien faite pour les empêcher d'apprendre à parler exclusivement avec l'hémisphère droit, qui jusqu'alors n'avait joué qu'un rôle accessoire dans la fonction de l'expression par le langage articulé. 
Puis, comment sait-on que l'aphémique n'est pas capable d'apprendre à parler avec l'hémisphère droit qui lui reste? A-t-on essayé de faire son éducation? lui a-t-on donné ces leçons de tous les jours, de toutes les heures, de tous les instants à l'aide desquelles on finit à la longue parfaire parler un enfant? Pour ma part, je suis convaincu que, sans rendre aux aphémiques la partie de leur intelligence qui a péri avec une partie de leur cerveau, on pourrait, en y mettant assez de persévérance, en les traitant avec l'infatigable constance de la mère qui apprend à parler à son enfant, on pourrait, dis-je, obtenir des résultats considérables. Lorsque j'étais à Bicêtre, j’ai gardé plusieurs mois un aphémique dans mes salles; souvent, à la visite, je lui consacrais quelques minutes, et j'avais fini par étendre notablement son vocabulaire. Mais, qu'est-ce qu'une leçon aussi courte? Et n'allez pas croire que, sous ce rapport, l'éducation d'un adulte soit plus facile que celle d'un enfant; elle est beaucoup plus difficile au contraire. Il y a des choses qu'on n'apprend jamais bien au-delà d'un certain âge. Les mouvements de l'écriture, quoiqu'ils soient dirigés par l'œil et par conséquent infiniment moins automatiques que ceux de l'articulation, sont dans ce cas. Un amputé du bras droit apprend à écrire de la main gauche, mais son écriture n'acquerra jamais la précision, la fermeté, la rapidité, qu'elle avait auparavant, et un homme qui serait aussi maladroit pour parler que cet amputé l'est pour écrire, passerait pour aphémique. Ce n'est pas seulement une question d'agilité musculaire ; il est probable que l'adulte et l'enfant suivent, pour arriver au but, des procédés différents. L'enfant se borne à imiter, il émet des sons au hasard jusqu'à ce qu'il trouve le son qu'on lui demande, et il recommence avec docilité aussi souvent qu'on le désire. L'adulte n'a pas cette patience; il ne peut faire abstraction de ce qu'il sait ou de ce qu'il croit savoir; il discute le procédé; au lieu d'obéir naïvement, il cherche en luimême les voies et moyens, et les connaissances qu'il possède déjà, loin de lui venir en aide, l'empêchent, au contraire, de profiter des leçons pratiques qu'on lui donne. Par exemple, il apprend beaucoup plus difficilement qu'un adolescent à s'exprimer dans une langue étrangère; souvent même, lorsque le sys- 
tème de prononciation des deux langues est essentiellement différent, il lui est tout à fait impossible d'apprendre à prononcer certaines consonnes.

L'aphémique de Bicêtre, dont je vous parlais tout à l'heure, avait perdu non seulement le langage parlé, mais encore le làngage écrit. Il n'était pas étonnant qu'il ne pût plus écrire, puisque sa main droite était paralysée; mais il ne savait plus lire, il ne connaissait même plus les lettres. Je lui présentai un alphabet, et pendant quelque temps nous essayâmes de le faire épeler. Il arriva bientôt à connaître toutes les lettres. Nous voulûmes alors les lui faire assembler et nous commençâmes par les syilabes. Au bout de quelques leçons, il connaissait bon nombre de syllabes simples; par exemple, quand nous lui demandions de nous montrer du doigt les syllabes $b a, p a, c o$, mo, etc., il se trompait rarement. Je pensai alors que le moment était venu de lui faire assembler les syllabes; mais ici j'échouai complètement. Je ne renonçai pas pour cela à lui faire lire des mots de plusieurs syllabes; j'essayai donc de lui montrer ces mots sans les décomposer, et je parvins à lui en faire connaître un bon nombre; mais. je m'aperçus bientôt qu'il ne les reconnaissait pas à leurs syllabes et à leurs lettres. C'était seulement leur forme générale, leur longueur, leur physionomie qui le frappaient; et par exemple, lorsque nous changions, au milieu d'un mot, une ou deux lettres en les remplaçant par des lettres de même longueur, comme $m$ pour $u, e$ pour $s, p$ pour $q, l$ pour $t$, il ne s'en apercevait même pas. En d'autres termes, il reconnaissait un mot comme on reconnaît un visage ou paysage dont on n'a jamais analysé les détails. Il est clair, par conséquent, que cet aphémique apprenait à lire par un procédé essentiellement différent de celui qu'il avait suivi dans sa jeunesse.

L'insuccès ordinaire des tentatives que l'on fait pour faire parler les aphémiques ne prouve donc pas que l'hémisphère sain ne puisse pas suppléer celui qui est malade. Et par exemple, je suis convaincu qu'une lésion de la troisième circonvolution frontale gauche, capable de produire chez un adulte une aphémie définitive, n'empêcherait pas un jeune enfant d'apprendre à parler. Voilà pourquoi, selon toute probabilité, la malade épi- 
leptique du service de M. Moreaı parlait, quoiqu'elle fût entièrement privée, dès sa naissance, non seulement de la troisième circonvolution frontale gauche, mais encore de tout le reste de la grande circonvolution d'enceinte de la scissure de Sylvius. Elle était comme ces individus qui viennent au monde sans braz, et qui apprennent à écrire, à coudre, à peindre avec les pieds; jamais un adulte qui aurait subi l'amputation des deux bras ne pourrait arriver à un pareil résultat.

Il n'est pas inutile de rappeler que cette femme était gauchère. L'hémisphère gauche du cerveau étant insuffisant, c'était avec l'hémisphère droit, et par conséquent avec la main gauche, qu'elle avait pris l'habitude de travailler comme de parler.

En résumé, les deux moitiés de l'encéphale étant parfaitement identiques, au point de vue anatomique, ne peuvent pas avoir des attributions différentes; mais le développement plus précoce de l'hémisphère gauche nous prédispose, dans nos premiers tâtonnements, à exécuter avec cette moitié du cerveau les actes matériels et intellectuels les plus compliqués, parmi lesquels il faut certainement compter l'expression des idées au moyen du langage, et, plus particulièrement, du langage articulé. Loin de moi la pensée de partager l'homme en deux êtres distincts, comme le fit, à un autre point de vue, Meinard Simon du Pui, dans sa dissertation intitulée : de Homine dextro et sinistro (Leyde, 1780). L'habitude que nous prenons, dès la première enfance, de répartir le travail entre nos deux hémisphères, et de demander de préférence les opérations les plus difficiles à notre hémisphère gauche, finit par devenir une seconde nature ; mais cette spécialisation des fonctions n'implique pas l'existence d'une disparité fonctionnelle entre les deux moitiés de l'encéphale.

A la suite de cette lecture, M. Gaussin souleva quelques objections. Il s'étendit prresque exclusivement sur la faculté d'expression en général, considérée non seulement chez l'homme, mais encore dans la série animale. Le langage en particulier n'est qu'une modalité de cette faculté générale. 


\section{DISCUSSION}

(Bulletins de la Société d'anthropologie, 1\$65, t. VI, p. 412 a 414 et 417.)

M. Broca. Je vais, tout d'abord, remercier M. Gaussin de la forme courtoise qu'il a donnée à ses objections, si je puis appeler ainsi des divergences d'opinions qui ne portent que sur des questions secondaires. Je n’ai, au surplıs, donné mon opinion que comme une hypothèse, et j’ai dit à la dernière séance, qu'une tout autre manière de voir m'ayant souvent été prêtée ailleurs, je ne pouvais m'empêchel' de rectifier le rôle que l'on m'attribue dans la question des localisations cérébrales, au sein même de la compagnie où elle est née. Sans nul doute, je crois à la symétrie fonctionnelle, non moins qu'à la symétrie anatomique du cerveau. Mais il y a plus d'une interprétation à donner aux actes physiologiques les plus réguliers qui, par une suite naturelle de l'exercice, peuvent prendre un caractère tout particulier. Ainsi, je suis complètement d'accord avec M. Gaussin sur l'existence d'une faculté générale que nous appellerons, si vous le voulez, la faculté d'expression, commune à l'homme et aux animaux, et qui ne comprend pas seulement le langage, mais la mimique et l'écriture; que si maintenant je me place au point de vue de M. Gaussin et que je considère le langage, comme une faculté innée, il n'y a aucune raison pour la localiser primitivement à gauche plutôt qu'à droite ; mais il est acquis que la manifestation de cette faculté est un résultat de l'exercice, et dès lors, de même que dans la vie extérieure nous accomplissons quatre-vingts actes sur cent à l'aide du bras droit, pareillement l'hémisphère gauche du cerveau finit, en suite d'une sorte d'habitude prise, par se charger exclusivement de la fonction du langage. Il y a là quelque chose d'artificiel, et il se peut que ce soit pour le plus grand avantage du cerveau qui aurait ainsi trouvé un mode de communication plus simple et plus rapide. 31 semble au surplus que l'on aurait de cette hypothèse une sorte de confirmation dans ce fait que des aphémiques recouvrent par l'éducation une certaine aptitude à un langage articulé, quoiqu'un peu différent du langage normal. J'en ai tout au moins un exemple. Je me demande, en ces cas, s'il 
ne se passe point un acte semblable à celui d'un homme qui aurait perdu l'usage du bras droit et se mettrait à écrire du gauche.

M. Gaussin me fait remarquer que l'exercice partiel de l'un des lobes du cerveau devrait entraîner des différences de volume sensibles et pareilles à celles de nos deux membres supérieurs. Mais d'abord ces différences ne sont point aussi considérables que paraît le croire notre collègue, et puis, il y a peutêtre lieu de distinguer, au point de vue de la nutrition, entre l'agent mécanique qui obéit, le muscle, seule partie du membre qui soit beaucoup plus volumineuse, et l'agent central qui ordonne. Enfin, si ces observations étaient insuffisantes, on pourrait dire tout simplement que ce qui est vrai pour les membres ne l'est point pour les hémisphères. De telle sorte que l'objection tirée de la symétrie anatomique et géométrique des hémisphères ne me paraît pas avoir autant de gravité que paraît lıi en donner notre savant collègue.

Je reviens maintenant à la faculté d'expression : elle appartient aux animaux comme à l'homme, et ici encore, je suis du même avis que M. Gaussin ; mais savoir en quoi l'intelligence des animaux ressemble à celle de l'homme, en quoi elle en diffère: voilà ce que la question de l'aphémie pourrait aider à trouver. Je souhaite que cette question, qui a déjà été effleurée, revienne devant nous, peut-être serons-nous mieux en mesure de l'éclairer, maintenant que nous possédons quelques faits précis sur les désordres cérébraux qui accompagnent la perte de la parole. La faculté d'expression une fois admise, c'est par l'éducation traditionnelle que l'on en développe les différentes ma. nières d'être et le degré d'avancement. Sans doute dans la faculté d'expression il y a à distinguer la mimique et la graphique, mais il m'a paru que ce qui était le plus circonscrit c'était le groupe des faits qui se rattachent à l'organisation des sons en langage. Je ne connais que cela qui manque aux animaux et que nous ayons, et c'est pourquoi j'ai disséqué les cerveaux des individus qui n'avaient perdu que cela; mon espoir était d'arriver à établir la relation qui existe entre la faculté centrale et la manifestation.

Mais en m'attribuant l'intention de distinguer radicalement 
de la mimique, la faculté d'articuler ces sons, M. Gaussin s'est mépris sur ma pensée. L'une et l'autre forme se rattachent à une seule faculté que je suis tout disposé à désigner, avec M. Gaussin, sous le nom de faculté d'expression.

Cette faculté est beatcoup plus nettement délimitée, beaucoup plus réelle que ne le sont la plupart de celles qu'admettent les psychologistes, beaucoup plus que la mémoire, par exemple, qui me paraît n'être qu'une propriété commune à toutes nos sensations ou aux moyens abstraits de l'intelligence. Il y a une mémoire de l'œil, une mémoire de l'oreille, une mémoire des lieux..., où est dans ces exemples multiples, l'unité nécessaire à une faculté ? Je la cherche en vain, tandis que la faculté d'attacher une idée à un signe vocal ou mimique est partout identique.

Maintenant, sans vouloir en rien juger l'explication que propose M. Gaussin, des causes qui empêchent certains aphémiques de prononcer les substantifs, je dois faire remarquer que dans les cas que j'ai pu observer, on arrivait facilement ì faire répéter cette classe de mots, en les intercalant dans des mots composés ou au milieu d'une locution rapide ou d'un juron.

M. Dalty cite un cas ou l'éducation nouvelle d'une aphémique avait donné des résultats assez nets, mais, dit-il, il faut repousser l'unité de la faculté d'expression au même titre que l'unité de la mémoire. Et avant de localiser les facultés, il faudrait les analyser et les définir, ce qui n'a encore été fait que métaphysiquement et non physiologiquement. Enfin, "l'uniformité de texture du cerveau autorise à croire que pour ce qui est de l'entendement proprement dit, toutes les parties similaires peuvent se suppléer; ce qui détermine la localisation fonctionnelle de l'entendement, ici ou là, n'est sûrement pas le fait du hasard, mais ce n'est pas non plus le fait d'organes comparables à ceux qu'accomplissent les fonctions intérieures de l'économie, tels que le cœur ou le foie. »

M. Broca. Je ne veux répondre qu'un mot à ce qu'a dit M. Dally, et ce sera sur un point anatomique qui, par conséquent, ne se prête pas à la discussion. M. Dally a avancé que la structure des circunvolutions était sensiblement uniforme. C'est là une erreur qu'il est important de relever. La face inférieure du lobe occipital se distingue par la présence du ruban rayé de Vicg d'Azyr qui n'existe pas sur les autres circonvolutions. (Voir t. II des Bulletins, p. 316.) 


\section{SUR}

\section{LA FAGULTÉ DU LANGAGE ARTIGULÉ}

(Bulletins de_la Socièté d'anthropologie, 1865, t. VI, p. 493-494.)

M. Broca. Quelques semaines après la séance du 15 juin, où j’ai soumis à l'attention de la Société une hypothèse tendant à expliquer l'influence spéciale de l'hémisphère gauche du cerveau sur la fonction du langage articulé, M. le professeur Bouillaud m'a fait l'honneur de m'envoyer un exemplaire du discours qu’il a prononcé à l'Académie de médecine, dans les séances du 4 et du 11 avril 1865 .

A la page 10 de cette brochure, on lit le passage suivant:

"Parmi nos organes doubles, il en est qui nous offrent une particularité qui ne diffère point, au fond, de celle dont il est question au sujet des hémisphères cérébraux. Qui ne sait, par exemple, que malgré l'identité de fonctions qui, d'une manière générale, existe entre nos membres gauches et nos membres droits, il est cependant un certain nombre des actes auxquels ils sont affectés que nous exécutons, soit ordinairement, soit même exclusivement, avec les membres droits, et de là cette expression si connue de droitiers (celle de gauchers est dunnée à ceux qui, par exception, se servent de leurs membres gauches dans l'exécution des actes spéciaux dont il est question)?

" N'est-ce pas ainsi, par exemple, que nous écrivons, nous dessinons, nous peignons, nous faisons des armes, etc., etc., de la main droite? Combien d'exemples du même genre ne pourrais-je pas rapporter, si je n'étais pressé par le temps!

« Eh bien, serait-il absolument impossible que pour certains actes auxquels sont affectés les hémisphères cérébraux, la parole, par exemple, nous fussions pour ainsi dire gauchers?

" Mais j'ai hâte d'en finir sur une question pour la solution 
de laquelle les observations me font défaut, et que j'aurais peutêtre dû ne pas aborder encore. J'ai toujours, en effet, présente à l'esprit la fameuse histoire de la Dent d'or, quand il s'agit d'expliquer des faits qui ne sont pas encore démontrés. "

Je regrette beaucoup, reprend M. Broca, de n'avoir pas eu connaissance de ce passage important lorsque j'ai fait ma communication à la Société. Il m’eût été précieux de pouvoir abriter mes hypothèses derrière la grande autorité de M. Bouillaud. Mais à l'époque où ce professeur a prononcé son discours, j'étais malade et absent de Paris ; je n'ai donc pas pul'entendre. Je n'ai connu, de la discussion de l'Académie, que les comptes rendus publiés par les journaux de médecine, et il s'est trouvé précisément qu'aucun journal n'a fait allusion à cette partie de l'argumentation de M. Bouillaud.

Je m'empresse, aujourd'hui, de réparer l'omission involontaire que j'ai commise. L'hypothèse que M. Bouillaud a le premier publiée a dû, sans doute, se présenter à l'esprit de plusieurs personnes. Je l'ai, pour ma part, émise en présence de mes élèves, à la Salpêtrière, le jour où nous étudiâmes le cerveau de la femme épileptique, mortè le 3 novembre 1864, dans le service de M. Moreau (voir plus haut, p. 92). Mais je ne l'avais pas publiée, et M. Bouillaud ne pouvait la connâttre lorsqu'il a été conduit, de son côté, à chercher devant l'Académie l'explication des relations spéciales qui existent entre le langage articulé et l'hémisphère gauche du cerveau. 


\title{
DISGUSSION
}

\section{SUR UNE OBSERVATION D'APHÉMIE}

\author{
DE M. VOISIN
}

(Bulletins de la Société d'anthropologie, 1S66, $2^{\circ}$ série, t. I, p. 377-384.)

M. Broca. Je remercie M. Voisin d'avoir apporté ici cette observation, qui confirme si bien l'opinion que j'ai déjà exposée, à plusieurs reprises devant la Société. Si ce fait était isolé, il ne serait sans doute pas suffisant pour servir de base à une conclusion ; mais, au point où en est aujourd'hui la question de l'aphémie, il acquiert, par sa netteté, une très grande importance. Mes recherches m'ont conduit à reconnaître que la faculté du langage est localisée dans la partie postérieure de la troisième circonvolution frontale gauche. Cette opinion découle d'une double série de faits établissant $: 1^{\circ}$ que les lésions de cette partie très limitée de l'hémisphère gauche produisent l'aphémie $; 2^{\circ}$ que les lésions de la même partie de l'hémisphère droit ne produisent pas l'aphémie.

Ces deux propositions ne souffrent que des exceptions très rares, et tout permet de croire que les individus chez lesquels la faculté du langage dépend de l'hémisphère droit sont en plus petit nombre que les gaucher's. Je ne reviendrai pas sur les explications que je vous ai soumises l'année 'dernière pour rendre compte du rôle spécial de l'hénisphère gauche dans l'exercice de la fonction du langage. (Voir Bulletins, t. VI, et dans ce volume, p. 84).

Les faits recueillis jusqu'ici rentraient soit dans l'une, soit dans l'autre des deux séries susmentionnées ; chacun d'eux, par conséquent, ne constituait qu'une demi-preuve et ne devenait probant que lorsqu'on le comparait avec les faits de la série 
inverse. Mais le fait de M. Voisin fournit à lui seul les deux parties de la démonstration. Deux attaques d'apoplexie, survenant à vingt mois d'intervalle chez le même individu, frappent successivement dans les parties postérieures, la troisième circonvolution frontale du côté droit et celle du côté gauche. La première attaque ne porte pas atteinte à la faculté du langage; la seconde produit une aphémie immédiate et complète. Il est impossible d'imaginer un ait plus conforme aux idées que je soutiens.

Le fait de M. Voisin se distingue, en outre, de la plupart des observations d'aphémie par cette circonstance que son malade, n'étant pas paralysé de la main droite, a pu tenir une plume et faire des efforts pour écrire. Cela a permis à M. Voisin d'étudier l'altération profonde, et même, pendant les premiers jours, l'abolition à peu près complète de l'écriture. Quoiqu'il n'y ait pas solidarité nécessaire entre la faculté de parler et celle d'écrire, quoiqu'il existe plusieurs observations d'individus qui, en devenant aphémiques, avaient conservé le pouvoir de lire et d'écrire couramment et correctement, il n'est pas douteux que, le plus souvent, les lésions qui détruisent la parole portent en même temps une atteinte plus ou moins grave aux autres modes, aux autres espèces de langage conventionnel. Beaucoup d'aphémiques ne savent plus lire; d'autres savent encore comprendre les phrases écrites, mais font des efforts impuissants pour écrire eux-mêmes. Ceux-là n'ont d'autre mode d'expression que la mimique spontanée, qui est le seul langage naturel, et qui, par conséquent, survit, plus ou moins, chez tous les individus qui n'ont pas encore entièrement perdu l'intelligence.

Ainsi que je l'ai dit dans mon premier mémoire, communiqué en 1861 à la Société anatomique, tous les langages de convention, tels que la parole articulée, les diverses espèces d'écriture, la dactylologie, la mimique artificielle usitée dans l'éducation des sourds-muets, etc., ne sont que des manifestations particulières de la faculté que nous possédons d'établir.une relation constante entre un signe et une idée. Cette faculté, que j’ai appelée la faculté générale du langage, ne doit donc pas être confondue avec la faculté spéciale du langage articulé qui, au point de vue psychologique, n’en est qu’une dépendance, mais qui, au 
point de vue de sa manifestation, constitue une fonction distincte de toutes les autres. L'aphémique nous comprend quand nous lui parlons; il a donc conservé la faculté d’associer les signes avec les idées, c'est-à-dire la faculté générale du langage; et pourtant, quoiqu'il ait encore le pouvoir de mouvoir sa langue à son gré, il ne peut pas nous répondre, parce qu’il ne sait plus articuler. Il est revenu à l'état où il était dans sa première enfance, lorsque, comprenant déjà ce qu'on lui disait, il n'avait pas encore acquis, à la suite de tâtonnements infinis, l'art très compliqué de faire concourir un grand nombre de muscles à l'articulation des sons. Cet art, qu'une longue habitude lui avait rendu familier, il l'a perdu, plus ou moins complètement, par suite de la lésion d'une partie très circonscrite de la masse circonvolutionnaire, et nous sommes, dès lors, autorisés à en conclure que cette partie très circonscrite est le siège de l'un des éléments indispensables de la fonction du langage articulé.

Les autres langages conventionnels peuvent se prêter à la mêne analyse. Comme le langage articulé, ils mettent en jeu la faculté générale du langage, telle que je viens de la définir, et, de plus, ils exigent, pour se manifester, l'exécution de certains mouvements combinés et coordonnés, et l'analogie permet de supposer que ces mouvements doivent être, comme ceux qui précèdent, régis par une partie déterminée et circonscrite du cerveau. G'est ce que les observations pathologiques ont déjà ètabli pour le langage écrit, et ce qu'elles établiront probablement un jour pour les autres espèces de langage.

Maintenant, on peut se demander s'il y a dans le cerveau autant d'organes régisseurs des mouvements de l'expression qu'il y a d'espèces de langage. Cela n'est pas admissible, car il dépend de nous de créer de nouveaux systèmes de langage, et personne n'imaginera que cela puisse avoir pour conséquence d'augmenter le nombre de nos organes cérébraux. Il est, dès lors, infiniment probable que la partie du cerveau qui tient sous sa dépendance la manifestation du langage articulé, régit aussi celle des autres langages conventionnels. L'association très fréquente de l'aphémie et de la perte de l'écriture ne doit donc pas nous surprendre; et s'il y a lieu d'être étonné d'une chose, c'est que l'écriture ne périsse pas toujours en même temps que la pa- 
role. Est-ce parce que, dans ces cas exceptionnels où l'aphémique continue à écrire couramment et correctement, un exercice continuel et prolongé des deux principaux modes du langage conventionnel aurait eu pour conséquence d'établir sinon une subdivision de l'organe commun de l'expression par le langage, du moins une sorte de répartition dans cet organe unique des deux fonctions qu'il dirige sans cesse? N'est-ce pas plutôt parce que les mouvements graphiques seraient plus simples et plus directement soumis à la volonté que ceux de l'articulation, de sorte qu'une lésion assez grave pour porter atteinte à ces derniers pourrait encore quelquefois laisser subsister les autres? Je me borne à poser ces questions, et je ferai remarquer seulement deux choses: c'est d'abord que l'intégrité de l'écriture dans l'aphémie est relativement beaucoup plus rare chez les individus qui écrivent peu ou difficilement que chez les gens de lettres, les médecins, les avocats, les notaires, les hommes qui, à force d'écrire, ont fini par écrire instinctivement, qui peuvent, par exemple, écrire en parlant, en écoutant. Ma seconde remarque, c'est que, si l'on connaît un certain nombre de faits d'aphémie avec conservation de l'écriture, il ńy en a pas, ou plutôt je n'en comnais pas encore, où l'écriture ait péri sans que la parole fût altérée.

On voit tout l'intérêt qui s'attache à l'étude chez les aphémiques, et je ne saurais trop féliciter M. Voisin des recherches utiles qu'il a faites dans cette voie; mais je ne puis partager l'opinion à laquelle paraît se rallier notre collègue, relativement à la nature du trouble intellectuel qui a eu pour conséquence d'altérer à la fois chez son malade la parole et l'écriture. Il est disposé à croire que ce trouble intellectuel consiste seulement en une perte de la mémoire des mots. Certes, il est bien évident que la perte de la mémoire des mots doit entraîner la perte de la parole ; cela constitue peut-être, je n'en sais rien, une variété d'alalie, mais ce n'est certainement pas la cause de l'aphémie. Si les aphémiques avaient perdu la mémoire des mots, ils ne comprendraient pas ce qu'on leur dit, et ils pourraient toujours, du moins, répéter un mot qu'on vient de prononcer à l'instant devant eux. 0r, personne n'ignore que beaucoup d'aphémiques ne peuvent y réussir. Ce n'est donc pas la mémoire des 
mots qui fait défaut, mais une autre faculté, peut-être une autre espèce de mémoire, celle du mécanisme compliqué de l'articulation.

Je puis ajouter enfin, sans m'écarter de la question de l'aphémie, que j’ai reçu ce matin une lettre de M. Vogt, de Genève, qui s'occupe actuellement de l'étude des microcéphales. Il a eu à sa disposition un assez grand nombre de pièces provenant de divers musées publics ou particuliers, et il a constaté que, dans tous les cas où les microcéphales n'avaient pu apprendre à parler, leur cerveau était, comme celui des singes, privé à droite et à gauche de la troisième circonvolution frontale. Ce renseignement, pour ce qui concerne les singes, m'a surpris, parce qu'il est en contradiction avec l'opinion reçue; mais je rappellerai à la Société que je lui ai présenté, il y a quelques années, le cerveau d'un adulte microcéphale, nommé Edern. Cet idiot n'avait jamais pu parler, quoiqu'il criât toute la journée, et la troisième circonvolution frontale faisait défaut chez lui d'une manière complète, comme dans les cas récemment étudiés par M. Vogt.

M. Lusıer. L'interprétation du fait de M. Voisin peut être contestée. En admettant avec lui et avec M. Broca que la faculté du langage articulé soit localisée dans la partie postérieure de la troisième circonvolution frontale, on peut se demander s'il est bien vrai que la troisième circonvolution frontale gauche soit seule, à l'exclusion de la droite, le siège de cette faculté. On pourrait, au contraire, conclure de ce fait que la fonction du langage est dévolue naturellement à deux organes pairs, qu'après la destruction de l'organe du côté droit, le gauche l'a suppléé, qu'après la destruction de ce second organe la fonction a été perdue, et que les effets auraient été les mêmes si l'organe gauche avait péri avant le droit. Pour établir les conclusions admises par notre collègue, il faudrait des faits plus simples, des lésions unilatérales; il faudrait, par exemple, constater que la même lésion qui, étant située à gauche, altère constamment la parole, ne l'affecte pas lorsqu'elle est située dans le point correspondant de l'hémisphère droit.

M. Broca. Ces faits, tant positifs que négatifs, existent déjà en grand nombre dans la science; je ne les ai pas mentionnés parce qu'ils ont déjà figuré souvent dans nos discussions précédentes.

M. Moreau (de Tours). Je pense, comme M. Broca, que ce n'est pas la mémoire des mots qui a péri chez les aphémiques. A l'argument qu'il vient 
d'invoquer, je puis en joindre un autre, tiré de l'étude de certains malades qui peuvent encore répondre à une question, et qui pourtant ne sont pas capables de répéter immédiatement après les mots de cette phrase. On leur demande un mot, ils ne peuvent le dire ; on leur fait une question sollicitant une réponse où ce mot doit se trouver, et ils répondent couramment. Par exemple, on leur dit : Comment allez-vous? - Cela va très bien. Redites les mots : Cela va trés bien. Ils s'efforcent en vain de prononcer cette phrase; ils s'impatientent, s'irritent, sans résultat. Puis, dès qu'ils cessent de penser aux mots particuliers qu'on leur demande, ils sont de nouveau en état de répondre à une autre question. Il est bien évident que ces malades-là n'ont pas perdu la mémoire des inots.

M. Dally. Il serait peut-être bon, dans les recherches sur lá faculté du langage, de définir nettement les limites de la mémoire des mots, de la faculté d'expression et du pouvoir locomoteur nécessaire à l'articulation des sons; on pourrait ensuite rechercher quel est de ces trois éléments du langage, celui qui est lésé. Dans les observations que l'on nous présente cette distinction n'est pas faite, en sorte qu'à cette heure, j'ignore encore ce qu'est, en dehors de la mémoire des mots, la faculté du langage. Maintenant notre éminent collègue, M. Moreau, introduit dans la question un nouvel élément : l'influence de l'effort mental (si l'on veut me passer cette expression), qui serait dans l'aphémic une condition de trouble; cette condition ne me paraît point spéciale et ne me parait pas pouvoir aider à établir que l'aphémie soit autre chose que la perte de la mémoire des mots; en effet, il arrive chaque jour que c'est en cessant de chercher que l'on trouve ce que l'on cherche. En sorte que, sans prendre parti sur le fond de la question (ce qui parait sage en l'ibsence de définitions que je réclame), l'observation de M. A. Voisin se rapporte à la localisation, non de la faculté complexe du langage, mais à celle de la mémoire.

M. Broca. M. Dally demande qu'avant de chercher à localiser la faculté du langage articulé, on détermine rigoureusement, par l'analyse, et qu'on définisse nettement les limites des diverses actions cérébrales qui concourent au langage. Il demande en d'autres termes qu'on procède $a$ priori, ce qui a toujours égaré les investigations dans les recherches sur les fonctions de l'encéphale. Je pense, au contraire, qu'il faut procéder à posteriori. L'analyse qu'il réclame découlera peu à peu des faits pathologiques. Lorsque nous connaîtrons toutes les conditions qui peuvent porter atteinte aux divers éléments de cette fonction complexe, nous l'analyserons avec sécurité, et nous"pourrons alors définir et circonscrire ces éléments.

A mon tour, M. Dally me permettra sans doute de lui renouveler un argument qui paraît ne l'avoir pas frappé, et auquel il 
n'a pas répondu. Je répète que les aphémiques conservent la mémoire des mots, puisqu'ils comprennent ce qu'on leur dit ; et puisque quelques-uns, n'ayant pas perdu l'écriture, peuvent répondre correctement par écrit. Il me paraît probable qu'ils ont perdu une espèce particulière de mémoire, mais il est impossible d'admettre que ce soit la mémoire des mots. Je serais reconnaissant à M. Dally de vouloir bien s'expliquer sur ce point. 


\section{APHASIE TRAUMATIQUE}

(Bulletins de la Société d'anthropologie, 1866, $2^{\circ}$ série, t. I, p. 396-399.)

M. Voisin nous a présenté dans la dernière séance une pièce anatomique importante pour la détermination de l'organe du langage articulé. Quelques jours après, le hasard a mis entre mes mains une pièce d'une autre nature non moins curieuse.

Une portion du cerveau a été détruite jusqu'aux limites de l'organe du langage ; celui-ci a été entamé, diminué, mais non détruit; la faculté a été altérée, diminuée, mais non anéantie.

Chev..., homme de quarante-sept ans, dans l'intention de se suicider, a placé sa tête sur le passage d'un balancier. Appliquant la tempe droite sur la table, il a exposé sa tempe gauche à l'action de cette puissante machine qui a produit une énorme lésion du cuir chevelu, de la voûte crânienne et du cerveau.

Le malheureux s'affaissa aussitôt et ne put se relever. On le transporta aussitôt à l'hôpital Saint-Antoine. Il n'avait pas perdu connaissance.

Ainsi, il a articulé à plusieurs reprises le juron qué nom de $D \ldots$, pour sacré $n$. de $D \ldots$, et il a répété au moins cinq ou six fois les mots ca hait mal, au lieu de ca fait mal, l'F du mot fait étant remplacé par une forte aspiration. L'interne qui a admis le blessé à l'hôpital, a nettement constaté ces particularités, dont il a été frappé d'autant plus.vivement que le lobe frontal gauche lui paraissait entièrement réduit en bouillie.

Le blessé était presque complètement hémiplégique; la jambe droite était tout à fait inerte; mais le bras droit a exécuté quelques petits mouvements partiels. Les deux membres gauches se mouvaient, au contraire, avec agitation. La sensibilité a paru anéantie dans les membres du côté droit.

Une énorme perte de substance, comprenant les deux tier's environ de la moitié gauche de l'écaille frontale, une partie notable 
du pariétal gauche et de la grande aile du sphénoïde du même côté, laissait échapper, à l'extérieur, une assez grande quantité de matière cérébrale réduite en bouillie. Le malade était furt agité. On le déposa difficilement dans son lit, et on appliqua une vessie de glace au voisinage de sa blessure. Dès que cette application fut faite il tomba dans le coma et mourut quelques instants après.

Je n'ai vu le blessé qu'à l'amphithéâtre; mais les détails qui précèdent m'ont été fournis bien nettement avant l'autopsie, par l'interne de garde, M. Paquet, par la religieuse et par les gens de service.

A l'autopsie, nous avons trouvé dans le cerveau des lésions énormes, mais beaucoup moins étendues cependant qu'on aurait pu le croire d'après l'aspect extérieur de la plaie. Vous pouvez en suivre la description sur les pièces que j'ai l'honneur de vous présenter. Ces lésions ont été produites principalement par un grand fragment d'os, long de 10 centimètres, large de 6 , qui, détaché d'une seule pièce, et refoulé vers la cavité crânienne, a d'abord écrasé la partie correspondante des circonvolutions subjacentes, et qui, ensuite, faisant bascule, a pénétré obliquement, de haut en bas et d'avant en arrière, dans l'épaisseur du lobe frontal. Le tiers moyen de la troisième circonvolution frontale est entièrement détruit, ainsi que la partie correspondante de la seconde. L'extrémité antérieure du lobe sphénoïdal est, en outre, profondément entamée. Le fragment d'os, pénétrant à travers cette large voie, a broyé le pli antérieur du lobule de l'insula, puis laissant au-dessus de lui le reste de ce lobule, s'est enfoncé dans le noyau extraventriculaire du corps strié qui est divisé dans la plus grande partie de son épaisseur.

Le tiers postérieur de la troisième circonvolution frontale a ainsi échappé à la destruction. En arrière, cette circonvolution a conservé tous ses rapports de continuité. Son méandre postérieur est parfaitement sain, mais sur son avant-dernier méandre on aperçoit un certain nombre de petits foyers de contusions moléculaires, formant une sorte de piqueté. D'arrière en avant, ces petits foyers deviennent de plus en plus nombreux, et notre circonvolution se terminait enfin, au niveau de la suture produite par le passage du fragment osseux, en une sorte de moignon écrasé et fortement contus. 
Enfin, le pédoncule cérébral gauche est le siège d'une contusion moléculaire, caractérisée par l'existence d'une douzaine de petils foyers, moins gros que des grains de mil.

Il n'existe absolument aucune lésion dans l'hémisphère droit.

Pour ne pas altérer la pièce, qui mérite d'être conservée, j'ai évité de pratiquer des coupes sur le corps strić. Je ne puis donc dire exactement dans quelle étendue la partie postérieure de ce corps a conservé sa continuité. Vous pouvez voir que la plaie produite par le fragment d'os s'arrête au milieu de sa substance. La continuité du corps strié n'est donc pas absolument interrompue, et cela suffit pour expliquer la conservation partielle de la mobilité du bras droit; vous serez, sans doute, frappés comme moi de la corrélation parfaite qui existe, dans ce cas, entre les lésions et les symptômes. Le tiers postérieur de la troisième circonvolution frontale gauche, c'est-à-dire, suivant moi, l'organe du langage articulé, est resté en place, et la faculté du langage, malgré l'énorme délabrement des parties environnantes, n'a pas été détruite; mais l'organe du langage, quoique relativement sain, ne l'est pas complètement. Sa partie antérieure est entamée, et de plus une contusion moléculaire a atteint l'un de ses méandres; il en est résulté que la parole a été cònsidérablement troublée. Il faut faire la part, sans doute, de l'état général produit par cette immense blessure. Notez, toutefois, qu'il n'y a pas eu de symptômes de commotion; qu'il n'est tombé dans le coma qu'après l'application de la glace; qu'il pouvait encore agir sur les muscles de l'articulation, puisqu'il prononçait, quelques mots, et que, par conséquent, il n'y avait rien, dans son état général ni dans l'état de ses nerfs moteurs, qui fût de nature à l'empêcher de parler. Notez surtout que l'altération du langage chez cetindividu était tout à fait semblable à celle qui caractérise l'aphémie incomplèłe. Il n'a prononcé qu'un juron de quatre syllabes, et un membre de phrase de trois syllabes, mais il les a répétés un certain nombre de fois, comme le font les aphémiques dont le langage est réduit à quelques mots. 


\section{REGHERCHES SUR LA LOCALISATION}

\section{DE LA \\ FAGULTÉ DU LANGAGE ARTIGULÉ}

(Exposé des titres et travaux scientifiques de Paul Broca, 1868.)

M. Broca croit avoir découvert que l'exercice de la faculté du langage articulé est subordonné à l'intégrité d'une partie très circonscrite des hémisphères cérébraux et plus spécialement de l'hémisphère gauche. Cette partie est située sur le bord supérieur de la scissure de Sylvius, vis-à-vis l'insula de Reil, et occupe la moitié postérieure, probablement même le tiers postérieur seulement de la troisième circonvolution frontale.

La faculté du langage articulé est jusqu'ici la seule faculté de l'ordre intellectuel dont la localisation ait pu être démontrée, car les localisations admises par l'école phrénologique reposaient sur des hypothèses que l'anatomie pathologique a renversées.

C'est, au contraire, l'anatomie pathologique qui a fourni à M. Broca les preuves du fait qu'il a découvert. L'importance de ce fait n'a pas besoin d'être démontrée; car il suffit qu'une seule faculté intellectuelle soit en rapport spécial avec une partie circonscrite du cerveau pour que la localisation des autres facultés devienne très probable.

Les preuves de l'opinion soutenue par M. Broca sont tirées de l'étude du cerveau des individus qui ont présenté pendant leur vie le symptôme de l'aphémie. Il désigne sous ce nom l'abolition ou l'altération de la faculté du langage articulé, survenant chez des individus qui n'ont aucune paralysie des muscles de l'articulation, qui comprennent ce qu'on leur dit, et peuvent se faire comprendre à leur tour par la mimique ou par l'écriture, qui, 
par conséquent, ont des idées et savent les exprimer, qui n’ont perdu, en un mot, que la faculté coordinatrice du langage articulé.

Les faits qui démontrent la localisation de cette faculté dans le point indiqué, sont de plusieurs ordres :

$1^{\circ} \mathrm{A}$ l'autopsie des aphémiques, on trouve à peu près constamment une lésion très évidente de la moitié postérieure de la troisième circonvolution frontale gauche ou droite. La lésion est quelquefois exclusivement limitée à cette partie du cerveau. Il existe un très petit nombre de cas où cette partie a paru saine, mais où une lésion très grave existait à une très petite distance, soit dans les circonvolutions adjacentes de l'insula, soit dans la partie de la circonvolution d'enceinte qui se continue sans interruption avec la troisième circonvolution frontale. Ces cas exceptionnels forment à peine la vingtième partie des faits observés depuis sept ans. D'autres exceptions ont été citées, mais elles sont relatives à des malades qui n'avaient pas présenté les véritables symptômes de l'aphémie.

$2^{\circ} \mathrm{La}$ lésion occupe, environ dix-neuf fois sur vingt, la troisième circonvolation frontale du côté gauche. Celle du côté droit est presque toujours parfaitement saine.

$3^{\circ}$ Il est sans exemple jusqu'ici que la faculté du langage soit restée intacte chez les individus qui avaient une lésion grave de la partie postérieure de la troisième circonvolution frontale gauche, à moins que cette lésion ne fût congénitale. Cette règle souffrira probablement plus tard quelques rares exceptions; mais on peut affirmer que, chez la très grande majorité des sujets, la troisième circonvolution du côté droit ne peut suffire à maintenir la fonction du langage articulé, lorsque celle du côté gauche est détruite ou altérée.

$4^{\circ} \mathrm{Il}$ existe, au contraire, un assez grand nombre de cas où la parole a persisté, malgré l'existence d'une très grave lésion ou même d'une destruction complète de la troisième circonvolution frontale droite.

$5^{\circ}$ On n'a jamais vu persister la faculté du langage articulé chez les individus qui ont présenté à l'autopsie une lésion profonde des deux circonvolutions en question.

$6^{\circ}$ Les lésions traumatiques démontrent, plus nettement 
encore que les lésions pathologiques, la localisation de la faculté du langage dans le point indiqué. Produisant quelquefois des désordres très limités, comparables à ceux que donneraient des vivisections méthodiques, les plaies, les contusions et les compressions de la partie postérieure de la troisième circonvolution frontale gauche permettent de constater que l'altération de la parole est, en quelque sorte, proportionnelle à l'étendue et à la profondeur de la blessure. Jusqu'ici les blessures de la troisième circonvolution frontale droite n'ont porté aucune atteinte à la parole; mais il est extrêmement probable que ces blessures peuvent, par exception, comme les lésions pathologiques, donner lieu aux symptômes de l'aphémie.

$7^{\circ}$ Enfin, à l'autopsie des idiots, plus ou moins microcéphales, qui n'avaient pas pu apprendre à parler, on a constaté plusieurs fois, des deux côtés du cerveau, l'absence de la troisième circonvolution frontale.

Considérant comme établie l'existence d'une relation directe et spéciale entre la faculté du langage articulé et la partie postérieure de la troisième circonvolution frontale des hémisphères, M. Broca a dû se demander pourquoi cette faculté est plus particulièrement en rapport avec la troisième circonvolution frontale gauche. Il pense que la circonvolution droite et celle de gauche ont, comme toutes les parties symétriques des organes pairs, les mêmes propriétés essentielles, et qu'au nombre de ces propriétés figure, pour chacune d'elles, celle de pouvoir présider à la fonction du langage ; mais le langage articulé étant en quelque sorte une fonction artificielle et conventionnelle, qui ne s'acquiert que par une éducation spéciale et par une longue habitude, on conçoit que l'enfant puisse contracter l'habitude de diriger de préférence avec l'une ou l'autre des deux circonvolutions en question la gymnastique toute spéciale de l'articulation. C'est ainsi que la plupart des actes qui exigent le plus de force ou d'adresse sont exécutés de préférence avec la main droite, et dirigés, par conséquent, par l'hémisphère gauche du cerveau. Mais, de même qu'il y a quelques gauchers qui dirigent ces mêmes actes avec l'hémisphère droit, de même il y a quelques individus qui dirigent de préférence le langage articulé avec la troisième circonvolution frontale droite. 
Les recherches de M. Broca sur le siège de la faculté du langage articulé ont provoqué, en France et à l'étranger, de nombreuses publications, et ont donné lieu à une importante discussion dans le sein de l'Académie de médecine. 


\section{SUR LE SIÈGE}

DE LA

\section{FACULTÉ DU LANGAGE ARTIGULÉ}

(La Tribune médicale, 1869 , nos 74 et $75-28$ février et 7 mars - p. 254-256 et 265-269.)

Toutes les parties de la masse circonvolutionnaire du cerveau ont-elles les mêmes fonctions, ou n'y a-t-il pas des parties plus ou moins circonscrites qui seraient douées d'attributions particulières? Tel est le problème de physiologie générale que je me suis efforcé de résoudre lorsque j'ai entrepris, il y a plus de sept ans, mes recherches sur le siège de la faculté du langage articulé.

Cette faculté, dont l'intégrité, l'altération ou la destruction sont faciles àconstater, se prêtait mieux que toute autre à ce genre de recherches; car elle est à la fois nettement déterminée et manifestement indépendante du reste de l'intelligence. Elle existe chez un grand nombre d'idiots, ou d'individus parvenus au degré le plus extrême de l'imbécillité et de la démence, et on la voit disparaître quelquefois chez certains individus qui semblent n'avoir perdu aucune autre aptitude intellectuelle.

Il semble que rien ne doive être plus facile que de constater l'état de cette faculté. Il y a cependant beaucoup de cas où il est plus ou moins difficile de savoir si le trouble ou l'abolition de la parole dépend d'une altération de la faculté propre du langage articulé ou de diverses autres causes. Sans parler des sourdsmuets, de certains idiots réduits à la condition de la brute, de certains maniaques qui, pareils aux disciples d'Apollonius de Tyanes, gardent quelquefois pendant plusieurs années un silence systématique, l'altération de la parole peut être la conséquence de quatre ordres d'affections bien distinctes, qui sont en rap- 
port avec les quatre éléments dont se compose la fonction complexe du langage. Pour parler : $1^{\circ}$ il faut a voir une idée à exprimer ; $2^{\circ}$ il faut connaître les rapports que la convention a établis entre les idées et les mots; $3^{\circ}$ il faut posséder l'art, acquis à la suite d'une longue et difficile éducation, de combiner avec régularité les mouvements délicats des organes de l'articulation, de manière à produire immédiatement et sans effort les mots convenables; $4^{\circ}$ enfin, il faut que les organes de l'articulation soient dans un état d'intégrité qui leur permette d'obéir aux ordres de la volonté.

Ces quatre éléments sont également nécessaires pour l'exercice de la fonction du langage articulé, et il suffit que l'un d'eux soit afteint pour que la parole soit altérée ou abolie.

La perte de la parole n'est pas une maladie particulière; mais ce serait bien à tort que, sous prétexte qu'elle n'est qu'un symptôme, on chẹrcherait à la désigner toujours sous le même nom; car ce symptôme peut appartenir à quatre affections, ou plutôt à quatre groupes d'affections essentiellement distin ctes. Il en résulte, pour l'analyse des faits comme pour leur synthèse, des confusions continuelles qui, pendant bien longtemps, ont empêché toute espèce de progrès. Pour dissiper ces confusions, la première condition serait d'adopter une nomenclature uniforme et rigoureuse.

Déjà, dans mes premiers travaux, je m'étais efforcé d'établir l'un des termes de cette nomenclature, en déterminant aussi bien que je l'avais pu l'un des quatre groupes, et en employant le nom d'aphémie pour caractériser la perte du langage spécialement afférente à ce groupe; mais, depuis lors, ceux qui ont écrit sur le même sujet, ont plus d'une fois rétabli la confusion que j'avais voulu éviter. Il me paraît done nécessaire de compléter aujourd'hui la nomenclature des divers groupes de cas où la parole est altérée ou abolie.

Le premier groupe comprend les cas où l'on ne parle pas, faute d'avoir des idées à exprimer; où l'on a perdu ce qu'on appelle la connaissance, et où l'individı est privé de cette espèce de " grand ressort " de l'intelligence que désignait, dans son acception la plus élevée, le mot grec $\lambda$ orós. Je propose de désigner dans ce cas la perte de la parole sous le nom d'alogic. 
Dans le second groupe, je range les cas où les malades ne connaissent plus les rapports conventionnels établis entre les idées et les mots. Ils peuvent encore, de diverses manières, comprendre les signes qu'on leur fait, et montrer qu'ils les comprennent; ils peuvent même prononcer des paroles confuses, qui souvent n'ont aucun rapport avec les idées qu'ils voudraient exprimer, mais ils ont oubliéle sens des mots qu'ils prononcent, et ne comprennent pas mieux ceux que l'on prononce autour d'eux. Ils peuvent, d'ailleurs, quelquefois, montrer qu'ils ne sont pas privés de toute espèce de mémoire; ils reconnaissent les objets, les visages, les localités ; on peut souvent, à l'aide de certains gestes, ou même de certains mots qu'ils n'ont pas oubliés, réveiller en eux des souvenirs déjà éloignés : on ne saurait donc prétendre qu'ils aient perdu la mémoire; mais ils ont perdu la mémoire spéciale des mots, le plus souvent celle des mots écrits aussi bien que des mots parlés. Pour désigner leur état, j'emploierail'expression d'amnésie verbale, déjà usitée par M. Lordat. Quoique cette dénomination ait été récemment, en vertu d'une théorie erronée, appliquée à des cas d'un ordre tout différent, je juge utile de la conserver, parce qu'elle a une acception bien évidente et qu'elle est d'ailleurs parfaitement exacte.

Le troisième groupe est celui pour lequel j'ai proposé le nom d'aphémie. L'aphémique a des idées, et les exprime par des gestes, ou même par. quelques mots qu'il sait encore prononcer et auxquels il attribue un sens plus ou moins bien déterminé; son vocabulaire est quelquefois tout à fait nul, d'autres fois réduit à quelques monosyllabes, ou à quelques jurons, ou à quelques mots étranges qui ne sont d'aucune langue; d'autres fois, enfin, il est plus étendu, et on a pu se demander si ces diverses atteintes du langage ne dépendaient pas d'un degré plus ou moins prononcé d'amnésie verbale. Mais ce qui distingue nettement ce cas du précédent, c'est que le malade comprend ce qu'on lui dit, qu'il reconnaît parfaitement le sens des mots et des phrases que l'on prononce, et qu'il ne peut pas répéter. Il n'a donc pas oublié les rapports qui existent entre les idées et les mots, et s'il n'a pas oublié le sens des mots, c'est la preuve qu'il est exempt de l'amnésie verbale. Il peut y avoir, sans doute, 
dans la clinique, des cas où le diagnostic de ce cas et du précédent peut être douteux, comme il y a des cas où le diagnostic entre un kyste et un lipôme peut rester indécis; mais les groupes pathologiques existent indépendamment des confusions qui peuvent se produire dans la pratique; et le diagnostic de l'aphémie et de l'amnésie verbale est d'ailleurs si aisé dans beaucoup de cas qu'il me paraît difficile de méconnaître la distinction de ces deux types.

Le quatrième groupe, enfin, comprend les cas où les agents mécaniques de l'articulation ont perdu leurs fonctions par suite d'une altération des muscles, des nerfs ou des parties de l'encéphale qui tiennent ces nerfs sous leur dépendance. Il n'est pas nécessaire pour cela que les muscles de l'articulation soient entièrement paralysés. Le mécanisme de la parole est si délicat, il exige des mouvements si rapides, si précis, si bien coordonnés, qu'il suffit souvent d'une hémiplégie même incomplète de la langue pour porter une profonde atteinte à l'articulation des sons. Bien que certaines complications cérébrales puissent rendre quelquefois assez difficile la distinction de ce cas et du précédent, il n'est personne qui n'ait.compris la nécessité de les séparer. On a proposé dans ces derniers temps de désigner sous le nom d'aphonie l'altération ou la perte du langage qui est la conséquence de la paralysie. C'était effectivement le sens que les anciens donnaient le plus souvent à ce mot. Mais on s'accorde généralement aujourd'hui à réserver le nom d'aphonie pour désigner le symptôme, essentiellement différent, de l'abolition de la voix. Or, il n'y a absolument aucun rapport entre la production du son vocal, qui rend l'articulation bruyante, et l'articulation elle-même, puisque les individus qui ont perdu la fonction laryngée, peuvent encore parler correctement à voix basse. Le nom d'aphonie ne convient donc pas ici. Il y a dans la science un mot qui exprime la perte de la parole, et qui a été appliqué à des cas extrêmement divers, mais qui, n'ayant jamais prévalu dans les nomenclatures médicales, peut, sans inconvénient, être restreint aujourd'hui à un sens particulier :

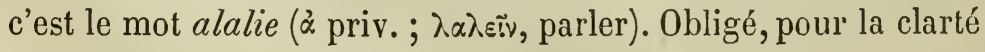
du langage, de donner un nom spécial au quatrième groupe des cas où la parole est altérée ou perdue, je propose d'appliquer 
l'expressien d'alalie à ce quatrième groupe, en y ajoutant l'épithète de mécanique qui lui donnera une acception plus précise. En résumé, la nomenclature que je propose est la suivante : $1^{\circ}$ Alogie, ou perte de la parole par suite de la perte de l'intelligence en général;

$2^{\circ}$ Amnésie verbale, ou perte de la parole par suite de la perte de la mémoire des mots ;

$3^{\circ}$ Aphémie, ou perte de la parole par suite de l'altération de la faculté spéciale du langage articulé ;

$4^{\circ}$ Alalie mécanique, ou perte de la parole par suite de l'impuissance des agents mécaniques de l'articulation ;

Ces quatre mots, formés à l'aide de l'A privatif, ne doivent pas faire naître l'idée que la parole soit toujours entièrement perdue. Elle l'est, en effet, et elle l'est complètement, dans les degrés les plus avancés, mais le plus souvent elle n'est pas complètement abolie, et quelquefois même l'affection se réduit à un simple trouble de la fonction du langage.

J'ai évité à dessein de faire figurer dans la nomenclature précédente le mot aphasie. Dans l'origine, ce mot, venu du grec, comme tous les autres, ne devait, dans la pensée de celui qui l'a promulgué, être qu'un synonyme du mot aphémie, que les hellénistes raffinés trouvaient trop béotien. La Béotie, si elle était quelque part, n'était pas de mon côté. On objectait que la seconde syllabe du verbe qnuí, étant une finale, ne pouvait entrer dans la composition des dérivés; mais on oubliait l'exis-

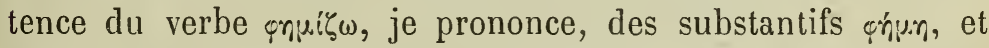
$\varphi \tilde{\eta}$ u. $\varsigma$, voix et parole, et enfin de l'adjectif äsnu.os et de l'adverbe aqnu. Grecs, les vrais Grecs de l'Hellade, employaient sans se douter que les Athéniens de Paris leur donneraient plus tard une leçon de grec. Moi aussi, lorsque, pour la première fois, je cherchai un nom pour désigner la perte de la faculté spéciale du langage articulé, j'avais d'abord songé au mot aphasie ; mais je l'avais écarté, parce que les lexiques lui donnaient une signification précise, une acception toute morale, exprimant l'état mental d'un raiscnneur embarrassé, qui reste court en attendant qu'il lui vienne un argument. Sachant combien les hellénistes sont difficiles, je n'avais pas osé, et plus je n'avais pas voulu, dé- 
tourner entièrement ce mot de son acception classique, et j'avais cru nécessaire de créer un mot nouveau pour désigner une catégorie nouvelle. Quoi qu'il en soit, je suis loin de regretter que feu le professeur Trousseau se soit servi du mot aphasie, car, après l'avoir appliqué d'abord à des cas semblables à ceux que j’avais rapportés et caractérisés dans mon premier mémoire, il n'a pas tardé à l'étendre à des cas tout différents, à l'amnésie verbale ou même générale, à des cas de congestion cérébrale passagère, et surtout à bon nombre de cas incertæ sedis, dont la symptomatologie n’a pas été suffisamment analysée. Il a ainsi rétabli dans la pathologie du langage, une partie des confusions que j'avais cherché à dissiper, et je préfère, pour ma part, que ces confusions se soient répandues sous l'étiquette de l'aphasie, plutôt que sous celle de l'aphémie, qui conserve, dès lors, sa première signification. Je ne propose point d'ailleurs de supprimer le mot aphasie. Son défaut de précision permet de l'utiliser dans les cas où diverses complications rendent le diagnostic incertain. Par exemple, lorsqu'on n'a pas pu déterminer, faute de renseignements suffisants, si un individu qui ne parle pas est aphémique ou amnésique, il est bon de pouvoir dire du moins qu'il est aphasique; mais si un nouvel examen ou de nouveaux renseignements, ou le développement ultérieur de la maladie, viennent donner au diagnostic la certitude qui lui manquait, alors il y a lieu de remplacer le terme vague d'aphasie par le mot précis qui exprime le diagnostic.

Sur un sujet aussi ardu, étudié depuis si peu d'années, relatif aux lésions d'un organe aussi complexe que le cerveau et dont la physiologie est si peu avancée, lorsqu'il s'agit de fonctions propres à l'homme, dont l'étude ne peut être éclairée par les vivisections, et lorsque enfin on n'a d'autre moyen d'information que l'observation des désordres morbides, lesquels, presque toujours, atteignent à la fois plusieurs parties de l'encéphale et troublent plusieurs fonctions, l'analyse des cas particuliers offre une difficulté exceptionnelle, et quelquefois insoluble dans l'état actuel de nos connaissances. Ne soyons donc pas étonnés si le diagnostic des diverses espèces de troubles du langage est souvent obscur et quelquefois impossible. Mais ne renonçons pas pour cela à l'espoir d'élucider cet important problème. Il est 
clair toutefois que si nous pouvons avoir quelque chance d'y réussir, ce n'est pas en nous heurtant de front contre les cas compliqués et incertains, c'est en nous attachant spécialement à ceux qui sont simples et évidents. Je suis loin, certes, de dédaigner les travaux qui ont été faits sur l'ensemble de la question; ils ont une grande importance pathologique, un grand intérêt psychologique, mais, pour le but que je me suis proposé, et qui consiste à chercher s'il existe dans le cerveau un siège particulier pour la fonction du langage, j'ai dû me restreindre à l'étude spéciale des cas d'aphémie bien déterminée. J'exposerai donc maintenant les notions à l'aide desquelles on peut établir pendant la vie le diagnostic de l'aphémie àvec assez de précision pour pouvoir ensuite, à l'autopsie, chercher la lésion qui a été la cause déterminante des troubles de la parole.

Le diagnostic est tout à fait certain lorsque les malades ont conservé toute leur intelligence, lorsqu'ils n'ont perdu que la faculté du langage articulé, qu'ils n'ont aucune paralysie de la langue, ni des membres, qu'ils peuvent continuer à lire, à écrire, à travailler du corps ou de l'esprit. Les cas de ce genre ne s'observent guère qu'à la suite de certaines lésions traumatiques; les lésions pathologiques peuvent cependant les produire aussi, mais c'est très exceptionnel. Le plus souvent (quoiqu'on m'ait fait dire le contraire) l'intelligence est plus ou moins altérée. Les malades ont perdu, en même temps que la parole, la lecture et l'écriture; ils sont, en outre, ordinairement atteints d'une hémiplégie qui, existant presque toujours à droite, les prive, pour le langage des gestes, de leur membre le plus expressif; enfin cette hémiplégie peut gagner la langue elle-même, et faire croire que l'altération de la parole n'est qu'une alalie mécanique. C'est alors que naissent les difficultés du diagnostic, et elles sont d'autant plus grandes, cela est évident, que l'intelligence est plus profondément troublée.

Il s'agit d'abord de distinguer l'aphémie de l'amnésie verbale. Lorsque le malade ne comprend plus du tout ce qu'on lui dit, lorsqu'il ne reconnaît ni son nom, comme cela arriva au grand orateur romain Messala Corvinus, ni celui de ses proches, ni celui de son pays, de sa résidence, de sa profession, et lorsqu'il montre cependant qu'il sait reconnaître, à première vue, les 
personnes et les choses, il est certain qu'il est amnésique. Mais il y a des degrés moins avancés d'amnésie verbale qui sont beaucoup moins évidents. L'amnésie peut être incomplète ; on peut se souvenir de certains mots et avoir oublié les autres. On peut ne pas comprendre certaines phrases et comprendre des phrases plus courtes, plus simples ou plus vulgaires. L'observateur' peut se demander alors si l'impossibilité où se trouve le malade de prononcer certains mots vient de ce qu'il en a oublié le sens, ou de ce qu'il a oublié comment on les prononce. Il serait amnésique dans le premier cas, aphémique dans le second. L'expérience à faire pour éclairer la question consiste, d'une part, à adresser au malade d'abord des paroles banales et usuelles, puis des phrases plus compliquées, et de voir à quel moment il cesse de comprendre; d'une autre part à essayer de lui faire prononcer les mots qui lui manquent; s'il les prononce immédiatement et sans effort il n'est pas aphémique, il est amnésique, puisqu'il a suffi pour le tirer d'embarras de lui rafraîchir la mémoire. Quant aux malades qui, dans le discours courant, disent un mot pour un autre, ils sont plus probablement amnésiques qu'aphémiques, quoiqu'il puisse cependant se faire, dans le début de l'aphémie, que la substitution des mots s'effectue par une sorte d'automatisme; mais alors, en général, le malade s'aperçoit de cette substitution et s'en afflige ou s'en impatiente; il recommence sa phrase, et arrivé au même point, il commet de nouveau la même erreur : ce caractère indique l'aphémie.

Il y a, comme on le voit, plusieurs traits distinctifs qui peuvent conduire à un diagnostic plus ou moins net; mais ils sont assez vagues au début; et ils deviennent de nouveau incertains dans une période plus avancée, lorsque la lésion, ayant fait des progrès, a porté une grave atteinte à l'intelligence. Il y a donc beaucoup de cas ambigus, qui ne peuvent, à cause de leur incertitude, peser d'un grand poids dans la balance lorsqu'on cherche, au moment de l'autopsie, à déterminer le siège de la faculté du langage. J'ajoute maintenant que si, au point de vue de l'analyse physiologique et pathologique, l'amnésie verbale et l'aphémie sont essentiellement différentes, il est assez probable qu'au point de vue du siège de la lésion qui les produit, elles doivent se rapprocher beaucoup, car il y a des raisons de supposer que 
la mémoire des mots doit avoir des relations étroites avec la partie de l'encéphale qui régit la prononciation des mots. Dans le fait, il m'est arrivé de trouver à l'autopsie d'une vieille femme que j'avais jugée atteinte d'amnésie verbale, une lésion qui avait le même siège que celles de l'aphémie; mais l'amnésie verbale est quelquefois si difficile à distinguer, surtout lorsqu'elle est très avancée, de l'amnésie générale qui dépend d'une atteinte générale de l'intelligence, - qu'il est prudent de ne pas faire intervenir les cas d'amnésie, quels qu'ils soient, dans les investigations qui ont pour but la recherche du siège de la faculté du langage articulé.

L'aphémie doit être distinguée, maintenant, des troubles de la parole qui rentrent dans le groupe désigné sous le nom d'alogie, et qui sont la conséquence de l'abolition plus ou moins complète de l'intelligence.

On voit souvent des individus presque entièrement abrutis et qui cependant expriment le peu d'idées faibles ou absurdes qui eur restent, par des mots convenablement articulés. On en voit même qui n'ont plus aucunẻ connaissance, qui ne comprennent rien, qui ne reconnaissent personne, qui unt évidemment perdu la totalité de leur intelligence, et qui cependant continuent à articuler aisément, quelquefois avec volubilité ; ils parlent tout à fait au hasard; ils débitent des enfilades de mots qui n'ont aucun sens, ou récitent hors de propos des phrases depuis longtemps stéréotypées dans leur esprit; mais, quoiqu'ils ne comprennent nullement ce qu'ils disent, ils continuent cependant à prononcer correctement. Telle était cependant cette vieille femme qui fut admise, en 1864, dans le service de M. Vulpian, à la Salpêtrière, et qui ne connaissait plus personne; qui ne répondait à aucune personne, et qui ne parlait jamais que pour réciter son credo en latin. Elle répétait presque sans erreur cette prière et l'articulait distinctement. A l'autopsie, on trouva un ramollissement diffluent du lobe occipital et du lobe pariétal du côté droit ; les deux lobes antérieurs étaient relativement sains, et on en a tiré une objection contre la localisation de la faculté du langage; or, ce qui avait péri chez cette femme, ce n'était certainement pas la faculté du langage qui seule avait survécu, lorsque toutesles autres facultés étaient éteintes. Elle était atteinte 
d'alogie sans aphémie. Mais les cas de ce genre sont rares. Le plus souvent la parole périt en même temps que l'intelligence, et, lorsque les lésions sont assez avancées pour que les malades cessent de comprendre ce qu'on leur dit, sans cesser pour cela d'émettre des mots sans suite ni sens, leur prononciation embarrassée, incertaine, ou même tout à fait inintelligible, rappelle plus ou moins celle des aphémiques.

D’un autre côté, les lésions qui produisent l'aphémie s'étendent souvent à un grand nombre de circonvolutions, et il en résulte que beaucoup d'aphémiques ont perdu une grande partie de leur intelligence. Ils ne pourraient manifester les idées qui leur restent que par le langage des signes, et ils n'ont plus assez de ressources dans l'esprit pour inventer ce nouveau langage ; on n'a donc aucun moyen de reconnaître l'état de leurs facultés, et on peut croire qu'ils n'ont plus d'idées à exprimer, quoique en réalité ils en aient encore.

Ainsi, l'alogie peut simuler l'aphémie et réciproquement. 11 y a beaucoup de cas où le diagnostic est à peu près impossible, et beaucoup d'autres où il ne repose que sur des probabilités; mais il y en a malheureusement un plus grand nombre où il est tout à fait certain. Toute espèce de doute disparaît lorsque le malade, malgré la perte de la parole, donne des signes bien manifestes d'une intelligence capable d'accomplir des actes plus difficiles que la parole elle-même, des actes que ne pourraient pas exécuter d'autres malades qui conservent encore la parole. En d'autres termes, le diagnostic est établi lorsqu'on constate que le malade a évidemment plus d'intelligence qu'il n'en faut pour parler. Cette constatation ne peut être soumise à aucune règle formulée; elle doit être laissée ì la sagacité de l'observateur. Elle est en général facile lorsque l'aphémie est incomplète. Lorsqu'elle est plus ou moins complète, et lorsqu'en outre la mimique est imparfaite, il est quelquefois difficile de savoir si le malade est encore capable de faire un raisonnement, et de concevoir des idées abstraites. Il faut alors varier les questions, choisir celles qui, tout en étant susceptibles de se prêter à une réponse très simple, exigent cependant un certain degré de réflexion préalable. Les questions de nombre sont celles qui peuvent donner les résultats les plus significatifs. Par exemple, lorsqu'un 
malade sait exprimer avec ses doigts des nombres au-dessus de dix, lorsqu'il peut, comme un de ceux dont j'ai publié l'observation, indiquer son âge en désignant d'abord, par un premier signe, le chiffre des dizaines, et par un second signe le chiffre des unités, toute incertitude se trouve aussitôt dissipée.

Parlons enfin du diagnostic de l'aphémie ou de l'alalie mécanique. On a cru qu'il suffisait de constater, pour résoudre la question, l'existence ou l'absence de l'hémiplégie de la langue; cette notion, si elle était admise sans réserve, serait tout à fait trompeuse. Certains aphémiques ont une hémiplégie plus ou moins complète de la langue; la pointe de cet organe est déviée ainsi que la luette, et, de plus, ils avalent maladroitement, ce qui indique un trouble de l'innervation des muscles du voile du palais et du pharynx. L'existence d'une paralysie de la langue ne suffit donc pas pour écarter l'idée de l'aphémie. D'un autre côté, on sait que beaucoup d'hémiplégiques, dont la langue est déviée depuis longtemps, finissent par parler assez bien; la paralysie partielle de la langue ne produit donc pas nécessairement une altération profonde et définitive de la parole. N'oublions pas enfin que les muscles de l'articulation sont souvent paralysés à un degré suffisant pour produire de grands troubles du langage, sans que la langue soit le moins du monde déviée, sans qu'elle ait même cessé d'exécuter à volonté les mouvements d'ensemble. C'est ce qui a lieu en particulier dans la paralysie générale. Les malades, lorsqu'on les en prie, peuvent tirer la langue, la porter à droite et à gauche, et cependant leur parole est très altérée; ils ne peuvent articuler distinctement certaines syllabes; ils suppriment souvent quelques-unes des syllabes intermédiaires des mots qui ont une certaine longueur. Et cela se conçoit aisément, car il ne suffit pas, pour parler, de pouvoir contracter les muscles de l'articulation, il faut pouvoir les maîtriser entièrement et leur imprimer, arec une très grande précision, des mouvements extrêmement délicats et combinés de mille manières; de sorte qu'un trouble d'innervation qui, sur les membres, ne produirait qu'un affaiblissement à peine appréciable, peut, lorsqu'il existe sur la langue, amener une altération très notable de l'articulation. Voilà pourquoi le tremblement de la voix et la difficulté de la parole constituent très fréquemment le premier symptôme 
physique, et quelquefois même le seul symptôme du début de la paralysie générale. Nous devons rappeler, toutefois, que M. Baillarger a constaté, dès le début de cette affection, l'existence d'une sclérose très manifeste de la substance blanche de toutes les circonvolutions des lobes frontaux, avant même que la substance grise environnante soit le siège d'aucune lésion apparente, et avant que les circonvolutions des autres lobes soient altérées. Il ne serait donc pas impossible que cette sclérose des circonvolutions frontales troublat le jeu de celle de ces circonvolutions que je crois affectée à la fonction du langage articulé; s'il en était ainsi, les troubles du langage qui accompagnent la paralysie générale ne seraient pas sans avoir quelque analogie avec ceux de l'aphémie; mais c'est une question que je ne veux pas aborder aujourd'hui, et dont la solution est peut-être encore éloignée.

Quoi qu'il en soit, on voit que le diagnostic de l'aphémie et de l'alalie mécanique est loin d'être aussi facile qu'on l'a dit. Si on ne s'en rapportait qu'à l'examen physique de la langue, on serait exposé à commettre souvent des erreurs. L'analyse physiologique du mécanisme de la parole ne fournit même pas des données certaines. Quoique ordinairement l'hémiplégie de la langue, la paralysie générale et les lésions de l'aphémie ne troublent pas le langage de la même manière, cependant il y a beaucoup de cas où ces trois formes se ressemblent assez pour donner le change. L'analyse psychologique, dans ces cas douteux, peut être d'un grand secours. Ainsi, la paralysie générale amène tôt ou tard un dérangement intellectuel bien caractérisé, et bien différent de celui qui accompagne habituellement l'aphémie. L'hémiplégie linguale, d'une autre part, peut exister chez des individus qui conservent toute leur intelligence, qui continuent à lire, à calculer, à écrire lorsque leur main droite est libre, qui apprennent même, lorsqu'elle est paralysée, à écrire de la main gauche. Ajoutons enfin que les uns et les autres, hémiplégiques ou paralytiques, ne cherchent généralement pas à suppléer par des signes à l'imperfection de leur parole. L'aphémique, au contraire, sentant les mots lui échapper, et reconnaissant, non sans impatience, l'impuissance des efforts qu'il fait pour parler, cherche à suppléer par la mimique à la faculté qui lui manque; il a des gestes expressifs, une physionomie mobile, souvent fine 
et même railleuse qui lui donne l'apparence d'un individu très intelligent, alors même que la plupart de ses facultés sont très affaiblies.

Je viens de comparer successivement l'aphémie avec les trois autres espèces de perte de la parole, etje crois avoir prouvé que, s'il est des cas où le diagnostic est certain, il y en a d'autres où il est plus ou moins douteux; et ceux-ci sont même plus fréquents que les autres. J'ai été appelé, il y a quelques années, à donner, le même jour, mon avis sur cinq malades qui avaient plus ou moins perdu la parole; et, après les avoir examinées avec le chef de service, je démontrai qu'une seule était réellement aphémique. Voilà pourquoi j’ai cru devoir insister si longuement sur la question du diagnostic. Le plus grand intérêt de l'étude des troubles de la parole est la recherche du siège de la faculté du langage articulé. G'est le but principal que je me suis proposé dès l'origine dans les recherches qui ont attiré l'attention des observateurs sur ce sujet, jusqu'alors trop peu exploré. Pour cela, sous peine de s'exposer à des erreurs continuelles, sous peine d'introduire, dans la science, des observations trompeuses, il faut ne faire figurer dans les relevés anatomiques que les cas où le diagnostic de l'aphémie a été tout à fait certain pendant la vie, et ces cas sont encore assez communs pour qu'on ait $\mathrm{pu}$, depuis sept ans, en recueillir un bon nombre.

Parmi ces cas choisis pour l'investigation anatomique et physiologique, j'en sigualerai quelques-uns qui sont parfaitement clairs. Il y a d'abord l'aphémie traumatique; lorsqu'un individu reçoit à la tête une blessure qui lui fait perdre tout à coup la parole, et qu'au bout de quelques heures ou de quelques jours, après avoir recouvré complètement sa connaissance et ses mouvements, il reste impuissant à parler, on peut le considérer comme aphémique. L'aphémie subite, produite par une attaque d'apoplexie ou par une embolie cérébrale, et qui se maintient ensuite définitivement, ou du moins pendant un temps assez long, après le retour de la connaissance; constitue encore un cas bien déterminé.

Un autre cas tout aussi clair est celui où l'aphémie, survenue peu à peu, a été observée dans sa progression graduelle, lorsqu'on a pu assister en quelque sorte à la destruction du langage, 
qu'on a vu les mots disparaître l'un après l'autre, et qu'enfin il ne reste plus au malade qu'un court vocabulaire, composé de quelques mots, de quelques monosyllabes, qu'il prononce assez bien, et qu'il applique dans un sens déterminé; si, en outre, par l'emploi de ces mots et par sa mimique expressive, il montre qu'il a encore une quantité notable d'intelligence, et qu'il comprend bien ce qu'on lui dit, on peut diagnostiquer l'aphémie avec certitude.

Je n'ai pas cru devoir, dans les considérations précédentes, m'occuper de la partie du diagnostic qui consiste à déterminer la nature de la lésion cérébrale. S'agit-il d'une apoplexie ou d'une embolie, ou d'un ramollissement aigu ou chronique, ou d'une atrophie circonscrite ou progressive, ou d'une tumeur de la pie-mère, ou de toute autre lésion? Cette partie du diagnostic n'est sans doute point sans intérêt; mais elle ne présente ici rien de bien spécial. Le trouble du langage peut bien servir à deviner le siège de la lésion, mais non à en diagnostiquer l'espèce. Je signalerai cependant un cas où la connaissance du siège ordinaire des lésions de l'aphémie peut amener le clinicien à la constatation d'un phénomène important. Sur plusieurs aphémiques j'ai reconnu, à la main et au thermomètre, l'existence d'une élévation de température au niveau de la région qu'occupe la troisième circonvolution frontale gauche, c'est-àdire vers le milieu d'une ligne tirée du pavillon de l'oreille à l'angle externe du sourcil. Dans un cas que j'ai observé cette année même, la température du côté gauche était supérieure de deux degrés et demi centigrades à celle du côté droit. Le malade avait depuis peu perdu la parole, à la suite d'une attaque qui d'ailleurs paraissait légère. D'après l'examen thermométrique, je diagnostiquai un ramollissement aigu et portai un pronostic fâcheux qui se réalisa au bout de quelques semaines. Dans un autre cas où l'aphémie dépendait d'une ancienne apoplexie, le thermomètre marqua exactement la même température des deux côtés. Il est clair que ces phénomènes thermométriques doivent varicr suivant la nature et l'étendue de la lésion. Il y a donc là un élément qui peut être utilisé dans le diagnostic, mais je me borne à le signaler, parce que les faits dont je dispose sont encore trop peu nombreux pour se prêter à des conclusions. 


\title{
SUR LE POIDS RELATIF
}

\section{DES DEUX HÉMISPHĖRES CÉRÉBRAUX}

\author{
ET DE LEURS LOBES FRONTAUX
}

(Bulletins de la Société d'anthropologie, $2^{\theta}$ série, 1875, t. X, p. 534-536.)

Depuis 186I, époque où j’ai reconnu que la faculté du langage est localisée dans la troisième sirconvolution frontale de l'hémisphère gauche, et que, par conséquent, les deux hémisphères cérébraux n'ont pas les mêmes attributions, j'ai pesé comparativement ces deux hémisphères et leurs trois principales régions chez tous les individus dont j'ai fait l'autopsie dans les hôpitaux. J'ai suivi le procédé suivant : je pèse d'abord, comme on le fait d'ordinaire : $1^{\circ}$ l'encéphale entier avec ses membranes, $2^{\circ}$ le bulbe, $3^{\circ}$ le cervelet, $4^{\circ}$ la protubérance; puis je prends séparément le poids des deux hémisphères cérébraux dépouillés de leurs membranes. Enfin, je pratique sur chaque hémisphère deux coupes qui détachent d'une part le lobe frontal, d'une autre part le lobe occipital, et j'obtiens trois tronçons que je pèse séparément. Le tronçon moyen comprend à la fois le lobe pariétal et le lobe temporo-sphénoïdal.

J'ai recueilli ainsi 440 observations détaillées qui remplissent trois grands registres et que je n'ai pas eu jusqu'ici le temps de dépouiller; mais il y a quelques mois, un candidat au doctorat m'ayant demandé des renseigrements sur cette question, j’ai fait deux petits relevés partiels comprenant 19 hommes de l'hospice de Bicêtre (à partir du $n^{\circ}$ ら้0) et 18 hommes de l'hôpital Saint-Antoine (à partir du n²00) et j'ai obtenu les chiffres moyens que je vous communique provisoirement aujourd'hui. 


\begin{tabular}{|c|c|c|c|c|c|}
\hline Hospice de Bicêt & & $\begin{array}{l}\text { Droit. } \\
531.31\end{array}$ & $\begin{array}{l}\text { Gauche. } \\
530.84\end{array}$ & $\begin{array}{l}\text { Différ. } \\
-0.47\end{array}$ & $\begin{array}{l}\text { Somme. } \\
1062.15\end{array}$ \\
\hline (Age moyen : 64 ans 6 & & 227.57 & 232.10 & +4.53 & 459.67 \\
\hline Hôpital Sai & Hémisphèr & 575.83 & 574.39 & -1.44 & 1150.22 \\
\hline (âge $n$ & Lobe fro & 245.05 & 248.50 & +3.45 & 493.55 \\
\hline
\end{tabular}

Le poids des hémisphères est beaucoup moindre à Bicêtre qu'à l'hôpital Saint-Antoine ; on pouvait s'y attendre, parce que les gens de l'hospice de Bicêtre sont plus âgés, et qu'ils ne prennent plus part à la vie active; mais ces deux catégories donnent des résultats analogues en ce qui concerne le poids relatif des deux hémisphères. Dans les deux cas, l'hémisphère droit, dans son ensemble, pèse un peu plus que le gauche, et cependant le lobe frontal gauche l'emporte sur le droit d'une quantité très notable. Si ce résultat est confirmé par des relevés plus étendus, il ne manquera pas d'importance.

\section{DISCUSSION.}

M. Auburtin demandis à M. Broca s'il se croit autorisé à conclure de ses recherches que cette différence est due à l'état de la troisième circonvolution frontale.

M. Broca. Je ne me crois pas en mesure d'étendre mes conclusions jusque-là. Il faudrait avoir recours ici à l'expérience directe, qui me paraît à peu près impossible. Lorsque nous séparons le lobe frontal du reste de l'hémisphère, le couteau est dirigé par le sillon de Rolando; il n'en serait pas de même si nous voulions isoler la troisième circonvolution. Les coupes ne pourraient jamais être assez précises. Cependant il y a un ordre de recherches qui peut permettre de faire le tour de la question. La troisième circonvolution est située au niveau et en arrière de la petite région crânienne que nous appelons le ptérion. Il paraît donc assez probable que les variations de largeur du ptérion dépendent en partie du volume de cette circonvolution. Or, le ptérion gauche est en moyenne un peu plus large que le droit.

M. LuNier fait remarquer que l'on sait déjà par d'autres observations que le lobe frontal gauche joue un rôle plus important et qu'on peut en déduire la présomption que l'observation de M. Auburtin est dûment motivée. 
M. Auburtin n'entendait pas demander à M. Broca des pesées; il ne voulait que le provoquer à donner son opinion sur l'influence qu'il attribue à la troisième circonvolution dans le développement du lobe frontal gauche.

M. Broca est tout disposé à croire à cette influence.

M. Delasiauve a presque toujours rencontré l'égalité entre les deux hémisphères. Un écrit d'un de nos collègues de Quimper avait affirmé leur inégalité chez les épileptiques. M. Delasiauve a vérifié et n’a pas trouvé les différences considérables annoncées. La règle, c'est l'égalité des deux luémisphères, et cette règle s'est trouvée confirmée même chez les épileptiques.

M. Broca a souvent trouvé entre les deux hémisphères, chez des adultes exempts de toute affection cérébrale, des différences de 5 à 6 grammes, et même de 10 à 14 grammes.

M. Lunier croit que le numbre des observations est insuffisant pour permettre des conclusions générales.

II. Bertillon rappelle que l'on peut supposer qu'il y a des gauchers et des droitiers du cerveau comme il ${ }^{-} \mathrm{y}$ en a pour la main. 


\title{
DIFFÉRENGE FONGTIONNELLE
}

DES

\author{
DEUX HÉMISPHĖRES CÉRÉBRAUX
}

(Bulletins de l'Académie de médecine, 1877, p. 508-539.)

Rapport à l'Académie de médecine sur un mémoire de $M$. Armand de Fleury, au nom d'une commission composée de MM. Baillarger, Gavarret et Broca, rapporteur (seance du 15 mai 1877).

\section{Messieurs,}

M. le docteur Armand de Fleury, professeur à l'Ecole de médecine de Bordeaux, a fait parvenir à l'Académie, au mois de février 1874, un mémoire très étendu intitulé : Recherches anatomiques, physiologiques et cliniques sur l'inégalité dynamique des deux hémisphères cérébraux.

Les premières études de l'auteur sur ce sujet datent déjà de douze ans. Elles remontent à une époque où le rôle spécial de l'hémisphère gauche du cerveau dans la fonction du langage était encore vivement contesté. L'idée d'attribuer des actions différentes à deux appareils aussi symétriques ou plutôt aussi peu dyssymétriques que le sont les deux hémisphères cérébraux, soulevait des résistances bien naturelles. Ce n'était pas sans hésiter que je l'avais émise. Après avoir, en moins de deux années, recueilli onze observations d'aphémie, suivies d'autopsie, et avoir constaté que dans tous ces cas la lésion cérébrale occupait constamment la troisième circonvolution frontale gauche, j'avais cru pouvoir signaler à la Société de biologie et à la Société d'anthropologie l'étrange prédilection des lésions de l'aphémie pour l'hémisphère gauche; mais je disais pourtant, 
quelques jours plus tard, dans une notice adressée à l'Académie: " J'espère encore que d'autres plus heureux que moi trouveront enfin un exemple d'aphémie produite par une lésion de l'hémisphère droit. Jusqu'ici, c'est toujours la troisième circonvolution frontale gauche qui a été atteinte; s'il fallait admettre que les deux moitiés symétriques de l'encéphale ont des attributions différentes, ce serait une véritable subversion de nos connaissances en physiologie cérébrale. Je pense donc qu'avant d'accepter les conséquences qui pourraient en découler, il faudrait prouver, par des observations suivies d'autopsie, que les lésions du tiers postérieur de la troisième circonvolution frontale droite ne portent pas atteinte à la faculté du langage (1) ».

Aujourd'hui les faits se sont considérablement multipliés. On a vu, dans un certain nombre de cas, la perte de la parole coïncider avec des lésions de l'hémisphère droit. 'Toutefois ces cas, dont la fréquence relative n'excède pas le nombre proportionnel des gauchers, fixé à 1 sur 12 ou 13 par les recherches statistiques de Malgaigne (2), ces cas, dis-je, sont restés à l'état d'exception, et l'existence d'une relation particulière entre Ia faculté du langage et l'hémisphère gauche, chez la très grande majorité des individus, est un fait maintenant bien établi.

Mais, en 1864, lorsque M. Armand de Fleury écrivit son Mémoire sur la pathogénie du langage articulé (3), la question était beaucoup moins avancée, et la différence fonctionnelle des deux hémisphères cérébraux était tellement contraire aux idées reçues, qu'elle fut souvent qualifiée de paradoxe physiologique. Les esprits n'y étaient pas encore habitués ; moi-même, comme on vient de le voir, je ne m'y étais pas rendu sans répugnance; et lorsqu'on invoquait contre elle " les lois immuables de la symétrie physiologique ", on croyait l'avoir suffisamment réfutés. M. Armand de Fleury fut donc tout d'abord porté à la rejeter. Il ne pouvait se dissimuler cependant que, dans la

(1) Exposé des titres scientifiques de $M$. Broca, avril 1863 , in- $\{0$, p. 67.

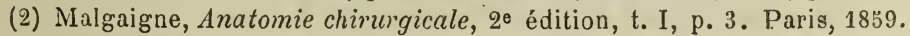

(3) Armand de Fleury, Sur la pathogénie du langage articulé, couronné le 9 février 1863 par l'Académie des sciences, belles-lettres et arts de Bordeaux. Paris, $\mathbf{1 8 6}$, brochure in-80 de 56 pages. Publié en partie dans la Grazette hebdomadaire, 1865, p. 229 et 244 . 
grande majorité des cas publiés par d'autres auteurs ou observés par lui-même, la perte de la parole avait coïncidé avec des lésions de l'hémisphère gauche. Cherchant l'explication de ce fait embarrassant, il se demanda s'il ne tenait pas à un autre fait plus général, à une plus grande susceptibilité pathologique de l'hémisphère gauche; un relevé qu'il fit dans les deux hospices de Bordeaux lui permit, en effet, de constater que, sur seize hémiplégiques qui existaient alors dans ces deux établissements, 11 étaient paralysés à droite et "̋ seulement à gauche; il put donc supposer que, d'une manière générale, l'hémisphère gauche était frappé beaucoup plus fréquemment que le droit, et il ajouta :

" $\mathrm{Si}$, comme nous avons lieu de le croire, ce rapport de proportion est en général exact, il n'y a plus à s'étonner si l'on trouve plus de lésions à gauche qu'à droite dans les pertes de la parole. Il y aurait seulement à chercher la cause physiologique de ce phénomène remarquable. Peut-être l'avons-nous trouvée dans la différence de calibre des vaisseaux artériels qui, de droite et de gauche, partent de la crosse de l'aorte pour monter au cerveau. C'est une loi de physique, que toutes les fois qu'une colonne liquide passe subitement d'un calibre plus fort dans un calibre moindre, la vitesse de la colonne liquide est proportionnellement augmentée en raison de l'augmentation des pressions égales et contraires des parois sur la colonne liquide. Or, chacun sait que, tandis qu'à droite le tronc brachio-céphalique établit une transition entre le diamètre de l'aorte et celui de la carotide, à gauche le sang artériel passe brusquement du diamètre de l'aorte à celui de la carotide primitive. Si les lois physiques sont applicables à la physiologie, il y aurait donc, d'une manière générale, plus d'activité dans la circulation cérébrale à gauche qu'à droite; de là, en vertu de l'effet croisé, une plus riche hématose du côté gauche de l'encéphale, et par suite la prééminence des membres droits sur ceux du côté gauche pour la force et l'adresse; de là aussi une plus grande tendance aux hémorrhagies à gauche qu'à droite de l'encéphale (1).

Les idées de l'auteur, en ce qui concerne la localisation du

(1) A. de Fleury, Essai sur la pathogénie du langage articulé, p. 53, et Gazette hebdomadaire, 1865, p. 249. 
langage, se sont considérablement modifiées depuis; il s'est rallié résolument à la doctrine de la différence fonctionnelle des deux hémisphères. J'ajoute que de nouveaux relevés faits en 1868 et en 1873 dans les hospices de Bordeaux lui ont fourni la preuve que la fréquence de l'hémiplégie est à peu près la même à droite et à gauche (1), et que le fait dont il arait cherché l'explication anatomique était par conséquent illusoire. J'ai cru deroir néanmoins reproduire le passage précédent, parce que l'auteur l'invoque à bon droit pour établir la priorité de son opinion sur l'inégale activité de la circulation dans les deux hémisphères cérébraux.

Peu de mois après la publication de son mémoire Sur la pathogénie du langage articulé, M. Armand de Fleury communiqua au Congrès médical de Bordeaux un travail intitulé : Des tentatives de lucalisation de la parole d'un seul côté du cerveau. Il y reproduisit les mêmes idées sur la symétrie fonctionnelle des hémisphères, sur la plus grande fréquence des hémorrhagies cérébrales dans l'hémisphère gauche, et sur la cause anatomophysiologique de ce fait, qu'il continuait à croire réel ; mais il fit intervenir dans cette explication un élément de plus. "Le tronc brachio-céphalique, dit-il, établit à droite une ligne brisée entre l'aorte et la carotide primitive. Cette ligne brisée et l'angulaison qui en résulte sont absentes à gauche (2). "

Il est donc parfaitement démontré que M. Armand de Fleury, dans deux publications qui datent de $\mathbf{1 8 6 5}$, a nettement exprimé l'opinion que la circulation est plus active dans l'hémisphère gauche du cerveau que dans l'hémisphère droit, par suite de la dyssymétrie des vaisseaux de la crosse aortique. C'est seulement en 1867 que M. William Ogle, de Saint-George's Hospital, a exprimé la même idée dans une courte note additionnelle qui suit son important mémoire intitulé : Aphasia and Agraphia. « Il doit y avoir, dit-il, entre les deux hémisphères, une diffé-

(1) Sur 57 cas, l'hémiplégie existait 29 fois à droite et 28 fois à gauche (voir Armand de Fleury, Du dynamisme comparé des hémisphères cérébraux chez l'homme. Paris, 1873, p. 142). A la page 170 du même volume, l'auteur produit un relevé extrait de l'ouvrage de $M$. Gintrac père, d'où il résulte que, sur 199 cas d'hémiplégie, il y a eu 102 hémiplégies à gauche et 97 à droite.

(2) Actes du Congrès médical de Bordeaux (1865), p. 474. 
rence qui détermine le choix de l'un d'eux pendant l'éducation du langage. Cette différence peut-elle être indiquée? Je pense que oui. D'après la disposition hien connue des artères qui naissent de la crosse aortique, l'hémisphère gauche reçoit le sang par la carotide plus directement et plus librement que le droit »(1). M. Ogle est revenu plus amplement sur ce sujet dans un travail très curieux, communiqué en 1871 à la Société médico-chirurgicale de Londres (2). Dans ce mémoire, qui runferme des études très intéressantes sur les droitiers et les gauchers, l'auteur attribue la prédominance habituelle des membres droits, et par conséquent du cerveau gauche, à la disposition asymétrique des troncs vasculaires sus aortiques; mais il ne cite pas les publications antérieures de M. Armand de Fleury. Celuici a donc tenu à faire valoir ses droits de priorité, et c'est dans cette pensée qu'il a adressé à l'Académie le mémoire qui fait le sujet de ce rapport. D'ailleurs il ne s'est pas borné à reproduire ses vues de 1865 ; il en a rectifié quelques-unes, il a précisé et développé les autres; il y a joint de nombreuses recherches d'anatomie humaine et comparée, de physiologie et de pathologie, qui témoignent d'un esprit original et plein de ressources, mais parfois dominé par des théories prématurées.

En ce qui concerne la question de priorité, les passages que j'ai reproduits établissent suffisamment les droits de M. Armand de Fleury. J'ai suivi avec intérêt les nombreuses publications relatives au rôle prépondérant de l'hémisphère gauche dans la fonction du langage et dans l'action des membres, et j'ai lieu de croire qu'aucun autre avant notre confrère bordelais n'a cherché dans les conditions, anatomiques de la circulation l'explication de cette prépondérance. Mais les deux causes qu'il invoque ne me paraissent pas également réelles ou, pour mieux dire, l'une d'elles seulement est réelle, tandis que l'autre est tout à fait imaginaire.

Ges deux causes, ainsi qu'on vient de le voir, sont: en premier lieu, l’inégale largeur des orifices du tronc brachio-céphalique à droite et de l'artère carotide primitive à gauche; en

(1) Saint Gecrge's Hospital Reports, vol. II, p. 121. I Londres, 1867. In-80.

(2) W. Ogle, On Dextral Preeminence (in Medico-Chirurgical Transuctions, vol. LIV, p. 279. Londres, 1871. In-80). 
second lieu, la bifurcation supplémentaire qui résulte, à droite, de la présence du tronc brachio-céphalique, et la déviation angulaire qu'y subit la colonne sanguine.

Sur le premier point, je ne saurais accepter les idées de l'auteur. 11 s'efforce de démontrer, par des arguments empruntés à l'hydrodynamique, que, de deux artères nées d'un même tronc, la plus étroite est celle où le sang se meut avec le plus de vitesse, et il en conclut que le cours du sang doit être plus rapide dans l'artère carotide gauche que dans le tronc brachio-céphalique. Je pourrais répondre que les raisons qu'il invoque ne sont pas applicables au cas qu'il considère; qu'un rétrécissement placé sur le trajet d'un tube, quoique produisant à ce niveau l'accélération du liquide, constitue un obstacle qui diminue le débit au lieu de l'augmenter ; qu'en outre, un embranchement latéral n'est nullement comparable à un rétrécissement, puisqu'il n'en résulte aucun obstacle à la progression du liquide; que, d'ailleurs, un système clos, comme le śystème vasculaire, diffère entièrement des systèmes hydrauliques ordinaires, où le liquide s'écoule à travers des orifices libres; qu'enfin l'élasticité des artères et leur contractilité, d'autant plus forte que les calibres sont plus petits, constituent des conditions toutes spéciales et impossibles à réaliser dans les expériences hydrodynamiques. L'ensemble de ces considérations permettrait déjà de rejeter sans hésitation la théorie en vertu de laquelle l'inégalité de calibre de la carotide gauche et du tronc brachio-céphalique rendrait la marche du sang plus rapide dans le vaisseau le plus étroit, c'est-à-dire dans la carotide gauche. Mais, alors même que l'auteur aurait raison sur ce point, il n'en résulterait aucune inégalité fonctionnelle entre les deux carotides primitives; car, en vertu du même principe, il devrait se produire, à l'origine de la carotide droite sur le tronc brachio-céphalique, une accélération qui compenserait le prétendu ralentissement de la circulation dans ce dernier tronc, et qui rétablirait ainsi l'égalité de la circulation dans ces deux carotides. Je dois dire que M. Armand de Fleury a prévu cette objection; il s'est efforcé d'y répondre en faisant remarquer que le calibre du tronc brachio-céphalique est supérieur à la somme des calibres de ses deux branches de bifurcation et que, par conséquent, le cours 
du sang doit être ralenti au-delà de la bifurcation; le fait est vrai, mais ce n'est qu'un cas particulier d'une règle générale applicable à tous les troncs artériels et formulée en ces termes par Cruveilhier : «Le calibre d'un tronc artériel ne diminue pas an proportion du calibre des branches qu'il fournit. ") G'est une des causes qui font décroître la rapidité du sang dans l'arbre artériel à mesure que l'on considère des rameaux plus éloignés du cœur, et cette cause agit sur la carotide gauche aussi bien que sur la droite.

La première cause invoquée par l'auteur n'est donc pas réelle; mais la seconde ne me paraît pas contestable. L'influence que M. Armand de Fleury et, après lui, M. Ogle ont attribuée au mode d'origine des deux carotides est bien certaine, et ce point de physiologie est assez important pour que vous vouliez bien me permettre d'y insister.

Tout le monde sait aujourd'hui que la rapidité du cours du sang est loin d'être uniforme dans toutes les artères; que des causes multiples, diversement combinées, contribuent à produire ce résultat, et que la plupart d'entre elles agissent en absorbant une partie de la force vive communiquée à la colonne sanguine par la contraction du ventricule gauche du cœur.

Parmi ces causes, il en est trois qui nous intéressent ici, ce sont : $1^{\circ}$ le frottement des molécules sanguines sur les parois de tous les vaisseaux, même des vaisseaux rectilignes; $2^{\circ}$ la décomposition de force qui se produit partout où la colonne sanguine subit un changement de direction; $3^{\circ}$ le choc qui s'effectue au niveau des embranchements sur l'éperon de bifurcation et qui amène la division de la colonne.

La première influence est proportionnelle, toutes choses égales d'ailleurs, à la longueur du trajet parcouru. Or, si l'on considère la distance comprise, suivant l'axe des vaisseaux, entre le cœur et l'extrémité supérieure des deux carotides primitives, on trouve qu'à son entrée dans les carotides internes le sang a parcouru de 8 à 10 millimètres de plus à droite qu'à gauche. En d'autres termes, l'hémisphère gauche est un peu plus rapproché du cœur que l'hémisphère droit. La différence est bien faible, sans doute, et, en soi, elle n'aurait aucune impor- 
tance, mais elle est due à une disposition anatomique qui en accroît notablement les effets en donnant prise à la seconde influence.

Il est clair, en effet, que les deux hémisphères cérébraux sont situés à la même hauteur au-dessus de la crosse de l'aorte; si donc la longueur des vaisseaux qui s'y rendent n'est pas la même des deux côtés, c'est parce que le trajet du sang est plus direct à gauche qu'à droite. Ce trajet étant.symétrique à la tête et au cou, il suffit de le considérer à la base du cou et dans la poitrine.

Là, on voit que la carotide gauche naît de l'aorte, directement au-dessous de la région où elle doit se rendre, tandis que le trone brachio-céphalique, quoique appelé à se distribuer dans la moitié droite du corps, ne naît pas à droite de la ligne médiane, comme on pouvait s'y attendre. C'est à gauche de cette ligne qu'il se détache de l'aorte, reculant ensuite vers la droite et traversant obliquement presque toute la largeur de la trachée, avant de fournir le tronc ascendant de la carotide droite. Il résulte de cette disposition que la colonne sanguine suit un trajet beaucoup plus indirect à droite, qu'elle y subit par conséquent davantage le ralentissement qui est la conséquence des changements de direction.

Toute force communiquée à un corps tend à lui donner un mouvement rectiligne. Sous ce rapport, les molécules d'un liquide en mouvement ne diffèrent pas des projectiles solides; la vis à tergo les pousse dans une direction déterminée qui est représentée, pour chaque partie de la colonne, par la direction de l'axe de la partie correspondante du cylindre vasculaire, et elles se meuvent dans cette direction tant que le vaisseau reste droit; mais s'il se recourbe tant soit peu, les molécules, obéissant à leur mouvement rectiligne, viennent rencontrer obliquement la paroi vasculaire du côté de la convexité de la courbure, et à ce niveau la force quiles anime se décompose en deux forces, l'une perpendiculaire à la paroi, l'autre parallèle à la nouvelle direction du vaisseau. La première se perd dans la paroi et le liquide n'est plus poussé que par la seconde. Quant à la quantité de force perdue, elle croît avec l'angle de la déviation; elle est donc d'autant plus considérable que la courbure est plus forte. 
Cette influence des courbures artérielles est plus grande qu'on ne le croit généralement. C'est à elle qu'il faut attribuer l'inégal développement des deux moitiés de la tête, que l'on observe constamment, sur la face comme sur le crâne, chez les individus atteints de torticolis depuis leur enfance. Les gibbosités latérales de la région cervicale produisent le même résultat. Les deux troncs carotidiens s'infléchissent l'un et l'autre dans le sens de la tête et du cou, mais celui qui est situé du côté de la déviation décrit nécessairement une courbe plus courte et par conséquent. plus rapide; cela suffit pour y rendre la circulation moins active et pour amoindrir, dans la moitié correspondante de la tête, le travail de l'accroissement (1).

La dyssymétrie normale des deux appareils carotidiens est bien loin sans doute d'être équivalente à celle que pròduisent les déviations pathologiques de la tête et du cou, et elle ne peut produire que des effets beaucoup moindres. J'ai néanmoins cru devoir citer cet exemple pour montrer que l'obstacle opposé au cours du sang par les changements de direction n'est pas seulement théorique, et qu'il est capable d'exercer une influence assez sérieuse sur la structure et les fonctions des organes.

De cette cause résulterait déjà un avantage physiologique en faveur du système de la carotide gauche; mais ce système ne diffère pas seulement de l'autre par une moindre longueur et par une moindre courbure, il en diffère encore par une plus grande simplicité. Le sang qui pénètre dans la carotide gauche ne se heurte que sur une seule bifurcation, au niveau de l'origine de ce vaisseau, tandis que la colonne sanguine se brise sur deux bifurcations successives, avant de s'engager dans la carotide droite.

Partout où une artère se divise, il se produit un changement de direction sur l'une au moins des deux branches de bifurca-

(1) On a signalé depuis Iongtemps l'inégalité des deux moitiés de la face chez les sujets atteints de torticolis ancien. Je crois avoir constaté le premier qu'une asymétrie du crâne coïncide toujours avec celle de la face. Cielte déformation crànienne rentre dans le type de la plagiocéphalic. J'ai, en outre, démontré, à l'aide de la couronne thermométrique, que la température frontale et la température temporale du côté qui correspond au torticolis sont abaissées, en général, d'un demi-degré environ. 
tion, et souvent sur toutes les deux. Les effets de ce changement de direction, en ce qui concerne les carotides, ont été exposés plus haut, et je n'ai plus à y revenir. Mais toute bifurcation fait naître une autre cause de ralentissement, en obligeant la colonne sanguine à se diviser en deux courants plus ou moins divergents. Au niveau de l'embranchement, à l'opposite du cœur, la paroi vasculaire constitue un angle ordinaire aigu, quelquefois droit ou même obtus, qui forme à l'intérieur du vaisseau une sorte d'éperon. C'est sur cet éperon que la colonne vient se briser en produisant un choc qui absorbe une certaine quantité de force; en outre, les molécules du liquide, avant de se répartir entre les deux branches de bifurcation, hésitent en quelque sorte et font un remous comparable à celui que l'on observe lorsque le courantd'un fleuve se divise sur une pile de pont. La marche du sang se trouve donc troublée et ralentie par le fait même de la bifurcation, abstraction faite du degré de divergence des deux branches qui se séparent; mais il est clair que la division de la coionne sanguine est d'autant plus laborieuse que la divergence est plus grande. Lorsque la bifurcation s'effectue sous un angle très aigu, le choc est faible et la perturbation minime; mais l'obstacle s'accroît de plus en plus lorsque l'angle s'ouvre davantage, lorsqu'il devient droit, enfin et surtout lorsqu'il devient obtus.

Ainsi, dans le parallèle des deux systèmes carotidiens, il y a à considérer à la fois le nombre des bifurcations et leur degré de divergence.

Sous le premier rapport, l'avantage appartient évidemment à la carotide gauche, puisque la.colonne sanguine y pénètre après une seule bifurcation, tandis qu'à droite elle est obligée de franchir deux bifurcations successives.

Sous le second rapport, la carotide gauche est bien plus favorisée encore; il suffit de jeter un coup d'œil sur la crosse de l'aorte pour voir que la carotide gauche s'en détache sous un angle très aigu, tandis que le tronc brachio-céphalique s'en détache sous un angle presque droit. Mais il y a plus :

Pour apprécier le degré de divergence de la carotide gauche par rapport à la crosse de l'aorte, il ne suffit pas de mesurer l'angle qu'elle fait avec la partie correspondante de la convexité 
de cette crosse ; il faut considérer surtout la direction du mouvement de la colonne sanguine. Pour cela, plaçons une flèche dans l'axe du tronc aortique au niveau du point où la partie ascendante de ce tronc se recourbe pour devenir horizontale. Cette portion, dite horizontale, ne l'est pas absolument : elle continue à monter encore un peu jusqu'à la naissance de la carotide primitive gauche; elle n'est vraiment horizontale que dans le court segment compris entre l'origine de cette artère et celle de la sous-clavière gauche, après quoi elle se recourbe vers le bas et devient descendante. La flèche que nous plaçons dans l'axe du vaisseau, au commencement de la portion dite horizontale, n'est donc pas dirigée seulement d'avant en arrière et de droite à gauche, elle est encore dirigée un peu de bas en haut, et, si on la prolonge en ligne droite, on voit qu'elle va aboutir directement à l'entrée de la carotide gauche, dont l'axe fait avec elle un angle très aigu et souvent même presque nul. Que les molécules de la colonne sanguine doivent suivre pour la plupart la direction de cette flèche, c'est ce qui découle nécessairement de cette loi générale de physique que tout mouvement est en soi rectiligne et demeure tel tant qu'aucune cause ne vient le déranger; et c'est ce que démontre d'ailleurs expérimentalement l'étude des embolies.

On sait, en effet, que les embolies cérébrales sont quatre ou cinq fois plus fréquentes à gauche qu'à droite. Les caillots détachés du cœur passent cependant sous la large embouchure du tronc brachio-céphalique avant d'arriver sur l'orifice bien plus petit de la carotide gauche; et s'ils s'engagent de préférence dans le canal le plus étroit et le plus éloigné, c'est parce qu'ils y sont portés plus directement par le courant sanguin.

Le mode d'origine de la carotide gauche est donc bien plus favorable à la circulation que celui du tronc brachio-céphalique. Celui-ci se détache de l'aorte sous un angle à peu près droi $i$. La colonne sanguine, avant d'y pénétrer, est dirigée de droite à gauche; pour y pénétrer, il faut qu'elle se dirige de gauche à droite, après s'être heurtée et divisée sur l'éperon obtus de la bifurcation, après avoir perdu ainsi une partie de sa force vive. Or, cette colonne déjà ralentie, et plus ralentie que celle de la carotide gauche, aura à subir encore, à l'extrémité supérieure 
du trone brachio-céphalique, les effets d'une seconde bifurcation; elle y éprouvera un nouveau ralentissement, et dès lors il est inévitable que la circulation carotidienne, à calibre égal, soit moins active à droite qu'à gauche.

Je ne pense pas qu'il y ait un rapport nécessaire et absolu entre le calibre d'une artère et la quantité de sang qui la traverse dans un temps donné, car le débit d'un tuyau de conduite dépend à la fois de la largeur de son diamètre et de la rapidité du liquide qui le parcourt. Je rappelle d'ailleurs que la rapidité du cours du sang présente, dans les diverses artères, des différences assez notables. Il serait donc possible que l'inégale activité de la circulation dans les deux carotides laissât persister l'égalité de leur calibre. Toutefois, si l'on songe que tout organe tend à se mettre en harmonie avec sa fonction, on est disposé à penser que l'inégalité fonctionnelle des deux carotides peut exercer quelque influence sur le calibre de ces vaisseaux.

Ceci me ramène au mémoire de $M$. Armand de Fleury. L'auteur, dans ses premières publications, s'était borné à invoquer à l'appui de sa thèse des arguments théoriques; mais il a compris que cela ne suffisait pas et qu'il était nécessaire de recourir à une vérification expérimentale. Il a fait, à ce sujet, deux ordres de recherches, les unes sur le vivani, les autres sur le cadavre.

Les premières concernent l'exploration sphygmographique des carotides. M. de Fleury a fait construire par M. Buchein (de Bordeaux) un appareil spécial qui permet d'obtenir simultanément, sur l'enregistreur de Marey, le tracé du pouls des deux carotides. Les deux ampoules exploratrices sont placées exactement sur le même niveau et fixées à A'aide d'un collier bien symétrique; on obtient ainsi les deux tracés dans des conditions identiques, et ces deux tracés cependant diffèrent constamment l'un de l'autre. On les compare d'autant plus aisément que la longueur des pulsations est, sur tous deux, rigoureusement la même. On constate ainsi que le pouls de la carotide droite donne une ligne ascensionnelle plus oblique, un plateau plus convexe et plus large, et par conséquent une ligne de descente plus rapide. Cela prouve que la diastole artérielle est moins su- 
bite et l'impulsion cardiaque plus atténuée dans ce vaisseau que dans la carotide gauche.

Après avoir ainsi étudié sur le vivant la circulation carotidienne, M. Armand de Fleury s'est efforcé de comparer sur le cadavie le volume relatif des deux carotides primitives. Cette recherche était assez délicate, car la différence, s’il y en avait une, ne pouvait être que légère, et rien n'est difficile comme la détermination rigoureuse du calibre d'une artère. Sur les tubes rigides, on peut mesurer les diamètres avec une grande précision; mais la mensuration directe du diamètre d'une artère, même d'une artère injectée, expose à des erreurs résultant du degré variable de pression exercé par les branches du compas sur la paroi qui est compressible et sur la matière à injection qui n'est jamais tout à fait dure; en outre, l'épaisseur de la paroi s'ajoute alors au diamètre interne. Pour éviter ces erreurs, mon vénéré et regretté maître, Martin-Magron, avait recours au procédé suivant: après avoir injecté dans les vaisseaux une substance solidifiable bien homogène, il coupait dans les artères qu'il comparait des tronçons d'égale longueur et pesait sur une balance de précision le cylindre de matière à injection extrait de chaque tronçon. Il pouvait apprécier ainsi des différences de volume que ne révélait pas la mensuration directe des diamètres. Ce procédé ne fait pas connaître le calibre même des artères; mais il se prête à des comparaisons très précises, et je pense qu'il permettrait mieux que tout autre de déterminer le volume relatif des deux tubes carotidiens. Je ne suis pas sûr qu'il ait été publié; en tous cas, il est peu connu, et M. Armand de Fleury ne l'a pas mentionné. Il avait songé d'abord à mesurer le diamètre extérieur des carotides injectées; mais il a craint que la distension forcée des vaisseaux donnât le change sur leur calibre réel et il a jugé préférable de mesurer la circonférence interne des vaisseaux non injectés. Son procédé consiste à exciser sur le cadavre un tronçon d'artère qu'il fend longitudinalement et qu'il déploie dans toute sa largeur sur une lame de carton. La largeur obtenue est égale à la circonférence interne du vaisseau. La circonférence une fois connue, on calcule aisément le rayon, et par le rayon on obtient l'aire du cercle qui représente le calibre de l'artère. 
Je ferai remarquer que, si le procédé de l'injection donne un calibre trop fort, le procédé du déploiement donne un calibre trop faible, car les parois élastiques des artères reviennent sur elles-mêmes, dès qu'elles sont soustraites à l'action du cœur. Mais ce qu'il s'agit de déierminer, ce ne sont pas les calibres absolus, ce sont les calibres relatifs : les deux procédés sont donc valables. Toutefois, celui de M. Armand de Fleury a l'avantage de ne pas exiger l'injection préalable des vaisseaux et d'être plus commode dans la pratique.

Quoi qu'il en soit, les recherches faites par l'auteur sur 22 sujets lui ont donné les résultats suivants :

La circonférence interne de la carotide primitive droite a été en moyenne de 20 millimètres, ce qui donne pour le calibre du vaisseau, c'est-à-dire pour l'aire d'une section perpendiculaire à son axe, une surface moyenne de 31,8 millimètres carrés:

A gauche, la moyenne a été de 21 millimètres pour la circonférence déployée et de 35 millimètres carrés pour l'aire de la section.

Le calibre de la carotide primitive gauche est donc supérieur à celui de la droite dans la proportion de 3500 à 318 ou de 110 à 100 .

J'ai licu de croire que ce rapport est un peu exagéré; je trouve, en effet, sur le tableau des observations qui ont fourni les moyennes deux cas où la crosse de l'aorte et les troncs qui en naissent étaient le siège d'une dilatation pathologique ; en retirant de la liste ces deux cas anormaux, la moyenne des 20 autres cas n'est plus que de 33,1 millimètres carrés pour la carotide gauche et de 30,0 millimètres carrés pour la droite, ce qui ne donne plus que le rapport de 107 à 100 .

Deux fois le calibre s'est trouvé exactement le même sur les deux carotides, et il est digne de remarque que l'un de ces deux sujets était gaucher. Dans tous les autres cas l'avantage est resté à la carotide gauche.

Ces résultats sont dignes d'attention, mais ils ne sauraient être considérés comme définitifs, car les faits sur lesquels ils reposent ne sont pas encore assez nombreux. Ils gagneraient à être établis sur des bases plus étendues et je ne saurais trop engager 
l'auteur à multiplier ses recherches. Il importe de grouper les faits en catégories suivant les sexes, les professions et les âges. Il faudrait distinguer, parmi les professions, celles qui ne nécessitent qu'un faible travail musculaire de celles qui exigent une grande dépense de forces; car il y a lieu de se demander si l'activité plus grande de la circulation dans la carotide gauche est due exclusivement à la disposition des vaisseaux de la crosse de l'aorte, et si, après avoir été dans l'origine la cause de la prédominance functionnelle de l'hémisphère cérébral gauche, elle ne s'accroîtrait pas ensuite par l'effet de l'excès de travail de cet hémisphère chez les droitiers. Il s'agit de savoir, en d'autres termes, si la différence de calibre des deux carotides est plus grande ou plus petite chez les enfants que chez les adultes, si elle va en croissant ou en décroissant avec l'âge et avec le travail. L'auteur est disposé à croire qu'elle est plus prononcée chez les jeunes enfants qui n'ont pas encore appris à marcher. Cette vue est intéressante, mais elle ne repose malheureusement que sur une seule observation, ce qui est tout à fait insuffisant.

La question étudiće par M. Armand de Fleury exige donc encore de nombreuses recherches. Mais, tout en faisant des réserves expresses sur le degré de constance du fait qu'il a constaté, tout en considérant comme très probable que la prédominance du calibre de la sarotide gauche doit subir quelques exceptions, même chez les droitiers, je pense que l'on peut admettre comme démontré que cette prédominance est habituelle.

A l'appui de son opinion sur ce point, l'auteur invoque un autre fait anatomique qui lui paraît de même ordre, mais qui est en réalité d'un ordre tout différent. Tous les anatomistes ont remarqué que les deux veines jugulaires internes sont très souvent inégales en calibre; quelques-uns ont ajouté que la prédominance appartient plus souvent à la jugulaire gauche qu'ì la droite, mais d'autres ont dit précisément le contraire, ce qui permet de croire qu'il n'y a pas de règle bien fixe à cet égard. Mes propres observations déposeraient plutôt en faveur de la première opinion : je suis done loin de blâmer M. Armand de Fleury de l'avoir admise. Mais, en supposant qu'elle soit exacte, pourrions-nous l'invoquer comme une nouvelle preuve de la 
prédominance circulatoire de l'hémisphère gauche ? Je ferai remarquer que la plus grande partie du sang veineux des deux hémisphères va aboutir au pressoir d'Hérophile, où les deux masses sanguines se confondent en une seule avant de se répartir entre les deux sinus latéraux; quant au sang qui revient de chaque côté par le sinus caverneux et par les deux sinus pétreux, il va, il est vrai, se rendre dans le sinus latéral correspondant, mais il représente à peine la sixième partie du sang veineux de l'hémisphère, et d'ailleurs les larges communications que fournissent en avant le sinus caverneux, en arrière le sinus transverse, rétablissent en tous cas l'équilibre de pression dans les deux systèmes. L'inégalité des veines jugulaires internes ne saurait donc avoir sa cause dans le crâne; elle tiendrait plutôt à la disposition des deux troncs brachio-céphaliques qui reçoivent les veines jugulaires. Le tronc droit, court et très peu oblique, transmet aisément au sang de la veine jugulaire l'aspiration thoracique, tandis qu'à gauche, où le tronc est deux fois plus long et deux fois plus oblique, l'action de l'aspiration thoracique est beaucoup plus faible. Il y a donc une raison pour que la veine jugulaire interne gauche se vide moins aisément que la droite; mais cela suffit-il pour amener une dilatation dans le premier de ces vaisseaux? La question est encore à l'étude (1).

M. Armand de Fleury n'est pas le seul auteur qui ait étudié comparativement le calibre des deux carotides. Cette question a préoccupé également M. Will. Ogle, auteur du mémoire déjà cité sur la prééminence du côté droit. M. Ogle a examiné les artères céphaliques chez 17 droitiers et chez 3 gauchers. "Sur 12 des droitiers, dit-il, soit la carotide primitive, soit la carotide interne, était plus grosse à gauche qu'à droite; cette différence de volume était très petite, mais une très petite différence de calibre implique une différence d'effet très considérable. Chez les 5 autres droitiers je n'ai pu découvrir aucune inégalité. On

(1) Le golfe de la jugulaire interne est reçu, comme on sait, dans une fossette du trou déchiré postérieur. M. A. Julien, élève de mon laboratoire d'anthropologie, a étudié comparativement la largeur de celte fossette à droite et à gauche sur une centaine de crânes, et il a trouvé que les cas où elle est plus large du côté droit sont en majorité. 
a si rarement l'occasion de disséquer le corps des individus qui ont été connus comme gauchers pendant leur vie, que je n'ai pu observer qu'un nombre très insuffisant de faits; j'en possède trois, néanmoins. Dans aucun de ces trois cas la carotide gauche n'était plus grosse que la droite, comme cela a lieu chez la grande majorité des droitiers ; deux fois il n'y avait aucune différence apparente, tandis que dans le troisième cas les deux carotides primitive et interne étaient presque deux fois plus volumineuses à droite qu'à gauche, et il y avait en outre une disproportion similaire, toujours en faveur du côté droit, entre les deux artères cérébrales moyennes. G'est le seul cas où j'aie trouvé une différence quelconque entre ces deux vaisseaux, soit sur les droitiers, soit sur les gauchers (1). ”

L'énorme différence constatée par M. Ogle sur son troisième gaucher ne peut être attribuée qu'à une anomalie, si l'on songe surtout que chez les deux autres gauchers il n'y avait pas de différence «apparente». Cela veut-il dire que l'égalité des vaisseaux de droite et de gauche fût parfaite? Il est permis de laisser la question dans le doute, car l'auteur ne fait pas connaître le procédé de comparaison auquel il a eu recours; et s'il s'est borné, comme cela paraît probable, à apprécier à l'œil le volume des vaisseaux, sans le secours de la mensuration, des différences légères ont nécessairement dû lui échapper.

En résumé, M. Will. Ogle attribue à la disposition des vaisseaux aortiques et à l'avantage qui en résulte pour la carotide gauche la prééminence fonctionnelle de l'hémisphère gauche et la tendance très générale de l'homme à être droitier. Cette opinion est aussi celle que M. Armand de Fleury a développée, et la concordance de ces résultats est d'autant plus significative que l'auteur français et l'auteur anglais ont faitleurs recherches à l'insu l'un de l'autre. Ici se présente une question de priorité assez compliquée. Dès 1864, et plusieurs années par conséquent avant M. Ogle, M. Armand de Fleury, ainsi que je l'ai dit, a soutenu que la circulation carotidienne est plus active à gauche qu'à droite; mais il n'invoquait alors que des raisons théori-

(1) W. Ogle, On Dextral Preeminence, in Medico-chirurgical Transactions, $2^{\circ}$ série, vol. XXXVI, p. 296. Londres, 1871. In-80. 
ques, dont l'une était d'ailleurs erronée. C'est seulement en 1868 qu'il a eu recours à la vérification anatomique, en étudiant comparativement le calibre des deux carotides, suivant le procédé que j'ai indiqué plus haut. Cette date n'est pas contestable: la première observation de son tableau de mensuration est celle du nommé Pradon, capitaine au long cours, mort de pleurésie double, le 27 septembre 1868, à l'hôpital Saint-André de Bordeaux.

Mais ces recherches n'ont été publiées qu'en 1872, dans un. mémoire Sur le dynamisme comparé des hémisplières cérébraux, lu à la section de médecine de l'Association française pour l'avancement des sciences (session de Bordeaux, p. 842); or, le mémoire de M. Ogle Sur la prééminence du côté droit a vait été lu un an auparavant, en 1871, à la Société médicochirurgicale de Londres. Ainsi, quoique l'antériorité de la recherche anatomique appartienne à M. Armand de Fleury, c'est en réalité M. Ogle qui, le premier, a publié le fait de l'inégalité du calibre des deux carotides. J'ajoute que ce dernier auteur n'a pas fait connaître son procédé de comparaison et qu'il. n'a pas donné les chiffres de ses mensurations ; ses recherches, par conséquent, sont moins précises que celles de M. Armand de Fleury.

Le mémoire qui fait le sujet de ce rapport renferme une seconde partie que je dois maintenant examiner. Obéissant à une tendance bien naturelle, M. Armand de Fleury s'est passionné pour son idée; il s'en est exagéré la portée et en a tiré des conséquences forcées. Les faits qu'il a étudiés sur l'homme ne sont qu'un cas particulier d'une loi générale de physiologie. L'activité des fonctions d'un organe est subordonnée à l'activité de la circulation dans les artères qui s'y rendent. Mais cela ne veut point dire que la nature de ces fonctions dépende de la même cause, et M. Armand de Fleury s'est bercé d'une vaine illusion lorsqu'il a cru que les instincts, les facultés, les mœurs, l'activité, en un mot toute la vie cérébrale des animaux, ou du moins des mammifères, était déterminée par le mode d'origine des vaisseaux carotidiens sur la crosse de l'aorte.

Ce qui a fait naître cette idée dans son esprit, c'est la relation qui existe, chez l'homme, entre la disparité fonctionnelle des 
deux hémisphères cérébraux, et la dyssymétrie des divisions de la crosse aortique.

Mais cette relation ne prouve qu'une chose : c'est que le mode d'origine des deux carotides exerce une certaine influence sur la répartition du travail entre les deux hémisphères; je dis " une certaine influence ", et non pas une influence décisive; car, si l'on a pu constater que les individus à inversion viscérale sont ordinairement gauchers, il est bien certain que, dans l'immense majorité des cas, les gauchers sont exempts de cette rare anomalie, et que, en ce qui concerne l'origine des vaisseaux aortiques, ils ne diffèrent pas sensiblement des droitiers. M. Ogle fait remarquer, il est vrai, que les angles d'insertion de la carotide gauche et du tronc brachio-céphalique sur l'aorte présentent des différences individuelles assez notables, et il se demande si les gauchers n'auraient pas sous ce rapport une disposition moins défavorable que de coutume à la circulation de la carotide droite; mais cette vue ingénieuse est, jusqu'ici, purement théorique. Il est clair, d'ailleurs, que la carotide droite, à moins d'une anomalie d'origine tout exceptionnelle qui n'est pas ici en question, ne saurait reconquérir l'avantage sur la carotide gauche; c'est ce qu'il faudrait pourtant si la qualité de droitier ou de gaucher dépendait exclusivement de la disposition deš vaisseaux carotidiens.

Si l'on devient ordinairement droitier, c'est parce que, au moment où l'enfant commence à exercer ses hémisphères cérébraux, l'hémisphère gauche est plus apte que le droit à diriger un travail difficile ou pénible. Que la légère inégalité de la circulation dans les deux carotides concoure à donner cette avance à l'hémisphère gauche et à rendre la plupart des hommes droitiers, c'est ce qu'il me semble difficile de nier; mais elle n'est pas assez grande pour surmonter à elle seule toutes les autres conditions héréditaires ou acquises qui peuvent influer sur le développement et la nutrition des organes.

Quant à la nature du travail que l'enfant, à mesure qu'il se livre à des actes de plus en plus compliqués, est appelé à distribuer entre ses deux hémisphères, elle est inhérente au cerveau lui-même, c'est-à-dire à sa masse, à sa forme, à sa constitution anatomique; les artères, en lui apportant du sang, main- 
tiennent sa structure et excitent son action, mais il ne leur appartient ni de lui donner telle ou telle faculté, ni de déterminer le degré de perfection ou de développement des facultés qui lui étaient dévolues par hérédité avant l'ébauche des premiers vaisseaux.

En vertu de cette loi d'hérédité, tout animal vertébré apporte en naissant un cerveau qui, à l'état normal, est apte à remplir ou à diriger les fonctions de relation indispensables à la vie. Ces facultés nécessaires et naturelles sont symétriquement réparties dans les deux moitiés de l'encéphale. Mais il s'y joint, chez un grand nombre d'animaux qui vivent en famille ou en société, d'autres facultés ou d'autres actes développés par l'éducation. Ce travail supplémentaire, imposé exclusivement à la partic de l'encéphale qui constitue le cerveau proprement dit, est plus ou moins compliqué suivant les espèces. Le plus souvent il est fort simple; il est probable qu'alors il se partage également entre les deux hémisphères, et l'on sait en effet que, dans la plupart des espèces, le cerveau droit et le cerveau gauche sont tout à fait symétriques; mais il est probable encore que, lorsque les facultés acquises par une espèce croissent en nombre et en importance, lorsque, par leur spécialisation, elles se distinguent de plus en plus des facultés générales communes à tous les cerveaux, il est probable, dis-je, que quelques-unes d'entre elles, celles qui sont le plus spéciales, peuvent se locaiiser de préférence dans l'un ou l'autre hémisphère et déterminer ainsi une disparité fonctionnelle qui se traduit, sur les cerveaux pourvus de circonvolutions par une diminution de la symétrie anatomique. Si l'on considère avec raison la dyssymétrie des circonvolutions comme un caractère de perfectionnement, ce n'est pas seulement parce qu'elle est très prononcée chez l'homme, c'est aussi parce qu'on la retrouve à un degré moindre sans doute, mais très manifeste encore, chez les grands singes anthropoïdes. J'ajoute que certaines espèces d'un rang moins élevé, mais perfectionnées parl'homme, ont le cerveau beaucoup plus dyssymétrique que les espèces congénères qui vivent à l'état sauvage. Ainsi, tandis que le cerveau du renard (canis vulpes) est presque symétrique, celui du chien, qui est construit sur le même type, l'est beaucoup moins. La différence entre les deux hémisphères 
est grande surtout chez les terre-neuve et les grands chiens de berger. Ce résultat serait inexplicable, si on ne l'attribuait à l'influence de l'homme, qui a développé par l'éducation, au gré de ses besoins ou de ses plaisirs, les facultés cérébrales du chien, son plus fidèle serviteur et son plus ancien allić.

La dyssymétrie dont il s'agit ici n'atteint d'ailleurs que les plis et les sillons secondaires; elle ne concerne ni le nombre ni les connexions des circonvolutions primaires, et elle respecte toujours - abstraction faite des cas tératologiques - les caractères essentiels de la constitution cérébrale de chaque espèce.

L'homme est, de tous les animaux, celıi dont le cerveau à l'état normal est le plus asymétrique. C'est aussi celui qui possède le plus de facultés acquises. Parmi ces facultés, que l'expérience et l'éducation ont développées chez ses ancêtres et dont l'hérédité lui transmet l'instrument, mais dont il n'acquiert l'exercice qu'à la suite d'une éducation individuelle longue et difficile, la faculté du langage articulé tient le premier rang. C'est elle qui nous distingue le plus nettement des animaux. Ge qui leur manque pour l'acquérir, ce n'est pas l'appareil de l'articulation, ce n'est pas non plus la circonvolution spéciale où elle se localise chez l'homme, car cette circonvolution existe chez la plupart des singes ; c'est le degré d'intelligence qui leur serait nécessaire pour analyser les éléments du discours, pour attacher un sens de convention à chacun des mots qui frappent leur oreille, et pour chercher par de longs tâtonnements à combiner le jeu de leurs muscles phonateurs, de manière à reproduire et à articuler les mêmes sons. A l'âge où l'enfant apprend à parler, au milieu des actes multiples auxquels il s'exerce et des connaissances variées qu'il acquiert chaque jour, la fonction du langage est certainement la plus compliquée de toutes celles que l'éducation développe en lui; c'est celle qui exige de lui le plus de travail. On conçoit donc que, si l'un des deux hémisphères cérébraux possède à ce moment quelque supériorité matérielle, l'enfant affecte de préférence à sa fonction la plus difficile son instrument le plus parfait; et je pense, comme M. Armand de Fleury, que l'inégale facilité de la circulation dans les deux carotides primitives contribue ainsi d'une manière très efficace à déterminer la localisation de la faculté du langage. 
dans l'hémisphère gauche. Mais l'auteur, sans doute, ne va pas jusqu'à croire que la faculté du langage ou toute autre faculté soit liée en quoi que ce soit à telle ou telle disposition des vaisseaux aortiques. Dès lors, l'étude de la disparité fonctionnelle des hémisphères cérébraux de l'homme n'établit pas la plus petite probabilité en faveur de l'idée qu'il y ait un rapport quelconque entre les facultés d'un animal, ses instincts, ses mœurs, son genre de vie - et le mode d'origine de ses carotides.

L'auteur s'est cependant pénétré de cette idée préconçue et s'est livré dès lors à de savantes recherches sur l'anatomie comparée de la crosse aortique et de ses quatre branches dans les onze ordres de mammifères. Cet appareil s'est présenté à lui sous douze formes différentes qu'il a ramenées aux cinq types suivants : dextérité (1), férocité, célérité, solidité, ambidextérité. Il a trouvé le type de la dextérité chez l'homme, celui de la férocité chez le tigre, de la célérité chez la gazelle, de la solidité chez l'éléphant, de l'ambidextérité chez la taupe, et il s'est efforcé d'expliquer comment ces divers résultats fonctionnels étaient produits par les rapports plus ou moins directs, plus ou moins favorables de la circulation centrale avec l'un ou l'autre hémisphère cérébral, avec l'un ou l'autre membre antérieur. L'énoncé de quelques faits suffira pour montrer combien ces interprétations sont hasardées. A part deux exceptions, sur lesquelles je vais revenir, tous les singes, même les plus doux, ont le même type aortique que les carnassiers, celui de la férocité. La plupart des rongeurs ont, comme l'homme et le phoque, le type de la dextérité ; mais parmi eux le lourd porc-épic a le type de la célérité, tandis que la marmotte obéissante et le cochon d'Inde inoffensif ont le type de la férocité. La similitude constatée entre les singes, qui sont herbivores, et les carnassiers, auxquels les instincts féroces ne peuvent être refusés, a quelque peu embarrassé l'auteur; il a répondu pourtant que les vieux magots deviennent méchants et taciturnes, tandis que le chimpanzé et l'orang, qui ont le type de dextérité, " sont essentiellement dextres (droitiers), sociables et généralement doux ». II est bien vrai que le chimpanzé a le type aortique de l'homme; il

(1) Dextérité n'exprime pas ici l'adresse, mais la qualité de droitier. 
a cela de commun avec le gorille, qui ne passe pas pour très doux. Mais, en ce qui concerne l'orang, l'auteur a emprunté à Siebold un renseignement erroné. Les vaisseaux aortiques de cet anthropoïde ne sont pas disposés comme ceux de l'homme; ses deux carotides naissent du tronc innominé, comme celles du tigre, et, de l'aveu même de l'auteur, ce type de férocité n'est. pas conforme aux mœurs de l'animal. Il est digne de remarque que l'orang est, de tous les animaux, celui dont le cerveau se rapproche le plus du cerveau de l'homme; par ce caractère, l'orang est bien supérieur au gorille, et même un peiı supéricur au chimpanzé; son cerveau ress̃emble d'une manière étonnante à celui d'un jeune enfant, et cela seul suffirait pour prouver que le type aortique n'exerce aucune influence sur la constitution anatomique du cerveau.

Je considère donc comme tout à fait illusoire la doctrine générale d'anatomie et de physiologie comparées que l'auteur, par un ingénieux effort d'imagination, a cru pouvoir déduire des faits constatés chez l'homme; mais nous ne devons méconnaître pour cela ni l'intérêt ni la portée de ces faits; car, si l'inégale activité de la circulation dans les deux carotides n'est pas la seule cause de la disparité fonctionnelle des deux hémisphères cérébraux de l'homme, elle y prend certainement une part importante, et c'est l'un des éléments dont on devra désormais tenir compte dans l'étude de cette grave question.

En conséquence, votre commission a l'honneur de vous proposer :

$1^{0}$ D'adresser une lettre de remerciement à M. Armand de Fleury;

$2^{\circ}$ De déposer honorablement son travail dans les archives;

$3^{\circ}$ D'inscrire son nom sur la liste des candidats au titre de membre correspondant dans la première section.

A la suite de ce rapport, MM. Bouillaud et Bouley ont successivement pris la parole. Une remarque incidente de M. Bouillaud a motivé la réponse suivante :

M. Broca. Je ne puis songer, à cette heure avancée, à discuter les questions que viennent de soulever nos deux éminents collègıres MM. Bouillaud et Bouley. Ge n'est pas à la fin d'une 
séance que l'on pourrait traiter un sujet comme celui de la psychologie comparée de l'homme et des animaux. Ce sujet est digne à coup sûr de fixer votre attention, mais si vous vous décidez à l'inscrire sur votre ordre du jour, il faudra vous décider en même temps à lui consacrer un grand nombre de séances.

Je ne demande donc la parole que pour répondre à un court passage de l'allocution de M. Bouillaud. Notre illustre collègue et maître, parlant de la ảécouverte du siège de la faculté du langage articulé dans l'hémisphère gauche du cerveau, a bien voulu me l'attribuer en grande partie. Il est juste cependant, a-t-il ajouté, de rappeler que cette opinion avait déjà été émise par le docteur Dax père, de Sommières (Gard).

Je n'aime pas à traiter les questions de priorité qui me concernent personnellement. Voilà pourquoi je n'ai pas mentionné le nom de Dax dans mon rapport; mais puisque, à la suite de mon rapport, cette question se trouve soulevée, je suis bien obligé de répondre.

Au mois d'avril 1865, lorsque eut lieu dans cette enceinte la grande discussion sur les localisations cérébrales, je n'avais pas encore l'honneur d'appartenir à l'Académie. Le bureau ne m'aurait peut-être pas refusé un tour de lecture, mais je ne pus même pas demander cette faveur, car l'état de ma santé m'avait contraint à faire un voyage dans le Midi. Arrivé à Montpellier, je lus quelques journaux de médecine, et ce fut ainsi que j'eus connaissance de la réclamation de priorité élevée par M. Dax fils en faveur de son père, mort depuis quelque temps déjà. Le mémoire où Dax père avait annoncé que l'hémisphère gauche est le siège exclusif de la faculté du langage, avait été communiqué, au dire de son fils, au Congrès méridional tenu en 1836 à Montpellier. Me trouvant précisément dans cette ville, j'eus la curiosité bien naturelle de chercher le texte de ce travail et de lire les discussions auxquelles l'annonce d'un pareil fait avait dû donner lieu dans une assemblée scientifique. Mais mes recherches, quoique faites avec le concours de M. le docteur Gordon, bibliothécaire de la Faculté de Montpellier, et gendre de notre collègue le professeur Charles Martins, ne produisirent que des résultats négatifs. Après avoir vainement cherché dans tous les 
journaux de 1836, médicaux ou autres, une trace quelconque du mémoire de Dax, je priai M. Gordon de vouloir bien, après mon départ, continuer l'enquête. Il le fit avec le plus grand zèle, mais sans le moindre succès, et m'adressa à ce sujet une lettre que je communiquai le 15 juin 1865 à la Société d'anthropologie (Bulletins de la Société d'anthropologie, $1^{\text {re }}$ série, t. VI, p. 380). "Le Congrès méridional, disait-il, a tenu sa troisième session à Montpellier du $1^{\text {er }}$ au 18 juillet 1836 . Il avait pour président le professeur Ribes et pour secrétaire le docteur Trinquier. Il n'a pas publié de travaux et il ne reste aucune trace de ses procèsverbaux. La Revue de Montpellier (1836, t. II, p. 51 et 53) a donné un aperçu des sujets de philosophie médicale qui y furent discutés; la question du langage n'y est pas mentionnée. J'ai interrogé personnellement vingt médecins qui étaient alors à Montpellier. Il n'est pas à leur connaissance que le mémoire en question ait été lu au Congrès ou publié quelque part. " Ainsi, l'existence du mémoire de Dax père était aussi inconnue à Montpellier qu'à Paris, lorsque son fils le communiqua à l'Académie de médecine et le publia en outre dans le numéro du 28 avril 1865 de la Gazette hebdomadaire.

A l'issue de la séance de la Société d'anthropologie où j'avais communiqué la lettre de M. Gordon, un de mes collègues m'annonça que les mémoires manuscrits de MM. Dax père et fils étaient encore entre les mains de Trousseau. Quelques jours après, le chef de clinique de Trousseau - c'était, je pense, mon collègue le professeur Peter - voulut bien m'apporter chez moi ces deux manuscrits, et je restai convaincu qu'ils émanaient de deux personnes différentes. Le style, la facture, le mode d'exposition et de discussion, tout démontrait la différence des origines. Ge contraste était plus frappant sur les manuscrits qu'il ne l'est dans la publication faite par la Gazette hebdomadaire, ce journal n'ayant donné qu'un extrait du manuscrit de M. Dax fils. Je ne gardai donc aucun doute sur l'authenticité du manuscrit de Dax père, ni sur la date qui lui était assignée. Dès lors, je n'avais aucune raison de douter que ce travail eût été préparé pour être communiqué au Congrès méridional de 1836 - mais il était certain que l'auteur ne l'avait pas présenté à ce congrès, et qu'il ne lui avait donné, ni là ni ailleurs, aucune publicité. Il 
est probable qu'au dernier moment, il ne s'était pas senti le courage d'affronter la discussion et les railleries, à une époque où les contestations soulevées par la doctrine de Gall étaient si vives, et où tout ce qui sentait plus ou moins la phrénologie était mis à l'index. Il ne disposait d'ailleurs que de moyens de défense bien insuffisants, car les rares faits qu'il avait observés dans sa pratique n’avaient pas été éclairés par l'autopsie.

Quoi qu'il en soit, le mémoire de Dax père n'avait pas vu le jour, et personne, excepté sans doute son fils, n'en connaissait l'existence, lorsque je commençai, en 1861 , mes recherches sur le siège de la faculté du langage articulé, recherches d'un ordre bien différent, puisqu'elles reposaient sur l'anatomie normale des circonvolutions et sur l'anatomie pathologique.

Ces recherches venaient à la suite d'uñe discussion de la Société d'anthropologie sur les localisations cérébrales; en voyant reparaître, sur des bases nouvelles il est vrai, la question des localisations que l'on croyait pour toujour's enterrée, et dont on ne parlait plus que pour en rire, beaucoup de personnes n'éprouvèrent que de la méfiance; beaucoup d'autres éprouvèrent quelque chose de plus. Ceux mêmes qui n'avaient aucune prévention contre le principe général des localisations repoussaient vivement l'idée qu'une localisation quelconque pût avoir lieu dans l'hémisphère gauche, à l'exclusion de l'hémisphère droit. Il y eut donc des discussions assez animées dans lesquelles je ne fus pas toujours ménagé, et je suis loin de m'en plaindre. Pourtant la question avança peu à peu, les faits se multiplièrent, les adhésions arrivèrent; on commença à accepter comme scientifique ce qui avait d'abord par'u si étrange. Ce fut alors que le mémoire de Dax père, enseveli dans un tiroir depuis 1836, fut exhumé par son fils et envoyé à l'Académie.

Ces explications suffiront, je l'espère, pour justifier le silence que j’ai gardé dans mon rapport sur les observations de Dax père. Appelé comme rapporteur à juger une question de priorité qui nous était soumise par M. Armand de Fleury, j'aurais eu mauvaise grâce à vous entretenir de mes propres revendications. Mais vous comprendrez que je ne pouvais me dispenser de répondre à l'observation, d'ailleurs si bienveillante, de M. Bouillaud. 
MÉMOIRES D'ANTHROPOLOGIE.

M. Boullaud. Je suis heureux d'avoir entendu les explications de M. Broca, qui tranchent définitivement à mes yeux la question de priorité. G'est donc à lui que revient tout l'honneur de l'importante découverte du siège de la faculté du langage. 


\section{SUR L'APHASIE}

(Bulletins de l'Académie de médecine, $2^{\circ}$ série, t. III, 1874, p. 933.)

M. Broca dépose sur le bureau une note de M. le docteur Wiart (de Caen) sur un cas d'aphasie. Il s'agit, dans cette observation, d'un homme de soixante ans, atteint depuis le mois de mai 1873 d'une hémiplégie incomplète du côté droit, a vec complication d'aphasie ; l'intelligence, à peu près intacte à son entrée à l'hôpital de Caen, s'affaiblit peu à peu, et la mort survint dans le courant du mois d'août après plusieur's accès épileptiformes. A l'autopsie, on trouva comme principales lésions : une petite cavité du volume d'une grosse noisette remplie de sérosité incolore, siégeant dans le noyau extra-ventriculaire du corps strié, au niveau du ventricule latéral gauche, en dehors et en avant. Cette cavité gagnait la troisième circonvolution frontale gauche dans la substance de laquelle elle pénétrait de 3 à 4 millimètres. Au-dessous de cette cavité principale et tout à fait indépendante, on trouvait encore dans l'épaisseur de cette troisième circonvolution une autre cavité du volume d'une petite lentille remplie également d'une sérosité incolore. 

ANATOMIE PATHOLOGIQUE DU CERVEAU

\section{PERTE DE SUBSTANCE DU CERVELET}

\section{A LA PARTIE POSTÉR0-INTÉRIEURE}

(Société d'anatomie, 1861, 20 série, t. VI, p. 35-36.)

I. Ввоса. Le malade présentait un degré d'hyperesthésie assez prononcé; la seule action de prendre et de soulever la verge pour pratiquer le cathétérisme paraissait éveiller des douleurs extrêmement vives.

Le poids du cerveau, constaté au moment de l'autopsie, était de 200 grammes environ inférieur à celui d'autres cerveaux de vieillards examinés comparativement. Les ventricules contenaient une grande quantité de sérosité, et, de plus, l'espace vide laissé par la perte de substance était occupé par un tissu cellulaire à mailles très larges, qui s'affaissa quand la sérosité qui les distendait fut écoulée, mais qu'il était facile de rendre de nouveau apparent par l'insufflation. Quant à la cause elle-même de l'excavation constatée, M. Broca est disposé ì la considérer comme le résultat d'un travail atrophique développé au niveau d'un ramollissement.

\section{PERTE DE SUBSTANCE DU CERVEAU}

(Société d'anatomie, $1861,2^{e}$ série, t. VI, p. 378.)

M. Broca montre un exemple de perte de substance du cerveau, observé sur un vieillard de quatre-vingt-neuf ans, qui n'avait présenté pendant la vie ni troubles intellectuels ni phénomènes paralytiques. Mais il avait un tremblement général. 
Cet homme, nommé Drévon, travaillait encore, comme tailleur d'habits, six mois avant sa mort; à partir de cette époque, le tremblement des membres, dont il était affecté auparavant, devint assez prononcé pour qu'il ne pût plus manier l'aiguille.

\title{
SUR
}

\section{LE VOLUIIE ET LA FORNE DU CERVEAU}

\author{
A L’ÉTAT NORMAL ET PATHOLOGIQUE
}

Gratiolet, à la séance de la Société d'anthropologie du 21 février 1861 (Bull., p. 66), présenta le moule intérieur d'un crâne de Totonaque. Sur ce moule se dessinent, ce qui n'a pas lieu dans la race caucasique, les empreintes des circonvolutions frontales, et il semble que ce soit là un caractère d'infériorité, observé également chez certains idiots (1). Les Totonaques sont, en effet, une race inférieure, et cependant ce cerveau est volumineux. Pour Gratiolet, "les recherches de la capacité de la cavité crìnienne n'ont presque aucune signification "); les pesées de Wagner prouvent qu'il n'y a pas de relation entre le développement de l'intelligeuce et celui de la masse encéphalique.

Auburtin contesta ces assertions; pour lui, le cerveau est un ensemble d'organes divers. L'intelligence ust en rapport avec le développement des lobes frontaux.

Gratiolet maintint ses opinions premières. Et il ajouta qu'on n'a pas de notions positives sur les diverses fonctions des diverses parties du cerveau. Le cerveau semble être un, quoiqu'il soit infiniment probable que toutes ses parties n'ont pas la même dignité.

Puis Broca prit la parole en ces termes:

(Bulletins de la Société d'anthropologie, 1861, t. II, p. 75-77.)

M. Broca. M. Gratiolet a critiqué les auteur's qui ont cherché à apprécier la masse de l'encéphale, en mesurant la capacité entière du crâne. Ces critiques ne sont pas sans fondement, et il est clair qu'il vaudrait mieux, si c'était possible, peser directement le cerveau, au lieu de recourir à un procédé indirect. Pourtant, il vaut micux connaître la capacité du crâne que de

(1) Voir plus loin, p. 176 
n'avoir aucune idée, même approximative, du volume du cerveau; et en attendant que les anatomistes aient trouvé le moyen d'aller étudier sur place, à l'état frais, le cerveau des hommes des diverses races, on ne peut que remercier les savants qui ont étudié avec une louable persévérance la contenance des crânes suivant les individus et suivant les races, comme nous remercions aujourd'hui M. Gratiolet d'avoir étudié sur des moules la forme de la cavité crânienne.

Au surplus, l'étude directe du cerveau, à l'état frais, est entravée par des obstacles de tout genre, car il ne suffit pas d'examiner le cerveau d'un Hottentot ou celui d'un Totonaque; il faudrait en examiner un grand nombre et les comparer entre eux, pour ne pas être induit en erreur par les variétés individuelles. M. Wagner, concentrant toute son attention sur les cerveaux de race caucasique, a voulu procéder ainsi ; il a réussi à se procurer quelques cerveaux d'hommes éminents; mais il a reconnu bientôt que ses propres recherches étaient insuffisantes; lorsqu'il a voulu étudier le poids du cerveau, il a été obligé de rassembler toutes les observations qu'il a pu trouver dans la science. Il en a réuni 964, chiffre imposant au premier abord; mais cette statistique pèche par la base, parce que la plupart des cerveaux qui ont été pesés étaient des cerveaux malades. La plupart de ces observations sont empruntées aux aliénistes, et on trouve dans le tableau un nombre très considérable de cas d'hydrocéphalie, d'idiotie, d'épilepsie, de manie, de démence, de paralysie générale, etc., confondus sans ordre avec les cas où le cerveau était parfaitement sain. Avec de pareilles données, il est impossible d'obtenir des relevés exacts. Il y a toutefois dans le tableau de M. Wagner une particularité frappante qui n'a pu échapper à l'attention de M. Gratiolet : les 964 cerveaux sont numérotés par ordre, depuis le plus lourd jusqu'au plus léger. Les numéros 1 et 2 sont des cerveaux malades; le cerveau numéro 1 provient d'un enfant de trois ans, hydrocéphale; le numéro 2 provient d'un homme de cinquante-quatre ans, mort d'un œdème du cerveau. Les numéros 3 et 4 sont des cerveaux sains. Le numéro 5 , pesant 1783 grammes, est le cerveau d'un aliéné mélancolique, etc. En réalité, par conséquent, les deux cerveaux sains les plus lourds sont les numéros 3 et 4 ; le pre- 
mier pèse 1861 grammes, c'est celui de Cuvier; le second pèse 1807 grammes, c'est celui de lord Byron.

Tout le monde connaît l'érudition de M. Wagner, et il est permis de croire que, s'il n'a pas réuni toutes les observations connues, il en a laissé échapper bien peu. Il est donc infiniment probable que, parmi tous les hommes dont on a pesé jusqu'ici le cerveau, Guvier et lord Byron, deux grands hommes, deux hommes de génie, qui marchent de front avec les premiers de notre siècle, sont ceux qui ont eu le plus de masse cérébrale. Il me paraît impossible d'attribuer ce résultat à une pure coïncidence, et de ne pas admettre que ces deux hommes ont dû la suprématie de leur intelligence à l'ampleur de leur cerveau.

La fin de la séance fut occupée par des répliques de Gratiolet soutenant toujours les mêmes doctrines.

Auburtin revint à la charge pour la doctrine de localisation, avec les faits pathologiques qui forcent à localiser la faculté du langage dans les lobes antérieurs.

Le 21 mars 1861 (Bull., p. 139), Broca reprit la parole et donna lecture d'un mémoire important qui a déjà été réimprimé dans les Mémoires d'anthropologie, t. I, p. 155 . 
SUR

\title{
LES PÉTRIFIGATIONS DE L'ENCÉPHALE
}

\author{
Bulletins de la Société d'anatomie, $2^{\circ}$ série, t. VII, 1862, p.103-105.)
}

M. Broca. Je présente une série de pièces qui s'éclairent un peu les unes les autres et qui nous révèlent une singulière lésion de l'intérieur du crâne. Cette énorme tumeur, que m'a

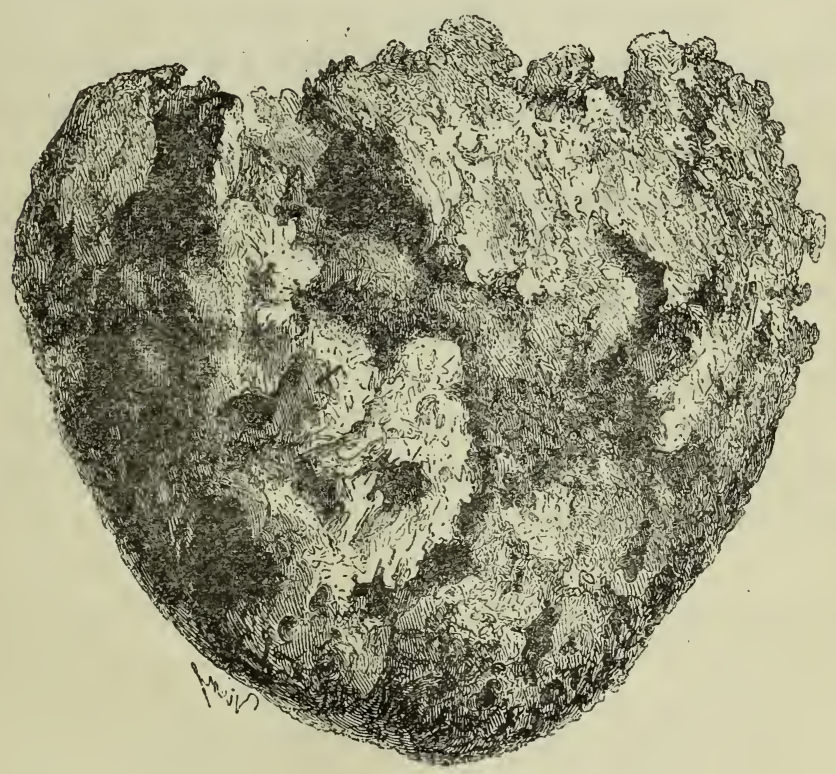

Fig. 1. Demi-grandeur.

remise il y a quelque temps M. Blain des Cormiers, offre à peu près le volume des deux poings; elle a l'aspect aréolaire de l'éponge, mais elle est d'une consistance pierreuse. Elle a été trouvée dans l'intérieur d'un crâne volumineux enseveli sous les dalles d'une église. J'ai rencontré depuis ces trois autres 
tumeurs qui jettent quelque jour sur le siège probable de ces productions.

M. Goubaud, vétérinaire distingué d'Alfort, a publié un savant mémoire sur les pétrifications de l'encéphale. L'opinion de ce vétérinaire paraît être que ces tumeurs dites pétrifications ne sont autres que des végétations insérées d'abord sur la base du crâne et dont le pédicule se détruit par la suite. Je ne partage pas cette opinion. Je regarde ces tumeurs poreuses sans structure organique, d'après mes premiers essais, comme s'étant développées au sein même de l'encéphale, et j'appuie ma manière de voir sur l'existence de cette substance grise, terreuse, que j’ai rencontrée au centre de cette pièce. Cette substance a tout à fait les caractères de celle en laquelle se convertit habituellement le cerveau déposé en terrain sec. Comparez, en effet, ces échantillons de résidu encéphalique avec le contenu de cette tumeur, et vous verrez que, déjà à la vue, ils sont identiques; il faut donc que cette concrétion pierreuse ait circonscrit une certaine partie de la substance cérébrale.

Les auteurs anciens avaient comparé ces tumeurs à des pétrifications post mortem. Mais la plus volumineuse des tumeurs que je présente a été recueillie dans une église, dans un cercueil parfaitement intact; on ne peut donc pas lui assigner une semblable origine. Quant à son volume étrange, je ne l'explique qu'en la faisant remonter à la naissance et coïncider avec une hydrocéphalie. Du reste, le crâne qui la renfermait était luimême très volumineux. 


\section{NOTES POUR SERVIR A L'HISTOIRE}

DU

\section{RAMOLLISSENENT CÉRÉBRAL PROGRESSIF}

SANS TROUbles DE L'intelligence NI DES FONGTiONS DE RELATION

(Bulletins de la Société d'anatomie, $2^{\circ}$ série, t. VIII, 1862, p. 268-275.)

M. Broca communique les notes suivantes:

$A$. Normant, âgé de soixante-quatre ans, mort le 23 janvier 1862, de rhumatisme goutteux (service de médecine de M. Léger). On n'avait constaté aucun trouble intellectuel. A l'autopsie, on a trouvé, à la partie postérieure de l'hémisphère gauche, sur la face interne, au niveau du sillon occipital interne, un foyer ressemblant à un kyste situé sous la première.

Ce foyer, plein de sérosité limpide, reposait sur la substance blanche de l'hémisphère; il avait le volume d'un œuf de pigeon. La circonvolution occipitale qui longe le sillon occipital interne était détruite; la suivante était fort amincie.

Il n'existait aucune paralysie; le malade n'était pas gâteux.

$B$. Méné, âgé de soixante-quatorze ans, mort le 14 juin 1862 , d'une pneumonie double.

Poids de l'encéphale entier, 1330 grammes; cervelet, bulbe et protubérance, 160 grammes; membranes et sérosité des hémisphères, 61; hémisphère droit, sans membranes, 567; hémisphère gauche, 542. L'hémisphère gauche pèse donc 25 grammes de moins que le droit.

Cet homme était intelligent, n'était atteint d'aucune paralysie ; il y a sur la table externe du pariétal gauche une longue dépression linéaire profonde (qui n'est pas le lit d'un vaisseau), et qui paraît le résultat d'une ancienne fracture de la table externe.

A la partie postéricure de la surface de l'insula, existe une 
perte de substance, de la substance grise, à bords presque abrupts, comme à l'emporte-pièce; à fond d'un jaune rougeâtre et de 2 millimètres de profondeur. Elle est large comme une pièce de 1 franc, à l'extrémité postérieure de la paroi de Sylvius, à 2 centimètres au moins en arrière de la lésion précédente, existe une autre lésion de même nature, mais plus étendue, qui empiète sur la partie postérieure de la circonvolution marginale inférieure et sur le pli de passage qu'elle ouvre dans le lobe pariétal. Cette circonvolution est très amincie, mais non interrompue. Ces deux lésions semblables paraissent dues à deux foyers d'hémorrhagie méningée. On notera que c'est du même côté, presque au même niveau, qu'existe sur le pariétal la trace probable d'une ancienne fracture incomplète du crâne. Le malade avait d'anciennes fractures sur les deux tibias, produites par deux chutes distinctes. Il avait perdu deux doigts de la main gauche par suite d'un autre accident. La fracture incomplète du pariétal (si elle a réellement existé) avait été la conséquence inaperçue d'un de ces accidents.

Cœur très volumineux. Insuffisance auriculo-ventriculaire gauche diagnostiquée pendant la vie; adhérence générale ancienne du péricarde, gros caillot ancien et adhérent dans le cœur gauche; embolie dans l'artère latérale antérieure gauche; gangrène sèche commençante du gros orteil gauche.

$C$. Cuvillier, âgé de soixante et onze ans, mort le $\mathbf{1 3}$ juin 1862, de phlegmon diffus de tout le membre inférieur atteint de chorée très grave (surtout des membres supérieurs), depuis ving tcinq ans. On n'a pu savoir si cette chorée était survenue peu à peu ou tout à coup.

Léger ramollissement rouge très superficiel et tout récent à la face inférieure des deux lobes temporaux; une très mince couche, à ce niveau, s'enlève avec la pie-mère. (ll y a eu du délire pendant les derniers jours.)

Sur la convexité de l'hémisphère gauche, à l'extrémité postérieure de la seconde parallèle, à l'union du lobe temporal, du lobe pariétal et du lobe occipital, existent les traces, soit d'un très ancien épanchement de sang, soit d'un ramollissement cortical guéri et cicatrisé. On n'apercevait_nullement ce foyer avant d'avoir enlevé la pie-mère. 
Cervelet peu consistant; le tiers postériéur du corps calleux s'est déchiré avec facilité, quoique l'autopsie ait été faite trente heures après la mort, par une température de 15 degrés. Le reste de l'encéphale est sain. La moelle épinière est saine, mais le renflement lombaire de cet organe est, ainsi que la queue de cheval, le siège d'une congestion récente assez intense, due probablement à la présence du phlegmon diffus du membre inférieur.

Les deux moitiés du cerveau ont le même poids ; hémisphère droit, sans membranes, 521 grammes; hémisphère gauche, sans membranes, 522 grammes.

$D$. Duchet (Louis), âgé de soixante-cinq ans, mort le 18 juin 1862, d'un cancroïde de la main.

Cet homme est entré dans le service le 150 octobre 1861. Il avait été admis à Bicêtre tout récemment, pour plusieurs affections incurables, savoir: $1^{\circ}$ un ulcère simple de la jambe droite, datant de trente ans; $2^{\circ}$ un abcès rongeant (polyadémie) de la face dorsale de la main droite, datant de trois ans et demi ; $3 \circ$ un autre ulcère rongeant, de même nature, mais plus récent, dans la région sternale. En outre, cet homme avait presque entièrement perdu l'intelligence; il ne put donner aucun renseignement sur ses antécédents; il nous dit qu'il demeurait rue Mignon; ce fut tout ce qu'on put tirer de lui. Il n'existait d'ailleurs chez lui aucune paralysie, ni de la sensibilité, ni du mouvement. Les quelques mots qu'il prononçait de temps en temps, soit pour se plaindre, soit pour répondre tant bien que mal à une question, étaient articulés, mais il était rare qu’il prononçât plus de quatre ou cinq mots de suite.

Ce malade a constamment gardé le lit depuis le jour de son entrée jusqu'au jour de sa mort. Il n'était pas gâteux; il restait couché continuellement sur le dos, et presque entièrement immobile; il fut pris, à plusieurs reprises, d'accidents assez graves : érysipèle, fièvre intermittente, phlegmon gangreneux, étendu sur le dos du pied, il y résista; mais affaibli de plus en plus, il s'éteignit graduellement le 18 juin 1862, sans avoir' jamais présenté ni délire, ni convulsion, ni paralysie.

La suppuration de ses ulcères était devenue très abondante dans les derniers temps, et cette cause a sans doute contribué 
à accélérer la mort; mais il n'a jamais eu ni la teinte jaunepaille, ni aucun autre symptôme en rapport avec un état eachectique.

Nous avions diagnostiqué un ramollissement de la couche corticale du cerveau.

Autopsie. Il s'écoule une grande quantité de sérosité à l'ouverture du crâne.

L'encéphale entier, avec ses membranes, pèse 1177 grammes, qui se décomposent ainsi : cervelet, bulbe et protubérance, avec leurs membranes, 1 ร๊̌ grammes; membranes et sérosité des hémisphères, 57 grammes; les deux hémisphères dépouillés de leur membrane, 965 grammes.

L'hémisphère droit pèse seul 48 grammes; le gauche, 480 grammes. Cette inégalité, quoique peu prononcée, doit être attribuée à l'inégale déperdition de poids qui a été la conséquence de l'affection cérébrale.

Il n'existe de lésions que dans les hémisphères cérébraux et seulement dans la couche corticale de ces hémisphères.

Avant d'avoir enlevé les membranes, on apercevait des deux côtés, à la surface convexe des hémisphères, plusieurs dépressions au niveau desquelles la pie-mère, légèrement affaissée et plissée, était séparée de la substance cérébrale par une certaine quantité de liquide transparent. Le fond de ces dépressions reposait sur la substance cérébrale affaissée, entamée comme par des altérations superficielles, ramollie dans une épaisseur de 2 millimètres tout au plus, et présentant une couleur d'un gris jaunâtre. Ces pertes de substance ne dépassaient pas l'épaisseur des circonvolutions, et s'arrêtaient à la substance blạnche; l'une d'elles, toutefois, pénétrait profondément dans le lobule orbitaire de l'un des lobes frontaux.

Je considère cette affection comme un ramollissement chronique progressif et à foyers multiples de l'écorce des hémisphères; j'ai déjà eu l'occasion de mentionner à la Société un certain nombre de pièces qui ont mis en évidence la marche de cette lésion. Le ramollissement débute en un point très circonscrit sous la pie-mère, il se propage ensuite autour de ce point, mais si lentement, qu'une partie actuellement, ramollie aura le temps de se dénouer, d'être entièrement résorbée et remplacée par un 
liquide séreux, avant que les parties adjacentes soient profondément lésées. Quoique ordinairement progressif, cette espèce de ramollissement cérébral peut s'arrêter dans sa marche; alors la couche ramollie se résorbe, le vide qu'elle laisse est remplacé par de la sérosité, ou comblé par la pie-mère, qui paraît déprimée et comme filtrée à ce niveau, et il reste à la surface du cerveau, tantôt une cicatrice, tantôt une collection de liquide qui a été l'effet et non la cause de la perte de substance.

Ces lésions sont très fréquentes chez les vieillards, au moins chez ceux du sexe masculin (je ne puis parler des autres). Elles s'observent assez souvent à Bicêtre, même chez des individus encore jeunes, mais il n'est pas douteux que les vieillards y soient plus particulièrement prédisposés. Elle échappent très souvent à l'attention des amateurs pathologistes, à moins qu'elles ne soient très avancées ou très étendues, parce qu'elles ne deviennent bien apparentes qu'après l'ablation de la pie-mère, et parce que dans la plupart des autopsies on se contente de pratiquer des coupes dans le cerveau sans le dépouiller de ses membranes.

Ayant l'habitude d'enlever constamment la pie-mère avant tout autre examen, j'ai eu l'occasion d'observer environ quinze fois les diverses phases de cette lésion sur une centaine d'autopsies que j'ai faites depuis que je suis chirurgien de Bicêtre. Je dois maintenant examiner et décrire rapidement les divers foyers qui existent dans les hémisphères cérébraux de mon malade, il y en a quatre dans chaque hémisphère.

I. Hémisphère gauche. $1^{\circ} \mathrm{La}$ circonvolution occipitale supérieure et interne est le siège d'une perte de substance assez étendue, qui occupe surtout la partie profonde de cette circonvolution, passe comme sous un pont au-dessous de la circonvolution adjacente, et va aboutir dans la partie la plus antérieure du sillon occipital interne; le fond de cette perte de substance est constitué par la substance blanche de l'hémisphère, qui n'est pas cutanée; $2^{\circ}$ un second foyer plus superficiel occupe la partie postérieure de la deuxième circonvolution frontale, tout près de son insertion sur le pli frontal ascendant (ou circonvolution frontale transverse). La perte de substance offre environ 1 centimètre et demi d'étendue d'avant en arrière: elle atteint toute 
la largeur et la plus grande partie de l'épaisseur de cette circonvolution; $3^{\circ}$ un troisième foyer, beaucoup plus considérable, occupe le bord inférieur de la scissure de Sylvius. La circonvolution marginale inférieure ou première circonvolution temporale est entièrement détruite dans ses deux tiers antérieurs; la perte de substance s'étend à toute la moitié antérieure de l'étage. supérieur du lobe temporo-sphénoïdal et s'arrête à la substance blanche. Il y avait une grande quantité de liquide dans ce foyer, qui ressemblait à un kyste, mais dont la paroi profonde était tapissée d'une couche de substance cérébrale ramollie, en voie de dissolution ; $4^{\circ}$ un quatrième foyer, très peu étendu, occupe la troisième circonvolution frontale en avant de la scissure de Sylvius.

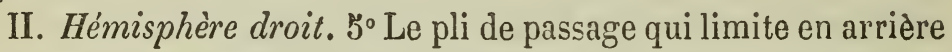
l'extrémité postérieure de la scissure de Sylvius est le siège d'une perte de substance partielle, qui empiète sur l'extrémité postérieure de la seconde circonvolution temporale; 6。 la deuxième circonvolution occipitale externe est entièrement coupée en travers; $7^{\circ}$ la première circonvolution occipitale interne et le pli de passage correspondant sont profondément excavés $; 8^{\circ}$ enfin, la troisième circonvolution frontale, dans son tiers antérieur en avant de l'extrémité antérieure de la scissure de Sylvius, est à peu près entièrement divisée en travers par une perte de substance qui pénètre jusqu'à la substance blanche, passe au-dessous de la deuxième et de la première circonvolution frontale et forme une cavité assez spacieuse, comprise entre l'étage supérieur du lobule orbitaire du lobe frontal. Il n'est pas certain qu'ici la substance blanche ne soit pas en partie détruite; elle paraît altérée, mais on n'aurait pu s'en assurer sans gâter la pièce.

Cette dernière lésion a divisé la troisième circonvolution fron. tale, c'est-à-dire celle qui était détruite chez les deıx individus aphémiques dont j'ai présenté les cerveaux à la Société l'année dernière. Or, quoique Duché ne parlât presque jamais, il n'était nullement aphémique, il ne parlait pas, parce qu'il n'avait pas d'idées à exprimer, mais les quelques mots qu'il prononçait prouvaient que la facultó du langage articulé n'était pas atteinte chez lui. 
Il semble donc qu'il y ait contradiction entre les résultats de cette autopsie et les conséquences qui avaient paru découler des autopsies de mes deux individus aphémiques; mais on devra, avant de tirer une conclusion, considérer que la lésion de mon dernier malade occupe une partie de la troisième circonvolution frontale située en avant de la scissure de Sylvius, tandis que chez les deux premiers la lésion était située bien plus en arrière. La physiologie et la pathologie des circonvolutions cérébrales sont à peu près complètement inconnues; en s'engageant sur un pareil terrain, on doit avant tout s'occuper de recueillir des faits, et n'admettre qu'avec une extrême prudence les interprétations positives ou négatives.

On notera en tous cas que chez mon dernier malade $: 1^{\circ}$ toutes les fonctions du système nerveux central étaient intactes, à l'exception de l'intelligence, qui était profondément altẻrée; $2^{\circ}$ toutes les parties du système nerveux central étaient saines, à l'exception des circonvolutions cérébrales, qui étaient le siège de lésions multiples et très étendues. C'était la substance grise périphérique, à peu près exclusivement, qui était lésée, et cette observation vient à l'appui de l'opinion de ceux qui pensent que l'intelligence réside dans la couche corticale des hémisphères. 


\title{
SUR LES EIIPREINTES CÉRÉBRALES
}

\author{
A L'ÉtAT NORMAL ET PATHOLOGIQUE
}

(Bulletins de la Socièté d'anthropologie, 1863, t. IV, p. 199-200.)

M. Broca. A l'occasion de la communication que nous fit M. Gratiolet sur le crâne d'un Totonaque, j'ai recherché les faits qui pouvaient être relatifs aux opinions de notre collègue sur les empreintes des circonvolutions cérébrales. M. Gratiolet avait dit que chez les idiots les circonvolutions écartées par suite d'un arrêt de déveioppement déterminaient des 'empreintes sur le crâne, et que cette disposition pouvait être observée aussi à l'état normal chez certaines races.

Voici plusieurs pièces qui démontrent ces propositions :

Sur ce crâne, ayant appartenu à un idiot appelé Edern, on voit des empreintes très larges et très profondes, en rapport avec des circonvolutions larges et médiocrement écartées.

Le crâne d'Abdallah, nègre de Tombouctou, porte les empreintes évidentes de circonvolutions, qui étaient surtout saillantes à la partie antérieure.

Une mulâtresse, Sarah, succomba à l'hôpital de Lourcine aux suites de brûlures graves; son cerveau était le plus léger de ceux que j’ai pesés : 890 grammes avec les membranes. Ici encore les empreintes circonvolutionnaires sont extrêmement marquées.

L'hypertrophie cérébrale produit le même résultat; les circonvolutions hypertrophiées creusent leur trace sur l'enveloppe osseuse. J'ai été conduit à reconnaître ce fait par l'examen d'un cerveau hypertrophié dont les circonvolutions sont très larges et peu nombreuses. Les sutures du crâne étaient ossifiées, et les empreintes fortement accusées.

Dans un second cas, j'ai pu vérifier ma première observation : j'ai fait l'autopsie d'un jeune homme de dix-neuf ans, très intel- 
ligent. Ses circonvolutions ont marqué de profondes empreintes sur le crâne, qui est très mince et dont les sutures sont ossifiées, tandis qu'en général l'ossification n'est complète que vers quarante-cinq ou cinquante ans.

M. Giraldès. Je dépose dans le même sens que MM. Gratiolet et Broca. Il y a deux ans, j'ai fait voir à la Société de biologie des voûtes de crâne ayant appartenu à des individus atteints d'hypertrophie cérébrale, et ces voûtes étaient extrêmement amincies, presque percées an niveau des circonvolutions. J'ai eu depuis l'occasion de répéter cette observation en différentes circonstances, sur des enfants. 


\title{
LE GRANE DE NÉANDERTHAL
}

EST-IL PATHOLOGIQUE?

\author{
DISGUSSION SUR LE VOLUME DU GERVEAL
}

(Bulletins de la Sociétè d'anthropologie, 1863, t. IV, p. 322-323.)

M. Broca. Toute l'argumentation de M. Pruner-Bey repose sur un point, à savoir, que les formes particulières du crâne de Néanderthal sont pathologiques. En effet, on n'a jamais vu un crâne pareil, et à moins d'admettre qu'il appartient à une race dont aucun vestige ne nous révèle l'existence passée, on est forcé d'en venir à l'origine morbide des particularités qu'il présente. Mais je crois pouvoir démontrer que ce crâne ne peut pas provenir d'un idiot : à quoi tient, chez l'idiot, de même que chez le gorille, la saillie des arcades orbitaires et le retrait du frontal? Cela tient à ce que la masse cérébrale n'est pas assez volumineuse; les lobes antérieurs et les lobes postérieurs convergent vers le centre idéal de la tête. Pareille chose se produit chez l'idiot, mais seulement chez l'idiot microcéphale. Or, le crâne de Néanderthal n'est point d'un microcéphale.

J'ai vu à Bicêtre, il y a deux ans, un idiot qui avait une tête énorme et dont l'aspect semblait contredire l'opinion de ceux qui attribuent une importance au volume du cerveau dans les manifestations intellectuelles. Mais à l'autopsie nous trouvâmes les téguments crâniens et la boîte osseuse singulièrement épais. Le cerveau était, en réalité, très petit; il pesait moins de 1100 grammes, mais son volume ne permettait point de le ranger dans les microcéphales. Pour trouver un crâne comparable à celui de Néanderthal, il faut aller chercher l'idiot microcéphale. Quant à ces larges impressions, indices de circonvolutions peu nombreuses, ce caractère existe, en effet, chez les idiots, mais il existe aussi chez tous les hommes qui ont de larges circonvolu- 
tions et chez les individus des races inférieures. En sorte que le cerveau de Néanderthal peut être simplement celui d'un individu de race inférieure.

Je résume mon objection en peu de mots : une idiotie capable de produire un pareil crâne est nécessairement microcéphalique; or, ce crâne n'est pas d'un microcéphale; donc il n'est pas d'un idiot.

\section{OSSIFICATION PRÉMATURÉE DES SUTURES}

(Bulletins de la Société d'anthropologie, 2e série, 1866, t. Ior, p. 441-442.)

II. Broca présente une pièce intéressante au point de vue des rapports qui existent entre le développement de l'intelligence et l'ossification des sutures du crâne. Il s'agit d'un garçon de seize ans, mort dernièrement à l'hôpital Saint-Antoine, dans un état de faiblesse intellectuelle, voisin de l'idiotie, à tel point que le malheureux a succombé aux suites d'une hernie étranglée, dont il ne se doutait pas et dont il ne s'était jamais plaint. Or, l'autopsie a montré, comme on peut le voir sur la pièce, que la suture sagittale était déjà fermée. 11 y a donc eu là concurremment ossification de la suture et par suite, arrêt dans le développement cérébral, entraînant l'idiotie du sujet. M. Broca rappelle en terminant que, d'après les recherches de Virchow, l'ossification prématurée des sutures est due souvent à des lésions cérébrales internes, mais que, dans l'espèce, rien de semblable n'a eu lieu. 


\title{
SUR L'ASSASSIN LEMAIRE
}

\author{
ET
}

\author{
SUR LA CRIMINALITÉ
}

(Bulletins de la Société d'anthropologie, 1867,' 2e série, t. II, p. 347-349.)

M. Broca offre à la Société, de la part de M. Vasseur, le crâne de Lemaire, un moule intracrânien, le cerveau de Lemaire et le moule de la tête entière. Il fait, à ce sujet, les remarques suivantes:

Aussitôt après l'exécution de l'assassin Lemaire, son cadavre a été transporté au laboratoire de M. le professeur Robin. Là on a pu constater les faits suivants :

Le corps était grêle, un des pieds difformes. Les organes génitaux, très pigmentés, offraient cet ensemble de caractères qui décèlent ordinairement la masturbation habituelle. La face était aussi prognathe que chez les nègres inférieurs, et le prognathisme tenait à la conformation des maxillaires supérieurs.

Le crâne est asymétrique; la région frontale est extrêmement petite, surtout dans le sens de la hauteur. On remarque à la partie moyenne du frontal et vers le bord supérieur des pariétaux un épaississement très considérable des os qui refoulent la substance cérébrale. Le cerveau est petit; il ne pesait que 1183 grammes, tandis que dans la race blanche, à l'âge de dixneuf ans, le poids moyen du cerveau de l'homme est supérieur à 1400 grammes.

Le cerveau et le crâne, extrêmement peu développés à la région frontale, le sont relativement beaucoup au niveau des lobes temporaux et occipitaux. A la face inférieure du cerveau, les circonvolutions sont très larges, tandis qu'à la face convexe elles sont irrégulièrement développées, les unes étant larges et d'autres non.

La suture sagittale est ossifiée, surtout à la partie antérieure, 
ce qui, à l'âge de dix-neuf ans, peut être considéré comme pathologique. La pie-mère, épaissie et injectée, adhérait tellement à la surface des hémisphères cérébraux, qu'on ne pouvait l'en détacher sans décortiquer les circonvolutions.

Ces désordres peuvent se grouper sous deux chefs: les uns accusent une organisation défectueuse, un développement imparfait du corps, surtout du crâne et surtout. du cerveau, dont ies lobes antérieurs rappellent ceux des idiots. Les autres sont la conséquence d'une méningite chronique diffuse, lésion surajoutée et relativement récente, mais pourtant bien antérieure au crime commis par Lemaire. Ce malheureux était donc, au moment où il a conçu et exécuté son crime, en proie à une maladie qui détruit la raison. Groyant punir un coupable, on a guillotiné un aliéné.

Cette conclusion découle invinciblement de l'autopsie, et elle est en parfait accord avec les symptômes observés pendant la vie, et en particulier avec ce fait, constaté à la préfecture de police, que, pendant les trois jours qui suivirent son arrestation, Lemaire ne fit que marcher avec agitation dans sa cellule, en crachotant sans cesse et sans manifester la moindre envie de dormir. Quelques jours après, il devint un peu moins agité, mais il ne dormait presque pas; en revanche, il se livrait à l'onanisme, et il conserva cette habitude même après sa condamnation. Pour un aliéniste, cette agitation, ce crachotement, cette insomnie sont des symptômes non douteux. Le crime luimême était l'acte d'un insensé; le mobile en était absurde, les moyens d'exécution ên étaient stupides. Mais l'imperturbable logique que l'accusé a montrée dans ses interrogatoires a donné le change aux magistrats et aux jurés; ils n'ont vu en lui qu'un criminel exécrable, et, lui appliquant la loi, ils n'ont pu faire autrement que de le condamner à mort. Je ne veux donc troubler la conscience de personne, car tout le monde a fait son devoir; mais il m'est bien permis de tirer de cette erreur judiciaire un argument contre la peine de mort. Avant de s'arroger le droit de tuer un homme, il faudrait que la société fût infaillible, et vous venez de voir qu'elle ne l'est pas.

M. Delasinuve. J'ai inséré dans mon Journal de médecine mentale une analyse des réponses de Lemaire pendant le cours des débats. Ces réponses 
ressemblent absolument à celles que font certains aliénés, et j'en ai conclu qu'il fallait classer Lemaire parmi les fous. J'offrirai à la Société un extrait de ce travail.

M. Pruner-Bey. Je demanderai si l'on a observé d'autres faits analogues et comparables à celui de Lemaire.

M. Broca. La population des hôpitaux d'aliénés compte un grand nombre d'assassins. Ce qui est de plus en plus rare, c'est que la société se trompe à un pareil degré, car ici la démonstration est aussi complète que possible. Mais néanmoins des centaines d'aliénés ont été exécutés.

mim. Brierre de Boismont, Delasiauve insistent sur la fréquence de l'aliénation judiciairement méconnue chez les criminels.

M. Dally soutient qu'au point de vue social peu importe l'existence de la folie : ce sont des sujets dangereux dont la société fait bien de se débarrasser, d'autant plus qu'on ne sait où commence la folie.

M. Moreau (de Tours) admet que Lemaire, atteint de lésions méningitiques, était vraisemblablement peu intelligent; il n'oserait affirmer la folie.

M. Broca. Je répondrai en quelques mots à MM. Moreau et Dally.

Les dissidences entre M. Moreau et moi me paraissent plus apparentes que réelles. Ainsi M. Moreau, après avoir admis que Lemaire était un idiot, n'a pas été éloigné de conclure qu'il était fou, et cette dernière opinion, l'anatomie pathologique l'a confirmée.

En réponse à l'étrange opinion de M. Dally, disant qu'il n'y a pas de fou, je demanderai si M. Dally considère la méningite comme un état normal.

Quant à l'erreur juridique, elle ne me paraît pas contestable. Il est bien évident que les jurés n'avaient pas l'intention de condamner un homme atteint de méningite.

Je conclus donc, encore une fois, que la faillibilité trop bien démontrée de la société est un argument contre la peine de mort.

A la séance suivante, la discussion fut reprise avec Pruner-Bey. Pour cet auteur, c'est trancher trop vite en ce qui concerne la corrélation des fonctions cérébrales avec leur substrateur anatomique. "Un instant, la doctrine de Gall lui paraissait ressuscitée. " Les caractères extérieurs du 
crâne de Lomaire ne suffisent pas à établir son infériorité. L'oblitération précoce de la suture sagittale est certaine, mais n'est pas un signe indéniable d'idiotic. Enfin la méningite est contestable, car on observe chezles suppliciés des méningites d’apparence.

(Bulletins de la Société d'anthropologie, 1867, $2^{\text {e }}$ série, t. II, p. 375, 376 et 378.)

M. Broca. Il faut que M. Pruner-Bey ou ne m'ait pas bien entendu ou ne m'ait pas bien compris, sans cela il ne m'accuserait pas de ressusciter la doctrine de Gall, qui consiste à établir un rapport constant, scientifique, entre certaines tubérosités crâniennes et certaines facultés. Rien de tout cela dans ce que j'ai dit. C'est la méningite que j'ai donnée comme preuve de la folie et non point la conformation du crâne et de la face. J'avais distingué ce que M. Pruner-Bey a confondu : l'idiotie et la folie ; car il n'est pas plus logique de classer un idiot parmi les aliénés que de considérer la synostose crânienne commə un signe ou comme une cause d'aliénation.

Je n'ai pas dit non plus que Lemaire fût d'une race inférieure; car, en ma qualité de polygéniste, je n'admets pas qu'une maladie puisse faire passer d'une race dans une autre.

Quant au poids du cerveau, je ne vois pas bien comment le genre de mort de Lemaire aurait pu l'alléger.

Mais le point essentiel, celui que nie M. Pruner-Bey, est la méningite, la lésion anatomique sur laquelle est basé le diagnostic de l'état maladif.

Je crains que M. Pruner-Bey ne se soit pas bien renseigné sur les caractères anatomo-pathologiques de la méningite. La méningite est la seule cause qui puisse faire adhérer la pie-mère au cerveau dans toute son étendue. Tous les anatomo-pathologistes qui ont examiné le cerveau de Lemaire ont reconnu sans hésitation les traces d'une méningite; traces tellement connues, tellement incontestables, qu'autant vaudrait nier une fracture en présence des fragments de l'os brisé.

Enfin, en terminant sa critique, M. Pruner-Bey m'a fort étonné, car après avoir attaqué toutes les conclusions tendant à relier le physique au moral, il a fait un vrai diagnostic phrénologique et a même été jusqu'à retrouver chez Lemaire la physionomie de deux parricides dont Gall nous a laissé les bustes. 
M. Pruner-Bey revient principalement sur la méningite d'apparence, constatée par Lelut et sur les suppliciés et sur les chiens décapités.

M. Broca. Je ne puis accepter comme un fait sérieux la méningite apparente des suppliciés, que M. Pruner-Bey invoque d'après l'autorité de M. Lélut. Chez Lemaire, les vaisseaux des méninges, au lieu d'être vides, étaient surpleins. Enfin, si le cerveau n'a point été soumis au lavage absolument comme le veut $M$. Lélut, il a été plongé dans un liquide, y a séjourné, et néanmoins les caractères anatomo-pathologiques de la méningite n'ont pas disparu. 


\section{SUR LA DÉFORIIATION TOULOUSAINE DU CRANE}

(Bulletins de la Société d'anthropologie, 2e série, 1871, t. VI, p. 103-131.)

Au mois de juillet dernier, en traversant la salle d'autopsie de l'hôpital de la Pitié, j’aperçus sur une table le corps d'une vieille femme morte dans le service de mon collègue le professeur Lasègue. La tête était entièrement chauve et présentait un exemple très évident de la déformation toulousaine. J'envoyai aussitôt prendre des renseignements au bureau et on me répondit que cette fermme était née à Toulouse même, en 1797.

Le corps n'étant pas réclamé, je priai M. Lasègue de vouloir bien me l'abandonner pour mes recherches et je le fis transporter à l'Ecole pratique, au laboratoire d'anthropologie.

Mon premier soin fut de faire faire le moule que je vous présente aujourd'hui, et que je dépose dans le musée de la Société. Après quoi, je procédai à l'examen du cerveau. Je me proposais surtout de constater l'état du lobe frontal et de voir si ce lobe avait conservé ses rapports ordinaires avec les os du crâne.

On a émis des opinions contradictoires relativement à l'influence que les déformations artificielles du crâne peuvent exercer sur l'intelligence. Que le cerveau doive se déformer en même temps que le crâne, c'est une chose nécessaire et admise par' tout le monde; mais tout le monde aussi a constaté que le crâne, déprimé artificiellement sur certains points, tend à se dilater dans les parties qui échappent à la compression; on peut donc se demander si le cerveau souffre réellement beaucoup de cette action mécanique, et s'il ne regagne pas dans un sens ce qu'il perd dans un autre. C'est sur ce point qu'ont roulé les discussions.

Il y a des cas où la déformation est tellement profonde, tellement générale, et où la capacité absolue du crâne est tellement réduite, que la diminution du volume du cerveau est tout à 
fait évidente. Mais, lorsque la déformation est moins grave, l'appréciation devient douteuse. La capacité crânienne peut être encore assez grande pour qu'on ne puisse pas savoir si elle a subi une réduction; et ce qu'il importe de constater alors, c'est moins le poids des hémisphères cérébraux, que le développement relatif de leurs lobes. Cette étude a été entièrement négligée jusqu'ici, faute d'occasion favorable. J'ai donc cherché à tirer le meilleur parti possible du fait que l'obligeance de mon collègue M. Lasègue mettait à ma disposition.

Je dirai d'abord quelques mots sur les rapports qu'affectent entre eux les os du crâne et les lobes cérébraux.

Les trois lobes de chaque hémisphère portent les noms des trois os qui les recouvrent: le frontal, le pariétal et l'occipital. Toutefois la correspondance des os et des lobes n'est pas rigoureuse.

Gratiolet, dont les travaux ont jeté tant de jour sur l'étude des circonvolutions cérébrales, avait cru pouvoir conclure de la comparaison des moules intracrâniens avec les cerveaux étalés sur la table, que le sillon de Rolande, qui établit la séparation du lobe frontal et du lobe pariétal, était situé directement sous la suture fronto-pariétale ou coronale; puis, en étudiant de la même manière les rapports du lobe occipital, il avait cru trouver que le sillon occipital, qui en établit la limite, était situé chez l'homme bien au-dessous de la suture occipito-pariétale ou lambdoïde (1).

C'était une double erreur. Lorsque je voulus à mon tour étudier cette question, je ne tardai pas à me convaincre de l'incertitude du procédé de recherches suivi par Gratiolet. Je reconnus que le cerveau, retiré de la boîte crânienne, subissait aussitôt une déformation considérable, qui ne permettait plus d'en retrouver exactement les rapports. Je cherchai done à déterminer ces rapports avant l'ouverture du crâne, à l'aide de petites chevilles de bois de diverses couleurs introduites à travers des trous de vrille, et poussées par un stylet jusque dans l'épaisseur du cerveau. Le crâne était alors ouvert à la scie, et,

(1) Gratiolet et Leuret, Anatomie comparée du système nerveux, t. II, p. 113 et 124. Paris, 1857 , in- $8^{\circ}$. 
le cerveau placé sur la table, il était facile de déterminer les rapports des diverses parties du cerveau avec les divers points du crâne. J'obtins ainsi deux résultats contraires à ceux qu'avait annoncés Gratiolet ; je constatai, d'une part, que le sillon occipital correspond presque toujours d'une manière assez exacte à la suture lambdoïde, et, d'une autre part, que le sillon de Rolando du cerveau humain est toujours situé bien en arrière de la suture coronale; qu'en d'autres termes l'étendue du lobe occipital est mesurée par celle de la fosse occipitale supérieure, tandis que le lobe frontal, beaucoup plus grand chez l'homme que la loge frontale, empiète considérablement sur la région pariétale (1). Je répétai ces expériences en présence de Gratiolet, et il se rendit aussitôt à ma démonstration.

Le sillon de Rolando, sur la ligne médiane, commence à 4 centimètres au moins en arrière de la suture coronale. Chez un épileptique dont le cerveau était très volumineux, la distance n'ćtait pas de moins de 63 millimètres; mais ce fait est tout à fait exceptionnel. Dans tous les autres cas que j’ai étudiés, l'écart du sillon et de la suture variait entre 40 et 56 millimètres, et il était en moyenue de 47 millimètres. A partir de la ligne médiane, le sillon de Rolando et la suture coronale descendent obliquement en avant et en bas sur les côtés du crâne; mais le sillon, beaucoup plus oblique que la suture, va toujours en s'en rapprochant, sans toutefois jamais l'atteindre, de sorte que l'extrémité inférieure du sillon est encore située à 15 millimètres environ en arrière de la suture.

Ces observations ont été recueillies à Bicêtre, où il n'y a que des hommes. Les cerveaux des femmes étant en général plus petits que ceux des hommes, il est probable que l'écart que je viens d'indiquer doit présenter chez les femmes une réduction proportionnelle.

J'ai dû rappeler ces résultats afin de pouvoir apprécier les effets produits par la déformation du crâne sur les lobes cérébraux de notre vieille Toulousaine, à laquelle je reviens maintenant.

(1) Voir Bulletins de la Societé anatomique, 2e série, t. VI, p. 340 (en note), août 1861 . 
Après avoir enfoncé plusieurs vrilles à travers les sutures coronale et lambdoïde, je poussai mes chevilles dans le cerveau, puis j'ouvris le crâne à la scie. Mais, lorsque le trait de scie fut terminé, je rencontrai un obstacle inattendu : la calotte, quoique entièrement détachée de la base et quoique devenue mobile sous la main, adhérait tellement à la dure-mère, qu'il fut impossible de la faire sauter. J'exerçai sur elle de fortes tractions à l'aide du crochet; mais je dus m'arrêter lorsque je vis la dure-mère se rompre sur un côté et la substance cérébrale faire hernie à travers cette ouverture. Il fallut donc renoncer au procédé ordinaire, inciser circulairement la dure-mère et détacher la faux de l'apophyse crista galli. Je pus ainsi enlever ensemble la voûte et la dure-mère, qui y adhérait fortement. L'adhérence occupait toute la région frontale et le tiers antérieur de la région pariétale, c'est-à-dire toute la partie du crâne qui avait été comprimée dans le jeune âge. Du reste, le tissu osseux n'était ni raréfié, ni cundensé, ni épaissi, ni aminci; il paraissait entièrement sain; et de même la pie-mère et le cerveau subjacent ne présentaient aucıne lésion; la seule altération appréciable était celle de la dure-mère, qui était non seulement adhérente, mais encore notablement épaissie. J'ai lieu de croire que ce résultat des déformations artificielles du crâne n'avait pas encore été signalé. Je ne puis évidemment tirer d'un seul fait une conclusion générale; il est probable que les effets des actions mécaniques doivent varier suivant le degré de la compression, et aussi suivant les dispositions individuelles. Il est clair toutefois que, si chez notre Toulousaine les agents de la déformation ont produit une lésion sur la dıre-mère, des causes analogues, agissant avec plus de force, peuvent produire des lésions sur les autres méninges, et ainsi s'explique peutêtre la grande fréquence des désordres intellectuels chez les individus dont le crâne a subi une déformation mécanique, fréquence constatée par les médecins aliénistes dans les départements de la Haute-Garonne, des Deux-Sèvres et de la SeineInférieure.

Le cerveau, quoique assez mou, comme il l'est souvent chez les vieillards, n'était d'ailleurs point malade; mais il était fort petit. L'encéphale entier, avec la pie-mère, ne pesait que 
1079 grammes. Cette exiguïté du cerveau ne dépendait point de la taille. On sait qu'en moyenne les individus petits ont le cerveau un peu moins gros que les individus plus grands. Mais notre Toulousaine avait $1^{\mathrm{m}}, 53$, ce qui est à peu près la taille moyenne des femmes. Comme elle avait soixante-quatorze ans, son cerveau avait pu diminuer un peu sous l'influence de la vieillesse. Toutefois les circonvolutions offraient un volume ordinaire; la sérosité qui, comme on sait, comble le vide laissé par l'atrophie sénile du cerveau n'était pas très abondante. Le poids de l'encéphale n'avait donc pas subi par cette cause une grande diminution ; vous pouvez voir d'ailleurs, sur le crâne sec que je vous présente, que la boîte crânienne est fort petite; la loge frontale est considérablement réduite et la capacité totale du crâne, jaugée avec du petit plomb, n'est que de 1198 centimètres cubes, inférieure de 125 à la capacité moyenne du crâne des femmes de notre race.

La galerie anthropologique du Muséum possède un autre crâne de Toulousaine, donné par M. le professeur Filhol, directeur de l'Ecole de médecine de Toulouse. La forme n'est pas sensiblement plus altérée que dans le cas actuel; mais la capa-

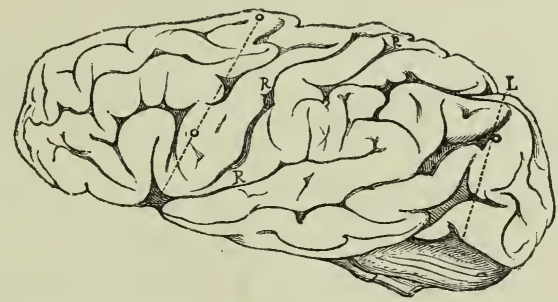

Fig. 2. Face latérale de l'hémisphère gauche (voir l'explication des lettres figure 3).

cité crânienne est encore plus faible : elle n'est que de 1043 centimètres cubes. C'est la plus petite capacité que j’aie observée jusqu'ici sur des crânes humains non microcéphales. La déformation toulousaine, lorsqu'elle est très prononcée, est donc capable de nuire au développement général de l'encéphale. On le conçoit sans peine lorsqu'on voit combien la capacité de la loge frontale est diminuée. Si le lobe frontal perdait tout ce que perd la loge frontale, l'idiotie en serait la conséquence presque 
inévitable. Les Toulousains déformés n'étant pas idiots, pouvant même être doués d'une intelligence très développée, il paraît probable que le lobe frontal doit regagner dans le sens de la longueur au moins une partie de la place qui lui est refusée dans le sens de la hauteur. C'est ce que j’ai constaté sur le cerveau de ma Toulousaine.

Les chevilles enfoncées à travers la suture coronale permettaient de retrouver sur le cerveau le trajet de cette suture. Or le

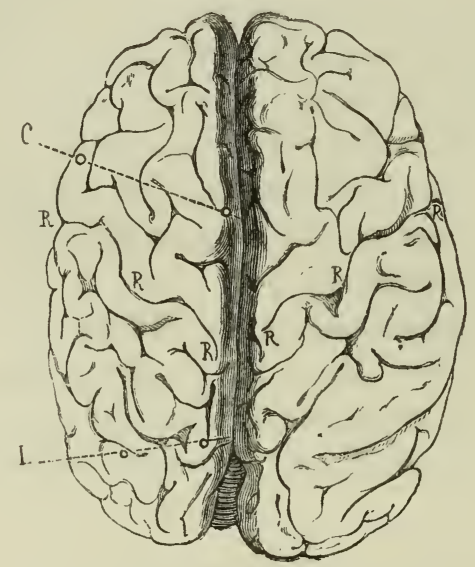

Fig. 3. C, ligne ponctuée passant par les deux trous de vrille de la suture coronale, I, ligne ponctuée passant par les deux trous de vrille de la suture lambdoïde ; R, R, R, silion de Rolando.

sillon de Rolando, beaucoup plus oblique que de coutume, commençait sur la ligne médiane à 57 millimètres en arrière du bregma. Cette distance excède de 1 centimètre la moyenne ordinaire; elle excède même de 1 millimètre le maximum que j'ai observé sur des hommes (à l'exception de l'épileptique dont j'ai parlé plus haut), et il ne faut nas oublier que le cerveau de l'homme est en général plus grand que celıi de la femme, plus grand à plus forte raison que le petit cerveau de notre Toulousaine. Toutes choses égales d'ailleurs, la distance du sillon de Rolando au bregma devrait donc être, chez cette dernière, inférieure non seulement au maximum, mais encore à la moyenne que l'on observe dans le sexe masculin. Elle est au contraire supérieure, même au maximum, et il est permis d'en conclure 
que le lobe frontal, sur la ligne médiane, a regagné au moins 1 centimètre en longueur. Mais sur les côtés le refoulement du lobe frontal est beaucoup muindre. J'ai déjà dit que le sillon de Rolando est très oblique; à sa partie inférieure et externe, il n'est plus qu'à 18 millimètres en arrière de la suture coronale; c'est donc à peine si, en ce point, le lobe frontal a gagné 3 ou 4 millimètres, et, somme toute, lorsqu'on compare ce qu'il a recouvré sur la longueur avec ce qu'il a perdu sur la hauteur, on est autorisé à en conclure que la déformation du crâne a nui d'une manière très notable au développement de ce lobe.

En arrière, les chevilles enfoncées dans la suture lambdoïde avaient pénétré exactement dans le sillon occipital des hémisphères cérébraux. Les rapports du lobe occipital n'étaient donc point changés; c'était exclusivement aux dépens du lobe pariétal que le lobe frontal s'était allongé.

Après avoir constaté la position relative des sillons et des chevilles, j'enlevai la pie-mère et j'étudiai la forme générale du cerveau. La région des lobes occipitaux était large, épaisse el paraissait plus ample que de coutume; celle des lobes pariétaux offrait une largeur et une épaisseur à peu près normales; c'était donc seulement dans le sens antéro-postérieur que la croissance de ces derniers lobes avait souffert. Les lobes temporo-sphénoïdaux, très aplatis en avant, étaient notablement atrophiés, résultat dû à la compression qui leur était transmise par les lobes frontaux. Ceux-ci enfin se faisaient remarquer par leur étroitesse, et surtout par leur peu de hauteur (voir les figures).

D’une manière générale, le cerveau était peu plissé, et la simplicité des circonvolutions annonçait peu d'intelligence.

J'aurais voulu pouvoir vous présenter le cerveau sur lequel j'ai fait ces observations; mais cet organe, déjà un peu ramolli par la vieillesse, plus altéré encore par la décomposition cadavérique (on était au mois de juillet), et soumis enfin, comme je l'ai déjà dit, à des pressions violentes au moment de l'autopsie, ne put être conservé. Au bout de peu de jours, des signes non équivoques m'annoncèrent que, sous l'écorce extérieure durcie par le liquide conservateur, les couches centrales commençaient à se liquéfier. Je me décidai alors à le mouler. J'ai tiré deux exemplaires de ce moule, l'un pour le musée de la Société, 
l'autre pour mon laboratoire. La forme générale de l'organe est malheureusement altérée; les lobes, en s'étalant sur la table, se sont inégalement affaissés, et le lobe pariétal, dont la hauteur excédait de beaucoup celle du lobe frontal, est maintenant presque aussi plat que lui. Mais, du moins, les circonvolutions sont bien dessinées, leurs connexions parfaitement conservées, et l'empreinte des chevilles permet de retrouver la position des sutures. On peut donc constater aisément tous les caractères que je viens de décrire.

Vous pouvez voir sur le moule que l'hémisphère gauche est sensiblement plus petit que le droit. Cette inégalité, que l'examen extérieur dı crâne ne faisait pas prévoir, a pu être constatée à la balance. L'espoir que j'avais eu d'abord de conserver le cerveau m'avait empêché de pratiquer les coupes et de faire, à l'état frais, les pesées partielles. Mais, après le moulage, avant de jeter le cerveau,j'ai déterminé le poids de ses diverses parties.

Cet organe avait séjourné d'abord pendant quelques jours dans l'alcool, puis dans le liquide Baudiau (eau, chlorure de zinc, alcool et glycérine). Il avait perdu dans ce liquide une notable partie de son poids.

Au sortir du crâne, il pesait, comme je l'ai déjà dit, 1079 grammes. Après l'ablation des membranes et l'écoulement de la sérosité ventriculaire, il pesait encore 1029 grammes. Après le séjour dans les liquides conservateurs, son poids était réduit à 825 grammes, c'est-à-dire aux huit dixièmes du poids précédent. La réduction due à l'absorption de l'eau de la substance cérébrale par le liquide conservateur n'est cependant pas uniforme; elle est toujours plus considérable sur la substance grise, qui prédomine dans les hémisphères et dans le cervelet, que sur la substance blanche, qui prédomine dans le bulbe et la protubérance annulaire. Les pesées partielles pratiquées sur les cerveaux conservés donnent donc des.résultats proportionnels un peu différents de ceux que l'on obtient sur les cerveaux frais; mais le poids relatif des deux hémisphères ou de leurs lohes isolés n'est pas sensiblement modifié, et le poids relatif du cervelet lui-même ne change pas beaucoup plus. Ces remarques permeltent de comprendre la signification des pesées partielles pratiquées sur le cerveau de la Toulousaine. 
Le cervelet pèse 109 grammes, et si l'on calcule le rapport de ce chiffre avec celui de 825 grammes qui représente le poids total de l'encéphale dépouillé de ses membranes, on trouve que le cerrelet constitue 13,21 pour 100 de la masse encéphalique. Sur 10 hommes âgés de plus de 40 ans (âge moyen, 51 ans), le rapport n'est que de 10,57 pour 100 , et sur 10 femmes âgées de plus de 60 ans (âge moyen, 72 ans), il est de 11,10 . Le cervelet est donc relativement plus volumineux chez la Toulousaine que chez les individus avec lesquels je la sompare, et la différence (plus de 2 pour 100) est assez grande pour qu'on ne puisse l'attribuer aux changements produits par l'action des liquides conservateurs. Cette prédominance du cervelet est d'ailleurs tout artificielle; elle ne tient pas à l'accroissement du volume de cet organe, mais à la diminution des parties antérieures de l'encéphale, et l'on verra d'ailleurs tout à l'heure que les lobes occipitaux des hémisphères sont exactement dans le même cas que le cervelct.

Le poids de l'hémisphère droit est de 351 grammes; celui de l'hémisphère gauche est de 339 grammes seulement. Cette différence de 12 grammes est déjà considérable; mais on remarquera qu'elle a subi une réduction proportionnelle à celle des hémisphères eux-mêmes, et puisqu'elle est de 12 grammes sur un cerveau réduit à 825 , elle était de 15 (exactement de $14^{\mathrm{g}}, 96$ lor'sque le cerveau pesait 1029 grammes. Si l'on songe que les différences de poids des deux hémisphères dépassent rarement ๖ ou 6 grammes (exception faite des cas pathologiques), on sera autorisé à attribuer ce résultat à la perturbation que la déformation du crâne a fait subir au développement du cerveau.

Chose remarquable, l'inégalité des deux hémisphères dépend à peu près exclusivement de l'inégalité des deux lobes frontaux. Dans les pesées cérébrales que j'ai faites depuis dix ans, et qui sont consignées sur un registre où la Toulousaine occupe le numéro 437, j’ai toujours noté séparément, pour chaque hémi. sphère, le poids du lobe frontal et celui du lobe occipital. Ce n'est pas ici le lieu d'indiquer le procédé que je suis pour séparer ces deux lobes du reste de l'hémisphère par deux coupes méthopiques. Ce procédé est d'unc exécution assez délicate; toutefois 
j'en ai acquis une assez grande habitude pour avoir le droit de considérer comme sûr's les résultats qu'il m'a fournis. Lorsque les deux hémisphères sont égaux, il est rare que je trouve plus de 3 ou 4 grammes de différence entre deux lobes de même nom. Or, sur la Toulousaine, le lobe frontal gauche ne pesait que 144 grammes, tandis que le droit en pesait 159. La différence est de 15 grammes. Celle des deux hémisphères n'est que de 12 grammes; mais, comme la coupe a été faite sur des sillons altérés et déformés, le couteau a bien pu faire un léger écart, et j'ai pu commettre ainsi une erreur de quelques grammes. Je pense donc que la différence réelle des deux lobes frontaux n'était pas supérieure à celle des deux hémisphères, mais qu'elle lui était à peu près égale. Ce que je crois pouvoir affirmer, en tous cas, c'est que l'inégalité des deux hémisphères est due principalement à l'atrophie du lobe frontal gauche.

Si maintenant nous passons aux autres lobes, nous verrons reparaître l'égalité. Les deux lobes occipitaux pèsent chacun 450 grammes, et le reste des hémisphères, comprenant à la fois le lobe pariétal et le lobe temporo-sphénoïdal, pèse 147 grammes à droite et $15 \%$ grammes à gauche, différence peu ou point significative.

Je signale le grand volume des lobes occipitaux. La somme de leurs poids s'élève à 90 grammes. Le poids total des deux hémisphères est de 690 grammes. Les lobes occipitaux forment donc un peu plus des treize centièmes de ce poids $(13,04$ pour 100). Or, sur les cerveaux normaux, le rapport du poids des lobes occipitaux au poids des hémisphères est toujours beaucoup moindre. Ce rapport est en moyenne de 9,50 pour 100 sur les 10 hommes et de 9,97 sur les 10 femmes que j'ai mis en parallèle avec la Toulousaine. La différence étant beaucoup plus grande que celle que j'ai constatée plus haut pour le cervelet, on est déjà disposé à croire que non seulement la dépression de la région frontale n'a pas nui au développement des lobes occipitaux, mais qu'elle a encore amené l'accroissement exagéré de ces lobes. C'est ce qu'il est d'ailleurs facile de démontrer en calculant le poids primitif des lohes occipitaux. L'encéphale entier ayant été réduit de 1029 grammes à 825 grammes par l'action des liquides conservateurs, il suffit, pour retrouver le poids 
réel des lobes occipitaux, de multiplier leur poids de 90 grammes par la fraction $\frac{823}{1029}$; ce qui donne un peu plus de 112 grammes. Or le poids moyen de ces lobes est de 108 chez nos 10 hommes, et de 95 seulement chez nos 10 femmes. Les lobes occipitaux de la Toulousaine avaient donc un poids absolu bien supérieur à la moyenne, quoique le cerveau de cette femme fût bien inféricur à la moyenne. Pour compléter la démonstration, j'ajoute que le cerveau moyen des 10 hommes pesait $135 \% 8$ grammes avec les membranes et 1307 sans les membranes; et que celui des 10 femmes pesait 1154 grammes avec les membranes et 1103 sans les membranes.

Ainsi, la déformation toulousaine ne se borne pas à refouler l'encéphale et à lui diminuer l'espace; elle modifie en outre le volume absolu et le volume relatif de ses diverses parties. Elle diminue beaucoup les lobes frontaux; elle produit également une atrophie notable sur l'extrémité antérieure des lobes temporo-sphénoïdaux; elle nuit encore, quoique à un moindre degré, à la croissance des lobes pariétaux, tandis qu'au contraire, en augmentant la courbure de l'écaille occipitale, elle agrandit la loge des lobes occipitaux, qui s'accroissent outre mesure. Quant au cervelet, plus éloigné de la région comprimée, et protégé d'ailleurs par le tentorium, il paraît échapper à l'influence qui agit sur les hémisphères, et s'il devient relativement plus grand, c'est parce que le cerveau proprement dit devient plus petit.

Quoique la physiologie des hémisphères cérébraux soit encore bien peu avancée, nous en savons assez cependant pour pouvoir dire que le développement relatif des divers iobes ne peut être modifié aussi manifestement qu'il l'a été chez notre Toulousaine, sans que les facultés intellectuelles s'en soient plus ou moins ressenties. J'aurais donc voulu recueillir quelques renseignements sur l'état mental de cette femme. Mais tout ce que j'ai pu apprendre dans le service où elle a été traitée, c'est qu'elle n'était ni idiote ni aliénée. Admise, peu de jours avant sa mort, pour une grave affection du cœur, atteinte d'un essoufflement qui lui rendait toute conversation pénible, elle parlait fort peu, mais ses paroles étaient sensées et son intelligence parut ordinaire. 
Je vous ai parlé en premier lieu de l'état du cerveau, et j'ai dû le faire avec quelques détails, parce que c'est l'étude de cette question jusqu'ici inexplorée qui a motivé ma communication. Mais je dois dire maintenant quelques mots de la conformation du crâne, et je serai bref sur ce point, qui est déjà connu.

La déformation principale et primordiale consiste en une dépression considérable de la voûte dı crâne au niveau du bragma. Le front commence à fuir à partir de la racine du nez; il remonte ainsi sous une inclinaison modérée jusqu'au niveau des bosses frontales; là il s'affaisse assez brusquement pour que ce changement de direction soit indiqué par une courbe d'un

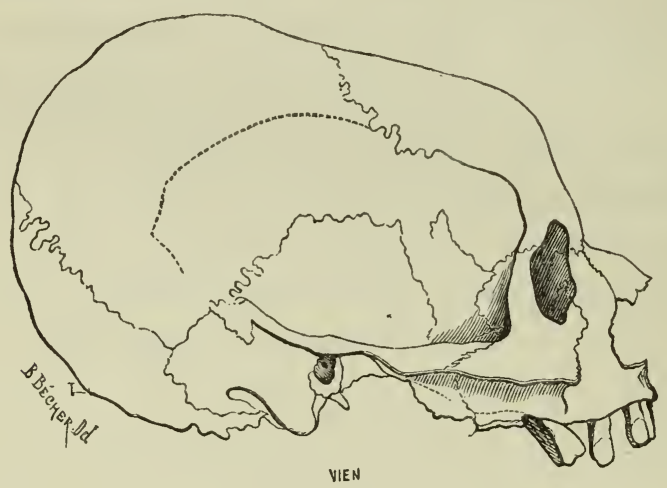

Fig. 4. Le protil de la Toulousaine dessiné au stéréographe.

rayon assez petit; devenu ainsi très oblique, il remonte sans nouvelle inflexion jusqu'au bregma. A partir du bregma, le profil du crâne se relève un peu, et continue à remonter, toujours très obliquement, jusqu'au vertex, situć à 4 centimètres environ en arrière dı bregma.

La mâchoire inféricure coïncide difficilement avec la supérieure; les dents incisives se répondent fort mal. Le menton n'est nullement fuyant. Lorsqu'on examine l'articulation temporo-maxillaire, on voit que la mastication devait être quelque peu gênée. La cause mécanique qui a repoussé la mâchoire supérieure en avant n'a pas eu prise sur la mâchoire inférieure. Ceelle-ci cst donc moins grande dans le sens antéro-postérieur que ne l'exigerait la coaptation des dents incisives; pour rendre 
la coaptation possible, il faut faire avancer les condyles jusque sous la partie la plus antérieure de la racine transverse de l'arcade zygomatique; aussi cette racine transverse est-elle très ćlargie et très aplatie. Ce qui rı'est pas moins curieux, c'est que l'écartement des condyles dı-maxillaire inférieur est notablement plus grand que celui des cavités glénoïdes des temporaux. Las condyles, de chaque côté, débordent de plus de 1 centimètre les cavités qui devtaient les recevoir, et l'impossibilité de les y adapter est telle, que, si la préparation des os n'avait pas ćté faite sous mes yeux, dans mon laboratoire, je pourrais douter que le crâne et la mâchoire inférieure eussent appartenu au mème individu. Ici encore nous pouvons reconnaître l'influence de la déformation crânienne, qui a entravé dans tous les sens le développement de la partie antérieure du crâne; le diamètre biauriculaire n'a pu s'accrô̂tre au degré voulu ; et la mâchoire inférieure, n'étant pas gênée dans sa croissance, a acquis une largeur qui paraît trop grande et qui pourtant n'est que normale.

On voit que la déformation toulousaine étend ses effets bien au-delà de la région qui est directement comprimée. Tous les rapports proportionnels sont changés, toute l'harmonie de la tête est détruite. Il est assez rare sans doute que les désordres du développement soient poussés jusqu'au degré que je viens de décrire. Lorsque la déformation est modérée, elle occasionne des troubles beaucoup moins profonds, mais pourtant de même nature, et on ne saurait nier les inconvénients d'une pratique qui date des temps barbares, et que les progrès des lumières n'ont pu extirper encore. Au surplus, je me hâte de dire que les efforts des médecins qui ont combattu la routine populaire ont déjà produit d'excellents effets. Dans la ville de Toulouse, les déformations du crâne sont devenues aujourd'hui assez rares chez les individus âgés de moins de quarante ans; mais elles sont toujours fréquentes dans les campagnes, et il s'écoulera sans doute encore plusieurs générations avant que ce dernier vestige des mœurs des anciens Tectosages ait entièrement disparu.

M. Rocher demande si l'on a quelques données établissant que la prd- 
tique des déformations ait exercé quelque influence sur l'intelligence de la population toulousaine.

M. Broca. Les Toulousains ont toujours passé pour très intelligents. Ils se sont distingués depuis longtemps dans les lettres, et il n'est peut-être pas de population en France qui ait une aussi grande aptitude pour la musique. Ils ont eu moins de succès dans les sciences. J'ai même entendu dire que la faculté des lettres se recrutait toujours dans le pays même, tandis que les professeur's de la faculté des sciences seraient souvent étrangers à la localité. Je ne sais jusqu'à quel point ce renseignement est exact, quoique je le tienne d'un homme très distingué, dont la famille est originaire de Toulouse. Quant à la femme dont je viens de présenter le cerveaı, je répète que je n'ai pu apprendre rien de précis sur l'état de son intelligence.

M. Delasiauve. M. Broca a signalé l'inégalité des deux hémisphères cérébraux de la Toulousaine. Cette inégalité est remarquable, car j’ai rarement trouvé entre les deux hémisphères plus de 5 à 6 grammes dle différence chez les sujets non épileptiques qui n’avaient pas de lésions cérébrales. J'ajoute que dans la très grande majorité des cas la différence ne dépasse pas 2 ou 3 grammes, et que les individus qui présentent des différences plus grandes ne sont ni plus ni moins intelligents que les autres.

M. Peltar'n demande à M. Broca s'il pense que les déforinations artificielles du crâne soient héréditaires.

M. Broca répond négativement. Il rappelle que les déformations artificielles, très communes à Toulouse à la fin du dernier siècle, y sont devenues de plus en plus rares à mesure qu'on a renoncé à la coiffure traditionnelle des jeunes enfants.

M. Rochet. J'ai observé dans les théâtres, dans les églises et dans tous les lieux publics où les hommes ont l'habitude de se découvrir, un grand nombre de têtes chauves. J'ai trouvé que chaque crâne, pour ainsi dire, avait sa déformation particulière, quelquefois poussée à un degré excessif, mais toujours visible et souvent très choquante. On pourrait conclure de là que la cause de ces déformations nous échappe souvent ou que chacun, selon son pays, comme le Toulousain, porte avec lui sa marque particulière et vient imprimer en quelque sorte une chance d'erreur aux découvertes de la science. Il n'y a pas quinze jours que, par une chaleur extrême, j'ai pu observer à la porte d'un café un vieillard qui, la tête découverte, cherchait à se rafraîchir. Il m’a semblé voir la calotte crânienne du Néanderthal. 
M. Delasiauve. Quelquefois dans les accouchements laborieux occasionnés par des bassins rétrécis, la déformation de la tête est très grande, et peut-être pourrait-on y trouver une cause des irrégularités crâniennes qu'a observées M. Rochet.

M. Prat ne pense pas qu'on puisse invoquer l'accouchement.

M. Genillier revient sur l'influence que la compression des lobes antérieurs doit exercer sur les fonctions cérébrales.

M. Broca. Je pense d'une manière générale qu'une petite déformation ne doit pas modifier notablement le développement des diverses parties des hémisphères cérébraux; mais il me paraît impossible que les grandes déformations, telles que celles que pratiquent les indigènes de l'Amérique, ne portent pas une atteinte très sérieuse aux fonctions cérébrales. Les auteurs qui ont dit le contraire ont constaté par exemple que les Indiens Têtes-Plates ne sont pas idiots; ils n'ont vu manquer chez eux aucune faculté fondainentale, et ils en ont conclu que les déformations crâniennes ne nuisaient pas à l'intelligence. Mais cette observation superficielle, qui ne repose pas sur une comparaison et sur une analyse méthodiques, est tout à fait sans valeur.

Si les renseignements recueillis par M. Grosse père sont exacts, les sauvages eux-mêmes auraient reconnu que les déformations artificielles exercent quelque influence sur l'intelligence, que l'aplatissement de la région antérieure du crâne développe le courage ou plutôt la brutalité des guerriers, et que la compression de la région occipitale développe au contraire la prudence. Il paraîtrait que, dans certaines îles de la mer du Sud, les famiiles aristocratiques employaient ces deux déformations inverses pour former dès le berceau des hommes pour la guerre ou pour le conseil. Je ne sais ce qu'il faut en croire; mais, si le fait était vrai, il ne serait nullement contraire à nos connaissances physiologiques.

La déformation toulousaine est peu de chose auprès de certaines déformations américaines et océaniennes; ne comprimant que la région frontale, à une époque ou les os sont souples et unis par des membranes, elle laisse au cerveau la possibilité de se réfugier en arrière; et si, dans les cas où elle est très prononcée, elle peut, comme je viens de le montrer, modifier notablement le développement absolu et relatif des divers lobes céré- 
braux, il est probable que, lorsqu'elle est légère, elle ne nuit pas au développement général de l'intelligence considérée dans son ensemble; mais je concevrais très bien qu'elle pût cxercer encore une certaine influence sur telle ou telle faculté, sur tello ou telle aptitude.

Toutefois ce serait peut-être aller bien vite que d'attribuer à la pratique de la déformation frontale la prédominance des aptitudes artistiques et littéraires sur les aptitudes scientifiques dans la population toulousaine. Quand même cette prédominance serait dûment constatée (ce qui n'est pas), elle pourrait tenir à d'autres causes, car on observe des particularités psychologiques analogues ou inverses dans des populations qui ne connaissent pas la pratique des déformations artificielles du crâne.

M. Jules GuÉrın. J'ai chez moi plusieurs crânes qui sont en apparence du même genre que celui que nous a présenté M. Broca, et dont la forme anormale est due à une maladie de tout le squelette. Dans le cas d'un squelette mal conformé, l'un des diamètres peut ètre allongé dans le sens antéro-postérieur, ou raccourci verticalement. Ces déformations appartiennent au rachitisme.

M. Broca. Les déformations pathologiques de certains crânes sont incontestables, et leur liaison avec certaines causes, comme le rachitisme, l'oblitération prématurée d'une suture ou la déformation d'une fontanelle, est bien connue; mais en même temps les individus sont plus ou moins atteints d'hydrocéphalie ou d'hypertrophie cérébrale, et on les reconnaît ordinairement à leur front projeté en avant.

MM. Pellarin et Rochet ont posé la question de savoir jusqu'à quel point cette déformation peut se continuer pendant plusieurś générations. On a cité l'autorité d'Hippocrate; Hippocrate raconte ce qu'il n'a pas vu, mais ce qu'il a entendu dire. On s'est occupé beaucoup de cette question dans la discussion des monogénistes et des polygénistes. Aujourd'hui la question a changé de face et doit être étudiée à un autre point de vue; on cherche dans le temps et non dans les lieux l'origine des races.

Il faut se défier des histoires toutes faites qui arrivent juste à point pour servir d'appui à une doctrine bâtie d'avance; que n'a-t-on pas avancé à propos des causes de la couleur des noirs et des blancs? 
Il a été reconnu par Hippocrate lui-même que, dans les pays où l'on abandonne la cause toute mécanique de la déformation, l'effet disparaît avec la cause. Ainsi la pratique ancienne qui distinguait les gens du peuple de Toulouse disparaît de plus en plus, et avec elle la déformation du crâne. Depuis une trentaine d’années, la très grande majorité des Toulousains échappe à la difformité. On a dit encore, en poussant cette théorie bien plus loin, que des individus privés accidentellement d'un ou de plusieurs doigts transmettaient leur difformité à leurs descendants. Mais c'est purement imaginaire, et autant il est certain que l'ectrodactylie de naissance est quelquefois héréditaire, autant il est démontré que l'amputation d'un doigt ou d'un membre ne l'est jamais.

Mi. Jules Guérix reprend la question de transmission héréditaire possible. Ainsi, dit-il, quand on coupe la queue ì un chien mâle, la transmission héréditaire peut s'observer si la section a été pratiquée avaut la puberté. A cela, M. Sanson répond par l'exemple des moutons mérinos, auxquels on coupe toujours la queue, et qui cependant naissent toujours pourvus de cet appendice.

M. Jules Guérin. C'est une question très générale que de savoir si l'action mécanique sur les organes a un effet retentissant sur les descendanls. Non seulement je crois que, dans certaines conditions, cela est possible, mais encore que c'est une loi de la génération. Les mutilations mécaniques accidentelles ne sont pas transmissibles, mais les modifications fonctionnelles avec influx nerveux sont transmissibles et se trouvent liées à l'âge tendre de l'individu avant la révolution qui s'opère par la puberté dans le système nerveux.

M. Broca. Il est fâcheux que M. Jules Guérin n'ait pas conservé des pièces qui auraient pu devenir pour nous si intéressantes. Je le prierai de les conserver à l'avenir si l'occasion se présentait à lui de nouveau. Cependant les chevaux et les moutons auxquels on coupe la queue dans le jeune âge ne transmettent pas leur infirmité par la génération et leur produit naît avec une queue entière, comme naissent avec un prépuce complet les Juifs circoncis de père en fils depuis Abraham. 


\title{
SUR UN CAS EXCESSIF DE MICROCÉPHALIE
}

\author{
(ENCÉPHALE DE 104 GRAMMES)
}

(Bulletins de la Société d'anthropologie, $2^{2}$ série, 1876, t. XI, p. 85 à 87.)

J'ai présenté à la Société, dans la séance du 18 novembre 1875 , une petite fille vivante atteinte de microcéphalie et d'imperforation de l'anus. Elle était alors âgée de trois mois et demi, et dans un état de dépérissement qui était la conséfquence de l'imperforation de l'anus, et qui présageait une fin très prochaine. J'annonçai à la Société, en lui présentant cette enfant, que je me proposais de l'opérer le lendemain, 19 novembre.

L'opération a été relativement facile. De fréquerites pesées montrèrent que l'enfant continuait à dépérir. La veille de l'opération, elle pesait 1723 grammes (le procès-verbal du 18 novembre porte par erreur d'impression 1825 grammes). Le 25 novembre elle ne pesait plus que 1650 grammes; ce poids descendit à 1640 grammes le 2 décembre, à 1530 grammes le 4 décembre; ce jour-là commença l'agonie, et la mort eut lieu le lendemain 5 décembre, dix-sept jours après l'opération. Le poids du corps n'était plus que de 1435 grammes.

L'enfant était née le 2 août 1885 ; elle avait donc vécu quatre mois et trois jours. Je rappelle qu'elle était née probablement à terme. La mère, en accouchant, se croyait à terme; en voyant naître un être si petit, la sage-femme soutint qu'elle devait se tromper. On consulta l'almanach, et il fut constaté qu'en aucun cas l'enfant ne pouvait être née avant la fin du huitième mois.

Le corps a été disséqué dans le laboratoire. Nous avons constaté l'existence d'anomalies nombreuses et diverses, dont la description détaillée sera le sujet d'un travail spécial. Je me borne aujourd'hui vous présenter sommairement celles con- 
cernant le cerveau. L'encéphale entier ne pèse que 104 grammes; c'est de beaucoup le plus faible poids qui ait jamais été constaté sur un être humain né vivant, et il est environ cinq fois moindre qu'il ne devrait l'être chez un enfant qui a vécu quatre mois.

Quelque petit que soit le crâne, ce cerveau minuscule était loin de le remplir entièrement. - Il s'est écoulé, au moment de l'ouverture des membranes, une assez grande quantité de sérosité parfaitement transparente, qui s'était accumulée sous l'arachnoïde, principalement dans la partie postérieure de la grande scissure médiane du cerveau. Il y avait là une loge assez spacieuse, mais ce n'était pas un kyste, car cette loge se continuait sans démarcation avec les autres espaces produits sous l'arachnoïde par l'infiltration du même liquide, et avec la cavité du troisième ventricule.

On ne peut donc pas supposer que l'accumulation du liquide ait été un phénomène primitif; elle n'a pas été la cause, mais l'effet du défaut de croissance du cerveau. Cet organe s'étant développé moins que le crâne, il s'est produit un vide qui a été comblé par l'exhalation de la sérosité.

On dit avec raison que l'accroissement du crâne est provoqué et régi par celui du cerveau; les os du crâne possèdent néanmoins en eux-mêmes un certain pouvoir d'accroissement, qui ne s'éteint pas entièrement lorsque le cerveau cesse de le solliciter. Ainsi s'explique ce fait très exceptionnel d'un cerveau resté trop petit pour la boîte qui le renferme.

Cet encéphale présente une forme étrange, qui rappelle celle de l'encéphale des ruminants. Les hémisphères cérébraux ne recouvrent nullement le cervelet; ils sont entièrement placés audevant de cet organe. La grande scissure inter-hémisphérique n'est pas parfaitement médiane; dans sa moitié antérieure elle empiète sur le côté droit, et présente une flexuosité assez prononcée ; dans sa moitié postérieure elle s'élargit heaucoup, les deux hémisphères s'écartent et laissent entre eux un espace large d'environ 6 millimètres qui était rempli par de la sérosité. Dans le fond de cet écartement on aperçoit les pédoncules supérieurs du cervelet, aboutissant à deux grosses saillies elliptiques qui paraissent formées par les tubercules quadrijumeaux postérieurs. Ceux-ci sont plus volumineux que sur les cerveaux 
d'adultes. Le genou postérieur du corps calleux manque, ainsi que la moitié postérieure de cet organe.

Les hémisphères cérébraux sont très courts, eu égard à leur largeur, et très asymétriques; ils ne sont d'aillẹurs le siège d'aucune lésion pathologique. Ils présentent de nombreuses circonvolutions, séparées par des sillons relativement profonds. La démarcation des lobes est très difficile à retrouver. La scissure de Sylvius, sur la face externe des hémisphères, ne suit pas unc direction longitudinale. A gauche elle est tout à fait verticale, et longue seulement de 1 centimètre; à droite, elle est trois fois plus longue, presque verticale encore, mais cependant légèrement inclinée en arrière, et occupe les trois quarts environ de la hauteur de l'hémisphère. Les sillons de Rolando sont asymétriques et très rapprochés de l'extrémité antérieure du cerveau. Les lobes frontaux sont donc très courts, mais leurs circonvolutions sont au complet et presque en ordre. Par contre, les circonvolutions pariétales et occipitales sont tout à fait désordonnées. A droite, avec un peu de complaisance, on retrouve deux sillons qui paraissent faire partie - de la scissure occipitale externe; mais à gauche rien de pareil, et rien n'indique les limites des lobes pariétal, occipital et temporal.

On sait que notre savant collègue $\mathrm{M}$. Vogt attribue la microcéphalie à un arrêt de développement du cerveau; le cerveau des microcéphales, d'après lui, présenterait la persistance d'un état transitoire chez l'homme et permanent chez les singes. Je suis loin de nier la réalité de l'arrêt de développement du cerveau chez certains microcéphales; mais je dois ajouter que dans la plupart des cas que j'ai étudiés, la morphologie du cerveau des microcéphales ne pouvait se rapporter ni à celle d'un cerveau humain en voie de développement, ni à celle d'un cerveaı de singe. Le cas actuel nous montre des dispositions qui ne correspondent à aucune phase de l'évolution du cerveau, soit chez l'homme, soit chez les autres animaux.

L'étude de ce cerveau précieux sera continuée : quelques coupes seront nécessaires pour constater la disposition des parties internes; le crâne et le squelette sont préparés pour le musée. Le résultat de ces recherches complémentaires vous sera communiqué ultérieurement. 


\section{DEFFORMATION GONGÉNITALE}

\section{DU GRANE ET DE LA FACE}

MICROCÉPHALIE FRONTALE

(Bulletins de la Societé d'anthropologie, 3e série, 1879, t. II, p. 256-258.)

M. Broca prézente à la Société une petite fille admise à son service d'hôpital pour une difformité congénitale propre à intéresser la Socićté.

Cette petite fille a les deux oreilles absolument oblitérées. Le pavillon de l'oreille est d'ailleurs très mal formé; quant au conduit auditif externe, il est très visiblement recouvert par la peau, en sorte qu'il est impossible d'y introduire même le stylet le plus fin. La conséquence naturelle de ce fait est que cette petite fille entend très mal; cependant, il est remarquable qu'elle distingue les sons articulés quand on parle à haute voix. Même, depuis deux ans (elle en a huit aujourd'hui), elle a fini par apprendre à parler. Cela prouve que, sans doute, son tympan, la chaîne des osselets et l'oreille interne sont dans un état suffisant de conformation. Je n'ai pas sondé la trompe d'Eustache. Le conduit auditif externe doit aussi exister; car, lorsqu'on appuie la doigt sur le lieu où il devrait s'ouvrir, on sent la peau qui le recouvre céder sous le doigt, et l'on sent, au-dessous, un anneau cartilagincux. Il y a donc lieu de se demander si une opération chirurgicale ne pourrait pas rétablir le conduit, et c'est pour cela que cette petite fille est entrée à l'hôpital.

Non seulement les oreilles de cette petite fille sont défectueuses, mais toute sa tête est plus ou moins mal conformée. Ses paupières se ferment mal, et il est visible que la paupière inférieure gauche a été divisée pendant la vie utérine.

Enfin, on observe sur elle un développement très inégal du 
crâne et de la face. La partie antérieure du crâne est très étroite et arrêtée dans sa croissance. La face, au contraire, a reçu son développement normal; mais la mâchoire inférieure a subi l'arrêt de développement qui a frappé le crâne et est visiblement trop petite pour la face. Il en résulte que la physionomie de cette petite fille ressemble absolument à un museau.

Cette petite fille n'est pas tout à fait idiote, mais elle est très peu intelligente. Elle ne peut fixer son attention. Elle connaît à peu près les lettres, mais elle n'a pu apprendre à assembler une syllabe. Il convient de la distinguer des microcéphales proprement dits. Ceux-ci, étant enfants, ont une disposition crânienne qui ne choque pas l'œil au premier abord; ce n'est que plus tard que l'exiguïté du crâne devient très visible. 11 en est dc même pour le développement de leur intelligence. Tant qu'ils sont enfants, elle n'apparait que comme médiocre; mais c'est quand ils se développent, qu'on s'aperçoit qu'elle est tout à fait défectueuse, car ils conservent toute leur vie une intelligence et des goûts dignes d'enfants de cinq ans. Tel était notamment l'individu exposé, à Paris, sous le nom d'Atzèque. Un jouet d'enfant causait une joie sans pareille à cet homme de trentesept ans !

Il en est à peu près de même pour la taille. Les microcéphales ordinaires conservent une taille inférieure, mais cet arrêt de développement est insensible dans la première enfance et ne se marque qu'au bout de quelques années.

Chez notre petite fille la taille est bien celle d'un enfant de huit ans. Il sera curieux d'examiner plus tard quelle sera la conséquence de la conformation que présente son crâne, et qui est la suivante : le front est très étroit, et la boîte osseuse s'est trouvée arrêtée, à ce niveau, dans son développement. Aussi le cerveau s'est trouvé refoulé dans la partie postérieure du crâne, qui se trouve, de ce fait, assez développée, en sorte que la nuque se trouve au milieu de la base du crâne. Le cerveau s'est également développé en hauteur, car vous voyez combien, chez cette enfant, la voûte du crâne est surélevée. Refoulé ainsi, comme dans le cas de déformation artificielle, le cerveau pourra néanmoins retrouver au moins en partie la place qui lui est nécessaire. 
Il y a donc lieu d'espérer que l'intelligence de l'enfant pourra s'élever au-dessus de celle des autres microcéphales.

Il faut remarquer toutefois que la région frontale est aussi petite que celle de certains microcéphales, et c'est pour cela sans doute que l'intelligence est si faible, quoique le volume total du cerveau ne soit pas inférieur à celui de certains enfants du même âge. Ce défaut de développement de la région frontale me paraît devoir être attribué à l'oblitération congénitale de la suture coronale. En d'autres termes, la conséquence exagérée qui s'est produite dans la première fente viscérale pendant le second mois de la vie intra-utérine et qui a fait oblitérer les conduits auditifs externes n'aurait pas atteint seulement les parties molles, et aurait gagné aussi les os voisins.

Le cas présent est désigné sous le nom de microcéphalie frontale, mais je m'empresse d'ajouter qu'il n'y a aucun rapport entre cette affection, dont la cause est purement crânienne, et la microcéphalie proprement dite, dont la cause est toute cérébrale. Chez les vrais microcéphales, les sutures sont libres, et si dans certains cas, d'ailleurs exceptionnels, quelques-unes d'entre elles peuvent se souder prématurément, cette tendance, loin d'être la cause de l'arrêt de développement du cerveau, en est au contraire l'effet. 


\title{
GRANE ET GERVEAU D'UN HOMME
}

\author{
ATTEINT DE LA DÉFORMATION TOULOUSAINE
}

(Bulletins de la Société d'anthropologie, $3^{\circ}$ série, t. II, 1879, p. 417-420.)

Je présente de la part de M. Nélaton, interne du service de M. Richet à l'Hôtel-Dieu, le crâne et le cerveau d'un homme de soixante ans, dont l'autopsie a été fäite ce matin; cet homme est né à Albi (Tarn), mais sa tête est déformée à la Toulousaine. Les Volskes-Tectosages, à qui on attribue l'importance de cette déformation, avaient leur capitale à Toulouse et ne s'étendaient, d'après les cartes, que jusqu'à la rivière d'Agout, à 8 lieues d'Albi. Mais on conçoit que la mọde qu'ils avaient introduite ait pu s'étendre plus loin, et jusque dans l'Aveyron, où l'on en connaît des exemples.

Par un hasard singulier, un autre homme âgé de trente-sept ans, atteint de la même déformation, est mort ce matin, dans le même service; il était né aussi dans le Tarn, mais sur la limite de la Haute-Garonne, et sur le territoire des anciens Tectosages.

Vous vous souvenez que j'ai fait en avril 1871 (voir les Bulletins de 1871, p. 100 et dans ce volume, p. 185) l'autopsie d'une vieille Toulousaine déformée dont le crâne et le mode cérébral sont déposés dans notre musée. L'autopsie d'aujourd'hui est donc la seconde qui ait été faite, et elle confirme les résultats de la première, à l'appui de l'opinion soutenue depuis une trentaine d'années par les médecins aliénistes de Toulouse.

La déformation toulousaine était volontiers regardée comme un fait indifférent, et restait ignorée, lorsque les médecins des asiles d'aliénés de cette région remarquèrent que la proportion des déformés était bien plus considérable dans la population de leurs asiles que dans la population libre. Ils en conclurent, avec 
raison, que cette pratique était dangereuse pour l'intelligence, et ils entreprirent une sorte de croisade à la suite de laquelle la plupart des familles renoncèrent à l'emploi des béguins qui déformaient d'une manière fâcheuse la tête des jeunes enfants.

Sur la Toulousaine que j’ai autopsiée naguère, j'ai décrit l'adhérence pathologique de la dure-mère avec la paroi du crâne. Vous savez qu'ordinairement, pour enlever la calotte du crâne, on fait un trait de scie circulaire, après quoi on détache la calotte sans aucune difficulté, en l'attirant avec un crochet. Lorsqu'on voulut faire sur la Toulousaine cette dernière opération, la calotte ne céda pas, et, lorsque je cherchai l'obstacle qui s'y opposait, je vis que c'était la dure-mère qui adhérait si fortement au crâne, que rien ne put l'en détacher; lorsque je vis que cette membrane menaçait de se déchirer, je la coupai tout autour de l'endroit où elle adhérait. Cette adhérence était visiblement due à une ancienne maladie des enveloppes du cerveau.

La même difficulté s'est présentée lorsqu'on a autopsié l'individu que je vous présente aujourd'hui, et dont voici le crâne préparé. Vous pouvez voir les traces de l'adhérence de la duremère; à ce niveau, on voit quantité de petites dépressions qu'on dirait tracées avec une aiguille et au fond desquelles la duremère est intimement adhérente au crâne.

Ainsi, la déformation artificielle n'est pas une opération innocente, ainsi qu'on l'a souvent écrit, même à l'occasion des déformations bien plus violentes des peuples de l'Amérique. On ne sait jusqu'à quel point elle a été nuisible aux deux individus dont je viens de vous décrire l'autopsie; mais il est certain que, chez tous les deux, elle a amené autrefois une lésion des enveloppes du cerveau et du crâne.

La forme extérieure du crâne du sujet dont nous avons les pièces sous les yeux est la suivante : le front monte jusqu'aux bosses frontales sans être très déformé. A partir de ce point, il est fortement abaissé, déprimé, jusqu'en arrière du bregma, et la tête finit par une sorte de pointe.

Les lobes frontaux du cerveau sont beaucoup plus déformés que ne le ferait croire l'aspect extérieur du crâne. La partie antérieure est très abaissée. Ils regagnent sur la longueur une 
partie de ce qu'ils ont perdu en hauteur, de sorte que la scissure de Rolando est refoulée en arrière ; mais, malgré cette compensation partielle, leur volume relatif est plus petit qu'à l'état normal. Les lobes occipitaux, au contraire, ont un volume exagéré, et les lobes pariétaux sont très réduits dans leur partie supérieure et externe.

La déformation toulousaine modifie donc d'une manière très notable le développement relatif des divers lobes du cerveau. Les mêmes faits ont été constatés par des pesées partielles sur le cerveau de la Toulousaine de 1871.

\section{DISCUSSION.}

M. D'AbBadie. Cette coutume du pays de Toulouse de déformer la tête des enfants était certainement plus fréquente autrefois qu'aujourd'hui. Cependant nous ne voyons pas que cette région soit particulièrement remarquable par le numbre de ses fous; ce qui se rencontre dans quelques contrées, et notamment dans un village du Guipuzcoa, où les aliénés, on ne sait pour quelle cause, sont prodigieusement nombreux. Toulouse, au contraire, a produit des hommes distingués, notamment le mathématicien Fermat, et la population toulousaine est intelligente.

M. Lunıer. Je présente à la Société une carte de l'aliénation mentale on France, et vous y voyez que le département de la Haute-Garonne est un de ceux où la folie fait le plus de victimes. Il se distingue sous ce rapport des autres départements du Midi, où elle ne se rencontre que rarement.

M. Broca. Le fait que M. d'Abbadie citait d'un village du Guipuzcoa, où les fous sont très fréquents sans qu'on sache pourquoi, peut sans doute s'expliquer par la seule hérédité, car il y a peu de maladies plus héréditaires que la folie. Heureusement que M. Foville, de Saint-Yon, a prouvé que les familles frappées de cette maladie ont une disposition à s'éteindre.

Quant à l'existence d'hommes distingués originaires de Toulouse, elle est bien connue et elle n'est pas en contradiction avec ce que je viens de dire. Outre que la mode de la déformation n'a jamais été générale, il est probable que les familles intelligentes, celles d'où proviennent généralement les hommes de mérite, ont dû le plus souvent s'opposer à l'application d'un u age aussi absurde que disgracieux, et il serait utile de savoir si le grand 
mathématicien Fermat avait ou non le crâne déformé. D’ailleurs, on conçoil que certaines intelligences puissent échapper ou résister aux dangers que leur fait courir la déformation artificielle.

La population de Toulouse est artiste et littéraire bien plus que scientifique : c'est à Toulouse qu'ont pris naissance les célèbres Jeux Floraux; la poésie et la musique y passionnent jusqu'aux dernières couches du peuple. Ces qualités ne sont pas incompatibles avec le moindre développement relatif du lobe frontal, car on les a rencontrées très souvent chez des Toulousains qui avaient le crâne déformé. 


\section{DU POIDS DU CERVEAU}

SUIVANT LA RACE ET LE SEXE

DISGUSSION

(Bulletins de la Soriété d'anthropologie, 3e série, 1879, t. 11, p. 500-503.)

M. Broca. La comparaison du poids du corps avec le cerveau est difficile à faire dans l'espèce humaine, mais Leuret l'a faite dans la série animale; il a trouvé que ce rapport varie d'une espèce à l'autre. Connaissant le poids du corps, on ne peut déduire le poids du cerveau. La quantité de cerveau nécessaire pour animer un poids donné de matière animale varie avec les animaux.

Il varie aussi avec les races humaines; car il est incontestable que les races mongoliques sont, toutes choses égales d'ailleurs, moins intelligentes que la nôtre. Et cependant la grosseur de leur cerveau ne rend pas compte de leur situation intellectuelle.

Le fait inverse se trouve pour certains nègres. Gratiolet l'avait remarqué : "La Vénus hottentote, disait-il, est suffisamment intelligente avec son petit cerveau; chez nous, avec un pareil cerveau, elle serait idiote! »

Ces problèmes sont d'ailleurs très complexes. Les campagnards ont le cerveau plus gros que les Parisiens, et surtout les femmes de la campagne ont le cerveau plus gros que les femmes de Paris. Mais il faut se rappeler l'influence fâcheuse que l'extrême division du travail peut exercer sur l'intelligence; c'est à Paris que l'on voit des ourriers consacrer leur vie entière à faire la pointe d'une aiguille, tandis que leur voisin n'a d'autre occupation que d'en aplatir la tête. $\Lambda$ la campagne, il faut qu'un homme soit autrement encyclopédiste pour pouvoir y gagner son pain. Peut-être est-ce pour ces raisons que j'ai trouvé que les campagnards de Bretagne et d'Auvergne ont la tête plus 
grosse que les Parisiens. Ces Bretons et ces Auvergnats sont d'ailleurs des hommes de petite taille, vaillants et intelligents. Ce sont leurs ancêtres qui ont combattu César et qui, plus tard, ont défendu pied à pied la civilisation gallo-romaine contre l'invasion des Barbares.

M. LE Bon ne partage pas du tout l'avis de M. Broca relativement à la grosseur de la tête chez les paysans. Si M. Broca l’a trouvée si considérable, c'est qu'il a mesuré des Auvergnats et des Bretons, c'est-à-dire des Celtes de pure race. Les recherches que M. Le Bon a faites sur les paysans de la Baauce lui ont donné des résultats opposés.

M. DE Mortillet. A Grenoble, les paysans de la montagne, qui pourtant sont des Celtes purs, avaient coutume de porter des chapeaux d'une forme spéciale, en feutre rigide. Ces chapeaux étaient faits d'avance, et les chapeliers avaient observé qu'il fallait leur donner une pointure plus faible gu’à ceux de la ville.

M. Pellarin rappelle qu'à Rodez, M. Durand (de Gros) a trouvé, d'après une enquête faite chez les chapeliers, que les citadins out la têle plus grosse que les campagnards.

M. Counereau. M. Broca nous expliquait tout à l'heure que la quantité de cerveau nécessaire pour les fonctions intellectuelles varie avec les races. Je lui demanderai donc si elle ne varierait pas avec le sexe, et si là ne serait pas le secret de la différence du poids du cerveau de la femme et de celui de l'homme.

M. Broca. Je n'ai comparé tout à l'heure les campagnards qu'aux Parisiens; Paris est dans des conditions industrielles spéciales. Nulle part la différence entre le poids du cerveau chez les deux sexes n'est aussi considérable que dans cette ville. Pourquoi cette différence? C'est qu'à Paris, les femmes peuvent gagner leur vie dans des positions où elles n'ont nullement à exercer leur cerveau. Il n'en est pas de même à la campagne.

J'examine à présent la question que in'a posée $M$. Goudereau. Elle est complexe. Il faut tenir compte, en effet, de la différence de taille qui sépare les deux sexes. Cette inflıence n'est pas très considérable. Elle est notable cependant. M. Le Bon, groupant ensemble un certain nombre d'individus de taille élevée et, d'autre part, un même nombre d'hommes petits, a trouvé qu'en moyenne le poids de leurs crânes présentait une différence de volume d'environ 100 centimètres cubes aux dépens des petits hommes. Cette différence ne représente pas la totalité de celle que nous trouvons entre les deux sexes. 
Le reste de cette différence ne me paraît pas trop élevé pour qu'on ne puisse l'attribuer à l'éducation.

On peut admettre comme démontré qu'un homme ayant reçu une instruction convenable a le crâne plus gros qu'un ignorant. Ainsi, j'ai pris la circonférence horizontale du crâne chez un certain nombre d'internes et d'externes en médecine et chez des infirmiers. J'ai trouvé que ce sont les étudiants qui avaient l'avantage; sans doute, ils le devaient à leur propre éducation et peut-être à ce qu'ils descendaient de parents également mieux élevés.

Quand on songe à la différence profonde qui sépare de notre temps l'éducation intellectuelle de l'homme de celle de la femme, on se demande si ce n'est pas cette influence qui rétrécit le cerveau et le crâne féminins, et si, les deux sexes étant livrés à leur spontanéité, leurs cerveaux ne tarderaient pas à se ressembler, ainsi qu'il arrive chez les sauvages. 


\title{
SUR UN GERVEAU HUMAIN
}

\author{
INGOMPLÈTEMENT DIVISÉ EN DEUX HÉMISPHĚRES ${ }^{1}$
}

(Revue d'anthropologie, 20 série, t. II, 1879, p. 538-546.)

L'étrange cerveau dont il s'agit a été expédié au professeur Turner par le docteur Wilkie Burman, qui l'avait recueilli en 1876, sur le corps d'un homme de quarante-huit ans, mort à l'asile des aliénés de Devires (Wiltshire).

L'observation clinique est malheureusement très incomplète. Nous la traduisons textuellement :

"Le malade fut admis dans l'asile le 7 décembre 1852 , à l'âge de vingt-trois ans. Il avait travaillé comme ouvrier inhabile dans une fabrique de toile. Il était sujet à des attaques d'épilepsie, et on dit, au moment de son admission, qu'il avait été fou pendant dix semaines. Les attaques étaient d'abord légères, mais il était dangereux et porté au suicide. Son père raconta que son attaque avait été causée par un coup de pierre reçu à la tête. Enfant, il n'avait jamais été intelligent, et n'avait pas été capable d'apprendre un état. Il savait seulement lire. Après son admission, les attaques devinrent plus fréquentes et plus graves, et l'intelligence s'affaiblit de plus en plus. Il était très irritable, et devenait très violent après les attaques. Il continua à avoir de temps en temps des périodes d'excitation et de violence jusqu'en 1863; mais alors il tomba dans un état de démence qui s'accentua ensuite de plus en plus. Il fut observé pour la première fois par le docteur Burman en décembre 1873. Il était alors habituellement hébété et dément, mais il avait parfois de violentes attaques d'épilepsie.

« La cause de la mort n'est pas indiquée. M. Burman a transmis

(1) Par le professeur Turner (d'Edimbourg). In Journal of Anat. and Physiol., janvier 1878 . 
sans commentaires les renseignements qui précèdent à $M$. Turner; mais nous rappellerons, eu égard à la cause traumatique mentionnée par le père, que les parents d'un épileptique sont toujours inclinés à attribuer à quelque accident la maladie de leur enfant.

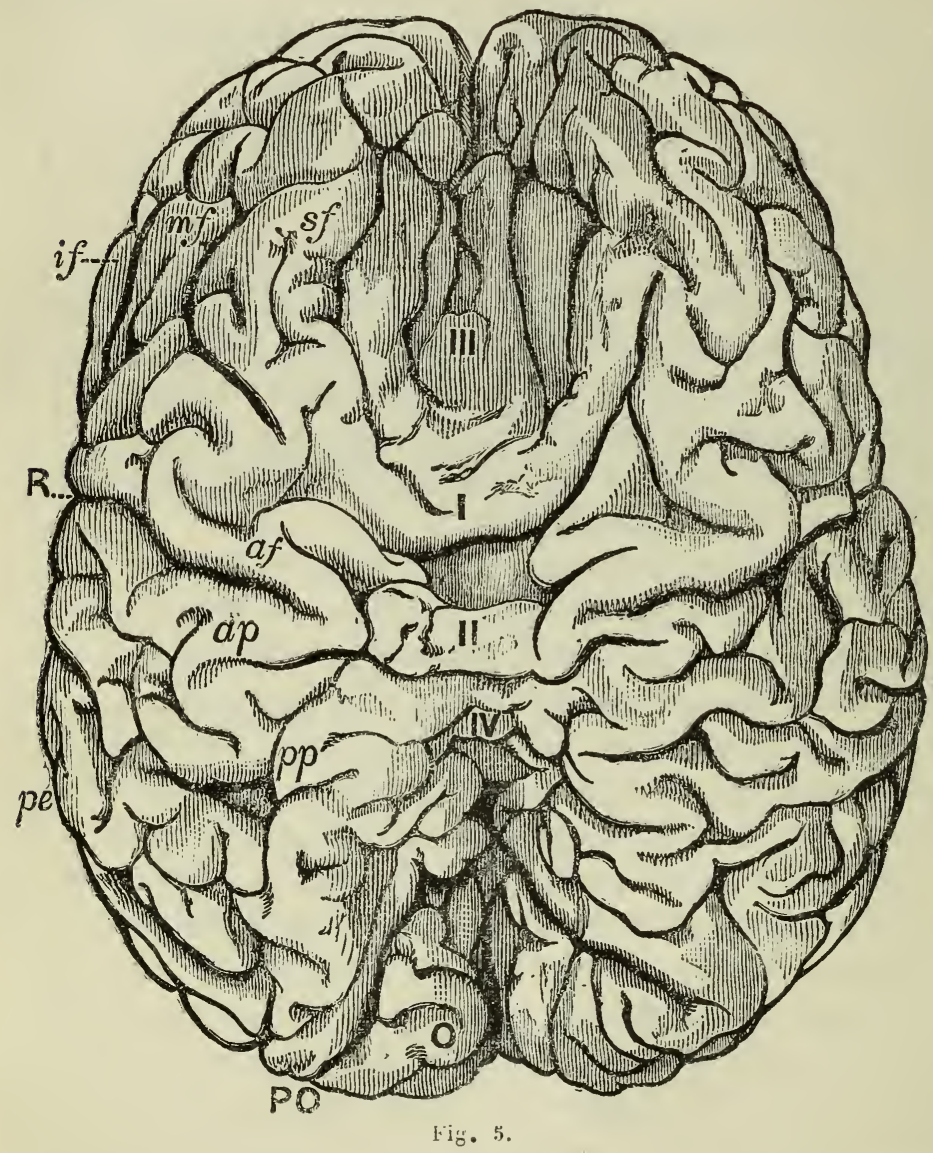

“ La taille du sujet était de $1^{\mathrm{m}}, 473$, son poids de 56 kilogrammes. Il avait un embonpoint assez notable. L'encéphale entier, au moment où il fut extrait du crâne, pesait 1113 grammes (39 onces un quart); le poids du cervelet était de 114 grammes ( 4 onces); celui de la protubérance et du bulbe, 21 grammes (3 quarts d'once); et enfin, celui des deux hémisphères, 978 grammes ( 34 onces et demi). 
"L’encéphale entier fut plongé dans l'alcoolet y séjourna quelques semaines sans être retourné. Il en résulta que la face inférieure des hémisphères, reposant sur le fond du vase, se ramollit pendant que la face convexe se raffermissait. Les deux hémisphères furent seuls envoyés à M. Turner, et leur face inférieure était malheureusement altérée.

" Les hémisphères, allongés par déformation, étaient longs dr 6 pouces ( $15 \% 2$ millimètres). La grande fente interhémisphérique était remplie dans une grande partie de sa longueur par une masse de substance grise disposée en circonvolutions, lesquelles, dans le tiers moyen de sa longueur, arrivaient presque au niveau du bord supérieur des hémisphères. Cette masse circonvolutionnaire mésiale (1), passant comme un pont d'un hémisphère à l'autre, occupait dans la fente interhémisphérique une étendue de 3 pouces ( 7 à 8 centimètres), laissant la fente libre au devant d'elle dans une longueur de 1 pouce 3 (33 millimètres), et en arrière d'elle dans une étendue de 1 pouce 7 (43 millimètres). On y distinguait superficiellement deux circonvolutions bien distinctes, séparées par un sillon transversal large et peu profond. La circonvolution antérieure unissait le bord sagittal de la première circonvolution frontale droite avec celui de la même circonvolution gauche, immédiatement en avant des frontales ascendantes; la circonvolution postérieure, transversale comme la première, unissait de la même manière les bords sagittaux des deux hémisphères au niveau de l'extrémité supérieure de la scissure de Rolando. Le sillon transversal, intermédiaire entre les deux hémisphères, était tapissé d'une couche de substance grise qui se continuait de chaque côté avec l'écorce de la circonvolution frontale ascendante.

“Immédiatement en avant de la circonvolution mésiale antérieure existait, dans une étendue de 28 millimètres d'avant en arrière (voir fig. $5, \mathrm{n}^{\circ} \mathrm{III}$ ), une surface grise et lisse, qui s'enfonçait entre les deux hémisphères.

«En bas et en avant, cette surface grise se continuait de chaque

(1) Nous conservons le mot anglais; l'adjectif français médian aurait à la rigueur la même acception, mais d'ordinaire il désigne des parties dirigées dans le sens de la ligne médiane, tandis que les circonvolutes dont il s'agit sont transversales. 
côté avec une circonvolution qui occupait la place de l'extrémité antérieure de la circonvolution du corps calleux, et se continuait en arrière et en haut avec la circonvolution mésiale antérieure et avec la circonvolution marginale située au-devant de celle-ci.

« En arrière de la circonvolution mésiale postérieure, trois plis circonvolutionnaires, se succédant de haut en bas et d'avant en arrière, traversaient le plan médiau, suivant une direction qui n'était pas tout à fait aussi transversale que celle des deux circonvolutions mésiales, et qui était un peu plus tortueuse. Le pli antérieur unissait entre eux les deux lobules pariétaux, tandis que les deux autres, situés plus bas et plus en arrière, unissaient la partie inférieure des deux lobules quadrilatères. Aucune circonvolution n'unissait entre eux les lobes occipitaux et les lobes temporaux.

«Les circonvolutions marginales des lobes frontaux et des lobes pariétaux étaient modifiées sans doute par suite de leur jonction sus-indiquée; mais en avant et en arrière de cette jonction des circonvolutions marginales de chaque hémisphère étaient distinctes et normales. De chaque côté, la circonvolution du corps calleux n'était pas développée, excepté en avant où elle paraissait représentée par une petite portion, qui se continuait avec la substance grise mésiale marquée $n^{\circ}$ III (fig. 5้).

( Les deux circonvolutions mésiales avaient chacune une étendue de 12 millimètres dans leur sens transversal aussi bien que dans le sens de leur largeur antéro-postérieure. Le sillon intermédiaire avait 10 millimètres d'avant en arrière et 12 en travers. Les trois plis circonvolutionnaires situés derrière la circonvolution mésiale postérieure avait respectivement une largeur antéro-postérieure de 10 , de 7 et de 7 millimètres et une étendue transversale de 10 millimètres. Les deux circonvolutions mésiales étaient un peu plus larges que les circonvolutions des lobes frontaux et des lobes pariétaux.

« Les $1^{\text {re }}, 2^{\mathrm{e}}$ et $3^{\mathrm{e}}$ circonvolutions frontales ne présentaient dans leur disposition rien de remarquable. La frontale ascendante de chaque hémisphère était subdivisée par un sillon profond en deux parties presque égales, l'une supérieure, l'autre inférieure. L'extrémité postérieure de la scissure de Rolando occupait le milieu de la longueur de l'hémisphère. Les circonvolutions de la 
face externe des lobes pariétal et occipital étaient normales. De chaque côté, la scissure occipitale interne était bien marquée, et les deux plis de passage pariéto-occipitaux étaient disposés comme à l'ordinaire. De l'extrémité inférieure de la scissure occipitale interne se détachait une anfractuosité qui pénétrait d'avant en arrière dans le lobe occipital, et qui paraissait être la scissure calcarine.

«Scissure de Sylvius normale. Le lobe de l'insula paraissait moins subdivisé qu'à l'état normal. Les circonvolutions de la face externe du lobe temporal étaient normales; quant à celles de la face inférieure, leır mauvais état de conservation, déjà signalé plus haut, n'a pas permis d'en constater la disposition d'une manière précise; circonstance très regrettable, car on n'a pu savoir si la circonvolution de l'hippocampe existait ou non. L'auteur pense toutefois qu'elle devait manquer, parce qu'en poursuivant la dissection du cerveau il a constaté l'absence de la corne inférieure des ventricules latéraux.

“ Pour constater l'état des parties situées profondément audessous des circonvolutions mésiales, M. Turner se décida à pratiquer une coupe transversale et verticale immédiatement en arrière de la circonvolution mésiale antérieure. Il pénétra ainsi dans une large cavité ventriculaire médiane, qui se continuait de chaque côté dans l'épaisseur des hémisphères et se prolongeait en avant et en arrière dans les lobes frontaux et occipitaux. Cette cavité, commune aux deux hémisphères, représentait les deux ventricules latéraux d'un cerveau normal; elle était large en tout de 48 millimètres, et son plus grand diamètre vertical était de 18 millimètres. D'après l'état de dilatation où elle se trouvait encore sur le cerveau durci, il était évident qu'elle était, pendant la vie, pleine de liquide céphalo-rachidien.

"Le toit de ce ventricule était voûté, et son point culminant était situé immédiatement au-dessous de la circonvolution mésiale antérieure; de ce point, il s'inclinait vers le bas aussi bien en avant et en arrière que sur les côtés. L'épaisseur de ce toit variait considérablement dans ses diverses parties. Sur la ligne médiane, où il était formé par les circonvolutions mésiales, il était plus mince que sur les côtés, où il était compris dans la substance même de l'hémisphère. Au niveau du large sillon 
transversal qui séparait les deux circonvolutions mésiales, était la partie la plus mince; là, le toit avait de 5 à 10 millimètres d'épaisseur, tandis que sur les côtés, au niveau de l'extrémité supérieure de la circonvolution frontale ascendante, cette épaisseur s'élevait à 25 millimètres.

" La partie la plus mince du toit consistait en un feuillet superficiel de substance grise, épais de 2 à $ّ$ millimètres, au-dessous duquel était un feuillet de substance blanche, dont l'épaisseur variait également de 2 à $ّ$ millimètres.

«Sur les parties latérales, où le toit était formé par la substance même des hémisphères, le feuillet de substance blanche s'épaississait et se confondait avec le centre ovale, ayant ainsi unc épaisseur de 12 à 15 millimètres. La limite externe du ventricule était constituée par la partie du centre ovale que forment les fibres pédonculaires de la couronne radiée, en se rendant des ganglions de la base aux circonvolutions. La paroi comprise entre le côté externe du ventricule et la surface externe de l'hémisphère était épaisse de 38 millimètres, et la plus grande partie de son épaisseur était formée par le centre ovale, que la substance grise des circonvolutions revêtait d'ailleurs comme à l'ordinaire.

" Un peu à droite de la ligne médiane, le toit, quelque peu affaissé, formait, du côté de la cavité ventriculaire, une saillie légère, irrégulière, qui occupait une étendue d'environ 25 millimètres dans le sens antéro-postérieur, et de 7 à 12 millimètres dans le sens transversal. Une incision pratiquée sur cette saillie montra que la substance blanche du toit était soulevée par une mince couche de substance grise.

( De la structure du toit, il résultait qu'il n'y avait pas de corps calleux. Les parties mésiales qui unissaient les deux hémisphères avaient la structure des circonvolutions. Leur écorce grise se continuait avec celle des circonvolutions adjacentes des deux hémisphères; quant à leur couche de substance blanche elle était si mince, qu'elle se bornait évidemment à supporter la substance grise des circonvolutions mésiales, et qu'elle ne constituait pas, comme le fait le corps calleux, une commissure entre les deux hémisphères.

«Le plancher des ventricules était formé par les faces supé- 
rieures des deux corps striés et des deux couches optiques; les connexions de ces parties, soit entre elles, soit avec les bandelettes demi-circulaires étaient comme dans les cerveaux normaux. Le corps strié avait ses rapports ordinaires avec l'insula. Il n'y avait aucun vestige de la voûte, ni du septum lucidum, ni de la toile choroïdienne, ni des plexus choroïdes. Les deux couches optiques étaient séparées l'une de l'autre par une fente médiane verticale qui représentait le troisième ventricule et qui communiquait librement avec le grand ventricule résultant de la fusion des deux ventricules latéraux.

"Une seconde section transversale et verticale pratiquée sur l'hémisphère gauche au niveau du lobe pariétal, et une section verticale et longitudinale sur l'hémisphère droit, permettait de reconnaître que la corne moyenne ou inférieure du ventricule latéral n'existait pas; la couche optiqque se continuait directement en arrière avec une masse de substance cérébrale qui formait le bord interne de la corne postérieure du ventricule et qui se continuait en dedans avec une circonvolution de la face interne de l'hémisphère, située immédiatement au-dessous des plis circonvolutionnaires mésiaux marqués IV sur notre figure.

a $M$. Turner ne put découvrir aucune trace de la commissure postérieure, ni de la glande pinéale. Il lui parut aussi qu'il n'y avait pas de commissure antérieure, mais le fait néanmoins ne lui parut pas absolument certain.

"En résumé, les points les plus remarquables de la description de ce cerveau extraordinaire sont les suivants : Oblitération partielle de la grande fente interhémisphérique; union des deux hémisphères par des circonvolutions mésiales; une seule cavité ventriculaire, avec absence de la corne inférieure ou moyenne des ventricules latéraux; absence du corps calleux, de la voûte, du septum lucidum, de la toile choroïdienne et des plexus choroïdes.

"Pour' se faire une idée de l'origine de cette série d'anomalies, continue M. Turner, il faut remonter aux premières phases du développement des hémisphères. On admet généralement que les hémisphères naissent comme deux prolongements vésiculaires distincts et latéraux de la partie antérieure de la paroi de la vésicule cérébrale antérieure, de sorte que, dès leur appari- 
tion, ils seraient dójà séparés et latéraux. Ce fait néanmoins a été récemment remis en question. Le professeur His a décrit en 1875 la vésicule primitive des hémisphères comme primitivement impaire et médiane, et comme contenant une seule cavité continue avec celle du cerveau intermédiaire (Zwischen. hirn) ou thalamencéphale. Cette vésicule impaire est bientôt divisée par un sillon, en deux moitiés qui sont en réalité des diverticules de la cavité médiane du cerveau antérieur. Mais depuis lors le professeur Mihalkovics (1877) a décrit plus minutieusement les premières phases du développement des hémisphères, d'après des embryons d'homme, de lapin, de veau, de poulet et d'oie. Suivant lui, un prolongement en forme de bourgeon, constituant le cerveau antérieur secondaire, naît de l'extrémité frontale de la vésicule cérébrale antérieure ou cerveau antérieur primaire, et forme une nouvelle grande vésicule, qui d'abord n'est séparée de la vésicule primaire par aucune limite nette, mais qui en est séparée plus tard par un pli falciforme, né de la paroi latérale, et dirigé de haut en bas et d'arrière en avant. Le cerveau antérieur secondaire est donc primitivement une vésicule impaire ou médiane. Sa partie supérieure forme le rudiment du manteau des deux hémisphères primitivement indivis, et sa partie inférieure forme le rudiment des ganglions de la base (corps striés et couches optiques). Il est creusé d'une seule cavité indivise, qui est le ventricule primitivement unique de la partie hémisphérique du cerveau. M. Balfour a fait la même remarque sur certains poissons. Le premier indice de la division de cette vésicule unique en deux moitiés droite et gauche, consiste en une faible dépression médiane, qui peu à peu se creuse en sillon, jusqu'à ce que la vésicule est enfin divisée en deux hémisphères, le sillon formant la grande fente interhémisphérique. Mihalkovics pense que la production de cette fente est due à la formation d'une bandelette médiane du tisau conjonctif qui deviendra la faux du cerveau. Chaque hémisphère est creusé d'une cavité qui deviendra le ventricule latéral et qui communique librement, sur la ligne médiane, avec celle de l'autre hémisphère, par un trou de Monro relativement très large. A cette époque, les deux hémisphères sont des corps de forme lenticulaire, dont la surface est lisse, leur face externe est convexe, 
leur face interne est verticale en avant, où elle est appliquée sur la face correspondante de l'autre hémisphère, et concave en arrière, où elle est appliquée sur la face externe du cerveau intermédiaire.

“ Plus tard, les ganglions de la base s'accroissent et font saillie dans la cavité du ventricule latéral; en même temps des fibres nerveuses se forment dans la paroi du manteau, se mettent en continuité avec la substance blanche des ganglions de la base et constituent la couronne radiée et le centre ovale. Alors, le primitif trou de Monro se rétrécit considérablement. Chaque ventricule latéral possède une corne antérieure et une corne inférieure, mais point de corne postérieure (celle-ci ne se formera que plus tard, avec le lobe occipital). Deux plis parallèles apparaissent sur la paroi interne de chaque hémisphère, faisait saillie dans le ventricule, et descendant du bord supérieur du trou de Monro vers l'extrémité temporo-sphénoïdale de la corne inférieure. Le pli supérieur, s'épaississant, devient le grand hippocampe, et l'inférieur, s'allongeant et s'amincissant, se change en un feuillet de cellules cylindriques qui forment l'épithélium des plexus choroïdes. Le feuillet épithélial de la face supérieure de ces plexus s'attache sur la voûte à trois piliers, et celui de leur face inférieure sur la couche optique.

a Sur le cerveau humain, vers le milieu du troisième mois, les parois internes des deux hémisphères, immédiatement en avant de la lamina terminalis du troisième ventricule, s'unissent ensemble et forment une expansion triangulaire.

"Dans la partie la plus postérieure de cette expansion, on voit apparaître à peu près simultanément les fibres de la commissure antérieure, et celles des piliers antérieurs de la voûte; et l'expan. sion elle-même devient le septum lucidım, formé de deux feuillets de substance grise, qui proviennent respectivement de la paroi interne de chaque hémisphère, et qui, incomplètement fusionnés, laissent entre eux, dans le plan médian, le cinquième ventricule.

“Celui-ci n'est qu'une partie de la grande fente interhémisphérique, partie circonscrite par suite de la fusion partielle des faces internes des hémisphères et du développement du corps calleux et de la voûte à trois piliers. Le cinquième ventricule 
n'est donc pas, comme les autres, dérivé du tube cérébro-spinal primordial.

«La surface extérieure du manteau est d'abord tout à fait lisse, mais il s'y forme ensuite des sillons qui la divisent en circonvolutions et en lobes. Le premier sillon qui apparaisse est la fissure de l'hippocampe, qui, vers le troisième mois, se forme sur la face externe de l'hémisphère, et correspond au grand hippocampe; puis apparaît la scissure calcarine, et, au commencement du quatrième mois, la scissure occipitale interne. Ces scissures ont été appelées primaires par Reichert, et les dépressions qu'elles forment à la surface de l'hémisphère correspondent à des saillies intérieures de la paroi du ventricule latéral, savoir : scissure de l'hippocampe au grand hippocampe, la calcarine au calcar avis ou petit hippocampe, et l'occipitale externe à une saillie de la paroi interne de la corne postérieure, nommée par Reil Zange ou forceps (pince) et par Henle bulbe de la corne postérieure. La scissure de Sylvius apparaît sur la face externe de l'hémisphère vers le quatrième mois; la scissure de Rolando se dessine visible à six mois, et de là jusqu'à la naissance, les circonvolutions de l'hémisphère se forment et acquièrent leur disposition caractéristique.

«Maintenant, si l'on voulait expliquer l'origine des circonvolutions mésiales du cerveau qu'on vient de décrire d'après l'idée courante que les deux hémisphères naissent de deux vésicules latérales et distinctes, il faudrait supposer qu'il s'est formé de la substance grise dans la fente interhémisphérique, là où il n'y en a jamais, qu'il s'est produit par conséquent un excès de développement.

“ Mais les vues plus récentes, et je pense, plus correctes, que je viens d'exposer, jettent un jour nouveau sur la formation des circonvolutions mésiales de ce cerveau; au lieu d'être dues à un excès de développement, elles sont dues à un arrêt de développement, car, la grande fente interhémisphérique ne s'étant formée qu'incomplètement, la division de la vésicule hémisphérale primitive en deux hémisphères distincts ne s'est faite que partiellement. La substance grise qui normalement, par suite de l'accroissement de la faux, forme la face interne des hémisphères, est restée mésiale et a constitué un pont unissant les 
deux hémisphères à travers la fente interhémisphérique. Cette vue sur la nature des circonvolutions mésiales est confirmée par la dissection des parties intérieures : l'existence d'une chambre ventriculaire unique, commune aux deux hémisphères par suite de la persistance du primitif trou de Monro, et la non-formation sur la ligne médiane du corps calleux, de la voûte et du septum lucidum, montrent que le développement de cette région a été arrêté prématurément; sur les côtés, l'absence de la corne descendante du ventricule latéral et l'absence du grand hippocampe et du plexus choroïde montrent que les plis de la paroi interne de l'hémisphère qui produisent l'hippocampe et le plexus choroïde ne se sont pas formés, circonstance due sans aucun doute au développement imparfait de cette paroi interne, et amenant dès lors un grave arrêt de développement dans le lobe temporal. Mais malgré le développement imparfait des parties médianes du cerveau, la substance grise corticale de l'hémisphère a revêtu la forme des lobes, des circonvolutions et des anfractuosités caractéristiques du cerveau humain, et la substance grise mésiale a revêtu également la forme de circonvolutions. Les grands ganglions de la base (corps strié et couche optique) se sont bien développés ainsi que la couronne radiée qui les unit aux circonvolutions de l'hémisphère, mais l'absence du corps calleux a amené une diminution de la masse de substance blanche qui constitue le centre ovale. Le déficit du développement a nécessairement diminué le volume total et le poids du cerveau, qui se trouvait réduit, au moment de l'autopsie à 1113 grammes (39 onces 1/4), poids inférieur d'environ 283 grammes (10 onces) au poids moyen du cerveau mâle de l'Européen adulte, et supérieur seulement de 57 grammes ( 2 onces) à celui qui établit la limite inférieure des cerveaux intelligents, car cette limite a été fixée par MI. Broca à 1049 grammes pour les hommes et à 907 grammes pour les femmes. Dès lors il n'est pas étonnant que cet homme ait été reconnu comme faible d'esprit bien longtemps avant son admission dans l'asile. "

M. Turner ajoute en terminant : «Le seul fait qui, à ma connaissance, se rapproche de celui-là, est cité par Mihalkovics qui l'emprunte à Bianchi (Storica del monstro die due corpi, Turin, 1749 , p. 100). Dans ce cas, dont je n'ai pu me procurer la des- 
cription originale, il est dit que les deux hémisphères étaient soudés sur la ligne médiane, et que les ventricules latéraux ne faisaient qu'une seule cavité. On sait aussi que, chez les monstres cyclopes, il n'y a qu'un hémisphère, avec un seul ventricule, mais la cyclopie s'accompagne d'arrêt de développement excessif du crâne, de la face, des yeux, du nez, etc. Dans certains cas d'hydrocéphalie chronique, il est dit que les hémisphères ne formaient qu'une seule poche; il est probable qu'alor's la production exagérée du liquide céphalo-rachidien avait commencé avant la séparation des deux hémisphères. D'autres cas où la séparation des deux hémisphères s'était effectuée, mais où le corps calleux était ou absent ou très rudimentaire, ont été décrits par Reil, Paget, Langdon Down, N. Knox et d'autres observateurs. On trouvera dans Glascow Medical Joumal, avril 1875, un travail où le docteur Knox, après avoir rapporté le fait qu'il a observé lui-même, donne un extrait des autres observations. ")

Nora. - Nous avons cru devoir reproduire parmi les mémoires originaux de Broca, celte traduction faite par lui-même, ce qui prouve bien la grande importance qu'il y attachait. 


\section{LOCALISATIONS GÉRÉBRALES}

\section{SUR LE \\ GERVEAU D'UN EGTROMÉLIEN}

(Bulletins de la Société d'anthropologie, $3^{e}$ série, t. II, 1879, p. 669-673.)

M. le professeur Verneuil, l'un des fondateurs de notre Société, a donné à notre laboratoire le moule et le cadavre d'un homme, ectromélien des deux bras, qui est mort dans son service au mois de juillet dernier. Ce malheureux gagnait sa vie en se montrant sur les places publiques.

L'ectromélie est complète à droite; à gauche, le membre supérieur est représenté seulement par un doigt et par un rudiment de métacarpien implanté sur l'épaule. C'est donc un état intermédiaire entre la phocomélie et l'ectromélie complète. Le squelette est en préparation; il vous sera prochainement présenté.

L'autopsie de cet individu offrait un grand intérêt pour les localisations cérébrales, question qui doit la plupart de ses progrès à l'étude des cas pathologiques plutôt qu'à l'expérimentation directe. Ce n'est pas que je récuse les expériences qui ont été faites sur le cerveau des singes. Mais c'est surtout en étudiant le cerveau d'individus frappés dans quelqu'une de leurs fonctions qu'on a jusqu'à présent obtenu les résultats les plus probants.

Par exemple, on a cherché quelles étaient les parties du cerveau qui s'étaient atrophiées chez des individus amputés depuis un certain nombre d'années; on supposait que la partie du cerveau qui sert à animer le membre perdu par ces amputés devait, faute d'usage, avoir diminué de volume, absolument comme un muscle réduit à l'inutilité s'atrophie au bout de quelque temps. 
Ce raisonnement, l'expérience l'a confirmé. Mais s'il est applicable à un individu qui a perdu ses membres par l'amputation, il l'est bien plus encore à un malheureux qui ne les a jamais eus.

L'autopsie de cet ectromélien a été faite dans le laboratoire en présence de plusieurs membres de la Société qu'attirait la curiosité scientifique.

Eclairé par les autopsies auxquelles je faisais allusion tout à l'heure, j'ai pu, avant l'ouverture du crâne, annoncer l'endroit précis du cerveau où nous devions trouver une diminution de volume, et l'autopsie a pleinement confirmé l'exactilude de ce diagnostic; si j'ai cru devoir ainsi me prononcer à l'avance, c'était pour convaincre les assistants de la réalité des localisations cérébrales: doctrine que j'ai soutenue depuis longtemps devant la Société d'anthropologie et ailleurs contre l'opinion très répandue et presque générale alors, que le cerveau est un organe indivis où les différentes facultés n'ont aucun siège déterminé.

J'ai donc annoncé d'avance que la partie atrophiée serait située au tiers supérieur de la première circonvolution frontale ascendante et au tiers postérieur de la première circonvolution frontale.

On ouvrit aussitôt le crâne, et le cerveau portait la lésion annoncée. Vous pouvez vous en convaincre sur ce moule, que M. Chudzinski a exécuté avec son habileté ordinaire; mais cet anatomiste a dû préalablement durcir le cerveau dans l'alcool, ce qui a notablement diminué la dépression des parties atrophiées. Cette dépression n'en est pas moins très apparente sur le moule.

Elle était remplie, au moment de l'autopsie, par une sorte de tumeur aqueuse transparente et tremblotante qui faisait corps avec la pie-mère et qu'on aurait pu, à la rigueur, prendre pour un kyste. Mais en détachant la pie-mère, on vit l'eau s'écouler goutte à goutte de sette membrane, ainsi qu'il arrive pour l'eau du corps vitré; au bout d'une heure, la pie-mère avait repris son apparence ordinaire, si ce n'est qu'elle restait un peu plus épaisse à ce niveau.

La coupe médiane du cerveau n'a pas encore été pratiquée, 
mais nous pouvons reconnaître, avant d'avoir examiné la face interne de l'hémisphère, que le lobule ovalaire (paracentral de Betz) est considérablement atrophié, car la dépression de la face convexe de l'hémisphère n'a pu se produire sans que la hauteur de ce lobule ait été diminuée d'autant.

Je viens de dire que l'atrophie de la face convexe porte sur le tiers supérieur de la frontale ascendante $F_{\text {。 }}$ et sur le tiers postérieur de la première frontale $\mathrm{F}_{1}$; il est plus exact de dire : sur la première portion de $\mathrm{F}_{\mathrm{o}}$ et sur la première portion de $\mathrm{F}_{1}$; ce n'est point, en effet, d'après leur longueur, mais d'après leur's caractères anatomiques, que se distinguent les diverses portions de ces circonvolutions.

La scissure de Rolando, dans son trajet oblique et sinueux, présente deux genoux qui la divisent en trois portions. La première portion est comprise entre le bord sagittal et le premier genou, qui correspond au niveau du premier sillon frontal, $f_{1}$; la seconde est comprise entre ce premier genou et le second, qui correspond au niveau du second sillon frontal $f_{s}$; la troisième enfin est située au-dessous du second genou. Comme la scissure de Rolando qu'elle longe, la circonvolution frontale ascendante se divise en trois portions : la première s'étend du bord sagittal à l'origine du sillon $f_{1}$, et donne insertion à la première frontale $F_{1}$; la seconde, comprise entre l'origine de $f_{1}$ et celle de $f_{s}$, donne insertion à la seconde frontale $\mathrm{F}_{\mathbf{s}}$; et la troisième enfin, comprise entre l'origine de $f_{\mathbf{s}}$ et la scissure de Sylvius, donne insertion à la troisième frontale $\mathrm{F}_{\mathrm{z}}$ (qui est la circonvolution du langage).

La première circonvolution, à son tour, est divisée anatomiquement en trois portions dont les limites sont établies par deux plis d'anastomose passant de $\mathrm{F}_{\text {, à }} \mathrm{F}_{\mathbf{s}}$ à travers le sillon $f_{1}$; de ces deux plis, tantôt superficiels, tantôt profonds, mais constants, l'un, l'antérieur, correspond à peu près au niveau de la bosse frontale; l'autre correspond à peu près à la partie supérieure de l'écaille frontale, un peu en avant du bregma. La première portion de la première circonvolution frontale est comprise entre sa racine et le pli d'anastomose postérieur; la seconde entre le pli d'anastomose antérieur et le postérieur; la troisième entre le pli antérieur et la pointe frontale de l'hémisphère. Cette troi- 
sième portion se continue avec la première circonvolution orbitaire, qui fait en réalité partie de la première circonvolution frontale et qui en constitue l'étage inférieur ou orbitaire. Sur la pointe de l'hémisphère, un troisième pli d'anastomose transversal unit la première frontale avec la seconde.

Ces dispositions anatomiques sont constantes; ce qui varie seulement, c'est la position superficielle ou profonde des deux premiers plis d'anastomose dont on vient de parler. La longueur relative ou absolue des trois portions de la première frontale est assez variable; chacune d'elles toutefois équivaut à peu près au tiers de la longueur de l'étage supérieur de cette circonvolution. De même, chacune des trois portions de la frontale ascendante en forme le tiers; mais elles sont souvent plus ou moins inégales entre elles. Il vaut donc mieux désigner ces portions par les numéros de première, seconde, troisième.

En résumé, sur le cerveau d'ectromélien que nous devons à M. Verneuil, il existe sur chaque hémisphère une atrophie très prononcée de la première portion de la circonvolution frontale ascendante, de la première portion de la première circonvolution frontale, et enfin du lobule ovalaire.

\section{DISCUSSION.}

M. Bertillon. Je demanderai à notre collègue s'il a quelque notion sur la région des circonvolutions qui seraient atrophiées sur les sourdsmuets et sur les aveugles de naissance.

M. Broca. En ce qui concerne les sourds-muets de naissance, j'ai pu, dans deux autopsies, constater que la portion postérieure de la troisième circonvolution frontale gauche était moins développée que d'habitude, et que l'insula apparaissait en partie entre les bords de la scissure de Sylvius. Rien de pareil n'existait à droite.

En outre, j'ai constaté un assez grand nombre de fois l'existence d'une plagiocéphalie droite (avec affaissement de la bosse frontale gauche) chez les sourds-muets de naissance, notamment dans un cas qui a été présenté il y a deux ans à la Société. 
Quant aux aveugles de naissance, je n'ai aucune observation à invoquer. Toutefois, il y a quelques motifs pour supposer que la partie postérieure du lobe pariétal est en rapport avec la fonction visuelle. Ce serait donc là qu'on pourrait chercher à constater l'existence d'un certain degré d'atrophie chez les aveugles-nés. 


\section{MOULE D'UN CERVEAU DE TOULOUSAIN}

(Bulletins de la Société d'anthropologie, 30 série, 1880, t. III, p. 163̈-167.)

M. Broca présente, de la part de M. Chudzinski, premier préparateur du laboratoire, le moule du cerveau d'un homme atteint de la déformation toulousaine. Cet homme est le nommé Cantié (Achille), trente-sept ans, dont le buste a été présenté à la Société dans une précédente séance (Bulletins de la Société d'anthropologie, 1879, p. 417, séance du 5ّ juin 1879).

Sur ce moule, que M. Chudzinski a exécuté avec son habileté ordinaire, il est aisé de voir que la scissure de Rolando est plus oblique que sur les cerveaux non déformés. Son extrémité inférieure occupe sa position normale, mais 'son extrémité supérieure est notablement refoulée en arrière. Il en résulte que l'étendue du lobe frontal le long de la suture sagittale est augmentée; mais ce lobe n'est pas accru pour cela, car ce qu'il a gagné dans ce sens, il l'a perdu et au delà dans le sens vertical, l'écaille frontale étant très fortement déprimée, comme on peut le voir sur le buste.

En mesurant, à l'aide d'un goniomètre flexible que je vous présenterai tout à l'heure, l'angle sous lequel la ligne de la scissure de Rolando rejoint le bord sagittal de l'hémisphère, on trouve que cet angle n'est que de 65 degrés. Sur les cerveaux normaux il descend rarement à 70 degrés et est en moyenne de 73 sur les cerveaux dolichocéphales, de 76 sur les cerveaux brachycéphales.

La scissure occipitale externe "ne paraît pas déplacée; il en résulte que l'étendue du lobe pariétal, dans sa partie supérieure, est diminuée, ainsi que celle du lobule quadrilatère, sur la face externe de l'hémisphère.

M. Marter demande si un individu dont le cràne aurait naturellement la région frontale aussi déprimée ne devrait pas être présumé idiot. 
M. Broca pense que non, pourvu que les conditions qui pourraient amener cette dépression ne soient pas de nature à empêcher le crâne de prendre dans une autre direction un accroisscment compensateur. G'est ce qui a lieu dans les déformations artificielles du crâne. A l'âge où on les pratique, les sutures sont encore membraneuses et se laissent distendre par le cerveau refoulé; celui-ci retrouve ainsi dans certains sens une grande partie de ce qu'il perd dans les autres, et son volume total reste considérable, encore bien qu'il puisse dans beaucoup de cas être plus ou moins réduit.

Sur deux autres cerveaux déformés à la toulousaine, et qui n'étaient pas en état d'être conservés, nous avons pratiqué des coupes pour peser séparément les divers lobes des hémisphères, et nous avons ipu constater que le poids relatif du lobe frontal était diminué, tandis que le poids relatif du lobe occipital était augmenté. La déformation modifie donc le développement relatif des diverses régions des hémisphères, et il paraît assez probable que lorsqu'elle est très prononcée, il doit en résulter des modifications psychologiques; mais celles-ci sont le plus souvent compatibles avec l'intégrité de l'intelligence. On sait toutefois qu'il n'en est pas toujours ainsi, et que, dans les départements où existent des déformations artificielles, la proportion des individus qui présentent ces déformations est beaucoup plus grande dans les asiles d'aliénés que dans le reste de la population de la région. 


\section{LE CERVEAU DE L'ASSASSIN PRÉVOST}

(Bulletins de la Société d’anthropologie, $3^{\circ}$ série, t. III, 1850, p. 233-243.)

Je vous présente le moule du cerveau d'un criminel qui avait, à quelques années d'intervalle, assassiné deux personnes pour les voler, et qui a été décapité à Paris il y a quelques semaines.

Ce moule sera conservé dans notre musée, et ceux qui voudront l'y étudier plus tard seront sans doute frappés comme vous de son volume extraordinaire, car il excède de beaucoup les plus grands cerveaux de notre vaste collection. On pourra donc être tenté de croire que le cerveau de Prévost était excessivement grand, tandis qu'en réalité il ne dépassait que fort peu le poids du cerveau des individus de même sexe, de même taille et de même âge. Je dois donc expliquer avant tout cette apparente contradiction.

Il est nécessaire, pour éviter les déformations, de durcir le cerveau avant de le mouler; à cet effet, on plonge ordinairement le cerveau dans l'alcool pendant une quinzaine de jours; il y perd une notable partie de son poids (un quart ou un tiers) et son volume se réduit en proportion. Tous les moules cérébraux du musée ont été faits sur des cerveaux ainsi rétractés.

Mais ce n'est pas dans l'alcool qu'a été préparé le cerveau du sergent de ville Prévost. Le corps a été transporté à l'Ecole pratique dans le laboratoire de mon collègue et ami Robin; le cer'veau, extrait le jour même par M. Cadiat, directeur adjoint de ce laboratoire, pesait avec ses membranes 1422 grammes, et ne présentait qu'un volume à peu près ordinaire. Désirant conserver la structure des éléments microscopiques, M. Cadiat plongea cet organe dans le liquide de Müller, qui, au lieu d'absorber l'eau et de faire rétracter les tissus, s'y infiltre au contraire de manière à augmenter le poids et le volume des parties. 
Il en est résulté que l'encéphale de Prévost, au moment où il a été moulé, pesait 1822 grammes, tandis qu'il aurait pesé tout au plus 1100 grammes s'il avait été durci dans l'alcool.

Prévost était très grand $\left(4^{\mathrm{m}}, 84\right)$ et très robuste. Il était né le 31 décembre 1836, et avait par conséquent un peu plus de quarante-trois ans. La soudure des sutures crâniennes était notablement plus avancée qu'elle ne l'est ordinairement à cet âge; elle était parvenue au degré que l'on observe généralement de cinquante à cinquante-cinq ans; mais l'état des muscles et des riscères montrait que le sujet était encore dans toute la force de l'âge mûr. En outre, la nature de sa mort l'a soustrait à l'amaigrissement cérébral qui est la conséquence des maladies chroniques. Dans ces conditions, le poids moyen du cerveau ne doit pas être différent de celui qui correspond à la moyenne de l'âge de quarante ans, soit 1410 grammes environ. La balance a donné 1422 grammes; on peut dire par conséquent que le cerveau de Prévost est un peu supérieur à la moyenne, et, quoique cette différence ne soit pas considérable, nous pouvons en tenir compte. Elle s'accorde d'ailleurs avec les observations de M. Bordier.

Nous savons que notre collègue, analysant avec beaucoup de sagacité l'histoire des cas où un crime est commis par deux individus, a établi une distinction entre le premier criminel qui conçoit le projet du crime, et le complice qui l'aide dans l'exécution. Celui-ci est presque toujours beaucoup moins intelligent que celui-]à. M. Bordier ayant eu, pendant l'Exposition des sciences anthropologiques, l'occasion de mesurer les crânes d'assassins qui avaient ainsi opéré à deux et qui avaient été décapités ensemble, a cherché et retrouvé dans la Gazette des tribunaux l'histoire de leurs crimes, et a pu constater ainsi que la capacité crânienne du premier criminel est généralement supérieure à la moyenne, et que celle du second, du complice, est gónéralement inférieure et souvent très inférieure à la moyenne.

Les criminels isolés sont aussi quelquefois des espèces de brutes au crâne bas et étroit; mais dans la grande majorité des cas ce sont des individus intelligents, et souvent même très intelligents, quoique mal équilibrés. On conçoit ainsi comment M. Bordier, après avoir mesuré un grand nombre de crânes 
d'assassins de toutes sortes, a trouvé que leur capacité cérébrale est supérieure en moyenne à la moyenne ordinaire.

Ce ne sont pas seulement les criminels qui présentent ce phénomène. Les aliénés non paralytiques et les suicidés sont très souvent dans le même cas. Le suicide n'est pas toujours, tant s'en faut, un acte d'aliénation. Il y a des suicidés parfaitement conscients et que je suis bien loin de comparer à des assassins; ils ont toutefois avec eux cela de commun qu'ils obéissent le plus souvent à une excitation cérébrale, à une passion violente ou à un faux jugement, et les cerveaux doués d'une grande activité sont plus sujets que les autres à cet état d'exaltation. Pour ces divers motifs, le poids moyen du cerveau des suicidés est supérieur à la moyenne. Nous possédons une collection de crânes extraits de la partie de l'ancien cimetière de l'Ouest (1790-1825) où l'on inhumait exclusivement les corps provenant de la Morgue. La capacité moyenne de ces crânes est supérieure non seulement à celle des crânes de la fosse commune, mais encore à celle des crânes des sépultures particulières. Parmi les corns qui sont exposés à la Morgue, on ne transporte au cimetière de la Morgue que ceux qui n'ont pas été reconnus, et la plupart de ceux-là sont des corps d'individus suicidés par submersion.

Je reviens à Prévost. Ce n'était pas un de ces assassins qui se laissent entraîner par de mauvais conseils ou par de mauvais exemples, ni de ceux qui obéissent à une excitation momentanée. Il a conçu lui-même ses crimes et les a exécutés seul avec autant de sang-froid que de précision. Il appartient donc à la catégorie des assassins intelligents, dont le cerveau est ordinairement plus grand que la moyenne; mais lorsqu'on étudie les cerveaux de ces individus, on y trouve presque toujours des défectuosités morphologiques qui ont été signalées par M. Benedickt de Vienne, et mises en évidence par la collection de cerveaux d'assassins qu'il a envoyée à notre Exposition des sciences anthropologiques. Ces cerveaux ne présentent généralement pas d'anomalies dans le sens anatomique du mot; leurs circonvolutions, leurs plis secondaires sont au complet et ont leurs connexions ordinaires; mais le développement relatif des diverses parties de certaines circonvolutions et de leurs plis de commu- 
nication est plus irrégulier que sur la plupart des cerveaux normaux. Tel pli de communication qui est habituellement assez volumineux pour être superficiel, est atrophié et reste profond; tel autre au contraire pourra être plus volumineux et plus superficiel que de coutume, mais le second cas est moins commun que le premier. Il en résulte que certains sillons ou certaines scissures, qui sont ordinairement séparés par des plis superficiels, se continuent ou plutôt semblent se continuer entre eux par suite de l'atrophie partielle de ces plis et de leur position profonde. Ce sont ces communications plus ou moins insolites d'anfractuosités ordinairement distinctes, qui ont été désignées par M. Benedickt sous le nom de communications anormales (et on se gardera bien de confondre ici la communication des anfractuosités avec celle des circonvolutions, qui est pour ainsi dire l'inverse). Ces communications anormales des anfractuosités, qu'il vaudrait mieux nommer insulites, se rencontrent soit à droite, soit à gauche, en un ou plusieurs points, sur la plupart des cerveaux ; c'est pour cela qu'aucun cerveau n'est absolument symétrique, ni absolument typique dans toutes ses parties. Une ou plusieurs de ces communications insolites n'empêchent pas un cerveau d'être à la fois très intelligent et très bien équilibré ; mais lorsqu'elles sont nombreuses, lorsqu'elles affectent des parties importantes, elles sont l'indice d'un développement défectueux. G'est ce qu'on voit souvent sur les cerveaux peu volumineux des pauvres d'esprit ou des imbéciles, et c'est ce qu'on voit aussi très fréquemment sur les cerveaux des assassins, avec cette différence que, dans le premier cas, le moindre développement des plis de passage ou d'anastomose est en rapport avec le peu de développement des circonvolutions en général et avec la faiblesse cérébrale, tandis que, dans le second cas, il coïncide au contraire avec l'ampleur de la plupart des circonvolutions et témoigne de l'irrégularité du développement du cerveau. C'est ainsi que M. Benedickt a pu constater, et que nous avons pu constater avec lui sur les pièces de sa collection, que les communications insolites des anfractuosités sont incom. parablement plus fréquentes, et plus nombreuses sur un même cerveau, chez les assassins que chez les individus ordinaires.

I.e cerveau de Prévost confirme pleinement ces observations 
et va même au delà. Lorsque M. Cadiat me l'a montré pour la première fois, le jour de l'autopsie, j'ai tout de suite demandé des pinces pour enlever la pie-mère au niveau de la scissure occipitale externe, et j'ai pu constater, comme vous le voyez sur le moule, que le premier pli de passage de Gratiolet (premier pli occipito-pariétal) est profond à droite comme à gauche, que par conséquent la scissure occipitale interne se continue directement avec la scissure occipitale externe, et que le lobe occipital se détache du lobe pariétal sous la forme de ce que Gratiolet a appclé, chez les singes, la calotle.

Les fonctions du lobe occipital ne sont pas encore connues; ce qu'on sait seulement, c'est qu'elles sont d'un ordre peu élevé, car les plus graves lésions de ce lobe ne portent pas atteinte à l'intelligence. L'étude de la disposition du lobe occipital reste donc jusqu'ici pour nous sans signification physiologique, mais elle offre un certain intérêt au point de vue morphologique.

Gratiolet toutefois en avait singulièrement exagéré l'importance. Après avoir vainement cherché, dans le reste du cerveau, des caractères anatomiques capables de distinguer, abstraction faite du volume, le cerveau de l'homme du cerveau des singes, il avait cru trourer ces caractères dans les connexions du lobe occipital. Chez l'homme, disait-il, les deux premiers plis de passage sont rapetissés, la scissure occipitale externe ne se continue pas avec l'interne, et le lobe occipital se continue directement, superficiellement, avec le lobe pariétal. Chez les singes, ajoutait-il, les deux plis de passage sont profonds (il avançait même que l'un d'eux manquait dans beaucoup d'espèces, mais ce n'est pas ici le lieu de discuter cette assertion erronée) et il en résulte que la scissure occipitale interne, se continuant avec l'externe, détache le lobe occipital sous la forme d'une calotte. Il reconnaissait toutefois que l'orang (anthropoïde) et l'atèle (cébien) ont le premier pli de passage superficiel, et n'ont pas la calotte; il aurait pu y ajouter le gorille, dont le cerveau était encore inconnu. Le caractère de la calotte n'était donc pas absolu; toutefois il avait l'avantage de distinguer l'homme de presque tous les singes.

Mais quoique ce caractère distinctif pût déjà paraître insuffisant, il était plus insuffisant encore que ne le pensait Gratiolet. 
Déjà dans une note de mon mémoire sur l'Ordre des primates (Bull. de la Soc. d'anthropologie, 1869, $2^{\mathrm{e}}$ série, t. IV, p. 387, et dans Mémoires d'anthropologie, t. III, p. 132 et 133) j'avais signalé plusieur's cas où j'avais vu sur des cerveaux hımains le premier pli de passage occuper une situation profonde, et la scissure occipitale interne se continuer ainsi avec l'externe. J'ajoutais que je n'avais jamais vu jusqu'alors cette anomalie sur les deux hémisphères à la fois, et qu'au surplus elle ne paraissait pas extrêmement rare. J'en ai effectivement recueilli depuis lors une quinzaine d'observations; et j'estime que le degré de fréquence de cette anomalie est, dans notre race, d'en-. viron 2 pour 100 ; j'ai pu constater en outre que, dans la majorité des cas, elle existe à la fois sur deux hémisphères.

L'un des cerveaux de blancs sur lesquels je l'ai observée est celui de notre regretté et distingué collègue Louis Asseline (voir dans les Bulletins de la Société d'anthropologie, 1878, p. 161, l'intéressant rapport de M. Thulié sur l'autopsie d'Asseline). Ce cerveau était d'ailleurs très bien développé dans tout le reste de sa surface, et pesait 1468 grammes, chiffre bien supérieur à la moyenne. La situation profonde des premiers plis de passage n'est donc pas, en soi, un obstacle au fonctionnement le plus régulier et le plus actif des facultés intellectuelles d'un ordre élevé, et cela semblera tout naturel, puisque le lobe occipital, tout permet de le croire, est étranger à ces facultés supérieures. Il est clair d'ailleurs que la situation profonde d'un pli de passage peut résulter de deux causes très différentes : ou bien de sa petitesse absolue, qui est une défectuosité, ou bien de sa petitesse relative, résultant de ce que, son volume étant ordinaire, celui des circonvolutions adjacentes est plus qu'ordinaire. Ce dernier cas, qui était celui d'Asseline, a évidemment une signification tout autre que celle du premier. On ne peut méconnaître, toutefois, que l'existence de "la calotte » chez l'homme est l'indice morphologique d'une certaine irrégularité du développement cérébral, et l'on conçoit que les cerveaux qui présentent cette disposition soient exposés plus que les autres à des imperfections fonctionnelles.

Le fait d'Asseline est certainement très exceptionnel. Tous les autres cas que j'ai recueillis se rangent sous deux chefs. Les 
premiers, qui sont les plus nombreux, concernent des cerveaux petits et assez pauvres : plusieurs sont déposés dans notre musée; ils proviennent de femmes très peu intelligentes ; l'une était même à peu près imbécile. Les autres, au nombre de trois, provenaient de jeunes hommes qui s'étaient suicidés pour des motifs sans importance sérieuse et qui étaient venus mourir à l'hôpital, dans mon service (l'un d'eux avait déjà fait antérieurement trois tentatives de suicide). Cies cerveaux de suicidés étaient d'ailleurs amples et riches en circonvolutions. Sans rattacher le cerveau de Prévost à celte dernière catégorie, on peut -jusqu'à un certain point l'en rapprocher, d'une part sous le rapport de son grand volume et d'une autre part sous le rapport du défaut d'équilibre fonctionnel; et c'est précisément parce que je prévoyais ce rapprochement, que, le jour de l'autopsie de Prévost, j'allai tout d'abord à la recherche des plis de passage pariéto-occipitaux.

La communication de la scissure occipitale externe avec l'interne n'est pas la seule communication insolite qui existe sur ce cerveau. Il y en a d'autres qui sont moins rares, sans doute, mais qui méritent cependant quelque attention, et d'autres enfin qui sont tout à fait extraordinaires.

De chaque côté, la racine de la troisième circonvolution frontale occupe une situation profonde, de sorte que la scissure prérolandique communique avec la scissure de Sylviust. En outre, du côté droit, la racine de la seconde circonvolution frontale est profonde, et il en résulte que le sillon prérolandique s'étend, sans interruption, de la scissure de Sylvius jusqu'à la racine de la première circonvolution frontale, près du bord sagittal de l'hémisphère.

Le pli de passage fronto-pariétal inférieur, dans lequel se termine la scissure de Rolando, est fort atrophié; il ne forme plus à la surface de l'hémisphère qu'une très mince languette, épaissc à peine de 1 à 2 millimètres; la scissure de Rolando ne communique donc pas avec la sylvienne, mais l'intervalle qui l'èn sépare est presque nul. Sur l'hémisphère gauche, la scissure de Rolando communique, dans sa troisième portion, avec le sillon postrolandique, et un peu plus haut, au niveau de son second genou, avec le sillon prérolandique. 
A droite, la scissure de Rolando ne présente pas de communication insolite, mais elle est très peu flexueuse, par suite du peu de développement des deux circonvolutions ascendantes; la pariétale ascendante surtout est très mince, et entamée en trois points par des incisures qui émanent du sillon postrolandique et qui arrivent presque jusqu'à la scissure de Rolando.

Sur les deux hémisphères, les connexions du lobe temporal avec les lobes pariétaux et occipitaux sont défigurées à tel point, qu'il faut une grande attention pour les reconnaître. A gauche, le pli courbe manque presque entièrement; on n'en retrouve que la partie supérieure, réduite à une épaisseur de 3 millimètres; et ce pli ne descend pas jusqu'au lobe temporal; il ne se continue pas avec la seconde circonvolution temporale; il en est séparé par un sillon, profond et très long, qui commence à 5 millimètres en arrière de la scissure de Sylvius et qui s'étend de là, d'avant en arrière, en ligne droite, jusqu'à la pointe du lobe occipital. Le lobe pariétal ne communique donc avec le lobe temporal que sur la marge de la scissure de Sylvius. Audessous de ce premier sillon rectiligne, il y en a un second qui s'étend tout droit, sans interruption, de la pointe du lobe temporal à la pointe du lobe occipital; il représente, en avant, le second sillon temporal $t_{\mathrm{s}}$; en arrière, il représente le troisième sillon occipital $o_{3}$; mais les extrémités de ces deux sillons sont ordinairement séparées l'une de l'autre par un intervalle de plusieurs centimètres, et jamais, jusqu'ici, je ne les avais vues se continuer directement l'une avec l'autre.

De cette même pointe du lobe occipital, au-dessus du premier de nos deux sillons rectilignes, part un troisième sillon rectiligne et très profond qui se continue directement en avant et en haut avec le sillon pariétal (intra-pariétal de Turner) et s'étend ainsi jusqu'au sillon postrolandique. Cette disposition est bien connue, et, quoique s'écartant du type ordinaire, elle n'est pas rare; mais, coïncidant ici avec l'existence des deux autres sillons rectilignes insolites que je viens de décrire, elle donne au lobe occipital une configuration tout à fait extraordinaire.

La même configuration se retrouve, à peu de chose près, sur le lobe occipital droit; mais, de plus, en avant de la partie externe de ce lobe, sur la base du lobe temporal, existe un profond 
sillon vertical, long de 4 centimètres, qui traverse la deuxième et la troisième circonvolution temporales et fait presque entièrement disparaître leur connexion avec le lobe occipital ; le pli courbe, passant en arrière et au-dessus de ce sillon, est entièrement dénaturé; il semble tout à fait étranger au lobe temporal ; il paraît ne se continuer qu'avec les deuxième et troisième circonvolutions occipitales, et, pour le reconnaître, il faut être fermement convaincu qu'il ne peut être ailleurs.

Il existe sur ce cerveau remarquable d'autres dispositions plus ou moins insolites, mais moins importantes et qu'il n'est pas nécessaire d'énumérer. Celles que je viens de décrire vous montrent suffisamment que le cerveau de Prévost est très anormal. Parmi les particularités qu'il présente, les unes sont fréquentes et ne tirent leur signification que de leur accumulation sur un même cerveau; les autres sont rares, mais déjà connues; d'autres enfin, et ce sont précisément les plus graves au point de vue morphologique, sont tellement rares, qu'elles n'ont pas encore été signalées.

Maintenant faut-il se demandẹ si ces anomalies cérébrales sont antérieures ou postérieures à l'époque où Prévost s'est engagé dans le crime? Il est certain qu'elles lui sont bien antérieure:, qu'elles datent des périodes de la formation ou du développement des circonvolutions, peut-être de la naissance, peut-être seulement de l'enfance. Je ne dis que cela, ct je laisse à chacun de vous le soin d'en tirer les conclusions. 


\section{LOGALISATIONS GÉRÉBRALES}

\section{SUR LE \\ GERVEAU D'UN GUL-DE-JATTE}

(Bulletins de la Société d'anthropologie, 3e série, 1880, t. III, p. 410-413.)

J'ai présenté à la Société, dans sa séance du 6 novembre 1879 , le cerveau d'un ectromélien qui ne présentait d'autre vestige des membres thoraciques qu'un doigt et un métacarpien rudimentaire implantés sur l'omoplate gauche. L'occasion était bonne pour vérifier ce que l'anatomie pathologique a enseigné sur la localisation du centre moteur cortical des membres thoraciques : il était probable, en effet, que cette partie du cerveau était atrophiée chez lui. Devant plusicurs personnes que cette intéressante autopsie avait attirées, j'annonçai que sans doute nous trouverions une anomalie par défaut dans une région circonscrite occupant le tiers supérieur de la circonvolution frontale ascendante et le tiers postérieur de la première circonvolution frontale. L'autopsie me donna raison, et sur le moule qui fut fait de ce cerveau, et que je remets sous vos yeux, vous voyez à droite et à gauche une dépression très apparente au niveau de la région indiquée. J'ajoute que sur le cerveau frais cette dépression était encore plus profonde; mais, pour le mouler, nous avons dû le durcir dans l'alcool, c'est-à-dire le faire rétracter, et par conséquent diminuer ainsi la dépression.

Cette dépression, vous vous en souvenez peut-être, était comblée par une petite tumeur aqueuse de la pie-mère. Une piqûre faite dans cette tumeur fit sortir goutte à goutte une sérosité transparente; la tumeur se vida, s'aplatit et se réduisit à une mince membrane.

J'avais besoin de vous rappeler en quelques mots cette observation, dont je vous ai donné naguère les détails, avant de vous 
présenter le cerveau d'un cul-de-jatte mort dans le service de M. Blachez, et que ce confrère a signalé à mon attention.

Il est probable qu'une paralysie essentielle de l'enfance fut la cause de l'infirmité de ce malade, qui, atteint de délire alcoolique lor's de son entrée à l'hôpital, et mort peu de jours après, n'a pu donner aucun renseignement sur l'époque à laquelle remontait son infirmité.

Quoi qu'il en soit, il avait une paralysie presque complète, mais nou pas complète, des membres inférieurs, qui étaient très atrophiés, plus courts et moins gros que les membres supérieurs, et qui ne pouvaient servir à la marche.

Les faits anatomo-pathologiques ayant établi que le centre moteur des membres inférieurs est situé sur le tiers supérieur de la parićtale ascendante (vis-à-vis le centre des membres tho raciques, dont il n'est séparé que par la scissure de Rolando), c'est sur ce point que devait se porter toute notre attention. Nous devions nous attendre à y trouver une atrophie, moindre toutefois que dans le cas précédent, puisqu'il ne s'agissait plus de l'absence congénitale et presque complète des membres, avec suppression totale de leurs fonctions, mais seulement d'une diminution de fonctions et de volume consécutive ă une maladie de l'enfance.

Nous pouvons voir, en effet, que sur le cerveau de notre culde-jatte il n'y a pas de dépression, pas de tumeur aqueuse remplissant une cavité ; la surface de la pariétale ascendante est partout de niveau, mais le tiers supérieur de cette circonvolution est considérablement aminci; il est réduit tout en haut au quart et plus bas au tiers de son épaisseur ordinaire, tandis qu'à la même hauteur la circonvolution frontale ascendante est au contraire plus épaisse que de coutume. Ce dernier fait coïncide avec la grande activité et la grande force des membres supérieurs de cet homme, qui marchait sur les mains.

La comparaison des cerveaux de l'ectromélien de M. Verneuil et du cul-de-jatte de M. Blachez est extrêmement intéressante et fournit un nouvel appui à la doctrine des localisations cérébrales. 
DISCUSSION.

M. Coudereau cite, à ce propos, une expérience faite en sa présence par M. Laborde sur un chien nouveau-né(1).

M. Broca. L'expérience de M. Laborde, citée par M. Coudereau, me paraît tout à fait sans valeur au point de vue des localisations cérébrales chez l'homme.

Le point du cerveau du jeune chien sur lequel cette expérience a été faite, n'est point indiqué; je suppose que ce doit être la région qui avoisine le sillon crucial de Leuret, car c'est là que d'autres expérimentateurs ont opéré. Mais ce point n'est nullement l'analogue, au point de vue anatomique, de celui où siègent chez l'homme les centres moteurs des membres.

Le type cérébral des chiens est tellement différent de celui de l'homme, que les expériences faites sur ces animaux, comme sur les autres vrais quadrupèdes, ne signifient absolument rien en ce qui nous concerne. C'est par ce motif que l'étude des localisations cérébrales est restée si longtemps infructueuse et contradictoire. Le type cérébral des singes, au contraire, est sem-

(1) M. Coudereau, qui avait cité cette expérience de mémoire, l'a rectifiée depuis dans les termes suivants, d'après une observation écrite (thèse de M. A. Lemoine) :

La calotte crânienne ayant été enlevée, ainsi que les méninges, avec beaucoup de précautions sur l'un des hémisphères d'abord (le gauche), après avoir attendu quelques instants, les extrémités de l'excitateur ont été portées très nettement et très superficiellement un peu en arrière du sillon crucial, au niveau du sillon de séparation du petit lobule de forme à peu près quadrilatère, qui borne en arrière le petit sillon et la scissure interhémisphérique, et nous obtenons immédiatement un soulèvement en totalité de la patte droite antérieure du côté opposé.

Lorsque l'excitateur est pareillement porté sur les circonvolutions postérieures selon une ligne parallèle à la scissure interhémisphérique et partant du petit lobule antérieur dont il vient d'être question, on n'obtient aucun effet moteur.

La répétition de l'expérience sul l'hémisphère du côté opposé donne des résultats absolument identiques. Lorsque l'excitateur est appliqué au fond de la scissure sur le corps calleux, on obtient des mouvements doubles des pattes antérieures et consécutivement des mouvements généraux des quatre membres. Il est d'ailleurs nécessaire pour cela, l'animal étant un peu épuisé, d'augmenter sensiblement l'intensité du courant. 
blable au nôtre; la correspondance des parties analogues est parfaitement établie. Les expériences faites par M. Ferrier sur des singes, ont donc une réelle importance. Toutefois, les excitations se propagent aisément au-delà des points attaqués, et les animaux dont on a ouvert le crâne se trouvent dans des conditions qui troublent beaucoup les résultats.

Somme toute, c'est toujours l'anatomie pathologique qui est le fondement le plus solide de l'étude des localisations cérébrales chez l'homme. On constate sur le vivant un trouble fonctionnel, et on en cherche la cause à l'autopsie. Cette méthode est lente sans doute, mais c'est elle qui nous a révélé le siège du langage, et c'est à elle que nous devons à peu près tout ce qui a été découvert depuis sur les autres centres corticaux. 


\section{ANATOMIE COMPAREE DU CER EEAU}

\section{SUR LE BEG DE L'ENCÉPHALE}

(Cette partie d'un travail sur l'endocrâne publié dans les Bulletins de la Société d'anthropologie, 1873, 2érie, t. VIII, p. 355-359, a été réimprimée avec lui dans le tome IV des Mémoires d'anthropologie, p. 201.)

\section{SUR LA GIRCONVOLUTION LIMBIQUE}

ET

\section{LA SGISSURE LIMBIQUE}

(Bulletins de la Socièté d'anthropologie, $2^{e}$ série, t. XII, 1877, p. 646-63̈7.)

M. Broca présente une série de pièces et de dessins relatifs ì l'anatomie comparée du grand lobe limbique des mammifères. Nous donnons ici une analyse de ses recherches qui seront publiées in extenso dans la Revue d'anthropologie.

Le lobe olfactif, réduit chez les primates à la bandelette olfactive (nerf olfactif) et au petit renflement qui la termine (ganglion olfactif), présente, chez tous les autres mammifères terrestres, un très grand développement. Son extrémité antérieure, reçue dans la fosse ethmoïdale, est libre; mais en arrière sa base se continue sans interruption avec la partie du manteau qui entoure le limbe de l'hémisphère, et il constitue avec elle un grand lobe que l'auteur appelle le grand lobe limbigue.

Le grand lobe limbique se compose de trois portions: $1^{\circ}$ la circonvolution du corps calleux ou lobe du corps calleux, qui 
en forme l'arc supérieur; $2^{\circ}$ le lobe de l'hippocampe, qui en forme l'arc inférieur; $3^{\circ}$ le lobe olfactif, qui en est le prolongement antérieur, et dont la base se continue en arrière avec le lobe de l'hippocampe, en haut avec l'extrémité antérjeure de la circonvolution du corps calleux, réfléchie au-devant et au-dessous du genou de ce corps. La circonvolution du corps calleux et le lobe de l'hippocampe se trouvent donc réunis en avant par la base du lobe olfactif; en arrière, sous le bourrelet du corps calleux, ils se continuent directement l'un avec l'autre, en cuntournant le pédoncule cérébral, dont ils sont séparés par la fente de Bichat. Les deux ares limbiques, ainsi réunis en avant et cn arrière, constituent donc autour du limbe de l'hémisphère un anneau complet, dont l'are inférieur forme presque toute la face inféricure du cerveau, et dont l'arc supérieur s'aperçoit sur la face interne de l'hémisphère.

Le grand lobe limbique, dont le bord concave entoure le limbe de l'hémisphère, est limité sur son bord convexe par une grande scissure qui le circonscrit presque entièrement; c'est la scissure limbique; celle-ci se compose aussi de deux arcs, l'un supérieur, l'autre inférieur. L’arc supérieur s'étend sur la face interne de l'hémisphère, parallèlement au bord convexe du corps calleux, entre ce bord et le bord supérieur ou sagittal de l'hémisphère. L'arc inférieur commence en avant, sur le bord externe du lobe olfactif, longe tout le bord externe de la base du cerveau, et va se terminer sur la face inférieure ou tentoriale de l'hémisphère, dans la partie qui représente le lobe occipital des primates.

Les deux ares de la scissure limbique ne se rejoignent pas comme les deux ares du grand lobe limbique; ils n'entourent pas entièrement ce lobe, parce que les deux extrémités de la circonvolution du corps calleux se continuent dans une étendue variable, en avant avec le lobe frontal, en arrière avec le lobe pariéto-occipital, ou plutôt avec la partie postérieure du lobe pariétal (le lobe occipital n'étant pas distinct chez les animaux autres que les primates).

Le grand lobe limbique, ainsi limité, constitue dans le manteau de l'hémisphère une partic entièrement différente de toutes les autres, et dont l'évolution dans la série animale est en 
quelque sorte inverse. Cette partie est la première qui commence à se distinguer du reste du manteau. Chez les mammifères lissencéphales, ou sans circonvolutions, alors qu'on n'aperçoit sur la face convexe du cerveau ni sillon ni scissure, on trouve cependant, sur le bord externe, l'arc inférieur plus ou moins complet de la scissure limbique, qui dessine la limite externe du lobe olfactif et du lobe de l'hippocampe, — et sur la face interne une légère dépression longitudinale, qui dessine la limite supérieure de la circonvolution du corps calleux. Exemple : le lapin, le castor. Si l'on cxamine ensuite les cerveaux gyrencéphales, ou à circonvolutions, on voit les deux parties de la scissure limbique se caractériser et se creuser de plus en plus; en même temps d'autres anfractuosités apparaissent à la surface, par suite d'un plissement qui donne lieu à la formation des circonvolutions. Ce plissement, dû à l'extension de l'écorce cérébrale, qui est le siége des facultés intellectuelles, s'effectue dans toutes les parties de l'hémisphère, excepté dans le grand lobe limbique. Celui-ci conserve son aspect lisse et sa simplicité, pendant que le reste du manteau se complique et se perfectionne. Chez les grands gyrencéphales, tels que le chameau, on voit, il est vrai, quelques légères incisures isolées, très superficielles, apparaître sur certains points de la surface $d u$ grand lobe limbique, mais celui-ci contraste toujours, par son extrême simplicité, avec tout le reste de l'hémisphère. Le contraste est tel, qu'il devient évident que ces deux parties de l'hémisphère, si différentes par leur structure, le sont aussi par la nature de leurs fonctions, et si l'on considère que l'une d'elles reste stationnaire et imperfectible, pendant que l'autre se perfectionne et se développe, que celle-là perd de son importance à mesure que celle-ci fait des progrès, on est conduit à reconnaître que la première est le siége des facultés inférieures qui prédominent chez la brute, que la seconde est le siége des facultés supérieures qui prédominent chez les animaux intelligents, et l'on peut exprimer cette opposition de leurs caractères respectifs, en disant que le manteau de l'hémisphère se compose de deux parties : l'une, brutale, représentée par le grand lobe limbique; l'autre, intellectuelle, représentée par le reste du manteau. 
La différence qui existe entre ces deux parties de l'hémisphère est incomparablement plus grande que celle qui existe entre les diverses divisions connues sous le nom de lobes. Le grand lobe limbique est donc plus qu'un lobe, et il ne suffirait pas de l'ap. peler le lobe limbique; il comprend d'ailleurs deux parties qui sont déjà désignées sous le nom de lobes (le lobe olfactif et le lobe de l'hippocampe), et il ne conviendrait pas de donner le même nom au tout et à ses parties; mais en le nommant le grand lobe limbique, on le caractérise suffisamment sans recourir au néologisme.

Gette partie de l'hémisphère est celle qui, dans la série des mammifères, se distingue la première du reste du manteau.

Chez les mammifères lissencéphales, c'est-à-dire sans circonvolutions, le lobe olfactif, prolongé en avant comme une sorte d'apophyse, constitue la seule saillie qui se détache du reste de l'hémisphère; néanmoins l'arc inférieur de la scissure limbique est déjà très bien dessiné, tantôt dans toute son étendue, tantôt seulement dans sa moitié antérieure, et on aperçoit en outre, au-dessus du corps calleux, une dépression, moins profonde et moins longue, réduite quelquefois à un léger sillon, mais qui représente manifestement l'arc supérieur de la scissure limbique. Le grand lobe limbique est donc déjà distinct, quoique le reste du manteau soit encore indivis. Mais cette division primaire devient plus apparente chez les mammifères gyrencéphales, par suite de la formation des plis circonvolutionnaires qui se développent dans tout le manteau, à l'exception du grand lobe limbique. La formation de ces plis résulte de l'extension de l'écorce cérébrale, qui s'accroît à la fois en longueur et en largeur, et qui, venant de toutes parts se fixer ou s'appuyer sur le pourtour du grand lobe limbique, ne peut ni s'élargir ni s'allonger sans se plisser. Le plissement dans le sens transversal produit les circonvolutions longitudinales ; celles-ci, étant devenues plus longues que le grand lobe limbique sur lequel leurs deux extrémités vont aboutir, décrivent des arcs dont ce lobe représcnte la corde. La plus élevée, celle qui longe la fente interhémisphérique sur le bord sagittal de l'hémisphère, et qu'on peut appeler la circonvolution sagittale, forme un grand arc peu flexueux; mais la plus inférieure et la plus externe, celle qui 
longe l'are inférieur de la scissure limbique, forme un are plus court, qui se replie en s'adossant à lui-même, et ce plissement donne naissance à la siissure de Sylvius. Lorsqu'il y a plus de deux circonvolutions longitudinales, les circonvolutions intermédiaires décrivent des arcs décroissants, entre la circonvolution supérieure ou sagittale et la circonvolution inférieure on sylvienne.

La scissure de Sylvius, due à un plissement qui s'effectue le long du bord externe du grand lobe limbique, se trouve toujours, ou du moins presque toujours, en continuité avec la scissure limbique, dont elle n'est en réalité qu'une branche. Partant de cette dernière scissure, vers le niveau qui correspond au bord antérieur du lobe de l'hippocampe, elle se termine d'autre part, en haut et en arrière, dans l'épaisseur de la circonvolution sylvienne. Inférieurement, elle ne dépasse pas la scissure limbique, et reste séparée de la ligne médiane de la base du cerveau par toute la largeur du grand lobe limbique, largeur toujours très considérable à ce niveau.

Tous les arcs circonvolutionnaires dont je viens de parler commencent à l'extrémité postérieure de l'hémisphère sur le pôle occipital, et cheminent de là d'arrière en avant, en parcourant presque toute la longueur de l'hémisphère; mais à leur partie la plus antérieure, au moment de se recourber pour rejoindre l'extrémité antérieure du grand lobe limbique, ces circonvolutions longitudinales subissent un second plissement; celui-ci n'est pas limité, comme le premier, à la circonvolution sylvienne : il traverse toute la largeur (minime, il est vrai, à ce niveau) de la face convexe de l'hémisphère, jusqu'à la circonvolution sagittale, dans laquelle il se termine. Il en résulte une scissure très oblique, qui est l'analogue de la scissure de Rolando des primates. Cette scissure de Rolando commence toujours bien en avant de la scissure de Sylvius; elle paraît communiquer avec la scissure limbique, mais ce n'est souvent qu'une apparence, la communication n'étant que superficielle et les deux scissures étant séparées dans leur profondeur par un pli flexueux de la circonvolution sylvienne. Née ainsi tout près de la scissure limbique, vers le point où le lobe olfactif commence à se réunir au reste de l'hémisphère, elle se dirige très obliquement en avant, 
puis se recourbe en haut et en dehors, ne laissant au-devant d'elle qu'une très petite partie de l'hémisphère.

Cette partie située au-devant de la scissure de Rolando représente le rudiment du lobe frontal des primates. Tout ce qui est en arrière représente l'ensemble des lobes appelés pariétal, occipital et temporal. Mais ces lobes ne forment qu'une seule masse, car il n'y a pas de scissure occipitale, de sorte que le lobe pariétal s'étend jusqu'à l'extrémité postérieure de l'hémisphère. On peut, toutefois, considérer comme le rudiment de notre lobe temporal la portion de la circonvolution sylvienne qui est située au-dessous et en arrière de la scissure de Sylvius. A l'exception de ce lobe temporal rudimentaire et du petit lobe frontal, plus rudimentaire encore, toute la face convexe de l'hémisphère est occupée par le lobe pariétal.

La circonvolution sagittale, qui forme le bord convexe de l'hémisphère, forme, en outre, une partie de la face interne. Elle longe, d'arrière en avant, l'arc supérieur du grand lobe limbique, ou circonvolution du corps calleux, dont elle est séparée par l’arc supérieur de la scissure limbique. Cette portion de la scissure limbique est donc située sous le lobe pariétal, et peut être appelée la scissure sous-pariétale. La scissure souspariétale commence tout en arrière du grand lobe limbique, et se porte de là en haut, puis en avant, parallèlement au bord convexe du corps calleux. En avant, elle se termine de deux manières différentes; quelquefois, elle reste parallèle au corps calleux, se recourbe au-devant du genou de ce corps et redescend vers la base du cerveau; c'est ce qui a lieu chez le cheval, l'éléphant, et chez les lémuriens ; mais, le plus souvent, au lieu de se diriger vers le bas, elle se dirige au contraire vers le haut, va gagner obliquement le bord sagittal de l'hémisphère et se prolonge même sur la face convexe, où elle prend une direction transversale, et où elle se termine en entaillant plus ou moins profondément la circonvolution sagittale. Cette extrémité antérieure de la scissure sous-pariétale, devenue apparente sur la face convexe de l'hémisphère, a été décrite par Leuret sous le nom de sillon crucial. Dans la partie située en avant de ce sillon crucial, legrand lobe limbique est en continuité avec la petite portion de l'hémisphère qui représente le lobe frontal des primates. 
La circonvolution du corps calleux forme à elle seule tout l'arc supérieur du grand lobe limbique. L'arc inférieur est formé de deux parties : l'une antérieure, qui est le lobe olfactif ; l'autre postérieure, qui est le lobe de l'hippocampe. Entre la base du lobe olfactif et la partie antérieure du lobe de l'hippocampe, existe une dépression transversale ordinairement très peu profonde, qui est l'analogue de la vallée de Sylvius des primates, mais qui n'interrompt nullement la continuité des deux lobes. Dans la base du lobe olfactif, on distingue trois parties, qu'on peut appeler racines; l'une externe, blanchâtre, formant une large et épaisse bandelette qui longe la scissure limbique et se continue avec la partie externe du lobe de l'hippocampe; l'autre interne, d'un gris pâle, qui passe sur la face interne de l'hémisphère et se continue avec l'extrémité antérieure ou origine de la circonvolution du corps calleux; la troisième, intermédiaire, d'un gris plus foncé, qui se porte directement en arrière et se jette sur la partie interne du lobe de l'hippocampe. Cette troisième racine n'est grise que dans sa couche superficielle; sa couche profonde, beaucoup plus épaisse, est formée de fibres blanches qui passent au-dessous de la bandelette optique, et se continuent directement avec les fibres de l'étage inférieur du pédoncule cérébral. Ces trois racines, tout à fait continues les unes avec les autres par leurs bords, à te point qu'il est quelquefois assez difficile de les distinguer, sont larges et plates; mais ont encore néanmoins une assez grande épaisseur.

La disposition que l'on vient de décrire existe chez tous les mammifères terrestres autres que les primates. Mais chez ceux qui vivent dans l'eau ou qui y cherchent leur proie, le grand lobe limbique subit de notables modifications. Leur appareil olfactif, dont l'utilité se trouve diminuée ou supprimée, subit une atrophie plus ou moins complète.

Un premier degré d'atrophie s'observe chez la loutre. Le lohe olfactif est encore assez grand pour rentrer dans le type ordinaire, mais il est déjà assez réduit pour se prêter à l'étude des caractères de transition. La distinction des trois racines à la base de ce lobe est facile, et la dépression transversale, que l'on observe principalement sur la racine grise, indique que celle-ci 
n'a plus qu'une médiocre épaisseur. Chez les amphibies, le lobe olfactif disparaît presque entièrement. Il paraît ne plus faire partie du cerveau, et se réduit à une bandelette qui a l'apparence d'un nerf, comme chez l'homme. La dépression qui est située en avant du lobe de l'hippocampe devient profonde, et constitue une véritable vallée de Sylvius. Cette vallée, dont le fond n'est plus traversé par la racine grise, aboutit en dehors à l'origine de la scissure de Sylvius, laquelle paraît ainsi se continuer transversalement sous la base du cerveau, jusqu'au bord externe du chiasma des nerfs optiques.

Chez beaucoup de cétacés, enfin, le lobe olfactif est entièrement anéanti. C'est ce qui a lieu, par exemple, chez les dauphins. La scissure de Sylvius continue directement avec la vallée de Sylvius, qui établit une séparation large et profonde entre la face inférieure du lobe frontal et l'extrémité antérieure du lobe de l'hippocampe; et le fond de cette vallée n'est plus constitué que par une mince lame grise criblée de trous, qui représente exactement l'espace perforé de Vicq-d'Azyr du cerveau humain.

La réduction de l'appareil olfactif chez les mammifères plus ou moins aquatiques, est la conséquence de leur genre de vie. Elle s'observe aussi chez les primates, mais elle est due, chez eux, à une tout autre cause; elle coïncide avec un accroissement excessif du lobe frontal. L'appareil olfactif de ces animaux fonctionne dans les mêmes conditions que chez les autres mammifères terrestres; mais il ne joue plus qu'un rôle tout à fait secondaire. Le guide de l'animal, maintenant, ce n'est plus son odorat, c'est son intelligence, éclairée à la fois par tous les sens, et il n'y a plus de raison pour que l'appareil de l'olfaction prédomine sur les autres appareils sensoriels. Cet appareil subit donc une réduction considérable, et, par suite, la constitution du grand lobe limbique se trouve profondément modifiée.

Le renflement terminal du lobe olfactif n'est plus représenté que par un petit corps appelé le ganglion olfactif; la partie moyenne du lobe se réduit à une petite bandelette olfactive, longue, étroite et mince, qu'on désigne à tort sous le nom de nerf olfactif; sa base enfin ne présente plus qu'à l'ćtat de vestige les trois larges et volumineuses racines qu'on observe chez 
les mammifères ordinaires, et une vallée de Sylvius large et très profonde s'étend transversalement du chiasma des nerfs optiques à l'origine de la scissure de Sylvius proprement dite, de manière à produire entre le bord postérieur du lobule orbitaire du lobe frontal et le bord antérieur du lobe temporal un vaste intervalle dont le fond est formé par l'espace perforé de Vicq-d'Azyr. Des trois racines de ce lobe olfactif atrophié, l'une interne, blanche, se recourbe en haut en se portant vers l'origine de la circonvolution du corps calleux; l'externe, blanche également, se porte en dehors sur le bord antérieur de la vallée de Sylvius, puis se recourbe en arrière, franchit le fond de cette vallée et va se jeter sur le lobule de l'hippocampe, c'est-à-dire sur l'extrémité antérieure de la dernière circonvolution temporale; la racine moyenne enfin, ou racine grise, très mince, très fragile, souvent difficile à voir, se fixe sur l'extrémité postérieure des deux premières circonvolutions orbitaires, là où vient aboutir la mince lame grise de l'espace perforé, lame sur laquelle elle ne se prolonge pas. Quant aux fibres blanches qui constituent la couche profonde de la racine grise des autres mammifères, et qui se continuent chez eux avec le pédoncule cérébral, elles ont entièrement disparu.

Le cercle du grand lobe limbique paraît donc complétement interrompu par la vallée de Sylvius; l'origine de la circonvolution du corps calleux n'est plus reliée directement au lobe de l'hippocampe par la base du lobe olfactif. Il reste cependant un vestige de cette connexion, puisque les deux racines blanches qui se détachent de la base de la bandelette olfactive s'étendent respectivement, l'une vers l'origine de la circonvolution du corps calleux, l'autre vers l'extrémité antérieure du lobe de l'hippocampe.

Ce dernier lobe ne s'atrophie pas au même degré que le lobe olfactif; mais il subit néanmoins une atrophie très notable; il ne forme plus qu'une simple circonvolution qui se fusionne avec le lobe temporal et qui devient ainsi la dernière circonvolution temporale (la cinquième chez l'homme et les anthropoïdes, la quatrième ou la troisième chez les singes ordinaires).

Cetic circonvolution est dite encore circonvolution de l'hippúcampe ou circonvolution à crochet; son bord externe est tou- 
jours plus ou moins fusionné avec le bord adjacent de l'avantdernière circonvolution temporale. Toujours cependant, à sa partie postérieure, elle en est plus ou moins séparée par un sillon longitudinal, qui se prolonge sur la face inférieure du lobe occipital, et qui est un vestige de la scissure limbique; en outre, chez tous les singes et chez beaucoup d'hommes (surtout dans les races inférieures), la partie antérieure de cette scissure limbique persiste sous la forme d'une incisure qui commence profondément sur la face antéro-supérieure de la pointe du lobe temporal, dans la vallée de Sylvius, et qui, parvenue sur cette pointe, se porte en arrière, dans une étendue variable, sur la face inférieure du lobe temporal. Chez les hommes de race blanche, cette portion de la scissure limbique est le plus souvent fusionnée, mais elle est toujours indiquée par une dépression longitudinale, en dedans de laquelle l'extrémité antérieure de la circonvolution de l'hippocampe dessine le contour d'un petit lobule arrondi.

Ainsi, les deux lobes qui forment l'arc inférieur du grand lobe limbique sont atrophiés chez les.primates. L'arc supérieur, formé par la circonvolution du corps calleux, participe également à ce travail d'atrophie, mais seulement dans sa partie antérieure, qui est la plus voisine du lobe olfactif atrophié. Il en résulte que cette circonvolution est moins large en arant qu'en arrière, tandis que chez les autres mammifères elle est, au contraire, plus large dans sa partie antérieure que dans sa partie postérieure. C'est la conséquence du grand développement du lobe frontal des primates. Ce lobe n'est plus rudimentaire; il s'est considérablement accru en largeur, en épaisseur et en longueur; devenu plus large, il donne à l'extrémité antérieure de l'hémisphère une forme plus arrondie; devenu plus épais, il déprime la partie antérieure de la circonvolution du corps calleux; devenu plus long enfin, il se développe aux dépens du lobe pariétal et le refoule d'avant en arrière, de telle sorte que la scissure de Rolando, jusqu'alors très oblique en avant et presque cachée sous la pointe de l'hémisphère, recule presque jusqu'au milieu de la longueur de l'hémisphère, se redresse et devient même un peu oblique de bas en haut et d'avant en arrière. Le lobe pariétal, ainsi refoulé ver's l'occiput, subit dans sa 
partie postérieure un plissement transversal qui produit la scissure occipitale, et la partie de ce lobe qui est située en arrière du plissement transversal constitue un lobe distinct appelé le lobe occipital. En même temps, les connexions de la circonvolution du corps calleux subissent une modification très remarquable. Chez les mammifères, autres que les primates, la partie postérieure de cette circonvolution ne communique avec le lobe pariétal que par une anastomose souvent très étroite, et quelquefois même profonde, située derrière le pédoncule cérébral, tandis que sa partie antérieure se continue largement et se fusionne même souvent avec le lobe frontal rudimentaire; chez les primates, au contraire, la continuité antérieure avec le lobe frontal, qui est devenu très grand, ne s'effectue que par des plis d'anastomose, souvent même par un seul, situé au-devant du genou du corps calleux, tandis que la communication postérieure, a vec le lobe pariétal, constitue une véritable fusion, audessus et en arrière du bourrelet du corps calleux. De là résulte un changement complet dans la situation et la direction de l'arc supérieur de la scissure limbique, qui longe le bord convexe de la circonvolution du corps calleux. Cette portion de la scissure limbique ne chemine plus d'arrière en avant, sur le lobe pariétal; elle n'est plus sous-pariétale; elle chemine, au contraire, d'avant en arrière, sous le lobe frontal, et elle est devenue sousfrontale.

Née au-devant de l'origine de la circonvolution du corps calleux, elle décrit, sur la partie antérieure de la face interne de l'hémisphère, une grande courbe qui se relève peu à peu et va rejoindre le bord supérieur de l'hémisphère, en arrière de l'origine de la scissure de Rolando, entre le lobule ovalaire (ou paracentral), qui appartient exclusivement au lobe frontal, et le lobule quadrilatère qui appartient au lobe pariétal.

Ce caractère résume, en quelque sorte, ceux qui viennent d'être indiqués. La scissure sous-frontale existe chez tous les primates, et chez eux seuls; la scissure sous-pariétale existe chez tous les autres mammifères. L'une et l'autre limitent le grand lobe limbique et font partie de la scissure limbique; la différence qui existe entre elles n'est donc pas essentielle; néanmoins, elle a une telle importance, qu'elle mérite d'être 
signalée par des dénominations distinctes. Les noms de scissure sous-frontale et de scissure sous-pariétale indiquent très bien cette différence et permettent d'exprimer en un seul mot la principale caractéristique du cerveau des primates. 


\title{
ANATOMIE COMPARÉE DU CERVEAU
}

\author{
LE GRAND LOBE LIMBIQUE
}

ET LA SGISSURE LIMBIQUE DANS LA SÉRIE DES MAMMIFÈRES

(Revue d'anthropologie, 1878, 20 série, t. I, p. 385-498.)

\section{$\S 1$ - la circonvolution limbigue du cerveau humain.}

L'hémisphère cérébral se compose d'une masse centrale très hétérogène où se trouvent groupées des parties extrêmement diverses, et d'une couche extérieure que j'appellerai avec Burdach le manteau de l'hémisphère. C'est par le plissement du manteau que se forment les circonvolutions. Chez les animaux lissencéphales (ou sans circonvolutions), il est lisse dans la plus grande partie de son étendue; chez les gyrencéphales, il est constitué par l'ensemble des circonvolutions ( 1 ).

Le manteau enveloppe de toutes parts la masse centrale ou corps de l'hémisphère, à l'exception d'une région très circonscrite que l'on aperçoit sur la face interne lorsqu'on a isolé l'hémisphère à l'aide d'une coupe médiane, suivie de la section du pédoncule cérébral. Cette région, de forme à peu près ova-

(1) Les mots lissencéphales et gyrencéphales sont dus à M. Owen, qui s'en est servi pour désigner deux sous-classes de la classe des mammifères. Cette classification n'est pas acceptable, parce qu'on peut trouver quelquefois dans un même ordre zoologique, des animaux à circonvolutions et des animaux sans circonvolutions (édentés, rongeurs, primates). Mais les mots lissencéphale et gyrencéphale, pris dans une acception purement descriptive et conforme à l'étymologie, sont très commodes pour distinguer les animaux qui ont des circonvolutions de ceux qui n'en ont pas. 
laire, limitée en avant et en haut par la rainure du corps cal. leux, enarrière et en bas par la grande fente de Bichat, a été appelée par Foville et par Gratiolet la grande ouverture de l'hémisphère, parce qu'on y voit au-dessus, en arrière et au-dessous de la couche optique et du pédoncule, une grande ouverture qui conduit dans la cavité du ventricule latéral; mais on y voit aussi une autre ouverture distincte, le trou de Monro, et ces deux ouvertures ne forment qu'une minime partie de la région dont il s'agit. Celle-ci comprend en effet la coupe du corps calleux, de la cloison transparente, de la partie antérieure de la voûte, des commissures, du pédoncule cérébral et du plancher du troisième ventricule, puis la paroi latérale de ce ventricule, le bord du corps godronné sur la marge inférieure de la fente de Bichat, enfin cette fente elle-même, qui conduit dans le ventricule latéral. C'est par là que passent toutes les fibres convergentes ou divergentes qui pénètrent dans l'hémisphère ou qui en sortent. La partie de la face interne qui n'est pas recouverte par le manteau n'est pas une ouverture, mais elle est en quelque sorte la porte d'entrée et de sortie de l'hémisphère, et je l'appelle dès lors le seuil de l'hémisphère, nom qui, dans la nomenclature latine, très usitée à l'étranger, peut être rendu par le mot limen.

Le seuil de l'hémisphère est circonscrit de toutes parts par le manteau, qui forme autour de lui un bord comparable à la bordure circulaire de l'entrée d'une bourse. Je donne donc à ce bord le nom de limbe de l'hémisphère (limbus, bordure), et à la circonvolution qui le forme le nom de circonvolution limbique.

Cette grande circonvolution se compose de deux arcs, l'un supérieur, qui longe dans toute son étendue la surface convexe du corps calleux, l'autre inférieur, qui longe la fente de Bichat jusqu'à la pointe du lobe temporal. L'arc supérieur porte le nom de circonvolution du corps calleux, l'arc inférieur s'appelle la circonvolution de l'hippocampe, ou circonvolution à crochet, et constitue la dernière circonvolution du lobe temporal. La distinction que l'on établit ordinairement entre ces deux arcs est motivée, jusqu'à un certain point, par leur situation dans deux régions différentes, et aussi par la ligne de démarcation qu'éta- 
blit entre eux le prolongement de la scissure calcarine (ou du petit hippocampe) (1). Mais cette ligne de démarcation n'est que superficielle; elle n'entame qu'une petite partie de l'épaisseur de la circonvolution limbique, le plus souvent même elle n'en occupe pas toute la largeur et n'en atteint pas le bord concave; en tout cas, la continuité de la circonvolution du corps calleux et de la circonvolution de l'hippocampe n'est jamais interrompue.

C'est ce que Gerdy et Foville ont parfaitement reconnu. En 1838, dans ses Recherches sur l'encéphale, trop dédaignées aujourd'hui, Gerdy décrivait en ces termes la circonvolution limbique : "Le côté interne des lobes latéraux (hémisphères) présente une circonvolution annulaire constante, mais d'une régularité variable; elle commence au-devant du corps calleux ou plafond du cerveau, en se continuant avec la circonvolution olfactive interne. De là elle se porte en arrière, le long de la face supérieure du plafond jusqu'à son bord postérieur qu'elle embrasse en se recourbant en bas pour aller se terminer vers la tubérosité interne obtuse du lobe temporal, à la partie inférieure du cerveau... Cette circonvolution forme par conséquent un long anneau ovalaire qui, se portant depuis la tubérosité du lobe temporal derrière, au-dessus et au-devant du corps calleux,

(1) Ce prolongement qui, chez l'homme et le gibbon, est commun à la scissure calcarine et à la scissure occipitale interne, appartient en réalité à la scissure calcarine, car chez tous les singes autres que les gibbons, il est séparé de l'extrémité inférieure de la scissure occipitale par un pli de passage superficiel étendu du lobule du cunéus à la circonvolution du corps calleux (pli cunéolimbique); le pli cunéo-limbique existe aussi chez l'homme et les gibbons, mais il est profond; il n'apparaît que lorsqu'on écarte les bords de la scissure occipitale interne, et celle-ci se trouve ainsi mise en communication avec la scissure calcarine. L'ensemble des scissures présente alors la forme d'un Y dont la queue s'étend jusque sur le bord concave de la circonvolution limbique. Gratiolet a très bien vu que cette queue de l'Y doit être rapportée à la scissure cal carine. Mais il a cru à tort qu'elle séparait entièrement la circonvolution de l'hippocampe de celle du corps calleux, qu'elle pénétrait dans la fente de Bichat et qu'elle se prolongeait sur le bord inférieur de cette fente, c'est-à-dire sur la face interne du lobe temporal, entre le grand hippocampe et le corps godronné. Il a ainsi réuni à tort en une seule scissure, appelée par lui scissure des hippocampes, deux anfractuosités entièrement différentes, et j'ajoute parfaitement distinctes: la scissure calcarine ou du petit hippocampe, qui se voit à l'extérieur, et la rainure du grand hippocampe, qui est cachée dans la fente de Bichat. 
l'embrasse dans son cercle et se continue en avant sous le lobe temporal, avec la circonvolution olfactive interne... Cet anneau n'est interrompu que par la partie interne de la scissure temporale (1). " La partie interne de la scissure temporale, ou de la scissure de Sylvius, est ce que nous appelons aujourd'hui la vallée de Sylvius. La tubérosité interne du lobe temporal n'est autre que le lobule de l'hippocampe. Remarquons en passant que Gerdy a fait un timide effort pour débarrasser la science de ce nom absurde et gênant de corps calleux, que le nom de plafond remplacerait avantageusement, mais qui est tellement enraciné dans le langage que je n'ose pas proposer de l'abandonner.

Un an après Gerdy, en 1839, dans un mémoire présenté à l'Académie de médecine et à l'Académie des sciences, Foville décrivit à son tour, comme unique circonvolution " de premier ordre ", la circonvolution annulaire, qu'il appela la circonvolution de l'ourlet. L'ourlet, suivant lui, était une bandelette de fibres blanches qui longeait toute la base de la circonvolution, et qu'il comparait à la lisière ou à l'ourlet de l'entrée d'une bourse. On trouve dans le premier volume (seul publié) de son ouvrage sur le système nerveux, la description suivante de la circonvolution de l'ourlet :

" La circonvolution unique du premier ordre, que nous appelons circonvolution de l'ourlet, a pour caractère principal de former un cercle complet sur la limite marginale, la lisière de la couche corticale de l'hémisphère. Arrondie et serrée autour du corps calleux, du tronçon pédonculaire et du quadrilatère perforé, elle enferme étroitement toutes ces parties dans sa circonférence. Considérée dans son ensemble, cette circonvolution peut être comparée à la coulisse d'un serre-tête, et à la petite échancrure de ce serre-tête par laquelle sortent les deux extrémités du ruban qui parcourt la coulisse. Toute la partie de cette circonvolution qui représente la coulisse dı serre-tête est située dans un plan vertical et se trouve serrée autour du corps calleux et du tronçon

(1) Gerdy, Recherches sur l'encéphale. Dans Joumal des connaissances riédico-chirurgicales, décembre 1848. Réimprimé dans ses Mélanges d'anatomie, de physiologie et de chirurgie, éd. Broca et Beaugrand. Paris, 1875, in-8, t. Ior, p. 337. 
pédonculaire; les endroits où sortiraient les deux cordons se trouvent l'un à la partie interne du bord antérieur du quadrilatère perforé, l'autre à la partie externe de son bord postérietrir. On pourrait même, en poussant plus loin la comparaison vulgaire, mais exacte que j'invoque, représenter l'un des deux cordons du serre-tête par le nerf optique, l'autre par le nerf olfactif(1).» Le nerf optique, ou plutôt la bandelette du nerf optique, car c'est d'elle seulement qu'il peut être question, n'a que des rapports de contiguité avec le système décrit par Foville; c'est donc à tort que l'auteur l'y a rattachée; mais il a très bien vu que ce que l'on désigne chez l'homme sous le nom défectueux de nerf olfactif, fait partie de ce système, que la racine olfactive externe, contournant le bord externe de l'espace perforé de Vicq d'Azyr pour aller se jeter dans la circonvolution de l'hippocampe, complète le cercle de la circonvolution de l'ourlet, que ce cercle, par conséquent, n'est pas interrompu par la vallée de Sylvius, comme le croyait Gerdy. Foville a très bien vu encore que l'espace perforé lui-même fait partie du système de la circonvolution de l'ourlet. Ce fait important est facile à constater lorsqu'on fait intervenir l'anatomie comparée, mais Foville n'étudiait que l'homme, et il lui a fallu beaucoup de sagacité pour faire cette découverte sur le cerveau humain. Chez les primates, en effet, par suite de l'atrophie du lobe olfactif, il se produit en avant du lobule de l'hippocampe une dépression transversale profonde qu'on appelle la vallée de Sylvius, et qui semble interrompre la continuité de la circonvolution " de l'ourlet ». Il en résulte que les successeurs de Foville n'ont pu retrouver les connexions qu'il avait décrites, et ont méconnu la signification et la nature de l'espace perforé. Sur ces entrefaites, Gratiolet étudiant le type cérébral des primates sur la guenon callitriche (cercopithecus sabæus) (2), crut remarquer que sur le cerveau de cet animal la scissure du petit hippocampe, ou scissure calcarine, s'étendait

(1) Foville, Anatomie, physiologie et pathologie du système nerveux. Paris, 1844 , in-8, t. Ior, p. 193.

(2) « M. de Blainville, mon illustre maître, ayant choisi le callitriche comme type ostéologique des primates, j'ai cru devoir suivre son exemple, et le cerveau du callitriche me servira de point de départ. ") (Gratiolet, Mémoire sur les plis cérébraux de l'homme et des primates, Paris, 1854, in-4, p. 22.) 
jusque dans la fente de Bichat, de manière à établir une séparation complète entre la circonvolutiou du corps calleux et la circonvolution de l'hippocampe ; c'était une fausse apparence, ainsi que je l'ai déjà dit (voir page 261, en note), mais la grande autorité de Gratiolet fit admettre que la circonvolution del'ourlet était interrompue en arrière par la scissure calcarine dite alors des hippocampes. On décrivit donc comme entièrement distinctes la circonvolution du corps calleux et celle de l'hippocampe, et telle est la force de l'habitude, que l'on continue encore aujourd'hui à considérer ces deux circonvolutions comme tout à fait indépendantes l'une de l'autre, quoique tout le monde pourtant s'accorde à reconnaître que la scissure calcarine ne pénètre pas dans la fente de Bichat.

Je me propose d'étudier, avec le concours de l'anatomie comparée, le grand appareil cérébral qui entoure le limbe de l'hémisphère, et dont l'importance, quoique considérablement atténuée chez l'homme, avait pourtant été reconnue depuis longtemps par Gerdy et par Foville. J'ai dû avant tout citer les travaux de ces deux anatomistes, et particulièrement du second, mais il m'a paru nécessaire de substituer aux dénominations dont ils se sont servis une dénomination mieux appropriée au sujet. Chez la plupart des mammifères, l'appareil dont il s'agit, complété en avant par un grand prolongement appelé le lobe olfactif, revêt une forme dont le mot annulaire (Gerdy) donnerait une idée inexacte, et chez les primates mêmes, ce nom manque d'exactitude, puisque le circuit circonvolutionnaire est interrompu par la vallée de Sylvius. Quant au nom de circonvolution de l'ourlet, il est tout à fait défectueux, car l'ourlet; auquel ce nom est emprunté, n'est pas une partie distincte : c'est le produit artificiel d'une dissection dirigée par une idée préconçue. Sur le bord antérointerne de l'espace perforé, la racine blanche interne du nerf olfactif se porte en dedans, puis en haut, où l'on peut la suivre jusque sur l'origine de la circonvolution du corps calleux, tandis que la racine blanche externe du même nerf, longeant le bord antérieur, puis le bord externe de l'espace perforé, franchit la vallée de Sylvius et se jette sur la circonvolution de l'hippocampe.

Les fibres blanches de ces deux racines pénètrent-elles dans l'épaisseur des circonvolutions auxquelles elles aboutissent, de 
manière à se continuer profondément avec la substance blanche de ces dernières ? La plupart des anatomistes en doutent; le fait est qu'elles semblent se perdre dans la couche la plus superficielle de la substance grise. La pulpe molle du cerveau se laisse si aisément diviser dans toutes les directions, que Foville, avec beaucoup d'habileté et un peu de complaisance, a pu produire sur le prolongement de ces racines des tractus qu'il a poursuivis à travers la couche corticale jusque dans la substance blanche; mais les anatomistes qui ont voulu répéter cette préparation n'y ont pas réussi, de sorte que la portion de l'ourlet qui correspond à l'espace.perforé est généralement considérée comme entièrement distincte de celle qui, dàtè Foville, longe la base de la circonvolution de l'ourlet. Cette seconde partie à son tour n'a pu être retrouvée avec les caractères que Foville lui a assignés. La continuité de la circonvolution de l'hippocampe et de celle du corps calleux implique nécessairement la continuité de leur substance blanche, comme cela a lieu partout où deux circonvolutions communiquent l'une avec l'autre. On peut donc très aisément, sur de simples coupes, sans aucune dissection, constater que la substance blanche se continue sans interruption depuis l'origine de la circonvolution du corps calleux jusqu'à la terminaison de la circonvolution de l'hippocampe, en formant sous le limbe de l'hémisphère un anueau presque complet, interrompu seulement au niveau de la vallée de Sylvius ; mais il est impossible de suivre dans cette masse blanche les fibres qui, d'après Foville, la parcouraient dans toute sa longueur, et qui constitueraient la bandelette annulaire de l'ourlet. La dénomination de la circonvolution qui forme le limbe de l'hémisphère ne peut donc pas être tirée du nom d'une bandelette purement théorique.

Le nom de crrconvontion limbrque que j'ai adopté indique les rapports constants de cette circonvolution avec le limbe de l'hémisphère; il n'implique aucune théorie; n'exprimant pas une forme déterminée, il est applicable à tous les cerveaux des mammifères, à ceux qui ont un vrai corps calleux comme à ceux dont le corps calleux est nul ou rudimentaire (lyencéphales d'Owen), à ceux qui ont un vrai lobe olfactif, comme à ceux dont le lobe olfactif n'est qu'à l'état de vestige. Enfin, il a l'avantage 
de permettre de désigner sans changement d'adjectif les parties qui se rattachent à la description de cette circonvolution : le grand lobe limbique, la scissure limbique, l'arc limbique supérieur ou inférieur, etc.

Je me suis exprimé jusqu'ici comme si la zone qui entoure là le limbe de l'hémisphère était une vraie circonvolution. J'ai dû le faire pour me conformer à la description donnée par Gerdy et par Foville. Mais après avoir reproduit leurs textes, et admis avec eux que la circonvolution du corps calleux et la circonvolution de l'hippocampe sont en continuité l'une avec l'autre, et ne font qu'un seul et même système, je dois ajouter que, dans la nomenclature cérébrale actuelle, te nom ¿de circonvolution n’est plus applicable à ce système. Le mot circonvolution, qu'on pouvait employer autrefois pour désigner une partie quelconque de la surface plissée de l'hémisphère, a aujourd'hui une acception déterminée; il s'applique aux subdivisions des lobes, et si dans certains cas un lobe peut (comme on le verra plus loin) n'être formé que d'une seule circonvolution, jamais du moins une circonvolution ne peut s'étendre au-delà des limites de son lobe, encore bien qu'elle puisse se continuer plus ou moins directement avec l'une des circonvolutions d'un lobe voisin. Or, chez l'homme, et j'ajoute chez tous les primates, la circonvolution «annulaire » ou " de l'ourlet », celle que j'appelle limbique, appartient, par la circonvolution de l'hippocampe qui forme son arc inférieur, au lobe temporal dont son arc supérieur, appelé circonvolution du corps calleux, ne fait évidemment pas partie. Ces deux circonvolutions, quoique continues l'une avec l'autré, ne peuvent donc pas être réunies en une seule. Je ferai remarquer en outre que la circonvolution du corps calleux forme dans l'hémisphère une région toute spéciale qui ne peut se rattacher à aucun des lobes admis jusqu'ici, et qui doit être considérée comme constituant à elle seule un lobe spécial, le lobe du corps calleux. Cette appréciation paraît déjà nécessaire chez les primates, mais elle le devient bien plus encore lorsqu'on considère les autres mammifères.

Je ne parlerai donc plus de la circonvolution limbique. C'est sous d'autres noms que j'étudierai séparément et que je grouperai en un seul système les parties dont elle se compose. 
Ce système ne présente chez les primates qu'un faible développement, mais il forme dans le cerveau des autres mammifères terrestres toute une vaste région, plus distincte du reste de l'hómisphère qu'aucune des divisions appelées lobes; il constitue dans le manteau une division primaire, une division fondamentale qui est plus qu'un lobe, qui renferme d'ailleurs plusieurs lobes, et que le simple nom de lobe ne caractériserait pas suffisamment : je l'appellerai donc le grand lobe limbique. Son évolution dans la série des mammifères est étroitement liée à celle du lobe olfactif. Il ne sera donc pas inutile de présenter d'abord quelques remarques sur le lobe olfactif et le sens de l'odorat.

\section{§ 2. - Le Lobe olfactif ET LE SENS de L'odorat.}

Les philosophes et les physiologistes qui ont nié que la vue fût le premier des sens, n'ont jamais mis en doute la supériorité des notions fournies par la vue; mais ils ont dit avec raison que ces notions seraient trompeuses et ne nous donneraient que des idées très imparfaites et mème très fausses des objets qui se peignent sur notre rétine, si d'autres sens et particulièrement le toucher ne venaient à notre aide. C'est seulement lorsque nous connaissonsla forme et les principalès propriétés d'un corps que nous parvenons à le reconnaître, non avec l'œil, qui ne reçoit qu'une image, ni avec les tubercules quadrijumeaux qui ne reçoivent que des impressions, mais avec l'intelligence qui redresse cette image, l'interprète et transforme en notions le plus souvent positives ces impressions toutes pleines d'illusions.

Ceux qui ont cru, par ces remarques parfaitement vraies, rabaisser le sens de la vue, en ont, sans le vouloir, proclamé la supériorité, car ils ont prouvé par là même que la vue est le plus intellectuel des sens. La vie vaut pour un animal ce que vaut son intelligence. Reconnaître de loin la proie ou l'ennemi, mesurer la distance pour se préparer à l'attaque, à la résistance ou à la fuite, n'est-ce pas l'un des premiers besoins de la lutte pour l'existence d'une intelligence très développée. Elle fournit à tous 
les animaux des notions très nombreuses et très importantes, mais ceux-là seuls en tirent des connaissances précises et complètes qui possèdent la faculté d'observer et de réfléchir, qui savent analyser et comparer entre elles les diverses parties d'une image, se mettre en garde contre les erreurs de la perspective, apprécier la distance d'après le volume apparent d'un objet dont le volume réel est connu, acquérir ainsi une idée exacte de l'étendue, etc. Chez ces animaux, la vue est à la fois la principale sentinelle et le principal guide; elle est le sens le plus utile; le rôle des autres est amoindri; l'odorat en particulier perd une grande partie de son importance et l'on sait que chez les primates l'appareil olfactif est considérablement atrophié.

Cet appareil'est au contraire extrêmement développé chez la plupart des autres mammifères. L'odorat joue chez eux un rôle souvent égal et même supérieur à celui de la vue. C'est lui qui les guide dans le choix de la nourriture, dans la poursuite de la proie, dans la fuite du danger, dans la recherche de la femelle, dans le retour au gîte. L'exercice de ce sens est simple et n'exige qu'une faible opération intellectuelle. L'odorat perçoit une certaine odeur, qui est propre à un certain corps; pour reconnaître ce corps il suffit d'un peu d'expérience; pour en apprécier la distance il suffit d'apprécier l'intensité de la sensation. L'animal qui fait le mieux ce diagnostic n'est pas le plus intelligent, c'est celui qui possède l'appareil olfactif le plus développé; et les quadrupèdes les plus stupides se trouvent souvent par là bien supérieurs à l'homme.

Ce fait seul suffit pour montrer toute l'infériorité du sens de l'odorat. On ne peut pas dire qu'il soit en raison inverse de l'intelligence, mais on peut dire du moins qu'il prédomine chez la brute, et on peut le gratifier de sens brutal, car il tire son importance du degré de perfection de l'appareil organique qui lui est attribué, bien plus que des actes intellectuels qu'il met en jeu dans l'ensemble du cerveau. Cet appareil peut même fonctionner indépendamment du cerveau proprement dit, puisque, chez les vertébrés inférieurs, il n'a aucune connexion avec la partie de l'encéphale qui représente l'hémisphère cérébral des mammifères.

L'appareil central de l'olfaction comprend deux parties: $1^{\circ}$ le 
lobe olfactif; $2^{\circ}$ une certaine portion de l'encéphale sur laquelle s'établissent les connexions de ce lobe.

Le lobe olfactif à son tour se compose de deux parties : $1^{\circ}$ un renflement terminal, amas de substance grise d'où émanent les filets nerveux de la membrane olfactive des fosses nasales; $2^{\circ}$ un pédoncule de longueur et de largeur très variables, formé en totalité et en partie par les fibres blanches qui mettent le lobe en continuité avec le reste de l'encéphale.

Le lobe olfactif, isolé dans la plus grande partie de sa surface, fait ordinairement saillie en avant de la base du cerveau. Il est presque toujours situé dans le crâne; mais chez certains poissons (cyprins, silures, etc.), il est en dehors de la cavité cérébrale, et en avant d'elle; son pédoncule est alors très long, et beaucoup plus long même que l'encéphale proprement dit. Chez la plupart des poissons, ce pédoncule passe sous le lobe cérébral sans y adhérer, et va s'insérer sur la commissure transversale que forment, à leur extrémité antérieure, les cordons inférieurs (ou cordons moteurs) de la moelle. Le congre est le seul poisson sur lequel Desmoulins ait vu ce pédoncule envoyer en outre un petit. filet dans le lobe cérébral (1). Cette exception n'est probablement pas unique, mais c'est du moins une règle très générale que, chez les poissons, le lobe olfactif ne communique pas avec les autres lobes du cerveau, qu'il communique seulement avec les faisceaux moteurs de la moelle. 11 tire de lui-même toute sa fonction, et les notions qu'il donne à l'animal se transforment immédiatement en volitions, qui deviennent effectives sans la participation des autres organes cérébraux.

Une disposition analogue s'observe chez certains oiseaux (oiseaux de proie, canards, etc.); le pédoncule olfactif n'a aucune connexion avec le lobe cérébral; il va aboutir à la face inférieure de la couche optique, qui est étrangère au lobe cérébral (2); il est même douteux qu'il pénètre dans cette couche, car il paraît

(1) Desmoulins, Anatomie du système nerveux des animaux à vertèbres. Paris, 1825, 2 vol. in-8, 1. Ier, p. 166.

(2) On sait que la couche optique des oiseaux forme la base du lobe optique, tandis que la base du lobe cérébral est formée par le corps strié. Chez les mammifères, le lobe optique, considérablement atrophié (tubercules quadrijumeaux), se sépare de la couche optique. Celle-ci s'unit au corps strié et se rattache comme lui au lobe cérébral, qui prend le nom d'hémisphère cérébral. 
s'insérer exclusivement sur une couche spéciale formée par les faisceaux inférieurs de la moelle. Mais chez les autres oiseaux, tels que les gallinacés et les passereaux, le pédoncule olfactif s'insère exclusivement sur le lobe cérébral, et il en est de même chez les batraciens et les reptiles.

Dans ce second type, si différent du premier, le vrai centre oliactif n'est plus le lobe olfactif : c'est le lobe cérébral. C'est celui-ci qui est le siège des actes psychiques mis en jeu par le sens de l'odorat. Le lobe olfactif ne descend pas pour cela au rang des simples organes de transmission. La grande quantité de substance grise qu'il renferme prouve que c'est aussi un organe d'élaboration. Il fait plus que recevoir l'impression, il la perçoit, il en tire des notions, mais pour les transmettre au lobe cérébral, qui les apprécie et prend des déterminations en conséquence.

Ainsi, dans les classes inférieures à la classe des mammifères, le lobe olfactif se rattache tantôt à la parlie de l'encéphale qui représente le pédoncule cérébral des mammifères, tantôt à celle qui représente leur hémisphèré cérébral. Ge n'est que très exceptionnellement que le lobe olfactif prend ses insertions sur ces deux parties à la fois (congre). Mais cette exception devient la règle chez les mammifères : leur lobe olfactif se continue à la fois avec le pédoncule cérébral et avec le manteau de l'hémisphère. De ces deux connexions, la seconde seule est constante (à moins que l'appareil olfactif ne soit entièrement anéanti, comme cela a lieu chez heaucoup de cétacés); la première, au contraire, peut disparaître; c'est ce qu'on voit chez les primates et chez les amphibies. Cette disposition prouve que le lobe olfactif a perdu une partie importante de sa fonction, celle qui faisait de lui un organe recteur, et on remarque effectivement qu'il est alors réduit à un très petit volume. Mais ce n'est là qu'une exception, dont nous chercherons tout à l'heure la raison d'être. Le type des mammifères en général comporte l'existence d'un grand lobe olfactif, qui d'une part communique directement avec les fibres motrices du pédoncule cérébral, qui d'une autre part communique avec le manteau de l'hémisphère, et qui par conséquent peut fonctionner tour à tour ou à la fois comme centre autonome, et comme vassal de l'hémisphère. 
De ce fait que le rôle de vrai centre olfactif est rempli chez les vertébrés les plus inférieurs et même chez certains oiseaux par un organe de second ordre, étranger au lobe cérébral, on peut conclure avec beaucoup de probabilité que lorsqu'une partie de ce rôle est attribuée au lobe cérébral, c'est-à-dire à l'hémisphère, l'exercice de la fonction olfactive n'exige de la part de l'intelligence qu'une assez faible intervention et que la portion de l'hémisphère qui en est le siège doit occuper un rang peu élevé dans la hiérarchie cérébrale.

Gette portion de l'hémisphère constitue le grand lobe limbique des mammifères. Elle diffère du reste du manteau de l'hémisphère par une évolution toute spéciale. G'est elle qui, dans les cerveaux les plus inférieurs (lissencéphales), se distingue la première ; ses contours se dessinent déjà alors qu'aucune autre division n'apparaît encore à la surface du manteau. Puis, lorsque le cerveau se perfectionne et se complique chez les gyrencéphales, elle reste étrangère au plissement qui produit les circonvolutions, et demeure stationnaire pendant que tout progresse autour d'elle. Enfin, elle rétrograde et s'atrophie en grande partie lorsque le grand développement des circonvolutions antérieures donne chez les primates la prééminence au lobe frontal, siège des facultés intellectuelles les plus élevées.

Envisagés au point de vue de la constitution du grand lobe limbique, les mammifères se divisent en deux catégories très inégales, que nous serons continuellement obligés de comparer l'une avec l'autre. La première comprend ceux dont le lobe olfactif est très développé et dont le grand lobe limbique est par conséquent au complet. La très grande majorité des mammifères rentrent dans cette première catégorie. La seconde, caractérisée par l'état rudimentaire ou par l'absence tutale du lobe olfactif, ne comprend que les cétacés, les carnassiers amphibies et les primates. 0 n voit qu'elle est très hétérogène. C'est parce que l'atrophie de l'appareil olfactif peut se produire sous deux influences très différentes, dont l'une concerne le milieu, tandis que l'autre concerne l'animal lui-même.

Le mammifère qui vit constamment ou habituellement dans l'eau ne tire de l'odorat que peu ou point d'utilité. L'eau est sans doute le véhicule de particules odorantes dont les qualités 
peuvent être appréciées par un appareil approprié ; aussi voyonsnous que, dans la classe des poissons, le lobe olfactif est à peu près constant, et souvent très développé; mais l'appareil olfactif des mammifères n'est pas approprié à l'odoration dans l'eau. En adoptant l'existence aquatique, les cétacés et les carnassiers amphibies ont conservé la structure anatomique des mammifères, et, de même que leurs poumons, leurs fosses nasales ne reçoivent que de l'air. L'odorat ne les renseigne donc pas sur les conditions du milieu où ils cherchent leur proie; ce n'est plus qu'un sens accessoire ou inutile, et le lobe olfactif s'atrophie en conséquence. Ghez la plupart ảes cétacés, il ne persiste aucun vestige de ce lobe; chez les autres cétacés et chez les carnassiers amphibies il est tellement réduit qu'on a pu le prendre pour un nerf. L'atrophie de l'appareil olfactif dans le cas qui précède est la conséquence d'une désuétude qui est due elle-même à l'influence du milieu. Chez les primates, elle est due à une tout autre cause, que j’ai déjà mentionnée. L'olfaction de ces animaux est aérienne, comme celle de tous les vertébrés terrestres, mais elle ne joue dans leur existence qu'un rôle accessoire. A la prépondérance de ce sens brutal a succédé celle de l'intelligence, éclairée à la fois par tous les sens, et ce changement physiologique est attesté par la coïncidence de deux faits anatomiques : le grand développement du lobe frontal et l'atrophie de l'appareil olfactif.

Les conditions qui amoindrissent l'importance du lobe olfactif sont donc très diverses; et l'on comprend ainsi pourquoi des primates se trouvent rapprochés par un caractère commun, qui les distingue de tous les autres mammifères. Ce caractère, comme on vient de le voir, n'est nullement zoologique. Les deux catégories qu'il établit parmi les mammifères ne correspondent à aucune division naturelle; elles n'existent que par rapport à l'étude du grand lobe limbique; il est nécessaire néanmoins de leur donner des noms qui permettent de les distinguer dans les descriptions. J'appellerai donc mammifères osmatiques (de żop.ŕ, odorat) les mammifères ordinaires, chez lesquels la prédominance du sens de l'odorat est attestée par le grand développement de l'appareil olfactif; et ceux chez lesquels ce sens, pour un motif quelconque, a perdu sa suprématie, peuvent, par opposition, être appelés anosmatiques. Ces épithètes peuvent d'ailleurs s'ap- 
pliquer aux cerveaux aussi bien qu'aux animaux eux-mêmes.

Le grand lobe limbique existe, plus ou moins distinct, plus ou moins volumineux et plus ou moins complet, chez tous les mammifères. Avant d'en étudier les diverses modifications, il convient d'en donner d'ahord une idée générale, et, à cet effet, je prendrai pour type le cerveau de la loutre. C'est celui qui se prête le mieux aux comparaisons, parce que c'est, de tous les cerveaux osmatiques, celui qui diffère le moins des autres. On sait que la loutre n'appartient pas à la famille des amphibies; c'est un carnassier de la famille des martres. Elle cherche surtout sa nourriture dans l'eau, mais elle vit sur la terre, et si l'odorat lui est d'un faible secours dans la poursuite de sa proie, il l'aide beaucoup à éviter ses nombreux ennemis. "En sortant de l'eau, dit Buffon, les loutres se secouent et se couchent en rond sur la terre, comme les chiens; mais avant de s'endormir elles cherchent à reconnaître par l'odorat plutôt que par la vue, qu'elies ont faible et courte, s'il n'y a pas d'ennemis à craindre dans les en. virons. " L'appareil olfactif de la loutre n'est donc pas atrophié comme celui des amphibies; son grand lobe limbique est encore au complet, mais ne présente plus qu'un développement médiocre, et la description de ce type intermédiaire nous fournira, pour nos comparaisons ultérieures, un excellent point de départ.

§ 3. - LE GRANd LOBE LIMbique DES MaMmifÈres décrit D'APRÈS LE CERVEAU DE LA LOUTRE.

Le grand lobe limbique peut être comparé à une raquette. Il entoure complètement, comme un anneau allongé, toute la région à peu près ovalaire que j'ai appelée le seuil de l'hémisplıère ; en outre, de l'extrémité antérieure de l'ovale, se détache un prolongement qui est constitué par le lobe olfactif et qui représente la queue de la raquette.

Mais les diverses parties du grand lobe limbique ne sont pas situées dans un même plan; sa partie supérieure se déploie sur la face interne de l'hémisphère, sa partie inférieure et son prolongement sur la face inférieure; il est donc impossible de les em- 
brasser d'un seul coup d'œil : c'est ce qui rend la description de l'ensemble très difficile. Le lecteur pourrait suivre aisément cette description sur le moule en plâtre de l'hémisphère d'un carnassier, ou sur l'hérnisphère lui-même, préalablement durci dans l'alcool (car les cerveaux mous se déforment lorsqu'on les manie). Les figures ordinaires, ne représentant qu’une seule norma de l'hémisphère, seraient insuffisantes. J'y joindrai donc une figure intermédiaire entre la norma médiane et la norma inférieure, et je la rendrai même quelque peu schématique en supposant les deux faces plus étalées qu'elles ne le sont en réalité. Le cerveau

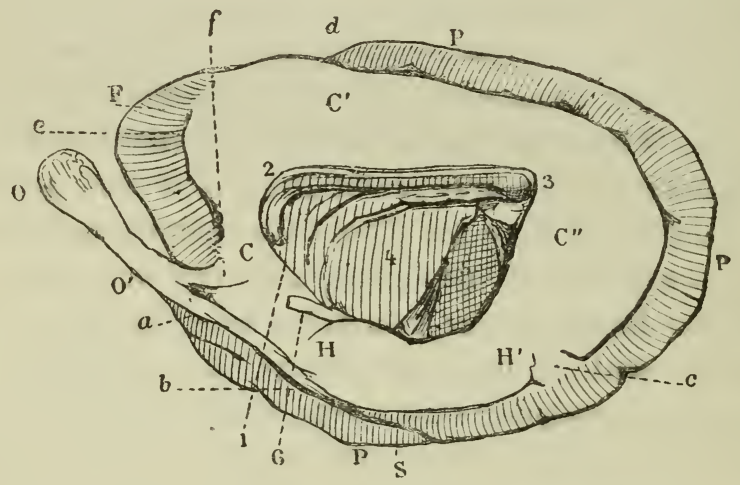

Fig. 6. Schćma de la face inféro-interne de l'hémisphère droit de la loutre. - 1, le bec du corps calleux; 2, son genou ; 3 , son bourrelet, au-dessous duquel on apercoit le pilicr pos. térieur de la voûte, et l'origine de la corne d'Ammon; 4, facc interne de la couche optique ; 5 , coupe du pédoncule cérébral, séparé du grand lobe limbique par la fente de Bichat; 6 , la bandelette optique, se dégageant, à la partie antérieure de la fente de Bichat, entre la couche optique et le lobe de l'hippocampe.

PPP, lobe pariétal; F, lobe frontal; S, scissure de Sylvius; O, lobe olfactif ; $\mathrm{O}^{\prime}$, pédoncule olfactif; C, origine du lobe du corps callcux : $\mathbf{C C}^{\prime} \mathbf{C}^{\prime \prime}$ le lobe du corps calleux; HH', le lobe de l'hippocampe.

$a b$, racine externe du lobe olfactif; $a b c$, l'are inférieur de la scissure limbique; . $c$, le pli de passage rétro-limbique; $c d$, scissure sous-pariétale; $e$, sillon sous-froutal; $f^{\prime}$, racine interne du lobe olfactif.

de la loutre se prête mieux que tout autre à cet artifice, parce que le grand lobe limbique, moins large que chez la plupart des autres mammifères, ne masque pas aussi complètement le reste du manteau.

La figure schématique ci-jointe (fig. 6) représente à la fois, dans sa partie supérieure, la face interne, el dans sa partie inférieure la face inférieure de l'hémisphère droit de la loutre. Elle montre tout le grand lobe limbique, qui a été laissé en blanc. On 
a teinté d'une ombre légère et uniforme tout le reste de la figure, savoir : au centre, le seuil de l'hémisphère, et à la périphérie les parties du manteau qui entourent le grand lobe limbique. Ce grand lobe, entourant tout le seuil de l'hémisphère, est partout continu avec lui-même, mais on y distingue trois portions qui constituent trois lobes. Ge sont : $1^{\circ}$ en haut, le lobe du corps calleux, qui forme l'arc supérieur $\mathrm{C}, \mathrm{C}^{\prime}, \mathrm{C}^{\prime \prime}$, du grand lobe $; 2^{\circ}$ en bas le lobe de l'hippocampe $\mathrm{H}, \mathrm{H}^{\prime}$, qui forme l'arc inférieur' $3^{\circ}$ en avant le lobe olfactif 0 , dont la base $0^{\prime}$ se continue avec la partie antérieure de l'hémisphère; le reste du grand lobe limbique est circonscrit par une scissure qui le sépare des circonvolutions cérébrales et que j'appelle la scissure limbique.

Les diverses parties de la scissure limbique seront étudiées sur les normas inférieure et latérale de l'hémisphère, mais on ne la voit dans son ensemble que sur notre figure schématique. Elle se compose de trois arcs. L'inférieur commence en $a$, sous le bord externe de la base du lobe olfactif, se porte en arrière et un peu en dehors le long de la racine externe de ce lobe, gagne en $b$ le bord externe (inférieur sur la figure) du lobe de l'hippocampe et s'étend en arrière jusqu'à une petite distance de l'extrémité postérieure de l'hémisphère $c$; là, s'arrête l'arc inférieur, et bientôt, après une courte interruption, commence l'arc supérieur qui, contournant le lobe du corps calleux, se relève, puis se recourbe en avant et remonte peu à peu sur la face interne de l'hémisphère, jusqu'au bord supérieur ou sagittal qu'il atteint en $d$ et qu'il dépasse, de manière à se prolonger plus ou moins sur la face convexe. Les deux arcs inférieur et supérieur, que nous venons de décrire, circonscrivent le grand lobe limbique en bas et en haut; ils le circonscrivent aussi en arrière où, il est vrai, ils ne se rejoignent pas, mais où ils ne sont séparés que par un court intervalle $c$, et où, sans cette petite interruption, ils ne formeraient qu'une même courbe. En avant, au contraire, ils s'écartent beaucoup et leurs deux extrémités antérieures $a$ et $d$ sont séparées l'une de l'autre par toute la hauteur de l'hémisphère; mais, dans le large espace compris entre ces deux extrémités, vis-à-vis le genou du corps calleux, la scissure limbique est représentée par un troisième arc, ou arc antérieur $e$, qui chez la loutre est assez court et très peu profond et qui n'établit 
en avant du lobe du corps calleux qu'une démarcation très incomplète.

Ce troisième arc, comme on le verra plus loin, n'est, à vrai dire, que la partie antérieure du système de l'are supérieur, avec lequel il est en continuité directe chez certains animaux. Il forme alors une scissure profonde; mais chez la loutre, comme chez les autres carnassiers, comme aussi chez beaucoup d'autres mammifères, ce vestige de la scissure limbique n'est plus qu'un léger sillon.

Après avoir montré, sur notre figure schématique, le grand

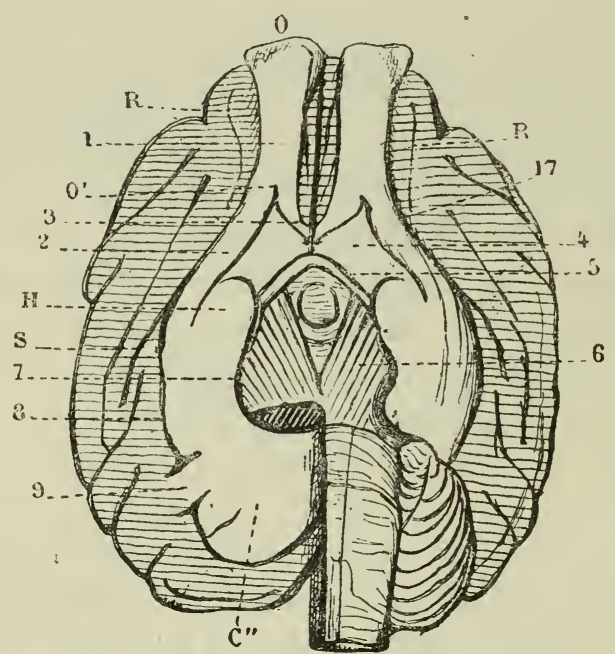

Fig. 7. Face inférieure du cerveau de la loutre. On a enlevé la moitié droite du cervelet, de la protubérance et du bulbe, pour montrer la face inférieure de l'hémisphère droit.

$\mathrm{O}$, lobe olfactif; $\mathrm{O}^{\prime}$, sa base; H, lobe de l'hippocampe; $\mathrm{C}^{\prime \prime}$, extrémité postérieure du lobe du corps calleux; R, scissure de Rolando; S, scissure de Sylvius.

2 , racine olfactive externe ; 3 , racine olfactive interne; 4 , espace quadrilatère ; 5 , bandelette optique aboutissant au chiasma qui est coupé ; 9 , le pli de passage rétro-limbique.

L'explication des autres chiffres se trouvera dans le texte.

lobe limbique dans son ensemble, nous étudierons sur des figures exactes, dessinées au diagraphe, les diverses parties dont il se compose.

Le lobe olfactif et le lobe de l'hippocampe se voient sur la norma inférieure (fig. 7).

Le lobe olfactif, situé au-dessous du lobe frontal, qu'il déborde un peu en avant, est constitué par un renflement antérieur 0 , 
peu volumineux, dont le pédoncule, 1, relativement très large, est libre et simplement appliqué sur la face inférieure du lobe frontal, mais dont la base, $0^{\prime}$, se continue avec le reste de l'hémisphère. Cette base s'élargit aussitôt, s'étale et on y distingue trois parties qui sont les trois racines du lobe olfactif.

La racine externe, volumineuse, 2 , se porte en dehors et en arrière et va s'appliquer sur le bord externe du lobe de l'hippocampe, avec lequel elle ne tarde pas à se confondre. Gette racine est blanchâtre et doit sa couleur aux fibres longitudinales super. ficielles qui viennent du pédoncule olfactif. Sous cette couche superficielle, les fibres longitudinales sont entourées d'une assez grande quantité de substance grise.

La racine interne, 3, moins grosse et beaucoup plus courte, se porte en dedans, atteint bientôt le bord inféro-interne de l'hémisphère, en avant du chiasma des nerfs optiques, et gagne la face interne, où elle se jette sur l'extrémité antérieure et inférieure du lobe du corps calleux (voy. fig. 9 et figure $6, f$ ); sa couleur est encore blanchâtre, mais un peu plus grise toutefois que celle de la racine externe.

La racine moyenne enfin ou racine grise occupe l'intervalle compris entre les deux autres, et remplit un espace quadrilatère (4, fig. 7), limité en arrière par le bord antérieur du lobe de l'hippocampe et par la bandelette optique, 5 , qui se dégage sous le bord interne de ce lobe pour se rendre au chiasma. Cet espace, un peu déprimé par rapport aux deux racines olfactives, et plus déprimé encore par rapport au lobe de l'hippocampe, forme la dépression connue sous le nom de vallée de Sylvius; il est revêtu d'une couche de substance corticale qui lui donne une couleur grise, d'où est venu le nom de racine grise. Mais au-dessous de cette couche grise, qui adhère aux bords des. deux racines blanches, et qui se continue sur la surface du lobe de l'hippocampe, on trouve une couche de fibres blanches qui, nées du pédoncule olfactif, parcourent d'avant en arrière l'espace quadrilatère, passent sous la bandelette optique comme sous un pont, et vont se continuer avec les fibres les plus inférieures de l'extrémité antérieure du pédoncule cérébral, 6 ; en outre les fibres les plus profondes de la racine olfactive moyenne vont se rendre à la commissure cérébrale antérieure, dans la partie de cette 
commissure qui traverse le corps strié. Desmoulins a cru remarquer que le volume de la commissure antérieure, dans la série des mammifères, est en rapport avec le degré de développement des lobes olfactifs (1). Plus profondément encore, la racine moyenne repose sur la face inférieure du corps strié, auquel elle adhère intimement, mais dans laquelle ses fibres ne semblent pas pénétrer.

Ainsi, le lobe olfactif, étudié de bas en haut, présente une double connexion, d'une part avec les deux autres lobes limbiques par ses deux racines latérales, d'une autre part avec le pédoncule cérébral et la commissure antérieure par sa racine moyenne.

. Mais lorsqu'on renverse ce lobe d'avant en arrière pour étudier la face supérieure de sa base, on découvre une troisième connexion, établie entre lui et le lobe frontal del'hémisphère par une insertion transversale que l'on peut appeler la racine olfactive supérieure. Cette insertion se fait sur l'extrémité postérieure de la face inférieure du lobe frontal, au moyen d'une courte lame formée d'une couche grise superficielle qui se continue avec la couche corticale du lobe frontal, et d'une couche blanche subjacente qui se continue avec la substance blanche du lobe frontal. Chez les animaux qui ont un grand lobe olfactif, il existe souvent dans l'épaisseur de ce lobe une cavité qui se relève en arrière, passe entre le lobe frontal et le corps strié et se continue avec l'extrémité antérieure du ventricule latéral. C'est ce qu'on peut voir sur la figure 8, représentant une coupe longitudinale du cerveau du cheval. La portion du pédoncule olfactif qui est au-dessus de cette cavité constitue la racine olfactive supérieure ou quatrième racine, et celle qui est au-deśsous fournit les trois autres racines (2). Mais revenons au cerveau de la loutre.

Le lobe de l'hippocampe (fig. 7) commence derrière l'espace quadrilatère par un renflement assez notable, $\mathrm{H}$, s'aplatit en

(1) Desmoulins, loc. cit., t. Jer, p. 264.

(2) Chez les primates, la racine supérieure, extrêmement réduite, se confond avec la racine grise, très réduite aussi. Elles forment ensemble une lamelle très courte, très mince, extrêmement fragile, qui s'insère sur la limite du lobe frontal et de l'espace quadrilatère perforé, et qui se continue avec tous les deux sous le nom de racine grise. 
arrière et se continue directement, au-dessous et en arrière du pédoncule cérébral, avec le lobe du corps calleux $\mathrm{C}^{\prime \prime}$. Il longe la partie latérale de la grande fente de Bichat, et occupe tout l'espace compris entre cette fente, 7 , et l'arc inférieur de la scissure limbique, 8. Cet espace, đssez étroit chez la loutre, est au contraire très large chez la plupart des autres mammifères, de sorte que, sur le cerveau vu en dessous, le lobe de l'hippocampe cache quelquefois entièrement la face latérale de l'hémisphère; mais

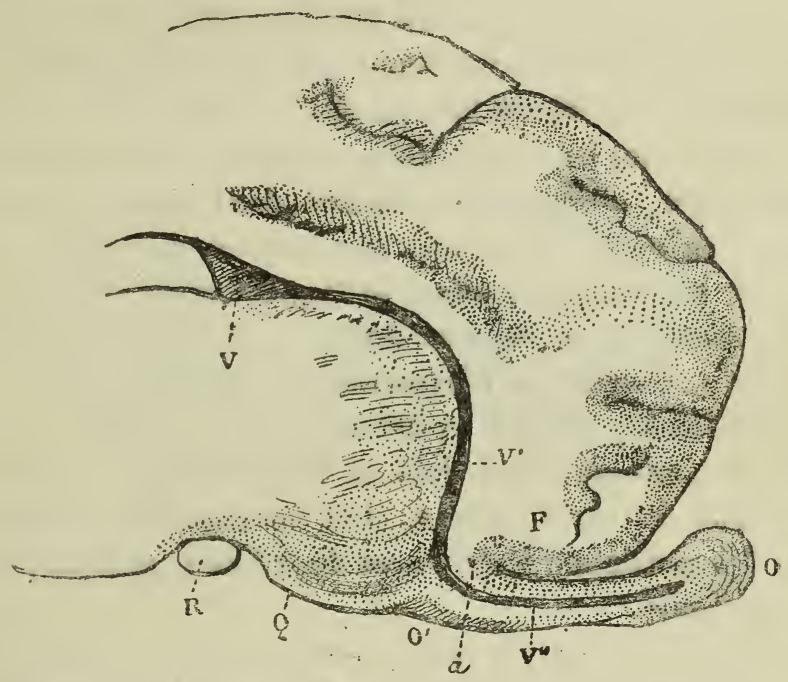

Fig. 8. Cheval. Coupe longitudinale de la partie antérieure de l'hémisphère gauche fà 6 millimètres de la ligne médiane : $O$, lobe olfactif; $O^{\prime}$, base de ce lobe; $Q$, coupe de l'espace quadrilatère et de la racine olfactive moyenne $; \mathrm{R}$, coupe de la bandelette optique ; $\mathrm{F}$, coupe du lobe frontal ; $\mathbf{S}$, coupe du corps strié ; V, coupe du ventricule latéral dont le prolongement antérieur $\mathrm{V}^{i}$, contuurnant le corps strié, se prolonge $\mathrm{V}^{\prime \prime}$ dans le lobe olfactif; $a$, racine supérieure ou frontale du lobe olfactif.

chez la loutre il laisse apercevoir, en dehors de lui, les circonvolutions inférieures de cette face externe, ainsi que la scissure de Sylvius $\mathrm{S}$, qui vient obliquement rejoindre la scissure limbique vers le niveau de l'extrémité antérieure du lobe de l'hippocampe. Lasurface de ce lobe est presque entièrementlisse; on y aperçoit cependant, en dehors, une légère dépression longitudinale, sorte de sillon très superficiel et bientôt effacé, qui fait suite au bord interne de la racine olfactive externe; quelquefois, en outre, il y a, en dedans de ce premier sillon, un second sillon longitudinal 
plus superficiel encore et surtout plus large, qui subdivise ou plutôt qui tend à subdiviser le lobe de l'hippocampe en deux parties, l'une interne, l'autre externe; mais cette subdivision, toujours très vague, n'existe pas chez la loutre.

Enfin le lobe du corps calleux $\mathrm{C}^{\prime \prime}$ commence sur la face inférieure de l'hémisphère, derrière le pédoncule cérébral, en se continuant directement, sans aucune ligne de démarcation, avec le lobe de l'hippocampe. Ce n'est donc pas sur ces lobes euxmêmes que leur limite se trouve établie; mais elle est indiquée, sur la scissure limbique, par un pli de passage, 9, qui fait communiquer le grand lobe limbique avec la partie postérieure du lobe pariétal, - je dis du lobe pariétal, parce qu'il n'y a pas de lobe occipital chez les mammifères autres que les primates. Ce pli de passage, absolument constant dans toute la série des mammifères, quoiqu'il ne soit pas toujours superficiel, mérite de recevoir un nom particulier; je l'appellerai le pli de passage rétro-limbique. Il est souvent simple, comme on le voit chez la loutre, mais souvent aussi divisé en deux plis juxtaposés plus ou moins parallèles; et dans ce dernier cas, on constate que l'antérieur se continue avec le lobe de l'hippocampe, le postérieur avec le lobe du corps calleux. Par conséquent, lorsqu'il est simple, il appartient à la fois à ces deux lobes, et il sert à indiquer leur limite.

A partir de ce point, le lobe du corps calleux longe, jusqu'à la ligne médiane, le bord postérieur de la grande fente de Bichat, puis contourne le bourrelet du corps calleux et parvient sur la face interne de l'hémisphère (voy. fig. 9), où il entoure complètement le corps calleux, reposant d'abord, $\mathrm{C}^{\prime}$, sur le bord supérieur de ce corps dont il est séparé par une rainure assez profonde (rainure du corps calleux), puis se recourbant audevant du genou, où s'arrête la rainure, et descendant enfin le long du bec du corps calleux jusqu'au bord inférieur de la face interne de l'hémisphère, où il se continue en $\mathrm{G}$, avec la racine interne du lobe olfactif. 0 n peut considérer ce point $\mathrm{C}$ comme l'origine du lobe du corps calleux, et on dira alors que ce lobe se termine en $\mathrm{C}^{\prime \prime}$, sur la face inférieure de l'hémisphère, en se continuant avec le lobe de l'hippocampe.

Le lobe du corps calleux, sur la face interne de l'hémisphère, 
s'élargit progressivement d'arrière en avant; en arrière, il n'occupe qu'une partie de la largeur de cette face, laissant apparaître au-dessus de lui la circonvolution la plus interne, ou circonvolution sagittale du lobe pariétal, P, P; mais en avant il occupe toute la largeur de la face interne de l'hémisphère jusqu'au bord convexe ou sagittal, et s'étend même un peu sur la face convexe, où nous le retrouverons tout à l'heure. Son extrémité antérieure, au-devant du genou du corps calleux, se continue largement avec le lobe frontal, $\mathrm{F}$; elle est séparée toutefois par une dépression très superficielle, 10 , qui est si peu marquée chez la loutre qu'on

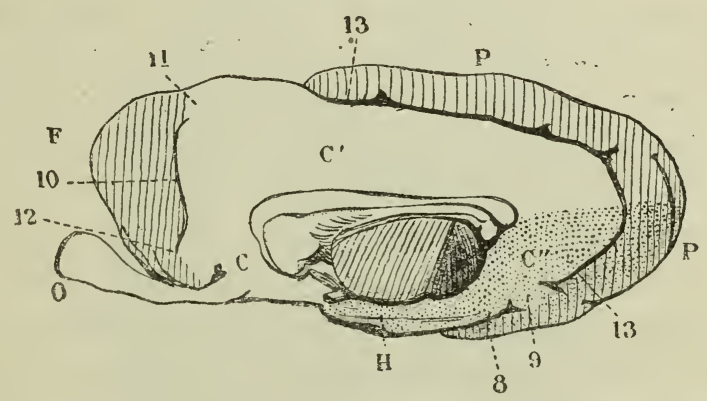

Fig. 9. Face interne de l'hémispli ire droit de la loutre. O, lobe olfactif; H, lobe de l'hippocampe; C, origine du lobe du corps calleux; $\mathrm{CG}^{\prime} \mathrm{G}^{\prime \prime}$, le lobe du corps calleux; F, lobe frontal ; P P, lobe pariétal.

9 , pli de passage rétro-limbique; 11 , pli de passage fronto-limbique. Voir le texte pour les autres lettres.

pourrait la prendre pour une empreinte vasculaire, mais qui est souvent beaucoup plus profonde, et qui constitue l'arc antérieur de la scissure limbique; c'est le vestige de la scissure sous-frontale des primates, mais elle est si rudimentaire chez les carnassiersqu'on peut tout au plus l'appeler le sillon sous-frontal. Audessus et au-dessous de ce sillon, le lobe frontal et le lobe du corps calleux communiquent largement l'un avec l'autre. La communication supérieure, $\mathbf{1 1}$, s'observe chez beaucoup de mammifères comme chez la loutre; je l'appellerai le pli de passage prélimbique ou fronto-limbique. Sa position et son volume peuvent varier, suivant l'étendue et la profondeur du sillon sousfrontal. Il est assez mince et assez profond, chez certains animaux, pour qu'on puisse croire qu'il fait entièrement défaut ; mais, avec un peu d'attention, on en retrouve au moins le ves- 
tige. Quant à la communication inférieure, 12, elle est constante et invariable, attendu que l'arc antérieur de la scissure ne s'étend jamais jusqu'au bord inférieur de l'hémisphère; toujours, par conséquent, la partie la plus inférieure et la plus interne du lobe frontal vient s'insérer directement sur l'origine du lobe du corps calleux, immédiatement au-dessus du point $\mathrm{C}$, où ce lobe reçoit la racine interne du lobe olfactif.

Entre le pli de passage rétro-limbique, 9 , et le pli de passage prélimbique, 11, le bord convexe du lobe du corps calleux est longé par l'arc supérieur de la scissure limbique, qui le sépare de la circonvolution pariétale la plus interne, P P.

Gette scissure, 13, 13, longue, profonde, ininterrompue, est donc tout entière sous le lobe pariétal, et mérite le nom de scissure sous-pariétale. Elle remonte jusqu'au bord sagittal de l'hémisphère, l'entaille profondément et se prolonge sur la face convexe. Ce prolongement terminal $(14,14$, fig. 10), dont la longueur et la direction sont très variables, est assez long chez la loutre; il est de plus très oblique; mais chez les autres carnassiers et chez beaucoup d'autres mammifères, il affecte une direction transversale, c'est-à-dire perperıdiculaire à la grande fente interhémisphérique, de sorte que les deux scissures, émergeant au même niveau sur le bord sagittal des deux hémisphères, forment avec la fente médiane qu'elles coupent perpendiculairement une figure en croix appelée par Leuret le sillon crucial. Cette épithète est défectueuse, puisque la figure n'est pas toujours cruciale, mais elle est très usitée, elle est commode; il serait peut-être difficile de la remplacer, et je continuerai à m'en servir; je ferai remarquer toutefois qu'il ne s'agit pas d'un simple sillon, mais d'une vraie scissure.

Plusieurs auteurs, considérant que la scissure cruciale établit la limite antérieure du lobe pariétal, ou du moins de sa circonvolution la plus interne, ont cru que cette scissure était, l'analogue de la scissure de Rolando des primates; mais la scissure de Rolando est propre à la face convexe, tandis que la scissure sous-pariétale, dont la cruciale n'est que le prolongement, appartient à la face interne. En outre, ce qui caractérise anatomiquement la scissure de Rolando, c'est la démarcation qu'elle établit entre le lobe frontal et le lobe pariétal; or, le bord anté- 
rieur de la scissure cruciale n'est pas formé par le lobe frontal, mais par la partie supérieure du lobe du corps calleux, qui, occupant àce niveau toute la hauteur de l'hémisphère, parvient jusqu'à la face convexe, 1 . Quant au lobe frontal, il est beaucoup plus en avant; c'est à peine s'il apparaît à la pointe de l'hémisphère, 16, au-devant d'une scissure, $R$, qui est la vraie scissure de Rolando, et qu’il faut étudier sur la norma latérale(voy. fig.11). Là, on voit que les circonvolutions pariétales, qui sont an nombre

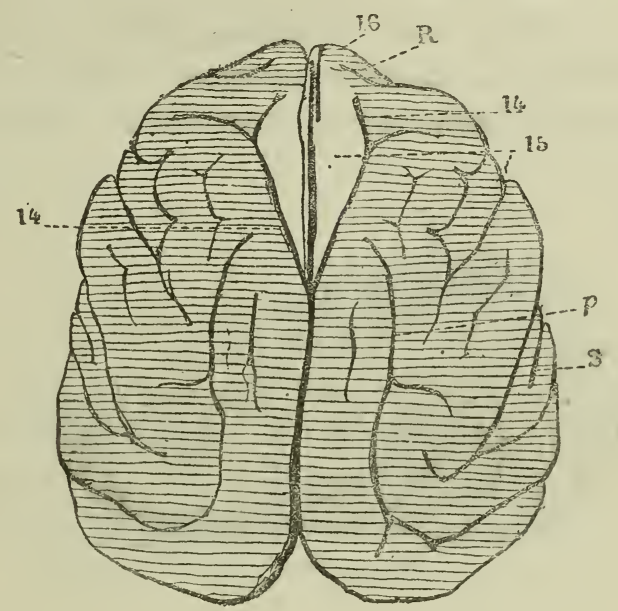

Fig. 10. Face supérieure du cerveau de la loutre. La portion laissée en blane, 15, est formée par le lobe du corps calleux; 14, le sillon crucial formé par l'extrémité antérieure de la scissure sous-pariétale; R, scissure de Rolando; S, scissure de Sylvius ; 16 , lobe frontal ; $p$, sillon pariétal primaire.

de trois chez la loutre, naissent sur la partie postérieure du bord externe de l'hémisphère, au-dessus de la scissure limbique, remontent vers le bord sagittal, s'infléchissent d'arrière en avant en décrivant autour de la scissure de Sylvius, S, trois al'cs plus ou moins brusquement fléchis, après quoi, prenant une direction postéro-antérieure, elles se prolongent jusqu’à une scissure très oblique, située tout en avant, et sur laquelle elles s'arrêtent toutes les trois, n’en étant séparée que par un pli, $\mathrm{P}^{\circ}$, qui est l'analogue de la circonvolution pariétale ascendante des primates. Cette scissure, $\mathrm{R}$, établit donc la limite antérieure du lobe pariétal, et le sépare du lobe frontal F, qui est extrêmement petit; c'est elle par conséquent qui représente la scissure de Ro- 
lando des primates. Elle naît sur le bord inféro-externe de l'hémisphère, immédiatement au-dessus de la scissure limbique, avec laquelle elle paraît communiquer au premier abord; mais ce n'est qu'une apparence, et en écartant les bords des deux scissures, on voit qu'elles sont séparées l'une de l'autre par un pli de passage, 17, étendu du lobe frontal à la circonvolution la plus inférieure ou circonvolution sylvienne du lobe pariétal $\mathrm{P}^{1}$. Ce point d'origine de la scissure de Rolando est situé bien en avant de l'origine de la scissure de Sylvius. Partant de là, la scissure de Rolando se porte d'abord très obliquement en avant, puis se relève en affectant une direction presque transversale, et se termine à une petite distance du bord sagittal de l'hémisphère; au-

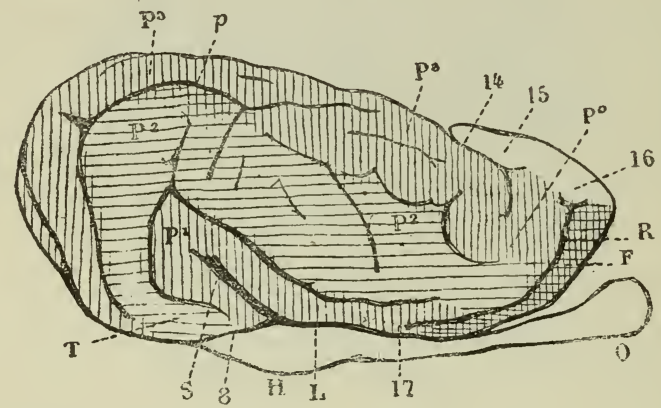

Fig. 11. Face externe de l'hémisphère droit de la loutre. O, lobe olfactif; H, lobe de l'hippo campe; L, arc inférieur de la scissure limbique; S, scissure de Sylvius; $R$, scissure de Rolando; F, lobe frontal ; T, lobule temporal du lobe pariétal; $p$, sillon pariétal primaire ; $\mathrm{P}^{4}$, circonvolution sylvienne ou première pariétale; $\mathrm{P}^{2}$, seconde pariétale; $\mathrm{P}^{3}$, troisièm $\mathrm{e}$ pariétale ou circonvolution sagittale; $\mathrm{P}^{\circ}$, circonvolution pariétale postrolandique.

Les parties laissejes en blanc appartiennent au grand lobe limbique.

dessus de cette terminaison, le lobe frontal communique à la fois, 16, avec la partie antéro-supérieure du lobe du corps calleux et avec la circonvolution pariétale supérieure $\mathrm{P}^{3}$, ou si l'on veut, avec la partie supérieure de la pariétale ascendante.

La scissure que nous venons de décrire comme l'analogue de la scissure de Rolando des primates a été interprétée tout autrement par M. Meynert. Cet auteur, n’ayant pas étudié dans son ensemble la scissure limbique, considère comme appartenant à la scissure sylvienne des carnassiers toute la partie de la scissure limbique qui est au-devant d'elle; en d'autres termes, il prolonge la scissure de Sylvius le long dı lobe de l'hippocampe et 
de la base du lobe olfactif jusqu'au-dessous du lobe frontal; en outre, n'ayant pas vu le pli de passage profond qui, chez la loutre et chez la plupart des mammifères, sépare la scissure limbique de notre scissure de Rolando, il considère celle-ci comme une branche de celle-là, c'est-à-dire comme une branche de la scissure de Sylvius, et il en fait l'analogue de la branche ascendante de la scissure de Sylvius de l'homme (1). Cette interprétation est déjà réfutée par ce qui précède. J'ajoute que la branche ascendante de la scissure de Sylvius est un effet du perfectionnement du lobe frontal, comme la branche antérieure de la même scissure; ces deux branches, formées dans les replis de la troisième circonvolution frontale, n'existent constamment que chez l'homme; elles se voient quelquefois toutes deux chez les orangs etles chimpanzés; les gorilles etles gibbons n'en ont qu'une seule, qui est l'antérieure; quant aux singes non anthropoïdes, pithéciens ou cébiens, ils n'ont ni l'une ni l'autre, quoique leur lobe frontal soit incomparablement plus développé que celui des carnassiers. Il serait donc très étrange que l'une ou l'autre de ces deux branches de perfectionnement se retrouvât chez les carnassiers (j'ajoute chez les ruminants et les pachydermes) lorsqu'elle manque chez les singes à queue. Je renverrai pour plus de détails à mon Mémoire sur le cerveau du gorille, où j'ai établi la distinction de la branche ascendante et de la branche antérieure ou horizontale de la scissure de Sylvius, et où j'ai discuté les principales questions qui s'y rattachent (2).

Pour revenir au cerveau de la loutre, je ferai remarquer que les trois circonvolutions pariétales prennent leur origine dans une petite région $T$, comprise entre le bord postérieur de la scissure de Sylvius, le bord supérieur de la scissure limbique, 8 , et le pli de passage rétro-limbique qui n'est pas visible sur la figure 11, mais qu'on a vu sur les figures 7 et $9\left(\mathrm{u}^{\circ} 9\right)$. Cette petite région renferme quelques-uns des éléments du lobe temporal des primates, et peut en être considérée comme le rudiment; mais c'est d'elle aussi que procèdent quelques-uns des éléments qui forment chez les primates le lobe occipital; elle fait

(1) Archiv für Psychiatrie, Berlin, 1877, t. VII, p. 262 et suiv.

(2) Revue d'anthropologie, janvier $1878,2^{\circ}$ série, t. Ior, p. 18-25 (et plus loin dans ce volume). 
d'ailleurs si manifestement partie du lobe pariétal qu'il est impossible de l'en distinguer. La loutre n'a donc ni lobe temporal ni lobe occipital. Il en est de même de tous les mammifères autres que les primates; leur lobe pariétal indivis comprend toute la masse des circonvolutions, à l'exception du petit lobe frontal.

Il est utile toutefois, pour faciliter les descriptions et les comparaisons, de distinguer par un nom spécial cette petite partie du lobe pariétal qui est située en T, entre la scissure de Sylvius et la partie postérieure de la scissure limbique; si elle ne forme pas encore un lobe distinct, on peut du moins la désigner sous le nom de lobule, et je l'appellerai le lobule temporal du lobe pariétal, ou, plus simplement, le lobule temporal, pour constater par cette épithète la part importante qu'il doit prendre chez les primates à la formation du lobe temporal.

En résumé, l'hémisphère de la loutre, que nous avons pris comme type dans la description précédente, se compose de deux parties bien distinctes : $1^{\circ}$ le grand lobe limbique, formé pa la réunion du lobe olfactif, du lobe de.l'hippocampe et du lobe du corps calleux; $2^{\circ}$ la masse circonvolutionnaire, qui constitue le reste du manteau, et dans laquelle on distingue seulement deux lobes, savoir : un lobe frontal tout à fait rudimentaire, et un immense lobe pariétal, séparés l'un de l'autre par la scissure de Rolando.

Maintenant que nous connaissons la constitution, les rapports et les connexions du grand lobe limbique, nous étudierons aisément les modifications qu'il présente dans la série des mammifères. Nous parlerons d'abord des mammifères osmatiques, en commençant par les lisseńcéphales.

\section{$\S$ 4. - cerveaux osmatiques lissencéphales.}

J'ai déjà dit (voy. page 259 , note 1) que j'emploie le mot lissencéphale dans un sens purement descriptif, pour désigner les mammifères qui n'ont pas de circonvolutions véritables, et sans y altacher l'acception zoologique que lui a donnée M. Owen.

Ce mot, pris au pied de la lettre, indiqueräit un hémisphère 
tout à fait lisse et sans aucune subdivision extérieure ; il ne serait donc applicable à aucun mammifère, puisque d'une part chez les osmatiques, il y a toujours un lobe olfactif bien distinct, et que, d'une autre part, chez les anosmatiques il y a toujours une séparation profonde, produite par la scissure et la vallée de Sylvius, entre le lobe frontal et le lobe temporal. Mais si l'on trouve constamment, sur la surface inférieure de l'hémisphère, certaines lignes de démarcation plus ou moins accusées, il n'en est pas de même de la face convexe, qui est tantôt plissée en circonvolutions bien dessinées, tantôt tout à fait lisse ou à peine empreinte çà et là de rares sillons très superficiels et très incomplets, qui n'y établissent pas de subdivisions véritables. Dans le premier cas, on dit que le cerveau est gyrencéphale; et dans le second cas, on dit, par opposition, il est lissencéphale.

Les cerveaux lissencéphales le sont plus ou moins; et de même les cerveaux gyrencéphales ont des circonvolutions plus ou moins nombreuses, plus ou moins distinctes. Il y a donc des cas où l'on hésite à dire si le cerveau est lissencéphale ou gyrencéphale. Ces faits intermédiaires s'observent surtout dans l'ordre des pri. mates, où l'on trouve d'ailleurs des genres tout à fait gyrencéphales et des genres tout à fait lissencéphales. Ils se retrouvent dans l'ordre des édentés, où le dionyx est tout à fait lissencéphale, tandis que l'unau et le pangolin sont gyrencéphales, et que l'oryctérope et le cachicame ont des circonvolutions rudimeritaires (1). On peut en conclure que la présence ou l'absence des circonvolıtions ne constitue pas un caractère essentiel de la clissification zoologique. Ce caractère, néanmoins, a une haute importance, et M. Owen a très justement remarqué qu'il se maintient habituellement dans tous les genres d'une même famille, et dans toutes les familles d'un même ordre. Voilà comment il a été conduit à constituer dans la classe des mammifères la sous-classe des lissencéphales, et celle des gyrencéphales (2).

(1) Voir les belles planches du Mémoire de George Pouchet sur l'encéphale des édentés. Thèse de la Faculté des sciences, Paris, 1869, in-4º, 6 planches (travail très important).

(2) Les deux autres sous-classes de mammifères sont, d'après M. Owen, les lyencéphales et les archencéphales. La première comprend les animaux dont le corps calleux est nul ou rudimentaire, savoir : les monotrêmes et les marsu- 
Il a rangé dans la première sous-classe les édentés, les rongeurs, les cheiroptères, les insectivores; dans la seconde, les cétacés, les ruminants, les pachydermes, les carnassiers et les primates (excepté l'homme). On remarquera que les ordres lissencéphales ne comprennent en génćral que des espèces de petite taille. Les ordres gyrencéphales, au contraire, renferment tous de très grands animaux, et si l'on y en trouve aussi de petits, il faut reconnaître du moins que les principaux représentants de ces ordres ont une taille bien supérieure à celle de la plupart des animaux lissencéphales.

Il y a plus : M. Dareste a constaté que, dans un même ordre, dans une même famille, la taille des diverses espèces exerce une influence très notable sur la présence ou l'absence des circonvolutions, et sur leur degré de développement (1). Cette découverte importante, qui date de $18 \% 2$, a été confirmée par les faits ultérieurs. Ainsi, quoique les rongeurs en général soient lissencéphales, le plus grand des rongeurs, le cabiai, est gyrencóphale (2), tandis que le chevrotain de Java, le plus petit des ruminants (taille d'un lapin), est presque lissencéphale (3). L'in. fluence de la taille se manifeste surtout dans l'ordre des primates où le type général, qui est gyrencéphale, fait place au type lissencéphale chez les plus petites espèces de la famille descébiens.

Ces différences cérébrales, en apparence si grandes, que l'on observe chez des animaux très analogues entre eux par leurs

piaux. La seconde ne comprend que l'homme; il est qualifié d'archencéphale, it cause de la perfection relative de son cerveau. Mais cette supériorité est pure. ment physiologique, et l'homme, par les caractères anatomiques, se rattachs manifestement à l'ordre des primates. En lous cas, il a de nombreuses circonvolutions, et doit être par conséquent qualitié de gyrencéphale. Quant aux lyencéphales, quelques-uns, comme l'échidné, ont de vraies circonvolutions; mais la plupart sont plus ou moins lissencéphales.

(1) Vojr surtout Dareste, Troisième mémoire sur les circonvolutions du cerveau chez les mammifères (Annales des sciences naturelles. Zoologie, 4e série, t. III, 18555). Les deux premiers mémoires se trouvent dans la même collection, $3^{\circ}$ série, t. XVII, 1852, et $4^{\mathrm{e}}$ série, t. Ir, 1854. Voir aussi Bulletins de la Société d'anthropologie, 1 re série, t. III, p. 26, 1\$62, et Comptes rendus de l'Acad. des sc., 1870, t. LXX, p. 193.

(2) Dareste, Nole sur le cerveau des rongeurs et particulièrement sur le cerveau du cabiai (Annales des sciences naturelles, $4^{\theta}$ série, Zcologre, t. III, p. 355 , pl. XI, fig. 1 et 3 . Paris, 1855 , in $\left.-8^{\circ}\right)$.

(3) Loc. cit., pl. II, fig. 4 à 6. 
caractères zoologiques, mais très inégaux par la taille, trouvent leur explication dans une remarque bien simple, faite par II. Baillarger à l'occasion d'une question très voisine de celle-là. Dans son mémoire sur l'étendue de la surface du cerveau et sur ses rapports avec l'intelligence, M. Baillarger, après avoir comparé la surface cérébrale de l'homme avec celle du lapin, ajoute: "La différence entre le rapport des volumes et des surfaces est le résultat de cette loi mathématique : que les volumes des corps semblables sont entre eux comme les cubes de leurs diamètres, tandis que leurs surfaces sont entre elles comme le carré de ces diamètres, ce qui donne des proportions très différentes. Le cerveau subit cette loi à laquelle il est cependant soustrait en partie par l'existence des circonvolutions (1)."

La proposition de géométrie invoquée par M. Baillarger est incontestable; elle fournit une explication très satisfaisante de l'influence de la taille sur le développement des circonvolutions.

Les circonvolutions sont formées par le plissement de l'écorce cérébrale, dont l'action est proportionnelle à sa surface ; un cerveau lisse est donc, toutes choses égales d'ailleurs, inférieur à un cerveau plissé, puisqu'il a moins de surface. Etant donnés deux animaux très analogues entre eux par leurs autres caractères, mais de taille très inégale, c'est le pltis grand des deux qui devra avoir le cerveau le plus volumineux. Ces deux cerveaux ayant à peu près la même forme, leurs volumes seront proportionnels aux cubes de leurs diamètres, suivant la loi des solides semblables. Par exemple, si les diamètres sont entre eux comme un est à deux, les volumes seront entre eux comme un est à huit. Quel sera maintenant le rapport des surfaces? On sait qu'elles sont entre elles comme les carrés des diamètres, par conséquent leur rapport ne sera pas un à huit, mais seulement un à quatre. La surface cérébrale, dans ce cas, s'est donc accrue deux fois moins que le volume cérébral, et le plus grand des deux cerveaux

(1) Baillarger, De l'étendue de la surface du cerveau et de ses rapports avec l'intelligence (Mémoire présenté à l'Académie de médecine le 1 š août 1845). Réimprimé dans le Recueil des Mémoires de l'auteur. (Recherches sur l'anıtomie, la physiologie et la pathologie du système nerveux, Paris, 1872, in-80, p. 54.) Voir aussi Bulletins de la Socièté d'anthropologie, 1re série, t. Il, p. 206, 1861. 
se trouvera par là très inférieur au plus petit, à moins que le plissement de sa couche corticale ne vienne compenser cette cause d'infériorité. En d'autres termes, un cerveau qui grandit doit se plisser sous peine de déchoir. Ainsi, à intelligence égale, et dans un même type zoologique, les animaux leṣ plus grands doivent avoir les cerveaux les plus plissés, et il peut en résulter tantôt que, dans un ordre où le type ordinaire est gyrencéphale, les plus petites espèces soient lissencéphales, tantôt que, dans un ordre où le type ordinaire est lissencéphale, les plus grandes espèces deviennent gyrencéphales. Cela ne veut nullement dire que, le manteau de l'hémisphère ne soit pas composé des mêmes parties dans les diverses espèces d'un même ordre, mais seulement que ces parties sont tantôt plates et tantôt soulevées en plis, tantôt diffuses à la surface, tantôt limitées par des sillons ou des scissures; car ce qui détermine la nature et les fonctions d'une partie du manteau, ce sont ses connexions profondes et non la forme extérieure qu'elle revêt.

Cetle explication de l'influence de la taille sur le développement des circonvolutions, diminue l'importance physiologique de la distinction des cerveaux lissencéphales et des cerveaux gyrencéphales, mais lui laisse toute son importance anatomique. Le plissement de la surface cérébrale, en effet, n'a point lieu au hasard ; la forme, la direction, la position et les rapports des circonvolutions sont déterminés par les connexions des divers points de l'écorce avec les parties centrales, connexions plus ou moins variables suivant les espèces, mais fixes dans la même espèce, de sorte que la morpholozie très diverse des circonvolutions fournit des caractères zoologiques d'une grande valeur. Toutefois, au milieu de ces diversités excessives, il y a une portion de l'hémisphère qui ne subit, chez les mammifères osmatiques, que des modifications très légères, et dont les principaux caractères sont indépendants de la présence ou de l'absence des circonvolutions. C'est le grand lobe limbique. Nous l'étudierons d'abord chez les lissencéphales.

Toujours, quelque lisse, quelque simple que soit le cerveau, une scissure limbique plus ou moins profonde et plus ou moins étendue permet de distinguer le grand lobe, alors même qu'aucune autre partie ne se laisse circonscrire sur le reste de l'hé- 
misphère. Cette scissure est toujours incomplète chez les lissencéphales; quelques-unes de ses parties peuvent n'être que rudimentaires, de sorte que les limites du grand lobe restent indécises en certains points.

La marmotte nous en fournit un exemple. Sur la norma inférieure, fig. $12, n^{\circ} I$, on aperçoit en avant le lobe olfactif 0 ,

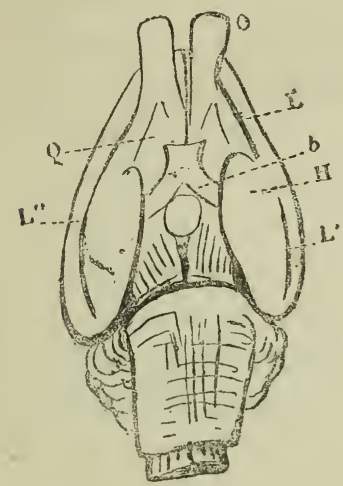

NoI.

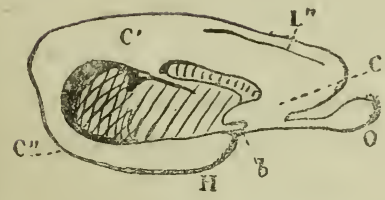

No II.

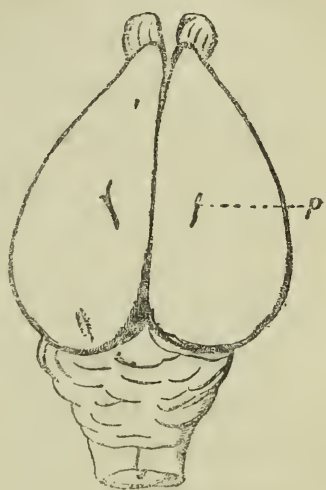

No IV.

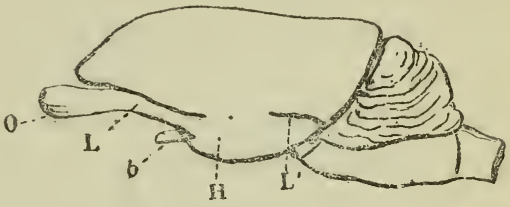

No III.

Fig. 12. Marmotte. No I, face inférieure; $n^{0} \mathbf{~ I}$, face interne de l'hémisphère gauche ; $n^{\circ}$ III, face externe de l'hémisphère gauche; $\mathrm{n}^{\circ} \mathrm{IV}$, face supérieure.

$\mathrm{O}$, lobe olfactif; H, lobe de l'hippocampe; Q, espace quadrilatère; $\mathrm{CC}^{\prime} \mathrm{C}^{\prime \prime}$, lobe du corps calleux; LL'L", la scissure limbique; $b$, la bandelette optique; $p$, vestige du sillon pariétal primairc

avec ses deux racines interne et externe, et sa racine moyenne ou grise qui occupe l'espace quadrilatère $Q$. Plus en arrière, un renflement assez fort constitue le lobe de l'hippocampe $\mathrm{H}$; de son bord interne se dégage la bandelette optique $b$, qui est très volumineuse, et qui se rend au chiasma en formant le bord postérieur et interne de l'espace quadrilatère.

Jusqu'ici, cette description ne diffère pas de celle du cerveau de la loutre. Mais l'arc inférieur de la scissure limbique n'est bien dessiné qu'en avant, $\mathrm{L}$, et en arrière, $\mathrm{L}^{\prime}$; sa partie moyenne qui 
correspond au tiers antérieur du lobe de l'hippocampe, est tantôt réduite à une ligne de dépression très superficielle comme on le voit en $L^{\prime \prime}$ sur l'hémisphère droit, tantôt tout à fait effacée comme on le voit du côté gauche, de sorte que le lobe de l'hippocampe se confond à ce niveau avec le reste du manteau. Sur un second cerveau de marmotte que nous possédons, l'arc inférieur de la limbique est continu des deux côtés, mais toujours il est très peu prononcé dans sa partie moyenne. La partie postérieure de cet arc, L, atteint à peine le bord postérieur de l'hémisphère. Elle ne se prolonge donc pas sur la surface interne ( $\left.\mathrm{n}^{\circ} \mathrm{II}\right)$, mais la scissure limbique reparaît en avant, $L^{\prime \prime}$, sous la forme d'une dépression longitudinale superficielle, située au-dessus du corps calleux et très rapprochée du bord sagittal de l'hémisphère. C'est à cela que se réduit l'arc supérieur de la scissure limbique. Il en résulte que le lobe du corps calleux, $\mathrm{G}, \mathrm{G}^{\prime}, \mathrm{G}^{\prime \prime}$, est très peu distinct, puisque sa partie postéro-supérieure se confond avec le reste du manteau. Il est bien moins distinct encore que le lobe de l'hippocampe. Seul, le lobe olfactif est bien caractérisé, et si l'on n'était pas guidé par l'anatomie comparée, on ne soupçonnerait pas la solidarité des trois lobes qui composent le grand lobe limbique. On remarquera toutefois que le lobe du corps calleux se continue en arrière et en bas, fig. $12, \mathrm{n}^{\circ} \mathrm{II}, \mathrm{C}^{\prime \prime}$, avec le lobe de l'hippocampe, en avant et en bas, $\mathrm{G}$, avec la racine interne du lobe olfactif, et que la réunion de ces trois parties forme un cercle complet autour du seuil de l'hémisphère (1), qu'elle représente par conséquent le grand lobe limbique.

La délimitation du grand lobe est un peu mieux marquée chez le castor, fig. 13. Sur la norma inférieure $\left(\mathrm{n}^{\circ} \mathrm{I}\right)$, on voit que le lobe olfactif et le lobe de l'hippocampe, tous deux très développés, sont limités en dehors par une scissure horizontale, $\mathrm{L}, \mathrm{L}^{\prime}$, interrompue seulement dans une étendue de quelques millimètres, vers la partie moyenne du lobe de l'hippocampe. La partie pos-

(1) On aperçoit dans la moitié postérieure du limbe l'hémisphère, fig. 12, no II, deux corps arrondis, presque égaux en volume. L'antérieur est la couche optique; le postérieur est le tubercule quadrijumeau antérieur, qui est très grand chez les l'ongeurs, et particulièrement chez la marmotte, et qui se creuse une loge sous le manteau de l'hémisphère. Le tubercule quadrijumeau postérieur, beaucoup plus petit, a été enlevé par la coupe. 
térieure $L^{\prime}$ est tout aussi profonde que l'autre, et constitue une véritable scissure; elle se continue $\left(\mathrm{n}^{\circ} \mathrm{II}\right)$ sur la face postérieure $\mathrm{L}^{\prime}$ et remonte jusqu’à la face interne, sur laquelle elle ne se prolonge que dans une étendue de quelques millimètres, mais après
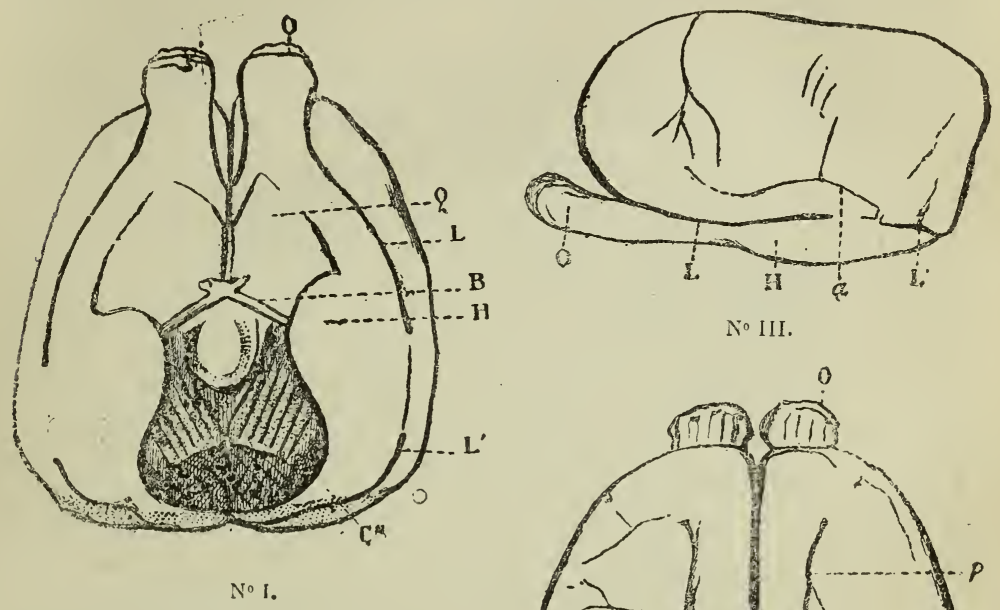

No III.

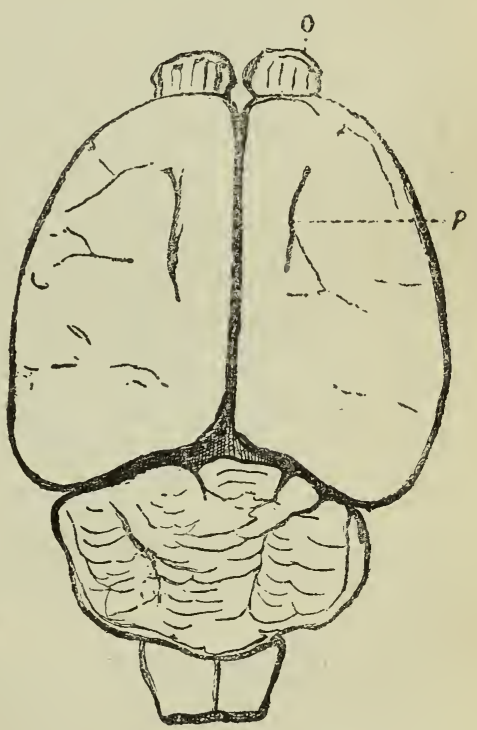

NoIV.

Fig. 13. Castor. No I, face inférieure; $n^{\circ}$ II, face interne de l'hémisphère gauche; $n^{\circ}$ III face externe de l'hémisphère gauche ; $\mathrm{n}^{0} \mathrm{IV}$, face supérieure.

$\mathrm{O}$, lobe olfaclif; $\mathrm{H}$, lobe de l'hippocampe; $\mathrm{Q}$, espace quadrilatère ; $\mathrm{B}$, bandelette optique ; C, origine du lobe du corps calleux ; $C^{\prime} C^{\prime \prime}$, lobe du corps calleux ; LL'L', la scissure limbique; L"', vestige du sillon sous-frontal; $p$, vestige du sillon pariétal primaire.

une seconde interruption elle reparaît en L", au-dessus du bourrelet du corps calleux. Cette portion supérieure de la scissure limbique est beaucoup moins profonde que les deux autres. Elle n'occupe que le tiers environ de la longueur de la face interne. . 
La scissure limbique est donc moins incomplète chez le castor que chez la marmotte; en outre on la retrouve encore, à l'état de vestige, au-devant du genou du corps calleux, sous la forme d'une légère dépression linéaire et curviligne $L^{\prime \prime \prime}$, qui est l'analogue du sillon sous-frontal de la loutre. C'est une limite qui commence à s'établir entre le lobe du corps calleux et la portion du manteau correspondant au lobe frontal. Ainsi, le lobe du corps calleux se trouve presque entièrement circonscrit en arrière $\mathrm{C}^{\prime \prime}$, en haut $\mathrm{C}^{\prime}$, et en avant $\mathrm{C}$. Enfin, une autre dépression linéaire longe quelquefois le bord externe dū lobe de l'hippocampe de manière à compléter l'arc inférieur de la scissure limbique. Cette disposition se voit sur l'hémisphère gauche $\left(n^{\circ}\right.$ I) du cerveau du castor mâle et adulte représenté sur la figure; elle manque du côté droit; elle manque aussi des deux côtés sur le cerveau du castor plus jeune de notre collection. La branche supérieure de la scissure limbique, $\mathrm{n}^{0} \mathrm{III}, \mathrm{L}^{\prime \prime}$, n'occupe pas toujours la position indiquée sur la figure 13. Elle est située beaucoup plus en avant sur l'autre hémisphère du même cerveau, ainsi que les deux hémisphères de notre cerveau de jeune castor. On peut conclure de ces variations que la scissure limbique existe virtuellement tout autour du grand lobe. Deux de ses parties, $L$ et $L^{\prime}$, sont constantes; elles sont assez profondes pour ne pas être subordonnées aux conditions du développement local; les autres se dessinent plus ou moins sous l'influence de ces conditions, qui peuvent varier non seulement d'individu à individu, mais encore sur le même cerveau, d'un hémisphère à l'autre.

Chez le lapin (fig. 14, $\mathrm{n}^{\circ} \mathrm{I}$ ), l'arc inférieur du grand lobe limbique est distinct dans toute son étendue. La scissure limbique, $\mathrm{L}$, L', longe sans interruption tout le bord externe du lobe olfactif et du lobe de l'hippocampe; elle se prolonge ensuite sur la face postérieure ( $\left.\mathrm{n}^{\circ} \mathrm{II}, \mathrm{L}^{\prime}\right)$, mais n'atteint pas la face externe, où l'are supérieur de la scissure est d'ailleurs représenté par une dépression linéaire $L^{\prime \prime}$, située au-dessous du genou du corps calleux, et par une autre empreinte longitudinale, courte et très superficielle, située au-dessus du bourrelet. Somme toute, la scissure limbique est un peu plus complète chez le lapin que chez le castor : mais la supériorité du cerveau de ce dernier ani- 
mal est rendue manifeste par la grande largeur et la grande épaisseur de l'extrémité antérieure de l'hémisphère, c'est-à-dire de la région qui colrespond au lobe frontal des animaux gyrencéphales. On remarquera que la même région est relativement beaucoup plus mince et beaucoup plus étroite chez la marmotte, et surtout chez le lapin.

Il serait superflu de passer en revue les autres cerveaux lissencéphales. Sur tous, même sur les plus petits, on retrouve aisé-

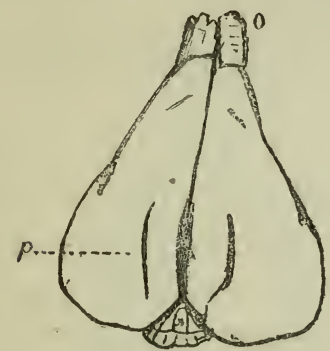

NoI.

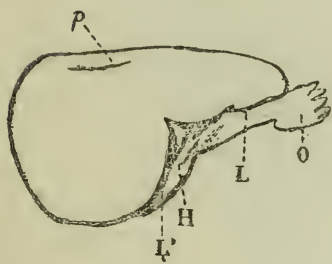

No 111 .

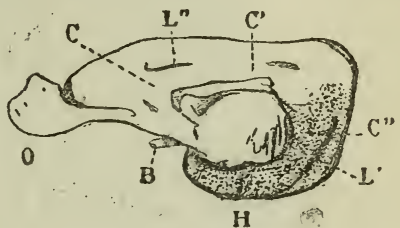

No II.

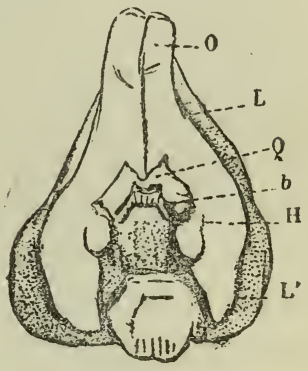

No IV

Fig. 14. Lapin. No I, face inférieure ; n II, face interne de l'hémisphère droit; nº III, face externe de l'hénisphère droit; $\mathrm{n}^{\circ} \mathrm{IV}$, face supérieure.

Les lettres comme sur la figure 13.

ment, à l'un des degrés que je viens de décrire, la scissure limbique, limitant le grand lobe limbique, qui offre quelquefois (par exemple, chez le hérisson) un volume relativement énorme.

J'ai pris mes exemples dans l'ordre des rongeurs; mais les mêmes dispositions s'observent chez les autres lissencéphales, insectivores, chéiroptères et édentés; elles se retrouvent même chez les marsupiaux, quoique la constitution du seuil de l'hémisphère soit notablement modifiée chez ces animaux par suite de l'absence presque complète du corps calleux (1).

(1) Le corps calleux, très rudimentaire chez les marsupiaux, nul même, diton, chez quelques-uns, fait entièrement défaut chez les monotrèmes. Ces deux 


\section{$\S$ Э. - CERVEAUX OSMATIRUES GYRENCÉPHALES.}

Le grand lobe limbique des cerveaux lissencéphales est toujours, comme on vient de le voir, suffisamment distinct pour qu'on puisse en déterminer la constitution et en reconnaître les connexions; toutefois cette recherche exige une certaine attention, parce que d'une part la scissure limbique est incomplète, et parce que, d'une autre part, la surface du grand lobe, à l'exception du lobe olfactif, présente à peu près la même apparence que la surface du reste du manteau.

Mais la distinction du grand lobe limbique et sa nature toute spéciale deviennent tout à fait évidentes sur les cerveaux gyrencéphales, par suite de l'apparition des circonvolutions. Le plissement qui produit ces dernières s'effectue dans toute l'étendue du manteau, à l'exception du grand lobe, et le manteau se décompose ainsi en deux parties essentiellement différentes, qui sont le grand lobe et la masse circonvolutionnaire. Dans les cerveaux très compliqués, c'est-à-dire très plissés, le lobe du corps calleux qui constitue l'arc supérieur du grand lobe, peut subir un léger degré de plissement, attesté par la présence d'une incisure longitudinale isolée et relativement très superficielle; mais il reste toujours beaucoup plus simple que la masse circonvolutionnaire; il ne revêt pas la forme flexueuse des circonvolutions. Quant à l'arc inférieur du grand lobe, il conserve toute la simplicité qu'il présente chez les lissencéphales; il reste complètement étranger au perfectionnement qui se manifeste dans les parties environnantes, perfectionnement auquel le lobe du corps calleux ne participe d'ailleurs qu'exceptionnellement, el à un degré relativement très faible. En même temps, la scissure limbique devient beaucoup plus profonde et aussi beaucoup plus complète; clle n'est plus interrompue qu'en certains points très limités. Il y a

groupes de mammifères forment la sous-classe des lyencéphales de M. Owen. Je n'ai pu jusqu'ici examiner moi-même le cerveau des monotrèmes, mais les dessins du cerveau de l'échidné, publiés par MM. Eydoux et Laurent dans le Magasin de zoologie ( $8^{\mathrm{e}}$ année, Paris, 1838 , in-8 $8^{\circ}, \mathrm{p} .174, \mathrm{pl} . \mathrm{XXX}$ ) me portent à croire que le grand lobe limbique de ces animaux est réduit à son arc inférieur, et ne comprend que le lobe olfactif et le lobe de l'hippocampe. 
donc une ligne de démarcation anatomique très évidente, tout autour du grand lobe, et, ce qui surtout frappe au premier coup d'œil, c'est le contraste qui existe entre l'aspect de cette partie

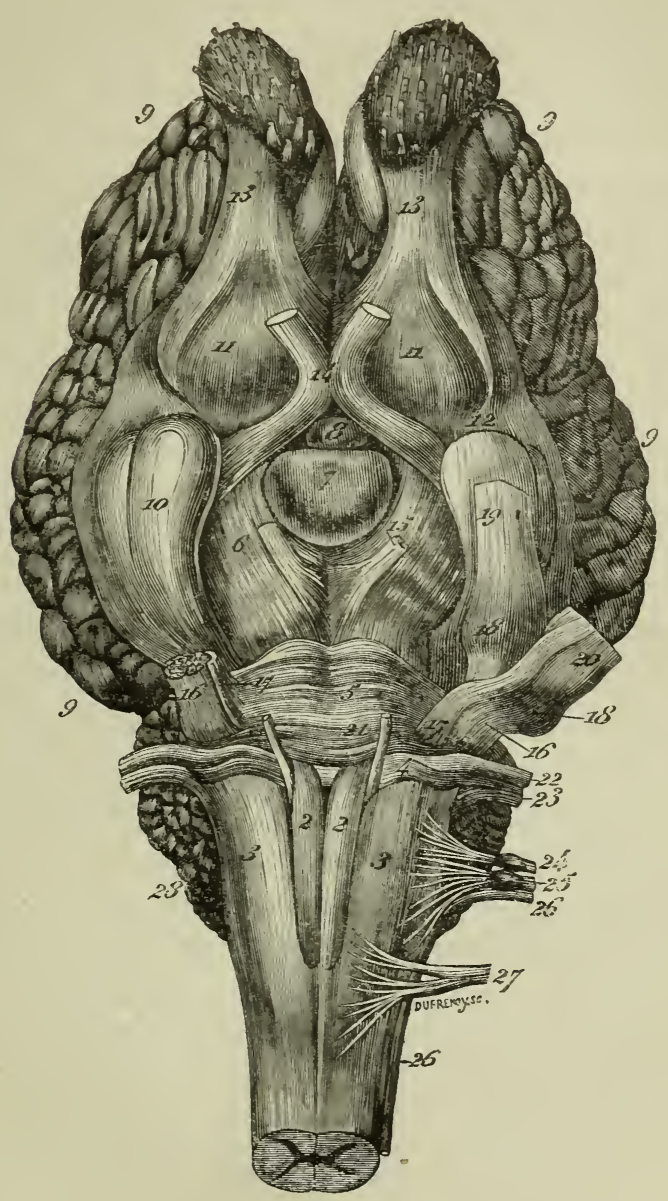

Fig. 10. La face inférieure du cerveau du cheval, figure extraite du Traité de l'anatome comparée des animaux domestiques, par MM. Chauveau et Arloing; 20 édition, p. 71!. (J.-B. Baillière et fils).

de l'hémisphère et celui de la masse circonvolutionnaire. Pour' en donner une idée, je reproduis ici une figure empruntée à l'excellent traité d'Anatomie comparée des animaux domestiques, de MINI. Chauveau et Arloing, figure 1ồ, qui a paru en 1871 
et qui, par conséquent, n'a pas été faite pour les besoins de la cause. Les contraste est tel qu'il devient évident que ces deux parties de l'hémisphère, si différentes par leur structure, le sont aussi par la nature de leurs fonctions; et si l'on considère que l'une d'elles reste stationnaire et imperfectible pendant que l'autre se perfectionne et se développe, que celle-là perd son importance à mesure que celle-ci fait des progrès, on est conduit à reconnaître que la première est le siège des facultés inférieures qui prédominent chez la brute, que la seconde est le siège des facultés supérieures qui prédominent chez l'animal intelligent, et l'on peut exprimer cette opposition de leurs caractères respectifs en disant que le manteau de l'hémisphère se compose de deux parties, l'une brutale reprósentée par le grand lobe limbique, l'autre intelligente, représentée par le reste du manteau. Je souligne ces épithètes pour en signaler l'importance physiologique, mais les dénominations anatomiques doivent être empruntées à l'anatomie elle-même. Des dèux portions du manteau de l'hémisphère que nous avons à distinguer, la première est suffisamment caractérisée par le nom de grand lobe limbique. L'autre, chez les gyrencéphales, peut être appelée la masse circonvolutionnaire; mais ce nom n'est pas applicable aux cerveaux qui n'ont pas de véritables circonvolutions; on peut alors le remplacer par celui de masse extra-limbique, puisque cette partie est située en dehors du grand lobe limbique.

Le grand lobe limbique présente chez tous les mammifères osmatiques les caractères qui ont été décrits plus liaut d'après le cerveau de la loutre, si ce n'est que les deux lobes de l'arc inférieur sont en général plus larges et plus épais. La largeur du lobe de l'hippocampe est quelquefois égale à celle de l'hémisphère lui-même, de sorte que, sur la norma inférieure, ce lobe masque entièrement la masse circonvolutionnaire. Le degré de saillie de la partie antérieure de ce lobe, et, par conséquent, le degré de profondeur de la dépression transversale qui est au-devant d'elle (vallée de Sylvius), présente aussi d'assez grandes variations. Il en est de même du volume de la racine olfactive externe qui, après avoir longé le bord externe de l'espace quadrilatère, va se jeter sur le bord externe du lobe de l'hippocampe.

Ce lobe, quoique paraissant presque toujours simple extérieu- 
rement, se compose cependant de trois parties, l'une externe, dans laquelle se prolonge la racine olfactive externe; l'autre interne, dans laquelle vient se rendre profondément la corne d'Ammon; l'autre moyenne, comprise entre les deux autres. Le plus souvent, rien n'indique à l'extérieur la succession de ces trois zones; c'est à peine si un relief latéral, faisant suite à la racine olfactive, marque, tout en avant, le prolongement de cette racine sur le bord externe du lobe de l'hippocampe; quelquefois cependant ce relief se dessine mieux, et dans une étendue plus considérable; il est alors limité par une sorte de sillon longitudinal superficiel, parallèle à la partie correspondante de la scissure limbique. C'est ce qui a lieu, par exemple, chez le cheval. Il y a même chez cet animal, en dedans de ce premier sillon, une seconde dépression longitudinale, qui indique la ligne de démarcation entre la zone moyenne et la zone interne. Malgré ces variations, le lobe de l'hippocampe ne présente, chez les mammifères osmatiques, que des différences assez légères.

Le lobe du corps calleux est presque aussi fixe. Chez les petits animaux, il est tout aussi simple que chez la loutre; chez les animaux de grande taille, il est plus ou moins suldivisé, suivant sa longueur, par un sillon longitudinal toujour's incomplet, et d'ailleurs très peu profond. Ces différences, on le voit, n'ont qu'une faible importance. Somme toute, le grand lobe limbique, au milieu des changements excessifs qui se produisent dans le leste du manteau, conserve, dans la série des mammifères osmatiques, tous ses caractères essentiels avec une constance remarquable, qui n'exclut pas certaines variations, d'ailleurs légères et d'ordre tout à fait secondaire. Mais ce qui varie beaucoup, extérieurement du moins, ce sont les connexions du grand lobe limbique avec la masse circonvolutionnaire.

Rappelons d'abord que, chez la loutre, la masse circonvolutionnaire ne forme que deux lobes, l'un antérieur très petit, situé tout en avant: c'est le lobe frontal; l'autre, comprenant tout le reste des circonvolutions : c'est le lobe pariétal. Ces deux lobes sont séparés l'un de l'autre par la scissure de Rolando. Quant à la scissure de Sylvius, qui pénètre dans le lobe pariétal, elle est contournée par les circonvolutions de ce lobe, mais elle 
ne les interrompt pas; elle ne les empêche pas de continuer leur trajet d'arrière en avant, jusqu'à la scissure de Rolando, ou plutôt jusqu'à un pli qui forme le bord postérieur de cette scissure, et qui est l'analogue de la circonvolution pariétale ascendante des primates.

La scissure de Rolando existe chez tous les gyrencéphales; tous, par conséquent, ont un lobe frontal distinct du lobe pariétal ; mais sur les cerveaux osmatiques, ce lobe frontal est presque constamment très petit, très simple, et réduit le plus souvent à une seule circonvolution. La seule subdivision que l'on y remarque consiste en une dépression longitudinale large et ordinairement peu profonde, située sur sa face inférieure, et dans laquelle s'applique le pédoncule du lobe olfactif. Toutefois, sur les cerveaux les plus compliqués (cheval, chameau, bœuf, tapir), le lobe frontal est un peu plus grand, et subdivisé par un ou deux sillons (1); quelquefois aussi la scissure de Rolando se relève plus ou moins, mais son extrémité inférieure est toujour dirigée en arrière ; et le point de la scissure limbique qui correspond à cette extrémité est toujours situé en avant de l'extrémité antérieure du lobe de l'hippocampe.

La scissure de Sylvius n'a pas la même constance que celle de Rolando. Elle existe chez tous les carnassiers, exactement comme chez la loutre; elle se retrouve, avec la même forme, sur plusieurs autres mammifères, tels que le sanglier; mais elle est disposée tout autrement chez les ruminants et la plupart des pachydermes; et quelquefois, enfin, elle manque tout à fait : c'est ce qui a lieu, par exemple, chez le tapir. Ces grandes différences indiquent que la scissure de Sylvius ne fait pas essentiellement partie de la constitution du cerveau des gyrencéphales. Elle est la conséquence ordinaire, mais non nécessaire, du mode de formation des circonvolutions.

J'ai déjà dit que le plissement du manteau est l'effet de l'extension de l'écorce cérébrale, dont la surface, devenue plus grande que ne le comporterait le volume de la masse subjacente, se soulève et se fronce plus ou moins profondément. Pour se

(1) Sur l'immense cerveau de l'éléphant, le lobe frontal est relativement beaucoup plus grand et beaucoup plus compliqué. 
faire une idée de ce mécanisme, on peut examiner chez l'homme la peau d'une région soumise pendant quelques jours à l'action d'un cataplasme. L'épiderme, gonflé par l'humidité, devient plus long et plus large que le derme qu'il recouvre; il se ride en formant de réritables circonvolutions, qui ressemblent quelquefois d'une manière frappante aux circonvolutions cérébrales. Mais ce plissement n'a pas lieu au hasard; la forme, la direction des plis et leurs anastomoses sont déterminées par les connexions de l'épiderme avec le derme subjacent, connexions qui varient suivant les régions, mais qui sont toujours les mêmes dans un même lieu. J'ai fait plusieurs fois mouler, dans ces conditions, la peau de la partie antérieure du genou; la disposition des circonvolutions flexueuses de l'épiderme est exactement pareille sur tous ces moules; elle est tout autre dans la région du coude-pied, du coude, de l'avant-bras, etc. Cet exemple nous montre comment l'ampliation de l'écorce cérébrale produit des circonvolutions.

La nature du plissement dépendant des connexions de l'écorce avec les parties subjacentes et environnantes, les circonvolutions doivent être, comme ces connexions elles-mêmes, fixes dans une même espèce, analogues dans les espèces voisines, différentes dans les espèces éloignées, et c'est ce qui a lieu en effet.

Le grand lobe limbique, ainsi que je l'ai déjà dit, reste à peu près étranger au plissement. Le reste du manteau forme dons toute la masse circonvolutionnaire, qui entoure de toutes parts la scissure limbique, et qui se continue toujours au moins en deux points avec le grand lobe limbique. D'une part, en effet, le lobe frontal se continue arec l'origine (voy. plus haut, p. 280) du lobe du corps calleux, et d'autre part le lobe pariétal se continue, par le pli du passage rétro-limbique (p. 280), avec la partie postérieure de ce même lobe et du lobe de l'hippocampe. Ainsi fixée à ses deux extrémités sur le grand lobe limbique, et appuyant ses deux bords interne et externe sur les bords de ce grand lobe, l'écorce de la masse circonvolutionnaire se continue en outre, par sa face profonde, avec les divers faisceaux de fibres blanches épanouies dans le corps de l'hémisphère. Par conséquent, lorsque l'extension de sa surface l'oblige à se plisser, elle ne se plisse pas au hasard; et le plissement se produit pour 
chaque espèce suivant un mode déterminé par ces connexions. $\mathrm{Au}$ surplus, cette extension de l'écorce ne s'effectue pas toujours au même degré dans le sens de la longueur et dans celui de la largeur. L'écorce agrandie produit, en s'allongeant, des plis plus ou moins transversaux, en s'élargissant, des plis plus ou moins longitudinaux. Chez les lissencéphales, l'extension de l'écorce n'est jamais bien grande, et il peut se faire que le plissement ait lieu presque exclusivement, ou même exclusivement dans une seule direction; je ne parle pas, bien entendu, des petits cerveaux tout à fait lisses des insectivores, des chéiroptères (excepté la roussette), et des plus petits rongeurs; les petites nervures qui existent parfois à leur surface sont des empreintes vasculaires et non pas des sillons (1). Mais chez les lissencéphales de plus grande taille, on trouve quelques sillons incomplets, isolés, ordinairement très espacés et très superficiels, qui marquent déjà l'ébauche des circonvolutions. Ces sillons, en se développant de plus en plus dans certaines espèces, établissent de nombreuses transitions entre les cerveaux lissencéphales et les cerveaux gyrencéphales.

Chez les gyrencépliales, l'écorce extra-limbique, très amplifiée en tous sens, se plisse à la fois en long et en large, mais elle s'élargit plus qu'elle ne s'allonge, et il en résulte que le plissement longitudinal est bien plus prononcé que le plissement transversal. Celui-ci existe toujours au moins en avant, près de la pointe de l'hémisphère. Il y produit la scissure de Rolando, qui est ordinairement très oblique dans sa partie externe, mais qui, par son autre extrémité, se relève toujours de manière à se diriger vers le bord interne de l'hémisphère. Ce qui caractérise le plissement transversal, ce n'est pas tant la direction absolue du pli, que ses connexions avec le grand lohe limbique, étranger

(1) Les nervures vasculaires, toujours extrêmement superficielles, corres poudent aux veines de la pie-mère. Elles ont souvent une forme rameuse qui les caractérise. Sur les cerveaux dépouillés à l'état frais elles 'sont très peu marquées et s'effacent même souvent au bout de peu de jours; mais sur les cerveaux durcis dans l'alcool avec leurs membranes, elles se dessinent beaucoup mieux parce que les veines, en se rétractant, dépriment la substance cérébrale. On les distingue aisément des sillons parce que, au moment où l'on enlève la pie-mere, on constile que chacune d'elles est recouverte par l'une des veines de cette membrane. 
au plissement. L'écorce extra-limbique, qui forme la face convexe du manteau, longe, par son bord supérieur et interne, l'arc supérieur du grand lobe, dont son bord inférieur et externe longe l'arc inférieur. Tout pli qui, partant de l'un de ces bords, se dirige vers l'autre soit directement, soit par un trajet plus ou moins oblique, interrompt ou dévie les plis longitudinaux et doit par conséquent être rapporté à un plissement par excès de longueur, c'est-à-dire à un plissement transversal. C'est ce plissement qui produit la scissure de Rolando, entre le petit lobe frontal qui forme la pointe de l'hémisphère, et l'immense lobe pariétal qui s'étend de lì, sans interruption, jusqu'à l'extrémité postérieure. Le plissement transversal se manifeste aussi le plus souvent sur le lobe pariétal, où il donne naissance à la scissure de Sylvius, comme on le verra plus loin.

Le plissement longitudinal est beaucoup plus prononcé; il est attesté par l'existence de sillons qui divisent le lobe pariétal dans toute sa longueur, en un certain nombre de circonvolutions parallèles. Le cerveau de l'unau présente cette disposition dans sa plus grande simplicité. L'unau est un édenté ; mais comme plısieurs autres représentants de cet ordre, il a de véritables circonvolutions pariétales. On voit dans la figure $16, n^{\circ} \mathrm{I}$, empruntée à l'excellent mémoire de M. G. Pouchet, que ces circonvolutions sont au nombre de quatre. La plus interne, $a$, que j’appelle la circonvolution sagittale, longe la fente inter-hémisphérique, qui correspond à la suture sagittale; elle se voit aussi sur la face interne de l'hémisphère, où elle repose sur le lobe du corps calleux. La plus externe, $b$, que j'appelle la circonvolution sylvienne, forme le bord externe de l'hémisphère. Si l'on aperçoit en dehors d'elle une portion de l'hémisphère, c'est parce qu'elle se relève en S en formant un arc à convexité supćrieure, premier indice d'un excès de longueur qui, en s'accentuant davantage, donne lieu, chez la plupart des gyrencéphales, à un vrai plissement et produit la scissure de Sylvius. Lorsque cette scissure n'existe pas, il y a toujours, du moins sous le bord concave de la circonvolution externe, une dépression plus ou moins profonde, analogue à celle qui est connue, en embryogénie humaine, sous le nom de fosse de Sylvius ( ${ }^{0}$ II, S). On verra plus loin que cette fosse est occupée par une portion du manteau appelée le lobule sous- 
sylvien. La circonvolution la plus externe, $b$, mérite donc le nom de circonvolution sylvienne que je viens de lui donner (1).

La circonvolution sagittale et la circonvolution sylvienne sont constantes chez les gyrencéphales. Entre elles se placent les circonvolutions intermédiaires, dont le nombre varie de un à trois. Il y en a ordinairement deux, comme chez l'unau, mais il n'y en a parfois qu'une seule, comme chez la loutre (voy. plus haut p.284, fig. 11, $\mathrm{P}^{2}$ ), et on en compte trois chez les grands chiens. On peut considérer ces circonvolutions intermédiaires comme des subdivisions des deux précédentes. Le lobe pariétal, en réa-

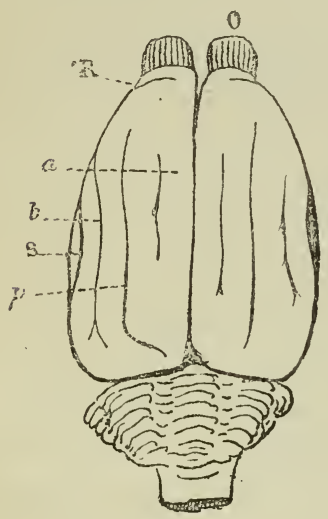

No I.

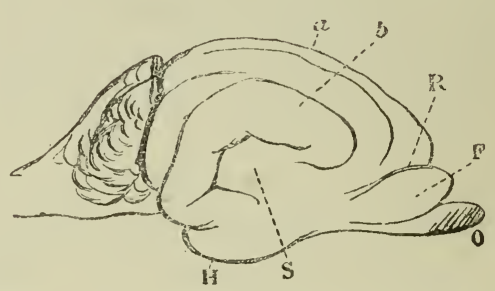

No Ii.

Fig. 16. Cerveau de l'unau. No I, face supérieure d'après Georges Pouchet; n II, face externe de l'hémisphère droit d'un unau plus jeune, d'après le mème auteur.

$\mathrm{O}$, lobe olfactif; H, lobe de l'hippocampe; $\mathrm{P}$, acissure de Rolando; F, lobe frontal; $\mathrm{S}$, fosse de Sylvius occupée par le lobule sous-sylvien; $b$, circonvolution sylvienne; $a$, circonvolution sagittale; $p$, sillon pariẹtal primaire.

lité, se décompose en deux zones longitudinales, séparées par un sillon que l'on peut appeler le sillon pariétal primaire. G'est le sillon $p$ de la figure $16, \mathrm{n}^{\circ} \mathrm{I}$. Ces deux zones sont simples chez certains lissencéphales qui, sans avoir de véritables circonvolutions, ont un sillon longitudinal unique, établissant une démarcation déjà manifeste, quoique encore incomplète, entre la région

(1) N'ayant pu me procurer le cerveau de l'unau, je suis obligé d'emprunter les deux numéros de la figure 16, au mémoire déjà cité de M. Pouchet (Mémoire sur l'encéphale des édentés, Paris, 1869, in-40, pl. II, fig. 6 et 7. On remarquera que ces deux dessins ont été faits d'après deux cerveaux différents, dont l'un ( $n^{\circ} \mathrm{II}$ ) provient d'un animal très jeune, voilà pourquoi les circonvolutions offrent quelques différences. 
interne, ou sylvienne, de la masse extra-limbique et sa région interne, ou sagittale. Ce vestige du sillon pariétal primaire se voit sur la marmotte (fig. $12, \mathrm{n}^{\circ} \mathrm{IV}, p$ ), le castor mâle et adulte (fig. 13, $\mathrm{n}^{\circ} \mathrm{IV}, p$ ), le lapin (fig. 14, $\mathrm{n}^{\circ} \mathrm{IV}, p$ ). Il est très prononcé chez l'agouti ainsi que chez le cochon d'Inde, mais il manque entièrement chez la taupe, le rat, le hérisson, etc. Chez les gyrencéphales, la zone sylvienne est toujours subdivisée en deux circonvolutions, dont l'inférieure est la circonvolution sylvienne proprement dite; quant à la zone sagittale, elle est quelquefois simple (la belette, le furet, la martre, la loutre, le blaireau), le plus souvent elle est divisée en deux circonvolutions parallèles, dont l'interne est la sagittale proprement dite, et dont l'externe est quelquefois dédoublée par un sillon longitudinal (1). C'est ainsi que le nombre des circonvolutions pariétales peut varier de 3 à 5 , et le nombre des sillons pariétaux de 2 à 4 . Par suite de ces subdivisions, la distinction primordiale des deux zones se trouve plus ou moins dissimulée; on la retrouve toutefois, en y regardant avec quelque attention, car le groupe des deux circonvolutions de la zone sylvienne est séparé du groupe sagittal par un sillon plus grand et plus profond que les autres; en outre, les circonvolutions de chaque groupe conservent une certaine tendance à s'unir entre elles. Ainsi, chez les chats, les deux circonvolutions de la zone sylvienne se fusionnent à leur partie moyenne. Une fusion moins étendue s'observe un peu plus en arrière dans certaines races de chiens. Chez les primates, où le lobe pariétal, comme on le verra plus loin, esí considérablement réduit par suite de l'extension du lobe frontal, cette tendance à la fusion s'accentue davantage; les deux circonvolutions de la zone sagittale n'en font plus qu'une seule qui est la première pariétale; les deux circonvolutions de la zone sylvienne, fusionnées en avant pour constituer la seconde pariétale, ne restent distinctes qu'en arrière, où elles se continuent avec les deux premières temporales; et il ne reste plus qu'un sillon longitudinal, le sillon pariétal, qui représente le sillon pariétal primaire

(1) La circonvolution sagittale peut elle-même être subdivisée en deux et même trois plis secondaires (exemple le cheval) par des sillons longitudinaux, mais ces plis ne se produisent que sur la partie postérieure de la circonvolution, et seulement sur sa face interne, qui est appliquée sur la grande faux. 
des gyrencéphales osmatiques, et le sillon pariétal unique de certains lissencéphales.

Mais revenons aux gyrencéphales osmatiques. Nous avons parlé jusqu'ici de leurs circonvolutions pariétales, comme si elles étaient parallèles; quelquefois, en effet, elles sont à peı près parallèles, comme on l'a vu sur le cerveau de l'unau, et elles le seraient toujours, si l'extension de l'écorce ne se faisait que dans le sens de la largeur'; mais le plus souvent elle se fait aussi dans le sens de la longueur. Alurs, les circonvolutions, sc trouvant plus longues que le grand lobe limbique sur lequel elles s'appuient, et qui représente la corde de leur arc, deviennent plus ou moins sinueuses; mais elles le deviennent inégalement, car n'ayant ni la même forme, ni la même longueur, ni les mêmes connexions, elles se trouvent placées, eu égard à l'allongement qu'elles doivent prendre, dans des conditions bien différentes. Il est évident que les effets de cet allongement, au point de vue morphologique, sont d'autant plus prononcés que les circonvolutions sont plus courtes, ou pour mieux dire, que les deux extrémités.sont plus rapprochées. Or, les circonvolutions pariétales sont d'autant plus courtes qu'elles sont plus externes. G'est donc la circonvolution sylvienne qui se replie le plus, et la circonvolution sagittale qui se replie le moins. C'est ce qu'on a déjà vu (p. 284, fig. 11) sur le cerveau de la loutre; et ce qu'on voit mieux encore sur le cerveau du renard (fig. 17), où la circonvolution sylvienne forme un pli brusque et profond, et où les suivantes décrivent des arcs de moins en moins fléchis, jusqu'à la circonvolution sagittale qui est la moins recourbée.

L'inflexion brusque de la circonvolution sylvienne produit la scissure de Sylvius (fig. 17, S) qui est tantôt presque verticale, tantôt très oblique en arrière et en haut, mais qui est toujours l'effet d'un plissement transversal, puisqu'elle est due à l'allongement de cette circonvolution.

La scissure de Sylvius est l'anfractuosité que forme le bord inférieur de la circonvolution sylvienne en se repliant tout à coup, de manière à se mettre en contact avec lui-même, ce qui lui permet de gagner beaucoup de longueur dans un petit espace. Cette scissure est presque constante, mais elle n'est pas torjours dis- 
posée de la même manière parce que ses connexions, sa direction, son existence même dépendent de la disposition des parties situées sous le bord inférieur de la circonvolution sylvienne.

La circonvolution sylvienne, comme les autres circonvolutions pariétales, s'arrête en avant sur le pli postrolandique, $\mathbf{P}^{\circ}$, qui est l'analogue de la circonvolution pariétale ascendante des primates et qui borde la scissure de Rolando; mais celle-ci, quoique séparant superficiellement le lobe pariétal du lobe frontal, n'interrompt pas, dans la profondeur, la continuité des deux lobes. Il y a d'ailleurs, au-dessus de l'arc inférieur de la scissure limbique, un pli de passage tantôt superficiel, tantôt profond (1), qui unit la circonvolution sylvienne avec le bord inférieur et

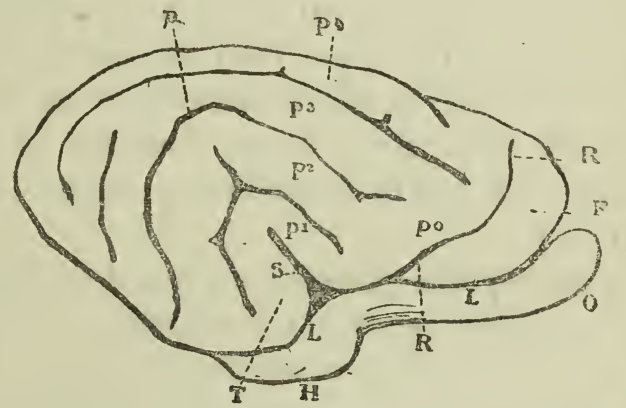

Fig. 17. Face externe de l'hémisphère droit du renard. O, lobe olfactif; II, lobe de l'hippocampe; LL, scissure limbique; RR, scissure de Rolando; S, scissure de Sylvius; F, lobe frontal; $\mathrm{P}$, circonvolution sylvienne, première pariétale de Leuret; $\mathrm{P}^{4}$, circonvolution sagittale, quatrième pariétale de Leuret; $p$, sillon pariétal primaire; Po, pli postrolandique, analogue de la circonvolution pariétale ascendante des primates; ' $\boldsymbol{T}$, lobule temporal du lobe pariétal.

externe du lobe frontal. Celui-ci se fixe sur le grand lobe, au niveau de la base du lobe olfactif. D'un autre côté, l'extrémitó postérieure de la circonvolution sylvienne est fixée, par le pli de passage rétro-limbique, sur l'extrémité postérieure du lobe de l'hippocampe; la circonvolution sylvienne forme done un arc complété en avant par le bord inférieur du lobe frontal, et fixé par ses deux extrémités sur le grand lobe limbique. J'appellerai cet arc l'arc sylvien.

(1) Ce pli de passage est quelquefois tout a fait superficiel ; il est presque superficiel chez la loutre (Voir plus haut, fig. 7 et $11, n^{\circ} 17$, p. 276 et 284 ). Lorsque ce pli est profond, la scissure de Rolando paraît communiquer avec la scissure limbique. 
Si l'arc sylvien n'était pas plus long que le bord correspondant du grand lobe, il s'appliquerait exactement sur lui, et la scissure limbique serait tout à fait simple, comme cela a lieu chez beaucoup de lissencéphales. Mais chez les gyrencéphales, l'arc sylvien présente un excès de longueur; il ne peut donc plus coïncider avec le grand lobe; il faut qu'il s'en sépare; et puisqu'il y est fixé par ses deux extrémités, il s'en sépare à sa partie moyenne, qui correspond à la partie antérieure du lobe de l'hippocampe.

De cet écartement résulte, chez le fœtus gyrencéphale, un intervalle déprimé qui a plus ou moins la forme d'une demiellipse, et qui est exactement l'analogue de la fosse de Sylvius des fœtus humains de cinq mois. Le bord inférieur de cette fosse est formé par le bord externe du grand lobe limbique, son bord supérieur par l'arc sylvien, son fond par une couche d'écorce cérébrale qui recouvre le noyau lenticulaire ou noyau extraventriculaire du corps strié, et qui constitue le lobule soussylvien, analogue au lobe de l'insula des primates. (Voy. p. 304, fig. $16, n^{\circ} \mathrm{II}, \mathrm{S}$.)

Le lobule sous-sylvien présente un degré de développement extrêmement variable. Lorsqu'il est volumineux, il reste plus ou moins apparent à l'extérieur et continue pendant toute la vie à séparer du grand lobe limbique la partie moyenne de l'arc sylvien; lorsqu'il est très petit, la circonvolution sylvienne, en s'accroissant, le recouvre, et revient s'appliquer sur le grand lobe après avoir décrit le méandre qui produit la scissure de Sylvius. Dans le premier cas, la fosse de Sylvius persiste, plus ou moins large, plus ou moins remplie par le lobule sous-sylvien, mais toujours plus ou moins déprimée au-dessous du niveau de l'arc sylvien qui l'entoure. Dans le second cas, elle se referme entièrement, et pour retrouver le lobule sous-sylvien rudimentaire, il faut écarter les bords de la scissure de Sylvius et de la partie de la scissure limbique qui est au-devant d'elle.

Les connexions du lobule sous-sylvien avec les parties qui l'entourent sont toujours les mêmes; toujours son écorce se continue sur son bord inférieur avec celle du grand lobe, sur son bord supérieur avec celle de l'arc sylvien; mais ces connexions se spécialisent en outre en certains points, lorsque le 
lobule est assez développé pour se diviser en plis secondaires. Ces plis sous-sylviens sont au nombre de deux. Ils sont longitudinaux. L'un est supérieur, l'autre inférieur. Il est rare qu'ils soient tous deux assez développés pour être superficiels l'un et l'autre; cette disposition s'observe pourtant quelquefois, par exemple, chez le tapir (fig. 18). J'appellerai l'inférieur le pli temporo-frontal, et le supérieur le pli temporo-pariétal.

Pour justifier ces dénominations, qui faciliteront beaucoup l'étude des analogies, je dirai par anticipation que le lobe temporal des primates résulte de la fusion du lobe de l'hippocampe atrophié, avec la portion du lobe pariétal qui est située en arrière de la scissure ou de la fosse de Sylvius, et que j'ai appelée le lobule temporal de ce lobe (voyez plus haut, p. 286); et comme, en étudiant les plis sous-sylviens des osmatiques, je me propose surtout de les comparer aux parties analogues du cerveau des primates, il est bon que les noms sous lesquels je les désigne ici puissent s'adapter ensuite à la description du cerveau des mammifères supérieurs. Or, l'enceinte sylvienne des primates se compose de trois parties, correspondant aux trois lobes temporal, pariétal et frontal; la portion temporale est située en arrière et au-dessous de la scissure de Sylvius; la partie frontale comprend la portion de la marge supérieure située en avant de la scissure de Rolando ; et la partie pariétale enfin forme le reste de la marge supérieure, entre la scissure de Rolando et l'extrémité terminale de la scissure de Sylvius. Je désignerai donc sous les mêmes noms les portions analogues de la région sylvienne des cerveaux osmatiques. Cela posé, je décrirai les deux plis sous-sylviens d'après le cerveau du tapir (fig. 18), où ils sont superficiels l'un et l'autre. Le pli inférieur ou temporo-frontal, $a a^{\prime}$, naît, en arrière, $a$, sur la partie postérieure du bord externe du lobe de l'hippocampe par une racine qui interrompt le trajet de la scissure limbique, $L L^{\prime} L^{\prime \prime}$; de là il se dirige horizontalement en avant, le long de la scissure limbique, et va joindre en $a^{\prime}$ le lobe frontal F, dans lequel il se termine; c'est ce pli qui chez les primates constitue l'insula. Le pli supérieur ou temporo-pariétal naît en $b$ de la partie postérieure de la circonvolution sylvienne, $\mathrm{P}^{1} \mathrm{P}^{1}$, longe le bord inférieur, $\mathrm{SS}$, de cette circonvolution, en se plaçant au-dessus du précédent, et va se terminer en $b^{\prime}$ 
sur la partie antérieure du lobe pariétal, en arrière de la scissure de Rolando. Ce second pli est l'analogue du pli de passage temporo-pariétal qui, chez les primates, traverse le fond de la scissure de Sylvius derrière l'insula, et qui s'étend de la première circonvolution temporale à la circonvolution pariétale externe ou sylvienne.

Ces deux plis étant grands et superficiels chez le tapir, le lobule sous-sylvien comble presque entièrement la fosse de Syl-

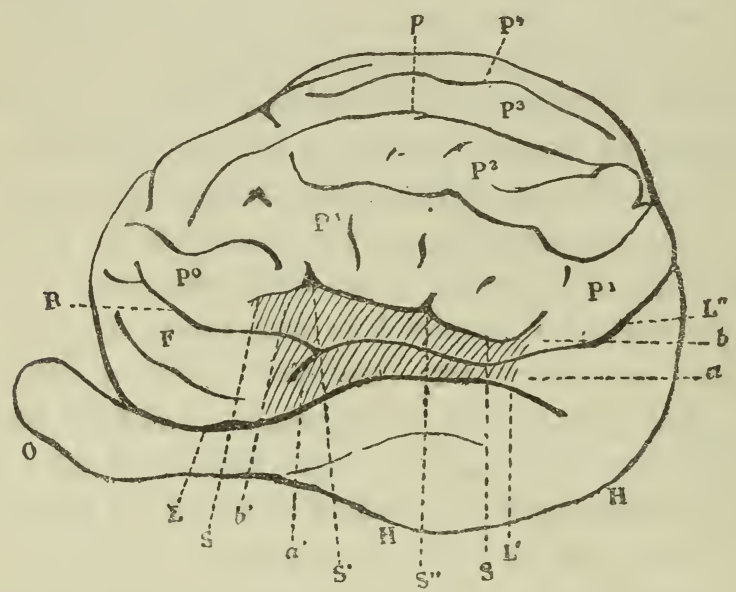

Fig. 18. Tapir, face exlerne de l'hémisphère gauche ; $\mathrm{O}$, lobe olfactif; HH, lobe de l'hippocampe ; $L L^{\prime} L^{\prime \prime}$, are inférieur de la scissure limbique, interrompu en a par le pli sous-sylvien inférieur; $R$, scissure de Rolando; $F$, lobe frontal; SS, bord inférieur de la circonvolution sylvienne ( $\left.\mathrm{P}^{\prime} \mathrm{P}^{\prime}\right)$ formant la marge supérieure de la fosse de Sylvius ; $a a^{\prime}$, pli sous sylvien inférieur ou temporo-frontal; $b b^{\prime}$, pli sous-sylvien supérieur, ou temporo-pariétal, allant aboutir au pli pariétal post-rolandique $\mathrm{P}^{0} ; p$, sillon pariétal primaire $; \mathrm{P}^{1} \mathrm{P}^{2} \mathrm{P}^{3} \mathrm{P}^{4}$, les quatre circonvolutions pariétales, numérotées d'après la nomenclature de Leuret.

vius, qui reste néanmoins un peu déprimée; il en résulte que la circonvolution sylvienne $\mathrm{P}^{1}$, fortement déviée vers le haut, décrit son arc autour d'un lobule volumineux, et que son excès de longueur ne se traduit que par le pli brusque et profond, qui, chez la plupart des gyrencéphales, constitue la scissure de Sylvius. Son bord inférieur, SS, est simplement sinueux ; on y remarque toutefois en $\mathrm{S}^{\prime}$ et $\mathrm{S}^{\prime \prime}$ deux incisures plus profondes que les autres, sans qu'on puisse considérer l'une d'elles, plutôt que l'autre, comme le rudiment de la scissure de Sylvius.

Chez le cheval, les deux plis sous-sylviens sont encore assez 
volumineux, mais ils ne sont superficiels que dans leur partie antérieure (voy. fig. 19). Le pli temporo-frontal, $a$, est moins développé que l'autre; en avant, à son extrémité frontale, il lorme un pli épais et bien distinct, appliqué en $\mathrm{L}^{\prime}$ sur le bord externe du grand lobe; mais un peu plus en arrière il s'amincit, se rétrécit et s'insère sur ce bord de $L^{\prime}$ en $L^{\prime \prime}$, à travers la scissure limbique qui à ce niveau n'a guère plus de 1 millimètre de profondeur. Cette insertion ne se fait pas au moyen d'une ra-

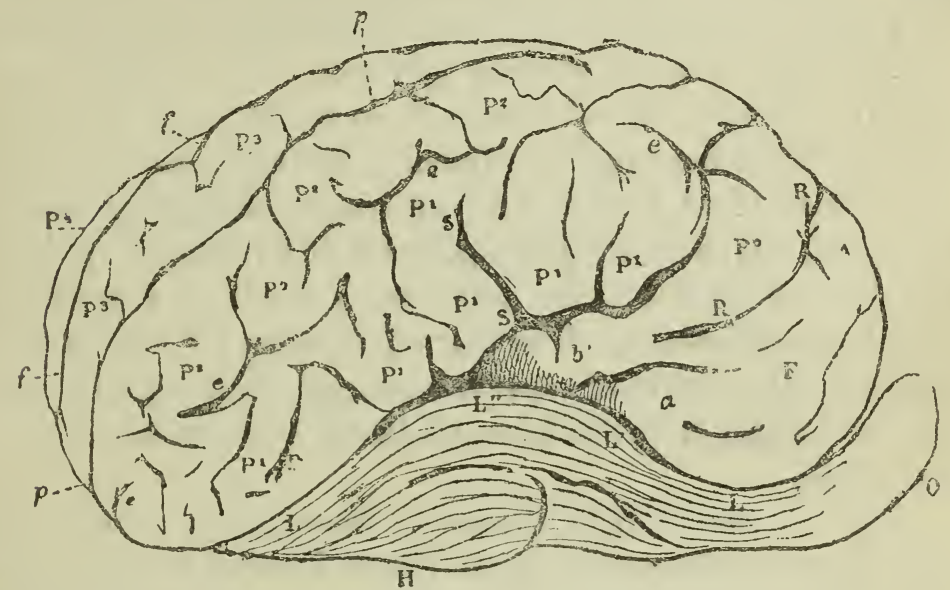

Fig. 19. Cheval, face externe de l'hémisphère droit. $\mathrm{O}$, lobe olfactif; H, lobe LL, are inférieur de la scissure limbique; RR, scissure de Polando; F, lobe frontal; $a b^{\prime}$; lobule sous-sylvien, superficiel en avant; $a$, pli temporo-frontal ; $b^{\prime}$, pli temporo-pariétal ; Ss, scissure de Sylvius ne communiquant pas avec la scissure limbique; $p p$, sillon pariétal primaire ou moyen; eeee, sillon pariétal externe; $f$, sillon pariétal interne; $\mathrm{P}^{\prime} \mathrm{P}^{\prime} \mathrm{P}^{\prime}$, circon volution sylvienne ou première de Leuret; $\mathrm{P}^{4}$, circonvolution sagittale ou quatrième de Leuret, cachée en avant par le profil de la circonvolution, $\mathrm{P}^{3}$. $\mathrm{P}^{\circ}$. circonvolution pariétale post-rolandique.

cine circonscrite, comme cela a lieu chez le tapir, mais par une large et mince lame de substance corticale, analogue à celle qui chez l'homme s'étend du lobe temporal au bord inférieur de l'insula, par-dessous la rigole inférieure de l'insula. Le pli temporopariétal, $b^{\prime}$, est plus gros et surtout plus long ; en avant, il aboutit à l'extrémité inférieure de la scissure de Rolando, RR, et se continue, derrière cette scissure, avec la partie antérieure du lobe pariétal $\mathrm{P}^{\circ}$, partie comparable à la circonvolution pariétale ascendante des primates; mais de plus il se continue avec le lobe frontal par une seconde racine antérieure, qui passe au-dessous et 
en avant de la scissure de Rolando. Cette racine frontale, d'ailleurs très étroite chez le cheval, manque sur tous les autres cerveaux que j'ai étudiés. De là, le pli temporo-pariétal, gros et un peu flexueux, se porte en arrière, entre l'arc sylvien, dont il est séparé par une rigole profonde (rigole supérieure de l'insula des primates), puis s'enfonce dans la fosse de Sylvius, et va s'insérer sur la face profonde du lobule temporal, derrière la scissure de Sylvius, SS. Quoique en partie caché dans le fond de la fosse de Sylvius, le lobule sous-sylvien du cheval est encore assez gros pour relever d'une manière notable le bord de l'arc sylvien; mais la circonvolution sylvienne est si longue, que, malgré ce détour, elle est obligée de se replier en formant une scissure de Sylvius longue et profonde, SS. Cette scissure, presque verticale (c'est à peine si elle est un peu oblique en haut et en arrière) émane de la rigole supérieure du lobule sous-sylvien : elle n'atteint pas la scissure limbique, dont elle est séparée par la racine temporale du pli temporo-pariétal ou pli sous-sylvien supérieur. On remarquera que la scissure de Rolando du cheval est peu oblique, et qu'elle laisse au-devvant d'elle un lobe frontal assez grand.

Jusqu'ici, chez le tapir et chez le cheval, nous avons vu les deux plis sous-sylviens développés l'un et l'autre à un degré suffisant pour subdiviser la fosse de Sylvius en deux régions dis. tinctes, l'une supérieure, l'autre inférieure. La constitution de cette fosse se simplifie chez les ruminants, par suite de l'atrophie du pli temporo-frontal; mais leur pli temporo-pariétal est très volumineux, et superficiel dans toute son étendue. La figure 20 représente la face externe de l'hémisphère dı chevreuil. La scissure de Rolando, RR, très oblique, ne laisse au devant d'elle qu'un lobe frontal extrêmement petit, $\mathrm{F}$, dont l'extrémité postérieure et inférieure, terminée en pointe, s'amincit et disparaît presque aussitôt dans le fond de la scissure limbique, L, en adhérant au grand lobe limbique sur le hord duquel elle se perd. Le lobule sous-sylvien est donc réduit, pour ainsi dire, au pli temporo-pariétal. Celui-ci, né en $a$, de la partie postérieure de la circonvolution sylvienne, par une racine superficielle, remplit toute la fosse de Sylvius qu'il parcourt d'arrière en avant en longeant la scissure limbique, $\mathrm{L}^{\prime} \mathrm{L}$, et va se terminer en $a^{\prime}$, 
derrière la scissure de Rolando, dans l'extrémité antérieure du lobe pariétal. La fosse de Sylvius étant très étroite, l'arc sylvien est très surbaissé, et ne fournit que très peu de développement à la circonvolution sylvienne, $\mathrm{P}^{1} \mathrm{P}^{1}$, qui se plisse très profondément en formant une grande scissure de Sylvius à peu près verticale, SS. Celle-ci est une émanation de la rigole sylvienne supérieure, qui est partout séparée de la scissure limbique (ou rigole inférieure) par le pli temporo-pariétal. Cette description est applicable à tous les ruminants, à l'exception du chevrotain de Java, le plus petit d'entre eux. Chez lui, les deux plis sous-

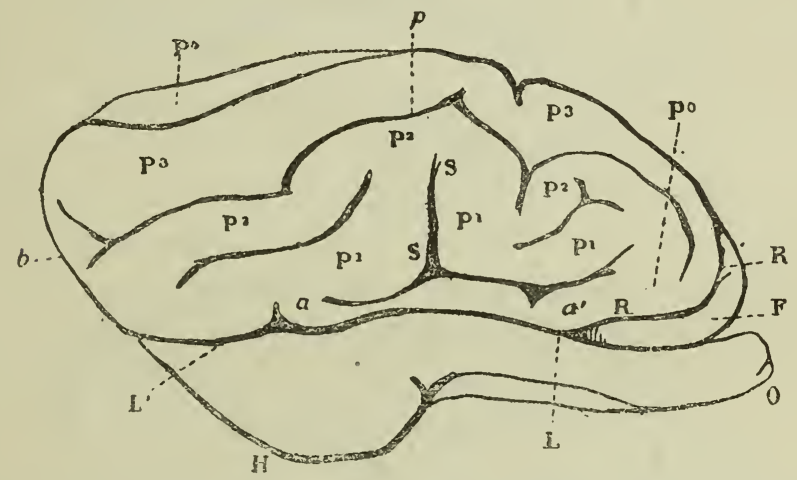

Fig. 20. Chevreuil, face externe de l'hémisphère droit; $\mathrm{O}$, lobe olfactif; $\mathrm{H}$, lobe de l'hippocampe ; LL', arc inférieur de la scissure limbique ; RR, scissure de Rolando; $F$, lobe frontal ; SS, scissure de Sylvius ne communiquant pas avec la scissure limbique ; $a a^{\prime}$, pli soussylvien supérieur ou temporo-pariétal; $b$, pôle de l'hémisphère ; $p$, sillon pariétal primaire ; $\mathrm{P}^{\prime} \mathrm{P}^{\prime}$, circonvolution sylvienne ou première pariétale de Leuret; $\mathrm{P}^{2} \mathrm{P}^{\mathbf{2}}$, seconde pariétale en partie fusionnée avec la précédente; P4, circonvolution sagittale, ou quatrième de Leuret; $\mathrm{P}^{\prime} \mathrm{P}^{\prime}$, troisième pariétale de Leuret; $\mathrm{P}$, circonvolution post-rolandique.

sylviens paraissent manquer, et, dès.lors, la scissure de Sylvius, très rudimentaire, aboutit à la scissure limbique. J'ai choisi comme type du cerveau des ruminants le cerveau très simple du chevreuil. Chez cet animal, la circonvolution sylvienne, $\mathrm{P}^{\mathbf{1}}$, et celle qui la surmonte, $\mathrm{P}^{2}$, sont fusionnées dans leur partie moyenne, tandis que la séparation du groupe sylvien, $\mathrm{P}^{1} \mathrm{P}^{2}$, et du groupe sagittal, $\mathrm{P}^{3} \mathrm{P}^{4}$, est établie complètement par un sillon long et profond, $p$, qui est le sillon pariétal primaire.

On vient de voir que le pli temporo-pariétal des ruminants est toujours volumineux et superficiel. La diminution du volume de ce pli amène dans la disposition de la fosse de Sylvius 
des modifications que l'on peut étudier dans la famille des porcs, de l'ordre des pachydermes. Chez le babiroussa, le pli temporopariétal est tout aussi volumineux que chez les ruminants, et il est superficiel dans toute son étendue. Chez le cochon domestique, il est moins développé et disparaît plus ou moins complè-

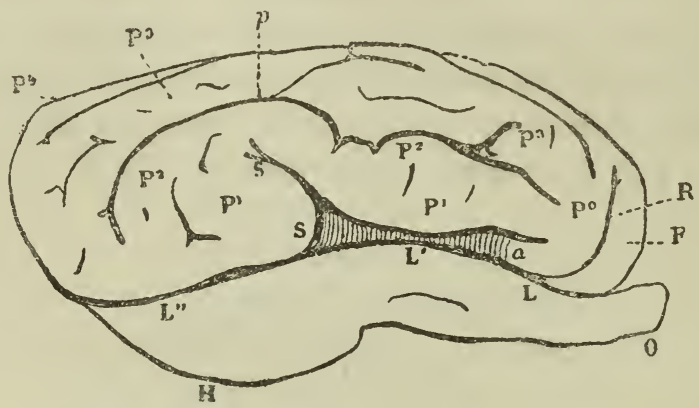

Fig. 21.

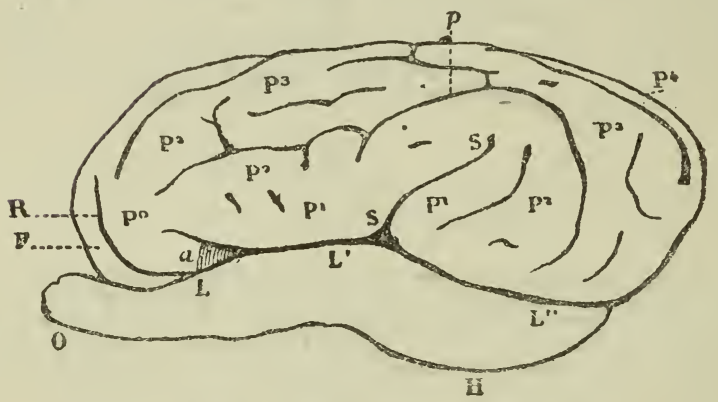

Fig. 22.

Fig. 21 et 22. Porc domestique. Fig. 21. Face externe de l'hémisphère droit; fig. 22, face externe de l'hémisphère gauche du même sujet.

Les lettres comme sur la figure 20. La fosse de Sylvius, entr'ouverte du còté droit, où le pli temporo-pariétal a est visible dans la plus grande partie de son étendue, est refermée du côté gauche (fig. 22), où l'on n'apercoit que l'extrémité antérieure de ce pli, et où la scissure de Sylvius rejoint la scissure limbique.

tement sous le bord de la circonvolution sylvienne. Sur l'hémisphère droit d'un porc domestique (voy. fig. 21), on voit que ce pli, superficiel à son extrémité antérieure ou pariétale, $a$, est presque entièrement caché dans sa partie moyenne par la circonvolution sylvienne qui est très abaissée, et qui arrive presque alı contact du grand lobe limbique; il reparaît un peu plus en arrière; on n'aperçoit pas son insertion temporale, qui se fait 
sur la face profonde de la circonvolution sylvienne, derrière la scissure de Sylvius, SS ; mais en écartant les bords de cette scissure, et en soulevant la partie antérieure de l'arc sylvien, on voit ce pli dans toute son étendue. On conçoit aisément que, si le pli temporo-pariétal était un peu plus réduit, la fosse de Sylvius se refermerait entièrement sur lui. C'est ce qui a lieu sur l'hémisphère gauche du même sujet (fig. 22). L'extrémité antérieure du pli temporo-pariétal, $a, \mathrm{y}$ est seule apparente ; elle disparaît aussitôt sous la circonvolution sylvienne, qui s'applique exactement sur le grand lobe limbique, en longeant la scissure limbique, $\mathrm{L}^{\prime}$, et il en résulte que la scissure de Sylvius descend jusqu'à cette dernière scissure, dont elle se détache comme une branche. Il n'y a donc pas une différence essentielle entre les cas où la scissure de Sylvius aboutit directement à la scissure limbique et ceux où elle s'arrête sur la rigole supérieure de la fosse sylvienne; il suffit pour produire ce léger changement que le pli temporo-pariétal soit un peu plus ou un peu moins volumineux, et la différence est si faible que les deux dispositions peuvent exister respectivement sur les deux hémisphères d'un même cerveau. J'ajoute que, sur deux cerveaux de sangliers que je possède, et qui sont d'ailleurs très semblables aux cerveaux des pores domestiques, le pli temporo-pariétal est profond à droite et à gauche, de sorte que la disposition de la région sylvienne est exactement la même que sur la figure 22.

Comme conséquence de l'amoindrissement du lobule soussylvien, la circonvolution sylvienne, n'étant plus déviée par ce lobule, ne peut plus s'allonger que par plissement; la scissure de Sylvius, qui est l'effet de ce.plissement transversal, devient donc relativement plus longue; et, ne trouvant plus assez d'espace dans le sens vertical (c'est-à-dire transversal), elle s'incline obliquement en arrière. Il y a donc corrélation entre la direction de cette scissure et ses connexions. Elle est oblique en haut et en arrière lorsqu'elle aboutit à la scissure limbique, c'est-à-dire lorsque la fosse sylvienne est refermée; elle est au contraire à peu près verticale, et reportée en outre un peu plus en avant lorsqu'elle aboutit à la rigole supérieure de la fosse sous-sylvienne, c'est-à-dire lorsque la fosse sylvienne est plus ou moins ouverte, et que le lobule sous-sylvien est apparent à l'extérieur. 
Cette corrélation est constante, et nous allons la retrouver chez les carnassiers.

On vient de voir que chez les porcs le pli temporo-pariétal, quoique déjà très atténué, n'est pas encore entièrement recouvert par l'arc sylvien, et que sa racine antérieure ou pariétale est encore superficielle. C'est elle qu'on aperçoit en $a$ (fig. '2 I et 22), au-dessus de la scissure limbique, L, en arrière de la scissure de Rolando, R, et au-dessous d'une anfractuosité horizontale qui se détache de la scissure limbique, et qui constitue l'extrémité antérieure de la rigole supérieure de la fosse de Sylvius.

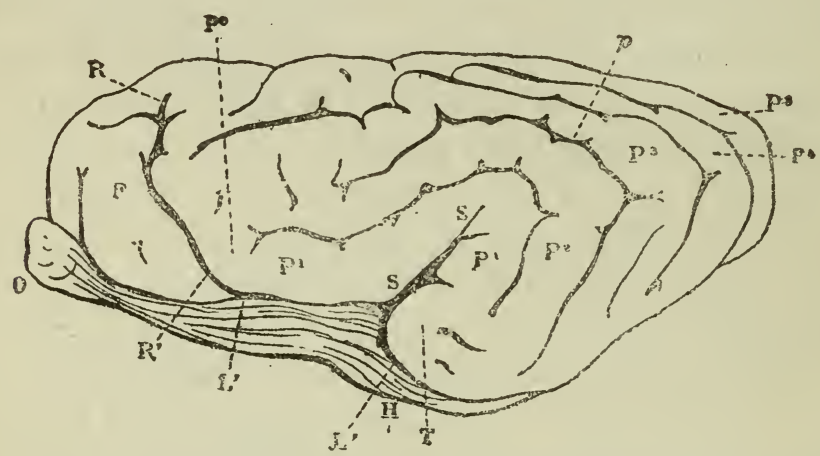

Fig. 23. Chien de berger; face externe de l'hémisphère gauche. $\mathrm{O}$, lobe olfactif; $H$, lobe de l'hippocampe; $\mathrm{LL}^{\prime}$, arc inférieur de la scissure limbique; $\mathrm{RK}^{\prime}$, scissure de Rolando; $\mathbf{F}$, lobe frontal; SS, scissure de Sylvius; T, lobule temporal du lobe pariétal ; $\mathrm{P}^{0}$, circonvolution pariétale post-rolandique ; $\mathrm{P}^{\prime} \mathrm{P}^{\prime}$, circonvolution sylvienne; $\mathrm{P}^{\mathbf{6}}$, circonvolution sagittale; $p$, sillon pariétal primaire. Les circonvolutions pariétales sont numérotées à partir de la scissure de Sylvius, suivant le procédé de Leuret. Les deux circonvolutions " et P' résultent de la subdivision $\mathrm{P}^{\prime}$ du renard (voir fig. 17, p. 307).

Cette racine antérieure du pli temporo-pariétal disparaît à son tour chez les carnassiers. Je représente ici la face externe du cerveau du chien de berger (fig. 23); on peut comparer cette figure avec la figure 11 (p. 284), qui représente le cerveau de la loutre, et avec la figure 17 (p. 307), qui représente celui du renard. Chez ces animaux, comme chez tous les autres carnassiers, le lobule sous-sylvien est entièrement profond. La scissure de Sylvius (fig. 23, SS), très oblique en haut et en arrière, se détache directement de la scissure limbique, LL'; la circonvolution sylvienne, $\mathrm{P}^{1} \mathrm{P}^{1}$, repliée autour de la scissure de Sylvius, s'applique exactement, dans le reste de son étendue, sur le bord 
externe du grand lobe limbique, et la fosse de Sylvius est entièrement refermée, de sorte que rien n'indique plus à l'extérieur la rigole supérieure de la fosse de Sylvius. La scissure de Rolando, $\mathrm{RR}^{\prime}$, est donc la première anfractuosité qui se détache de la scissure limbique en avant de la scissure de Sylvius; mais la scissure de Rolando n'est pas pour cela une branche de la scis. sure limbique. On a déjà vu que chez la loutre (voy. plus haut, p. 284 et fig. 11, $n^{\circ} 17$ ) elle en est séparée par un pli presque superficiel, qu’on aperçoit aisément après avoir enlevé la pie.

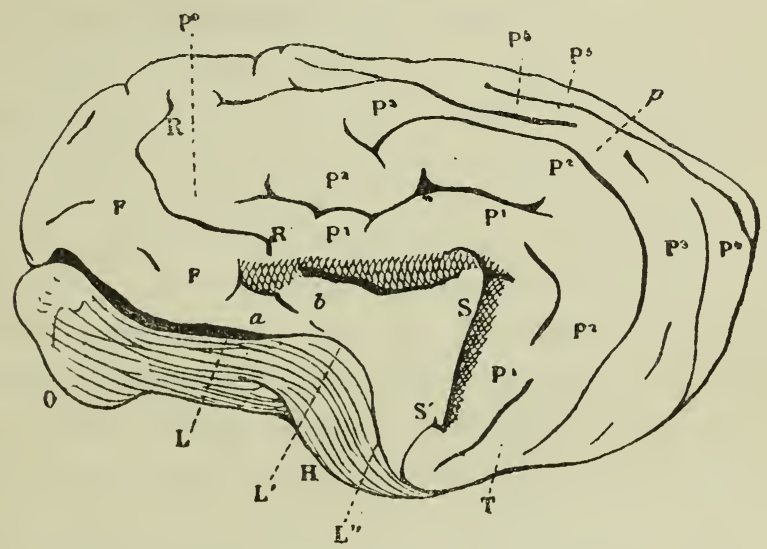

Fig. 24. Chien ratier, face esterne de l'hémisphère gauche, sur laquelle on a dilaté la scissure de Sylvius et relevé l'arc sylvien pour mettre à découvert la fosse de Sylvius. La face profonde de la circonvolution sylvienne, $\mathrm{P}^{\prime} \mathrm{P}^{\prime}$, devenue apparente, est ombrée.

$\mathbf{S}^{\prime} a$, pli sous-sylvien inférieur ou temporo-frontal; $\mathbf{S}$ 'S $b$, pli sous-sylvien supérieur ou temporo-pariétal.

Les autres lettres comme sur la figure 23.

mère. Ce même pli existe chez le chien ; seulement il est plus profond. Pour le rendre apparent, il faut prendre un cerveau frais ou à peine durci pendant vingt-quatre heures dans l'alcool ; la circonvolution sylvienne, encore souple, se laisse aisément soulever avec le manche d'un scalpel; on arrache délicatement avec une pince fine les prolongements profonds de la pie-mère, et, dilatant ainsi la scissure de Sylvius et la partie antérieure de la scissure limbique, on met à découvert tout le lobule soussylvien, c'est-à-dire tout le fond de la fosse de Sylvius. La pièce ainsi disposée peut être aisément moulée. La figure 24 représente l'un de ces moules pris sur l'hémisphère gauche d'un chien 
raticr. Un léger relief linéaire, $L L^{\prime} L^{\prime}$, formé par le bord externe du grand lobe limbique, représente la lèvre inférieure de la scissure limbique, et forme le bord inférieur de la fosse de Sylvius. Celle-ci est triangulaire; ses deux autres bords sont entourés par la circonvolution sylvienne, $\mathrm{P}^{1} \mathrm{P}^{1}$, dont la face profonde, retournée ver's l'extérieur, est indiquée sur la figure par une ombre légère. On voit en $a b$ que cette circonvolution se continue avec le lobe frontal, par un pli qui passe an-dessous de l'extrémité inférieure de la scissure de Rolando, $\mathrm{R}$, et qui la sépare de la fosse de Sylvius (1). Le lobe sous-sylvien, $a b S^{\prime}$, qui forme tout le fond de la fosse, est un peu déprimé par rapport au bord limbique, $\mathrm{LL}^{\prime} \mathrm{L}^{\prime}$, sur lequel il s'insère régulièrement dans toute sa longueur. Il se continue avec la circonvolution sylvienne, en arrière, par-dessous le fond de la scissure de Sylvius, $\mathrm{SS}^{\prime}$, en haut, par-dessous le fond de la rigole supérieure de la fosse sylvienne, $a b \mathrm{~S}$; mais de plus il est uni à l'extrémité postérieure et inférieure de cette circonvolution par un pli large, court, et relativement peu profond, $S^{\prime}$, et à son extrémité antérieure par un autre pli, $b$. Ces deux plis $b$ et. $\mathrm{S}^{\prime}$ représentent les deux racines du pli temporo-pariétal, dont la partie moyenne ne fait aucune saillie. Enfin, un dernier pli, $a$, situé tout en avant, fait communiquer le lobule sous-sylvien avec le lobe frontal; c'est la racine antérieure du pli temporo-frontal. Nous retrouvons donc dans le lobule sous-sylvien du chien le vestige des deux plis sous-sylviens observés chez les ruminants et les pachydermes. Ce lobule est très étalé et assez large, mais tout à fait plat; il ne soulève pas les bords de la circonvolution sylvienne, et celle-ci, s'appliquant sur le grand lobe limbique, referme entièrement la scissure de Sylvius.

Ce type, en ce qui concerne la région sylvienne, se reproduit chez tous les carnassiers, quoique le nombre des circonvolutions pariétales de ces animaux soit variable, ainsi que leurs connexions. Chez les primates gyrencéphales, le lobule sous-sylvien

(1) Ce pli très prononcé sur la figure 24, est souvent très rudimentaire, et il faut alors une certaine altention pour en découvrir le vestige. Il est quelquefois très inégalement développé à droite et à gauche. Ainsi il est presque nul sur l'hémisphère droit du cerveau dont l'hémisphère gauche est représenté sur la figure 24. Les moules de ces deux hémisphères sont déposés dans le Musée (Musce Broca). 
grandit d'une manière notable; celui des primates supérieurs devient assez volumineux et assez compliqué pour constituer un véritable lobe, qui est celui de l'insula; mais ce lobe, malgré son volume, ne devient pas superficiel; il reste caché au fond d'une profonde scissure, parce que la circonvolution sylvienne, encore plus développée que lui, le recouvre entièrement et referme au-dessus de lui la fosse de Sylvius.

Nous venons d'étudier, chez les gyrencéphales osmatiques, les connexions de l'arc inférieur du grand lobe limbique avec la masse circonvolutionnaire. Passons maintenant à l'arc supérieur, formé par le lobe du corps calleux.

On a vu plus haut que, chez la loutre, la partie postérieure du grand lobe est unie à la masse circonvolutionnaire par le pli de passage rétro-limbique ( $\mathrm{p}$. 280). Ce pli est constant. Il est presque toujours superficiel et il interrompt alors la scissure limbique qu'il divise en deux arcs, l'un inférieur, longeant le lobe de l'hippocampe, l'autre supérieur, contournant le lobe du corps calleux. Il parait au premier abord mănquer chez le chien domestique et chez le loup; mais on le retrouve aisément en écartant les bords de la scissure limbique. Sur l'un de nos cerveaux de tapir, ce pli est superficiel à gauche et profond à droite; sur l'autre, il est superficiel des deux côtés; sa position superficielle ou profonde n'a donc que peu d'importance.

$1 l$ naît du grand lobe limbique là où le lobe de l'hippocampe se continue avec le lobe du corps calleux. Il en naît par une racine plus ou moins large, quelquefois assez étroite, qui est tantôt simple, tantôt subdivisée en deux. On a vu (fig. $7, n^{\circ} 9$, p. 276 , et fig. $9, \mathrm{n}^{\circ} 9, \mathrm{p} .281$, et fig. $6, c, \mathrm{p} .274$ ) qu'il est simple chez la loutre; il l'est aussi chez le tapir, chez le chien où il est profond, chez la martre, le renard, le chat, etc. Il est alors très court et n'alteint pas le bord de l'hémisphère vers lequel il se dirige; mais en retournant l'hémisphère on voit que sur ce bord, au même niveau à peu près, commence le grand sillon pariétal primaire (voy. p. 304), qui se porte de là sur la face convexe de l'hémisphère jusqu’à son extrémité antérieure, en séparant la zone sagittale de la zone sylvienne. Cette partie du bord postérieur de l'hémisphère peut être considérée comine un pôle, autour duquel les circonvolutions longitudinales prennent leur ori- 
gine, et par l'intermédiaire duquel elles communiquent toutes plus ou moins directement les unes avec les autres et avec le pli rétro-limbique. Les normas latérale, supérieure et inférieure de l'hémisphère, ne montrent pas cette disposition; mais elle peut s'apercevoir sur la norma postérieure, - car on sait que chez la plupart des mammifères autres que les primates, la face cérébelleuse ou tentoriale de l'hémisphère est très relevée et devient plus ou moins postérieure, le cervelet étant situé plus ou moins en arrière du cerveau. Chez le tapir, cette face est tout à fait

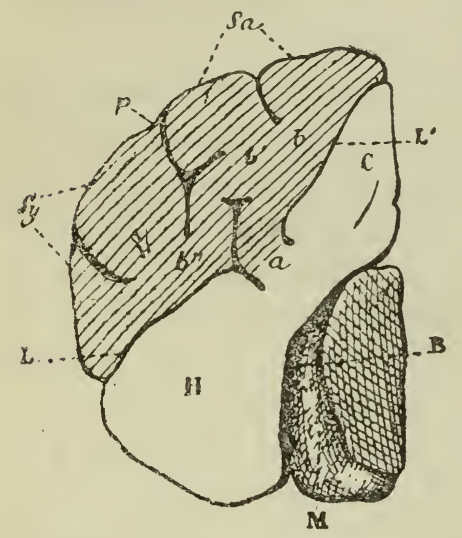

Fig. 23. Face postérieure et tentoriale de l'hémisphère gauche du tapir. $M$, le pédoncule cérébral coupé près de son entrée dans l'hémisphère; $\mathrm{B}$, la grande fente de Bichat; $H$, extrémité postérieure du lobe de l'hippocampe; C, extrémité postérieure du lobe du corps calleux; $L$ ', arc inférieur de la scissure limbique; $L$, son arc supérieur ; $a$, pli de passage rétro-limbique; $b b^{\prime} b^{\prime \prime}$, pli du pôle de l'hémisphère; $p$, sillon pariétal primaire; $S a$, origine des deux circonvolutions du groupe sagittal; $S y$, origine des deux circonvolutions du groupe sylvien. postérieure, et on peut y étudier facilement la constitution du pôle de l'hémisphère. La figure 25 représente la face postérieure de l'hémisphère gauche du tapir. On y voit l'extrémité postérieure du grand lobe limbique, $\mathrm{CH}$, contournant le pédoncule cérébral, $M$, qu'on a coupé un peu en dedans de son entrée dans l'hémisphère. Le lobe du corps calleux, $\mathrm{G}$, et le lobe de l'hippocampe, $\mathrm{H}$, se continuent l'un avec l'autre au niveau du pli rétrolimbique, $a$; celui-ci interrompt la scissure limbique, dont l'arc inférieur, $\mathrm{L}$, se dirige en bas et en dehors et dont l'arc supérieur, $L^{\prime}$, se dirige en haut et en dedans pour se portér sur la face interne de l'hémisphère (où on le retrouvera sur la figure 28 ci-après, p. 326).

Le pli rétro-limbique aboutit au pôle de l'hémisphère, $b$, $b^{\prime}, b^{\prime \prime}$, sur lequel naissent les trois sillons longitudinaux qui divisent le lobe pariétal en quatre circonvolutions. Le sillon moyen ou sillon pariétal primaire, $p$, sépare les deux circonvolutions internes, $S a$ (groupe sagittal), des deux circonvolutions externes, $S y$ (groupe sylvien). Il est situé vis-à-vis le pli rétro-limbique, mais il ne l'atteint pas; il en est séparé par un 
pli transversal, $b^{\prime} b^{\prime \prime}$, qui fait communiquer l'origine des deux circonvolutions internes avec celle des deux circonvolutions externes. Lorsque la face postérieure du cerveau est moins relevée, lorsque la masse circonvolutionnaire se prolonge audessus du cervelet, les divers sillons qui aboutissent au pôle se contournent et se compliquent plus ou moins, mais jamais ces sillons ne se prolongent jusqu'à la scissure limbique; ils en sont séparés par un pli transversal plus ou moins sinueux qui établit la continuité entre toutes les circonvolutions pariétales à leur extrémité postérieure, et qui constitue leur origine, et toujours

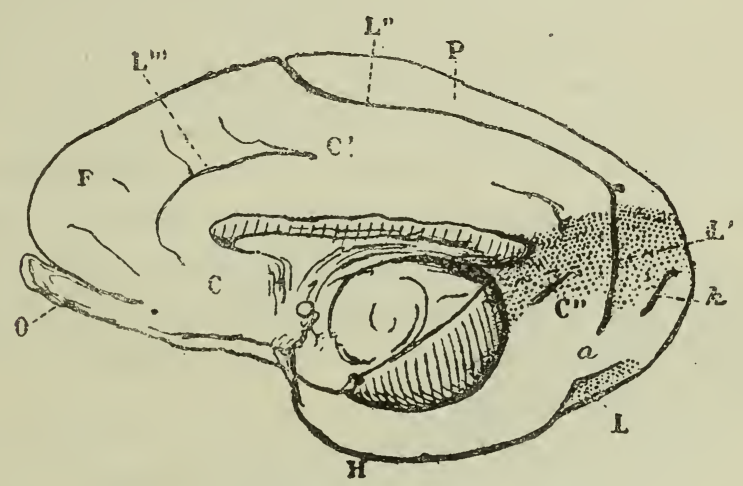

Fig.(26. Cherreuil, face interne de l'hémisphère droit. O, lobe olfactif; H, lobe de l'hippocampe; $\mathrm{CC}^{\prime} \mathrm{C}^{\prime \prime}$, lobe du corps calleux; F, lobe frontal ; P, lobe pariétal; L, arc inférieur de la scissure limbique ; $\mathbf{L}^{\prime} \mathrm{L}^{\prime \prime}$, son arc supérieur, ou scissure sous-pariétale; $\mathrm{L}^{\prime \prime \prime}$, sillon sous-frontal ; $a$, pli de passage rétro-limbique; $k$, sillon calcarin très court.

ce pli communique avec le grand lobe limbique par le pli rétrolimbique, qui peut dès lors être considéré comme la racine commune des circonvolutions pariétales.

Je viens de dire que le pli rétro-limbique est simple chez les carnassiers, comme chez le tapir ; mais il ne l'est pas toujours ; il est quelqu^fois subdivisé dans sa longueur par un sillon longitudinal qui est l'analogue de l'importante scissure calcarine des primates, ce qui mérite par conséquent quelque attention.

Ce sillon existe, plus ou moins développé, chez la plupart des pachydermes que j'ai pu me procurer, et chez tous les ruminants. Il n'est que rudimentaire chez le chevreuil (fig. 26). Le pli rétro-limbique $a$, interrompant la scissure limbique, LL', se porte vers le bord postérieur de l'hémisphère, mais avant de

T. $v$. 
l'atteindre il est subdivisé par un petit sillon, $k$, en deux parties, l'une supérieure, comprise entre $k$ et L', qui va, sur la portion adjacente de la face convexe, fournir l'origine des deux circonvolutions du groupe sagittal, l'autre inférieure, comprise entre $k$ et $\mathrm{L}$, qui va fournir de même l'origine des deux circonvolutions du groupe sylvien. Du reste, ce petit sillon, $k$, ne s'étend ni jusqu'à la base du pli, ni jusqu'au bord de l'hémisphère ; en arrière de lui la racine sagittale et la racine sylvienne communiquent ensemble, et, si l'on retourne l'hémisphère, on voit que le point vers lequel il se dirige est le pôle, marqué par la lettre $b$, sur la figure 20 (p. 313), qui représente la face externe de l'hémisphère droit du chevreuil. Là vient aboutir aussi l'extrémité postérieure bifurquée du grand sillon pariétal primaire, $p$, qui sépare le groupe sagittal du groupe sylvien. Le sillon, $k$, marque la même séparation sur la face postéro-inférieure de l'hémisphère. Les deux sillons ne forment donc qu'un même système, néanmoins ils ne se continuent jamais l'un avec l'autre, parce que toujours le pôle de l'hémisphère s'interpose entre eux sous la forme d'un pli qui s'étend de la racine sagittale à la racine sylvienne.

Je prouverai plus loin que le petit sillon, $k$, est l'analogue de la grande scissure calcarine des primates. Je demande donc la permission de l'appeler le sillon calcarin, quoique l'ergot (calcar) ou petit hippocampe, d'où son nom est tiré, n'existe que chez les primates. Le sillon calcarin est à peine plus long chez la chèvre que chez le chevreuil, et chez le mouton que chez la chèvre. Chez le daim et l'antilope il atteint une longueur de 15 millimètres, en se prolongeant de plus en plus vers la base du pli rétro-limbique. Ghez le chameau enfin, et surtout chez le cheval (fig. $27, k$ ), il subdivise entièrement ce pli jusqu'à sa base, de sorte qu'il atteint le grand lobe limbique, comme le fait la scissure calcarine des primates. Il y a donc alors deux plis rétrolimbiques, l'un supérieur, $a$, pour le groupe sagittal, l'autre infórieur, $a^{\prime}$, pour le groupe sylvien. Le premier représente le pli cunéo-limbique des primates (lequel est profond chez l'homme et les gibbons), le second représente la circonvolution qui forme chez ces animaux le bord inférieur de la scissure calcarine (et qui est la cinquième occipitale chez l'homme et les anthro- 
poïdes). A son extrémité postérieure, le sillon calcarin du cheval arrive jusqu'à la pointe de l'hémisphère; là il est contourné par le pli, $b$, qui joint le groupe sagittal au groupe sylvien. Sur ce pli long et sinueux viennent se terminer ou plutôt commencer les trois sillons longitudinaux de la face convexe, savoir : $1^{\circ} \mathrm{le}$ grand sillon pariétal primaire,' $p$, qui sépare le groupe sylvien du groupe sagittal (c'est le sillon $p p$ de la figure 19, p. 311); $2^{\circ}$ le sillon pariétal externe, $e$, qui sépare l'une de l'autre les deux circonvolutions du groupe sylvien (c'est le sillon e e e e de la fi-

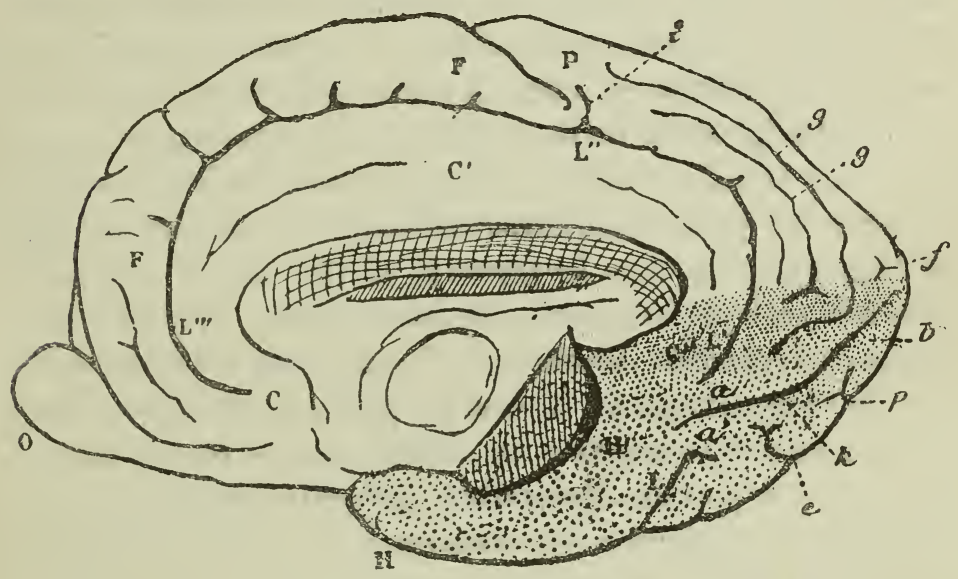

Fig. 27. Cheval, face interne de l'hémisphère droit. O, lobe olfactif; $\mathrm{HH}^{\prime}$, lobe de l'hippocampe ; $\mathrm{CC}^{\prime} \mathrm{C}^{\prime \prime}$, lobe du corps calleux; $\mathbf{F F}$, face interne du lobe frontal ; $\mathrm{P}$, face interne du lobe pariétal; $L$, arc inférieur de la scissure limbique; $L^{\prime} L^{\prime \prime} L^{\prime \prime \prime}$, arc supérieur; $L^{\prime} L^{\prime \prime}$, sa portion pariétale ou scissure sous-pariétale; $\mathrm{L}^{\prime \prime} \mathrm{L}^{\prime \prime \prime}$, sa portion frontale ou scissure sousfrontale; $a a^{\prime}$, pli de passage rétro-limbique, subdivisé en deux par le sillon calcarin $k$; $b$, le póle de l'hémisphère; $p$, origine du sillon pariétal primaire; $e$, origine du sillon pariélal externe; $f$, origine du sillon pariétal interne; $i$, incisure fronto-pariétale interne.

gure 19, où il est interrompu par plusieurs plis de passage); $3^{0}$ le sillon pariétal interne, $f$, qui sépare l'une de l'autre les deux circonvolutions du groupe sagittal (c'est le sillon $f f$ de la figure 19). Indépendamment des sillons de la face externe, il existe chez le cheval plusieurs sillons longitudinaux $g g$ (fig. 27), qui subdivisent en plusieurs plis secondaires la face interne de la circonvolution sagittale, et qui naissent tous plus ou moins au-dessus du sillon calcarin. L'extrémité postérieure du cerseau de cet animal est donc très compliquée, mais en définitive toutes les circonvolutions pariétales naissent autour du sillon 
calcarin, et prennent par conséquent leur origine sur la partie postérieure et inférieure du grand lobe limbique, par l'intermédiaire des deux branches du pli de passage rétro-limbique.

La scissure limbique, interrompue par ce pli, reprend aussitôt son trajet autour de l'arc supérieur du grand lobe, c'est-à-dire autour du lobe du corps calleux, dont elle suit la convexité, et qu'elle sépare plus ou moins complètement des circonvolutions environnantes.

On a déjà vu (p. 280) que ce lobe prend son origine à la partie inférieure de la face interne de l'hémisphère, en avant du chiasma des nerfs optiques, en arrière de la base du lobe olfactif; la racine olfactive interne aboutit à cette origine, sur laquelle vient se fixer en outre la partie inférieure et interne du lobe frontal (p. 282). De là, le lobe du corps calleux se porte en haut et en avant, en formant une portion ascendante, puis il se recourbe sur le genou du corps calleux pour se diriger en arrière en formant une portion horizontale, $\mathrm{G}^{\prime}$ (fig. 27); il se recourbe enfin une seconde fois derrière le bourrelet du corps calleux, en formant une portion descendante $\mathrm{C}^{\prime \prime}$, qui va se continuer avec le lobe de l'hippocampe au niveau du pli rétro-limbique. La portion ascendante va toujours en s'élargissant de bas en haut. La largeur de la portion descendante présente des variations peu significatives quoique assez étendues; mais celle de la portion horizontale donne lieu à une remarque importante : chez les mammifères osmatiques, cette portion va en s'élargissant d'arrière en avant (1), tandis que chez les primates elle va au contraire en s'élargissant d'avant en arrière.

La portion ascendante du lobe du corps calleux est toujours en rapport avec le lobe frontal, qui se prolonge en outre, dans une étendue variable, sůr la partie antérieure de la portion horizontale; le lobe pariétal recouvre le reste de cette portion horizontale et toute la portion descendante. La scissure limbique se compose donc de deux parties, qui correspondent respectivement au lobe frontal et au lobe pariétal, et qu'on peut appeler

(1) Ciette règle est à peu près sans exception, car si, chez le tapir, cette parlie horizontale conserve dans la plus grande partie de son étendue une largeur à peu près uniforme, tout en avant du moins, au-dessus du genou du corps calleux, elle présente un certain élargissement (voir plus loin fig. 28). 
la scissure sous-frontale et la scissure sous-pariétale. Ces deux parties de la scissure limbique sont tantôt en continuité l'une a vec l'autre, tantôt séparées par une interruption plus ou moins étendue. Dans le premier cas, la scissure limbique forme autour du lobe du corps calleux un arc complet; dans le second cas, l'arc est incomplet. De là, deux types distincts que j'étudierai successivement.

$1^{\circ}$ Le premier type s'observe chez le cheval, le tapir et l'éléphant : je le décrirai d'abord d'après le cheval, qui le présente dans toute sa netteté (voy. fig. 27). L'arc inférieur de la scissure limbique L vient s'arrêter sur le pli de passage rétro-limbique $a a^{\prime}$, au-dessus duquel la scissure reprend son trajet. Elle remonte de $\mathrm{L}^{\prime}$ en $\mathrm{L}^{\prime \prime}$ sous la circonvolution sagittale du lobe pariétal ; c'est sa partie sous-pariétale; puis de L" en L'" elle chemine sous la face interne du lobe frontal; c'est sa partie sous-frontale. Ces deux parties de la scissure limbique se continuent sans interruption l'une avec l'autre; elles sont toutes deux très profondes. Je les ai étudiées sur un cerveau divisé en seize conpes transtersales, dont je conserve les dessins; leur profondeur n'a jamais moins de 6 millimètres, et peut s'élever jusqu'à 12 millimètres. Il n'y a sous ce rapport aucune différence entre la partie sous-pariétale et la sous-frontale. La scissure limbique longe donc tout le bord convexe du lobe du corps calleux ; ce bord est très légèrement sinueux, mais ne présente aucune incisure, tandis que plusieurs incisures, émanées de la scissure limbique, s'observent sur le bord correspondant de la masse extra-limbique. Parmi ces incisures, il en est une située en $\mathrm{L}^{\prime \prime}$, sur la limite du lobe frontal et du lobe pariétal; c'est l'incisure fronto-pariétale interne. Elle est située à une grande distance de l'extrémité antérieure de l'hémisphère, parce que le lobe frontal du cheval, déjà grand sur la face externe, devient très grand sur la face interne. Cette incisure est dirigée en haut et en avant; elle est courte et n'atteint pas le bord sagittal de l'hémisphère; elle s'arrête sur un petit pli de passage, étendu du lobe frontal au lobe pariétal, et qui n'existe, à ce niveau, que chez les solipèdes ; mais au-dessus de ce pli, elle reparaît sous la forme d'une grande scissure oblique qui gagne le bord supérieur, et va se terminer sur la face convexe où elle prend une direction transversale, en pré- 
sentant une disposition analogue à celle du "sillon crucial » des carnassiers.

Le lobe du corps calleux est subdivisé par un sillon longitudinal assez long quoique incomplet; ce sillon montre que le lobe du corps calleux du cheval n'a pas échappé entièrement au plissement, mais il est très superficiel; sa profondeur n'est en moyenne que de 2 millimètres, tandis que les sillons de la masse circonvolutionnaire ont souvent plus de 2 centimètres de profondeur. Ainsi, même chez cct animal très riche en circonvolu-

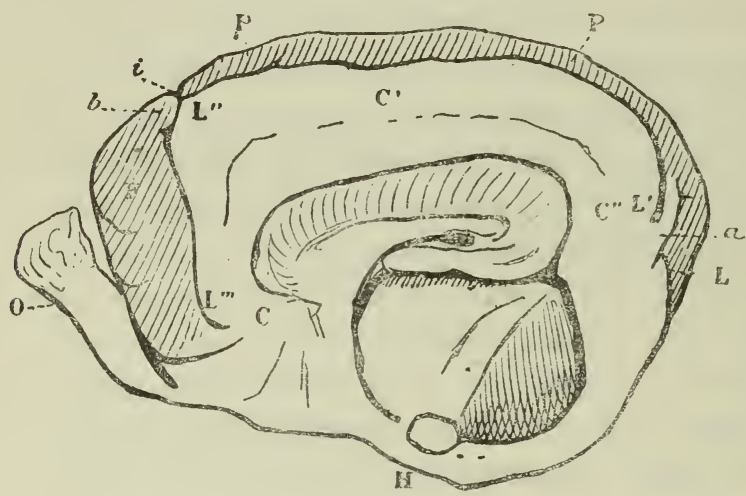

Fig. 28. Tapir, face interne de l'hémisphère droit. O, lobe olfactif; H, lobe de l'hippocampe CC.' $^{\prime \prime}$, lobe du corps calleux; L, arc inférieur de la scissure limbique; $a$, pli de passage rétro-limbique; $L^{\prime} L^{\prime \prime} L^{\prime \prime \prime}$, arc supérieur de la scissure limbique; $F$, lobe frontal ; PP, lobe pariétal; $b$, pli de passage profond fronto-limbique; $i$, incisure fronto-pariétale interne.

tions, le lobe du corps calleux se monţre réfractaire au plissement du manteau.

Par suite du grand développement de la face interne du lole frontal, la scissure sous-frontale du cheval est non seulement aussi longue, mais même plus longue que la scissure sous-parićtale. L'inverse s'observe chez le tapir et l'éléphant. Chez le tapir (fig. 28), le lobe du corps calleux, très large, $\mathrm{GC}^{\prime} \mathrm{C}^{\prime \prime}$, forme presque toute la face interne de l'hémisphère; en outre, le lobe frontal, très petit, ne repose que sur sa partie ascendante, et ne s'étend que sur sa portion horizontale. L'incisure fronto-pariétale interne $i$ est ainsi reportée tout en avant, un peu plus en avant même que le genou antérieur du corps calleux : elle atteint presque aussitôt le bord sagittal, et va former sur la face con- 
vexe un «sillon crucial » situé tout près de l'extrémité antérieure de l'hémisphère. Chez l'éléphant, le lobe du corps calleux est au contraire très étroit; il est en quelque sorte étouffé sous la masse énorme des circonvolutions fronto-pariétales qui se pressent sur lui. En outre, l'arc supérieur de la scissure limbique est interrompu en plusieurs points en avant, en haut, en arrière, par plusieurs plis de passage qui s'étendent du lobe du corps calleux à la masse circonvolutionnaire adjacente. Le nombre, la position, le volume de ces plis de passage et des incisures limbiques qui existent à leur base, sont très variables. Je les ai étudiés sur les deux hémisphères du cerveau d'un jeune éléphant des Indes (1), et sur l'hémisphère droit d'un animal adulte de la même espèce, pièce momifiée par l'acide nitrique, et donnée au laboratoire par M. Mathias Duval : ils sont très différents sur ces trois pièces, ainsi que sur le cerveau publié par Leuret. Ces plis ne constituent donc que des détails tout à fait secondaires, et la variabilité de leur siège ne m'a pas permis de déterminer exactement quelle est celle des incisures limbiques qui représente sur le cerveau de l'éléphant l'incisure fronto-pariétale interne du cheval et du tapir; mais, quoique l'arc supérieur de la scissure limbique de l'éléphant ne soit pas continu, il ne forme en réalité qu'un seul trajet, et il rentre par conséquent dans le même type que celui du cheval.

Chez les animaux de ce type, la largeur relative du lobe du corps calleux est très variable, mais elle est toujours moindre que celle de la face interne de l'hémisphère, de sorte que ce lobe et la scissure limbique qui l'entoure n'arrivent jamais jusqu'au bord sagittal ; chez le tapir, toutefois, ils s'en rapprochent beaucoup (roy. fig. 28); il suffirait d'ajouter au corps calleux 2 ou 3 millimètres en avant et en haut, pour qu'il atteignît le bord sagittal, et qu'il devînt apparent sur' la face supérieure de l'hémisphère; le second type de la scissure limbique se trouverait ainsi réalisé.

(1) Ce cerveau a été donné au laboratoire par M. Tramond, naturaliste, rue de l'École-de-Médecine. Je profite de cette occasion pour remercier bien sincèrement $M$. Tramond du concours empressé et désintéressé qu'il prête à nos recherches; nous lui devons une partie importante de notre collection de cerveaux. 
$2^{\circ}$ Ce second type, incomparablement plus fréquent que le premier, s'observe chez beaucoup de pachydermes, chez tous les ruminants que j’ai étudiés (même chez le bœuf, malgré les apparences), et enfin chez tous les carnassiers, sans aucune exception. Il est caractérisé, $1^{\circ}$ par un pli de passage frontolimbique qui sépare la portion sous-pariétale de la scissure limbique de sa portion sous-frontale; $2^{\circ}$ par ce fait que la scissure sous-frontale est très incomplète et très superficielle, tandis que la scissure sous-pariétale est très grande et très profonde ; $3^{\circ}$ enfin par cet autre fait que la partie antérieure du lobe du corps calleux devient assez large pour s'élever jusqu'au bord sagittal et pour aller former au-delà de ce bord une partie de la face supérieure de l'hémisphère (voy. p. 283, fig. 10, la face supérieure du cerveau de la loutre). Il en résulte que la scissure sous-pariétale remonte au moins jusqu'au bord sagittal, et qu'elle dépasse même presque toujours, en s'étendant plus ou moins sur la face supérieure de l'hémisphère où elle forme le « sillon crucial » de Leuret.

Tous ces caractères sont très manifestes chez tous les carnassiers. Ils ne le sont pas moins chez les porcs, parmi les pachydermes et, parmi les ruminants, chez la chèvre, le mouton, le daim, l'antilope. Mais ils sont quelque peu modifiés chez d'autres ruminants. La scissure sous-pariétale du chevreuil (fig. 26) remonte jusqu'au bord sagittal, mais ne le dépasse pas et ne produit pas le "sillon crucial "; du reste, elle est encore séparée, par un très large pli de passage, de la scissure sousfrontale, qui est très superficielle et qui n'eșt plus qu'un léger sillon L'", sillon sous-frontal. Chez le chameau, la partie antérosupérieure du lobe du corps calleux, quoique très volumineuse et subdivisée, arrive à peine jusqu'au bord sagittal et seulement tout en avant, c'est-à-dire presque sur la pointe de l'hémisphère. Toutefois, la scissure sous-pariétale, qui est très longue, se pro longe transversalement sur cette pointe. Le lobe frontal es! excessivement réduit, et communique néanmoins avec le lobe du corps calleux par deux plis de passage superposés, qui séparent la scissure sous-pariétale de la scissure sous-frontale; celle-ci est courte, mais assez profonde. La même disposition se retrouve chez le bouf, si ce n'est qu'il n'y a qu'un seul pli 
de passage fronto-limbique, qu'il est étroit et peu volumineux, et que la scissure sous-pariétale ne se prolonge pas sur la face convexe. Il en résulte que cette scissure et la scissure sous-frontale décrivent sur la face interne de l'hémisphère une courbe presque complète, interrompue seulement par un petit pli superficiel, situé exactement au point où l'on aperçoit sur le cerveau du tapir (fig. $28, b$ ) un pli fronto-limbique rudimentaire et profond. Il paraîtrait même que ce pli ne serait pas toujours superficiel chez le bœuf, car il n'est pas représenté sur le dessin de Leuret (1). Quoi qu'il en soit, le tapir et le bœuf présentent

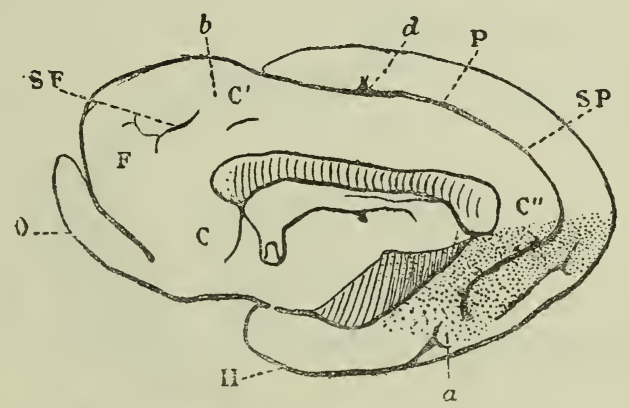

Fig. 29. Renard, face interne de l'hémisphère droit. $\mathrm{CC}^{\prime} \mathrm{C}^{\prime \prime}$, lobe du corps calleux; $a$, pli de passage rétro-limbique; $\mathrm{F}$, lobe frontal; $\mathrm{P}$, lobe pariétal ; SF, sillon sous-frontal ; SP, scissure sous-pariétale ; $b$, pli de passage fronto-limbique ; $d$, position du pli de passage pariéto-limbique antérieur, qui est profond.

une double transition entre le type limbique du cheval et celui des carnassiers.

C'est chez les carnassiers surtout qu'on voit le lobe du corps calleux s'élargir d'arrière en avant, et se fusionner à sa partie antérieure avec le lobe frontal. Le grand développement de cette partie antérieure paraît en rapport avec le rôle très important que joue l'appareil olfactif chez les carnassiers. La scissure sousfrontale n'est représentée que par un sillon très superficiel qui est le sillon sous-frontal (fig. 29, SF); elle est tellement atténuée qu'on n'y reconnaîtrait pas le vestige de la scissure limbique, si l'on n'était guidé par l'étude des cerveaux des pachydermes et des ruminants. Le pli de passage fronto limbique ne revêt donc pas la forme d'un pli ; c'est une fusion pure et simple du lobe du corps calleux et du lobe frontal, dont les surfaces

(1) Ana'yse comparée du système nerveux, atlas in-fol., pl. IX. 
lisses et plates se continuent directement l'une avec l'autre.

La scissure sous-pariétale, SP, au contraire, est grande et profonde. Pour bien l'étudier, il faut la dilater de manière à refouler vers l'extérieur le bord oblique et imbriqué de la circonvolution sagittale. La figure 30 représente la face interne du cerveau d'un chien ratier, qui a été moulée dans cet état. Je rappelle que le chien et le loup paraissent privés du pli de passage rétro-limbique : chez eux, l'arc inférieur de la scissure limbique se continue sans interruption apparente avec la scissure sous-

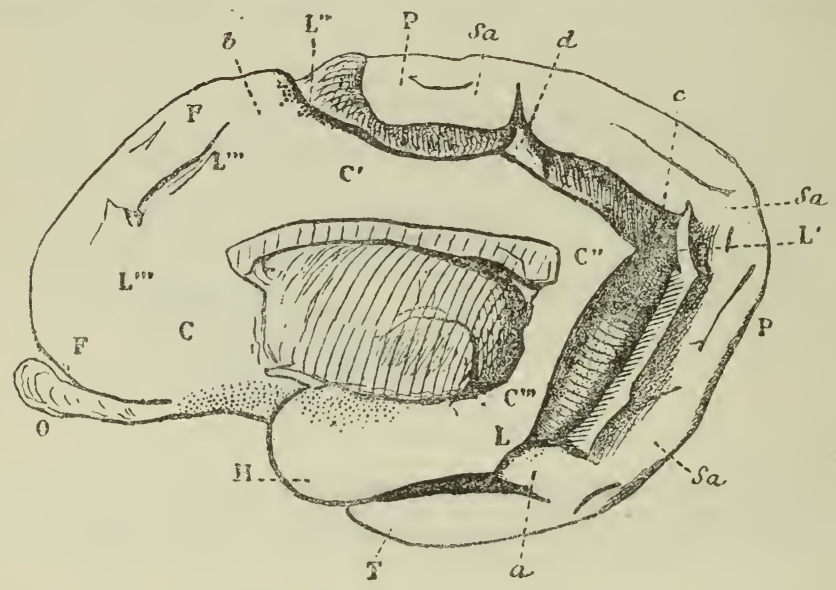

Fig. 30. Chien ratier, face interne de l'hémisphère droit. La scissure pariétalo est déployée pour montrer les plis de passage profonds.

O, lobe olfaetif; H, lobe de l'hippocampe; $\mathrm{CG}^{\prime} \mathrm{C}^{\prime \prime}$, lobe du corps calleux; FF, lobe frontal; PP, lobe pariétal ; T, lobule temporal du lobe pariétal ; L, are inférieur de la scissure limbique, se eontinuant avee l'are supérieur 'eette eontinuité n'existe que ehez le ehien, Je loup et le dauphin); LL'L", la seissure sous-pariétale; L'", le sillon sous-frontal ; $S a, S a$, free interne de la cireonvolution sagittale; $a$, pli de passage rétro-limbique; il est profond; $c$, pli de passage pariéto-limbique postérieur; il est profond; $d$, pli de passage pariétolimbique antérieur; il est profond; $b$, pli de passage fronto-limbique.

pariétale; mais lorsqu'on a dilaté cette dernière scissure, on aperçoit le pli rétro-limbique, $a$, qui existe, comme toujours, à l'union du lobe du colps calleux et du lobe de l'hippocampe, et qui présente ses connexions ordinaires, à cela près qu'il est profond (1). De $\mathrm{L}$ en $\mathrm{L}^{\prime}$, la scissure sous-pariétale longe la portion descendainte du lobe du corps calleux; en $\mathrm{L}^{\prime}$ elle se réfléchit sur la portion horizontale de ce lobe; à ce niveau existe un pli de

(1) Le pli de passage rétro-limbique est profond aussi chez certains cétacés (dauphin), mais il est superficiel chez d'autres cétacés (marsouin). 
passage profond, $c$, qui s'étend du lobe du corps calleux à la circonvolution sagittale. Ce pli, très profond chez les grands chiens, se développe assez chez les petits chiens (petit bull et havanais) pour devenir superficiel. Plus en avant, au niveau d'une incisure pariétale qui est constante chez les chiens et qui se retrouve aussi chez le renard, un autre pli de passage profond, $d$, traverse la scissure limbique, et unit le lobe du corps calleux à la circonvolution sagittale, $S a$. Ce pli est superficiel chez les chats, mais il est profond chez tous les autres carnassiers que j'ai pu examiner. Les deux plis de passage, $c$ et $d$, peuvent être appelés les plis pariéto-limbiques postérieur et antérieur. Leur existence chez les carnassiers est le premier degré de la fusion qui s'effectue chez les primates entre le lobe du corps calleux et la scissure sagittale, et qui fait disparaître presque entièrement la scissure sous-pariétale.

Après avoir fourni le pli pariéto-limbique antérieur, la scissure sous-pariétale continue son trajet jusqu'en $L^{\prime \prime}$ en s'élevant de plus en plus, de manière à dépasser le bord sagittal et à former sur la face convexe le sillon crucial de Leuret (scissure cruciale).

Le sillon crucial existe chez tous les carnassiers. Il est limité en arrière par la circonvolution sagittale, en avant par la partie supérieure et antérieure du lobe du corps calleux (voy. fig. 31, cerveau du renard). Cette portion du grand lobe limbique qui apparaît sur la face convexe de l'hémisphère, et qui est laissée en blanc sur la figure, se continue en avant avec le lobe frontal, F, en dehors avec l'extrémité antérieure de la circonvoiution sagittale, $\mathrm{PP}^{\prime}$, et avec le pli post-rolandique, sur lequel viennent se terminer successivement toutes les circonvolutions pariétales. Le pli post-rolandique est l'analogue de la circonvolution pariétale ascendante des primates.

L'extrémité antérieure de la scissure sous-pariétale forme également un "sillon crucial » chez les porcs, chez le daim, la chèvre, le mouton, l'antilope ; il y a aussi un sillon crucial chez le tapir et le chameau, mais il est formé par l'incisure frontopariétale de la scissure sous-pariétale, et non par cette scissure elle-même.

Nous avons dû étudier en détail, chez les gyrencéphales osma- 
tiques, les connexions du grand lobe limbique et de la scissure limbique, ainsi que les circonvolutions qui viennent y aboutir. Ces développements étaient nécessaires pour apprécier la nature des différences qui apparaissent lorsque l'on s'élève au cerveau des primates. Celles-ci sont de deux ordres : les unes résultent de l'atrophie de l'appareil olfactif; les autres résultent de l'agrandissement énorme du lobe frontal. Chez les primates, la réunion de ces deux causes pourrait rendre difficile la déter-

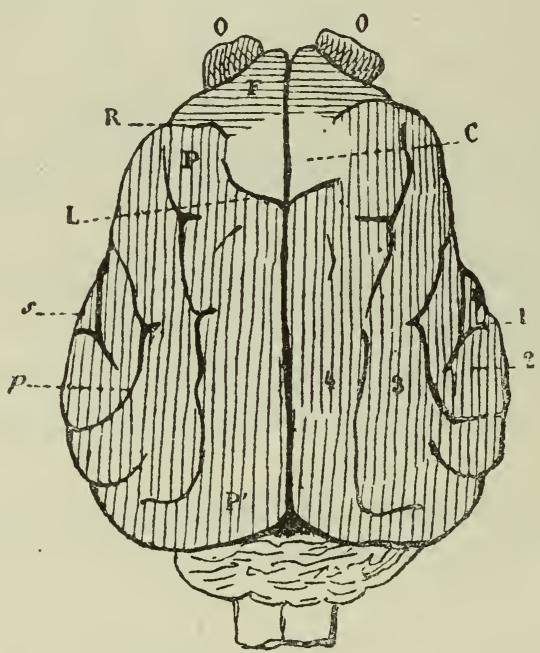

Fig. 31. Face supérieure du cerveau du renard. O, lobe olfactif; R, scissure de Rolando; F, lobe frontal; $\mathrm{PP}^{\prime}$, lobe pariétal ; $\mathrm{C}$, extrémité antéro-supérieure du lobe du crps calleux, laissée en blanc; L, scissure cruciale, formée par l'extrémité antéro-supérieure de la scissure sous-pariétale; $p$, sillon pariétal primaire; $\mathrm{S}$, extrémité supérieure et postérieurc de la scissure de Sylvius, 1 à 4 les quatre circonvolutions pariétales, numérotées d'après Leuret.

mination de la part qui revient à chacune d'elles; mais on peut étudier la première à l'état d'isolement chez les animaux dont l'appareil olfactif est nul ou rudimentaire sans que le volume de leur lobe frontal soit exagéré. Ces animaux sont les mammifères aquatiques.

§ 6. - cerveaux anosmatiques des hammifères aruatiques.

Je ne me propose nullement de décrire ici le cerveau des mammifères aquatiques, ni d'établir une comparaison entre 
leurs circonvolutions et celles des autres mammifères. Il est possible, je pense, de prouver que les circonvolutions si compliquées des cétacés, quelque spéciales qu'elles soient, peuvent se ramener à un type peu différent de celui des solipèdes et des grands pachydermes, et que celles des amphibies (famille des phoques) se rattachent au type des carnassiers. Mais cette double démonstration exigerait de longs développements qui, d'ailleurs, nous éloigneraient beaucoup de notre sujet, car nous n'avons à nous occuper des circonvolutions qu'au point de vue de leurs rapports avec le grand lobe limbique. Il nous suffira donc d'étudier les modifications qui sont la conséquence de l'atrophie ou de l'anéantissement de l'appareil olfactif.

Cet appareil est entièrement nul chez les cétacés de la famille des Dauphins. Tout a disparu, non seulement le lobe olfactif, mais même les filaments des nerfs olfactifs, de sorte que la " lame criblée " de l'ethmoïde est imperforée. Le grand lobe limbique ne se compose donc plus que de deux parties : le lobe de l'hippocampe et le lobe du corps calleux.

Les anatomistes qui ont méconnu la solidarité anatomique et fonctionnelle du lobe de l'hippocampe et du lobe olfactif en auraient jugé autrement s'ils avaient étudié les cerveaux des cétacés delphiniens. Chez ces animaux, qui n'ont pas d'appareil olfactif, le lobe de l'hippocampe est réduit au minimum de volume; il est plus petit non seulement que chez les amphibies, mais même que chez les primates eux-mèmes; il est très court, et tellement étroit (fig. 32, H), qu'on serait tenté de le confondre avec les minces et nombreuses subdivisions des circonvolutions adjacentes. Toutefois, son bord externe est nettement limité par la scissure limbique, $L^{\prime}$, qui se continue directement de $\mathrm{L}^{\prime}$ en $\mathrm{L}^{\prime \prime}$ autour du lobe du corps calleux, $\mathrm{C}^{\prime \prime}$; le pli de passage rétro-limbique existe, comme toujours, à l'union des deux lobes, en $L^{\prime}$, mais il est profond chez le dauphin, comme il l'est chez le chien et le loup. Ce pli est, au contraire, superficiel chez le marsouin, dont le cerveau est d'ailleurs très analogue à celui du dauphin.

Le lobe de l'hippocampe du dauphin n'est pas seulement atrophié ; il a, en outre, en partie perdu son indépendance. La scissure limbique qui le limite ne se prolonge pas sur son extrémité 
antérieure, qui se fusionne en ' $\mathrm{T}$ ' avec les circonvolutions adjacentes. Cette fusion est en quelque sorte le premier degré de la formation du lobe temporal, dont la constitution ne s'achève

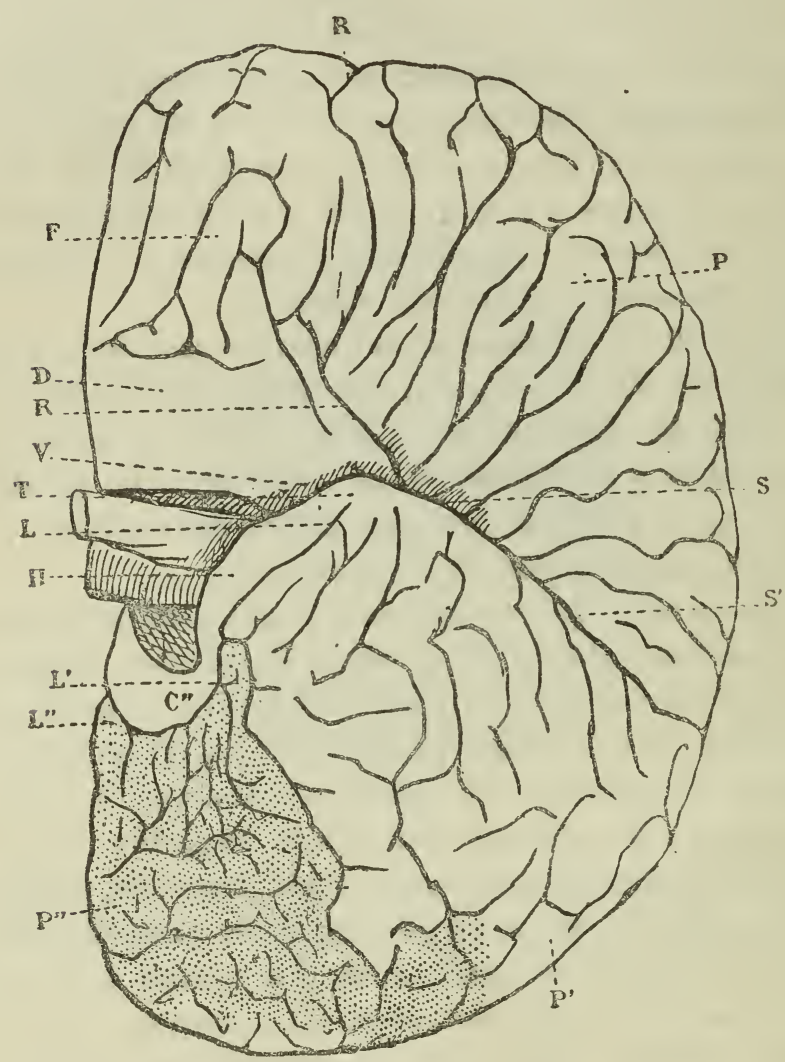

Fig. 32. Dauplin, face inférieure de l'hémisplı̀̀re gatehe. RR, scissure de Rolando; SS'. scissure de Sylvius; $\mathrm{PP}^{\prime} \mathrm{P}^{\prime \prime}$, lobe pariétal ; F, face inférieure du lobe frontal ; D, lobule désert du lobe frontal (désert olfactif); V, vallée de Sylvius; H, lobe de l'hippocampe atrophié : $\mathrm{C}^{\prime \prime}$, lobe du corps calleux; LL', are inférieur de la scissure limbique; $\mathrm{L}^{\prime} \mathrm{L}^{\prime \prime}$, son arc supéricur vu en raccourci ; T, le pôle temporal du lobule (ou lobe) temporal; $B$, bandelette optique. (Cette lettre B, omise par le graveur, était placée entre T et L ) .

que chez les primates. Elle s'effectue sur le bord postérieur et inférieur de la scissure de Sylvius, $\mathrm{S}$, dans la partie qui correspond chez les osmatiques au lobule temporal du lobe pariétal. G'est une question de savoir si cette partie du manteau doit 
continuer à s'appeler chez le dauphin lobule temporal, ou s'il ne conviendrait pas de l'appeler déjà lobe temporal, car elle est certainement très voisine du type que l'on observe chez les primates. Il suffirait que l'extrémité antérieure T se prolongeât un peu plus en avant, par-dessus la vallée de Sylvius dont il va être question, pour que le type du lobe temporal des primates fût réalisé. Du reste, on peut voir que les sillons situés en dehors de la scissure limbique, L, convergent comme elle vers le point T, qui est par conséquent l'analogue du pôle temporal des primates.

La scissure de Sylvius, très oblique en arrière, $\mathrm{SS}^{\prime}$, se relève à sa partie postérieure pour se prolonger sur la face externe. A sa partie antérieure, un peu en dehors du pôle temporal, une grande et profonde scissure s'en détache pour se porter en avant et un peu en dehors : c'est la scissure de Rolando, $R$, qui sépare le grand lobe pariétal, $\mathrm{PP}^{\prime} \mathrm{P}^{\prime \prime}$, du lobe frontal, F. Celui-ci, quoique assez large, n'occupe pas toute la largeur de la face inférieure de l'hémisphère. Il est subdivisé en avant par de nombreux sillons; mais en arrière, dans une étendue qui représente environ le tiers de sa longueur totale, il est tout à fait lisse. Il y a là une large surface dont la simplicité absolue contraste d'une manière très frappante avec la grande complication de tout le reste du manteau, surface comparable à celle que forme, sur une carte de géographie, un désert entourẻ de pays fertiles. Cette portion du lobe frontal des cétacés peut donc être appelée le lobule désert ou encore le désert olfactif. Il n'est pas douteux, en effet, que l'absence de toute espèce de plis et de sillons est la conséquence de l'absence totale de l'appareil olfactif. Je rappelle que le lobe olfactif, toutes les fois qu'il existe, s'insère sur l'extrémité postérieure de la face orbitaire du lobe frontal, immédiatement en avant de l'espace quadrilatère, au moyen d'une double lame (grise et blanche) de stibstance cérébrale que j'ai appelée la racine olfactive supérieure (p. 278). La portion postérieure du lobe frontal, sur laquelle se fait cette insertion, doit done participer à la fonction olfactive, puisque c'est par là, et par là seulement que le lobe olfactif communique directement avec le cerveau intellectuel, et il est probable que son rôle consiste à interpréter, à discuter, à transformer en idées les im- 
pressions olfactives qui lui sont transmises. Ce rôle est indépendant du volume de l'appareil olfactif, et nous voyons en effet que chez les amphibies et les primates, dont l'appareil olfactif est très atrophié, la portion postérieure de la face orbitaire du lobe frontal n'est ni plus ni moins compliquée que le reste du manteau; les plis et les sillons s'y prolongent, et elle ne forme pas un lobule spécial (1), car sa constitution ne diffère pas de celle de la partie antérieure. Mais, chez les cétacés, cette portion du lobe frontal, dont la fonction olfactive est anéantie, subit une atrophie aussi complète que possible : sa couche corticale, amincie et étalée, ne montre plus le moindre pli, et sa surface, devenue entièrement lisse, paraît indiquer qu'elle a perdu son activité, qu'aucune fonction nouvelle n'est venue prendre la place de celle qui a été supprimée. La nature de cette modification est exprimée par le nom de désert olfactif.

Ghez le marsouin, le désert olfactif a plus d'étendue d'avant en arrière que chez le dauphin; toutefois il n'occupe pas toute la longueur de la face orbitaire, car un sillon longitudinal règne sur la moitié antérieure de cette face.

De même que l'absence de la racine olfactive supérieure amène l'aplanissement du lobule désert, de même l'absence des trois racines inférieures, et particulièrement de la racine grise ou moyenne, amène la dépression de la vallée de Sylvius. Cette dépression transversale, qui correspond chez les osmatiques à l'espace quadrilatère, est chez eux très peu profonde; ce n'est pas, à proprement parler, une dépression ; ce n'est qu'une différence de niveau résultant de la grande épaisseur du lobe de l'hippocampe, qui forme une saillie sur le bord postérieur de l'espace quadrilatère. Mais chez les cétacés, le lobe de l'hippocampe, atrophié, n'a qu'une faible épaisseur, et si la vallée de Sylvius se creuse, c'est parce que l'espace quadrilatère s'est profondément affaissé. L'épaisse couche de substance grise et de fibres blanches qui formait la racine olfactive moyenne, se réduit maintenant à une très mince couche grise, qui se confond

(1) On notera, toutefois, chez les primates gyrencéphales l'existence constante de l'incisure transversale appelée "le sillon en $\mathrm{H}$ ). Il est permis de supposer que la partie du lobule orbitaire située en arrière de cette incisure est affectée à l'olfaction, et représente le lobule désert des cétacés delphiniens. 
avec la face inférieure du corps strié, et qui est traversée comme un crible par les vaisseaux de ce corps. L'espace quadrilatère est ainsi devenu l'espace perforé. La vallée de Sylvius est donc large et profonde; en dedans, elle s'étend, comme toujours, jusqu'au bord interne du chiasma des nerfs optiques; mais à son extrémité externe, ses connexions sont changées. La racine olfactive externe qui la iimitait chez les osmatiques, et qui la séparait de la scissure de Sylvius, est absente ; par conséquent, la grande et profonde vallée de Sylvius se trouve en continuité directe, sous la pointe du lobule temporal, avec la grande et profonde scissure de Sylvius, et ces deux anfractuosités n'en forment plus qu'une seule qui, commençant en arrière sur la face externe de l'hémisphère, descend obliquement jusqu'à la base $d u$ cerveau, la traverse transversalement et va se terminer sur le bord externe du chiasma.

Sur la face interne du cerveau des cétacés, le lobe du corps calleux contourne comme d'habitude le limbe de l'hémisphère. Il est limité, dans toute sa longueur, par une scissure limbique grande et profonde qui passe sans interruption sous le lobe pariétal et sous le lobe frontal, en décrivant un arc complet, suivant le premier type limbique, décrit plus haut (p. 446), chez le cheval, le tapir et l'éléphant. Par ce caractère et par quelques autres aussi, le t,ype cérébral des cétacés se rattache à celui des pachydermes, et on pourrait se demander si les cétacés ne seraient pas des pachydermes modifiés, comme les amphibies sont des carnassiers modifiés. Je dois signaler ici un fait qui est assez difficile à concilier avec la solidarité qui me paraît exister, chez les autres animaux, entre l'appareil olfactif et la partie antérieure du lobe du corps calleux. Cette partie antérieure, voisine de l'origine de la racine olfactive interne, est très développée chez les osmatiques et plus large que la partie postérieure du lobe ; chez les primates, au contraire, c'est cette partie qui est la plus étroite, et il paraît vraisemblable que l'atrophie dont elle est le siège est en rapport avec l'atrophie de la racine olfactive interne. Or, quoique, chez les cétacés, cette racine soit non seulement atrophiée, mais anéantie, la partie antérieure du lobe du corps calleux n'est nullement atrophiée; elle est aussi développée que chez les osmatiques; elle est même sillonnée 
d'incisures assez nombreuses, indices d'une activité fonctionnelle très prononcée. Le grand développement antérieur du lobe du corps calleux est-il dû à l'extrême petitesse du lobe frontal, qui est presque réduit à son étage orbitaire, et qui ne se prolonge que très peu sur' la face convexe de l'hémisphère? Le lobe du corps calleux prendrait, par une sorte de compensation, la place laissée vacante par suite de cette extrême réduction du lobe frontal. On pourrait admettre encore que quelque autre fonction se serait développée dans cette portion du lobe du corps calleux, devenue disponible par suite de la disparition de sa fonction olfactive? Quoi qu'il en soit, il est digne de remarque, que par sa conformation générale, aussi bien que par la disposition de la scissure limbique qui l'entoure, le lobe du corps calleux des cétacés présente une grande analogie avec celui des pachydermes.

Occupons-nous maintenant des amphibies. Leur appareil olfactif n'est pas anéanti ; mais il est considérablement atrophié. Toutefois, il est moins rudimentaire que chez les primates, et l'atrophie de la portion du lobe antérieur du corps calleux est un peu moindre aussi. Pour apprécier cette réduction, il faut songer que le cerveau des phoques se rattache au type des carnassiers; c'est donc avec le cerveau des carnassiers que nous devons le comparer. La face interne de l'hémisphère du phoque est représentée sur la figure 33. On n'a pas oublié que chez les carnassiers le lobe du corps calleux croît considérablement d'arrière en avant ; sa portion antérieure est si développée qu'elle s'élève jusqu'au bord sagittal de l'hémisphère, et qu'elle apparaît même sur la face convexe, de sorte que la scissure sous-pariétale va former sur cette face convexe le « sillon crucial » (voy. p. 332, fig. 31, le cerveau du renard, et p. 288, fig. 10, le cerveau de la loutre); en outre la scissure sous-frontale, L'" (fig. 30, p. 331) n'est plus qu'un léger sillon (SF, fig. 29). Elle ne communique pas avec la scissure sous-pariétale, $L^{\prime \prime}$; elle en est séparée par le pli du passage fronto-limbique, $b$. Ces dispositions se retrouvent, dans ce qu'elles ont d'essentiel, chez les amphibies, mais la scissure sous-pariétale ne s'élève pas jusqu'au bord sagittal, par' conséquent, et ne va pas former le " sillon crucial "; la portion antérieuro du lobe du corps calleux ne s'étend donc pas jusqu'à 
la face convexe de l'hémisphère; cela prouve qu'elle a subi une grande diminution de volume. Malgré cette réduction, elle reste encore aussi large, chez le phoque, que le reste du lobe du corps calleux ; mais chez l'otarie, elle est plus étroite, et le lobe du corps calleux va en diminuant d'arrière en avant comme chez les primates. Quant au pli de passage fronto-limbique, il est subdivisé en deux plis secondaires, $b$ et $b^{\prime}$, par un sillon assez grand, $d d^{\prime}$, qui se prolonge dans l'épaisseur du lobe du corps calleux, jusqu'à une petite distance de la rainure du corps cal-

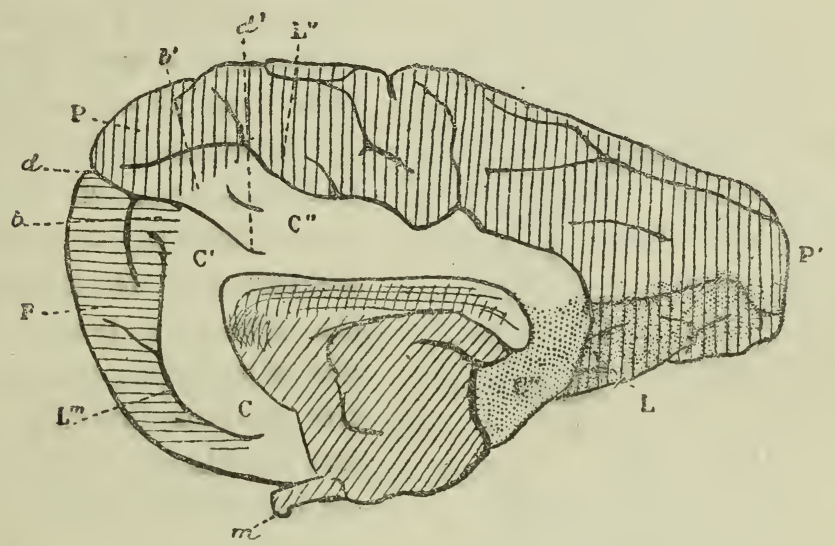

Fig. 33. Plıoque, face interne de l'hémisphère droit. C, origine du lobe du corps calleux ; $\mathrm{CC}^{\prime}$, portion sous-frontale de ce lobe, séparée de sa portion sous-pariétale, $\mathrm{C}^{\prime \prime}$, par le sillon $d d^{\prime} ; b^{\prime}$, le pli de passage pré-limbique, subdivisé par le mème sillon en deux plis : l'un inférieur, $b$, ou fronto-limbique, l'autre supérieur, $b$, ou pariéto-limbique ; $L^{\prime} L^{\prime \prime}$, scissure sous-pariétale; L'", scissure sous-frontale; F, lobe frontal; $\mathrm{PP}$, lobe pariétal; $m$, bandelette optique.

Le lobe de l'hippocampe, atrophié, n'est pas visible sur la face interne.

leux, et qui par conséquent divise ce lobe dans presque toute son épaisseur. Chez le phoque, il reste encore entre la rainure et l'extrémité postérieure du sillon $d d^{\prime}$ une languette d'une certaine largeur; mais cette languette est déjà plus mince sur l'hémisphère droit de notre cerveau d'otarie, et elle manque entièrement sur l'hémisphère gauche, où le sillon $d d^{\prime}$ rejoint la rainure du corps calleux. Ce dernier cas, qui est peut-être anormal, est le seul où j'aie vu la continuité du lobe du corps calleux interrompue par un sillon (qui n'est d'ailleurs que superficiel). Quoi qu'il en soit, le sillon $d d^{\prime}$ prolongé dans l'épaisseur du lobe du corps calleux, constitue un caractère propre aux am- 
phibies; il établit une ligne de démarcation bien tranchée entre la portion sous-frontale de ce lobe, $\mathrm{CG}^{\prime}$, et sa portion souspariétale $\mathrm{G}^{\prime \prime}$, et, je suis enclin à supposer que cette disposition se rattache à l'atrophie du lobe olfactif. S'il en était ainsi, on pourrait conjecturer que la démarcation établie par le sillon $d d^{\prime}$ entre les deux portions du lobe du corps calleux des amphibies

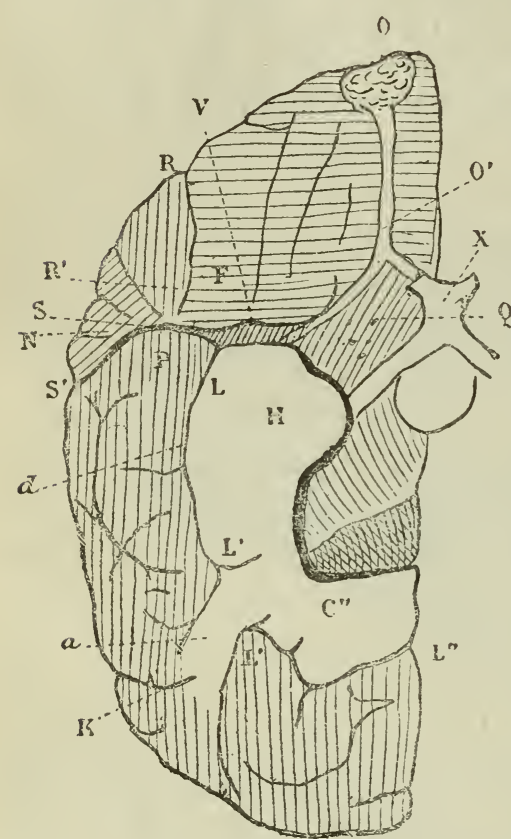

Fig. 34. Phoque, face inférieure de l'hémisphère droit. $\mathrm{O}$, ganglion (lobe) olfactif ; $\mathrm{O}^{\prime}$, ruban (pédoncule) olfactif avec ses deux racines blanches; H, lobe de l'hippocampe ; C'", extrémité postérieure du lobe du corps calleux; LL', arc inférieur de la scissure limbique; $L^{\prime} L^{\prime \prime}$, son arc supérieur ou scissure sous-pariétale; $a$, pli de passage rétro-]imbique; $k$, sillon calcarin; $d$, position d'un pli de passage profond (qui est superficiel chez l'otarie); $\mathrm{X}$, chiasma; $\mathrm{Q}$, espace perforé; $\mathrm{RR}^{\prime}$, scissure de Rolando; V, vallée de Sylvius, se continuant en dehors avec la scissure de Sylvius, SS'; F, lobe frontal; P, lobe pariétal; N, lobule sous-sylvien, en partie superficiel.

n'est pas seulement anatomique, qu'elle est fonctionnelle, et que la portion sousfrontale, CG', est affectée, à l'exclusion de l'autre, à la fonction olfactive. Enfin, on serait conduit par analogie à présumer que chez les autres animaux, où la ligne de démarcation anatomique n'existe pas, la répartition des fonctions reste néanmoins la même, et que chez eux aussi la fonction olfactive du lobe du corps calleux est limitée à sa portion sousfrontale.

L'étude de la face inférieure du cerveau des cétacés nous a déjà montré comment l'absence du lobe olfactif modifie la constitution de la vallée de Sylvius et la transforme en une scissure transversale très profonde, qui se continue en dehors avec la scissure de Sylvius. La simple atrophie du lobe olfactif amène chez les amphibies une modification analogue. La figure 34 représente la face inférieure de l'hémisphère droit du phoque. Le lobe olfactif ne forme plus qu'un petit ganglion, 0 , dont le pédoncule étroit se divise en arrière en deux racines divergentes, 
l'une interne, l'autre externe. Celle-ci se porte très obliquement en dehors et va rejoindre la partie externe de l'extrémité antérieure du lobe de l'hippocampe $\mathrm{H}$. Le point où s'effectue cette jonction n'est pas visible sur la figure; il est situé profondément sur le bord interne de l'extrémité antérieure de la scissure limbique LL'. Entre ces deux racines olfactives d'une part, le chiasma $\mathrm{X}$ et l'extrémité antérieure du lobe de l'hippocampe, existe l'espace quadrilatère $Q$. Chez les osmatiques, cet espace quadrilatère est formé par la racine olfactive moyenne, couche épaisse de substance grise, traversée par les fibres pédonculaires les plus antérieures et reposant sur la face inférieure du corps strié. Chez les amphibies, il n'y a plus de fibres pédonculaires ; la couche de substance grise s'amincit extrêmement, et se confond avec la face inférieure du corps strié, et un certain nombre de petits vaisseaux (veineux, je pense), émanés de ce corps, la traversent comme un crible. L'espace quadrilatère prend alors, comme chez les cétacés et les primates, le nom d'espace perforé. La mince couche grise qui le tapisse reçoit tout en avant une petite languette grise, très mince, très courte, très fragile, émanée de la face supérieure du pédoncule olfactif au niveau de la réunion des deux racines blanches (1); c'est tout ce qui reste de la racine olfactive grise des osmatiques. L'espace perforé, très déprimé, se trouve ainsi bien au-dessous du niveau du lobe de l'hippocampe, et forme au-devant de lui une vallée de Sylvius très profonde. Chez les osmatiques, cette vallée transversale n'aboutit pas à la scissure limbique; elle en est séparée, en dehor's, par la racine olfactive externe; mais chez les amphibies, celle-ci, étant atrophiée, n'établit plus la séparation ; la vallée $V$ se prolonge donc en dehors jusqu'à la scissure limbique L, qui est rejointe dans le même point par la scissure de Sylvius SS'; il elı résulte que la scissure et la vallée de Sylvius se continuent directement l'une avec l'autre; mais lorsqu'on écarte les bords de cette anfractuosité, on voit que le vestige de leur séparation

(1) Cette petite racine grise n'est pas visible sur la figure; pour l'apercevoir il faut soulever d'avarit en arrière, avec les plus grandes précautions, le pédoncule olfactif. On voit alors qu'elle s'insère transversalement sur le bord postérieur du lobe frontal; elle représente donc ì la fois la racine olfactive grise et la racine olfactive supérieure du cerveau des osmatiques. Cette disposition est exactement la mème chez les primates. 
subsiste encore; il est représenté par la mince et longue racine olfactive externe, qui descend dans le fond de la vallée, le traverse, et va se jeter enfin sur la partie la plus externe et la plus antérieure du lobe de l'hippocampe.

Cette modification de la vallée de Sylvius donne lieu à une disposition exactement semblable à celle qui existe chez les primates en général, et chez l'homme en particulier, à cela près que, le lobe olfactif des primates étant ordinairement plus atrophié encore que celui des amphibies, la racine olfactive externe est plus atténuée en arrière, et que son insertion sur le lobe de l'hippocampe est plus difficile à voir ; mais on l'aperçoit toujours sur les cerveaux légèrement durcis par l’acide nitrique, ou même seulement par l'alcool. Si l'on songe que le cerveau des primates diffère beaucoup par ses autres caractères de celui des amphibies, on est obligé de reconnaître que la similitude complète de leur vallée de Sylvius est la conséquence d'une cause commune, et que cette cause est l'atrophie de l'appareil olfactif.

Le bord antérieur de la vallée de Sylvius, V, et de la partie la plus inférieure de la scissure de Sylvius, est formé chez les amphibies par le lobe frontal F, comme cela a lieu chez les primates. Ce lobe s'étend en dehors jusqu'à la scissure de Rolando RR', qui s'étend presque jusqu'à la scissure de Sylvius, mais qui cependant ne l'atteint pas. La lèvre postérieure de l'anfractuosité sylvienne est formée en dehors par la circonvolution pariétale sylvienne, $\mathrm{P}$, qui est l'analogue de la première circonvolution temporale des primates, et en dedans par le lobe de l'hippocampe, H, qui est l'analogue de la circonvolution de l'hippocampe des primates.

Le lobe de l'hippocampe des amphibies est peu volumineux, aplati, et si on le compare à celui des osmatiques, on voit qu'il a perdu une grande partie de son importance. En outre, il est moins distinct de la masse circonvolutionnaire, car la scissure limbique, LL', est très peu profonde; sa profondeur diminue surtout au point $d$, en avant d'un petit sillon transversal qui limite un petit pli de passage, profond chez le phoque, mais volumineux et superficiel chez l'otarie. Le lobe de l'hippocampe de ce dernier amphibie est moins large encore que celui du 
phoque; le pli de passage dont je viens de parler prouve qu'il est moins distinct aussi. Nous voyons donc que le lobe de l'hippocampe tend à se fusionner avec la másse circonvolutionnaire à mesure qu'il s'atrophie, et cette tendance à la fusion nous indique que sa fonction devient de moins en moins spéciale. On verra tout à l'heure que, chez les primates, le lobe de l'hippocampe, de plus en plus réduit, ne forme qu'une circonvolution du lobe'temporal, et qu'il se fusionne de plus en plus avec la circonvolution adjacente.

Je ne crois pas devoir décrire en détail le reste de la face inférieure du cerveau du phoque. Le sens des autres lettres explicatives est déjà connu. J'ajoute cependant que les trois replis fuyants indiqués par la lettre $\mathrm{N}$ font partie des plis soussylviens. Ces plis, profonds chez les vrais carnassiers, sont en partie superficiels chez les amphibies.

\section{\$ 7. - CERVEAU dES PRIVATES.}

Nous possédons maintenant tous les éléments nécessaires pour déterminer les caractères distinctifs du cerveau des primates.

Lordre des primates, comprenant les quatre familles des hominiens, des anthropoïdes, des pithéciens et des cébiens (1), constitue un groupe parfaitement naturel, caractérisé par l'analogie de structure bien plus encore que par la similitude morphologique. Les naturalistes qui ont voulu en retirer l'homme pour en faire un ordre à part ont obéi à une idée toute sentimentale, qu'aucun anatomiste ne saurait admettre. Si l'on voulait, au nom de l'anatomie, établir une coupure dans ce groupe, ce serait entre la quatrième famille (celle des cébiens) et les trois familles supérieures qu'il faudrait la placer ; mais les caractères qui distinguent les cébiens des autres primates sont si peu de chose auprès de ceux qui les en rapprochent, et sont si loin

(1) La famille des lémuriens, rattachée à cet ordre depuis Linnœus, ne peut plus y être maintenue. Elle s'en sépare, ainsi que l'a établi M. Alphonse Milne Edwards, par des caractères embryologiques d'une valeur décisive. 
d'avoir une valeur ordinale, que l'ordre des primates se pré.sente à nous comme l'un des plus naturels de la zoologie.

Dans cet ordre, pourtant, les caractères cérébraux présentent des différences qui, au premier abord, paraissent excessives. On y trouve, en effet, à côté des cerveaux lissencéphales les plus simples, les gyrencéphales les plus compliqués; puis, entre ces degrés extrêmes, tous les degrés intermédiaires du développement des circonvolutions. Certes, si l'on se contentait de jeter un coup d'œil rapide sur le cerveau lisse du ouistiti, on serait tenté de croire qu'il est plus semblable à celui d'un petit rongeur qu'à celui des grands singes, et même qu'à celui des sapajous, et l'on pourrait en conclure non seulement que la conformation du cerveau n'est pas au nombre des caractères ordinaux, mais encore qu'elle se trouve avec eux en contradiction flagrante. Cette conclusion peu satisfaisante s'est présentée à beaucoup d'esprits, et elle serait sans réplique si l'on continuait à considérer les circonvolutions comme l'élément le plus essentiel, comme l'élément primaire de la constitution du manteau de l'hémisphère. Mais nous savons maintenant qu'il n'en est rien. On a vu plus haut (p. 290 et 303 et suiv.) que le plissement du manteau n'est pas un phénomène primaire; qu'il est la conséquence de l'extension de l'écorce cérébrale; ;que, s'il est dirigé par les connexions de l'écorce avec le corps et avec le limbe de l'hémisphère, il est déterminé par la loi géométrique du rapport des surfaces avec les volumes, et qu'à ce titre il est influencé à un haut degré par les variations de la taille parmi les genres d'une même famille, parmi les familles d'un même ordre. Cette influence de la taille n'est sans doute pas la seule qui exerce son action sur le plissement du manteau; elle se borne à agir sur un type donné, de manière à en exagérer ou en diminuer les caractères, sans jamais pouvoir le transformer en un autre type; elle n'a donc que des effets limités; mais elle suffit pour prouver' que la présence ou l'absence des circonvolutions, leur degré de simplicité ou de complication, ne font pas essentiellement partie de la constitution du type cérébral.

La division primaire de l'hémisphère n'est pas celle qui s'effectue dans la masse extra-limbique et qui y produit les circonvolutions; c'est celle qui s'établit entre le grand lobe limbique 
et le reste de l'écorce cérébrale. Il ne peut donc pas y avoir de plus grande différence entre deux cerveaux que celles qui concernent la constitution, les connexions et le degré d'indépendance du grand lobe.

Ces remarques permettent d'établir une distinction radicale entre le cerveau des primates lissencéphales et celui des autres lissencéphales. Chez ceux-ci, le lobe olfactif est très développé, et le lobe de l'hippocampe, relativement énorme, est séparé de la masse extra-limbique par une scissure limbique toujours très apparente, très étendue, qui existe souvent en l'absence de toute autre anfractuosité cérébrale, de sorte que le lobe de l'hippocampe est déjà distinct du reste du manteau alors que toutes les autres parties sont encore confondues entre elles. Or, chez les primates lissencéphales, ces caractères sont remplacés par des caractères tout différents. Il n'y a plus de vrai lobe olfactif, mais seulement un tout petit ganglion qui n'en est que le rudiment; il n'y a plus de lobe de l'hippocampe distinct, c'est-à-dire que ce lobe est fusionné avec la masse extra-limbique, et que la partie correspondante de la scissure limbique a complètement disparu. Cette fusion prouve que le lobe de l'hippocampe, en perdant son individualité, a perdu son importance; on ne peut admettre, en effet, qu'elle soit l'expression d'une simplicité plus grande de l'hémisphère, puisque celui-ci présente deux grandes scissures qui manquent chez les lissencéphales osmatiques, savoir : la scissure calcarine et la scissure de Sylvius, qui est en continuité directe avec la vallée de Sylvius. Le cerveau des primates lissencéphales appartient donc à un type tout autre que celui des lissencéphales osmatiques. Il en diffère par des caractères de premier ordre, et ces caractères le rattachent absolument au type du cerveau des primates gyrencéphales.

En remontant de bas en haut la série des singes, en passant du ouistiti au saïmiri, du saïmiri ail cébus, au saki, puis aux cébiens supérieurs, aux pithéciens; enfin, aux anthropoïdes et à l'homme, il serait aisé de suivre dans cette longue série le développement des lobes et des circonvolutions de l'hémisphère. Mais ce n'est pas là le but que je me suis tracé. L'anatomie comparée du cerveau des primates est déjà assez bien connue; les faits que je pourrais y ajouter pourront faire l'objet d'un travail 
spécial, mais ne sauraient trouver place ici. Ce que je me propose d'établir, c'est que les caractères essentiels du cerveau restent les mêmes dans toute la série des primates, au milieu des grandes variations produites par le degré de plissement du manteau, et que ce type cérébral des primates est entièrement différent de celui des autres mammifères (1); mais je montrerai en même temps que toutes les parties du cerveau des primates ont leurs analogies dans les autres cerveaux, et vice versâ, et la détermination de ces analogies permettra de constater qu'il existe un type commun à tous les mammifères, type général dont les divers types spéciaux ne sont que des dérivés.

J'ai déjà posé les premières bases de ce parallèle en comparant les primates lissencéphales avec les lissencéphales osmatiques. Pour le compléter, il faut comparer les primates gyrencéphales avec les autres mammifères gyrencéphales, et plus particulièrement avec les carnassiers terrestres qui, par l'ensemble de leurs caractères anatomiques, sont, de tous les gyrencéphales, les plus rapprochés des primates.

Lorsqu'on passe de l'ordre des carnassiers (non compris les amphibies) à l'ordre des primates, on voit le type cérébral se modifier tout à coup, et se distinguer par une double série de caractères, dont les uns se manifestent dans le grand lobe limbique, et les autres dans le reste du manteau. Les premiers sont la conséquence de l'atrophie de l'appareil olfactif; ils ont déjà été étudiés chez les amphibies; il suffit donc de les énumérer :

$1^{\circ}$ Le lobe olfastif, devenu rudimentaire, se réduit à un petit renflement (ganglion olfactif), et son pédoncule, devenu long et grêle, ne forme plus qu'un petit ruban (ruban olfactif), improprement appelé nerf olfactif;

$2^{\circ}$ Le lobe de l'hippocampe, considérablement atrophié, perd son indépendance et se fusionne plus ou moins avec la circonvolution adjacente; il cesse de constituer un lobe distinct, et ne forme plus que la dernière circonvolution du lobe temporal ;

$3^{\circ}$ Le lobe du corps calleux est bien développé; il est beau-

(1) Je ne parle pas des lémuriéns, n'ayant pu me procurer jusqu'ici qu'un seul cerveau de maki, qui est même incomplet. 
coup moins réduit que les deux autres portions du grand lobe; sa partie postérieure n'a rien perdu de son volume, mais sa partie antérieure est relativement atrophiée et sa largeur par conséquent va en croissant d'avant en arrière ;

$4^{\circ}$ Par suite de l'atrophie des racines olfactives, l'espace quadrilatère se déprime et devient l'espace perforé; la vallée de Sylvius se creuse profondément et son extrémité externe se met en continuité avec la scissure de Sylvius, de sorte que cette delnière paraît se prolonger transversalement, sur la face inférieure de l'hémisphère, jusqu'au chiasma des nerfs optiques. Il semble donc que le grand lobe ne forme plus un circuit complet autour du limbe de l'hémisphère; néanmoins, la continuité du lobe du corps calleux et du lobe (ou circonvolution) de l'hippocampe est maintenue en avant par les deux racines olfactives blanches, dont l'interne se dirige ver's l'origine du lobe du corps calleux, tandis que l'externe traverse le fond de la vallée de Sylvius, pour aller se jeier sur l'extrémité antéro-externe de la circonvolution de l'hippocampe.

Ces quatre premiers caractères ne sont pas exclusivement propres aux primates; ils se retrouvent, comme on l'a vu plus haut, chez les carnassiers amphibies. Chez les primates, toutefois, les racines olfactives, surtout l'externe, sont plus atrophiées; le lobe de l'hippocampe est plus réduit, plus confondu avec la circonvolution adjacente. J'ajoute que le lobe temporal, dans lequel il se fusionne, est constitué autrement que chez les amphibies ; mais cette dernière différence, sur laquelle je reviendrai tout à l'heure, ne concerne pas le grand lobe limbique.

Je passe maintenant aux caractères cérébraux qui n'appartiennent qu'aux primates. Ge sont ceux qui se montrent dans la masse extra-limbique, c'est-à-dire dans les lobes et les circonvolutions. Ils se rattachent néanmoins, dans une certaine mesure, aux modifications du grand lobe limbique, considéré sinon au point de vue de sa constitution, du moins all point de vue de ses connexions.

Ces caractères sont les suivants :

$1^{\circ}$ Développement énorme du lobe frontal, d'où résultent le recul et le changement de direction de la scissure de Rolando;

$2^{\circ}$ Subdivision du lobe pariétal en trois lobes, qui sont : le 
lobe occipital, le lobe temporal et le lohe pariétal proprement dit;

$3^{\circ}$ Constitution du lobe occipital, par suite de la formation de la scissure occipitale; agrandissement du sillon calcarin, qui devient la scissure calcarine;

$4^{\circ}$ Constitution du lobe temporal, par suite de l'allongement du lobule temporal et de la disparition partielle de l'arc inférieur de la scissure limbique;

$5^{\circ}$ Constitution du lobe pariétal proprement dit, par suite de la formation des deux lobes précédents;

$6^{\circ}$ Constitution particulière du lobe de l'insula dans la fosse de Sylvius ;

$7^{\circ}$ Développement considérable de la scissure sous-frontale; $8^{\circ}$ Effacement presque complet de la scissure sous-pariétale. Nous avons maintenant à étudier successivement ces divers caractères :

$1^{\circ}$ Agrandissement du lobe frontal. - Le premier de ces caractères, celui qui prime tous les autres en importance et qui est le point de déparl de la plupart l'entre eux, peut-être de tous, est constitué par le développement excessif du lobe frontal des primates. Ce lobe présente chez les autres mammifères un volume très variable; mais, même chez le cheval et l'éléphant, qui sont les plus favorisés sous ce rapport, il n'a qu'un volume relatif médiocre. Chez les carnassiers, que nous comparons avec les primates, il est très rudimentaire; la scissure de Rolando qui le limite est située tout en avant, bien en avant de l'extrémité antérieure de la scissure de Sylvius; elle est toujours très oblique, au moins dans sa partie inférieure et externe, et cette obliquité est dirigée de bas en haut et d'arrière en avant. Il en résulte que le lobe frontal, déjà très court dans sa partie externe, est plus court encore dans sa partie interne, qui ne forme qu'une très petite portion du bord sagittal de l'hémisphère. Chez les primates, au contraire, ce lobe est très grand dans toutes ses parties, mais surtout dans sa partie interne, qui forme plus de la moitié du bord sagittal. La scissure de Rolando est reportée beaucoup plus en arrière. Son extrémité inférieure et externe n'est plus située en avant de l'extrémité antérieure de la scissure de Sylvius, mais au-dessus de la partie moyenne de cette 
scissure, et son extrémité supérieure est beaucoup plus reculée encore, de sorte que sa direction n'est plus oblique en haut et en avant, mais en haut et en arrière.

L'agrandissement du lobe frontal des primates s'effectue à la fois en longueur, en largeur et en épaisseur. L'épaississement du lobe relève l'écaille de l'os frontal, son élargissement la dilate transversalement, et l'extrémité antérieure dủ cerveau devient plus volumineuse, plus arrondie que chez la plupart des autres animaux; mais l'élargissement de la loge frontale ne fournit, dans le sens transversal, qu'un espace insuffisant, eu égard à la grande ampliation transversale de l'écorce du lobe frontal, et celle-ci, dès lors, devient le siège d'un plissement longitudinal, qui produit les circonvolutions frontales.

Les effets de l'agrandissement en longueur sont plus remarquables encore. Cet agrandissement, gêné en avant par la résistance de la paroi crânienne, se traduit par une poussée antéropostérieure exercée sur le reste de l'hémisphère; mais, sur la face inférieure ou orbitaire du lobe frontal, les connexions de la base du cerveau avec la base du crâne s'opposent au recul du bord postérieur du lobule orbitaire. C'est donc presque exclusivement sur la face convexe du lobe frontal que se manifestent les effets de l'allongement des circonvolutions frontales. Là, cet allongement ne rencontre d'autre obstacle que la résistance des circonvolutions pariétales, résistance inférieure à la poussée frontale, et le lobe frontal se développe ainsi aux dépens du lobe pariétal.

Chez la plupart des mammifères, le lobe frontal ne se prolonge que très peu sur la convexité de l'hémisphère, et c'est à peine si, dans quelques cas, il recouvre quelque peu la partie la plus antérieure du ventricule latéral : sa portion orbitaire est donc généralement beaucoup plus grande que sa partie supérieure; celle-ci n'est pour ainsi dire que l'extrémité de celle-là, et il n'y a pas lieu de distinguer deux étages dans le lobe frontal. Mais chez les primates la portion supérieure de ce lobe, énormément agrandie, s'étend au loin sur la face convexe de l'hémisphère, au-dessus du ventricule latéral, et le lobe frontal, dès lors, comprend deux étages : l'un supérieur, très grand, situé au-dessus du centre ovale; l'autre inférieur ou orbitaire, 
situé au-dessous de ce centre, et beaucoup plus petit que le premier. Les circonvolutions se continuent d'ailleurs directement d'un étage à l'autre en se réfléchissant sur la partie antérieure du lobe.

$2^{\circ}$ Subdivision du lobe pariétal. - Par suite du développement excessif du lobe frontal, le lobe pariétal, qui formait jusqu'ici presque toute la masse circonvolutionnaire, se trouve considérablement refoulé. Perdant ainsi une très grande partie de leur longueur, ses circonvolutions se replient, se prolongent en arrière et en bas, et forment trois groupes qui constituent trois lobes distincts, savoir : le lobe occipital, le lobe temporal et le lobe pariétal proprement dit ou lobe pariétal des primates.

$3^{\circ}$ Constitution du lobe occipital. - La séparation du lobe occipital résulte d'un plissement transversal, rendu nécessaire par l'insuffisance de l'espace dans le sens de la longueur. La portion postrolandique de l'hémisphère, repoussée en arrière par le lobe frontal, regagne une partie de l'espace perdu en se prolongeant au-dessus du cervelet, qu'elle recouvre entièrement et que parfois même elle déborde. Mais ce n'est pas assez : les circonvolutions longitudinales se trouvent trop longues encore pour l'espace qui leur est laissé, et elles deviennent le siège d'un plissement transversal, qui s'effectue à la fois sur la face convexe et sur la face interne, et qui produit la scissire occipitale (ou scissure perpendiculaire de Gratiolet). La partie de cette scissure qui s'étend sur la face interne se nomme scissure occipitale interne; celle qui s'étend sur la face externe se nomme scissure occipitale externe; mais ces deux portions se continuent le plus souvent l'une avec l'autre sans interruption.

Ce qui est en arrière de la scissure occipitale forme le lobe occipital.

On a vu plus haut (p. 319) que les circonvolutions pariétales des cerveaux osmatiques prennent naissance sur la partie postérieure de l'hémisphère, daus une petite région où elles communiquent les unes avec les autres, et où elles communiquent en outre avec le pli de passage rétro-limbique. Cette région, que nous avons appelée le pôle de l'hémisphère, fait naturellement partie du lobe occipital des primates, et prend chez eux le nom de jồle occipital. On a vu encore que le pli rétro-limbique, 
ordinairement simple chez les carnassiers, est subdivisé dans sa longueur chez la plupart des ruminants et des pachydermes par un sillon que nous avons considéré par anticipation comme l'analogue de la scissure calcarine des primates, et que nous avons nommé le sillon calcarin. Chez les primates, il devient grand, profond, fixe, typique, et acquiert l'importance des véritables scissures. L'extrémité antérieure de la scissure calcarine, droite ou très peu flexueuse, aboutit au grand lobe limbique, dans l'épaisseur duquel elle se prolonge et où elle établit une séparation très marquée entre le.lobe de l'hippocampe (devenu la circonvolution de l'hippocampe) et le lobe du corps calleux, séparation souvent si profonde qu'elle paraît complète et que la scissure calcarine semble se continuer jusque dans la grande fente de Bichat. C'est ce qu'on voit en particulier chez le cercopithecus sabæus, que Gratiolet avait pris pour type dans la description du cerveau des primates. Cet auteur en a conclu que la scissure calcarine se continuait dans la fente de Bichat avec la scissure de l'hippocampe, tout le long du corps godronné, jusqu'au crochet de la circonvolution de l'hippocampe, et il a ainsi réuni deux anfractuosités essentiellement distinctes en une seule anfractuosité qu'il a appelée la scissure des hippocampes. Cela n'a pas peu contribué à faire méconnaître la continuité de la circonvolution de l'hippocampe et de celle du corps calleux, déjà démontrée cependant par les recherches de Gerdy et de Foville (voy. plus haut, p. 264). Le fait est que chez la plupart des singes, quelquefois chez l'homme même, lorsqu'on examine la scissure calcarine avant l'ablation des membranes, on croit la voir aboutir à la fente de Bichat B (voy. fig. 35 , K); mais, si on enlève la pie-mère, si l'on dilate la fente de Bichat et si l'on écarte les bords de la scissure calcarine, on voit (fig. 36) que celle-ci n'entame que superficiellement le grand lobe limbique, et qu'un tractus plus ou moins épais de substance cérébrale maintient la continuité de la circonvolution de l'hippocampe, $\mathrm{H}^{\prime}$, et du lobe du corps calleux, C'. Les deux figures 35 et 36 représentent la face interne de l'hémisphère droit du lagotriche de Humboldt, moulée avant et après la dilatation des scissures.

L'isthme de communication est ici assez étroit; il est souvent beaucoup plus large; chez l'homme, il est généralement assez 
volumineux pour être superficiel, et il devient apparent dès que la pie-mère est enlevée, sans qu'il soit nécessaire de dilater la

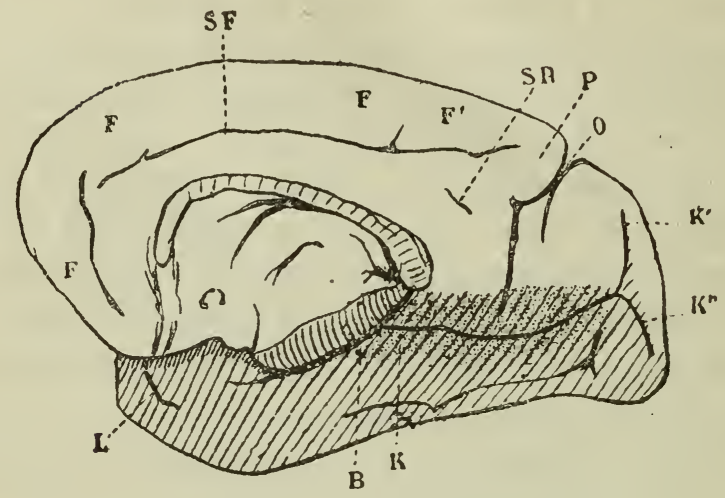

Fig, 3̈̈.

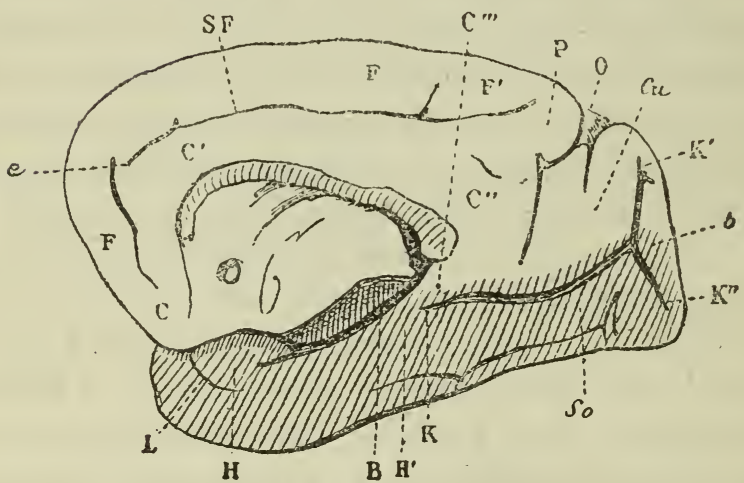

Fig. 36.

Face interne de l'hémisphère droit du lagotriche de Humboldt (singe cébien). Sur la figure 35 , la scissure calcarinc, $\mathrm{K}$, et la fente de Bichat, $\mathrm{B}$, sont dans leur état naturel; sur la figure 36 , elles sont dilatées, pour montrer la continuité de la circonvolution de l'hippocampe, $\mathrm{H}^{\prime}$, et du lobe du corps callcux, C'"'.

IH' $^{\prime}$, circonvolution de l'hippocampe; L, vestige de l'arc inférieur de la scissure limbique (sillon limbique); $\mathrm{CC}^{\prime} \mathrm{C}^{\prime \prime} \mathrm{C}^{\prime \prime \prime}$, le lobe du corps calleux; $\mathrm{SF}$, scissure sous-frontale; $\mathrm{FFF}^{\prime}$, face interne du lobe frontal; 'F', lobule ovalairc du lobe frontal; SP, sillon sous-pariétal ; $\mathrm{P}$, lobule quadrilatère, on face internc de la première circonvolution pariétale; $\mathrm{O}$, scissure occipitale; $\mathrm{C} u$, cunéus ou lobule occipital interne; $\mathbf{K}$, scissure calcarine; $\mathrm{K}^{\prime}$, sa branche supérieure; $\mathrm{K}^{\prime \prime}$, sa branche iıférieure; So, lobule sous-occipital; $b$, le pôle occipital.

scissure. Chez le gorille, il est également volumineux et super ficiel ; mais chez beaucoup de singes il est profond, et chez certains cébiens (cebus) il est tellement mince et tellement profond 
qu'il faut une certaine attention prour le découvrir; enfin, il manque chez les primates lissencéphales, tels que les ouistitis et les tarnarins. Dans ce dernier cas, la scissure calcarine paraît n'être qu'une émanation de la fente de Bichat, et la continuité des deux parties adjacentes du grand lobe limbique n'est établie que par une lame de substance grise qui passe sous l'extrémité antérieure de la scissure calcarine. Les fœtus humains de trois ¿ cinq mois présentent quelquefois la même disposition : ce n'est pas le cas le plus ordinaire; le plus souvent un petit pli profond ou même superficiel s'étend de la circonvolution de l'hippocampe à celle du corps calleux. J'ai toujours trouvé ce pli plus ou moins développé chez les fœtus âgés de plus de cinq mois, et il se dessine de plus en plus à mesure que les circonvolutions se forment dans le reste du manteau.

Quoi qu'il en soit, il faut reconnaître que la scissure calcarine entame beaucoup plus profondément le grand lobe limbique chez les primates que chez les autres animaux. Cela prouve que la solidarité anatomique et fonctionnelle du lobe de l'hippocampe et du lobe du corps calleux est devenue beaucoup moindre. On peut conjecturer que cet isolement relatif des deux arcs du grand lobe limbique à leur extrémité postérieure, comme l'isolement plus complet qui s'effectue à leur partie antérieure, au niveau de l'espace perforé, est la conséquence de l'atrophie de l'appareil olfactif et de la décadence de la fonction olfactive. Le lobe de l'hippocampe et le lobe du corps calleux sont très différents l'un de l'autre par leur structure et leurs connexions, et il ne paraît pas vraisemblable qu'ils n'aient qu'une seule et même fonction : chacun d'eux doit avoir une fonction spéciale encore inconnue; mais ils ont en outre chez les osmatiques une fonction olfactive commune, attestée par la continuité de leurs extrémités antérieures avec le lobe olfactif, et par la fusion de leurs extrémités postérieures l'une avec l'autre. Chez les primates, cette fonction olfactive commune perd presque toute son importance, et les deux lobes qu'elle unissait tendent à se séparer. Mais je n'insiste pas sur' cette conjecture un peu hasardée, et je reviens à la scissure calcarine.

Née, comme on vient de le voir, sur le grand lobe limbique, la scissure calcarine des primates devient bientôt très profonde, 
se porte à peu près directement en arrière le long du bord très émoussé qui sépare la face interne de l'hémisphère de sa face inférieure ou tentoriale, et gagne la pointe de l'hémisphère, c'est-à-dire le pôle occipital (fig. $36, b$ ), où elle se termine en se divisant en deux branches, l'une ascendante $\mathrm{K}^{\prime}$, l'autre descendante $K^{\prime \prime}$. Dans son trajet, elle affecte un rapport constant avec le prolongement postérieur du ventricule latéral, connu sous le nom de cavité ancyroïde. Cette cavité est exclusivement propre aux primates, comme le lobe occipital dont elle fait partie; la portion de l'hémisphère qui est refoulée en arrière et qui va former au-dessus du cervelet le lobe occipital, entraîne avec elle la partie postérieure de la paroi du ventricule latéral. Ge ventricule s'y prolonge done sous la forme d'un diverticule long et étroit, que l'on a comparé à une corne (corne postérieure) ou à la branche récurrente d'une ancre (cavité ancyroïde). La formation de la cavité ancyroïde est donc la conséquence de la formation du lobe occipital. La direction de cette cavité est longitudinale; son bord inférieur est soulevé par une saillie longitudinale, décrite par Morand sous le nom d'ergot, en latin calcar, et appelée encore le petit hippocampe. Or, le fond de la scissure calcarine correspond précisément à cette saillie inférieure : de là est venu le nom de scissure calcurine qui a été proposé par Huxley, et que nous adoptons (1).

La scissure calcarine subdivise le lobe occipital en deux parties, l'une supérieure, l'autre inférieure. L’inférieure ou lobule sous-occipital (So, fig. 36) forme la face inférieure du lobe; la supérieure ou lobule occipital interne, ou cunéus, en forme la face interne $(C u)$.Le cunéus, ainsi nommé parce qu'il a souvent la forme d'un coin, est compris entre la branche ascendante de la scissure calcarine $\mathrm{K}^{\prime}$ et la partie de la scissure occipitale 0 , qui s'étend sur la face interne. En avant, le cunéus et le lobule

(1) Gratiolet a eu le mérite de découvrir la scissure calcarine, d'en déterminer les rapports et d'en signaler l'importance; mais il a cru à tort qu'elle se prolongeait dans la fente de Bichat, le long du grand hippocampe, que par conséquent elle se trouvait successivement en rapport avec les deux hippocampes, en arrière avec le petit, en avant aveo le grand. Il l'appelait donc scissure des hippocampes, dénomination reconnue inexacte aujourd'hui, puisqu'on sait que la portion postérieure et la portion antérieure de cette prétendue scissure constituent deux anfractuosités distinctes. 
sous-occipital communiquent avec le grand lobe limbique par deux plis que sépare la partie antérieure de la scissure calcarine, et qui représentent à eux deux le pli de passage rétro-limbique des osmatiques. Chez ces animaux, le pli de passage rétro-limbique fait communiquer le grand lobe avec la partie postérieure du lobe pariétal, laquelle est représentée chez les primates par le lobe occipital. Celui-ci est en quelque sorte l'épanouissement du pli rétro-limbique, et la scissure calcarine qui le subdivise est l'analogue du sillon calcarin que nous arons étudié plus haut chez le cheval (p. 323, fig. 27) et chez le chevreuil (p. 321, fig. 26). La profonde subdivision du pli rétrolimbique des primates reproduit donc, à peine modifiée, une disposition qui se trouve chez beaucoup d'autres animaux. La branche inférieure de la subdivision fait communiquer le lobe sous-occipital avec la partie postérieure de la circonvolution de l'hippocampe (fig. $36, \mathrm{H}^{\prime}$ ), c'est-à-dire avec l'arc inférieur du grand lobe limbique; sa forme habituelle lui a fait donner le nom de lobule lingual; je l'appellerai le pli de passage occipitohippocampique. La branche supérieure de la subdivision du pli rétro-limbique fait communiquer le cunéus avec l'extrémité postérieure et inférieure, $\mathrm{C}^{\prime \prime \prime}$, du lobe du corps calleux : je l'appelle donc le pli de passage cunéo-limbique. Ce pli passe au-dessus de la scissure calcarine et au-dessous de l'extrémité inférieure de la scissure occipitale 0 . Il est superficiel chez presque tous les primates, et alors la scissure occipitale ne communique pas avec la scissure calcarine; chez l'homme et les gibbons il est profond, et les deux scissures se réunissent en $\mathrm{Y}$; mais si l'on écarte les bords au niveau de leur confluent, on aperçoit constamment le pli cunéo-limbique, dont les connexions ne sont nullement modifiées.

La scissure calcarine, avons-nous dit, se termine en arrière, sur l'extrémité postérieure de l'hémisphère, en se divisant en deux branches, l'une ascendante $\mathrm{K}^{\prime}$ (fig. 36), et l'autre descendante $K^{\prime \prime}$. La longueur relative de ces deux branches varie suivant les espèces. L'inférieure $K^{\prime}$ aboutit à la pointe de l'hémisphère, c'est-à-dire à la partie la plus inférieure et la plus postérieure du lobe occipital; elle peut même quelquefois dépasser cette pointe, et se prolonger quelque peu sur la face con- 
vexe du lobe. Entre les deux branches $\mathrm{K}^{\prime}$ et $\mathrm{K}^{\prime \prime}$ se trouve un pli plus ou moins contourné $b$, qui se continue à la fois avec toutes les circonvolutions occipitales, c'est-à-dire : en haut et en dedans avec le cunéus, en bas avec les circonvolutions du lobule sous-occipital, en dehors avec les circonvolutions plus ou moins rudimentaires de la face externe. C'est sur ce pli que nous avons vu, chez les osmatiques (voy. p. 320, fig. 25, face postérieure de l'hémisphère du tapir'), aboutir les sillons longitudinaux du lobe pariétal, et nous lui avons donné dès lors le nom de pôle, nom qui, dans ce cas, manque peut-être de justesse, parce que, si tous les sillons pariétaux se rendent sur un même pli, ils ne convergent pas vers un même point; ils peuvent même quelquefois être un peu divergents, attendu que l'extrémité de l'hémisphère sur laquelle se déploie transversalement le pli en question $b b^{\prime} b^{\prime \prime}$, fig. 25) a toujours une assez grande largeur. Mais chez les primates cette extrémité postérieure se termine en une pointe vers laquelle convergent plus ou moins directement les sillons occipitaux et qui mérite réellement le nom de pôle. Le pôle occipital, origine de toutes les circonvolutions occipitales, correspond donc à la pointe de l'hémisphère vis-à-vis de l'extrémité de la branche descendante de la scissure calcarine ( $\mathrm{K}^{\prime \prime}$, fig. 36). Ces circonvolutions, toujours bien distinctes sur les faces interne et inférieure du lobe occipital, le sont beaucoup moins, dans la plupart des cas, sur la face externe, où elles sont souvent fusionnées dans la plus grande partie de leur étendue; mais toujours, sur la limite de la face externe et de la face inférieure, existe un grand sillon longitudinal $l$ (fig. 37) qui se dirige vers le pôle et qui sépare le lobule sous-occipital $S o$ du lobule sus-occipital $S u$. Je donne à ce sillon, qui est constant chez les primates gyrencéphales, le nom de sillon occipital latéral (c'est le troisième sillon occipital de l'homme et des anthropoïdes).

Le lobule sous-occipital est quelquefois presque indivis, surtout dans sa partie antérieure, qui borde la scissure occipitale externe et qui se prolonge même un peu au-dessus d'elle en manière d'opercule (opercule occipital de Gratiolet $m$, fig. 37 ). On peut croire alors qu'il n'y a pas de vraies circonvolutions sus-occipitales, et que le lobule sus-occipital ne se continue pas 
avec les circonvolutions pariétales, situées au-devant de la scissure. Quelquefois cependant ces dernières circonvolutions, qui sont au nombre de deux, franchissent la scissure occipitale en formant les plis de passage supérieur's de Gratiolet, ou plis de passage sus-occipitaux. Gratiolet accordait une très haute importance à ces plis de passage ; il en faisait un caractère de pre-

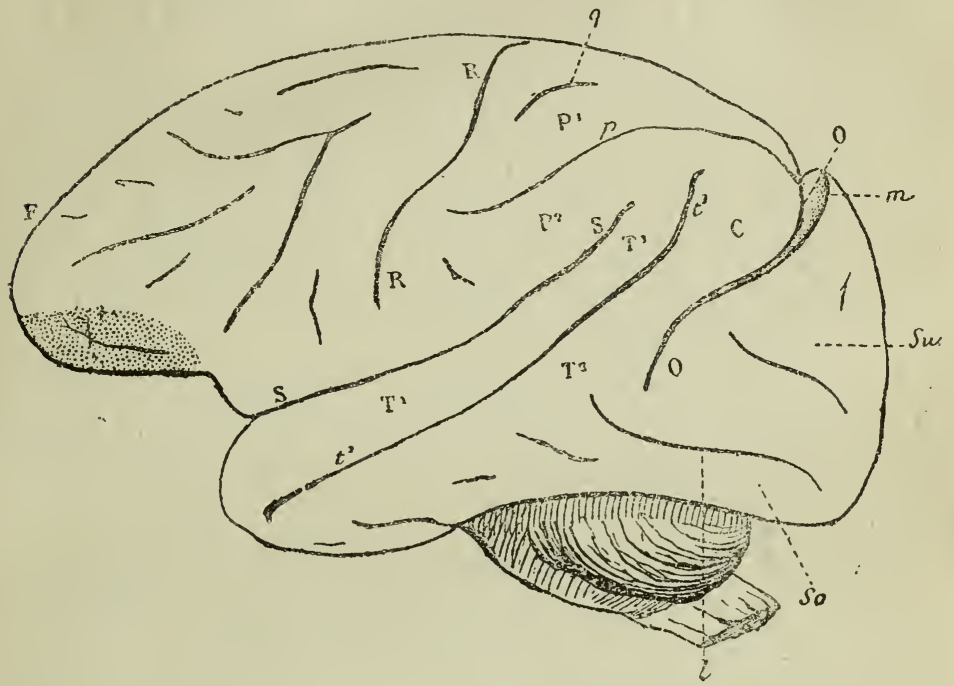

Fig. 37. Cynocéphale papion (pithécien); face externe de l'hémisphère gauche.

SS, scissure de Sylvius; RR, scissure de Rolando; OO, scissure occipitale externe; F, lobe frontal; $\mu^{2}$, première circonvolution pariétale; $q$, scissure subdivisant cette circonvolution; $\mathrm{P}^{\prime}$, seconde circonvolution pariétale; $p$, sillon pariétal; $\mathrm{T}^{\prime} \mathrm{T}^{\mathbf{\prime}}$, première circonvolution temporale; $\mathrm{T}^{2}$, seconde circonvolution temporale; $t^{\prime} t^{\prime}$, premier sillon temporal, dit scissure parallèle; $l$, sillon occipital latéral; So, lobule sous-occipital; $S u$, lobule sus-occipital; $m$, opercule occipital,

mier ordre, et s'en servait même pour diviser les primates en trois groupes, suivant qu'ils avaient deux plis de passage ou un seul, ou qu'ils n'en avaient aucun; mais, lorsqu'on dilate la scissure occipitale jusque dans sa profondeur pour en faire le moulage à la cire, suivant le procédé que j’ai décrit dans mon mémoire sur le cerveau du gorille (1), on trouve toujours au moins le rudiment des deux plis de passage (2); on reconnaît ainsi

(1) Revue d'anthropologie, 1878,p. 38, et plus loin dans ce volume.

(2) Le premier pli de passage est ordinairement situé sur le bord sagittal, la où la scissure occipitale interne se continue avec l'externe; quelquefois il est situé un peu plus au dehors, et alors on le trouve aisément; mais quelquefois il 
que les deux circonvolutions pariétales se continüent toujours dans le lobule sus-occipital ; que celui-ci, par conséquent, comprend toujours deux circonvolutions plus ou moins fusionnées. Par suite de cette fusion, le lobule sus-occipital de la plupart des primates paraît très simple ; on peut même dire que la simplicité absolue ou relative de ce lobule constitue un caractère typique du cerveau des primates; c'est seulement dans les genres les plus élevés que le lobule sus-occipital, participant à son tour au perfectionnement du reste de l'hémisphère, se couvre de véritables circonvolutions; celles-ci sont en réalité au nombre de deux, comme les plis de passage, mais leur nombre est porté à trois, par suite de la subdivision de la seconde, dont la partie antérieure et externe se continue avec le lobe temporal. Toutes trois d'ailleurs, après s'être plus ou moins contournées, vont aboutir au pôle occipital.

$4^{\circ}$ La formation du lobe temporal constitue le troisième caractère du cerveau des primates, et peut être, comme celle du lobe occipital, attribuée en grande partie au développement exagéré du lobe frontal.

L'obliquité déjà indiquée de la scissure de Rolando nous a montré que ce dernier lobe s'accroît dans sa partie supérieure et interne beaucoup plus que dans sa partie inférieure et externe. Le lobe pariétal, aux dépens duquel il s'amplifie, se trouve donc refoulé à la fois en arrière et en bas. On a vu que le refoulement vers l'arrière donne lieu à la formation du lobe occipital; celle du lobe temporal résulte du refoulement vers le bas. La circonvolution externe ou sylvienne du lobe pariétal descend donc vers la base du cerveau, où l'atrophie du lobe de l'hippocampe laisse une place disponible. On n'a pas oublié que, sur le cerveau des carnassiers, la partie postérieure des circonvolutions du groupe sylvien forme, au-dessus du lobe de l'hippocampe, en arrière et au-dessous de la scissure de Sylvius (qui est oblique), une sorte de lobule que nous avons appelé le lobule temporal du lobe pariétal (voy. plus haut, p. 286 ; voyez aussi p. 307 , fig. 17, T, le cerveau du renard, et p. 316 , fig. 23 , T, le

est plus en dedans, de manière à traverser la scissure occipitale interne. Les cas où il occupe cette dernière position sont ceux où Gratiolet croyait qu'il manquait entièrement. 
cerveau du chien). Ge lobule, dirigé vers le bas, est aussi dirigé un peu en avant, comme la scissure de Sylvius elle-même. Si l'on suppose que, chez ces animaux, le lobe de l'hippocampe s'atrophie graduellement, le lobule temporal, s'avançant à sa place, tendra à se porter non seulement en bas, mais aussi en avant, et trouvera d'autant plus de facilité à s'étendre dans cette dernière direction, que la région inféricure et externe qu’il occupe correspond à la partie la moins volumineuse du lobe frontal. C'est ce qui a lieu chez les primates, et la partie du lobe pariétal qui s'étend ainsi en bas et en avant, jusqu'audessous de la partie postérieure du lobe frontal, constitue alors un lobe distinct, qui est le lobe temporal. La scissure de Sylvius, devenue ainsi très longue et très oblique, forme la limite supérieure de ce lobe, qui conserve d'ailleurs ses connexions de continuité, sur sa face externe avec le lobe pariétal, et sur sa face inférieure avec le lobe occipital (qui est un autre prolongement du même lobe pariétal). Enfin le lobe temporal est complété en bas et en dedans par ce qui reste du lobe de l'hippocampe atrophié, c'est-à-dire par la petite circonvolution de l'hippocampe, devenue la dernière circonvolution temporale.

J'ai déjà indiqué la fusion qui s'effectue entre la circonvolution de l'hippocampe et l'avant-dernière circonvolution temporale. Cette fusion, toutefois, n'est jamais complète, elle laisse toujours subsister un vestige de la scissure limbique. Chez tous les singes, cébiens, pithéciens et anthropoïdes, la partie antérieure de la scissure limbique reste ouverte, et ordinairement même assez profonde. Elle entaille dans une étendue variable la pointe du lobe temporal (voy. p. 3ร̌2, fig. $36, \mathrm{~L}$ ) jusqu’à la vallée de Sylvius où elle semble s'arrêter; mais, si l'on soulève le bord postérieur de cette vallée, on voit que la scissure limbique se prolonge en dehors et en haut, en se recourbant sous la pointe du lobe temporal, de manière à atteindre l'extrémité externe de cette vallée, pour se terminer dans l'entrée de la fosse de Sylvius. Ce vestige de la scissure limbique (sillon limbique) tend à s'effacer chez l'homme. Il se retrouve habituellement dans les races inférieures, et quelquefois aussi prononcé que chez les singes. Chez la plupart des blancs, il disparaît tout à fait; la position du sillon limbique est néanmoins indiquée en- 
core, sur la pointe du lobe temporal, par une dépression longitudinale superficielle qui permet de rèconnaître la limite externe de la circonvolution de l'hippocampe. L'extrémité antérieure de cette circonvolution reste donc toujours plus ou moins distincte. Elle se dessine sous la forme d'un petit lobule arrondi qu'on appelle le lobule de l'hippocampe.

La présence du sillon limbique sur la pointe du lobe temporal doit être considérée chez l'homme comme un caractère d'infériorité. J'ai trouvé ce sillon sur tous les cerveaux de nègres que j’ai étudiés jusqu'ici, mais je suppose qu'il doit manquer quelquefois dans cette race. Je l'ai vu aussi chez quelques blancs, qui, pour la plupart, mais non tous, étaient idiots ou imbéciles, et j'ajoute que je possède plusieurs cerveaux d'idiots et de microcéphales sur lesquels il n'existe pas.

En somme, l'homme est, de tous les primates, celui sur lequel on observe le degré le plus avancé de la fusion de l'extrémité antérieure du lobe de l'hippocampe : c'est donc celui qui s'éloigne le plus du type des mammifères osmatiques; chez lui néanmoins la partie postérieure de ce lobe est toujours longée par un sillon qui est le dernier sillon temporal, et qui est l'analogue de la partie correspondante de la scissure limbique des osmatiques.

Ce dernier sillon temporal, prolongé ou non jusqu'à la pointe du lohe, est constant chez les primates gyrencéphales; le premier sillon temporal qui longe le bord sylvien du lobe (scissure parallèle de Gratiolet) est constant aussi. La portion du lobe temporal qui est comprise entre ces deux sillons est ordinairement subdivisée en deux ou trois plis par un ou deux sillons dont la direction est également longitudinale. Tous ces sillons se dirigent vers la pointe du lobe, qui, comme on l'a vu plus haut, est le plus souvent indivise chez l'homme ou du moins chez l'homme blanc, et qui mérite dès lors, dans la description du cerveau humain, le nom de pôle temporal.

$5^{\circ}$ Constitution du lobe pariétal. - On vient de voir comment la partie postérieure et la partie inférieure des circonvolutions pariétales se modifient chez les primates de manière à former deux lobes distincts, le lobe occipital et le lobe temporal. Par suite de ce démembrement, dû à la poussée qu'excrce 
d'avant en arrière et aussi de haut en bas le lobe frontal amplifié, le lobe pariétal des primates se trouve considérablement réduit. De ce vaste lobe pariétal qui, chez les autres mammifères, formait presque toute la face convexe de l'hémisphère, un tiers environ est devenu chez les primates le lobe occipital, un autre tiers est devenu le lobe temporal, et le nom de lobe pariétal ne s'applique plus maintenant qu'à la portion antérieure et supérieure, comprise d'une part entre la scissure de Rolando et la scissure occipitale, d'une autre part entre le bord sagittal et la scissure de Sylvius. Ainsi réduit dans ses dimensions, le lobe pariétal est en outre notablement modifié dans ses formes. Il subit une évolution inverse de celle du lobe frontal. Ce dernier lobe, si petit et si simple chez les autres mammifères, où il était manifestement primé par le lobe pariétal, est devenu, chez les primates, le principal lobe de l'hémisphère; il s'est en quelque sorte emparé de l'hégémonie cérébrale, et l'importance croissante de ses fonctions est attestée par le plissement longitudinal qui le subdivise d'abord en deux, puis en trois circonvolutions. Le lobe pariétal se comporte tout autrement : loin de se compliquer, il se simplifie an contraire, et les circonvolutions qui le composent tendent à se fusionner. Il y avait chez les gyrencéphales osmatiques de trois à cinq circonvolutions pariétales: il n'y en a plus que deux chez les primates.

Ce changement est important sans doute, mais il l'est moins qu'on ne pourrait le croire au premier abord. Souvenons-nous que, chez les gyrencéphales autres que les primates, les circonvolutions pariétales, quel qu'en soit le nombre, se répartissent en deux groupes, le groupe sagittal et le groupe sylvien, séparés l'un de l'autre par le sillon pariétal primaire (voy. plus haut, p. 304). Chez les animaux osmatiques qui n'ont que des circonvolutions rudimentaires, ce sillon indique déjà la séparation des deux zones sagittale et sylvienne, qui, d'ailleurs, ne sont pas subdivisées; mais chez ceux qui ont de vraies circonvolutions la zone sylvienne est toujours subdivisée en deux circonvolutions longitudinales. Quant à la zone sagittale, elle est aussi, le plus ordinairement, subdivisée en deux circonvolutions parallèles; mais cela est moins constant, car elle peut être divisée en trois, ce qui est d'ailleurs très exceptionnel, et elle peut rester' 
tout à fait indivise, comme on le voit, par exemple, chez le blaireau, la loutre, et chez la plupart des petits carnassiers. La subdivision de la zone sagittale est donc moins essentielle que celle de la zone sylvienne, puisqu'elle est plus variable.

Chez les primates, le lobe pariétal, en se simplifiant, tend à revenir au type rudimentaire. La zone sagittale se réduit à une seule circonvolution, qui est la première pariétale; la zone sylvienne se réduit également à une seule circonvolution, qui est la seconde pariétale, et il ne reste plus qu'un seul sillon longitudinal, qui est le sillon pariétal (intra-pariétal de Turner).

Malgré cette double modification, il est facile de montrer l'analogie des circonvolutions pariétales chez les primates et chez les autres gyrencéphales, car la seconde circonvolution des primates n'est simple qu'en avant ; en arrière, elle est subdivisée en deux plis parfaitement distincts par un sillon parfaitement constant (fig. $37, t^{\prime} t^{\prime}$, p. 357 ) qui de là, se dirigeant en bas, puis en avant, se continue directement avec le premier sillon temporal (ou scissure parallèle de Gratiolet). Les deux plis qui résultent de ce dédoublement se recourbent pour se porter en bas, puis en avant, comme le sillon qui les sépare ; le pli antérieur, qui est en même temps supérieur, contourne l'extrémité de la scissure de Sylvius, S, pour aller constituer la première circonvolution temporale, $\mathrm{T}^{\mathrm{T}}$; le pli postérieur, qui est en même temps inférieur, $\mathrm{C}$, mérite parfaitement le nom de pli courbe, qui lui a été donné par Gratiolet; il se recourbe autour de l'extrémité du premier sillon temporal, passe au-devant de la scissure occipitale externe, 00 , et va se continuer à la fois avec la seconde circonvolution temporale, $\mathrm{T}^{2}$, et avec la circonvolution sus-occipitale la plus externe. Cette bifurcation de la seconde circonvolution pariétale est constante chez tous les primates gyrencéphales; elle se montre même déjà chez certains cébiens qui n'ont presque aucune autre division dans leurs hémisphères.

Quelquefois, il est vrai, l'extrémité postérieure de la scissure de Sylvius va aboutir obliquement au premier sillon temporal : c'est ce qui a lieu, par exemple, chez les cébus (fig. 38) et, parmi les pithéciens, chez beaucoup de cercopithèques, de macaques, ainsi que chez le cynocéphale mandrill. On pourrait croire alors que la seconde pariétale (fig. $38, \mathrm{P}^{2}$ ) ne donne que 
le pli courbe, $\mathrm{C}$, et ne se continue qu'avec la seconde circonvolution temporale, $T^{2}$, puisque la scissure de Sylvius, $\mathrm{S}$, va rejoindre le premier sillon temporal, $t^{1}$; mais, si l'on écarte les bords de

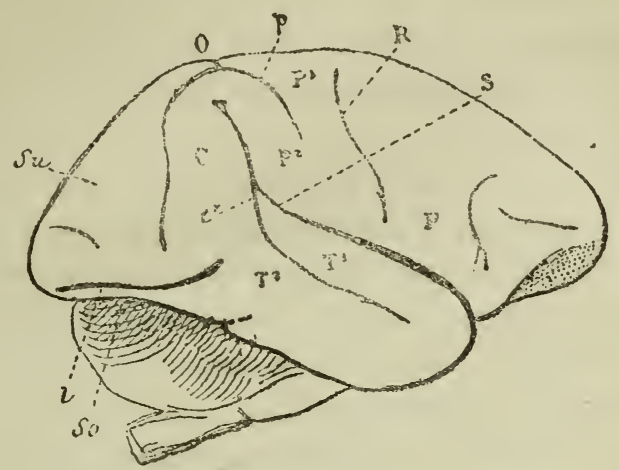

Fig. 38 .

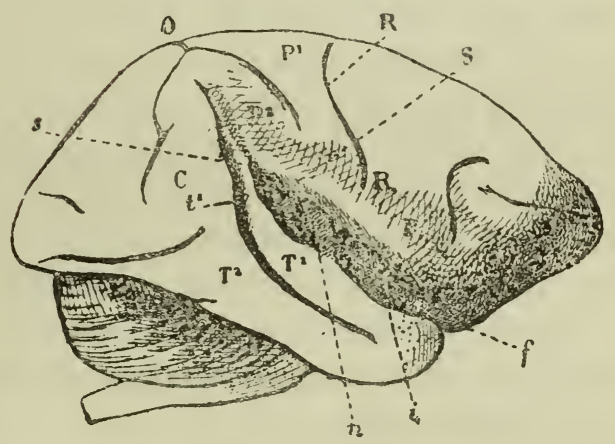

Fig. 39

Cebus apella; face externe de l'hémisphère droit.

Fig. 38. Les parties sont dans leurs rapports naturels. R, scissure de Rolando; O, scissure occipitale; S, scissure de Sylvius, communiquant superficiellement en arrière avec le premier sillon temporal, $t^{4} ; \mathrm{F}$, lobe frontal; $\mathrm{P}^{\prime}$. première circonvolution pariétale; $\mathrm{P}^{2}$, seconde circonvolution pariétale; $\mathrm{C}$, pli courbe; $\mathrm{T}^{\mathbf{}}$, première temporale; $\mathrm{T}^{2}$, seconde temporale ; $l$, sillon occipital latéral; $S u$, lobule sus-nccipital; $S o$, lobule sous-occipital; $p$, sillon pariétal.

Fig. 39. Le mème hémisphère moulé à la cire, la scissure de Sylvius ayant été préalablement dilatée pour montrer la fosse de Sylvius et le lobe de l'insula; $s$, pli profond établissant la continuité de $\mathrm{P}^{2}$ et de $\mathrm{T}^{\mathbf{3}}$ entre la scissure de Sylvius et le premier sillon temporal; nif, lobe de l'insula dans la fosse de Sylvius ; $n$, pli de passage temporo-pariétal profond; $i$, l'insula proprement dite; $f$, pli fálciforme temporo-frontal, à l'entrée de la fosse de Sylvius.

Les autres lettres comme sur la figure 38 .

la scissure de Sylvius pour mettre à découvert la fosse de Sylvius - comme on l'a fait sur la figure 39 , qui représente le même hémisphère moulé à la cire - on voit qu'un pli parfaitement distinct, $s$, sépare du premier sillon temporal l'extrémité de la 
scissure de Sylvius, et fait par conséquent communiquer la seconde circonvolution pariétale avec la première circonvolution temporale (1).

La seconde circonvolution pariétale des primates est donc réellement double en arrière, et il est aisé de reconnaître qu'elle représente les deux circonvolutions de la zone sylvienne des autres gyrencéphales.

L'analogie de la première circonvolution pariétale des primates avec la zone pariétale sagittale des autres gyrencéphales est plus facile à démontrer encore. $0 \mathrm{n}$ a vu, en effet, que cette zone est indivise chez la loutre, le blaireau, et chez la plupart des petits carnassiers, qui, sous ce rapport, ne diffèrent pas des primates inférieurs. Elle est ordinairciuent subdivisée, chez les carnassiers, en deux plis parallèles, tantôt en partie fusionnés, comme chez l'ours, tantôt parfaitement distincts, comme chez le renard. Cette subdivision complète ne s'observe jamais chez les primates : voilà pourquoi nous ne comptons chez eux qu'une seule circonvolution sagittale ; celle-ci, néanmoins, montre une tendance à la subdivision lorsque le cerveau grandit et se complique. Chez le cynocéphale papion (fig. 37, p. 3557), une incisure longitudinale, $q$, dessine sur la première circonvolution pariétale, $\mathrm{P}^{1}$, une subdivision rudimentaire; chez les'grands anthropoïdes, cette incisure se retrouve toujours, ordinairement assez grande, quelquefois plus ou moins sinueuse et même bifurquée ; elle peut se prolonger assez pour se mettre en communication avec le sillon pariétal ; la première circonvolution pariétale n'est donc plus simple. Chez l'homme, l'incisure qui la divise est quelquefois formée de plusieurs branches tortueuses, dont l'ensemble assez compliqué a suggéré le nom, d'ailleurs inutile, de lobule pariétal, donné par Gratiolet à cette circonvolution. La première circonvolution pariétale de l'homme et des grands anthropoïdes peut donc être considérée comme l'analogue des deux circonvolutions sagittales des carnassiers, comme le ré-

(1) La position superficielle ou profonde du pli $m$, c'est-à-dire de la branche de bifurcation supérieure de la seconde circonvolution pariétale, a si peu d'importance qu'elle ne constitue pas même un caractère générique. Aussi ce pli est maigre et profond chez le cynocéphale mandrill, volumineux et superficiel chez le cynocéphale papion. 
sultat de la fusion de ces deux circonvolutions. La fusion est ici plus prononcée que sur les deux circonvolutions de la zone sylvienne; et il n'y a pas lieu de s'étonner que la zone sagittale du cerveau des primates soit relativement plus atrophiée que la zone sylvienne, puisque le lobe frontal, dont la poussée produit cette atrophie, est beaucoup plus développé dans sa partie supérieure que dans sa partie externe.

$6^{\circ}$ Constitution du lobe de l'insula. - En étudiant plus haut la formation de la fosse de Sylvius chez les osmatiques (voir p. 308 et sq.), nous avons vu que cette fosse est l'espace compris, sur la face externe de l'hémisphère, entre le bord externe du grand lobe limbique (p. 317, fig. 24, L, L', L') et la concavité de l'arc sylvien, $\mathrm{P}^{1} \mathrm{P}^{1}$. Plus ou moins superficielle chez beaucoup de pachydermes et de ruminants, elle est toujours profonde chez les carnassiers, où elle est cachée par le bord de l'arc sylvien; mais on la met à découvert en soulevant ce bord et en dilatant la scissure de Sylvius, comme sur la figure 24 . On voit alors que l'arc sylvien est constitué tout en avant par le bord inférieur et externe du petit lobe frontal $\mathrm{F}$, et dans le reste de son étendue par la circonrolution sylvienne du lobe pariétal, $\mathrm{P}^{1} \mathrm{P}^{1} \mathrm{P}^{1}$. Celle-ci se compose de deux parties qui, avant la dilatation, formaient les deux bords de la scissure de Sylvius. Quant au bord inférieur ou limbique de la fosse de Sylvius, il est formé en arrière par le lobe de l'hippocampe, en avant par la racine olfactive externe.

Chez les primates, la fosse de Sylvius est profonde comme chez les carnassiers, et pour la mettre à découvert il faut encore dilater la scissure de Sylvius (voir p. 363, fig. 39). Le lobe de l'hippocampe, atrophié, refoulé vers la base de l'hémisphère par les autres circonvolutions temporales qui se prolongent audessus de lui, n'est plus en rapport avec la fosse de Sylvius, et la racine olfactive externe, plus atrophiée encore, se réduit à un petit tractus blanc qui s'enfonce dans la partie externe de la vallée de Sylvius, la traverse et va se jeter profondément sur l'extrémité antéro-externe de la circonvolution de l'hippocampe. La fosse de Sylvius n'est donc plus séparée de la vallée de Sylvius que par un pli très court et très profond, $f$, qui unit l'extrémité antéro-externe de la circonvolution de l'hippocampe à la circon- 
volution la plus externe du lobe frontal, et sur lequel chemine la racine olfactive externe rudimentaire. Ce pli profond, que j'appelle le bord falciforme, est tout ce qui reste, chez les primates, du bord limbique de la fosse de Sylvius. Il correspond à l'entrée de la fosse de Sylvius, c'est-à-dire au point où cette fosse se met en continuité avec la vallée de Sylvius.

Par suite de la disparition du bord limbique, la fosse de Sylvius des primates n'est plus entourée que par l'arc sylvien. Celui-ci forme un circuit presque complet, interrompu seulement, en bas et en avant, par la vallée de Sylvius, circuit très allongé suivant une direction presque longitudinale, et composé par conséquent de deux marges, l'une supérieure, l'autre inférieure. La marge supérieure (fig. 39) est formée en avant par le bord inférieur du lobe frontal, $\mathrm{F}$, en arrière par celui du lobe pariétal, $\mathrm{P}^{2}$; la marge inférieure est formée dans toute sa longueur par la première circonvolution du lobe temporal, $\mathrm{T}^{1}$; les deux marges se continuent l'une avec l'autre en arrière, $s$, sur l'extrénité terminale de la scissure de Sylvius. Il est aisé de voir que cette constitution de l'arc sylvien des primates est tout à fait analogue à celle de l'arc sylvien des osmatiques. Le lobe frontal agrandi forme une plus grande partie de la marge supérieure; ce n'est qu'une différence de degré. En outre, la circonvolution sylvienne, appelée pariétale sur la marge supérieure, a pris le nom de temporale sur la marge inférieure; ce n'est qu'une différence de nom. Enfin les extrémités antérieures des deux marges se sont rapprochées l'une de l'autre et ne sont plus séparées que par la largeur de l'entrée de la fosse de Sylvius, $f$; ce n'est qu'une différence de forme. Somme toute, le circuit qui entoure la fosse de Sylvius des primates ne diffère par aucun caractère essentiel de celui qui a été décrit chez les osmatiques.

Le fond de cette fosse est occupé par une portion du manteau qui est assez développée chez les primates, surtout chez les primates supérieurs, pour mériter le nom de lobe, et qu'on appelle le lobe de l'insula, mais qui correspond exactement par sa position et par sa constitution au lobule sous-sylvien déjà décrit chez les osmatiques (voir plus haut, p. 308-318). Comme ce dernier lobule, le lobe de l'insula repose sur le noyau lenticulaire ou noyau extra-ventriculaire du corps strié; comme lui il se com- 
pose de deux parties, l'une temporo-frontale, l'autre temporopariétale.

On a vu que le lobule sous-sylvien des osmatiques est formé de deux plis que nous avons appelés le pli temporo-frontal et le pli temporo-pariétal. Nés l'un et l'autre du lobule temporal, c'est-à-dire de la portion du lobe pariétal qui est située derrière la scissure de Sylvius, ils vont se rendre tous deux à la marge supéricure de la fosse de Sylvius, le premier à la portion frontale de cette marge, le second à sa portion pariétale. Le lobule temporal formant la limite postérieure de la fosse de Sylvius, les deux plis cheminent d'arrière en avant, et le pli temporo-frontal est situé au-dessous de l'autre (voy. fig. 18 et 19, p. 310 et p. 311).

Chez les primates, le lobule temporal devient le lobe temporal et se prolonge en bas et en avant jusqu'au-dessous du lobe frontal ; la direction des plis sous-sylviens se trouve donc nécessairement changée. Le pli temporo-frontal se dirige en haut pour atteindre la portion frontale de la marge sylvienne supérieure; d'horizontal, il est devenu ascendant. Le pli temporopariétal est plus dévié encore : il se dirige non seulement de bas en haut, mais encore d'avant en arrière; c'est parce que d'une part son insertion temporale s'est portée en bas et en avant, et parce que d'une autre part son insertion pariétale est considérablement refoulée vers l'arrière, par suite de l'agrandissement du lobe frontal et du recul de la scissure de. Rolando. Dès lors, le pli temporo-frontal n'est plus situé au-deśsous, mais en avant du pli temporo-pariétal. D'ailleurs ce cliangement de direction ne modifie en rien les connexions des deux plis sous-sylviens.

Pour retrouver ces deux plis chez les primates, il faut dilater la scissure de Sylvius, de manière à abaisser la marge inférieure, formée par la première circonvolution temporale et à élever la marge supérieure, formée en arrière par la seconde circonvolution pariétale, en avant par le bord externe ou sylvien du lobe frontal (voy. p. 363, fig. 39). On aperçoit alors dans toute son étendue le fond de la fosse de Sylvius, c'est-à-dire le lobe de l'insula, et on y distingue aussitôt deux parties, l'une postérieure et supérieure, $n$, l'autre antérieure et inférieure, $i$.

La partie postérieure, $n$, est formée par un pli qui se détache 
profondément du bord supérieur de la première circonvolution temporale, gagne la rigole supérieure de la fosse de Sylvius, puis se relève et se rejette sur la face profonde de la seconde circonvolution pariétale. Ce pli, unissant deux circonvolutions qui appartiennent à deux lobes différents, est au nombre de ceux qu'il faut appeler plis de passage. Je le nomme donc le $p l i$ de passage temporo-pariétal profond. Je dis profond, parce qu'il l'est toujours chez les primates, mais il ne l'est pas toujours chez les autres animaux; car on n'a pas oublié que le pli sous-sylvien temporo-pariétal, dont il est l'analogue, est superficiel chez les ruminants et chez le tapir, qu'il est superficiel en avant chez le cheval, qu'il l'est parfois chez le porc (voir plus haut p. 310-314, fig. 18 à 22).

Le pli de passage temporo-pariétal est simple et sans flexuosités chez la plupart des primates; il se complique un peu plus chez les primates supérieurs et particulièrement chez l'homme, où il forme une circonvolution assez flexueuse et assez volumineuse, mais toujours très profonde.

En avant de ce pli de passage, le reste du lobe de l'insula est constitué par une saillie convexe, $i$, presque toujours lisse, qui s'étend jusqu'à l'entrée de la fosse de Sylvius, où elle est limitée par le pli falciforme, $f$. Cette saillie constitue l'insula proprement dite. C'est elle que l'on désigne lorsqu'on dit simplement l'insula. En d'autres termes, le lobe de l'insula comprend deux parties : l'insula et le pli de passage temporo-pariétal.

L'insula est séparée du bord externe et inférieur du lobe frontal par une rigole supérieure, et de la partie antérieure du hord supérieur du lobe temporal par une rigole inférieure; en outre, elle est séparée du bord antéro-supérieur du pli de passage temporo-pariétal par un sillon étendu obliquement de la rigole supérieure à la rigole inférieure ; ce dernier sillon est ordinairement très peu déprimé, mais les deux rigoles sont très profondes : de là est venu le nom d'insula, donné par Reil à la partie qu'elles circonscrivent presque entièrement.

La couche de substance grise qui revêt l'insula se prolonge sans interruption, par-dessous les deux rigoles, sur les bords respectifs des deux lobes frontal et temporal; mais, outre cette continuité très profonde et purement corticale, l'extrémité an- 
térieure et infér: :ure de l'insula présente immédiatement en dehors du pli falciforme, à l'entrée de la fosse de Sylvius, des connexions plus directes avec les deux lobes voisins. De cette extrémité, que nous appellerons le pôle de l'insula, se déta. chent d'une part un pli court et épais qui se continue aussitôt avec l'extrémité terminale de la circonvolution frontale la plus externe, d'une autre part un tractus large et court qui se confond avec le pli falciforme, et va se jeter profondément sur la face supérieure du lobe temporal. L’insula établit donc, dans le fond et surtout à l'entrée de la fosse de Sylvius, une communication entre le lobe temporal et le lobe frontal, et, d'après ces connexions, nous devons la considérer comme l'analogue du pli temporo-frontal des osmatiques.

L'insula est toujours simple chez les cébiens et les pithéciens, ainsi que chez les gibbons. Ghez les grands anthropoïdes et chez l'homme, elle se subdivise en un certain nombre de plis qui convergent vers le pôle et vont gagner successivement les divers points de la rigole supérieure, qu'ils traversent pour se jeter profondément dans le lobe frontal.

Chez les animaux autres que les primates, le lobule sous-sylvien est tantôt superficiel, tantôt profond, et sa position dépend principalement de son volume. Chez les carnassiers, où il est très petit, il est entièrement caché sous la circonvolution sylvienne; chez les ruminants, chez la plupart des pachydermes, il est plus volumineux, et il apparaît en tout ou en partie à la surface du cerveau. Le lobe de l'insula des primates, toujours beaucoup plus grand que le lobule sous-sylvien des carnassiers, atteint, chez les primates supérieurs, un volume non seulement égal, mais même supérieur (toutes proportions gardées) à celui du lobule sous-sylvien superficiel des ruminants et des pachydermes. Il reste profond cependant, et il est même d'autant plus profond qu'il est plus volumineux. Ce résultat, en apparence contradictoire, est la conséquence du grand développement des circonvolutions qui forment la marge supérieure de la fosse de Sylvius. En avant, le lobe frontal élargi abaisse son bord externe comme une sorte de toit, par-dessus l'insula; c'est la conséquence naturelle de l'agrandissement général de ce lobe; en arrière, la marge supérieure de la fosse de Sylvius est formée par le lobe 
pariétal, qui, loin d'être agrandi, est au contraire diminué; mais sil'on se souvient que l'extrémité supérieure et postérieure du lobe frontal exerce sur le lobe pariétal une poussée qui le refoule non seulement eri arrière, mais encore en dehors et en bas, on comprendra que le bord inféro-externe de la seconde circonvolution pariétale doit être abaissé de manière à recouvrir en arrière le pli de passage temporo-pariétal, comme le lobe frontal recouvre en avant l'insula proprement dite. La marge supérieure de la fosse de Sylvius descend donc tout entière pardessus le lobe de l'insula, dont elle constitue l'opercule, et vient s'appliquer sur le bord supérieur presque rectiligne de la première circonvolution temporale. Cette ligne de contact est la scissure de Sylvius. La fosse de Sylvius se trouve ainsi refermée. Pour la mettre à découvert, il faut écarter les bords de la scissure et soulever l'opercule.

La formation de la portion frontale de l'opercule est la conséquence du développement du lobe frontal en largeur. Chez les anthropoïdes, le bord externe de ce lobe se développe aussi en longueur; et l'excès de la longueur de la circonvolution frontale la plus externe (qui est chez eux la troisième) donne lieu à un plissement qui s'effectue au devant de l'insula, et qui forme la branche antérieure de la scissure de Sylvius. Un second plissement, situé au-dessus du premier, produit chez l'homme, et parfois aussi chez le chimpanzé et l'orang, la branche ascendante de la scissure de Sylvius (1). La troisième circonvolution frontale, ainsi repliée, ne longe plus seulement le bord supérieur de l'insula : elle contourne en outre son bord antérieur, dont elle est séparée par une troisième rigole, la rigole antérieure de l'insula. En même temps, l'insula, devenue plus grande, s'est recouverte de plusieurs plis radiés. La fosse de Sylvius, la scissure de Sylvius et le lobe de l'insula, ont acquis alors leur maximum de complication et de développement. Néanmoins, la constitution de ces parties et leurs connexions sont restées exactement les mêmes que chez les primates inférieurs, et sont restées en

(1) Pour plus de détails sur les branches antérieures de la scissure de Sylvius, voir mon Mémoire sur le cerveau du gorille, p. 18-22 (Revue d'anthropologie, 1878), et plus loin dans ce volume. 
outre, dans ce qu'elles ont d'essentiel, les mêmes que chez les autres mammifères.

$7^{\circ}$ Développement de la scissure sous-frontale et effacement de la scissure sous-pariétale. - Nous venons d'étudier les modifications que subissent chez les primates les parties qui composent la face externe et la face inférieure de l'hémisphère. Des modifications non moins remarquables se montrent sur la face interne de l'hémisphère, dans les parties qui sont en rapport avec l'arc supérieur du grand lobe limbique, c'est-à-dire avec le lobe du corps calleux.

Ge lobe, quoique restant toujours relativement plus simple que le reste du manteau, se ressent cependant, au moins chez les primates supérieurs, de la complication générale de l'hémisphère; son bord convexe devient plus ou moins onduleux ou sinueux, de manière à revêtir, jusqu'à un certain point, l'aspect des circonvolutions proprement dites : on le désigne donc sous le nom de circonvolution du corps calleux. Mais sa constitution est restée la même, et le nom de circonvolution ne lui convient pas, car le propre d'une circconvolution est d'être l'une des parties d'un lobe:or, la circonvolution du corps calleux ne peut être rattachée ni au lobe frontal, ni au lobe pariétal, ni au lobe occipital, ni au lobe temporal, puisqu'elle passe successivement sous les trois premiers pour aller se continuer avec le quatrième. Elle constitue donc à elle seule un lobe distinct de tous les autres, et qui est manifestement l'analogue du corps calleux des mammifères osmatiques. Le nom de lobe du corps calleux que nous lui donnons constate cette analogie.

Le lobe du corps calleux passe en avant sous le lobe frontal et en arrière sous le lobe pariétal. L'état de ses connexions avec ces deux lobes constitue l'un des caractères distinctifs les plus remarquables du cerveau des primates.

Chez les osmatiques, l'arc supérieur de la scissure limbique, qui circonscrit plus ou moins complètement le lobe du corps calleux, se compose de deux parties, l'une sous-frontale, l'autre sous-pariétale (voy. plus haut, p. 32う-330). Ces deux portions de la scissure limbique sont quelquefois continues l'une avec l'autre, mais le plus souvent séparées par le pli de passage fronto-limbique (fig. $29, b$, p. 329). Il y a alors une scissure 
sous-frontale, entièrement distincte de la scissure sous-pariétale. Dans l'ordre des carnassiers, qui est le plus proche voisin de celui des primates, et qu'il convient dès lors de prendre pour terme de comparaison, la scissure frontale n'existe qu'à l'état de vestige (voy. fig. $29, \mathrm{SF}$ ) ; le lobe frontal, $\mathrm{F}$, qui est très petit, est presque entièrement fusionné avec le lobe du corps calleux, GG'. L'anatomie comparée nous montre que la petite anfractuosité rudimentaire, $\mathrm{SF}$, est l'analogue de la scissure sousfrontale; mais, si cette anfractuosité indique à un observateur attentif la limite des deux lobes, on ne peut pas dire qu'elle les sépare, car elle se réduit à une courte dépression à peine plus marquée que les empreintes vasculaires, et elle est si peu apparente que Leuret ne l'a pas vue sur le cerveau du renard, étudié pourtant par lui avec une prédilection toute particulière (1). C'est donc seulement par analogie que je l'ai appelée scissure frontale dans la description précédente; mais, en réalité, chez les carnassiers, ce n'est pas une scissure, le propre d'une scissure étant de séparer deux lobes; ce n'est qu'un léger sillon, que nous appellerons le sillon sous-frontal. La scissure sous-pariétale des carnassiers est, au contraire, une vraie scissure (fig. 29, $\mathrm{SP})$, une grande et profonde scissure, qui commence au pli rétro-limbique, $a$, décrit une grande courbe sous le lobe pariétal, $\mathrm{P}$, remonte jusqu'au bord sagittal de l'hémisphère, et va former sur la face convéxe le sillon crucial de Leuret. Je rappelle que cette scissure sépare très profondément le lobe du corps calleux de la circonvolution pariétale sagittale; mais que, toutefois, deux plis de passage ordinairement profonds, l'un antérieur situé en $d$, fig. 30, l'autre postérieur situé en $c$, font communiquer entre eux ces deux lobes (voy. plus haut, fig. 30 , et p. 331).

En résumé, le lobe du corps calleux des carnassiers se trouve en rapport avec un lobe frontal très petit et avec un lobe pariétal très grand. Il se fusionne avec le petit, et reste entière-

(1) Voir l'atlas de Leuret (Anatomie comparée du système nerveux, atlas in-folio, pl. IV, fig. 3). Sur cette figure le lobe du corps calleux, dont la surface est absolument plate, se continue sans aucune ligne de démarcation jusqu'au bord sagittal du lobe frontal. Le vestige de la scissure sous-frontale, qui a échappé à l'attention d'un observateur aussi habile que Leuret, se retrouve constamment sur le cerveau des carnassiers, même sur les plus simples et les plus petits, tels que le cerveau de la belette. 
ment distinct du grand. La scissure sous-frontale n'existe qu'à l'état de vestige, il n'en reste qu'un léger sillon: la scissure sous - pariétale, au contraire, est extrêmement grande.

Ghez les primates, la scène change tout à coup. Le lobe frontal devient très grand, le lobe pariétal très petit; le sillon sous-frontal devient une grande et profonde scissure, et la scissure sous-pariétale s'efface presque entièrement; il n'en reste

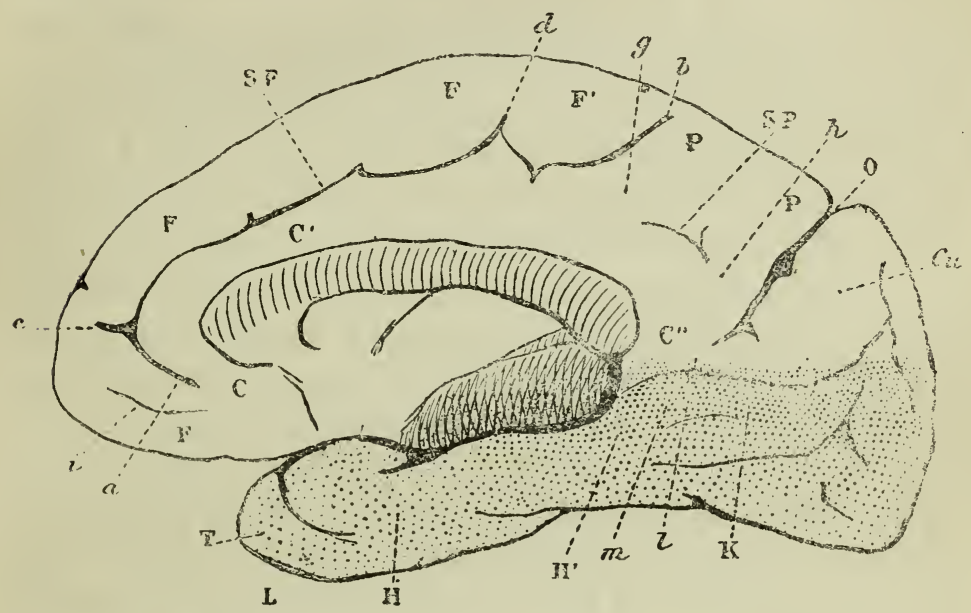

Fig. 40. Cynocéphale papion; face interne de l'hémisphère droit.

HH', circonvolution de l'hippocampe avec son crochet; T, pointe du lobe temporal ; L, vestige de l'arc inférieur de la scissure limbique (sillon limbique); $O$, scissure occipitale interne ; $\mathrm{K}$, scissure calcarine; $\mathrm{SF}$, scissure sous-frontale ; $a$, son origine; $b$, sa terminaison; $e$, incisure fronto-limbique marquant la position du pli de passage fronto-limbique, qui est profond; $i$, incisure sous-frontale. $\mathrm{CC}^{\prime} \mathrm{C}^{\prime \prime}$, circonvolution ou lobe du corps calleux ; FFF, face interne de la première circonvolution frontale, dont l'étage supérieur est séparé de l'étage inférieur ou orbitaire par l'incisure fronto-limbique $e ; \mathrm{F}^{\prime}$, lobule ovalaire, ou face interne de la circonvolution frontale ascendante; PP, lobule quadrilatère ou face interne de la première circonvolution pariétale; $g, h$, les deux plis de passage pariéto-limbiques; $C u$, le cunéus ou lobule triangulaire du lobe oceipital ; $l$, pli de passage cunéo-limbique ou pli rétro-limbique supérieur; $m$, pli rétro-limbique inférieur ; $i$, incisure sous-orbitaire.

plus qu'un rudiment qui mérite tout au plus d'être appelé le sillon sous-pariétal. Ici, d'ailleurs, comme chez les carnassiers, les connexions du lobe du corps calleux avec les deux lobes voisins sont déterminées par le degré d'importance respective dẹ ces deux derniers lobes; le lobe le plus petit se fusionne avec le lobe du corps calleux; le lobe le plus grand en reste entièrement distinct.

La scissure sous-frontale des primates (SF, fig. 40) commence en $a$, près de l'origine $\mathrm{C}$ du lobe du corps calleux, contourne la 
partie antérieure de ce lobe, parcourt d'avant en arrière une grande partie de la face interne de l'hémisphère, en se rapprochant de plus en plus du bord sagittal, que son extrémité postérieure $b$ atteint souvent et dépasse quelquefois. Toute la portion de la face interne qui est comprise entre cette scissure et la circonférence de l'hémisphère est formée parlelobe frontal FFFF' savoir : en arrière, $\mathrm{F}^{\prime}$, par l'extrémité épanouie de la circonvolution frontale ascendante (ou prérolandique), en avant et en bas par la face interne de la première circonvolution frontale. Une incisure à peu près constante (incisure sous-frontale), $d$, tantôt réduite à un simple feston, tantôt plus profonde, le plus souvent émanée directement de la scissure, quelquefois isolée, toujours dirigée vers le bord sagittal, marque la limite antérieure de la partie $\mathrm{F}^{\prime}$, qui constitue le lobule ovalaire de Pozzi (ou lobule paracentral de Meynert). Cette incisure ne manque que sur les cerveaux des primates les plus inférieurs. Elle est encore apparente chez le lagotriche (voy. plus haut, fig. 35, p. 352), mais elle ne l'est plus chez les cébus. L'extrémité antérieure et inférieure de la scissure sous-f̈rontale s'arrête toujours à une certaine distance du bord inférieur de l'hémisphère ; et toujours, au-dessous d'elle, la première circonvolution frontale vient s'insérer sur l'origine du lobe du corps calleux, par une portion réfléchie qui appartient au lobule orbitaire. C'est la seule communication superficielle qui existe constamment entre le lobe frontal et le lobe du corps calleux; mais il y en a une autre qui est tantôt superficielle, tantôt profonde, et qui est constituée par le pli de passage fronto-limbique. Ce pli traverse la scissure sous-frontale au niveau du genou du corps calleux. Chez le cynocéphale papion, représenté sur la figure 40 , il est profond, et sa position n'est indiquée extérieurement que par l'incisure $e$. Lorsqu'il est superficiel, ce qui est le cas le plus ordinaire, il subdivise la scissure sous-frontale en deux portions correspondant respectivement à l'étage supérieur et à l'étage inférieur ou orbitaire de la première circonvolution frontale; il est quelquefois très mince et très court, comme on peut le voir sur notre lagotriche (p. 352, fig. $36, c)$. Il est d'autres fois plus large et plus long, comme sur le chimpanzé, représenté plus loin (p. 377, fig. $4 i, e)$. Au reste, le volume dı pli fronto-limbique est très 
variable dans la même espèce : ainsi, chez l'homme, chez l'orang, il est tantôt superficiel, tantôt profond, mais toujours, lorsqu'il est profond, sa position est indiquée par une incisure qui marque la séparation de l'étage supérieur et de l'étage orbitaire de la première circonvolution frontale. Il n'est pas inutile d'ajouter que ce pli fronto-limbique des primates n'est pas l'analogue de celui des carnassiers (celui-ci, en effet, est situé à l'extrémité de la scissure sous-frontale, qu'il sépare de la scissure souspariétale).

L'extrémité postérieure de la scissure sous-frontale, relevée vers le haut, atteint et dépasse le bord sagittal de l'hémisphère chez l'homme et les anthropoïdes; chez les autres primates, elle ne dépasse pas ce bord et ne l'atteint même pas toujours, mais toujours du moins elle s'en rapproche beaucoup. Ce qui est absolument constant, c'est que cette extrémité postérieure est toujours un peu en arrière de l'extrémité postérieure et supérieure de la scissure de Rolando. Ces deux scissures, qui limitent le lobe frontal, l'une sur la face interne, l'autre sur la face externe de l'hémisphère, ne se rejoignent pas sur le bord sagittal, auquel elles aboutissent toutes deux, parce que leurs deux extrémités sont séparées par un pli qui établit la communication entre la circonvolution frontale ascendante et son lobule ovalaire d'une part (fig. $40, \mathrm{~F}^{\prime}$ ) et la pariétale ascendante; il est d'ailleurs aisé de constater que le lobule ovalaire appartient au lobe frontal et non au lobe pariétal, car, si l'on tire sur le bord sagittal et sur la face interne de l'hémisphère une ligne qui prolonge la direction de l'extrémité postérieure de la scissure de Rolando, on voit que cette ligne laisse au-dessous d'elle tout le lobule ovalaire.

Pendant que la scissure sous-frontale des primates grandit et se développe, comme le lobe frontal dont elle est solidaire, un changement inverse se produit dans la scissure sous-pariétale. On se souvient que le lobe pariétal de ces animaux est considérablement réduit dans toutes ses parties, mais qu'il l'est bien plus dans sa portion sagittale que dans sa portion sylvienne. Nous avons déjà constaté cette atrophie sur la face convexe de la première circonvolution pariétale; nous allons maintenant la retrouver, bien plus prononcée encore, sur la face interne de la même circonvolution. De même que le lobe frontal présente son 
maximum de longueur sur la face interne de l'hémisphère, le lobe pariétal y présente son minimum de longueur. La première circonvolution pariétale n'y occupe que le court espace PP (fig. 40) compris entre l'extrémité postérieure, $b$, de la scissure sous-frontale et la scissure occipitale interne, 0 , espace rarement aussi grand qu'il l'est chez le cynocéphale, et quelquefois beaucoup plus petit (voy. p. 352, fig. 35 , P, la face interne de l'hémisphère du lagotriche). Cet espace a été désigné par Foville sous le nom de lobule quadrilatère.

$\mathrm{La}$ base du lobule quadrilatère est presque entièrement fusionnée avec le lobe du corps calleux, et a même été considérée comme faisant partie de ce lobe, qui a été, d'après cela, désigné par Rolando sous le nom de circonvolution crêtée; la circonvolution du corps calleux, surmontée du lobule quadrilatère, offre en effet une certaine ressemblance avec la crête de certains gallinacés, étroite en avant et large en arrière, comme le cimier d'un casque. Mais le Jobule quadrilatère ne fait pas partie du lobe du corps calleux ; il n'appartient qu'au lobe pariétal, et quoique sa base soit en grande partie fusionnée avec le lobe du corps calleux, on y trouve toujours encore, chez tous les primates gyrencéphales, depuis le cébus jusqu'à l'homme, le vestige de la grande scissure sous-pariétale des autres mammifères.

Ce vestige n'est plus une scissure : ce n'est plus qu'un court sillon, quelquefois très superficiel, plus ou moins parallèle à la convexité du corps calleux. C'est le sillon SP de la figure 40 (cynocéphale); on le retrouvera beaucoup plus courtsur le lagotriche (fig. 35, p. 352) et sur le chimpanzé (fig. 41, ci-contre). Chez l'homme, il est quelquefois plus flexueux, et compliqué d'une ou deux incisures, mais il occupe toujours la même position et son analogie ne peut être méconnue.

Le sillon sous-parićtal, par ses deux extrémités, se rapproche plus ou moins, en avant, de la scissure sous-frontale, en arrière, de la scissure occipitale, mais ne communique pas avec elles. Il en est séparê par deux plis, l'un antérieur, $g$, l'autre postérieur, $h$ (fig. 40), qui établissent une double et large communication entre le lobe pariétal et le lobe du corps calleux et qui méritent dès lors le nom de plis de passage pariéto-limbiques.

Ces deux plis de passage ne sont nullement propres aux pri- 
mates; ils sont les analogues de ceux que nous avons déjà reconnus chez les carnassiers. Les plis pariéto-limbiques des carnassiers sont ordinairement profonds (voy. plus haut, p. 331 et p. 372 , et fig. $30, c$ et $d$ ) ; mais nous ne saurions trop répéter que la position superficielle ou profonde des plis de passage, quoique pouvant amener de grandes différences morphologiques, ne constitue pas une différence anatomique. Rappelons d'ailleurs que le pli pariéto-limbique antérieur est large et superfi-

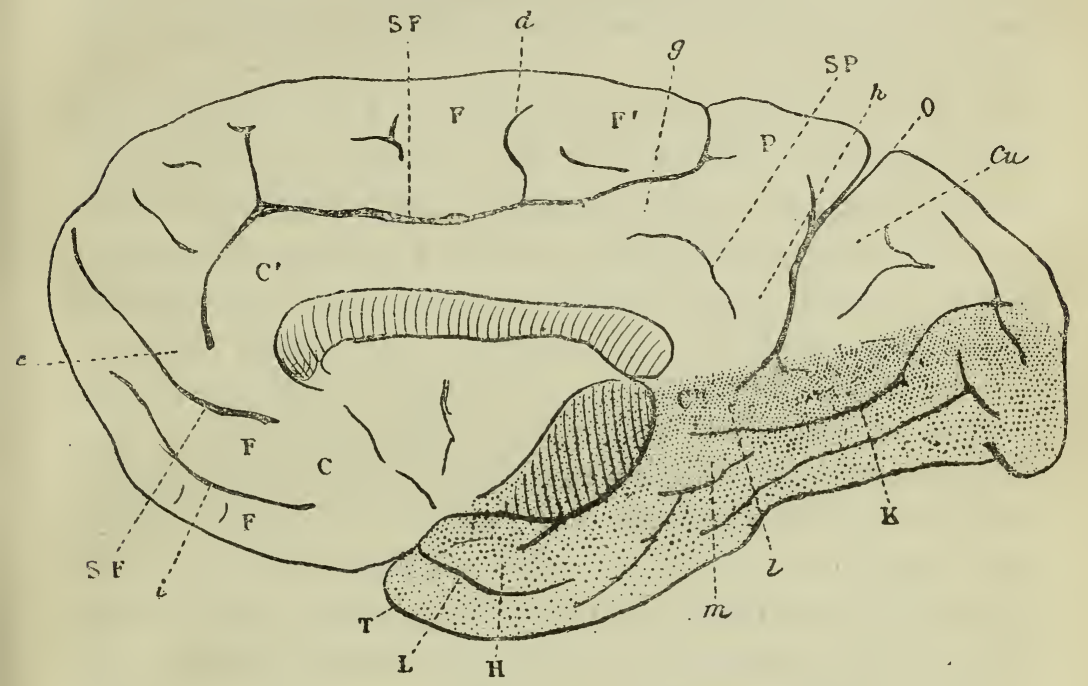

Fig. 41. Chimpanzé ; face interne de l'hémisphère droit.

Les lettres comme sur la figure 38 , si ce n'est que le pli fronto-limbique est superficiel et interrompt la scissure sous-frontale.

ciel chez les chats, que le pli pariéto-limbique postérieur est superficiel chez certains chiens. Tous deux sont larges et superficiels chez les primates. Voilà toute la différence.

La constitution de la face interne de l'hémisphère reste la même dans toute la série des primates gyrencéphales. On peut s'en assurer en comparant les figures déjà décrites du lagotriche, qui est un cébien (p. 352, fig. 35), et du cynocéphale papion, qui est un pithécien (p. 373, fig. 40), a vec la figure ci-dessus du chimpanzé, qui est un anthropoïde (fig. 41). Le cerveau de l'homme est trop connu pour qu'il soit nécessaire de le représenter, mais tout le monde y reconnaitra les parties suivantes, 
qui composent la face $\bullet$ interne de l'hémisphère des autres primates :

A. Autour du seuil de l'hémisphère, une première zone, formée par le lobe du corps calleux, CG'C' (fig. 40), et dont la limite est établie en arrière par le sillon sous-pariétal, SP, en avant par la scissure sous-frontale, SF. Celle-ci est souvent subdivisée, par le pli fronto-limbique, e, en deux portions, l'une supérieure, l'autre inférieure (ou réfléchie, ou orbitaire). Le pli de passage fronto-limbique, $e$, est quelquefois profond, et alors les deux portions de la scissure sous-frontale se continuent l'une avec l'autre.

B. Autour de cette première zone, une zone excentrique qui s'étend jusqu'à la limite de la face externe et qui est formée: $1^{\circ}$ en arrière de la scissure occipitale interne par le cunéus $(\mathrm{Cu})$ ou lobule triangulaire du lobe occipital; $2^{\circ}$ entre cette scissure et la sous-frontale par le lobule quadrilatère, $\mathrm{P}$, du lobe pariétal ; $3^{\circ}$ en avant et au-dessus de la scissure sous-frontale par la face interne du lobe frontal, qui se décompose à son tour en trois portions, savoir : a) en arrière le lobule ovalaire, qui est l'épanouissement de la circonvolution frontale ascendante ou prérolandique et dont l'incesure sous-frontale (d) marque la limite antérieure; $b$ ) en avant et en haut par l'étage supérieur de la première circonvolution frontale; $c$ ) en avant et en bas par l'étage inférieur ou orbitaire de la même circonvolution; le pli fronto-limbique, $e$, qui est quelquefois profond, marque la limite de ces deux étages chez les primates supérieurs, et l'incisure sus-orbitaire, $i$, subdivise en deux plis la face interne du lobule orbitaire.

La continuité du lobe du corps calleux avec les lobes environnants est établie : $1^{\circ}$ par l'insertion du lobe frontal sur l'origine, $\mathrm{C}$, du lobe du corps calleux; $2^{\circ}$ par le pli fronto-limbique, $e$, ordinairement superficiel ; $3^{\circ}$ par le pli pariéto-limbique antérieur, $g$; $4^{\circ}$ par le pli pariéto-limbique postérieur, $h$; 5० enfin par le pli cunéo-limbique, $l$, qui passe entre la scissure occipitale, 0 , et la scissure calcarine, K. Ce pli est profond chez l'homme et les gibbons, superficiel chez tous les autres primates; il représente la moitié supérieure du pli de passage rétro-limbique des autres mammifères, la moitié inférieure du même pli de passage étant 
représentée par le pli, $m$, qui s'étend du lobule sous-occipital à l'extrémité postérieure de la circonvolution de l'hippocampe.

\section{§8. CONCLUSIONS.}

Nous venons de passer en revue les caractères qui distinguent le type cérébral des primates de celui des autres mammifères et particulièrement des carnassiers. Ces caractères sont nombreux et très frappants; mais, lorsqu'on en étudie l'évolution, on voit qu'ils se rattachent tous directement ou indirectement à un fait fondamental, peut-être même initial : la prédominance du lobe frontal. Ces caractères, en effet, se divisent en deux séries : les uns se manifestent dans la région du grand lobe limbique, les autres se manifestent dans la masse circonvolutionnaire ou extra-limbique. Ceux-ci sont la conséquence de l'agrandissement du lobe frontal, qui, se développant en longueur, refoule les circonvolutions pariétales et amène la formation du lobe occipital et du lobe temporal, et qui, se développant en largeur, emprisonne le lobe de l'insula dans la fosse de Sylvius. Ceux-là sont la conséquence de l'atrophie de l'appareil olfactif et sont analogues à ceux qui s'observent chez les cétacés et surtout chez les amphibies; mais, tandis que, dans ce dernier cas, l'atrophie de l'appareil olfactif est due à un mode d'existence qui amène la diminution ou la suppression de la fonction olfactive, elle est due, chez les primates, à une cause toute différente; leur appareil olfactif a perdu son autonomie, il est retombé au même niveau que les organes des autres sens; comme eux, il se borne à recevoir et à transmettre des impressions qu'il n'élabore plus. Il n'est plus que l'un des vassaux du cerveau intellectuel, et même le plus humble, car les renseignements qu'il apporte ne valent pas ceux que l'intelligence agrandie sait tirer des autres sens. Les facultés d'observation, d'analyse, d'interprétation, se sont développées avec le lobe frontal et ont rendu inutile toute la portion de l'appareil olfactif qui excédait les besoins de la simple transmission sensorielle. Cet appareil s'est donc atrophié. J'ai déjà exposé ces vues dans un précédent paragraphe (voy. plus haut, p. 268). Je les rappelle ici pour montrer que ce n'est pas 
une simple coïncidence, mais une vraie corrélation qui existe entre l'agrandissement du lobe frontal des primates et l'évolution inverse du grand lobe limbique(1).

Tous les caractères distinctifs du type cérébral des primates sont donc subordonnés à un caractère fondamental qui, en quelque sorte, les résume tous, et qui peut se formuler en deux mots : prédominance frontale. L'apparition simultanée de ces nombreux caractères amène de grandes modifications extérieures et bouleverse toutela morphologie cérébrale, à tel point quel'on croit entrer dans un ordre de choses tout nouveau, comme si la chaîne des êtres était interrompue, comme si la nature, après a voir brisé ses anciens moules, s'était remise à l'œiıve avec des plans tout différents. D'après ces apparences, la plupart des auteurs ont renoncé à établir la détermination des parties similaires du manteau de l'hémisphère chez les primates et les autres mammifères. Quelques-uns cependant, plus convaincus de la continuité de la série, ont essayé de découvrir au moins quelques analogies entre les circonvolutions et les scissures des primates et celles des carnassiers (2). Ces tentatives n'ont pas réussi parce qu'elles reposaient sur la morphologie extérieure, qui ne montre que des dif-

(1) L'évolution des lobes optiques dans la série des vertébrés nous fournit un exemple de même nature. Ces lobes, très développés dans les classes inférieures, depuis les poissons jusqu'aux oiseaux, et si grand parfois qu'on les a pris pour les lobes cérébraux dont ils peuvent excéder le volume, ne sont plus représentés chez les mammifères que par les tubercules quadrijumeaux. Leur atrophie coïncide avec le développement des lobes cérébraux proprement dits, c'est-à-dire avec la constitution des hémisphères. Grands encore chez les monotrèmes, chez les didelphes, chez les monodelphes lissencéphales, ils perdent leur volume et leur importance à mesure que l'hémisphère grandit et se perfectionne. De même que, chez les mammifères ordinaires, la décadence des lobes optiques et la fin de leur autonomie sont la conséquence de la prépondérance des liémisphères, où s'effectue la centralisation céríbrale, de même, chez les primates, un nouveau perfectionnement de l'hémisphère, allesté par l'amplification énorme du lobe frontal, amène la décadence des lobes olfactifs.

(2) Benedikt (Moriz), Der Raublhiertypus am menschlichen Gehirne (Le type des carnassiers dans le cerveau humain), dans Centralblatt für die Med. Wissenschaftcn, 1876, p. 930. - Le même, Der Hinterhaupts-Lappen der Säuge. thiere (Le lobe occipital des Mammifères), dans le même recueil, 1877, p. 161.-Meynert (Théod.), Die Windungen der convexen Oberflxche des VorderHirnes bei Menichen, Affen und Ruubthieren (Les circonvolutions de la surface convexe du cerveau chez l'homme, les singes et les carnassiers), dans Archiv für Psychiatrie, Berlin, 1877, in-8u, t. I, p. 257-286. 
férences. Mais l'étude des connexions anatomiques conduit à de tout autres résultats. Elle permet de suivre, dans toute la série des mammitères, la formation, l'évolution, les modifications des diverses parties du manteau, de retrouver toutes ces parties dans le cerveau des primates, de reconnaître la raison d'être des caractères qui le distinguent et d'en déterminer, en quelque sorte, la filiation.

Leuret, cet auteur éminent qui a eu la gloire de constater le premier la fixité des circonvolutions dans chaque espèce, et d'être l'inspirateur posthume des travaux de Gratiolet, Leuret, dis-je, après avoir étudié les circonvolutions chez un grand nombre de mammifères, fut frappé de la grande diversité de leur nombre, de leur forme et de leur disposition; et, procédant d'abord par voie d'analyse, il fut conduit à établir, parmi les mammifères, jusqu'à quatorze groupes, caractérisés par autant de types cérébraux distincts, et à se demander mème s'il n'existait pas d'autres types parmi les animaux qu'il n'avait pas examinés. Puis, en y réfléchissant davantage, en voyant que les groupes basés sur les circonvolutions différaient souvent beaucoup des groupes naturels de la zoologie, il se demanda si ces nombreux types cérébraux étaient séparés les uns des autres par des limites absolues, s'il n'y avait pas entre eux, malgré leur dissemblance, des affinités et des transitions. Et il posa ainsi les deux questions suivantes:

" Y a-t-il des degrés intermédiaires entre tous les cerveaux? Y a-t-il des formes de cerveaux différentes de celles que j'ai décrites? Une observation plus étendue pourra seule résoudre ces questions, qui sont du plus haut intérêt pour l'anatomie et pour la physiologie (1). »

Ces deux questions n'en font, à vrai dire, qu'une seule, car la seconde n'est qu'une paraphrase de la première. Leuret écrivait en 1839 , à une époque où la doctrine de Lamark n'inquiétait plus les esprits et où celle de Darwin ne les agitait pas encore. Il est donc bien probable que le problème du transformisme ne le préoccupait pas, lorsqu'il posait la question de savoir si

(1) Leuret, Anatomie comparée du système nerveux considérée dans ses rapports avec l'intelligence, t. 1, p. 400. Paris, 1839, in-8. 
les divers types cérébraux des mammifères peuvent dériver les uns des autres.

Cette question, dont il signalait la haute importance anatomique et physiologique, était restée douteuse pour lui. Elle peut recevoir aujourd'hui, je pense, une réponse affirmative. Il y a, dans le manteau de l'hémisphère, une partie commune à tous les types cérébraux des mammifères : c'est le grand lobe limbique, qui, tantôt grand, développé et distinct dans toutes ses parties, tantôt plus ou moins modifié par l'atrophie et par la fusion, conserve toujours, néanmoins, son identité anatomique. Peu variable chez les osmatiques, presque étranger aux changements qui s'effectuent autour de lui, il reste toujours assez semblable à lui-même pour que la détermination de ses diverses parties et la démonstration de leur analogie soient de la dernière évidence; il fournit donc une base certaine pour la comparaison et le classement des plis qui se produisent dans le reste du manteau et qui, tout en revêtant des formes protéiques, conservent avec lui leurs connexions caractéristiques ; puis, lorsqu'il devient chez les anosmatiques le siège d'une atrophie qui modifie notablement quelques-uns de ses caractères, ces mêmes connexions permettent, d'une part, de retrouver en lui tous les éléments essentiels de sa constitution, de déterminer, d'une autre part, les rapports analogiques des circonvolutions qui l'entourent et des scissures qui y aboutissent, et de constater ainsi que tous les types cérébraux, jusques et y compris celui des primates, ne diffèrent les uns des autres que par des caractères d'évolution, c'est-à-dire par la forme et le volume relatif, et non par la nature de leurs parties constituantes.

Cette conclusion offre assez d'intérêt pour justifier l'importance que j'attache à l'étude du grand lobe limbique et de ses connexions dans la série des mammifères. Elle est peu conforme aux apparences, et j'ai dû, pour l'établir, entrer dans des détails de description qui ont pu paraître bien minutieux. Mais ceux de mes lecteurs qui ont eu la patience de me suivre jusqu'au bout reconnaîtront peut-être que ces détails n'étaient pas inutiles, et j'espère qu'ils voudront bien me pardonner la sécheresseet la longueur du présent mémoire. 
LOGALISATIONS CÉRÉBRALES

\section{RECHERCHES SUR LES CENTRES OLFACTIFS}

(Revue d'anthropologie, 1879, 2 e série, t. II, p. 385-455.)

\section{$\S$ 1. DU RंOLE DE L'ANATOMIE COMPARÉE DANS L'ÉTUDE DES LOCALISATIONS CÉRÉBRALES.}

La méthode expérimentale a rendu, dans notre siècle, de tels services à la physiologie, qu'elle a pour ainsi dire éclipsé toutes les autres méthodes. Il est bien vrai que les démonstrations les plus éclatantes sont celles qui sont établies ou confirmées par les vivisections; mais beaucoup de problèmes physiologiques échappent à cette méthode ou n'en reçoivent que des solutions incomplètes. Geux qui concernent les organes encéphaliques et leurs diverses parties sont bien souvent dans ce cas. Alors même que l'on réussit à produire la mutilation limitée de l'une de ces parties sans altérer les fonctions des autres, l'impossibilité où l'on se trouve d'interroger l'animal s'oppose à l'exacte détermination des troubles fonctionnels que l'on a provoqués, en même temps que la différence des types cérébraux et de la vie cérébrale ne permet pas d'appliquer à l'homme les conséquences qui paraissent découler d'expériences faites sur les autres animaux, même sur les singes.

A défaut de la méthode des vivisections, l'étude des phénomènes produits chez l'homme par les lésions traumatiques ou pathologiques du cerveau, et la comparaison de ces phénomènes, constatés pendant la vie, avec ces lésions, constatées après la mort, fournissent de précieuses ressources; c'est grâce à la mé- 
thode anatomo-pathologique que le siège de la faculté du langage a pu être déterminé, et que, par là même, le principe général des localisations cérébrales s'est trouvé établi. D'autres applications de ce principe sont aujourd'hui à l'étude, et quelques-unes paraissent en voie de démonstration. Mais la méthode anatomo-pathologique est bien lente : car les cas où les lésions sont assez circonscrites pour équivaloir à une vivisection sont très rares ; lorsqu'un fait de ce genre se présente à l'observation, il faut attendre bien longtemps encore pour que le hasard reproduise plusieurs fois la même lésion dans le même lieu et dans les mêmes limites, et pour qu'il soit permis de considérer comme réelle la relation qu'on suppose exister entre la faculté altérée ou abolie et le siège de la lésion.

Cette méthode enfin est très épineuse : car elle est subordonnée à l'étude préalable, faite pendant la vie de l'individu, de l'état de chacune de ses facultés cérébrales, non seulement quant à leur existence, mais encore quant à leur degré, étude souvent douteuse, quelquefois impossible et toujours extrêmement difficile. La faculté du langage est la seule qui se prête à des constatations suffisamment certaines ; c'est ce qui m'a permis d'en reconnaître le siège; mais dix-huit années se sont écoulées depuis lors, et quoique la méthode anatomo-pathologique ait été appliquée avec la plus grande persévérance par un grand nombre d'observateurs, les questions qui concernent les autres loca. lisations cérébrales n'ont pas encore reçı de solutions définitives.

Ainsi, d'une part, la méthode expérimentale n'est pas applicable à l'homme ; d'une autre part, la méthode anatomo-pathologique, qui en tient lieu chez l'homme, ne répond que très incomplètement aux besoins de la physiologie cérébrale. Dans cette pénurie, il est permis de se retourner vers une troisième méthode, qui est l'interprétation des faits anatomiques. Elle est assez dédaignée aujourd'hui ; mais pendant longtemps elle fut la source presque exclusive des connaissances physiologiques. De là esí venue lá définition classique de la physiologie : anatome animata. La méthode de l'interprétation anatomique ne doit pas être rendue responsable des erreurs des physiologistes des temps passés : car ces erreurs sont imputables avant tout à 
l'imperfection des notions anatomiques qu'ils s'elforçaient d'interpréter. N'oublions point, d'ailleurs, qu'elle est aujourd'hui cncore notre guide principal dans l'étude de certaines fonctions, de celles par exemple qui concernent la mécanique animale. Cela est si vrai que ce n'est pas dans les traités de physiologie, mais dans les traités d'anatomie, que se trouve indiquée l'action de chaque muscle; c'est qu'en effet, lorsqu'on connaît exactement les insertions d'un muscle, sa direction, la longueur des leviers sur lesquels il agit et leurs connexions, la détermination de son action n'est plus qu'une question de mécanique. De même la connaissance des mouvements d'une articulation découle de l'examen des surfaces et des moyens d'union; le seul examen des surfaces y suffit même dans les cas si nombreux où l'on ne possède l'animal qu'à l'état de squelette fossile.

Ces exemples prouvent que la méthode anatomique est très efficace, lorsque la structure et les fonctions de l'organe que l'on étudie sont assez simples pour ne relever que d'un petit nombre de facteurs. Lorsque cette structure et ces fonctions sont plus compliquées, l'application de la méthode devient beaucoup plus délicate; les conditions multiples dont il faut tenir compte sont combinées de telle sorte qu'il est difficile de faire la part de chacune d'elles. C'est alors que se manifeste toute la supériorité de la méthode expérimentale, dont les moyens variés permettent de procéder à une analyse régulière, en agissant séparément sur les divers éléments de la structure et en troublant ou détruisant leur action. Plus sûre dans ses résultats, plus claire dans ses démonstrations, allant droit au but, elle justifie pleinement la préférence qu'on lui donne; mais la méthode anatomique, quoique reléguée alors sur le dernier plan, n'a pas pour cela perdu sa valeur, et l'on sait qu'elle a souvent réalisé des découvertes importantes, dont l'exactitude a ensuite été confirmée par l'expérimentation. Elle n'est donc jamais à dédaigner; son utilité se manifeste surtout dans les cas où la méthode expérimentale n'est pas applicable, et j'ai déjà dit que l'étude des localisations cérébrales chez l'homme échappe le plus souvent à cette dernière méthode.

Mais la valeur de l'interprétation anatomique dépend avant tout du degré de perfection des connaissances que l'on possède 
sur la structure et les connexions des organes mis à l'étude. Or, l'anatomie du cerveau est bien éloignée encore de la perfection. Si l'on commence à connaître assez bien la morphologie des circonvolutions, s'il a été possible de poursuivre certaines racines nerveuses dans l'épaisseur des centres nerveux un peu au-delà de leurs origines apparentes, et si l'on a pu ainsi se flatter quelquefois d'avoir découvert leurs origines réelles, il faut bien avouer que la plupart des connexions établies par la continuité des fibres nerveuses, sensitives ou motrices, entre les diverses parties de l'encéphale, entre les agents de transmission et le point de l'écorce cérébrale où ils vont aboutir, ne sont pas directement démontrables par les procédés de préparation et de dissection connus jusqu'à ce jour; et c'est cette insuffisance de l'anatomie actuelle qui paralyse les efforts des physiologistes.

Mais, en attendant le jour, peut-être encore bien éloigné, où de nouveaux procédés permettront de compléter l'étude de l'anatomie cérébrale, on peut déterminer certaines connexions en invoquant le concours de l'anatomie comparée.

Après avoir constaté que la morphologie cérébrale présente dans les divers groupes de mammifères des différences excessives, l'anatomie comparée m'a montré que ces différences ne sont pas absolues, qu'elles laissent persister un certain nombre de caractères fondamentaux variables dans leurs degrés, mais non dans leur essence. Chacune des parties constituantes de l'hémisphère peut croître ou décroître à tel point que la forme de la région correspondante ou même celle de l'hémisphère entier soit considérablement modifiée; et l’on méconnaîtrait bien souvent les analogies, si l'on se bornait à comparer entre eux deux cerveaux pris dans des groupes différents. Mais lorsqu'on passe en revue toute la série, on observe des transitions qui permettent de déterminer sûrement les parties analogues et de reconnaître que leurs connexions sont invariables. L'anatomie comparée peut donc nous révéler certaines connexions, certaines solidarités anatomiques et fonctionnelles, que la dissection la plus altentive du cerveau n'a pu constater jusqu'ici, et l'interprétation de ces faits anatomiques peut nous être d'un grand secours dans la recherche des localisations cérébrales.

De toutes les parties de l'hémisphère cérébral, les plus varia- 
bles sans contredit sont celles qui sont affectées à la fonction olfactive. Les différences que présente l'appareil olfactif sont tellement grandes qu'elles justifient la division des nıammifères en deux groupes, ou plutôt en deux catégories que j'ai distinguées sous les noms de mammifères osmatiques et anosmatiques. Chez les premiers, cet appareil est très développé dans toutes ses parties; chez les autres, il est tantôt considérablement amoindri, tantôt complètement anéanti. Le développement de la fonction olfactive, l'importance du rôle qu'elle joue dans la vie de l'animal, croissent et décroissent dans le même sens. Nous pouvons donc étudier comparativement la fonction et l'organe à tous leurs degrés, depuis leur maximum jusqu'à zéro, en passant par l'avant-dernier degré qui s'observe chez l'homme, et dans ces conditions favorables nous pouvons espérer que la méthode de l'interprétation des faits anatomiques trouvera son utilité.

\section{§ 2. - L'Appareil olfactif des maymifÈres.}

Dans mon mémoire sur le grand lobe limbique des mammifères (1), j'ai décrit les caractères extérieurs et les connexions du lobe olfactif, en me plaçant principalement au point de vue morphologique; j'ai dû y joindre quelques considérations générales sur le sens de l'olfaction, pour donner, en quelque sorte, la clef des faits anatomiques que j'avais à exposer ; mais j'ai négligé, à dessein et non sans regret, les détails physiologiques, qui m'auraient éloigné de mon but. J'ai omis également tout ce qui concerne la structure du lobe olfactif, me bornant à dire que ce lobe renferme de la substance grise et de la substance blanche, qu'il est, par conséquent, à la fois un organe d'élaboration et un organe de transmission.

Je reprends aujourd'hui la question de l'appareil olfactif à un point de vue tout différent. L'étude anatomique de cet appareil interviendra ici comme base de l'étude de ses fonctions. J'aurai donc à la compléter en y ajoutant des notions plus précises sur

(1) Anatomie comparée des circonvolutions cérébrales. Le grand lobe limbique et la scrssure limbique dans la série des mammifères. Dans Revue d'anthropologie, $3^{\circ}$ série, t. I, p. 38 š-498 (1878), et dans ce volume, p. 259. 
la structure du lobe olfactif. Je devrai m'appuyer aussi sur les descriptions anatomiques et sur les considérations physiologiques qui ont été exposées dans mon précédent mémoire. Ce travail, qui a paru, il y a quelques mois, dans la Revue d'anthropologie, est entre les mains de mes lecteurs; mais, comme il est fort étendu et comme, en outre, les faits qui concernent l'appareil olfactif y sont disséminés un peu partout, je demanderai la permission d'en présenter ici le résumé.

Je rappellerai d'abord sommairement que, dans la catégorie des osmatiques, qui comprend la très grande majorité des mammifères, le sens de l'olfaction joue un rôle prépondérant; il est. la principale sentinelle et le principal guide de l'animal. L'appareil nerveux olfactif est au complet; il est très développé, et toutes ses connexions sont faciles à reconnaître. Il se distingue de tous les autres appareils nerveux sensoriaux par son énorme volume, par sa complexité, par la multiplicité et la diversité de ses parties, et enfin par la propriété qu'il possède d'agir, à de certains moments, comme centre autonome. Cette prédominance du vo. lume de l'organe, cette prépondérance et cette autonomie de sa fonction disparaissent chez les anosmatiques. Ce nom n'implique pas nécessairement l'idée que l'olfaction soit abolie, car elle ne l'est que rarement. Elle persiste le plus souvent; mais elle ne joue plus le rôle de sens recteur; elle retombe au niveau et même aı-dessous des autres sens; son appareil organique est réduit à un volume extrêmement petit, et toute la morphologie de l'hémisphère se trouve par là considérablement modifiée. Sous ce dernier rapport, il'n'y a que d'assez légères différences entre le cas où l'appareil olfactif est anéanti et celui où il n'est que rudimentaire. Dans l'un et l'autre cas, la vallée de Sylvius, profondément déprimée, forme, à la base de l'hémisphère, une grande anfractuosité transversale, qui se continue directement en dehors avec la scissure de Sylvius, tandis que, chez les osmatiques, la vallée de Sylvius, très peu déprimée, ne constitue pas une anfractuosité et reste, d'ailleurs, séparée de la scissure de Sylvius par la racine olfactive externe.

La catégorie des anosmatiques comprend les cétacés, les carnassiers amplibies et les primates. Chez les cétacés, qui vivent exclusivement dans l'eau, l'olfaction est inutile, et l'appareil 
olfactif est tantôt seulement atrophié (baleines), tantôt entièrement anéanti (delphiniens). Les amphibies fréquentent les rivages, où ils peuvent tirer quelque parti du sens de l'odorat; mais ils ne cherchent leur proie que dans l'eau, et, par suite de ce genre de vie, leurs organes olfactifs, perdant la plus grande partie de leur importance, subissent une atrophie considérable.

Une atrophic plus prononcée encore s'observe chez les primates, mais pour un tout autre motif. Elle coïncide avec le développement excessif du lobe frontal. Ce lobe, agrandi aux dépens des autres, s'est emparé de l'hégémonie cérébrale; la vie intellectuelle s'y est centralisée; ce n'est plus le sens de l'odorat qui guide l'animal, c'est l'intelligence éclairée à la fois par tous les sens, entre autres par le sens du toucher, énormément perfectionné et devenu la source d'une multitude de connaissances, grâce à la constitution de la main. Ayant perdu sa fonction rectrice, son action autonome, l'appareil olfactif est réduit au rôle modestc d'organe de transmission. Tout ce qui excéderait les besoins de cette humble fonction est devenu inutile. Telle est la cause de l'atrophic de l'appareil olfactif des primates. Elle diffèro cntièrement de celle qui produit le même résultat chez les amphibies; elle dépend de l'animal lui-même et non du milieu où il vit; mais elle entraîne, à peu de chose près, les mêmes conséquences anatomiques et physiologiques en ce qui concerne, d'une part, la structure du lobe olfactif, et, d'une autre part, l'état des parties sur lesquelles il établit ses connexions : c'est ce qui nous autorise à constituer parmi les mammifères, en nous plaçant à un point de vue anatomique tout spécial, la catégorie zoologiquement si disparate des anosmatiques, catégorie que nous aurons sans cesse à comparer avec ‘la catégorie infiniment plus nombreuse des osmatiques.

On peut dire que les anosmatiques font exception au type général des mammifères. La règle est que les mammifères sont osmatiques. Leur type cérébral comporte un appareil olfactif volumineux et complet, dont les caractères anatomiques et physiologiques se maintiennent dans les ordres les plus divers, avec une remarquable fixité, au milieu des modifications excessives qui se produisent dans le reste du manteau de l'hémisphère. 11 
convient donc de présenter d'abord une description sommaire de l'appareil olfactif des osmatiques.

Le lobe olfactif des mammifères est un des trois lobes qui composent le grand lobe limbique. Celui-ci constitue dans l'hémisphère une partie entièrement distincte du reste du manteau. Il forme à la fois une portion de la face inférieure et une portion de la face interne de l'hémisphère; on ne peut donc pas le voir dans son ensemble sur les dessins qui représentent l'une ou l'autre de ces faces; mais on peut, par un artifice de dessin, supposer la face inférieure quelque peu rabattue sur la face interne et représenter ainsi, pour la facilité de la description, tout le grand lobe limbique sur une figure schématique. C'est ce qui a été fait sur la figure 42, qui montre le schérna de la face inférointerne de l'hémisphère droit de la loutre (1).

Le grand lobe limbique comprend toute la partie qui a été laissée en blanc sur cette figure. Il a la forme d'une raquette, et il se décompose en trois lobes, qui sont: $1^{\circ}$ le lobe du corpps calleux, $\mathrm{C} \mathrm{C}^{\prime} \mathrm{C}^{\prime \prime}$, qui prend son origine en $\mathrm{C}$, au-devant du bec du corps calleux, 1 , qui contourne le genou de ce corps, 2 , puis sa face supérieure jusqu'au bourrelet, 3 , et qui descend derrière le pédoncule cérébral, 5 , jusqu'à la rencontre du lobe de l'hippocampe; $2^{\circ}$ le lobe de l'hippocampe, $\mathrm{HH}^{\prime}$, qui commence en avant, H, par un lobule arrondi (au niveau du point où la bandelette optique 6 apparait au-dessous de la couche optique 4, pour se rendre au chiasma), qui, de là, se porte d'avant en arrière, le long de la fente de Bichat, et qui va se continuer en $\mathrm{H}^{\prime}$ avec la portion descendante du lobe du corps calleux; $3^{\circ}$ le lobe olfactif, 0 , formé en avant par un renflement volumineux, le renflement olfactif, qui est libre au-dessous et en avant du lobe frontal, F; plus en arrière, par une portion moins large et moins épaisse, $0^{\prime}$, qui est libre encore et qui est le pédoncule olfactif, et, plus en arrière enfin, par une base adhérente, qui fournit les racines olfactives. Les, deux principales racines, blanches et

(1) Il est inutile de rappeler que la loutre n'est pas un amphibie, c'est un carnassier de la famille des martes; mais elle a des habitudes aquatiques; son appareil olfactif est moins développé que celui des autres osmatiques et fournit un excellent terme pour la comparaison des cerveaux osmatiques et des cerveaux anosmatiques. 
épaisses, se séparent en divergeant : l'interne, $f$, se porte sur la face interne de l'hémisphère et se rend sur l'origine du lobe du corps calleux, C; l'externe $a$ se porte en arrière, sur la face inférieure de l'hémisphère, et va se jeter en $b$ sur le bord externe du lobule de l'hippocampe, $\mathrm{H}$, avec lequel elle ne tarde pas à se fusionner.

Le grand lobe limbique peut donc être comparé à une raquette dont l'anneau, entourant le seuil de l'hémisphère, est formé en

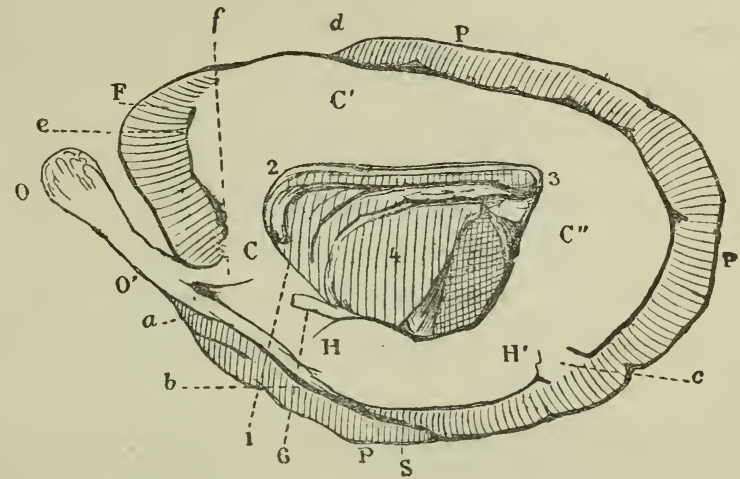

Fig. 42. Le grand "lobe limbique dans son ensemble. Schéma de la face inféro-interne de l'hémisphère droit de la loulre.

1, Bec du corps calleux; 2, son genou; 3, son bourrelet ; 4, face interne de la couche optique; 5 , coupe du pédoncule cérébral ; 6 , la bandelette optique se dégageant, à la partie antérieure de la fente de Bichat, entre la couclie optique et le lobe de l'hippocampe.

PPP, lobe pariétal ; F, lobe frontal ; S, scissure de Sylvius.

Le grand lobe limbique (il est laissé en blane). O, lobe olfactif; $\mathrm{O}^{\prime}$, son pédoncule ; $a$, sa raeine externe; $f$, sa racine interne ; $\mathrm{HH}^{\prime}$, le lobe de l'hippocampe; $\mathrm{H}$, son lobule ; $\mathrm{CC}^{\prime} \mathrm{C}^{\prime \prime}$,

le lobe du corps calleux; C, son origine; abc, are inférieur de la scissure limbique ; $c$, pli de passage rétro-limbique; $c d$, scissure sous-pariétale; $e$, sillon sous-frontal.

haut par le lobe du corps calleux, en bas par le lobe de l'hippocampe, et dont la queue est formée par le lobe olfactif.

Le lobe olfactif est libre jusqu'à sa base; les deux lobes de l'hippocampe et du corps calleux font corps avec l'hémisphère; mais ils sont séparés du reste du manteau, c'est-à-dire de la masse extra-limbigue, FPPP, par une scissure annulaire, la scissure limbique, qui les entoure presque complètement; celle-ci est toutefois traversée par divers plis de passage superficiels ou profonds qui unissent le grand lobe limbique avec le lobe pariétal, PP, et avec le lobe frontal, F. La partie antérieure de la scissure limbique, $e$, ne descend jamais jusqu'au bord inférieur de l’hémisphère; toujours, par conséquent, l'origine du lobe du 
corps calleux, $\mathrm{G}$, communique directement avec la partie inf rieure et interne du lobe frontal, au-dessus de la racine olfactivc interne; c'est la seule connexion du grand lobe limbique qui soit invariablement superficielle. Les autres sont tantôt superficielles, tantôt profondes ; celles qui sont superficielles interrompent plus ou moins la continuité de la scissure limbique, et il en résulte des différences morphologiques qui prennent une part importante à la constitution des divers types cérébraux. Ce sujet a été exposé en détail dans mon Mémoire sur le grand lobe limbique, et je n'y reviendrai pas ici.

Outre les deux racines blanches qui ont été indiquées plus haut, le lobe olfactif possède une troisième racine décrite depuis longtemps sous le nom de racine grise ou moyenne, et une quatrièrne racine, que je crois avoir déterminée le premier et que j'appelle la racine supérieure. Cette racine supérieure a échappé à l'attention, parce qu'on l'a confondue avec la racine grise; mais il sera aisé de prouver qu'elle en diffère essentiellement.

Lorsqu'on examine la face inférieure de l'hémisphère (fig.43), on y aperçoit l'espace quadrilatère, 4 , limité en avant par les deux racines olfactives blanches, 2 et 3 , qui se séparent en divergeant sous un angle plus ou moins aigu, en arrière par le lobule de l'hippocampe, $\mathrm{H}$, en arrière et en dedans par la bandelette optique, 5, qui se rend au chiasma. Cet espace quadrilatère (1), légèrement déprimé par rapport à la racine externe et surtoul par rapport à la saillie du lobule de l'hippocampe, est l'analogue de l'espace décrit par Vicq d'Azyr, dans le cerveau humain, sous le nom d'espace perforé. Il est occupé, chez les animaux osmatiques, par une substance grise assez épaisse, qui s’insère à la fois sur l'angle de séparation, $0^{\prime}$, des deux racines blanches, et sur les bords de ces deux racines; qui, en arrière, se continue avec l'écorce grise du lobule de l'hippocampe; qui, en dedans, cufin, passant sur la face interne de l'hémisphère, tapisse une petite surface plate que nous décrirons plus loin

(1) Le nom d'espace quadrilatère est généralement adopté, et je pense qu'il doit être corıservé: je ferai remarquer toutefois que cet espace est ell réalité penlagonal. Il a un cinquième côté très court formé par le bord interne de l'hémisphère, entre la racine olfactive interne et le chiasma. 
sous le nom de carrefour de l'hémisphère, et se continue avec l'écorce grise de l'origine du lobe du corps calleux (vers le point G de la figure 42). Je dirai tout à l'heure que cette couche n'est pas uniformément grise dans toutes les parties de la surface ; mais elle l'est presque partout, et l'est surtout par rapport à la coloration blanchâtre des deux racines blanches; de là est venu le nom de racine grise qu'on lui a donné.

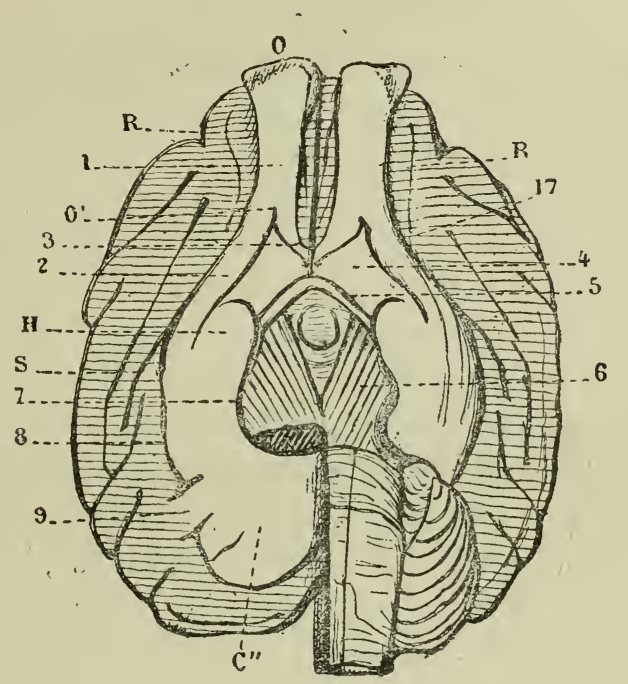

Fig. 43. Face inféricure du cerveau de la loutre. On a enlevé la moitié droite du cervelct, de la protubérance et du bulbe, pour montrer la face inférieure de l'hémisphère droit.

$\mathrm{O}$, lobe olfactif; $\mathrm{O}^{\prime}$, sa base; $\mathrm{H}$, lobe de l'hippocampe; $\mathrm{C}^{\prime \prime}$. extrémité postérieure du lobe du corps calleux; R, scissure de Rolando; S, scissure de Sylvius.

2, racine olfactive externe; 3 , racine olfactive interne; 4, espace quadrilatère; 5 , bandelette optique aboutissant au chiasma qui est coupé ; 9 , le pli de passage rétro-limbique.

Mais lorsqu'on pratique une coupe dans cette racine, on voit qu'il existe, au-dessous de son écorce grise, une couche blanche de fibres longitudinales qui émanent de la base du lobe olfactif, entre les deux racines latérales, et qui se continuent avec la substance blanche du pédoncule olfactif. La face profonde de cette couche blanche repose sur la face inférieure du corps strié, avec lequel elle semble se confondre, et quelques auteurs en ont conclu que la racine olfactive moyenne se rendait au corps strié. Mais Desmoulins a reconnu que ce n'est là qu'une apparence. Parmi les fibres blanches dont il s'agit, les unes forment un 
petit faisceau qui se jette dans la commissure antérieure; les autres se portent horizontalement en arrière et un peu en dedans, atteignent le bord postéro-interne de l'espace quadrilatère, formé par la bandelette optique ( lette comme sous un pont, et vont se continuer avec les fibres les plus inférieures du pédoncule cérébral, qui émanent des cordons antérieurs de la moelle (1). Le lobe olfactif se trouve ainsi mis en communication directe avec les fibres motrices de la moelle.

Cette continuité ne s'observe que chez les osmatiques; elle fait entièrement défaut chez les anosmatiques, dont la racine olfactive moyenne disparaît presque entièrement. On sait que plusieurs auteurs ont nié l'existence de cette racine moyenne chez l'homme; elle est si mince et si fragile qu'elle se déchire avec la plus grande facilité. Pour la découvrir il faut durcir le cerveau dans l'alcool pendant deux ou trois jours, et renverser délicatement d'avant en arrière le ruban olfactif (2), qui représente le pédoncule du lobe olfactif des osmatiques : on voit alors se détacher de la base de ce pédoncule, entre les deux racines blanches qui sont très grêles, une très mince et très courte lamelle de substance cérébrale, grise en dessous, blanchâtre en dessus, qui va s'implanter sur la limite même du lobule orbitaire et de l'espace perforé. Je reviendrai bientôt sur l'interprétation de cette racine olfactive moyenne des anosmatiques. Je me borne à constater ici qu'elle s'insère sur le bord antérieur de l'espace quadrilatère, qu'elle ne va pas plus loin, qu'elle ne recouvre pas cet espace; que celui-ci, par conséquent, est profondément déprimé, et que la face inférieure du corps strié,

(1) Willis connaissait déjà cette connexion des lobes olfactifs. Décrivant les nervi olfactorii, vulgo processus mamillares, il dit: Hi nervi è cruribus medullo oblongatce (pédoncules cérébraux) inter corpora striata et thalamos nervorum opticorum profisiscuntur. Cette description, qu'il appliquait à tort au cerveau de l'homme, était faite évidemment d'après le cerveau des quadrupèdes, car il ajoutait aussitôt que les nerf́s olfactifs sont creusés d'une cavité manifeste (cavitate manifesta proditi), qui va s'ouvrir dans le ventricule latéral (Th. Willis, Nervorum descriptio et usus, dans la Biblioth. anatom., de Manget, Genève, 1699, in-fol., t. II, p. 587).

(2) Le ruban olfactif est improprement nommé par beaucoup d'auteurs nerf olfactif. 
criblée de petits trous pour le passage des veines de la pie-mère, s'y trouve à découvert (1).

Revenons aux racines olfactives des osmatiques. Nous venons d'en décrire trois qui apparaissent à l'extérieur sans aucune préparation, et qui ont été indiquées par tous les auteurs ; mais il y en a une quatrième qui méritele nom de racine olfactive supérieure ou frontale, et que j'ai décrite dans mon mémoire sur le grand lobe limbique.

Lorsqu'on abaisse le lobe olfactif et son pédoncule, on voit que leur face supérieure, reçue dans unc anfractuosité rectiligne et profonde dı lobule orbitaire du lobe frontal, est libre jusqu'à la base du pédoncule. Cette base, assez épaisse, s’insère transversalement sur la partie postérieure du lobule orbitaire, à laquelle elle adhère solidement. Elle ne se continue donc pas seulement avec les trois racines précédemment décrites, qui sont visibles sur sa face inférieure; elle sc continue, en outre, par sa face supérieure, avec le lobe frontal. On peut se demander maintenant si cette continuité indique une simple adhérence ou l'implantaticn d'une véritable racine. On ne peut rien conclure de ce fait que la mince couche grise qui recouvre la fiice supérieure du pédoncule olfactif se réfléchiit à sa base pour se confondre avec la couche corticale du lobule orbitaire: car c'est seulement par les fibres blanches que s'établissent les connexions des organes cérébraux. Mais si l'on enlève eette couche grise, ou mieux encore si l'on pratique une coupe verticale, on roit qu’l existe dans le pédoncule olfactif, entre les deux couches grises superficielles qui tapissent ses deux faces, une couche de substance blanche, et que celle-ci, parvenue à la base du pédoncule, se continue, d'une part, en arrière, avec la couche blanche de la racine grise, ou moyenne, d'une autre part, en haut, avec la substance blanche de la circonvolution frontale orbitaire. La figure 4 représente une coupe pratiquée sur l'hémisphère gauche d'un mouton, à cinq millimètres de la ligne médiane, sur l'angle de séparation des deux racines olfactives blanches. On voit que la couche blanche du pédoncule olfactif, parvenue en $a$, à la base

(1) On doit admettre, théoriquement, que la couche grise qui tapisse toute la surface du manteau, recouvre aussi la face inférieure du corps strié, mais elle y est si mince qu'elle se confond avec la substance même de ce corps. 
de ce pédoncule, y aboutit à une masse de substance blanche qui se continue à la fois en $c$, avec l'axe médullaire de la circonvolution orbitaire, et en $b$, avec les fibres blanches de la racine moyenne. Celles-ci, se portant en arrière, parcourent toute l'étendue antéro-postérieure de l'espace quadrilatère, Q, et vont se rendre au pédoncule cérébral, $P e d$ (tronqué sur la figure 44), en passant au-dessus de la bandelette optique, $0 p$. Quant au prolongement supérieur, $c$, il constitue une racine olfactive dis-

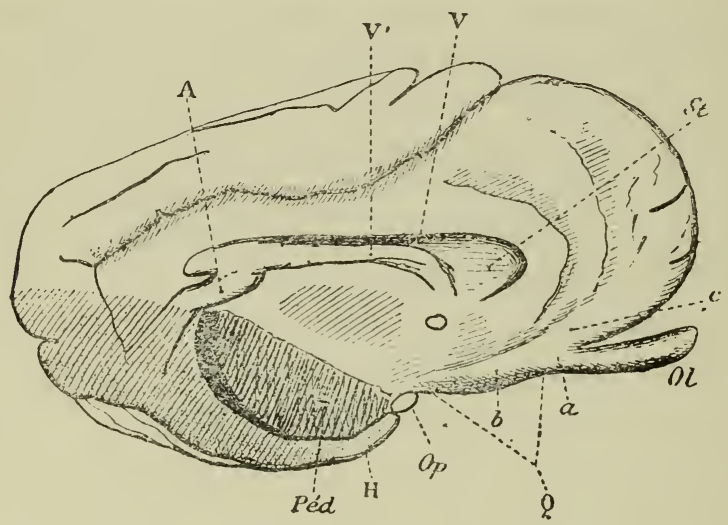

Fig. 44. Mouton. Coupe longitudinale verticale pratiquée sur l'lémisphère gauche, à 5 millimètres de la ligne médiane.

Ped, le pédoncule cérébral, tronqué; A, pilier postérieur de la voûte ; $\mathrm{V}^{\prime}$, la voûte à trois piliers ; V, coupe du ventricule latéral ; St, corps strié; $\mathbf{H}$, lobe de l'hippocampe ; $O p$, bandelette optique; $Q$, coupe et limites de l'espace quadrilatère.

$0 l$, coupe du lobe olfactif et de son pédoncule ; $a$, axe blanc du pédoncule se continuant en arrière, $b$, pour former la racine olfactive moyenne, et en haut, $c$, pour former la racine olfactive supérieure ou frontale.

tincte, qui mérite le nom de racine supérieure ou frontale. On pourrait objecter, il est vrai, que, si nous pouvons suivre aisément sur cette coupe la continuité de la substance blanche, nous ne pouvons y constater la continuité des fibres de cette substance; il est bien vrai, en effet, que, sur les coupes ordinaires, la direction des fibres blanches est souvent incertaine; les coupes microscopiques mêmes laissent souvent persister cette incertitude, et c'est ce qui rend si difficile la détermination des connexions des divers organes cérébraux. Majs, dans ce cas particulier, il y a une circonstance qui rend la démonstration tout à fait positive. 
A l'état embryonnaire, le lobe olfactif est creusé d'une cavité qui se prolonge jusqu'à sa base, et qui de là, remontant audevant du corps strié, va se continuer avec l'extrémité anté. rieure du ventricule latéral. Chez beaucoup d'animaux, cette communication n'est que transitoire; le canal qui s'étend du ventricule latéral à la cavité olfactive s'oblitère entièrement; il ne reste aucune trace de son existence embryonnaire: c'est ce

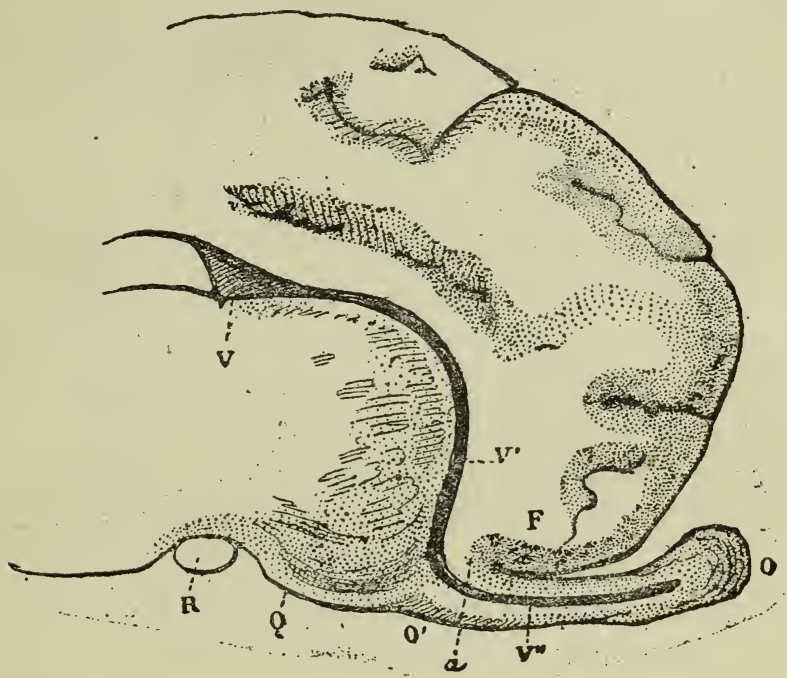

Fig. 45. Gheval. Coupe longitudinale de la partie antérieure de l'hémisphère gauche, à 6 millimètres de la ligne médiane.

$O$, lobe olfactif; $\mathrm{O}^{\prime}$, base de ce lobe; $Q$, coupe de l'espace quadrilatère et de la racine olfactive moyenne; R, coupe de la bandelette optique; F, coupe du lobe frontal ; S, coupe du corps strié ; V, coupe du ventricule latéral dont le prolongement antérieur, $V^{\prime}$, contournant le corps strié, se prolonge, $V^{\prime \prime}$, dans le lobe olfactif ; $a$, racine supérieure ou frontale du lobe olfactif.

qui a lieu, par exemple, chez le mouton, représenté sur la figure 44. Quant à la cavité olfactive elle-même, tantôt elle s'efface complètement, tantôt il en reste un vestige reconnaissable à l'œil nu ou au microscope. Mais il y a des animaux chez lesquels la cavité olfactive et son prolongement ventriculaire persistent pendant toute la vie. C'est ce qu'on voit sur la figure $45^{\circ}$, représentant la partie antérieure d'une coupe verticale et longitudinale pratiquée sur l'hémisphère gauche du cheval, à 6 millimètres de la ligne médiane. Le ventricule latéral, $\mathrm{V}$, après a voir recouvert toute la face supérieure du corps strié, se recourbe en 
se rétrécissant et constitue un canal qui descend verticalement, $\mathrm{V}^{\prime}$, au-devant du corps strié, entre ce corps et le lobe frontal, F; parvenu au niveau de la base du lobe olfactif, $0^{\prime}$, ce canal se recourbe de nouveau pour se porter en avant et pour se continuer avec la cavité olfactive, $V^{\prime \prime}$. Le canal de communication, $V^{\prime}$, est cylindrique et assez étroit ; la cavité olfactive, au contraire, est relativement très grande: elle est plate et paraît linéaire sur les coupes verticales; mais elle occupe presque toute la largeur du pédoncule olfactif qui est, comme on le sait, très large chez le cheval (plus de 1 centimètre), et elle divise ce pédoncule en deux feuillets, l'un supérieur, l'autre inférieur. Ces deux feuillets ont d'ailleurs une structure analogue; chacun d'eux se compose d'une couche corticale grise et d'une couche médullaire blanche qui forme la paroi de la cavité. Il est évident, d'après cette disposition, que le feuillet inférieur est le seul qui forme la racine moyenne, et que le feuillet supérieur, $a$, entièrement étranger à la formation de cette racine, se continue exclusivement, par sa couche corticale comme par sa couche médullaire, avec la circonvolution orbitaire du lobe frontal, F, ou plutôt avec les deux circonvolutions orbitaires: car, quelque simple que soit en général le lobe frontal des osmatiques, on peut le considérer comme subdivisé en deux circonvolutions par le sillon longitudinal dans lequel est couché le pédoncule olfactif.

Dans ce cas, l'existence d'une racine olfactive supérieure ou frontale ne peut être mise en doute. Pour rendre cette démonstration applicable à tous les mammifères, il suffit de se reporter aux périodes embryonnaires. A l'époque où se forme le lobe olfactif, la cavité olfactive n'est qu'un prolongement du ventricule latéral, comme cela a lieu chez le cheval adulte; la paroi supérieure de cette cavité s'implante donc exclusivement sur le lobe frontal, et les connexions qui s'établissent alors persistent évidemment après l'oblitération du canal ventriculaire. Le pédoncule olfactif reste d'ailleurs presque toujours divisé en deux feuillets, l'un supérieur, l'autre inférieur, que l'on distingue quelquefois à l'œil nu, quelquefois seulement au microscope. Ghez le chien, par exemple, la division du pédoncule olfactif en deux feuillets n'est pas apparente sur une simple coupe; on n'y 
voit qu'une lame unique de substance blanche, recouverte sur ses deux faces d'une couche grise corticale; mais sur les coupes microscopiques durcies et colorées au carmin, on voit que cette lame blanche est réellement subdivisée en deux couches par une très mince couche de tissu conjonctif et de cellules endothéliales qui représentent la cavité olfactive oblitérée. (Voy. fig. 46 et 47.$)$

L'existence de la racine olfactive supérieure ou frontale chez les mammifères osmatiques est donc clairement démontrée. Le pédoncule olfactif de ces animaux se compose de deux feuillets ou, si l'on veut, de deux couches, l'une supérieure qui ne fournit qu'une seule racine, la racine supérierure, implantée sur le lobule orbitaire du lobe frontal ; l'autre inférieure, qui fournit les trois racines inférieures, savoir: l'externe aboutissant au lobe de l'hippocampe, l'interne aboutissant à l'origine du lobe du corps calleux, et la moyenne, aboutissant ì la fois à la commissure antérieure et aux faisceaux inférieurs du pédoncule cérébral. Les connexions de ces quatre racines sont établies par les fibres de leur substance blanche. Toutes les quatre renferment, en outre, de la substance grise, qui dans les deux racines interne et externe est interstitielle, c'est-à-dire disséminée au milieu des fibres blanches, et qui est corticale dans les deux autres racines. La couche corticale de la racine supérieure est très mince et se continue exclusivement avec l'écorce de la circonvolution frontale orbitaire. Celle de la racine moyenne est beaucoup plus épaisse. Insérée sur l'angle de bifurcation et sur les bords correspondants des deux racines blanches, elle remplit tout l'espace quadrilatère ; j'ai déjà dit qu'elle se continue à la fois, en arrière avec l'écorce du lobule de l'hippocampe, et en dedans, sur la face interne de l'hémisphère, avec l'écorce du lobe du corps calleux; pour gagner cette face interne, elle passe entre la racine olfactive interne qui est en avant, et le chiasma des nerfs optiques qui est en arrière (1). Elle s'étend donc sans

(1) On sait que le bord profond ou postérieur du chiasma est adhérent. Cette adhérence est établie par une mince lame grise, dite racine grise des nerfs optiques, qui se détache de ce bord concave, et qui prend part à la formation du plancher du troisième ventricule. La même lame grise se continue aussi, en avant avec la couche grise de l'espace quadrilatère; mais on ne peut pas dire que cettecontinuité établisse une connexion entre l'appareil olfactif et le chiasma; 
interruption de l'extrémité antérieure du lobe de l'hiprocampe à l'origine du lobe du corps calleux, et complète ainsi, en avant, le circuit formé par le grand lobe limbique autour du scuil de l'hémisphère, comme on le voit sur la figure 42 (p. 391).

L'origine du lobe du corps calleux (G, fig. 42), où se rend et se perd la racine olfactive interne, est évidemment affectée à la fonction olfactive, ainsi que le lobule de l'hippocampe, $\mathrm{H}$, où se rend la racine olfactive externe ; tout l'espace quadrilatère compris entre ces deux points est occupé par la racine olfactive moyenne et est évidemment encore affecté à la même fonction. Il est donc tout naturel que toutes ces parties soient reliées entre elles par une même couche corticale. Il ne s'agit point ici de cette continuité insignifiante, et en quelque sorte banale, que la substance grise extérieure établit entre toutes les parties de la surface plus ou moins anfractueuse, mais ininterrompue, de l'hémisphère. La substance grise de l'espace quadrilatère fait bien réellement partie intégrante de l'appareil olfactif; car elle disparaît, en même temps que les fibres blanches de la racine moyenne, chez tous les anosmatiques; elle établit donc chez les osmatiques une continuité véritable, à la fois anatomique et fonctionnelle, entru les deux centres olfactifs où aboutissent les deux racines blanches, c'est-à-dire entre le lobe de l'hippocampe et l'origine du lobe du corps calleux. Mais n'existe-t-il pas en outre, entre ces deux centres, une communication directe, par des fibres nerveuses étendues de l'un à l'autre? G'est ce qu'il s'agit maintenant d'examiner.

La surface de l'espace quadrilatère, occupée par la couche grise de la racine moyenne, est grise dans toute son étendue, mais ne l'est pas uniformément. A sa partie postérieure, en arrière d'une dépression transversale sur laquelle repose l'artère cérébrale moyenne, existe une zone plus pâle, quelquefois blanchâtre, qui correspond à une sorte de ruban de fibres nerveuses transversales. Ces fibres, traversant la couche la plus superficielle de la substance grise, en atténuent plus ou moins la couleur. Elles sont quelquefois rares, dispersées et peu visi-

car j'ai déjà fait remarquer que les connexions des diverses parties de l'appareil cérébral ne sont constituées que par la continuité des fibres nerveuses. 

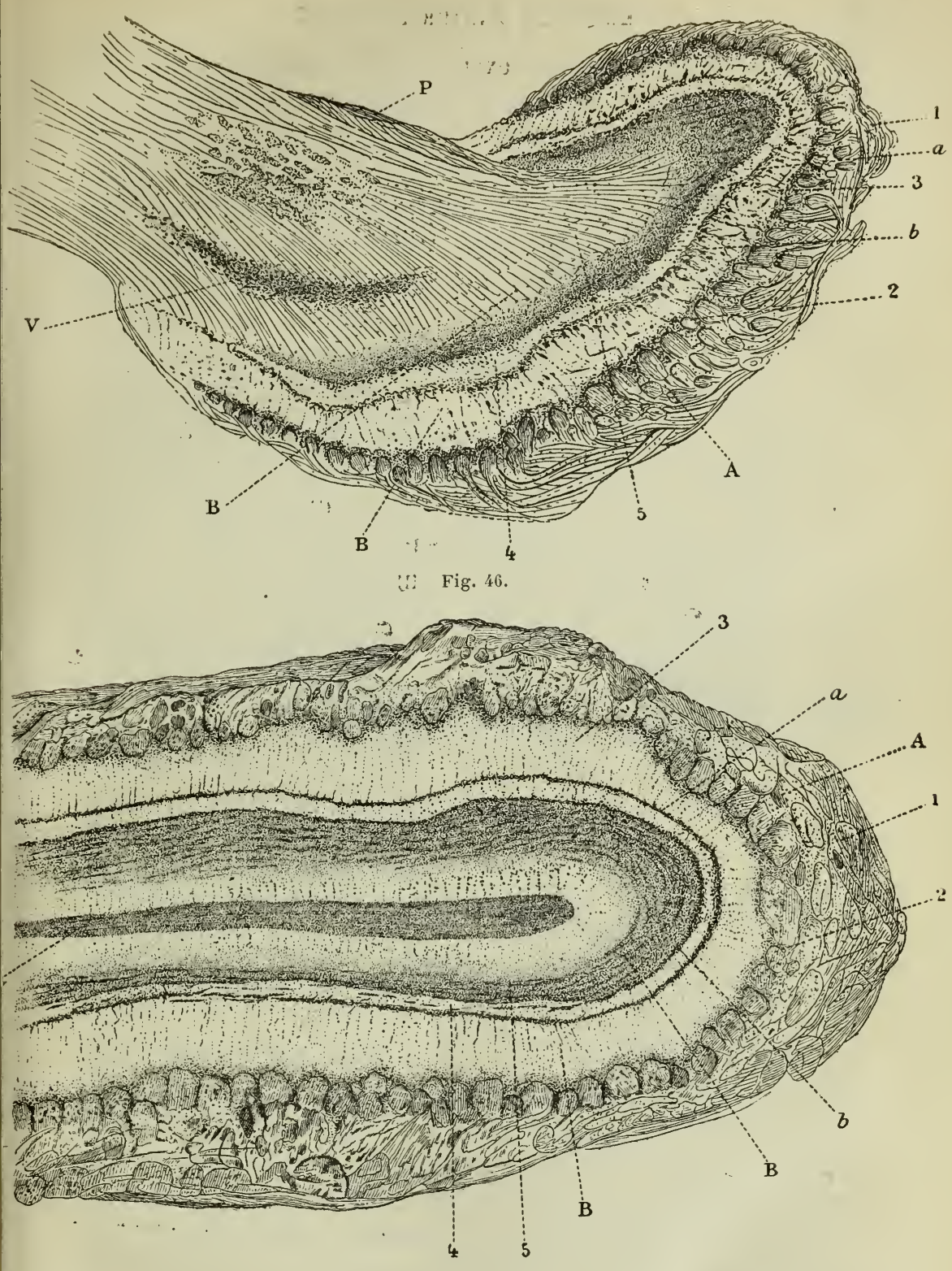

Fig. 47.

Fig. 46. Coupe vertico-longitudinale du lobe olfactif du chien, 12 diamètres; figure demi-schématique. Les lettres comme sur la figure 47.

Fig. 47. Coupe horizontale du lobe olfactif du chien, 15 diamètres. V, l'axe ventriculaire; A, couche corticale ; BB, couche médullaire ; P (fig. 46), origine du pédoncule olfactif.

1, zone des faisceaux nerveux; 2 , zone des papilles ; 3 , première zone médullaire; $a$, rangée formée par les cellules profondes de cette zone, et la séparant nettement de la suivante ; 4 , seconde zone médullaire; $b$, rangée formée par les cellules profondes de cette zone, et la séparant nettement de la suivante; 5 , troisième zone médullaire s'étendant jusqu'à l'axe ventriculaire.

T. V. 
bles : c'est ce qui a lieu, par exemple, chez la loutre, qui est, de

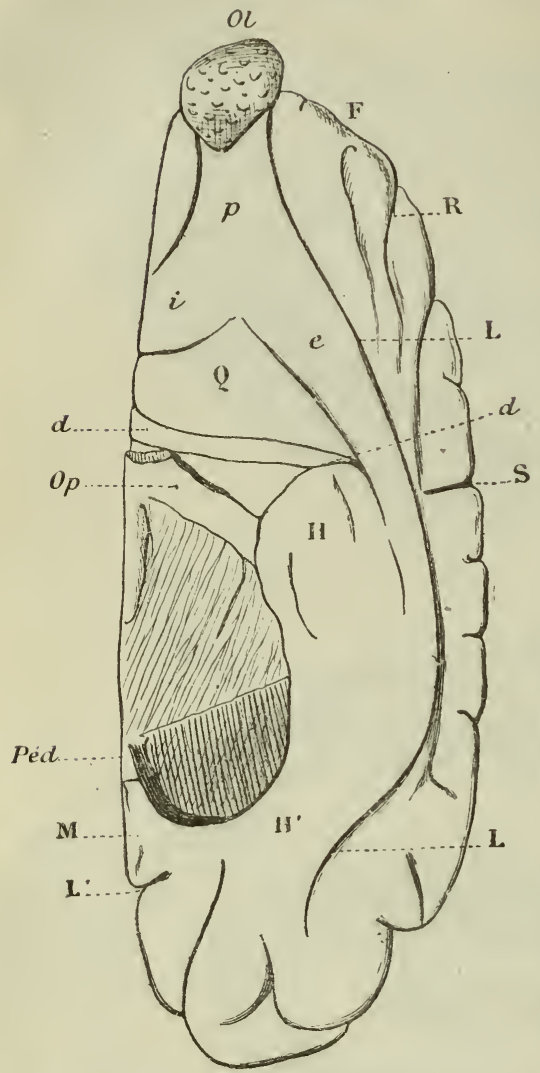

[Fig. 4S. Face inférieure de l'hémisphère gauche $\rightarrow+\infty$ de l'âne.

Ped. Coupe, du pédoncule cérébral; F, lobe frontal; R, scissure de Rolando; S, scissure de Sylvius.

Grand lobe : $O l$, lobe olfactif; $p$, son pédoncule. $\mathrm{HH}^{\prime}$, lobe de l'hippocampe; H, son lobule; $\mathrm{I}$, extrémité postérieure du lobe du corps calleux; L, L, arc inférieur de la scissure limbique; $\mathrm{L}^{\prime}$, terminaison de son arc supérieur.

$Q$, l'espace quadrilatere; $i$, racine olfactive externe; $e$, l'extcrne; $O p$, la bandelette optique, aboutistissant au chiasma qui est coupé ; $d d^{\prime}$, la bandelctte diagonale de l'espace quadrilatère, née de la partic externe du lobule de l'hippocampe et se portant vers le bord interne de l'hemisphère, en avant du chiasma, pour gagner la face interne. tous les mammifères osmatiques, celui dont l'appareil olfactif est le moins développé; mais ordinairement elles sont assez serrées pour former une bandelette que j'appellerai la bandelette diagonale de l'espace quadrilatère, ou plus simplement la bandelette diago. nale, puisque le lecteur est averti qu'il s'agit de l'espace quadrilatère. (Voy. fig. 48.)

La bandelette diagonale naît en $d$ de la partie la plus antérieure et la plus externe du lobe de l'hippocampe, dans l'angle externe du quadrilatère, angle aigu formé par la rencontre du lobe de l'hippocampe, $\mathrm{H}$, et de la racine olfactive externe, $e$. Sur une figure du cerveau du cheval publiée par MM. Chauveau et Arloing, et reproduite dans mon $M e ́$ moire sur le grand lobe limbique (1), cette bandelette est dessinée comme étant une émanation de la racine olfactive externe; mais elle n'y aboutit pas

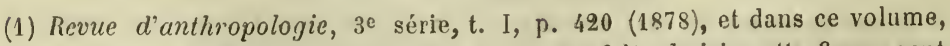
fig. 15, p. 297. Les raisons particulières qui m'ont fait choisir cette figure sont exposées dans mon mémoire. Elles concernent la constitution générale du grand lobe limbique et non sa structure. 
réellement, elle se recourbe en arrière pour se jeter sur le lobe de l'hiprocampe. Chez la plupart des animaux, elle se confond avec ce lobe; mais chez les solipèdes, comme on le voit sur la figure 49 qui représente l'hémisphère gauche d'un âne, l'origine de la bandelette diagonale s'aperçoit dans une étendue de plus de 1 centimètre sur la face inférieure du lobe de l'hippocampe, où elle forme un léger relief longitudinal.

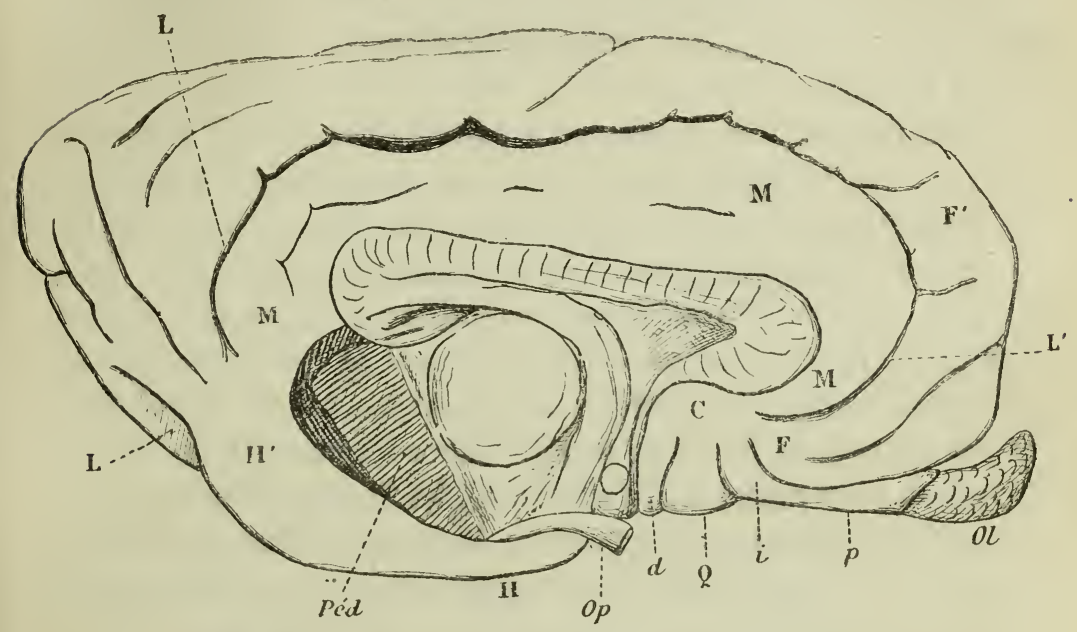

Fig. 49. Face interne de l'hémisphère ganche de l'àne.

Ped, coupe du pédoncule cérébral; HH', lobe de l'hippocampe; MMM, lobe du corps calleux; $L$, fin de l'arc inférieur de da scissure limbique; $\mathrm{L}^{\prime} \mathrm{L}^{\prime}$, son arc supérieur; $\mathrm{FF}^{\prime}$, face interne du lobe frontal; $O l$, le renflement olfactif; $p$, le pédoncule olfactif; $i$, racine olfactive interne; $Q$, bord interne de l'espace quadrilatère; $O p$, bandelette optique; $d$, la bandelette diagonale de l'espace quadrilatère, gagnant la face interne où clle se termine au carrefour.

C, le carrefour de l'bémisphère, communiquant avec l'origine du lobe du corps calleux; M, avec le lobe frontal ; $\mathrm{F}$, avec lc lobe olfactif par la racine interne; $i$, avec l'espace quadrilatère; $Q$, enfin avec le lobc de l'hippocampe par la bandelette diagonale, $d$.

Partant de l'angle externe de l'espace quadrilatère, $d$, la bandelette diagonale se porte en dedans et quelquefois aussi un peu en avantjusqu'au bord interne de l'hémisphère, $d$ ', où elle passe au-devant du chiasma pour atteindre la face interne; elle traverse ainsi toute la largeur du quadrilatère, laissant en arrière d'elle un petit triangle de couleur un peu plus grise. Parvenue sur la face interne de l'hémisphère, elle remonte vers le bec du corps calleux en se portant un peu en avant et en se rapprochant de plus en plus de la racine blanche interne, qui s'est réfléchie aussi sur la même face; dans ce trajet ascendant (voy. fig. $49, d$ ), la bandelette diagonale passe au-devant de la commis- 
sure antérieure et du pilier antérieur de la voûte. A mesure qu'elle remonte, elle s'étale et se confond de plus en plus avec la substance grise, de sorte qu'il n'est pas facile de la suivre jusqu'à sa terminaison. Le point vers lequel elle se dirige est celui où l'origine du lobe du corps calleux vient se confondre avec le bec du corps calleux, et il y a lieu de se demander quelle cst de ces deux parties celle où va se terminer la bandelette diagonale. Je suis disposé à admettre qu'elle se termine à la fois sur l'une et l'autre, mais principalement sur le lobe du corps calleux.

Cette dernière connexion m'a paru évidente dans beaucoup de cas, et, lorsqu'elle ne l'était pas, la direction des fibres la rendait au moins très probable; j'ai donc lieu de croire qu'elle est constante. Quant à la terminaison sur le bec du corps calleux, je crois l'avoir reconnue en même temps que l'autre sur plusieurs cerveaux de pachydermes et de ruminants; d'autres fois, notamment chez les carnassiers, elle est restée dans le vague, et la question de savoir si elle est constante est encore douteuse pour moi. Lorsqu'elle existe, les fibres correspondantes de la bandelette diagonale peuvent être considérées comme formant, par l'intermédiaire du corps calleux, une commissure entre les deux lobes de l'hippocampe; mais je pense que la plupart des fibres de cette bandelette se rendent à l'origine du lobe du corps calleux, et qu'elles établissent une connexion directe cntre ce lobe et le lobe de l'hippocampe. Il y a donc une certaine association entre les deux parties de l'hémisphère d'où naissent les deux racines olfactives blanches, et qui peuvent être considérées comme deux centres olfactifs, communiquant l'un avec l'autre dans l'intérêt d'une fonction partagée entre eux.

Un troisième centre olfactif, bien distinct des deux précédents, existe dans la circonvolution orbitaire, où aboutit la racine oliactive supérieure.

Enfin, puisque toute racine suppose un centre, nous savons qu'il doit $\mathrm{y}$ avoir un quatrième centre olfactif correspondant à la racine moyenne; mais celle-ci ne se rend pas à l'écorce cérébrale; elle aboutit au pédoncule cérébral, qui n'est qu'un organe de transmission; elle est donc centrifuge et non centripète, et le centre olfactif qui lui correspond doit être situé, par consé- 
quent, à son extrémité centrale, c'est-à-dire dans le lobe olfactif lui-même.

Cette proposition est assez importante pour exiger quelque:s développements. Je viens de dire que les fibres de la racine moyenne se continuent avec le pédoncule cérébral (à l'exception de celles qui se jettent dans la commissure antérieure); j’ajoute qu'elles font partie de la couche la plus inférieure de ce pédoncule, couche provenant des cordons antérieurs (ou mieux inférieurs) de la moelle, qui sont affectés à la motilité ; cela suffit déjà pour nous indiquer que les fibres de la racine olfactive moyenne sont motrices. Il est d'ailleurs évident qu'elles ne peuvent pas être sensitives : car si elles l'étaient, si en d'autres termes elles marchaient de la moelle au lobe olfactif, au lieu de marcher du lobe olfactif à la moelle, il faudrait admettre de deux choses l'une: ou bien que la moelle transmet au lobe olfactif des impressions olfactives et que celles-ci peuvent naître ailleurs que dans les fosses nasales, ce qui est contraire à la physiologie ; ou bien que le lobe olfactif reçoit, par l'intermédiaire de la moelle, des impressions non olfactives, et qu'il n'est pas affecté exclusivement à l'olfaction, ce qui n'est pas moins contraire à la physiologie. Les fibres blanches de la racine olfactive moyenne ne sont donc pas sensitives; elles sont, par conséquent, motrices. En d'autres termes, le lobe olfactif n'est pas seulement uri organe de réception et de transmission; c'est aussi un organe excito-moteur, capable de transformer directement en action motrice les impressions olfactives que lui apportent directement, à travers la lame criblée, les nerfs des fosses nasales.

A l'appui de cette conclusion, on peut invoquer l'anatomie microscopique du lobe olfactif des animaux osmatiques.

Les importantes recherches faites sur ce sujet, il y a une quinzaine d'années (de 1860 à 1863̋), par MM. Owsiannikow, Walter et Lockhard Clarke, n'obtinrent pas alors toute l'aitention qu'elles méritaient (1). Ces auteurs avaient décrit et figuré

(1) Owsiannikow, Ueber die feinere Structur der Lobi olfactorii der. Saügethiere, dans Muller's Archiv, 1860, p. 469-477. - George Walter, Ueber den feineren Bau des Bulbus olfactorius, !dans Virchow's Archiv, 1861, p. 241-259 et pl. III et IV. - J. Lockhart Clarke, Ueber den Bau des Bulbus olfactorius und der Geruchsschleimhaut, (traduit par Kolliker sur le manuscrit anglais) dans 
les grandes cellules multipolaires qui existent dans le lobe olfactif des mammifères ordinaires (chien, chat, lapin, cochon, mouton, bœuf, etc.) et qui se retrouvent dans celui des poissons; mais la signification des cellules géantes multipolaires, leur propriété excito-motrice, n'avaient pas encore été suffisamment reconnues, et leur présence dans le lobe olfactif ne fut pas appréciée à sa juste valeur. Ge qui résultait de ces travaux, c'était la complexité exceptionnelle du lobe olfacijf, la diversité de ses éléments, l'intrication de ses fibres, la multiplicité des couches stratifiées qu'on y observe; au milieu de tant de difficultés, beaucoup de points relatifs au trajet et aux connexions des fibres nerveuses étaient restés douteux, ou avaient donné lieu à des interprétations contradictoires, et on en avait conclu, avec raison, que la question du lobe olfactif était loin d'être complètement résolue. Je suis le premier à le reconnaître; j'ajoute que mes propres recherches sont loin d'avoir dissipé mes incertitudes, et s'il pouvait entrer dans le plan de ce travail d'exposer dans tous ses détails la structure du lobe olfactif, j'ạurais à y introduire plus d'un point d'interrogation. Mais il me suffit d'établir, pour le but que je me propose ici, que le lobe olfactif n'est pas seulement sensitif ; que c'est en outre un centre moteur, et je pourrai le faire avec d'autant plus d'assurance, que les observations de mes prédécesseurs s'accordent pleinement avec les miennes, en ce qui concerne du moins l'existence de grandes cellules multipolaires, que la physiologie actuelle nous permet de considérer comme motrices.

Lorsqu'on examine au microscope une coupe verticale et longitudinale du renflement olfactif du chien, après avoir préalablement durci la pièce et l'avoir colorée à l'aide du picro-carminate d'ammoniaque, on y reconnaît une structure compliquée annoncée déjà, à l'œil nu et à la loupe, par l'existence de plusieurs zones assez régulièrement superposées et inégalement coloriées. Nous possédons dans le laboratoire un grand nombre de ces coupes qui ont été faites, suivant des directions variées, par M. le docteur Planteau, aide de clinique chirurgicale à

Zeitschrift für Wissenschafliche Zoologie de Siebold et Kölliker, 1862, t. XI, p. 31-42, et pl. V. Ce dernier travail est trìs important. 
l'hôpital Necker. Les deux figures 46 et 47 , qu'il a dessinées lui-même, représentent: $1^{\circ}$ une coupe vertico-longitudinale, pratiquée sur le milieu de la largeur du lobe olfactif du chien $; 2^{\circ}$ une coupe horizontale pratiquée vers le milieu de la hauteur du lobe. La première coupe est quelque peu schématique; après l'avoir dessinée d'après nature, sous un grossissement de 12 diamètres, on y a indiqué, en exagérantleur volume, des fibres et des cellules qui n'apparaissent que sous des grossissements beaucoup plus forts, afin de montrer la situation de celles-ci et la direction présumée de celles-là. La seconde coupe est figurée sous son aspect naturel, à 15 diamètres : c'est donc surtout sur la figure 47 qu'on devra suivre notre description, en se reportant au besoin à la figure 46 , où les lettres et les chiffres ont les mêmes significations.

Dans l'axe de la coupe $V$, existe le vestige du ventricule olfactif oblitéré (1) et représenté par des éléments conjonctifs (cellules et fibres). Cet axe ventriculaire se prolonge en arrière dans toute la longueur du pédoncule olfactif; autour de lui se replient de droite à gauche, de haut en has, les diverses zones superposées du renflement olfactif.

Ces zones multiples peurent se ramener à deux couches: l'une superficielle ou corticale, $\Lambda$, l'autre profonde ou médullaire, B.

(1) Sur certaines coupes, cet axe ventriculaire ne présente aucune lacune, et il est évident alors que la cavité olfactive est complètement oblitérée. D'autres fois, on y voit des solutions de continuité particlles, irrégulières, manifestement dues à l'action du rasoir. D'autres fois enfin, il reste une lacune plus étendue, dont l'interprétation est douteuse. J'ai lieu de croire, néanmoins, que, sur les chiens adultes qui ont servi à nos préparations, la cavité olfactive était tout à fait effacée. Ces chiens étaient tous des ratiers; peut-être la cavité persiste-t-elle dans d'autres races de chiens. Toutes nos préparations ont été faites sur des lobes olfactits extraits et plongés dans l'alcool absolu immédiatement après la mort de l'animal. La décomposition cadavérique amène très promptement le ramollissement de la substance cellulo-fibrillaire qui a pris la place du ventricule olfactif. Lorsqu'on ne durcit la pièce qu'au bout de quelques heures, cette partic centrale a déjà perdu sa consistance, de sorte que, sur les coupes pratiquées ultérieurement, elle se dissocie et produit une lacune qui simule un ventricule.

La cavité ventriculaire persiste d'ailleurs chez beaucoup d'animaux adultes, notamment chez le mouton, où elle a été étudiée avec soin par M. Lockhardt Clarke. Elle est tapissée d'un épillélium cylindrique. 
La couche corticale, très molle et grisâtre à l'état frais, présente sa plus grande épaisseur à la partie antérieure de la face inférieure du renflement olfaclif, c'est-à-dire dans la région qui repose sur la lame criblée et qui reçoit l'insertion des filets olfactifs venus des fosses nasales (1j; à ce niveau, l'épaisseur de la couche corticale dépasse souvent 1 millimètre et demi, et peut s'élever jusqu'à $1^{\mathrm{mm}}, 77$; elle est moindre sur le reste du renflement, où elle n'a, en moyenne, que $1^{\mathrm{mm}}, 15$ à $1^{\mathrm{mm}}, 20$; elle est moindre encore sur la face supérieure, où elle s'atténue rapidement en approchant de l'origine du pédoncule (P, fig. 46).

Lorsqu'on examine la couche corticale sous de faibles grossissements, de 12 à 15 diamètres, on reconnaît déjà qu'elle n'est pas homogène. Dans sa partie profonde, elle paraît exclusivement formée de petites masses arrondies, que j'appellerai les papilles du lobe olfactif. Serrées les unes contre les autres, comme des grains de mûres, elles forment, sur la limite de la couche médullaire, une rangée continue, par-dessus laquelle d'autres papilles, moins serrées, se superposent le plus souvent, mais d'une manière peu régulière et sans former des rangées distinctes ; lorsqu'on approche de la surface de la couche corticale, on voit les papilles, moins nombreuses, se disperser dans une substance qui forme à elle seule la couche la plus superficielle, et dont les éléments ne sont pas distincts sous ces faibles grossissements.

Les papilles 2,2 sont de petits corps bien réguliers, quelquefois à peu près ronds, plus souvent un peu elliptiques, dont le diamètre moyen est de $0^{\mathrm{mm}}, 20$ à $0^{\mathrm{mm}}, 25$; mais quelques-unes n'ont que $0^{\mathrm{mm}}, 12$ de large, et d'autres peuvent aller jusqu'à

(1) Les filets olfactifs sont les vrais nerfs olfactifs. Ils sont les analogues des autres nerfs sensoriels; étendus comme eux de la membrane sensitive au centre nerveux, ils en diffèrent seulement en ce que, au lieu de se réunir pour ne former qu'un seul cordon et pour pénétrer dans le crâne à travers un trou unique, ils forment un certain nombre de faisceaux très petits, qui pénètrent isolément dans le crâne par les trous de la la me criblée, et qui restent distincts jusqu'à leur insertion sur le lobe olfactif. Quant au cordon qu'on a appelé chez l'homme le nerf olfactif, ce n'est pas un nerf, mais un organe encéphalique absolument analogue au gros pédoncule olfactif des osmatiques, on n'aurait jamais eu l'idée d'en faire un nerf si on l'avait étudiẻ chez les osmatiques; où il fait manifestement partie de l'encéphale. 
$0^{\mathrm{mm}}, 33$. Lorsqu'elles sont elliptiques, leur grand axe est disposé suivant l'épaisseur de la couche; leur contour est tonjours très net dans toute leur surface, excepté du côté qui est tourné vers la périphérie; là, leur substance paraît souvent se continuer avec la substance qui les entoure.

Sous des grossissements plus forts, de 400 diamètres, par exemple, les contours des papilles conservent encore leur netteté, quoiqu'ils ne soient pas limités par une enveloppe membraneuse. On y aperçoit, au milieu d'un grenu assez abondant et d'une gangue quelque peu fibrillaire qui en diminuentla transparence, un grand nombre de petites cellules nerveuses arrondies, de volume uniforme, larges de 8 à $10 \mu$. et à peine plus grosses que leur noyau (1); on peut, en outre, quelquefois, y voir aboutir un filament nerveux, émané de la substance environnante.

Gelle-ci est constituée principalement par des faisceaux nerveux qui proviennent des filets olfactifs et qui se comportent de la manière suivante : les nombreux filets olfactifs qui pénètrent dans le crâne à travers les trous de la lame criblée se jettent aussitôt, après un très court trajet, dans la face inférieure du renflement olfactif et paraissent s'y implanter; mais peu d'entre eux y pénètrent directement; la plupart s'éparpillent sur toute la surface du renflement, de manière à se répandre aussi bien sur son extrémité antérieure que sur ses bords et sur sa face supérieure. Divisés en un grand nombre de petits faisceaux reliés et soutenus par des éléments de substance conjonctive, ils forment par-dessus les papilles une sorte d'enveloppe commune, qui toutefois ne constitue pas une couche distincte, parce que les filaments nerveux qui s'en détachent continuellement, pour se rendre aux papilles subjacentes, et les prolongements de substance conjonctive qui pénètrent entre ces papilles ne permettent pas de l'isoler. Mais on peut dire, du moins, que la couche corticale se compose de deux zones: l'une, superficielle, 1, 1, formée par les faisceaux nerveux ; l'autre, profonde, 2, 2, formée par les papilles, avec une zone intermédiaire qui participe à la fois de la nature des deux autres.

(1) Nous rappelons que le signe $\mu$ désigne les millièmes de millimètre. 
Nous avons déjà dit que les papilles sont des amas de petites cellules nerveuses. Par leur petitesse, leur forme arrondie, leur régularité, elles présentent tous les caractères des cellules nerveuses sensitives (1). Les papilles sont done les organes de réception des impressions olfactives. Ce sont des organes exclusivement sensitifs. C'est là que, pour la première fois, l'impression transmise par les filets olfactifs est mise en rapport avec les cellules sensitives; elle y subit une première élaboration et une première répartition ; c'est le premier acte de la sensation olfactive, qui va se compléter ensuite dans les autres centres olfactifs.

Telles sont les deux zones de la couche corticale du renflement olfactif. Les trois zones suivantes forment la couche médullaire B, qui s'étend jusqu'à l'axe ventriculaire, et qui constitue les deux tiers environ de l'épaisseur du renflement.

La troisième zone, 3, qui est la première zone de la couche médullaire, offre une épaisseur à peu près égale, en moyenne, à celle de la couche corticale. Elle renferme des cellules nerveuses de deux ordres : les unes très petites, arrondies, uniformes et tout à fait semblables à celles des papilles; les autres beaucoup plus grandes, très irrégulières, triangulaires ou polygonales, pour

(1) Ce n'est pas ici le lieu de discuter la constitution des cellules nerveuses. Je suis de ceux qui pensent qu'elles ne sont jamais apolaires, que toutes ont au moins un filament de communication; elles ne sont donc jamais absolument rondes, puisqu'il y a toujours au moins un point de leur contour d'où se détache un prolongement. Mais lorsque la cellule est très petite et son prolongement très fin, son contour ne présente aucun angle appréciable, il est arrondi et presque régulier. Cette forme ne s'observe que dans les cellules sensitives. Sur un champ de cellules non dissociées, il est alors très difficile, souvent même impossible d'apercevoir les filaments de communication; en outre, lorsqu'on dissocie les cellules, ces filaments se brisent à leur base, et la cellule paraît apolaire. Mais théoriquement, il est difficile d'admettre qu'une cellule nerveuse puisse fonctionner sans communiquer avec les filaments nerveux, et il paraît même assez probable que cette communication doit être au moins double, qu'en d'autres termes, toutes les cellules, anguleuses ou arrondies, sont au moins bipolaires, quoiqu'il ne soit pas toujours possible de le constater.

Les cellules sensitives, lorsqu'elles sont bien développées, ont souvent une forme pyramidale avec trois prolongements visibles jusqu'à une petite distance de leur contour; ce n'est alors que par leur plus faible volume qu'elles se distinguent des cellules motrices, et peut-être aussi par l'inégale facilité avec laquelle elles sümprègnent de certaines matières colorantes; mais les cellules sensitives du lobe olfactif ne présentent pas cette forme pyramidale, et diffèrent par conséquent de la manière la plus évidente des cellules motrices du même lobe, qui sont à la fois multipolaires et très grandes. 
la plupart pyramidales, c'est-à-dire plus larges à leur extrémité profonde qu'à leur extrémité périphérique, et terminées par les prolongements qui caractérisent les cellules multipolaires.

Les cellules du premier type sont de beaucoup les plus nombreuses; elles forment, à elles seules, toute la partie de la zone qui avoisine la zone des papilles; à mesure qu'on s'éloigne de cette dernière zone, on voit apparaître çà et là quelques cellules de la seconde espèce, d'abord très espacées, puis plus rapprochées, et se serrant enfin les unes contre les autres, de manière à former, dans la couche la plus profonde de la troisième zone, une rangée ininterrompue qui se dessine, sous la forme d'une ligne très nette, $a$, entre cette zone et la șsuivante. Pour montrer cette répartition des grandes cellules de la troisième zone, on a un peu exagéré leur volume sur la figure 46 , où elles sont représentées par de petites taches noires, allongées suivant, "une direction perpendiculaire à la surface du renflement olfactif. Outre ces deux éléments, on aperçoit, dans la même zone, des fibres nerveuses, mais en nombre relativement très petit, et dont les connexions restent douteuses pour moi ; quelques-unes paraissent manifestement des prolongements des grandes cellules polygonales; mais je ne puis dire si elles ont toutes cette origine. $\mathrm{Ya}$ a-t-il, en outre, des fibres nerveuses émanées de la zone des papilles et traversant toute l'épaisseur de la troisième zone? Cela me paraît possible; mais je ne saurais l'affirmer.

Quant aux deux espèces de cellules nerveuses de cette zone, elles appartiennent évidemment à deux types entièrement différents. Les petites cellules n'ont que 8 à $10 \mu$ de diamètre. Elles sont très régulières et uniformes; elles sont semblables à celles des papilles, à celles de la corne postérieure de la moelle; elles sont par conséquent sensitives. Les grandes cellules, au contraire, sont très diverses dans leur forme et dans leur volume; nous avons déjà dit qu'elles sont multipolaires. Celles qui sont représentées sur la figure 50 peuvent donner une idée de leur grande diversité, qui contraste de la manière la plus remạrquable avec l'uniformité des cellules sensitives. Il est difficile d'en trouver deux qui se ressemblent. Elles sont tantôt triangulaires, tantôt quadrilatères, plus rarement pentagonales. Elles se composent d'un corps qui renferme le noyau et qui, au niveau 
de chaque angle, se rétrécit pour se continuer avec des prolongements souvent beaucoup plus longs que le corps. Ces prolon. gements, ordinairement assez larges à leur base, s'atténuent peu à peu et se terminent en une extrémité effilée, qui se continue avec une fibre nerveuse à simple contour; quelquefois ils se bifurquent avant leur terminaison et se continuent alors avec deux fibres. Au milieu de ces variétés presque illimitées, il y a un élément qui est à peu près fixe : c'est le noyau, toujours bien régulier et toujour's assez gros; il a, en moyenne, $12 \mu$ à $13 \mu$. de diamètre, pouvant toutefois descendre jusqu'à $10 \mu$ et aller jusqu'à 15 \%.. Le corps de la cellule, quelquefois à peine plus large que le noyau, peut atteindre un diamètre de $25 \%$, $40 \mu$ et $50 \mu$;

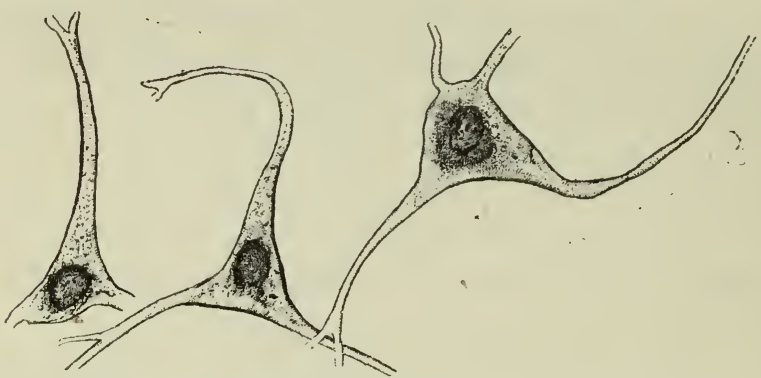

Fig. 50. Grandes cellules multipolaires (motrices) du lobe olfactif du chien, provenant de la rangée $a$, fig. 46, 400 diamètrcs.

en y joignant la partie des prolongements, qui est assez large pour faire manifestement partie de la cellule, certains diamètres peuvent aller jusqu'à $60 \mu, 80 \mu$ et $90 \mu$; enfin, en mesurant les prolongements jusqu'à leur extrémité effilée, la longueur totale peut s'élever à $150 \mu, 200 \mu$ et même $250 \mu$.

Tous ceux qui ont étudié la structure des centres nerveux reconnaîtront dans ces grandes cellules multipolaires des cellules motrices. Elles sont, en effet, tout à fait pareilles à celles que l'on observe dans les cornes motrices de la substance grise de la moelle et dans les couches profondes de l'écorce des circonvolutions cérébrales. Elles sont même notablement plus grandes que ces dernières, ainsi qu'on peut le voir sur la figure $ّ 1$, où sont représentées, sous le même grossissement de 400 diamètres, les cellules motrices des circonvolutions 
qui aroisinent le sillon crucial du chien, c'est-à-dire l'extrémité antérieure, supérieure et externe de la scissure souspariétale. L'existence de cellules motrices bien caractérisées l.ans le lobe olfactif du chien est donc tout à fait incontestable.

Ces cellules motrices appartiennent exclusivement à notre troisième zone. Nous avons déjà dit qu'à la limite profonde de cette zone, elles forment une rangée serrée qui repose directement sur la quatrième zone, et qui, examinée à de faibles grossissements, se détache sous la forme d'une ligne très régulière (fig. 47,a.)

La quatrième zone ou deuxième zone médullaire (fig 47, $\mathrm{n}^{\circ} 4$ ), séparée très nettement de la précédente par cette rangée de cellules motrices, est très mince, formée de petites cellules rondes, et dont les plus profondes constituent une seconde rangée cellulaire, $b$, tout aussi régulière tout aussi nette que la première.

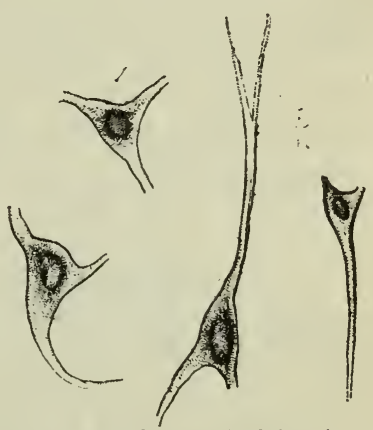

Fig. 51. Cellules multipolaires (motrices) des circonvolutions qui bordent le sillon crucial du chien, 400 diamètres.

La cinquième zone enfin (fig. $47, n^{\circ} 5$ ) ne paraît pas être purement nerveuse; elle renferme une assez grande quantité d'éléments conjonctifs, au milieu desquels se trouvent de petites cellules nerveuses sensitives, irrégulièrement disséminées, formant par places des groupes diffus; en outre, on y voit une certaine quantité de fibres nerveuses, qui deviennent de plus en plus nombreuses lorsqu'on approche de l'extrémité postérieure du renflement olfactif, c'est-à-dire de sa base. Là on voit s'amincir et disparaître les quatre premières zones; la cinquième seule persiste et constitue le pédoncule olfactif. Celui-ci est formé principalement de fibres nerveuses longitudinales et parallèles; mais il renferme aussi dans toute son épaisseur de petites cellules nerveuses arrondies qui, plus nombreuses à sa surface, y constituent une sorte de couche corticale d'ailleurs extrêmement mince.

Parvenues à la base du pédoncule, ces fibres se répartissent entre les quatre racines qui ont été décrites plus haut. Celles 
de la racine supérieure, de la racine externe, de la racine interne, vont aboutir à diverses parties de l'hémisphère et sont évidemment centripètes, et par conséquent sensitives (1). Mais celles qui constituent les faisceaux blancs de la racine grise, et qui vont se rendre dans la couche inférieure du pédoncule cérébral, pour gagner de là les faisceaux antérieurs ou moteurs de la moelle, ne peuvent être que centrifuges et motrices; e, comme toute fibre motrice vient d'un centre moteur, commet en outre, ces fibres centrifuges viennent du renflement olfactif, nous pouvons en conclure que celui-ci est un centre moteur. C'est ce que nous savions déjà, puisque l'examen microscopique nous a révélé l'existence de cellules motrices dans la troisième zone du renflement olfactif.

G'est ce oue confirme surtout l'étude du renflement olfactif des anosmatiques, et de

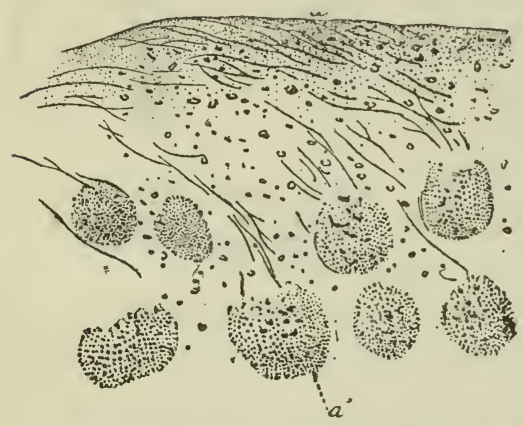

Fig. 52. Coupe de la couche corticale du renflement olfactif de l'homme, 100 diamètres,

$a a^{\prime}$, épaisseur de la couche corticale $\left(0^{\mathrm{mm}}, 3 \%\right) ; a$; zone superficielle ou des faisceaux nerveux; $a^{\prime}$, zone profonde ou des papilles. Diamètre de la papille $a^{\prime}, 0^{\mathrm{mm}}, 10$.

régulière ; elle est épaisse de 3 à 4 dixièmes de millimètre et beaucoup plus mince par conséquent que chez le chien; les papilles ont de $0^{\mathrm{mm}}, 06$ à $0^{\mathrm{mm}}, 10\left(0^{\mathrm{m} m}, 20\right.$ à $0^{\mathrm{mm}}, 25$ chez le chien $)$. Quant

(1) L'histologie n’a pu établir jusqu'ici aucune différence appréciable entre les fibres sensitives et les fibres motrices. J'ai lieu de croire toutefois, ou plutôt de conjecturer que la racine olfactive supérieure doit contenir, outre les fibres sensitives ou centripètes dirigées du lobe olfactif vers le lobe frontal, des fibres centrifuges dirigées du lobe frontal vers le lobe olfactif; car je chercherai à montrer plus loin que la fonction de celte racine supérieure consiste à placer sous l'action directe du lobe frontal, c'est-à-dire de la volonté, le centre olfactif moteur dont l'anatomie établit l'existence dans le lobe olfactif des osmatiques. 
à la couche médullaire, on y observe bien quelques traînées de cellules, mais qui ne forment pas des stratifications régulières. Cette couche renferme, comme les papilles, de petites cellules nerveuses arrondies (fig. §ొ3, a) larges de 10 à $12 \mu$, avec un noyau de 5 à $6 \mu$. (cellules sensitives). $0 \mathrm{n}$ y voit en outre des fibres nerveuses, et beaucoup de substance conjonctive; mais on $\mathrm{y}$ cherche en vain les grandes cellules multipolaires de la troisième zone du chien. A leur place, mais en nombre beaucoup moindre, existent des cellules assez grandes (fig. $53, b$ ) longues de $33 \mu$, larges de $20 \mu$, avec un noyau de $ّ$ à $7 \mu$, et entièrement différentes des cellules sensitives; mais plus différentes encore des cellules motrices, car elles sont régulièrement elliptiques, sans pôles ni prolongements. Sur des cellules aussi volumineuses, les prolongements, s'ils existaient, seraient certaine-

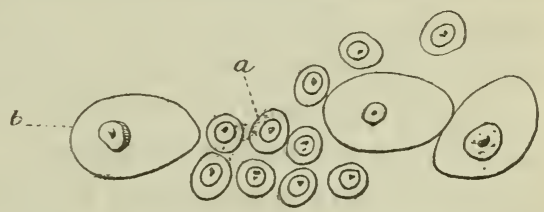

Fig. 53. Cellules du renflement olfactif de l'homme (400 diamètres).

$a$, cellules nerveuses; $b$, les grandes cellules elliptiques.

ment très visibles. J'ajoute que je n'ai jamais rencontré de cellules semblables dans les autres parties du système nerveux central que j'ai examinées. Leur situation dans l'épaisseur de la couche médullaire du lobe olfactif montre qu'elles ne peuvent remplir aucune fonction étrangère à l'action nerveuse; en outre, leur état de complet isolement prouve qu'elles ne peurent prendre aucune part à cette action. Je pense donc qu'elles n'ont aucune fonction, et comme elles sont situées là où se trourent les cellules multipolaires des osmatiques, on peut les considérer comme étant le vestige de ces dernières. Elles se sont formées pendant la période embryonnaire, comme les autres cellules; mais elles n'ont pas subi l'évolution spéciale en rapport avec la fonction motrice qui n'existe plus, et en perdant cette fonction, elles ont perdu leur structure caractéristique. Quoi qu'il en soit, le renflement olfactif de l'homme ne renferme pas de grandes cellules multipolaires; l'absence de cet élément coïncide avec la disparition de la racine moyenne et des fibres centrifuges. Il est clair dès lors que les fibres centrifuges et les cellules motrices du lobe olfactif sont solidaires les unes des autres, que celles-ci 
donnent naissance à celles-là, et que le lobe olfactif des osmatiques agit comme centre moteur.

Il est aussi centre sensitif, puisque les impressions qu'il reçoit sont le point de départ d'une action motrice, qui diftère très notablement, comme on le verra plus loin, des actions simplement réflexes; mais cette sensation n'est pas complète encore, ce n'est qu'un rudiment de sensation, dont nous chercherons tout à l'heure à apprécier le degré, et la sensation véritable, celle qui donne à l'animal des notions précises, celle qui met en jeu son attention, son discernement, sa décision, ne se produit que dans l'hémisphère proprement dit, auquel vont aboutir, en trois points différents, les trois racines supérieure, externe et interne, émanées des couches sensitives du lobe olfactif.

On sait que ces trois points de l'hémisphère sont : $1^{\circ}$ la partie postérieure du lobule orbitaire du lobe frontal, qui reçoit la racine olfactive supérieure; $2^{\circ}$ la partie antérieure du lobe de l'hippocampe, qui reçoit la racine externe ; $3^{\circ}$ la partie antéroinférieure du lobe du corps calleux, qui reçoit la racine interne. Les points où aboutissent ces racines paraissent être leurs origines réelles, car elles disparaissent aussitôt, sans qu'on puisse les suivre plus loin. Gela permet déjà de considérer comme probable que les trois parties du manteau de l'hémisphère où se rendent les trois racines sont affectées à la fonction olfactive, et constituent autant de centres olfactifs. La preuve ne serait pourtant pas suflisante, car on sait qu'il existe dans le cerveau des connexions de continuité établies par la physiologie, quoique la dissection ne puisse pas les démontrer directement. On pourrait se demander si les points d'origine des trois racines olfactives ne seraient pas seulement des lieux de passage, au-delà desquels ces racines iraient se rendre à un ou plusieurs centres olfactifs encore inconnus. La question resterait donc douteuse, si l'anatomie comparée ne nous fournissait une démonstration décisive. On va voir, en effet, que nos trois centres olfactifs subissent, chez les anosmatiques, une atrophie qui marche de front avec celle du lobe olfactif. 


\section{\$ 3. - LES CENTRES OLFACTIFS CHEZ LES ANOSMATIQUES.}

Nous désignerons nos trois centres olfactifs sous les noms suivants :

$1{ }^{\circ}$ Centre olfactif antérieur ou orbitaire (racine supérieure);

$2^{\circ}$ Centre olfactif postérieur ou du lobe de l'hippocampe (racine externe);

$3^{\circ}$ Centre olfactif supérieur ou du lobe du corps calleux (racine interne).

$1^{\circ}$ Occupons-nous d'abord du centre olfactif antérieur. La racine supérieure des osmatiques s'insère sur l'extrémité postérieure du lobule orbitaire ; ce lobule n'est jamais simple: il est subdivisé en deux parties, l'une interne, l'autre externe, séparées par un large sillon longitudinal, dans lequel s'applique le pédoncule du lobe olfactif ; il se compose donc de deux circonvolutions longitudinales et parallèles, qui s'étendent de sa base à sa pointe. La racine olfactive supérieure s'insère à la fois sur l'extrémité postérieure de ces deux circonvolutions; il paraît probable dès lors qu'elles sont olfactives l'une et l'autre, au moins dans leur partie postérieure, sans qu'on puisse savoir jusqu'où s'étend, d'arrière en avant, chez les osmatiques, la zone affectée à cette fonction.

Voici maintenant ce que l'on observe chez les cétacés delphiniens, dont l'appareil olfactif est entièrement anéanti. Chez ces animaux, la surface de l'hémisphère présente, sans qu'on puisse savoir pourquoi, une complication excessive. Le nombre des circonvolutions fondamentales n'excède pas celui qu'on observe chez les pachydermes; mais ces circonvolutions sont divisées et subdivisées à un degré qui ne se retrouve dans aucun autre type animal. Ce n'est donc pas sans étonnement qu'on aperçoit, sur ces cerveaux extraordinairement compliqués, une région entièrement lisse, qui occupe la partie postérieure du lobule orbitaire, et qui se distingue du reste de la surface de l'hémisphère, comme se dessine sur une carte de géographie un désert entouré de pays fertiles. Cette zone déserte, que j'appelle le désert olfactif, n'occupe chez le dauphin (roy. fig. $54, \mathrm{D}$ ) que le tiers postérieur de la surface, d'ailleurs très grande, du lobule orbitaire; mais 
chez le marsouin, dont le cerveau est moins riche, quoique très riche encore, elle en occupe environ la moitié. Il est impossible de méconnaître le lien qui existe entre cette suppression des sillons et des plis de la surface cérébrale, et l'anéantissement du

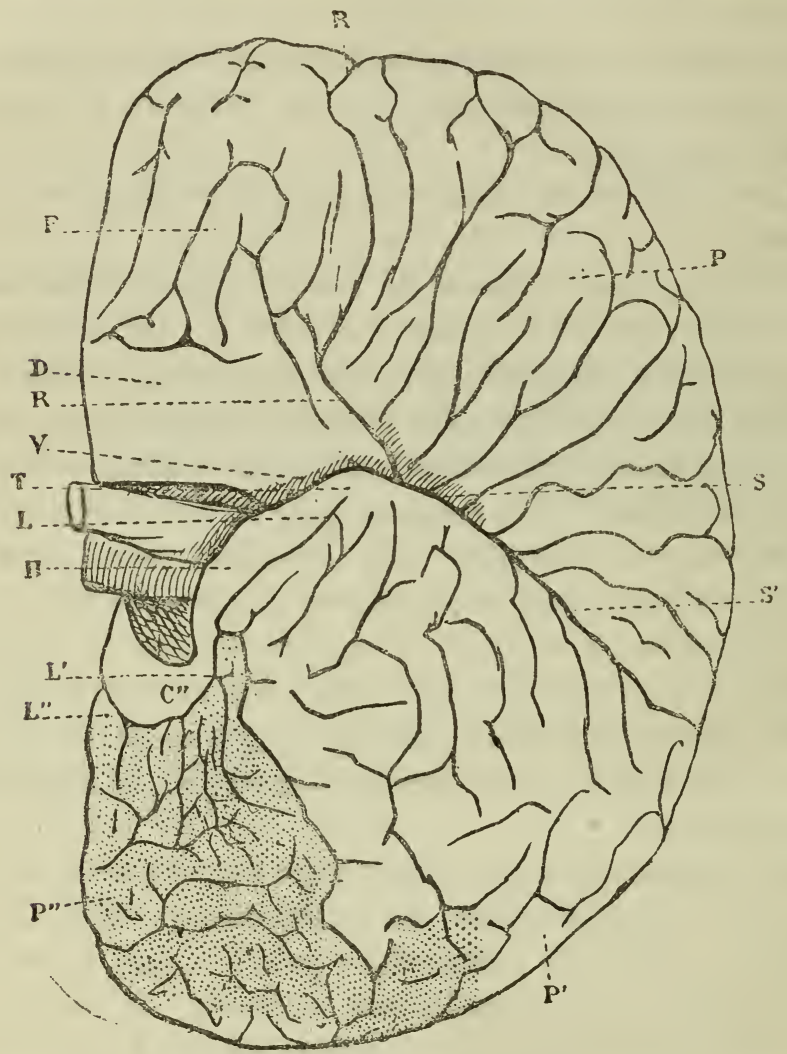

Fig. 54. Dauphin'; face inférieure de l'hémisphère gauche.

RR, scissure de Rolando; SS', scissure de Sylvius; PP'P', lobe pariétal; $F$, face inférieure du lobe frontal; D, lobule désert du lobe frontal (désert olfactif); V, vallée de Sylvius; H, lobe de l'hippocampe atrophié ; $\mathrm{C}^{\prime \prime}$, lobe du corps ealleux"; L. L', arc inférieur de la scissure limbique; $L^{\prime} L^{\prime \prime}$, son are supérieur vu en raccourci; $T$, le póle temporal du lobule (ou lobe) temporal ; B, bandelette optique (cette lettre B, omise par le graveur, était placée entre $\mathrm{T}$ et $\mathrm{L})$.

lobe olfactif. C'est là que ce lobe, s'il existait, implanterait sa racine supérieure; celle-ci manquant tout à fait, comme le reste du lobe, l'atrophie de l'organe a suivi la disparition de la fonction, et toute la portion affectée à cette fonction s'est affaissée, déplissée, et s'est réduite à la mince couche banale de substance 
grise qui revêt sans interruption toute la surface du manteau.

L'étendue de la surface déserte nous fait donc connaître celle de la région olfactive du lobule orbitaire; nous voyons ainsi que cette région ne comprend que la partie postérieure du lobule, dont la partie antérieure a conservé chez les delphiniens son activité fonctionnelle, attestée par le plissement de sa surface. Sur le cerveau du marsouin ce plissement n'est que longitudinal, et la limite des deux portions du lobule orbitaire n'est indiquée que par la terminaison graduelle des sillons longitudinaux; mais chez le dauphin ceux-ci viennent aboutir à un sillon transversal qui établit très nettement la démarcation de la portion plissée et de la portion déserte.

L'existence du centre olfactif antérieur ou orbitaire chez les osmatiques se trouve par là démontrée. Si la racine olfactive supérieure ne prenait sur l'extrémité postérieure du lobule orbitaire qu'une origine apparente, sa disparition n'exercerait aucune influence sur cette portion de l'écorce cérébrale, qu'elle ne ferait que traverser pour aller prendre ailleurs son origine réelle. Il est clair, par conséquent, que la région qui devient déserte chez les delphiniens est, chez les osmatiques, le vrai centre de la racine olfactive supérieure.

Cette région n'est déserte que chez les animaux complètement anosmatiques, dont l'appareil olfactif est entièrement anéanti. Les autres anosmatiques (amphibies et primates) ont un lobe olfactif rudimentaire, qui possède, comme on l'a dit plus haut (p. 394), une racine supérieure rudimentaire, mais constante. La région que nous appelons le centre olfactif antérieur n'est donc pas privée de fonctions, et, dès lors, il n'y a aucun motif pour qu'elle se distingue, par une apparence spéciale, du reste de la surface de l'hémisphère; si sa fonction a moins d'importance, son étendue peut devenir moindre; mais sa structure, l'épaisseur de sa substance corticale, doivent conserver les caractères ordinaires des autres circonvolutions, comme cela a lieu chez les osmatiques, où rien n'indique une démarcation entre la portion olfactive du lobule orbitaire et le reste de ce lobule. Mais, de plus que chez les osmatiques, dont le lobe frontal est très petit et très simple, il y a, chez les primates, une disposition qui permet de reconnaître les limites du centre olfactif. 
Les primates, ainsi que nous l'avons amplement démontré dans un autre travail(1), ont un type cérébral tout particulier, dont les nombreux caractères distinctifs paraissent dépendre plus ou moins d'un caractère fondamental, qui est le grand développement du lobe frontal. Ce lobe se subdivise en trois circonvolutions longitudinales, qui, se réfléchissant en avant, se prolongent sur le lobule orbitaire. La troisième circonvolution frontale, rudimentaire chez les primates inférieurs, n'apparaît, à la partie externe du lobule orbitaire, que chez l'homme et les anthropoïdes. La première, volumineuse dans son étage supérieur, se réduit, dans sa portion orbitaire, à un pli droit et étroit (gyrus rectus), compris entre le sillon olfactif et le rebord interne du lobule. C'est donc la seconde circonvolution frontale, devenue la seconde circonvolution orbitaire, qui forme, à elle seule, la plus grande partie de la largeur du lobule orbitaire. Or, chez tous les primates (chez tous ceux, du moins, qui ont des circonvolutions), cette seconde circonvolution orbitaire est plus ou moins subdivisée par des incisures, qui, bien que très variables dans leurs dispositions, se rattachent cependant à un type caractérisé par Gratiolet sous le nom de sillon en $H$; le nom de sillon devant être réservé pour désigner les anfractuosités qui séparent deux circonvolutions, et, celles qui subdivisent une circonvolution n'étant que des incisures, nous ne retiendrons que l'épithète et nous dirons, par conséquent, l'incisure en $H$. Le plus souvent, en effet, elle se compose de deux branches plus ou moins longitudinales, tantôt presque droites, tantôt quelque peu curvilignes el se regardant alors par leur convexité ; et d'une branche transversale, plus ou moins complète, qui s'étend de l'une à l'autre comme le trait transversal de l'H (fig. 5̌). D'autres fois, la branche transversale étant très courte, ou même nulle, la figure est celle d'un $\mathrm{K}$ ou d'un $\mathrm{X}$, dont le point de tangence se développe en une ligne longue de quelques millimètres; ce cas est assez commun chez l'homme (fig. 56). D'autres fois, enfin, les deux branches restent isolées; mais, dans le point où existe ordinairement la branche transversale, on aperçoit une dépression, une sorte de fossette qui la remplace (fig. 57); ou encore l'une des branches n'occupe que la partie antérieure du lobule,

(1) Revue d'anthrop., 1878, p. 462 et suiv., et dans ce volume, p. 343 et suiv. 
et la seconde se divise, en arrière, en deux branches divergentes, qui se portent l'une en dedans, l'autre en dehors, de manière à intercepter une surface triangulaire dont la base correspond au bord antérieur de l'espace perforé et qui représente la portion marquée $\mathrm{M}$ sur la figure 50 (voy. aussi, plus loin, fig. 59, à la page 432). Ces variations, dont nous n'avons pas épuisé la liste, s'observent surtout chez l'homme et chez les anthropoïdes ; mais sur les cerveaux plus simples des pithéciens et des cébiens, la

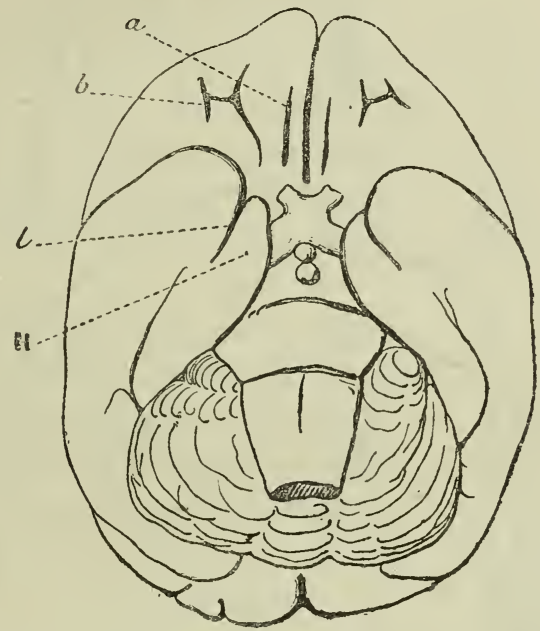

Fig. 53. Face inférieure de l'encéphale du Cebus apella.

$a$, le sillon olfactif, séparant la première circonvolution orbitaire de la seconde; $b$, l'incisure en $\mathrm{H}$ sur la face inférieure de la seconde eireonvolution orbitaire.

H, le lobule de l'hippocampe; $l$, le sillon limbique, séparant ce lobule de la pointe du lobe temporal.

forme en $\mathrm{H}$ est ordinairement bien dessinée, et le nom sous lequel Gratiolet a désigné cette incisure prouve qu'elle subdivise la seconde circonvolution orbitaire en deux portions : l'une, antérieure, qui se continue jusqu'à la pointe du lobe; l'autre, postérieure, qui s'étend jusqu'au bord de la vallée de Sylvius et qui reçoit, sur ce bord, l'insertion de la racine olfactive supérieure.

Il paraîtra très probable, dès lors, que cette partie postérieure, le plus souvent distincte de l'antérieure, est l'analogue de celle qui reste déserte chez les delphiniens; - que la branche transversale qui la limite ordinairement est l'aualogue de celle qui 
limite le désert olfactif sur le cerveau du dauphin. Il serait difficile d'expliquer autrement la subdivision transversale de la seconde circonvolution orbitaire, subdivision qui n'existe que chez les primates, et qui ne peut être attribuée, comme les autres

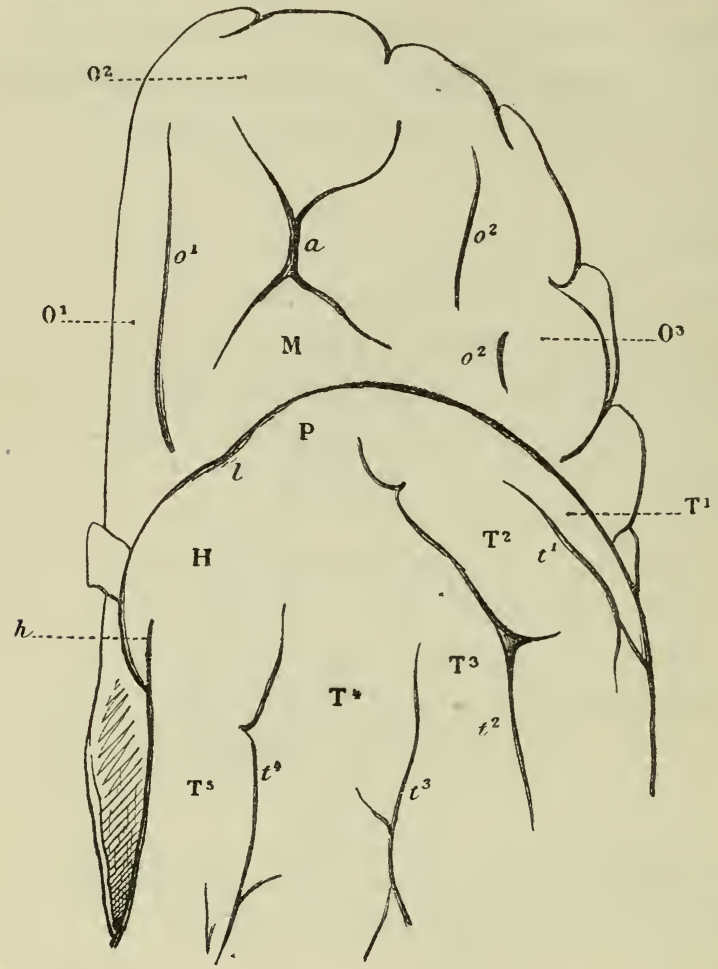

Fig. 56. Homme; face inférieure de l'hémisphère gauche.

$\mathrm{O}^{1}, \mathrm{O}^{2}, \mathrm{O}^{3}$, les trois circonvolutions orbitaires $; o^{1}$, le premier sillon orbitaire $; o^{2}, o^{2}$, le second sillon orbitaire; $a$, le sillon en $\mathrm{H}$, présentant la forme d'un X allongé; $\mathrm{M}$, le centre olfactif de la seconde circonvolution orbitaire.

H, le lobule de l'hippocampe ; $\mathrm{T}^{1}, \mathrm{~T}^{3}, \ldots \mathrm{T}^{3}$, les cinq circonvolutions temporales ; $t^{1}, t^{3}, t^{3}, t^{4}$, les quatre sillons temporaux; $\mathrm{P}$, le pôle temporal; $l$, dépression qui limite l'extrémité antérieure du lobule de l'hippocampe, et qui représente chez l'homme blanc le sillon limbique des singes; $h$, le crochet de la circonvolution de l'hippocampe.

plissements secondaires des circonvolutions, à la complication générale du cerveau; car elle est plus nette et plus fixe chez les primates inférieurs que chez les primates supérieurs. La complication croissante que l'on observe chez ces derniers, loin de rendre cette subdivision plus apparente, tend, au contraire, à la rendre plus confuse, par suite du développement et de l'allon- 
gement des plis de la portion antérieure, qui peuvent empiéter plus ou moins sur la portion postérieure; mais, quoique pouvant être ainsi plus ou moins dissimulée, la ligne de démarcation n'en reste pas moins reconnaissable sur la très grande majorité des cerveaux humains et tout à fait évidente sur beaucoup d'entre eux. Ce qui prouve, d'ailleurs, que l'incisure en $\mathrm{H}$ n'est pas, comme les autres incisures, le résultat d'un plissement de complication, c'est la remarquable précocité de son apparition chez le fœtus humain. On en aperçoit déjà le rudiment au cinquième mois, lorsque la plupart des circonvolutions ne sont pas même encore dessinées, et bien longtemps avant l'époque où un plissement secondaire produit les incisures ordinaires.

Nous dirons donc que le sens olfactif antérieur des primates en général et de l'homme en particulier occupe, sur la deuxième circonvolution orbitaire, l'espace compris entre le bord antérieur de la vallée de Sylvius, où se termine le lobule orbitaire, et les branches de l'incisure en $\mathrm{H}$, jusqu'au niveau du point où ces branches sont ordinairement unies par une incisure transversale.

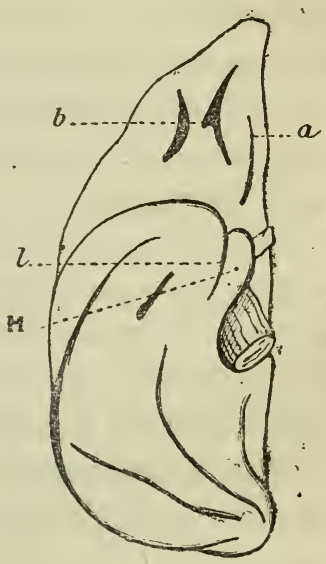

Fig. 57. Face interne de l'hémisphère droit du macaque Resus. Mèmes lettres que sur la figure 5 s.

Le sillon en H n'est pas complet, mais une dépression transversale, $b$, représente la branche transversale de l'H.

Ce centre olfactif n'est pas limité à la seconde circonvolution orbitaire; il s'étend aussi à la première, qui représente la partie interne du désert olfactif des delphiniens et qui reçoit, d'ailleurs, comme la seconde, sur son extrémité postérieure, l'insertion de la racine olfactive supérieure; mais la première circonvolution orbitaire, mince el droite, ne se subdivise pas; rien n'y indique donc la limite du centre olfactif; il est permis de supposer, néanmoins, que ce centre s'étend sur elle jusqu'au même niveau que la seconde.

Nous avons déjà dit que chez les osmatiques il n'existe aucune démarcation analogue à celle qui est établic chez-les primates par l'incisure en H. Serait-ce parce que leur centre olfactif anté- 
rieur occuperait toute la région orbitaire et s'étendrait jusqu'à la pointe du lobe frontal? C'est fort probable, et si l'on songe à l'importance prépondérante du rôle que joue chez ces animaux le sens de l'olfaction, on comprendra peut-être qu'une grande partie, ou même la plus grande partie de leur lobe frontal puisse être affectée aux actes intellectuels que ce sens met en jeu; ct on pourra même se demander si ce ne serait pas là la raison d'être de la supériorité intellectuelle du lobe frontal, appelé, par ses connexions avec le lobe olfactif, à interpréter, à discuter sans cesse les sensations qui ont le plus d'utilité pour l'existence de l'animal. Devenu ainsi le siège des déterminations les plus importantes et constamment tenu en éveil, ce lobe acquerrait dans l'hémisphère une sorte d'hégémonie, et sa prépondérance intellectuelle, une fois étahlie, une fois devenue la loi du type cérébral des mammifères, se maintiendrait et se développerait ensuite par elle-même, en dépit des vicissitudes et de la décadence du lobe olfactif chez les anosmatiques. Mais laissons là ces conjectures et passons aux autres centres olfactifs.

$2^{\circ}$ Le centre olfactif postérieur est le lobe de l'hippocampe, où va se rendre la racine olfactive externe. L'anatomie comparée nous montre, en effet, que le lobe de l'hippocampe croît et décroît en même temps que cette racine. Large et volumineux chez les osmatiques, où on le voit ordinairement occuper toute la largeur de la face inférieure de l'hémisphère, et déborder même quelquefois sur la face externe, il est déjà beaucoup moins large chez la loutre, dont l'appareil olfactif, réduit au rôle de sentinelle défensive, pour annoncer sur terre l'approche de l'ennemi, est sans aucun usage dans l'eau, où l'animal cherche sa proie; et il se réduit bien plus encore chez les anosmatiques, dont l'appareil olfactif est atrophié ou anéanti. Jamais toutefois il ne s'efface entièrement; il diffère en cela du centre antérieur, et on peut en conclure que, s'il est affecté principalement à la fonction olfactive, il sert aussi à quelque autre usage encore inconnu. Son minimum de volume s'observe chez les cétacés, où il ne forme, autour du pédoncule cérébral et de l'émergence des bandelettes optiques, qu'une mince bordure, et où il n'occupe pas même la dixième partie de la largeur de la face inférieure de l'hémisphère (voy. fig, 54, H, p. 418); il est moins 
atrophié chez les primates (voy. fig. 55, 56 et 57, H, p. 421 à 423) qui ont un lobe olfactif rudimentaire, et moins encore che\% les amphibies (fig. 58), qui ont l'appareil olfactif un peu plus développé que les primates; son volume est donc manifestement solidaire de celui du lobe olfactif, et plus spécialement de la racine olfactive externe.

Ce n'est pas seulement par son petit volume que le lobe de l'hippocampe des anosmatiques se distingue de celui des osmatiques. Chez ces derniers, il constitue un lobe spécial, entièrenient différent, par sa structure et son apparence extérieure, de la partie adjacente du manteau de l'hémisphère, dont il est séparé par la scissure limbique (voy. fig. 43, p. 393, et fig. 48, p. 402). Chez les osmatiques, au contraire, son aspect est exactement le même que celui des circonvolutions voisines, avec lesquelles il se fusionne plus ou moins, de sorte qu'il cesse de constituer un lobe distinct; ce n'est plus qu'une simple circonvolution, dite circonvolution de l'hippocampe, et annexée au lobe temporal, dont elle forme le bord inférieur et interne (1). Par suite de cette fusion, la scissure limbique devient moins profonde et cesse d'être continue; elle ne diffère plus des sillons ordinaires qui séparent les circonvolutions d'un même lobe. J'ai décrit ailleurs les modifications que présente chez les cétacés, les amphibies et les primates la disposition de ce sillon, vestige de la scissure limbique des osmatiques (2). Je rappellerai seulement ici que chez les cétacés (voy. p. 418, fig. 54, P) l'extrémité antérieure de la circonvolution de l'hippocampe, $\mathrm{H}$, se continue directement, en T, sur le bord postérieur de la vallée de Sylvius, avec le reste du lobe (ou lobule) temporal; qu'en d'autres termes la pointe de ce lobe reste indivise, tandis que chez les amphibies et les singes cette pointe est subdivisée plus ou moins profondément par un sillon qui, se détachant de la partie externe de la vallée de Sylvius, à l'entrée de la scissure de Sylvius, pénètre,

(1) Le lobe temporal n'est bien caractérisé que chez les primates; chez les amphibies et les cétacés, il n'est pas assez distinct du lobe pariétal pour constituer un vrai lobe, et le nom qui lui convient le mieux est celui de lobule tempora! du lobe pariétal; mais cette différence de dénomination est ici sans importance.

(2) Revue d'anthrop., 1878, p. 4วั5, 462 et 477., et dans ce volume, p. 33 s̆, 343 et 359 . 
d'avant en arrière, plus ou moins profondément dans le lobe temporal. Ce sillon, étant un reste de la scissure limbique des osmatiques, mérite le nom de sillon limbique. Il est d'autant

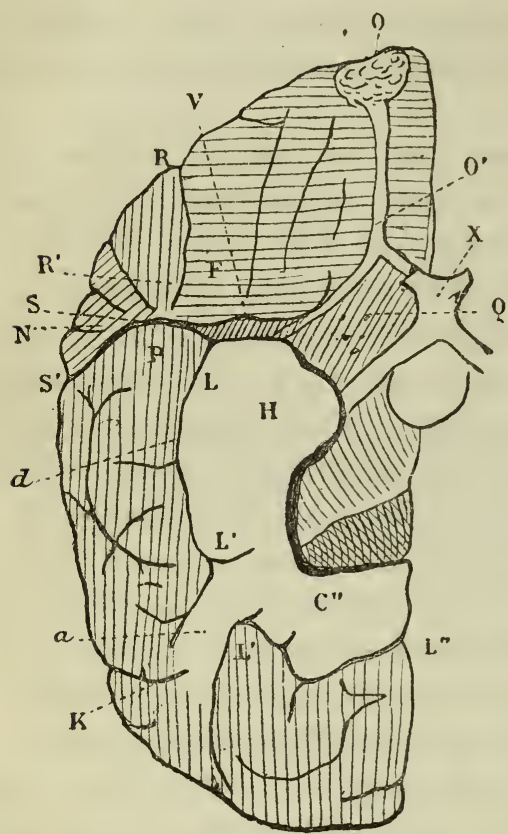

Fig. 58. Phoque, face inférieure de l'hémisphère droit.

$O$, renflement (lobe) olfactif; $O^{\prime}$, ruban (pédoncule) olfactif avec ses deux racines blanches. H, lobule de l'hippocampe ; C', extrémité postérieure du lobe du corps calleux ; LL'. arc inférieur de la scissure limbique ou sillon limbique; $\mathrm{L}^{\prime} \mathrm{L}^{\prime \prime}$, son arc supérieur ou scissure pariétale; $a$, pli de passage rétro-limbique ; $\mathrm{K}$, sillon calcarin; $d$, position d'un pli de passage profond (qui est superficicl chez l'otaric); $X$, chiasma; $Q$, espace perforé ; RR, scissure de Rolando; V, vallée de Sylvius se continuant en dehors avec la scissure de Sylvius $\mathrm{SS}^{\prime} ; \mathrm{F}$, Tobe frontal; $N$, lobule sous-sylvien en partie superficiel. plus grand et plus profond que la circonvolution de l'hippocampe et la racine olfactive externe, qui traverse pour s'y rendre le fond de la vallée de Sylvius, sont moins atrophiées.

On remarque, en effet, que ce sillon est grand chez les amphibies ( fig. 58, LL'), moindre chez les singes (fig. 55 et $57, b$ ), moindre et souvent nul chez l'homme, toujours nul enfin chez les cétacés. Il s'atténue donc à mesure que décroissent le volume de l'appareil olfactif et l'importance de la fonction olfactive. C'est parce qu'un organe reste d'autant plus indépendant, résiste d'autant, mieux à la fusion, que sa fonction est plus importante. Nous tirerons plus loin de ce fait des conséquences qui ne seront peut-être pas sans intérêt.

On vient de voir que, chez les anosmatiques, les divers degrés de fusion indiqués par l'absence, la présence et l'étendue du sillon limbique, se montrent sur la partie antérieure du lobe de l'hippocampe, ou, si l'on veut, de la circonvolution de l'hippocampe; il parait donc probable, d'après cela, que c'est cette partie antérieure seulement qui est le centre olfactif externe.

L'étude du volume relatif de cette partie antérieure dépose dans le même sens. Chez les cétacés, la circonvolution de l'hip- 
pocampe va en s'amincissant d'arrière en avant, et se termine antérieurement en une extrémité effilée (fig. 54); tandis que chez les autres anosmatiques, hommes, singes ou amphibies, cette circonvolution se renfle à sa partie antérieure en un lobule arrondi, qu'on appelle le lobule de l'hippocampe, et qui est plus grand chez les amphibies que chez les primates (fig. こૅ à ら8, H). C'est le volume de ce lobule, bien plus que celui de la circonvolution de l'hippocampe considérée dans son ensemble, qui est proportionnei au volume de la racine olfactive externe. Chez les cétacés, la racine manque, et le lobule est nul; chez les primates, la racine est très grêle, à tel point que beaucoup d'anatomistes ont cru qu'elle se perdait dans le fond de la vallée de Sylvius et qu'elle n'aboutissait pas à la circonvolution de l'hippocampe; mais lorsqu'on l'étudie sur des cerveaux durcis dans l'acide nitrique, on voit qu'elle traverse entièrement la vallée de Sylvius, sur le bord externe de l'espace perforé, et qu'elle va se rendre à la partie la plus externe de la face profonde de cette circonvolution. Elle est donc constante, quoique très petite, et le lobule de l'hippocampe, qui est constant comme elle, n'offre qu'un volume médiocre (fig. 55,56 et $57, \mathrm{H}$ ). Ghez les amphibies enfin la racine, qui suit exactement le même trajet, est notablement plus forte, et le lobe de l'hippocampe est aussi naturellement plus grand que chez les primates : car il occupe près de la moitié de la largeur de la face inférieure de l'hémisphère, et la moitié au moins de la longueur de la circonvolution de l'hippocampe (fig. 58, H). De ces faits on peut conclure que c'est bien réellement le lobule de l'hippocampe qui est le centre olfactif postérieur, correspondant à la racine olfactive externe; il ne paraît pas probable que ce centre s'étende, en arrière, au-delà de la limite postérieure de ce lobule, limite qui correspond chez les primates à une ligne transversale passant par l'extrémité postérieure du crochet de l'hippocampe (fig. $56, h$ ).

Il y a aussi, chez les osmatiques, une partie que l'on peut appeler le lobule de l'hippocampe; car l'extrémité antérieure du lobe de l'hippocampe forme constamment, en arrière de l'espace quadrilatère occupé par la racine grise ou moyenne, une saillie plus ou moins forte; mais en arrière rien n'indique la limite de ce lobule, qui se continue entièrement, sans ligne de démarca- 
tion, sans renflement ni étranglement, avec le reste du lobe; d'où il résulte que le centre olfactif s'étend très probablement à tout le lobe; et il est bien naturel, en effet, que ce centre olfactif ait une grande étendue : car la racine olfactive externe est très volumineuse; elle est si grossequ'elle est superficielle dans toute sa longueur, et qu'elle forme une large barrière, qui sépare entièrement la scissure de Sylvius de la vallée de!Sylvius ou espace perforé.

On pourra remarquer que le centre olfactif postérieur ou de l'hippocampe, et le centre olfactif antérieur ou orbitaire, présentent des modifications analogues lorsqu'on les étudie comparativement chez les osmatiques et chez les primates. Tous deux sont en quelque sorte diffus chez les osmatiques, où rien n'indique leur limite, le premier s'étendant probablement à tout le lobe de l'hippocampe, et le second à tout le lobule orbitaire; tandis que chez les primates ces deux centres, moins étendus, sont en même temps plus distincts, en ce sens que l'on peut retrouver à la surface de l'hémisphère l'indication de leur limite, savoir : sur le lobule orbitaire l'incisure en $\mathrm{H}$, et sur la circonvolution de l'hippocampe l'étranglement situé en arrière du lobule, au niveau du bord postérieur du crochet.

Ceci n'est nullement en contradiction avec ce qui a été dit plus haut (p. 426), relativement it la fusion du lobe de l'hippocampe des anosmatiques avec leur lobule temporal, fusion que j'ai attribuée à l'atrophie du lobe de l'hippocampe et à la diminution de son importance fonctionnelle. Ce cas, en effet, est bien différent de ceux que je viens d'examiner. Je crois avoir démontré, dans mon mémoire sur l'anatomie comparée des circonvolutions, que le grand lobe limbique, dont le lobule de l'hippocampe fait partie, constitue l'une des deux divisions primaires de l'hémisphère des osmatiques, et qu'il se distingue de la masse circonvolutionnaire ou extra-limbique (qui forme tout le reste du manteau) par une constitution morphologique et anatomique toute spéciale, et aussi par une évolution inverse, car il reste étranger aux perfectionnements qui amènent la formation et le développement des circonvolutions dans la masse extralimbique. Je lui ai même, pour exprimer ce contraste, donné le nom de cerveau brutal, en donnant à l'autre division primaire de 
l'hémisphère le nom de cerveau intellectuel (1). Le grand lobe limbique est le siège principal de toutes les fonctions qui sont en rapport direct avec l'olfaction ou qui en découlent, et ces fonctions jouent un si grand rôle dans la vie de l'animal, dans ses connaissances, dans ses délibérations et dans ses déterminations, que to!ttes les autres parties du manteau viennent prendre sur lui leurs connexions, et que ces connexions sont même ce qu'il y a de plus constant dans le manteau des mammifères. Mais pour être ainsi relié à l'ensemble du cerveau, le grand lobe n'en conserve pas moins une spécialité d'action démontrée par la spécialité de sa structure, et une certaine indépendance fonctionnelle, attestée par la grande scissure limbique qui l'entoure presque entièrement de tous côtés. Telle est la raison d'être de la division primaire du manteau des osmatiques en deux régions tout à fait distinctes, l'une limbique et l'autre extra-limbique. Mais les motifs de cette division primaire n'existent plus, lorsque le sens olfactif cesse d'être prépondérant, lorsqu'il n'est plus autonome, lorsqu'il descend au niveau des autres sens. Alors l'appareil central de l'olfaction, complètement annexé à l'hémisphère, rentre dans la règle commune; il revêt la même apparence et la même structure que le reste du manteau. Le lobe de l'hippocampe, en particulier, n'est plus qu'une circonvolution du lobe temporal, et cette circonvolution communique avec sa voisine comme celle-ci avec la suivante: car il est naturel qu'un organe, en perdant son indépendance fonctionnelle perde aussi son indépendance anatomique. Mais il n'y a rien de commun entre ce fait général d'évolution et les modifications qui peuvent se produire dans l'organe même, par suite du nouvel état de ses fonctions. Si une fonction qui était assez importante pour occuper toute l'étendue de l'organe, s'atténue de manière à n'en occuper maintenant qu'une portion, il y aura entre cette portion et le reste de l'organe une différence fonctionnelle qui n'existait pas auparavant, et qui pourra se traduire extérieurement par une démarcation plus ou moins nette. C'est ainsi que, chez les amphibies et les primates, le centre olfactif postérieur se dessine sur la partie antérieure de la cir-

(1) Revue d'anthrop., 1878, p. 420 et dans ce volume, p. 298. 
convolution de l'hippocampe, sous la forme d'un lobule moins atrophié que le reste de la circonvolution, et c'est ainsi encore que, sur la seconde circonvolution orbitaire des primates, une dépression ou une incisure transversale marque la limite du centre olfactif antérieur, qui, ayant cessé de s'étendre à tout le lobule orbitaire, a rétrogradé jusque-là.

$3^{\circ}$ Le centre olfactif supérieur, enfin, correspond à la racine olfactive interne, qui, après avoir formé le côté antérieur et interne de l'espace quadrilatère, et atteint le bord interne du lobule orbitaire, remonte sur la face interne de l'hémisphère, et va se perdre dans la couche de substance grise qui revêt l'origine du lobe du corps calleux (voy. plus haut, fig. 42, G, p. 391, et surtout fig. 49, C, p. 403). Il y a là une petite région plane qui communique en avant avec la face interne du lobe frontal, en haut avec le lobe du corps calleux, en bas avec la partie interne de l'espace quadrilatère, et sur laquelle vient se terminer insensiblement, en bas et en arrière, l'extrémité effilée ou bec du corps calleux. Ajoutons, enfin, que c'est là que viennent aboutir, en bas et en avant, la racine olfactive interne, en bas et en arrière, la bandelette diagonale de l'espace quadrilatère, qui s'étend, comme on l'a vu plus haut, du lobule de l'hippocampe à l'origine du lobe du corps calleux (voy. p. 401 et fig. $45, d$, p. 403).

Ces communications multiples justifient le nom de carrefour de l'hémisphère que j'ai donné à cette petite région. La constitution essentielle du carrefour de l'hémisphère reste la même chez tous les mammifères, à l'exception des cétacés delphiniens, qui n'ont ni racine olfactive ni bandelette diagonale; mais le carrefour est relativement beaucoup plus large chez les osmatiques que chez les anosmatiques; sa largeur est à peu près celle de l'origine du lobe du corps calleux; or, ce lobe, chez les osmatiques, présente toujours, même à son origine, une assez grande largeur, tandis que, chez les amphibies et les primates, il naît par une extrémité étroite, et quelquefois presque effilée.

Nous venons de dire que le carrefour est constitué de la même manière chez tous les mammifères qui ont un appareil olfactif grand ou petit. Cela ne veut pas dire toutefois qu'il ait toujours la même apparence. Chez les osmatiques, la racine olfactive in- 
terne, quoique constamment beaucoup plus petite que l'externe, est toujours assez visible sur la face interne de l'hémisphère pour qu'on puisse la suivre sur le carrefour jusqu'à l'origine du lobe du corps calleux. La bandelette diagonale, bien apparente dàns l'espace quadrilatère, devient plus confuse au niveau du carrefour; mais on l'y suit cependant, de bas en haut dans une certaine étendue, et quelquefois même on la voit atteindre manifestement l'origine du lobe du corps calleux, en même temps que quelques-unes de ses fibres se jettent dans le bec du corps calleux (voy. plus haut, p. 40').

Mais chez les amphibies et les primates ces caractères sont considérablement atténués, disons même presque effacés. On retrouve difficilement sur la face interne de l'hémisphère le trajet de la racine olfactive interne; on la voit se perdre dans la substance grise du carrefour, sans pouvoir la suivre jusqu'au lobe du corps calleux, et ses rapports avec ce lobe ne pourraient être établis sans le secours de l'anatomie comparée.

Plus vague encore est, chez ces animaux, la bandelette diagonale. J'ai même pu croire pendant quelque temps qu'elle n'existait pas, et je le croirais encore si l'étude de deux cerveaux pathologiques ne m'avait permis de la reconnaître chez l'homme.

Nous avons déjà dit que, chez les anosmatiques, la racine olfactive moyenne ou grise, qui occupe tout l'espace quadrilatère des osmatiques, n'existe plus. Par suite de cette disparition, l'espace quadrilatère se creuse en une profonde vallée, et prend le nom d'espace perforé, parce que la très mince couche de substance grise qui le tapisse est criblée de trous pour le passage de veines émanées du corps strié subjacent.

La bandelette diagonale, qui traverse l'espace quadrilatère, continue à traverser de la même manière l'espace perforé; mais, considérablement atrophiée, réduite à quelques filaments peu serrés les uns contre les autres, elle ne se distingue pas de la substance grise dans l'épaisseur de laquelle ses fibres cheminent. Il en reste toutefois un vestige qui est quelquefois bien apparent chez l'homme, et qui a été décrit par Vicq d'Azir sous le nom de pédoncule antérieur du corps calleux. G'est un petit tractus blanchâtre qui, né du bec du corps calleux sur la ligne 
médiane, chemine d'avant en arrière le long de la ligne médiane jusqu'au niveau de la lame grise dite racine grise du chiasma, et qui alors, se dirigeant en dehors le long du bord

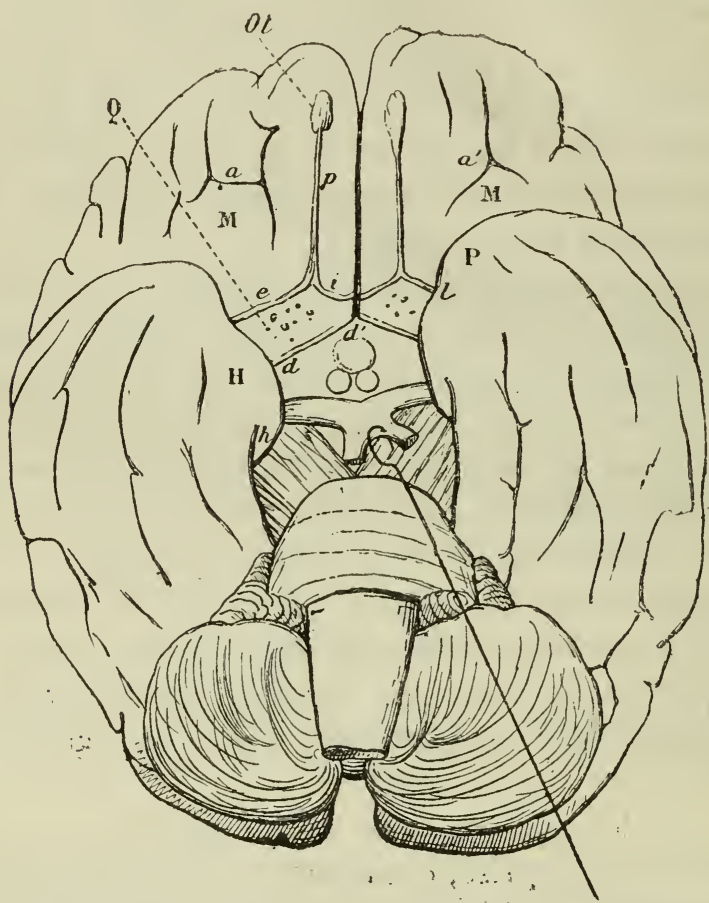

Fig. 59. Face inférieure du cerveau d'un homme mort de la paralysie générale des aliénés. (1/2 grandeur).

Pour montrer la bandelette diagonale on a détaché et attiré en arrière avec un crochet le chiasma et les bandelettes optiques, qui se trouvent ainsi reportées en arrière des tubercules mamillaires.

$\mathrm{O} l$, le renflement olfactif; $p$, son pédoncule ; $i$, racine olfactive interne ; $e$, l'externe, disparaissent dans la vallée de Sylvius, sous la pointe du lobe temporal; $Q$, l'espace quadrilatère perforé $; d d^{\prime}$, la bandelette diagonale, son extrémité externe et postérieure $d$ se jette sur la face profonde du lobule de l'hippocampe $\mathrm{H}$; son extrémité antérieure et interne atteint le bord interne de l'hémisphère en avant de la racine grise du chiasma, et se jette sur la face interne, où elle se perd dans le carrefour.

$\mathrm{P}$, le pôle temporal; $l$, dépression représentant le sillon limbique des singes.

$M, M^{\prime}$, le centre olfactif antérieur ou orbitaire, limité antérieurement par l'iucisure en $H$, qui à droite, $a$, a la forme d'une $\mathrm{H}$, et qui présente à gauche, $a^{\prime}$, une forme un peu différente.

antéro-externe de la bandelette optique, va se perdre, après un court trajet, dans la substance grise de l'espace perforé, sans atteindre le lobule de l'hippocampe. Telle est la description classique, et, d'après cela, le pédoncule du corps calleux, restant tout à fait étranger au lobule de l'hippocampe ainsi qu'au lobe 
du corps calleux, représenterait tout au plus la portion la plus interne des quelques fibres diagonales qui vont, sinon chez tous les osmatiques, du moins chez quelques-uns, se rendre au bec du corps calleux; et rien ne représenterait chez l'homme la véritable bandelette diagonale, qui se porte du lobule de l'hippocampe à l'origine du lobe du corps calleux.

Mais cette connexion, que l'on n'aperçoit pas à l'état normal, peut être rendue apparente dans certains cas pathologiques. Il y a quelques mois, en faisant l'autopsie d'un homme de trentesept ans, qui avait succombé à une paralysie générale avec délire ambitieux, je découvris enfin la bandelette diagonale, que j’avais vainement cherchée sur les cerveaux sains. Je dessinai la pièce et j'en donnai la démonstration aux élèves de ma clinique (fig. 56). Née de la partie moyenne de la face profonde du lobule de l'hippocampe, et cheminant à la surface de l'espace perforé, la bandelette diagonale formait un petit ruban très net qui se portait obliquement en dedans et en avant, gagnait le bord antéro-externe de la bandelette optique, le suivait ensuite jusqu'au niveau de la "racine grise » du chiasma, atteignait ainsi le bord interne de l'hémisphère au-devant du chiasma et en arrière de la racine olfactive interne (qui était très apparente ainsi que l'externe), et passait enfin sur la face interne, où elle se jetait dans le carrefour. Là, on pouvait suivre encore quelques-unes de ses fibres, qui se dirigeaient vers l'origine du lobe du corps calleux, et se perdaient dans la substance grise la plus superficielle, en même temps que quelques autres fibres, les plus postérieures, se dirigeaient vers le bec du corps calleux.

Pour montrer sur la figure le trajet de la bandelette diagonale, $d d$, j’ai détaché et renversé en arrière le chiasma et les deux bandelettes optiques, de sorte que le chiasma se trouve reporté en arrière des tubercules mamillaires.

L'apparition de la bandelette diagonale, chez ce paralytique, a été la conséquence de l'atrophie de la substance grise environnante. On sait que la paralysie générale des aliénés consiste principalement en une altération de la substance grise corticale des hémisphères ; cette substance corticale perd une partie de sa consistance, se décolore el s'amincit, tandis que la substance 
blanche subjacente devient au contraire plus dense et plus ferme, sinon pendant toute la durée de la maladie, du moins pendant les premières périodes. On conçoit donc que, lorsque cette lésion s'étend à l'espace perforé, l'atrophie de la substance blanche puisse rendre apparentes les fibres blanches de la bandelette diagonale. J'ai effectivement pu constater l'existence de cette bandelette sur un second cerveau d'aliéné paralytique; mais je n'ai pu l'apercevoir sur un troisième cerveau provenant d'une femme atteinte de la même affection. Les conditions favorables à l'apparition de cette disposition anatomique doivent varier suivant les individus et suivant le degré d’intensité et le degré d'évolution de la maladie; on ne peut donc s'attendre à la retrouver sur tous les aliénés paralytiques, et peut-être même ne l'observera-t-on que dans des cas plus ou moins rares; mais comme il est évident qu'une affection qui désorganise le cerveau ne peut avoir pour conséquence de produire des fibres nouvelles, il suffit que l'on puisse constater quelquefois, ne fût-ce qu'exceptionnellement, la présence et le trajet des fibres de la bandelette diagonale, pour qu'on soit en droit de considérer 'comme normale la connexion établie par ces fibres entre le lobule de l'hippocampe et le carrefour de l'hémisphère.

Et si cette connexion, constante chez les osmatiques, existe aussi chez l'homme, il est clair qu'elle ne doit pas manquer chez les autres anosmatiques (abstraction faite, bien entendu, des cétacés), puisque leur appareil olfactif, dont la bandelette diagonale fait partie, est moins rudimentaire que celui de l'homme.

Répétons donc que la constitution essentielle du carrefour de l'hémisphère est la même chez tous les mammifères doués de l'odorat.

Ce carrefour, où se rend la racine olfactive interne, et où aboutit en outre la bandelette diagonale venue du centre olfactif postérieur, est évidemment affecté à l'olfaction; il fait partie par conséquent du centre olfactif supérieur; mais il est non moins évident qu'il n'en constitue, chez les osmatiques, qu'une faible partie : car ce n'est pas sur lui que les fibres de la racine olfactive interne prennent leur origine réelle; on les suit chez ces animaux jusque sur l'origine du lobe du corps cal- 
leux, et, dès lors, la participation de ce lobe à la fonction olfactive est tout aussi certaine que celle du lobe de l'hippocampe.

L'existence d'une fonction commune à ces deux lobes était d'ailleurs tout indiquée déjà par ce fait que, constituant respectivement les deux arcs du grand lobe limbique, et séparés du reste du manteau dans la plus grande partie de leur étendue par la scissure limbique, ils se continuent directement l'un avec l'autre, derrière le pédoncule cérébral, de manière à former autour du seuil de l'hémisphère un circuit ininterrompu, complété en avant par la bandelette diagonale de l'espace quadrila-

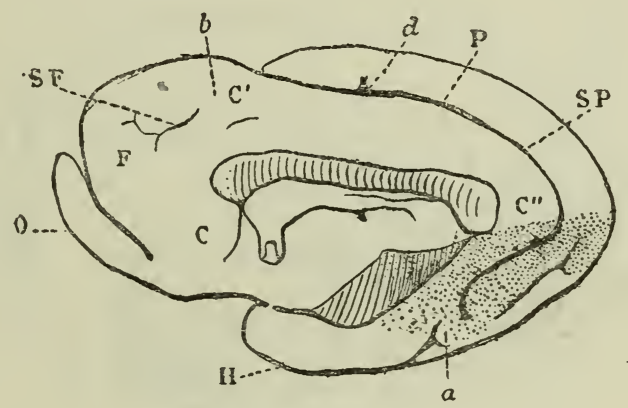

Fiğ. 60. Renard. Face interne de l'hémisphère droit.

C, $\mathrm{C}^{\prime}, \mathrm{C}^{\prime \prime}$, lobe du corps calleux; $a$, pli de passage rétro-limbique; $\mathrm{F}$, lobe frontal ; P, lobe pariétal; SF, sillon sous-frontal; SP, scissure sous-pariétale; $b$, pli de passage frontolimbique.

tère. Que cette fonction commune soit l'olfaction, c'est ce que nous montre l'étude des deux racines olfactives qui en naissent.

Mais il n'en résulte nullement que ces deux lobes soient $e x$ clusivement affectés à l'olfaction. L'anatomie comparée nous a déjà montré que le lobe de l'hippocampe possède certainement, en sus de la fonction olfactive qui est démontrée, d'autres fonctions encore inconnues, et on va voir que le lobe du corps calleux est dans le même cas.

Cette dernière conclusion ne serait peut-être pas légitime, si l'on n'examinait que les cerveaux des osmatiques. On verrait chez eux le lobe du corps calleux, relativement assez étroit à son origine, s'élargir bientôt au-devant et au-dessus du genou du corps calleux, acquérir en ce lieu son maximum de volume, et 
diminuer ensuite d'avant en arrière, jusqu'au-dessous du bourrelet du corps calleux, de manière à se continuer par sa partie la plus étroite avec le lobe de l'hippocampe (voy. fig. 60, Renard, et fig. 61, Tapir). La largeur plus grande de sa portion antérieure s'expliquerait très bien par la plus grande proximité de la racine olfactive interne, et rien n'autoriserait à admettre que le lobe fût affecté à quelque fonction autre que l'olfaction.

Mais si nous passons à l'examen du cerveau des primates (fig. 62, Chimpanzé), nous voyons la forme du lobe du corps calleux se modifier entièrement. Ici, ce n'est plus l'extrémité

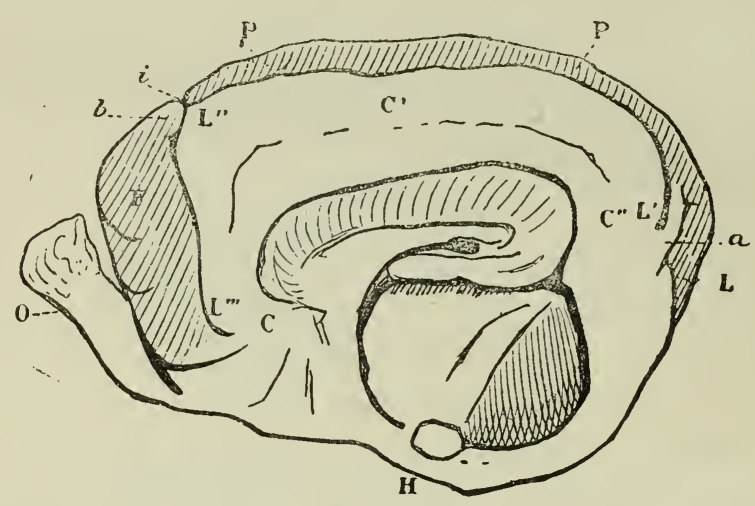

Fig. 61. Tapir. Face interne de l'hémisphère droit.

O, lobe olfactif; H, lobe de l'hippocampe; C, C', C', lobe du corps calleux ; L, arc inférieur de la scissure limbique; F, lobe frontal; PP, lobe pariétal ; $b$, pli de passage profond fronto-limbique; $i$, incisure fronto-pariétale interne.

antérieure qui est la plus large: elle est au contraire la plus étroite, de sorte que la scissure sous-frontale qui la limite remonte d'avant en arrière jusqu'au bord sagittal de l'hémisphère (1). Quant à la partie postérieure du lobe, elle est relativement aussi développée que chez les osmatiques, et quelquefois même plus développée que chez plusieurs d'entre eux. La différence entre les primates et les osmatiques consiste donc en ceci, que la partie antérieure du lobe du corps calleux est atrophiée

(1) J'énonce ici sommairement ce caractère que j'ai exposé en détail dans mon mémoire sur l'Anat. comp. des circonvolutions, et qui constitue le caractère distinctif le plus remarquable du cerveau des primates (Revule d'anthr., 1878, 2c sér., t. Ier, p. 487, et dans ce volume, p. 371). 
chez les primates, et, comme ce fait coïncide avec l'atrophie du lobe olfactif et de sa racine interne, on peut en 'conclure que la différence anatomique est en rapport avec la différence fonctionnelle, que par conséquent le centre olfactif supérieur s'étend au lobe du corps calleux; mais le fait que l'atrophie ne s'étend pas à la partie postérieure du lobe, prouve clairement que cette partie postérieure n'a pas été gravement atteinte dans ses fonc-

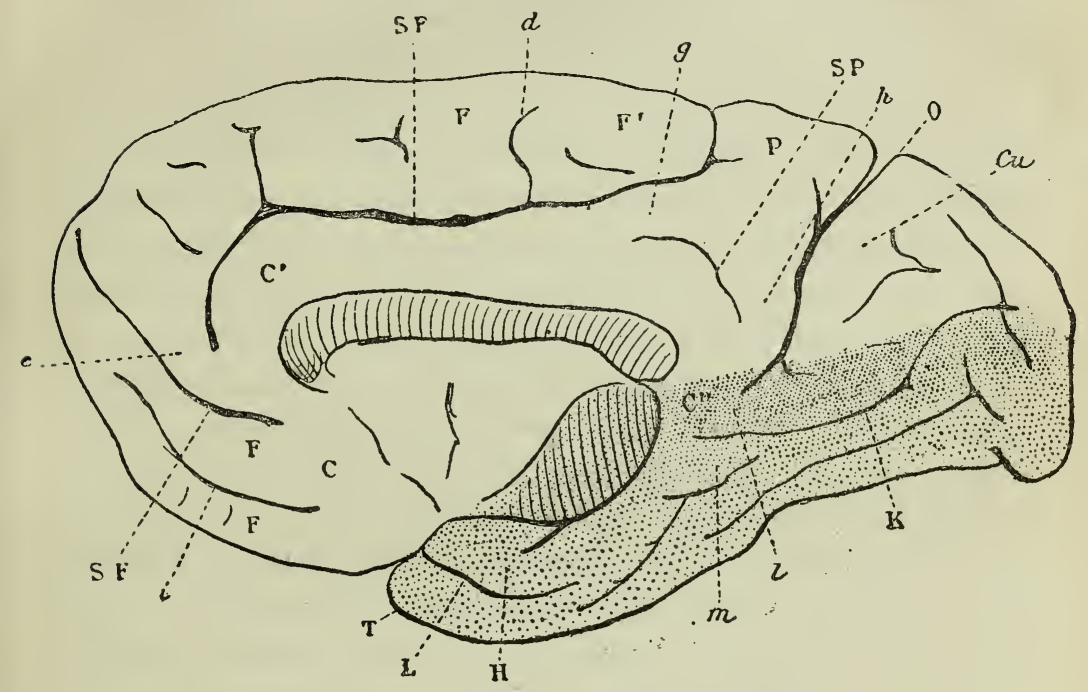

Fig. 62. Chimpanzé. Face interne de l'hémisphère droit.

C, C', C', le lobe du corps calleux; C, son origine au carrefour de l'hémisphère ; SF, scissure sous-frontale, s'élevant en arrière jusqu'au bord sagittal, interrompue en avant par le pli fronto-limbique, $e$.

H, lobe de I'hippocampe; L, le sillon limbique. (Il est inntile d'expliquer ici les autres lettres de cette figure, qui est extraite de la Revue d'anthrop., 1878, p. 493, et dans ce volume, p. 377.)

tions, qu'elle possède par conséquent des fonctions autres que l'olfaction.

Ceci permet d'interpréter un fait qu'on observe chez les cétacés, et qui, au premier abord, semble en opposition flagrante avec l'existence d'un centre olfactif dans le lobe du corps calleux. On a vu que, chez les cétacés, la zone du centre olfactif supérieur reste déserte par suite de l'absence de la racine olfactive supérieure; qu'en outre, par suite de l'absence de la racine externe, la partie antérieure du lobe de l'hippocampe est extrêmement atrophiée, de sorte que le lobule de l'hippocampe fait 
entièrement défaut. Il semble donc, d'après cela, que l'absence de la racine interne devrait amener, sur la partie antérieure du lobe du corps calleux, une atrophie bien plus prononcée encore que chez les primates. Il n'en est rien. Chez les cétacés, comme chez les osmatiques (et en particulier comme chez les pachy. dermes, avec lesquels les cétacés delphiniens ont plus d'une analogie cérébrale), c'est à sa partie antérieure que le lobe du corps calleux présente sa plus grande largeur. En outre, cette partie antérieure, loin d'être déserte, est creusée de sillons qui la subdivisent en circonvolutions ou en plis secondaires, ni plus ni moins larges, ni plus ni moins serrés que ceux qui recouvrent le reste du manteau, caractère d'autant plus frappant que, chez tous les autres mammifères osmatiques ou anosmatiques, le lobe du corps calleux est toujours beaucoup plus simple que les lobes extra-limbiques, et est même presque toujours tout à fait lisse. Cette partie se présente donc à nous avec tous les caractères qui dénotent une pleine activité fonctionnelle, et ce fait serait tout à fait inexplicable si le lobe du corps calleux n'avait d'autre fonction que l'olfaction. La psychologie des cétacés est actuellement (et pour longtemps sans doute) trop inconnue pour que l'on puisse savoir ou présumer quelles sont ces fonctions cérébrales qui se sont développées chez eux au-delà du degré que l'anatomie permet d'admettre chez les autres animaux. On accordera, toutefois, que leur mode d'existence est assez différent de celui des mammifères ordinaires pour motiver de notables modifications dans le degré de développement de leurs fonctions cérébrales. Je ferai remarquer d'ailleurs que, chez eux, l'origine du lobe du corps calleux est toute spéciale, si même on peut dire que ce lobe ait une origine : car la scissure limbique quil'entoure, au lieu de se recourber au-devant du genou du corps calleux, et de reculer pour se diriger vers le carrefour, continue à se porter en avant jusqu'au bord sagittal de l'hémisphère, qu'elle rejoint au-dessus de la région orbitaire, de telle sorte que les circonvolutions ou plis secondaires du lobe du corps calleux se continuent largement et se confondent pourla plupart avec ceux du lobe frontal et de son lobule orbitaire. On n'observe rien de pareil chez les autres animaux, soit qu'ils aient comme les cétacés une scissure sous-frontale (cheval, tapir, élé- 
phant, primates, etc.), soit qu'ils aient simplement un petit sillon sous-frontal (carnassiers, etc.).

Revenons maintenant aux mammifères pourvus de l'odorat. Nous venons de voir que, chez eux, le lobe du corps calleux possède, en sus de certaines fonctions inconnues, une fonction olfactive, qu'il joue par conséquent le rôle de centre olfactif supérieur. Ce centre commence, en avant et en bas, sur l'origine même du lobe du corps calleux, et nous savons qu'il occupe au moins la partie antérieure du lobe; mais jusqu'où s'étend-il d'avant en arrière? Il est bien probable qu'il se prolonge plus loin chez les osmatiques que chez les anosmatiques; toutefois, on n'y aperçoit chez les premiers ni plissement transversal, ni rétrécissement plus ou moins brusque, ni rien qui puisse en indiquer la limite. La question du degré d'extension du centre olfactif supérieur reste donc douteuse. Mais, en ce qui concerne les anosmatiques, elle est susceptible de recevoir une solution assez probable, dont les amphibies vont nous donner la clef.

Nous avons déjà dit que le lobe olfactif de ces animaux est moins rudimentaire que celui des primates (1). Leurs racines olfactives interne et externe sont moins grêles aussi, et on a vu déjà que le lobule de l'hippocampe, où aboutit la racine externe, est plus volumineux chez eux que chez les primates. Une différence analogue s'observe sur la partie antérieure du lobe du corps calleux. Cette partie antérieure est sans doute moins large que chez les carnassiers terrestres; au lieu d'atteindre, comme chez eux, le bord sagittal de l'hémisphère, au-dessus du genou du corps calleux, elle n'occupe pas même la moitié de la largeur de la face interne du manteau; elle a donc subi une réduction considérable (2), mais moindre que chez les primates,

(1) Cette proposition est vraie d'une manière générale. Toutefois chez les petits singes hapaliens de l'Amérique, qui sont les primates les plus inférieurs, et qui sont presque lissencéphales, le lobe olfactif est quelquefois aussi développé que chez les amphibies.

(2) Pour apprécier le degré de réduction de la partie antérieure du lobe du corps calleux des amphibies, il faut comparer ces animaux, qui font partie de l'ordre des carnassiers, avec les carnassiers terrestres, leurs voisins zoologiques. J'ai établi cette comparaison dans mon Mémoire sur l'anatomie comparée des circonvolutions, auquel je demande la permission de renvoyer le lecteur (Voir Revue d'anthrop., 1878, p. 40\%, et dans ce volume, p. 338). 
de sorte que le lobe du corps calleux présente une forme intermédiaire entre les deux formes qui caractérisent respectivement le type des primates et celui des osmatiques (voy. fig. 63, Phoque). Tandis que, chez les osmatiques, ce lobe va en s'élargissant d'arrière en avant, et qu'il va au contraire en se rétrécissant d'arrière en avant chez les primates, il conserve chez les amphibies, ou du moins chez les phoques, une largeur à peu près la même dans toute son étendue (1). Nous reconnaissons ainsi que la partie antérieure du lobe du corps calleux crôit et

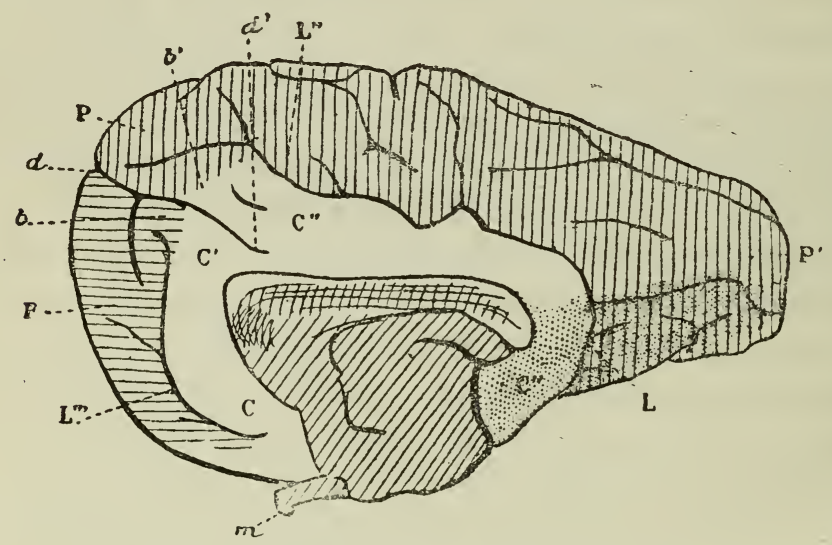

Fig. 63. Phoque. Face inlerne de l'hémisphère droit.

C, origine du lobe du corps calleux; $\mathrm{CG}^{\prime}{ }_{\text {, }}$ portion sous-frontale de ce lobe, séparée de sa portion sous-pariétale $\mathrm{C}$, par le sillon $d d^{\prime} ; b b^{\prime}$, le pli de passage pré-limbique, subdivisé par le mème sillon en deux plis, l'un inférieur $b$ ou fronto-limbique, l'autre supérieur $b^{\prime}$ ou pariéto-limbique; $L, L^{\prime}$, scissure pariétale; $L^{\prime \prime \prime}$, scissure sous-frontale; F, lobe frontal ; $\mathrm{PP}^{\prime}$, lobe pariétal; $m$, bandelette optique.

Le lobe de l'hippocampe peu volumineux, n'est pas visible sur la face interne.

décroît, chez tous les mammifères pourvus de l'odorat, dans le même sens que la racine olfactive interne et joue un rôle de centre olfactif. Cette conclusion, que nous avait déjà fournie la comparaison des osmatiques et des primates, est confirmée par l'étude de l'état intermédiaire que l'on observe chez les phoques.

Mais le cerveau des amphibies nous montre une autre disposition non moins instructive, disposition qui n'existe, ou plutôt

(1) Chez l'otarie, qui appartient à la même famille, mais non à la même tribu que les phoques, la parlie antérieure du lobe du corps calleux se rétrécit comme chez les primates. 
qui ne se montre que chez eux : c'est la subdivision du lobe du corps calleux en deux parties, l'une antérieure ou sous-frontale ( $\mathrm{CC}^{\prime}$, fig. 63), l'autre postérieure ou sous-pariétale, $\mathrm{C}^{\prime \prime}, \mathrm{G}^{\prime \prime \prime}$, par un sillon, $d d^{\prime}$, qui, venu du bord sagittal de l'hémisphère, s'enfonce dans le lobe, en traverse presque toute la largeur et ne s'arrête qu'à une très petite distance de la rainure du corps calleux. La continuité des deux parties du lobe du corps calleux n'est donc plus maintenue que par une mince languette, plus mince encore chez l'otarie que chez le phoque. J'ai même vu, sur l'hémisphère gauche (mais non sur le droit) d'un cerveau d'otarie, le sillon $d d^{\prime}$ se prolonger superficiellement jusqu'à la rainure du corps calleux, de sorte qu'il fallait écarter les bords du sillon pour apercevoir la mince languette de communication.

Il me paraît, d'après cela, très probable que le sillon $d d^{\prime}$ marque chez les amphibies la limite du centre olfactif supérieur, qu'en d'autres termes ce centre occupe la portion du lobe du corps calleux comprise entre son origine $\mathrm{G}$ et le sillon $d d^{\prime}$. Que ce centre s'étende plus loin chez les usmatiques, c'est ce qui paraîtra assez vraisemblable, et l'on conçoit que, lorsqu'il rétrograde par suite de la diminution de la fonction olfactive, la limite sur laquelle il s'arrête puisse être indiquée par une démarcation apparente, analogue à celle qui est indiquée sur la seconde circonvolution orbitaire des primates par l'incisure en $\mathrm{H}$ dont il a été question plus haut.

Ge sillon, $d d^{\prime}$, que j'appelle le sillon fronto-limbique, n'existe que chez les amphibies, ou plutôt c'est chez eux seulement qu'il pénètre dans le lobe du corps calleux. Chez les primates, cette ligne de démarcation n'existe pas; mais on peut retrouver néanmoins la position qu'elle occupe chez les amphibies.

On remarque, en effet, que le sillon fronto-limbique des amphibies accompagne un pli de passage (fig. $63, b$ ) qui unit le lobe du corps calleux ati lobe frontal, et quej'ai décrit sous le nom de pli de passage fronto-limbique. Ce pli n'est pas propre aux amphibies. Il est constant chez les mammifères; mais il n'est pas toujours apparent; il est souvent caché dans le fond de la scissure limbique, ou de l'anfractuosité (scissure sous-frontale) qui représente la partie antérieure de cette scissure chez les primates; il est, suivant les espèces, tantôt superficiel, tantôt pro- 
fond: ou plutôt il est toujours superficiel dans certaines espèces, toujours profond dans quelques autres; dans d'autres, enfin, et en particulier chez l'homme, on peut rencontrer ces deux dispositions non seulement chez des individus différents, mais même sur les deux hémisphères d'un même cerveau. Lorsqu'il est caché, il suffit, pour l'apercevoir, d'écarter les bords de la scissure sous-frontale vis-à-vis le genou du corps calleux; on le voit alors se détacher de la convexité du lobe du corps calleux,

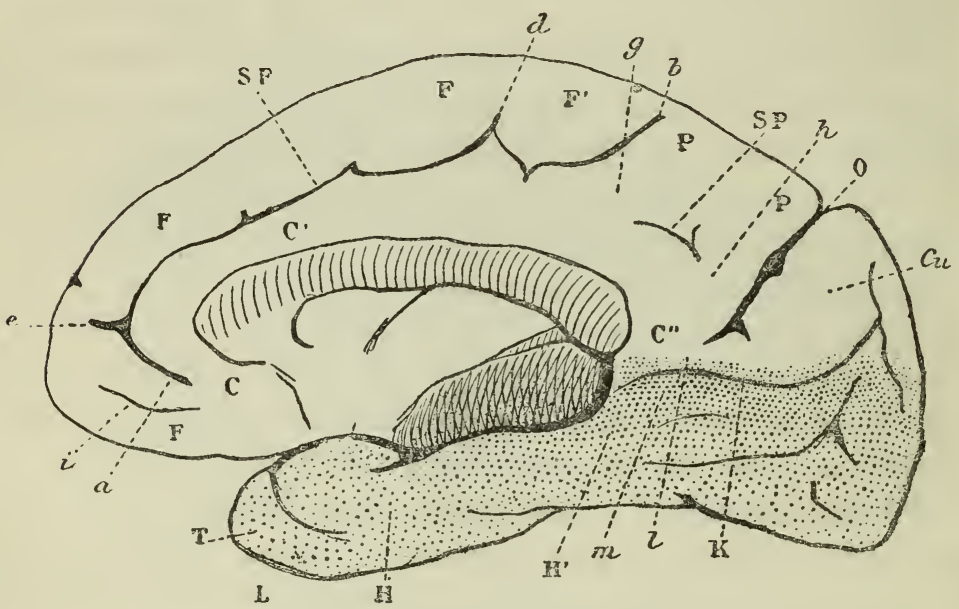

Fig. 64. Gynocéphale papion. Face interne de l'hémisphère droit.

Les lettres comme sur la figure 62, si ce n'est que le pli de passage fronto-limbique, étant profond, n'interrompt pas la scissure sous-frontale; mais sa position est indiquée par l'incisure fronto-limbique, $e$, qui marque, sur la face interne de l'hémisphère, la limite de l'étage supérieur et de l'étage inférieur du lobe frontal.

se porter en avant, et se jeter dans la face interne de la première circonvolution frontale. D’ailleurs, sa présence est toujours indiquée extérieurement par une incisure horizontale ou à peu près horizontale (fig. $64, e$ ), tantôt assez longue pour atteindre et même dépasser le bord sagittal de l'hémisphère, tantôt réduite à une longueur de quelques millimètres, mais toujours parfaitement visible. Cette incisure, qui accompagne le pli fronto-limbique, est l'incisure fronto-limbique, constante chez les primates, où elle établit, sur la face interne de la première circonvolution frontale, la limite de l'étage supérieur et de l'étage inférieur ou orbitaire. Quoiqu'elle ne pénètre pas dans 
le lobe du corps calleux, elle est évidemment l'analogue du sillon fronto-limbique des amphibies; elle indique, comme lui, le niveau du pli de passage fronto-limbique, et marque très probablement, comme lui, la limite du centre olfactif supérieur.

Lnn résumé, les anosmatiques pourvus de l'odorat ne diffèrent des osmatiques d'une manière absolue que par l'absence de la racine olfactive moyenne et du centre qui lui correspond dans le lobe olfactif. Ils possèdent les trois autres centres olfactifs, savoir : l'antérieur ou orbitaire, qui correspond à la racine supérieure; le postérieur ou de l'hippocampe, qui correspond à la racine externe, et le supérieur ou du lobe du corps calleux, qui correspond à la racine interne; mais ces trois centres ont subi, ainsi que leurs racines, une atrophie considérable, et ils cessent entièrement d'exister chez les cétacés delphiniens qui sont privés du sens de l'odorat.

\section{§ 4. - REMARqUeS SUR LES Fonctions 'RESPECTIVES DES DIVERS CENTRES OLFACTIFS.}

Les faits anatomiques que nous venons d'exposer nous permettent de dire que l'appareil central de l'olfaction est le plus compliqué de tous les appareils sensoriels. Chez tous les mammifères pourvus de l'odorat cet appareil comprend trois racines et trois centres; il comprend en outre, chez la plupart d'entre cux, un quatrième centrê, qui est le lobe olfactif, et une quatrième racine, qui est la racine grise ou moyenne. C'est par l'existence de la quatrième racine que les osmatiques se distingilent des anosmatiques.

La multiplicité de ces racines et de ces centres témoigne de l'extrême complexité des fonctions olfactives, et soulève d'intéressantes questions de physiologie que nous étudierons d'abord chez les osmatiques.

Tout appareil sensoriel remplit une double fonction, se rapportant d'une part à la serssation elle-même, considérée comme source de notions sur les qualités des corps; d'une autre part à la détermination des actes que l'animal, ainsi averti, peut éprouver le besoin d'exécuter pour sa sécurité, son utilité ou 
son plaisir. C'est ce que l'on exprime en disant que les sensations se transforment en volitions; celles-ci, toutefois, ne suivent pas nécessairement celles-là : car, dans la très grande ma. jorité des cas, la sensation ne provoque aucun acte extérieur.

Mais, chez l'animal osmatique, le sens de l'odorat joue un rôle si important, si prédominant, et d'une utilité si immédiate dans la lutte pour l'existence, lorsqu'il s'agit d'éviter un ennemi ou de suivre une proie à la piste et de prendre des déterminations soudaines dans la fuite ou dans la poursuite, - il est si nécessaire, en un mot, que l'acte suive instantanément la sensation, qu'il existe une union très étroite entre les deux faits que nous venons d'analyser. Cette union s'effectue dans le lobe olfactif, que ses connexions multiples mettent directement en rapport à la fois avec les parties de l'encéphale qui étudient les odeurs, et avec celles qui déterminent les actes provoqués par l'olfaction.

Ces diverses parties et le lobe lui-même sont autant de centres affectés à des opérations distinctes. Pour faciliter mon exposé, je choisirai mes expressions comme si le problème était déjà résolu. Considérant le centre postérieur (lobe de l'hippocampe) qui reçoit la racine externe, et le centre supérieur (lobe du corps calleux) qui reçoit la racine interne, comme affectés à la sensation olfactive proprement dite, je les appellerai tous deux ensemble le centre sensoriel, me réservant de chercher ensuite s'il n'y aurait pas à établir entre eux deux une répartition secondaire. J'appellerai centre de direction le centre antérieur ou orbitaire, qui fait partie du lobe frontal et qui reçoit la racine olfactive supérieure. Cette racine est la seule qui se rende à la partie extra-limbique de l'hémisphère, à celle que j'ai nommée le cerveau intellectuel; c'est donc par elle que les impressions olfactives sont mises directement en rapport avec l'intelligence, et je considère comme très probable que le centre olfactif antérieur où elle aboutit, est chargé de diriger les actes motivés par le sens olfactif. Mais ce n'est pas seulement par la racine olfactive supéricure que le lobe frontal est mis en communication avec l'appareil olfactif. Il communique en outre avec lui par les connexions fronto-limbiques. D'une part, en effet, sur la face interne de l'hémisphère il aboutit au carrefour, où il se continue 
avec l'origine du lobe du corps calleux, reliée au lobe de l'hippocampe par la bandelette diagonale de l'espace quadrilatère; en outre, le pli fronto-limbique, superficiel ou profond, le fait communiquer plus ou moins largement avec la partie antérieure et supérieure du lobe du corps calleux. Le centre sensoriel peut donc lui transmettre et lui soumettre les sensations qu'il a perçues.

Le quatrième centre enfin, ou plutôt le premier, puisque les impressions le traversent avant d'aboutir aux autres, est le lobe olfactif, que l'on peut appeler chez les osmatiques centre moteur; nous avons reconnu en effet qu'il possède une action motrice, démontrée à la fois par les cellules motrices qu'il renferme, et par la direction centrifuge des fibres de la racine moyenne.

Nous choisirons maintenant un exemple pour montrer comment s'enchaînent les fonctions de ces divers centres olfactifs.

Considérons un chien qui part pour la chasse. Il va d'abord à l'aventure, explorant les buissons, flairant les sentiers, aspirant sur son chemin une foule d'odeurs végétales, animales, minérales, qui produisent sur son lobe olfactif des impressions aussitôt transmises au centre sensoriel. Celui-ci les transforme en sensations qui sont transmises au lobe frontal par les connexions fronto-limbiques.

Tout à coup notre chien s'arrête. Parmi ces odeurs, il en a distingué une qui révèle la présence ou le passage d'un certain animal. Est-ce un ami, un ennemi, ou une proie? Si c'est une proie, est-elle digne d'être poursuivie, et la piste est-elle assez fraîche pour qu'il y ait chance de succès? Le chien délibère, il juge qu'il n'y a pas lieu de se mettre en chasse, reprend sa marche, puis s'arrête de nouveau. Cette fois, c'est plus sérieux : il a cru reconnaître l'odeur d'un lièvre. Il flaire attentivement, il étudie sa sensation; décidément il ne se trompe pas; il s'agit bien d'un lièvre, et la chasse va commencer.

Jusqu'ici l'appareil olfactif a fonctionné comme aurait pu le faire tout autre appareil sensoriel. Il a reçu une impression, l'a transformée en sensation, et a transmis à l'intelligence une notion qui a été suivie d'une détermination. Le chien aurait vu le lièvre, ou l'aurait entendu, au lieu de le sentir, les choses 
se seraient passées de la même manière. Jusqu'ici donc rien de spécial.

Mais la chasse commence. Voici le chien sur la piste, et il n'y a point de temps à perdre : car le lièvre, lui aussi, est pourvu d'une sentinelle olfactive; il sentira venir l'ennemi; il prendra de l'avance. Le succès dépend donc de la célérité. La question d'ailleurs est maintenant étudiée. L'odeur spéciale de la piste est reconnue, elle est classée ; il n'y a plus à craindre de la confondre avec une autre; le centre sensoriel n'a plus à intervenir ; en outre, la détermination est prise, l'intelligence n'a plus à délibérer.

La consigne est donnée : suivre la piste; c'est un acte simple et les cellules nerveuses du lobe olfactif y suffiront. Elles ne feront point face aux difficultés, aux complications qui pourront surgir; si le lièvre a bondi, rebroussé, fait un détour brusque, recroisé son chemin (il est si rusé!), si dans ce même lieu a passé quelque autre gibier, si la piste devient ainsi plus ou moins incertaine, le chien hésitera : une nouvelle étude deviendra nécessaire, le centre sensoriel fonctionnera de nouveau, et l'intelligence, délibérant encore une fois, pourra modifier le plan de la chasse. Mais ce moment d'hésitation passé et la piste retrouvée, la poursuite recommence, et elle consiste comme la première fois à courir le plus rapidement possible sur une trace indiquée par l'impression spéciale que r’eçoit le lobe olfactif. Tant que les cellules nerveuses de ce lobe recevront la même impression, elles guideront notre chasseur, sans qu'il soit obligé de retarder sa course en consultant continuellement le vrai centre sensoriel.

Le lobe olfactif jouit donc de la propriété d'agir à de certains moments comme organe recteur de mouvements presque automatiques, qui ne peuvent souffrir le moindre retard. Il fonctionne alors à la manière d'un centre à la fois sensitif et moteur : sensitif, jl faut bien qu'il le soit dans une certaine mesure, puisqu'il est moteur, et nous savons qu'il est moteur, puisque d'une part il renferme un grand nombre de cellules motrices énormes, et que d'une autre part il émet par sa racine moyenne des fibres centrifuges qui, pour la plupart, se continuent avec les fibres motrices du pédoncule cérébral (les autres se jettent dans la 
commissure antérieure, et servent probablement à établir l'unité d'action entre les deux lobes olfactifs).

Mais, quoique possédant une certaine autonomie, le lobe olfactif du chien n'en reste pas moins subordonné à la volonté de l'animal, c'est-à-dire au cerveau intelligent, ou encore au lobe frontal, avec lequel il communique directement par sa racine supérieure. Le chien reste toujours maître d'accélérer sa course, de la ralentir, de la dévier; ses autres sens sont en éveil : sa vue, son ouïe le renseignent aussi sur les mouvements, la position, la distance de la proie qu'il poursuit; s'il voit le lièvre faire un détour, il peut juger utile de se jeter à la traverse, d'abandonner un moment la piste pour la retrouver au demi-cercle; il ne s'agit plus ici d'étudier, d'analyser, d'interpréter la sensation olfactive, mais de prendre des déterminations instantanées dont le lobe olfactif est l'un des principaux facteurs, et il faut pour cela que ce lobe soit en communication immédiate avec les circonvolutions cérébrales. Cette communication est établie par la racine olfactive supérieure, racine large et $t$ 'ès courte, qui relie directement la base du pédoncule olfactif à la partie postérieure et inférieure du lobe frontal, et dans laquelle existent probablement à la fois, comme je l'ai dit plus haut (p. 414, en note) des fibres centripètes et des fibres centrifuges.

Nous venons de prendre pour exemple le mammifère chasseur à la poursuite de sa proie. Une analyse pareille nous montrerait aisément que l'animal poursuivi use des mêmes ressources, qu'il a besoin d'une association non moins prompte, non moins immédiate, du lobe olfactif qui le guide et du lobe frontal qui le gouverne. Prenons maintenant l'un ou l'autre animal dans les autres actes de sa vie, choisissant ses aliments, courant à l'amour, cherchant et suivant après de longues courses le chemin du logis, toujours son appareil olfactif fonctionne de deux manières, d'abord comme moyen d'étude, puis comme moyen d'action.

La première fonction se nomme le flairer; elle s'effectue dans le centre sensoriel auquel le lobe olfactif transmet les impressions par ses deux racines blanches interne et externe. Elle n'a pas besoin d'être précipitée, elle ne perd rien à être ralentie par 
la longueur des voies de transmission, et elle ne pourrait se simplifier qu'au détriment de sa délicatesse et de son utilité. Au contraire, la seconde fonction, que nous appellerons l'action olfactive, celle qui comprend les actes provoqués ct guidés par l'olfaction, exige souvent la promptitude extrême; tout ce qui la complique l'entrave, tout ce qui la retarde lui nuit; l'impression ne va donc pas faire un détour inutile à travers les racines blanches, le centre sensoriel et les connexions fronto-limbiques; reçue dans le lobe olfactif, elle s'y arrête, elle y produit une sensation incomplète, mais suffisante pour le but à atteindre. Les cellules sensitives qui la reçoivent agissent immédiatement sur les cellules motrices adjacentes, et celles-ci agissent aussitôt sur les cordons moteurs de la moelle par les fibres blanches de la racine grise. L'action est donc aussi prompte que possible; elle a la simplicité et l'instantanéité des actions réflexes, et elle en a jusqu'à un certain point le caractère : car ce qui constitue l'action réflexe, c'est la transformation directe de la sensation en mouvement sans l'intervention de la volonté. Or chez les mammifères, où les divers centres cérébraux sont fusionnés dans l'hémisphère, et où l'intelligence est centralisée et unifiée dans l'écorce de cet hémisphère, toute impression qui met en jeu les cellules motrices avant d'avoir atteint l'écorce cérébrale, ne produit qu'une action réflexe. L'action motrice du lobe olfactif participe donc, jusqu'à un certain point, de la nature des actions réflexes ; mais ce qui l'en distingue, c'est qu'elle n'est pas entièrement automatique, puisqu'elle est surveillée par le lobe frontal qui, communiquant directement avec le lobe olfactif par la racine olfactive supérieure, peut à tout instant modifier et dominer l'action de son subordonné. Ce qui l'en distingue surtout, c'est l'absence de toute régularité et de toute proportion entre la cause et l'effet. La véritable action réflexe est fixe et aveugle. L'excitation, transmise par certains nerfs sensitifs ou centripètes, se réfléchit sur certains nerfs moteurs et provoque par eux, sur une partie déterminée de l'appareil musculaire, des contractions proportionnelles à l'intensité de l'excitation, mais toujours de même nature, toujours réglées par les connexions anatomiques et toujours limitées aux mêmes parties. Sous ce rapport, l'action du lobe olfactif diffère entièrement des actions 
réflexes : car elle n'est ni proportionnelle à l'intensité de l'impression olfactive, ni distribuée par tels ou tels nerfs aux muscles d'une région déterminée, mais répartie dans tout le corps et traduite en mouvements généraux qui changent à chaque instant.

L'action olfactive, qui joue un rôle si important dans la vie des mammifères osmatiques, possède ainsi le double avantage d'être tour à tour et à la fois rapide comme les actes réflexes et dirigée comme les actes volontaires, grâce à la double connexion du lobe olfactif avec les cordons moteurs de la moelle et avec le lobe frontal.

Parlons maintenant des deux autres connexions de ce lobe, c'est-à-dire des deux racines blanches externe et interne qui le font communiquer avec le "centre sensoriel ". et qui sont en rapport avec le flairer. Je dis le centre sensoriel; mais le moment est venu de rappeler qu'il y en a deux : car les deux racines s'écartent en divergeant, et vont aboutir l'une au lobe du corps calleux, l'autre au lobe de l'hippocampe. Ces deux lobes, il est vrai, sont en continuité l'un avec l'autre; ils font partie tous deux du grand lobe limbique, qui se comporte chez les osmatiques comme un organe indivis. La solidarité des deux ares du grand lobe limbique, leur continuité directe en arrière, l'union établie entre leurs extrémités antérieures par la bandelette diagonale de l'espace quadrilatère, tout cela permet d'admettre que, sans préjudice de l'action propre à chacun d'eux, ils remplissent une fonction commune, et l'atrophie qu'ils subissent tous deux chez les anosmatiques prouve que cette fonction commune est l'olfaction. On peut donc, si l'on veut, considérer ces deux centres olfactifs comme réunis en un seul; mais il faut bien reconnaître que les deux parties du grand lobe limbique auxquelles se rendent les deux racines blanches, diffèrent notablement par leur structure, qu'elles sont séparées l'une de l'autre par toute la largeur de l'espace quadrilatère, et que dès lors leurs propriétés olfactives sont probablement plus ou moins différentes.

En quoi peut consister cette différence fonctionnelle? L'anatomie, qui nous conduit à poser la question, ne nous fournit pas le moyen de la résoudre. Elle nous suggère la pensée que 
les impressions olfactives doivent produire deux sortes de sensations, et donner à l'animal deux sortes de notions; mais la recherche de ces deux modes de la sensation olfactive est entièrement conjecturale.

On peut se demander d'abord si le sens de l'olfaction ne serait pas sous ce rapport comparable au sens du goût. On sait que les impressions sapides sont transmises aux centres nerveux par deux nerfs entièrement différents, savoir : le lingual, branche de la cinquième paire, et le glosso-pharyngien qui fait partie de la huitième paire. Ils diffèrent l'un de l'autre par leur origine, par leur trajet, par leur terminaison, enfin et surtout par leurs fonctions. Tous deux joignent à leur propriété de sensibilité générale la propriété de recueillir et de transmettre les impressions gustatives. Mais celles-ci se divisent en deux groupes bien distincts : les unes, comme la saveur sucrée, n'agissent que sur la partie antérieure de la langue, et ne sont transmises que par le nerf lingual, qui seul s'y distribue; les autres, comme la saveur amère, n'agissent que sur la base de la langue, et ne sont transmises que par le nerf glosso-pharyngien. Il y a donc deux régions gustatives, deux nerfs gustatifs, deux centres gustatifs. Ce dédoublement de la fonction gustative a longtemps tourmenté les physiologistes; il n'embarrasse plus personne depuis le jour où l'on a découvert qu'il y a deux catégories de saveurs. N'y aurait-il pas de même deux catégories d'odeurs? Et les deux racines olfactives blanches ne joueraient-elles pas le rôle des deux nerfs du goût, chacune transmettant exclusivement au centre olfactif correspondant les odeurs de l'une des deux catégories? Telle est la question qu'on peut d'abord se poser.

Mais cette conjecture ne peut supporter l'examen. Elle repose sur un rapprochement tout à fait faux. Les nerfs du goût ne sont pas les analogues des racines olfactives; ils sont les analogues des nombreux petits filets nerveux qui, de la membrane pituitaire, se rendent au lobe olfactif à travers les trous de la lame criblée. Parvenus au lobe olfactif, ils cessent d'être des nerfs; ils s'y dissocient en fibres nerveuses qui se confondent avec la masse du lobe. Il n'y a donc pas deux nerfs olfactifs, il y en a un très grand nombre : ils proviennent de toute la surface de la membrane olfactive, et tout permet de croire que cha- 
cun d'eux transmet indistinctement toutes les odeurs. Remarquons d'ailleurs que toute la théorie du goût repose sur ce fait que la muqueuse gustative se divise en deux régions distinctes par leurs propriétés. Il faudrait, pour que le rapprochement fût possible, qu'il y eût aussi dans la muqueuse nasale deux régions olfactives distinctes, ce qui est contraire à toutes les notions admises jusqu'ici.

On ne peut donc pas accepter l'idée que le dédoublement olfactif indiqué par la divergence des deux racines blanches soit en rapport avec la nature des odeurs transmises respectivement par les deux racines blanches. Il est probable dès lors que chaque odeur est transmise à la fois par la racine interne et par la racine externe, et que, si une même odeur aboutit ainsi à deux centres différents, c'est parce que l'animal peut l'apprécier à deux points de vue différents.

En admettant cette hypothèse, on est conduit à se demander comment une même odeur peut être perçue de deux manières, et en quoi diffèrent l'une de l'autre les deux sensations qu'elle produit. Il serait possible, par exemple, que l'une des sensations fût relative à la détermination de la nature des odeurs, et l'autre à l'appréciation de leur intensité. Il sérait possible encore que l'une fût en rappor't avec l'olfaction en général, et l'autre avec l'étroite corrélation qui existe entre le sens du goût et celui de l'odorat (corrélation bien connue de tous les physiologistes), ou a vec la corrélation non moins connue qui existe entre l'olfaction et la génération. A ces diverses conjectures, on peut en ajouter une autre qui me paraît plus plausible, et qui est conforme à l'analyse de la sensation en général.

Les impressions agissent sur le sensorium de deux manières : d'une part, elles provoquent une sensation agréable ou désagréable; d'une autre part, elles fournissent des notions sur les qualités des corps. Ces deux modes d'action sont associés sans doute, mais cependant séparables. Ainsi, lorsque nous appliquons les doigts sur un corps trop chaud, nous n'éprouvons d'autre sensation que celle de la brûlure; si ce même corps est refroidi, le contact n'est plus douloureux et nous révèle les qualités de forme, de volume, de consistance, etc., qui sont perçues par le sens du toucher. Enfin, si la température est intermé- 
diaire, elle pourra produire encore une certaine douleur sans empêcher pour cela la fonction tactile des doigts. Ce sont d'ailleurs les mêmes nerfs qui, dans tous ces cas, transmettent l'impression, et l'on distingue leurs deux modes d'action en disant qu'ils agissent, à la fois ou séparément, comme nerfs de la sensibilité générale et comme nerfs de la sensibilité spéciale du tact.

Plus élevé que le sens du toucher (si imparfait chez les animaux qui n'ont pas une véritable main) et ayant sur lui l'incomparable avantage d'agir à distance, le sens de l'odorat fournit des notions beaucoup plus nombreuses, beaucoup plus variées, qui jouent un rôle prépondérant dans la vie de l'animal osmatique. Il est pour lui la source des connaissances pratiques les plus utiles; et il est en outre la source de sensations agréables qui peuvent aller jusqu'à la volupté, ou de sensations désagréables qui peuvent aller jusqu'à la douleur. Nous ne saurions mesurer par nous-mêmes toute l'intensité de ces sensations, car nous ne possédons qu'un appareil olfactif rudimentaire; elles sont cependant encore très vives chez nous. On sait, en effet, que c'est par l'odorat, bien plus que par le goût, que nous apprécions les qualités de nos aliments, depuis celles qui plongent les gourmands dans une véritable jouissance jusqu'à celles qui provoquent la répugnance, la nausée et même le vomissement. Bien plus encore que nous, l'animal osmatique se trouve naturellement attiré vers l'odeur agréable et repoussé par l'odeur désagréable; mais cette attraction et cette répulsion qu'il éprouve sont indépendantes de l'utilité qu'il peut retirer du sens de l'odorat considéré comme moyen de recherche; elles paraissent même de nature à le troubler dans l'étude, la comparaison et la détermination des odeurs. Il semble donc qu'il y aurait un certain avantage à ce que ces deux modes de la sensation olfactive ne fussent pas confondus dans un même centre, à ce que chaque odeur fût transmise par deux racines distinctes à deux centres olfactifs différents, ou si l'on veut à deux parties différentes du centre sensoriel, et j'ai quelque tendance à supposer que telle est la raison d'être de la séparation et de la divergence des deux racines blanches du lobe olfactif.

Cette hypothèse est tellement dénuée de preuves que j'hésite 
à y insister. Si toutefois des études ultérieures lui donnaient quelque consistance, il y aurait lieu de se demander quelle est la répartition des deux modes de la sensation olfactive entre les deux racines blanches olfactives. On pourrait remarquer alors que la racine externe est toujours beaucoup plus volumineuse que l'interne, et il paraîtrait probable que c'est elle qui "est affectée à la partie la plus importante ou plutôt à la partie la plus complexe de la sensation olfactive. Ce serait le lobe de l'hippocampe qui serait chargé de distinguer les odeurs les unes des autres, et le lobe du corps calleux les apprécierait seulement au point de vue du plaisir ou de la peine qui en résulte pour l'animal. D'après cette conjecture, la délicatesse, la perfection du sens de l'odorat dépendraient du lobe de l'hippocampe, et l'on expliquerait peut-être ainsi, par une influence fonctionnelle moins vague que l'idée générale de l'évolution, les différences que présẹntent chez l'homme les connexions du lobule de l'hippocampe.

J'ai déjà exposé plus haut (p. 423 et suiv.) les modifications que subit chez les anosmatiques le centre olfactif postérieur ou de l'hippocampe. Par suite de l'atrophie du lobe olfactif, le lobe de l'hippocampe, très atrophié aussi, perd son indépendance et ne forme plus qu'une circonvolution (la dernière) du lobe temporal, dans lequel il se fusionne. En outre, le centre olfactif n'occupe plus toute l'étendue de cette circonvolution, mais seulement sa partie antérieure, où il se dessine sous la forme d'un renflement appelé le lobule de l'hippocampe. Encore assez volumineux chez les amphibies, le lobule de l'hippocampe est relativement beaucoup plus petit chez les primates, et il fait entièrement défaut chez les cétacés, qui n'ont pas d'odorat. Dans ce dernier cas, la circonvolution de l'hippocampe se termine antérieurement en une extrémité très rétrécie qui, parvenue à la pointe du lobe temporal, se porte en dehors et se continue sans interruption ni démarcation avec les circonvolutions qui forment le bord postérieur de la vallée de Sylvius. La partie correspondante de la scissure limbique se trouve entièrement effacée par suite de cette fusion, et la pointe du lobe temporal reste indivise.

Mais chez les amphibies et chez les singes, la présence du lobule de l'hippocampe prouve que la partie antérieure de la 
circonvolution de l'hippocampe n'a pas perdu sa fonction olfactive; elle est loin sans doute d'avoir l'importance qu'elle avait chez les osmatiques; toutefois, elle conserve encore en partie son indépendance, et, pendant que des plis de fusion effacent plus ou moins la partie postérieure de la scissure limbique, sa partie antérieure, celle qui correspond au lobule, c'est-à-dire au centre olfactif, persiste sous la forme d'un sillon, le sillon limbique, qui, partant de la vallée de Sylvius, entaille plus ou moins profondément, d'avant en arrière, la pointe du lobe temporal. (Voy., p. 420 et suivantes, les figures 5็, 5̌7, $l$, et fig. $58, \mathrm{~L}$.)

Or, chez l'homme ou du moins chez l'homme de race blanche, le sillon limbique s'efface le plus souvent. Le lobule de l'hippocampe existe toujours: sa fonction n'est pas supprimée, mais elle est devenue moins importante (car l'homme trouve dans ses autres sens et dans son intelligence des ressources qui diminuent notablement l'utilité de l'odorat), et le lobule de l'hippocampe se fusionne, sur la pointe du lobe temporal, avec la circonvolution adjacente. Mais sa fusion toutefois n'est pas tout à fait aussi complète que chez les cétacés; à défaut du sillon limbique et à sa place une légère dépression longitudinale, plus large que profonde, et sur laquelle la pie-mère s'applique sans former un repli, indique encore la limite externe du lobule de l'hippocampe. C'est à ce vestige que se réduit, chez la plupart des hommes de race blanche, le sillon limbique des singes et des amphibies. (Voy. plus haut, p. 422, fig. 56, l, et p. 432, fig. 59, l.)

J'ai donc pu croire, il y a quelques années, que l'absence du sillon limbique établissait un caractère distinctif entre le cerveau humain et les cerveaux des singes. L'ayant retrouvé depuis lors sur tous les cerveaux des nègres (au nombre de quatorze) que j'ai examinés - l'ayant retrouvé en outre sur les cerveaux d'un Annamite, d'un Chinois, d'un noir de l'Hindoustan et d'un indigène du Pérou - ne l'ayant vu manquer en un mot sur aucun des cerveaux non caucasiques que j'ai pu étudier (sans préjudice d'exceptions probables, mais que je n'ai pas encore rencontrées), j'ai été conduit à considérer la présence de ce sillon chez l'homme comme un caractère d'infériorité, comme l'indice 
d'une évolution incomplète arrêtée au degré simien. Mais en poursuivant mes recherches, j'ai reconnu que le sillon limbique existe aussi, par exception, chez quelques blancs, un peu plus souvent peut-être sur les cerveaux de qualité inférieure que sur les cerveaux riches en circonvolutions, mais quelquefois aussi (bien rarement, il est vrai), sur ces derniers. Il manque d'ailleurs chez la majorité des idiots et chez presque tous les microcéphales, même chez ceux qui présentent de nombreux caractères simiens. Cela ne prouve rien contre l'idée de l'évolution; un cerveau très développé et très perfectionné dans son ensemble peut conserver néanmoins en une de ses parties un caractère d'infériorité; et de même un cerveau très inférieur dans son ensemble peut offrir quelque part un caractère de supériorité. Il est digne de remarque toutefois que l'évolution du sillon limbique est l'inverse de celle des autres sillons. Ceux-ci se développent et se multiplient d'autant plus que le cerveau se perfectionne davantage ; tandis que dans les mêmes conditions le sillon limbique tend au contraire à s'effacer; cela permet de croire que la présence ou l'absence de ce sillon, ou, en d'autres termes, le degré de développement ou d'atrophie du lobule de l'hippocampe n'est pas en rapport avec la dynamique générale du cerveau, mais avec une cause fonctionnelle spéciale et locale.

Cette cause physiologique, ainsi que je crois l'avoir établi ailleurs (1), e'est la diminution de l'importance de la fonction olfactive. J'ai montré comment chez les primates le grand développement du lobe frontal, où la vie intellectuelle se centralise, amène la décadence de l'appareil olfactif. Le sens de l'odorat a perdu sa prépondérance et son autonomie; il n'est plus la source principale des connaissances pratiques de l'animal. Ce n'est plus qu'un sens ordinaire; les renseignements qu'il fournit sont même beaucoup moins utiles que ceux que l'intelligence agrandie reçoit de la vue, de l'ouïe et enfin du toucher, immensément perfectionné grâce à la constitution de la main.

Déchu du rôle prédominant qu'il jouait chez les animaux osmatiques, il n'en continue pas moins à recueillir les impres-

(1) Revue d'anthropologie, 1878, p. 393, 397 et 496 et dans ce volume, p. 268, 272 et 380. 
sions olfactives, et à les transmettre aux centres olfactifs par les deux racines blanches, dont le volume est singulièrement réduit, mais dont flles connexions et les fonctions restent les mêmes.

Si maintenant nous nous plaçons dans l'hypothèse exposée plus haut; si nous supposons que les impressions olfactives soient perçues par le lobe du corps calleux au point de vue du plaisir ou de la peine qu'elles provoquent, et par le lobe de l'hippocampe au point de vue de l'étude comparative des odeurs, nous serons conduits à nous demander quelle est chez l'homme l'importance relative de ces deux parties de la sensation olfactive, et nous reconnaîtrons qu'il y a sous ce rapport de très grandes différences entre l'homme de la nature et l'homme de la civilisation. Le civilisé, par suite de son existence raffinée, savoure mieux les odeurs agréables et souffre davantage des mauvaises odeurs; mais la délicatesse de son odorat ne lui est, pour ainsi dire, d'aucune utilité dans la vie, et s'il y a tel cas particulier où il peut tirer quelque bénéfice de. la perfection de ce sens, d'une manière très générale il n'a pas intérêt à le cultiver. Il en est autrement de l'homme aux prises avec la nature : à la chasse, à la guerre, l'odorat lui rend des services continuels. Celui qui a le meilleur odorat possède un avantage réel dans la lutte pour l'existence; il peut suivre la proie ou l'ennemi à la piste sans le secours de ses chiens; on sait que certaines tribus chasseresses ont des chiens domestiques pour divers usages, mais non pour la chasse.

L'utilité du sens de l'odorat considéré comme source de notions pratiques est done au maximum dans la vie de nature; elle s'attéuue dans les sociétés moins rudimentaires, pour tomber au minimum chez les nations civilisées, et si, parmi les deux centres olfactifs sensoriels, il y en avait un qui fût influencé parallèlement par les mêmes conditions, on pourrait admettre avec quelque probabilité que c'est celui-là qui est le siège de l'analyse olfactive. Or, ce que j'ai dit plus haut sur l'état com-paratif du sillon limbique et du lobule de l'hippocampe chez les Européens et chez les peuples moins civilisés, permet de supposer que les odeurs sont étudiées et analysées par ce lobule. L'absence presque constante du sillon limbique chez les blancs 
s'expliquerait ainsi par l'influence de l'état social, de l'éducation, de la désuétude, et non par l'influence typique de la race; on ne pourrait plus la considérer comme un caractère de perfectionnement, puisqu'elle serait au contraire l'indice de l'affaiblissement d'un de nos sens, affaiblissement qui n'est suivi d'aucun avantage et qui, par conséquent, ne nous donne aucune supériorité.

L'hypothèse que nous venons d'exposer n'en reste pas moins très conjecturale; mais elle n'est que secondaire, et on peut l'écarter sans porter atteinte à nos autres conclusions. Quelle que soit la répartition de la fonction olfactive entre les deux centres olfactifs sensoriels, l'existence de ces deux centres associés, mais distincts, et affectés l'un et l'autre à la sensation olfactive, est un fait établi par l'anatomie comparée; et un autre fait non moins solidement établi, c'est l'existence d'un troisième centre olfactif antérieur ou orbitaire qui, comme les deux précédents, se retrouve chez tous les mammifères pourvus à un degré quelconque de la fonction olfactive.

Si l'on ne considérait que l'homme, on pourrait s'étonner à bon droit de la complexité tout à fait extraordinaire et de la nature tout à fait exceptionnelle de son appareil olfactif. On se demanderait par quelle fantaisie de la nature le moins utile de nos sens est précisément celui qui possède les ressources anatomiques les plus nombreuses, les plus spéciales et les plus variées; pourquoi les filaments nerveux de sa membrane sensitive, au lieu de se réunir en un seul tronc, comme ceux de la vue et de l'ouïe, et de pénétrer dans le crâne par un seul trou, restent distincts et pénètrent isolément par un grand nombre de trous, à travers un crible très fragile; pourquoi la solidité du crâne se trouve ainsi sacrifiée (les chirurgiens ne le savent que trop) et sacrifiée sans compensation; pourquoi ces filaments olfactifs, au lieu de se rendre directement à leur destination, se jettent et se fusionnent dans un amas de substance grise, le renflement olfactif, organe tout spécial, sorte d'avant-cerveau qui semble affecté à une première élaboration, et dont les autres sens, bien que plus importants, sont privés. Ce n'est pas moi qui pose ces questions; elles ont depuis longtemps embarrassé les anatomistes; pour y échapper, ils ont essayé de faire du 
renflement olfactif un ganglion comparable à ceux des nerfs sensitifs ordinaires, et du pédoncule olfactif un simple nerf intracrânien : mais en vain; car il a bien fallu reconnaître que ce prétendu nerf, qui n'est pas divergent comme les autres, qui n'est pas rond, qui n'a pas de névrilème, qui est creux chez le $\mathbf{f}_{œ \mathrm{c}}$ 'us, est un organe sans analogue, et que ce prétendu ganglion, qui ne communique pas avec les autres, qui ne reçoit ni n'émet aucune branche anastomotique, et qui est tapissé par la piemère et l'arachnoïde, n'a absolument rien de commun avec les ganglions de nerfs.

Mais ce n'est pas tout; et, après avoir constaté ces dispositions étranges, compliquées, inexplicables, réalisées au bénéfice d'un sens qui n'est pour nous qu'accessoire, on s'étonnerait bien plus encore de l'importance sans égale qui lui est donnée par ses relations avec l'hémisphère cérébral. Les autres appareils sensoriels ne communiquent qu'indirectement avec le sensorium commun, qui est l'écorce cérébrale; leurs nerfs viennent s'insérer sur le bulbe, sur la protubérance, ou sur la partie postérieure de la couche optique, derrière les pédoncules cérébraux; ce n'est qu'après un trajet long et détourné, dans la profondeur des parties, que les impressions qu'ils apportent parviennent au sensorium. Seul, l'appareil olfactif échappe à ce détour et se rend directement à l'écorce cérébrale; seul encore, il a le privilège d'y aboutir par trois racines, d'y établir ses connexions sur trois centres distincts, situés tous les trois en avant des pédoncules; et seul enfin il a l'honneur de prendre une insertion directe sur le noble lobe frontal.

Si un anatomiste, descendu de quelque autre monde, comme le héros de Micromégas, commençait ici-bas ses études par l'homme, à voir l'appareil olfactif entouré de tant de faveurs, à le comparer avec la constitution bien plus modeste des autres appareils sensoriels, il n'hésiterait pas à dire que ce sens privilégié doit être le plus précieux de tous, le plus immédiatement utile, le plus intimement associé à la vie intellectuelle; et lorsqu'il apprendrait qu'au contraire l'odorat ne joue dans l'existence de l'homme qu'un rôle tout à fait secondaire, n'ajoutant presque rien à ses connaissances, ne prenant qu'une faible part à ses plaisirs, lui procurant même peut-être plus de désagrément 
que de jouissance, et lui rendant si peu de services dans la vie civilisée que sa perte n'est pas même considérée comme une -infirmité, alors notre anatomiste exotique serait tenté d'attribuer cette antinomie à une étourderie de la nature, qui se serait tracé un but sans pouvoir l'atteindre, comme ces souverains maladroits qui élèvent au premier rang un homme médiocre, le comblent de titres et de distinctions extérieures et s'évertuent à lui donner les apparences d'un personnage important, sans pouvoir réussir à faire de cet ambitieux autre chose qu'un sot.

Mais en poursuivant ses recherches, et les étendant à toute la série des mammifères, l'anatomiste dont nous parlons s'élèverait à des conceptions plus hautes, et ses critiques mesquines feraient place à l'admiration. Il verrait apparaître devant lui, dans leur simplicité majestueuse les lois de l'évolution organique. Il trouverait chez les osmatiques, qui représentent le type commun des mammifères, la raison d'être des caractères de supériorité de l'appareil olfactif, de sa structure exceptionnelle, de ses connexions multiples, et il comprendrait comment ces caractères, une fois établis, se sont maintenus chez les anosmatiques dans les limites compatibles avec le mode d'existence et les besoins de ces animaux; car si les parties devenues tout à fait inutiles peuvent finir par disparaître entièrement, jamais elles ne se dénaturent au point de changer leurs connexions, suivant le principe d'Etienne Geoffroy-Saint-Hilaire qu'un organe est plutôt anéanti que transposé.

En perdant son rôle de sens autonome, en cessant d'être un centre d'action, le lobe olfactif des osmatiques subit une modification profonde; ses cellules motrices, sa racine motrice, n'ont aucune raison d'être; elles seraient même nuisibles, puisque leur action troublerait, sans compensation, l'unité de la vie cérébrale; elles disparaissent donc. Mais les racines interne et externe, qui sont les racines sensitives proprement dites, continuent à fonctionner utilement, quoique leur importance soit devenue beaucoup plus faible; elles persistent donc sous un volume amoindri, en conservant d'ailleurs leurs connexions directes avec leurs centres respectifs, dont l'étendue est réduite, mais dont le siège reste le même. L'insertion directe de l'appareil olfactif sur l'écorce cérébrale a cessé, il est vrai, chez l'anos- 
matique d'être motivée par un besoin physiologique, mais elle ne saurait lui être désavantageuse, et il n'y a dès lors aucune raison pour qu'elle se modifie. Quant à la racine supérieure, l'anosmatique pourrait sans doute s'en passer, puisque les conditions de son existence n'exigent plus que le lobe frontal prenne directement et immédiatement connaissance des impressions olfactives; mais cette communication existe, elle ne lui est pas nuisible, il peut même l'utiliser comme un objet de luxe, et cela suffit pour qu'elle ne s'atrophie pas entièrement.

$\S$ §๊. - conclusions relatives auX centres olfactifs de l'homin.

C'est l'homme qui est le but de nos études; les faits d'anatomie comparée que nous venons d'exposer n'auraient peut-être pas mérité d'aussi longs développements s'ils ne convergeaient vers ce but. Il ne sera donc pas inutile de résumer en terminant, sous forme de conclusions, les notions que l'anatomie comparée nous permet d'ajouter à l'anatomie et à la physiologie du cerveau de l'homme.

$1^{\circ}$ La racine olfactive externe traverse la vallée de Sylvius et va se rendre dans la partie supérieure et externe du lobule de l'hippocampe. Elle prend son origine réelle dans l'écorce de ce lobule.

$2^{\circ}$ La racine olfactive interne se rend au carrefour de l'hémisphère et va se perdre dans l'origine du lobe (circonvolution) du corps calleux.

$3^{\circ} \mathrm{La}$ racine olfactive supérieure, connue sous le nom de $\mathrm{ra}$ cine grise, doit ce nom à la mince couche de substance grise qui tapisse sa face inférieure et qui se continue avec la couche grise de l'espace perforé, mais elle est constituée, comme les deux autres racines, par des fibres blanches qui après un trajet extrêmement court, vont se rendre sur le bord postérieur des deux premières circonvolutions orbitaires.

$4^{\circ}$ Il existe dans le manteau de l'hémisphère trois centres olfactifs distincts, correspondant respectivement aux trois racines olfactives. 
$5^{\circ}$ Le centre olfactif antérieur comprend la partie postérieure des deux premières circonvolutions orbitaires. Il commence, en arrière, sur le bord antérieur de l'espace perforé, et s'étend, d'arrière en avant, sur la deuxième circonvolution orbitaire jusqu'au niveau de l'incisure en $\mathrm{H}$. Sur la première circonvolution orbitaire sa limite antérieure n'est pas indiquée anatomiquement; mais il est probable qu'elle correspond au même niveau. D'après la position moyenne de l'incisure en $\mathrm{H}$ ou de la dépression qui la représente, on peut dire que le centre olfactif orbitaire comprend, en moyenne, le tiers postérieur des deux premières circonvolutions orbitaires.

$6^{\circ}$ Le centre olfactif postérieur occupe le lobule de l'hippocampe, qui forme environ le tier's antérieur de la circonvolution de l'hippocampe; une ligne transversale menée par l'extrémité du crochet de l'hippocampe indique assez bien la limite de ce centre olfactif.

$7^{\circ}$ Le centre olfactif supérieur est situé sur la face interne de l'hémisphère. Il comprend le carrefour de l'hémisphère et la première portion du lobe du corps calleux (ou circonvolution du corps calleux), depuis son origine au carrefour jusqu'au niveau du pli de passage fronto-limbique, ou de l'incisure fronto-limbique, qui indique, au-devant du genou du corps calleux, la position de ce pli de passage lorsqu'il est profond.

P.S. - Au moment de donner le bon à tirer de cette feuille, je reçois de M. le docteur Fallot, de Marseille, le cerveau d'une négresse de douze ans, sur lequel le sillon limbique est effacé comme chez les blancs (voir p. 454). 


\section{ANATOMIE DU LOBE OLFACTIF}

(Bulletins de la Société d'anatomie, 40 série, t. IV, 1879, p. 596-598)

M. Broca fait une communication sur l'anatomie du lobe olfactif, considéré au point de vue de l'anatomie comparée.

Les différences que présente l'appareil olfactif sont tellement grandes qu'elles justifient la division des mammifères en deux groupes, ou plutôt en deux catégories que M. Broca a distinguées sous les noms de mammifères osmatiques et anosmatiques. Chez les osmatiques, le sens de l'olfaction joue un rôle prépondérant. L'homme est anosmatique, par exception, car la règle est que les mammifères soient osmåtiques.

Le lobe olfactif des mammifères est l'un des trois lobes qui composent le grand lobe limbique; celui-ci constitue, dans l'hémisphère cérébral, une partie entièrement distincte du reste du manteau. Il est constitué par trois lobes, qui sont : $1^{\circ}$ le lobe du corps calleux; $2^{\circ}$ le lobe de l'hippocampe, et $3^{\circ}$ le lobe olfactif.

Ce lobe olfactif est rattaché au cerveau par un pédoncule qui fournit à sa base quatre racines; les deux principales sont blanches et se séparent en divergeant : l'interne se porte sur la face interne de l'hémisphère et se rend sur l'origine du lobe du corps calleux; l'externe, qui se porte en arrière sur la face inférieure de l'hémisphère et va se jeter sur le bord externe du lobule de l'hippocampe. La troisième racine, racine grise ou moyenne, occupe l'espace quadrilatère ou espace perforé et consiste en une substance grise assez épaisse, qui s'insère sur l'angle de séparation des deux racines blanches, sur leurs bords, se continue en arrière avec l'écorce grise du lobule de l'hippocampe, et en dedans passe sur la face interne de l'hémisphère pour tapisser' une petite surface désignée par M. Broca sous le nom de carre- 
four. Mais cette racine grise contient au-dessous de son écorce grise une couche de fibres blanches longitudinales qui, passant sous la bandelette optique comme sous un pont, vont se continuer avec les fibres les plus inférieures du pédoncule cérébral. Le lobe olfactif, chez les osmatiques, se trouve donc mis en communication directe avec les fibres motrices de la moelle. Chez l'homme, cette racine moyenne existe.

La quatrième racine olfactive a été désignée par M. Broca sous le nom de racine supérieure ou frontale. Lorsqu'on enlève la mince couche de substance grise qui recouvre à la partie supérieure l'implantation du pédoncule olfactif sur le lobe frontal, on aperçoit une couche de substance blanche qui se continue en haut avec la substance blanche de la circonvolution frontale orbitaire.

D'ailleurs, à l'état embryonnaire, le lobe olfactif est creusé d'une cavité qui se continue avec l'extrémité antérieure du ventricule latéral. Chez quelques animaux, le cheval en particulier, la cavité persiste; on voit alors, facilement, qu'il existe deux feuillets au pédoncule olfactif, l'un supérieur, l'autre inférieur, et chacun d'eux est composé d'une couche blanche recouverte d'une couche corticale grise. On voit de la sorte que le feuillet supérieur se continue, par sa substance blanche, arec la circonvolution orbitaire du lobe frontal.

La surface de l'espace quadrilatère occupée par la couche grise de la racine moyenne est souvent coupée par un amas de substance blanche, que M. Broca appelle la bandelette diagonale de l'espace quadrilatère. Elle part de l'angle externe de l'espace et vient se perdre à la face interne de l'hémisphère, probablement dans le bec et dans le lobe du corps calleux.

Chez les animaux anosmatiques, les cellules motrices décrites dans la substance grise du lobe olfactif disparaissent. La racine moyenne disparaît également. Il en résulte que cette région s'affaisse, s'atrophie; elle devient une vallée profonde. Mais la racine externe s'atrophie de même. Il en résulte une scissure constante, qui est la scissure ou mieux la vallée de Sylvius. La mise en communication de la vallée avec la scissure est le résultat de la disparition de l'appareil olfactif. L'anatomie comparée le montre clairement. Ghez les primates qui ont le sens du tou- 
cher à leur service, chez les amphibies où l'appareil olfactif n'est plus qu'appareil deficiens, la vallée se creuse.

D'après les considérations anatomiques qui précèdent, il est évident que le lobe olfactif affecte des connexions avec trois centres : $1^{\circ}$ avec le lobe de l'hippocampe, qui lui sert peut-être à étudier, à reconnaître les odeurs; $2^{\circ}$ avec le lobe du corps calleux, qui lui servirait à reconnaître les sensations désagréables; enfin, $3^{\circ}$ avec le lobe frontal. 


\section{CERVEAU D'UN NÈGRE}

(Bulletins de la Société d'anthropologie, 1859, t. I, p. 53-55.)

Avant de donner lecture d'un travail manuscrit, qui a été adressé à la Société par M. Gubler, professeur agrégé à la Faculté de médecine, M. Broca donne quelques indications sur les circonstances qui ont provoqué la rédaction de ce travail. Un nègre étant mort à l'hôpital de la Pitié, et ayant été transporté à l'amphithéâtre de Clamart, M. Broca demanda à M. Bastien, prosecteur des hôpitaux, l'autorisation d'examiner le cerveau de ce cadavre. C'était pendant les grandes chaleurs du mois d'août, et le corps comınençait déjà à se putréfier lorsque l'autopsie fut faite. La pulpe cérébrale était trop molle pour se prêter à l'étude des circonvolutions; elle était même en quelques points tout à fait diffluente. M. Broca dut donc se borner à examiner la couleur des substances du cerveau, et, pour rendre celte étude plus facile, il ouvrit simultanément le crâne d'un individu de race blanche, qui était arrivé à l'amphithéâtre le même jour que le cadavre du nègre.

La pie-mère du nègre présentait une couleur brunâtre en certains points; rien de pareil n'existait chez le blanc. La substance blanche du cerveau du nègre présentait une teinte légèrement fumeuse; mais c'était surtout la substance grise qui se faisait remarquer par sa coloration brunâtre. Les deux cerveaux 
furent reçus dans deux vases de même capacité, renfermant la même quantité d'alcool. Au bout de trois jours, ils parurent assez fermes pour être examinés; la différence de coloration était alors aussi tranchée et même plus tranchée que le premier jour. N'ayant pu prendre le poids absolu du cerveau du nègre, qui, à l'état frais, n'aurait pu être dépouillé de ses membranes sans se dissocier entièrement, M. Broca voulut du moins apprécier approximativement le poids relatif des deux cerveaux, après trois jours de séjour dans l'alcool. Les membranes furent enlevées, et les deux organes, après avoir été égouttés sur un linge, pendant le même nombre de minutes, furent placés dans la balance. Celui du blanc pesait 1003 grammes, et celui du nègre pesait seulement $925^{\circ}, 5$. C'était une différence de 8,3 pour 100 . Un seul fait, qui n'a même pas donné le poids absolu des masses cérébrales, serait presque insignifiant, s'il ne s'accordait avec les données déjà acquises à la science. On sait que la mesure de la capacité du crâne, laite par M. Meigs, suivant le procédé de Morton, sur les crânes très nombreux de la collection mortonienne, a donné une moyenne de 93 pouces cubes et demi (mesure anglaise) pour les crânes des Européens et des AngloAméricains, et une moyenne de 82 un quart seulement pour les crânes de nègres; c'est une différence de 11 pouces cubes un quart, c'est-à-dire que, la capacité crânienne du nègre étant représentée par 100, celle des Européens serait représentée par 112. La différence de 8,3 pour 100 trouvée par M. Broca, dans un cas particulier, est donc inférieure à la moyenne de 12 pour 100.

M. Broca a conservé dans l'alcool la partie la moins altérée du cerveau du nègre, et l'a présentée aujourd'hui à la Société d'anthropologie; mais, craignant qu'à la longue le contact de l'alcool ne modifiât la coloration de ce cerveau (ce qui d'ailleurs n'est pas arrivé), il montra la pièce fraîche à la Société de biologie. M. Rayer avait déjà, il ỳ a une dizaine d'années, fait à la même Société une présentation analogue; et on sait que depuis Meckel, qui publia sur ce point, en 1753 , un travail dans les Mémoires de l'Académie des sciences de Prusse, un grand nombre d'auteurs ont constaté que le cerveau des nègres est notablement plus foncé que celuj des blancs. 
A l'occasion de la présentation faite par M. Broca à la Société de biologie, M. Gubler exposa sommairement le résultat de ses recherches sur la coloration des centres nerveux chez les individus appartenant aux races blanches, et, à la suite de cette communication, il rédigea, pour la Société d'anthropologie, un mémoire dont M. le secrétaire donne lecture.

\section{SUR LA COLORATION NOIRATRE DES CENTRES NERVEUX CHEZ LES INDIVIDUS}

DE RACE BLANCHE,

REMARQUABLES PAR L'ABONDANCE DU PIGMENT EXTÉRIEUR

PAR M. GUBLER.

Nous donnons ici une analyse de ce travail, qui sera publié dans les Mémoires de la Société. L'auteur ayant eu l'occasion, il y a quelques années, d'examiner le cerveau d'un nègre mort à la Charité dans le service de M. Rayer, et ayant constaté sur le cerveau la coloration brunâtre dont on vient de parler, a eu l'idée de chercher si des modifications analogues ne se présenteraient pas chez les individus bruns des races blanches. En procédant par voie de comparaison, et en plaçant sur la même table des cerveaux provenant de sujets blonds et des cerveaux provenant de sujets très bruns, il a pu constater que les substances cérébrales étaient plus pâles chez les premiers que chez les derniers. Il a répété cette observation assez souvent pour être convaincu que cette différence ne tient pas à des variétés individuelles, et qu'il y a vraiment une certaine connexité entre la couleur plus ou moins foncée des centres nerveux et l'abondance du pigment extérieur. Ce n'est pas seulement dans les substances cérébrales que se dépose chez les individus très. bruns la matière colorunte; des dépôts analogues se retrouvent aussi quelquefois sur la pie-mère qui entoure la protubérance, et $\mathrm{M}$. Gubler, en faisant cette remarque, a eu soin de ne pas s'en laisser imposer par les colorations pathologiques plus ou moins ardoisées qui se produisent quelquefois dans les membranes cérébrales. Il résulte de ces intéressantes recherches que le pigment ne se dépose pas seulement dans les parties exposées à la lumière.

M. BRown-SÉQUard rappelle à ce propos un travail publié dèrnièrement par M. Ehrmann dans les Annales de Virchow sur le pigment des membranes cérébrales chez les Nalais. Cet auteur a vu dans la pie-mère, non seulement du pigment grenu, mais encore des̃ cellules pigmentaires. Il n'a pas parlé de la couleur des substances cérébrales; cette couleur ne l'a pas frappé, probablement parce qu'il n'a pas eu soin de co: fronter les cerveaux de Javanais avec les cerveaux d'Européens. A la suite du travail de M. Ehrmann, M. Virchow a ajouté qu'on trouve souvent des colorations pigmentaires sur la pie-mère des blancs, et notamment autour de la moelle allongée ; mais il n’a pas cherché, comme M. Gubler, à rattacher cette particularité à l'état du pigment dans le reste du corps. 


\section{SUR LE GERVEAU DU GORILLE}

(Bulletins de la Sociclé d'anthropologie, 2e série, 1866, t. Ier, p. 648.)

M. de Quatrefages. J'appellerai l'attention de la Société sur un fait anatomique concernant le gorille et qui m'a été communiqué par l'amiral Fleuriot de l'Angle. Le gorille, dans sa jeunesse, serait doux, intelligent, contrairement à ce qu'll est d’ns l'àge adulte; celui qui a fait le sujet de l'observation, a présenté cette différence de caractère à ce point même qu'il est mort de chagrin en laissant le navire où il avait d'abord vécu. En rapprochant ce fait d'autres qu'a bien voulu me faire connaître M. Fleuriot de l'Augle, ces nuances de caractère semblent coïncider avec une dif férence d'état du cerveau. Ainsi, l'amiral a tué un gorille adulte dont la taille s'élevait à $1^{\mathrm{m}}, 63$ ou $\mathbf{1}^{\mathrm{m}}, 70$, et qui, d'après les renseignements qu'on a pu recueillir, aurait été àgé de dix ans : sọn cerveau pesait 400 grammes, tandis que chez un gorille mâle très adulte dont la taille s'élevait à $1 \mathrm{~m}, 90$, le cerveau ne pesait plus que 300 granmes; il est possible que ces chiffres n'expriment que des différences individuelles, mais cette diminution cérébrale correspond peut-être aussi au changernent d'instincts de l'animal; c'est là une question à approfondir.

M. Вrоса. A popos du fait que vient de signaler M. de Quatrefages, je rappellerai qu'il existe plusieurs espèces de gorilles, ce qui, je crois, explique la différence considérable des chiffres qui viennent de nous être communiqués avec ceux établis par M. Du Chaillu, qui portent le poids du cerveau du gorille jusqu'à 560 grammes.

\section{SUR LE CERVEAU DES PRIMATES}

Voir le mémoire sur l'Ordre des primates, Bulletins de la Société d'anthropologie, $1 \mathrm{e}^{\mathrm{r}}$ avril 1869 , $2^{\mathrm{e}}$ série, t. IV, p. 374 à 395 .

Ce travail a déjà été réimprimé dans les Mémoires d'anthropologie de Paul Broca, t. lII, p. 122 à 139. 


\title{
SUR LE TYPE CÉRÉBRAL DES PRIMATES
}

\author{
RÉPONSE AUX OBSERVATIONS
}

DE M. LE PROFESSEUR RICHARD OWEN

(Bulletins de la Socièté d'anthropologie, 1870, t. V, p. 592-603.)

Ceux d'entre vous qui m'ont fait l'honneur d'écouter, l'année dernière, mon discours sur l'Ordre des primates, ou qui ont bien voulu le lire depuis qu'il est publié, me rendront peut-être cette justice que je n'ai jamais eu l'intention de déprécier les travaux de M. le professeur Richard Owen. Obligé d'examiner la doctrine de ce savant anatomiste sur la sous-classe des archencéphales, et ne pouvant l'admettre, j'ai essayé de la réfuter, et je crois l'avoir fait avec toute la déférence due à son auteur. J'ai évité avec soin de faire la moindre allusion aux polémiques longues et acérées que cetle question a soulevées dans la GrandeBretagne; j'ai même commis volontairement envers M. Huxley (qu'il veuille bien me le pardonner) la petite injustice de ne pas prononcer son nom, de ne pas renvoyer à son ouvrage, dont la traduction, due à notre collègue M. Dally, est entre les mains de la plupart d'entre vous, et où les diverses phases du débat sont exposées avec quelque sévérité.

Aujourd'hui encore, quoique M. Owen me fournisse l'occasion de prendre part à mon tour à cette polémique, je m'abstiendrai de le faire, et je me bornerai à répondre à la mise en demeure qui m'est adressée par le directeur du British Museum.

" Cela n'empêche pas, dit-il, que M. Broca n'eût dû citer l'ouvrage et l'endroit où se trouve la déclaration qu'il m'attribue. Je lui impose l'onus probandi par une simple dénégation; je n'ai jamais fait de déclaration semblable. ')

Ce n'est pas une fois, mais plusieurs fois que M, le professeur 
Owen a formulé les traits caractéristiques du cerveau de l'homme. Il me suffira de citer un passage du mémoire où sa doctrine s'est produite, je pense, pour la première fois (1).

L'auteur, après avoir parlé de la sous-classe des gyrencéphales, ou mammifères à circonvulutions cérébrales, arrive à l'homme et s'exprime ainsi :

"In man, the brain presents an ascensive step in development higher and more strongly marked than that by which the precedent sub-class was distinguished from the one below it. Not only do the cerebral hemispheres overlap the olfactory lobes and cerebellum, but they extend in advance of the one and further back than the other. The posterior development is so marked, that anatomists have assigned to that part the character of a third lobe; it is peculiar to the genus homo, and equally peculiar is the posterior horn of the lateral ventricle and the " hippocampus minor", which characterize the hind lobe of each hemisphere. The superficial grey matter of the cerebrum, through the number and depth of the convolutions, attains its maximum of extent in man.

"Peculiar mental powers are associated with this highest form of brain, and their consequences wonderfully illustrate the value of the cerebral character, according to my estimate of which I am led to regard the genus homo as not merely a representative of a distinct order, but of a distinct sub-class of the mammalia, for which I propose the name of archencephala.»

Voici la traduction de ce passage :

"Chez l'homme, le cerveau présente dans son développement un degré d'ascension plus élevé et plus fortement marqué que celui par lequel j’ai distingué la sous-classe précédente de celle qui est au-dessous d'elle. Non seulement les hémisphères cérébraux recouvrent les lobes olfactifs et le cervelet, mais encore ils s'étendent en avant de ceux-là et en arrière de celui-ci. Le développement postérieur est si marqué, que les anatomistes ont assigné à cette partie de l'hémisphère le caractère d'un

(1) On the Characters, Principles of Division and Primary Groups of the Class mammalia, read feb. 17th and 21 st april 1857, dans Journal of Proceedings of the Linnæen Society of London, vol. II, Zoology, nu V, p. 19 (London, 1857, in-80, June 21st.). 
troisième lobe. Cela est propre au genre homo; la corne postérieure du ventricule latéral et l'hippocampus minor, qui caractérisent le lobe postérieur de chaque hémisphère, sont également propres à l'homme. La substance grise superficielle du cerveau, grâce au nombre et à la profondeur des circonvolutions, atteint chez l'homme son maximum de développement.

“ Des pouvoirs intellectuels particuliers sont associés à cette forme plus élevée du cerveau, et leurs conséquences confirment merveilleusement la valeur du caractère cérébral, d'après l'évaluation duquel je suis conduit à considérer le genre homo comme représentant non seulement un ordre distinct, mais encore une sous-classe distincte de mammifères, sous-classe pour laquelle je propose le nom d'archencéphales. ")

Je me suis efforcé de traduire le plus littéralement possible, au risque de sacrifier parfois la forme à l'exactitude.

J'avais ce passage sous les yeux lor'sque j'ai rédigé la partie de mon mémoire sur l'Ordre des primates qui concerne la doctrine de M. Owen sur la sous-classe des archencéphales. En l'analysant, j'ai trouvé que l'auteur invoquait cinq arguments à l'appui de la distinction des archencéphales : $1^{\circ}$ le lobe postérieur des hémisphères n'existe que chez l'homme; $2^{\circ}$ la corne postérieure du ventricule latéral n'existe que chez l'homme; $3^{\circ}$ le petit hippocampus n'existe que chez l'homme; $4^{\circ}$ la substance grise superficielle atteint chez l'homme son maximum de développement; $5^{\circ}$ enfin l'homme possède des pouvoirs intellectuels particuliers.

Mais, ce dernier trait distinctif de l'homme n'étant pas de l'ordre anatomique, l'auteur ne le range pas au nombre des caractères sur lesquels repose la distinction des archencéphales; il le mentionne seulement comme confirmant la valeur des caractères anatomiques qu'il vient d'invoquer.

Si maintenant on considère les faits anatomiques, qui sont au nombre de quatre, on trouve que le dernier, celui qui concerne le grand developpement de la substance grise corticale, ne constitue pas en faveur de l'homme ce qu'on appelle en zoologie un caractère distinctif; ce n'est qu'une différence de volume cormme on peut en observer, dans la plupart des organes, chez des animaux appartenant à un même groupe. Et, 
après cette seconde élimination, il m'a paru que la sous-classe des archencéphales proposée par l'auteur ne reposait plus que sur trois caractères : $1^{\circ}$ l'existence du lobe postérieur des hémisphères $; 2^{\circ}$ celle de la corne postérieure des ventricules $; 3^{\circ}$ celle du petit hippocampe.

Si je m'étais borné à cette énonciation sommaire, M. le professeur Owen n'aurait probablement élevé aucune réclamation. Et le fait est que, lorsque j'ai prononcé mon discours, dans la discussion sur les primates, m'adressant à des collègues qui connaissent parfaitement la structure du cerveau, je n'ai fait qu'énumérer, sans aroir besoin de les expliquer, les trois caractères distinctifs de la sous-classe des archencéphales. Mais, lorsque ensuite j'ai rédigé mon discours, j'ai cru devoir, pour les lecteurs ordinaires, donner la définition et la synonymie de la corne postérieure, du petit hippocampe et du lobe postérieur. Voilà pourquoi, au lieu de citer textuellement, comme je viens de le faire, le texte de M. Owen, j'ai formulé à ma manière les trois caractères que j'avais à examiner. Je ne reproduirai pas cet alinéa, déjà reproduit en tête de la Iettre de M. Owen. Mais je rappellerai que cet alinéa dans mon texte est sans guillemets; que l'alinéa suivant, où j'annonce que "l'homme seul, d'après le professeur Owen, posséderait ces trois caractères "), est également sans guillemets; que, par conséquent, je n'ai nullement cherché à faire passer ma rédaction pour une reproduction du texte de l'auteur.

Je n'ai donc pas «attribué » à M. Owen une "déclaration » inexacte. Je n'ai pas prétendu qu’il eût écrit textuellement la phrase suivante : "L'homme seul possède ces trois caractères, et se distingue par là de tout le reste de la série des mammifères, et spécialement des singes. " Cette phrase n'est pas de lui, elle est de moi; et il n'a pas tort de dire " qu'il n'a jamais fait une déclaration semblable ". Non, mais il a dit, en parlant du lobe postérieur: It is peculiar to the genus homo, and equally peculiar (sous-entendu to the genus homo) is the posterior horn of the lateral ventricle and the "hippocampus minor ", which characterize the hind lobe of each hemisphere. Ce qui veut dire que le lobe postérieur, la corne postérieure et le petit hippocampe sont exclusivement propres à l'homme. Je ne vois done 
pas en quoi M. Owen peut me reprocher d'avoir travesti sa pensée. Est-ce parce que j'ai ajouté que «l'homme se distinguerait par là de toute la série des mammifères, et spécialement des singes "? Mais, s'il ne s'en distinguait pas, les trois caractères en question ne seraient pas propres au genre homme, peculiar to the genus homo, et M. Owen, qui non seulement disting'ue l'homme, mais encore en fait une sous-classe spéciale, ne peut pas dire que j’aie dénaturé son opinion.

Il me fait remarquer, il est vrai, que, dans ses cours de 1842 devant le Collège des chirurgiens de Londres, il a admis l'existence de la cavité ancyroïde dans le cerveau des singes, et qu'il a accepté depuis lors l'opinion de MM. Schrœder van der Kolk et Vrolik sur l'existence d'un petit hippocampe dans la cavité ancyroïde du chimpanzé et de l'orang. Je diraii, sur le premier point, que, puisque, de l'aveu de M. Owen, les singes ont une cavité ancyroïde, ce caractère cesse d'être propre aux archencéphales; et, quand même la cavité ancyroïde des singes serait rudimentaire, ce ne serait qu'une différence de degré tout à fait insuffisante pour servir de base à l'institution d'une sous-classe. Mais est-il vrai que cette cavité soit rudimentaire chez les singes? Elle est sans doute, absolument parlant, plus petite dans leur cerveau que dans le cerveau humain; elle a cela de commun avec toutes les autres parties de l'hémisphère, parce que le plus grand cerveau de singe est beaucoup plus petit que le plus petit cerveau d'homme sain. Mais si l'on compare la cavité ancyroïde à la cavité ventriculaire totale, dont elle n'est qu'une partie, on trouve, au contraire, qu'elle est relativement plus grande chez les singes que chez l'homme. "Ce prolongement, dit Gratiolet, a une grandeur énorme chez les singes, eu égard à l'ensemble du ventricule latéral, dont l'arc est fort petit. Dans l'homme, la prédominance passe à celui-ci (1). " Et il ajoute en note :

" Ce prolongement occipital du ventricule est particulier aux singes et à l'homme, et, par conséquent, il caractérise fort bien le type de ces êtres. Toutefois il ne peut être considéré comme un signe d'élévation, car il est beaucoup plus grand, eu égard à la partie enroulée du ventricule, dans les singes, oì son déve-

(1) Anatomie compàrée du système nerveux, t. II, p. 7ว̆. Paris, 183̈7, in-8. 
loppement est énorme, que dans l'homme, où la partie enroulée l'emporte évidemment sur lui. »

Sur le second point, qui concerne l'existence du petit hippocampe chez l'orang et le chimpanzé, il paraît que M. Owen ne la nie plus; mais le passage de son mémoire de 1857, que j'ai reproduit textuellement plus haut, prouve qu'à cette époque il ne l'admettait, que chez l'homme. J'apprends avec plaisir, par sa lettre de ce jour, que depuis lors il a changé d'avis, et qu'il a reconnu l'exactitude de l'opinion de MMI. Schrœder van der Kolk et Vrolik; mais j'ignore encore, faute de renvoi, dans quel passage de ses écrits il a fait cette déclaration. C'est certainement depuis 1862, car à cette époque, dans leur Note sur l'encéphale de l'orang-outang, communiquée à l'Académie royale des sciences d'Amsterdam, les deux éminents anatomistes hollandais reproduisirent avec une médiocre satisfaction le passage suivant publié en 1861 par M. Owen : " Je n'ai aucun doute que mes confrères faillibles (MII. Schrœder et Vrolik) n'aient dit la vérité, telle qu'ils l'entendent, en affirmant que les singes d'un ordre supérieur ont un lobe postérieur à l'hémisphère de leur cerveau, une corne postérieure dans leur ventricule latéral, et dans celle-ci un pes hippocampi minor; mais, de mon côté, je crois aussi prononcer une vérité strictement scientifique, d'accord avec les définitions de ces parties, en affirmant qu'elles ne sont propres qu'à l'espèce humaine (1). »

G'est donc seulement depuis 1862 que M. Owen a pu reconnaître que les parties décrites par les deux savants hollandais, chez le chimpanzé et l'orang, sous le nom de lobe postérieur, de corne postérieure et de petit hippocampe, étaient viraiment dignes de ces noms. J'ignore dans quels termes il a pu le faire; il nous dit qu'il l'a fait, et cela me suffit; et ce qui me suffit surtout, c'est de savoir que le petit hippocampe des singes n'est plus mis en question. Quant au peu de volume de cet organe, j'en suis peu touché. La comparaison des mammifères ongulés et onguiculés, invoquée par H. Owen, ne me semble pas venir à l'appui de sa thèse, puisque le caractère du sabot n'a pas la prétention de servir à distinguer une sous-classe. Or les ongles

(1) Versl. en Meder . afd. Natuurk, deel XIII, bl. 3. Le mémoire étant écrit en français, j'ai emprunté aux auteurs la traduction des paroles de M. Owen. 
et les sabots remplissent des fonctions connues, importantes, en rapports directs avec le mode d'existence des animaux, tandis que le petit hippocampe est le plus humble de tous les plis de l'encéphale; bien hardi celui qui essayerait de lui attribuer une fonction, et, dans une classification basée sur les caractères encéphaliques, il n'a vraiment qu'un rôle bien modeste à jouer. Au surplus, le correctif que, par cette comparaison même, M. Owen apporte à sa concession réduit à l'épaisseur. d'un cheveu la différence qu'il expose entre son opinion actuelle et l'opinion que je lui ai attribuée d'après ses autres publications. J'ai dit qu'il faisait du petit hippocampe un caractère propre aux archencéphales, et c'est encore ce qu'il répète aujourd'hui.

Sa seconde réclamation peut parâtre plus fondée, puisque j'ai inscrit, à côté de son nom, une définition du lobe occipital où une conjonction disjonctive a pris la place d'une conjonction copulative. Je ne m'inspirerai pas, pour me défendre, du plaidoyer de Figaro, et je déclare tout d'abord qu'à l'avenir, lorsque j'aurai l'occasion de dire ce que M. Owen entend par " le lobe occipital du cerveau ", je ne dirai plus que c'est la partie de l'hémisphère qui recouvre ou déborde la partie postérieure du cervelet, mais que c'est celle qui recouvre et déborde la partie postérieure du cervelet. Je dois ajouter toutefois que, si j'ai choisi la conjonction ou, ce n'est pas " par inadvertance », comme il paraît le croire, et encore moins pour " tourner sa pensée à l'absurde », comme un lecteur malveillant pourrait le supposer.

G'est simplement parce que je voulais éviter d'entrer dans une controverse dont mon argumentation pouvait se passer, et qui n'aurait pu y être introduite sans détourner l'attention du lecteur sur un fait à mes yeux tout à fait secondaire. Cette controverse, $\mathrm{j}$ 'ai à peine besoin de la rappeler ici. M. Owen sait mieux que personne que des dénégations s'élevèrent de toutes parts lorsqu'il annonça que l'homme était le seul animal dont le cervelet fût débordé en arrière par le cerveau. Je ne dirai pas, comme M. Huxley, que "pas un seul anatomiste, grand ou petit, n'a donné son appui au professeur Owen (1) ", car j'ignore

(1) Voir Huxley, Evidence as to Man's Place in Nature. London, 1863, in-8, 
si, depuis le jour où M. Huxley s'exprimait ainsi, M. Owen n'a réussi à convaincre quelque anatomiste; mais ce qui est certain, c'est que je possède plusieurs cerveaux de singes (un chimpanzé, un gibbon, trois papions, un macaque rhésus; un mandrill, un cébus) où le cerveau s'étend notablement en arrière du cervelet, et que même, sur l'un de mes papions, la saillie postérieure de l'hémisphère est relativement beaucoup plus considérable qu'elle ne l'est habituellement chez l'homme. Je considère donc comme tout à fait illusoire le caractère de la saillie du cerveau en arrière du servelet, et je vais mêrne plus loin, car je nie que de l'étude d'un fait de cet ordre il puisse découler un caractère anatonique. Que signifie, en effet, la saillie plus ou moins forte des circonvolutions occipitales? Une seule chose : le degré de volume relatif de ces circonvolutions et du cervelet qu'elles recouvrent; mais ce qui conslitue un caractère réellement anatomique, ce n'est pas le volume absolu ou relatif d'un organe, c'est sa structure; et si les anatomistes ont décrit dans les hémisphères un lobe occipital, ce n'est pas parce qu'il affecte tel ou tel rapport avec le cervelet, mais parce qu'il est séparé du lobe pariétal par un sillon transrersal, parce qu'il reçoit un prolongement spécial du ventricule latéral, et parce qu'enfin on y trouve, chez plusieurs espèces, un petit hippocampe plus ou moins développé. Si le troisième lobe était caractérisé par la saillie qu’il fait en arrière du cervelet, il suffirait donc de quelques millimètres de plus ou de moins dans le sens de la longueur pour que le cerveau eût ou n'eût pas de troisième lobe; on prendrait l'encéphale, on le mettrait en position, on ferait passer par le bord postérieur du cervelet un plan vertical, et si ce plan laissait en arrière de lui une partie quelconque de l'hémisphère, grande ou petite, il y aurait un troisième lobe; sinon non. De sorte que, dans certaines espèces de singes dont le cerveau s'arrête tantôt sur la limite du cervelet, tantôt un peu en arrière, on trouverait des individus qui auraient un lobe occipital et d'autres qui n'en au-

p. 118. On trouvera dans cet ouvrage, de la page 113 à la page 118 , un chapitre intitulé : Histoire surcincte de la controverse relative à la structure cérébrale de l'homme et des singes. (Voir la traduction française de Dally. Paris, 1868, in- 8 , 1. 250-257. Les dessins de Schrœder van der Kolk et Vrolik et ceux de Gratiolet sont reproduits dans cette dernière édition, 
raient pas. Pour ma part, il m'importe peu que les circonvolutions occipitales ne fassent que recouvrir le cervelet, ou qu'elles le débordent; elles existent ou elles n'existent pas; ce qui les caractérise, ce sont leur structure et leurs connexions. Voilà ce que j'aurais objecté à $M$. Owen si j'avais cru nécessaire d'aborder, dans mon mémoire sur les primates, la question de la saillie du troisième lobe, et peut-être alors aurait-il pu croire que je cherchais à « tourner sa pensée à l'absurde ». Mais j'en avais tellement peu le désir, que j'ai voulu éviter de discuter ce point, et que j'ai glissé sur le caractère morphologique de la saillie du troisième lobe pour m'arrêter sur le principal caractère anatomique de ce lobe, savoir : la corne ventriculaire qui s'y prolonge. C'est pourquoi, lorsque je me suis demandé de quelle manière $\mathrm{M}$. Owen pouvait concevoir la distinction du lobe occipital, je suis arrivé à penser qu'un esprit aussi familiarisé que le sien avec les principes de l'anatomie comparée devait attacher moins d'importance à la position d'un organe qu'à ses connexions et à sa constitution anatomique; et comme il était évident pour moi que ce qu'il appelait le lobe occipital n'était pas la région séparée du lobe pariétal par la scissure perpendiculaire (car elle existe chez tous les primates qui ont le cerveau plissé), je me suis dit : Puisque le lobe occipital, dans la pensée de M. Owen, n'est pas caractérisé par ses connexions, il doit l'être par sa structure; et j'en ai conclu que, pour lui, la véritable caractéristique du lobe occipital devait être le prolongement ventriculaire appelé corne postérieure. C'est après avoir ainsi raisonné (à tort ou à raison) que j'ai passé en revue, dans les termes suivauts, les trois caractères du cerveau des archencéphales qui ont été énumérés plus haut :

"Je ferai d'abord une première remarque relativement au lobe occipital. Si l'on désigne sous ce nom la partie de l'hémisphère... qui est située en arrière de la scissure perpendiculaire, alors il faudra dire que tous les primates, sans exception, ont un lobe occipital. Ce n'est donc pas ainsi que M. le professeur Owen a voulu caractériser le lobe occipital. Pour lui, ce lobe est la purtie du cerveau qui recouvre ou déborde la partie postérieure du cervelet, et dans laquelle se prolonge la corne postérieure du ventricule. Si la corne manque, le lobe postérieur n'existe pas. 
Le troisième des caractères indiqués plus haut se confond donc déjà a vec le premier.

"Et j'en puis dire autant du second, car le petit hippocampe n'est pas dans le cerveau un organe spécial, ce n'est qu'une circonvolution retournée dans la cavité ancyroïde (suit la preuve de cette assertion).

" Le petit hippocampe n'est donc qu'une conséquence de l'existence de la cavité ancyroïde; et le second caractère, comme le troisième, se confond avec le premier; de sorte que, en réalité, la thèse du célèbre professeur de Londres se réduit à ces termes fort simples : Il y a dans le cerveau de l'homme une cavité ancyroïde qui ne se retrouve sur aucun autre animal. »

It résulte de ce passage que je n'ai pas eu l'intention de citer une définition du lobe occipital par Owen, et encore moins celle de faire croire au lecteur que la définition fût de lui. Il n'y a dans mon texte ni renvoi, ni guillemets, ni lettres italiques (car le passage que je viens de souligner n'est pas souligné dans mon mémoire), rien en un mot qui puisse indiquer que je cite l'auteur, tandis qu'au contraire la question qui précède et le raisonnement qui suit prouvent que je cherche quelle est sa pensée. Cette pensée, il paraît que j'ai eu le malheur de ne pas la découvrir; mais, si je n'ai pas su la dégager exactement, du moins je ne l'ai pas altérée par un changement de conjonction.

On comprendra maintenant pourquoi j'ai employé la conjonction ou. Je ne voulais pas discuter la question de la position du lobe occipital. Pour moi (et je ne citais pas, c'était moi qui parlais), pour moi, que le lobe occipital recouvre seulement la partie postérieure du cervelet, ou qu'il la déborde, cela est sans importance réelle. Tantôt il ne fait que la recouvrir, tantôt en outre il la déborde; mais, pourvu que le ventricule s'y prolonge en forme de corne, c'est toujours un lobe occipital. De sorte que, dans mon texte, les mots qui recouvre ou déborde le cervelet signifiaient que ce n'était pas là le point que je voulais discuter. Je ne voulais discuter que les points qui étaient absolument nécessaires à mon argumentation. Ce n'était pas sans un vif regret que je me voyais contraint de réfuter la doctrine d'un savant tel que M. Owen; je réduisais donc ma réfutation au strict nécessaire, et j'écartais une question qui m'eût mis une fois de 
plus en opposition avec lui. Si j'ai obéi à ce sentiment de déférence envers M. Owen, ce n'est pas seulement parce que j'ai eu l'honneur d'être reçu dans sa maison et d'être admis à sa table, mais parce que je respectais en lui un des plus illustres maîtres de la science; s'il en pouvait douter, je pourrais remettre sous ses yeux un passage qu'il a pu lire dans mon mémoire sur les primates, et que je me plais à reproduire ici comme conclusion de ma réponse : "Quelles que soient mon estime pour le caractère et mon admiration pour les travaux du grand anatomiste Richard 0 wen, je crois être autorisé à dire que les caractères anatomiques qu'il a invoqués à l'appui de la distinction de la sous-classe des archencéphales sont illusoires. ») (Bull. de la Soc. d'anthrop., 1869, p. 378, et dans Broca, Mémoires d'anthropologie, t. III, p. 125.). 


\title{
A PROPOS DU CERVEAU D'UNE IMBÉGILE
}

\author{
PRÉSENTÉ PAR M. S. POZZI
}

(Bulletins de la Société d'anthropologie, 1874, 2e série, t. IX, p. 793.)

M. Broca. En décrivant le cerveau qui est sous nos yeux, M. Pozzi a indiqué un certain nombre de caractères qu'il considère les uns comme des arrêts de développement, les autres comme des exemples de réversion; or, en fait de cerveau, arrêts de développement et réversion sont un. Si l'on étudie, en effet, le cerveau de l'homme et celui des anthropoïdes, l'on est forcé d'avouer qu'il n'y a aucun organe important appartenant au cerveau de l'homme qui ne se retrouve dans le cerveau des singes supérieurs, et réciproquement; il ne peut donc être question de cas de réversion appliquée à certains cerveaux humains, ceux-ci ne différant des cerveaux des anthropomorphes que par un plus grand développement.

Toutes les autres différences que l'on a cru exister entre les cerveaux de l'homme et des anthropoïdes n'existent réellement pas. Gratiolet, par exemple, pensait que le cerveau de l'homme était essentiellement caractérisé par la présence des premier et deuxième plis de passage, tandis que chez l'orang l'on ne retrouverait que le second pli, celui-ci manquant chez le chimpanzé; mais le premier pli de passage peut, chez l'homme, manquer assez souvent d'un côté et parfois même des deux côtés, de même que, mais cela est plus rare, les deux premiers plis peuvent faire défaut ou d'un côté ou des deux côtés ; cetle particularité se remarque surtout chez des individus imbéciles ou très peu intelligents. Le premier pli de passage existe ordinairement d'un côté chez le chimpanzé. 


\section{SUR}

\section{IAA TOPOGRAPHIE CRANIO-CÉRÉBRALE}

OU

LES RAPPORTS ANATOMIQUES DU GRANE ET DU CERVEAU

(Revue d'anthropologie, 1876, $2^{\mathrm{e}}$ série, t. V, p. 193-248 et 278.)

§ 1. HISTORIQUE DE LA QUESTION. - EXPOSÉ DES PROCÉDÉS.

L'étude de la topographie cranio-cérébrale intéresse à la fois les anthropologistes et les physiologistes, les médecins et les chirurgiens, et il est permis de s'étonner du peu d'attention qu'on lui a accordé jusqu'ici. Il semhle que c'est sur cette base que Gall el Spurzheim auraient dû faire reposer tout leur édifice, car ce n'est pas dans les bosses du crâne, c'est dans les organes cérébraux subjacents qu'ils se proposaient de localiser les facultés. On ne conçoit donc pas qu'ils ne se soient jamais demandé quelles étaient exaciement les parties du cerveau recouvertes respectivement par les nombreux districts de leurs cartes phrénologiques. Cette lacune fondamentale fut la cause principale de l'impuissance de l'école phrénologique, car un système phy:iolngique qui ne repose pas sur des déterminations anatomiques précises ne peut résister à la critique.

Aujourd'hui la question des localisations cérébrales, remise à l'ordre du jour par la découverte du siège de la faculté du langage, se développe sous une forme plus scientifique. Ce n'est plus sur le crâne, c'est sur le cerveau même que l'on cherche à établir les circonscriptions. Ces circonvolutions compliquées, qui firent si longtemps le désespoir des anatomistes, et que l'on 
croyait aussi désordonnées que celles de l'intestin grêle, on sait maintenant qu'elles sont soumises à une répartition régulière, qu'au point de vue de leur nombre, de leurs rapports, de leurs connexions, de leurs vaisseaux, elles présentent une fixité égale à celle des autres organes, supérieure même à celle de beaucoup d'organes, et qu'elles constituent plusieurs groupes, plusieurs lobes distincts, séparés par des scissures constantes. Nombreux sont les anatomistes qui, depuis Rolando, ont contribué à élucider cette question importante. Mais il en est un qui prime tous les autres : c'est Gratiolet. D'autres avant lui, Leuret surtout, dont il acheva l'ouvrage, avaient interrogé l'anatornie comparée; mais ce fut lui qui, le premier, s'attachant surtout à l'ordre des primates, suivant de bas en haut, dans toute la série des singes, l'évolution des plis cérébraux, et s'élevant de là jusqu'à l'homme, débrouilla définitivement le chaos des circonvolutions humaines. Les recherches ultérieures ont permis de compléter quelques-unes dé ses déterminations, mais ont confirmé de plus en plus la division générale qu'il a établie. Tout $1^{e}$ monde sait qu'il a ramené à cinq 'groupes, formant autant de lobes, toutes les circonvolutions de l'hémisphère, savoir : le lobe centra! ou l'insula de Reil, caché dans le fond de la scissure de Sylvius; le lobe temporo-sphénoüdal, situé au-dessous de cette scissure; puis, au-dessus d'elle, le lobe frontal et le lobe pariétal, séparés l'un de l'autre par la scissure de Rolando ; et enfin, en arrière, le lobe occipital, séparé du pariétal et du temporo-sphénoïdal, en dedans par la scissure occipitale interne, en dehors par la scissure occipitale externe (1).

(1) M. Bischoff dit, à la page 5 de son mémoire sur les circonvolutions (Die Grosshirnwinaunyen des Menschen, Munich, 1868, in-4"), que Gratiolet a admis dans chaque hémisphère les cinq lobes habituels (die gewœhnlichen fünf Lappen); et il ajoute, p. 18, que Gratiolet a conservé la division habituelle (die gewœhnliche Eintheilung beibehalten). Il est assez étrange de qualifier cette division d'habituelle, dans un chapitre historique d'où il résulte que si Arnold, avant Gratiolel, avait admis cinq lobes dans l'hémisphère, d'Arnold (1838) à Gratiolet (1830-1857) personne ne les avait acceptés. Quant aux cinq lobes d'Arnold, si l'on en excepte le lobe central de l'insula, ils diffèrent entièrement de ceux de Gratiolet; les seules lignes de démarcation qu'Arnold ait reconnues sur le cerveau sont la scissure de Sylvius et la scissure occipitale interne, dejà connues depuis longtemps; ses autres délimitations ne sont pas anatomiques, mais seulement topographiques; il ne connaît ni la scissure occipitale externe, ni la 
Les trois lobes frontal, pariétal et occipital, qui se succèdent d'avant en arrière sur la convexité de l'hémisphère, tirent respectivement leurs noms des trois os, des trois vertèbres crâniennes qui les recouvrent.

En adoptant ces dénominations déjà employées par Arnold dans une acception très différente, Gratiolet pensait qu'il y avait non seulement un rapport de superposition, mais encore une certaine solidarité entre les segments du crâne et les lobes du cerveau. Il supposa donc que le degré de développement relatif de ces lobes devait se refléter à l'extérieur, et qu'il suffisait, pour l'apprécier, de constater celui des trois vertèbres crâniennes correspondantes.

De là, la célèbre distinction craniologique qu'il établit entre les races frontales, pariétales et occipitales, caractérisées respectivement par la prédominance de la vertèbre dont elles portaient le nom.

Cela impliquait l'idée qu'il devait y avoir une correspondance sinon rigoureuse, du moins approximative, entre les sutures qui limitent les vertèbres du crâne et les scissures qui limitent les lobes du cerveau, c'est-à-dire, d'une part entre la suture coronale et la scissure de Rolando, d'autre part entre la suture lambdoïde et la scissure occipitale. Gratiolet fut donc conduit à étu-

scissure de Rolando; le lobe temporal, pour lui, n'est pas distinct du lobe occipital, dont il n'est qu'un prolongement; son lobe frontal n'est que la partie de l'hémisphère qui surplombe la voûte orbitaire, et ne form pas même la moitié du lobe frontal de Gratiolet. Au surplus, si l'on veut s'assurer qu'Arnold, en 1838, ne pouvait songer à établir des divisions méthodiques, jl suffira de lire dans son traité d'anatomie publié en 1851 (t. II, p. 730) le passage où il déclare qu'il ne connaît aucune règle, ni pour la direction des circonvolutions primaires ni pour leurs subdivisions. Les déterminations faites par Gratiolet étaient donc tout à fait originales. Personne avant lui n'avait fait reposer exclusivement la distinction des lobes des hémisphères sur l'étude des circonvolutions et des scissures cérébrales. M. Bischoff se demande par deux fois si (iratiolet a compris ou non dans le lobe frontal le "premier pli ascendant » qui forme le bord antérieur de la scissure de Rolando. Il est bien vrai que Gratiolet a émis à ce sujet deux opinions différentes. Ses premières observations, consignées en 1854 dans son célèbre mémoire Sur les plis cérébraux de l'homme et des primates, l'avaient conduit à penser que le premier pli ascendant se ruttachait au lobe pariétal; mais, en continuant ses recherches, il reconnut que le lobe frontal s'étend jus. qu'à la scissure de Rolando, et cette opinion définitive fut exposée sous la forme la plus claire et la plus didactique dans le deuxième volume de son Anatomie com. parée du système nerveux (t. II, p. 111 et suiv. Paris, 18ว̈7, in-8). 
dier les rapports de ces sutures et de ces scissures. Il eut recours pour cela à un procédé qu'il n'a pas publié lui-même, mais qu'il m'a communiqué verbalement en 1862 , dans une discussion particulière, et que j'ai fait connaître depuis lors dans mon mémoire sur la Déformation toulousaine du crâne (Bulletins de la Société d'anthropologie, 1871, p.104, et dans ce volume, p. 185). Il plaçait devant lui sur une table lecerveau déponillé de ses membranes, le moule intérieur du crâne, et enfin le crâne lui-même. Il marquait au crayon sur le moule la position de la suture coronale et de la suture lambdoïde, puis il y reportait proportionnellement les longueurs respectives du lobe frontal, du lobe pariétal, du lobe occipital, qu il mesurait sur le cerveau étalé devant lui. Il crut reconnaître ainsi que, chez l'homme et chez les singes, la scissure de Rolando correspondait à la suture coronale, et que par conségueut l'étude de l'écaille frontale faisait connaître exactement l'étendue des lobes frontaux des hémisphères. C'était bien là ce qu'il attendait; mais, en ce qui concerne la scissure occipitale, qui sépare le lobe occipital du lobe pariétal, son attente fut trompée; il trouva en effet, ou plutôt il crut trouver, qu'elle ne correspondait pas à la suture lambdoïde, et qu'elle était toujours située chez l'homme bien au-dessous de cette suture.

Ces résultats, consignés en 1857 dans le deuxième volume de l'Anatomie comparée du système nerveux de Leuret et Gratiolet, étaient admis sans contestation, lorsque je fis, en 1861, mes premières recherches sur la localisation du langage articulé (1). Ayant reconnu que la faculté du langage siège dans la partie postérieure de la troisième circonvolution frontale, au-dessus de la scissure de Sylvius, je dus naturellement chercher quel était le point de la paroi crânienne qui recouvrait cette partie toute spéciale du cerveau. Quelques coupes pratiquées sur des têtes fraîches suffirent pour me démontrer l'inexactitude des rapports indiqués par Gratiolet; je ne connaissais pas encore le procédé dont il s'était servi, mais il était évident pour moi que ce procédé devait être défectueux.

Le procédé des coupes que j'avais employé tout d'abord ne

(1) P. Broca, Sur le siège de la faculté du langage articu'é, dans Bulletins de la Société anatomique, 1861, $2^{\mathrm{e}}$ série, t. VI, p. 330-357. Voir la note de la page 340 , et dans ce volume, p. 4-32, note p. 1 \%. 
manquait pas d'une certaine précision; mais il ne pouvait donner sur chaque cerreau qu'une seule indication; il était d'ailleurs peu pratique ; j'eus donc recours à un procédé simple et facile que je décrirai plus loin dans tous ses détails et que je n'indique ici que sommairement. Il consiste à pratiquer, sur les divers points du crâne dont on veut déterminer les rapports, de petites perforations à travers lesquelles on introduit de petites fiches ou chevilles de bois. Celles-ci sont poussées dans le cerveau à l'aide d'un stylet qui les accompagne jusqu'au-delà de la dure-mère. On pratique alors la coupe des autopsies, on extrait le cerveau suivant le procédé ordinaire, et les fiches qu'on y aperçoit aisément montrent sur cet organe la situation exacte des divers points du crâne qui ont été perforés. En pratiquant deux perforations sur le trajet de chacune des principales sutures, on obtient des repères suffisants pour retrouver ce trajet à la surface du cerveau; on peut voir alors quels sont ceux des points du cerveau qui correspondent à chaque suture, et quant à ceux qui s'en éloignent plus ou moins, on en détermine aisément la position en mesurant au compas et exprimant en millimèires la distance qui les sépare de telle ou telle suture. Ces mensurations, pour être comparables entre elles, doivent toijours être faites parallèlement ou perpendiculairement à l'axe longitudinal des hémisphères cérébraux; on obtient ainsi, après un certain nombre d'observations, des chiffres qui se prêtent à la recherche du maximum, du minimum et de la moyenne de chaque distance topographique.

Pour étudier les rapports de la surface du cerveau, on se sert de chevilles de 2 à 3 centimètres de longueur; il suffit d'enployer des chevilles plus longues pour étudier de la même manière les rapports des parties profondes.

Ce procédé à la fois simple, sûr, rapide, facile, ne mutile ni le crâne ni le cerreau; il peut être appliqué dans toutes les autopsies; il se prête à des études partielles aussi bien qu'à des études d'ensemble; il permet d'étudier sur le même sujet les rapports de toutes les parties du crâne et de toutes les parties du cerveau, et de constater les différences topographiques qui peuvent exister entre le côté droit et le côté gauche. Il satisfait donc à tous les besoins. J'aurais pu me borner à en donner ici 
une description sommaire, comme je l'ai fait en 1861 dans mon mémoire Sur le siège de la faculté du langage, et en 1871 dans mon mémoire déjà cité sur la Déformation toulousaine du crâne; mais l'intérêt croissant qui s'attache aujourd'hui aux localisations cérébrales et à la détermination des rapports cérébrocrâniens, me fait croire qu'il ne sera pas inutile de préciser les détails opératoires qui permettent de faire aisément et rapidement des constatations exactes et de multiplier les observations. Je me réserve donc de revenir plus loin sur ce procédé, et de l'exposer avec tous les développements et toutes les applications qu'il comporte.

Lorsque je le communiquai à la Société anatomique, au mois d'août 1861 , je ne l'avais encore appliqué que sur onze sujets du sexe masculin, morts à l'hôpital de Bicêtre, et ayant presque tous dépassé l'âge adulte; mais l'inexactitude des deux résultats obtenus par Gratiolet ressortait déjà avec évidence de ces premières recherches. L'origine de la scissure de Rolando, loin de coïncider avec le bregma, comme le croyait Gratiolet, s'était toujours trouvée à 40 millimètres au moins en arrière de ce point; la distance s'était même élevée une fois à 63 millimètres, mais le sujet était un épileptique, dont le cerveau était d'ailleurs très grand, et dans les dix autres cas la distance avait été comprise entre 40 et 522 millimètres. (Depuis lors, j'ai vu ce maximum normal atteindre des chiffres beaucoup plus élevés.) Quant à la scissure occipitale, je l'avais vue au contraire coïncider parfaitement ou à quelques millimètres près avec la suture lambdoïde. La seconde assertion de Gratiolet n'était donc pas plus exacte que la première.

J'eus l'occasion, l'année suivante, de causer à ce sujet avec Gratiolet. Il manifesta quelque surprise; mais, comme il ne cherchait que la vérité, il me donna rendez-vous à l'amphithéâtre de Clamart pour une séance de vérification, et, après trois expériences successives, il se rendit à l'évidence de la démunstration. Ce fut dans cette séance qu'il me donna l'explication de son procédé, tel que je l'ai décrit plus haut.

Depuis lors, j'ai souvent appliqué le procédé des fiches, soit dans mon laboratoire, soit dans les salles d'autopsie, pour démontrer aux élèves la position des deux scissures transversales 
du cerveau et pour constater que la ligne sus-orbitaire établit la vraie limite entre la région cérébrale et la région faciale du crâne; je m'en suis servi pour quelques études comparatives faites sur des têtes de nègres ou sur des têtes déformées: j’y ai eu recours encore pour chercher à déterminer la situation de la partie de la troisième circonvolution frontale qui est le siège de la faculté dı langage; et enfin, dans ces derniers temps, j’ai préparé, pour le musée de l'Institut anthropologique, une assez grande série de crânes sur lesquels j'ai pu, grâce aux points de repère fournis par de nombreuses fiches, dessiner dans leur position exacte, et jusque dans leurs moindres détails, toutes les scissures et les sillons de la surface du cerveau, et peindre en couleurs différentes les circonvolutions de chaque lobe. Mais je n'ai jamais trouvé le temps de mettre à exécution le projet, que j'avais conçu dès l'origine, d'entreprendre une série méthodique et. complète de recherches sur l'ensemble de la topographie cérébrale; - et lorsque, en 1871, j'eus l'occasion d'étudier la tête d'une vieille femme qui avait subi dans son enfance la déformation toulousaine, je ne pus, faute de termes de comparaison suffisants, constater que d'une manière incomplète l'influence exercée par cette déformation sur les rapports cérébro-crâniens. Je pus reconnaître toutefois (1) que d'une part la scissure occipitale externe était située sous la suture lambdoïde, qu'elle n’était par conséquent pas déplacée, - mais que d'une autre part la scissure de Rolando, beaucoup plus oblique que de coutume, se trouvait reportée, à sa partie supérieure, à 57 millimètres en arrière de la suture coronale. Pour apprécier la signification de ce fait, j'éprouvai quelque embarras, car toutes mes observations antérieures avaient été recueillies sur des hommes. En laissant de côté les cas pathologiques, j’avais trouvé que la distance de la fiche bregmatique au sillon de Rolando, comprise entre le minimum de 40 millimètres et le maximum de 50 (2), était en moyenne de 47 millimètres, et comme il était probable

(1) Bull. de la Société d'anthrop., 1871, 2e série, t. VI, p. 100-120, et dans ce volume, p. 18 à 201.

(2) J'ai vu depuis lors, sur la tête d'un vieillard de soixante-cinq ans, dont le cerveau était très grand (1460 grammes), Ia distance rolando-bregmatique s'élever du côté droit à 64 millimètres, et du côté gauche à 70 millimètres. Elle a atteint 67 millimètres sur un crâne d'Arabe. 
que cette distance devait être un peu moindre chez les femmes, moindre surtout chez ma Toulousaine, dont l'encéphale ne pesait que 1094 grammes, je crus pouvoir en conclure qıe la déformation frontale avait produit sur le lobe frontal un allongement de 1 centimètre au moins. Deux figures jointes à mon mémoire, et que je reproduis ici (fig. 65,66 et 67 ), montraient la position des fiches coronales et lambdoïdiennes sur la face supérieure et la face latérale du cerveau de cette femme. J'ai cru devoir rappeler cette observation, parce que c'est jusqu'ici la seule qui concerne l'influence des déformations du crâne sur la topographie cérébrale. Mais je viens d'anticiper un peu sur les dates, car mon mémoire sur la Déformation toulousaine n'a paru qu'en 1871, trois ans après les recherches publiées par M. Bischoff dans. son important ouvrage sur les circonvolutions cérébrales de l'homme (1).

Le procédé de cet auteur ne différait pas du mien, si ce n'est qu'il s'était servi de fiches métalliques au lieu de fiches de bois. Il avait vu, comme moi, les fiches de la suture lambdoïde pénétrer assez exactement dans la scissure occipitale externe, qui limite le lobe occipital; mais la limite postéro-supérieure du lobe frontal, que j'avais placée à 4 centimètres au moins en arrière du bregma, n'était située, d'après lui, qu'à « 2 centimètres et plus » de ce point. Cette différence de résultats, qui a paru contradictoire, ne l'est nullement; elle est tout entière dans les mots. M. Bischoff, contrairement à l'opinion de la plupart des auteurs contemporains, ne comprend pas dans le lobe frontal la circonvolution frontale transverse ou ascendante, qu'il nomme la circonvolution centrale antérieure; il diminue donc la longueur du lobe frontal de toute l'épaisseur de cette circonvolution, épaisseur qui est partout considérable, et qui, près de la ligne médiane, atteint souvent 2 centimètres. En réalité, par conséquent, la détermination faite par M. Bischoff est en parfait accord avec la mienne.

M. Bischoff n'a pas étudié seulement les rapports de la suture coronale et de la suture lambdoïde; il a reconnu en outre que la partie antérieure de la suture écailleuse correspond à la scis-

(1) Die Grosshirnwindungen des Menschen. Munich, 1868, in-4º, p. 20 et 21. 
MORPHOLOGIE DU CERVEAU DE L'HOMME ET DES PRIMATES. 489 sure de Sylvius; mais quoiqu'il ait fait ses recherches sur " plusieurs crânes d'adultes ", il ne les a pas multipliées assez pour se rendre compte des variations individuelles; il s'est d'ailleurs

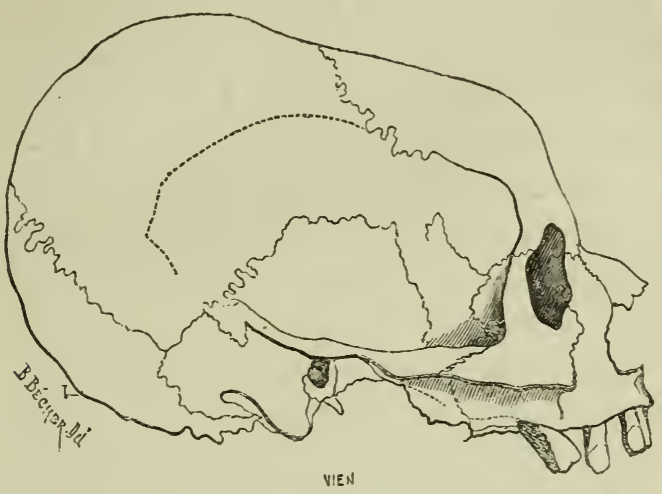

Fig. 63. Le profil de la Toulousaine dessiné au stéréographe
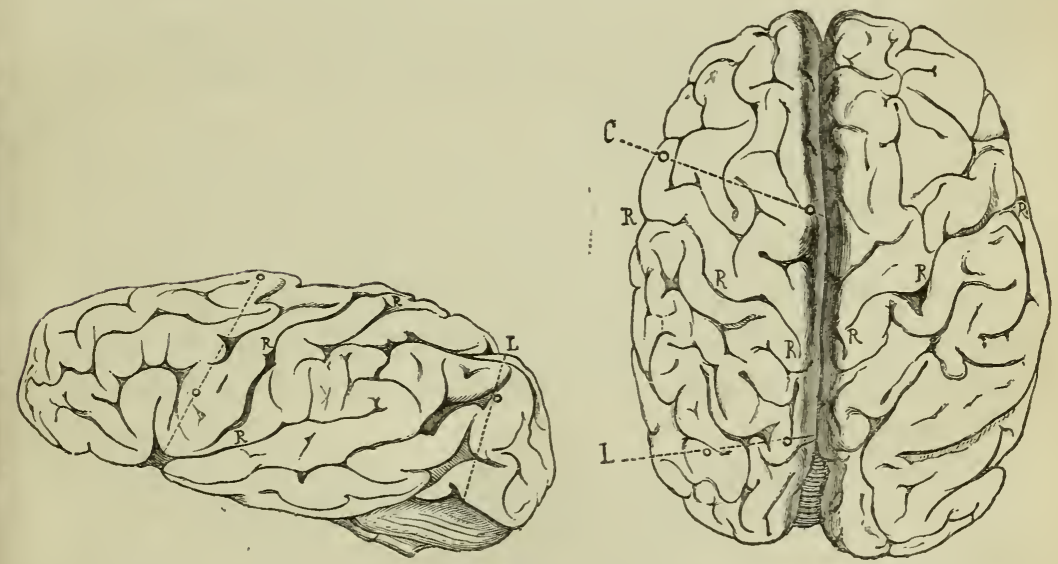

Fig. 66 et 67 . Le cerveau de la Toulousaine dessiné d'après le moule en plâtre : les lignes ponctuées passant par les fiches coronales et lambdoïdiennes indiquent le trajet des deux sutures du mème nom; 'RR, scissure de Rolando; C, ligne indiquant la position de la suture coronale; L, ligne indiquant la position de la suture lambdoïde.

borné comme moi à la détermination d'un petit nombre de points, et n'a pas envisagé la question dans son ensemble.

C'est M. Ferdinand Heftler qui a eu le mérite d'exécuter le premier un travail d'ensemble sur la topographie cérébrale. 
L'importance de cette étude lui a été signalée par son mâtre le professeur Landzert, de Saint-Pétersbourg, qui a institué à cette occasion un procédé de recherches assez compliqué, mais d'une exactitude tout ì fait rigoureuse. M. Heftler s'est acquitté de sa tâche avec un zèle et une habileté dignes des plus grands éloges. Les résultats de ses longues études ont été consignés dans sa thèse inaugurale, soutenue le ら̌ mai 1873 devant l'Académie médico-chirurgicale de Saint-Pétersbuırg (1).

L’ingénieux procédé de M. Landzert consiste à superposer sur une même figure plane trois dessins au trait, de couleurs différentes, représentant : $1^{\circ}$ le contour extérieur des parties molles de la tête; $2^{\circ}$ la surface des os du crâne aıec toutes leurs sutures; $3^{\circ}$ la surface du cerveau avec ses circonvolutions, ses scissures et ses sillons. Les trois dessins sont faits successivement, en grandeur naturelle, à l'aide de l'appareil de Lucæ, qui donne des projections dites géométrales, et évite les erreurs de la perspective. La superposition une fois faite, la situation respective des diverses parties du crâne et du cerveau devient aussi évidente qu'elle le serait sur une tête à parois transparentes, et il est aisé de mesurer en millimètres la distance de telle ou tello scissure à telle ou telle suture.

Mais, pour que ce procédé soit exact, il faut en premier lieis que le cerveau soit assez ferme pour conserver exactement sa forme, lorsqu'on le dessine après l'avoir dépouillé de ses membranes; de là résulte la nécessité de durcir préalablement cet organe en puussant quelques jours à l'avance, dans les artères carotides, une injection de chlorure de zinc, de glycérine et d'acide phénique.

Il faut en second lieu que la pièce, présentée trois fois de suite à l'appareil de Lucæ, soit replacée chaque fois dans une attitude absolument constante. Sans cela la superposition des dessins serait tout à fait trompeuse. Pour atteindre ce but, on commence par couler autour de la tête une épaisse couche de plâtre, qu'on enlève ensuite en partie, de manière à mettre à

(1) Ferd. Heftler, Izviliny golovnavo morga ou tchelovielia $i$ olnochenia ich $K$ 'svodou tcherepa (Des circonvolutions cérćbrales chez l'homme et de leur's rapports avec le crâne), thèse inaugurale, Saint-Pétersbourg, in-8, 50 pages avec planches. 
découvert celle des faces crâniennes que l'on se propose d'étudier; le reste du moule extérieur fournit les points de repère dont on a besoin pour l'orientation de la tête. Les deux premiers dessins une fois faits, on enlève délicatement, à l'aide de traits de scie convenablement dirigés, la partie correspondante de la voûte du crâne, c'est-à-dire la moitié latérale, s'il s'agit d'un dessin de profil; la moitié postérieure, s'il s'agit de la norma occipitale, etc.

Ce procédé est parfaitement correct; mais il est long et difficile. L'opération préalable du moulage est lente et pénible, et il faut beaucoup d'attention et d'habileté pour replacer la tête, à trois reprises différentes, dans une attitude invariable. Le procédé n'est donc valable qu'entre les mains d'un anatomiste expert, qui en a fait une étude spéciale, et qui a en outre beaucoup de temps à y consacrer. Ces conditions sont de nature à en restreindre beaucoup l'application; et nous devons apprécier d'autant plus l'importance des recherches de M. Heftler, que peu de personnes, sans doute, pourront les reprendre avec la même compétence et la même persévérance.

M. Heftler a appliqué ce procédé sur qunrante têtes, de manière à obtenir dix dessins topographiques de chacune des quatre norma du crâne, et si l'on songe que chacun de ces dessins a dû lui coûter au moins une journée de travail, on lui saura gré d'avoir recueilli pour la science et consigné dans sa thèse inaugurale tant de matériaux utiles. Grâce à la complaisance de mon ami le docteur Mierzejewski, de Saint-Pétersbourg, j’ai pu donner, dans la Revue d'anthropologie, 1876, t. V, p. 279, une analyse de cette thèse intéressante, et reproduire les figures qui l'accompagnent.

Le peu de publicité des thèses inaugurales et le peu de diffusion de la langue russe expliquent pourquoi les recherches de M. Heftler n'étaient pas encore connues dans l'Occident lor'squ'à son tour M. le professeur Turner (d'Edimbourg) publia, dans les numéros de novembre 1873 et de mai 1874 du Journal of Anatomy and Physiology, les résultats de ses études sur la topographie cérébrale (1).

(1) IV. Turner, On the relations of the Convolution of the Human Cerebrum to the outer Surface of the Skull and Head, dans Journ. of Anat. and Phys., sér. II, no XIII, p. 142, novembre ${ }_{\dot{\alpha}}$ 1873. - Le même, An lllustration of the 
Le procédé de M. Turner est un procédé graphique, comme celui de M. Landzert. Il consiste à superposer le dessin du cerveau sur celui du crâne, mais avec ceci de plus, que des lignes auxiliaires tracées sur le crâne multiplient les points de repère et permettent d'obtenir les rapports avec plus de détails.

On établit d'abord à la surface du crâne des circonscriptions déterminées par les lignes des sutures et par un certain nombre de lignes artificielles tracées suivant une règle uniforme. Chaque moitié latérale de la voûte du crâne se trouve ainsi divisée en dix districts que l'auteur appelle des aires (areas), et qu'il désigne chacun sous un nom spécial. On trouvera plus loin, dans l'analyse que nous donnons du travail de M. Turner, l'indication de ces lignes auxiliaires et la nomenclature des dix aires crâniennes (voir plus loin, p. 5้'4).

Lorsque les lignes sont tracées, on fait le dessin du crâne et de ses diverses aires, dont les contours fourniront, pour le dessin des circonvolutions, des lignes de repère comparables aux méridiens et aux parallèles des carteș de géographie. Il ne s'agit plus que de reporter sur ce dessin le tracé des circonvolutions qui correspondent respectivement à chacune des aires du crâne.

A cet effet, on circonscrit de toutes parts à l'aide d'une scie fine les contours d'une première aire; on fait sauter la pièce, on excise la dure-mère subjacente, on enlève en outre la pie-mère, et les circonvolutions qui sont ainsi mises à nu sont dessinées dans la case correspondante du dessin crânien. On passe alors à une seconde aire, puis à une troisième jusqu'à la dixième, et on finit par obtenir un dessin complet de toutes les circonvolutions de la norma crânienne que l'on se propose de représenter.

Ces dessins sont faits à la main, et sont par conséquent sujets à erreur, mais l'erreur ne saurait jamais être grave, puisque le dessinateur se guide sur les limites, toujours très rapprochées, du district qu'il représente. L'exactitude du procédé, à ce point de vue, est donc parfaitement suffisante. Mais il y a à se préoccuper d'une cause d'erreur plus sérieuse : c'est la déformation du cerveau, qui tend à se produire lorsque, de district en district, on a mis à découvert une grande partie de la surface de the Skull, dans le même journal, nºXIV, p. 359, may 1874. 
cet organe; les parties dénudées, n'étant plus fixées ni par la paroi osseuse, ni par la dure-mère, ni par la pie-mère, qu'on est obligé d'enlever pour mettre à jour les sillons, s'affaissent sous l'influence de la pesanteur et tiraillent plus ou moins, en les déformant, les circonvolutions des districts environnants. Un anatorisise attentif peut, en soutenant convenablement les circonvolutions dénudées, obvier à cet inconvénient; il serait préférable toutefois de durcir le cerveau à l'aide d'une injection préalable, comme le fait M. Landzert.

M. Turner n'a figuré ainsi que la norma latérale, ou vue de profil. Mais son procédé est applicable aux autres norma du crîne. On remarqnera d'ailleurs que la norma latérale est de beaucoup la plus utile; car elle montre les détails les plus importants de la topographie cérébrale.

L'exécution de ce procédé exige une certaine habileté anatomique et une assez grande dépense de temps; c'est. un obstacle à la multiplicité des recherches. M. Turner ne dit pas quel est le nombre des sujets sur lesquels ses observations ont été faites; il ne paraît pas que ce nombre ait été bien grand, et cependant il a pu constater que certains rapports présentent des variations assez étendues. Ainsi, quoiqu'il n'ait étudié que des sujets adultes, et du sexe masculin, il a vu la distance de la suture coronale à la scissure de Rolando varier sur la ligne médiane entre 1 pouce et demi et 2 pouces ( 38 millimètres et 51 millimètres) et sur les parties latérales entre 1 ponce un tiers et un pouce et demi (34 millimèlres et 38 millimètres). D'autres rapports lui ont présenté plus de fixité, mais les variations qu'il a constatées suffisent pour montrer la nécessité de faire reposer l'étude de la topographie cérébrale sur de très nombreuses observations. Ce but ne peut être atteint qu'à l'aided'un procédé d'une exécution facile et rapide, double condition que le procédé de M. Turner ne réalise pas et que celui de M. Landzert ne réalise pas davantage.

Je n'insisterai pas ici sur les résultats obtenus par M. Turner, ils sont exposés en détail dans l'analyse que j'en donne plus loin (voir p. ๖̌44), et personne n'en méconnaîtra l'importance. L'idée de multiplier les districts de la surface du crâne, en ajoutant aux lignes des sutures un certain nombre de lignes artificielles, mérite une sérieuse attention. 11 est clair, par 
exemple, que, pour déterminer et décrire les rapports du pariétal, il serait avantageux de pouvoir subdiviser méthodiquement en plusieurs parties la vaste surface de cet os. Mais il est à craindre que ces subdivisions et la nomenclature compliquée qu'elles entraînent ne paraissent gênantes pour la mémoire, et n'ajoutent une difficulté de plus aux recherches topographiques. Dans l'état actuel de la question, il vaut mieux la' simplifier que la compliquer, et les belles recherches de M. Ferdinand Heftler prouvent d'ailleurs que l'on peut obtenir des déterminations très satisfaisantes sans recourir à l'emploi des lignes artificielles.

Le mémoire de M. Turner était encore sous presse, et la thèse de M. Heftler était encore tout à fait inconnue en France, lorsque nous eûmes l'occasion de signaler la question de la topographie cérébrale à l'attention de M. Charles Féré, élève distingué des hôpitaux, qui avait travaillé quelque temps dans le laboratoire d'anthropologie. Placé comme externe à l'hospice de la Salpêtrière, où les autopsies sont si fréquentes, M. Féré se mit à l'œuvre au mois de janvier $187 \%$, et recueillit pendant le courant de cette année de nombreuses observations, dont il a consigné les résultats dans un mémoire communiqué à la Société anatomique en décembre 1875 , et à la Société de biologie au commencement de janvier 1876. Voir le résumé, dans une analyse écrite par l'auteur lui-même (1).

M. Féré a étudié principalement la topographie des circonvolutions, et s'est servi pour cela du procédé des fiches. Mais il s'est occupé en outre d'une autre que.tion que ses prédécesseurs n'avaient pas abordée. Elève de mon savant collègue le professeur Charcot, qui poursuit avec tant de talent l'étude pathologique et fonctionnelle des parties profondes des hémisphères, il s'est attaché à déterminer la position de ces parties profondes par rapport aux parois crâniennes. J'ai déjà dit que le procédé des fiches est applicable à ce genre de recherches, pourvu qu'on ait le soin de prendre des fiches très longues et de les enfoncer suivant une direction exactement perpendiculaire à la surface du crâne; mais il ne donne que des résultats partiels, parce qu'on ne peut étudier le trajet profond d'une fiche sans pratiquer

(1) Revue d'anthropologie, t. V, p. 289. 
des incisions qui déforment gravement les parties. M. Féré a donc eu recours à des coupes pratiquées sur des têtes préalablement congelées. Ce procédé doit porter son nom, car personne avant lui n'avait appliqué la méthode, déjà ancienne, de la congélation à l'étude de la topographie cérébrale.

Le procédé de M. Féré donne à la fois les rapports des organes cérébraux profonds et des circonvolutions tant externes qu'internes. Il est bien supérieur à celui des coupes ordinaires. Gelles-ci fournissent de bons résultats lorsqu'on n'en pratique qu'une seule et lorsqu'en outre le liquide sous-arachnoïdien est peu abondant; alors, en effet, le cerveau, remplissant presque exactement la cavité de la dure-mère, se laisse traverser par la scie sans aucun déplacement, et conserve tous ses rapports avec la paroi du crâne; mais il n'en est plus de même lorsqu'on veut pratiquer une nouvelle coupe à quelque distance de la première, parce que la substance cérébrale, trop molle pour résister par elle-même, et n'étant plus suffisamment fixée dans une cavité déjà ouverte, se déforme assez pour rendre incertaine la détermination des rapports. Les coupes ordinaires ne peuvent donc donner, sur chaque tête, que des notions limitées à un seul plan, et c'est pour cela qu'après avoir commencé mes recherches à l'aide de ce procédé, j'y ai bientôt renoncé pour le remplacer par le procédé des fiches. Mais le procédé de M. Féré n'a pas le même inconvénient; il permet de pratiquer sur une même tête plusieurs coupes parallèles, d'étudier successivement les rappurts cérébro-craniens sur chaque tronçon, et de rapprocher ensuite les tronçons cérébraux de manière à reconstituer le cerveau entier sans aucune déformation. Il peut donc donner des résultats très complets, non seulement pour ce qui concerne les rapports de la surface des hémisphères, mais encore pour ce qui concerne les rapports des parties profondes du cerveau. Ce dernier avantage est précieux. Mais l'inconvénient du procédé de M. Féré, c'est qu'il n'est applicable dans nos climats que dans des conditions de température assez exceptionnelles. Il faut un froid très intense pour congeler la tête jusqu'au centre du cerveau, et la congélation artificielle, d'ailleurs assez difficile à obtenir sur une aussi grande masse, ne pourrait réussir que dans une chambre refroidie au-dessous de zéro, car il ne suffit 
pas de faire des coupes sur le cerveau gelé, il faut encore avoir le temps de les étudier et de les décrire avant le dégel. Le procédé de $M$. Féré ne peut donc se généraliser dans la pratique. L'auteur, au surplus, ne s'en est servi que pour étudier, sur un petit nombre d'individus, les rapports des parties profondes; mais ce n'est là que la moindre partie de ses recherches. Il s'est attaché surtout à la topographie superficielle des circonvolutions, qu'il a étudiée exclusivement par le procédé des fiches.

M. Féré n'ignore pas que les formes et les dimensions du crâne présentent des variations individuelles très étendues, que toute détermination craniométrique doit, pour être valable, reposer sur la moyenne d'une série nombreuse d'observations de même ordre, et que par conséquent les rapports cérébro-crâniens, étant influencés à la fois par les variations du crâne et par celles du cerveau, sont sujets à de grandes oscillations, sinon dans la région de la base, où ces rapports sont maintenus par des conditions anatomiques particulières, du moins dans la région de la voûte, où chaque lobe peut se développer librement aux dépens de ses voisins. Il s'est donc proposé avant tout de multiplier ses observations autant que possible, afin de pouvoir constater avec sécurité le maximum, le minimum et la moyenne de chaque résultat millimétrique. Sous ce rapport, ses recherches topographiques offrent toute garantie, car le schéma sur lequel il a représenté les rapports cérébro-crâniens de la femme repose sur la moyenne de cinquante-quatre observations. M. Féré a en outre recueilli huit observations sur des hommes, mais ce n'est que le commencement d'une étude qu'il s'occupe actuellement de compléter, et il n'a pas fait entrer en ligne de compte, dans son travail actuel, cette trop courte série masculine.

Les déterminations faites par M. Féré ne concernent donc que les femmes, tanảis que celles de M. Heftler ont été faites sur vingt-huit hommes et douze femmes, et celles de M. Turner sur des hommes seulement. Ceux qui compareront les résultats publiés par ces trois auteurs devront tenir compte de cette différence sexuelle, car, le cerveau de la femme étant en moyenne moins volumineux que celui de l'homme, il est naturel que les distances que l'on y mesure soient un peu moindres. En réalité, ces résultats, quoique obtenus par des procédés très divers, 
concordent d'une manière assez satisfaisante, du moins dans les points principaux; les divergences qu'on y pourra trouver ne sunt que secondaires, et ne sont imputables ni aux observateurs, ni aux procédés qu'ils ont suivis, mais aux conditions dans lesquelles ils ont fait leurs recherches. Les sujets qu'ils ont ćludiés n'étaient ni du même sexe ni de même race; en outre II. Heftler n'a mis en œuvre que dix observations pour chaque norma du crâne; le nombre des faits recueillis par M. Turner cst probablement moindre encore ; ce n'est pas assez pour soustraire les résultats à l'influence des variations individuelles, tandis que les cinquante-quatre cas étudiés par M. Féré forment IIn groupe nombreux et homogène dont les moyennes méritent toute confiance.

Je rappelle que M. Féré ne s'est pas borné, comme ses prédécesseurs, à l'étude des rapports superficiels des hémisphères. Il a le premier poussé les recherches topographiques jusque dans les parties profondes. La partie de son travail qui concerne cette topographie profonde est encore peu avancée; mais la voie est tracée, et je ne saurais trop inviter l'auteur à y persévérer.

Je viens d'exposer les divers procédés dont on s'est servi jusqu'ici dans l'étude des rapports cérébro-crâniens. Sans parler des coupes ordinaires, qui ne fournissent que des notions très incomplètes, ces procédés sont au nombre de cinq :

$1^{\circ}$ Le procédé des moules intra-crâniens de Gratiolet (1857);

$2^{\circ}$ Le procédé des fiches, dont je me sers depuis 1861, et qui a été employé depuis par MM. Bischoff (1868) et Féré (1875);

$3^{\circ}$ Le procédé graphique de M. Landzert, employé par M. Heítler (1873);

$4^{\circ}$ Le procédé graphique de M. Turner (1874);

$5^{\circ}$ Le procédé des coupes sur des têtes congelées, dû à M. Féré (1875).

Le premier procédé est insuffisant et trompeur, puisqu'il a conduit un homme aussi habile que Gratiolet à des conclusions très fausses. Le dernier n'est qu'un moyen d'exception. Restent donc les trois autres. Ils sont également corrects, mais d'une valeur pratique très inégale. Pour les comparer à ce point de vue, nous devons d'abord nous demander quelles sont les 
questions que la topographie cérébrale est appelée à éclairer ou à résoudre.

§ 2. application dE la topographie cérébrale a la médecine ET A L'ANTHROPOLOGIE.

Le but des déterminations topographiques est double ; il està la fois anthropologique et médical, et je comprends à la fois, sous cette seconde détermination, tout ce qui se rapporte à la physiologie, à la pathologie et à la chirurgie. Or, dans l'étude des faits anatomiques qui sont sujets à des variations de quelque importance, les recherches de l'ordre médical diffèrent notablement des recherches anthropologiques. Les premières, en effet, ne concernent que des individus isolés, tandis que les dernières concernent des collections d'individus.

L'intérêt médical de la topographie du cerveau gît tout entier dans la question des localisations cérébrales. L'homme n'est pas une matière à expériences, mais les hasards de la maladie ou du traumatisme produisent quelquefois des lésions cérébrales aussi circonscrites et aussi démonstratives que pourraient l'être des vivisections, et l'interprétation des troubles fonctionnels qui en résultent est la seule base sur laquelle puisse reposer l'étude des localisations. Lorsque le cas se termine par la mort, et lorsque en outre l'autopsie est possible, la détermination du siège de la lésion dont on a constaté les symptômes se fait anatomiquement sur le cerveau même, sans que les notions topographiques aient à'intervenir. Ces faits sont les plus probants; mais les autres, quoique moins décisifs, peuvent avoir aussi leur utilité, et par exemple, étant donnée une plaie pénétrante du crâne, la connaissance des rapports cérébro-crâniens fournit le moyen d'apprécier avec une certaine probabilité le siège de la lésion cérébrale subjacente.

Ce n'est pas seulement dans les recherches physiologiques et pathologiques que la topographie cérébrale peut être utilisée. Il est des cas, rares jusqu'ici, mais qui pourront se multiplier avec les progrès de nos connaissances, où elle peut servir de guide au chirurgien dans l'opération du trépan. J'en citerai plus loin un 
exemple qui m'est personnel. On verra que j'ai pu tomber du premier coup sur un abcès intra-crânien qui comprimait la partie postérieure de la troisième circonvolution frontale gauche, et dont le siège était annoncé par l'abolition du langage. Mais ces déterminations topographiques, faites sur l'homme vivant, manquent très souvent de certitude; elles ne donnent ordinairement que des probabilités. Supposons par exemple qu'il existe une blessure pénétrante sur la partie supérieure de la région pariétale; certains symptômes spéciaux se manifestent; on présume que leur nature spéciale dépend du siège anatomique de la lésion cérébrale, et on se demande si celle-ci a atteint le lobe frontal ou le lobe pariétal de l'hémisphère, en d'autres termes, si elle est située en avant ou en arrière de la scissure de Rolando. Supposons encore, pour simplifier la question, que le sujet soit de ceux chez lesquels la position du bregma et celle du lambda peuvent être reconnues à travers l'épaisseur du tégument. Si la blessure est à moins de 4 centimètres du bregma, nous dirons avec certitude qu'elle est sur le lobe frontal, sans savoir d'ailleurs à quelle distance d'elle se trouve la scissure de Rolando; si elle est à plus de 6 centimètres du bregma, nous dirons encore, avec une probabilité voisine de la certitude, qu'elle a atteint le lobe pariétal, car il est très exceptionnel que la distance du bregma à la scissure excède ce chiffre; mais si la blessure est comprise entre ces deux limites, si elle est par exemple à 5 centimètres du bregma, notre diagnostic sera incertain. C'est en vain que nous saurons que, chez l'homme adulte, la distance rolando-bregmatique est en moyenne de 47 à 48 millimètres; nous ne saurons pas si, chez notre blessé, elle est supérieure ou inférieure à la moyenne; tout au plus pourronsnous le supposer d'après la forme brachycéphale ou dolichocéphale et d'après les dimensions de son crâne; et tout cela ne nous conduira qu'à une probabilité. Cela ne veut pas dire qu'il soit inutile de connaître cette distance moyenne ; il est bon d'en avoir une idée, pour apprécier les chances du diagnostic; mais si, au lieu de la connaître à 1 millimètre près, nous ne la connaissions qu'à 2 ou 3 ou 4 millimètres près, l'utilité pratique que nous en retirerions serait à peu près la même.

Ainsi, lorsqu'on se place au point de vue des études de l'ordre 
médical, la détermination exacte des moyennes topographiques n'est pas absolument nécessaire ; une description topographique qui résulterait de l'examen d'une seule tête pourrait être trompeuse sans doute; il faut se méfier des exceptions, et pour cela prendre la moyenne d'un certain nombre de faits; mais on n'a pas besoin de multiplier beaucoup les observations pour atteindre le but pratique que l'on se propose. Dès lors le degré de difficulté ou de lenteur d'un procédé de recherches n'a plus que peu d'importance; il n’est pas nécessaire que tout le monde puisse l'appliquer; les faits constatés une bonne fois par un ana-. tomiste digne de confiance suffisent aux besoins de la pratique, et par exemple on pourrait très bien s'en tenir aux résultats que M. Heftler a publiés dans sa thèse.

Mais tout autre est le but anthropologique. L'anthropologie ne se propose pas seulement, comme la médecine, de connaitre l'individu; elle se propose surtout d'étudier les groupes, et d'apprécier l'influence que peuvent exercer sur chaque caractère l'âge, le sexe, la race et le milieu. Plus un caractère est variable, et plus il importe de chercher les conditions qui le font varier. Sous ce rapport, la topographie cérébro-crânienne appelle toute l'attention des anthropologistes, car, à côté de certains faits qui sont à peu près constants, elle en montre d'autres qui présentent des écarts considérables.

On peut objecter, il est vrai, que les caractères anthropologiques doivent être tirés de la constitution propre des organes plutôt que de leurs rapports; mais si ces derniers sont de nature à être modifiés par des conditions de développement ou d'accroissement, ils peuvent fournir des indications d'une importance égale à celle de certains caractères simples; or, les rapports cérébro-crâniens sont dans ce cas.

La formation des os du crâne est indépendante du cerveau, puisqu'elle s'effectue chez les notencéphales et chez les anencéphales; mais l'état rudimentaire où ils restent alors, et les formes étranges qui les rendent presque méconnaissables, prouvent très manifestement que leur développement est régi par le cerveau. Il ne faut pas croire pour cela que chaque os du crâne soit solidaire du lobe cérébral correspondant. L’embryologie ne se prête pas à cette supposition, car les os du crâne sont formés 
et régulièrement répartis, longtemps avant l'apparition des deux scissures transversales qui marquent la distinction des trois lobes de la convexité des hémisphères. L'anatomie comparée ne s'y prête pas davantage. Il est bien vrai que, dans l'ordre des primates, les scissures transversales des hémisphères sont au nombre de deux, comme dans les sutures transversales du crâne, et que par conséquent trois lobes se succèdent d'avant en arrière sur la convexité des hémisphères, comme trois os se succèdent d'avant en arrière sur la convexité du crâne ; mais on ne peut y voir qu'une pure coïncidence, car le nombre et les connexions des os du crâne restent les mêmes dans les autres ordres de mammifères, quoique la disposition, le nombre et jusqu'à la nature des lobes des hémisphères soient très variables.

Il est donc impossible d'admettre que, dans le développement du crâne, chaque lobe du cerveau tienne directement sous sa dépendance l'os dont il porte le nom. Tout ce que l'on peut dire, c'est que l'accroissement du crâne est provoqué par l'expansion cérébrale, en raison de la poussée exercée sur les divers os qui le composent. Si la masse cérébrale était liquide, cette poussée serait partout la même ; si elle était dure ou très ferme, la pression exercée sur chaque os serait presque proportionnelle au développement du lobe cérébral subjacent; mais la consistance du cerveau n'est ni assez faible pour répartir uniformément sur tous les points de sa surface la pression qui résulte de l'accroissement d'un lobe, ni assez forte pour que cette pression reste limitée à la partie correspondante de la boîte crânienne; par conséquent, lorsqu'un lobe s'accroît d'une certaine quantité, il tend à dilater tout le crâne, mais la dilatation qu'il produit est à son maximum dans la partie du crâne qui le recouvre.

De cette action expansive du cerveau qui s'accrô̂t, et de la résistance modératrice de la paroi ostéo-membraneuse (puis osseuse) qui l'enveloppe, résultent, lorsque le développement est achevé, un certain état du crâne et de ses sutures, un certain état du cerveau et de ses sutures, et par conséquent une certaine topographie crânio-cérébrale. Qu'arrivera-t-il maintenant si l'un des lobes du cerveau avorte complètement? La partie correspondante du crâne ne sera pas arrêtée pour cela dans son développement; elle recevra toujours une certaine poussée, qui 
lui sera transmise par l'action expansive du reste du cerveau; elle continuera done à prendre un certain accroissement, mais cet accroissement sera moindre qu'à l'état normal. De même, si ce lobe, sans avorter, reste au-dessous de son volume ordinaire, l'accroissement local du crâne sera diminué aussi, mais il ne le sera pas au même degré, et les rapports cérébro-crâniens seront par conséquent modifiés. Enfin, si ce lobe acquiert un volume exagéré, l'accroissement local du crâne sera exagéré, mais il ne le sera pas dans la même proportion, et les rapports cérébrocrâniens seront modifiés en sens inverse.

Ainsi, l'étude du développement relatif des diverses régions du crâne ne donne qu'une notion très imparfaite du développement relatif des diverses parties du cerveau ; cette notion, certes, quelque imparfaite qu'elle soit, est loin d'être sans utilité ; car c'est elle qui fait le principal intérêt de l'analyse craniologique. $\mathrm{Si}$, par exemple, on attache tant d'importance à la mensuration de l'écaille de l'os frontal, c'est parce que l'on sait que le volurrie des lobes frontaux exerce une grande influence sur l'ampleur de la loge frontale; mais, lorsgu'on a mesuré celle-ci, on ne connaît pas l'étendue de ceux-là, puisqu'ils se prolongent toujours considérablement en arrière de la suture coronale. Les résultats craniométriques perdent ainsi une grande partie de leur signification. Pour la leur rendre, il faudrait pouvoir évaluer les dimensions des lobes frontaux d'après celles de l'os frontal; en d'autres termes, il faudrait savoir de combien de centimètres ou de millimètres la région frontale du cerveau dépasse la région frontale du crâne. Or la topographie cérébrale nous fournit cette notion précieuse, ainsi que les autres notions de même ordre. Grâce à elle, l'examen du crâne ne fait plus connaître seulement les dimensions et la forme générale de l'encéphale, il fait connaître en outre approximativement le développement relatif des principales subdivisions des hémisphères. Les faits topographiques deviennent ainsi le complément des faits craniométriques, et comme ils peuvent, aussi bien que ces derniers, être exprimés en mesures millimétriques, ils réclament l'application des principes ordinaires des recherches craniologiques.

Ces principes sont connus. Il faut en premier lieu que les observations soient groupées en séries assez grandes pour que 
l'on puisse connaitre le maximum, le minimum et la moyenne de chaque caractère, et pour que cette moyenne ne soit pas faussée par les variations individuelles. L'expérience a prouvé que les séries de moins de vingt crânes n'atteignent pas ce but avec certitude.

Il faut en second lieu que chaque série ne comprenne que des crânes d'une même catégorie, de manière à séparer non seulement les races, mais encore, dans chaque race, les sexes et les âges. Ces catégories suffisent dans les études craniologiques ordinaires; mais les recherches topographiques appellent en outre d'autres subdivisions basées sur les divers degrés de la dolichocéphalie et de la brachycéphalie, car ces conditions exercent une influence notable sur les rapports cérébro-crâniens. Et il faut enfin que tous les faits anormaux ou exceptionnels, tous les cas de déformation tératologique, pathologique ou accidentelle, tous les cas où des troubles fonctionnels constatés pendant la vie ont rendu probable l'existence d'une constitution vicieuse du cerveau, soient exclus des séries précédentes, et étudiés isolément, avec d'autant plus de soin qu'il ne dépend pas de l'observateur de retrouver des faits analogues.

L'application de la topographie cérébrale à l'anthropologie exige donc des observations très multipliées et très variées, et si l'on veut atteindre ce but, il faut choisir un procédé simple, facile, rapide, applicable à tous les cas, dans les salles d'autopsie aussi bien que dans les amphithéâtres, et mis à la portée de tout le monde. Toute condition de nature à diminuer le nombre des observateurs ou à restreindre le champ de leurs recherches, doit être écartée autant que possible. Ainsi, les sujets qui succombent dans les hôpitaux sont pour la plupart réclamés par leurs familles ou par leurs amis, mais n'en sont pas moins soumis à l'autopsie ; les procédés qui ne peuvent être mis en pratique que dans les laboratoires d'anatomie excluent donc la grande majorité des cas qu'il serait intéressant d'étudier. De même, ceux qui mutilent gravement le crâne excluent presque nécessairement les recherches faites sur les sujets exotiques ou anormaux, dont les crânes, toutes les fois que cela est possible, doivent être conservés pour les collections anthropologiques. Enfin, tout médecin d'hôpital, tout élève des hôpitaux a accès dans les salles 
d'autopsie; mais peu d'observateurs disposent d'un laboratoire d'anatomie convenablement installé et outillé, et beaucoup n'ont ni les loisirs nécessaires pour consacrer plusieurs henres à l'étude d'un seul fait, ni l'habileté toute spéciale qu'exige l'application de certains procédés.

Il ne suffit donc pas qu'un procédé de recherches topographiques soit exact; il faut en outre qu'il soit prompt, commode $t$ t facile; là est l'avenir de la question au point de vue anthropologique. C'est en nous plaçant à ce point de vue que nous allons comparer entre eux les trois procédés dont nous avoris reconnu l'exactitude, savoir : le procédé des fiches et les procédés gira. phiques de MM. Landzert et Turner.

\section{§ 3. de la valeur pratique des procédés.}

Le procédé des fiches, comme on le verra plus loin, permet de recueillir tous les éléments nécessaires pour représenter sur le papier ou sur le crâne lui-même toute la surface du cerveau, dans ses rapports avec le crâne; mais ce dessin, où l'on peut reporter, pour la facilité de la démonstration, les résultats obtenus sur quelques sujets de choix, ne fait nullement partie du procédé; la constatation des rapports, la détermination des distances se font sur les pièces mêmes, et se font en quelques instants.

Dans les procédés graphiques, au contraire, les dessins sont la base même des recherches; il faut d'abord dessiner complètement la surface du crâne, puis dessiner complètement par superposition la surface du cerveau, et c'est sur ce double dessin que l'on applique le compas pour mesurer les distances. La longue opération du dessin ne peut donc être évitée, non plus que les préparations délicates qui doivent la précéder; ce n'est qu'après tout ce travail lent, difficile et pénible, qui ne peut exiger moins d'une journée, si même il n'en exige pas plusieurs, que l'on peut commencer à déterminer et à inscrire sur' la feuille d'observations les rapports cérébro-crâniens.

Lorsqu'on applique le procédé des fiches avec l'intention de s'en servir pour obtenir un dessin topographique, on est obligé 
d'augmenter le nombre des fiches, et l'exécution du dessin, quoique facilitée par de nombreux points de repère, est toujours assez longue. Mais, lorsqu'on se propose seulement de constater les faits topographiques, il suffit de pratiquer un petit nombre de perforations, et l'opération tout entière n'ajoute que quelques minutes à la durée de l'autopsie ordinaire du cerveau. Il est donc facile de multiplier indéfiniment les observations, tandis que les recherches faites à l'aide des procédés graphiques ne pourront jamais être que très peu nombreuses. C'est le principal avantage du procédé des fiches; mais il en présente plusieurs autres qui ont aussi une grande importance.

Il est à la portée de tous les observateur's, tandis que les procédés graphiques ne peuvent être exécutés avec exactitude que par des anatomistes très experts, secondés par un mouleur ou par un dessinateur habile.

Il ne détériore nullement le crâne, qui doit être mutilé ou sacrifié dans les procédés graphiques. Il est done seul applicable aux sujets de races étrangères, aux sujets atteints d'anomalies ou de déformations, à tous ceux en un mot qui sont exceptionnels à un titre quelconque, et dont les crânes doivent par conséquent être conservés.

Il est le seul qui puisse donner la topographie de toutes les régions crânio-cérébrales d'un même sujet. Les procédés graphiques, en effet, ne peuvent représenter qu'une seule norma du crâne et du cerveau. La norma latérale ou de profil montre, il est vrai, la plupart des rapports de l'hémisphère correspondant, mais ceux de ces rapports qui concernent la partie supérieure et les deux extrémités de l'hémisphère ne sont vus qu'en raccourci, et se prêtent difficilement à l'étude; c'est pour cela que M. Heftler a jugé nécessaire de représenter en outre la norma supérieure, la norma antérieure et la norma postérieure; l'étude complète des rapports topographiques exige ainsi quatre préparations, faites sur quatre têtes différentes, et ne saurait dès lors donner un résultat d'ensemble, puisque la plupart des rapports varient plus ou moins dans chaque sujet. Il y a d'ailleurs une étude à laquelle les procédés graphiques se refusent absolument : c'est la comparaison des deux hémisphères du même sujet; cette comparaison, devenue importante depuis que 
l'on sait que les deux hémisphères n'ont pas les mêmes attributions fonctionnelles, est rendue très facile par le procédé des fiches.

J'insiste encore sur ce fait que les procédés graphiques, exigeant une installation spéciale, des opérations longues, et des mutilations graves, précédées avant tout de la décapitation, ne peuvent être appliqués que dans les laboratoires d'anatomie, sur les corps abandonnés aux dissections. On ne peut y avoir recours dans les salles d'autopsie. Le champ des observations se trouve ainsi considérablement rétréci, si l'on songe surtout que, parmi les corps apportés à l'amphithéâtre, beaucoup ont déjà subi dans les hôpitaux l'autopsie du cerveau, et sont devenus par conséquent impropres aux recherches topographiques. Ajoutons que l'étude et l'interprétation des cas anormaux ou exceptionnels à un titre quelconque, gagnent beaucoup à être faites par les médecins qui ont connu les sujets pendant leur vie. Ce résultat ne peut être atteint qu'à l'aide du procédé des fiches.

'Ainsi, lorsqu'on se place au point de vue de l'investigation anthropologique, la supériorité du procédé des fiches devient tout à fait évidente. C'est seulement lorsqu'on se propose de démontrer sur une figure les rapports cérébro-crâniens, que l'avantage des procédés graphiques se manifeste. Ceux-ci donnent de vrais dessins, tracés d'un trait continu et copiés directement, sans déformation, sur le cerveau en place. Le procédé des fiches permet, lui aussi, d'ubtenir des dessins qui sont parfaitement exacts dans leurs points essentiels, mais dont les détails secondaires laissent place à quelques incertitudes; on ne dessine le cerveau que lorsqu'il est placé sur la table, et par conséquent déformé; les points de repère fournis par les fiches sont seuls rapportés rigoureusement à leur véritable position; le dessinateur, en se guidant sur ces jalons, qui peuvent d'ailleurs être nombreux, met aisément à leur place les circonvolutions et les sillons; il est clair, toutefois, que cette restitution des formes ne peut jamais être parfaite. Si donc on se propose seulement de représenter la topographie cérébrale d'un indivichu, il est naturel de donner la préférence aux procédés graphiques. 
On peut se demander toutefois quel est le degré d'utilité de ce portrait topographiqne. Si les rapports cérébro-crâniens étaient invariables ou seulement peu variables, le dessin fait sur un individu pourrait être montré ou publié comme un type, et il serait nécessaire que ce type fût dessiné d'après nature; mais on sait qu'il n'en est rien; on sail que beaucoup de rapports cérébro-crâniens présentent des oscillations extrêmement étendues, de sorte que la publication ou l'exhibition d'un dessin topographique individuel sera tout à fait trompeuse, si l'on n'y joint la mention expresse que ce dessin pourrait être tout différent. Choisira-t-on à cet effet, parmi les divers dessins dont on dispose, celui qui paraît s'écarter le moins des formes moyennes? Mais combien de dessins ne faudra-t-il pas comparer entre eux, a vant de connaître l'état moyen de chaque rapport, surtout avant de trouverun cas qui soit par hasard, dans toutes ses parties, exempt de toute excentricité ? En supposant même qu'on le trouve, on n'en sera jamais pleinement satisfait; on regrettera que telle scissure ne soit pas un peu plus oblique, que telle autre ne soit pas un peu plus longue; on voudrait que tel trait fût un peu plus haut, tel autre un peu plus bas. Il suffira sans doute, pour satisfaire à ces desiderata, d'introduire dans le dessin quelques corrections légères; mais alors le dessin ne sera plus naturel, il deviendra schématique. C'est qu'en effet, si l'on veut obtenir, comme moyen de démonstration, une figure vraiment typique, on est obligé de recourir à un schéma sur lequel on reporte les positions et distances moyennes relevées sur un nombre suffisant de cas individuels.

Pour construire ce schéma, qui doit représenter la superposition de la surface crânienne et de la surface cérébrale, on fait d'abord le dessin complet de la première, puis, avant de dessiner l'autre, on détermine par des mensurätions méthodiques la position des points du cerveau qui sont reconnus comme les plus significatifs; enfin, lorsque ces divers points sont marqués, on groupe autour d'eux les éléments du second dessin, que l'on raccorde de manière à respecter la morphologie, tout en l'assujettissant aux exigences topographiques. Ce second dessin est nécessairement schématique; quant au premier, on peut le faire d'après nature, en choisissant, parmi les crânes de la 
race, ou de la catégorie que l'on étudie, un spécimen convenable, dont il est d'ailleurs toujours permis de corriger certains détails.

L'exécution de ce schéma cranio-cérébral peut se faire aisément à l'aide des dessins fournis par les procédés graphiques, pourvu qu'ils soient suffisamment nombreux; comme ils sont en grandeur naturelle, et orientés d'une manière identique, on détermine sur chacun d'eux, à l'aide d'un compas, les diverses distances horizontales ou verticales qui fixent la position des points caractéristiques; et on prend la moyenne do chacune de ces distances pour la reporter proportionnellement sur le schéma. Cela ne souffre aucune difficulté, si ce n'est que l'exécution des nombreux dessins topographiques dont on prend ainsi la moyenne nécessite une énorme dépense de temps.

Or, les déterminations et mensurations faites sur le cerveaı même à l'aide du procédé des fiches donnent, avec infiniment moins de travail, un résultat tout aussi exact; les moyennes offrent même plus de sécurité, car on peut les faire reposer sur des séries beaucoup plus nombreuses. Il y a d'ailleurs ici, comme dans le cas précédent, une réduction proportionnelle à établir, pour que l'ensemble des dimensions moyennes obtenues s'accommode exactement avec les dimensions du dessin crânien sur lequel on établit le schéma du cerveau.

Dans l'un et l'autre cas, le schéma est obtenu en grandeur naturelle; on peut ensuite, à l'aide du pantographe, le grandir pour le montrer dans un cours, ou le réduire pour le publier.

Ainsi, le principal avantage des procédés graphiques, qui consiste à représenter sur des dessins les rapports cérébro-crâniens, peut être réalisé aisément par le procédé des fiches; il y a même un mode de représentation très intéressant que ce dernier procédé permet seul de réaliser : c'est celui qui consiste à reproduire, sur le crâne même de l'individu étudié, le dessin noir ou polychrome de toutes les circonvolutions, de toutes les scissures et sillons de son cerveau.

La supériorité du procédé des fiches dans les recherches anthropologiques ressort du parallèle que je viens d'établir entre 
MORPHOLOGIE DU CERVEAU DE L'HOMME ET DES PRIMATES. 50g

les divers procédés topographiques. Mais je suis bien loin de méconnaître l'intérêt et l'utilité des procédés graphiques. Il est heureux que des recherches de cette importance puissent être contrôlées, car la concordance de résultats obtenus par des voies diverses est la meilleure garantie de l'exactitude. MM. Landzert et Turner ont donc rendu un vrai service à la science par l'institution de leurs procédés, et M. Heftler mérite tout spéciale. ment nos éloges pour la persévérance et le talent qu'il a montrés en exécutant dix dessins topographiques dans chacune des quatre norma du crâne. Rappelons, en outre, que cet auteur a eu le mérite d'entreprendre _le premier et de mener à bonne fin un travail d'ensemble sur la topographie cérébrale.

Quelques mots enfin sur la valeur relative des deux procédés graphiques. Ils donnent l'un et l'autre des résultats parfaitement exacts, lorsqu'ils sont appliqués par des opérateurs habiles. Ils diffèrent peu sous le rapport de la difficulté et de la durée de l'exécution. Les lignes auxiliaires de M. Turner permettent de multiplier les repères topographiques et de donner plus de précision à la détermination de certains rapports ; mais, si l'on considère ce résultat comme avantageux, on peut très bien l'obtenir au moyen du procédé de M. Landzert, en traçant sur le crâne, avant de le dessiner, les mêmes lignes auxiliaires. Le procédé de M. Turner est certainement plus simple que l'autre; il n'exige, en fait d'instruments, que les scies variées dont se servent les anatomistes; mais les nombreuses sections à l'aide desquelles on circonscrit successivement chacun des districts topographiques du crâne, afin d'enlever isolément chacune des pièces correspondantes, ne peuvent être faites que par la main d'un opérateur très adroit, et l'exécution des dessins, quoique facilitée par les lignes auxiliaires, reste toujours assez difficile. Le procédé de M. Landzert exige l'opération préalable du moulage extérieur; c'est une complication gênante sans doute, mais qui procure un avantage tout à fait décisif, car elle permet de donner à la tête, dans les dessins successifs du crâne et du cerveau, une attitude invariable, et d'exécuter ces dessins, à l'aide de l'appareil de Lucæ, du diagraphe ou du dessinateur mécanique, avec une exactitude que l'artiste le plus habile ne saurait atteindre et avec une rapidité qui compense largement la perte de temps néces- 
sitée par le moulage. Somme toute, je pense que le meilleur procédé graphique est celıi de M. Landzert.

\section{§ 4. DESCRIPTION DU PROCÉDÉ DES FICHES ET DE SES DIVERSES}

\section{APPLICATIONS.}

Je crois avoir établi, dans les deux paragraphes qui précèdent, que le procédé des fiches est le seul qui réponde complètement aux exigences de l'anthropologie. Il me paraît donc utile de le décrire en détail, afin d'en faciliter et d'en régulariser l'application. On me pardonnera cet exposé minutieux, si l'on songe que les procédés anthropologiques doivent être soumis à des règles invariables. Il est impossible, en effet, qu'un observateur, quelque laborieux qu'il soit, et quelque favorisé qu'il soit par les circonstances, puisse remplir à lui seul tout le programme des recherches anthropologiques. S'il n'y avait qu'une seule population à étudier, on y parviendrait peut-être, quoique les cas anormaux ne soient fournis que par. le hasard; mais l'occasion d'ouvrir des corps de races étrangères ne s'offre que rarement à chaque anatomiste, et, si l'on veut arriver à connaître les influences ethniques, il faut que l'on puisse grouper ensemble des faits recueillis en des lieux et par des observateurs différents. Gela suppose que les résultats sont exprimés en chiffres, car c'est sous cette forme seulement qu'ils peuvent être régulièrement comparés ; dès lors, il est nécessaire que le procédé de recherches. soit parfaitement uniforme.

Je décrirai d'abord le procédé des fiches dans sa généralité. J'en indiquerai ensuite les diverses applications.

Après avoir enlevé les téguments et le péricrâne, on marque au crayon chacun des points du crâne dont on se propose d'étudier les rapports. On a préparé d'avance de petites fiches de bois bien sec, larges de 2 millimètres, et terminées en pointe à l'une de leurs extrémités. Dans les recherches relatives à la topographie des circonvolutions superficielles, on se sert de fiches longues de 2 à 3 centimètres; mais, pour déterminer la position des parties profondes, il faut employer des chevilles de 3 à $\breve{~ c e n-~}$ timètres et plus.

Lorsque les points que l'on explore sont peu nombreux et très 
espacés, il n'y a aucune précaution à prendre pour éviter de les confondre entre eux. Mais, lor'squ'on les multiplie davantage, et surtout lorsque quelques-uns d'entre eux sont très rapprochés, il faut, d'une part, en dresser la liste et, d'une autre part, distinguer les fiches les unes des autres, d'après leur longueur, leur couleur, la nature du bois, etc.

On pratique alors, à l'aide d'un instrument quelconque, une petite perforation sur chacun des points du crâne que l'on a marqués. Un simple poinçon suffit pour perforer ainsi les crânes d'enfants.L'instrument le plus commode, sur les crânes d'adultes, est le petit foret à hélice et à mèche d'acier, dont les ouvriers se servent pour percer des trous dans les substances dures. Cet outil, connu sous le nom de drille, se trouve à bas prix chez tous les quincailliers. On choisit une mèche longue de 3 centimètres environ et large de 2 à 2 millimètres et demi ; on tourne rapidement, mais avec une pression très modérée, pour éviter de fausser la mèche et de décentrer l'instrument. En quelques secondes, on a percé toute l'épaisseur de la paroi crânienne; alors, la résistance cessant tout à coup, la mèche perce la duremère et s'enfonce jusqu'à la garde. On la retire et on introduit à sa place l'une des fiches de bois. Celle-ci s'engage dans le trou de la dure-mère, et on la pousse avec un stylet, jusqu'à ce qu'elle ait complètement disparu sous cette membrane. Ce temps de l'opération exige quelque attention; si la cheville n'était pas entièrement dégagée de la dure-mère, on pourrait l'arracher en enlevant cette membrane, et si, pour éviter cet inconvénient, on la poussait trop profondément, elle disparaîtrait dans la substance cérébrale, et il pourrait être difficile de la retrouver. Il faut donc, avant d'introduire la cheville, mesurer avec le stylet l'épaisseur de l'os; en ajoutant à cette épaisseur 2 millimètres pour les membranes, on détermine la profondeur à laquelle le stylet qui pousse la cheville doit être enfoncé.

Lorsque le crâne est épais, le canal osseux dans lequel on engage la cheville est assez long pour la maintenir dans la bonne direction. Mais, chez les sujets dont le crâne est mince, et particulièrement chez les enfants, la fiche peut manquer le trou de la dure-mère et glisser entre cette membrane fibreuse et la paroi crânienne, ou ne pénétrer qu'obliquement dans le cerveau. On 
évite cet inconvénient en explorant le trajet avec le stylet avant d'enfoncer la cheville, et en maintenant attentivement celle-ci dans une direction exactement perpendiculaire à la surface du crâne.

Les perforations ne doivent jamais être faites sur la ligne médiane, qui correspond au sinus longitudinal supérieur; il faut donc, pour atteindre les hémisphères près de leur bord interne, enfoncer les chevilles à $\mathbf{1 5}$ millimètres de la ligne médiane.

Lorsque toutes les chevilles sont en place, on ouvre le crâne à la scie, en faisant passer la coupe circulaire aussi bas que possible. Chez l'adulte, on peut recourir au procédé ordinaire en faisant sauter la calotte; mais, chez les enfants encore jeunes et souvent même jusqu'à l'âge de huit à dix ans, l'adhérence de la dure-mère sur les sutures est tellement forte, que les tractions exercées sur la calotte compriment, déforment et déchirent le cerveau avant de la détacher; il faut donc, après avoir achevé le trait de scie, couper circulairement la dure-mère d'abord à la base du front, puis sur les côtés; on enlève alors à ìa fois le ceryeau, la dure-mère de la voûte, la faux du cerveau et la calotte crânienne, en suivant d'avant en arrière la base du cerveau, comme dans l'extraction ordinaire. Cela fait, il ne reste plus qu'à retourner la pièce et à extraire doucement le cerveau de la calotte. Ce procédé d'extraction, nécessaire chez les enfants, est parfaitement applicable chez les adultes; je le préfère, pour ma part, au procédé ordinaire.

Le cerveau, une fois extrait, est placé surr la table, à côté de la calotte, et on va à la recherche des fiches qui correspondent respectivement aux diverses perforations de la paroi crânienne.

Cette recherche doit se faire d'abord avant l'ablation de la pie-mère, car souvent la fiche est encole en partie engagée dans l'épaisseur de cette membrane; c'est ce qui arrive surtout lorsqu'elle a pénétré dans un sillon ou dans une scissure, et on risquerait alors de l'entraîner en enlevant la pie-mère.

Lorsque les fiches ont été introduites avec un soin suffisant, on les retrouve, pour la plupart, sans difficulté. Mais celles sur lesquelles on a exercé, sans le vouloir, une certaine pression pendant l'extraction du cerveau, peuvent n'être plus apparentes à l'extérieur, et il faut un certain soin pour les retrouver. On sait 
d'ailleurs approximativement, d'après la position des perforations du crâne, quelle doit être la position des chevilles correspondantes. En examinant en cet endroit la surface du cerveau, après l'ablation de la pie-mère, on y aperçoit le trou fait par la cheville, et, d'ailleurs, une légère pression, faite avec la pulpe du doigt, permet de sentir la résistance du bois. Il est facile alors de ramener la fiche à l'extérieur, à l'aide d'une pince fine, introduite dans le trou du cerveau.

En procédant ainsi, on retrouve ordinairement toutes les chevilles; si toutefois l'une d'elles s'était perdue, on y suppléerait aisément à l'aide des autres, car il suffit, à la rigueur, de trois points disposés en triangle pour en déterminer un quatrième, puis un cinquième, et ainsi de suite.

Les fiches doivent rester en place pendant toute la durée de l'observation. On les retire à demi pour qu'elles soient plus apparentes.

Par suite de l'ablation de la pie-mère, les sillons cérébraux se sont écartés, et l'hémisphère, quoique attenant toujours au reste de l'encéphale, s'est notablement étalé. On lui rend à peu près sa forme et ses dimensions en repliant au-dessous de lui un linge épais, de manière à soulever ses deux extrémités, et en prenant pour modèle l'autre hémisphère, dont la pie-mère est encore en place et dont la forme est peu altérée. Malgré cette précaution, la surface du cerveau reste encore un peu plus étalée et, par conséquent, un peu plus grande qu'elle ne l'était dans le crâne; de sorte que les mesures que l'on y prendra seront un peu accrues; mais la surface extérieure dı crâne, avec laquelle on se propose de la comparer, est, elle aussi, un peu plus grande que la surface du cerveau encore en place; il en résulte que les distances comprises entre les fiches cérébrales ne diffèrent pas ou ne diffèrent que très peu des distances comprises entre les trous extérieurs du crâne, et que les mesures prises sur le cerveau peuvent être, sans erreur notable, reportées sur le crâne, sans recourir aux réductions proportionnelles, qui seraient nécessaires, toutefois, si l'on n'avait pas su s'opposer à la déformation de l'hémisphère.

Les fiches représentant sur le cerveau la position des principaux points de la paroi crânienne, il suffit, pour déterminer les 
rapports d'un point quelconque du cerveau, de mesurer en millimètres la distance qui les sépare de telle ou telle fiche; mais, pour que ces mensurations aient une signification réelle, il est indispensable qu'elles soient faites suivant, des directions fixes. Les lignes non orientées seraient tout à fait trompeuses. On ne prendra donc que des distances longitudinales et des distances transversales. Les premières se mesurent dans les plans parallèles au plan médian de la tête. Les autres sont comprises dans des plans perpendiculaires à ce plan médian, sur des arcs transversaux qui partent du bord supérieur et médian de l'hémisphère et se portent de là vers le bord inférieur et externe, et dont la direction, presque horizontale en haut, devient à peu près verticale en bas. Les distances transversales n'expriment donc pas nécessairement l'idée de largeur; ceiles qui sont prises sur les côtés de la tête expriment, au contraire, l'idée de hauteur.

Les petites plaies qui résultent de l'introduction des fiches ne gâtent en rien le cerveau et ne nuisent ni à l'étude ni à la conservation de cet organe; d'un autre côté, les petites perforations de la voûte osseuse ne détériorent nullement le crâne, double avantage, que l'on apprécie surtout lorsqu'on a l'occasion d'étudier des individus appartenant à des races étrangères ou des sujets atteints d'anomalies cérébrales ou de déformations crâniennes, car, dans ces cas, on doit rejeter toute recherche qui entraînerait la mutilation du crâne ou celle du cerveau.

Le nombre et la position des fiches varient suivant le but que l'on se propose d'atteindre. Il peut convenir à un observateur de limiter ses recherches à l'étude d'une région particulière ou même d'un point tout spécial; alors, il n'a besoin de pratiquer qu'une ou deux perforations; si, par exemple, on veut seulement déterminer la position et la direction de la scissure de Rolando, pour connaître les limites du lobe frontal, il suffit d'enfoncer deux fiches dans la suture coronale, l'une à son extrémité inférieure, l'autre près du bregma, et de mesurer des distances longitudinales : l'une rolando-coronale supérieure $(\mathrm{C} r)$, l'autre rolando-coronale inférieure $\left(r^{\prime \prime} k\right)$ (voir fig. 68). Si l'on veut, en outre, connaître la position de la scissure occipitale externe, quilimite le lobe occipital, une troisième cheville, enfoncée dans 
la partie supérieure de la lambdoïde, devient nëcessaire. Enfin, une quatrième perforation, pratiquée sur le point culminant de la suture écailleuse, permet de déterminer, par une mesure transversale, qui est ici une hauteur, la position de la scissure de Sylvius.

Ces quatre fiches suffisent parfaitement lorsque l'on se propose seulement de constater les limites des lobes de l'hémisphère. Les points de repère qu'elles fournissent permettent même de déterminer ensuite, par des mesures longitudinales ou transversales, la position de tout autre point soit du crâne, soit du cerveau, et d'obtenir ainsi, point par point, toute la topographie cranio-cérébrale. Mais ces déterminations successives seraient laborieuses; il est plus simple, plus sûr et surtout plus prompt de les faire directement, à l'aide de nouvelles perforations; on y gagne beaucoup de temps, car, lorsqu'on se sert du drille, l'introduction de chaque fiche se fait en quelques secondes.

Le plan à suivre, dans les recherches partielles, varie donc suivant la nature des questions spéciales que l'on veut étudier. Mais les recherches d'ensemble sont celles qui ont le plus d'importance, et, si l'on veut qu'elles soient comparables entre elles, conformément aux exigences de l'anthropologie, il faut qu'elles soient faites suivant des règles précises et uniformes. Le plan que j’ai adopté dans mes recherches me paraissant répondre à tous les besoins, je crois pouvoir le recommander aux observateurs, après en avoir mainte fois constaté les avantages.

Les divisions naturelles de la surface du crâne sont établies par les lignes des sutures. D'un autre côté, les divisions naturelles de la surface de l'hémisphère sont établies par des dépressions étroites et profondes, qui portent le nom de scissures, lorsqu'elles séparent les lobes, et de sillons, lorsqu'elles séparent deux circonvolutions voisines (1).

(1) Il y a à distinguer les sillons primaires qui limitent les circonvolutions proprement dites ou fondamentales, et les sillons secondaires ou accessoires qui subdivisent plus ou moins certaines circonvolutions. Les sillons primaires sont constants, tandis que les sillons secondaires sont extrêmement variables, non seulement d'individu à individu, mais encore d'un hémisphère à l'autre. Les sillons secondaires n'étant assujettis à aucune règle, ni quant à leur nombre, ni quant à leur disposition, ne peuvent donner lieu à aucune détermination topographique. Nous n'avons donc pas à nous en occuper, et les sillons dont il est question dans le texte sont les sillons primaires. 
L'étude topographique consiste donc à déterminer la situation des scissures et des sillons par rapport aux sutures crâniennes, et on y parvient en reportant sur la surface du cerveau les points les plus propres à indiquer la position des sutures.

La partie la plus essentielle de cette étude est celle qui concerne la limitation des lobes. La recherche des rapports de chaque circonvolution en particulier est un complément intéressant, mais non indispensable. Je parlerai donc d'abord de la topographie des scissures; j'ajouterai ensuite quelques indications sur la topographie des sillons.

$1^{\circ}$ Topographie des scissures. - Les scissures de la surface convexe de l'hémisphère sont au nombre de trois : $1^{\circ}$ la scissure de Rolando, $r, r^{\prime}, r^{\prime \prime}$, qui sépare le lobe frontal du lobe pariétal; $2^{\circ}$ la scissure occipitale externe, $o, o^{\prime}$, qui sépare le lobe pariétal dụ lobe occipital; $3^{\circ}$ la scissure de Sylvius, $s, s^{\prime}, s^{\prime \prime}$, qui sépare le lobe temporal du frontal et du pariétal, et au fond de laquelle est caché le cinquième lobe, celui de l'insula (1).

Six fiches suffisent pour déterminer la position de ces trois scissures, savoir : trois fiches coronales, deux fiches lambdoïdiennes et une fiche temporale.

Les trois fiches coronales $\mathrm{C}, \mathrm{C}^{\prime}, \mathrm{C}^{\prime \prime}$, se placent de la manière suivante : la première, $\mathrm{C}$, fiche coronale supérieure ou bregmatique, pénètre dans la suture coronale, à 15 millimètres environ de la ligne médiane, pour éviter le sinus longitudinal supérieur; la troisième, $\mathrm{G}^{\prime \prime}$, fiche coronale inférieure ou ptérique, correspond à l'extrémité inférieure de cette suture, à l'union du frontal, du pariétal et de la ptère (grande aile du sphénoïde), dans la région désignée sous le nom de ptérion. Quant à la seconde fiche coronale, $\mathrm{C}^{\prime}$, elle se place dans le point appelé stéphanion, et se nomme la fiche stéphanique ou coronale moyenne (2).

(1) Il n'est question ici que de la partie antéro-postérieure de la scissure de Sylvius; la partie transversale ou inférieure de cette scissure appartient à la face inférieure du cerveau, où elle correspond au bord postérieur de la petite aile du sphénoïde.

(2) Les mots ptérion, stéphanion, ainsi que les mots astêrion et obélion, dont il sera question plus loin, font partie de la nomenclature adoptée dans les Instruclions craniologiques de la Société d'anthropologie, Paris, 1875, 1 vol. in-80 de 204 pages, avec figures, planches et tableaux. Voir surtout p. 25 et p. 31. 
Le stéphanion est un point anatomique parfaitement déterminé; c'est le point où la ligne courbe temporale supérieure du pariétal croise obliquement la suture coronale, pour se continuer

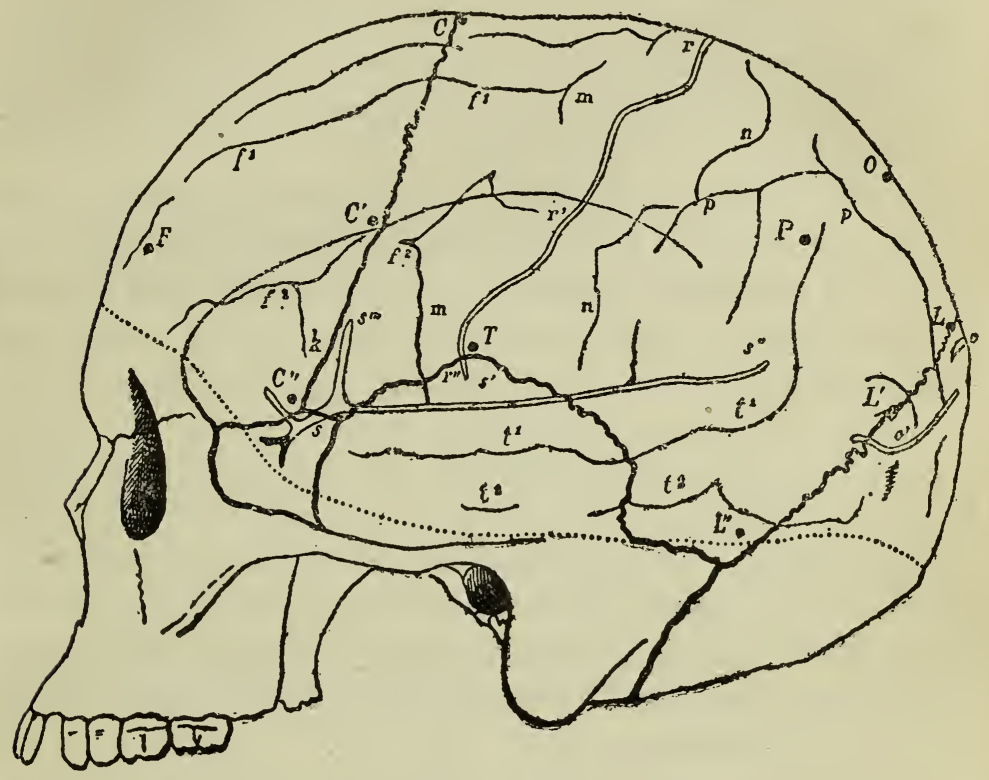

Fig. 68. Topographie cérébrale d'un nègre de l'Afrique orientale (rẻgion égyptienne). Environ quarante ans. Demi-grandeur. Les gros traits représentent les sutures et la ligne temporale. La ligne ponetuée représente la limite inférieure de l'hémis $\rho$ hère. Les scissures eérébrales sont indiquées par des lignes doubles, et les sillons par des lignes simples. On a marqué tous les sillons primaires. On a omis, pour la clarté du dessin, la plupart des sillons secondaires. Les lettres majuseules indiquent la position des fiches. Les petites lettres indiquent les seissures et les sillons.

C, C', C' ', fiches coronales : C, bregmatique; C', stéphanique; C', ptérique ; L, L', L", fiches lambdoïdiennes ; L, interne ; L', moyenne; L", externe ou astérique ; T, fiche temporale ; $F$, frontale ; $P$, pariétale ; $O$, obélique.

Scissures : $r, r^{\prime}, r^{\prime \prime}$, scissure de Rolando ; $o, o^{\prime}$, scissure occipitale externe; $s, s^{\prime}, s^{\prime \prime}$, scissure de Sylvius ; $s^{\prime \prime \prime}$, branche ascendante de la scissure de Sylvius.

Sillons : $f^{\prime}$, premier sillon frontal, séparant la première circonvolution frontale de la seconde ; $f^{2}$, second sillon frontal, séparant la seconde frontale de la troisième : $p$, sillon pariétal, séparant la première circonvolution pariétale de la seconde $t^{4}$, premier sillon temporal ou scissure parallèle; $t^{2}$, second sillon temporal; $m, m$, sillon pré-rolandique, limitant en avant la circonvolution frontale ascendante; chez ce sujet, le sillon pré-rolandique descend jusqu'à la seissure de sylvius; $n, n$, sillon post-rolandique, limitant en arrière la circonvolution pariétale ascendante.

sur le frontal avec la crête temporale. A ce niveau, la disposition de la suture coronale change subitement; cette suture, toujours plus ou moins compliquée au-dessus du stéphanion,

Voir aussi mon mémoire sur l'Ostéologie du crâne et la nomenclalure craniolo. gique, dans Bulletins de la Société d'anthropologie, 1875, p. 349-369. 
devient tout à coup très simple et presque linéaire en descendant vers le ptérion.

La fiche bregmatique, $\mathrm{G}$, pénètre constamment dans la première circonvolution frontale, et la fiche ptérique dans la troisième, immédiatement en avant de la scissure de Sylvius. Quant à la fiche stéphanique, $\mathrm{G}^{\prime}$, elle correspond à peu près, chez l'adulte, à la limite de la deuxième et de la troisième circonvolution frontale.

Les deux fiches lambdoïdiennes, $L, L^{\prime}$, se placent dans la suture lambdoïde, la première à $155^{\circ}$ millimètres de la ligne médiane; la seconde sur le milieu de cette suture, c'est-à-dire à égale distance du lambda, où elle commence sur la ligne médiane, et de l'astérion, où elle se termine sur l'angle externe de l'occipital.

La fiche temporale enfin, $\mathrm{T}$, se place sur le point culminant de la suture écailleuse.

Cela posé, la situation de la scissure de Rolando se détermine par rapport aux fiches coronales : celles de la scissure occipitale par rapport aux fiches lambdoïdiennes; celles de la scissure de Sylvius par rapport à la fiche temporale et à la fiche coronale inférieure ou ptérique.

a. La position de la scissure de Rolando est fixée par la mensuration millimétrique de trois distances longitudinales, savoir :

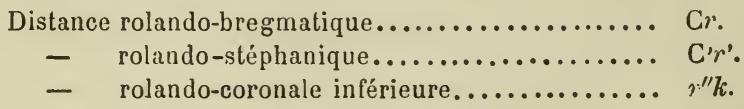

Tous les points de la scissure de Rolando étant constamment situés en arrière de la suture coronale, ces distances sont toujours dans le même sens, et il est dès lors inutile de les affecter d'un signe positif ou négatif.

La distance $\mathrm{Cr}$, qui est sans contredit la plus importante de toutes, pourrait donner lieu, si l'on n'y prenait garde, à de très graves erreurs. C'est la distance comprise entre le bregma et la scissure de Rolando sur le bord supérieur de l'hémisphère. La fiche dite bregmatique n'est jamais située sur ce bord même, mais elle en est très rapprochée, et l'on peut toujours, sans aucune hésitation, placer l'une des pointes du compas sur le vrai 
bregma de l'hémisphère. De ce côté, il n'y a aucune difficulté ; ce qui demande quelque attention, c'est la recherche du point $r$, c'est-à-dire de l'extrémité supérieure de la scissure de Rolando. Lorsque celle-ci n'atteint pas tout à fait le bord de l'hémisphère (ce qui est d'ailleur's assez rare), ou lorsqu'elle se termine exactement sur ce bord (ce qui est beaucoup plus commun), la seconde branche du compas s'applique sur l'extrémité même de la scissure; mais il arrive très souvent que la scissure, parvenue au bord de l'hémisphère, suivant une direction presque transversale - quoique toujours un peu oblique - se réfléchit brusquement, devient antéro-postérieure et se prolonge sur ce bord dans une étendue qui peut varier de 1 à 2 et même 3 centimètres. Or ce prolongement horizontal, tantôt nul, tantôt très long, reporte l'origine de la scissure de Rolando bien en arrière de la limite du lobe frontal, de sorte que, si l'on plaçait le compas sur l'origine même de la scissure, on ajouterait au lobe frontal, aux dépens du lobe pariétal, une longueur très variable et souvent très considérable. C'est cette erreur qu'il s'agit d'éviter ; on y parvient d'ailleurs très aisément, en faisant aboutir la distance rolando-bregmatique sur le point où la scissure de Rolando se détache du bord de l'hémisphère. Quant au prolongement horizontal de la scissure, on peut, si l'on veut, le mesurer, en le dísignant, par exemple, sous le nom de $r$; mais cette mesure n'est pas indispensable; elle a toutefois l'avantage de donner plus de valeur à l'observation, en prouvant qu'on n'a rien négligé pour obtenir correctement la distance $\mathrm{Cr}$.

La distance $\mathrm{C}^{\prime} r^{\prime}$ part directement de la fiche stéphanique et se mesure suivant une ligne horizontale antéro-postérieure, menée de ce point jusqu'à la scissure de Rolando.

La troisième distance, $r^{\prime \prime} k$, que j'appelle rolando-coronale inférieure, sert à déterminer la position du point $r^{\prime \prime}$, où se termine la scissure de Rolando. C'est la longueur d'une ligne horizontale tirée de ce point jusqu'à la rencontre de la suture coronale; elle n'aboutit ni à la fiche stéphanique $\mathrm{C}^{\prime}$, qui, chez l'adulte, est située plus haut, ni à la fiche ptérique $\mathrm{G}^{\prime \prime}$, qui est toujours située plus bas; mais on sait qu'entre ces deux fiches la direction de la suture coronale est à peu près rectiligne, de sorte qu'un fil tendu de l'une à l'autre donne sans erreur la ligne de la suture. Il est 
donc facile de trouver la position du point $k$ (qui varie suivant les sujets) et de mesurer la distance $r^{\prime \prime} k$, qui indique l'étendue du lobe frontal en arrière de la partie inférieure de l'écaille de l'os frontal. Cette mesure, comparée à la mesure $\mathrm{C} r$, qui est toujours beaucoup plus longue, fait connaître le degré d"obliquité de la scissure de Rolando. Quant à la mesure intermédiaire C' $r^{\prime}$, elle pourrait, au premier abord, paraittre superflue ; elle a cependant son utilité, attendu que la scissure de Rolando n'est pas rectiligne; elle décrit ordinairement, vers le niveau de la ligne $\mathrm{C}^{\prime} r^{\prime}$, qui correspond à l'origine de la seconde circonvolution frontale, une flexuosité assez prononcée, à convexité antérieure, de sorte que la distance $\mathrm{C}^{\prime} r^{\prime}$ peut quelquefois devenir moindre que la distance $r^{\prime \prime} k$.

La position du point $r^{\prime \prime}$ n'est pas complètement déterminée par la mensuration de la distance horizontale $r^{\prime \prime} k$; on sait par là jusqu'où s'avance l'extrémité inférieure de la scissure de Rolando; mais on ne sait pas encore jusqu'où elle descend. Cette notion pourrait être obtenue à l'aide d'une mesure transversale donnant la hauteur du point $r^{\prime \prime}$ au-dessus d'une horizontale menée par la fiche ptérique $\mathrm{C}^{\prime \prime}$; mais cela n'est pas nécessaire, car on déterminera tout à l'heure la position de la scissure de Sylvius, qui, comme on sait, passe à quelques millimètres au-dessous de la terminaison de la scissure de Rolando.

$b$. La scissure occipitale externe correspond assez ordinairement, chez les adultes de notre race, à la suture lambdoïde, à quelques millimètres près; toutefois elle peut s'en écarter davantage soit en dessus, soit en dessous. On la détermine à l'aide de deux distances longitudinales, savoir :

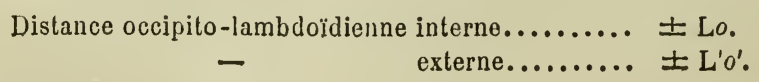

On emploie le signe + lorsque la scissure est en avant de la suture, et le signe - lorsqu'elle est en arrière. Quoique la scissure et la suture soient, en général, à peu près parallèles, il peut arriver qu'elles ne le soient pas exactement et que les deux distances $\mathrm{L} o, \mathrm{~L}^{\prime} o^{\prime}$ ne soient ni égales ni de même signe. Chez les jeunes enfants, la distance Lo est presque toujours positive; elle l'est, en outre, très souvent chez les individus des races infórieures. 
La difficulté de cette détermination résulte des deux plis de passage qui interrompent la scissure occipitale externe et qui souvent la masquent en grande partie. Lorsque l'un des plis de passage est profond, ce qui n'est pas rare, et surtout lorsqu'ils le sont tous deux, ce qui est tout a fait exceptionnel, la scissure est très apparente ; mais le plus souvent ils sont l'un et l'autre superficiels, et il faut quelque attention pour reconnaitre la position de la scissure : on cherche d'abord, sur la face interne de l'hémisphère, la scissure occipitale interne, qui est toujours très manifeste; le point où elle aboutit sur le bord interne de l'hémisphère marque très nettement le commencement de la scissure occipitale externe. Celle-ci est bientôt interrompue par le premier pli de passage, puis par le second, qui tantôt est adossé au premier et tantôt en est séparé par un certain intervalle; dans ce dernier cas, la scissure occipitale reparaît entre les deux plis; dans l'autre cas, elle ne reparaît qu'en dehors du second, après quoi elle s'arrête définitivement sur le troisième pli de passage. C'est ce défaut de fixité de la partie externe de la scissure en question qui en rend la détermination difficile; mais on la retrouve toujours en examinant attentivement la surface du cerveau sur le trajet (ou très près du trajet) d'une ligne transversale menée perpendiculairement au bord interne de l'hémisphère, à partir du point où la scissure occipitale externe vient y aboutir. Si l'on ne tenait pas compte de ces indications, on serait souvent exposé à reporter trop en avant l'origine de la scissure occipitale externe; il arrive, en effet, dans beaucoup de cas, que le premier pli de passage décrit une sinuosité oblique, au-dessus de laquelle la partie interne de cette scissure s'avance obliquement de 1 à 2 centimètres dans le lobe pariétal. Il faut donc savoir que ce n'est pas dans ce prolongement, mais à son origine sur le bord interne de l'hémisphère, que doit aboutir l'extrémité interne de la scissure occipitale externe.

Les distances Lo et $\mathrm{L}^{\prime} O^{\prime}$ sont des distances longitudinales, c'est-à-dire parallèles au bord interne de l'hémisphère ; longitudinales, ici, ne veut pas dire horizontales, car cette partie du bord de l'hémisphère se recourbe vers le bas. Nus deux distances Lo et $\mathrm{L}^{\prime} o^{\prime}$ sont donc perpendiculaires à la direction de la suture lambdoïde LL'. 
$c$. Il reste à déterminer la position de la scissure de Sylvius. La partie inférieure ou basilaire de cette scissure n'est pas en cause ici. Elle apparait sur la face convexe de l'hémisphère, à 4 ou 5 millimètres environ en arrière du point ptérique; c'est donc là que nous placerons son origine. A ce niveau, elle se bifurque en deux branches : l'une courte ou ascendante, l'autre longue ou antéro-postérieure.

La première s'élève dans un repli de la troisième circonvolution frontale; elle n'a plus l'importance qu'on lui attribuait à l'époque où l'on y plaçait la limite du lobe frontal; mais elle a acquis un intérêt d'un autre ordre, depuis que l'on connaît le siège de la faculté du langage, car on sait aujourd'hui que cette faculté est localisée dans la partie de la troisième circonvolution frontale, qui est située en arrière de la branche ascendante de la scissure de Sylvius. Il est facile de constater que cette branche ascendante coïncide assez exactement avec la partie inférieure de la suture coronale. La distance qui l'en sépare est au plus de quelques millimètres, et ce rapport est assez évident, surtout assez constant, pour qu'il ne me paraisse pas indispensable de l'étudier' au moyen de la mensuration.

Il importe, au contraire, beaucoup d'étudier la position et la direction très variables de la branche antéro-postérieure de la scissure de Sylvius, $s, s^{\prime}, s^{\prime \prime}$, qui sépare le lobe temporal du lobe frontal et du lobe pariétal.

Le point d'origine de cette branche, $s$, est déjà connu ; on a vu qu'il est placé à 5 millimètres environ en arrière de la fiche ptérique (1); mais il s'agit de déterminer la direction de la scissure, à l'aide d'un point $s^{\prime}$ pris sur son trajet. On obtient ce point en mesurant la distance sylvio-temporale $\mathrm{Ts}^{\prime}$, distance transversale, qui est comprise entre la scissure et la fiche tem. porale $T$, et qui est ici une hauteur. Le point $s^{\prime}$ est tantôt situé au niveau même du point T, tantôt au-dessus, tantôt enfin audessous de lui ; la distance est nulle dans le premier cas, positive dans le second, négative dans le troisième.

La scissure de Sylvius étant à peu près rectiligne, les deux

(1) Cette distance peut aller à 10 et même 15 millimètres chez les individus qui ont l'os frontal très petit. Je l'ai vue s'élever à 15 millimètres chez un nègre. 
points $s$ et $s^{\prime}$ en indiquent suffisamment la direction. Mais il reste à trouver la position du point $s^{\prime \prime}$, où elle se termine. Or, si l'on mène une ligne de la fiche stéphanique $\mathrm{G}^{\prime}$ à la seconde fich lambdoïdienne $L^{\prime}$, on voit que le point $s^{\prime \prime}$ est toujours situé, à peu de chose près, sur le trajet de cette ligne, qui, d'ailleurs, est à peu près longitudinale; de sorte que les deux lignes $\mathrm{C}^{\prime} s^{\prime \prime}$ et $s^{\prime \prime} L^{\prime}$ sont à peu près longitudinales aussi. On détermine donc la position du point $s^{\prime \prime}$ avec une approximation suffisante, en mesurant la distance sylvio-stéphanique $\mathrm{C}^{\prime} s^{\prime \prime}$ et la distance sylvio-lambdoïdienne $s^{\prime \prime} \mathrm{L}^{\prime}$.

Le tableau des distances à mesurer, pour obtenir la position des trois scissures qui séparent les quatre lobes de la convexité des hémisphères, est donc le suivant :

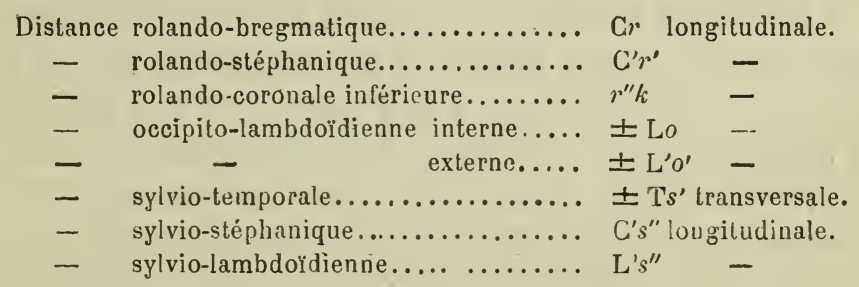

Il faut joindre à ce tableau, pour compléter chaque observation : l'indication de l'âge, du sexe et de la race du sujet; la mesure du diamètre antéro-postérieur maximum du crâne, celle du diamètre transversal maximum et, enfin, la longueur des trois parties de la courbe inio-frontale, savoir : la courbe frontale, la courbe pariétale et la courbe sous-occipitale (ou iniolambdoïdienne).

L'indication de la longueur du prolongement horizontal de la scissure de Rolando $(r r)$ n'est pas indispensable; mais j'ai dit plus haut qu'elle n'est pas sans utilité.

Les observations relatives à la topographie des scissures cérébrales, c'est-à-dire des lobes cérébraux, se trouvent ainsi ramenées à des chiffres rigoureux, qui permettent de comparer les observations, de les grouper en séries et de prendre des moyennes correctes; elles ont surtout l'avantage de rendre comparables entre eux les faits recueillis par des observateurs différents, ce qui est une des premières conditions des recherches anthropologiques. 
$2^{\circ}$ Topographie des sillons cérébraux. - Le procédé d'orientation que j'ai adopté pour constater la position des scissures permet de constater de la même manière la position des sillons cérébraux et, en général, la position d'un point quelconque de la surface du cerveau. De même que, sur un plan, on détermine un point, à l'aide d'une abscisse et d'une ordonnée, et que, sur une sphère, on détermine un point à l'aide d'une longitude et d'une latitude, de même, sur la surface d'un hémisphère où des fiches sont implantées, une mesure longitudinale et une mesure transversale donnent la position d'un point quelconque par rapport à la fiche la plus voisine; je dis la plus voisine, parce que l'hémisphère est toujours déformé et que les effets de cette déformation croissent avec la distance des points que l'on considère.

Ce procédé est excellent lorsqu'on se propose d'obtenir un dessin topographique, comme on le verra tout à l'heure ; mais je doute qu'il soit avantageux de l'appliquer à une étude collective des sillons et à la recherche des moyennes. Les sillons, en effet, sont beaucoup plus variables que les scissures; ils sont, en outre, très souvent flexueux, et, si leur direction générale peut être appréciée, leur direction en un point donné échappe à toute règle. Dans ces conditions, des mensurations rigoureuses feraient perdre beaucoup de temps sans beaucoup de profit.

Je pense donc que l'étude complémentaire des sillons doit se limiter à l'étude d'un petit nombre de rapports. Il n'y a, d'ailleurs, sur la surface crânienne, abstraction faite des sutures, déjà reportées sur la surface cérébrale par nos premières fiches, qu'un très petit nombre de points ou de lignes anatomiques auxquels on puisse rapporter les circonvolutions subjacentes.

Il y a bien, sur l'occipital, une ligne courbe transversale, la ligne occipitale, qui s'étend de l'angle externe de l'occipital, ou astérion, à la protubérance occipitale, ou inion; mais il n'y a pas à chercher les rapports de cette ligne; ils sont déjà connus, puisqu'on sait qu'elle correspond intérieurement à la tente du cervelet, c'est-à-dire à la limite du cerveau (1).

(1) La correspondance de la gouttière latérale de l'occipital, sur laquelle s'insère la tente, et de la ligne courbe occipitale supérieure n'est pas toujours parfaite, et on peut avoir le désir d'étudier les variations de ce rapport; mais cette 
Il y a, en outre, à la base de.l'os frontal, une ligne craniologique, la ligne sous-orbitaire, qui passe transversalement audessus des arcades orbitaires, et qui aboutit aux deux extrémités du diamètre frontal minimum. Gette ligne établit la démarcation entre le crâne cérébral et le crâne facial; elle donne donc la limite antérieure du cerveau, excepté sur la ligne médiane, où la circonvolution olfactive descend dans la fosse olfactive, à une profondeur qu'on reconnaît immédiatement sur le crâne sec, sans avoir besoin de l'étudier sur le cerveau même.

Après cette double élimination, on ne trouve plus, sur le crâne, que deux points qu'il soit utile de reporter sur le cerveau, savoir : le centre de la bosse frontale, F, et celui de la bosse pariétale, $\mathrm{P}$.

Sur les jeunes enfants, ces deux points sont très bien marqués; il y a, en outre, quelques crânes d'adultes, sur lesquels on les retrouve assez approximativement; mais, le plus souvent, leur position reste indécise à plusieurs millimètres près, et souvent même à 1 centimètre près. Pour ce qui concerne la bosse frontale, l'incertitude est ordinairement assez limitée; cette bosse est rarement tout à fait effacée; elle s'annonce par une courbure plus forte, dont on peut marquer le centre sans courir la chance de se tromper de plus de 4 à 5 millimètres; mais la bosse pariétale est souvent tout à fait nulle, et, plus souvent encore, quoique apparente, elle est tellement vague, surtout dans le sens antéropostérieur, que, si l'on n'avait recours à une ligne auxiliaire, on devrait renoncer à y marquer un point de repère.

Pour obtenir cette ligne auxiliaire, on cherche sur la suture sagittale le point le plus élevé de la petite région spéciale que j'ai décrite sous le nom d'obélion (1). Lorsque les bosses pariétales sont bien accusées, elles se trouvent assez exactement sur le trajet d'une ligne menée, par ce point, perpendiculairement à la suture sagittale; par conséquent, lorsqu'elles ne sont pas apparentes, on est certain de ne pas se tromper beaucoup en les

recherche peut se faire sur le cràne sec, et il est dès lors inutile d'en surcharger l'étude de la topographie cérébrale.

(1) Voir Instructions craniologiques, p. 25 ; voir en outre mon mémoire Sur les trous parietaux, dans Bulletins de la Société d'anthropologie, 1875, p. 329, et mon mémoire sur la Nomenclature craniologique, même volume, p. 356. 
supposant placées sur cette ligne. L'incertitude est ainsi considérablement diminuée, puisque ce n'est plus sur une grande surface, mais seulement sur une ligne, que la recherche doit se faire. Rappelons maintenant que la bosse pariétale est toujours située au-dessus de la ligne courbe temporale supérieure du pariétal, ligne qui, à ce niveau, n'est pas toujours bien visible sur le crâne sec, mais qui, sur le crâne frais, est toujours indiquée par l'insertion de l'aponévrose temporale. On examine donc la ligne transversale de l'obélion au-dessus du point où elle vient couper la ligne courbe temporale supérieure du pariétal, et on y marque, comme le centre probable de la bosse pariétale, le point où la courbure est la plus forte.

L'étude complémentaire des sillons cérébraux se fait à l'aide des deux fiches $\mathrm{F}$ et $\mathrm{P}$, placées sur le centre des deux bosses frontale et pariétale; ces deux fiches sont, à la rigueur, suffisantes; toutefois, il est commode de placer en outre une fiche 0 au niveau du point le plus élevé de l'obélion, puisque c'est à l'aide de ce dernier point que l'on est obligé, dans beaucoup de cas, de déterminer la position du point pariétal.

Cela posé, quels sont les sillons dont on peut étudier les rapports topographiques? J'ai déjà dit qu'il ne peut être question des sillons secondaires, mais seulement des sillons primaires, qui séparent les unes des autres les circonvolutions fondamentales; or, ces circonvolutions sont en assez petit nombre et plusieurs d'entre elles sont déjà connues, par cela seul que l'on connaît la position des scissures.

Ainsi, connaissant la situation de la scissure de Rolando, on met aussitôt en place la circonvolution frontale transverse et la circonvolution pariétale transverse (premier et deuxième pli ascendant), parce que ces deux circonvolutions:bordent le sillon de Rolando.

De même, connaissant la scissure de Sylvius, "on connaît trùs bien la position de la première circonvolution"temporale qui la longe, et aussi celle de la seconde temporale, qui est exactement parallèle à la première, n'en étant séparée que par le sillon soussylvien ou scissure parallèle de Gratiolet. La largeur de la première circonvolution temporale, comprise entre la scissure de Sylvius et la scissure parallèle, est peu variable; on peut, néan- 
moins, si l'on veut, la mesurer au compas, et alors la position de la seconde circonvolution temporale sera tout à fait certaine. Quant à la troisième circonvolution temporale, qui est parallèle aux deux précédentes, il n'est pas nécessaire de s'en occuper, car on sait que cette circonvolution forme le bord externe de l'hémisphère et qu'elle correspond, par conséquent, à la base de l'écaille temporale.

Mais il reste à déterminer la position des trois circonvolutions frontale : et des deux circonvolutions pariétales.

Les trois circonvolutions frontales, numérotées de 1 à 3 , naissent, en arrière, sur la frontale transverse et, de là, se portent vers l'extrémité antérieure du cerveau, suivant une direction longitudinale. $\mathrm{La}$ première frontale longe le bord interne de l'hémisphère ; la troisième repose sur la partie antérieure de la scissure de Sylvius; la seconde est comprise entre les deux précédentes, dont elle est séparée par deux sillons longitudinaux que nous appellerons $f^{1}$ et $f^{2}$ (voir la figure 68, p. 517 ). Il suffit donc de connaître la position de ces deux sillons pour connaître la position des trois circonvolutions frontales. Le premier, quoique interrompu par une et quelquefois par deux anastomoses, est toujours facile à reconnaître jusqu'à l'extrémité antérieure de l'hémisphère; il est, d'ailleurs, presque rectiligne et facilement accessible aux mensurations. Le second, plus tortueux, plus interrompu, beaucoup moins net et surtout beaucoup plus variable, donne des mesures moins certaines.

On détermine la position de ces deux sillons à l'aide de la fiche de la bosse frontale $\mathrm{F}$. On mesure la distance $\mathrm{F} f^{1}$ comprise entre la fiche $\mathrm{F}$ et le sillon $f^{1}$; la fiche $\mathrm{F}$ tombe le plus souvent sur la deuxième circonvolution frontale; on donne alors à la mesure $\mathrm{F} f^{1}$ le signe +; on lui donne le signe - lorsque la fiche a pénétré dans la première circonvolution; enfin, le chiffre zéro indique que la fiche est entrée directement dans le sillon (ce qui est assez commun).

La mesure $\mathrm{F} f^{2}$, qui s'exprime sans signe, parce qu'elle est toujours prise dans le même sens, donne la distance de la fiche frontale au second sillon frontal; cette mesure, comme je viens de le dire, manque de précision; elle permet toutefois de reconnaître que le second sillon frontal correspond presque toujours 
d'une manière assez exacte à la crête temporale de l'os frontal. J'ai déjà dit que, plus en arrière, ce sillon passe à peu près sous le stéphanion.

Les deux circonvolutions pariétales, première et seconde, naissent, en avant, sur la pariétale transverse et se dirigent d'avant en arrière; la première, ou supérieure, longe le bord interne de l'hémisphère jusqu'à la scissure occipitale externe, qu'elle franchit sur le premier pli de passage; la seconde, ou inférieure, longe le bord supérieur de la scissure de Sylvius, en arrière de laquelle elle se recourbe, et se divise en deux parties pour se continuer, d'une part, avec la première temporale, et, d'une autre part, par le second pli de passage, avec la seconde circonvolution occipitale.

Entre ces deux circonvolutions pariétales existe le sillon pariétal, sillon profond, longitudinal, quoique un peu oblique en arrière et en dedans, quelquefois interrompu par un pont, souvent étendu, sans interruption, jusqu'à la scissure occipitale (souvent même ce sillon se prolonge à travers la scissure occipitale jusque près de la pointe du lobe occipital, de manière à séparer la première circonvolution occipitale de la seconde). Nous désignerons ce sillon pariétal sous le nom de $p$ et nous en déterminerons la position, par rapport à la fiche pariétale $\mathrm{P}$, à l'aide de la mesure $\mathrm{P} p$, prise sur une ligne menée de cette fiche $\mathrm{P}$ à la fiche de l'obélion 0 .

La fiche pariétale tombe à peu près constamment en dehors du sillon pariétal. Il n'est pas impossible toutefois qu'elle tombe en dedans, sur la première circonvolution pariétale. Si ce cas se présentait, on donnerait à la mesure $\mathrm{P} p$ le signe -, mais il est, à coup sûr, très exceptionnel, de sorte qu'il est inutile d'employer le signe + . On peut donc convenir que l'absence de signe indiique une valeur positive.

Les circonvolutions occipitales sont trop étroites et trop peu importantes pour mériter de donner lieu à des recherches topographiques spéciales. On peut, d'ailleurs, en obtenir approximativement la position à l'aide des données précédentes. Le sillon longitudinal, qui sépare la première circonvolution occipitale de la seconde, se trouve, en effet, sur le prolongement de la ligne qui indique le trajet du sillon pariétal, $p$, et on sait, d'autre part, 
qu'il va aboutir à la pointe du lobe occipital. Si donc, du point $p$ déjà reporté sur le crâne, on mène une ligne oblique aboutissant à l'inion, cette ligne représentera à la fois, sur l'os pariétal, le sillon pariétal, $p$, qui sépare la première circonvolution pariétale de la seconde, et, sur l'os occipital, le sillon occipital, qui sépare la première circonvolution occipitale de la seconde.

Ainsi, il suffit d'ajouter aux six fiches des sutures les trois fiches de la bosse frontale, de la bosse pariétale et de l'obélion pour mettre en place tous les sillons primaires et, par conséquent, toutes les circonvolutions de la face externe de l'hémisphère.

$3^{\circ}$ Dessins topographiques. - J'ai dit déjà que les dessins topographiques, qui sont la base nécessaire de toute recherche faite suivant les procédés graphiques, ne jouent aucun rôle dans les recherches faites suivant, le procédé des fiches. C'est l'avantage principal de ce dernier procédé de ne pas exiger l'intervention de dessins compliqués, dont l'exécution impose à l'observateur une très grande perte de temps. Mais l'utilité des dessins topographiques, comme moyen de démonstration, est incontestable, et sous ce rapport, comme on va le voir, le procédé des fiches répond à tous les besoins.

Ces dessins peuvent être de deux ordres: ils peuvent être schématiques ou d'après nature.

Les dessins schématiques sont destinés à représenter l'état moyen des rapports cérébro-crâniens qui ont été constatés sur une série d'individus. J'ai dit plus haut de quelle manière on les obtient; j'ai montré, en même temps, que le procédé des fiches en facilite beaucoup l'exécution (voir plus haut, p. 507 et 508 ). Je n'y reviendrai donc pas ici ; je ne parlerai que des dessins individuels, faits d'après nature.

Le procédé des fiches permet de les obtenir sous deux formes bien différentes, soit sur un plan, soit sur le relief du crâne.

a. Dessins plans. - On dessine d'abord, par un procédé quelconque, suivant la norma que l'on a choisie, la surface du crâne de l'individu mis à l'étude. Sur cette surface, on marque la position des fiches. Les six fiches des sutures et les trois fiches complémentaires destinées à l'étude des sillons cérébraux pourraient parfaitement suffire; il y a avantage toutefois à enfoncer une dixième fiche dans l'angle postérieur et inférieur du parié- 
tal, à 1 centimètre environ au-dessus de l'astérion. (Si l'on enfonçait la fiche astérique dans l'astérion même, on n'atteindrait pas le cerveau, on tomberait entre le cerveau et le cervelet.)

En se guidant sur les fiches et en marquant les distances, on reporte sur le dessin crânien tous les points significatifs de la surface du cerveau. Toutefois, comme le dessin est plan, tandis que le crâne et le cerveau sont convexes, les distances doivent être réduites proportionnellement aux raccourcis de la perspective ou des projections; le degré de cette réduction est indiqué dans chaque région par la comparaison des distances réelles, comprises entre les fiches de la région, et des distances apparentes, mesurées sur le dessin entre ces mêmes fiches.

Pour dessiner une scissure, on en marque l'origine et la terminaison; au besoin, si elle est onduleuse, on marque un ou deux points sur son trajet ; alors on la dessine en prenant le eerveau pour modèle, de manière à reproduire entre les points marqués les détails de conformation de la scissure.

On dessine d'abord les scissures, puis les sillons primaires, et sur ce canevas on inscrit ensuite aisément jusqu'aux moindres détails des sillons et plis secondaires.

Ces dessins n'ont pas l'exactitude absolue de ceux que donne le procédé de Landzert, mais ils sont tout aussi précis que ceux du procédé de Turner.

b. Dessins sur le relief du crâne. - Voici maintenant une espèce de dessin qui ne peut être obtenue que par le procédé des fiches, et qui constitue en faveur de ce procédé une supériorité tout à fait hors ligne. Les dessins plans ne montrent bien que la partie centrale de la surface qu'ils représentent;le reste se dénature de plus en plus à mesure que la surface se recourbe; aussi M. Heftler s'est-il trouvé obligé de dessiner séparément, sur autant d'individus différents, les quatre norma du crâne, pour muntrer tous les rapports topographiques. Les dessins sur le relief du crâne ont, sur les dessins plans, toute la supériorité que possèdent les mappemondes sphériques sur les cartes géographiques. Une seule pièce permet d'étudier tous les rapports et d'apprécier partout les formes et les dimensions.

Ces dessins en relief se font sur le crâne même, ou plutôt sur la calotte crânienne. J'ai déjà dit que la coupe des autopsies doit 
être faite très bas, de sorte que toutes les fiches soient situées sur la calotte. Celle-ci, bien dépouillée de son périoste, bien essuyée, séchée au besoin pendant quelques instants devant le feu ou au soleil, fournit une surface sur laquelle on dessine très bien avec un crayon tendre. Les traits manqués s'effacent avec la plus grande facilité, comme sur une ardoise. On a déjà, comme points de repère, les perforations sur le crâne et les fiches sur le cerveau ; la position des autres points s'obtient au compas, et presque sans réduction des distances ; car il n'y a à tenir compte que de la déformation du cerveau, toujours légère en un point donné, sans se préoccuper des raccourcis. Dès lors, le dessin des scissures, puis des sillons, soit primaires, soit secondaires, s'effectue aisément et sans grande perte de temps. J'ai fait un grand nombre de fois ces dessins en relief sans dépenser plus d'une heure et demie pour représenter complètement un hémisphère cérébral.

En repassant le dessin à la plume, on pourrait conserver la calotte dans cet état; mais on sait que les os non macérés deviennent gras et malpropres, et la macération effacerait le dessin ; il faudrait donc, avant de faire macérer la pièce, graver le dessin au burin ; je l'ai fait quelquefois ; c'est un travail assez long.

Il est bien préférable de faire le dessin en relief, non pas sur la calotte même, mais sur un moule en plâtre de cette calotte. Rien de plus facile à faire que ce moule. Le dessin une fois fait, on peint chaque lobe d'uue couleur particulière et chaque circonvolution d'une nuance particulière. Les scissures et sillons sont marqués en noir; enfin on peint en blanc le trajet des sutures. Puis on vernit la pièce, et elle peut servir indéfiniment pour les démonstrations aussi bien que pour les recherches.

J'ai fait pour le musée d'anthropologie une assez grande série de ces moules topographiques. Ils nous rendent les plus grands services. Les procédés graphiques ne peuvent donner rien de pareil, puisqu'ils exigent le sacrifice des parois crâniennes. M. Heftler, après avoir relevé sur ses divers dessins les distances moyennes des sutures et des scissures, les a reportées sur un crâne ordinaire, et à l'aide des points de repère qu'il a ainsi obtenus, il a pu faire sur le crâne un dessin schématique des 
circonvolutions cérébrales. G'est un bon moyen de démonstration, auquel d'ailleurs tous les procédés se prêtent également. Mais le procédé des fiches est le seul qui permette de montrer les rapports réels, en représentant le cerveau d'un individu sur son propre crâne.

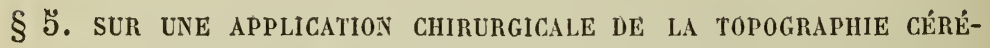
brale (trépanation du crane). — topographie de l'organe dU LANGAGE.

En signalant plus haut les divers genres d'utilité des connaissances topographiques (voir p.498), j'ai fait allusion à un cas où j'ai pu, grâce à elles, ouvrir du premier coup, avec une couronne de trépan, un abcès intra-crânien. Quoique l'issue ait été défavorable, l'indication opératoire a été atteinte avec la plus grande précision. Le lecteur me permettra donc de lui présenter ici cette observation, mais aupararant il me paraît utile de donner quelques explications qui en feront ressortir l'importance.

Je rappellerai d'abord qu'il y a dans le cerveau un petit district très circonscrit, une portion de circonvolution, qui préside à la fonction du langage articulé, et que j'appelle l'organe du langage. Cet organe occupe environ les deux cinquièmes postérieurs de la troisième circonvolution frontale de l'hémisphère gauche. C'est la partie de cette circonvolution qui est limitée en avant par la branche verticale de la scissure de Sylvius, en arrière par la partie inférieure de la scissure de Rolando, en bas par la branche horizontale de la scissure de Sylvius, en haut enfin par le deuxième sillon frontal $\left(f^{2}\right)$, qui sépare la troisième circonvolution frontale de la seconde. L'étendue de cet espace quadrilatère est de 3 à 4 centimètres de haut en bas; il n'est que de 25 à 35 millimètres dans le sens antéro-postérieur; mais la partie correspondante de la troisième circonvolution décrit des méandres profonds et serrés, et elle est beaucoup plus longue que la région qu'elle occupe.

Connaissant la forme générale et les dimensions de la région du langage, nous connaîtrons la position de ce quadrilatère si nous déterminons celle de son bord antérieur et de son bord inférieur. Son bord antérieur, formé par la branche verticale de 
la scissure de Sylvius, correspond assez exactement, à mètres près, à la partie inférieure de la suture coronale. Son bord inférieur est formé par la branche horizontale de la scissure de Sylvius. Celle-ci, dans sa partic antérieure, n'est pas rectiligne; elle se recourbe pour gagner transversalement la base du cerveau, en arrière de la fiche ptérique, $\mathrm{C}^{\prime \prime}$. Mais, si l'on marque un point à 1 centimètre au-dessus de la fiche ptérique, et si de là on mène une ligne droite tangente à la suture ćcailleuse, cette ligne représente assez exactement la direction et la position de la scissure de Sylvius.

Sur le cadavre une couronne de trépan dont le centre est appliqué sur le pariétal, à 1 centimètre et demi en arrière de la suture coronale, et à 2 centimètres au-dessus de la scissure de Sylvius, tombe invariablement sur la région du langage, et le plus souvent très près du centre de cette région.

Mais ce procédé n'est pas applicable à l'lomme vivant, car le chirurgien ne peut mettre à nu la surface du crâne dans une étendue suffisante pour s'assurer de la position des diverses sutures du voisinage. C'est donc par un autre procédé qu'on doit aller à la recherche de la région du langage, lorsqu'on juge nécessaire d'y pratiquer la trépanation.

On trouve très bien, sur le vivant, le point où la base de l'apophyse orbitaire externe se recourbe et se relève pour se continuer avec la crête temporale de l'os frontal. Ce point est celui où aboutit la ligne sus-orbitaire, et oû l'on mesure, en céphalomé. trie, le diamètre frontal minimum.

Menons, par ce point, à travers la fosse temporale, une ligne horizontale sur laquelle nous prenons une longueur de 5 centimètres. Nous obtenons ainsi un second point, qui correspond à peu près à la pointe du lobe temporal. Par ce second point élevons sur la ligne précédente une perpendiculaire sur laquelle nous mesurons 2 centimètres, et nous aboutissons à un troisième point qui correspond à peu près au centre de la région du langage, qui en d'autres termes est situé, dans le sens horizontal, à égale distance de la scissure de Rolando et de la branche montante de la scissure de Sylvius, et, dans le sens vertical, à égale distance du second sillon frontal $\left(f^{2}\right)$ et de la branche horizontale de la scissure de Sylvius. 
Les variations individuelles, qui sont très grandes dans la partie supérieure des hémisphères, sont au contraire assez limitées dans leur partie inférieure, où elles n'excèdent pas 1 centimètre, où elles sont par conséquent inférieures au diamètre d'une couronne de trépan. Un procédé opératoire basé sur les indications précédentes permet donc de tomber sûrement sur l'organe du langage.

ll s'agit de savoir maintenant s'il peut se présenter des cas où cette trépanation soit opportune. Ce n'est pas ici le lieu de discuter la doctrine chirurgicale du trépan dans les plaies de tête. Au dix-huitième siècle, on trépanait sans cesse, « même dans les cas douteux ॥; on trépanait pour les accidents primitifs comme pour les accidents consécutifs, pour les simples fêlures comme pour les fractures avec enfoncement ; on trépanait même pour prévenir des accidents qui n'existaient pas encore. La chirurgie actuelle a rejeté ces opérations dangereuses et aventureuses; elle n'admet plus le trépan que dans des cas très exceptionnels, parce qu'on a reconnu d'une part que la plupart des anciennes "indications » du trépan sont illusoires, et d'une autre part que le diagnostic du lieu affecté est le plus souvent incertain.

Pourtant, parmi ces anciennes " indications », il en est une qui, en principe, n'est pas contestable : c'est celle qui résulte de l'existence d'un abcès traumatique dans la cavité du crâne. Les épanchements de sang peuvent se résorber et se résorbent trèssouvent, mais les épanchements de pus sont presque nécessairement mortels, et tout le monde reconnaît qu'il serait très désirable de pouvoir leur donner issue ; le blessé, sans doute, ne serait pas guéri pour cela ; il aurait encore bien des dangers à courir ; toutefois on aurait supprimé une cause de mort et obtenu une chance de salut. Pourquoi donc a-t-on renoncé à trépaner les blessés chez lesquels on diagnostique un abcès intra-crânien ? Parce que le siège des abcès intra-crâniens, de ceux surtout qui sont tardifs, n'est assujetti à aucune règle, parce que ces abcès peuvent se former en un point quelconque, près ou loin de la plaie ou du point qui a reçu la violence extérieure, et parce que le hasard seul pourrait faire tomber la couronne de trépan sur le lieu où le pus est collecté. C'est cette incertitude qui a fait bannir presque entièrement de la chirurgie actuelle la trépana- 
tion des abcès intra-crâniens. Mais, s'il arrivait qu'un symptôme particulier fît connaître le siège du foyer de suppuration, ou le rendît du moins assez probable pour ne laisser subsister que de faibles chances d'erreur, l'abstention ne serait plus justifiée.

Or les notions que nous possédons aujourd'hui sur le sièg'e du langage articulé dans un point bien déterminé du cerveau fournissent à la chirurgie un moyen de diagnostic d'une grande valeur. Lorsqu'une blessure de la tête abolit ou altère la parole sans abolir l'intelligence et sans paralyser les muscles de la langue, on peut être certain que l'organe spécial du langage est lésé; et lorsque ce symptôme survient seulement au bout de quelques jours ou de quelques semaines, avec d'autres symptômes qui révèlent l'existence d'une suppuration intra-crânienne, on doit considérer comme très probable que l'abcès occupe, ou comprime, ou irrite l'organe du langage. On est donc autorisé à pratiquer en ce point la trépanation.

C'est dans un cas de ce genre que j'ai été conduit à faire une application chirurgicale des notions de la topographie cérébrale. En voici l'observation :

\section{Diagnostic d'un abcès situé au niveau de la région du langage;} trépanation de cet abcès.

Pierre Baron, trente-huit ans, charretier, fut admis le 27 juin 1871 à l'hôpital de la Pitié et placé dans mon service, salle Saint-Louis, $\mathrm{n}^{\circ} 56$.

Il avait reçu, le 26 juin, dans la région fronto-pariétale gauche, un coup de pied de cheval qui avait produit une plaie contuse et une dénudation des os. Légèrement étourdi sur le coup, il n'avait pas perdu connaissance, il n'était même pas tombé. Laplaie avait peu saigné, le blessé était rentré chez lui à pied, et le lendemain il était venu à pied à l'hôpital.

Je constatai une plaie contuse longue de 6 à 7 centimètres. Dans la partie moyenne de cette plaie, l'os était à nu dans une étendue de 25 millimètres. La partie dénudée ne présentait ni trait de fracture ni ligne de suture; elle me parut correspondre au pariétal, près de son bord antérieur, vers le niveau de la ligne temporale. 
La plaie fut pansée avec de l'eau frâtche; elle se comporta très bien pendant les premiers jours; les bourgeons charnus se formèrent régulièrement et recouvrirent bientôt entièrement la surface osseuse dénudée. Le blessé n'avait pas eu la fièvre un seul instant ; il se levait ; il passait la plus grande partie de ses journées sous les arbres de la cour. Les bords de la plaie étaient déjà en voie de cicatrisation rapide, lorsque, le 11 juillet, quinzième jour de l'accident, il survint un érysipèle du cuir chevelu, sous l'influence d'une épidémie d'érysipèle qui régnait dans la salle.

Cet érysipèle provoqua une fièvre intense, sans aucun délire, gagna rapidement toute la tête, puis la nuque et une partie du dos; au bout de onze jours, l'érysipèle était guéri, et l'état géné. ral paraissait excellent. Ce qui restait de la plaie était recouvert de bourgeons charnus exubérants. Mais le samedi 24 juillet une hémorrhagie artérielle se déclara. Le sang venait de la partie inférieure et postérieure de la plaie, et paraissait fourni par une branche de la temporale profonde. L'interne de garde appliqua un bouton de feu, et l'hémorrhagie ne reparut plus.

Le dimanche 24 juillet, vingt-neuvième jour après l'accident, journée excellente.

Le lundi, le malade est inquiet, impatient; il s'ennuie à l'hôpital; il accuse un peu de céphalalgie. Point de fièvre.

Le mardi 25 juillet, même état; dans la journée plusieurs vomissements.

Le mercredi matin, 26 juillet, il est dans un grand abattement: 84 pulsations; température, 38 degrés. Depuis la veille il ne répond plus aux questions, et les infirmiers croient qu'il est devenu sourd; mais nous nous assurons qu'il entend très bien le bruit de la montre. Lorsqu'on l'interroge, le plus souvent il ne répond pas du tout; si on le presse davantage, il répond invariablement: Ca ne va pas mal, et il répète cette phrase plusieurs fois de suite. Par exemple : « D. Savez-vous où vous êtes? R. Ca ne va pas mal. - D. Savez-vous quelle heure il est? R. Ca ne va pas mal. "Il est évident, d'après cela, que la faculté du langage est altérée et je diagnostique une altération de la troisième circonvolution frontale gauche.

Le mercredi soir, stupeur. Il ne répond que par des sons inar- 
ticulés, mais il comprend ce qu'on lui dit ; quand on le lui demande, il serre la main, ouvre la bouche, tire la langue. La face est déviée à gauche; la main droite est paralysée du mouvement; la sensibilité du membre supérieur droit est considérablement diminuée. La langue n'est pas déviée et se meut librement en tous sens. Les membres inférieurs et le membre supérieur gauche ont conservé leurs mouvements et leur sensibilité. Pouls à 72 ; température, $38^{\circ}, 2$.

Le jeudi matin, 27 juillet, trente-deuxième jour après l'accident, le malade est dans le coma depuis minuit. Les deux membres gauches ont conservé leur sensibilité et se meuvent lorsqu'on les pince ; les deux membres droits sont tout à fait insensibles; ils sont dans le relâchement; toutefois, de légers mouvements qui s'y produisent lorsqu'on excite les parties sensibles du reste du corps prouvent que l'action motrice n'est pas complètement abolie dans ces membres. La paralysie faciale persiste. Respiration stertoreuse. Pupilles égales et contractées. Pouls à 60 .

Ces symptômes tardifs, développés seulement à partir du vingt-neuvième jour, chez un blessé qui n'avait pas éprouvé d'accidents primitifs appréciables, révélaient l'existence d'une suppuration intra-crânienne, survenue probablement sous l'influence pyogénique de l'érysipèle. Le siège de cette suppuration était évidemment du côté gauche, puisque la paralysie n'affectait que les membres du côté droit. En outre, il y avait eu, avant l'apparition de cette paralysie, une période courte, mais décisive, pendant laquelle l'altération de la faculté du langage, coïncidant avec la conservation de l'intelligence, avait été constatée bien nettement, sous une forme qui se présente fréquemment à l'observation.

Je crus donc devoir admettre que le début du travail de suppuration s'était effectué au niveau de l'organe du langage articulé, c'est-à-dire au niveau de la partie postérieure de la troisième circonvolution frontale gauche, et je résolus de trépaner le crâne en ce point pour aller à la recherche du pus. L'abcès était-il situé sur la dure-mère, sous la dure-mère, sous la pie-mère, ou enfin dans l'épaisseur même de la circonvolution? Cette question restait douteuse, et j'annonçai aux élèves que si le pus n'apparaissait pas après l'ablation de la rondelle crâ- 
nienne, je n'hésiterais pas à ponctionner la dure-m̧ère pour aller le chercher dans les parties plus profondes.

Je déterminai d'abord, d'après les données topographiques indiquées ci-dessus (p. 532 et 533), le lieu où devait être appliquée la couronne du trépan. Sur une ligne horizontale menée par la base de l'apophyse orbitaire externe, je pris une longueur de ๖ centimètres; puis, sur l'extrémité postérieure de cette ligne, j'élevai une perpendiculaire longue de 2 centimètres et $\mathrm{j}$ 'arrivai ainsi sur le point à trépaner. Ce point était situé sur la partie de la plaie extérieure qui était encore en suppuration, et sur la limite inférieure de la surface qui avait été mise à nu par le coup de pied de cheval. La plaie était devenue trop étroite pour donner passage à une couronne de trépan; je l'élargis à l'aide d'une incision. Pour mettre l'os à nu, je n'eus, vers le haut, qu'à enlever les bourgeons charnus qui avaient recouvert, sans y adhérer, la surface dénudée par l'accident, mais vers le bas le péricrâne était adhérent, et je dus me servir de la rugine. Ma couronne de trépan empiétait donc par moitié sur la partie de l'os qui n'avait pas été atteinte par le coup. La trépanation ne présenta rien de particulier. Au moment où j'enlevai la rondelle, il s'écoula une assez grande quantité de pus crémeux et blanc. En explorant le foyer que je venais d'ouvrir, 'je reconnus qu'il s'étendait surtout vers le bas, et, pour faciliter l'écoulement du pus, j'appliquai une seconde couronne au-dessous de la première, en empiétant un peu sur celle-ci, de manière à n'obtenir qu'une seule ouverture. La face externe de la dure-mère fut nettoyée ; elle parut intacte.

La quantité de pus qui s'écoula en tout représentait le volume d'un œuf de pigeon ; mais, quelque satisfaisant que fût le résultat opératoire, je ne me dissimulais pas qu'un épancliement de ce volume, situé en dehors de la dure-mère, n'était pas suffisant pour expliquer, à lui seul, toute la gravité des symptômes cérébraux ; je supposai donc qu'il devait exister des altérations plus profondes; toutefois je ne crus pas devoir aller plus loin et j'attendis l'événement.

Le blessé, pendant l'opération, avait, par ses mouvements, manifesté quelque sensibilité ; mais il n'avait poussé aucun cri et n’avait donné aucun signe de connaissance. Quand je le 
quittai, à onze heures du matin, il était encore dans le coma.

L'interne du rang, M. Mauquier, resta auprès de lui toute la juurnée. Vers midi, l’opéré reprit connaissance. Il répondait par signes à l'appel de son nom ; il ne parlait pas, mais il faisait des efforts pour parler et il montra plusieurs fois qu'il comprenait les questions qu'on lui adressait. On lui uffrit à boire; il prit son verre de la main gauche et le porta à sa bouche. Le bras droit n'était plus relâché, mais légèrement contracturé. Le pouls était remonté à 80 . Cette amélioration dura jusqu'à quatre heures de l'après-midi, mais alors le blessé retomba dans le coma, et il y était encore à six heures du soir, lorsque je vins le voir avec mon collègue et ami M. Trélat, dont j’avais demandé le concours.

Il était alors à peu près dans le même état qu'avant l'opération. Nous supposâmes donc qu'il devait y avoir un autre foyer de suppuration sous la dure-mère, peut-être dans la scissure de Sylvius; quoique le blessé fût dans un état où il n'y avait plus rien à perdre, je n'osai pas, sur cette hypothèse, enfoncel un bistouri dans la dure-mère, et $\mathrm{M}$. Trélat pensa comme moi qu'avant de prendre une aussi grave détermination il convenait de pratiquer une ponction exploratrice.

Je pris donc un trocart fin, large de 1 millimètre, et je l'enfonçai à travers la dure-mère, dans l'axe de la seconde couronne de trépan, en un point qui devait correspondre à la scissure de Sylvius. J'avançai lentement, retirant fréquemment le poinçon ; il ne s'écoula aucun liquide, si ce n'est une gouttelette de sang, à la profondeur de క̆ millimètres. Au delà, plus rien; à 15 millimètres, je m'arrêtai etje retirai la canule.

Cette exploration ne produisit aucun effet immédiat. A neuf heures du soir, frisson assez intense. Dans la nuit, quelques gémissements, sans parole articulée.

Le vendredi 28 juillet, à sept heures du matin, il survient une convulsion épileptiforme surtout dans les membres du côté droit: deux autres convulsions semblables dans l'heure suivante.

A neuf heures du matin, heure de la visite, coma et stertor. Paralysie complète de la sensibilité et du mouvement dans les deux membres droits. Pouls, 130. Température, $39^{\circ}, 5$. Respiration inégale et embarrassée. 
Dans la journée, les symptômes s'aggravent, et le blessé meurt le samedi 29 juillet à une heure du matin.

Autopsie. - Le crâne est ouvert à la scie. Il ne présente ni fracture ni fèlure. Il n'y a entre le crâne et la dure-mère aucun foyer de suppuration autre que celui qui a été ouvert par le trépan.

L'hémisphère droit est sain, ainsi que ses membranes. Il existe au contraire, sur l'hémisphère gauche, des lésions très étendues.

La partie de la dure-mère qui était en contact avec le pus de l'abcès trépané présente, sur sa face externe, une coloration noirâtre; à ce niveau, et à ce niveau seulement, l'arachnoïde pariétale est unie à l'arachnoïde viscérale par des adhérences molles et récentes. La pie-mère est épaissie, friable et adhérente dans toute la partie qui recouvre les deuxième et troisième circonvolutions frontales, la frontale et la pariétale transverses et les deux premières circonvolutions temporo-sphénoïdales; elle est, en outre, rendue opaque par une infiltration de pus qui, partant de la scissure de Sylvius, s'étend le long des vaisseaux sur la face externe des circonvolutions voisines. Dans la partie antérieure de la scissure de Sylvius cette infiltration est assez abondante pour former une nappe continue qui ressemble à un abcès ; mais, lorsqu'on l'incise, le pus ne coule pas ; il est infiltré dans les membranes et on ne peut l'en extraire que par pression. Ces membranes, épaissies et purulentes, qui remplissent et dilatent la partie antérieure de la scissure de Sylvius, adhèrent à la troisième circonvolution frontale, et lorsqu'on les enlève, on enlève en même temps la couche corticale de cette circonvolution, qui est rouge, enflammée et ramollie. L'encéphalite superficielle diffuse dont cette circonvolution est le siège s'étend, en s'atténuant avec la distance, sur toute la seconde circonvolution frontale, mais non sur la première, qui est saine. En avant, sur la pointe du lobe frontal, les lésions de la méningo-encéphalite s'aggravent de nouveau, sans toutefois atteindre le degré de la suppuration. En ce point, lorsqu'on enlève la pie-mère, on aperçoit dans la couche corticale des détritus de sang et de matière cérébrale, formant plusieurs foyerš irréguliers dus à une contusion superficielle du cerveau.

En examinant attentivement lá surface interne de la dure- 
mère, nous y retrouvons la trace de la ponction exploratrice pratiquée trente et une heures avant la mort ; mais rien, ni sur les membranes viscérales, ni dans la substance cérébrale, n'indique la position du trajet parcour'u par l'aiguille. Celle-ci, d'après la situation du trou de la dure-mère, doit avoir pénétré dans la scissure de Sylvius sans atteindre les circonvolutions voisines.

L'étude comparative des faits anatomo-pathologiques et des faits cliniques conduit à l'interprétation suivante. Le coup de pied de cheval a produit à la fois une plaie avec dénudation du pariétal gauche et une secousse cérébrale, d'où est résultée la contusion superficielle de la pointe du lobe frontal gauche. Cette contusion, comme on le voit souvent, n'a donné lieu à aucun accident primitif et n'a été le point de départ d'aucun accident consécutif. L'érysipèle qui s'est développẻ le quinzième jour autour de la plaie a fait naître des conditions pyogéniques et provoqué la formation d'un foyer purulent entre le crâne et la duremère ; l'irritation, partie de ce foyer, s'est propagée à travers la dure-mère et l'arachnoïde, a atteint ainsi la pie-mère de la scissure de Sylvius et s'est étendue de là à la surface du cerveau, sous forme de méningo-encéphalite diffuse. C'est donc l'abcès formé entre les os et la dure-mère qui a été le point de départ des accidents cérébraux, et, en effet, le premier organe cérébral qui ait manifesté son altération a été l'organe du langage, situé directement sous l'abcès. La trépanation est venue trop tard; l'abcès qu'elle a permis de vider avait déjà produit des complications irréparables; c'est pourquoi l'évacuation du pus n'a amené qu'une amélioration passagère, et le blessé a succombé à la méningo-encéphalite diffuse, que rien ne pouvait plus conjurer.

Les cliniciens apprécieront l'intérêt chirurgical de cette observation, mais je dois faire ressortir les deux points qui intéressent les anthropologistes. Ce sont :

$1^{\circ}$ Le diagnostic du siège de l'abcès intra-crânien, basé sur la connaissance du siège de la faculté du langage articulé ;

$2^{\circ}$ La trépanation de cet abcès, basée sur la connaissance des rapports cérébro-crâniens. 


\section{TOPOGRAPHIE GRANIO-GÉRÉBRALE}

\section{POSITION RELATIVE DES DIVERSES PARTIES DU CRANE}

\section{ET DES DIVERSES PARTIES DU GERVEAU}

(Revue d'anthropologie (Revue critique), 1876, t. V, p. 278-279.)

Anatomie comparée du système nerveux, par Gratiolet, t. II, p. 11 ๖̆ et 124. Paris, 1857, in-8 ${ }^{\circ}$ - Sur le siège de la faculté du langage articulé, par P. Broca, dans Bulletins de la Société anatomique, 1861, $2^{\mathrm{e}}$ série, t.VI, p. 340 , en note. Die Grosshirnwindungen des Menschen, par Th. Bischow ; Munich, 1868, in $-4^{\circ}$, p. 20. - Sur la déformation toulousaine du crâne, par P. Broca, dans Bulletins de la Société d'anthropologie, $2^{\mathrm{e}}$ série, t. VI, p. 104, août 1871. - Des circonvolutions chez l'homme et de leurs rapports avec le crâne (en russe), par Ferd. Heftler. Dissertation inaugurale à l'Académie médico-chirurgicale de Saint-Pétersbourg, 5 mai 1873, in- $8^{\circ}$. - On the Relations of the Convolutions of the Human Cerebrum to the outer Surface of the Skull and Head, par W. Turner, dans the Journal of Anatomy and Physiology, série II, $\mathrm{n}^{\circ}$ xIII, p. 142, novembre 1873. - An Illustration of the Relations of the Convolutions of the Human Cerebrum to the outer Surface of the Skull, par W. Turner, dans le même journal, no xiv, p. 3ò9, may 1874. - Note sur quelques points de la topographie du cerveau, par Ch. Féré, dans Bulletins de la Société anatomique, 24 décembre 1875. Sur un cas de lésion probable du pli courbe, par le même. Comptes rendus, Société de biologie, 26 février 1876. - Die topographischen Beziehungen zwischen Schædel und Gehirn im normalen Zustand, in Gratulationsprogramm Hern Louis Stromeyer, par Alexandre Ecker; Brunswick, 1876, in- $8^{\circ}$.

L'intérêt croissant qui s'attache à l'étude des localisations cérébrales justifie l'étendue de l'article que j'ai consacré, dans ce numéro, à la question de la topographie cérébro-crânienne. Je me suis surtout proposé, dans cet article, de faire connaître les procédés de recherche usités jusqu'ici et d'en apprécier la valeur pratique, afin de vulgariser ces études et de les mettre à la portée de tout le monde. Quant aux résultats obtenus, soit par les autres observateur's, soit par moi-même, j’ai pu, accessoire- 
ment, en indiquer quelques-uns, mais je ne les ai ni exposés ni discutés, parce que, au point de vue anthropologique où je me suis placé, ces résultats, basés sur des observations trop peu nombreuses ou trop peu variées, sont tout à fait insuffisants. Il ne s'agit pas, en effet, de mettre à l'étude tel ou tel cas particulier, ni de prendre les moyennes d'un certain nombre de faits quelconques, mais d'établir méthodiquement des séries suivant les âges, les sexes, les races, et suivant les degrés de dolichocéphalie ou de brachycéphalie du crâne. J'ai recueilli depuis quinze ans un grand nombre de faits topographiques; j'en ai vu assez pour savoir que ces diverses conditions exercent une grande influence sur certains rapports cérébro-crâniens et notamment sur la position des trois scissures de la face convexe de l'hémisphère; mais, pour déterminer exactement cette influence, il faudrait disposer, dans chaque catégorie spéciale, d'une série suffisamment nombreuse. Je n'en suis pas encore là. Les moyennes que je pourrais tirer de quelques-unes de mes séries seraient acceptables, mais les autres manqueraient de solidité, et ce qui serait trompeur surtout, ce seraient les moyennes générales obtenues par la fusion de ces diverses séries. Voilà pourquoi, dans le mémoire publié en tête de ce nuinéro, je me suis borné à présenter le plan de mes recherches, sans y ajouter l'exposé des résultats que j'ai obtenus et que je ne puis considérer que comme provisoires. Je me suis abstenu, pour le même motif, d'y consigner les descriptions publiées par les autres observateurs ; je n'aurais pu le faire, d'ailleurs, sans sortir du cadre que je m'étais tracé. Il m'a paru bien préférable de leur faire une place à part dans cet article complémentaire, où le lecteur pourra plus aisément les étudier et les comparer. Je donnerai donc successivement l'analyse des publications de MM. Heftler, Turner et Féré. J'y joindrai l'extrait d'un travail tout récent de $M$. le professeur Alex. Ecker, de Fribourg en Brisgau, publié le 6 avril 1876, à l'occasion du jubilé du professeur Louis Stromeyer, de Hanovre. Les feuilles de mon mémoire sur la topographie cérébrale étaient déjà tirées lorsque j’ai reçu ce travail. Je n'ai donc pu faire figurer dans mon historique le procédé de l'auteur. Je suis heureux, du moins, de pouvoir combler ici cette lacune. 
On the Relations of the Convolutions of the Human Cerebrum to the outer Surface of the Skull and Head, par W. Turner (Sur les rapports des circonvolutions du cerveau humain avec la surface externe du crane ct de la tête), dans Journal of Anatomy and Physiology, série II, no xirr, novembre 1873, p. 142-148, et no ${ }^{\circ}$ Iv, may 1874, p. 359-361.

(Revue d'anthropologie (Revue critique), 1876, t. V, p. 285-289.)

L'auteur a conçu, depuis plusieurs années, le plan de ces recherches; le temps lui a manqué pour les exécuter complètement; il se borne donc à rapporter quelques faits principaux, en attendant le mémoire plus étendu qu'il se propose de publier ultérieurement. Il parlera alors des particularités qu'il a constatées chez les femmes et des différences individuelles dues à des conformations particulières du crâne et du cerveau ; aujourd'hui, il ne traitera que des rapports observés sur des têtes d'hommes adultes.

Il est, avant tout, nécessaire de cencevoir clairement, sur la surface externe des crânes et des têtes que l'on examine, des lignes de démarcation bien définies. La protubérance occipitale externe, les bosses pariétales et frontales, l'apophyse orbitaire externe, se reconnaissent aisément à travers les chairs de la tête et, plus aisément encore, sur le crâne dénudé. Les sutures coronales et lambdoïdes peuvent, en outre, être reconnues sur beaucoup de têtes. Sur le crâne, enfin, on aperçoit non seulement toutes les sutures, mais encore la ligne courbe temporale.

A l'aide de ces dispositions anatomiques, on peut subdiviser chaque courbe latérale de la tête ou du crâne en dix régions ou aires bien définies, de manière à localiser dans ces aires les circonvolutions de la face externe de l'hémisphère.

Ces dix aires sont circonscrites par les lignes des sutures, auxquelles on ajoute les lignes suivantes: $1^{\circ}$ la ligne courbe tem. porale, qui part de l'apophyse orbitaire externe, s'étend sur le frontal, puis sur le pariétal et va aboutir à l'angle externe de l'occipital; $2^{\circ}$ la ligne verticale du pariétal, menée par la bosse pariétale et prolongée, en haut, jusqu'à la suture sagittale, en bas, jusqu'à la suture squameuse; $3^{\circ}$ la ligne frontale, menée par la bosse frontale parallèlement à la ligne médiane et se 
MORPHOLOGIE DU CERVEAU DE L'HOMME ET DES PRIMATES. 545

terminant, en haut, à la suture coronale, en bas, au bord supé. rieur de l'orbite.

Sur la figure ci-jointe, dont M. Turner a bien voulı nous envoyer le cliché, ces lignes auxiliaires sont marquées par des traits pleins; des traits sinueux plus fins indiquent les sutures; les lettres inscrites sur chaque aire sont parfois masquées par

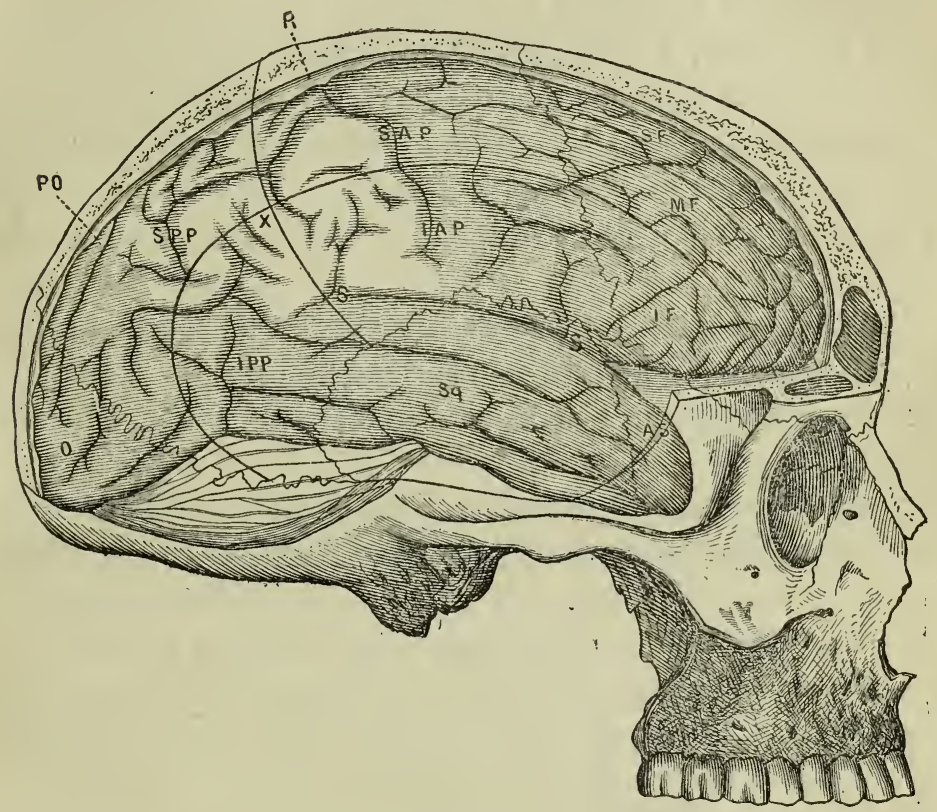

Fig. 69.

les ombres du dessin, mais on les retrouvera avec un peu d'attention, surtout à l'aide d'une loupe.

Cela posé, la région frontale ou précoronale se trouve divisée en trois aires, savoir : $1^{\circ}$ aire frontale supérieure (SF), comprise entre la ligne médiane et la ligne frontale; $2^{\circ}$ aire frontale moyenne (MF), entre la ligne frontale et la ligne temporale; $3^{\circ}$ aire frontale inférieure (IF), entre la ligne temporale et la suture fronto-sphénoïdale. Ces trois aires se terminent en arrière sur la suture coronale.

La région pariétale est subdivisée par la ligne pariétale en deuxzones, l'une pariétale antérieure ou post-coronale, l'autre 
pariétale postérieure ou prélambdoïdienne. Chacune de ces deux zones est subdivisée à son tour par la ligne courbe temporale en deux parties, l'une supérieure, l'autre inférieure; de sorte que la surface du pariétal se décompose en quatre aires, savoir: $4^{\circ}$ pariétale antéro-supérieure $(\mathrm{SAP}) ; 5^{\circ}$ pariétale antéro-inférieure (IAP) ; $6^{\circ}$ pariétale postéro-supérieure $(\mathrm{SPP}) ; 7^{\circ}$ pariétale postéro-inférieure (IPP).

Le frontal et le pariétal sont les seuls os subdivisés par les lignes auxiliaires. Les trois dernières aires correspondent donc chacune à un os différent, savoir : $8^{\circ}$ aire occipitale $(0)$, formant la région occipitale ou post-lambdoïdienne; $9^{\circ}$ aire squameuse $(\mathrm{S} q)$, correspondant à l'écaille du temporal; $10^{\circ}$ aire sphénoödale ou ali-sphénoüdale (AS), correspondant à la grande aire du sphénoïde.

Les deux dernières aires, étant recouvertes par le muscle temporal, ne peuvent être déterminées que sur le crâne; les autres peuvent le plus souvent être limitées sur la tête ellemême:

Position des scissures cérébrales. - La scissure de Sylvius commence immédiatement derrière le bord postérieur de la grande aile du sphénoïde et, dans son trajet en arrière et en haut, est recouverte par la suture sphéno-pariétale ; elle pa:se alors obliquement sous la partie antérieure et supérieure du temporal et apparaît dans la partie inférieure de l'aire pariétale antéroinférieure, qu'elle traverse de manière à aboutir, soit par ellemême, soit par l'une des petites branches dans lesquelles elle se divise souvent, à l'aire pariétale postéro-inférieure.

La scissure de Rolando est située dans la région post-coronale ou pariétale antérieure; elle traverse obliquement, de haut en bas et d'arrière en avant, les deux aires supérieure et inférieure de cette région. La distance de cette scissure à la suture coronale varie suivant les sujets. Je l'ai vue, en haut, s'élever à 2 pouces et descendre à 1 pouce et demi; en bas, je l'ai vue varier entre 1 pouce et demi et 1 pouce trois quarts.

La scissure pariéto-occipitale (ou occipitale externe) est située dans l'aire pariétale postéro-supérieure, près de son bord sagittal. La distance qui la sépare du sommet de la suture lambdoïde varie, d'une part, suivant les variations du cerveau lui-même; 
d'une autre part, suivant les fréquentes variations de l'ossification du sommet de l'écaille occipitale; cette distance est, en moyenne, de 7 à 8 dixièmes de pouce.

Les rapports de la bosse pariétale avec l'hémisphère paraissent très constants. Dans les pièces que j’ai examinées, celte bosse correspondait à la circonvolution supéro-marginale, qui, d'après cela, pourrait être appelée la circonvolution de la bosse pariétale.

L'auteur donne alors les rapports de chacune des aires crâniennes:

$1{ }^{\circ}$ L'aire pariétale antéro-inférieure recouvre le tiers inférieur. des deux circonvolutions ascendantes (pariétale et frontale), puis, en avant, l'origine de la frontale inférieure (troisième circonvolution frontale), qui naît de la frontale ascendante à un peu moins de 1 pouce en arrière de la suture coronale, et, enfin en arrière, l'origine de la circonvolution de la bosse pariétale. Dans la partie inférieure de cette aire, au-dessous des circonvolutions ascendantes, apparaît un autre segment de la scissure de Sylvius, et, en outre, tout en arrière, une petite partie de la circonvolution temporo-sphénoïdale supérieure apparaît au-dessus de la suture squameuse (1);

$2^{\circ}$ L'aire pariétale antéro-supérieure comprend les deux tiers supérieurs des deux circonvolutions ascendantes, puis, en avant, l'origine des circonvolutions frontales supérieure et moyenne (première et deuxième circonvolution frontale); la première commence à 1 pouce et 2 ou 3 dixièmes et la seconde à 1 pouce environ en arrière de la suture coronale. Derrière la circonvolution pariétale ascendante, on trouve en dedans une petite partie du lobule pariétal, et en dehors une petite partie de la circonvolution de la bosse pariétale;

$3^{\circ}$ L'aire pariétale postéro-inférieure comprend la partie postérieure de la circonvolution de la bosse pariétale, derrière elle le gyrus angulaire (pli courbe), et plus bas l'extrémité postérieure des trois circonvolutions du lobe temporo-sphénoïdal ;

(1) Ces deux derniers rapports sont indiqués dans le texte publié en novembre 1873 ; mais ils ne se retrouvent pas sur la figure, qui représente un individu dessiné plus tard et qui a été publiée en mai 1874. On peut voir sur cette figure que la scissure de Sylvius et la circonvolution temporale supérieure ne se rencontrent pas au-dessous de la suture squameuse. 


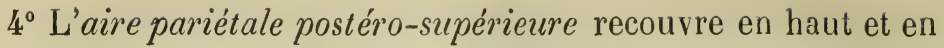
dedans le lobule pariétal, plus en dehors le gyrus angulaire et une partie de la circonvolution de la bosse pariétale;

$5^{\circ}$ Dans l'aire occipitale ou post-lambdoïdienne, qui est relativement petite, les trois circonvolutions du lobe occipital se succèdent de dedans en dehors;

$6^{\circ}$ L'aire frontale inférieure comprend principalement la circonvolution frontale inférieure (troisième frontale), et en outre, à son angle supérieur et postérieur, une petite partie de la circonvolution frontale moyenne;

$7^{\circ}$ L'aire frontale moyenne comprend la circonvolution frontale moyenne (deuxième frontale);

$8^{\circ}$ L'aire frontale supérieure correspond exactement à la circonvolution frontale supérieure (première frontale);

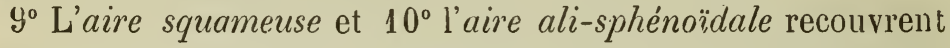
les deux tiers antérieurs des circonvolutions du lobe temporosphénnïdal.

Les lobes de l'hémisphère ne correspondent pas exactement aux os crâniens dont ils portent les noms. Le lobe frontal déborde l'os frontal et empiète considérablement sur l'os pariétal. Le lobe occipital déburde en outre l'os occipital, en empiétant un peu sur la partie postérieuredel'aire pariétale postéro-supérieure.

La circonvolution temporo-sphénoïdale supérieure, quoique recouverte par l'écaille du temporal, remonte un peu sur les deux aires pariétales inférieures.

La surface de l'os pariétal est donc entamée en avant, en arrière et en bas par les trois lobes qui entourent le lobe pariétal.

Le lobe central de l'insula, caché au fond de la scissure de Sylvius, est placé vis-à-vis la partie supérieure de la grande aile du sphénoïde et des sutures qui unissent celle-ci au pariétal et à l'écaille temporale.

L'auteur rappelle, en terminant ce premier travail, qu'il n'a pas tenu compte des variations qui peuvent être en rapport avec le sexe, la race et les particularités individuelles.

Dans un second travail pulié en mai 1874, M. Turner expose le procédé qu'il a suivi dans ses recherches. Il connaissait le procédé des fiches, employé par M. Broca et par M. Bischoff. Ce procéde, dit-il, très convenable lorsqu'on ne se propose que de 
déterminer les limites des lobes, demanderait beaucoup trop de temps et de travail si l'on voulait déterminer la position des circonvolutions en particulier. Il a donc procédé autrement. A l'aide d'une scie fine, il découpe successivement les contours des divcr'ses aires, et, après l'ablation de chaque pièce il fait dessiner les circonvolutions correspondantes. Il donne ensuite une figure topographique qu'il a fait dessiner par M. C. Berjeau, d'après une préparation qu'il a faite pendant le courant de l'hiver. Cette figure, qui a été reproduite plus haut (page 545"), est accompagnée de l'explication suivante:

$\mathrm{R}$, scissure de Rolando, séparant le lobe pariétal du lobe frontal.

Po, scissure pariéto-occipitale (occipitale externe), séparant le lobe pariétal du lobe occipital.

SS, seissure de Sylvius, séparant le lobe temporo-sphénoïdal des lobes frontal et pariétal.

$\mathrm{SF}, \mathrm{MF}$, IF, les trois aires supérieure, moyenne et inférieure de la région frontale. Les lettres sont placées respectivement sur les trois circonvolutions frontales.

SAP, aire pariétale antéro-supérieure. La lettre $\mathrm{S}$ est placée sur la circonvolution pariétale ascendante; les lettres $\mathrm{AP}$, sur la frontale ascendante.

IAP, aire pariétale antéro-inférieure. La lettre I est placée sur la circonvoJution pariétale ascendante; les lettres $\mathrm{AP}$, sur la frontale ascendante.

SPP, aire pariétale postéro-supérieure. Las lettres sont placées sur la circonvolution angulaire.

IPP, aire pariétale postéro-inférieure. Les lettres sont placées sur la circonvolution temporo-sphénoïdale moyenne.

$\mathrm{X}$, circonvolution de la bosse pariétale, ou sus-marginale.

0 , aire occipitale. La lettre est placée sur la circonvolution occipitale moyenne.

$\mathrm{S} q$, aire squameuse. Les lettres sont placées sur la circonvolution temporosphénoïdale moyenne.

AS, aire ali-sphénoïdale. Les lettres sont placées sur la pointe de la circonvolution temporo-sphénoïdale supérieure. 


\section{SUR UN CAS}

\section{DE LÉSION PROBABLE DU PLI COURBE}

(Comptes rendus de la Société de biologie, 1876 , et Revue d'anthropologie (Revue critique), 1876, t. V, p. 295-296.)

Le pli courbe de Gratiolet forme la partie postérieure et externe du lobe pariétal. On sait que M. Ferrier, dans ses expériences sur les animaux, a vu l'excitation électrique du pli courbe produire la contraction des muscles des paupières et de l'œil.

Une observation recueillie par M. Féré à l'hospice de Bicêtre tend à confirmer jusqu'à un certain point cette opinion.

Il s'agit d'un homme de cinquante-huit ans observé à l'infirmerie de l'hospice, dans le service de M. Bouchard.

Cet homme, étant en état d'ivresse, fit au mois de mai 1871 une chute dans un escalier. La partie postérieure de la tête heurta sur l'angle d'une marche. Il en résulta une plaie profonde des téguments et une fracture du crâne avec enfoncement. Sur le coup, le blessé perdit connaissance : il ne revint à lui qu'au bout de dix à douze heures. Au réveil, il s'aperçut que son œil gauche était le siège d'un mouvement convulsif intermittent ; bientôt il remarqua que ce mouvement convulsif s'étendait en outre à la commissure labiale du même côté. Au bout de six semaines, la plaie était guérie, mais le tic persista.

Quelque temps après, cet homme fut atteint de rhumatisme articulaire généralisé et d'une douleur cervicale qui rendit les mouvements de rotation du cou très difficiles. Cette affection devint chronique, et au bout de onze mois le malade fut admis comme infirme à l'hospice de Bicêtre. C'est là que M. Féré a eı récemment l'occasion de l'observer.

Le tic facial a persisté depuis près de cinq ans sans changr ment notable. Les mouvements convulsifs viennent par accè, qui durent de dix à trente minutes, et qui sont séparés par ds intervalles de calme d'une durée à peu près égale. Ces intervalis 
sont plus longs lorsque le malade reste immobile. Les accès ne sont accompagnés d'aucune douleur ; ils sont constitués par des contractions convulsives, involontaires, très rapprochées et très courtes, comme des battements; ces contractions occupent à la fois l'orbiculaire des paupières de l'œil gauche et le muscle grand zygomatique du même côté ; elles s'accompagnent d'une certaine agitation du globe de l'œil, mais ces mouvements de l'œil sont peu étendus; il n'est pas certain qu'ils soient produits par les muscles de l'œil ; ils peuvent dépendre seulement du spasme de l'orbiculaire. Le muscle grand zygomatique est, avec l'orbiculaire, le seul muscle de la face qui prenne part à la convulsion.

Ce tic ayant paru en mai 1871 , immédiatement après la fracture du crâne, M. Féré a pensé qu'il était la conséquence d'une lésion cérébrale produite par cette fracture.

En examinant la région sur laquelle le coup a porté, il y a trouvé une dépression, irrégulièrement quadrilatère, longue et large d'environ 15 millimètres et profonde de 2 à 3 millimètres. Cet enfoncement est situé sur la partie droite et postérieure du crâne, à la l'encontre d'une ligne transversale menée par le lambda (qui est très apparent chez cet homme) et d'une ligne antéro-postérieure menée par la base de l'apophyse orbitaire externe; il se trouve à environ 1 centimètre en avant d'une ligne verticale passant par le bord postérieur de l'apophyse mastoïde. En reportant ces lignes sur le schéma topographique du crâne, M. Féré a reconnu que l'enfoncement produit par le coup correspondait à la partie postérieure du pli courbe. Pour s'en assurer, il a fait, sur sept cadavres, une perforation dans le point désigné, et chaque fois la fiche a pénétré dans la partie postérieure du pli courbe. Un de ses collègues, M. Mayor, a répété cette expérience avec le même résultat.

Il est donc extrêmement probable que le tic facial a été produit ar une lésion du pli courbe. Cette conclusion est presque aussi ûre que si elle était déduite directement d'une autopsie.

En rapprochant ce fait intéressant des recherches expérimentles de M. Ferrier, on comprendra toute l'étendue des services que la topographie crânio-cérébrale est appelée à rendre à la pysiologie et à la médecine. 


\title{
LES RAPPORTS TOPOGRAPHIQUES
}

\author{
ENTRE
}

\section{LE GRANE ET LE GERVEAU}

\author{
A L'ÉTAT NORMAL ${ }^{2}$
}

(Revue d'anthropologie (Revue critique), 1876, t. V, p. 296-298.)

M. le professeur Ecker, chargé par la Faculté de médecine de Fribourg-en-Brisgau de présenter un " gratulationsprogramm " à l'illustre professeur Louis Stromeyer, à l'occasion de son jubilé de doctorat, qui a été célébré le 6 avril 1876, a choisi, comme sujet de dissertation, “l'influence des déformations artificielles du crâne sur le volume, la forme et la situation du cerveau et de ses diverses parties ». Dans ce mémoire, adressé à un chirurgien qui a fait faire de grands progrès à l'orthopédie, M. Ecker a désigné les déformations artificielles du crâne sous le nom de scoliopédie du crâne. Orthopédie signifie redressement des enfants, et scoliopédie, d'après l'étymologie grecque, signifie courbure des enfants. Cette opposition de mots est un hommage délicat rendu aux travaux de M. Stromeyer; c'est un contraste entre la chirurgie savante et bienfaisante qui rétablit les formes naturelles et la coutume barbare qui les altère d'une manière aussi nuisible que ridicule; et à ce point de vue le titre du "gratulationsprogramm " de M. Ecker est heureusement choisi. Mais il est douteux que ce néologisme d circonstance paraisse utile aux anthropologistes, car, comme le mots usités jusqu'ici, il exprime seulement l'idée de déform:

(1) Die topographischen Beziehungen zwischen Schxdel und Gehirn im n'malen Zustand, par Alexandre Ecker. C'est le chapitre Iv, p. 16-18, d'un rémoire de l'auteur Sur les déformations artificielles du cräne. Brunswick, 18c், in $\cdot 4^{\circ}$. 
tion, et il faut de toute nécessité y joindre une épithète, afin que l'on puisse savoir s'il s'agit de déformations pathologiques ou de déformations artificielles.

Le mémoire de M. Ecker repose sur l'examen de six crânes déformés de l'Orégon et d'un crâne de l'Alaska, qu'il a étudiés et décrits avec sa précision et sa sagacité habituelles. Ces crânes étaient déformés suivant le type connu sous le nom anglais de flatheads, en français têtes plates. L'auteur s'est naturellement demandé quelle influence ces graves déformations exerçaient sur le développement et le volume relatifs des divers lobes des hémisphères cérébraux ; et, à défaut du cerveau lui-même, il a étudié la conformation générale de cet organe sur le moule intra-crânien de l'un des crânes les plus déformés. Reportant alors sur le moule, suivant le procédé de Gratiolet, le dessin des sutures du crâne, il a pu reconnaître approximativement la situation et les rapports des principales parties des hémisphères.

Il est bien malheureux que plusieurs cerveaux de flatheads, recueillis à Vancouver par le docteur Bessels, aient été perdus dans un naufrage. M. Ecker, à qui ces pièces précieuses étaient destinées, aurait pu ajouter à l'étude des déformations artificielles un chapitre important et entièrement nouveau ; car l'observation que j'ai pu faire sur un cas de déformation toulousaine ne peut donner qu'une idée tout à fait insuffisante des effets prodıits sur le cerveau par les grandes déformations américaines.

Pour apprécier, sur son moule intra-crânien, le degré de changement des rapports des lobes cérébraux, M. Ecker s'est occupé d'abord de l'étude de ces rapports à l'état normal. Il a consacré à cette question, dans son mémoire, un chapitre dont je donne ici l'analyse.

L'auteur rappelle d'abord, dans un court historique, les travaux de ses devanciers, à l'exception toutefois de la thèse de M. Heftler, qui n'est pas encore parvenue à sa connaissance, et qui, avant le présent numéro de la Revue d'anthropologie, n'avait reçu aucune publicité en dehors de la Russie. M. Ecker mentionne donc seulement les recherches de M. Bischoff, les miennes et celles de M. Turner. Il en donne un résumé sommaire qu'il serait superflu de reproduire ici, puisque ces travaux ont été analysés plus haut (p: 485,488 et 492 ); j'aurai toutefois 
une petite remarque àfaire concernant les dates. M. Ecker attribue la priorité des recherches topographiques et l'invention du procédé des fiches à M. Bischoff, dans le mémoire daté de 1868, et il ajoute: "Broca a suivi un procédé semblable à celui de Bischoff, dont il n'a point d'ailleurs cité le travail. " A cela je réponds que le procédé des fiches et les premiers résultats qu'il m'a fournis ont été publiés dans mon Mémoire sur le siège de la faculté du langage, qui a paru en 1861, sept ans avant le mémoire de M. Bischoff.

Après avoir exposé le procédé de M. Turner, M. Ecker continue en ces termes:

« La méthode suivante donne, je pense, un contrôle plus sûr : j'ai scié une tête fraîche sur la ligne médiane, après avoir indiqué la position des sutures à l'aide de fiches introduites suivant le procédé de Bischoff-Broca (une tête congelée conviendrait encore mieux) ; alors j’ai extrait les deux moitiés du cerveau : je dessine au moyen du diopter (de Lucæ) les principaux sillons et circonvolutions avec les fiches, et, faisant reposer les deux hémisphères horizontalement sur leur plan de section, je les conserve dans une solution de chlorure de zinc et, plus tard, dans l'alcool. Prenant alors les deux moitiés du crâne, j'enlève la dure-mère, je dessine sur leur face interne, avec de la laque blanche, le trajet des sutures, et j'y coule du plâtre (probablement du plâtre coloré). Sur le moule intra-crânien ainsi obtenu, les sutures se dessinent en blanc, et lorsqu'on compare le moule avec le cerveau, les rapports de celui-ci avec la surface de celuilà se laissent apprécier avec toute l'exactitude désirable. Sur le crâne bien développé d'un jeune homme de ce pays, j’ai constaté ainsi que l'extrémité médiane du sillon cérébral (scissure de Rolando) était à 38 millimètres en arrière du point médian de la suture coronale, et que son extrémité externe était à 17 millimètres en arrière de la même suture. Celle-ci passait sur l'opercule, au niveau du commencement de la scissure de Sylvius. De là, pendant que cette scissure se portait en haut et en arrière, la suture écailleuse, s'en séparant à angle aigu, se dirigeait à peu près horizontalement en arrière en passant sur le lobe temporal, et en suivant, dans une petite étendue, le trajet du sillon temporal. La suture lambdoïde venait la rejoindre au niveau 
MORPHOLOGIE DU CERVEAU DE L'HOMME ET DES PRIMATES. 5ว5 environ de la limite du lobe temporal et du lobe occipital, et remontait à peu près sur la scissure pariéto-occipitale (scissure occipitale externe). Le sommet de la suture lambdoïde se trouvait à 7 millimètres en arrière de l'extrémité médiane de cette scissure. ")

La partie du mémoire de M. Ecker qui concerne la déformation des flatheads, présente beaucoup d'intérêt, mais ce n'est pas ici le lieu d'en donner l'analyse. 


\section{NOTE}

SUR

\section{LA TOPOGRAPHIE CÉRÉBRALE}

ET SUR

QUELQUES POINTS DE L'HISTOIRE DES CIRCONVOLUTIONS

(Bulletins de l'Académie de médecine, séance du 8 août_1876, p. 824-834.)

J'ai publié récemment, dans la. Revue d'anthropologie, un mémoire sur la topographie cérébrale ou sur les rapports anato. miques du crîne et du cerveau. Ce mémoire s'adresse surtout aux anthropologistes, mais le sujet qui y est traité a aussi un intérêt médical, et c'est à ce dernier point de vue que je viens en entretenir l'Académie.

Cette étude est toute récente. Si l'on s'en rapportait aux apparences, on pourrait la croire plus ancienne, car il y a déjà bien longtemps que certains lobes cérébraux ont été désignés sous le nom des os qui les recouvrent ; mais ces apparences sont trompeuses, car ce qu'on appelait alors le lobe frontal, ce n'était pas une partie déterminée du cerveau, c'était simplement la portion de cet organe qui se trouvait au-dessous de l'os frontal; quant ì la limitation sur le cerveau même, il ne pouvait en être question à une époque où on ne connaissait ni le nombre ni les connexions des circonvolutions cérébrales, et où l'on croyait que celles-ci étaient aussi désordonnées que les circonvolutions de l'intestin grêle. Il eût été complètement superflu de chercher à déterminer les rapports de ces circonvolutions que l'on croyait essentiellement variables et qui n'avaient pas encore reçu de nom. On possédait cependant déjà quelques notions de nature à 
indiquer que les plis et les sillons de la surface du cerveau étaient assujettis à certaines règles. La partie antérieure de la scissure de Sylvius était connue depuis le seizième siècle. A la fin du dix-huitième siècle (1786), Vicq-d'Azyr avait figuré et décrit comme constantes les circonvolutions du corps calleux, les deux circonvolutions du sillon olfactif, et aussi les deux circonvolutions ascendantes que nous nommons la frontale transverse et la pariétale transverse. Depuis lors, plusieurs anatomistes, parmi lesquels il faut citer surtout Rolando, Burdach, Valentin, Foville, avaient commencé à débrouiller le chaos des circonvolutions, en signalant parmi les détails de la surface du cerveau ceux qui paraissent les moins variables; Leuret enfin, ouvrant la véritable voie, celle de l'anatomie comparée, avait passé en revue toute la série des mammifères. Il avait constaté d'abord que les caractères des circonvolutions sont invariables dans chaque famille d'animaux, puis il avait reconnu que le type cérébral des singes était le même que celui de l'homme, qu'il en était en quelque sorte l'ébauche ou l'embryon. "Le cerveau du papion, disait-il (pl. XV), est un petit encéphale d'homme, ou plutôt un très grand encéphale de fœetus humain ; les circonvolutions cérébrales y sont en même nombre que chez l'homme, mais elles n'ont pas plus d'ondulations que celles d'un fœetus de six à sept mois. ) Il avait donc classé et décrit les circonvolutions du papion, et, passant aussitôt à l'homme (́pl. XVI), il avait pu lui appliquer la même description, la même nomenclature, les mêmes lettres, les mêmes signes. Mais la mort ne lui permit pas de compléter son œuvre; ce parallèle fécond de l'homme et des primates, il n'eut pas le temps de le pousser jusqu'au bout; il ne vit pas la scissure occipitale, il ne distingua pas le lobe occipital ; enfin plusieurs circonvolutions, primaires ou secondaires, d'une grande importance, échappèrent à son attention, et s'il eut le mérite de frayer la voie, ce ne fut pas lui qui eut la gloire d'atteindre le but.

Cette gloire était réservée à Gratiolet. Leuret n'avait étudié qu'un seul singe, le papion, Gratiolet étudia toute la série des singes et surtout les anthropoïdes, d'autant plus précieux à observer qu'ils sont plus voisins de l'homme. Pour rendre les comparaisons plus faciles et plus claires il plaça, au-dessous du 
cerveau compliqué de l'homme blanc adulte et normal, les cerveaux plus simples de la Vénus hottentote, des idiots, des microcéphales et des fotus, et de ce parallèle lumineux il dégagea définitivement la synthèse des circonvolutions cérébrales. Il en donna le premier une nomenclature régulière et complète, il en démontra la fixité, en détermina le nombre, la position, les connexions et les groupa enfin dans chaque hémisphère, en cinq lobes désormais classiques.

Depuis lors, les noms des lobes du cerveau, quoique empruntés encore (à l'exception d'un seul) aux noms des os crâniens correspondants, ne désignent plus des régions limitées sur le crâne par des sutures, mais de vrais lobes, limités sur le cerveau même par des scissures; c'est donc depuis lors seulement qu'on a pu éprouver le besoin de chercher quels sont les rapports des divers points du crâne avec les diverses circonscriptions, désormais connues, de la surface du cerveau.

L'étude de la topographie cérébrale intéresse beaucoup les anthropologistes, puisque la craniologie tire une grande partie de son importance de ce fait que l'examen extérieur du crâne fournit des données plus ou moins approximatives sur le degré de développement des diverses régions du cerveau; mais cette étude est aussi de l'ordre médical, aux divers points de vue de l'anatomie proprement dite, de la physiologie, de la pathologie médicale ou chirurgicale et, enfin, de la médecine opératoire, car elle est l'auxiliaire indispensable de toutes les recherches que l'on peut faire sur l'homme blessé ou malade, relativement à la grande question des localisations cérébrales.

Dans le mémoire que j'ai mentionné au début de cette note, j’ai donné l'exposé complet des travaux qui ont èté faits jusqu'ici sur ce sujet, et la description des divers procédés et recherches qui ont été suivis par ces divers auteurs. Gratiolet, qui a le premier déterminé anatomiquement les limites des lobes cérébraux, est aussi le premier qui ait cherché à reconnaître les rapports de ces lobes avec les parois crâniennes. Il crut y parvenir en reportant sur des moules intra-crâniens, d'une part, le trajet des scissures cérébrales; d'une autre part, celui des sutures du crâne. En opérant ainsi, il reconnut, ou plutôt, il crut reconnaître que la scissure de Rolando, qui sépare le lobe frontal du lobe 
pariétal, coïncidait avec la suture coronale; pour lui, par conséquent, le lobe frontal était entièrement contenu sous l'écaille de l'os frontal. Ainsi, quoique Gratiolet eût procédé tout autrement que ses prédécesseur's, quoiqu'il eût cherché les limites des lobes cérébraux non sur le crâne, mais sur le cerveau, il se trouvait que son lobe frontal morphologique était exactement le même que le lobe frontal ostéologique admis jusqu'alors. Cela explique la facilité avec laquelle fut acceptée la détermination topographique faite par ce célèbre auteur.

Les choses en étaient là, en 1861, lorsque je commençai mes recherches sur le siège de la faculté du langage articulé. Ayant reconnu que l'exercice de cette faculté était subordonné à l'intégrité de la partie postérieure de la troisième circonvolution frontale gauche, je fus conduit à chercher la position exacte de la petite région très circonscrite dont les lésions troublent ou abolissent le langage. Cette petite portion du cerveau était située au-dessus de la ${ }^{\circ}$ scissure de Sylvius et en avant de la scissure de Rolando; elle faisait donc partie du lobe frontal proprement dit et, dès lors, elle aurait dû, d'après la détermination faite par Gratiolet, se trouver sous l'écaille frontale. G'était bien là, en effet, que M. Bouillaud avait placé la faculté du langage ; mais, dans les discussions que cette opinion avait provoquées, on avait produit un certain nombre de faits d'où il résultait que les lésions les plus graves de ce qu'on appelait alor's les lobes frontaux, c'est-à-dire des lobes frontaux ostéologiques, pouvaient laisser la parole parfaitement intacte. J'avais observé moi-même deux faits de ce genre; je fus donc conduit à penser que la petite région du langage devait être située en arrière de l'os frontal, qu'en d'autres termes, malgré l'autorité de Gratiolet, la scissure de Rolando devait être placée en arrière de la suture coronale.

Pour m'en assurer, j'eus recours à un procédé fort simple, qui consiste à pratiquer sur les parois crâniennes, au niveau des principales sutures, des trous de vrille et à enfoncer à travers ces trous de petites fiches de bois dont on détermine ensuite la position sur le cerveau retiré du crâne. Je constatais ainsi que le lobe frontal s'étend bien au-delà des limites de l'os frontal, qu'il occupe environ le tiers de la région du pariétal et que la scissure de Rolando, loin de coüncider avec la suture coronale, commence 
sur la ligne médiane à 47 millimètres, en moyenne, en arrière de cette suture, pour descendre obliquement de là vers la partie antérieure de la suture écailleuse. J'ajoute que ce chiffre moyen de 47 millimètres, qui se dégageait de mes premières observations, est même un peu inférieur à la moyenne réelle et que, si la distance de la scissure de Rolando au bregma, point médian de la suture coronale, peut descendre à 40 et même à 39 millimètres, elle peut s'élever quelquefois à 60 ou même à 70 millimètres. Je passe sous silence les faits moins importants relatifs aux rapports de la suture lambdoïde et de la suture occipitale externe, qui sépare le lobe occipital du lobe pariétal.

Le procédé des fiches, dont je viens de parler, fut communiqué à la Société anatomique, en 1861 , et consigné, avec les premiers résultats qu'il m'avait fournis, dans une note de mon premier mémoire sur le siège de la faculté du langage. Gratiolet, à la suite d'une séance de vérification, se rendit de bonne grâce à l'évidence des faits. Depuis lors, la question des limites topographiques du lobe frontal n'a plus été controversée (1); tout le monde a admis sans contestation qu'une très grande partie du lobe frontal, et notamment la partie affectée au langage, est située en arrière de la suture coronale; et, en ce qui concerne cette petite région du langage, $j$ 'ai pu en déterminer les rapports avec assez de précision pour instituer un procédé de trépanation qui m'a permis de tomber du premier coup sur un abcès intracrânien, dont le siège était indiqué par l'abolition du langage. Mais les rapports du lobe frontal ne constituent qu'une partie de la topographie cérébrale; ceux des autres lobes, pour être moins importants, n'en sont pas moins dignes d'attention, et ils ont donné lieu, depuis trois ans, à des recherches assez nombreuses, faites en Russie, en Grande-Bretagne, en France et en Allemagne, par MM. Heftler, Turner, Féré, de la Foulhouze et Ecker. Les auteurs de ces recherches ont suivi tantôt le procédé des fiches, qui est le plus rapide et le plus commode de tous,

(1) M. Bischoff, en 1868, a placé la limite postérieure et /supérieure du lobe frontal à 20 et quelques millimètres seulement en arrière de la suture coronale. Il y a loin de là aux 47 millimètres que j'ai indiqués; mais la contradiction n'est qu'apparente; elle tient uniquement à la nomenclature adoptée par l'auteur, qui ne comprend pas dans le lobe frontal la circonvolution frontale postérieure ou ascendante. M. Bischoff s'est servi comme moi du procédé des fiches. 
tantôt le procédé de M. Landzert (de Saint-Pétersbourg), qui consiste à superposer des dessins de projections obtenus par des moyens mécaniques, tantôt le procédé de M. Turner (d'Edimbourg), qui consiste à dépecer chaque moitié du crâne en dix aires, dont les rapports sont successivement reportés sur le même dessin.

En outre, M. Féré a déterminé certains rapports profonds à l'aide de coupes pratiquées sur des têtes congelées. M. Ecker a combiné le procédé des fiches avec celui des moules intracrâniens de Gratiolet; et moi-même enfin j'ai complété le procédé des fiches en y ajoutant quelques règles qui permettent de représenter exactement sur le crâne même toutes les scissures et tous les sillons et, par conséquent, tous les lobes et toutes les circonvolutions de la convexité des hémisphères, ainsi qu'on peut le voir sur une pièce que je présente à l'Académie.

Malgré la diversité et la multiplicité de ces procédés, les résultats obtenus ont présenté une concordance très satisfaisante. Les divergences, d'ailleurs légères et relatives à des détails secondaires, sont imputables aux différences du sexe, de l'âge et de la race des sujets observés. L'âge surtout influe à un haut degré sur les rapports cérébro-crâniens; ce point spécial de la topographie cérébrale a été étudié avec le plus grand soin par M. de la Foulhouze, dans une thèse remarquable soutenue, il y a quelques jours, devant la Faculté de médecine. L'influence du sexe et celle de la race sont beaucoup moins connues; mais je n'ai pas l'intention de me placer ici au point de vue anthropologique et je crois pouvoir dire que les notions topographiques acquises jusqu'ici suffisent aux besoins de la physiologie, de la pathologie et de la médecine opératoire.

L'utilité de ces notions ne me semble pas douteuse. Depuis que le siège de la faculté du langage articulé est connu, la question des localisations cérébrales a été remise à l'étude et des recherches récentes ont mis les physiologistes sur la voie de plusieurs autres localisations. Or, l'homme n'est pas un sujet d'expériences, mais seulement un sujet d'observations; il ne comporte d'autres vivisections que celles que produisent les accidents et, si l'on veut tirer parti des observations faites sur le vivant, il faut que l'on puisse savoir quelle est la partie du 
cerveau qui correspond à une plaie pénétrante ou à un enfoncement de la paroi crânienne.

En parlant des limites du lobe frontal, j’ai mentionné plusieurs fois la scissure de Rolando, qui sépare ce lobe du lobe pariétal. Permettez-moi de profiter de cette occasion pour vous présenter une remarque historique et bibliographique au sujet de la découverte de la scissure de Rolando.

Rolando est, je pense, le premier anatomiste qui ait eu la notion de la fixité des circonvolutions cérébrales et qui se soit efforcé de les soumettre à une description méthodique. Il n'a pas toujours été heureux dans ses déterminations; il ne s'est pas inspiré, comme Leuret et Gratiolet, de l'anatomie comparée, et, ne procédant pas du simple au compliqué, il n'a pas su distinguer les caractères fondamentaux et invariables des caractères secondaires et variables; il n'a donc pas atteint son but; il n'a pu déterminer ni le nombre des circonvolutions, nileurs limites, ni leurs connexions, ni leur groupement en lobes; et la morphologie cérébrale est restée, après lui, presque aussi confuse qu'elle l'était avant lui ; mais, lorsqu'on étudie les travaux antérieurs à la période moderne, on doit reconnaître que, de tous les prédécesseurs de Leuret et de Gratiolet. Rolando est celui qui mérite le plus d'éloges et je trouve bon que le nom de cet homme éminent reste inscrit d'une manière ineffaçable dans l'histoire des circonvolutions cérébrales.

Je suis donc bien loin de songer à débaptiser la scissure à laquelle Leuret, le premier, a donné le nom de Rolando. Mais il me paraît utile et juste, cependant, de constater que notre Vicq-d'Azyr a connu cette scissure ni plus ni moins que Rolando lui-même et plus de quarante ans avant lui.

A vrai dire, elle n'a été décrite ni par Rolando, ni par Vicqd'Azyr ; ils ont indiqué seulement les deux circonvolutions ascendantes et parallèles qui la limitent, et ce n'est pas tout à fait la mêmé chose. Maintenant que la morphologie cérébrale est bien connue, on peut, à volonté, décrire la surface du cerveau de deux manières, soit en considérant les circonvolutions elles-mêmes, soit en considérant les sillons ou scissures qui les séparent, car le sillon implique la distinction des deux circonvolutions voisines et réciproquement. On trouve avantageux, dans 
l'intérêt de la clarté, de combiner ces deux procédés de description, mais ils sont absolument équivalents et chacun d'eux pourrait très bien remplacer l'autre. On remarquera toutefois que le second procédé, celui des sillons, est bien plus délicat que l'autre; il exige des connaissances plus précises et plus complètes; car il ne suffit pas, pour l'appliquer, de constater l'existence des diverses circonvolutions; il faut suivre les scissures dans tout leur trajet, connaître toutes leurs connexions, et déterminer exactement leurs limites, détermination rendue souvent très difficile par le développement si variable des plis secondaires qui interrompent les sillons. La description des sillons ne pouvait donc venir qu'après celle des circonvolutions, et voilà pourquoi Vicq-d'Azyr et Rolando ont connu les deux circonvolutions ascendantes, sans décrire le sillon, si remarquable pourtant et si spécial, qui existe entre elles.

C'est Leuret qui, en 1839 , a reconnu le premier toute l'importance de ce sillon. Il a dès lors éprouvé le besoin de lui donner un nom, et, dans sa justice, il a voulu que ce nom fât celui de l'anatomiste qui avait découvert les deux circonvolutions ascendantes. Pourquoi a-t-il choisi le nom de Rolando plutôt que celui de Vicq-d'Azyr? Est.ce parce qu'il avait négligé de consulter l'ouvrage de ce dernier ? De la part d'un homme aussi savant que Leuret, ce serait incompréhensible. D'ailleurs Rolando, que Leuret avait sous les yeux, avait eu soin lui-même de rapporter à Vicq-d'Azyr la découverte des deux circonvolutions ascendantes. Il l'avait fait à deux reprises, une première fois dans le texte de son Mémoire sur la structure des hémisphères cérébraux, une seconde fois dans l'explication des planches (1).

On lit en effet dans le texte de Rolando (p. 118): « De la partie supérieure de la circonvolution qui entoure la scissure de Sylvius, s'élèvent quatre circonvolutions presque verticales : les deux du milieu sont les plus longues et ont déjà été observées par Vicq-d'Azyr. " Et on lit en outre dans l'explication de la planche I, $\mathrm{n}^{\mathrm{os}} 12,13,14,15$ : " Des quatre circonvolutions verticales, les deux du milieu (13 et 14) ont été considérées par

(1) Memorian della reale Academia delle scienze di Torino, t. XXXV, p. 103146. Turin, 1831; in-40. Le mémoire de Rolando a été lu à la dite Académie dans la séance du 18 janvier 1829. 
Vicq-d'Azyr comme constantes. Elles sont aussi représentées, grâce à l'exactitude du dessinateur, sur les planches VIII et IX de l'Anatomie et physiologie du système nerveux, de Gall et Spernheim ; mais ces anatomistes semblent n'y avoir fait aucune attention." "

Or, des quatre circonvolutions verticales admises par Rolando, il y en a deux, l'antérieure et Ia postérieure, qui ne sauraient être admises aujourd'hui ; il n'en reste donc que deux qui soient réelles, ce sont les deux circonvolutions que nous nommons aujourd'hui ascendantes, celles qui limitent notre sillon de Rolando, et dont Rolando, comme on vient de le voir, attribue par deux fois la découverte à Vicq-d'Azyr.

Leuret n'eut garde de dédaigner cette double citation de Rolando. Il possédait l'édition complète des œuvres de Vicqd'Azyr, publiée en 1805 par Moreau (de la Sarthe). Il s'y reporta, mais il y chercha vainement la planche signalée par Rolando. Il n'y trouva qu'une seule planche où les circonvolutions de la face externe de l'hémisphère fussent représentées; c'était la planche I, et par malheur on n'y voit que les circonvolutions postérieures; quant aux circonvolutions moyennes, indiquées par Rolando, elles y sont entièrement masquées sous la dure-mère. Leuret dut en croire ses yeux ; il pensa donc que Rolando avait commis quelque méprise, et que c'était lui qui, en réalité, avait découvert les deux circonvolutions ascendantes.

Pouvait-il faire autrement? Oui, sans doute, il aurait pu recourir à l'édition originale. Mais comment supposer que Moreau (de la Sarthe), un homme de bibliothèque, élevé dans le respect des livres, eût défiguré ou dénaturé l'œuvre de Vicqd'Azyr sous le prétexte, pompeusement annoncé dans la préface, d'élever " le plus beau monument que l'on puisse consacrer à sa mémoire " ? G'est cependant ce qui est arrivé.

Moreau, sans en avertir le lecteur, a réduit de 35 à 32 le nombre des planches de l'Anatomie du cerveau. La réduction a porté sur les premières planches et sur la XVIII ${ }^{\circ}$. Les numéros de toutes les planches sont donc changés, de sorte que toutes les citations bibliographiques faites d'après cette édition sont nécessairement fausses. Cette première conséquence ne manque pas de gravité. 
Voyons maintenant comment Moreau s'y est pris pour réaliser, aux dépens de son monument, cetle économie inavouée de trois planches. Il a d'abord supprimé purement et simplement les planches II et XVIII, reproduites par Vicq-d'Azyr d'après Rysch et d'après Sœmmering. Puis, comme il voulait gagner encore une troisième planche, il a eu l'idée étrange de fusionner en une seule la planche I, qui représentait les vaisseaux de la dure-mère, et la planche III, qui représentait les circonvolutions de la face externe de l'hémisphère. A cet effet il a, sur la planche I, effacé la partie postérieure de la dure-mère, et il a obtenu ainsi un espace blanc sur lequel il a figuré quelques circonvolutions. Par suite de ce procédé inqualifiable, les deux circonvolutions ascendantes découvertes par Vicq-d'Azyr ne se trouvent plus dans les prétendues œuvres complètes de Vicq-d'Azyr. Il est bien entendu que le profanateur n'a pas seulement mutilé les planches, il a aussi dû mutiler le texte, en supprimant tout ce qui aurait pu faire deviner la première fraude. Mais a-t-il du moins respecté les circonvolutions particulières qu'il a montrées sur cette malheureuse planche? Pas même, et jugeant parfaitement inutile de les calquer sur Vicq-d'Azyr, il a eu la sottise de les remplacer par des circonvolutions ridicules qu'on pourrait croire copiées sur un intestin de chat. C'est là pourtant que Vicq-d'Azyr a représenté et décrit, sous le nom de bourrelet, le petit groupe de circonvolutions que l'on nomme aujourd'hui le lobule pariétal.

Ce n'est pas la seule falsification que cet éditeur, aussi peu scrupuleux que peu intelligent, a fait subir à l'œure de Vicqd'Azyr. On dirait qu'il s'est plu à dénaturer systématiquement tout ce qui concerne les circonvolutions cérébrales. J'en pourrais citer de nombreux exemples : le plus triste, après celui qui précède, est celui qui concerne la planche XXV de Vicq-d'Azyr, devenue la planche XXII dans le "monument " de Moreau. Notre illustre anatomiste y avait représenté les circonvolutions de la face interne de l'hémisphère. On y voyait plusieurs dispositions importantes dont la découverte lui appartient : la circonvolution du corps calleux et la scissure qui la surmonte, le lobule quadrilatère, la scissure occipitale interne séparant le lobe moyen du lobe postérieur (découverte de premier ordre); on y voyait 
aussi la scissure des hippocampes et le lobule triangulaire désigné aujourd'hui sous le nom de cuneus : tout cela a disparu et le dessin si étudié de Vicq-d'Azyr a été remplacé par une abominable caricature.

Voilà comment le nom de Vicq-d'Azyr, qui aurait dû figurer au premier rang parmi ceux des fondateurs de la morphologie cérébrale, a été omis ou à peine indiqué par les auteurs qui ont écrit sur ce sujet. - Les amis comme Moreau rappellent l'ours de la fable. Mieux vaudrait un sage ennemi. 


\section{GERVEAU DE GORILLE MALE ET ADULTE}

(Bulletins de la Société d'anthropologie, 20 série, 1876, p. 426-431.)

Cette pièce, dont la conservation est parfaite, a été rapportée du Gabon par M. le docteur Nègre, médecin de la marine de l'Etat, et donnée par lui au laboratoire d'anthropologie. Nous en avons fait un moule qui est déposé dans le musée.

Jusqu'ici les anatomistes et les naturalistes avaient fait de vains efforts pour se procurer un cerveau de gorille. Les notions que l'on possédail sur le cerveau de cet anthropoïde étaient tout à fait insuffisantes. On connaissait, d'après les moules intracrâniens, la conformation générale de l'organe. Quant à la conformation des circonvolutions, on n'en avait qu'une idée très imparfaite. Les corps de gorilles qui ont été envoyés en Europe, et sur lesquels on a pu étudier l'anatomie de cet animal, avaient été soigneusement enfermés dans des tonneaux pleins de tafia ; leurs muscles, leurs viscères étaient en bon état et se prètaient très bien à la dissection ; mais le cerveau était toujours gravement altéré.

Le liquide conservateur ne filtre que lentement à travers les parois crâniennes, surtout à travers la dure-mère; lorsqu'il atteint graduellement la substance cérébrale, celle-ci est déjà en voie de décomposition, et le contact de l'alcool ne saurait lui rendre la consistance qu'elle a perdue. Elle se réduit à l'état d'une pâte mollasse, et, quelque soin qu'on y mette ensuite, on ne retire du crâne qu'un organe défiguré, dont les sillons sont pour la plupart effacés, et qui s'écroule promptement lorsqu'on enlève la pie-mère. 
Gratiolet lui-même, malgré son habileté bien connue, ne put surmonter ces difficultés. Le Muséum d'histoire naturelle ayant reçu le corps d'un gorille tué au Gabon et expédié dans le tafia, Gratiolet procéda avec les plus grandes précautions à l'extraction du cerveau, mais il le trouva dans un tel état, qu'il ne put ni le conserver ni même le décrire; il dut se borner à faire photographier cette masse confuse, où l'on distinguait à peine quelques parties des sillons cérébraux.

L'étude de cette photographie ne put lui fournir que des notions assez vagues. Il constata pourtant que le cerveau du gorille est plus pauvre en circonvolutions que ceux de l'orang et du chimpanzé. Il crut même, d'après certains traits généraux, pouvoir le rattacher au type cérébral des cynocéphales et non pas à celui des macaques, auquel il rattachait, comme on sait, le cerveau de l'orang et celui du chimpanzé.

C'est à cela que se réduisent toutes les notions que l'on possédait jusqu'ici sur le cerveau du plus grand des anthropoïdes, et on reconnaîtra que c'était tout à fait insuffisant.

Le seul procédé qui permette de conserver convenablement le cerveau sans le déformer, est celui qui consiste à l'extraire promptement du crâne à l'aide d'une coupe méthodique, à le déposer sur un fond de ouate dans un vase plein d'alcool, et à le retourner tous les deux ou trois jours jusqu'à ce qu'il soit bien durci. Quoique ce procédé soit familier à tous les médecins, j’ai cru devoir l'exposer en détail dans les Instructions générales sur l'anthropologie publiées en 1865 ; j'y ai même joint une mention spéciale concernant les cerveaux des anthropoïdes (1). Cet appel toutefois n'a pas été entendu. Plusieurs fois, les médecins de la marine ont bien voulu me rapporter des singes très bien conservés dans l'alcool, et très bons pour la dissection; mais lorsque j'ai ouvert le erâne, je n’y ai trouvé qu'une masse cérébrale altérée et informe. Il m'a donc paru nécessaire de leur faire parvenir, au nom du laboratoire d'anthropologie et par l'intermédiaire du ministère de la marine, des instructions particulières sur ce sujet. J'y ai joint une annexe concernant spécialement les squelettes et cerveaux de gorille, et destinée à notre station

(1) Némoires de la Société d'anthropologie, t. II, p. 85 (1865), 
MORPHOLOGIE DU CERVEAU DE L'HOMME E'T DES PRIMATES. 669 du Gabon. Il y a six ans déjà que ces instructions ont été expédiées, et cette lois ce n'a pas été en vain, car j’ai reçu depuis lors trois squelettes de gorille et quatre têtes isolées qui sont aujour.

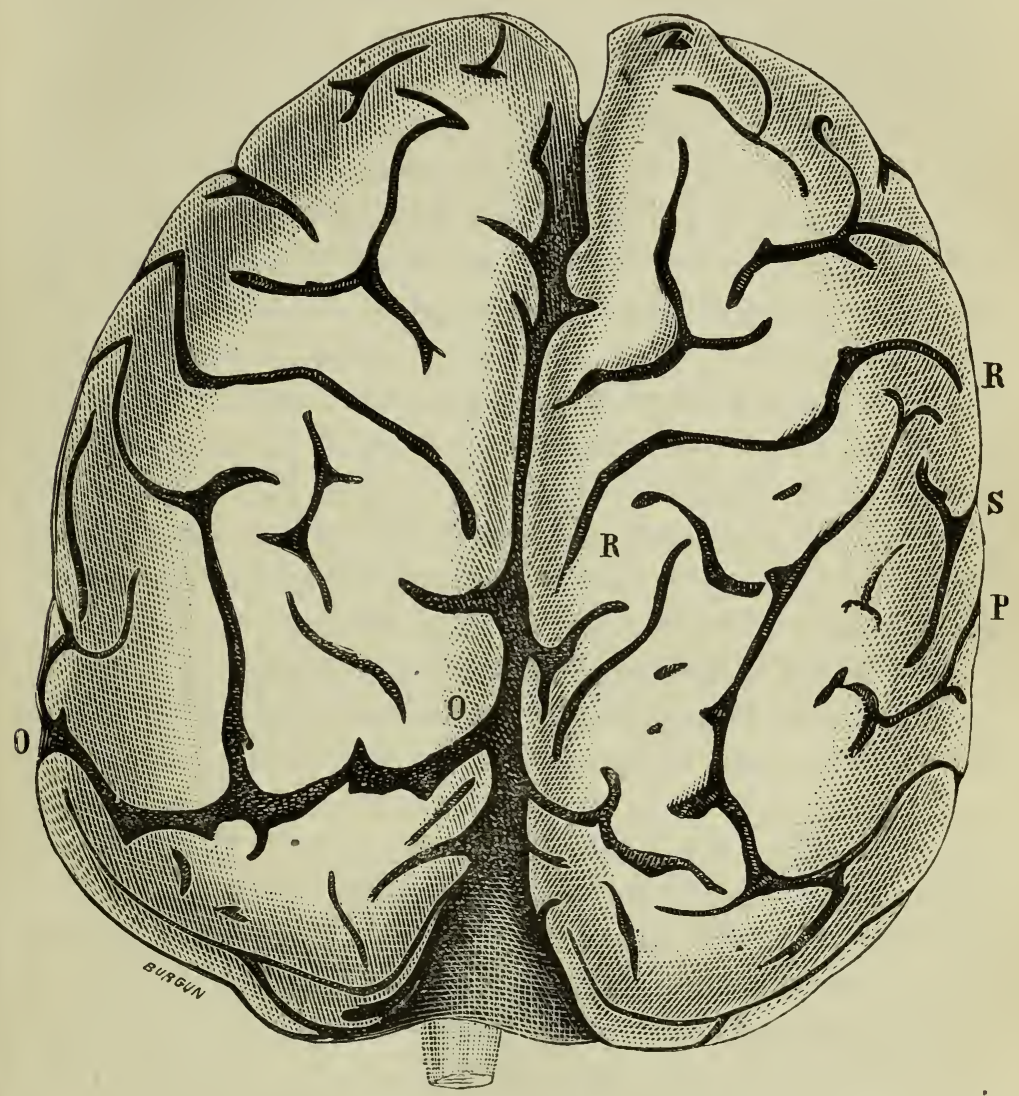

Fig. 70. - Face supérieure du cerveau d'un gorille male et adulte.

Figure de grandeur naturelle, dessinée au diagraphe; la pièce s'étant rétractée dans l'alcool, les dimensions primitires devaiert ètre un peu plus grandes.

RR, scissure rolandique; $\mathrm{S}$, scissure sylvienne droite; $\mathrm{P}$, scissure parallèle; $\mathrm{OO}$, scissure occipitale de l'hémisphère gauche; du còté droit, le second pli de passage est superficiel et interrompt la scissure.

On peut voir que les deux hémisphères sont peu symétriques.

d'hui déposés dans notre musée; mais c'étaient surtout les cerveaux que j'avais demandés, et mes confrères de la flotte, malgré tout leur zèle, n'arrivaient jamais à temps pour pouvoir conserver cet organe. La décomposition cadavérique en général, 
et celle du cerveau en particulier, survient si promptement dans l'Afrique équatoriale, que les cas où l'autopsie méthodique du crâne peut être faite avant le début de la décomposition du cerveau ne peuvent être que très exceptionnels.

Cette année enfin, M. le docteur Nègre a heureusement saisi une occasion favorable. Ayant appris le jour même qu'un gorille avait été tué dans les bois à quelque distance de la station, il s'est transporté sur les lieux dès le lendemain et a pu extraire un cerveau en très bon état. Ne disposant pas de moyens de transport suffisants, et voulant néanmoins préparer le squelette, il fit creuser une fosse peu profonde où le corps du gorille fut déposé; mais lorsqu'il revint quelque temps après, il ne retrouva plus que quelques ossements dispersés autour de la fosse ; le crâne avait disparu, des animaux carnassiers avaient fouillé la fosse, dépecé le cadavre et emporté dans leurs tanières tout ce qu'ils n'avaient pu dévorer sur place. M. Nègre n'a donc pu rapporter le squelette, ni même la tête de ce gorille, et je le regrette bien vivement, car si la détermination du genre est certaine, celle de l'espèce est restée douteuse.

M. Nègre a vu des chimpanzés et des gorilles, il a donc pu reconnaître par lui-même qu'il s'agissait d'un gorille et non d'un chimpanzé. C'était d'ailleurs l'opinion bien formelle des nègres qui l'accompagnaient et des chasseurs qui avaient tué l'animal. Mais, quoique cet anthropoïde fût de grande taille, qu'il fût màle et adulte (les quatre dents de sagesse étaient en place), la crête sagittale et la crête occipitale existaient à peine, et elles n'opposèrent aucun obstacle à l'autopsie régulière du crâne.

Ce renseignement, que M. Nègre nous avait donné avant l'ouyerture de la boîte soudée où il avait renfermé le cerveau, avait fait naître dans notre esprit quelques doutes sur le genre de l'animal. Nous savions bien que le genre gorille comprend au moins deux et probablement trois espèces, et que le crâne du gorilla Savagii est le seul qui porte de très grandes crêtes. Nous avions cependant quelque crainte de voir sortir de la boîte le cerveau d'un chimpanzé. Mais lorsque la boîte a été ouverte, tous les doutes se sont dissipés, et il a été évident au premier coup d'œil que ce cerveau ne peut être celui d'un chimpanzé.

Je vous le présente à côté de trois cerveaux de chimpanzé et 
d'un cerveau d'orang. Il n'y a pas à s'y méprendre. Le cerveau du gorille est plus simple, les circonvolutions y sont moins nombreuses et plus volumineuses, elles sont aussi grosses que celles des grands cynocéphales. Il est beaucoup moins simple cependant que ne le faisait supposer la photographie de Gratiolet. Quant au rapprochement que notre collègue toujours regretté avait cru pouvoir établir entre le cerveau du gorille et celui des cynocéphales, je puis jusqu'à un certain point l'admettre, mais je n'en tirerai pas la même conséquence que lui, car je pense qu'il n'y a sous ce rapport aucune différence entre les anthropoïdes. Tous ces animaux, les orangs et les chimpanzés, aussi bien que les gibbons et les gorilles, se rattachent au type cérébral des cynocéphales, auquel se rattache aussi le cerveau de l'homme, ainsi que Leuret l'avait déjà reconnu. Quant au type cérébral des macaques, d'où dériverait, d'après Gratiolet, celui des orangs et des chimpanzés, il s'en distingue complètement par la disposition du lobe frontal, par la communication de la scissure parallèle avec la scissure de Sylvius, et par la constitution plus rudimentaire du lobe occipital. Je me borne à signaler ici ces faits, me réservant de les développer plus amplement dans un mémoire que je présenterai dans trois semaines à la section d'anthropologie del'Association française pour l'avancement des sciences.

Le poids du cerveau n'a pu être déterminé à l'état frais. Aujourd'hui, après six mois de séjour dans l'alcool, cet organe ne pèse plus que 252 grammes. Il résulte de diverses observations que j'ai eu l'occasion de faire dans le laboratoire, que le cerveau, à la suite de l'action prolongée de l'alcool, perd en moyenne de 28 à 30 pour 100 de son poids. Ce chiffre n'a rien de rigoureux; il présente des variations assez étendues suivant les espèces et suivant les individus; il dépend pour beaucoup de la proportion relative de la substance blanche et de la substance grise. C'est donc sous toutes réserves que j'évalue à environ 360 grammes le poids primitif de notre cerveau de gorille; et ce chiffre paraîtra assez faible si l'on songe que l'animal était mâle, adulte et de grande taille. 


\section{SUR LE GERVEAU A L'ÉTAT FOETAL}

(Bulletins de la Société d'anthropologie, 2e série, 1877, t. XII, p. 216-222.)

M. Alix présente à la Société le croquis de la face externe de l'hémisphère gauche d'un fœetus de quatre mois passés. Sur ce,cerveau, on voit nettement l'indication du sillon de Rolando, et en avant de ce sillon, troi.s autres sillons indiquant les premiers indices des circonvolutions frontales.

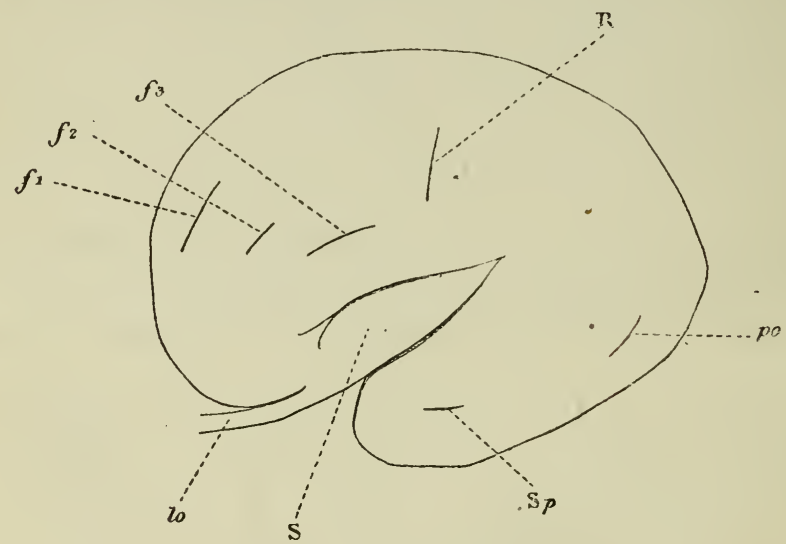

Fig. 71. - Cerveau d'un fœtus humain de quatre mois passés.

lo, lobe olfactif ; $f^{1}, f^{2}, f^{3}$, sillons frontaux ; R, sillon de Roląndo; $p o$, dépression préoccipitale; $\mathrm{S} p$, sillon sphénoïdal à direction antéro-postérieure; $\mathrm{S}$, fossette de Sylvius.

Il n'y a rien sur le lobe pariétal, et la scissure parallèle (à la scissure de Sylvius) a fait complètement défaut. En un mot, ce cerveau est presque semblable à celui qui a été figuré par Gratiolet dans l'Anatomie comparée du système nerveux, et l'observation faite par M. Alix est la confirmation de celle de Gratiolet.

\section{DISCUSSION.}

M. Broca. L'ordre suivant lequel se développent les circonvolutions dans le cerveau humain n'est pas assez fixe pour pouvoir être formulé en loi. Gratiolet, d'après l'examen d'un trop petit 
nombre de faits, avait cl'u pouvoir annoncer que, chez l'homme, les circonvolutions antérieures apparaissent les premières, tandis que chez les singes elles apparaissent les dernières ; c'était pour lui un caractère distinctif absolu de l'homme et il formulait sa pensée en ces termes: "Le cerveau de l'homme se développe d’alpha en oméga, celui des singes d'oméga en alpha. "

Il attachait d'autant plus d'importance à ce caractère du développement inverse qu'il ne trouvait dans la morphologie du cerveau tout formé aucune différence décisive. Une étude attentive des deux premiers plis de passage lui avait montré, il est vrai, que le cerveau de l'homme est le seul sur lequel ces plis soient tous deux superficiels; mais il comprenait bien que la position plus ou moins superficielle d'un pli cérébral ne constitue qu'un caractère de peu de valeur, et nous savons, d'ailleurs, aujourd'hui, que chez certains individus peu intelligents, mais nullement idiots, les deux plis de passage sont assez profonds pour ne pas interrompre la scissure occipitale externe. Gratiolet espéra donc que l'étude du développement suppléerait à l'insuffisance de la caracléristique morphologique, et il se laissa aller avec un peu trop de facilité à énoncer ce qu'il appelait la loi du développement inverse.

A l'appui de cette loi, M. Alix nous présente aujourd'hui un fait qu'il a eu l'occasion d'observer récemment. 11 aurait pu en trouver quatre autres dans l'une des vitrines de notre musée; mais il y en aurait trouvé en outre un assez bon nombre qui déposent dans le sens opposé.

Voici une série de moules qui ont été faits depuis huit ans dans mon laboratoire par M. Chudzinski et moi. Ce n'est pas ma faute si la série n'est pas plus nombreuse; je n'ai rien négligé pour l'accroître, et grâce surtout au concours des chefs de clinique du service d'accouchement, j'ai reçu au moins une trentaine d'embryons ou de fœtus humains âgés de trois à six mois; mais M. Alix n’ignore pas avec quelle rapidité la pulpe cérébrale de ces produits d'avortement se dissocie et se réduit en une bouillie informe. Il ne suffit inême pas de plonger la petite tête dans l'alcool immédiatement après l'avortement, l'alcool ne s'imbibe pas assez vite à travers les membranes; le plus souvent la pulpe cérébrale est déjà altérée sans retour avant de subir l'action de 
ce liquide. Il faut donc, le plus tôt possible, enlever les téguments, et inciser de chaque côté la membrane des sutures ainsi que la dure-mère, afin que le cerveau puisse baigner directement dans l'alcool. Lorsqu'on arrive à temps, l'organe se raffermit ; on peut l'extraire au bout de quelques jours, on le replonge aussitôt dans l'alcool sur une épaisse couche de ouate, et on l'y laisse jusqu'à
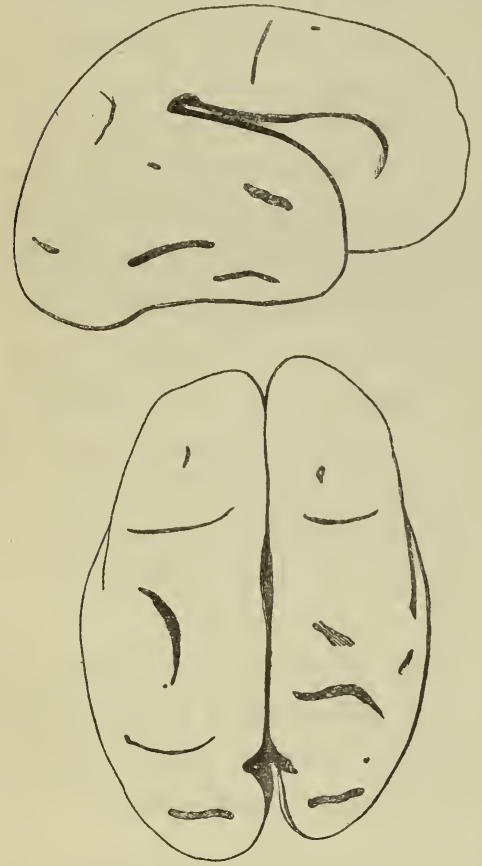

Fig. 72. - Fœtus mâle de cinq et demi à six mois $\left(n^{0} 16\right)$. ce qu'il soit assez ferme pour être moulé. Mais, bien souvent, toutes ces précautions sont inutiles parce que les pièces ne nous parviennent pas assez promptement. Voilà comment, parmi les cerveaux de fœtus âgés de moins de six mois, que j'ai pu obtenir jusqu'ici, il ne s'en est trouvé que quinze qui fussent en état d'être convenablemeṇt étudiés.

Je vous en présente les moules. Quatre d'entre eux ne se rapportent pas à la question, attendu qu'on n'y voit encore aucun vestige de circonvolution. Il n'y en a donc que onze qui puissent nous servir. Cette série, quelque petite qu'elle soit, permet de constater que l'ordre suivant lequel se dessinent et se forment les circonvolutions n'est assujetti à aucune règle. Il n'y a que deux faits absolument constants : c'est que, d'une part, la scissure de Sylvius précède toujours de beaucoup l'apparition de toutes les autres scissures et que, d'une autre part, la scissure occipitale interne et la scissure des hippocampes apparaissent sur la face interne avant les sillons de la surface convexe. Un autre fait assez général, c'est que la scissure de Rolando se dessine ordinairement avant les autres dépressions de la convexité des hémisphères ; on rencontre ici, toutefois, quelques exceptions, je vous en présente deux et même trois; mais, dans ce 
troisième cas, la scissure de Rolando ne manque que du côté gauche.

Quant aux sillons qui subdivisent chaque lobe en circonvolutions, l'ordre de leur apparition est très variable. Voici quatre
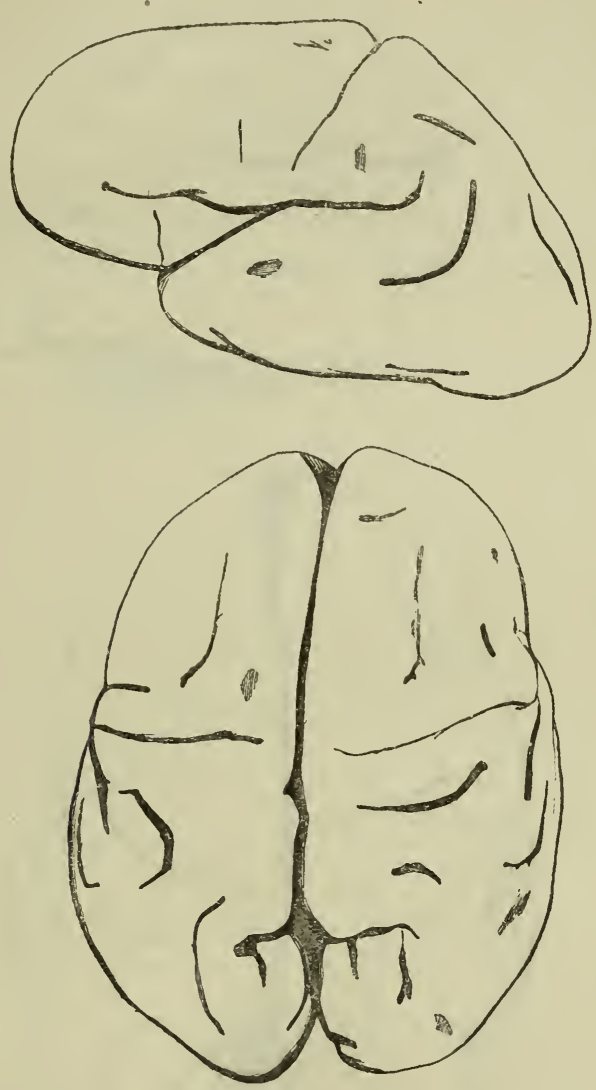

Fig. 73, Fœtus mâle de six mois environ (n० 8 ).

pièces sur lesquelles les circonvolutions du lobe frontal sont manifestement en avance, mais en voici trois sur lesquelles il est non moins évident que l'évolution des circonvolutions pariétales et temporales a été plus avancée que celle des circonvolutions frontales (voir les figures 72,73 et 74 ); sur les quatre dernières, enfin, il est aisé de voir que la formation des circonvolutions 
s'est effectuée simultanément dans la région frontale et dans la région temporo-pariétale.

Le fait que Gratiolet avait érigé en loi est donc bien loin d'être

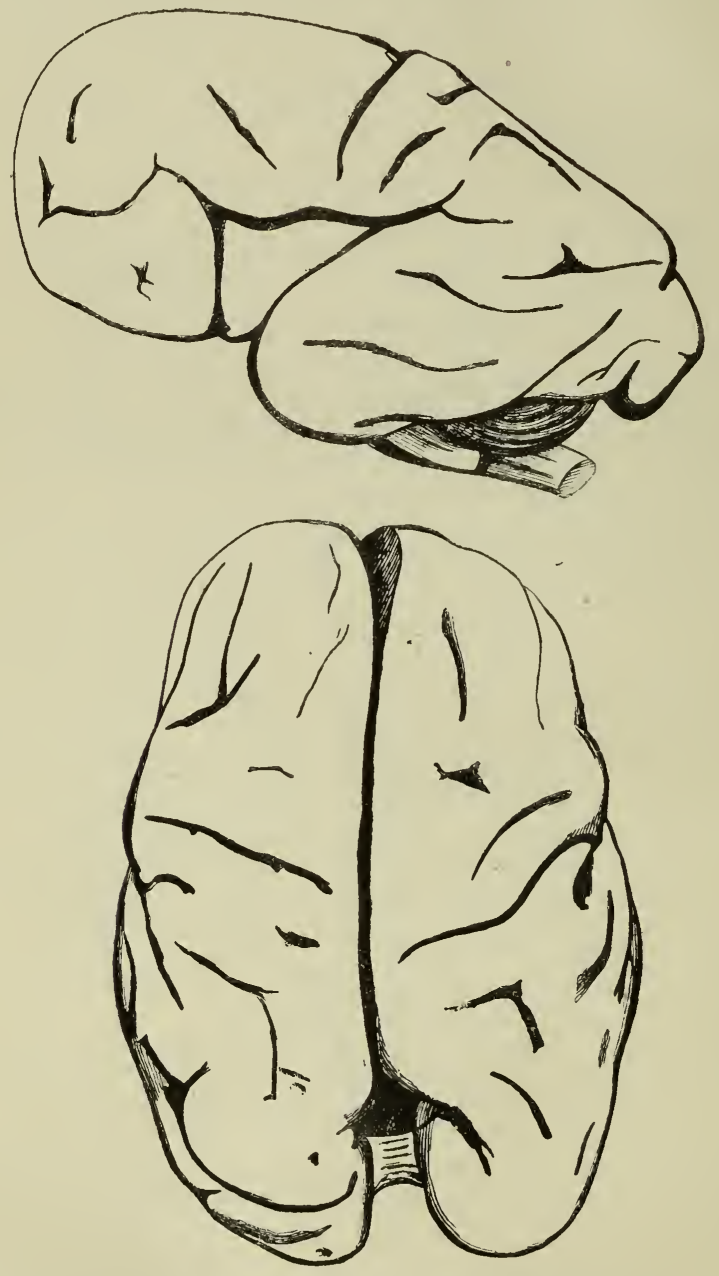

Fig. 74. Fœtus mâle de cent quatre-vingt-un jours ( $\left.n^{\circ} 1\right\}$ ?

général, puisqu'il ne paraît pas même exister une fois sur deux. M. Alix peut s'en convaincre de visu.

L'ordre d'apparition des circonvolutions est-il plus fixe chez les singes que chez l'homme? Je l'ignore, n'ayant pas eul'occa- 
MORPHOLOGIE DU CERVEAU DE L'HOMME ET DES PRIMATES. 377

sion d'étudier, sous ce rapport, les fœtus des singes. Je pense que Gratiolet lui-même n'en a étudié qu'un très petit nombre. Il nous dit que, chez ces animaux, les circonvolutions du lobe frontal apparaissent les dernières. C'est possible; mais ce qui est certain, c'est qu'il n'a pu examiner que des fœtus de singes pithéciens, ou de gibbons tout au plus; ni lui ni personne jusqu'ici n'a étudié le développement du cerveau des grands anthropoïdes. C'est là pourtant le nœud de la question ; car, en admettant même qu'il y eût, sous ce rapport, une différence entre l'homme et les singes ordinaires, rien ne prouverait encore que la même différence existât entre l'homme et les anthropoïdes. 


\title{
SUR
}

\section{I.A TOPOGRAPHIE GÉRÉBRALE GOMPARÉE}

\author{
DE L'HOMME ET DU GYNOGÉPHALE SPIINX
}

(Bullelins de la Socièté d'anthropologie, 2e série, t. XII, 1877, p. 262-270.)

J'ai publié l'année dernière, dans la Revue d'anthropologie, un mémoire sur la topographie cérébrale chez l'homme. La topographie cérébrale est l'étude des rapports qui existent entre les diverses circonvolutions cérébrales et les divers points de la surface du crâne. Cette détermination peut se faire suivant plusieurs procédés. Le plus simple et le plus pratique est celui dont je me sers depuis 1861 , et que j'appelle le procédé des fiches. Il consiste à pratiquer sur le crâne frais un certain nombre de trous de vrille, à travers lesquels on pousse de petites chevilles de bois piquées dans le cerveau. Le cerveau une fois extrait et déposé sur la table, on enlève la pie-mère en laissant les fiches en place ; on peut alors reconnaître par la mensuration et exprimer en chiffres la position de tel ou tel sillon cérébral par rapport à telle ou telle suture crânienne. Pour rendre la démonstration plus commode, on peut, en se guidant sur les points de correspondance, dessiner à la surface externe de la calotte crânienne, ou d'un moule en plâtre qui la représente, le trajet de toutes les scissures et de tous les sillons de la surface des hémisphères.

Toutes les circonvolutions étant ainsi reportées sur le crâne, on en étudie les rapports comme on pourrait le faire si le crâne était transparent; et cette étude devient facile, surtout lorsqu'on peint la pièce en donnant une couleur particulière à chaque 
lobe, et dans chaque lobe, une nuance particulière à chaque circonvolution.

J'ai déposé dans notre musée un grand nombre de moules

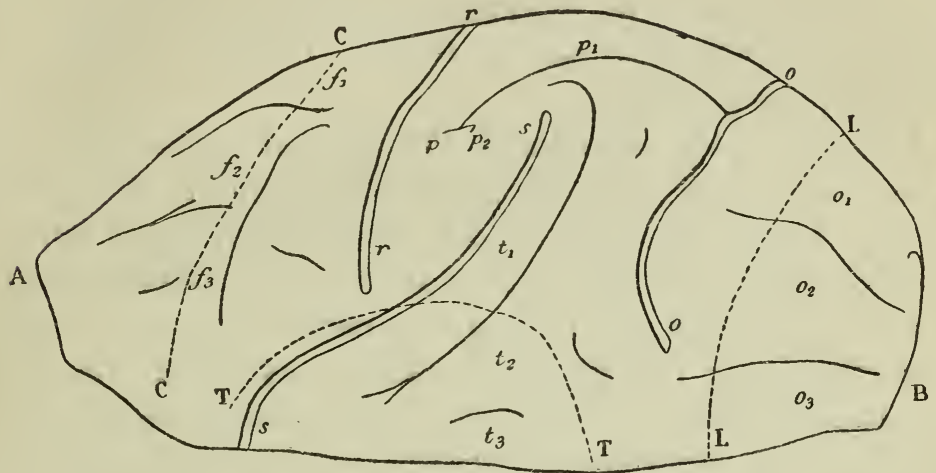

Fig. 75. Topographie cérébrale d'un cynocéphale sphinx.

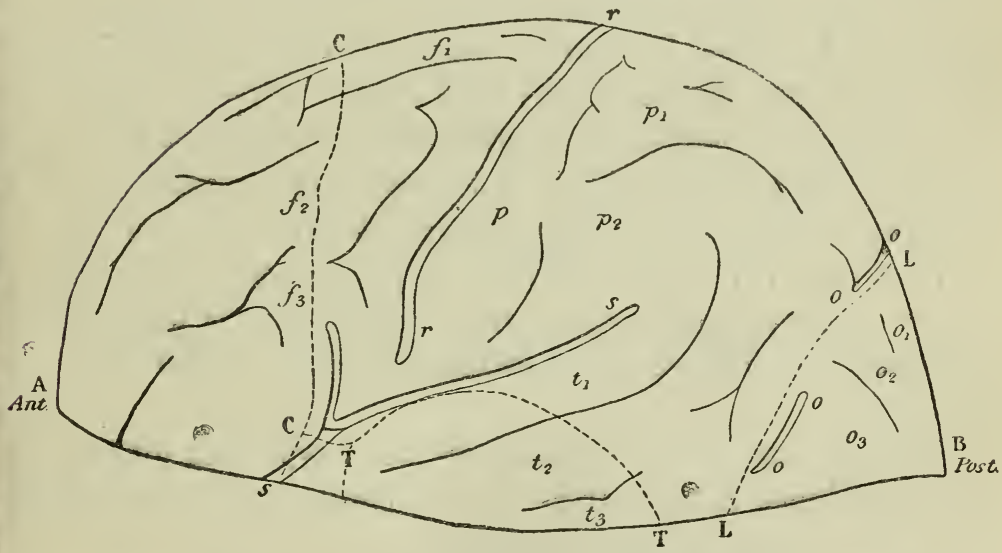

Fig. 76. Topographie cérébrale de l'homme (Parisien).

Explication commune aux deux figures : les traits pleins et doubles représentent des scissures qui séparent les lobes cérébraux; les traits pleins et simples représentent les sillons séparant les circonvolutions ; les traits ponctués représentent les sutures crâniennes.

A. extrémité antérieure de la calotte crânienne ; B, extrémité postérieure ; C, C, suturecoronale ; L, L, suture lambdoïde; T, T, suture temporale ; $r, r$, scissure de Rolando ; $o, o$, scissure occipitale externe; $s, s$, scissure de Sylvius; $f, f_{1}, f_{2}, f_{3}$, circonvolutions frontales; $p, p_{1}, p_{2}$, circonvolutions pariétales $t_{1}, t_{2}, t_{3}$, circonvolutions temporales $; o_{1}, o_{2}, o_{3}$, circonvolutions occipitales.

crâniens coloriés représentant la topographie cérébrale de l'homme, suivant les sexes, les âges et les races. Cette collection, qui, en ce qui concerne les races, est loin d'être comlète, remplit déjà toute une vitrine. Quelques-unes des pièces 
relatives aux enfants sont dues à $M$. de la Foulhouze, auteur d'une thèse très intéressante sur la topographie cérébrale chez les enfants.

J'ai décrit en détail, dans le mémoire que je viens de citer, les autres procédés de topographie cérébrale qui ont été employés par MM. Landzert et Heftler, par M. Turner, par M. Féré, par M. Ecker.

La topographie cérébrale de l'homme est aujourd'hui assez bien connue, mais celle des singes n'a pas encore été étudiée. Cette recherche offre pourtant un grand intérêt, car on sait que le cerveau des singes est dressé et subdivisé de la même manière que celui de l'homme. On y trouve les mêmes lobes et les mêmes circonvolutions; la différence consiste dans le degré de complication de celles-ci, et dans le volume relatif de ceux-là, et il en résulte des changements notables dans les rapports cérébrocrâniens.

J'ai reçu cette semaine, dans mon laboratoire, le corps d'un jeune papion dont la dent de sagesse n'est pas encore sortie. Il n'a donc pas encore atteint l'âge adulté, mais le volume de son cerveau ne paraît pas sensiblement inférieur à celui des papions adultes. J'ai profité de l'occasion pour étudier, par le procédé des fiches, la topographie cérébrale de cet animal.

Voici (fig. 75̆) le plâtre colorié que j'ai obtenu; j’y joins plusieurs autres moules sur lesquels est représentée la topographie cérébrale de l'homme. L'un de ces moules est reproduit sur la figure 76.

On peut voir qu'il existe entre les deux types des différences topographiques considérables, qui se montrent sur les quatre lobes de la convexité des hémisphères.

$1^{\circ}$ Le lobe frontal, dans les deux cas, s'étend bien en arrière de la suture coronale, et empiète par conséquent beaucoup sur la région pariétale. Gratiolet avait admis, d'après des comparaisons basées sur un procédé très défectueux, que la scissure de Rolando, qui sépare le lobe frontal du lobe pariétal, coïncidait exactement chez l'homme comme chez le singe, avec la suture coronale; qu'en d'autres termes le lobe frontal était situé tout entier sous l'os frontal. En ce qui concerne l'homme, j'ai constaté depuis 1861 que cette détermination est tout à fait 
fausse. - La scissure rolandique de l'homme, toujours très oblique de haut en bas et d'arrière en avant, commence sur la ligne médiane, à 48 millimètres en moyenne du bregma, et vient se terminer au-dessus de la scissure sylvienne, à 2 centimètres environ en arrière de l'extrémité inférieure de la suture coronale. On pouvait supposer qu'en commettant une aussi grande erreur, Gratiolet s'était laissé influencer par les résultats qu'il avait constatés chez les singes, et qu'il avait ensuite étendus à l'homme par généralisation, sans recourir à une constatation directe. Mais la règle qu'il avait cru pouvoir poser n'est pas même applicable aux singes. Le lobe pariétal du papion se prolonge sous l'os pariétal, bien en arrière de la suture coronale ; sa partie inférieure et externe empiète même beaucoup plus sur la région pariétale chez le singe que chez l'homme (toutes proportions gardées), mais tandis que la scissure rolandique de l'homme remonte très obliquement en arrière, celle du papion est beaucoup moins oblique, presque transversale; il en résulte que, près de la région médiane, le lobe frontal occupe chez l'homme environ les deux cinquièmes du bord sagittal du pariétal, tandis que chez notre singe il n'en occupe que le tiers. En tenant compte de cette disposition, et aussi de l'unique développement des écailles frontales, on voit que le lobe frontal tient relativement beaucoup plus de place dans le crâne de l'homme que dans celui du singe.

$2^{\circ}$ Le lobe occipital, au contraire, est considérablement plus grand chez le singe. La scissure occipitale externe qui le limite est située bien au-dessus de la suture lambdoïde, de sorte que le tiers au moins du lobe occipital empiète sur le pariétal. Ghez l'homme, la partie de la scissure occipitale externe présente quelque variation, mais elle ne remonte jamais aussi haut; dans la très grande majorité des cas, elle correspond exactement à la suture lambdoïde, c'est-à-dire que le lobe occipital est situé tout entier sous l'écaille occipitale.

$3^{\circ}$ Le lobe temporal du papion est plus développé que celui de l'homme, au moins dans le sens de celui de la hauteur. C'est la conséquence de la direction de la scissure de Sylvius. Cette scissure, dans ces deux cas, se place d'abord sous la partie antérieure de la suture temporale, et s'en écarte ensuite pour se 
porter en arrière et en haut; mais celle de l'homme se dirige surtout en arrière, tandis que celle du papion se dirige surtout en haut en devenant presque parallèle à la scissure rolandique, et en traversant ainsi plus des deux tiers de la hauteur de l'os pariétai. Le pli courbe, déjà refoulé en avant par suite du grand volume du lobe occipital, se trouve donc, en outre, reporté très haut.

$4^{\circ}$ Le lobe pariétal du papion se trouve ainsi réduit à un très petit volume. Il n'occupe pas même le tiers de l'étendue de l'os pariétal, tandis que chez l'homme il en occupe plus de la moitié. 


\section{SUR LE GERVEAU DU GORILLE}

(Bulletins de la Socićté d’anthropologie, $2^{\mathrm{e}}$ série, t. XII, 1877, p. 432-439.)

La Société n'a pas oublié que je lui ai présenté l'année dernière un cerveau de gorille mâle et adulte, rapporté du Gabon par' le docteur Nègre, médecin de la marine.

Tout ce que l'on savait jusgu'alors sur le cerveau du gorille se réduisait à quelques observations très incomplètes faites par Gratiolet sur une pièce extrêmement altérée, dont tout autre que lui n'aurait pu tirer aucun parti. Le cerveau recueilli par le docteur Nègre est donc en réalité le premier sur lequel on ait pu étudier les circonvolutions du gorille, et c'est aujourd'hui encore le seul cerveau de gorille adulte que l'on ait pu se procurer.

Je vous ai présenté cette pièce importante dans la séance du 3 août 1876 . Je ne remis au secrétaire qu'une note très abrégée, me proposant de faire une communication plus étendue à la section d'anthropologie de l'Association française; mais étant tombé malade pendant la durée de la session de Clermont-Ferrand, je ne pus faire cette communication.

Ma note du 3 août dernier a paru dans le quatrième et dernier fascicule de nos Bulletins de 1876 (p. 426) et dans ce volume, p. 567, avec un dessin représentant la face supérieure du cerveau en question. Mais vous savez que l'apparition de ce fascicule a été retardée jusqu'au commencement du mois d'avril, parce qu'on avait eu l'intention d'y joindre le catalogue de la bibliothèque (dont l'impression n'est malheureusement pas encore terminée); il en est résulté que M. Pansch et M. Bischoff ne connaissaient pas encore ma présentation du mois d'août dernier, lorsqu'ils ont publié, l'un en septembre 1876, l'autre en mars 1877, la description du cerveau d'un jeune gorille, 
d'environ six mois, rapporté du Gabon, pour le musée de Hambourg, par le gardien de la ménagerie de cette ville.

En recevant tardivement, comme tout le monde, notre $4^{\circ}$ fas cicule de 1876, M. Bischoff a été frappé de la différence considérable qui existe entre le cerveau du gorille de Hambourg et celui du gorille du docteur Nègre. Il m'a écrit aussitôt pour me signaler cette différence, qui est évidente, et que m'avaient déjà fait connaître les dessins photographiques publiés par M. Pansch. D'après cela, M. Bischoff pense que l'animal dont $M$. Nègre a rapporté le cerveau n'est pas un gorille, mais un chimpanzé. Je reviendrai tout à l'heure sur cette opinion, que je ne partage pas.

La pièce que je vous présente m'a été envoyée par M. Bischoff en échange du moule du cerveau de gorille du docteur Nègre. Ce n'est pas un moule, mais un modelage en cire d'une exécution très soignée. Pour obtenir cette pièce, M. Bischoff a procédé de la manière suivante. Il a choisi dans sa collection le plus grand crâne de gorille mâle et adulte, et il en a fait le moule intra-crânien en cire, puis, sur ce moule, il a reporté par le modelage, en les grandissant proportionnellement, les sillons et circonvolutions du petit gorille de Hambourg. Cette pièce est donc schématique. Exécutée par M. Bischoff lui-même, elle ne peut être que très conforme au petit modèle qu'il avait sous les yeux. Mais ce schéma représente-t-il réellement l'état du cerveau du gorille adulte? Pour qu'il en fût ainsi, il faudrait que la morphologie cérébrale de cet animal fût déjà parvenue à son état définitif six mois après la naissance, ce qui est fort peu probable. Il est très probable, au contraire, que chez les anthropoïdes, comme chez l'homme, le volume relatif des divers lobes cérébraux et des diverses circonvolutions subit pendant l'accrois. sement du cerveau des modifications assez notables, et qu'il ne suffit pas de grandir un jeune cerveau pour obtenir un cerveau adulte. J'ajoute qu'un moule intra-crânien ne peut reproduire exactement ni le volume ni la forme du cerveau correspondant ; car, d'une part, son volume se trouve accru de toute l'épaisseur des méninges, et, d'une autre part, ses contours du côté de la base ne sont pas adoucis comme ceux du cerveau par l'interposition de ces mêmes méninges. Par exemple, l'extrémité anté- 
rieure des moules intra-crâniens présente chez tous les anthropoïdes un prolongement très saillant, presque pointu, que Gratiolet a désigné sous le nom de bec, et qui correspond à la fosse olfactive. Sur les cerveaux ce bec est presque effacé, tandis qu'il conserve toute sa saillie, avec sa forme pointue et recourbée, sur le cerveau modelé par M. Bischoff.

D'un autre côté, le cerveau rapporté par le docteur Nègre n'a été étudié, dessiné et moulé qu'après avoir séjourné plusieurs mois dans l'alcool, où il s'est rétracté et déformé. J'ai lieu de croire que, reposant sur sa base dans le fond du vase, il a dû s'aplatir et s'élargir d'une manière notable. Il est certain, en tous cas, qu'il a perdu une grande partie de son volume. Le modelage de $\mathrm{M}$. Bischoff est au contraire plus grand que nature, et si l'on songe que ce savant a opéré sur le moule intra-crânien le plus volumineux qu'il ait pu se procurer, il paraîtra probable que le modèle en cire qu'il a bien voulu nous envoyer doit être plus grand que le cerveau naturel de n'importe quel anthropoïde.

Nous devons tenir compte de ces diverses conditions avant de comparer cette pièce avec le cerveau que nous devons à M. Nègre. Cela atténue un peu la différence qui existe entre les deux pièces. Néanmoins cette différence est si grande, qu ïl est impossible de la méconnaître, et j'admets, comme M. Bischoff, que l'animal de Hambourg et celui de MI. Nègre ne sont pas de même espèce. Mais cela ne veut pas dire qu'ils ne soient pas de même genre.

Que l'animal de Hambourg soit un vrai gorille, c'est ce que j'accepte comme suffisamment établi. Le corps, rapporté en enticr, a été examiné et décrit par M. Bolau, naturaliste très compétent; et quoique l'extrême jeunesse du sujet soit peu favorable à la détermination de l'espèce, celle du genre, je le répète, ne doit pas être mise en doute.

Quant à l'autre animal, nous n'avons ni sa peau, ni son squelette, ni même son crâne, et nous savons en outre que, quoique mâle et adulte (il avait ses dents de sagesse), il ne portait pas les grandes crêtes sagittale et lambdoïdienne qui caractérisent, à cet âge et chez les mâles, le crâne du gorille de Savage. D'après l'absence de ce caractère, $M$. Bischoff pense que le sujet 
devait être un chimpanzé. Mais je ferai remarquer que la description classique du crâne du gorille ne se rapporte qu'à l'espèce découverte par Savage; qu'on a découvert depuis lors une et probablement deux autres espèces du même genre gorille, caractérisées précisément par l'absence des crêtes crâniennes chez les mâles adultes. Si j'ajoute maintenant que les chasseurs du Gabon savent parfaitement distinguer les gorilles des chimpanzés, et que le docteur Nègre, appelé par eux auprès d'un animal récemment tué, a reconnu, comme eux, un gorille et non un chimpanzé, il deviendra très probable que l'animal était vraiment un gorille. C'est ce qui résulte d'ailleurs bien claire. ment de l'examen du cerveau.

Je rappelle d'abord que nous avons un terme de comparaison. G'est une photographie représentant la masse encéphalique d'un gorille adulte étudié par Gratiolet. L'animal, rapporté du Gabon dans un tonneau de tafia, était en assez bon état de conservation, en ce qui concerne les téguments et les muscles; mais le cerveau, très altéré, réduit en une pulpe mollasse, ne put être sauvé; il s'écroulait, pour ainsi dire, à mesure qu'on enlevait les membranes. Gratiolet retrouva toutefois les principaux sillons de la surface convexe des hémisphères; et, comme il était évident que la pièce allait très promptement tomber en putrilage, il se hâta de la faire photographier. Cette photographic, quelque insuffisante qu'elle soit pour l'étude des détails, nons montre pourtant d'une manière évidente que le cerveau du gorille a des circonvolutions très larges, très simples, très peu sinueuses, et qu'il est beaucoup plus simple que celui des chimpanzés. Vous pouvez vous en assurer en jetant les yeux sur les trois cerveaux de chimpanzés noirs que je vous présente.

Maintenant, entre cette photographie et ces trois cerreaux de chimpanzé, plaçons le cerveau rapporté par le docteur Nègre. Il saute aux yeux que ce dernier cerveau, par sa simplicité, par la largeur et le peu de sinuosité de ses circonvolutions, reproduit aussi exactement que possible le type du cerveau de gorille photographié par Gratiolet, et que, par ces nêmes caractères, il diffère entièrement des cerveaux de chimpanzé.

Quant au cerveau du jeune gorille de Hambourg, modelé par 
M. Bischoff, il est aisé de vuir qu'il s'écarte beaucoup plus que le précédent du type représenté sur la photographie de Gratiolet, tandis qu'à beaucoup d'égards, il se rapproche du cerveau des chimpanzés. Il en diffère toutefois par la simplicité des circonvolutions temporales, par l'obliquité plus grande de la scissure de Rolando, d'où résulte une diminution notable du lobe frontal, et surtout par l'écartement des bords de la scissure sylvienne, une partie de l'insula restant à découvert, comme chez nos enfants nouveau-nés.

Mais, pour apprécier la valeur de ce dernier caractère distinctif, il faudrait pouvoir comparer ce cerveau avec celui d'un chimpanzé de six mois; tous nos cerveaux de chimpanzé, et aussi, je pense, tous ceux qui ont été décrits jusqu'ici, proviennent d'animaux beaucoup plus âgés. Chez l'homme, le lobe frontal est relativement moindre dans les premiers mois de la vie que dans les périodes suivantes, et il n'est pas invraisemblable qu'il en soit de même chez les anthropoïdes. G'est pour cela, probablement, que le lobe frontal du gorille de Hambourg est relativement bien plus petit que celui de nos chimpanzés et que celui du gorille adulte du docteur Nègre; et la même cause explique sans doute aussi en partie les autres différences qui existent entre ce dernier cervean et celui qui a été modelé par M. Bischoff.

Je ne prétends pas pour cela que le cerveau du très jeune gorille de Hambourg fût destiné à devenir, par les progrès de la croissance, semblable à celui du gorille adulte du docteur Nègre. Tout annonce que ces deux gorilles n'appartiennent pas à la même espèce; il est donc naturel qu'ils n'aient pas le même cerveau; mais la différence des âges explique peut-ĉtre pourquoi la différence de leurs cerveaux paraît plus grande que celle qu'on observe ordinairement entre deux espèces du même genre.

Gratiolet, d'après les observations très incomplètes qu'il avait pu faire sur une pièce unique et très altérée, avait cru pouvoir rattacher le cerveau du gorille au type cérébral des cynocéphales. Le cerveau du gorille du docteur Nègre donne au premier abord une impression favorable à cette opinion. Voici plusieurs cerveaux de cynocéphales. Si l'on ne considérait 
que l'aspect général, on trouverait que le cerveau en question ressemble beaucoup plus au cerveau des cynocéphales qu'à celui des chimpanzés; mais, en pénétrant dans les détails, il est aisé de reconnaître qu'il présente plusieurs caractères qui sont propres aux anthropoïdes et à l'homme, à l'exclusion des pithéciens. Parmi ces caractères, je signale spécialement l'incisure horizontale de la troisième circonvolution frontale; incisure que j'appelle la branche horizontale antérieure de la scissure de Sylvius. Chez les singes ordinaires, la troisième circonvolution frontale est simple; son bord inférieur limite, sans aucune flexuosité, la partie correspondante de la scissure de Sylvius; mais, chez les anthropoïdes et chez l'homme, cette circonvolution, se développant davantage, et devenue trop longue pour l'espace qu'elle occupe, est obligée de se replier, et elle se replie d'autant plus qu'elle est plus développée. Au degré le plus simple, qui s'observe chez les gibbons (1), ce repli, unique, est peu profond, se produit vis-à-vis la pointe du lobe temporal, là où la troisième circonvolution frontale se porte en bas pour se continuer avec la troisième circonvolution orbitaire; il en résulte une incisure horizontale qui se détache de la scissure de Sylvius pour se porter en avant, et qui constitue la branche horizontale antérieure de cette scissure. Au degré le plus compliqué, qui s'observe chez l'homme, le repli est double, et audessus de cette branche horizontale antérieure on voit se détacher de la scissure de Sylvius une seconde branche, qui se porte obliquement en haut et un peu en avant. G'est la branche ascenlante de la scissure de Sylvius. Ces deux branches divergentes interceptent sur la troisième circonvolution frontale un pelit lobule triangulaire en forme de cap, que j'appelle le cap de la troisième circonvolution frontale.

(1) Sur l'un de nos cerveaux de semnopithèques, une toute petite incisure horizontale constitue, du côté droit, un rudiment de la branche horizontale antérieure. On sait que les semnopithèques forment le genre le plus élevé de la famille des pithéciens.

On a souvent confondu cette branche ascendante, qui est constante, avec un autre sillon ascendant qui manque dans la majorité des cas et qui se produit lorsque le sillon prérolandique, se prolongeant sur la base de la troisième circonvolution frontale (sans la diviser d'ailleurs dans toute son épaisseur), va aboutir a la scissure de Sylvius. 
Quoique la troisième circonvolution frontale des orangs et des chimpanzés soit toujours bien moins flexueuse et bien moins compliquée que celle de l'homme, on retrouve presque toujours sur le cerveau de ces animaux les deux branches antérieures de la scissure de Sylvius et le cap de la troisième circonvolution frontale; cela, toutefois, n'est probablement pas constant, car la branche ascendante fait presque entièrement défaut sur l'un dé nos cerveaux de chimpanzé.

Cela posé, le cerveau du gorille du docteur Nègre ne présente, ni à droite ni à gauche, la branche ascendante de la scissure de Sylvius; mais la branche horizontale antérieure y est très prononcée des deux côtés et beaucoup plus longue que chez les gibbons. Ce cerveau est donc, sous ce rapport comme sous les autres, constitué sur le type des anthropoïdes et se distingue absolument par là du cerveau des cynocéphales. Il s'en distingue aussi par le nombre des circonvolutions temporo-sphénoïdales, qui est de cinq au lieu de quatre; par le moindre volume du lobe occipital, par la position moins profonde des deux plis de passage supérieur (le pli interne est même superficiel du côté droit); et enfin, d'une manière générale, par une moindre simplicité des circonvolutions. Il vient donc contredire l'opinion qu'avait suggérée à Gratiolet l'examen d'une pièce altérée, où les détails morphologiques ne pouvaient être reconnus. 


\section{SUR LA NOMENGLATURE GÉRÉBRALE}

(Bulletins de la Société d'anthropologie, $2^{\text {e }}$ série, t. XII, 1877, p. 614-618.)

M. Broca fait remarquer que les termes dont on se sert dans la description des circonvolutions cérébrales datent d'une époque où l'on considérait ces circonvolutions comme des plis désordonnés et où l'on n'avait aucune idée de la valeur relative des nombreuses divisions et subdivisions de la surface des hémisphères. Il est naturel qu'alors on n'attachât aucune importance au choix des mots; chaque auteur s'exprimait à sa manière, et le langage, surchargé de synonymes, était vague comme les idées elles-mêmes. Mais maintenant que les progrès de l'anatomie ont permis de débrouiller le labyrinthe des circonvolutions, de distinguer dans l'hémisphère un certain nombre de lobes, et dans chaque lobe un certain nombre de circonvolutions fixes chez les animaux de même espèce, la clarté des découvertes exige l'emploi de termes précis, dont l'acception soit suffisamment déterminée pour que chaque chose ait un nom, qu'elle n'en ait qu'un seul, et que ce nom ne désigne qu'une chose.

Pour alteindre ce but, il n'est pas nécessaire de créer des mots nouveaux, car il y a déjà plus de mots dans le vocabulaire cérébral qu'il n'y a de choses à désigner. Il suffit de convenir qu'on donnera une acception spéciale et précise à certains mots qui, pris dans un sens vague et général, étaient considérés comme synonymes. Cette réforme du langage a déjà été faite en partie par l'usage, et elle continue à se faire peu à peu; mais il reste à la compléter. Les noms auxquels un usage général a donnó une acception anatomique bien précise doivent être conservés, alors même qu'ils ne seraient pas parfaitement appropriés. Ainsi les lobes n'ont pas toujour's une forme lobée, et les 
circonvolutions ne sont pas toujours contournées; mais tout le monde s'entend aujourd'hui sur la signification anatomique de ces dénominations, et cela suffit. Des mots beaucoup plus nombreux, dont le sens n'est pas encore fixé, n’imposent pas la même réserve; il est bon, toutefois, de tenir compte, autant que possible, des habitudes déjà acquises, à moins qu'elles ne soient incompatibles avec la clarté, auquel cas on ne doit pas reculer devant une innovation reconnue nécessaire. En se pénétrant de ces principes, M. Broca a passé en revue toutes les parties de la surface de l'hémisphère, et il s'est effor'cé de donner à la nomenclature cérébrale une précision qui a fait défaut jusqu'ici. Le travail de revision et de critique auquel il s'est livré est consigné dans un mémoire sur la nomenclature cérébrale, qui paraîtra prochainement dans la Revue d'Anthropologie (voir dans ce volume page 652). 11 y a, dans ce travail, un grand nombre de détails relatifs à des faits spéciaux, dont l'exposé fatiguerait la Société ; mais il y a certains faits plus généraux, qui peuvent être résumés en peu de motsetqui sont le sujet de la présente communication.

L'ensemble des circonvolutions de l'hémisphère constitue ce que Burdach a appelé le manteau. Le manteau recouvre do toutes parts la masse cérébrale ou corps de l'hémisphère, à l'exception d'une portion de la face interne, limitée en haut par le corps calleux, en bas par la branche latérale de la grande fente de Bichat. C'est par là que passent toutes les fibres divergentes ou convergentes qui font communiquer l'hémisphère avec le reste de l'encéphale. Cette portion de la face interne est donc le seuil de l'hémisphère et le bord du manteau qui l'entoure est le limbe de l'hémisphère.

Les divisions primaires du manteau sont appelées lobes. Le nombre des lobes et le nom spécial de chacun d'eux ne sont plus en question.

Les divisions secondaires sont appelées circonvolutions. Chaque lobe comprend un certain nombre de circonvolutions, qui sont des organes fixes, déterminés anatomiquement par leur origine, leur terminaison et leurs connexions. Tous les auteurs donnent aujourd'hui cette acception anatomique au mot circonvolution, qui ne doit plus être employé pour désigner indistinctement une portion quelconque de la surface anfractuaire 
de l'hémisphère. Ghaque circonvolution a reçu un nom particulier, qui varie beaucoup dans les diverses nomenclatures. La nomenclature numérique est la meilleure. La règle la plus ordinaire, et à laquelle il faut par conséquent se conformer, consiste à placer dans chaque lobe le numéro 1 sur la circonvolution la plus rapprochée du bord supérieur ou même sagittal de l'hémisphère, en distance comptée sur la face convexe. La nomenclature numérique est applicable à toutes les circonvolutions longitudinales, c'est-à-dire à toutes les circonvolutions, exception faite des deux circonvolutions frontale ascendante et pariétale ascendante qui longent la scissure de Rolando.

Le nom de $p l i$, employé d'abord par Gratiolet comme synonyme de circonvolution, doit être pris dans une acception plus restreinte pour désigner certaines portions de circonvolution. Les parties auxquelles on peut l'appliquer sont de diverses natures. Il faut distinguer les plis de communication et les plis de complication. $1^{\circ}$ Les plis de communication sont des prolongements superficiels ou profonds, quị s'étendent d'une circonvolution à l'autre. Si les deux circonvolutions qui communiquent ainsi appartiennent à deux lobes différents, le pli est appelé pli de passage (Gratiolet); si elles appartiennent à ces mêmes lobes, c'est un pli d'anastomose. $2^{\circ}$ Les plis de complication sont aussi de deux espèces : les plis d'inflexion et les plis de subdivision. Les premiers résultent des sinuosités et des méandres que décrivent les circonvolutions, lorsque celles-ci sont plus longues que la distance comprise entre leurs deux extrémités. Les plis de subdivision sont ceux qui se produisent à la surface d'une circonvolution entre ses deux bords.

Les limites des lobes, des circonvolutions et des plis sont établies par des lignes de dépression dont la longueur, la largeur et la forme sont extrêmement variables et qui ont été désignées dès l'origine sous le nom général d'anfractuosités de l'hémisphère. Ce nom, peu usité aujourd'hui, doit être remis en vigueur, car c'est le seul qui puisse s'appliquer correctement à toutes les dépressions de la surface centrale, et c'est aussi le seul qui ait l'avantage de n'avoir jamais été employé dans un sens spécial.

Les anfractuosités, étant très nombreuses et très diverses, 
doivent être classées d'après la nature des divisions qu'elles établissent daus le cerveau. Parmi ces divisions il en est qui sont spéciales, qui n'existent qu'en un seul lieu; il y a par conséquent des anfractuosités qui devraient rester en dehors de la nomenclature générale et recevoir des noms particuliers, mais la plupart des anfractuosités se reproduisent un grand nombre de fois à la surface de l'hémisphère. Ce sont celles-là qu'il s'agit de classer ou de soumettre à des dénominations régulières.

Il y a dans l'hémisphère trois ordres de divisions ou de subdivisions : $1^{\circ}$ les lobes; $2^{\circ}$ les circonvolutions; $3^{\circ}$ les plis de complication. Il y a donc aussi trois ordres d'anfractuosités : $1^{\circ}$ celles qui séparent les lobes, ce sont les scissures; $2^{\circ}$ celles qui séparent les circonvolutions, ce sont les sillons; $3^{\circ}$ celles qui subdivisent ou compliquent les circonvolutions, ce sont les incisures.

Ces trois noms, surtout les deux premiers, ont été souvent employés comme synonymes les uns des autres, et même dans le sens général qu'exprime le mot anfractuosité. On remarque toutefois que beaucoup d'auteurs tendent à considérer la scissure comme plus importante que le sillon, et celui-ci comme plus important que l'incisure. La détermination précédente est en accord avec cette tendance quant à la valeur relative des mots, mais non quant à l'importance relative des choses qu'ils désignent. Partant de cette idée que le nom de scissure doit ètre réservé pour les anfractuosités les plus grandes et les plus profondes, on a appliqué ce nom à la fois à la grande fente interhémisphérique (scissure inter-liémisphérique), qui sépare les deux hémisphères, à la scissure de Sylvius qui sépare réellement deux lobes, et au premier sillon temporal (scissure parallèle) qui sépare seulement deux circonvolutions d'un même lobe, tandis que la scissure de Rolando qui sépare deux lobes est ai pelée le plus souvent le sillon de Rolando. Cet emploi est arbitraire. Ce qui fait l'importance d'une anfractuosité, ce u'est ni sa longueur ni sa profondeur, c'est la nature de la division qu'elle établit, et puisqu'on s'accorde à donner au mot scissure plus de valeur qu'au mot sillon, il convient de l'employer exclusivement pour désigner les divisions primaires qui séparent les lobes, et d'appeler sillons les divisions secondaires qui séparent 
les circonvolutions. Quant au mot incisure, qui exprime une idée moindre, il servira à désigner les divisions tertiaires qui se produisent dans une même circonvolution, de manière à la compliquer ou à la subdiviser.

Les incisures correspondent aux plis de complication, présentant comme ces plis eux-mêmes deux variétés, qui sont les incisures continues et les incisures isolées. Les premières correspondent aux plis d'inflexion; elles pénètrent dans les méandres d'une circonvolution, elles émanent du sillon ou de la scissure qui la limitent, elles sont donc continues avec cette anfractuosité. Les incisures qui séparent les plis de subdivision sont ap. pelées par opposition incisures isolées.

M. Broca se borne à ces indications générales, qu'il présente ici sans discussion, et dont les motifs sont exposés dans son mémoire susmentionné : Sur la nomenclature cérébrale. 


\section{SUR LE GERVEAU DU GORILLE}

(Association francaise pour l'avancement des sciences, 1877, p. 706-710.)

M. Broca rappelle qu'il s'était fait inscrire l'année dernière pour faire à la section d'anthropologie une communication sur le cerveau du gorille. Etant tombé malade pendant la durée du Congrès de Clermont-Ferrand, il ne put tenir sa promesse. La question n'a plus aujourd'hui le caractère de nouveauté qu'elle avait alors; il pense néanmoins que l'examen des pièces qui s'y rapportent offre encore quelque intérêt.

Il montre d'abord le moule du cerveau d'un gorille mâle et adulte rapporté du Gabon, au mois de juillet 1876, par M. le docteur Nègre, médecin de la marine de l'Etat. Tout ce que l'on savait jusqu'alors sur le cerveau de cet animal se réduisait à quelques notions très incomplètes et très imparfaites, recueillies pour ainsi dire au vol par Gratiolet, à l'ouverture du crâne d'un gorille adulte envoyé au Muséum dans un tonneau de tafia. Le cerveau, entièrement altéré et réduit à la consistance d'un mastic, tombait en miettes à mesure qu'on enlevait la pie-mère. Désespérant de conserver cet organe qui s'écroulait sous ses yeux, Gratiolet en fit aussitôt photographier la face supérieure.

Cette photographie, que M. Broca présente à la section, était le seul document que l'on possédât sur le cerveau du gorille, lorsque M. le docteur Nègre rapporta le cerveau dont le moule est mis sous les yeux de la section.

Ayant appris que les chasseurs indigènes venaient de tuer un gorille dans les bois, à une assez grande distance de la côte, M. Nègre monta immédiatement à cheval pour aller extraire le cerveau avant l'heure de la putréfaction, si hâtive sous les tropiques. Sous ce rapport, il réussit à souhait. Ne disposant pas 
ce jour-là, de moyens de transport, il ne put emporter avec lui que le cerveau. Il déposa le corps du gorille dans une fosse peu profonde, creusée à la hâte et recouverte d'un peu de terre; mais lorsqu'il revint, quelques jours après, pour prendre le squelette, il trouva la fosse vide soit que le corps eût été dépecé et emporté par les animaux carnassiers, soit qu'il eût été enlevé par les nègres. Par suite de cette circonstance malheureuse, on ne peut déterminer l'espèce de l'animal. Il appartient certainement au genre gorille; les chasseurs indigènes, qui s'y connaissent, n'auraient pu s'y tromper. Il n'y a dans cette région que deux genres d'anthropoïdes : les chimpanzés et les gorilles, et $M$. Nègre, qui connaissait parfaitement les chimpanzés, a pu s'assurer que ce n'était pas un chimpanzé. C'était donc un gorille. Quoiqu'il fût mâle et adulte, il n'avait pas les grandes crètes sagittales et occipitales du gorille, du Gorilla Savagii. Mais on sait que le genre gorille comprend une et probablement deux autres espèces qui se distinguent précisément du Gorilla Savagii par l'absence des grandes crêtes crâniennes. Il serait possible d'ailleurs que le gorille ảu docteur Nègre ne fût pas complètement adulte, quoiqu'il fût d'une grande taille et qu'il eût ses quatre dents de sagesse.

En tout cas, son cerveau suffit pour démontrer que cet animal n'était pas un chimpanzé, comme l'a supposé M. le professeur Bischoff, de Munich. M. Broca a présenté cette pièce à la Śociété d'anthropologie dans la séance du 3 août 1876, à côté de toute une série de cerveaux de singe, et tout le monde a constaté que ce cerveau différait entièrement non seulement de celui des chimpanzés, mais encore de tous les cerveaux connus. Une figure représentant la face supérieure de ce cerveau a été publiée avec le procès-verbal de la séance (fig. 77).

Quelques semaines plus tard, un autre cerveau de gorille fut présenté par MM. Pansch et Bolau au Congrès des naturalistes et médecins allemands, dans sa $49^{\circ}$ session, tenue à Hambourg le 20 septembre 1876. Cette pièce provenait d'un jeune gorille âgé d'environ six mois, mort au Gabon, en captivité, et rapporté en Europe dans un baril de rhum. Une injection de chlorure de zinc avait été poussée dans la carotide quelques heures après la mort, de sorte que le cerveau s'était parfaitement conservé. Cette 
MORPHOLOGIE DU CERVEAU DE L HOMME ET DES PRIMATES.

pièce fut décrite avec soin par M. Pansch, qui en publia la photographie. Elle fut étudiée ensuite par M. le professeur Bischoff, de Munich, qui la décrivit à son tour, dans un mémoire publié au mois de mars 1877.

M. Bischoff a en outre représenté les formes de ce cerveau sur

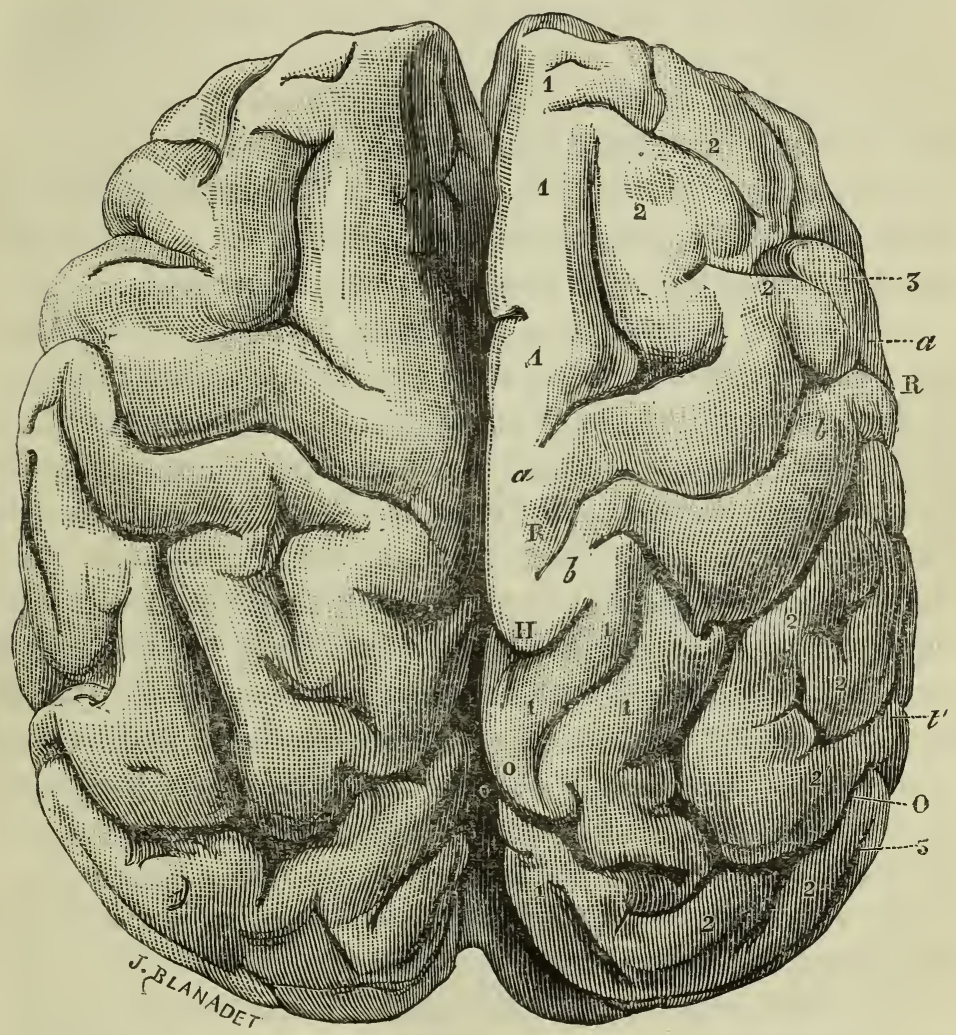

Fig. 77. Corveau du gorille mâle et adulte du docteur Nègre.

un modelage en cire, que M. Broca a reçu par voie d'échange, et qu'il présente à la section.

Ce modelage a été fait de la manière suivante : M. Bischoff a d'abord fait le moule en cire de la cavité crânienne d'un gorille mâle et adulte; puis il y a gravé, en les grandissant proportionnellement, toutes les saillies et sillons du petit cerveau du gorille de Hambourg. Il a voulu représcnter sur ce schéma le cer- 
veau du gorille tel qu'il est dans l'âge adulte, mais il aurait mieux valu s'en tenir à un moule ou fac-similé de grandeur naturelle, car il est très probable que chez le gorille, comme chez l'homme, la morphologie des lobes et des circonvolutions du cerveau doit subir, de la première enfance à l'âge adulte, des modifications très notables.

L'influence de l'âge explique sans doute en grande partie la grande différence qui existe entre le cerveau du gorille adulte du docteur Nègre et celui du jeune gorille de Hambourg, mais cette différence tient sans doute aussi en partie à l'influence de l'espèce, car tout permet de croire que l'animal de Hambourg est un Gorilla Savagii, tandis que le gorille du docteur Nègre appartient selon toutes probabilités à une autre espèce.

M. Bischoff, ne tenant pas compte de la différence des âges, pense que ces deux cerveaux sont trop dissemblables pour provenir d'animaux d'un même genre, et comme l'animal de Hambourg est certainement un gorille, il en conclut que l'animal du docteur Nègre doit être un chimpanzé. Mais l'examen des cerveaux n'autorise pas cette conclusion, car le cerveau de Hambourg est beaucoup plus riche que l'autre et beaucoup plus semblable au cerveau compliqué du chimpanzé, tandis que le cerveau recueilli par $M$. Nègre, incomparablement plus simple qu'aucun cerveau de chimpanzé, présente des circonvolutions pauvres, larges et très peu sinueuses, qui le font ressembler autant que possible à la photographie du gorille adulte de Gratiolet.

M. Broca pense donc qu'il convient de décrire le cerveau du gorille d'après le spécimen rapporté par M. Nègre.

Le poids de ce cerveau, réduit à 252 grammes après un séjour de plusieurs mois dans l'alcool, était certainement. plus granl à l'état frais, et devait s'élever à einviron 360 grammes. Le cervelet, quoique grand, est entièrement couvert. Les hémisphères ont très peu de hauteur, mais il est probable que l'organe s'est aplati par suite d'un séjour prolongé sur sa base dans le fond du vase. Ce qui frappe au premier abord, c'est la largeur et la simplicité des circonvolutions. Aucun antlıropoïde n'en présente de pareilles, et le seul cerveau de singe qui ait la même apparence est celui des cynocéphales. Cette ressemblance, constatée par 
Gratiolet sur le cerveau très altéré qu'il fit photographier, le conduisit à supposer que le cerveau du gorille se rattachait au type des cynocéphales, et non à celui des chimpanzés et des orangs ; mais il eût conclu autrement, s'il eût pu se livrer à une étude plus complète.

Le cerveau du gorille appartient au même type que celui du chimpanzé et de l'orang; et ce type n'est autre que le type humain.

Si l'on compare spécialement.le cerveau du gorille avec celui du chimpanzé, on reconnaît que le volume relatif du lobe frontal est plus grand chez le gorille; que celui du lobe pariétal est plus petit ; et que celui du lobe temporal est à peu près le même dans les deux cas. Le lobe occipital que Gratiolet croyait très volumineux chez le gorille, est au contraire très peu développé, car il est plus petit même que celui de l'orang, et n'est comparable sous ce rapport qu'à celui de l'homme. On sait quelle importance Gratiolet accordait à la position superficielle ou profonde des deux premiers plis de passage qui traversent la scissure occipitale externe. Lorsque ces plis sont petits, ils restent cachés dans le fond de la scissure; lorsqu'ils sont plus volumineux, ils deviennent superficiels, et le lobe occipital se trouve ainsi plus intimement lié au lobe pariétal. Quoiqu'il n'y ait pas lieu de donner à ce caractère une valeur de premier ordre, comme l'a fait Gratiolet, on peut du moins s'en servir pour apprécier le degré de perfectionnement de la région occipitale du cerveau. Le second pli de passage est profond, c'est-à-dire peu volumineux chez tous les anthropoïdes. Mais le premier, qui est toujours superficiel chez l'orang, est ordinairement profond chez le chimpanzé, sinon sur les deux côtés à la fois, du moins sur l'un des hémisphères. Sous ce rapport, le gorille paraît prendre place entre l'orang et le chimpanzé. On peut voir, en effet, sur le gorille du docteur Nègre que le premier pli de passage est superficiel à droite et peu profond à gauche; en outre, sur le gorille de Hambourg, il est superficiel des deux côtés, comme cela a lieu chez l'orang.

La branche horizontale antérieure à la scissure de Sylvius est très prononcée chez le gorille. Ce caractère est commun à l'homme et à tous les anthropoïdes, et n'existe que chez eux. C'est la con- 
séquence de l'allongement de la troisième circonvolution frontale qui, avant de se porter vers le bas pour se continuer avec la troisième circonvolution orbitaire, décrit une sinuosité au-dessus de la partie antérieure du lobe temporal. Chez l'homme, une seconde sinuosité, située au-dessus de la précédente, donne lieu à la formation de la branche ascendante de la scissure de Sylvius. Cette branche ascendante existe assez souvent chez l'orang et chez le chimpanzé. Elle manque chez le gibbon, et elle manque aussi chez le gorille.

En terminant, M. Broca signale les différences qui existent entre le cerveau du gorille de M. Nègre et celui du gorille de Hambourg. Sur ce dernier cerveau, le lobe temporo-sphẻnoïdal est relativement beaucoup plus simple; les cinq circonvolutions temporales y sont nettement séparées par des sillons parallèles et presque rectilignes, tandis que sur l'autre, la deuxième, la troisième et la quatrième, plus ou moins confondues par suite de leurs anastomoses, ne sont bien distinctes qu'en arrière. Par contre, le lobe frontal est relativement plus petit sur le gorille de Hambourg que sur le gorille de M. Nègre, ce qui est dû presque exclusivement à l'extrême petitesse de la troisième circonrolution frontale, car la seconde est beaucoup moins réduite, et la première est très développée en longueur et en largeur. Il en résulte que la scissure de Rolando est beaucoup plus oblique.

Les différences qui viennent d'être indiquées n'excèdent pas celles qui peuvent exister entre deux espèces d'un même genre ; mais il est très probable qu'elles tiennent surtout à la différence des âges, le gorille de Hambourg étant très jeune, tandis quie celui de M. le docteur Nègre est adulte. 


\section{ÉTUDE}

SUR

\section{LE GERVEAU DU GORILLE}

(Revue d'anthropologie, 1878, $2^{\text {e }}$ série, t. Ier, p. 1-46.)

Il y a déjà trente ans que le docteur Savage a découvert le gorille et ccpendant, l'anuée dernière encore, on ne possédait que des notions très vagues et très incomplètes sur le cerveau de cet anthropoïde. Les observations faites d'abord sur le squelette, puis sur le système musculaire, avaient établi qu'il existe entre les gorilles et les chimpanzés des différences non seulement spécifiques, mais encore génériques. Le groupe des grands singes anthropoïdes comprenait donc désormais, à côté des genres orang et chimpanzé, un troisième genre, le genre gorille. On se demanda aussitôt quel était celui de ces trois genres qui devait être considéré comme le plus élevé dans l'échelle, c'est-à-dire comme le plus voisin de l'homme, et l'importance de cette question s'accrut encore lorsque la doctrine transformiste se développa dans la science. On sait que Darwin, dans son traité de l'Origine des espèces (1859), avait évité de parler des origines de l'homme. A la faveur de ce silence, quelques-uns de ses disciples avaient pensé que l'ancêtre de l'homme devait être cherché parmi les genres actuels, et le gorille qui, par sa haute taille, par la conformation de ses extrémités et les proportions de ses membres, se rapprochait plus que tout autre du type humain, eut, pendant plusieurs années, l'honneur d'éclipser tous ses rivaux. 
Aujourd'hui la question a changé de face. Les transformistes s'accordent généralement à reconnaître que l'homme ne peut descendre d'aucun des anthropoïdes connus, ni même d'aucun autre genre vivant. Le parallèle hiérarchique des anthropoïdes se trouve donc dégagé de toute préoccupation doctrinale, mais. il conserve toujours un haut intérêt anatomique. Ce qui résulte de l'ensemble des faits connus jusqu'ici, c'est l'inégale répartition des caractères de supériorité ou d'infériorité dans le groupe des grands anthropoïdes. Ainsi l'orang, que son cerveau élève un peu au-dessus du chimpanzé, reste bien au-dessous de lui par plusieurs caractères ostéologiques, myologiques et splanchnologiques. Le gorille, à qui son squelette et ses muscles assigneraient le premier rang, ainsi que la plupart de ses viscères, a prourtant le foie divisé comme celui des pithéciens et se troure par là remis au troisième rang. Je cite sommairement ces résultats acquis, afin qu'on ne soit pas surpris si l'étude du cerveau du gorille ne confirme pas l'opinion de ceux qui considèrent cet animal comme le premier des anthropoïdes.

Avant de pénétrer dans notre sujet, il n'est pas inutile de rappeler que la détermination du genre gorille a été faite dans l'origine d'après les crânes et squelettes de l'espèce qui a reçu le nom de Gorilla Savagii ; espèce caractérisée principalement par les grandes crêtes osseuses qui surmontẹt le crâne des individus mâles et adultes, à l'exclusion des jeunes mâles et des femelles. Mais depuis lors de nombreux documents fournis par les chasseurs de l'Afrique équatoriale ont rendu très probable l'existence d'une autre espèce de gorille, différenciée de la première par une taille moins élevée, par la couleur rousse des poils de la région dorsale, et par la petitesse des crêtes crâniennes des mâles adultes. Si la question est encore douteuse, c'est parce que, dans les cas extrêmement rares où l'on a pu expédier en Europe le corps entier d'un gorille mâle et adulte, on a naturellement choisi, pour ces envois coûteux, des sujets appartenant à l'espèce demandée, c'est-ì-dire présentant bien nettement les caractères du Gorilla Savagii. Quant aux crânes, aujourd'hui très nombreux, qui sont déposés dans les musées, on a l'habitude de les déterminer en se basant, non sur les certificats d'origine ou sur des renscignements précis qui font le plus souvent 
MORPHOLOGLE DU CERVEAU DE L'HOMME ET DES PRIMATES. 603

défaut, mais sur les caractères ostéologiques. Il en résulte que tout crâne de gorille adulte dont les crêtes ne sont que rudimentaires est généralement considéré comme appartenant à une femelle. C'est simple et commode, et de la sorte on peut continuer à croire qu'il n'y a qu'une seule espèce de gorille. Mais il faut y mettre cependant une certaine complaisance, car il y a des cas où la crête sagittale est dessinée et formée à un degré suffisant pour rendre le sexe féminin invraisemblable, et où elle est néanmoins incomparablement plus petite que chez les mâles adultes de l'espèce Gorilla Savagii. Voici d'ailleurs un fait qui me paraît de nature à établir l'existence d'une seconde espèce de gorille. Parmi les trois squelettes entiers de gorilles que possède le musée de l'Institut anthropologique, il en est un, le numéro 3, qui nous a été donné par M. le docteur BérengerFéraud, et qui est incontestablement masculin : la taille, les os longs, le bassin, ne laissent à cet égard aucun doute. En outre, les dents sont fortement usées et toutes les sutures du crâne et de la face sont effacées, à l'exception de la suture naso-maxillaire dont le vestige est encore apparent. Le sujet est donc non seulement adulte, mais vieux, et plus vieux qu'aucun des sept autres gorilles de la collection. Ce serait donc celui qui, d'après la règle, devrait avoir la plus grande crête sagittale, c'est au contraire celui qui a la plus petite. Les deux crêtes temporales, qui se rejoignent ordinairement au niveau ou même en avant du bregma pour donner naissance à la crête sagittale, se recourbent en arrière, se rapprochent lentement de la ligne médiane ct ne l'atteignent qu'à 16 centimètres de la glabelle, au lieu de 7 qui est la distance ordinaire; il en résulte que la crête sagittale est extrêmement courte; elle n'a que 39 nillimètres de longueur, tandis que sur tous les autres gorilles mâles elle atteint une longueur de 103 à 134 millimètres. Si l'on représente par 100 la longueur totale du crâne, comprise entre la glabelle et la crête occipitale, on trouve que la longueur de la crête occipitale n'est que de 19,8 pour 100 sur le gorille $n^{\circ} 3$, tandis qu'elle varie chez les autres gorilles entre 56,2 et 64,5 pour 100 . La plus grande hauteur de cette crête, au niveau du lambda, n'est que de 20 millimètres au lieu de 32 à 43 qu'elle mesure sur les autres gorilles. Des différences analogues s'observent sur la crête 
lambdoïdienne, dont la saillie est de 37 à 43 millimètres chez les gorilles ordinaires (1) et de 22 millimètres seulement sur le squelette $\mathrm{n}^{0} 3$. Je pense donc que ce dernier gorille appartient à une espèce autre que le Gorilla Savagii, et caractérisée par la petitesse des crêtes crâniennes; si l'on n'admettait pas cette conclusion, il faudrait admettre du moins que certains gorilles déjà adultes, et même vieux, peuvent n'avoir que des crêtes rudimentaires, ce qui est tout un, d'ailleurs, au point de vue de la question que soulèvera tout à l'heure l'observation du gorille du docteur Nègre.

On a pu étudier sans difficulté les cerveaux de l'orang et du chimpanzé, parce que ces animaux, lorsqu'ils sont encore jeunes, se laissent aisément réduire en captivité. On les amène en Europe, où leur vie est généralement assez courte, et à leur mort les anatomistes peuvent recueillir leur cerveau tout frais encore, et en parfait état. Mais les jeunes gorilles, dont le naturel est beaucoup plus sauvage, meurent presque toujours très peu de temps après avoir été capturés; un seul jusqu'ici a pu être amené vivant en Europe; c'est celui qu'on élève précieusement à l'aquarium de Berlin, et à qui je souhaite de longs jours.

Privés de cette ressource, les naturalistes espéraient du moins pouvoir étudier les cerveaux des gorilles tués au Gabon et conservés dans le rhum; mais ce moyen de conservation, parfaitement suffisant pour les chairs et pour les viscères ordinaires, ne l'est pas pour le cerveau; le liquide alcoolique ne s'imbibe que lentement à travers les os du crâne et surtout à travers la duremère, et lorsqu'il arrive au contact de la substance cérébrale, celle-ci a déjà subi une altération irrémédiable; - d'autant mieux que le plus souvent, sous ce climat équatorial, la putréfaction est déjà commencée avant même que le corps de l'animal

(1) Sur le crâne no 1, qui a ses quatre dents de sagesse, mais qui est encore preaque jeune, car toutes les sutures, y compris la basilaire, sont ouverles, la crête sagittale n'a que 27 millimètres de hauteur, et la lambdoïde 28 . Ces mesures, malgré la jeunesse du sujet, sont encore bien supérieures à celles que donne le crine du squelette no 3 . 
soit amené à la station européenne. L'action du liquide conservateur met un terme à cette décomposition cadavérique, et la plupart des organes reviennent à un état qui se prête très bien aux recherches anatomiques; mais la substance friable du cerveau, une fois ramollie, ne se raffermit plus, et se dissocie de plus en plus, même au sein du liquide conservateur. Il en résulte qu'à l'ouverture du crâne, pratiquée dans les laboratoires d'Europe, au bout d'un ou plusieurs mois, on trouve à la place du cerveau, tantôt une bouillie sans aucune consistance, tantôt une matière mollasse qui se déforme et s'écroule dès qu'on y touche. Une. fuis seulement, en 1860, Gratiolet put, à force de précautions, utiliser cette masse pulpeuse, y reconnaître la disposition des principales circonvolutions et en faire faire la photographie ; mais ces observations très incomplètes, dont je donnerai plus loin les résultats, étaient tout à fait insuffisantes et montraient la nécessité de recourir à des moyens de conservation plus efficaces.

Pour conserver convenablement le cerveau sans le déformer; on peut recourir au procédé recommandé par M. Bischoff et qui consiste à pousser dans la carotide une injection de chlorure de zinc, puis à entamer le crâne avec la scie avant de plonger l'animal dans l'alcool. Ce procédé a été appliqué avec succès dans un cas tout spécial dont je parlerai tout à l'heure, et il est d'une exécution assez facile; mais il exige un outillage qui en restreint beaucoup l'application dans les conditions ordinaires. Il est plus simple d'ouvrir le crâne avec la scie, d'extraire le cerveau, de le déposer sur un fond de ouate dans un vase plein d'alcool et de le retourner tous les deux ou trois jours jusqu'à ce qu'il soit bien durci. Quoique ce procédé soit familier à tous les médecins, j'ai cr'u devoir l'exposer en détail dans les Instructions générales sur l'anthropologie, publiées en $186 \%$ par la Société d'anthropologie de Paris; j'y ai même joint une mention spéciale cuncernant les cerveaux des anthropoïdes. Toutes ces recommandations sont restées longtemps sans effet. Plusieurs fois les médecins de la flotte ont bien voulu me rapporter des singes très bien conservés dans le tafia, et très bons pour la dissection, - à l'exception des cerveaux, qui étaient réduits en putrilage; il m'a donc paru nécessaire de leur faire parvenir, en 1870, au nom du Laboratoire d'anthropologie, par l'intermédiaire du mi- 
nistère de la marine, des instructions particulières sur ce sujet. Peu de temps après, mon ami, le docteur Bérenger-Féraud, médecin principal de la marine, qui, en sa qualité de médecin en chef de nos stations du Sénégal, entretenait des relations fréquentes avec les médecins de la station du Gabon, voulut bien exciter leur zèle en faveur de nos collections, et je ne saurais trop l'en remercier, car j'ai reçu depuis lors trois squelettes de gorilles et quatre crânes isolés qui sont aujourd'hui déposés dans notre musée. Mais c'étaient surtout des cerveaux que j'avais demandés, et mes confrères de la marine, malgré tout leur empressement, n'arrivaient jamais à temps. Les altérations cadavériques surviennent si promptement dans l'Afrique intertropicale, que les cas où l'autopsie méthodique du crâne peut être faite avant le début de la décomposition du cerveau ne peuvent être que très exceptionnels. On sait en effet que les gorilles adultes ne sont jamais pris vivants. On les tue dans les bois, et il s'écoule presque toujours plusieurs jours avant qu'on transporte leur corps à la station.

Pour la première fois enfin, pendant l'hiver de 1876, M. le docteur Nègre, médecin de la marine au Gabon, a pu et su saisir une occasion favorable. Ayant appris, le jour même, qu'un gorille avait été tué dans les bois à quelque distance de la station, il monta à cheval dès le lendemain matin et se rendit sur les lieux, emportant avec lui un scalpel, une scie et un bocal de tafia. Il pratiqua une coupe méthodique, et fit l'extraction du cerveau, qui était en parfait état. Il constata que cet anthropoïde était de grande taille, qu'il était mâle et qu'il avait ses quatre dents de sagesse. Ne disposant ce jour-là d'aucun moyen de transport, et voulant néanmoins préparer le squelette, il fit creuser une fosse peu profonde, où le corps du gorille fut déposé; il le recouvrit d'un peu de terre et de branchages, mais lorsqu'il revint quelque temps après, il ne retrouva plus que quelques ossements dispersés autour de la fosse. Il battit vainement les buissons sans pouvoir découvrir le crâne. Des animaux carnassiers avaient fouillé la fosse, dépecé le cadavre et emporté dans leurs tanières ce qu'ils n'avaient pu dévorer sur place. G'est une perte très fâcheuse, car si la détermination du genre est certaine, celle de l'espèce reste douteuse. 
M. le docteur Nègre a vu des chimpanzés et des gorilles; il a donc pu reconnaître par lui-même qu'il s'agissait d'un gorille et non d'un chimpanzé. C'était d'ailleur's l'opinion bien formelle des nègres qui l'accompagnaient et des chasseurs qui avaient tué l'animal. Mais, quoique cet anthropoïde fût mále et adulte, les crêtes crâniennes étaient rudimentaires, et elles n'opposèrent aucun obstacle à l'autopsie régulière du crâne.

Ce renseignement, que $M$. Nègre nous avait donné dans le laboratoire avant l'ouverture de la boîte soudée où était renfermé le cerveau, avait fait naître dans notre esprit quelques doutes sur le genre de l'animal. Nous avions déjà étudié le gorille $n^{\circ} 3$ dont il a été question plus haut, et nous admettions déjà, dans le genre gorille, l'existence d'une seconde espèce, distinguée du Gorilla Savagii par l'absence des grandes crêtes crâniennes. Nous avions bien cependant quelque crainte de voir sortir de la boîte le cerveau d'un chimpanzé. Mais lorsque la pièce fut sous nos yeux, tous nos doutes se dissipèrent, et mes deux préparateurs, MM. Ghudzinski et Kuhff, qui ont moulé, dessiné et étudié plusieurs cerveaux de chimpanzé, s'écrièrent en même temps que moi que ce cerveau ne pouvait pas être celui d'un chimpanzé.

La pièce fut présentée à la Société d'anthropologie, dans la séance du 3 août 1876 (1). A côté d'elle je plaçai trois cerveaux de chimpanzé et un cerveau d'orang. On put constater que le cerveau rapporté par le docteur Nègre différait de la manière la plus évidente de ceux de tous les anthropoïdes connus jusqu'alors, mais qu'il présentait néanmoins tous les caractères distinctifs des cerveaux de cette famille. Cette présentation ne souleva aucune discussion, et je ne remis au secrétaire qu'une note très abrégée, me proposant de faire une communication plus étendue à la section d'anthropologie de l'Association française, dont la réunion était prochaine. Mais étant tọmbó malade pendant la durée de la session de Clermont-Ferrand, je ne pus faire cette communication, qui a été reportée sur la session de cette année (Session dı Havre, séance du 25 auût 1877).

(1) P. Broca, Présentation du cerveau d'un gorille mâle et adulte, dans Bul letins de la Société d'anthropologie, 1876, p. 426-431, avec une planche représentant la face supérieure du cerveau du gorille, et dans ce volume, p. 567 . 
Ma note du 3 août 1876 n'était pas encore publiée, lorsque le Congrès des naturalistes et médecins allemands tint à HambourgAltona, le 18 septembre 1876 , sa quarante-neuvième session. Les mémoires d'apparat (festgabe) présentés aux membres du Congrès par M. Bolau, renfermaient des études zoologiques et anatomiques très intéressantes faites sur les corps de trois gorilles appartenant au musée de Hambourg, et M. Pansch y avait joint la description du cerveau d'un de ces animaux, récemment rapporté par M. Freckmann, que la Société zoologique de Hambourg avait envoyé en mission au Gabon pendant l'hiver 1875 -1876.

Cet animal, âgé d'environ six mois, fut amené à la mission américaine du Gabon, où il mourut trois jours après. Le corps fut donné aussitôt à M. Freckmann, qui, avant de le plonger dans le rhum, poussa dans la carotide, suivant le procédé de M. Bischoff, une injection de chlorure de zinc, et qui en outre entama de chaque côté le crâne avec la scie, pour faire pénétrer le rhum dans la boîte crânienne. Grâce à ces opérations, le cerveau se conserva parfaitement et put être étudić complètenıent par M. Pansch, qui en publia une description très soignée, accompagnée de trois photographies (1).

Cette publication reçue à Londres dans la première semaine de décembre, donna lieu à des observations importantes consignées par M. G.-D. Thane, dans le numéro du 14 décembre 1876 du journal Nature (2). Mais ce fut surtout M. Bischoff, de Munich, qui s'en préoccupa. M. Pansch, dans sa description, avait émis, relativement à la troisième circonvolution frontale des singes en général et du gorille en particulier, une opinion contraire aux idées soutenues par le savant professeur de Munich. Celui-ci désirant contrôler les résultats communiqués au Gongrès de Hambourg, s'adressa à M. Bolau, qui voulut bien lui envoyer la pièce originale; il put ainsi la décrire et l'interpréter à son tour dans un mémoire très étendu, communiqué le 10 mars 1877

(1) Bolau (Heinrich), Die Menschenæhnlichen Affen der Hamburger Nuseums, Hamburg, 1876, in-4. Dans Abhandlungen aus dem Gebiete der Naturwissenschaften, herausgegeben vom Naturwissenschaftlichen Verein zu Hamburg-Altona. A la page 20 commence le mémoire de M. Ad. Pansch, intitulé : Ueber die Furchen und Windungen am Gehrm eines Gorilla.

(2) Nature, vol. XV, p. 142. 
à la classe mathématico-physique de l'Académie des sciences de Munich (1). Il fit en outre exécuter un modelage en cire; mais comme la pièce provenait d'un animal très jeune, M. Bischoff voulut rendre à ce petit cerveau les formes et les dimensions du cerveau de l'animal adulte. A cet effet, il fit faire le moule en cire de la cavité crânienne d'un gorille adulte, et ce fut sur ce bloc de cire que furent reportés aussi exactement que possible les sillons et les circonvolutions des hémisphères du jeune gorille de Hambourg (2).

La publication du quatrième et dernier fascicule des Bulletins de la Société d'anthropologie pour $\mathbf{1 8 7 6}$ ayant été retardée pendant plusieurs mois (3), M. Bischoff ne pouvait savoir encore, à la date du 10 mars 1877, que j'avais présenté au mois d'août précédent à cette Société le cerveau d'un autre gorille, rapporté par le docteur Nègre. En recevant le fascicule en retard, et en jetant les yeux sur le dessin de la page 429, M. Bischoff fut frappé de la différence qui existe entre ce dernier cerveau et celui du gorille de Hambourg. Il m'écrivit aussitôt pour me signaler cette différence, que les photographies de M. Pansch m'avaient déjà permis d'apprécier, et pour m'exprimer l'idée que l'animal du docteur Nègre ne devait pas être un gorille, mais un chimpanzé. J'aurai bientôt à revenir sur cette opinion, que je ne saurais partager.

Telles sont les sources des connaissances que nous possédons aujourd'hui sur le cerveau du gorille.

\section{III}

Avant de pouvoir étudier directement le cerveau du gorille, on en était réduit aux observations faites sur les moules intra-

(1) Bischoff, Ueber das Gehivn eines Gorilla und die untere oder dritte Stirnwindung der Affen, in Sitzung der Math. Phys. Classe von 10 Mirs 1877, p. 96-139, avec 4 pl. lith.

(2) Un exemplaire de ce modelage, obtenu par voie d'échange, est actuellement déposé dans le musée de l'Institut anthropologique de Paris.

(3) On se proposait d'y joindre le catalogue de la Bibliothèque, dont l'impression n'a pu être achevée en temps utile. Le dernier fascicule de 1876, au lieu de paraître en janvier de l'année suivante, comme d'habitude, n'a paru qu'au commencement d'avril, près d'un mois après la date du mémoire de M. Bischoff. 
crâniens et aux renseignements fournis par le cubage des crânes. Gratiolet n'eut pas recours à ce dernier moyen, mais il publia, en 1854, dans son célèbre mémoire sur les plis cérébraux de l'homme et des primates (1), les résultats de ses études comparatives sur les moules intracrâniens d'un gorille, d'un chimpanzé et d'un orang. Par un hasard malheureux et extraordinaire, il se trouva que le crâne de son gorille était d'une petitesse exceptionnelle et celui de son orang d'une grandeur exceptionnelle. Les deux moules avaient la même longueur., mais celui de l'orang était plus large et surtout plus haut. Gratiolet fut donc conduit à admettre que le cerveau du gorille était moins volumineux que celui de l'orang, résultat tout accidentel qu'il se laissa aller trop facilement à généraliser et que tous les faits ultérieurs sont venus contredire. Mais l'observation relative à la plus grande hauteur du cerveau de l'orang a été pleinement confirmée.

Le cerveau du gorille, dit Gratiolet, est plus déprimé, plus plat; le lobe frontal est moins haut, mais par une sorte de compensation, il est moins atténué à son extrémité antérieure, en sorte que le cerveau, vu par sa face supérieure, n'est point ovale, comme celui de l'orang, mais elliptique, forme qui rappelle celle du cerveau des cynocéphales. Ajoutons que les plis cérébraux laissent sur la boìte du crâne des impressions un peu plus marquées, circonstance qui semble indiquer une richesse un peu moindre du développement de ces plis.

Passant ensuite au cerveau du chimpanzé, Gratiolet ajouta :

Sa forme s'éloigne beaucoup de celle qu'on observe chez les orangs et rappelle davantage celle des gorilles. Nous remarquerons, néanmoins, une saillie plus grande du lobe sphénoïdal dans le chimpanzé, un développement plus apparent du lobe frontal, en un mot, et malgré la dépression générale du cerveau, un aspect plus globuleux, plus arrondi dans toutes ses parties.. . Je crois pouvoir assurer l'avance que dans le gorille, le lobe occipital est beaucoup plus grand que dans le chimpanzé. Cette circonstance bien apparente d'une réduction relative du lobe frontal, correspondant à un plus grand développement du lobe occipital, établirait entre le gorille et le chimpanzé une différence semblable à celle 'qu'on observe entre le cerveau des cynocéphales et celui des macaques; et ce fait, rapproché de la forme particulière du crâne des gorilles, forme qui rappelle au premier abord, bien que dans des proportions plus massives, celle des

(1) Paris, 1854 , in $-4^{\circ}$, p. 54. 
baboins, rend très probable la vérité de cette proposition : que le gorille est un baboin (cynocéphale); que le chimpanzé est un macaque, au même titre que l'orang est un gibbon.

On trouvera peut-être que c'était aller bien vite d'édifier, sur des observations aussi peu nombreuses et aussi incomplètes, cette doctrine des trois séries simiennes, développées parallèlement et ayant chacune pour chef l'un des trois grands anthropoïdes. On a dit que ce jour-là Gratiolet fut transformiste sans le savoir; mais il avait conclu avant de connaître les faits. Le rapprochement qu'il avait établi entre le cerveau du gorille et celui des cynocéphales ne reposait en réalité que sur la grandeur supposée du lobe occipital du gorille; or il se trouve précisément que ce lobe est très petit et qu'il est relativement beaucoup moins développé que le lobe frontal.

Gratiolet put croire cependant que ses prévisions s'étaient réalisées. Le Muséum d'histoire naturelle reçut en 1860 le corps d'un gorille femelle, envoyé par M. Sennal, lieutenant de vaisseau. Gratiolet ouvrit le crâne avec le plus grand soin. Il parvint, à force de précautions, à extraire " une masse cérébrale caséiforme au centre, pultacée à la surface, mais sur laquelle une insuffla. tion ménagée a fait, en ouvrant un peu les anfractuosités, apparaître tous les détails essentiels des plis cérébraux ». Quant au cervelet, il était réduit en une « boue liquide ». Comme on ne pouvait guère espérer qu'une pièce aussi décomposée pût se conserver, on en fit une photographie de grandeur naturelle, qu'il me paraît utile de publier ici (fig. 78). Gratiolet put en outre faire quelques observations sur les faces externe et inférieure, qui ne peuvent se voir sur la photographie. La note qu'il communiqua le 30 avril 1860 à l'Académie des sciences renfermait une description nécessairement très incomplète de ce cerveau altéré (1). Nous y lisons que le cerveau du gorille est comparativement plus plat que celui du chimpanzé, mais qu'il est « moins atténué en avant ", c'est-à-dire qu'il a les lobes frontaux plus larges. L'auteur signale la brièveté de la face orbitaire, « qui est en outre très peu excavée », et s'efforce de prouver, par des arguments extrinsèques, que ce dernier caractère, qui constitue 
chez l'homme un signe de supériorité, constitue au contraire chez le gorille un signe d'infériorité; mais il aurait peut-être fallu se demander si cet aplatissement de la surface orbitaire était naturel, ou s'il n'était pas dû simplement à l'affaissement de la substance cérébrale ramollie.

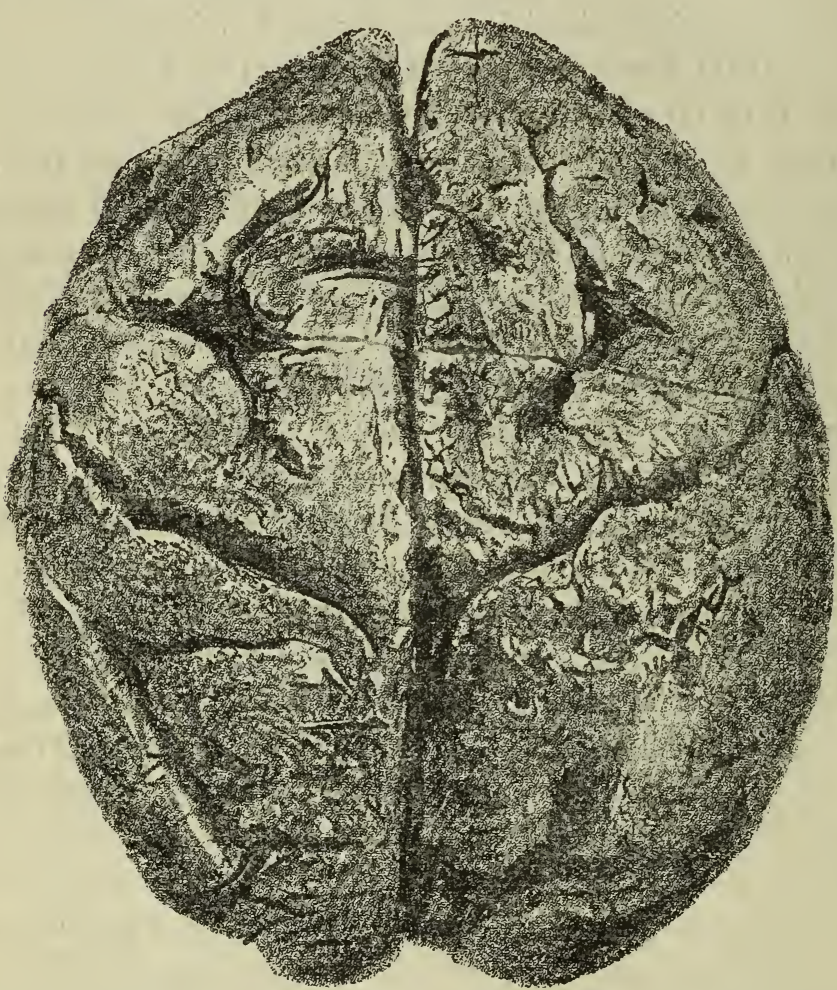

Fig. 78. - Reproduction de la photographie du cerveau très altéré extrait en 1860, par Gratiolet, du crâne d'un gorille femelle adulte, expédié du Gabon dans un tonneau de tafia.

Les plis cérébraux, continue Gratiolet, sont larges, à peine flexueux, et d'une simplicité remarquable. L'étage supérieur du lobe frontal (première circonvolution frontale) est divisé en deux gros plis par une scissure à troi $i_{\mathbf{S}}$ branches, assez semblable à celle du chimpanzé, mais beaucoup moins compliquée. Les étages moyen et inférieur (deuxième et troisième circonvolution frontale) sont également très peu divisés. Le premier pli ascendant est très épais, à peıne flexueux et très incliné en arrière; le second pli ascendant a une obliquité plus grande encore et se termine supérieurement par un petit lobule triangulaire à peine divisé, fait digne d'être noté, parce que, dans le chimpanzé, ce même lobule est grand, quadrilatère, et chargé 
de plis nombreux. Il n'y a, entre ce lobule et le sommet du lobe occipital, aucune trace d'un pli supérieur de passage. Le pli courbe présente un sommet très aigu ; il émane, comme dans les macaques et les cynocéphales, du sommet de la scissure de Sylvius; un gros pli de passage caché sous l'opercule l'unit au lobe occipital, qui est peu développé.

La scissure de Sylvius est longue, très couchée en arrière; sa marge inféricure (première circonvolution temporcle) est limitée par une scissure parallèle assez profonde. Les autres divisions du lobe inférieur (lobe temporul) sont très simples, et il en est de même de toutes celles que présente lit face médiane des hémisphères. Elles présentent d'ailleurs le type commun à tous les singes.

Cette fois, Gratiolet ne pouvait plus se baser sur le grand développement du lobe occipital pour établir un rapprochement entre le cerveau du gorille et celui du cynocéphale; il trouvait, contrairement à son attente, que ce lobe, si grand chez les cynocéphales, et grand encore chez le chimpanzé, était, chez le gorille, réduit à un petit volume; il resta néanmoins fidèle à sa précédente conclusion, parce que les circonvolutions du gorille, par leur largeur, leur simplicité et leur peu de flexuosité, lui rappelaient celles des cynocéphales, et aussi parce qu'il croyait que le pli supérieur de passage (ou premier pli de passage) faisait cntièrement défaut chez le gorille. Je ne m'explique pas comment cette dernière assertion a pu glisser sous sa plume, car il suffit de jeter les yeux sur la photographie du cerveau qu'il a décrit, pour voir que du côté gauche la scissure occipitale externe ne s'étend pas tout à fait jusqu'au bord interne de l'hémisphère, que par conséquent le premier pli de passage existe, et qu'il est même superficiel; à droite, la scissure occipitale externe, manifestement altérée et très largement ouverte, se continue sans interruption avec la scissure occipitale interne, mais, de ce côté même, on ne peut conclure à l'absence du pli de passage, car il est évident qu'à ce niveau une portion notable de la substance cérébrale a été détruite. On remarquera que, dans cette nouvelle description, il n'est plus question de la petitesse du lobe frontal, signalée dans le premier travail de l'auteur, d'après l'examen trompeur du moule intracrânien. On peut voir, en effet, sur la photographie, que le lobe frontal du gorille est très long et très large, et que les deux scissures rolandiques, fort peu obliques, interceptent un angle très obtus. Quant à la 
pauvreté des circunvolutions, elle est réelle, mais exagérée très probablement par suite de l'altération de la pièce. On conçoit, en effet, que, sur cette écorce cérébrale ramollie et pultacée, les sillons superficiels se soient effacés, et que les sillons grands et profonds soient seuls restés distincts.

Malgré ces imperfections, la photographie que nous devons à Gratiolet nous montre quelques-uns des traits les plus essentiels du cerveau du gorille, et j'ai tenu à la reproduire ici, parce qu'elle pourra nous fournir tout à l'heure d'utiles points de comparaison, et surtout parce qu'elle est le témoin d'une observation qui a été la première en date et qui a été pendant seize ans, jusqu'en 1876, la source de tout ce que l'on savait sur le cerreau du gorille.

\section{IV}

J'arrive maintenant à la description du premier cerveau de gorille qui ait été réellement étudié; c'est celui qui a été rapporté, en 1876, par M. le docteur Nègre. Il provient d'un sujet mâle et adulte.

Cette pièce est complète et parfaitement intacte, mais elle a subi, dans le vase où elle a été conservée, une certaine déformation. M. Nègre, obligé de s'absenter pour les besoins du service, ne put la surveiller lui-même ; on négligea de la retourner de temps en temps, pendant les premiers jours, de sorte qu'elle s'affaissa un peu sous son propre poids avant d'être durcie. Cette circonstance a dû exagérer quelque peu la largeur des hémisphères et en diminuer la hauteur. En outre, les deux hémisphères, inégalement déprimés, sont devenus asymétriques, le droit étant moins large et moins plat que le gauche. Cette asymétrie d'ailleurs concerne seulement les formes générales et elle ne participe en rien à l'asymétrie des sillons et des circonvolutions, dont il sera question plus loin.

Le poids de l'encéphale n'a pu être déterminé à l'état frais. Au sortir de la boîte, après six mois de séjour dans l'alcool à 36 degrés, cet organe ne pesail plus que 252 grammes. En communiquant ce chiffre à la Sosiété d'anthropologie, j'ajoutai : "Il résulte de diverses observations que j'ai eu l'occasion de faire dans le laboratoire, que le cerveau, à la suite de 
l'action prolongée de l'alcool, perd en moyenne de 28 à 30 pour 100 de son poids. Ce chiffre n'a rien de rigoureux; il présente des variations assez étendues suivant les espèces et suivant les individus; il dépend pour beaucoup de la proportion relative de la substance blanche et de la substance. grise. G'est donc sous toutes réserves que j'évalue à environ 360 grammes le poids primitif de notre cerveau de gorille, chiffre qui paraîtra assez faible si l'on songe que l'animal était mâle, adulte et de grande taille (1). »

Dans l'important mémoire que j'ai déjà cité, et sur lequel j'aurai plusieurs fois l'occasion de revenir, M. Bischoff évalue à 25 pour 100 seulement la déperdition de poids due à l'action combinée de l'alcool et du chlorure de zinc (2). D'après cette évaluation, le poids réel du cerveau en question descendrait à 336 grammes.

L'encéphale, dans son ensemble, a été moulé par M. Chudzinski avant qu'on eût pratiqué la section médiane pour étudier la face interne des hémisphères (3). Les dimensions de ce moule sont les suivantes :

Longueur totale..................... 108 millimètres.

Largeur?maxima, à la base des lobes temporaux. $\quad 96 \quad$ -

Largeur des hémisphères, au niveau de la pointe des lobes temporaux....................

Hauteur maxima, au niveau de la partie moyenne

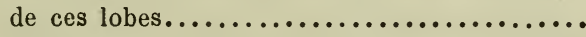

Longueur de la surface orbitaire en avant de la

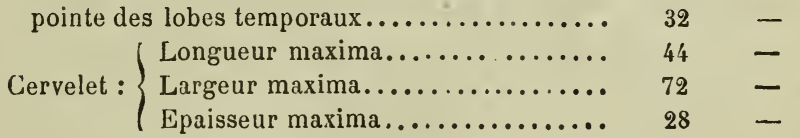

Sur la photographie de Gratiolet, représentant le cerveau d'une femelle adulte, la longueur n'est que de 102 millimètres et la largeur maxima de 87 millimètres.

Par suite de la déformation signalée plus haut, l'hémisphère

(1) Bulletins de la Société d'anthropologie, 1876, $2^{\text {e }}$ série, t. XI, p. 431 et dans ce volume, p. 571 .

(2) Bischoff, Ueber das Gehirn eines Gorilla, etc. Munich, 1877, in-8. Dans Sitz. der Math. Phys. Classe, p. 98.

Ł (3) M. Tramond, naturaliste, rue de l'Ecole-de-Médecine, a fait; pour nos échanges, un bon creux de cè moule et du moule de l'un des hémisphères. Je l'ai autorisé à en mettre des exemplaires dans le commerce. 
droit l'emporte sur le gauche de 4 millimètres en longueur, et de 2 millimètres en hauteur; mais celui-ci, en revanche, est plus large que l'autre d'environ 8 millimètres.

Le cervelet est d'une petitesse remarquable; il est entièrement recouvert et même un peu dépassé par les lobes occipitaux, quoique ceux-ci soient très petits.

La protubérance annulaire, peu saillante, est longue de 20 millimètres et large de 21 millimètres. Les pédoncules cérébraux sont volumineux; le plancher du troisième ventricule était un peu déchiré et est mal venu sur le moule ; mais, avant le moutlage, j'ai pu constater la présence des deux tubercules mamillaires, du tuber cinereum et de la base de l'infundibulum; ces parties étaient disposées comme chez l'homme.

Sur la coupe médiane, le corps calleux a 41 millimètres de longueur, il est court et massif ; l'épaisseur du bourrelet s'élève à 7 millimètres.

Avant de passer à la description des circonvolutions des hémisphères, je demande la permission de déterminer le sens des mots que j'emploierai pour désigner les diverses espèces d'anfractuosités de l'écorce cérébrale connues sous les noms généraux de sillons, scissures, fissures, incisures, en latin sulci, en allemand furchen, etc. Ces dénominations étaient employées indistinctement à l'époque où l'on croyait que les circonvolutions étaient dispersées sans aucun ordre ; cela n'avait alors aucun inconvénient. Mais on sait aujourd'hui que les plis du cerveau ne sont nullement désordonnés, qu'ils forment certains groupes appelés lobes, que chaque lobe est formé d'un certain nombre de circonvolutions, et que ces circonvolutions enfin sont rendues plus ou moins compliquées par la présence et l'absence de plis secondaires. Puisque la surface des hémisphères présente des subdivisions de trois ordres, les "sillons " qui établissent ces subdivisions sont également de trois ordres et ne doivent pas être confondus sous un même nom. Les dénominations de sillons primaires, secondaires et tertiaires seraient très commodes, si elles n'avaient déjà reçu d'autres acceptions; on les a employées pour indiquer tantôt l'ordre réel ou supposé suivant lequel les plis apparaissent sur le cerveau du fœtus, tantôt pour indiquer le degré d'importance qu'on y attache d'après leurs 
connexions avec telle ou telle partie des hémisphères. Leur donner une nouvelle acception serait introduire dans le langage une confusion fâcheuse. Mais, sans recourir au néologisme, on peut convenir de donner une signification particulière aux trois mots scissure, sillon et incisure, qui sont déjà usités.

J'appelle donc scissures les lignes de dépression qui séparent les lobes, sillons celles qui séparent les circonvolutions de chaque lobe, et incisures celles qui subdivisent les circonvolutions. Les incisures sont tantôt isolées, tantôt continues avec un sillon ou une scissure. Dans ce dernier cas, elles peuvent quelquefois être considérées comme formant une branche du sillon ou de la scissure.

D'après cette nomenclature, la scissure parallèle de Gratiolet ne sera plus que le sillon parallèle (ou le premier sillon tem-. poral), car elle sépare seulement deux circonvolutions d'un même lobe; mais je continuerai à considérer comme une scissure la scissure du petit hippocampe ou scissure calcarine d'Huxley (1), parce que l'anatomie comparée et l'embryologie lui donnent une valeur égale à celle des scissures proprement dites, et parce que le lobule triangulaire (cuneus) qu'elle sépare du reste du lobe occipital présente une constance remarquable, et mériterait peut-être d'être élevé au rang des lobes.

Enfin, je rangerai aussi parmi les scissures le grand sillon longitudinal qui s'étend au-dessus de la circonvolution du corps calleux et qui la sépare du lobe frontal. G'est le grand sillon du lobe fronto-pariétal de Gratiolet, la scissure calloso-marginale d'Huxley, le sillon fronto-pariétal interne de Pansch. C'est une scissure, parce qu'elle établit la limite inférieure du lobe frontal sur la face interne de l'hémisphère ; elle a d'ailleurs, en ana-

(1) La scissure des hippocampes, de Gratiolet, correspond en arrière au petit hippocampe ou ergot de Morand (calcar avis), et en avant au grand hippocampe ou corne d'Ammon. Mais les deux parties de cette prétendue scissure des hippocampes sont entièrement étrangères l'une à l'autre. L'antérieure, située dans la corne inférieure du ventricule latéral, sépare le grand hippocampe du corps godronné. La partie postérieure seule appartient aux lobes de l'hémisphère. Le nom de scissure du petit hippocampe conviendrait très bien à celte parlie postérieure, mais le noin de scissure calcarine (de calcar, ergot) proposé par M. Huxley' a l'avantage d'être plus court et d'être d'ailleur's adoplé déjà par beaucoup d'auteurs. 
tomie comparée, une importance considérable, parce qu'elle est exclusivement propre aux primates, et parce qu'elle constitue l'un des traits les plus caractéristiques de leur cerveau. Chez eux, en effet, elle chemine d'avant en arrière au-dessous du lobe frontal, et mérite le nom de scissure sous-frontale; tandis que, chez les autres mammifères, elle chemine d'arrière en avant au-dessous du lobe pariétal, et prend dès lors le nom de scissure sous-pariétale. Cette différence remarquable se manifeste tout à coup, sans transition, lorsque, après avoir parcouru de bas en haut toute la série, on arrive à l'ordre des primates; elle est la conséquence du grand développement du lobe frontal de ces animaux. Ce caractère, le plus important de ceux qui distinguent le cerveau des primates, se trouve exprimé par la seule opposition des mots scissure sous-frontale et scissure sous-pariétale (1).

Mais revenons au cerveau de notre gorille. Ce qui frappe tout d'abord le regard, c'est la grande simplicité des circonvolutions, qui sont très grosses, très peu flexueuses et très peu subdivisées. Ces caractères, qui se retrouvent sur la photographie de Gratiolet, contrastent d'une manière remarquable avec la complication et les sinuosités des circonvolutions de l'orang et du chimpanzé, et ils expliquent jusqu'à un certain point le rapprochement que Gratiolet a établi entre le cerveau du gorille et celui des cynocéphales. Il faut donc montrer avant tout que tous les caractères essentiels du cerveau des anthropoïdes existent sur le cerveau du gorille, savoir : $1^{\circ}$ le grand développement du lobe frontal et surtout la grande largeur de sa partie antérieure; par ce dernier caractère, le gorille est même supérieur à tous les autres anthropoïdes; $2^{\circ} l^{\prime}$ 'existence de la branche horizontale antérieure de la scissure de Sylvius; $3^{\circ}$ la subdivision du lobule de l'insula en circonvolutions radiées $; 4^{\circ}$ la petitesse et la complication du lobe occipital $; 5^{\circ}$ la terminaison de la scissure sous-frontale, qui

(1) La formation de ces deux scissures se rattache à l'évolution de la grande circonvolution limbique et de la scissure limbique qui l'entoure. Ne pouvant exposer ici cette question que j'ai développée dans mon cours d'anthropologie anatomique de l'année dernière, je renverrai au résumé sommaire que j’ai communiqué à la Société d'anthropologie dans la séance du 6 décembre 1877, et dans ce volume, p. 247. Celte rédaction faisait d'abord partie du présent mémoire; mais c'était une digression un peu trop longue, et il m'a paru préférable de la publier ailleurs. 
de Sylvius), est situé comme toujours sous la pointe du lobe temporal; parvenu au niveau de la première circonvolution de l'insula, il se divise en deux branches inégales, l'une antérieure, l'autre postérieure.

La grande branche, ou branche postérieure, celle qui longe le bord supérieur du lobe temporal, est très inclinée en arrière. Elle l'est notablement plus que chez les autres singes anthropoïdes, à plus forte raison que chez les pithéciens, et elle l'est tout autant que chez l'homme. Il faut tenir compte, il est vrai, de la déformation qui a aplati le cerveau, et qui a pu exagérer un peu ce caractère; mais je remarque que sur l'hémisphère gauche, qui est le plus aplati, la scissure est un pel moins couchée que dı côté droit; l'influence de la déformation n'a donc pu être que très faible, et il faut reconnaîlre que la direction de la branche postérieure de la scissure de Sylvius constitue en faveur du gorille un caractère de supériorité.

Le bord supérieur de cette branche présente, au niveau du lobe pariétal, une incisure profonde. Il y en a presque toujours deux chez l'homme, quelquefois même trois ; chez le chimpanzé et l'orang, tantôt une, tantôt deux. Cette incisure pariétale ne se retrouve qu'exceptionnellement et à l'état rudimentaire chez les gibbons et les pithéciens. Sur notre gorille, elle a 1 centimètre de profondeur (fig. 79, i).

La branche horizontale antérieure de la scissure de Sylvius (S", fig. 79 et 80 ) mérite quelques moments d'attention. Elle naît au niveau de la première circonvolution de l'insula, et se porte horizontalement en avant sous la forme d'une incisure droite et simple, longue de 10 millimètres à droite, de 12 à gauche, qui s'enfonce dans la troisième circonvolution frontale.

La portion de cette circonvolution qui est située au-dessus et en arrière de la branche dont il s'agit fait partie de l'opercule; la portion qui est située au-dessous et en avant de la même branche fait partie du lobule orbitaire du lobe frontal et y forme la troisième circonvolution orbitaire, $0^{3}$.

La discussion qui s'est élevée récemment entre MM. Bischoff et Pansch au sujet de la troisième circonvolution frontale des singes et à la "branche antérieure ou ascendante » de la scissure 
MORPHOLOGIE DU CERVEAU DE L'HOMME ET DES PRIMATES. 621

de Sylvius m’oblige à donner quelques explications générales sur la constitution de cette région de l'hémisphère.

Le nom de branche ascendante de la scissure de Sylvius a été donné à trois choses bien différentes, savoir : $1^{\circ}$ au prolonge-

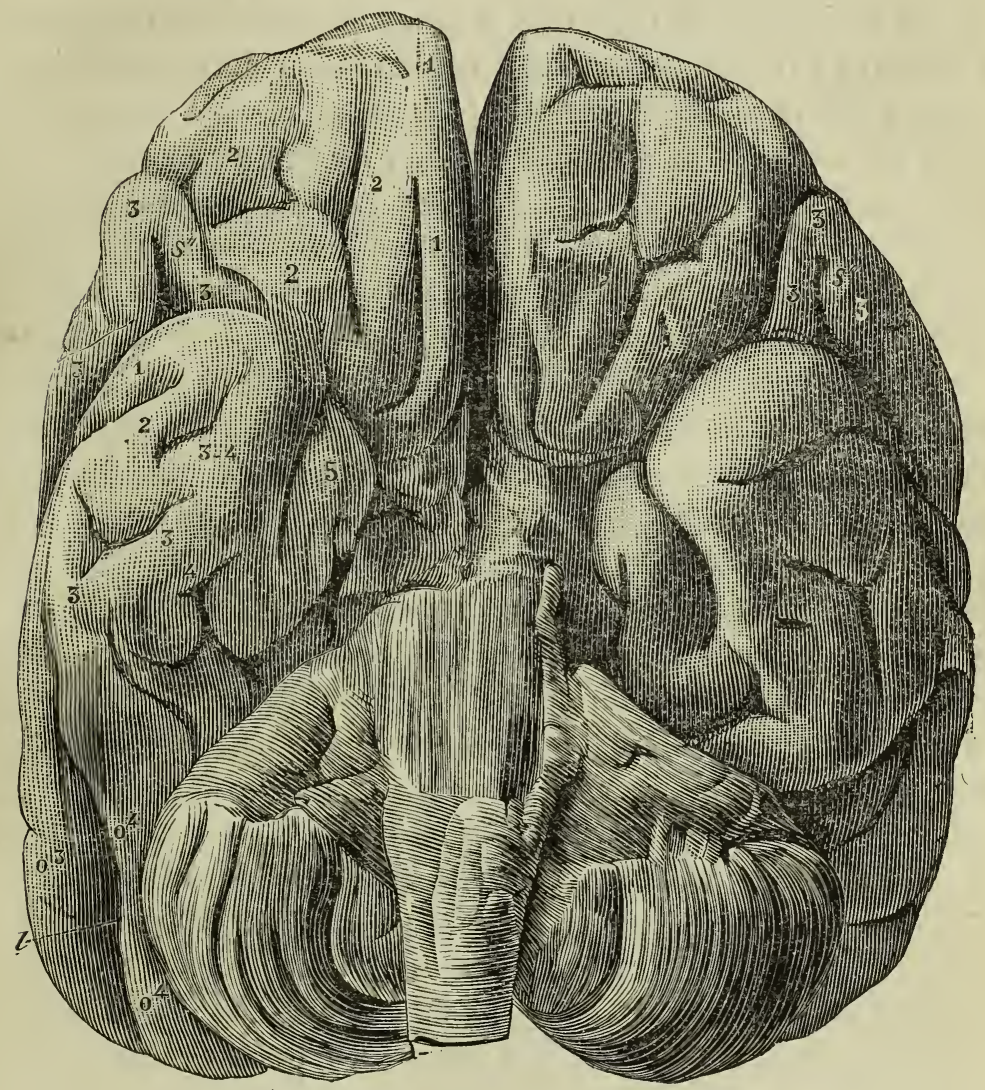

Fig. 80. Cerveau du gorille du docteur Nègre (face inférieure).

ment que le sillon prérolandique envoie quelquefois, sur la racine de la troisième circonvolution frontale, jusqu'au niveau de la scissure de Sylvius; $2^{\circ}$ à la branche horizontale antérieure de la scissure de Sylvius; $3^{\circ}$ à la vraie branche ascendante de cette scissure. Les auteurs qui ont admis la première détermination, entre autres le savant anatomiste d'Edimbourg, M. Will. Tur- 
ner, ont ajouté que la branche ascendante n'est pas constante (1). Le sillon prérolandique ou précentral, qui limite antérieurement la circonvolution frontale ascendante et qui est à peu près parallèle à la scissure de Rolando, commence sur la racine de la première circonvolution frontale; il est interrompu, vers le milieu de son trajet, par la racine de la seconde, reparaît au-dessous d'elle et va se terminer sur la racine de la troisième, qu'il entaille plus ou moins profondément; le prolongement qu'il envoie sur cette dernière racine n'occupe ordinairement qu'une partie de sa largeur, et s'arrête ainsi à quelques millimètres au-dessus de la scissure de Sylvius; mais sur un certain nombre de cerveaux humains, et même sur quelques très rares cerveaux de singes, il se prolonge un peu plus bas et paraît se terminer dans la scissure de Sylvius. Il semble alors que cette scissure émette une branche ascendante très longue, qui remonte jusqu'à la racine de la deuxième circonvolution frontale. Mais ce n'est qu'une apparence, car le prolongement dont il s'agit n'est qu'un plissement superficiel de la racine de la troisième circonvolution frontale; il n'occupe pas toute l'épaisseur de cette racine, il ne communique pas avec le fond de la scissure de Sylvius, et il ne peut être considéré comme une émanation de cette scissure. M. Bischoff a depuis longtemps formulé la même conclusion, reproduite dans son mémoire sur le cerveau du gorille (2).

La branche ascendante et la branche horizontale antérieure sont au contraire de véritables branches de la scissure de Sylvius. Les auteurs qui en ont parlé jusqu'ici n'en ont décrit qu'une seule, l'ascendante, et lorsqu'ils ont vu la seconde, ils l'ont confondue avec la première. Mais il y en a constamment deux chez l'homme, et la plus essentielle des deux n'est pas celle qui est ascendante.

On connaît aujourd'hui la haute importance de la troisième circonvolution frontale, qui est chez l'homme le siège de la faculté du langage articulé. Chez les singes pithéciens, cette circonvolution est très simple : née de la partie inférieure de la

(1) W. Turner, The Convolutions of the Human Brain, in Edimburgh Medical Journal, vol. XI, part. II, 1866, in-8, p. 1108. Voir aussi fig. 1, S'S'.

(2) Bischoff, Ueber die Grosshirnwindungen der Menschen, Munich, 1868, in-4, p. 19 et 27. - Ueber das Gehirn eines Gorilla, Munich, 1877, in-8, p. 112. 
frontale ascendante, elle longe en ligne droite le bord supérieur de la scissure de Sylvius, et, parvenue au-dessus de la partie antérieure du lobe temporal, elle se réfléchit sans aucune sinuo-

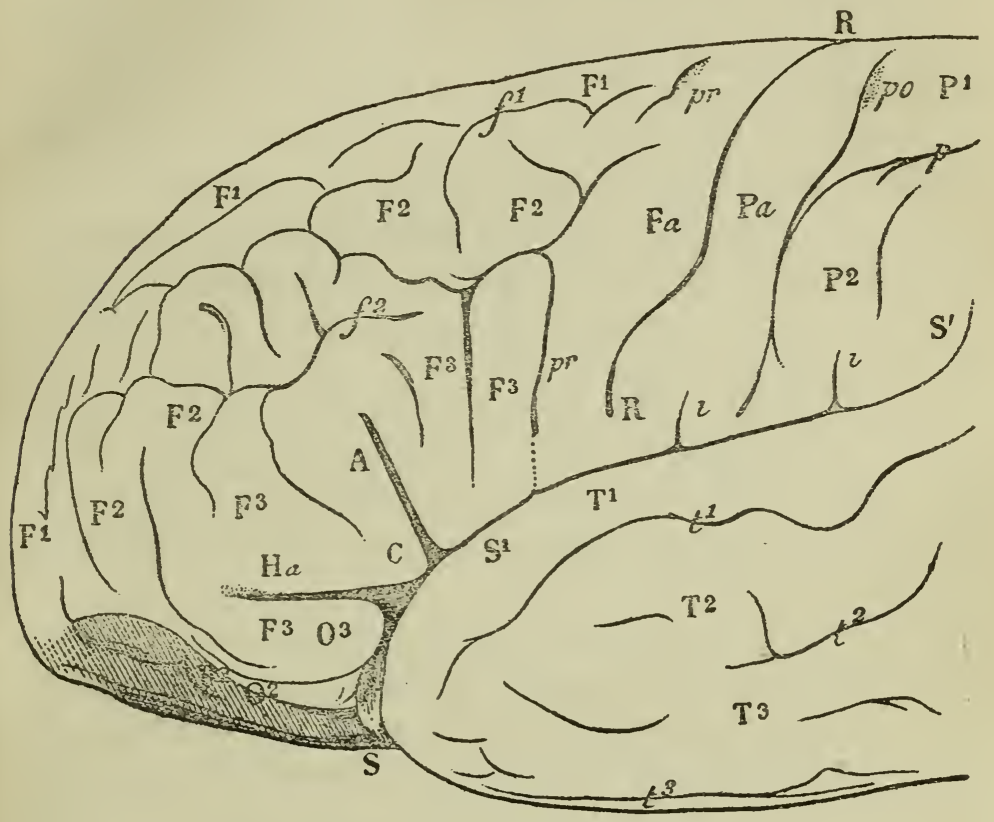

Fig. S1. F, lobe frontal ; T, lobe temporal; RR, scissure de Rolando ; Fa, circonvolution frontale ascendante; $\mathrm{F}^{4}, \mathrm{~F}^{2}, \mathrm{~F}^{3}$, les trois circonvolutions frontales; $\mathrm{F}^{3}, \mathrm{O}^{3}$, portion orbitaire de la troisième circonvolution frontale; $\mathrm{P} a$, circonvolution pariétale ascendante; $\mathrm{P}^{\imath}$, $\mathrm{P}^{2}$, les deux circonvolutions pariétales; $\mathrm{T}^{1}, \mathrm{~T}^{2}, \mathrm{~T}^{\mathbf{3}}$, circonvolutions temporales; $t^{1}$, premier sillon temporal ou sillon parallèle; $t^{2}$, le second; $p$, le sillon pariétal ; $p o$, le sillon postrolandique; $f^{2}$, le premier sillon frontal; $f^{2}$, le second; $p r$, le sillon prérolandique; sa terminaison inférieure sur la racine de la troisième circonvolution frontale présente quelquefois un prolongement superficiel qui est indiqué par une ligne ponctuée et qui, aboutissant à la scissure de Sylvius, a été considéré à tort comme la branche ascendante de cette scissure; $\mathrm{S}$, le tronc de la scissure de Sylvius, ou vallée de Sylvius; $\mathbf{S}^{\prime} \mathrm{S}^{\prime}$, la branche postérieure, ou branche principale de la scissure de Sylrius ; $i, i$, ses incisures pariétales; $A$, la vraie $b r a n-$ che ascendante de la scissure de Sylvius; $\mathrm{H} a$, branche horizontale antérieure de cette scissure, placée sur le bord externe de l'hémisphère et séparant la portion frontale de la troisième circonvolution $\mathrm{F}^{\mathbf{3}}$ de la portion orbitaire $\mathrm{F}^{3} \mathrm{O}^{3} ; \mathrm{C}$, le cap de la troisième circonvolution frontale, petit lobule triangulaire, compris entre la branche ascendante de la scissure de Sylvius et la branche horizontale antérieure.

sité pour se continuer avec la troisième circonvolution orbitaire, qui n'en est que la terminaison.

Chez l'homme, cette circonvolution commence et finit de la même manière, mais devenue beaucoup plus longue, elle ne peut trouver place entre le lobule orbitaire et la frontale ascendante, qu'en se repliant plusieurs fois, en décrivant des méan- 
dres plus ou moins compliqués et par conséquent assez variables ; cette région présente néanmoins deux particularités absolument constantes. Ce sont : $1^{\circ}$ la branche horizontale $(\mathrm{H} a) ; 2^{\circ}$ la branche ascendante de la scissure de Sylvius (A). (Voir. fig. 81.)

La première naît de l'extrémité externe de la vallée de Sylvius, et par conséquent de l'extrémité antérieure de la branche postérieure de la scissure de Sylvius, au niveau du bord antérieur du premier pli de l'insula, et un peu au-dessus du bord falciforme qui s'étend de la dernière circonvolution temporale à la troisième circonvolution orbitaire. Elle se porte horizontalement en avant, dans l'épaisseur de la troisième circonvolution frontale, à l'union de la face externe et de la face inférieure du lobe frontal; c'est elle qui établit la démarcation entre la portion supérieure ou frontale de cette circonvolution et sa portion orbitaire. Elle est ordinairement simple; quelquefois cependant elle se termine en une courte bifurcation. Sa longueur est assez variable; elle peut n'être que de 1 centimètre et s'élever à 3 et même 4 centimètres.

La branche ascendante naît du bord supéricur de la scissure de Sylvius un peu au-dessus de la précédente; elle se dirige en haut, et quelquefois aussi un peu en avant, suivant une ligne qui, chez l'homme, correspond assez exactement au trajet de la suture coronale; elle est souvent plus longue que la branche horizontale, elle pénètre comme elle dans la troisième circonvolution frontale.

Ces deux branches, nées très près l'une de l'autre sur la scissure de Sylvius, et s'écartant l'une de l'autre sous un angle à peu près droit, interceptent dans la troisième circonvolution frontale, dont elles occupent toute l'épaisseur, un petit lobule de forme triangulaire, dont le sommet aboutit à la scissure de Sylvius : je nomme ce petit lobule le cap de la troisième circonvolution frontale (C). Son volume et sa forme sont peu variables; toutefois il est plus ou moins aigu suivant que les deux branches qui le limitent naissent plus ou moins près l'une de l'autre. Ordinairement elles sont séparées à leur origine par une distance de 4 à 5 millimètres seulement, alors le sommet du cap est quelque peu arrondi; quelquefois la distance est plus grande et le sommet s'élargit en proportion; mais quelquefois aussi 
MORPHOLOGIE DU CERVEAU DE L'HOMME ET DES PRIMATES. 623 cette distance est nulle ; les deux branches, quoique divergentes, se confondent à leur origine, avant de se séparer ; leur ensemble présente la forme d'un Y à courte queue, et le cap se termine en pointe. Cette forme en Y s'observe surtout sur les cerveaux peu riches en circonvolutions.

Telle est la disposition des deux branches antérieures de la scissure de Sylvius dans le cerveau humain ; elle est constante, exception faite d'un petit nombre de cerveaux d'imbéciles et d'idiots, et de quelques cerveaux de microcéphales. Elle est la conséquence du grand développement qu'acquiert chez l'homme la troisième circonvolution frontale.

Entre l'homme, qui possède les deux branches antérieures de la scissure de Sylvius, et les pithéciens qui n'ell ont aucune (1), se placent les anthropoïdes qui en ont toujours au moins une. Il n'y en a jamais qu'une seule chez les gibbons. Chez l'orang et le chimpanzé, au contraire, il y en a souvent deux, tantôt d'un seul côté, tantôt des deux côtés. Il paraît résulter de cette première notion que l'une des branches est constante, essentielle, tandis que l'autre n'est qu'accessoire. Il s'agit de savoir maintenant quelle est celle qui est constante; or, si l'on examine le cerveau des gibbons, qui n'ont jamais qu'une seule branche sylvienne antérieure, on voit que cette branche est horizontale, qu'elle se place sur la limite de la face externe du lobe frontal et de sa face orbitaire, que par conséquent elle représente exactement la branche horizontale antérieure de l'homme. Si maintenant nous passons aux chimpanzés et aux orangs, nous constatons que, lorsqu'ils n'ont qu'une seule branche, elle correspond à

(1) Sur l'un de nos cerveaux de mandrill, il y a dans la troisième circonvolution frontale une petite incisure superficielle qui occupe à peu près la position de la branche horizontale antérieure et qui, sur le moule et sur les dessins de notre album, paraît représenter cette branche; mais en examinant le cerveau lui-même, qui est conservé dans l'alcool, on voit que c'est un sillon superficiel, n'occupant qu'une faible partie de l'épaisseur de la circonvolution et n'atteignant par conséquent pas le fond de la scissure de Sylvius. J'ai lieu de croire que le sillon ascendant représenté sur le cerveau de mandrill et le cerveau d'atèle de l'atlas de Gratiolet, est de même nature que le précédent, et n'est pas réellement une branche de la scissure de Sylvius. Je considère, au contraire, comme un rudiment de la branche horizontale antérieure un tout petit feston que l'on apercoit sur l'hémisphère gauche du cerveau de l'un de nos semnopithèques ( $S$. entelles), dans le point où s'observe chez les gibbons la branclie horizontale antérieure.

T. v. 
notre branche horizontale antérieure, et que lorsqu'ils en ont deux, la seconde, située plus ou moins au-dessus et en avant de la première, dont elle est séparée par un cap plus ou moins arrondi, représente notre branche ascendante. Cette seconde branche, quelquefois aussi grande et même plus grande que la première, est quelquefois, au contraire, très petite (1).

Lorsque l'insula n'est pas entièrement recouvert, ce qui s'observe sur deux de nos chimpanzés $\left(\mathrm{n}^{\circ} 1\right.$ et $\left.\mathrm{n}^{\circ} 2\right)$, c'est le pli antérieur de l'insula qui apparaît au-dessus de la pointe du lobe temporal; dans ce cas, la branche horizontale antérieure de la scissure de Sylvius est écartée en triangle, et c'est entre ses deux bords que s'effectue la petite saillie de l'insula.

Nous possédons maintenant les notions nécessaires pour apprécier la disposition qui existe chez notre gorille. Il y a de chaque côté une branche antérieure unique, mais de chaque côté aussi, au-dessus et en arrière de cette branche, entre elle et le niveau de la scissure de Rolando, le bord supérieur de la scissure de Sylvius présente une ondulation légère (fig. $79, \mathrm{~m}$ ) correspondant au point où existe éventuellement la branche ascendante des orangs et des chimpanzés. Quant à la branche antérieure, $\mathrm{S}^{\prime \prime}$, elle se porte horizontalement en avant vis-à-vis le sommet du pli antérieur de l'insula; elle sépare la portion supérieure de la troisième circonvolution frontale de sa portion réfléchie, ou inférieure, ou orbitaire; elle chemine sur le bord externe de la face orbitaire du lobe frontal; il est donc tout à fait certain qu'elle représente non pas la branche ascendante, mais la branche horizontale antérieure de la scissure de Sylvius. Sa longueur est de 10 millimètres à droite, de 12 à gauche; du côté gauche ses deux bords se touchent dans toute leur longueur, mais du côté droit ils s'écartent un peu en arrière, laissant un petit espace triangulaire au fond duquel on peut, en inclinant le cerveau, apercevoir la pointe du pli antérieur de l'insula.

J'ajoute, avant de quitter ce sujet, que sur le cerveau du jeune

(1) La branche ascendante de la scissure de Sylvius est nulle à droite et à gauche sur notre orang no 1 , grande des deux côtés sur le numéro 2 ; chez le chimpanzé no 1 , elle est nulle à droite et très grande à gauche; chez le numéro 2 , nulle à droite, très petite à gauche; chez le numéro 3 , grande à droite, très petite à gauche. 
gorille décrit par MM. Pansch et Bischoff, il existe une branche horizontale antérieure très grande, dont la base élargie laisse apercevoir dans une étendue de 8 à 10 millimètres le premier pli de l'insula. M. Pansch pense, avec raison, que cette branche est une émanation de la scissure de Sylvius; et il l'appelle branche antérieure; mais ce nom est synonyme de branche ascendante, les auteurs n'ayant connu jusqu'ici qu'une seule branche sylvienne frontale, appelée indifféremment antérieure ou ascendante (Ramus ascendens sive anterior, Ecker). M. Bischoff admet, au contraire, que la branche en question est étrangère à la scissure de Sylvius; ce u'est pour lui que le sillon orbitaire externe, ou sillon latéral, prolongé en arrière jusqu'à la scissure de Sylvius. Pour établir cette interprétation, il fait remarquer qu'au-dessus et en arrière du sillon dont il s'agit, le bord de l'opercule présente un léger feston limité par une dépression légère qui correspond au siège habituel de la branche antérieure ou ascendante de la scissure de Sylvius; cette dépression est donc pour lui l'analogue de la branche ascendante ou antérieure ; et puisque la branche antérieure est là, et que, d'ailleurs, il n'y en a qu'une seule, le sillon décrit par Pansch comme une branche antérieure de la scissure de Sylvius, n'est pas une branche de cette scissure. Cette dissidence entre les deux anatomistes allemands semble au premier abord de peu d'importance ; on pourrait croire qu'il ne s'agit que d'une discussion de mots ; mais ce sont bien les faits eux-mêmes qui sont en question : si le grand sillon appelé par M. Pansch branche antérieure de la scissure de Sylvius est réellement une branche de cette scissure, la circonvolution qui l'entoure est la troisième circonvolution frontale, et celle-ci, par conséquent, est assez grande. Si, au contraire, ce sillon n'est pas une branche de la scissure de Sylvius, si c'est le sillon orbitaire externe, la circonvolution qui le borde n'est pas la troisième circonvolution frontale, mais la seconde, et dès lors, il ne reste plus, pour représenter sur le cerveau du gorille la troisième circonvolution frontale, que le tout petit feston signalé par M. Bischoff. Ce dernier auteur applique, d'ailleurs, la même interprétation au cerveau des autres anthropoïdes, et il en conclut que la troisième circonvolution frontale, si développée chez l'homme, est très rudimentaire chez l'orang et le chim- 
panzé, et presque nulle chez le gorille. Cette conclusion, si elle était exacte, me plairait beaucoup, car je crois avoir démontré que la troisième circonvolution frontale est le siège de la faculté du langage articulé, et ce serait un argument bien précieux pour moi, si l'organe du langage articulé n'existait que chez l'homme. Mais tout en constatant que la troisième circonvolution frontale est beaucoup plus développée chez l'homme, je suis obligé de reconraître qu'elle existe aussi chez les singes, et qu'elle présente $\mathrm{m}$ me chez les anthropoïdes un développement très notable, attesté par la présence constante de la branche horizontale antérieure de la scissure de Sylvius, attestée, en outre, sur quelques cerveaux d'orang et de chimpanzé par l'existence d'une seconde branche antérieure, analogue à la branche ascendante du cerveau de l'homme.

On le voit, toute la discussion qui s'est élevée entre MM. Pansch et Bischoff porte sur l'idée que la scissure de Sylvius du cerveau le plus compliqué, c'est-à-dire du cerveau humain, n'émet qu'une seule branche antérieure. Sï M. Pansch avait connu la distinction des deux branches antérieures de la scissure de Sylvius, il y aurait aisément constaté que le sillon dont l'interprétation est contestée par M. Bischoff correspond à la branche horizontale antérieure, et non pas à la branche ascendante, et il n'aurait pas donné prise à l'argumentation très serrée de son adversaire (1).

(1) M. le profeseur Theod. Meynert, de Vienne, dans un travail récent sur les circonvolutions de la face convexe du cerveau chez l'homme, les singes et les carnassiers, vient d'introduire une nouvelle complication et une nouvelle confusion dans l'étude de la branche ascendante de la scissure de Sylvius (dans Archiv für Psychiatrie, Berlin, 1877, t. VII, p. 262 et suiv.). Il croit avoir retrouvé cette branche chez les carnassiers, où elle serait même relativement beaucoup plus grande que chez l'homme. Il serait déjà assez étrange que cette branche, qui manque chez tous les singes non anthropoïdes, existât au-dessus d'eux, chez l'homme, et au-dessous d'eux, chez les carnassiers. Ce serait tout à fait contraire aux lois de l'évolution sériaire. L'auteur aurait dû considérer que la branche ascendante de la scissure de Sylvius est le prolongement de la scissure sylvienne dans le lobe frontal, et l'indice du perfectionnement de ce lobe; que le lobe frontal, grand chez tous les primates, n'est que rudimentaire chez les carnassiers, et ne saurait dès lors présenter chez eux un caractère de perfectionnement. Le grand sillon considéré par M. Meynert comme la branche sylvienne ascendante des carnassiers, n'est pas situé dans le lobe frontal, mais au-dessus et en arrière de lui; il en établit la limite sur la face externe de l'lémisphère, 
En résumé, la scissure de Sylvius de notre gorille présente une branche postérieure presque horizontale, avec une incisure pariétale simple et profonde, et une branche horizontale antérieure très prononcée, tout à fait fermée à gauche, légèrement

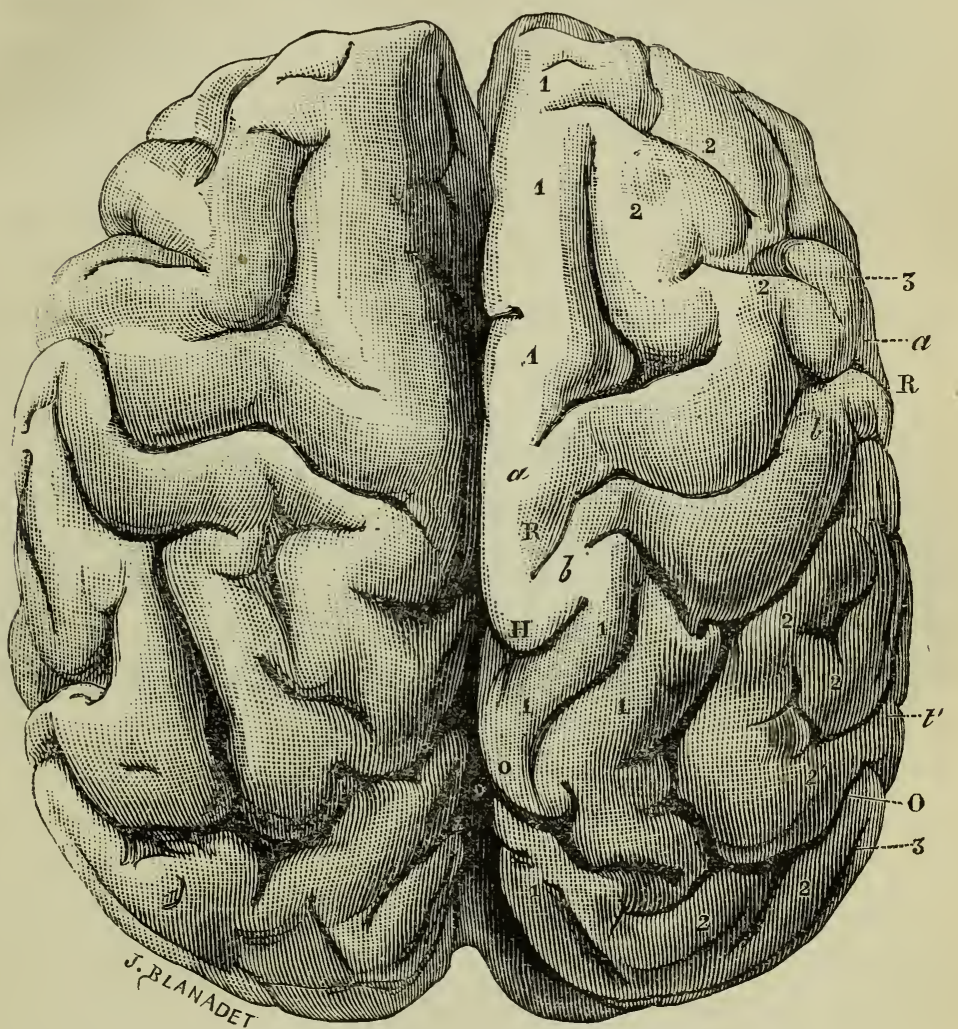

Fig. 82. Cerveau du gorille du docteur Nègre (face supérieure).

entr'ouverte à droite, où ses bords sont quelque peu écartés par l'extrémité antérieure du premier pli de l'insula.

le séparant du lobe pariétal; il est, par conséquent, l'analogue de la scissure de Rolando. D'ailleurs, il n'émane pas de la scissure, le plus souvent même il n'y aboutit pas; il en est séparé par un rebord ordinairement assez épais. Chez quelques carnassier's (la loutre, l'ours, par exemple), ce rebord se replie un peu vers la profondeur et la scissure de Rolando paraît ainsi se mettre superficiellement en communication avec la scissure de Sylvius (comme on le voit chez certains hommes); mais ce n'est qu'une apparence, et il suffit pour s'en assurer de passer en revue la série des cerveaux des carnassiers, ainsi que des pachydermes et des ruminants, où l'on constate la même disposition. 
Les autres scissures ne nous entraîneront pas dans des développements aussi étendus.

La scissure de Rolando (RR, fig. 79 et 82 ) commence presque sur le bord sagittal de l'hémisphère et se termine à environ 4 millimètres au-dessus de la scissure de Sylvius. Sa direction générale est peu oblique. Son origine, un peu plus reculée à droite qu'à gauche (peut-être par suite de la déformation des hémisphères), est notablement plus rapprochée de l'extrémité postérieure du cerveau que de l'extrémité antérieure. Elle est sinueuse et présente deux coudes (ou convexités antérieures), l'un interne, l'autre externe. L'interne est situé vis-à-vis de l'espace qui sépare la racine de la première circonvolution frontale de celle de la seconde, l'autre vis-à-vis de l'espace qui sépare la racine de la seconde de celle de la troisième. Ces deux coudes occupent donc la même position que chez l'homme, l'orang et le chimpanzé, et ils sont dus à la même cause, mais ils sont moins saillants, par suite de la moindre complication des hémisphères. En dehors du second coude, la scissure de Rolando n'avance plus, elle recule même légèrement, de sorte qu'elle se termine, au-dessus de la scissure de Sylvius, bien en arrière de la pointe du lobe temporal et de l'origine de la branche horizontale anté rieure de la scissure. La distance comprise entre l'origine de cette branche et l'extrémité inférieure de la scissure de Rolando, indique l'étendue de la base du lobe frontal en arrière du lobule orbitaire. Elle s'élève à droite à 18 millimètres, à gauche à 16 millimètres et ne le cède en rien à celle qu'on mesure sur les cerveaux d'orang et de chimpanzé.

La scissure occipitale interne $(00$, fig. 83) ne mériterait point ici le nom de scissure perpendiculaire interne que lui a donné Gratiolet, car elle est très oblique, et se détache du bord sagittal de l'hémisphère sous un angle très aigu. Cette obliquité est la conséquence de l'exiguïté du lobe occipital ; commençant très loin en arrière, la scissure occipitale interne est obligée de suivre un trajet très oblique pour atteindre la circonvolution du corps calleux, dans laquelle elle se termine. Ses connexions sont d'ailleurs les mêmes que chez l'orang et le chimpanzé, c'està-dire qu'elle ne communique pas avec la scissure calcarine ( $K$ ) dont elle est séparée par un gros pli, étendu du cumeus (M) à la 
circonvolution du corps calleux. On sait que chez l'homme (et j'ajoute chez les gibbons) la scissure occipitale interne et la scissure calcarine se réunissent en Y.

La scissure occipitale externe (fig. 82 et 79,00 ) forme sur la face convexe de l'hémisphère Ie prolongement direct de la précédente ; elle a une direction générale à peu près transversale ; elle décrit d'abord une concavité dirigée en avant, puis, parvenue derrière le pli courbe $(2 ')$, elle décrit une concavité inverse, se portant à la fois en dehors, en bas, et même un peu en

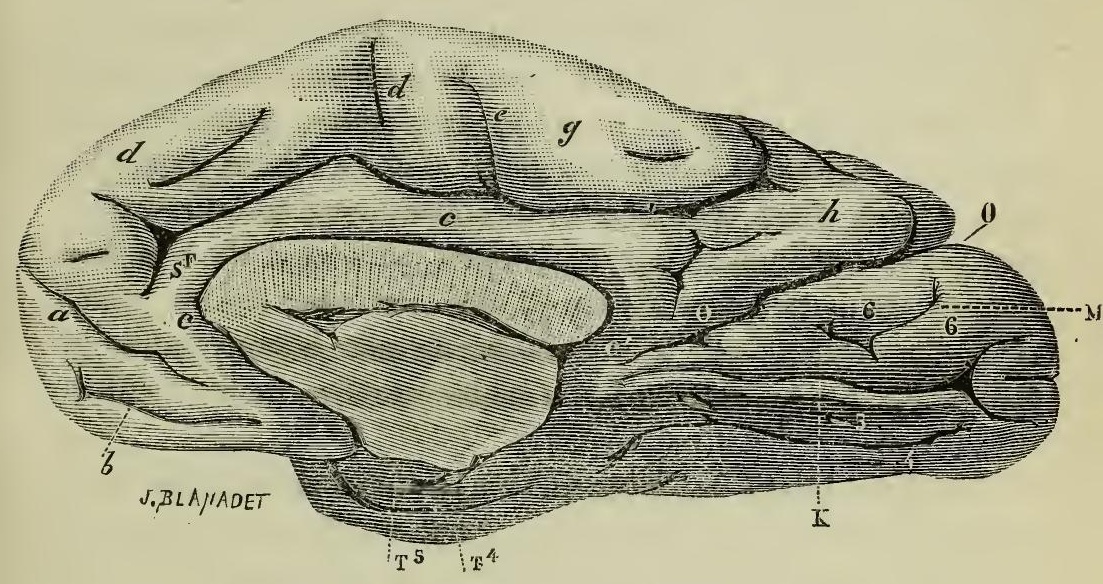

Fig. 83. Cerveaı du gorille du docteur Nègre (face interne).

arrière, sur le bord externe de l'hémisphère, où elle se termine. En suivant les sinuosités, sa longueur totale est de 72 millimètres; elle est profonde, mais, à 18 millimètres environ de la ligne médiane, elle est traversée par le premier pli de passage supérieur, qui est volumineux, peu profond à gauche et superficiel a droite. En dehors de ce premier pli existele second pli de passage qui est profond et va se rendre dans la deuxième circonvolution pariétale, immédiatement en dehors du sillon pariétal. On sait que le premier pli de passage est constamment superficiel chez l'orang ; chez le chimpanzé, il l'est quelquefois aussi, mais il est ordinairement profond, et même si profond dans certains cas qu'il paraît manquer (1). Il est très prononcé, et super-

(1) Gratiolet pensait que le premier pli de passage n'existait pas chez le chim- 
ficiel à droite et à gauche sur le jeune gorille de Hambourg. Il semble donc que, sous ce rapport, le gorille soit supérieur au chimparzé.

Parlons enfin de la scissure sous-frontale, dont j'ai plus haut (p. 618) justifié le nom et signalé l'importance (fig. 83, SF). Elle commence en avant du genou du corps calleux, se porte obliquement en arrière, puis en haut, en décrivant deux flexuosités assez faibles, atteint le bord sagittal de l'hémisphère, bien en arrière du bourrelet du corps calleux, et se prolonge sur la face convexe de l'hémisphère dans une étendue de 12 millimètres, derrière le pli dans lequel la scissure de Rolando prend naissance (fig. 82, H). Le bord inférieur de la scissure sous-frontale ne présente qu'une seule incisure, très petite, située tout en arrière et pénétrant horizontalement dans le lobule quadrilatère. De son bord supérieur se détachent deux incisures simples, mais profondes, l'une antérieure, pénétrant dans la face interne de la première circonvolution frontale; l'autre postérieure, verticale, située sous la racine de cette circonvolution et formant la limite antérieure du lobule ovalaire ou paracentral (fig. 83,e).

Le volume relatif des lobes limités par les scissures que nous venons de décrire est peut-être ce qu'il y a de plus caractéristique dans le cerveau de notre gorille. Le lobe frontal est très grand, plus grand relativement que chez aucun autre singe; il n'a, il est vrai, qu'une épaisseur médiocre, mais sa longueur excède en haut la moitié et en bas le tiers de la longueur totale panzé ; faute d’avoir contrôlé cette assertion, j’ai mal interprété les plis de passage du cerveau de chimpanzé représenté dans mon mémoire Sur l'ordre des primates (Bulletins de la Société d'anthropologie, 1869, 2 série, t. IV, p. 391 , fig. 14, 2, 2, et dans P. Broca, Mémoires d'anthropologie, t. III, p. 137. J'ai appelé second pli de passage un pli superficiel qui, en réalité, est le premier. Le premier pli de passage est celui qui aboutit à la première circonvolution pariétale, le deuxième est celui qui aboutit à la seconde. J'ai toujours trouvé ces deux plis cliez le chimpanzé, en écartant suffisamment les bords de la scissure occipitale externe. Je les ai même retrouvés chez le cynocéphale, mais le premier pli de passage de cet animal est très petit, très profond et retourné en dedans, de sorte qu'il empiète sur la scissure occipitale interne. C'est pour cela que Gratiolet ne l'a pas vu, et qu'il a fait de l'absence de ce pli un caractère du cerveau des cynocéphales. L'étude des plis de passage profonds ne se fait bien que sur les moules préparés par le procédé du moulage à la cire, dont il sera question plus loin. 
MORPHOLOGIE DU CERVEAU DE L'HOMME ET DES PRIMATBS. 633

de l'hémisphère, et sa largeur, partout considérable, surtout dans la région sus-orbitaire, lui donne une forme générale tout à fait humaine. Tous les autres lobes au contraire sont plus ou moins sacrifiés. Le lobe occipital est plus petit non seulement que chez le chimpanzé, ce qui ne serait pas beaucoup dire, mais que chez l'orang, et même que chez l'homme. Le lobe pariétal est assez développé dans sa partie latérale, mais extrêmement réduit dans sa partie supérieure et interne, qui est relativement plus petite que chez aucun autre singe. Le lobe temporal enfin est assez large, mais peu épais, par suite de la grande inclinaison de la scissure de Sylvius, et, somme toute, il est peu volumineux.

Parlons maintenant des circonvolutions de chaque lobe. Elles sont très peu compliquées et cependant très peu symétriques, contrairement à la règle ordinaire ; car on sait que d'une manière très générale, chez les primates (je ne parle pas des autres animaux), la symétrie des hémisphères est en raison inverse de leur degré de complication.

Dans le lobe frontal, la circonvolution ascendante (fig. 82, $a a)$, très grosse et peu flexueuse, fournit, en haut, une très grosse racine pour la première circonvolution frontale; au milieu, une racine très grosse encore pour la seconde; en bas enfin, sur la scissure de Sylvius, une racine plus mince, mais assez large, pour la troisième circonvolution frontale.

La racine de la deuxième circonvolution interrompt dans son milieu le sillon prérolandique, qui est d'ailleurs très profond, et assez exactement parallèle à la scissure rolandique. Divisé ainsi en deux parties, le sillon prérolandique fournit, dans sa partie interne ou supérieure, le premier sillon frontal, qui est à peu près rectiligne, et dans sa partie externe et inférieure, le deuxième sillon frontal, ou sillon latéral du lobe frontal.

La première circonvolution frontale, sur la face convexe, est large et simple. On la retrouve très exactement pareille sur la photographie de Gratiolet (fig. 78). Cet auteur a dit, il est vrai, dans un passage reproduit plus haut(p.612), que la première circonvolution frontale de son gorille était " divisée en deux gros plis par une scissure à trois branches assez semblable à celle du chimpanzé "); mais cette scissure à trois branches n'est autre 
que le $\mathrm{I}$ formé par la portion interne du sillon prérolandique et par le premier sillon frontal qui s'en détache. Gratiolet, dans la détermination de la première circonvolution frontale (étage supérieur) des anthropoïdes, a commis une erreur qu'il importe de signaler. Chez l'homme, cette circonvolution est assez souvent subdivisée en deux par une incisure longitudinale plus ou moins longue; on trouve alors, de dedans en dehors, deux plis parallèles, avant d'arriver à la seconde circonvolution frontale. Cette subdivision étant très marquée chez la Vénus hottentote, qui lui servait de terme de comparaison, Gratiolet a cru que la même disposition devait se retrouver sur le cerveau des anthropoïdes (où elle n'existe jamais) et d'après cela, comptant deux plis à partir du bord interne de l'hémisphère, il en a formé sa première circonvolution frontale, qui naturellement s'est trouvée subdivisée en deux; en d'autres termes, il a rattaché à la première circonvolution frontale toute la partie postérieure de la seconde. Il est tout simple qu'ayant interprété ainsi le cerveau de l'orang et celui du chimpanzé, il ait appliqué le même procédé de détermination au cerveau du gorille. Mais en cela il n'a pas été fidèle au grand principe qui a fait tout le succès de ses recherches, savoir que, pour débrouiller les circonvolutions il faut aller du simple au composé en s'élevant du singe à l'homme, au lieu de suivre la marche inverse, en descendant de l'homme au singe.

Le nombre et la distinction des circonvolutions frontales sont déterminés par leurs racines, c'est-à-dire par les insertions qu'elles prennent sur la circonvolution frontale ascendante. La première racine, située sur le bord sagittal de l'hémisphère, ne peut donner lieu à aucune difficulté. La troisième, au-dessus de la scissure de Sylvius, bien que quelquefois cachée au fond de la fausse "branche ascendante " de Turner (1), se retrouve toujour's aisément lorsqu'on soulève l'opercule ; quant à la seconde, clle est plus évidente encore, surtout chez les anthropoïdes, où elle est constamment volumineuse et superficielle.

Je répète donc que la première circonvolution frontale de notre gorille est très simple du côté de sa face convexe, et dans

(1) Voir plus haut, p. 621. 
sa partie postérieure ; en avant une grosse anastomose transversale l'unit à la deuxième circonvolution; plus en avant encore elle s'élargit un peu, puis se rétrécit rapidement pour se réfléchir sur la pointe du lobe frontal et former le pli étroit et simple qui constitue la première circonvolution orbitaire.

La face interne de la première circonvolution frontale sera décrite avec la face interne de l'hémisphère.

La seconde circonvolution frontale sera plus facile à décrire quand nous connaîtrons la troisième. Celle-ci naît de la frontale ascendante par une racine large mais courte, qui va former presque aussitôt le bord supérieur de la branche horizontale antérieure de la scissure de Sylvius; elle se replie brusquement sur l'extrémité de cette branche en s'amincissant beaucoup, et après cette réflexion, passe dans le lobule orbitaire en formant la troisième circonvolution orbitaire (voir fig. $79, \mathrm{n}^{\mathrm{cs}} 3,3,3^{\prime}$ ). Elle se termine sur la pointe du lobe temporal, dans la vallée de Sylvius, où elle se continue avec l'extrémité antérieure du premier pli de l'insula. Dans sa portion orbitaire, elle est longée par un sillon profond qui est incontestablement le sillon orbitaire externe. Ce sillon très long se prolonge, en prenant une direction transversale, sur la face externe de l'hémisphère.

La partie de la troisième circonvolution frontale qui est située au-dessus de la branche horizontale antérieure est courte, mais très épaisse; en haut elle émet une branche anastomotiqu qui longe le second sillon frontal, contourne l'extrémité supérieure du sillon orbitaire externe, et va se jeter dans la seconde circonvolution.

La seconde circonvolution frontale, enfin, occupe sur la face convexe de l'hémisphère, aussi bien que sur sa face orbitaire, tout l'espace compris entre les deux précédentes. Née, par une très grosse racine, du milieu de la frontale ascendante, elle se porte d'abord en dedans, puis s'élargit, devenant aussi large à elle seule que les deux autres ensemble, et se replie enfin sur le bord de l'hémisphère, pour aller constituer la seconde circonvolution orbitaire, qui forme comme toujours la plus grande partie du lobule orbitaire.

Ce lobule (fig. 79 et 80), limité en dehors par la branche horizontale antérieure de la scissure de Sylvius, en dedans par 
la scissure interhémisphérique, présente deux sillons et diverses incisures. Le sillon orbitaire interne, ou sillon droit, ou sillon du nerf olfactif, est disposé comme chez tous les primates; le sillon orbitaire externe a déjà été décrit. Entre ces deux sillons existe la surface aplatie de la deuxième circonvolution orbitaire, rétrécie en arrière et très élargie en avant, par suite de la direction divergente du sillon orbitaire externe. Sur cette surface on remarque les incisures dont l'ensemble porte le nom de sillon en $\boldsymbol{H}$. Ces incisures, très peu symétriques à droite et à gauche, sont plus profondes que chez le chimpanzé et l'orang, mais plus simples.

Lobe pariétal. - La circonvolution pariétale ascendante $(b b$, fig. 79 et 82), mince à ses deux extrémités, est beaucoup plus large à sa partie moyenne, qui envoie, comme d'habitude, un large pli d'anastomose à la première circonvolution pariétale. Dı côté droit ce pli d'anastomose est déprimé à sa base, il n'arrive pas jusqu'à la surface de l'hémisphère, et le sillon postrolandique n'est pas interrompu; mais à gauche ce pli, tout à fait superficiel, divise le sillon post-rolandique en deux parties, l'une interne assez courte, l'autre externe, beaucoup plus longue, qui se continue en arrière, sous un angle très obtus, avec le sillon pariétal (intra-pariétal, Turner). Celui-ci, très peu sinueux, va se jeter directement dans la scissure occipitale externe, et sa disposition rappelle celle que l'on observe sur le cerveau des cynocéphales.

Il est digne de remarque que, sur la photographie de Gratiolet (fig. 78), les deux sillons post-rolandiques présentent la même asymétrie; seulement c'est celui du côté gauche qui est ininterrompu, tandis qu'à droite l'anastomose de la circonvolution pariétale ascendante et de la première pariétale est large et superficielle.

La première circonvolution pariétale $(1$, fig. 82$)$ est très petite et très simple. Sa racine naît de la pariétale ascendante, sur l'extrémité de la profonde découpure qui marque sur la face convexe de l'hémisphère la terminaison de la scissure sousfrontale(H, fig. 82). Entre cette scissure et la scissure occipitale externe, il n'y a qu'une distance de 12 millimètres environ; c'est à cela que se réduit la partie de la première circonvolution 
pariétale qui est si développée chez l'homme, si notable encore chez le chimpanzé et l'orang, et qui a reçu le nom de lobule pariétal. Ce lobule n'est pas seulement très petit, il est encore extrêmement simple : on n'y remarque à droite qu'une seule incisure isolée; à gauche cette incisure isolée est plus petite, mais une incisure émanée du sillon post-rolandique complique un peu plus le lobule.

En arrière, la deuxième circonvolution pariétale communique avec le lobe occipital par le premier pli de passage déjà décrit.

La deuxième circonvolution pariétale est très simple, mais complète. Née de la partie inférieure de la pariétale ascendante, elle se replie presque aussitôt autour de l'incisure pariétale unique de la scissure de Sylvius (fig. $79, i)$, présente vers le milieu de sa largeur une très petite incisure isolée à trois branches, puis se divise en deux plis, séparés par l'extrémité postérieure et supérieure du premier sillon temporal (scissure parallèle); le pli antérieur, plus petit, contourne l'extrémité de la scissure de Sylvius pour se continuer avec la première circonvolution temporale ; le second, beaucoup plus large, communique en arrière et en dedans avec le lobe occipital, par un pli de passage caché au fond de la scissure occipitale externe, et forme aussitôt après le pli courbe ( 2 ', fig. 79 et 82$)$ qui va se continuer avec la seconde circonvolution temporale.

Lobe occipitul. - Ge lobe est très petit et assez peu compliqué ; on y trouve cependant toutes les parties qui le composent chez l'homme.

Pour décrire le lobe occipital il convient de le diviser en trois lobules, savoir : le supérieur ou sus-occipital, l'inférieur ou sousoccipital, l'interne ou cuneus. Le premier forme la face convexe du lobe. Sous le bord inférieur de la circonvolution qui contourne l'extrémité externe el inférieure de la scissure occipitale externe existe un sillon longitudinal qui se dirige en arrière vers la pointe du lobe occipital, et qui se prolonge en avant dans le lobe temporal : c'est le sillon occipito-temporal latéral $(l$, fig. 79 et 80). Dans le lobe temporal, il sépare la deuxième circonvolution temporale de la troisième; dans le lobe occipital, il sépare le lobule sus-occipital du lobule sous-occipital. Celui-ci 
s'étend de là jusqu'à la scissure calcarine ou du petit hippocampe ( $k$, fig. 83), qui prend son origine sur le pôle du lobe occipital, c'est-à-dire sur son extrémité postérieure, et qui se porte en avant et en bas jusqu'au niveau du point où la partie réfléchie de la circonvolution du corps calleux se continue avec la circonvolution de l'hippocampe. Le lobule sous-occipital, compris entre le sillon latéral et la scissure calcarine, forme la face inférieure ou tentoriale du lobe occipital. Enfin, entre la scissure calcarine et la scissure occipitale interne, il reste un in. tervalle ordinairement triangulaire, occupé par un lobule bien distinct, décrit par Burdach sous le nom de zwickel (coin) et connu aujourd'hui sous le nom de cuneus. Le cuneus forme la face interne du lobe occipital (M, fig. 83); sa base correspond au bord supérieur de l'hémisphère, et se continue à ce niveau avec le lobule sus-occipital.

Cela posé, le sillon latéral occipito-temporal est extrêmement marqué sur le cerveau du gorille ( $l$, fig. 79 et 80 ). Il est long, ininterrompu, profond et presque droit. En arrière, il se prolonge jusqu'à une petite distance du pôle. La limite externe du lobule sus-occipital est donc parfaitement tranchée.

Ce lobule est large et court et son bord antérieur, qui correspond à la scissure occipitale externe, ne présente aucune trace du prolongement superficiel qui, chez tous les pithéciens, constitue l'opercule occipizal, qui se retrouve encore, quoique atténué, chez le chimpanzé, et qui n'existe ni chez l'homme ni chez l'orang. On y remarque, en dedans, un petit sillon à peu près longitudinal, en dehors un grand sillon très proforid, qui, à sa partie postérieure, devient presque transversal et se divise en deux branches, l'une grande et transver'sale dirigée en dedans, l'autre postérieure, plus courte, dirigée vers le pôle. La partie du lobule située en dedans du premier sillon sus-occipital constitue la première circonvolution occipitale; la partie beaucoup plus large comprise entre le premier sillon sus-occipital et le deuxième constitue la deuxième circonvolution occipitale; on y voit une petite incisure qui aboutit à la scissure occipitale externe; enfin la partie comprise entre le deuxième sillon susoccipital et le sillon latéral occipito-temporal constitue la troisième circonvolution occipitale (fig. $\left.79,0^{3}\right)$. Celle-ci, assez 
mince en arrière, très large en avant, où elle présente une incisure jsolée, passe au-dessous de l'extrémité externe de la scissure occipitale externe, et se continue à la fois avec le pli courbe et avec la deuxième circonvolution temporale.

Le lobule sous-occipital (fig. 8., $n^{\text {os }} 4$ et 5้) est large et plat. Un grand sillon longitudinal, profond, très simple et presque droit, né, en arrière, à peu de distance du pôle, et s'étendant en avant jusque dans le lobe temporal où il se bifurque, divise ce lobule en deux circonvolutions parallèles et à peu près égales en largeur; la plus externe est la quatrième circonvolution occipitale, désignée quelquefois sous le nom de circonvolution fusiforme; elle se continue en avant avec les troisième et quatrième circonvolutions temporales; la plus interne est la cinquième circonvolution occipitale, appelée aussi circonvolution linguale (1); elle se continue en avant avec la cinquième temporale ou circonvolution de l'hippocampe.

Le lobule du cuneus (M, fig. 83), enfin, est formé comme d'habitude par une seule circonvolution, qui est la sixième circonvolution occipitale. Il est très simple, n'étant subdivisé que par une incisure isolée à trois branches. Il n'affecte pas la direction transversale qu'on observe chez l'homme et chez presque tous les singes; il est très oblique, très long; en arrière et en haut, dans sa partie la plus volumineuse, il n'a que 15 millimètres de large, puis il se rétrécit graduellement, et va se terminer sur la partie descendante de la circonvolution du corps calleux, en établissant une séparation large de 6 millimètres entre la scissure occipitale interne 0,0 et la scissure calcarine $K$. On sait que chez l'homme et les gibbons les deux scissures se terminent en $\mathrm{Y}$, et qu'elles restent séparées chez l'orang et le chimpanzé comme chez le gorille, et aussi comme chez les pithéciens et les cébiens.

(1) Les dénominations tirées de la forme des circonvolutions me paraissent de nature à faire naître des confusions, parce que cette forme est très variablesuivant les espèces et, dans la même espèce, suivant les individus. Les dénominations ordinales me paraissent bien préférables. Je fais toutefois une exception pour le cuneus, parce qu'il présente invariablement chez l'homme la forme d'un coin, et parce qu'il la conserve chez beaucoup de singes, même lorsque la scissure calcarine ne communique pas avec la scissure occipitale externe; mais il faut bien reconnaître pourtant que le cuneus du gorille n'a aucune ressemblance avec la forme d'un coin. 
Lobe temporal. - Le lobe temporal du gorille se compose de cinq circonvolutions, comme celui de l'homme. On n'en compterait que quatre si l'on n'examinait que l'extrémité antérieure du lobe, où l'on ne voit aboutir que trois sillons; mais plus en arrière apparaît un quatrième sillon qui porte à cinq le nombre des circonvolutions temporales.

Le premier sillon temporal (scissure parallèle de Gratiolet) est tout à fait simple et presque sans sinuosités dans sa partie soussylvienne. En arrière il présente d'abord une incisure inférieure, puis se relève derrière l'extrémité postérieure de la scissure de Sylvius, et va se terminer dans la seconde circonvolution pariétale, à gauche sans bifurcation, à droite avec une courte bifurcation.

Le deuxième sillon temporal n'est autre que le sillon occipitotemporal latéral déjà décrit ( $l$, fig. 79 et 80$)$; interrompu deux fois par les anastomoses de la deuxième et de la troisième circonvolution temporale, il reparait ensuite, et on peut le suivre jusqu'à la pointe du lobe.

Le troisième sillon temporal n'existe qu'en arrière; il est longitudinal et subdivise en deux gros plis la partie de la quatrième circonvolution uccipitale qui se continue avec le lobe temporal.

Le quatrième sillon temporal enfin mérite une description plus étendue et exige quelques explications. C'est le vestige de la grande scissure limbique qui, chez les mammifères inférieurs aux primates, entoure presque complètement l'immense circonvolution limbique. Chez les primates, la partie supérieure de la circonvolution limbique reste distincte et constitue la circonvolution du corps calleux; mais sa partie inférieure, considérablement atrophiée par suite de la disparition presque complète du lobe olfactif qui en est une dépendance, se fusionne avec le lobe temporal, et ne constitue plus que la circonvolution de l'hippocampe, ou circonvolution à crochet (gyrus uncinatus). Cette fusion, toutefois, n'est pas complète, et certains sillons, qui sont les restes de la scissure limbique, continuent à marquer la limite externe de la circonvolution de l'hippocampe. Mais il y a sous ce rapport une différence très remarquable entre le type de l'homme et celui des singes. 
Chez l'homme, ou du moins dans les races supérieures, la fusion de la circonvolution de l'hippocampe avec le lobe temporal est complète en avant; on aperçoit constamment à ce niveau une dépression longitudinale très superficielle, mais point de sillon véritable. G'est en arrière seulement que la circonvolution de l'hippocampe est séparée de la quatrième circonvolution temporale par un sillon longitudinal qui fait suite au sillon compris entre la quatrième et la cinquième circonvolution occipitale (1).

La disposition est tout autre chez les singes : c'est au contraire datis sa partie postérieure que la circonrolution de l'hippocampe ou dernière circonvolution temporale se fusionne avec l'avant-dernière, tandis que la séparation de ces deux circonvolutions est effectuée en avant par un sillon profond, long, linéaire, qui ne présente jamais la moindre flexuosité, et qui, parvenu sur la pointe du lobe temporal, pénètre dans la scissure de Sylvius. En soulevant la pointe du lobe, on voit, sur la paroi inférieure de la scissure de Sylvius, une incisure horizontale qui se porte en dehors dans une étendue très notable, détachant ainsi la partie antérieure de la circonvolution de l'hippocampe sous la forme d'un petit lobule parfaitement distinct.

Ce caractère établit donc entre le cerveau de l'homme et celui des singes une différence très remarquable, mais qui n'est pourtant pas absolue, car sur beaucoup de cerveaux de nègres, et chez beaucoup d'idiots, la partie antérieure de la scissure limbique reste ouverte, comme chez lez singes.

Chez notre gorille, elle constitue un sillon profond, long de 2 centimètres, en arrière duquel la circonvolution de l'hippocampe est unie à la quatrième circonvolution temporale par un large pli anastomotique (fig. 80 et 83 ).

J'aurai peu de chose à ajouter relativement aux cinq circonvolutions temporales, limitées par les quatre sillons que je viens d'indiquer.

(1) Ce sillon peut, par suite d'un plissement, communiquer en arrière avec la scissure calcarine; mais la communication n'est jamais que très superficielle, et n'interrompt réellement pas la continuité de la cinguième circonvolution temporale et de la cinquième occipitale.

T. V. 
La première circonvolution temporale est d'une minceur remarquable et tout à fait simple. La seconde, mince en avant, s'épaissit en arrière, où elle se continue à la fois avec le pli courbe et avec la troisième circonvolution occipitale (2, fig. 79); dans sa partie moyenne, elle communique avec la suivante par un pli anastomotique assez volumineux. La troisième et la quatrième circonvolution temporale, peu distinctes en avant, sont très distinctes en arrière, où elles se continuent toutes deux avec l'extrémité antérieure de la quatrième circonvolution occipitale. Enfin, la cinquième et dernière circonvolution temporale, ou circonvolution de l'hippocampe $\left(\mathrm{T}^{5}\right)$, est assez large; elle présente en avant un crochet volumineux; en arrière, elle se continue d'une part avec la cinquième circouvolution occipitale, d'une autre part avec la partie descendante de la circonvolution du corps calleux.

Parlons maintenant de la face interne de l'hémisphère. On y remarque d'abord la scissure sous-frontale (SF, fig. 83) et la scissure occipitale interne (00) qui ont déjà été décrites plus haut. J'ai décrit, en outre, le cuneus ou sixième circonvolution occipitale qui forme l'extrémité postérieure de cette surface interne. Il reste à étudier les parties situées en avant de la scissure occipitale interne, et d'abord la circonvolution du corps calleux (c, c). Elle est mince, peu ondulée, et tout à fait simple.

Au-dessous du genou antérieur du corps calleux, elle présente ses connexions ordinaires avec la face interne de la première circonvolution frontale. En arrière, elle se réfléchit comme d'habitude $\left(c^{\prime}\right)$ derrière le bourrelet du corps calleux, et à ce niveau elle se continue avec le lobule quadrilatère, c'est-à-dire avec la face interne de la première circonvolution pariétale ( $h$, fig. 83). Ge lobule, compris entre la scissure sous-frontale et ì scissure occipitale interne est extrêmement petit; près du bord de l'hémisphère il n'a que 10 millimètres de large. Il est relativement plus petit que sur tout autre cerveau de primate. On n'y aperçoit qu'une seule incisure à quatre branches, très peu sinueuse.

Le reste de la face interne de l'hémisphère appartient au lobe frontal. En avant el en bas, les incisures sus-orbitaires $(a, b$, 
MORPHOLOGIE DU CERVEAU DE L'HOMME ET DES PRIMATES. 6:3

fig. 83) sont au nombre de deux (1). La supérieure, qui a presque l'importance d'un sillon, aboutit à la pointe du lobe frontal, et établit, sur la face interne de la première circonvolution frontale, la ligne de démarcation entre l'étage supérieur de cette circonvolution et son étage inférieur ou sus-orbitaire. Audessus de l'incisure sus-orbitaire supérieure, att-devant du genou du corps calleux, un pont anastomotique unit la première circonvolution frontale à la circonvolution du corps calleux. On a vu plus haut (p. 632) que deux incisures émanées de la scissure sous-frontale pénètrent profondément dans la face interne du lobe frontal. L'une d'elles, situće en avant et très oblique, s'enfonce dans la première circonvolution frontale; l'autre, presque verticale $(e$, fig. 83$)$, est située à 30 millimètres en arrière de la première, et à $2: 3$ millimètres en avant de l'extrémité postérieure de la scissure sous-frontale. Cette seconde incisure marque, sur la surface interne de l'hémisphère, la limite postérieure de la première circonvolution frontale. La partie de la face interne comprise entre cette incisure verticale et la scissure sous-frontale constitue le lobule appelé par M. Meynert le lobule paracentral, et, par M. Pozzi, le lobule ovalaire ( $g$, fig. 833); il est formé comme d'habitude par l'épanouissement de la partie supérieure de la circonvolution frontale ascendante sur la face interne de l'hémisphère, et il se termine postérieurement en une extrémité pointue, sur laquelle vient s'insérer la circonvolution pariétale ascendante, derrière l'origine de la scissure de Rolando.

Arrivons enfin au lobe de l'insula. L'étude de ce lobe, caché au fond de la scissure de Sylvius et recourert par l'opercule, est facile sur les cerveaux frais, dont les circonvolutions, encore souples, se laissent écarter sans se rompre. Sur les cerveaux durcis par l'alcool, on ne pourrait écarter suffisamment les bords de la scissure de Sylvius sans risquer de produire des ruptures; aussi préfère-t-on pratiquer, à l'aide d'une coupe, l'ablation de l'opercule; le fond de la scissure se trouve alors exposé au regard. Mais cette coupe, qui mutile gravement la pièce, ne doit pas être faite sur les cerveaux rares; il n'cst done pas inutile d'in-

(1) Il y a deux incisures sus-orbitaires chez l'homme, l'orang et le chimpanzé; la supérieure correspond à l'incisure sus-orbitaire unique des pilhéciens. Chez les gibbons il y en a deux, mais l'inféricure est rudimentaire. 
diquer le procédé de dilatation graduelle auquel j'ai recours pour étudier, sans crainte d'accident, l'insula des anthropoïdes. La pièce, retirée de l'alcool, est d'abord plongée dans l'eau pendant vingt-quatre heures. Alors, avec deux manches de scalpel, introduits avec précaution dans la partie antérieure de la scissure, on produit un léger écartement qui permet d'introdıire un petit tampon de ouate mouillée; la pièce est remise dans l'eau; le lendemain, on enlève le tampon, on réintroduit les deux manches de scalpel, on augmente un peu l'écartement, on le maintient aver un tampon plus gros et plus long et ainsi de suite. Les tampons finissent par remplir toute la longueur de la scissure, mais on doit éviter de les introduire dans la profondeur, et surtout de les pousser sous l'opercule, afin de ne pas comprimer et déformer l'insula. Au bout de quatre ou cinq jours, la dilatation est suffisante, et on peut apercevoir, en inclinant la pièce, toute la surface de l'insula. Mais on ne peut conserver le cerveau dans cet état; un séjour plus prolongé dans l'eau deviendrait nuisible, et lorsque, après avoir enlevé les tampons, on remet la pièce dans l'alcool, les bords de la scissure se referment promptement. L'étude de l'insula devrait donc être faite en une seule fois, ce qui rendrait les vérifications et les comparaisons très difficiles. Le moulage à la cire nous fournit heureusement le moyen de conserver définitivement l'empreinte du fond de la scissure de Sylvius. Lorsque la dilatation est jugée suffisante, on dispose convenablement la pièce, et on verse dans la scissure de Sylvius de la cire d'abeille fondue, mais ramenée à une température très voisine du degré de la fusion (68 degrés). On sait que la cire, au moment où elle se solidifie, se rétracte rapidement; il faut donc remplir en une seule fois toute la scissure de Sylvius et maintenir avec les doigts l'écartement du lobe temporal abaissé et de l'opercule relevé, jusqu'à ce que le liquide soit solidifié. Le reste de l'hémisphère est moulé au plâtre, par le procédé ordinaire; on enlève ce moule dès que le platre est chaud, afin que la cire n'ait pas le temps de devenir trop dure; la masse flexible qui remplit toutes les anfractuosités de la scissure se laisse donc aisément extraire sans endommager les parties (ce que ne manquerait pas de faire une masse de plattre). On obtient ainsi un creux constitué en partie 
par de la cire, en partie par du plâtre. On le remplit de plâtre et on en extrait aisément le moule; mais les parties profondes de la scissure de Sylvius retiennent toujours de petits prolongements de cire détachés du creux; on les extrait avec une pointe recourbée (1). Ce moule montre tout ce qu'on peut étudier sur la pièce fraîche, et on pcut remettre aussitôt celle-ci dans l'alcool. Le procédé du moulage à la cire m’a permis de déposer dans notre musée des moules représentant l'insula des divers anthropoïdes et de quelques autres singes; j’ajoute que j'ai reproduit par le même procédé tous les détails de la scissure occipitale externe et des plis de passage profonds qui la traversent. Ces plis profonds ne sont visibles que lorsque la scissure est largement dilatée, et sur les cerveaux de chimpanzé, qui ont un opercule occipital, on ne peut les mettre à découvert que par la dilatation graduelle, à moins de côuper l'opercule; voilà pourquoi Gratiolet, qui n'avait pas voulu recourir à ce dernier moyen, ne vit pas le premier pli de passage de ses chimpanzés et crut que ce pli faisait défaut.

Mais revenons à l'insula de notre gorille. On n'y voit que trois plis rayonnés comme chez l'orang (2), tandis qu'il y en a quatre chez le chimpanzé et cinq au plus chez l'homme. Tous trois se dirigent vers la pointe de l'insula, mais le premier seul y aboutit tout à fait. Le troisième ou postérieur va communiquer en arrière avec la face profonde de la première circonvolution pariétale; le deuxième ou moyen est directement audessous de l'extrémité inférieure de la scissure de Rolando et communique, dans le fond de la rigole supérieure de l'insula, avec la face profonde de l'extrémité inférieure des deux circonvolutions ascendantes. Ce pli moyen correspond donc aux deuxième et troisième plis de l'insula du chimpanzé, lesquels

(1) Le moulage à la cire est une opération délicate. Je passe sous silence beaucoup de détails qu'il serait trop long d'indiquer, mais j'en ai dit assez pour que tout mouleur expérimenté puisse appliquer ce procédé avec succès.

(2) Sur notre orang no 1, qui est très jeune (environ un an), il n'y a sur l'insula que deux plis assez faib!es; sur le numéro 2, qui est beaucoup moins jeune (on le suppose âgé d'environ trois ans), il y en a trois, et ils sont beaucoup plus prononcés. Peut-être, chez l'orang adulte, le nombre des plis atteint-il le chiffre de quatre, comme chez le chimpanzé. On sait d'ailleurs que la surface de l'insula est d'abord tout à fait lisse, et que les plis ne se produisent qu'au cours du développement. 
communiquent respectivement avec les deux circonvolutions ascendantes. Enfin le premier pli ou pli antérieur communique par ses deux extrémités avec les dẹx extrémités de la troisième circonvolution frontale. Son extrémité supérieure et externe se réfléchit au fond de la rigole supérieure de l'insula, pour se jeter dans la face profonde de la racine de celte circonvolution, et son extrémité inférieure et interne, qui forme la pointe de l’insula, aboutit à l'extrémité orbitaire de la même circonvolution. Le pli, qui est droit, n'a que quinze millimètres de longueur; la circonvolution est environ trois fois plus longue que lui, elle ne peut douc venir le rejoindre qu'après s'être repliée sur ellemême, et ce méandre donne lieu à la formation de la branche horizontale antérieure de la scissure de Sylvius.

Ces relations du premier pli de l'insula sont d'ailleurs exactement les mêmes chez les autres anthropoïdes.

En arrière de l'insula, le fond de la scissure de Sylvius ne constitue plus qu'une rigole simple au-dessous de laquelle s'effectue le passage d'un pli profond, étendu de la première circonvolution temporale à la première circonvolution pariétale. Ce pli de passage, large et même ordinairement subdivisé chez l'homme, est encore très bien dessiné chez le chimpanzé; il est plus faible chez l'orang et n'est que rudimentaire chez le gorille. Il existe cependant, et c'est à son niveau qu'est située l'incisure pariétale de la scissure de Sylvius.

Il résulte de la description précédente que le cerveau du gorille possède tous les caractères de supériorité qui distinguent les grands anthropoïles de tous les autres singes; que, par la grandeur du lobe frontal et la petitesse du lobe occipital, il se rapproche du type humain plus qu'aucun autre cerveau, mais que ses circonvolutions sont plus simples, moins tortueuses et plus larges que chez les deux autres grauds anthropoïdes. On sait que sous ce rapport le chimpanzé est, en moyenne, un peu inférieur à l'orang; le gorille prendrait rlonc la troisièıne place, mais rien ne justifie le rapprochement établi par Gratiolet entre le cerveau du gorille et celui des cynocéphales. Par les caractères cérébraux, comme par les autres caractères, la vraie place du gorille est auprès du chimpanzé, qui est à la fois son voisin géographique et son voisin zoologique; toutefois leurs cerveaux 
diffèrent d'une manière notable: d'abord par leur degré de com. plication, ensuite, et surtout par le volume et la constitution des lobes occipitaux. Cette dernière différence, supérieure à celle qu'on observe parmi des espèces d'un même genre, confirme pleinement la distinction qui a été établie entre le genre gorilla et le genre troglodytes.

\section{V}

Les remarques qui précèdent surprendront probablement beaucoup ceux qui ont pris connaissance des mémoires de MII. Pansch et Bischoff sur le jeune gorille de Hambourg. M. Pansch s'exprime ainsi : "Ce qui frappera tout le monde au premier coup d'œil jeté sur les figures, c'est la richesse des sillons sur toute la surface. Le cerveau est, comme on dit habituellement, "très riche en circonvolutions", beaucoup plus que celui des autres anthropoïdes. Cette différence, considérable sur le lobe pariétal, l'est bien plus encore sur le lobe occipital (1). » M. Bischoff dit, de son côté : " En comparant les cerveaux des trois anthropoïdes, sur trois animaux jeunes il est vrai, mais probablement du même âge, je trouve que le cerveau du gorille est indubitablement le plus riche en circonvolutions, et cela dans tous les lobes, à l'exception du lobe temporal (2). " Ainsi ces deux auteurs s'accordent à dire que le cerveau du gorille de Hambourg est plus compliqué que celui des autres grands anthropoïdes. C'est précisément le contraire de ce que j'ai constaté sur le gorille du docteur Nègre, et c'est pour cela sans doute que M. Bischoff, en examinant le dessin annexé à la note que j’ai publiée sur le cerveau de cet animal, a pensé que ce devait être le cerveau d'un chimpanzé et non pas d'un gorille.

Avant de discuter cette appréciation, il faut d'abord voir s'il est bien vrai que le cerveau du gorille de Hambourg soit plus riche en circonvolutions que celui des autres anthropoïdes. Les photographies réduites publiées par M. Pansch peuvent à cet

(1) Pansch, Ueber die Furchen und Winchungen am Gehirn eines Gorilla, loc. cit., p. 25.

(2) Bischoff, Mémoire cité dans Acad. de Munich, Classe Math. Phys., p. 135 (1877). 
égard donner une impression trompeuse, parce que, par suite de la diminution des dimensions, les circonvolutions paraissent naturellement plus étroites et plus serrées que sur les cerveaux de grandeur naturelle auxquels on les compare. Le modelage en cire exécuté par M. Bischolf, étant plus grand que nature, pourrait tromper l'œil en sens inverse. Il faut donc se reporter aux figures lithographiées qui accompagnent le Mémoire de M. Bischoff, et qui ont été faites d'après des photographies de grandeur naturelle. $0 r$, cn comparant ces figures avec les cerveaux de chimpanzé dont les dessins ont été publiés, et avec ceux que je conserve dans l'alcool, je trouve que le cerveau du gorille de Hambourg n'est plus ni moins riche que le cerveau du chimpanzé dans ses parties frontale et pariétale; que son lobe occipital est plus compliqué, son lobe temporal plus simple, et qu'en somme le degré de complication des hémisphères est à peu près le même dans les deux cas.

Dès lors, il n'y aurait pas plus de raison de rapprocher notre cerveau de gorille, à cause de sa simplicité, du cerveau des chimpanzés que de celui du gorille de Hambourg. J'ajoute que, si le degré de richesse des circonvolutions devait suffire pour déterminer les analogies et les différences, on devrait dire que le cerveau du gorille de Hambourg ressemble beaucoup plus à celui d'un chimpanzé que celui du gorille du docteur Nègre. Je suis très loin d'en conclure que le cerveau de Hambourg soit semblable à celıi des chimpanzés; il en diffère essentiellement par la constitution du lobe occipital et de la scissure occipitale externe, et ces caractères le rattachent tout à fait au type du cerveau de notre gorille.

Malgré ces atténuations, il reste entre ce dernier cerveau et celui du gorille de Hambourg une différence très grande et très frappante, l'un étant dans la plus grande partie de son étendue aussi compliqué que celui du chimpanzé, et l'autre étant beaucoup plus simple. Comment expliquerons-nous celte différence? On peut tenir compte ici de trois influences : 10 l'influence des variations individuelles ; $2^{\circ}$ celle de l'espèce $; 3^{\circ}$ celle de l'âge.

La première produit chez l'homme des différences très grandes; on observe dans la même race des cerveaux très simples et des cerveaux très compliqués, chez des individus inégalement 
intelligents, mais d'ailleurs normaux (car je ne parle ici ni des imbéciles ni des idiots). Cette influence est bien moindre chez les anthropoïdes, mais elle est encore très notable ; il suffit, pour cela, de comparer entre eux plusieurs cerveaux de chimpanzé ou plusieurs cerveaux d'orang. Qu'il y ait entre les cerveaux des gorilles des différences analogues, c'est ce qui doit paraître fort probable. On peut donc tenir compte de ce premier élément, sans d'ailleurs en exagérer l'importance.

Nous pouvons invoquer en second lieu l'influence de l'espèce. Le gorille du docteur Nègre, ainsi qu'on l'a vu plus haut, n'appartient pas à l'espèce Gorilla Savagii, à laquelle appartient très probablement le gorille de Hambourg. Entre espèces d'un même genre, le type cérébral ne varie que très peu, mais le degré de complication des circonvolutions peut présenter de grandes différences, qui sont en rapport à la fois avec le degré d'intelligence des espèces et avec leur taille, les espèces les plus petites ayarst, toutes choses égales d'ailleurs, le cerveau le plus simple. Gratiolet a pu tirer de l'étude des circonvolutious cérébrales de l'homme un argument en faveur de la doctrine polygéniste, c'est-à-dire de la multiplicité des espèces d'hommes, en faisant remarquer que le cerveau de la "Vénus hottentote ", femme d'une intelligence ordinaire, présente un degré de simplicité qu'on n'observe dans la race blanche que chez les idiots. Or, les cerveaux de nos deux gorilles ne diffèrent pas beaucoup plus sous ce rapport que le cerveau de la Vénus hottentote ne diffère de celui d'un blanc bien intelligent. Ce second élément du problème mérite donc d'être pris en grande considération.

Mais nous devons nous occuper surtout de la troisième influence, celle de l'âge. Le jeune gorille de Hambourg paraissait âgé d'environ six mois, tandis que le gorille du docteur Nègre était adulte. En reproduisant la morphologie cérébrale du jeune gorille sur le moule intra-crânien d'un gorille adulte, M. Bischoff n'avait sans doute d'autre but que de restituer à l'encéphale, plus ou moins rétracté et déformé par l'action des liquides conservateurs, la forme générale de la boîte crânienne; car il savait mieux que personne que le développement du cerveau n'est pas achevé à la naissance, que le volume relatif des lobes et des circonvolutions se modifie beaucoup pendant la croissance, et qu'il ne suffit 
pas de grandir le cerveau d'un jeune animal pour obtenir le cerveau de l'animal adulte. On sait par exemple que chez l'homme le volume relatif des lobes temporal et occipital est au maximum dans l'enfance, et celui du lobe frontal aı minimum; il en résulte des différences topographiques importantes qui ont été exposées dans la thèse de $\mathbf{M}$. de la Foulhouze (1). Ces différences s'atténuent par les progrès de l'âge, et elles ne sont pas même encore effacées à la fin de l'adolescence. Les périodes du développement sont certainement beaucoup plus courtes chez le gorille que chez l'homme. Il est clair toutefois qu'un gorille de six mois est encore bien loin du terme de l'accroissement de son cerveau.

Ainsi s'expliquent certainement les principales différences qui existent entre le cerveau de notre gorille et celui du gorille de Hambourg. Sur ce dernier, le lobe occipital et surtout le lobe temporal sont beaucoup plus volumineux, et le lobe frontal est au contraire beaucoup plus petit. Je șuis surpris que MM. Pansch et Bischoff n'aient pas été frappés de cette petitesse du lobe fron. tal. L'origine des scissures de Rolando, sur le bord sagittal de l'hémisphère, est, il est vrai, très reculée; mais ces scissures sont très obliques, et si celle du côté droit, représentée par M. Bischoff sur la figure 2 de son Mémoire, se termine inférieurement à quelque distance en arrière de la pointe du lobe temporal, celle du côté gauche, représentée sur la seconde photographie de M. Pansch, et facile à étudier surtout sur le modelage en cire, se termine au niveau et même un peu en avant de cette pointe, de sorte qu'il faudrait la reporter à 20 millimètres au moins en arrière pour lui donner la position qu'elle occupe sur les cerveaux d'orang et de chimpanzé, et sur le cerveau de notre gorille adulte. Il est donc évident que le développement de la partie inférieure du lobe frontal du gorille de Hambourg est encore très éloigné de son terme; la région de l'opercule est encore rudimentaire, et c'est pour cela que l'extrémité antérieure de l'insula apparaît, des deux côtés, dans une étendue considérable, à la base de la branche horizontale antérieure de la scissure de Syl-

(1) Paul de la Foulhouze, Recherches sur les rapports anatomiques du cerveau et de la voûte du crîne chez les enfants, Thèse inang. Paris, 1876. In-4. 
vius. Celte position superficielle de l'extrémité antérieure de l'insula s'obscric plus ou moins, chez l'homme, sur' un grand nombre d'enfants nouveau-nés ou âgés de quelques mois; passé la seconde année, elle ne se rencontre plus que sur les cerveaux d'idiots ou d'imbéciles (et aussi chez quelques sourds-muets). Il est donc extrêmement probable que, sur le gorille de Hambourg, elle ne constitue pas un caractère typique, comme on a pu le croire, mais simplement un caractère d'âge, qui se serait effacé si l'animal avait vécu seulement quelques années.

Ces remarques suffisent, je pense, pour résoudre les difficultés que pourrait faire naître la comparaison des cerveaux de notre gorille adulte et du jeune gorille de Hambourg, considérés au point de vue du volume relatif des lobes, et de la richesse des plis secondaires. Quant aux caractères essentiels de la constitution cérébrale, ils sont les mêmes dans les deux cas.

Au moment de livrer ce travail à l'impression, j'apprends par les journaux politiques - qui ne sont pas infaillibles - que le jeune gorille élevé avec tant de soins à l'aquarium de Berlin vient de mourir. Si cette nouvelle se confirme, ce que je regretterais beaucoup, les journaux scientifiques de Berlin nous apporteront bientôt un nouveau terme de comparaison, et j'ai lieu de croire que le cerveau de cet intéressant animal, jeune encore, mais bien moins jeune que le gorille de Hambourg, servira de trait d'union entre les deux cerveaux de gorille qui sont connus jusqu'ici. 


\section{NOMENCLATURE GÉRÉBRALE}

\section{DÉNOMINATION DES DIVISIONS ET SUBDIVISION DES HÉMISPHÈRES}

ET DES ANFRACTUOSITÉS DE LEUR SURFACE

(Revue d'anthopologie, 1878, $2^{\text {e }}$ série, f. III, p. 193-236.)

\section{§ 1. NÉGEssitÉ J'Une NOMENCLATURE FIXE.}

Une des principales difficultés de l'étude des circonvolutions cérébrales résulte du défaut de précision des termes dont on se sert dans cette description. A l'époque, bien peu éloignée de nous, où l'on croyait que les détails de la morphologie cérébrale n’avaient aucune fixité, où l'on répétait que les saillies contournées de la surface du cerveau étaient aussi désordonnées que les circonvolutions de l'intestin grêle, et où l'on jugeait par conséquent qu'il était inutile de les classer et les décrire, il était naturel qu'on n'éprouvât pas le besoin de déterminer le sens des mots dont on se servait pour en parler.

La seule distinction qu'on eût cherché à établir était celle des lobes, et comme ce nom de lobe implique l'idée d'un renflement arrondi et distinct, on n'admit d'abord l'existence des lobes qu'à la face inférieure de l'hémisphère, les autres faces ne présentant évidemment pas la forme lobée. Certains auteurs ne reconnaissaient que deux lobes, l'antérieur et le postérieur, séparés par la profonde dépression transversale qu'on appelle aujourd'hui la vallée de Sylvius. D'autres, avec un peu de complaisarce, en reconnurent trois, en appelant lobe moyen notre lobe temporal, ct lobe postérieur la partie postérieure de la face inférieure, qui repose sur le cervelet et qui se trouve par là un peu excavée. Le nom de lobe se trouvant ainsi détourné de son acception primi- 
tive, on put s'en servir pour désigner les diverses régions de la face convexe, sans croire pour cela qu'elles fussent réellement distinctes les unes des autres, car on se proposait surtout, en les dénommant, d'indiquer leurs rapports avec les divers os de la voûte du crâne. Ce fut bien tard seulement qu'on chercha s'il n'y aurait pas sur le cerveau lui-même des lignes de démarcations anatomiques. A partir de la fin du dernier siècle on commença à reconnaître la fixité de certains sillons ou scissures, et Gratiolet put enfin, en 1854 , établir dans l'hémisphère de grandes divisions bien déterminées. Pour les désigner il employa le nom de lobes, depuis longtemps usité dans la description du cerveau, sous une acception analogue, quoique beaucoup moins précise.

Mais, pendant que la plupart des auteur's appliquaient ce nom aux subdivisions de l'hémisphère, d'autres, considérant que toutes les parties de l'hémisphère sont en continuité avec leurs voisines et quaucune d'elles ne se détache sous la forme d'un véritable lobe, jugèrent que chaque hémisphère ne devait former qu'un seul lobe; le mot lobe devint ainsi pour eus synonyme d'hémisphère. Ce fut ainsi que Chaussier divisa le cerveau entier en deux lobes seulement, le droit et le gauche, séparés par la grande fente médiane qu'il appela la scissure interlobaire, et unis par la grande commissure du corps calleux, qu'il nomma le mésolobe. Quant à l'idée exprimée ordinairement par le mot lobe, il l'exprima par le mot lobule. Cette nomenclature, à l'exception du nom de mésolobe qui fut jugé inutile, fut acceptée pendant la première moitié de ce siècle par un assez grand nombre d'auteurs, et notamment par Longet ; elle est aujourd'hui abandonnée, mais le mot lobule est resté, et il a été employé dans des acceptions diverses, tantôt pour désigner un vrai lobe, mais pour indiquer en même temps que ce lobe est plus petit qu'un lobe ordinaire, exemple : le lobule de l'insula ; tantôt pour désigner une portion particulière de tel ou tel lobe, exemple : le lobule orbitaire du lobe frontal, le lobule triangulaire du lobe occipital, etc.

Le mot circonvolution a eu aussi ses vicissitudes. Ce mot, emprunté à la description de l'intestin, désignait dans l'origine, ainsi que ses divers synonymes, toute partie quelconque de la 
surface plissée et contournée des hémisphères. Les auteurs qui les premiers reconnurent la fixité de quelques-unes de ces parties, continuèrent à les désigner sous le nom de circonvolutions, qui eut aiusi deux sens, l'un général et indéterminé, l'autre propre à certains cas déjà précisés; exprimant deux idées différentes, il donna lieu à des confusions qui ne contribuèrent pas peu à embrouiller la description du cerveau. Une autre difficulté se présenta lorsque Leuret s'efforça d'arriver à la connaissance du cerveau de l'homme en se basant sur l'anatomie comparée, et surtout lorsque Gratiolet, son continuateur, s'attacha spécialement à l'étude du cerveau des primates. Certaines circonvolutions, compliquées et très repliées chez l'homme, sont droites ou à peine flexueuses chez beaucoup de singes; Gratiolet pensa donc que le nom de circonvolutions n'était pas exact, et il le remplaça par celui de plis cérébraux. Il assigna à chaque pli ses connexions et ses limites et donna ainsi pour la première fois un classement méthodique et complet des circonvolutions cérébrales; mais la dénomination qu'il avait employée - et à laquelle d'ailleurs il ne se conformait pas toujours dans ses descriptions - ne fut pas adoptée par ses successeurs. Quelques-uns l'acceptèrent en donnant au mot $p l i$ une signification anatomique déterminée, et en continuant à employer le mot circonvolution dans un sens vague et général. D'autres, au contraire, prenant ce dernier mot dans le sens anatomique, pour désigner des partiez circonscrites correspondant aux plis de Gratiolet, se servirent dı mot pli, accompagné ou non de l'épithète de secondaire, pour désigner les diverses portions dont une circonrolution se compose. On sait que Gratiolet avait appelé plis de passage certains prolongements courts et parfois cachés, qui font communiquer entre elles deux circonvolutions voisines, et qui n'occupent qu'une étendue très limitée. Cette dénomination, qui fut promptement adoptée, fit prévaloir l'idée qu'un pli était moins qu'une circonvolution; ce fut ainsi que le mot pli fut détourné de l'acception première que Gratiolet lui avait donnée, et il y a lieu de se mettre en garde contre les confusions qui pourraient en découler.

Mais ce qui demande surtout à être déterminé, c'est le sens des mots dont on se sert pour désigner les anfractuosités si nom- 
breuses et si irrégulières de la surface de l'hémisphère. Ces lignes de dépression, qui forment comme un labyrinthe autour des circonvolutions, ont reçu les noms les plus divers : anfractuosités (en latin anfractus, en italien anfratti, Malacarne), sillons, sulci (lat.), Furchen (all.), scissures, fissures, incisures, fentes, rigoles, vallées et vallécules, etc. A l'époque où l'on croyait que les circonvolutions étaient disposées sans aucun ordre, on employait ces expressions indistinctement, comme des synonymes commodes pour éviter les répétitions ; c'était parfaitement naturel; il n'y avait alors aucune raison pour diviser les anfractuosités cérébrales en plusieurs espèces, puisqu'elles n'étaient pas plus significatives les unes que les autres. Mais depuis que l'on a établi la distinction anatomique des lobes, puis celle des circouvolutions, dont la fixité se retrouve sous le degré de complication variable de leur's plis secondaires, il est devenu nécessaire de classer les anfractuosités comme on a classé les parties qu'elles limitent. La clarté des descriptions y gagnera beaucoup. On a pu dire que la science n'était qu'une langue bien faite: c'est une exagération; mais il est bien certain qu'une nomenclature régulière est un grand moyen de simplification, dont l'utilité se manifeste surtout dans une étude aussi compliquée que celle du cerveau.

Une nomenclature est régulière Iorsque le rapport des choses que l'on veut distinguer et des noms qu'on leur donne est nettement déterminé, et il n'est nullement nécessaire que ces noms soient noureaux et assujettis à certaines règles de dérivation et de composition. On est obligé de recourir au néologisme et à la réglementation dans les grandes nomenclatures, où doivent trouver place des faits très nombreux, et même les faits encore inconnus. Mais il s'agit ici d'une nomenclature partielle et très restreinte, concernant des choses peu nombreuses et obscurcies par une synonymie déjà trop riche, que des dénominations nouvelles viendraient surcharger inutilement. Il est bien préférable de choisir, parmi les termes déjà usités, ceux qui s’adaptent le mieux, soit par leur sens littéral, soit par leur acception la plus répandue, aux choses que l'on se propose de désigner. Un mot dont la signification est déjà fixée par l'usage doit être conservé quand même il ne serait pas en parfait accord avec l'idée qu'il 
exprime. Ainsi le nom de lobe, tel qu'on l'entend aujourd'hui dans la description du cerveau, est évidemment détourné de son vrai sens ; mais il est généralement accepté, il ne donne plus lieu maintenant à aucune confusion ; dès lors il n'y aurait aucun avantage à le remplacer. De même, le mot circonvolution, dans son serıs anatomique actuel, désigne des parties qui souvent ne sont ni enroulées ni même flexueuses, et c'est pour cela que Gratiolet avait voulu lui substituer le mot pli, qui est certainement plus exact; mais cette innovation n'a pas prévalu; et le sens du mot circonvolution est fixé aujourd'hui par un usage universel, auquel nous devons nous conformer.

Ce n'est donc pas une nomenclature en règle que je viens proposer, mais plutôt une réforme du langage usité dans la description des hémisphères, réforme consistant à atteindre ce but: que chaque chose ait un nom, qu'elle n'en ait qu'un seul, et que ce nom ne désigne qu'une seule chose. Pour cela il suffira de restreindre à un sens déterminé les tẻrmes déjà usités. Ce ne sera que très exceptionnellement que j'aurai un terme nouveau à proposer.

\section{§ 2. L'HÉMISPHÈre DANS SON ENSEMBLE.}

L'écorce et le manteau. - Le corps de l'hémisphère. - Le seuil et le limbe de l'hémisphère.

L'hémisphère se compose d'un groupe de parties très diverses qui en occupent le centre, et d'une couche superficielle qui enveloppe cette masse centrale. Dans les cerveaux plissés, comme celıi de l'homme, la couche enveloppante revêt la forme de circonvolutions.

On la nomme ordinairement couche corticale ou écorce cérébrale ; mais ce nom n'est pas parfaitement exact, car il ne désigne anatomiquement que la couche grise des circonvolutions; or une circonvolution se compose de deux parties : $1^{\circ}$ un prolongement de la masse blanche centrale, constituant en quelque sorte l'axe de la circonvolution, et présentant une longueur et une largeur très variables; $2^{\circ}$ une couche superficielle assez uriforme, présentant une couleur grise due à la prédominance de la sub- 
stance grise et constituant à proprement parler l'écorce de la circonvolution.

Le mot écorce ou couche corticale désignant ainsi, d'un accord unanime, une partie déterminée des circonvolutions, ne doit pas désigner en même temps les circonvolutions elles-mêmes.

L'enveloppe que l'ensemble des circonvolutions forme, autour du groupe des parties centrales de l'hémisphère, doit donc recevoir un autre nom. Celui de manteau (Mantel, all., pallium, lat.) qui lui a été donné par Burdach est parfaitement convenable et mérite d'être conservé.

Ge nom est peu usité et même peu connu en France, où on le remplace tantôt par le nom défectueux d'écorce, tantôt par des expressions comme celles-ci : les lobes de l'hémisphère, la masse lobaire, les circonvolutions de l'hémisphère, la masse circonvolutionnaire. Ces dénominations, déjà gênantes par leur longueur, ont l'inconvénient de ne viser que les cerveaux plus ou moins plissés comme celui de l'homme. Mais beaucoup d'animaux, appelés par 0 wen lissencéphales, n'ont pas de circonvolutions; chez eux le manteau de l'hémisphère est lisse ou homogène dans la plus grande partie de son étendue, et toute dénomination dans laquelle figure l'idée de lobe ou celle de circonvolution ne leur est pas applicable. Le nom de manteau doit donc être préféré.

Ainsi, le manteau de l'hémisphère comprend à la fois l'écorce cérébrale, et les prolongements que la substance blanche centrale envoie dans l'épaisseur de la plupart des circonvolutions, chez les animaux gyrencéphales ou à circonvolutions.

Le manteau enveloppe tout l'hémisphère, à l'exception d'une partie de la face interne, limitée en haut par le corps calleux, en bas par la branche latérale de la grande fente de Bichat. Sur l'hémisphère isolé, séparé de l'hémisphère opposé par une coupe médiane et de la protubérance par la coupe du pédoncule cérébral, cette partie non recouverte par le manteau présente une forme à peu près ovalaire. On l'a appelée la grande ouverture de l'hémisphère, parce qu'on y voit effectivement au-dessus, en arrière et au-dessous de la couche optique une grande ouverture qui conduit dans la cavité du ventricule latéral ; mais on y voit aussi la face interne de la couche optique, la coupe du corps calleux, de la cloison transparente, de la voûte, des commissures, 
du pédoncule cérébral. C'est par là que passent toutes les fibres convergentes ou divergentes qui pénètrent dans l'hémisphère ou qui en sortent. Cette portion de la face interne de l'hémisphère n'est donc pas une ouverture. Mais elle est en quelque sorte la porte d'entrée et de sortie de l'hémisphère et je l'appellerai dès lors le seuil de l'hémisphère, nom qui, dans la nomenclature latine, très usitée à l'étranger, peut être rendu par le mot limen.

Le seuil ou limen de l'hémisphère est circonscrit de toutes parts par le manteau qui forme autour de lui un bord comparable à la bordure circulaire de l'entrée d'une bourse. Je donne donc à ce bord le nom de limbe (limbus, bordure); et à la circonvolution qui le forme le nom de circonvolution limbique (1). Ghez tous les mammifères qui ont un vrai lobe olfactif, le limbe est complet et ininterrompu ; mais chez les primates, les amphibies et les cétacés, qui n'ont qu'un lobe olfactif tout à fait rudimentaire (quelquefois nul), la circonvolution limbique est interrompue en bas par la vallée de Sylvius.

Il s'agit maintenant de donner un nom à l'ensemble des parties de l'hémisphère qui sont recouvertes par le manteau. Burdach a appelé cet ensemble le noyau (K $\mathrm{ern})$ de l'hémisphère; mais ce nom ne saurait convenir à un groupe aussi disparate, qui comprend non seulement la couche optique, le corps strié et leurs dépendances, mais encore le corps calleux, la voûte, la cloison, les commissures, la grande masse de substance blanche qui, sur les coupes, forme le centre ovale, etc. Il y a là un mélange inextricable de substance blanche et de substance grise, de fibres divergentes et de fibres convergentes, et cette masse si complexe, qui n'a d'ailleurs du côté du manteau ni ligne de démarcation ni limite d'autre sorte, ne peut, en aucune façon, être comparée à un noyau. Le nom de noyau donnerait d'ailleurs lieu à des confusions, parce qu'il y a dans les masses centrales de l'hémisphère plusieurs amas de substance grise, plus ou moins arrondis et plus ou moins circonscrits, qui portent déjà ce nom de noyau. J'ajoute que Foville appelle le noyau

(1) Foville l'a appelée circonvolution de l'ourlet parce qu'il croyait que sa base était formée par une bandelette de fibres circulaires, qu'il nommait l'ourlet. Cette bandelette est artificielle. 
cérébral, ou le noyau central du cerveau l'ensemble de toutes les parties centrales des deux hémisphères, réunies en un seul tout par le corps calleux et les autres commissures (1). En d'autres termes, si l'on divise en deux par une coupe médiane le noyau cérébral de Foville, chacune des moitiés sera ce que Burdach a appelé le noyau. Ce nom de noyau est donc à la fois inexact, confus et amphibologique, et il faut chercher une autre expression.

Le nom de medullum, proposé par Gerdy, manque de clarté; il désigne une masse médullaire ainsi appelée par opposition avec la masse corticale que forment les circonvolutions; mais les mots substance médullaire et substance corticale sont souvent employés comme synonymes de substance blanche et de substance grise. Le mot medullum ferait donc naître l'idée erronée que la masse centrale de l'hémisphère est exclusivement composée de substance blanche.

N'ayant trouvé dans la science aucune dénomination satisfaisante pour désigner l'ensemble des parties de l'hémisphère qui sont cachées sous le manteau, je propose d'appeler cette masse complexe le corps de l'hémisphère, pour continuer l'image exprimée par le mot manteau. Le mot tronc aurait peut-être indiqué plus clairement ce qui est recouvert par le manteau, mais ce mot (en allemand Stamm), très usité en Allemagne, désigne depuis Burdach un ensemble qui, d'une part, ne comprend qu'une portion du corps de l'hémisphère, et qui, d'une autre part, comprend diverses parties étrangères à l'hémisphère.

Nous dirons donc que l'hémisphère se compose de deux parties, l'une enveloppante, qui est le manteau, l'autre enveloppée, qui est le corps de l'hémisphère. Le manteau est tantôt lisse, tantôt plissé et subdivisé en lobes et circonvolutions. La partie qu'il ne recouvre pas, et qui est située sur la face interne, constitue le seuil de l'hémisphère, et le bord du manteau qui entoure le seuil est le limbe de l'hémisphère.

(1) Pour éviter la confusion produite par le nom de noyau cérébral, Foville appelait cotylédons les deux noyaux extra et intraventriculaires du corps strié. 


\section{§ 3. LES DIVISIONS DE L'HÉMISPHÈRE.}

Lobes et lobules. - Circonvolutions et plis.

A. Lobes et lobules. - Les divisions primaires du manteau sont désignées sous le nom de lobes. Ce ne sont point de vrais lobes, dans le sens propre du mot; ce sont plutôt des districts que des lobes; mais l'usage ici doit faire loi. Nous conservons donc une dénomination aujourd'hui universellement adoptée, et qui dès lors ne peut plus, comme autrefois, donner lieu à des confusions.

Les lobes de l'hémisphère ont leurs limites marquées, sur le cerveau même, par des lignes anatomiques; leur nombre et leurs rapports avec les os du crâne variant beaucoup suivant les espèces, il serait, sinon impossible, du moins très difficile de donner à chacun d'eux un nom parrticulier qui restât exact chez tous les mammifères; mais on est convenu d'étendre à toute la série les dénominations adoptées dans la description du cerveau humain. Elles expriment tantôt la forme des lobes (L. de l'insula), tantôt leur position sous tel ou tel os du crâne (L. frontal, pariétal, etc.), et elles ne donnent lieu à aucune difficulté sérieuse lorsqu'on ne considère que l'ordre des primates. Dans les ordres moins élevés, quelques-unes d'entre elles cessent d'être exactes, mais tout le monde s'entend sur l'acception qu'il faut leur donner, et cela suffit parfaitement pour la clarté du langage. Quant aux discussions qui peuvent s'élever sur la détermination des parties homologues dans les divers ordres de mammifères, elles concernent l'anatomie et non la nomenclature. Ajoutons toutefois que la nomenclature humaine ne répond pas à tous les besoins de la description du cerveau des mammifères en général ; car certaines parties qui, dans les ordres inférieurs aux primates, constituent de véritables lobes, sont tellement atrophiées ou effacées chez l'homme et les singes, que ce nom de lobes ne leur est plus applicable. Ainsi le grand lobe olfactif des autres mammifères n'est plus représenté, chez les primates, que par une bandelette que termine un petit renflement appelé le ganglion olfactif. De même, le lobe de l'hippocampe, qui 
est étroitement associé au lobe olfactif, se réduit chez les primates à une circonvolution peu volumineuse dite de l'hippocampe, qui se fusionne avec le lobe temporal. On ne peut donc pas imposer des dénominations absolument fixes à des parties dont la constitution, le volume et l'importance présentent, dans la série des Imammifères, des différences aussi grandes; mais si le nombre variable des lobes et le nom particulier de chacun d'eux peuvent introduire des complications dans la nomenclature, le terme général de lobe n'en conserve pas moins une acceptation très simple et parfaitement déterminée, puisque tout le monde s'accorde maintenant à désigner sous ce nom les divisions primaires dı manteau de l'hémisphère.

Les divisions secondaires ou subdivisions des lobes sont aussi bien caractérisées que les lobes eux-mêmes, et sont décrites aujourd'hui sous le nom de circonvolutions. Tout lobe se compose d'un certain nombre de circonvolutions (quelquefois d'une seule dans les cerveaux très simples de certains animaux), et ces circonvolutions, fixes dans chaque espèce, sont déterminées par leur position et leurs connexions, de telle sorte que chacune d'elles peut être décrite isolément. Toute partie du manteau de l'hémisphère se rattache à une certaine circonvolution, et l'ensemble des circonvolutions comprend le manteau tout entier. Il ne reste donc aucune place pour les divisions connues sous le nom de lobules.

Ce nom cependant est d'un usage très fréquent et il est bon à conserver, car il est commode et utile. Dans la description des cerveaux, on a besoin avant tout d'établir les divisions anatomiques appelées lobes et circonvolutions; mais cela fait, on éprouve en outre, dans beaucoup de cas, le besoin de désigner certaines régions qui font partie d'un lobe ou d'une circonvolution. On se sert pour cela du mot lobule, qui, souvent, n'est pas anatomique, mais seulement topographique. Ainsi, la région de la face inférieure du cerveau qui est située en avant de la vallée de Sylvius appartient anatomiquement au lobe frontal; toutefois, les rapports particuliers qu'elle affecte avec la voûte orbitaire, et les connexions terminales très importantes des trois circonvolutions frontales réfléchies qui la composent, la distinguent assez du reste du lobe frontal pour qu'on ne puisse 
se passer de la désigner sous le nom spécial de lobule orbitaire. Ce lobule comprend une portion de chacune des trois circonvolutions frontales. D'autres fois, la région que l'on juge utile de distinguer sous le nom de lobule est beaucoup plus restreinte ; elle ne dépend que d'une seule circonvolution, et ne comprend même le plus souvent qu'une portion de circonvolution unique. Ainsi, le lobule de l'hippocampe n'est que la partie antérieure de la circonvolution de l'hippocampe; le lobule quadrilatère n'est que la face interne de la première circonvolution pariétale; le lobule paracentral de Meynert, ovalaire de Pozzi, n'est que la portion de la circonvolution frontale ascendante qui s'épanouit sur la face interne de l'hémisphère. Parmi les régions appelées lobules, il en est une toutefois qui comprend une circonvolution entière : c'est le lobule occipital interne ou lobule triangulaire, ou cuneus; il est formé par la dernière circonvolution occipitale (qui est la sixième chez l'homme et les anthropoïdes). Ce dernier lobule est le seul qui soit très bien limité et qui soit, à proprement parler, un lobule. Les autres ne se détachent pas assez des parties. environnantes pour répondre réellement à l'idée exprimée par le mot lobule. Mais leur dénomination est parfaitement consacrée par l'usage, et puisqu'on est convenu d'appeler lobes des parties qui, pour la plupart, n'ont pas la forme lobée, on peut tout aussi bien appeler lobules des parties qui, pour la plupart, ne sont pas lobulées. Il faut se souvenir seulement que ces noms de lobes et de lobules ont reçu autrefois des acceptions différentes; car on a vu que Chaussier et ses successeurs appelaient lobe l'hémisphère entier, et lobules ce que nous appelons aujourd'hui des lobes.

J'ai déjà dit que l'acception du mot lobule est surtout topographique. Il en est de même du mot étage, qui facilite beaucoup la description du lobe frontal, et dont il faut maintenant déterminer le sens. J'ai dit que la portion du lobe frontal qui se réfléchit à la base du front et se porte horizontalement en arrière, en reposant sur la voûte orbitaire, est distinguée sous le nom de lobule orbitaire. Il est indispensable de distinguer aussi le reste du lobe sous un nom spécial ; on le désigne souvent sous le nom de lobule frontal, et on dit alors que tout le lobe frontal se compose de deux lobules, l'un orbitaire, qui en 
forme la face inférieure, l'autre frontal, qui en forme la face convexe; mais cette face convexe, qui occupe près de la moitié de la longueur de l'hémisphère, est vraiment trop grande pour être appelée un lobule. Il me paraît bien préférable de la nommer étage supérieur du lobe frontal. Le lobule orbitaire pourrait être appelé par opposition l'étage inférieur du même lobe ; mais cette symétrie n'est pas nécessaire, car le nom de lobule orbitaire est déjà classique et est d'ailleurs parfaitement clair.

Le nom d'étage est ici pleinement justifié, puisque les deux portions du lobe frontal sont superposées, l'une étant située audessous, l'autre au-dessus du prolongement antérieur du centre ovale de Vieussens. Ce nom, déjà utile dans la description de tout le lobe, s'applique avec plus d'avantage encore à la description particulière des circonvolutions qui le composent.

Chacune des trois circonvolutions frontales longitudinales se réfléchit en avant pour aller prendre part à la formation du lobule orbitaire. Dans la description de ces circonvolutions, il est nécessaire de pouvoir distinguer leur portion supérieure ou directe de leur portion réfléchie ou inférieure; celle-ci s'appelle naturellement portion orbitaire, ce qui est commode et clair ; l'autre est appelée portion frontale, ce qui est beaucoup moins satisfaisant, car lorsqu'on dit : la portion frontale de la deuxième circonvolution frontale, on donne la même qualification au tout ou à la partie, au détriment de la clarté. Le nom de portion supérieure manquerait aussi de clarté; on croirait qu'il s'agit seulement de la partie la plus élevée de la circonvolution, de celle qui avoisine la circonvolution frontale ascen. dante; je pense donc qu'il vaut mieux dire l'étage supérieur de telle ou telle circonvolution frontale.

Cette dénomination aurait pu donner lieu à une confusion sérieuse lorsque la nomenclature de Gratiolet était encore en vigueur. Cet auteur employait le nom d'étages comme synonyme de plis ou de circonvolutions, pour indiquer la position plus ou moins élevée des circonvolutions d'un même lobe. Ainsi, il appelait la première circonvolution frontale l'étage supérieur du lobe frontal, la seconde l'étage moyen, la troisième l'étage inférieur. Ces expressions étaient inexactes, car les trois circonvolutions dont il s'agit ne sont nullement superposées; elles ont 
donc été abandonnées, et dès lors le mot étage, pris dans un sens topographique et non plus dans un sens anatomique, reste à notre disposition pour indiquer la superposition de la portion directe et de la portion réfléchie du lobe frontal et de ses circonvolutions.

On peut se demander s'il n'y aurait pas lieu de distinguer aussi deux étages dans le lobe occipital, dont les circonvolutions supérieures sont situées au-dessus et les inférieures au-dessous du prolongement postérieur du centre ovale de Vieussens. Mais cela est tout à fait inutile, attendu que ce ne sont pas les mêmes circonvolutions qui passent d'un étage à l'autre, comme cela a lieu dans le lobe frontal. Les circonvolutions occipitales supérieures, au nombre de trois chez l'homme et les anthropoïdes, forment un lobule, le lobule sus-accipital; les circonvolutions inférieures, au nombre de deux, forment un second lobule, le lobule sous-occipital. Ces deux lobules sont séparés sur le bord externe du lobe par un sillon longitudinal (sillon occipital latéral), qui est le troisième chez l'homme et les anthropoïdes. La dernière circonvolution occipitale, enfin, située sur la face interne du lobe et formée d'une seule circonvolution, constitue le lobule triangulaire ou cuneus, parfaitement limité. Le lobe occipital se trouve ainsi décomposé en trois lobules, ce qui est simple et clair, tandis que la division en étages couperait en deux le lobule triangulaire, étendu du bord supérieur au bord inférieur du lobe occipital.

Un mot enfin sur deux parties qui ont été désignées sous le nom d'opercules, l'une par Reil, l'autre par Gratiolet. Reil a appelé opercule (Dach all., operculum lat.) la portion du bord supérieur de la scissure de Sylvius, qui recouvre et cache le lobe de l'insula. Gratiolet a appliqué le même nom à la portion du lobe occipital qui, chez la plupart des singes, se prolonge en avant sur la scissure occipitale externe, de manière à cacher le fond de cette scissure.

Ces deux noms sont très usités et très convenables; mais il faut y joindre une qualification pour éviter les confusions. Nous dirons donc l'opercule de l'insula et l'opercule occipital.

B. Circonvolutions et plis. - Revenons maintenant aux circonvolutions (en latin gyri). J'ai déjà dit comment le nom 
qu'on leur donne maintenant avait eu autrefois un autre sens ; l'acception morphologique a fait place à l'acception anatomique. Ce nom est très inexact, puisqu'il y a chez la plupart des animaux et chez l'hornme niême des circonvolutions sans aucune sinuosité ; l'usage unanime qu'on en'fait aujourd'hui nous oblige toutefois à le conserver, malgré sa longueur gênante. Les Anglais l'ont déjà réduit d'une syllabe (convolution), et on pourrait très bien le réduire encore, car le mot volution exprimerait suffisamment la forme tortueuse des saillies de l'hémisphère ; il l'exprimerait même mieux que le mot circonvolution, qui fait naître l'idée très fausse d'un enroulement autour d'un centre (circum), comme celui d'un serpent roulé sur lui-même (1); mais je ne me propose pas de renouveler toute la nomenclature cérébrale; je me borne à chercher la précision du langage ; toute dénomination dont le sens est assez bien déterminé pour ne donner lieu à aucune confusion doit être maintenue, et le mot circonvolution est dans ce cas.

Les circonvolutions sont des organes fixes dans chaque espèce; mais cette fixité concerne leur position et leurs connexions plutôt que leur forme, qui peut présenter des différences notables non-seulement chez deux individus d'une même espèce; mais encore sur les deux hémisphères d'un même cerveau. Ces variations, qui ont si longtemps donné le change aux anatomistes, sont dues d'une part au degré de complication des circonvolutions, c'est-à-dire au nombre de leurs détours et de leurs subdivisions, d'une autre part au degré de développement et à la situation plus ou moins superficielle ou profonde des prolongements qui les mettent en communication avec leurs voisines. Il est impossible de décrire une circonvolution sans indiquer ces détails, et la clarté exige que les parties qui la composent ne portent pas le même nom que la circonvolution elle-même.

A cet effet, on a proposé d'appeler circonvolutions primaires les circonvolutions proprement dites, et circonvolutions secondaires ou même tertiaires les parties plus ou moins accessoires qui viennent les compliquer. Mais ces dénominations, qui ont

(1) La plupart des anatomistes du dix-septième siècle qui ont écrit en latin ne disaient pas circumvolutiones, mais convolutiones. 
déjà le tort d'être trop longues, ont en outre le désavantage d'être ambiguës, parce qu'elles ont reçu plusieurs acceptions très différentes, en rapport avec les vues théoriques des auteurs qui les ont employées. On y a donc assez généralement renoncé, et je ne fais que me conformer à un usage qui tend déjà à s'établir, en proposant de désigner sous le nom de plis ces parties secondaires d'une circonvolution.

On a vu plus haut que ce nom de pli, employé d'abord par Gratiolet comme synonyme de circonvolution, avait reçu depuis une acception plus restreinte, et ne désignait plus maintenant que certaines portions de circonvolution. Mais les parties auxquelles on peut l'appliquer sont de diverses natures. Il faut donc distinguer plusieurs espèces de plis. Je les diviserai en deux groupes : $1^{\circ}$ les plis de communication, comprenant les plis de passage et les plis d'anastomose; $2^{\circ}$ les plis de complication, comprenant les plis de subdivision et les plis d'inflexion ou méandres.

$1^{\circ}$ Les plis de communication sont des prolongements qui s'étendent d'une circonvolution aux circonvolutions voisines, $\cdot$ a travers les anfractuosités intermédiaires. Ils peuvent être profonds, c'est-ì-dire cachés en tout ou en partie au fond de l'anfractuosité qu'ils traversent, ou superficiels, c'est-à-dire apparents à l'extérieur dans toute leur étendue. Les circonvolutions qu'ils unissent peuvent appartenir à un même lobe ou à deux lobes voisins; dans le premier cas, je les nomme plis d'anastomose; dans le second, plis de passage. Cette distinction est très commode dans les descriptions, et elle est pleinement justifiée par l'inégale importance des deux espèces de plis de communication.

Les plis de passage ont été ainsi nommés par Gratiolet, qui les a décrits le premier, et qui les a rangés au nombre des principaux caractères distinctifs des cerveaux des primates. La valeur de ce caractère est moindre qu'il ne l'a cru, mais elle est grande encore, car la position superficielle ou profonde des plis de passage, qui dépend de leur volume, amène des différences très remarquables dans la disposition du lobe occipital et des scissures occipitales. L'étude des plis de passage est donc très importante, et le nom que Gratiolet leur a donné est aujourd'hui 
généralement adopté, soit sous sa forme française, soit sous la forme équivalente qu'il a revêtue en passant dans les lảngues étrangères.

Quant aux plis d'anastomose, ils méritent plus d'attention qu'on ne leur accorde généralement. On ne s'est guère occupé jusqu'ici que de ceux qui sont superficiels et qui interrompent en certains points, d'ailleurs variables, les sillons intercirconvolutionnaires, de manière à rendre quelquefois assez confuses les limites des circonvolutions. Tous les auteurs qui ont eu à décrire complétement un cerveau les ont mentionnés à l'occasion, en indiquant parfois leur position, leur forme et leur volume, mais sans leur accorder assez d'importance pour les caractériser par un nom spécial. Il semble en effet que ces connexions latérales de deux circonvolutions d'un même lobe ne soient soumises à aucune règle, et que leur présence ou leur absence ne soient que des phénomènes fortuits; mais si l'on écarte les bords des sillons pour en examiner le fond, on reconnaît que ce désordre n'est qu'apparent.

Deux grandes circonvolutions séparées par un sillon communiquent toujours entre elles par un certain nombre de prolongements latéraux qui, en passant de l'une à l'autre, soulèvent plus ou moins le fond du sillon, formant tantôt une simple colline transversale, tantôt un pli plus saillant et plus ou moins contourné, tantôt enfin un gros pli qui monte jusqu'à la surface et qui interrompt le sillon. Le nombre de ces prolongements latéraux varie un peu dans chaque sillon, suivant que le cerveau est très simple ou très compliqué; mais il en est qui sont constants, sinon quant à leur volume, du moins quant à leur's points d'insertion, et ce sont ceux-là précisément qui font varier la morphologie extérieure de l'hémisphère, car ce sont les seuls qui soient susceptibles de prendre assez de développement pour arriver jusqu'à la surface du cerveau. Lorsqu'ils sont peu volumineux, ils restent cachés dans le sillon, dont la profondeur est diminuée à ce niveau, mais dont la continuité n'est pas interrompue; lorsqu'ils sont forts, ils deviennent apparents ; mais qu'ils soient superficiels ou profonds, leurs connexions restent les mêmes. lls se comportent donc comme les plis de passage, et il est nécessaire de les connaître pour apprécier les différences 
que l'on observe non-seulement sur les cerveaux de deux individus de 'même espèce, mais encore sur les deux hémisphères d'un même cerveau. C'est pour cela que j'ai jugé utile de leur faire une place dans la nomenclature, et le nom de plis d'anastomose que je leur ai donné, les distingue suffisamment des plis de passage, avec lesquels on ne doit pas les confondre, quoiqu'ils soient comme eux des plis de communication.

$2^{\circ}$ Les plis de complication sont également de deux espères. Beaucoup de circonvolutions sont plus longues que la distance comprise entre leurs deux extrémités; elles se replient donc une ou plusieurs fois dans leur trajet en décrivant des plis d'inflexion qui sont tantôt de simples simuosités, tantôt de véritables méandres. Il y a sinuosité lorsque le bord de la circonvolution est alternativement concave et convexe; il y a méandre lors: que le bord se recourbe assez fortement pour revenir en contact avec lui-même avant de continuer son trajet; il y a alors entre les deux bords de ce brusque contour une incisure plus ou moins profonde qui communique arec le sillon adjacent comme une petite branche avec son tronc. Les méandres ont été encore appelés des arcs; mais ce nom n'indique nullement leır forme, puisqu'un arc ne se recourbe jamais à ce point. J'ajoute qu'il y aurait quelque inconvénient à donner au mot arc une acception anatomique spéciale, parce qu'on est souvent obligé de l'employer dans son sens vulgaire, comme terme purement descriptif, pour exprimer la forme générale de certaines circonvolutions ou de certaines scissures.

Les plis d'inflexion ne sont que l'un des deux éléments de la complication du cerveau; l'autre élément est constitué par les plis de subdivision. Beaucoup de circonvolutions présentent à leur surface, c'est-à-dire entre leurs deux bords, des dépressions qui sont des fossettes lor'squ'elles sont très superficielles, et des incisures lorsqu'elles sont plus profondes. Les incisures peuvent être longues ou courtes, simples ou divisées, droites ou sinueuses, transversales, longitudinales ou obliques. Celles qui sont longitudinales, c'est-ì-dire à peu près parallèles à l'axe de la circonvolution, et qui, en outre, ont une certaine longueur, subdivisent la circonvolution en deux parties, que nous appelons les plis de subdivision. Dans les cerveaux très compliqués, la 
présence des plis de subdivision peut quelquefois rendre assez difficile la détermination des vraies limites de certaines circonvolutions. Fixons enfin le sens de quelques mots dont on est souvent obligé de se servir dans la desçription des circonvolutions.

Toute circonvolution commence et finit quelque part; elle a done une origine et une terminaison; mais il est clair que l'on pourrait la faire commencer ou finir vers l'une ou l'autre extrémité, et l'on voit effectivement que les auteurs ne s'accordent pas toujours à cet égard. Les appréciations sont tantôt arbitraires, tantôt basées sur l'idée que l'on se fait, à tort ou à raison, de l'importance relative des connexions des parties centrales avec les deux extrémités de la circonvolution, tantôt enfin tirées des observations que l'on peut faire soit en embryogénie, soit en anatomie comparée, sur le mode de formation des circonvolutions. Au milieu de ces incertitudes, il faut reconnaître d'abord qu'il n'y a pas de règle fixe, et prendre ensuite le parti de se conformer à l'usage le plus répandu.

Nous dirons donc que les circonvolutions du lobe frontal et du lobe pariétal, ont leur origine sur les deux circonvolutions ascendantes qui limitent la scissure de Rolando. Les origines, dans ce cas, portent encore le nom de racines. Daus les cerveaux simples, chacune des trois circonvolutions frontales et des deux pariétales n'a qu'une seule racine, généralement moins large que la circonvolution elle-même; mais sur les cerveaux plus compliqués, on voit quelquefois une racine s'élargir et se subdiviser à sa base. Le nombre des racines paraît alors augmenté, et il faut une certaine attention pour le ramener au nombre typique. On devra en outre se garder de prendre pour une troisième racine pariétale le pli d'anastomose qui s'étend constamment du milieu de la pariétale ascendante à la partie antéricure et externe de la première circonvolution pariétale, et qui est souvent assez gros pour devenir superficiel et simuler une racine.

Le mot racine est légitimé par le mode d'insertion des circonvolutions frontales et pariétales sur les circonvolutions ascendantes; il n'est nullement applicable aux origines des circonvolutions des autres lobes. Celles-ci portent donc purement et simplement le nom d'origines. 
On est convenu de placer l'origine des deux circonvolutions ascendantes sur le bord supérieur ou sagittal de l'hémisphère, et leur terminaison à leur extrémité inférieure, qui aboutit à la scissure de Sylvius.

Toutes les circonvolutions du lobe occipital ont leur origine sur l'extrémité postérieure ou sur la pointe de ce lobe. Il y a là un point vers lequel convergent tous les sillons, comme les méridiens vers le pôle. Ces sillons, à leur extrémité postérieure, sont plus ou moins contournés, mais ne communiquent pas les uns avec les autres, et il reste entre eux une petite surface qui se continue à la fois avec toutes les circonvolutions occipitales, et que je propose d'appeler le pôle occipital.

On s'accorde généralement à placer l'origine des circonvolutions temporales sur la pointe du lobe temporal. L'anatomiecomparée conduirait peut-être à une interprétation opposée, mais je pense qu'il y a lieu de se conformer à l'usage.

La partie de la pointe de ce lobe.qui communique avec toutes les circonvolutions temporales, peut être appelée pôle temporal, quoique chez tous les animaux autres que l'homme, et même aussi chez certains hommes (races inférieures), la dernière circonvolution temporale ou circonvolution de l'hippocampe soit séparée du pôle temporal par une scissure assez profonde.

On peut également appeler le pôle de l'insula le sommet de l'éventail formé par des circonvolutions du lobe de l'insula. On sait que ce sommet occupe l'extrémité antérieure et inférieure du lobe. De là les circonvolutions se portent en divergeant vers la rigole supérieure de l'insula, où elles se terminent en se continuant avec la face profonde des circon volutions fronto-pariétales, par des plis de passage très profonds. Il ne faut pas confondre avec ces circonvolutions de l'insula un gros pli de passage temporo-pariétal, qui existe dans le fond de la scissure de Sylvius, en arrière de l'insula, et qui est également très profond.

Il reste à parler de la circonvolution du corps calleux, qui n'appartient à aucun des lobes de l'hémisphère. L'origine de cette circonvolution doit être placée à son extrémité antérieure, au-dessous du genou et au niveau du bec du corps calleux.

En indiquant l'origine des diverses circonvolutions de l'hémisphère, j'ai par là même indiqué celle de leur extrémité qui 
doit être considérée comme leur terminaison. Les circonvolutions peuvent se terminer de trois manières : $1^{\circ}$ tantôt en aboutissant à une surface sans circonvolution; c'est ainsi que se terminent les deux premières circonvolutions frontales sur le bord postérieur du lobule orbitaire (la troisième se termine en se continuant avec la première circonvolution de l'insula); $2^{\circ}$ tantôt en aboutissant à une.scissure, où elles peuvent d'ailleurs se continuer, par des plis de passage superficiels ou profonds, avec les circonvolutions d'un autre lobe (exemple, la première circonvolution pariétale et la première occipitale); $3^{\circ} \operatorname{tantôt~enfin~en~se~continuant~directement,~sans~ligne~de~}$ démarcation et d'une manière insensible, avec les circonvolutions d'un lobe voisin. Dans ce dernier cas, la terminaison n'est que virtuelle, ou plutôt de pure convention : c'est une même circonvolution qui change simplement de nom en passant d'un lobe à l'autre, sans qu'on puisse indiquer le lieu où se fait le partage; la continuité des circonvolutions est la conséquence de la continuité des lobes. La base du lobe temporal se continue ainsi avec le lobe pariétal et avec le lobe occipital.

Je devrais maintenant exposer les règles sur lesquelles repose la nomenclature des circonvolutions d'un même lobe; mais il sera préférable, pour éviter les répétitions, de réserver la question pour le moment où je parlerai de la nomenclature des sillons.

\section{§ 4. LES aNfractuosités de L'HÉMISPHÈre.}

Scissures, sillons et incisures.

On pourrait, à la rigueur, décrire le manteau de l'hémisphère en se bornant à décrire les circonvolutions, car, celles-ci étant une fois connues jusque dans leurs moindres détails, la connaissance des anfractuosités qui les séparent devrait en découler nécessairement.

On pourrait encore, à la rigueur, se borner à décrire les anfractuosités du manteau, car celui qui les connaîtrait complétement connaîtrait par là même les diverses saillies dont elles établissent les limites. 
De là, deux méthodes de description, dont tous les auteurs n'ont pas apprécié la valeur de la même manière. Presque tous ont reconnu qu'il était utile de les combiner. Mais les uns ont donné la primauté à la première, en ne faisant de la seconde qu'un usage tout à fait accessoire; d'autres ont appliqué surtout la seconde, supposant que l'esprit du lecteur pourrait toujours se représenter nettement des circonvolutions dont les limites sont bien déterminées; d'autres, enfin, et je suis du nombre, pensent que les deux méthodes sont également nécessaires.

Les circonvolutions sont des organes, et les lobes sont des groupes d'organes, tandis que les anfractuosités ne sont que des intervalles; l'étude des circonvolutions est le but, celle des. anfractuosités n'est que le moyen; mais ce moyen est le seul qui conduise logiquement au but, car il est clair qu'avant de décrire correctement un organe, il faut d'abord le limiter. La description du manteau de l'hémisphère se compose donc de deux parties qui doivent se succéder dans l'ordre suivant: $1^{\circ}$ description des anfractuosités; $2^{\circ}$ description des lobes et des circonvolutions.

L'étude des anfractuosités acquiert par là une importance égale à celle des circonvolutions elles-mêmes, et elle exige au même degré l'emploi de termes précis. Il faut reconnaître, toutefois, que jusqu'ici les anatomistes ne se sont pas préoccupés du choix à faire, pour chaque cas, parmi les noms très-nombreux qui servent à désigner les anfractuosités cérébrales. S'il n'y avait qu'une seule espèce d'anfractuosités, si l'on croyait encore que toutes ont la même signification etla même importance, ce choix serait indifférent comme il l'était autrefois ; l'un pourrait appeler sillon ce que l'autre appelle scissure, et un autre pourrait même, comme on le voit bien souvent, apppliquer tour à tour ces deux noms à la même chose, sans donner le change au lecteur. Mais la multiplicité des espèces d'anfractuosités résulte nécessairement de la multiplicité des espèces de divisions ou de subdivisions qu'elles établissent, et là où il y a des idées distinctes à exprimer, la clarté exige qu'il y ait aussi des dénominations distinctes.

Il faut d'abord un terme général pour désigner toutes les dépressions de la surface de l'hémisphère. Je pense que le mot anfractuosité, en latin anfractus, mot depuis longtemps classique, 
exprime mieux que tout autre cetteidée générale. Il a l'avantage de llavoir jamais été employé dans un sens spécial, et d'être applicable à toutes les dépressions du manteau, qu'elles soient longrues ou courtes, larges ou étroites, superficielles ou profondes.

Il faut ensuite des termes particuliers pour désigner chaque espèce d'anfractuosité. A part quelques cas spéciaux, sur lesquels je devrai revenir, toutes les anfractuosités peuvent se ramener anatomiquement à trois groupes.

Il y a dans l'hémisphère trois ordres de divisions et de subdivisions, savoir: $1^{\circ}$ les lobes $; 2^{\circ}$ les circonvolutions; $3^{\circ}$ les plis de complication. Il y a donc aussi trois ordres d'anfractuosités: $1^{\circ}$ celles qui séparent les lobes; $2^{\circ}$ celles qui séparent les circonvolutions; $3^{\circ}$ celles qui subdivisent ou compliquent les circonvolutions.

Les noms de sillons primaires, secondaires et tertiaires pourraient désigner ces trois espèces d'anfractuosités, s'ils n'avaient déjà reçu d'autres acceptions; on les a employés tantôt pour désigner l'ordre réel ou supposé suivant lequel les anfractuosités apparaissent sur le cerveau du fotus, tantôt pour indiquer le degré d'importance qu'on croit devoir y attacher, d'après leurs rapports avec telle ou telle partie du corps de l'hémisphère, tantôt enfin pour signaler leur valeur plus ou moins grande au point de vue de l'anatomie comparée (1). Leur donner une nouvelle acception serait introduire dans le langage une complication et une confusion fâcheuses. Faut-il donc recourir au néologisme? Cela n'est nullement nécessaire, et il vaut bien mieux convenir de donner une signification particulière aux trois mots scissure, sillon et incisure, qui sont déjà usités, mais dont l'acception n'est pas encore fixée.

J'appellerai donc scissures les anfractuosités qui séparent les lobes, sillons, celles qui séparent les circonvolutions de chaque lobe, et incisures, celles qui subdivisent ou compliquent une circonvolution.

Quoique les mots scissure et sillon aient été bien souvent et

(1) Ces dénominations des sillons primaires, secondaires et tertiaires sont en rapport avec la distinction établie par quelques auteurs entre les circonvolutionz primaires, secondaires et terliaires, classification purement théorique, et qui varie énormément suivant les idées de chaque auteur. La classificalion analomique est la seule qui réponde aux besoins d'une description claire et précise. 
soient bien souvent encore employés comme synonymes, on peut remarquer chez beaucoup d'auteurs une tendance à préférer le premier, lorsqu'ils veulent désigner une anfractuosité grande et profonde. C'est qu'en effet le mot scissure, d'après son étymologie, fait naître l'idée d'une division plus complète et plus importante. Ainsi la scissure de Sylvius, qui est la plus vaste de toutes, n’a jamais été appelée un sillon, pas même par les auteurs qui ne voient que des sillons dans le reste du cerveau. L'acception précise que je propose de donner désormais à ces deux mots est donc conforme à l'importance respective des deux espèces d'anfractuosités qu'ils désignent. Il est bien entendu que cette importance n'est pas déterminée par la profondeur ou par la longueur des anfractuosités, mais par la nature des divisions qu'elles établissent, les unes entre deux lobes, les autres seulement entre deux circonvolutions, car certains sillons peuvent être plus longs et même plus profonds que certaines scissures.

A. Scissures. - D'après celte nomenclature, nous devons ranger parmi les scissures:

$1^{\circ}$ La scissure de Sylvius, qui sépare le lobe temporal des lobes frontal et pariétal;

$2^{\circ}$ La scissure de Rolando, qui sépare sur la face convexe de l'hémisphère le lobe frontal du lobe pariétal ;

$3^{\circ}$ La scissure occipitale, qui sépare le lobe occipital du lobe pariétal; elle se compose de deux parties, l'une interne, l'autre externe;

$4^{\circ}$ La scissure sous-frontale qui, sur la face interne de l'hémisphère, établit la limite inférieure et postérieure du lobe frontal, et qui fait partie dela grande scissure limbique des mammifères inférieurs aux primates.

$5^{\circ}$ A ces quatre dernières scissures, on peut en ajouter une cinquième, la scissure calcarine, ou du petit hippocampe, qui, rigoureusement parlant, ne serait qu'un sillon, subdivisant le lobe occipital, mais qu'il vaut mieux ranger parmi les scissures pour des raisons qui seront exposées plus loin.

Je crois devoir joindre à cette énumération quelques remarques sur la nature et les dénominations particulières de ces diverses scissures. 
$1^{0}$ Scissure de Sylvinus. - Le nom de fissure (grande fissure de Sylvius) qu'on lui donnait autrefois en France a fait place chez nous à celui de scissure; il s'est maintenu en Angleterre (où on l'applique d'ailleurs indistinctement à tous les sillons). Ces deux mots se ressemblent trop pour qu'on puisse y attacher des idées différentes; on n'en doit done conserver qu'un seul dans une même langue, et lorsqu'on les rencontrera dans des langues différentes, ils ne donneront lieu à aucune ambiguïté.

Mais quelques auteurs ont substitué à ce nom de scissure (ou de fissure) de Sylvius, celui de fosse (fossa Sylvii, sylvische Grube). C'est une complication inutile et une dénomination inexacte. Le nom de fosse, qui indique une excavation à larše ouverture, a été employé d'abord en embryologie, pour désigner la dépression triangulaire qui, chez les foetus humains de cinq à six mois, représente la future scissure de Sylvius ; par suite du développement des circonvolutions, le bord supérieur et le bord inférieur de cette fosse se rapproclient peu à peu l'un de l'autre, et, lorsqu'ils viennent à se toucher, la fosse de Sylvius devient la scissure de Sylvius. Ges deux noms, par conséquent, ne sont nullement synonymes, puisqu'ils indiquent deux formes absolument différentes, correspondant à deux phases de développement.

$2^{\circ}$ Scissure de Rolando. - Elle a été appelée jusqu'ici plus souvent sillon que scissure, non-seulement par lez auteurs qui ne se préoccupent pas de la valeur de ces deux noms, mais encore par ceux qui, sans y attacher l'acception anatomique que je leur donne, emploient le mot scissure pour désigner les anfractuosités les plus importantes, et le mot sillon pour désigner les autres. Or, même ì ce dernier point de vue, la scissure de Rolando ne peut pas être laissée parmi les sillons, car elle a une importance de premier ordre. C'est seulement depuis qu'on la connaît qu'on a pu débrouiller le chaos des circonvolutions. Leuret, qui le premier en a reconnu la signification, lui a donné le nom de l'anatomiste italien qui en avait reconnu la fixité (1),

(1) Rolando n'a pas décrit la scissure même, mais, ce qui revient à peu près au même, il a décrit les deux circonvolutions ascendantes qui la longent, et dont il a constaté la fixité. J'ai dit ailleurs que Rolando dans son Mémoire de 1829 avait à deux reprises rapporté à Vicq d'Azyr la découverte des deux cir- 
et ce nom, il est juste de le conserver. Puisque le nom de Sylvius reste attaché à la scissure qu'il a découverte (quoiqu'il l'ait assez mal connue), pourquoi voudrait-on débaptiser la scissure de Rolando? Ce nom, qui a l'avantage de fournir dans les descriptions l'adjectif rolandique, et ses dérivés pré et postrolandique, est plus commode et plus court que celui de scissure fronto-pariétale, qu'on cherche à lui substituer, et il est plus clair, car il y a, sur la fa ze interne de l'hémisphère, une seconde scissure fronto-pariétale qui sépare le lobule ovalaire (frontal) du lobule quadrilatère (pariétal). Quant au nom de sillon central, introduit par Huschke, et qui est très usité aujourd'hui en Allemagne, il indique une position qui, il est vrai, n'est pas très éloignée d'être centrale chez les primates, mais qui est beaucoup plus antérieure chez les autres mammifères à circonvolutions, par suite de l'extrême petitesse du lobe frontal de ces animaux. Le nom de sillon central est donc très défectueux; il est, de plus, très trompeur, car il n'a pas peu contribué à faire croire que ce sillon, ou plutôt cette scissure, n'existait que chez les primates, tandis qu'en la cherchant là où elle est chez les autres mammifères, c'est-à-dire tout près de l'extrémité antérieure de l'hémisphère, on la trouve constamment sur tous les cerveaux à circonvolutions.

$3^{\circ}$ Scissure occipitale. - La scissure occipitale interne et la scissure occipitale externe n'en font, à vrai dire, qu'une seule, car chez la plupart des singes, par suite de la position profonde

convolutions ascendantes, mais que cette découverte, consignée dans l'édition originale de Vicq d'Azyr (1786), avait été, après sa mort, sottement supprimée par l'éditeur de ses OEuvres complètes (1805). Leuret (1839), trompé par cette édition prétendue complète, crut que les citations de Rolando étaient inexactes et que c'était Rolando lui-même qui avait découvert les deux circonvolutions ascendantes (voir ma note communiquée à l'Académie de médecine dans le Bulletin de l'Académie de médecine, 8 avril 1876, p. 830 et suiv., et dans ce volume, p. 556 et suiv.). C'est donc par hasard que le nom de Rolando a été donné à la scissure qui sépare ces deux circonvolutions; mais le hasard cette fois n'a pas été injuste, car l'œuvre de Rolando, en ce qui concerne les circonvolutions, est très supérieure à celle de Vicq d'Azyr. Rolando est le premier anatomiste qui ait eu la notion de la fixité des circonvolutions cérébrales en général, et qui ait essayé de les soumettre à une description méthodique. De tous les prédécesseurs de Leuret et de Gratiolet, c'est celui qui mérite le plus d'éloges, et il est juste que le nom de cet homme éminent reste inscrit d'une marière ineffaçable dans l'histoire des circonvolutions cérébrales. 
du premier pli de passage, elles sont en continuité l'une avec l'autre; cette continuité s'observe même tantôt d'un seul côté, tantôt des deux côtés à la fois, sur certains cerveaux humains peu riches en circonvolutions; il est bon néanmoins, pour la commodité des descriptions, de distinguer les deux parties de la scissure occipitale par les noms d'interne et d'externe. Gratiolet les appelait perpendiculaires parce qu'elles sont souvent (mais pas toujours, cependant) perpendiculaires à l'axe longitudinal de l'hémisphère; mais le nom de scissures occipitales, qui fait connaître leur rapport avec le lobe occipital, est plus topique et a généralement prévalu. Celui de scissures pariéto-occipitales, adopté par plusieurs auteurs, est bon également, mais il est moins commode parce qu'il est plus long.

$4^{\circ}$ Scissure sous-frontale. - Sur la face interne de l'hémisphère, la grande circonvolution du corps calleux est longée, dans la plus grande partie de son étendue, par une longue anfractuosité qui la sépare du lobe frontal chez les primates, et du lobe pariétal chez les autres mammifères. Cette circonvolution, la plus grande et la plus fixe de toutes, commence au-dessous du genou du corps calleux, s'étend au-dessus de ce corps et se recourbe derrière son bourrelet pour se continuer avec la circonvolution de l'hippocampe ou dernière circonvolution du lobe temporal. Elle se met ainsi successivement en rapport avec tous les lobes de la surface de l'hémisphère et n'appartient par conséquent à aucun d'eux (1). La grande anfractuositélongitudinale qui la sépare du lobe frontal établit donc, sur la face interne de l'hémisphère, la limite du lobe frontal. En outre, à sa partie postérieure, elle se relève vers le bord sagittal de l'hémisphère, de manière à séparer le lobule ovalaire (paracentral) qui appartient au lobe frontal, du lobule quadrilatère qui appartient au lobe pariétal. Ces rapports nous montrent déjà que cette anfractuosité doit

(1) La circonvolution du corps calleux, d'après le sens que l'on donne aujourd'hui au mot lobe, forme en réalité dans le manteau de l'hémisphère un lobe spécial, que l'on peut appeler le lobe du corps calleux, ou encore le lobe limbique, parce qu'il forme la plus grande parlie du limbe de l'hémisphère, et parce qu'il représente seul chez les primates le grand lobe limbique des mammifères moins élevés. Chez les primates, en effet, les autres partios de ce lobe s'effacent par atrophie ou par fusion, 
être considéróe comme une vraie scissure et non pas comme un simple sillon. Mais l'anatomie comparée rend cette conclusion bien plus nécessaire encore, en nous montrant que la scissure sous-frontale représente chez les primates l'arc supérieur de la scissure limbique. Ce n'est pas ici le lieu d'exposer l'histoire de l'immense circonvolution limbique ou grand lobe limbique dont la circonvolution du corps calleux forme l'arc supérieur; elle constitue chez les mammifères autres que les primates, une division de l'hémisphère beaucoup plus distincte et beaucoup plus importante qu'aucune de celles que nous appelons lohes. L'anfractuosité qui l'entoure et qui la sépare du reste de l'hémisphère est donc par excellence une scissure. La scissure limbique cst formée de deux ares, l'un supérieur, l'autre inférieur. Le supérieur, qui seul nous intéresse ici, longe le bord supérieur de la circonvolution du corps calleux; il établit la séparation entre cette circonvolution et le lobe pariétal, qui la recouvre presque entièrement, et mérite ainsi le nom de scissure sous-pariétale. Voilà ce que l'on observe chez tous les mammifères inférieurs aux primates; mais chez les primates le lobe frontal devient très grand et refoule le lobe pariétal en arrière ; c'est lui maintenant qui recouvre la plus grande partie -de la circonvolution du corps calleux, et l'arc supérieur de la scissure limbique se trouvant ainsi placé au-dessous de ce lobe, dont il établit la limite sur la face interne de l'hémisphère, mérite le nom de scissure sousfrontale. Par la seule opposition des mots scissure sous-pariétale et scissure sous-frontale, on exprime le plus important des caractères qui distinguent le type cérébral des primates de celui de tous les autres mammifères. Je ne puis insister plus longtemps sur l'évolution de la circonvolution limbique (1); ce que j’en ai dit suffit pour prouver que la scissure sous-frontale des primates, commela sous-pariétale des mammifères moins élevés, n'est pas seulement un sillon, mais une véritable scissure.

La scissure sous-frontale a été appelée par Gratiolet le grand sillon du lobe fronto-puriétal; par Huxley, la scissure (fissure) calloso-marginale; par Pansch, le sillon fronto-pariétal interne. Ces trois noms sont bien plus difficiles à manier que celui que

(1) Bulletins de la Société d'anthropologie, décembre 1877, et dans ce volume, p. 247. 
je propose ; j'ajoute que le premicr et le troisième sont anatomiquement inexacts, et que le second manque de clarté, car il fait naître l'idée d'une scissure placée sur le bord du corps calleux lui-même, et l'on est portó à croire qu'il désigne la raimure du corps calleux.

$5^{\circ}$ Scissure calcarine. - Si l'on appliquait rigoureusement le principe sur lequel repose la distinction des sillons et des scissures, il faudrait ranger parmi les sillons la scissure du petit hippocampe, ou scissure calcarine de Huxley, qui s'observe sur le bord inféro-interne du lobe occipital, et qui sépare très nettement du reste de ce lobe le lobule triangulaire ou cuneus. Je continuerai néanmoins à l'appeler scissure, parce que l'anatomie comparée et l'embryologie lui donnent une valeur égale à celle des scissures proprement dites; le lobule triangulaire, dont elle établit la limite, présente, en cffet, chez les primates, une constance très remarquable, et mériterait, n'était sa petitesse, d'être élevé au rang des lobes.

Le fond de la scissure calcarine correspond chez les primates au petit hippocampe, ou ergot de Morand. Gratiolel, d'après ses observatious sur le cervenu du cercopithecus sabæus, qu'il avait pris pour type, a cru qu'elle se prolongeait sur la face interneou rentriculaire du lobe temporal, en se continuant arec la rainure qui sépare le grand hippocampe du corps godronné, et il l'a appelće la scissure des hippocampes, parce que, d'après lui, olle se trouvait successivement en rapport avec les deux hippocampes, dans sa partie postéricure avec le petit, dans sa partie antérieure avec le grand. Mais ces deux parties sont absolument distinctes et tout à fait indépendantes l'une de l'autre; la partie antérieure ou ventriculaire est d'ailleurs étrangère à la description des circonvolutions proprement dites. Quant ì la partie postćrieure, elle ne doit plus s'appeler que la scissure du petit hippocampe. Mais ce nom, ayant trop de ressemblance avec celui dont s'est servi Gratiolet, pourrait donner lieu à quelque confusion. Je pense donc qu'il est préférable d'adopter la dénomination de scissure calcarine, qui a été proposée par M. Huxley, et qui cxprime la mêrne idée; on sait, en effet, que le petit hippocampe, ou ergot de Morand, porte, dans la nonienclature latine, le nom de calcar avis (de calcar, ergot). 
Je n'ai pas fait figurer au nombre des scissures la célèbre scissure parallèle, de Gratiolet, située entre la première circonvolution temporale et la seconde. Ce n'est qu'un sillon, puisqu'elle sépare seulement deux circonvolutions d'un même lobe. Pour s'écarter le moins possible du langage reçu, on pourrait l'appeler le sillon parallèle; mais cette épithète même est défectueuse, car si, chez l'homme et les anthropoïdes, le sillon est parallèle (ou peu s'en faut) à la scissure de Sylvius, on le voit converger vers elle et la rejoindre chez beaucoup de singes pithéciens et cébiens. Le nom de premier sillon temporal est donc bien préférable.

B. Sillons. - Les sillons, ou anfractuosités de second ordre, se trouvant, par ce qui précède, distingués des scissures, il n'y a plus à les distinguer que des incisures. La distinction est suffisamment établie par notre définition : les sillons sont les anfracluosités qui séparent deux circonvolutions d'un même lobe. Ils sont donc déterminés par l'anatomie des circonvolutions ; mais nous devons nous occuper des règles à suivre dans la dénomination de chaque sillon.

Certains sillons ont reçu des noms spéciatix tirés de leur direction ou de leur forme : tel est le sillon parallèle dont nous venons de parler; tel est encore le sillon droit, dans lequel s'applique la bandelette olfactive. Il est bien vrai que chezl'homme et les anthropoïdes, ce dernier sillon se distingue de tous les autres par ssa rectitude; mais chez les singes moins élevés, d'autres sillons peuvent être rectilignes; ce nom est donc défectueux.

Ge qui caractérise les sillons, ce n'est, ni leur forme ni leur direction, c'est leur position. Ce principe, d'ailleurs, est généralement adopté, et je n'ai signalé les exceptions que pour les faire disparaître. Le nom d'un sillon doit comprendre au moins implicitement l'indication du lobe auquel il appartient; mais ce n'est pas assez, car il y a ordinairement plusieurs sillons dans un même lobe; il faut donc une indication de plus pour désigner complétement un sillon. La désignation la plus usitée et la plus commode est celle qui procède par numéro d'ordre. On sait que la plupart des circonvolutions ont aussi leurs numéros d'ordre, on donne à un sillon le plus faible des deux numéros 
inscrits sur les deux circonvolutions qu'il sépare. Cette règle n'est évidemment pas applicable lorsque les circonvolutions elles-mêmes n'ont pas de numéros d'ordre; ce cas est exceptionnel; j'y reviendrai tout à l'heure, mais je dois d'abord exposer la règle, et puisque la nomenclature des sillons se trouve ainsi rattachée à celles des circonvolutions, c'est par cette dernière que je commencerai.

Toutes les circonvolutions, à l'exception des deux circonvolutions ascendantes, ont une direction plus ou moins longitudinale. Celles d'un même lobe se succèdent donc de dedans en dehors ou de dehors en dedans, suivant que l'on commence par l'un ou l'autre bord de l'hémisphère. Le numéro de la première impliquant ainsi celui des suivantes, il suffit de savoir quelle est la circonvolution qui sera étiquetée comme la première. Ce choix ne peut être fixé que par une convention. Or, un usage déjà accepté par la très grande majoritó des auteurs, permet de formuler la règle suivante : Le numéro 1 doit être inscrit, dans chaque lobe, sur la circonvolution la plus rapprochée du bord supérieur ou sagittal de l'hémisphère, en distance comptée sur la face convexe de l'hémisphère.

Ainsi, dans les lobes frontal, pariétal et occipital, la première circonvolution est celle qui, sur la face convexe, forme le bord sagittal. Dans le lobe temporal, c'est celle qui longe la scissure de Sylvius. Dans le lobe de l'insula, les circonvolutions sont disposées en éventail, et la règle, faite pour les lobes dont les circonvolutions sont à peu près parallèles, ne saurait s'appliquer dans toute sa rigueur. On remarquera toutefois quela circonvolution la plus inférieure, celle qui longe la rigole inférieure de l'insula, est plus éloignée du bord sagittal que celles qui la précèdent; elle doit donc, conformément à la règle, être numérotée comme la dernière, et dès lors le numéro 1 doit être placé sur la circonvolution la plus antérieure qui forme l'autre bord de l'éventail.

Cette nomenclature est admise par la très grande majorité des auteurs contemporains. Quelques-uns toutefois appliquent autrement la règle numérique en comptant les numéros à partir de la scissure de Sylvius. De la sorte, la troisième circonvolution frontale devient la pręmière, et la première devient la troisième. 
C'était ainsi que comptait Huschke, et on pourrait emprunter à l'embryologie, ainsi qu'à l'anatomie comparée, quelques arguments en faveur de ce procédé; mais il n'est pas applicable au lobe occipital. L'autre procédé permet d'adopter une règle fixe; c'est ce qui en fait l'avantage, et c'est ce qui l'a fait prévaloir.

Gratiolet, qui ne se servait pas encore des désignations numériques, nommait les circonvolutions d'un même lobe d'après leur position plus ou moins élevée au-dessus de la base du cerveau. Ainsi il appelait supérieure, moyenne et inférieure, les trois circonvolutions longitudinales du lobe frontal, que nous appelons première, seconde et troisième. Ce procédé serait excellent si le nombre des circonvolutions d'un même lobe ne dépassait jamais le chiffre trois; mais le lobe temporal et le lobe occipital ont plus de trois circonvolutions, de sorte que, pour appliquer sa nomenclature, Gratiolet fut obligé de diviser la surface de ces lobes en une région externe et une région interne; il put ainsi désigner toutes les circonvolutions en ajoutant aux trois épithètes de supérieure, moyenne et inférieure, celles d'interne ou d'externe; mais l'emploi de ces doubles adjectifs était si gênant et si nuisible à la clarté qu'on a été contraint d'y renoncer. Quelques auteurs, il est vrai, continuent encore à désigner les trois circonvolutions longitudinales du lobe frontal sous les noms que leur a donnés Gratiolet, noms qui, dans ce cas particulier, sont à la fois simples et clairs; mais il est plus simple et plus clair de se rattacher à une règle générale, et la nomenclature numérique, qui est seule applicable à tous les lobes, doit recevoir la préférence.

Il reste toutefois des circonvolutions qui ne rentrent pas dans cette nomenclature, attendu qu'elles ne sont pas longitudinales, mais plus ou moins transversales; ce sont les deux circonvolutions ascendantes (Gratiolet) qui longent la scissure de Rolando. L'antérieure appartient au lobe frontal, la postérieure au lobe pariétal. La première a reçu quelquefois le nom de quatrième circonvolution frontale, et la seconde celui de première (ou de troisième) circonvolution pariétale; mais les chiffres ici sont tout à fait trompeurs, parce qu'ils font croire que les circonvolutions se succèdent dans le même ordre que les chiffres, que la 
quatrième frontale par exemple, est placée en dehors ou au-dessous de la troisième, comme celle-ci l'est par rapport à la seconde, et la seconde par rapport à la première. Les deux circonvolutions ascendantes ont encore été désignées sous le nom de frontales transverses, mais leur direction s'éloigne ordinairement beaucoup de la direction transversale ; l'épithète d'ascendante est donc plus exacte. Huschke les avait appelées les deux circonvolutions centrales, l'une centrale antérieure, l'autre centrale postérieure, séparées par la scissure de Rolando, qu'il nommait le sillon central. Plusieurs auteurs allemands donnent encore la préférence à ces dénominations (1) dont j’ai déjà signalé l'inexactitude à l'occasion de la scissure de Rolando. Si les noms de circonvolutions ascendantes, auxquelles on est obligé d'ajouter l'épithète d'antérieure et de postérieure, ou de frontale et de pariétale paraissaient trop longs, je proposerais de les remplacer par ceux de circonvolution prérolandique et de circonvolution postrolandique, qui sont plus courts et qui sont parfaitement clairs, puisque tout le monde est obligé de connaître la scissure de Rolando avant de passer à l'étude des circonvolutions.

La nomenclature ordinaire des sillons découle d'une manière très simple de celle des circonvolutions.

Parlons d'abord des sillons longitudinaux : il est clair que leur nombre, dans chaque lobe, est inférieur d'une unité au nombre des circonvolutions longitudinales. Celles-ci ayant déjà leurs numéros, on donne à chaque sillon le nom de la circonvolution adjacente dont le numéro est le plus faible.

Ainsi, il y a trois circonvolutions frontales, et par conséquent deux sillons frontaux; le premier sillon frontal est compris entre la première et la deuxième circonvolution, le second entre la deuxième et la troisième.

En se réfléchissant pour passer sur la face inférieure du lobe

(1) M. Bischoff admet même l'existence d'un lobe central formé par l'ensemble des deux circonvolutions centrales. Je ne crois pas devoir discuter cette manière de voir, qui a peu de partisans, mais je ferai remarquer en tout cas que le nom de lobe central est de nature à introduire une confusion dans le langage, ce nom ayant déjà élé appliqué par beaucoup d'auteurs au lobe de i’insula, dont la position d'ailleurs est beaucoup plus centrale. 
frontal, les trois circonvolutions frontales prennent le nom de circonvolutions orbitaires $\left(1^{\mathrm{re}}, 2^{\circ}\right.$ et $\left.3^{\mathrm{e}}\right)$, et de même on appelle sillons orbitaires $\left(1^{\mathrm{er}}\right.$ et $2^{\mathrm{c}}$ ) la partic orbitaire des deux sillons frontaux.

Dans le lobe pariétal, où il n'y a que deux circonvolutions longitudinales ( $1^{\text {re }}$ et $2^{\circ}$ pariétale), il n'y a qu'un seul sillon longitudinal, qui, dès lors, n'a pas besoin de numéro d'ordre. M. Turner l'a appelé le sillon intra-pariétal; mais le nom de sillon pariétal est aussi clair, plus court et plus conforme à la nomenclature.

Le lobe temporal comprend chez l'homme et les anthropoïdes cinq circonvolutions parallèles, dont la première longe la scissure de Sylvius, tandis que la cinquième (circonvolution de l'hippocampe) longe la grande fente cérébrale de Bichat. Il y a donc quatre sillons temporaux, numérotés de 1 à 4 .

Le lobe occipital comprend six circonvolutions, dont la première forme le bord sagittal de ce lobe; la seconde et la troisième lui succèdent sur la face convexe; puis la quatrième et la cinquième sur la face inférieure; la sixième enfin, formant sur la face interne le lobule triangulaire ou cuneus, remonte jusqu'au bord sagittal, où sa base se confond avec la première circonvolution occipitale. Il y a donc cinq sillons occipitaux : les deux premiers sur la face convexe du lobe, le troisième sur son bord externe, le quatrième sur sa face inférieure, et le cinquième enfin sur son bord inféro-interne; mais ce cinquième sillon est celui qui sépare la cinquièrne circonvolution occipitale du lobule du cuneus, c'est celui qui porte le nom de scissure calcarine ou du petit hippocampe; j'ai exposé plus haut les motifs pour lesquels ce cinquième sillon occipital doit être rangé parmi les scissures; il ne reste donc à numéroter que quatre sillons occipitaux.

Ces désignations numériques sont très simples, très commodes et très claires, tant que l'on reste dans le groupe supérieur formé par l'homme et les grands anthropoïdes. Mais, en descendant dans la série des singes, on voit diminuer le nombre des circonvolutions occipitales, temporales ou même frontales. Les numéros d'ordre se trouvent ainsi changés, ceux des sillons aussi bien que ceux des circonvolutions, Il semble que 
MORPHOLOGIE DU CERVEAU DE L'HOMME ET DES PRIMATES. 6SF

ce soit une objection grave contre la nomenclature numérique; mais aucune nomenclature ne peut avoirla prétention de s'appli. quer sans modificationà des parties dont la constitution présente d'aussi grandes différences. Si l'homme possède plus de circonvolutions que les singes inférieurs, ce n'est pas parce qu'il s'estproduit chez lui des circonvolutions réellement nouvelles, mais parce que certaines parties qui, chez ces singes, ne forment qu'une seule circonvolution, se sont divisées de manière à en former plusieurs, de sorte qu'aucune de celles-ci ne représente celle-là, et qu'aucun de leurs noms ne lui est applicable. Tout ce que l'on peut exiger, c'est que la nomenclature permette de signaler aisément cette correspondance de parties analogues, mais diversement constituées, et la nomenclature numérique, grâce à sa concision, répond très bien à cette exigence. Elle possède d'ailleurs l'avantage sans rival de se prêter à une notation trèssimple et très claire, qui abrège singulièrement le discours, et qui est précieuse surtout pour rendre les dessins intelligibles, sans qu'on soit obligé de recourir continuellement à l'explication des planches. Cette notation, déjà très usitée, sera exposée plus loin.

Je n'ai parlé jusqu'ici que des sillons longitudinaux; quant à ceux qui ont une direction plus ou moins transversale, ils sont seulement au nombre de deux. Ils sont à peu près parallèles à la scissure de Rolando: l'un limite en avant la circonvolution frontale ascendante ou prérolandique, l'autre limite en arrière la pariétale ascendante ou postrolandique. Je les désigne sous les noms de sillon prérolandique et postrolandique. (lls sont nommés sillon précenträl et postcentral par les auteurs qui appellent scissure centrale la scissure de Rolando.)

C. Incisures. - Les incisures ou anfractuosités de troisième ordre, c'est-à-dire les anfractuosités qui compliquent ou subdivisent les circonvolutions, doivent être réparties en deux groupes : celles qui correspondent aux plis d'inflexion et celles qui séparent les plis de subdivision.

Les premières sont celles qui pénètrent dans les méandres d'une circonvolution; elles émanent de la scissure ou du sillon qui la limitent; elles sont donc en continuité avec cette scissure ou ce sillon, et dès lors je les appelle incisures continues. 
Je dis, par exemple : il y a en tel point une incisure continue avec tel sillon ou telle scissure.

Les incisures de subdivision sont appelées par opposition les incisures isolées.

Certaines incisures sont à peu près constantes, au moins dans la même espèce animale, et doivent trouver place dans la description générale du cerveau de l'espèce; j'y reviendrai tout à l'heure. Mais la plupart sont très variables; elles le sont surtout chez l'homme et les anthropoïdes, non seulement d'individu à individu, mais même de droite à gauche. G'est d'elles que dépendent principalement les variétés individuelies et les différences qui existent entre deux espèces voisines ou entre deux genres voisins. Il faut donc leur accorder beaucoup d'attention dans les descriptions particulières. A cet effet, il est bon de les caractériser par des épithètes indiquant leur direction, leur longueur, leur degré de profondeur superficielles et profondes), et enfin leur forme. Certaines incisures isolées sont dites bifurquées, à trois branches, à quatre branches. Le sons de ces termes descriptifs n'a pas besoin d'être fixé. Mais il y a une forme, ou, si l'on veut, un degré qui mérite un nom spécial. On trouve souvent sur le milieu de la largeur d'une circonvolution, une dépression plus large que profonde, sur laquelle la pie-mère s'applique sans se plisser, sans s'adosser à elle-mêmc. Ces dépressions appartiennent à la catégorie des incisures isolées; elles en sont en quelque sorte le premier degré ; ce degré est très bien indiqué par le nom de fossettes.

Les incisures variables - et c'est le plus grand nombre ne peuvent pas recevoir de dénominations particulières. On les désigne, en décrivant le cerveau, d'après leur position et lcurs connexions. Mais il en est quelques-unes qui se retrouvent constamment dans une même espèce ou même dans toutes les espèces d'un même genre, ou d'une même famille, ou d'un même ordre zoologique; elles acquièrent ainsi une importance presque égale à celle des sillons, et il est nécessaire, par conséquent, de leur donner des noms spéciaux.

Celles qui me paraissent mériter cet honneur sont les suivantes :

$1^{\circ}$ L'incisure en II. - Chez tous les primates, la face infé- 
rieure ou orbitaire de la deuxième circonvolution frontale, qui occupe presque toute la largeur du lobule orbitaire, présente un certain nombre d'incisures qui sont de la nature des incisures isolées, parce qu'elles ne communiquent pas avec les sillons voisins, mais qui communiquent le plus souvent entre elles de manière à dessiner la forme d'un $\mathrm{H}$, ou d'un $\mathrm{X}$, ou d'un $\mathrm{K}$; on les a désignées quelquefois sous le nom de sillon crucial, qui doit être rejeté, parce qu'il a déjà une autre acception toute différente, très connue dans la description du cerveau des carnassiers et d'un grand nombre de ruminants, de pachydermes, etc. Le nom de sillon en $I I$ est beaucoup plus usité et c'est celui que nous adoptons, à cela près que cette anfractuosité, ne faisant que subdiviser une circonvolution, doit être rangée parmi les incisures et non parmi les sillons. Le nom d'incisures sous-orbitaires serait peut-être préférable, car il y a quelquefois plus de deux incisures longitudinales; en outre, la branche transversale de l'H fait quelquefois défaut. Mais nous nous conformerons à l'usage.

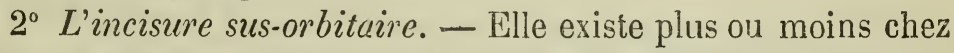
tous les primates. Elle se voit sur la face interne de l'hémisphère, où elle établit la démarcation entre le lobule orbitaire du lobe frontal et l'étage supérieur de ce lobe. En arrière, elle commence sous l'origine de la circonvolution du corps calleux ; de là elle s'étend horizontalement en avant jusqu'à l'extrémité antérieur du lobe frontal, de manière à atteindre et quelquefois même à dépasser le bord convexe de la face interne de l'hémisphère. Sa partie supérieure se confond souvent et même le plus souvent avec la portion inférieure ou réfléchie de la scissure sousfrontale. Sa partie antérieure se trouve alors en continuité directe avec cette scissure, qui semble ainsi se prolonger jusque surle bord de l'hémisphère; mais quelquefois aussi l'incisure sus-orbitaire reste isolée dans toute son étendue. Ces diversités de la disposition de l'incisure sus-orbitaire tiennent en grande partie à la situation superficielle ou profonde du pli de passage qui se détache de la circonvolution du corps calleux au-devant du genou de ce corps, et qui se jette dans la partie adjacente de la face interne de la première circonvolution frontale.

Au-dessous de cette incisure sus-orbitaire, on en trouve chez 
l'homme et les anthropoïdes une seconde qui lui est parallèle, et qui subdivise la face interne de la première circonvolution orbitaire. Il y en a même quelquefois une troisième chez l'homme. Les seconde et troisième incisures sus-orbitaires peuvent être appelées inférieures. Ce sont des incisures isolées. Elles ne sont qu'accessoires. Le nom d'incisure sus-orlitaire au singulier et sans épithète, désignera suffisamment la supérieure, qui est commune à tous les primates.

$3^{\circ}$ Les incisures sous-frontales. - Ce sont les incisures plus ou moins verticales qui, sur la face interne de l'hémisphère, se détachent de la scissure sous-frontale en pénétrant de bas en haut dans la face interne de la première circonvolution frontale. Elles s'étendent souvent chez l'homme jusqu'au bord sagittal de l'hémisphère. Il y en a toujours une, et souvent deux, en avant et au-dessous du genou du cnrps calleux. La plus importante est celle que j'appelle préovalaire, parce qu'elle établit la limite antérieure du lobule ovalaire (Pozzi) ou lobule paracentral (Meynert). Ge lobule est formé par l'épanouissement de la circonvolution frontale ascendante sur la face interne de l'hémisphère. L'incisure préovalaire est, située au-dessous de la racine de la première circonvolution ; ce qui est en avant appartient ä la première circonvolution frontale; ce qui est en arrière appartient à la frontale ascendante. L'incisure préovalaire communique ordinairement avec la scissure sous-frontale; quelquefois cependant elle en est séparée par un pli d'anastomose superficiel.

$4^{\circ}$ Incisures pariétales. - Ce sont des incisures qui naissent du bord supérieur de la scissure de Sylvius en arrière de la scissure de Rolando, et qui pénètrent plus ou moins profondément dans le lobe pariétal, tantôt dans le bord inférieur de la deuxième circonvolutión pariétale, tantôt dans sa racine ou dans la partie inférieure de la circonvolution pariétale ascendante. Ce sont les prolongements des anfractuosités qui limitent, dans le fond de la scissure de Sylvius, en arrière de l'insula, le pli de passage profond temporo-pariétal. Chez l'homme, il y a toujours au moins une incisure pariétale, ordinairement deux, quelquefois trois; chez l'orang et le chimpanzé, il y en a au moins une, souvent deux; chez le gorille, il n'y en a qu'une. On 
MORPHOLOGIE DU CERVEAU DE L'HOMME ET DES PRIMATES. 689 ne la retrouve qu'exceptionnellement, et à l'état rudimentaire, chez les gibbons et chez les pithéciens.

\section{§ כ. AUTRES ANFraCtUOSITÉS CÉRÉBRALES.}

Les scissures, les sillons et les incisures sont les trois espèces d'anfractuosités qui se répètent plusieurs fois dans l'hémisphère avec des caractères déterminés, et qui se prêtent par conséquent à une nomenclature générale. Mais il y a d'autres séparations d'une nature ou d'une forme plus spéciale, et qui doivent recevoir des dénominations particulières.

Ce sont : $1^{\circ} \mathrm{La}$ grande fente inter-hémisphérique danslaquelle pénètre la grande faux de la dure-mère. On la nomme généralement la scissure inter-hémisphérique, mais le mot scissure, désignant les anfractuosités qui séparent' les lobes de l'hémisphère, ne peut désigner en même temps celle qui sépare les hémisphères eux-mêmes.

$2^{\circ}$ La grande fente cérébrale de Bichat qui, après l'ablation des membranes, fait communiquer avec l'extérieur du cerveau le ventricule moyen et les cornes inférieures des ventricules lațéraux. Elle ne subdivise pas le manteau; elle le sépare des tubercules quadrijumeaux et de la partie supérieure des pédoncules; elle s'étend sans interruption d'un hémisphère à l'autre ; quoiqu'elle soit entièrement différente de la précédente, elle est, comme elle, commune aux deux hémisphères. Ces points d'analogie légitiment l'analogie des noms sous lesquels nous les désignons.

Les auteurs allemands emploient souvent le mot fente (Spalte) pour désigner chez les singes l'ensemble des deux scissures occipitales interne et externe qui, se continuant l'une avec l'autre, détachent très nettement le lobe occipital sur la face interne et la face convexe de l'hémisphère (Affenspalte, fente des singes). Mais d'une part cette fente n'existe pas chez tous les singes, le premier pli de passage étant superficiel chez l'orang, le gorille et même chez un assez bon nombre de chimpanzés; d'une autre part, les scissures occipitales ne diffèrent en rien des autres scissures ; elles établissent comme elles une séparation lobaire, 
rien de plus, rien de moins; elles doivent par conséquent recevoir une désignation commune dans une nomenclature régulière.

$3^{\circ}$ La rainure du corps calleux. - C'est l'anfractuosité qui longe la face convexe du corps calleux, et qui la sépare de la face inférieure ou concave de la circonvolution du corps calleux. Elle formel'arc supérieur du limbe de l'hémisphère. On l'a appelée le sinus, le sillon, la scissure, le ventricule du corps calleux, le sillon battard (Foville). Ce n'est pas un ventricule, ce n'est ni un sillon ni une scissure, puisque le corps adjacent n'est ni un lobe ni une circonvolution et n'appartient pas même au manteau. Le nom de sinus est trop vague; il n'est d'ailleurs nullement approprié à la forme d'une anfractuosité linéaire et qui n'est nullement sinueuse. Le nom de . vinure a l'avantage d'exprimer exactement cette forme, et de distinguer nettement cette anfractuosité de toutes celles qui apparaissent à la surface du manteau.

$4^{\circ}$ La rainure du grand hippocampe. - C'est l'anfractuosité longue, étroite et profonde que l'on aperçoit sur le bord inférieur de la fente de Bichat, entre le corps godronné et le grand hippocampe. C'est celle que Gratiolet considérait comme le prolongement de la scissure calcarine, réunissant ainsi en une seule scissure, qu'il nommaitscissure des hippocampes, deux anfractuosités entièrement distinctes et de nature différente.

$5^{\circ}$ La fosse de Sylvius. - On décrit sous ce nom, dans l'histoire du développement du cerveau des primates, une dépression triangulaire, large et relativement peu profonde, et dont les bords, en se rapprochant plus tard, formeront la scissure de Sylvius. J'ai déjà dit que, par une extension fâcheuse, on avait appliqué ce nom à la scissure de Sylvius elle-même. On l'a appliqué aussi, d'une manière tout aussi fâcheuse, à la vallée de Sylvius, dont nous allons parler.

$6^{\circ}$ La vallée de Sylvius. - La grande anfrastuosité décrite pour la première fois par François Deleboe, dit Sylvius, se compose de deux parties essentiellement distinctes, quoique continues l'une avec l'autre chez les primates: l'une transversale et largement ouverte, qui commence à la base du cerveau, en dehors du chiasma des nerfs optiques et qui correspond à l'espace perforé, entre le lobule orbitaire et le lobe temporal, - l'autre 
refermée et située sur la face latérale de l'hémisphère, entre le lobe temporal d'une part, le lobe frontal et le lobe pariétal d'autre part. La première partie est désignée par beaucoup d'auteurs sous le nom de vallée de Sylvius, que nous adoptons. La seconde partie lui succède au point appelé par Gratiolet le coude de la scissure de Sylvius, au niveau du bord falciforme qui s'étend du lobe temporal au lobule orbitaire et qui sépare l'espace perforé de la région de l'insula; elle se divise chez l'homme et les anthropoïdes en deux ou trois branches dont il sera question plus loin; on a donc comparé la vallée de Sylvius à un tronc, dont l'extrémité externe se diviserait en branches, et de là est venu le nom de tronc (Stamm) de la scissure de Sylvius donné à la vallée de Sylvius par M. Bischoff et quelques auteurs allemands. Ce nom me semble défectueux, parce qu'il établit entre la vallée de Sylvius et la grande scissure latérale de l'hémisphère une solidarité que l'anatomie comparée repousse. Ces deux anfractuosités sont mises en communication chez les primates, les amphibies et les cétacés, par suite de l'atrophie du lobe olfactif et du lobe de l'hippocampe; mais elles sont de nature absolument différente. La première existe plus ou moins chez tous les mammifères, la seconde n'existe que chez ceux qui ont des circonvolutions. La première est largement ouverte, la seconde est refermée par les circonvolutions qui se pressent sur ses bords et présente seule les caractères d'une scissure. Je propose donc résolument de restreindre le nom de scissure de Sylvius à la partie latérale de la grande anfractuosité sylvienne, dont la partie transversale portera le nom de vallée de Sylvius, où le mot trompeur de scissure ne figure pas.

Chez les animaux qui ont un lobe olfactif, c'est-à-dire chez la grande majorité des mammifères, ce lobe, situé en avant du lobe de l'hippocampe avec lequel il se continue, en est séparé cependant par une dépression transversale large et superficielle qui n'est qu'une légère ondulation: c'est la vallée de Sylvius. Lorsqu'il existe en outre des circonvolutions, celles-ci se forment par suite d'un plissement auquel le grand lobe limbique (dont le lobe olfactif et le lobe de l'hippocampe font partie) reste tout à fait étranger; le plissement dans le sens longitudinal donne les circonvolutions, et le plissement de celles-ci dans le 
sens transversal donne la scissure de Sylvius. Cette scissure profonde s'arrêle brusquement sur le bord externe du grand lobe limbique et ne communique par conséquent pas avec la vallée de Sylvius. La nécessité de distinguer par des substantifs différents deux choses aussi évidemment distinctes est d'autant plus grande qu'aucun auteur n'a songé à les confondre. Ainsi on dit généralement que, chez les mammifères qui ont un lobe olfactif, la scissure de Sylvius n'est que latérale, qu'elle ne s'étend pas jusqu'au chiasma, qu'elle n'a pas de portion transversale; on ajoute, il est vrai, que chez les primates elle se prolonge transversalement à la base du cerveau jusqu'au chiasma, mais pour cela il faut $\mathrm{y}$ joindre une chose que, chez les autres animaux, on considère comme lui étant étrangère. Tant que le lobe olfactif persiste, sa base : $t$ unie au lobe de l'hippocampe par une couche de substance cérébrale épaisse quoique déprimée ; lorsqu'il s'atrophie, cette substance s'amincit au point de disparaître presque entièrement; il n'en reste plus que la mince lanıe grise de l'espace perforé de Vicq d'Azyr, et alors la vallée de Sylvius devient assez profonde pour se mettre en communication avec la scissure de Sylvius; mais ces deux anfractuosités n'en sont pas moins indépendantes l'une de l'autre ; elles résultent de deux évolutions entièrement différentes, et il est nécessaire qu'elles soient distinguées dans le langage comme elles le sont dans la réalité. J'appelle donc scissure de Sylvius celle qui est une vraie scissure résultant, comme toutes les autres, de l'accroissement et du plissement du manteau, et vallée de Sylvius celle qui est au contraire une dépression véritable, produite par atrophie.

$7^{\circ}$ Les branches de la scissure de Sylvius. - La vraie scissure de Sylvius, telle que nous l'avons définie plus haut, est simple, c'est-à-dire réduite à ure seule branche, chez tous les animaux autres que l'homme et les anthropoïdes. S'il arrive parfois qu'une autre scissure ou un sillon voisin semble se mettre en communication avec elle, on peut reconnaître avec un peu d'attention que cette communication n'est que superficielle, que le bord de la scissure n'est pas interrompu dans sa partie profonde.

Chez l'homme et les anthropoïdes, les communications superficielles s'observent quelquefois, mais de plus il se produit, sur 
le bord fronto-pariétal de la scissure, des incisures qui en occupent toute l'épaisseur. Sur la partie pariétale de ce bord, les incisures sont assez variables; elles ont peu d'importance, et il suffit de les nommer incisures pariétales; mais sur la partie frontale il en est tantôt une, tantôt deux qui sont beaucoup mieux caractérisées, et qui méritent le nom de branches antérieures de la scissure de Sylvius.

L'une d'elles est constante: c'est celle que j'ai décrite sous le nom de branche horizontale antérieure de la scissure de Sylvius. Elle existe chez tous les hommes, chez tous les anthropoïdes, et probablement chez eux seuls. L'autre, située au-dessus et en arrière, a été décrite depuis longtemps sous le nom de branche ascendante de la scissure de Sylvius. Elle est à peu près constante chez l'homme, et se retrouve fréquemment chez l'orang et le chimpanzé. Le nom de branche ascendante lui convient très bien, mais non celui de branche antérieure, qu'on lui donne souvent, puisqu'il y a deux branches antérieures et non une seule. Lorsque ces deux branches existent, elles interceptent dans la troisième circonvolution frontale une espèce de petit lobule disposé en forme de cap (cap de la troisième circonvolution frontale). Je renvoie pour plus de détails aux faits que j'ai exposés dans mon ménıoire sur le cerveau du gorille (Revue d'anthrop., 1878, p. 20, et dans ce volume, p. 601).

Quant à la scissure principale, qui s'étend au loin sur la face latérale de l'hémisphère en longeant le bord supérieur du lobe temporal, on l'a nommée jusqu'ici la branche postérieure de la scissure de Sylvius : ce nom, usité avant que l'on eût étudié et dénommé les branches antérieures, était opposé à celui de branche transversale, sous lequel on désignait la vallée de Sylvius.

La nomenclature des branches de la scissure de Sylvius est donc assez compliquée, mais on peut la simplifier beaucoup si l'on cesse de confondre la vallée de Sylvius avec la scissure de Sylvius. Alors la branche postérieure, qui est très grande, qui est constante non seulement chez tous les primates, mais chez tous les mammifères à circonvolutions, s'appellera purement et simplement la scissure de Sylvius; on ne l'appellera plus branche, puisque ce sera la scissure elle-mẹme, telle qqu'elle existe, à l'état 
de simplicité, chez la plupart des mammifêres; on réservera exclusivement le nom de branches pour les deux branches que je viens d'appeler antérieures, et pour distinguer ces deux branches, il suffira d'appeler l'une la branche antérieure, l'autre la branche ascendante de la scissure de Sylvius.

En résumé, la grande anfractuosité décrite par Sylvius se compose de la vallée de Sylvius et de la scissure de Sylvius. La scissure de Sylvius est la scissure qui longe le bord supérieur du lobe temporal dans toute son étendue.

Cette scissure, dans les cerveaux les plus élevés, émet dans sa partie antérieure, en avant de l'insula, deux branches, l'une antérieure, qui est horizontale, l'autre ascendante. Lorsqu'il n'y en a qu'une seule, c'est l'ascendante qui fait défaut.

$8^{\circ}$ Les rigoles de l'insula. - Le lobe de l'insula de Reil, situé au fond de la scissure de Sylvius, entre les lobes frontal, pariétal et temporal, a été désigné souvent sous le nom de lobe central. Son mode de formation, sa position profonde, ses rapports de continuité avec les trois principaux lobes de l'hémisphère, et surtout ses connexions immédiates avec le noyau extraventriculaire du corps strié, légitimeraient parfaitement ce nom, s'il y avait une raison quelconque pour abandonner celui sous lequel Reil a le premier fait connaître ce lobe. La découverte de Reil est certainement l'une de celles qui ont le plus contribué aux progrès de l'anatomie cérébrale, et il est convenable de maintenir le nom de l'insula de Reil.

La base de l'insula est limitée par une anfractuosité profonde qui l'entoure de toutes parts, et que Reil a appelée la rigole (Rinne), conformément à l'image d'une île entourée d'eau. Plus tard, Burdach a complété cette description, en distinguant dans ce circuit trois parties, l'une supérieure, l'autre inférieure, l'autre antérieure, et il les a distinguées sous le nom de fentes (Spalte); on les a appelées encore fossés, vallécules, sillons. Mais aucun de ces noms ne vaut celui que Reil a employé. Nous devons toutefois, comme l'a fait Burdach avec avantage, diviser la rigole de Reil en trois parties que nous nommerons la rigole supérieure de l'insula, la rigole inférieure et la rigole antérieure.

Je justifie l'emploi de ce nom spécial en faisant remarquer d'abord que les rigoles de l'insula ne peuvent être appelées sil- 
lons puisqu'elles sont interlobaires. Elles ne pourraient donc être nommées que scissures si l'on appliquait rigoureusement la nomenclature. Mais il y aurait ainsi trois scissures dans le fond de la scissure de Sylvius, et ces scissures incluses dans une autre embrouilleraient le discours. On conviendra d'ailleurs que la disposition des parties autour de l'insula de Reil est sans analogue dans le reste du manteau, et qu'il y a tout avantage à constater ce fait par une dénomination particulière.

$9^{\circ}$ Fossettes. - J'ai déjà, en parlant des incisures, défini les fossettes, dépressions plus larges que profondes, qui représentent le premier degré de la formation des incisures isolées. Elles sont tantôt très superficielles, tantôt plus profondes. On passe de la fossette à l'incisure par des transitions insensibles. Le nom d'incisure est applicable lorsque la pie-mère, qui tapisse la dépression, forme un pli en s'adossant à elle-même. On trouve souvent sur un hémisphère une simple fossette là où, sur l'autre hémisphère du mème cerveau, existe une véritable incisure.

$10^{\circ}$ Nervures. - Ce sont des dépressions linéaires et superficielles produites sur la surface des circonvolutions par les vaisseaux les plus gros de la pie-mère. Elles méritent d'être signalées, parce que, sur les dessins, sur les moules non retouchés et sur les cerveaux durcis dans les liquides, elles simulent des incisures et paraissent mettre en communication deux sillons voisins. Souvent, en effet, elles passent d'un sillon à l'autre, suivant une direction à peu près perpendiculaire à celle de la circonvolution intermédiaire, et elles donnent le change d'autant plus aisément qu'elles vont très fréquemment aboutir à l'extrémité d'une incisure véritable. Sur les cerveaux frais, et sur ceux qui ont été dépouillés de leurs membranes avant d'être plongés dans l'alcool, les nervures ne sont que des empreintes légères et ne peuvent tromper personne; mais sur les cerveaux durcis avec leurs membranes, les gros vaisseaux de la pie-mère, se rétractant plus fortement que les circonvolutions subjacentes, creusent des nervures assez profondes, et, pour distinguer celles-ci des incisures, ik faut écarter avec précaution les bords des sillons voisins. Les nervures peuvent être simples ou ramifiées. Le nom que je leur donne est celui dont on se sert pour désigner les divisions de la feuille de figuier, qui sont aussi des empreintes vasculaires. 


\section{$\S 6$. DE LA NOTATION DES CIRCONVOLUTIONS ET DES SILLONS.}

La nomenclature numérique a permis de recourir à des notations très simples pour désigner rapidement les diverses circonvolutions et anfractuosités de l'hémisphère. Ces notations, déjà très commodes dans les descriptions, sont utiles surtout pour donner de la clarté aux dessins. Des figures aussi compliquées que celles du cerveau ne peuvent être bien comprises sans le secours des chiffres ou lettres de renvoi. Mais le passage des renvois au texte explicatif et vice versâ fait perdre beaucoup de temps; il est bien préférable d'inscrire l'explication sur la figure même, en choisissant des lettres significatives au lieu de lettres arbitraires. C'est ce que font maintenant presque tous les auteurs. Quelques détails cependant restent encore à régler.

Les scissures sont désignées par des lettres majuscules.

La lettre $R$, répétée au besoin sur les deux extrémités, indique la scissure de Rolando.

La lettre 0 indique la scissure occipitale. Il n'est pas nécessaire de distinguer par deux lettres différentes la scissure occipitale interne et la scissure occipitale externe, puisqu'on voit au premier coup d'œil quelle est la face de l'hémisphère qui est représentée sur le dessin.

Les lettres $\mathrm{S}, \mathrm{S}^{\prime}, \mathrm{S}^{\prime \prime}$ servent à désigner les diverses parties de la "scissure de Sylvius ). La lettre $\mathrm{S}$ se place dans la vallée de Sylvius, et la lettre $\mathrm{S}^{\prime}$ sur l'extrémité postérieure ou supérieure de la scissure de Sylvius proprement dite (branche postérieure de la prétendue scissure de Sylvius). Ces deux lettres suffisent sur les cerveaux où il n'y a pas de branches antérieures. Lorsque celles-ci existent, on se sert de la lettre $\mathrm{S}^{\prime \prime}$; mais il y a ici une confusion à éviter, puisqu'il y a deux branches antérieures chez l'homme et chez quelques anthropoïdes. Les auteurs, n'en ayant décrit jusqu'ici qu'une seule, l'ont marquée de la lettre S", qu'ils placent sur la branche ascendante lorsqu'il y a deux branches antérieures, et sur la branche antérieure lorsqu'il n'y en a qu'une. Maintenant que la distinction de ces deux branches est établie, l'emploi de deux lettres distinctes est devenu nécessaire, et si je rappelle que la branche antérieure existe chez tous les anthro- 
poïdes comme chez l'homme, tandis que la branche ascendante manque chez les gibbons, chez les gorilles, chez un certain nombre de chimpanzés et d'orangs, et n'est constante (ou à peu près) que chez l'homme, on reconnaîtra que la branche antérieure est la plus essentielle, et que c'est sur elle qu'il convient d'inscrire la lettre $\mathrm{S}^{\prime \prime}$. Quant à la branche ascendante, on peut se dispenser de l'indiquer, puisque, lorsqu'elle existe, elle est toujours facile à retrouver au-dessus de la précédente. Si l'on voulait toutefois la signaler, la lettre italique $s$ serait plus commode que le signe $\mathrm{S}^{\prime \prime \prime}$, trop volumineux pour un si petit espace.

La scissure calcarine, étant maintenue parmi les scissures, doit recevoir aussi une lettre majuscule, qui sera l'initiale K (la lettre $\mathrm{C}$ étant réservée pour la circonvolution du corps calleux). Reste la scissure sous-frontale des primates et sous-pariétale des autres animaux. Toutes deux faisant partie de la scissure limbique, je propose de les désigner sous l'initiale L. Je me suis servi jusqu'ici des doubles lettres SF, sous-frontale, et SP, souspariétale. Mais ces lettres doubles sont gênantes, et le signe commun L a l'avantage de constater l'analogie de ces deux scissures.

Les lettres des scissures font connaître immédiatement les divisions lobaires, sans qu'on soit obligé de se préoccuper de la notation des lobes. Je passe donc à celle des circonvolutions et des sillons.

La notation des scissures ne concerne que les dessins ; celle des circonvolutions et des sillons peut être utilisée aussi dans le langage descriptif, qu'elle abrège beaucoup.

On désigne les circonvolutions et les sillons par l'initiale du lobe auquel ils appartiennent, avec un exposant numérique indiquant le rang qu'ils occupent dans ce lobe. Pour distinguer les sillons des circonvolutions, on emploie pour celles-ci les lettres majuscules, pour ceux-là les lettres italiques. Ainsi $\mathrm{F}^{1}$ signifie la première circonvolution frontale, et $f^{1}$ le premier sillon frontal. Les lettres $\mathrm{P}, p, \mathrm{~T}, t, 0, o, \mathrm{I}, i$, avec les divers exposants, désignent respectivement les circonvolutions et les sillons des lobes pariétal, temporal, occipital et de l'insula. Sur les figures, on peut simplifier encore cette notation, en supprimant les initiales majuscules des circonvolutions et en n'inscrivant que le chiffre 
de leur exposant, puisque l'on voit au premier coup d'œil à quel lobe ces circonvolutions appartiennent; on peut en outre, le plus souvent, se dispenser d'écrire un numéro sur les sillons, puisque ce numéro est suffisamment indiqué par celui des circonvolutions voisines; toutefois, si le trajet exact de certains sillons n'était pas assez évident sur la figure, on l'indiquerait au moyen de la lettre italique avec son exposant.

Dans le langage écrit, les majuscules et les italiques ne peuvent se confondre; mais, dans le langage parlé (où l'emploi des notations est d'ailleurs beaucoup moins utile, quoique commode encore), il faut faire précéder la lettre de l'adjectif grand ou petit.

Quant à la circonvolution du corps calleux, qui n'appartient à aucun lobe, la lettre G lui revient naturellement, et on l'emploie sans exposant, puisqu'elle est unique.

La majuscule 0 , employée à la fois pour désigner la scissure occipitale et les circonvolutions occipitales, ne donnera lieu à aucune confusion, puisque, appliquée à la scissure, elle n'a pas d'exposant, et qu'elle en a un lorsqu'elle s'applique aux circonvolutions.

On a souvent marqué de la lettre 0 les trois circonvolutions orbitaires, qui, bien que continues sans interruption avec les trois circonvolutions frontales, dont elles font réellement partie, forment cependant par leur ensemble une région spéciale appelée le lobule orbitaire. L’initiale 0 désignant déjà la région du lobe occipital, il est préférable de ne pas la reproduire à l'autre extrémité de l'hémisphère. Les chiffres 1, 2, 3, sans lettre, suffisent d'ailleurs pleinement; on verra aisément qu'il s'agit du lobule orbitaire.

Les notations précédentes suffisent pour les sillons et circonvolutions dont la direction est longitudinale et qui sont désignés par la nomenclature numérique. Mais les deux circonvolutions ascendantes, la prérolandique et la postrolandique, ne rentrent pas dans cette nomenclature, et il faut convenir, pour ce qui les concerne, d'une notation particulière.

La lettre $\mathrm{F}$ sans exposant désigne et distingue parfaitement la circonvolution frontale ascendante ou prérolandique; de même la pariétale ascendante ou postrolandique est très bien désignée par la lettre $P$ sans exposant. 
Le sillon prérolandique pourra ainsi être marqué $f$, et le sillon postrolandique $p$; ce dernier sillon sera suffisamment distingué par l'absence de l'exposant, du sillon pariétal (intra-pariétal de Turner), qui sera marqué $p^{1}$, puisqu'il est compris entre la première circonvolution pariétale $\mathrm{P}^{1}$ et la seconde $\mathrm{P}^{2}$.

Quelques mots en terminant sur la polychromie cérébrale. Gratiolet a adopté pour les planches coloriées un procédé très commode, qui consiste à distinguer chaque lobe par une certaine couleur,'dont les diverses nuances servent à distinguer entre elles les circonvolutions de ce lobe. Ce procédé augmente notablement les frais de publication des planches, et les notations dont nous venons de parler le remplacent suffisamment; mais on l'applique avec grand avantage aux moules cérébraux destinés à la démonstration, et il facilite singulièrement pour les élèves l'étude des lobes et des circonvolutions. Dans le musée de l'Institut anthropologique, les couleurs adoptées sont le rouge pour le lobe frontal, le bleu pour le pariétal, le vert pour l'occipital, le jaune pour le temporal, et enfin le brun pour la circonvolution du corps calleux.

Lorsque l'insula est à découvert, on peut également le colorier en brun, ce lobe étant suffisamment éloigné de la circonvolution du corps calleux. Dans la répartition des nuances d'un même lobe, il n'y a pas à se préoccuper de mettre la graduation chromatique en harmonie avec la notation numérique des circonvolutions. Il est désirable au contraire de chercher le contraste, afin que la distinctiơn de deux circonvolutions voisines soit tout à fait évidente; cela permet d'ailleurs de diminuer le nombre des nuances, ce qui simplifie beaucoup la tâche des peintres. Ainsi, deux nuances seulement, le vert clair et le vert foncé, suffisent par leur alternance à distinguer les six circonvolutions occipitales; de même, deux jaunes, l'un clair, l'autre foncé, suffisent pour les cinq circonvolutions temporales. Sur le lobe frontal, le même rose s'applique sur la première et la troisième circonvolution, et un seul rouge plus vif peut s'appliquer sur la deuvième frontale et sur la frontale ascendante, qu'on ne craint pas de confondre l'une avec l'autre. Mais trois nuances de bleu sont nécessaires pour distinguer nettement les trois circonvolutions pariétales, et pour éviter de confondre, comme l'ont 
fait Gratiolet et plusieurs autres auteurs, la pariétale ascendante avec la première pariétale.

Gratiolet, sur ses dessins coloriés, laissait en blanc les plis de passage. Cela n'est nullement nécessaire sur les moules, où la rencontre de deux couleurs, effectuée brusquement ou par fusion, fait reconnaître ces plis au premier coup d'œil. 


\title{
GERVEAU D'UN GORILLE
}

\author{
DE DEUX ANS ET DEMI
}

(Bulletins de la Société d'anthropologie, 3e série, t. II, 1879, p. 114-116.)

M. le docteur Bestion, voyageur de retour du Gabon, a offert à la Société un cerveau de gorille que M. Broca présente en ces termes:

La Société sait combien de difficultés s'opposent à l'envoi en Europe de cerveaux de gorille en état suffisant de conservation. Aussi, malgré les instructions que le ministre de la marine a bien voulu envoyer à ce sujet, ces envois ont jusqu'ici été très rares.

La Société d'anthropologie a eu l'avantage de recevoir le premier cerveau de gorille qui soit parvenu en Europe. C'est celui qu'a expédié le docteur Nègre. Ce cerveau n'appartenait pas à l'espèce Gorilla Savagii, mais à une espèce différente parfaitement distincte aujourd'hui, notamment par ce caractère que les mâles adultes n'ont que des crêtes beaucoup plus petites. Il existe encore d'autres différences moins importantes.

Le cerveau que je vous présente appartient à l'espèce Gorilla Savagii. Le sujet n'avait que deux ans et demi. L'âge est ici un élément dont l'importance est facile à saisir. Vous savez que, deux mois après l'arrivée du cerveau envoyé par M. Nègre, on envoyait à Hambourg le cerveau d'un jeune gorille de six mois. La comparaison des figures qui furent gravées de ces deux pièces montra des différences remarquables dans le volume relatif des lobes; le lobe frontal était plus petit dans le cerveau de Hambourg que dans le nôtre et le lobe temporal plus grand. Ces différences tenaient presque entièrement à l'âge, et lorsque M. Bischoff soutint que le cerveau recueilli par M. Nègre 
n'appartenait pas à un gorille, mais à un chimpanzé, je pus démontrer que les différences remarquées entre les deux cerveaux tenaient à l'âge très différent des deux sujets, et qu'on trouve exactement les mêmes différences entre l'homme adulte et l'enfant.

L'âge de deux ans et demi qu'avait le sujet dont nous recevons aujourd'hui le cerveau correspond sans doute à une époque plus avancée de la vie humaine, le développement du gorille étant certainement plus rapide que celui de l'homme. Nous pouvons évaluer (d'une façon assez peu exacte sans doute) que cet âge correspond chez le gorille à ce que serait à peu près la cinquième année chez nous. Le sujet pesait $6^{\mathrm{k}}, 711$, et son encéphale, que M. Bestion a pesé à l'état frais, pesait 416 grammes. Aujourd'hui il a naturellement perdu beaucoup de son poids, ainsi qu'il fallait s'y attendre après le séjour prolongé qu'il a fait dans l'alcool: il ne pèse plus que $361^{\mathrm{g}}, 50$. Le poids de cet encéphale est donc plus élevé que celui que $M$. Nègre nous a donné naguère. En effet, d'après le poids que cet encéphale pesait après une longue macération dans l'alcool, on peut évaluer qu'à l'état frais sor volume ne dépassait pas 360 grammes. Or celui-ci pesait 416 grammes étant frais. D'où vient cette différence? Vient-elle de l'âge? Je crois plutôt qu'elle vient de ce que l'espèce est différente. Rappelons pourtant que, chez l'homme, le cerveau diminue de poids dans la vieillesse. L'homme peut évidemment combattre plus longtemps que l'animal le mouvement de recul et de dégradation de sa substance cérébrale. Cependant il est probable que la différence constatée entre le poids de l'encéphale rapporté par M. Nègre et celui de M. Bestion tient surtout à la différence des deux espèces.

Il nous reste à examiner sa forme et le degré de complication de ses plis cérébraux. Remarquons, relativement à sa forme, qu'elle est visiblement très dolichocéphale. Quant aux caractères des circonvolutions, ils sont encore difficiles à constater, parce que cet encéphale a été aplati dans le transport. Il en résulte que la grande fente inter-hémisphérique est écartée considérablement. Il faudra donc le replacer quelque temps dans l'alcool en relevant avec des tampons de ouate les parties déprimées, afin de lui rendre sa forme avant de le dépouiller de ses membranes. 
Un autre cerveau de gorille que nous connaîtrons bientôt, il faut l'espérer, est celui du gorille mort au Jardin zoologique de Berlin. Il est mort, il y a un an, à l'âge de deux ans environ, mais jusqu'à présent son cerveau n'est pas publié. On ne peut douter qu'il n'ait été étudié avec soin.

\section{SUR LE GERVEAU DU GORILLE}

(Bulletins de la Société d'anthropologie, 3e série, t. II, 1879, p. 610.)

M. Le secrétatre général. Le musée de la Société possède depuis longtemps déjà une belle collection de crânes de gorille. Nous avons aussi trois squelettes de ces grands singes et une collection de moules de cerveaux plus rare et plus précieuse encore. Enfin, nous avons, pour démontrer dans les cours l'anatomie de ces animaux, le mannequin si utile de M. Auzoux; mais nous n'avions pu encore nous procurer le cadavre même du gorille. Pendant les vacances dernières, cette lacune a été réparée, et l'Ecole a fait, moyennant 1000 francs, l'acquisition de deux cadavres de gorille parfaitement bien conservés dans des tonneaux d'alcool. Le plus grand de ces deux animaux mesure $1^{\mathrm{m}}, 30$. C'est donc un jeıne presque adulte, l'autre est beallcoup plus jeune. Les deux cerveaux ont été préparés à part et sont admirablement conservés.

M. le secrétaire général se propose de faire une communication ultérieure sur ces cerveaux. Il annonce dès aujourd'hui que celui du grand gorille est tout à fait semblable à celui du gorille mâle et adulte qui rous a été procuré en 1877 par M. le docteur Nègre. M. Bischoff avait supposé que ce dernier cerveau devait provenir d'un chimpanzé, parce qu'il différait notablement de celui du jeune gorille de Hambourg. Ces différences sont réelles, mais elles sont dues seulement à la différence des âges ; c'est ce que M. Broca avait répondu à M. Bischoff, et c'est ce que con- 
firme l'examen du nouveau cerveau de gorille adulte ou presque adulte qui vient d'enrichir nos collections.

M. Broca rappelle que le musée possède maintenant cinq cerveaux de gorille : celui du docteur Nègre, deux autres qui ont été donnés dans le courant de l'année par M. le docteur Bestion, médecin de la marine au Gabon, et enfin les deux cerveaux, l'un d'adulte, l'autre de jeune, dont nous venons de faire l'acquisition. Il se trouve précisément que c'est encore le docteur Bestion qui a bien voulu se charger de préparer ces deux cerveaux pour rendre service au commerçant qui nous les a cédés.

\section{SUR UN FETUS EXENGÉPHALE}

(Bulletins de la Société d'anthropologie, $3^{\text {e série, }}$ II, 1879, p. 467-468.)

M. Tarnier, chirurgien à la Maternité, a offert au laboratoire un fœtus exencéphale que j'ai fait mouler avec d'autant plus d'empressement qu'il contribue à résoudre une question digne d'intérêt.

La Société s'est souvent occupée de l'influence qu'exercent l'un sur l'autre le développement du cerveau et celui du crâne. Nous savons que si le cerveau ne se développe pas, le crâne reste petit, et, d'autre part, les effets de la résistance offerte par le crâne au développement du cerveau ne sont pas douteux.

Mais voici la question qui se pose; on a pensé que si le cerveau se plissait chez les animaux supérieurs, c'était pour prendre moins de place.

On peut donner du fait une autre explication empruntée à la géométrie: c'est que la suiface du cerveau et la grosseur de la masse ne croissent pas suivant la même progression. C'est grâce aux plis qu'elle forme que la surface peut néanmoins s'adapter à la masse centrale du cerveau.

L'exencéphale que nous avons sous les yeux donne raison à 
MORPHOLOGIE DU CERVEAU DE L'HOMME ET DES PRIMATES. 705

cette dernière théorie. On sait que l'exencéphalie est une monstruosité caractérisée par ce fait, que les os du crâne se forment, mais ne se referment pas, en sorte que le cerveau se développe en dehors du crâne. Donc, si les plis du cerveau sont dus à ce qu'ordinairement il manque de place, il est clair qu'ici cet organe ne doit pas en présenter, car il jouit de tout l'espace nécessaire.

Ge n'est pas ce que nous observons : non seulement le cerveau de ce fœtus est plissé, mais il l'est plus que celui d'un enfant ordinaire à terme; les plis sont plus nombreux et plus étroits ; ils présentent une irrégularité extrême; on s'y reconnaît à peine. Je crois avoir reconnu la scissure de Rolando; la scissure de Sylvius se retrouve sur la face intérieure, mais elle est très dénaturée.

La présence de ces plissements nombreux rend ce fœtus exencéphale très intéressant.

\section{SUR TROIS GERVEAUX D'ORANG}

(Bulletins de la Société d'anthropologie, 3e série, t. II, 1879, p. 607-608.)

M. Broca présente trois moules de cerveaux d'orang, qui viennent d'être ajoutés à la collection déjà și riche que possède le musée de la Société. Il n'existe nulle part une collection de cerveaux aussi complète.

Les trois nouveaux moules de cerveaux d'orang ont été exécutés par M. Chudzinski avec son habileté ordinaire. Deux de ces cerveaux sont ceux des deux animaux qui viennent de mourir au Jardin d'acclimatation. Leur propriétaire n'a gardé que leur peau et leurs squelettes et M. Tramond nous a permis de prendre et de garder leurs cerveaux et leurs viscères.

On sait que les deux orangs du Jardin d'acclimatation étaient d'âges très différents ; on les faisait passer (à tort, comme nous allons le voir) comme étant le père et le fils, celui-ci étant encore tout à fait enfant. 
La différence de volume de leurs cerveaux ne répondait pas à la différence considérable de leurs âges, ce qui est en rapport avec ce que nous savons de l'intelligence précoce des jeunes singes. Plus tard, ils deviennent, sinon moins intelligents, du moins plus brutes et plus intraitables.

Mais ces deux cerveaux diffèrent sous un rapport plus important. On sait que le premier pli de passage de Gratiolet est ordinairement superficiel chez l'orang. C'est par là que le cerveau de l'orang se rapproche du cerveau de l'homme, et se distingue de celui du chimpanzé, chez qui ce même pli de passage n'est superficiel que par exception.

Or, sur le cerveau du plus jeune orang du Jardin d'acclimatation, nous trouvons le premier pli de passage, profond, comme l'est ordinairement celui du chimpanzé. Dira-t-on que cette exception est due au jeune âge du sujet? Mais d'autres exemples semblent indiquer que l'âge n'a pas d'influence sur cette disposition anatomique.

Je croirais plutôt qu'elle est due à une différence d'espèce. Le plus vieux des deux orangs était incontestablement un orang roux ou de Bornéo. Le plus jeune, un orang bicolor ou de Sumatra, et le dessin qu'a exécuté M. Verneau, et que je vous présente, vous en convaincra. 


\title{
DESCRIPTION ÉLÉMENTAIRE
}

\section{DES \\ CIRCONVOLUTIONS CÉRÉBRALES DE L'HOMME}

\author{
D'APRÈS LE GERVEAU SCHÉMATIQUE 1
}

(Revue d'anthropologie, $2^{\text {e }}$ série, 1883 , t. VI, p. 1-34, 193-210;385-405;

et $1884, \mathrm{t}$. VII, p. 1-21.)

GHAP. I. - introdugtion. Utilité du Cervead Schématioue.

La grande difficulté de l'étude des circonvolutions cérébrales de l'homme est due aux plis secondaires qui les compliquent et qui en masquent plus ou moins les caractères essentiels. Ces plis secondaires sont tellement variables qu'il est impossible de trouver deux cerveaux semblables l'un à l'autre, et même de trouver un cerveau normal dont les deux hérnisphères soient symétriques. Voilà pourquoi, pendant longtemps, les anatomistes ont cru que les circonvolutions cérébrales manquaient entièrement de fixité et qu'elles ne se prêtaient pas à la description.

Cette idée a prévalu, tant que l'on s'est borné à examiner le cerveau de l'homme. C'est seulement depuis quarante ans que l'intervention de l'anatomie comparée a permis de retrouver l'ordre au milieu de ce désordre apparent. Leuret comprit le premier, en 1839, que pour résoudre le problème il fallait le simplifier.

(1) Cet important mémoire, auquel Broca travaillait au moment où il a élé surpris par la mort, était demeuré inachevé. Il a été complété pour la partie morphologique par M. S. Pozzi, qui a aussi présidé à l'exécution des figures.

Tonte addition au texte de l'auteur sera soigneusement indiquée par un caractère spécial. 
La symétrie et la fixité des circonvolutions, dissimulées chez l'homme par leur extrême complication, sont évidentes sur les cerveaux plus simples des autres mammifères. Leuret put donc constater aisément que le nombre et la disposition des circonvolutions sont invariables dans chaque espèce. Il constata, en outre, que les conformations si diverses du cerveau des mammifères peuvent se ramener à un certain nombre de types, et que les cerveaux d'un même type peuvent différer les uns des autres par leurs caractères secondaires, mais en conservant toujours leurs caractères fondamentaux. Guidé par ces principes, il put reconnaître que le. type cérébral de l'homme est le même que celui des singes; ce rapprochement était d'autant plus remarquable qu'il ne connaissait pas les cerveaux des singes supérieurs.

C'était le cerveau du cynocéphale papion qui lui servait de terme de comparaison, et quoique l'absence des jalons intermédiaires rendît la détermination des analogies plus difficile pour lui qu'elle ne l'est devenue pour nous, il eut assez de sagacité pour retrouver chez l'homme tou tes les circonvolutions du papion; il put même dire que le cerveau de cet animal était en quelque sorte un petit cerveau d'homme, et que la loi générale de fixité et de symétrie qui préside à la constitution et à la répartition des circonvolutions est applicable à l'homme aussi bien qu'aux autres mammifères.

Tous les progrès modernes de la morphologie cérébrale étaient contenus en germe dans cette découverte de Leuret. ll ne lui fut pas donné de les réaliser lui-même. Après avoir publié le premier volume de son Anatomie comparée du système nerveux, il fut arrêté dans ses travaux par la maladie qui devait dix ans plus tard le conduire au tombeau, et il ne put rédiger son second volume, qui devait être consacré à l'étude du cerveau de l'homme. Ce soin fut confié après sa mort à l'illustre Gratiolet, qui, se pénétrant de la méthode comparative inaugurée par Leuret, et étendant ses études à toute la série des primates pour multiplier les termes de comparaison, réussit enfin à débrouiller pour la première fois le chaos des circonvolutions du cerveau humain.

Je ne me propose pas, dans ce travail élémentaire, de tracer l'historique de la question ; je n'ai donc à parler ni des résultats 
partiels obtenus par les prédécesseurs de Leuret et Gratiolet, ni des recherches complémentaires et des rectifications dues à leurs successeurs. J'ai voulu rappeler seulement quelle est la méthode qui a enfin conduit à la connaissance des circonvolutions du cerveau de l'homme. Celte méthode consiste à réduire les circonvolutions à l'état de simplicité en éliminant les complications très variables qui les surchargent. Aucun cerveau humain normal n'offrant dans toutes ses parties ce degré de simplicité, il a fallu, pour ramener les circonvolutions à leurs caractères essentiels, procéder par voie de comparaison en descendant de l'homme au singe, en considérant surtout les cerveaux d'anthropoïdes, dont la description générale est applicable, presque sans changement, au cerveau de l'homme.

Les déterminations une fois faites grâce à ce moyen d'investigation, il est devenu possible d'établir dans le cerveau humain des divisions et des subdivisions réellement anatomiques que tout observateur expérimenté sait reconnaître même dans les cas les plus compliqués, mais que les commençants retiennent difficilement, même dans les cas les plus simples. Les meilleures descriptions ne sont en effet que des guides insuffisants; elles se rapportent à un type qui est en quelque sorte abstrait, car il n'est jamais complètement réalisé dans toutes ses parties sur un même cerveau. Pour pouvoir suivre la description des circonvolutions, pour en comprendre tous les détails, il faudrait que l'élève eût sous les yeux toute une série de cerveaux maniables, c'est-àdire durcis ou moulés, et choisis de telle sorte que chaque caractère fût à l'état typique au moins sur l'un d'eux. Or, ce sont là des matériaux qu'un anatomiste déjà instruit peut trouver aisément pour ses recherches, mais que la plupart des élèves ne peuvent évidemment pas se procurer pour leurs études. Il leur reste, il est vrai, la ressource de suivre les descriptions sur des figures qu'on peut rendre plus claires en supprimant les détails inutiles; mais cela même ne suffit pas, parce que les circonvolutions qui passent d'une face sur une autre, ou celles qui sont situées sur la limite de deux faces, ne peuvent être figurées dans leur ensemble; aucun dessin, par exemple, ne peut donner une idée de la pointe du lobe occipital où viennent aboutir les circonvolutions de la face supérieure, de la face inférieure et de la face 
interne. En d'autres termes, on ne peut se faire une idée exacte et complète de la position des circonvolutions, de leurs rapports, de leurs connexions qu'en tenant soi-même l'hémisphère dans sa main et en le retournant continuellement au cours de la description. C'est de cette manière qu'on étudie les os. Qui pourrait comprendre l'os iliaque, ou les vertèbres ou seulement le tibia sans le secours des squelettes? Ces os cependant sont incomparablement moins compliqués que la surface de l'hémisphère.

Il m’a donc paru que le meilleur moyen de faciliter l'étude des circonvolutions cérébrales consistait à mettre entre les mains des élèves des moules cérébraux. A cet effet, j'avais, dès 1861, pendant que j'étais chirurgien de l'hôpital de Bicêtre, choisi un certain nombre de cerveaux, que j'avais moulés de manière à rendre les circonvolutions aussi distinctes que possible. Ces moules, sur lesquels j'avais peint chaque circonvolution d'une couleur particulière, me servirent alors à démontrer la fixité des divisions et subdivisions de l'hémisphère non seulement à mes élèves, mais encore à un certain nombre de mes confrères; car à cette époque les travaux de Leuret et Gratiolet, déjà célèbres à l'étranger, étaient presque inconnus en France; les traités d'anatomie ne mentionnaient les circonvolutions cérébrales que pour en signaler l'irrégularité désordonnée; et les médecins qui voulaient décrire une lésion de l'écorce cérébrale n'avaient aucun guide pour en déterminer le siège anatomique, détermination qu'ils faisaienten un clin d'œil en examinant un moule colorié. En 1867, lorsque je fondai à l'École pratique le laboratoire qui devint l'année suivante le laboratoire d'anthropologie de l'École des hautes études, je m'attachai à augmenter ma collection de moules cérébraux, qui depuis lors, grâce à l'habile concours et au zèle infatigable de M. Chudzinski, préparateur du laboratoire, est devenue très considérable. C'est, je pense, la plus riche collection de ce genre qui ait été faite jusqu'ici; elle est déposée dans le musée de l'Institut anthropologique, qui est ouvert au public, et dont les pièces sont mises entre les mains de tous ceux qui désirent les étudier. Ces pièces ont servi de base à la plupart des travaux qui ont été publiés en France depuis dix ans sur les circonvolutions cérébrales. Toutes les variétés de circonvolutions, leurs anomalies, les phases de leur développement y sont repré- 
sentées; on y trouve un bon nombre de cerveaux de races humaines étrangères à l'Europe, la série complète des cerveaux d'anthropoïdes, la plupart des cerveaux des autres singes, et enfin plus de cent moules relatifs aux autres ordres de mammifères. Mais, parmi ces nombreuses pièces, si utiles aux travailleurs pour leurs recherches, il n'en est aucune qui puisse être indiquée aux commençants comme présentant complètement, sans complications ni lacunes, sans exagération ni défectuosité, le type de toutes les circonvolutions, de toutes les scissures, de tous les sillons et incisures du cerveau de l'homme.

Ce cerveau humain à la fois élémentaire et typique, c'est-àdire à la fois simple et complet, et pouvant dès lors suffire aux élèves pour leurs études, je l'ai vainement cherché depuis dix ans, quoique j’aie passé en revue, soit dans le laboratoire, soit dansles salles d'autopsie, plusieurs centaines de cerveaux. Sur les cerveaux même les plus simples, il y avait toujours en certains points quelque chose de trop, quelque complication nuisible à la clarté, tandis qu'en d'autres points la petitesse exagérée et la situation profonde de certains plis laissaient établir entre les anfractuosités adjacentes des communications anormales. J'ai choisi quelques cerveaux qui approchaient du but, sans toutefois l'atteindre(1); ils ont été moulés, et ce ne sont pas les pièces les moins intéressantes de notre collection; mais aucun ne m'a paru remplir l'indication cherchée. Si j'avais trouvé cette pièce introuvable, mon intention était d'en donner le moule à M. Tramond, avec l'autorisation d'en mettre des exemplaires dans le commerce à un prix minime, accessible à tous les étudiants. J'y tenais d'autant plus que c'était, suivant moi, le meilleur moyen de vulgariser l'étude si négligée des circonvolutions cérébrales. Mais je viens de dire que mes recherches n'ont pas abouti.

J'aurais dû le prévoir du reste, car je n'ignorais pas que la description des circonvolutions de l'homme est en partie schématique; elle n'a pas été faite d'après tel et tel cerveau, mais d'après des comparaisons qui ont abouti à la détermination d'un

(1) Cescerveaux provenaient d'individus très peu intelligents ou même imbéciles, mais non idiots. On ne confondra pas les imbéciles, dontlecerveau n'est qu'insuffisant, sans anomalie véritable, avec les idiots, dont le cerveau est toujours plus ou moins anormal. 
type en quelque sorte idéal. Je n'ai jamais espéré que ce type pût se réaliser complètement sur un individu quelconque; j'espérais seulement que le hasard m'en donnerait une approximation suffisante; mais à cela même il a fallu renoncer, et j'ai dû reconnaître qu'un type arbitraire ne pouvait être exprimé que par une pièce schématique.

Je me suis donc décidé à modeler en cire un hémisphère céré. bral sur lequel j'ai cherché à reproduire dans ce qu'elles ont d'essentiel et de typique, toutes les dispositions qui caractérisent le cerveau humain, en excluant les particularités dues aux variations individuelles. Pour faciliter ce travail, dont la bonne exécution aurait exigé la main d'un artiste, mais que j'étais obligé cependant de faire moi-même, j'ai pensé qu'il suffirait de faire des retouches sur le moule d'un cerveau naturel. J'ai donc choisi, dans notre collection, parmi les cerveaux les moins compliqués, l'un de ceux qui s'écartaient le moins des caractères typiques ; j'en ai moulé en cire l'hémisphère gauche, et j'ai fait sur ce moule, à l'aide de l'ébauchoir, les corrections qui m'ont paru nécessaires. Je suis loin d'être satisfait du résultat obtenu ; je me suis aperçu, au cours de l'exécution, qu'une correction destinée à rétablir un caractère compromettait souvent les caractères des parties adjacentes, à tel point qu'il aurait fallu, de proche en proche, refaire entièrement toutes les circonvolutions, toutes les racines, tous les sillons. J'ai donc plus d'une fois laissé subsister certaines formes, certaines dispositions plus ou moins insolites, lorsqu'elles n'étaient que d'ordre secondaire, lorsqu'elles ne portaient pas atteinte aux caractères vraiment typiques.

Ces caractères concernent, d'une part, la délimitation et les connexions des lobes de l'hémisphère et des circonvolutions; d'une autre part, la position et le trajet des scissures, des sillons proprement dits et de certaines incisures. Ils sont donc anatomiques plutôt que morphologiques. On les retrouvera tous, je pense, sur mon hémisphère schématique. Quant aux autres caractères qui concernent la forme ou le volume relatif des parties, j'ai cru pouvoir me dispenser de les modifier. Ainsi on verra aisément que la partie antérieure du lobe temporal présente sur la face externe une épaisseur un peu supérieure à la moyenne, que le lobule du cunéus est au contraire un peu trop 
petit, d'où résulte une courbure exagérée de la scissure calcarine. En d'autres termes, notre moule conserve donc au point de vue de la morphologie générale les particularités propres à l'individu qui en a fourni le modèle. Il n'est schématique qu'au point de vue des caractères anatomiques. C'est tout ce qui est nécessaire pour la description des circonvolutions et des anfractuosités cérébrales, et c'est tout ce que je pouvais me proposer d'obtenir, car il n'y a que cela qui soit typique; le reste varie 'suivant les individus et suivant les races, suivant que le crâne est dolichocéphale ou brachycéphale, acrocéphale ou platycéphale, etc.

Sur un hémisphère entier et non déformé, les circonvolutions sont étroitement pressées les unes contre les autres, les anfractuosités, quelque profondes qu'elles soient, sont ordinairement très étroites; elles sont en outre souvent plus ou moins obliques par rapport à la surface, de sorte que, pour en apprécier la profondeur et pour en étudier le fond, on est obligé d'écarter les circonvolutions voisines. Sur la pièce en cire que j'ai modelée, j'ai pu rendre ces détails visibles sans élargir beaucoup les anfractuosités, en creusant avec un ébauchoir mince et légèrement recourbé. Cette pièce, qui a figuré à l'Exposition, et qui est conservée dans le Musée, se prête donc à l'étude des parties profondes des anfractuosités, sans que l'apparence antérieure des circonvolutions soit sensiblement dénaturée : mais lorsque $\mathrm{M}$. Tramond a voulu en faire le moule à bon creux, pour en tirer des exemplaires en plâtre, il a reconnu que, même en employant le procédé de la gélatine, ces parties étroites, profondes, obliques, des anfractuosités ne pouvaient être reproduites; que, suivant l'expression des mouleur's, elles n'étaient pas de dépouille. J'ai dû par conséquent l'autoriser à élargir notablement certains sillons ou scissures et à en évaser les bords. Cette modification, faite au grattoir sur une première épreuve en plâtre tirée à la gélatine, a permis de préparer le bon creux pour le tirage. Sans nuire aux caractères anatomiques, elle a quelque peu altéré le modelé naturel des circonvolutions et leur aspect général; les formes sont devenues plus lourdes, les contours moins élégants, mais il a bien fallu se résoudre à sacrifier les qualités artistiques à l'utilité pratique.

Pour faciliter l'étude des diver'ses parties de l'hémisphère, j'ai 
fait peindre chaque circonvolıtion d'une couleur particulière, suivant un procédé dont quelques planches del'album de Gratiolet ont montré l'utilité. Toutes les circonvolutions d'un même lobe sont teintées des diverses mances d'une même couleur, et cette couleur varie suivant les lobes : tout lobe se distingue donc des autres par sa couleur, et les circonvolutions qui le composent se distinguent entre elles par leur nuance. Les couleurs adoptées depuis longtemps dans notre musée sont les suivantes:

Pour le lobe frontal, le rouge (du rose clair au rouge vif).

Pour le lobe pariétal, le bleu.

Pour le lobe occipital, le rert.

Pour le lobe temporal, le jaune (et l'orangé).

Enfin les circonvolutions du corps calleux et la circonvolution de l'hippocampe, qui représentent, chez l'homme et chez les singes, le grand lobe limbique des autres mammifères, sont peints en couleur chamois. Gette couleur a été choisie parce qu'elle se rapproche des tons jaunes du lobe temporal, avec lequel se fusionne, comme on le verra plus loin, la circonvolution de l'hippocampe.

Le présent travail, destiné à guider les élèves dans l'étude du cerveau schématique, est essenticllement élémentaire. On n’y trouvera done ni détails historiques ni discussions critiques; je ne m'attacherai même pas à donner la synonymie complète de chaque circonvolution et de chaque anfractuosité.

CHAP. II. - NOMENCLATURE.

La plupart des termes dont on se sert dans la description de la surface du cerveau datent d'une époque où l'on croyait que les circonvolutions cérébrales étaient aussi instables, aussi désordonnées que les circonvolutions de l'intestin grêle. Jugeant inutile de les classer et de les décrire, mais voulant seulement en donner une idée vague et générale, les auteurs qui en parlaient ne s'attachaient pas à préciser le sens des expressions dont ils se servaient. Chacun employait à sa guise les mots qui lui paraissaient les plus commodes ou les plus significatifs. De là, une multitude de synonymes appliqués indistinctement aux mêmes choses, et, 
pour accroître encore la confusion, on donnait souvent le inême nom à des choses très différentes.

Depuis que l’on a reconnu la possibilité et par conséquent la nécessité de décrire méthodiquement, suivant les règles de l'anatomie descriptive, les saillies et les anfractuosités de la surface de l'hémisphère, on: a été conduit à préciser un peu mieux le langage : ainsi tout le monde est d'accord aujourd'hui sur l'acception du mol lobe et du mot circonvolution; mais la plupart des autres termes descriptifs ont conservé leur sens vague ou multiple et leurs synonymies confuses. Il m'a donc paru nécessaire d'en préciser l'acception et d'en régulariser l'emploi. C'est ce que je me suis efforcé de faire dans mon mémoire sur la Nomenclature cérébrale (1). J'ai développé amplement dans ce travail les motifs généraux de la réforme que j’ai cherché à introduire dans le langage, et les conditions particulières qui m’ont dirigé dans le choix de chaque mot. Je ne crois pas devoir les répéter ici; je reproduis toutefois un passage qui les résume : "Ce n'est donc pas une nomenclature en règle que je viens proposer, mais plutôt une réforme du langage usité dans la description des hémisphères, réforme consistant à atteindre ce but : que chaque chose ait un nom, qu'elle n'en ait qu'un seul, et que ce nom ne désigne qu'une seule chose. Pour cela, il suffira le plus souvent de restreindre à un seus déterminé les termes déjà usités. Ge ne sera qu'exceptionnellement que j'aurai un terme nouveau à proposer (2). is

Si les définitions qui vont suivre ne paraissaient pas suffisamment motivées, je prierais lelecteur de vouloir bien se reporter au mémoire dont je viens de parler.

ART. I. L'hémisphère dans son ensemble.

Nous supposons connue la forme générale de l'hémisphère ainsi que ses connexions, soit avec l'hémisphère opposé, soit avec les autres parties de l'encéphale. Mais, pour préciser les descriptions, il est bon de s'entendre sur quelques points relatifs à la délimitation et à la dénomination des faces et des bords de cette masse irrégulière.

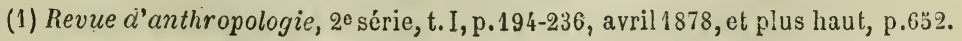

(2) Loc. cit., p. 197. 
Lorsque, sur un cerveau frais, on isole un hémisphère à l'aide d'une coupe médiane suivie de la section du pédoncule cérébral, lorsqu'on le dépouille ensuite de ses membranes et qu'on le dépose sur une table, il subit une déformation qui dénature considérablement la position des parties; il s'étale et s'aplatit, et sa face inférieure, à l'exception de la portion qui reposait sur la voûte orbitaire, se confond avec sa face interne. C'est donc sur le cerveau encore en place, ou mieux encore sur un encéphale entier, convenablement durci dans un liquide, que les faces et bords de l'hémisphère doivent être déterminés. Les personnes qui moullent ou dessinent un hémisphère sans l'avoir préalablement durci n'obtiennent que des résultats tout à fait incorrects (1).

Dans la description générale de l'encéphale ainsi durci, on distingue souvent la face supérieure des faces latérales, afin de nieux apprécier les rapports des diverses régions cérébrales avec les diverses régions crâniennes. Mais, au point de vue de la description particulière de l'hémisphère, ces deux faces, dont rien n'établit la limite, n'en font qu'une seule, que l'on désigne sous le nom de face convexe.

Cela posé, l'hémisphère présente trois faces et trois bords. Ces trois faces sont : $1^{\circ}$ la face interne, plane, verticale, médiane, appliquée sur la grande faux qui la sépare de la face correspondante de l'hémisphère opposé ; $2^{\circ}$ la face convexe, qui correspond à toute une moitié de la surface interne de la voûte crânienne; $3^{\circ}$ la face inférieure, qui se compose, d'avant en arrière, de trois portions, l'une antérieure ou orbitaire, presque plane, reposant sur la voûte orbitaire; l'autre moyenne ou temporale, convexe, reposant sur la fosse moyenne de la base du crâne; l'autre postérieure ou tentoriale, légèrement concave et reposant sur la tente du cervelet.

Les trois bords sont : $1^{\circ}$ le bord externe, qui est en même temps inférieur, séparant la face convexe de la face inférieure; $2 \circ$ le bord sagittal, qui sépare la face convexe de la face interne, tout le long de la base de la grande faux; $3^{\circ}$ le bord inféro-in-

(1) Il ne sufft pas de plonger le cerveau dans un liquide durcissant pour lui conserver sa forme. Il faut, en outre, le déposer sur un fond de ouate et le retourner tous les jours ou tous̀ les deux jours, jusqu'à ce qu'il soit tout à fait raffermi. 
terne, qui est comme le précédent, sur la ligne médiane, et qui sépare la face interne de la face inférieure.

Le nom de bord externe est parfaitement clair et ne peut donner lieu à aucune amphibologie.

Le bord inféro-interne est quelquefois appelé simplement bord inférieur; mais cela ne suffit pas, puisqu'il n'est situé ni plus haut ni plus bas que le bord externe, a vec lequel on pourrait le confondre si l'on n'ajoutait pas qu'il est interne en même temps qu'inférieur. Il se compose de trois portions qui correspondent aux trois portions déjà nommées de la face inférieure, savoir : une portion antérieure ou orbitaire, une postérieure ou tentoriale, et une portion moyenne ou temporale, ainsi nommée parce qu'elle longe le lobe temporal, quoique celui-ci ne parvienne pas tout à fait jusqu'au bord interne. Cette portion moyenne s'étend du chiasma des nerfs optiques au bourrelet du corps calleux.

Quelques mols enfin sur le bord sagittal. On l'appelle souveni bord supérieur, et il est supérieur en effet dans sa portion moyenne. Mais en avant et en arrière il descend jusqu'à la base, et l'épithète de supérieur, appliquée à ces deux portions descendantes, rend les descriptions très obscures. Ainsi certaines inci sures du lobe frontal vont rejoindre ce bord ou se dirigent vers lui, quoiqu'elles soient à peu près horizontales, et quelquefois même un peu descendantes; dire qu'elles se dirigent vers le bord supérieur, c'est donner une idée tout à fait trompeuse de leur direction. Le nom de bord sagittal, tiré de la suture sagittale qui longe la partie moyenne de ce bord, n'a pas le même inconvénient. Il est parfaitement conforme au langage anatomique, qui permet de désigner certaines parties d'après l'un de leurs rapports.

Par exemple, le nerf radial porte ce nom depuis l'aisselle jusqu'à la main, quoiqu'il ne soit en rapport avec la région ra diale que dans sa partie moyenne.

Ceci dit sur les dénominations relatives à la forme de l'hémisphère, et avant de nous occuper des divisions et subdivisions de sa surface, nous devons distinguer d'une manière générale les deux groupes d'organes qui composent sa masse.

L'hémisphère comprend deux groupes d'organes formant deux 
parties, l'une centrale, qui est le corps de l'hémisphère, l'autre périphérique, qui en est le manteau.

Le manteau comprend l'ensemble des circonvolutions. Le corps de l'hémisphère comprend tout le reste de l'hémisphère.

Le corps est de toutes parts recouvert par le manteau, à l'exception d'une région de forme à peu près elliptique qui est située vers le milieu de la face interne, et que nous nommerons le seuil de l'hémisphère. C'est par le seuil de l'hémisphère que s'effectuent toutes les connexions de l'hémisphère soit avec l'autre hémisphère, soit avec le reste de l'encéphale. Il est limité en haut par la rainure que longe la face convere du corps calleux, en bas par la grande fente de Bichat.

Le bord du manteau qui entoure le seuil est le limbe de l'hémisphère. Les parties qui forment ce bord sont donc appelées limbiques. Nous y reviendrons tout à l'heure.

ARt. Ir. Les divisions du manteau de l'hémisphère.

Les divisions du manteau sont établies par des anfractuosités de divers ordres, sur lesquelles on reviendra tout à l'heure.

Les divisions primaires du manteau sont désignées sous le nom de lobes. Les limites des lobes sont marquées par des lignes anatomiques qui se trouvent sur le cerveau même, et non sur les os du crâne, comme pourraient le faire croire les noms des principaux lobes.

Les divisions secondaires du manteau, c'est-à-dire les subdivisions des lobes, sont les circonvolutions. Le mot " circonvolution ") ne désigne plus, comme autrefois, toute partie plus ou moins contournée de la surface du cerveau, mais des parties déterminées par des limites anatomiques fixes. Le nombre des circonvolutions de chaque lobe est donc absolument constant. La plupart des lobes comprennent plusieurs circonvolutions ; l'un d'eux, toutefois (le lobe du corps calleux), se réduit à une seule circonvolution.

On donne le nom de lobules à certaines portions de lobes ou de circonvolutions formant certaines régions de peu d'étendue que l'on juge dignes d'une attention spéciale. Ce nom a une accention topographique plutôt qu'anatomique. Certains lobules 
cependant ont des limites bien déterminées. Un lobule peut être formé par une circonvolution entière (lobule du cunéus), ou seulement par une portion de circonvolution (lobule ovalaire), ou des portions de plusieurs circonvolutions adjacentes (lobule orbitaire).

Lorsqu'une ou plusieurs circonvolutions se réfléchissent pour passer de la face supérieure d'un lobe sur sa face inférieure, on dit qu'elles forment deux étages, l'un supérieur, l'autre inférieur. L'acception du mot "étage » est donc topographique et non anatomique. Les trois circonvolutions longitudinales du lobe frontal sont à deux étages; la réunion de leurs étages inférieurs forme l'étage inférieur, ou lobule orbitaire du lobe frontal.

On donne le nom d'opercules à certaines portions de lobes ou de circonvolutions qui se prolongent plus ou moins obliquement au-dessus d'une anfractuosité profonde. Il y a deux opercules : l'opercule de l'insula et l'opercule occipital.

Toute circonvolution commence et finit dans le lobe dont elle fait partie, mais elle se continue souvent par des plis de passage avec une ou plusieurs circonvolutions appartenant aux lobes roisins. On reviendra tout à l'heure sur les plis de passage.

Les deux extrémités d'une circonvolution constituent son origine et sa terminaison. C'est ordinairement l'usage, plutôt que la raison anatomique, qui détermine l'application de ces deux noms aux extrémités qu'ils désignent respectivement. Le nom spécial de racines désigneles origines des circonvolutions frontales et des circonvolutions pariétales sur les deux circonvolutions ascendantes.

Lorsque toutes les circonvolutions d'un même lobe prennent leur origine en un même point sur une petite région qui leur est commune, ce point prend le nom de pôle. Il y a trois pôles: l'occipital, le temporal et celui de l'insula.

Les parties secondaires d'une circonvolution portent le nom de plis. Ce mot, employé par Gratiolet comme synonyme de circonvolution, ne désigne plus que certaines portions de circonvolutions.

Il y a deux espèces de plis : les plis de communication et les plis de complication. 
a) Les plis de communication sont des prolongements qui s'étendent d'une circonvolution aux circonvolutions voisines à travers les anfractuosités intermédiaires. Lorsque les circonvolutions qu'ils unissent appartiennent à un même lobe, ils se nomment plis d'anastomose; lorsqu'ils appartiennent à deux lobes différents, les plis de communication, passant d'un lobe à l'autre, prennent le nom de plis de passage.

Les plis de communication peuvent être superficiels, c'est-àdire apparents à l'extérieur dans toute leur étendue, ou profonds, c'est-à-dire cachés en tout ou en partie dans le fond des anfractuosités qu'ils traversent. Cette différence de position dépend de leur volume, et n'a que peu d'importance anatomique, mais elle modifie considérablement les caractères morphologiques.

b) Les plis de complication sont de deux sortes : les uns sont des plis d'inflexion, les autres des plis de subdivision.

I.es plis d'inflexion se produisent lorsqu'une circonvolution est plus longue que l'espace compris entre ses deux extrémités; la circonvolution se replie alors une ou plusieurs fois dans son trajet en formant de simples sinuosités ou de véritables méandres. Il y a sinuosité lorsque le bord est alternativement concave et convexe ; il y a méandre, lorsque le bord se recourbe assez fortement pour revenir en contact avec lui-même avant de continuer son trajet.

Les plis de subdivision sont des dédoublements plus ou moins longitudinaux produits par des incisures plus ou moins longues, plus ou moins profondes.

ART.III. Les anfractuosités de l'hémisphère (scissures, anfractuosités, incisures).

Les divisions et les subdivisions du manteau sont établies par des dépressions ordinairement étroites et profondes que nous désignerons, abstraction faite de leurs caractères anatomiques, sous le nom général d'anfractuosités.

Il est nécessaire de diviser ces anfractuosités en plusieurs espèces, suivant la nature anatomique des divisions qu'elles établissent dans le manteau. Il y a trois ordres de divisions dans le manteau : $1^{\circ}$ les lobes; $2^{\circ}$ les circonvolutions; $3^{\circ}$ les plis de com- 
plication. Il y a donc aussi trois ordres d'anfractuosités : $1^{\circ}$ celles qui séparent les lobes, ce sont les scissures; $2^{\circ}$ celles qui séparent les circonvolutions d'un même lobe, ce sont les sillons; $3^{\circ}$ celles qui produisent les plis de complication, ce sont les incisures.

Il faut se garder d'employer ces trois termes comme synonymes, ou de croire qu'ils expriment les divers degrés de longueur ou de profondeur des anfractuosités; certains sillons pouvant être plus grands ou plus profonds que certaines scissures. Ce qui les distingue, ce n'est pas leur apparence grossière, c'est leur position et leurs rapports anatomiques.

Il y a toutefois dans le lube occipital une importante anfractuosité dont l'importance anatomique est bien supérieure à celle des sillons ordinaires, et à laquelle nous conserverons le nom de scissure. C'est la scissure calcarine. Cette explication est justifiée par l'embryologie et par l'anatomie comparée des primates, car le lobule triangulaire ou cuneus, dont la scissure calcarine établit la limite, présente chez les primates une précocité et une constance remarquables, et mériterait, n'était sa petitesse, d'être élevé au rang des lobes. Je prouverai d'ailleurs que si la partie postérieure de cette anfractuosité se borne à séparer l'une de l'autre deux circonvolutions du lobe occipital, et joue en cela le rôle d'un sillon, sa partie antérieure, dans une étendue de plus de 2 centimètres, sépare le lobe du corps calleux de la partie postérieure du lobe temporal, et se comporte par conséquent comme une véritable scissure.

Comme les plis de complication qu'elles déterminent, les incisures se divisent en deux espèces que l'on peut appeler les incisures continues et les incisures isolées. Ces dernières forment les plis de subdivision des circonvolutions; tantôt réduites à une petite dépression plus large que profonde appelée fossette (1), tantôt profondes, étroites et plus ou moins longues, elles ont pour caractère de ne jamais commencer réellement qu'avec les sillons

(1) La fossette est de même nature que l'incisure; on trouve souvent sur un hémisphère une fossette là où sur l'autre hémisphère du même cerveau, existo une véritable incisure. La fossette est évasée et simplement tapissée par la pie. mère ; l'incisure est plus étroite et plus profonde, et la pie-mère qui y pénètre forme un pli en s'adossant à elle-même. 
véritables; il peut se faire que l'une de leurs extrémités aboutisse jusqu'au sillon voisin, sous la forme d'une gouttière très superficielle qui loge un vaisseau de la pie-mère émané de ce sillon, mais on reconnaît aisément, en écartant les bords de cette gouttière, que l'incisure est en réalité indépendante du sillon, qu'elle est due à un plissement effectué dans une circonvolution dont elle augmente la surface sans en augmenter la longueur. Les incisures continues, au contraire, sont des prolongements directs, des branches d'un sillon ou d'une scissure, prolongements qui pénètrent dans les méandres d'une circonvolution. Parmi les incisures continues il en est qui sont très variables, d'autres sont constantes et figurent au nombre des caractéristiques du type de certaines espèces animales; dans ce dernier cas, elles peuvent acquérir assez d'importance pour mériter d'être appelées branches. Telle est par exemple la branche horizontale de la scissure de Sylvius.

Les incisures isolées revêtent des formes très diverses. Celles qui sont simples, c'est-à-dire formées d'une seule branche droite ou ondulée, sont dites longitudinales, transversales, ou obliques, non pas d'après leur direction par rapport à l'hémisphère, mais d'après leur direction par rapport à l'axe de la circonvolution. Gelles qui sont composées de plusieurs branches portent, suivant leur forme, les noms d'incisures en T, en $\mathrm{L}$, en $\mathrm{H}$, en Y. L'incisure en étoile est formée de trois petites branches qui s'irradient à partir d'un point commun. L'incisure en double fourche se compose d'une branche moyenne, terminée en fourche à ses deux extrémités.

On trouve fréquemment à la surface des circonvolutions, surtout lorsque le cerveau a été durci dans les liquides aree ses membranes, de petites dépressions linéaires, très ótroites, très superficielles, quelquefois bifurquées, qui sont les empreintes des plus gros vaisseaux de la pie-mère. Co sont des nervures, qu'on ne doit pas confondre avec les incisures, car ce ne sont pas des anfractuosités.

Presque toutes les anfractuosités de l'hémisphère rentrent daus les trois catégories que nous venons de désigner sous les noms de scissures, de sillons et d'incisures. Mais il en est quelques-unes qui sont d'une nature spéciale et qui, par con- 
séquent, ne rentrent pas dans la nomenclature lgénérale. Ge sont :

$1^{\circ}$ Dans le manteau : la vallée de Sylvius, la fosse de Sylvius et les rigoles de l'insula;

$2^{\circ}$ Sur les limbes de l'hémisphère, la rainure du corps calleux et la rainure du grand hippocampe;

3. Dans le seuil de l'hémisphère, la fente de Bichat et, entre les deux hémisphères, la fente interhémisphérique.

Celle-ci est appelée ordinairement scissure interhémisphérique, mais le nom de scissure, désignant les anfractuosités qui sóparent les lobes de l'hémisphère, ne peut désigner en même temps l'intervalle médian qui sépare les deux hémisphères l'un de l'autre.

Art. Iv. Quelques mots sur le grand lobe limbique et la scissure limbique.

L'anatomie comparée ne saurait trouver place dans ce travail élémentaire sur le cerveau de l'homme; il me paraît nécessaire toutefois de donner un aperçu du grand lobe limbique des mammifères, en vue des dénominations de certaines parties dont l'anatomie comparée a établi l’importance, et qui, ayant leurs analogues chez l'homme, doivent conserver chez lui le nom qu'elles portent chez les autres mammifères.

La très grande majorité des mammifères sont osmatiques, c'est-à-dire pourvus d'un grand lobe olfactif dont les primates n'ont que le rudiment. Chez tous les osmatiques, le seuil de l'hémisphère (voir plus haut, p. 718) est entièrement circonscrit par le grand lobe limbique, formé en haut par le lobe du corps calleux, en bas par le lobe de l'hippocampe, en avant par le lobe olfactif. Ce grand lobe, à son tour, est circonserit presque entièrement par une très grande scissure qu'interrompent seulement certains plis de passage; c'est la scissure limbique. Chez les primates, le lobe olfactif s'atrophie et se détache de l'hémisphère à tel point qu'il semble n'en plus faire partie; le lobe de l'hippocampe s'atrophie aussi, et se fusionne avec le lobe temporal. Le lobe du corps calleux est donc le seul qui conserve ses caractères; il conserve aussi ses principales connexions, quoique celles-ci semblent au premier abord notablement modifiées par suite du 


\section{volume plus ou moins grand et de la situation plus ou moins}

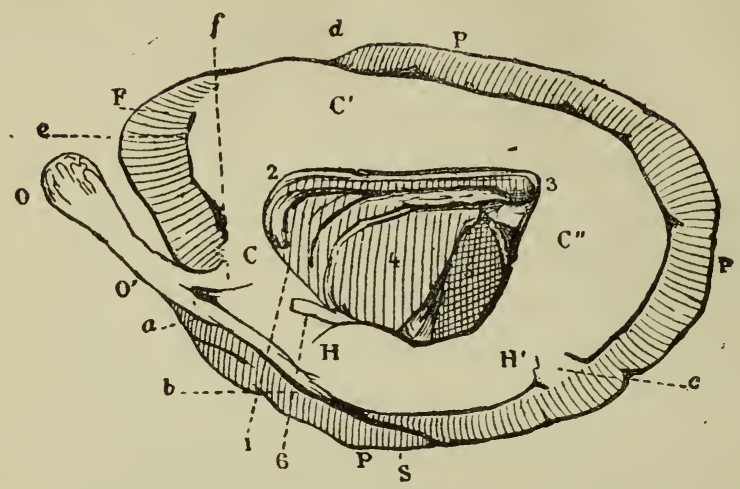

Fig. 84. Schéma de la face inféro-interne de l'hémisphère droit de la loutre.

1, le bec du corps calleux : 2 , son genou ; 3 , son bourrelet, au dessous duquel on aperçoit le pilier postérieur de la voûte, et l'origine de la corne d'Ammon; 4, face interne de la couche optique; 5 , coupe du pédoncule cérébral, séparé du grand lobe limbique par la fenle de Bichat ; 6 , la bandelette optique, se dégageant, à la partie antérieure de la fente de Bichat, entre la couche optique et le lobe de l'hippocampe; PPP, lobe pariétal; F, lobe frontal; $\mathrm{S}$, scissure de Sylvius ; O, lobe olfactif ; $\mathrm{O}^{\prime}$, pédoncule olfactif ; $\mathrm{C}$, origine du lobe du corps calleux ; $C^{\prime} C^{\prime \prime}$, le lobe du corps calleux; $H^{\prime}$, le lobe de l'hippocampe; $a b$, racine externe du lobe olfactif; $a b c$, l'arc inférieur de la scissure limbique ; c, lo pli de passage rétrolimbique; $c d$, scissure sous-pariétale; $e$, sillon sous-frontal; $f$, racine interne du lobe olfactif.

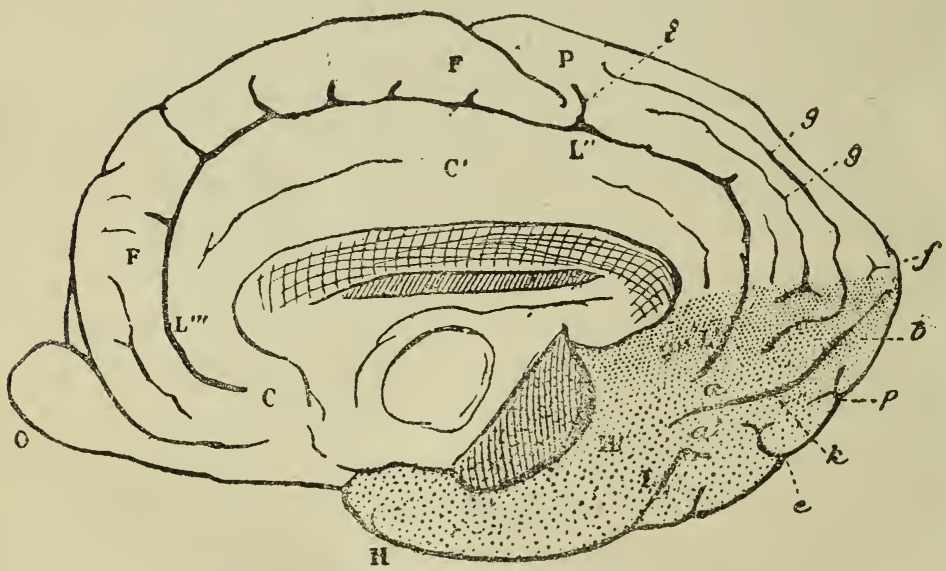

Fig. 85. Cheval, face interne de l'hémisphère droit.

O, lobe olfactif; $\mathrm{HH}^{\prime}$, lobe de l'hippocampe; $\mathrm{CC}^{\prime} \mathrm{C}^{\prime \prime}$, lobe du corps calleux ; FF, face interne du lobe frontal; $P$, face interne du lobe pariétal ; $L$, arc inférieur de la scissure limbique ; $\mathrm{L}^{\prime} \mathrm{L} \prime \mathrm{L}^{\prime \prime \prime}$, arc supérieur; $\mathrm{L}^{\prime} \mathrm{L}^{\prime \prime}$, sa portion pariétale ou scissure sous-pariétale; $\mathrm{L}^{\prime \prime} \mathrm{L}^{\prime \prime \prime}, \mathbf{s a}^{\prime}$ portion frontale ou scissure sous-frontale; $a a^{\prime}$, pli de passage rétro-limbique, subdivisé en deux par le sillon calcarin $k ; b$, le pôle de l'hémisphère; $p$, origine du sillon pariétal primaire ; $e$, origine du sillon pariétal externe; $f$, origine du sillon pariétal interne ; $i$, incisure fronto-pariétale interne. 
scissure limbique que traversent ces plis de passage, elle est bien plus modifiée encore. Quelques-unes de ses parties s'effacent entièrement, d'autres ne sont plus représentées que par de légers sillons, d'autres au contraire s'exagèrent énormément. Il en résulte que le type cérébral des primates diffère considérablement de celui des mammifères osmatiques; il en diffère à tel point qu'on a cru pendant longtemps qu'il n'y avait rien de commun entre ces deux types; mais l'étude des connexions du grand lobe limbique permet de constater au contraire que toutes les parties essentielles du cerveau des osmatiques ont leurs analogues chez les primates (fig. 84 et 85 ). On ne saurait méconnaître l'importance de cette conclusion.

Je ne puis songer à appliquer à la description du cerveau humain toute la nomenclature de l'anatomie comparée. Ce serait troubler toutes les habitudes du langage. Mais, lorsque j'aurai à décrire certaines parties qui n'ont pas encore reçu de nom chez l'homme parce qu'on n'en avait pas reconnu la fixité ou la signification, je leur appliquerai de préférence les noms tirés de l'anatomie comparée. Ces parties concernent pour la plupart les connexions du grand lobe limbique, et plus particulièrement du lobe du corps calleux. En leur donnant l'épithète de limbique (ou ses composés) je constaterai leur nature et leur analogie, et j'y trouverai un autre avantage, celui de la simplicité des mots, car le nom de "lobe du corps calleux » est trop long et trop complexe par lui-même pour entrer en composition. Ainsi les mots prélimbique, rétro-limbique désignent très clairement les connexions antérieures ou postérieures du lobe du corps calleux; de même on verra tout de suite que le pli de passage cunéolimbique s'étend du cunéus au lobe du corps calleux, etc. Pour comprendre immédiatement la signification de ces noms, il suffira de savoir une fois pour toutes que le lobe du corps calleux fait partie du grand lobe limbique des mammifères en général.

GHAP. III. - notATION.

Les notations inscrites sur notre cerveau schématique ne concernent que les circonvolutions. Sur les dessins, on emploie les 
mêmes notations, mais on y en ajoute d'autres qui se rapportent aux scissures et aux sillons.

Parlons d'abord de la notation des circonvolutions.

Toute circonvolution est désignée par une lettre majuscule avec ou sans exposant. La lettre indique le lobe auquel la circonvolution appartient; l'exposant indique le rang qu'elle y occupe.

$\mathrm{F}$ désigne les circonvolutions du lobe frontal; $\mathrm{P}$, celles du lobe pariétal ; 0 , celles du lobe occipital ; T, celles du lobe temporal, et $\mathrm{C}$, enfin, désigne la circonvolution unique du lobe du corps calleux.

Toutes ces circonvolutions sont longitudinales, à l'exception des deux ascendantes sur lesquelles je vais revenir.

Les circonvolutions longitudinales sont comptées de chaque lobe, à partir du bord supérieur ou sagittal de l'hémisphère. La première est donc celle qui est la plus rapprochée de ce bord en distance comptée sur la face convexe de l'hémisphère; elle porte donc l'exposant 1 ; la suivante porte l'exposant 2 , et ainsi de suite.

La circonvolution du lobe du corps calleux, étant unique, n'a pas d'exposant.

Les deux circonvolutions ascendantes qui longent la scissure de Rolando appartiennent respectivement au lobe frontal et au lohe pariétal, et doivent par conséquent être marquées, l'une par la lettre F, l'autre par la lettre P. Entièrement différentes par leur direction, leur position, leurs connexions, des circonvolutions longitudinales qui viennent s'ouvrir sur elles, elles ne peuvent prendre rang dans la série ordinale. C'est done à tort - que quelques personnes ont noté la frontale $\mathrm{F}^{*}$ et la pariétale $\mathrm{P}^{3}$; si l'on veut leur donner un exposant, ce ne peut être que zéro; on peut donc les marquer $\mathrm{F}^{0}$ et $\mathrm{P}^{0}$; mais on peut aussj les inscrire sans exposant.

La notation des scissures et des sillons ne se fait que sur les dessins. On marque les scissures par des lettres majuscules et les sillons par des lettres italiques, avec ou sans exposants.

La scissure de Rolando se marque R.

La lettre 0 désigne la scissure occipitale ; la même lettre désigne aussi les circonvolutions occipitales, mais, dans ce dernier cas, elle porte un exposant. L'absence de l'exposant indique qu'il s'agit de la scissure et non d'une circonvolution. 
La majuscule $\mathrm{S}$ indique l'anfractuosité sylvienne; celle-ci, comme on le verra plus loin, se compose de trois parties : la vallée de Sylvius, la scissure de Sylvius proprement dite et la branche antérieure de la scissure de Sylvius. La vallée se marque S, la scissure proprement dite $\mathrm{S}^{\prime}$, et la branche antérieure $\mathrm{S}^{\prime \prime}$. Cette dernière est absolument.constante chez l'homme et les anthropoïdes. Au-dessus d'elle, existe normalement chez l'homme et quelquefois chez les grands anthropoïdes, la branche ascendante de la scissure de Sylvius; le signe $\mathbf{S}^{\prime \prime \prime}$, qu'on devrait lui appliquer, serait gênant dans un si petit espace; il est donc plus commode de le remplacer par la lettre italique $s$.

La scissure calcarine ou du petit hippocampe est désignée par la lettre $\mathrm{K}$, au lieu de l'initiale $\mathrm{C}$, qui désigne déjà la circonvolution ou lobe du corps calleix.

Reste une dernière scissure, qui sépare, sur la face interne, le lobe frontal du lobe du corps calleux et que nous appelons la scissure sous-frontale. On pourrait la désigner par la double lettre $\mathrm{SF}$, mais la lettre L, initiale de la scissure limbique, est préférable. La scissure sous-frontale, en effet, représente, chez les primates, l'arc supérieur de la scissure limbique des autres mammifères.

Les sillons sont désignés par des lettres (petites italiques), arec ou sans exposants. La lettre est celle du lobe dans lequel se trouve le sillon: $f$ pour le lobe frontal, $p$ pour le lobe pariétal, etc. Elle est donc la même que celle des circonvolutions adjacentes, si ce n'est qu'elle est d'un autre caractère. On donne à cette lettre l'exposant de celle des deux circonvolutions adjacentes dont le numéro est le plus faible. Ainsi on nomme premier sillon frontal $f^{1}$ celui qui est compris entre les circonvolutions $\mathrm{F}^{1}$ et $\mathrm{F}^{2}$, second sillon frontal $f^{2}$ celui qui est compris entre $\mathrm{F}^{2}$ et $\mathrm{F}^{3}$.

Dans le lobe pariétal, qui n'a que deux circonvolutions longitudinales, il n'y a qu'un seul sillon longitudinal, nommé le sillon pariétal; on n'y ajoute pas une appellation ordinale, puisque ce sillon est seul en son genre ; mais, dans la notation, on le marque $p^{1}$, conformément à sa position entre $\mathrm{P}^{1}$ et $\mathrm{P}^{2}$. L'addition d'un exposant est nécessaire pour distinguer le sillon pariétal du sillon postrolandique, qui appartient aussi au lobe pariétal et 
qui, longeant le bord postérieur de la circonvolution pariétale ascendante, se marque $p^{0}$.

De même, dans le lobe frontal, la circonvolution ascendante $\mathrm{F}^{0}$ est longée par le sillon prérolandique, qui longe son bord antérieur et qui se marque $f^{\circ}$.

Les exposants 0 s'appliquent donc aux circonvolutions et aux sillons parallèles à la scissure de Rolando, dont la direction est plutôt transversale que longitudinale, tandis que les autres circonvolutions et les autres sillons dont la direction est longitudinale portent les exposants $1,2,3 \ldots$

Il y a, sur la face interne de l'hémisphère, à la base du lobulc quadrilatère, un petit sillon dont l'existence est constante, mais dont la forme est assez variahle. G'est le vestige de la scissure limbique, qui, chez les mammifères autres que les primates, sépare le lobe dı corps calleux du lobe pariétal. La lettre $l$ indique cette analogie. On n'a pas oublié que la lettre L marque la scissure sous-frontale, qui procède aussi de la scissure limbique, mais qui, ayant conservé le caractère d'une vraie scissure, doit être indiquée par une majuscule.

On emploie quelquefois la notation $0, o$ pour marquer les circonvolutions et les sillons du lobule orbitaire; mais il en résulte une confusion, parce que ces lettres indiquent déjà le lobe occipital. Les éléments du lobule orbitaire n'étant que le prolongement de ceux de l'étage supérieur' du lobe frontal, il suffit parfaitement de les marquer des lettres $\mathrm{F}$, et $f$, avec leurs exposants. G'est conformeà l'anatomie etl'on verra bien toujours si la figure représente la face supérieure ou la face inférieure du cerveau.

\section{GHAP. IV. - MÉTHODE DE DESCRIPTION.}

La surface de l'hémisphère présente des parties en relief qui sont les circonvolutions, et des anfractuosités qui les séparent en formant une sorte de lacis très compliqué.

On peut décrire cet ensemble de deux manières, soit en décrivant les circonvolutions elles-mêmes, soit en décrivant les anfractuosités.

Il est clair, en effet, que, connaître les circonvolutions, c'est 
MORPHOLOGIF DU CERVEAU DE L'HOMME ET DES PRIMATES. 729

connaître leurs lignes de démarcation. Une circonvolution étant une fois complètement décrite, la connaissance des sillons ou scissures qui la limitent en découle nécessairement.

Mais il est clair encore que toutes les anfractuosités étant une fois décrites, les parties qu'elles circonscrivent le sont aussi par là même.

S'il fallait choisir entre ces deux méthodes, la première devrait évidemment recevoir la préférence, car le but à atteindre est la connaissance des circonvolutions, qui sont des organes et non des anfractuosités qui ne sont que des intervalles. Or, la première méthode va droit au but, tandis que la seconde n'y conduit qu'indirectement.

Aucun auteur n'a pensé que l'une ou l'autre méthode dût recevoir une préférence exclusive; tous reconnaissent que se borner à une seule serait exiger de l'esprit du lecteur un trop grand effort, et qu'il est par conséquent nécessaire de les combiner. Mais, ce qui donne lieu à des divergences, c'est le degré d'importance des deux méthodes et l'ordre suivant lequel elles doivent être appliquées. Les uns décrivent d'abord les circonvolutions, et ne donnent les descriptions des anfractuosités que comme un complément accessoire ; d'autres, au contraire, s'attachent surtout à décrire les anfractuosités, et se bornent ensuite à indiquer sommairement les circonvolutions intermédiaires ; d'autres encore s'efforcent de faire marcher les deux descriptions de front; ce qui est conforme à la logique plus qu'à la clarté. La plupart, enfin, pensent que les deux descriptions doivent être faites séparément, complètement, et avec le même soin, mais ils discutent sur la question de savoir quelle est celle qui doit précéder l'autre.

Si l'on songe qu'avant d'étudier correctement un organe il faut d'abord en établir les limites, on reconnaîtra qu'il y a tout avantage à commencer par l'étude des anfractuosités. G'est ainsi qu'en géographie on détermine les frontières d'un pays avant d'en décrire la surface.

C'est cette méthode que nous allons suivre. Nous l'appliquerons d'abord à l'étude des lobes, puis à celle des circonvolutions. 


\section{LES LOBES ET LES SCISSURES.}

Les lobes, divisions primaires du manteau, sont des groupes de circonvolutions. Leurs limites sont établies par les scissures cérébrales.

Ils sont au nombre de six, dont cinq sont superficiels et un profond.

Les lobes superficiels sont: le lobe frontal (peint en rouge sur le moule), le lobe pariétal (bleu), le lobe occipital (vert), le lobe temporal (jaune) et le lobe du corps calleux (couleur chamois).

Le dernier lohe est le lobe de l'insula. Il est caché dans le fond de la scissure de Sylvius. Pour le rendre apparent, il faut écarter largement les bords de cette scissure et déformer considérablement l'hémisphère. Il n'est donc pas visible sur notre cerveau schématique et nous sommes obligés, pour le décrire, de recourir à une figure spéciale (1).

Conformément à la méthode indiquée dans le chapitre précédent, nous décrirons d'abord les scissures qui jdéterminent les limites de ces lobes.

ART. I. Description des scissures.

$1^{\circ} \mathrm{La}$ scissure de Sylvius et l'anfractuosité sylvienne ( $\mathrm{pl}$. I, $\left.\mathrm{S}^{\prime}, \mathrm{S}^{\prime \prime} s\right)$. La plus grande, la plus évidente et aussi la plus compliquée des anfractuosités cérébrales est celle qui est connue sous le nom de scissure Sylvius, et que nous appellerons l'anfractuosité sylvienne. Elle a ćté décrite ou plutôt indiquée pour la pre. mière fois au dix-septième siècle par François de Le Boë, qui écrivait en latin sous le nom de Sylvius. Elle s'étend à la fois sur la face inférieure et sur la face externe de l'hémisphère; clle sépare le lobe temporal du lobe frontal et du lobe pariétal.

Elle se compose de deux parties qui sont la vallée de Sylvius et la scissure de Sylvius. On considère généralement ces deux parties comme ne formant qu'une seule scissure; on nomme la première branche horizontale de la scissure de Sylvius, et la

(1) On trouvera chez M. Tramond un moule spécial reproduisant le lobe de l'insula et montrant ses connexions avec les lobes voisins. 
seconde branche postérieure de la même scissure. Cette manière de voir, déjà peu conforme à certains faits de l'anatomie humaine, est tout à fait incompatible avec les faits de l'anatomie comparée; car l'étude du cerveau dans la série des mammifères nous montre que la vallée et la scissure de Sylvius sont complètement différentes par leur nature et par leur mode de formation (1).

Lorsqu'on écarte les bords de la scissure de Sylvius on pénètre dans une anfractuosité large et profonde qui est la fosse de Sylvius, et qui renferme le lobe de l'insula.

Nous aurons donc à décrire successivement la vallée, la fosse et la scissure de Sylvius.

a. La vallée de Sylvius se voit sur la surface inférieure du cerveau : c'est une anfractuosité assez profonde, transversale, dont le bord postérieur, très saillant, est formé par l'extrémité antérieure du lobe temporal, et dont le bord antérieur se continue sans aucune saillie et presque sans ligne de démarcation, avec le bord postérieur du lobule orbitaire du lobe frontal. Elle commence, sur le bord inféro-interne de l'hémisphère, immédiatement en dehors du chiasma des nerfs optiques, et se porte de là transversalement en dehors jusqu'au bord externe de l'hémisphère, où elle se continue avec la scissure de Sylvius.

Elle n'existe à l'état d'anfractuosité véritable, avec des caractères plus ou moins analogues à ceux des scissures, que chez les animaux anosmatiques, c'est-ì-dire chez ceux dont l'appareil olfactif est rudimentaire ou nul. Ces animaux sont les primates, les carnassiers amphibies et les cétacés. Chez tous les autres mammifères, dits osmatiques, elle se réduit à une simple dépression, très large, peu profonde, plus large que profonde, de forme à peu près quadrilatère (espace quadrilatère). Elle 's'arrête en dehors, sur la racine olfactive externe qui est très grosse, et qui la sépare complètement de la scissure de Sylvius. :Elle est donc tout à fait indépendante de cette dernière scissure, qui appartient exclusivement à la face externe de l'hémisphère, et qui ne communique avec elle, chez les anosmatiques, que par suite de l'atro-

(1) J'ai donné cette démonstration dans mon Mémoire sur la nomenclature cérébrale (Revue d'anthropologie, 1878, p. 228, et dans ce volume, p. 690) et dans mon mémoire sur l'Anatomie comparée des circonvolutions, même volume, p. 402, 428, 456, 460, 466, et dans ce volume, p. 277, 305, 336, 340, 347 . 
phie de la racine olfactive externe. Désignée chez la plupart des mammifères sous le nom de vallée de Sylvius, elle doit conserver ce même nom dans le cerveau humain.

Ge qui la différencie essentiellement des scissures, des sillons et généralement de toutes les autres anfractuosités, c'est que celles-ci se produisent par suite de l'élévation de leurs bords et se creusent d'autant plus que la partie correspondante du manteau se développe davantage, tandis que la vallée de Sylvius se creuse par suite de l'atrophie des parties qui forment sa base.

Pour examiner chez l'homme le fond de la vallée de Sylvius il faut soulever la pointe du lobe temporal; on voit alors qu'il existe entre le bord postérieur du lobule orbitaire du lobe frontal et la partie profonde de l'extrémité antérieure du lobe temporal, un intervalle assez large et criblé de petits trous vasculaires. C'est l'espace perforé de Vicq-d'Azyr. Sa partie interne, qui est la plus large, s'étend jusqu'au bord externe du chiasma. Sa partic externe, plus étroite, aboutit au pli falciforme, pli profond étendu de la face profonde de la première circonvolution temporale ou de la pointe du lobe temporal à l'extrémité postérieure de la circonvolution la plus externe du lobule orbitaire. Le bord antérieur de la vallée de Sylvius est limité par les deux racines olfactives blanches. Ces deux racines, très grêles et adhérentes à la substance cérébrale, se séparent en divergeant sous un angle très obtus; l'interne se dirige vers la face interne de l'hémisphère, l'externe se porte transversalement en dehors jusqu'au niveau du pli falciforme sur lequel elle s'applique en se recourbant et en se portant en arrière pour se jeter sur la pointe du lobe temp oral. G'est cette dernière racine qui, très volumineuse chez les osmatiques, sépare la vallée de Sylvius, très peu profonde, de la scissure de Sylvius. Le pli falciforme constitue donc la ligne de démarcation entre la vallée et la scissure et correspond à ce que nous appellerons l'entrée de la scissure de Sylvius.

b. La scissure de Sylvius commence au niveau de cette entrée, sur le pli falciforme, qui est caché dans sa profondeur, sur le bord externe de l'hémisphère; de là elle se porte en arrière et un peu en haut sur la face externe de l'hémisphère, où elle se termine, après un trajet légèrement curviligne, long en moyenne de 
8 centimètres. On peut la subdiviser en trois portions : l'une antérieure, longue de 1 à 2 centimètres, qui remonte assez fortement, en décrivant une courbe convexe vers l'avant; une partie moyenne beaucoup plus longue, presque rectiligne, presque horizontale, quoique continuant pourtant à remonter un peu, et une partie postérieure qui se relève assez fortement de manière à former avec la précédente une courbe dont la concavité est tournée en avant. Cette portion relevée, dont la longueur et la direction varient beaucoup, se termine sur la convexité de l'hémisphère vers l'uuion du tiers postérieur avec le tiers moyen, un peu plus en arrière cependant; car, la longueur totale de l'hemisphère étant représentée par 100 , la partie située en arrière de l'extrémité postérieure de la scissure de Sylvius est en moyenne de 36 environ. Dans son ensemble, la scissure présente la lur'me d'une 's italique couchée et très allongee.

Son bord inférieur est formé par le bord supérieur du lobe temporal; son bord supérieur est formé en avant par le lobe frontal et en arrière par le lobe pariétal. Cies deux bords sont rapprochés jusqu'au contact, si ce n'est tout en avant, où s'interpose entre eux un prolongement de la pie-mère d'une épaisseur insolite.

A la partie antérieure du bord supérieur existent les deux branches antérieures de la scissure de Sylvius, l'une horizontale, l'autre ascendante.

La branche horizontale (pl. I, S") naît très près de l'entrée de la scissure de Sylvius. Elle se porte directement en avant, dans l'épaisseur de la circonvolution frontale la plus externe $\left(\mathrm{F}^{3}\right)$. Eille est absolument constante chez l'homme et chez les anthropoïdes. Elle manque chez tous les autres animaux. Elle a généralement 2 centimètres de long, quelquefois 3 et même un peu plus; eile occupe toute l'épaisseur de la circonvolution, elle est donc très profonde et communique directement avec le fond de la scissure de Sylvius, c'est-à-dire a vec la fosse de Sylvius.

La branche horizontale est presque toujours visible sur la face externe du lobe frontal; quelquefois cependant elle se trouve reportée sur le bord qui sépare cette face externe de la face inférieure ou orbitaire, et quelquefois enfin elle appartient plutôt à la face inférieure qu'à la face externe ; cette dernière disposition, 
commune chez certains singes anthropoïdes, est assez rare chez l'homme.

A quelques millimètres au-dessus de la branche horizontale, quelquefois au même niveau, en formant avec elle un $\mathrm{V}$, quelquefois même par une branche commune bifurquée en $\mathrm{Y}$, naît la branche ascendante de la scissure de Sylvius (pl. I, s), qui se dirige en haut et un peu en avant. Cette seconde branche est à peu près de la même longueur que la précédente ; elle a la même profondeur, les mêmes connexions, la même constance. Toutes deux sont de même nature; ce sont des anfractuosités produites sur le bord d'une circonvolution $\left(F^{3}\right)$, qui se replie deux fois sur elle-même en formant deux méandres (voir pl. I); ce sont donc en réalité des incisures, mais elles ne doivent pas être confondues avec les incisures ordinaires, car elles ont l'importance des caractères typiques. G'est par elles qu'on apprécie le mieux le perfectionnement du cerveau dans les rangs les plus élevés de la série des primates, car leur formation est due à l'allongement de la troisième circonvolution frontale, qui, devenue plus longue que la région qu'elle occupe, se replie en décrivant un ou deax méandres. Elles manquent toutes deux chez les singes cébiens eł pithéciens. Lorsqu'on arrive à la famille des anthropoïdes, on voit apparaître la branche horizontale, qui est constante chez eux comme chez l'homme. L'existence de cette branche est le principal et presque le seul caractère qui distingue le type cérébral des anthropoïdes de celui des autres singes. La branche horizontale existe seule chez les gibbons et les gorilles. La branche ascendante apparaît dans les deux genres orang et chimpanzé, tantôt rudimentaire et représentée par un simple feston, tantôt à l'état d'incisure véritable, mais elle est loin d'être constante chez ces animaux; elle manque environ une fois sur deux, tantôt d'un seul côté et tantôt, ce qui est moins commun, des deux côtés à la fois. Ghez l'homme enfin on peut dire qu'elle est constante, les cas où elle manque peuvent être considérés comme anormaux, car ils sont tout à fait exceptionnels, et ils s'observent pour la plupart, non tous cependant, sur les cerveaux d’imbéciles, d’idiots ou de microcéphales; mais, même sur ces cerveaux imparfaits, l'existence des deux branches antérieures de la scissure de Sylvius est la règle la plus ordinaire. 
La troisième circonvolution frontale de l'homme décrit quelquefois plus de deux méandres, et on peut dès lors observer au-dessus ou en arrière des deux branches antérieures, dont la pointe est fixe, une ou même deux incisures supplémentaires dont la position est variable. Mais il faut se garder de prendre pour des incisures émanées de la scissure de Sylvius certaines anfractuosités voisines qui viennent quelquefois y aboutir par un prolongement superficiel. C'est ainsi que le sillon prérolandique, qui sera décrit plus loin, prolonge quelquefois son extrémité inférieure jusque sur la marge supérieure de la scissure de Sylvius, marge qu'elle n'entame d'ailleurs que dans sa couche superficielle, sans jamais pénétrer jusqu'au fond de la scissure, On s'en assure en écartant les bords de l'anfractuosité. Mais lorsqu'on se borne à considérer l'apparence extérieure, on peut dire que ce sillon est une branche de la scissure; et quelques auteurs ont cru que c'étaitlà la branche ascendante de la scissure de Sylvius, ajoutant naturellement que cette branche n'était pas constante, car ils ne la retrouvaient pas dans la très grande majorité des cas. La vraie branche ascendante, au contraire, est constante; clle est situće heancoup plus en avant, très près de la branche antérieure, et la petite portion de la troisième circonvolution frontale qui est comprise entre ces deux branches a une forme triangulaire qui lui a valu le nom de cap de la troisième circonvolution frontale (1).

Le bord supérieur de la scissure de Sylvius présente dans sa moitié postérieure, qui correspond au lobe pariétal, des incisures ordinaires assez petites et en nombre variable qui pénètrent dan 3 la seconde circonvolution pariétale, et qui sont les incisures pariétales. Il y en a toujours au moins une, ordinairement deux, quelquefois trois. On en retrouve toujours au moins une, et quelquefois deux, chez l'orang et le chimpanzé. Il n'y en a qu'une seule chez le gorille; elle n'existe qu'exceptionnellement, et à l'état rudimentaire chez les gibbons et les pithéciens. Ces détails permettent d'apprécier l'importance des incisures pariétales.

(1) J'ai donné dans mon Mémoire sur le cerveau du gorille (Revue d'anthropologie, $2^{e}$ série, t. I, p. 18-22, 1878, et dans ce volume, p. 620-625), une description et une histoire plus complètes des branches de la scissure de Sylvius. Voir aussi mon Mémoire sur la nomenclature cérébrale, même volume, p. 229-231, et dans ce volume, p. 692-696. 
Une ou deux incisures, moins importantes, s'observent souvent à peu près au même niveau que les précédentes sur le bord inférieur ou temporal de la scissure de Sylvius. Ces incisures temporales sont le prolongement de deux sillons obliques qui existent constamment dans le fond de la scissure de Sylvius, mais qui ne s'étendent pas toujours jusqu'à la surface extérieure.

c. La fosse de Sylvius. Lor'squ'on examine la face externe de l'hémisphère sur un fotus humain de quatre à cinq mois, on $\mathrm{y}$ voit une dépression triangulaire nettement limitée par trois bords inégaux : c'est la fosse de Sylvius (fig. 86).

Le bord inférieur $a b$, obliquement dirigé en arrière et un peu

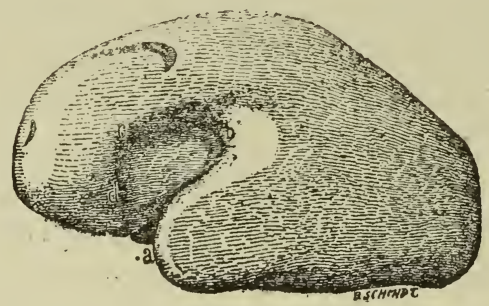

Fig. 86. Cerveau d'un fotus humain de cinq mois, face externe.

ab, bord inférieur de la fosse de Sylvius ; cb, " bord supérieur; cd, bord antérieur ad, entrée de la fosse de Sylvius.

en haut, est formé par le lobe temporal. Il est rejoint en $b$, sous un angle très aigu, par le bord supérieur cb qui est un peu plus court que lui, et qui est horizontal, et qui est formé en arrière par le lobe pariétal, en avant par le lobe frontal. Le troisième bord, $c d$, beaucoup plus court que les deux autres, est vertical, et formé en entier par le lobe frontal. Ce bord ne va pas rejoindre directement le bord inférieur; il reste entre $a$ et $d$ un petitintervalle, occupé par ce qui deviendra le pli falciforme; le petit intervalle ad constitue l'entrée de la fosse de Sylvius; c'est par là que celte fosse communique à la vallée de Sylvius, qui est déjà très prononcée à la face inférieure de l'hémisphère; c'est par là que passe l'artère cérébrale moyenne, ou artère sylvienne.

A cet âge, le fond de la fosse de Sylvius est formé par une surface lisse, légèrement convexe, qui est le lobe de l'insula encore tout à fait simple. Sur les limites de ce lobe la substance céré- 
brale se relève brusquement pour constituer les trois bords de la fosse, qui est entièrement à découvert. La base de chaque bord est séparée du lobe de l'insula par une ligne très nette, qui est l'une des rigoles de l'insula. Il y a done trois rigoles, l'une inférieure, l'autre supérieure, l'autre antérieure, correspondant respectivement aux trois bords de la fosse, et circonscrivant de toutes parts le lobe de l'insula, excepté à l'entrée de la fosse, ad.

Quant au reste de la surface de l'hémisphère, on n'y aperçoit qu'un petit nombre d'anfractuosités rudimentaires, mais pas encore de circonvolutions véritables. Les circonvolutions se développent pendant les mois suivants; on voit alors les bords de la

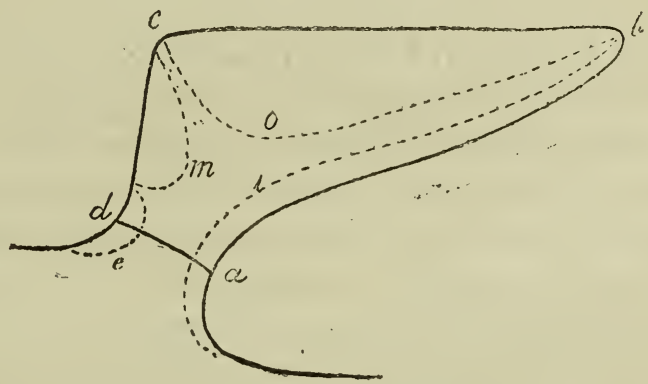

Fig. 87. Figure s chématique pour montrer le mode de disparition de la fosse de Sylvius. $a d$, entrée de la fosse et pli falciforme; $e$, renflement du lobe frontal ; $i$, élévation du bord inférieur ; $o$, abaissement du bord supérieur ; $m$, rétrogradation du bord antérieur (allant former le cap de la troisième frontale); $c o b$, opercule de l'insula.

fosse de Sylvius devenir de plus en plus épais; en même temps, les lobes environnants s'élargissent, se subdivisent en circonvolutions, et celles de ces circonvolutions qui bordent la fosse de Sylvius, refoulées par leurs voisines, empiètent peu à peu sur l'aire de la fosse qu'elles finissent par recouvrir entièrement en passant pardessus le lobe de l'insula.

Ciet empiètement graduel se produit simultanément, mais non au même degré, sur tout le pourtour de la fosse. Au niveau de l'entrée de la fosse $d a$ (fig. 87), la pointe du lube temporal $a$ se porte en avant, et la portion $d$ du lobe frontal qui lui fait face se renfle en un feston $e$ qui se porte en arrière, en recouvrant de plus en plus le pli falciforme ad qui finira par être entièrement masqué. Quant à la fosse elle-même, elle est recouverte à la fois par le bord inférieur qui s'élève en $i$, par le bord supérieur qui 
s'abaisse en $o$, et par le bord antérieur qui rétrograde en $m$. Les trois rigoles de l'insula deviennent ainsi très profondes.

Le bord inférieur ou temporal est celui qui se déplace le moins, il ne s'élève qu'assez peu au-dessus du niveau de la rigole inférieure, mais le bord supérieur ou bord fronto-pariétal s'abaisse considérablement au-dessous du niveau de la rigole supérieure; c'est lui qui recouvre la plus grande partie de la fosse de Sylvius. Il forme une sorte de lambeau, cob, qui retombe par-dessus le lobe de l'insula, et qui constitue l'opercule sylvien ou de l'insula:

Les deux bords $o b$ et $i b$ se rapprochent aisément jusqu'au contact, mais en avant ils ne peuvent se rejoindre, et la partie antérieure du lobe de l'insula resterait à découvert si le bord antérieur $c d$ ne se développait à son tour en formant une saillie en $m$ qui est en réalité un petit opercule, et qui comble peu à peu l'espace vide compris entre l'opercule proprement dit et la partie antérieure du lobe temporal. Cette saillie $m$, que nous appelons le cap de la troisième circonvolution frontale, se développe assez lentement. Le plus souvent, au moment de la naissance, il reste entre les trois bords un petit espace triangulaire, à travers lequel on aperçoit encore une portion du lobe de l'insula, mais bientôt ces bords arrivent au contact, n'étant plus séparés que par le prolongement de la pie-mère. L'insula est alors complètement recouvert, et l'anfractuosité sylvienne est parvenue, dans toutes ses parties, à son état définitif.

Les lignes suivant lesquelles les divers bords se touchent constituent la scissure de Sylvius proprement dite et ses deux branches antérieures. La scissure elle-mêmelonge le bord supérieur du lobe temporal, $i$, sur lequel viennent s'appliquer tout en avant, à l'entrée, le petit lobule terminal $e$ de la troisième circonvolution frontale, immédiatement au-dessus de la pointe $m$ du cap de la même circonvolution, et de là, entin, jusqu'à son extrémité postérieure le bord inférieur ob de l'opercule. De la rencontre du même lobule terminal $e$ avec le cap $m$ résulte la branche horizontale de la scissure, et la branche ascendante enfin résulte du contact du cap avec le bord antéricur' co de l'opercule.

Lorsqu'on écarte les bords de cette scissure, on pénètre dans la fosse de Sylvius. Pour apercevoir dans son entier le lobe de 
l'insula et ses trois rigoles, il faut abaisser le bord temporal, relever l'opercule et renverser le cap en avant.

Les rigoles del'insula, établissant les limites d'un lobe, rentreraient à la rigueur dans la définition générale des scissures. Toutefois leur constitution, comme on vient dele voir, est toute spéciale. La disposition des parties qui les entourent, celles du lobe de l'insula qu'ellescirconscrivent, sontsans analogue dans le reste du manteau, et il y a tout avantage à constater ce fait par une dénomination particulière. D'ailleurs, elles sont situées dans le fond de la scissure de Sylvius, et si on les appelait scissures, ces trois scissures incluses dans une autre qui est déjà très compli-quée embrouilleraient beaucoup les descriptions. Le nom de rigoles (Rinne), qui leur a été donné par Reil, est donc préférable.

$2^{\circ}$ La scissure de Rolando (pl. I et II, RR). La scissure de Rolando a été décrite pour la première fois en 1839 par Leuret, qui lui donna le nom de sillon de Rolando (1829). Rolando n'avait pas parlé de cette anfractuosité, mais, après Vicq-d'Azyr(1786), et plus nettement que lui, il avait décrit comme constantes les deux circonvolutions ascendantes qui la limitent (1). Leuret est d'ailleurs le premier qui en ait reconnu l'importance. C'est lui en réalité qui l'a découverte. Cette découverte a été le trait de lumière qui a éclairé la question jusque-là ténébreuse des circonvolutions cérébrales de l'homme.

La scissure de Rolando établit sur la face convexe de l'hémisphère la séparation du lobe frontal et du lobe pariétal (2).

(1) L'éditeur posthume des OEuvres complètes de Vicq-d'Azyr ayant sottement supprimé et remplacé par une sorte de caricature la planche originale sur laquelle Vicq-d'Azyr a, en 1786, représenté les deux circonvolutions ascendantes, Leuret a cru que Rolando seétait trompé en attribuant à Vicq-d'Azyr la découverte de ces deux circonvolutions, et il a cru que c'était Rolando qui les avait fát connaître le premier. (Voir ma communication à l'Académie de médecine, séance du 8 août 1876, p. 83 1-833, et dans ce volume, p. 556.)

(2) La limite postérieure du lobe frontal avait d'abord été placée par Gratiolet (1854) en avant de la scissure de Rolando, sur le sillon que nous appelons prérolandique; mais en 1837 , il fixa défnitivement cette limite sur la scissure de Rolando.

Leuret, Gratiolet et la plupart des auteurs qui les ont suivis ont décrit cette scissure comme exclusivement propre aux primates. Quelques autres ont cru la reconnaitre chez les carnassiers, dans le sillon crucial de Leuret; mais j'ai démontré dans un travail récent qu'elle existe, quoique très modifiée dans sa position et dans sa direction, chez tous les mammifères à circonvolutions, et qu'elle 
Elle est rétrécie vers le milieu de la face convexe de l'hémisphère, qu'elle traverse dans la plus grande partie de sa largeur. Elle n'est pas transversale, sa direction est oblique de haut en bas et d'arrière en avant. Elle est comprise entre deux circonvolutions dites ascendantes, qui appartiennent respectivement au lobe frontal et au lobe pariétal (circonvolution frontale ascendante et circonvolution pariétale ascendante).

Elle commence sur le bord sagittal, un peu en arrière du milieu de la longueur totale de l'hémisphère, et elle va se terminer en bas et en avant, au-dessus de la partie moyenne de la scissure de Sylvius, un peu en avant du milieu de la longueur totale de l'hémisphère.

Si l'on représente par 100 la longueur totale FO de l'hémisphère, mesurée en ligne droite de l'extrémité antérieure du lobe frontal à l'extrémité postérieure du lobe occipital, on trouve que la portion FR de cette longueur qui est en avant de l'origine $R$ de la scissure de Rolando représente environ 60 pour 100 de FO, et que la partie $\mathrm{FR}^{\prime}$ qui est en avant de la terminaison $\mathrm{R}^{\prime}$, de la scissure représente environ 43 pour 100 de FO. Ces chiffres moyens donnent une idée de l'obliquité générale de la scissure.

Son extrémité supérieure ou postérieure naît dans le pli de passage fronto-pariétal supérieur, sur le bord sagittal de l'hémisphère, en un point qui présente des rapports assez tixes avec le bourrelet du corps calleux. Une ligne verticale, menée par le bord postérieur de ce bourrelet, passe tantôt (1 fois sur 4) sur l'origine même de la scissure, tantôt un peu en avant (maximum +5 millimètres), tantôt un peu en arrière (maximum -8 ) et en moyenne à 1 millimètre et demi en arrière. Ce dernier chiffre étant assez petit pour être négligé, on peut dire que la scissure de Rolando commence au-dessus du bord postérieur du bourrelet du corps calleux.

Le tracé qu'elle suit, toujours très sinueux, semble au premier abord tout à fait capricieux. Ces sinuosités toutefois présentent, au milieu de leurs variétés individuelles, quelques caractères constants. 
A son origine, sur le bord sagittal, la scissure de Rolando suit ordinairement, dans une étendue d'environ 1 centimètre, quelquefois de 2 centimètres et plus, une direction à peu près longitudinale, puis elle se détache du bord sagittal, pour se porter plus ou moins directement en dehors ou en avant. Après avoir traversé ainsi le tiers environ de la largeur de la face convexe, elle se recourbe vers le bas, et quelquefois même un peu vers l'arrière, en décrivant une flexuosité à convexité antérieure, qui est le genou supérieur de la scissure de Rolando. A cette direction descendante succède bientôt une direction oblique en avant, aboutissant au genou inférieur, au-dessous duquel la scissure se porte de nouveau vers le bas, tantôt directement, tantôt en inclinant un peu vers l'avant ou vers l'arrière. Entre les deux genoux, qui forment deux courbes convexes vers l'avant, est comprise une portion moyenne dont la courbe est inverse, c'est-à-dire concave vers l'avant. La scissure se termine ordinairement à quelques millimètres au-dessus du bord supérieur de la scissure de Sylvius, dont elle est séparée par le pli de passage frontopariétal inférieur. Ce pli, qui met en continuité les deux circonvolutions ascendantes à leur extrémité inférieure, fait partie de l'opercule. 11 est plus ou moins épais. Quelquefois il est très mince, et la scissure de Rolando se prolonge sur lui sous la forme d'une sorte de queue superficielle. qui arrive jusqu'à la scissure de Sylvius. Les deux scissures paraissent alors communiquer l'une avec l'autre; mais ce n'est qu'une apparence : si l'on écarte légèrement les bords des deux scissures, on voit qu'elles sont toujours très nettement séparées par le pli de passage frontopariétal inférieur, ce pli occupant seulement une situation profonde, et que l'opercule reste indivis.

Disons à cette occasion que la plupart des variétés de l'hémisphère, celles mêmes qui paraissent porter atteinte aux connexions des parties et constituent par conséquent des anomalies, dépendent, comme celle qui vient d'être décrite, de la position superficielle ou profonde des plis de communication, différence qui peut être importante au point de vue morphologique, mais qui, au point de vue anatomique, est insignifiante. Certains plis de communication sont toujours superficiels, d'autres sont toujours profonds, mais la plupart peuvent être tantôt superficiels, tantôt 
profonds, soit que l'une des positions soit ordinaire et l'autre exceptionnelle, soit que toutes deux aient à peu près la même fréquence. Nous aurons souvent l'occasion de rappeler cette remarque.

On vient de voir que le pli de passage fronto-pariétal inférieur est presque toujours superficiel, quoique pouvant être par exception plus ou moins profond. Il y a deux autres plis fronto-pariétaux dont la position est invariable. L'un, le supérieur, est toujours superficiel; l'autre, le moyen, est toujours profond.

Le pli de passage fronto-pariétal supérieur est situé sur le bord sagittal de l'hémisphère ; il s'étend de l'extrémité supérieure de la circonvolution frontale ascendante à celle de la pariétale ascendante, en contournant l'origine de la scissure de Rolando, qui l'entaille plus ou moins profondément, mais jamais dans toute son épaisseur.

Le pli de passage fronto-pariétal moyen, toujours très profond, ne se voit que lorsqu'on dilate complètement la scissure de Rolando, dont il traverse le fond, au niveau et au-dessous du genou supérieur de la scissure. Il n'approche jamais de la surface; je ne l'ai vu devenir superficiel qu'une seule fois sur un cerveau d'idiot rendu méconnaissable par un grand nombre d'anomalies très graves. Le moule de cette pièce unique est déposé dans le musée.

Les deux genoux de la scissure de Rolando ne sont pas dus à des inflexions fortuites; leur formation est en rapport avec la constitution du lobe frontal. On verra plus loin que la circonvolution frontale ascendante $\left(\mathrm{F}^{\circ}\right)$, qui forme le bord antérieur de la scissure, donne insertion aux trois racines des trois circonvolutions frontales longitudinales. Or la portion concave de la scissure qui est comprise entre les deux genoux correspond à la portion de $\mathrm{F}^{0}$ qui fournit la racine de la seconde circonvolution frontale. Le genou supérieur correspond à l'espace compris entre cette scissure et celle de la première frontale, vis-à-vis l'origine du premier sillon frontal. Le genou inférieur est situé de même entre les racines de la deuxième etde la troisième frontale, et vis-à-vis l'origine du second sillon frontal. Ainsi s'expliquent l'existence constante et la position fixe des deux genoux de la scissure de Rolando. lls permettent de diviser la scissure et la 
circonvolution frontale ascendante en trois portions, supérieure, moyenne et inférieure, correspondant respectivement aux trois circonvolutions frontales, divisions importantes au point de vue de l'étude des localisations.

$3^{\circ}$ Scissure sous-frontale. La scissure sous-frontale déjà vue et représentée par Vicq-d'Azyr, sépare, sur la face interne de l'hémisphère, le lobe frontal du lobe du corps calleux et du lobe pariétal; elle se prolonge en outre un peu sur la face convexe, où elle entaille quelque peu la circonvolution pariétale ascendante. Elle a été décrite jusqu'ici comme un simple sillon. Elle a été appelée grand sillon du lobe fronto-pariétal (Gratiolet), sillon fronto-pariétal interne (Pansch), fissura calloso marginalis (Huxley) (1). D'après notre nomenclature elle doit porter le nom de scissure parce qu'elle étạblit une division lobaire.

Il est facile de montrer en effet que la circonvolution du corps calleux, qu'elle circonscrit dans la plus grande partie de son étendue, constitue à elle seule un lobe de l'hémisphère. Nous n'avons pas besoin, pour cela, de recourir à l'anatomie comparée qui rend cette détermination tout à fait évidente. Il suffira de dire qu'aucun 'auteur jusqu'ici ne l'a comprise ni dans le lobe frontal ni dans le lobe pariétal sous lesquels elle chemine, ni à plus forte raison dans les autres lobes de l'hémisphère. On l'a toujours décrite à part, comme une circonvolution toute spéciale, et puisqu'il est de règle que toute circonvolution doit appartenir à un lobe, nous sommes obligés de dire que la circonvolution du corps calleux forme une des divisions primaires de l'hémisphère, c'est-à-dire un lobe qui est le lobe du corps calleux (2).

La scissure sous-frontale est de beaucoup la plus longue de toutes les anfractuosités cérébrales. Si elle était redressée, sa longueur égalerait la longueur totale de l'hémisphère. Elle prend son origine sur la partie inférieure de la face interne, un peu au-dessous du bec du corps calleux, en avant de la commissure antérieure, sur un petit espace plan qui sera décrit plus loin et

(1) M. Huxley, comme les autres auteurs anglais, désigne indistinctement sous le nom de fissura ou de fissure toutes les anfractuosités du manteau.

(2) On trouvera des preuves multipliées et évidentes de cette détermination dans mon mémoire déjà cité sur l'Anatomie comparée des circonvolutions (1878). Voir aussi Bull. de la Société d'anthropologie, séance du 6 décembre 1877 et dans ce volume, p. 590 . 
que j'appelle le carrefour de l'hémisphère, se porte de là horizontalement en avant, dans une étendue de 2 à 3 centimètres, puis se relève en décrivant une forte courbe à convexité antérieure au-devant du genou du corps calleux, se réfléchit au-dessus de ce genou' en' se recourbant vers l'arrière, suivant une direction à peu près horizontale qui est d’'abord presque parallèle à celle de la rainure du corps calleux, mais qui ensuite s'en écarte peu à peu; parvenue vers le niveau du tiers postérieur du corps calleux, la scissure sous-frontale se relève une seconde fois en décrivant une seconde courbe dont la concavité est antérieure, atteint le bord sagittal de l'hémisphère, à quelques millimètres en arrière de l'extrémité supérieure et postérieure de la scissure de Rolando, et se prolonge enfin sur la face convexe, dans une étendue d'environ 1 centimètre, en pénétrant dans l'extrémité supérieure de la circonvolution pariétale ascendante, où elle se termine.

L'espace étroit compris, sur le bord sagittal de l'hémisphère, entre la terminaison de la scissure sous-frontale et l'origine de la scissure de Rolando, est occupé par le pli de passage frontopariétal supérieur déjà mentionné. Ce pli étant toujours superficiel, les deux scissures ne communiquent jamais l'une avec l'autre.

A l'exception du petit prolongement que nous venons d'indiquer, la scissure sous-frontale appartient exclusivement à la face interne de l'hémisphère, où elle établit, dans toute sa longueur, la limite du lobe frontal. Ses rapports essentiels sont ceux qu'elle affecte avec ce lobe, dont elle mesure en quelque sorte le développement dåns toute la série des mammifères. Lorsqu'il est rudimentaire, elle se réduit à une dépression courte et légère qui marque entre lui et le lobe du corps calleux une démarcation plutôt qu'une séparation véritable; c'est le type le plus ordinaire (voir les figures 84 et 85 ); mais chez les primates, le lobe frontal, énormément agrandi, s'étend considérablement en arrière aux dépens du lobe pariétal qu'il refoule (1),

(1) J'ai montré ailleurs que la plupart des caractères qui constituent le type cérébral des primates et qui le distinguent profondément de tous les autres sont la conséquence plus ou moins directe de cet agrandissement énorme du lobe frontal. Le type cérébral des primates peut donc être défini en deux mots : prédominance frontale. (Voir mon mémoire surl'Anatomie comparée des circon. volutions,) 
et la scissure sous-frontale, devenue grande et profonde, se prolonge commelui, de telle sorte que, le séparant en avant du lobe du corps calleux, elle le sépare en outre en arrière, du lobe pariétal. Ce dernier rapport n'est donc pas essentiel, puisqu'il n'existe que dans un seul ordre de mammifères; et le vrai caractère de la scissure sous-frontale, celui qui légitime son nom, c'est qu'elle limite, sur la face interne, le lobe frontal, comme le font, sur la face convexe, les scissures de Sylvius et de Rolando.

Nous allons étudier, d'après cette notion, le trajet long et compliqué de la scissure sous-frontale, en nous basant sur les rapports qu'elle affecte avec les diverses parties de la face interne du lobe frontal. Disons d'abord, par anticipation, que cette face interne est formée en haut et en arrière par le lobule ovalaire qui appartient à la circonvolution frontale ascendante, et dans le reste de son étendue par la face interne de la première circonvolution frontale, qui se décompose en deux parties correspondant à ses deux étages, car on verra plus loin que le lobe frontal, se réfléchissant sur la partie antérieure du centre ovale de Vieussens, de manière à passer de la région de la voûte à la région de la base, forme deux étages: l'un supérieur ou métopique placé

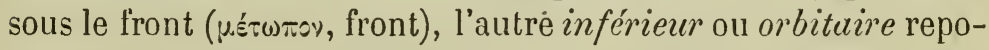
sant sur la voûte orbitaire et constituant le lobule orbitaire. Les deux étages de la première circonvolution frontale forment donc sur la face interne de l'hémisphère deux portions distinctes. Nous nommerons la supérieure le lobule métopique, l'inférieure le lobule sus-orbitaire. La face interne du lobe frontal se décompose donc en trois lobules qui sont, en procédant de bas en haut et d'avant en arrière, le lobule sus-orbitaire, le lobule métopique et le lobule ovalaire.

Cela posé, la scissure sous-frontale se décompose en trois arcs qui correspondent à ces trois lobules et dont la délimitation est établie sur la scissure elle-même par deux changements de direction, correspondant respectivement à deux plis de passage et à deux incisures, à deux points nommés le point de réflexion et le point d'inflexion.

Pour apprécier ces changements de direction on ne doit considérer que la direction générale de la scissure, en négligeant les plis de passage qui Ja traversent, et les festons qui rendent plus 
ou moins onduleuses certaines parties de son trajet. On voit alors que, dans toute la portion qui correspond à la première circonvolution frontale, la scissure décrit une grande courbe dont la convexité est dirigée en avant et dont la forme est comparable à celle d'un $ص$ couché; le point le plus antérieur de cette courbe correspondant au maximum de courbure est situé au-devant du genou du corps calleux. C'estle point de réflexion (fig. $88 \mathrm{~A}$ ) de la scissure, qui, après avoil, depuis son origine, longé le bord infé-

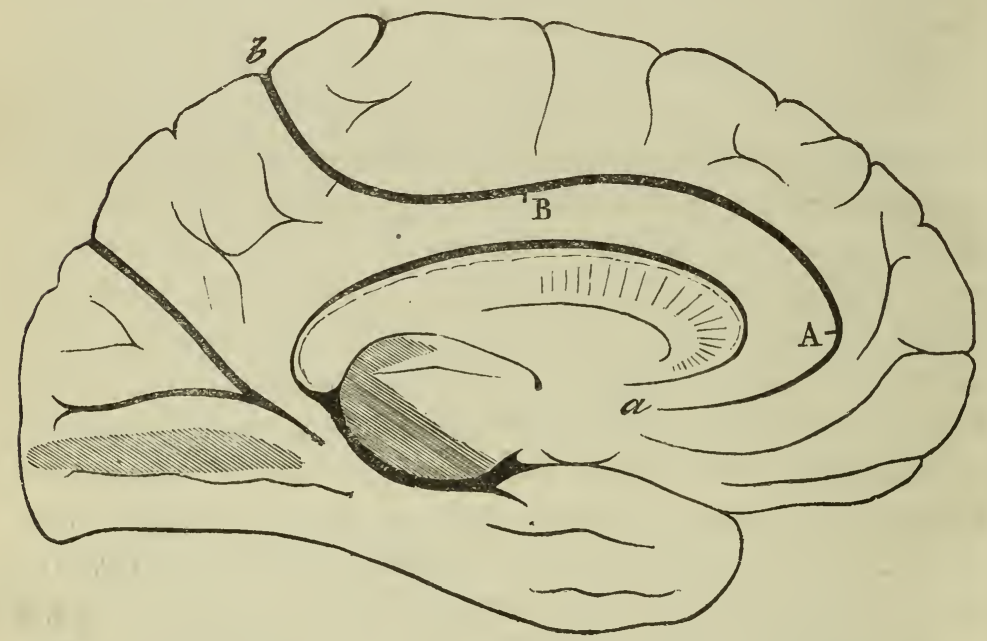

Fig. 88. Les trois ares de la scissure sous-frontale (figure schématique).

$A$, point de réflexion; $\mathrm{B}$, point d'inflexion; $a \mathrm{~A}$, are sus-orbitaire; $\mathrm{AB}$, are métopique ; $\mathrm{bB}$, are sous-ovalaire.

rieur du lobe du corps calleux, se réfléchit en ce point et se porte en arrière sur le bord supérieur de ce lobe. Parvenue sous l'extrémité postérieure du lobule métopique, la scissure, tout en continuant à cheminer vers l'arrière, change une secunde fois de direction en subissant le changement de courbure connu en géométrie sous le nom d'inflexion; jusque-là, en effet, la scissure avait décrit une courbe dont la convexité était tournée vers le lobe frontal, mais de là jusqu'à sa terminaison la courbure est dirigée en sens inverse, c'est-à-dire que la scissure embrasse dans sa concavité la partie postérieure du lobe frontal. Nous nommerons point d'inflexion ce point (B), où la concavité succède à la convexité, en ajoutant toutefois qu'il ne s'agit pas ici d'un point géo- 
métrique, mais plutôt d'une petite portion intermédiaire où la courbure génórale n'est ni concave ni convexe (1), cela ne veut point dire qu'elle soit rectiligne, car il y a au contraire presque toujours en ce lieu un feston assez prononcé qui correspond à la base d'un pli de passage.

Le point d'inflexion est situé tantôt un peu en arrière d'une verticale passant par le milieu de la longueur du corps calleux, tantôt sur cette verticale même.

Le point de réflexion et le point d'inflexion subdivisent la scissure sous-frontale en trois arcs.

Le premier arc, arc inférieur ou sus-orbitaire $(a \mathrm{~A})$, est compris entre l'origine de la scissure et le point de réflexion. Il sépare le lobule sus-orbitaire, qui est au-dessous de lui, de la première portion (ou portion ascendante) du lobe du corps calleux.

Le second arc, arc moyen ou métopique (AB), est compris entre le point de réflexion et le point d'inflexion. Il sépare le lobule métopique, qui le recouvre, de la seconde portion ou portion horizontale du lobe du corps calleux; mais tandis qu'il suit toute la longueur du lobule, il ne s'étend guère au-delà de la moitié antérieure du lobe du corps calleux.

Le troisième arc enfin, arc postérieur ou sous-ovalaire (bB), est compris entre le point d'inflexion et la terminaison de la scissure sous-frontale. Il correspond au lobule ovalaire qui remplit sa concavité. Il longe d'abord le bord inférieur de ce lobule qu’il sépare du lobe du corps calleux, puis son bord postérieur, qu'il sépare du lobule quadrilatère du lobe pariétal; alors, parvenu sur le bord sagittal de l'hémisphère, il envoie, comme on l'a vu plus haut, un court prolongement terminal sur la face convexe de l'hémisphère dans la circonvolution pariétale ascendante. Du côté de sa convexité, le lobe du corps calleux, graduellement élargi, se continue insensiblement avec le lobule quadrilatère, de sorte que rien n'iudique le lieu où elle passe de l'un sur l'autre.

La division de la scissure sous-frontale en trois ares est donc

(1) En géométrie, le point d'inflexion est celui où la courbe traverse sa tangente ; c'est un point absolu, mais dans le voisinage de ce point, les deux portions de la courbe se confondent presque entièrement avec leur tangente commune, de sorte qu'il y a toujours une portion intermédiaire qui paraît rectiligne 
établie à la fois par ses changements de direction au point de réflexion et au point d'inflexion, et par ses rapports avec les trois lobules de la face interne du lobe frontal. Elle est établie en outre par deux plis de passage et par deux incisures dont nous allons maintenant nous occuper.

Ces plis de passage sont constants. Ils s'étendent, à travers la scissure sous-frontale, du lobe du corps calleux aux deux extrémités du lobule métopique.

Le premier naît du lobe du corps calleux, au niveau du point d'inflexion dont la position est déjà connue; il se porte en haut en décrivant une petite sinuosité, et va se jeter sur l'extrémité supérieure et postérieure du lobule métopique. Il est donc situé au-devant du lobule ovalaire et nous le nommerons le $p l i$ de passage préovalaire. Son épaisseur est variable; il en résulte qu'il est tantôt superficiel, tantôt profond; dans le premier cas il interrompt tout à fait le trajet de la scissure sous-frontale et l'are sous-ovalaire se trouve séparé de l'arc métopique. Dans le second cas, qui est plus fréquent, les deux arcs se continuent l'un avec l'autre, mais en écartant les bords de la scissure, on aperçoit le pli de passage, toujours très prononcé et toujours bien caractérisé par ses connexions. Le cas lo plus commun est celui où il est presque entièrement superficiel, mais où cependant la partie moyenne est déprimée de 2 ou 3 millimètres seulement, comme on le voit sur notre cerveau schématique. L’insertion supérieure du pli de passage est indiquée sur la partie correspondante dı lobe frontal par deux incisures plus ou moins longues et à peu près verticales correspondant, l'une au bord antérieur du pli, l'autre à son bord postérieur. La disposition de ces deux incisures varie nécessairement beaucoup, suivant que le pli de passage préovalaire est superficiel ou profond. Il y en a toujours une, et une seule, tantôt l'antérieure, tantôt la postérieure, qui communique avec la scissure sous-frontale; l'autre reste à l'état d'incisure isolée. L'antérieure a peu d'importance; ce n'est que l'une des incisures du lobule métopique; mais la postérieure mérite plus d'attention, car c'est elle qui établit la ligne de démarcation entre ce lobule et le lobule ovalaire. Nous la nommerons l'incisure préovalaire. Je répète qu'elle est tantôt isolée, tantôt continue avec la scissure sous-frontale. Elle est 
constante chez l'homme et la plupart des singes, elle ne manque que chez les primates les plus inférieurs.

Le second pli de passage de la scissure sous-frontale est situé au point de réflexion. Il est presque toujours superficiel. Il naît de la portion la plus antérieure du lobe du corps calleux et se porte en avant, souvent aussi un peu en haut, pour se confondre avec le lobule métopique dontil constitue l'extrémité inférieure. Nous le nommerons le pli de passage prélimbique (1). Son bord inférieur est longé par l'incisure prélimbique qui fait suite à l'extrémité antérieure de l'arc inférieur ou sous-orbitaire de la scissure sous-frontale. Cette incisure, qui se dirige d'abord en avant, mais qui ensuite peut se relever plus ou moins, traverse la plus grande partie de la face interne du lobe frontal et s'étend même quelquefois jusqu'au bord sagittal de l'hémisphère. Elle établit la séparation du lobule métopique, dont elle longe le bord inférieur, et du lobule sus-orbitaire dont elle longe le bord supérieur.

Le pli de passage prélimbique est ordinairement superficiel chez l'homme et les anthropoïdes, il est profond chez les pithéciens, et il l'est quelquefois aussi chez l'homme; mais il est constant, on l'aperçoit toujours en écartant les bords de la scissure sous-frontale, et sa position est toujours indiquée à l'extérieur par l'incisure prélimbique. Lorsqu'il est superficiel, l'arc susorbitaire de la scissure sous-frontale ne se continue pas avec l'arc métopique, et paraît se confondre avec l'incisure prélimbique qui continue sa direction; on pourrait donc être tenté de croire que l'arc sus-orbitaire ne se rattache pas au reste de la scissure sous-frontale. Mais les cas où le pli de passage est profond font disparaître cette supposition: on voit alors que l'arc sus-orbitaire de la scissure se continue pleinement avec l'arc métopique en formant une seule et même courbe comparable à un $ص$ couché; la limite des deux arcs n'est plus indiquée que par l'incisure prélimbique qui se détache horizontalement du point le plus antérieur de la courbe et se porte en avant dans le lobe frontal.

Lorsqu'on écarte les bords de la scissure sous-frontale entre les deux plis de passage que nous venons de décrire, on y aperçoit

(1) Voir p. 723, l'explication de cette épithete de prélimbique. 
ordinairement plusieurs autres plis moins importants qui s'étendent du lobe du corps calleux au lobule métopique; quelquefois aussi un pli de même nature s'observe en arrière du point d'inflexion et se rend du même lobe au lobule ovalaire. Ces plis sont presque toujours petits et profonds; il peut se faire toutefois que l'un d'eux devienne superficiel et produise une interruption dans l'arc métopique, mais ils ne sont qu'accessoires; leur nombre et leur position sont variables suivant le degré de complication de l'hémisphère, tandis que les deux vrais plis de passage préovalaire et prélimbique sont fixes et constants.

Les divisions que nous avons établies dans la scissure sousfroutale résultent de ses rapports avec le lobe frontal, qui constitue l'un de ses bords. Les rapports de l'autre bord ont fourni à M. le professeur Giacomini, de Turin, la base d'une autre division. Cet anatomiste éminent, dans son travail Sur les circonvo-lutions cérébrales de l'homme, a distingué dans la scissure sousfrontale (qu'il appelle fronto-pariétale interne) deux portions, l'une horizontale, comprise entre la circonvolution (lobe) du corps calleux et le lobe frontal, l'autre ascendante, séparant sur la face interne de l'hémisphère le même lobe frontal du lobe pariétal (1). Cette dernière portion comprend la moitié postérieure environ de notre arc sous-ovalaire; l'autre comprend tout le reste de la scissure sous-frontale. Ainsi, la seconde portion succède à la première là où le lobe du corps calleux se continue avec le lobule quadrilatère du lobe pariétal; mais j'ai déjà dit que cette continuation se faitd'une manière insensible, sans limite anatomique; le point de démarcation est donc incertain. Reste le changement de direction. Il est bien vrai qu'au niveau du lobe pariétal la scissure devient plus concave, plus ascendante; mais tout le reste de l'arc sous-ovalaire est concave et ascendant, quoique à un moindre degré, et le point où la concavité s'exagère assez pour qu'on puisse dire que la direction est changée demeure encore incertain. Si enfin on voulait prendre pour limite le point où la scissure cesse de reculer tout en continuant à monter, il n'y aurait plus d'incertitude, mais on verrait cette limite se déplacer consi-

(1) C. Giacomini, Guida allo studio delle circonvoluzione cerebrali del' uomo. Turin, 1878, gr. in-8, p. 81 . 
dérablement vers le haut ou vers le bas suivantles individus, car la forme de l'extrémité postérieure du lobule ovalaire est assez variable, et le moindre changement de forme suffit pour que le point le plus reculé de ce lobe se rapproche du bord sagittal ou de la base du lobule quadrilatère. La division proposée par le professeur Giacomini me paraît donc défectueuse, tandis que celle qui résulte des rapports de la scissure sous-frontale avec le lobe frontal, a le double avantage de correspondre aux trois lobules de la face interne de ce lobe, et d'être déterminée par des points anatomiques précis.

Le point où la scissure sous-frontale rejoint le bord sagittal de l'hémisphère, et où s'arrête par conséquent le lobe frontal, est situé en moyenne à 6 ou 7 millimètres en arrière d'une verticale menée par le bord postérieur du bourrelet du corps calleux; la verticale passe quelquefois sur ce point même, jamais en avant. On n'a pas oublié que l'origine de la scissure de Rolando est située en moyenne à $1^{\mathrm{mm}}, \breve{g}$ en arrière de cette ligne; la différence de $ّ$ millimètres environ qui existe entre ces deux mesures représente l'épaisseur moyenne du pli de passage frontopariétal supérieur, qui sépare la scissure sous-frontale de la scissure de Rolando.

$4^{\circ}$ Scissure calcarine. La scissure calcarine est une scissure à peu près horizontale qui naît sur la limbe de lhémisphère, audessous du bourrelet du corps calleux, et qui de là se porte en arrière, jusqu'à l'extrémité postérieure de l'hémisphère, où elle se recourbe plus ou moins, pour se terminer sur le pôle occipital (voyez p. 670). Sa portion antérieure sépare le lobe calleux du lobe temporal et du lobe occipital ; elle a donc le caractère des vraies scissures, puisqu'elle établit une séparation lobaire. Sa portion postérieure, pénétrant dans le lobe occipital, où elle sépare seulement deux lobules, rentrerait dans la catégorie des sillons; mais ces deux anfractuosités n'en font manifestement qu'une seule, qui doit être décrite en une seule fois, et qui doit par conséquent prendre rang parmi les scissures.

Le fond de cette profonde scissure correspond, dans une partie de son étendue, à la partie inférieure de la cavité digitale ou ancyroïde, ou corne postérieure du ventricule latéral, et repose 
par conséquent sur l'ergot de Morand, ou petit hippocampe, qui forme cette partie inférieure. Ergot se dit en latin calcar, de là le nom de scissure calcarine. Cruveilhier, qui, le premier, je pense, en 1852, en reconnut la constance et en détermina les rapports, la décrivit sous le nom de anfractuosité de la cavité digitale(1). Gratiolet, en 1854, montra toute l'importance de cette scissure, il en fit avec raison l'un des principaux caractères du type cérébral des primates, mais, croyant à tort qu'elle se continuait avec la rainure du grand hippocampe, qui se trouve dans la fente de Bichat, il donna à ces deux anfractuosités, réunies par lui en une seule, le nom de scissure des hippocampes. D'un autre côté, plusieurs auteurs, à l'exemple de Vicq-d'Azyr, au lieu de la prolonger en avant, en avaient au contraire retranché la partie antérieure, qu'ils avaient ajoutée à la scissure occipitale interne. Enfin M. Huxley, corrigeant à la fin cette erreur et celle de Gratiolet et restituant à cette scissure ses vraies limites, telles que Gruveilhier les avait indiquées, lui donna le nom de scissure calcarine, pour constater qu'elle est étrangère au grand hippocampe et qu'elle est en rapport seulement avec le petit (2).

La scissure calcarine se voit sur la norma interne de l'hémisphère. Cette norma montre à la fois une vue de champ de toute

(1) Cruveilhier, Anatomie descriptive, 1re édition, 1836, t. IV, p. 663. Il est bien étonnant que cet auteur n'ait pas vu en même temps la scissure occipitale externe, et ait continué à nier l'existence du lobe postérieur. La scissure calcarine est assez bien représentée sur la planche XXV du grand ouvrage de Vicqd'Azyr, mais elle n'est pas mentionnée dans le texte, à l'exception de la première portion que l'auteur confond avec la scissure occipitale. Celte planche doit être étudiée dans l'édition originale in-folio (1786); dans les prétendues OEuvres complètes de Vicq-d'Azyr, publiées après sa mort, elle porte !e numéro XXII; elle y est défigurée et remplacée par une abominable caricature où l'on ne retrouve plus ni la scissure calcarine ni la scissure sous-frontale.

(2) Je conserve cette dénomination qui est assez généralement adoptée au jourd'hui et qui est exacte chez tous les primates. Il existe chez beaucoup d'autres mammifères (exemple les ruminants, les solipèdes, etc.), un sillon qui est manifestement l'analogue de la scissure calcarine, quoique ces animaux n'aient. pas de lobe occipital el qu'ils n'aient par conséquent ni cavité digitale ni petit hippocampe; j'ai décrit néanmoins ce sillon sous le nom de sillon calcarin, pou: faciliter l'étude de son évolution dans la série des mammifères (Revue d'anthro. pologie, 1878, p. 442 et dans ce volume, p. 320-321). Le seul nom qui serait correctement applicable à tous les cas serait celui de sillon ou de scissure rétro-lim. lique,car cette anfractuosité prend naissance dans le pli de passage rétro-limbique, qu'elle dédouble et qui existe, simple ou dédoublé, chez tous les mammifères. 
la face interne et une vue fuyante des portions temporale et tentoriale de la face inférieure. La face interne, en arrière du bourrelet du corps calleux, se continue avec la surface tentoriale par un bord très émoussé, qui est le bord tentorial. La scissure calcarine longe approximativement ce bord.

Lorsqu'on examine cette région sur un hémisphère encore revêtu de ses membranes, on peut y reconnaître, malgré la présence de la pie-mère, la position de certaines anfractuosités. Deux d'entre elles, beaucoup plus marquées que les autres, dessinent une figure comparable à un خ couché. La branche inférieure et la queue de l'Y sont formées par la scissure calcarine, la branche supérieure par la scissure occipitale interne. Les deux branches de l'Y limitent un lobule triangulaire qui forme la face interne du lobe occipital et qui porte le nom de cunéus (coin).

Dans cet état, c'est-à-dire avant l'ablation des membranes, la queue de l'Y semble souvent se continuer plutôt avec la branche supérieure qu'avec la branche inférieure; voilà pourquoi quelques auteurs l'ont rattachée à la scissure occipitale, et non à la scissure calcarine; en outre, elle semble provenir directement de la fente de Bichat, et se prolonger par conséquent dans le ventricule latéral, en séparant complètement le lobe du corps calleux du lobe temporal ; voilà pourquoi Gratiolet a cru qu'elle se combinait avec les rainures de l'hippocampe. Mais, lorsqu'on enlève les membranes et qu'on écarte les bords des scissures, on reconnaît que ces apparences sont trompeuses.

D’une part, en effet, on voit se détacher de la pointe du cunéus un pli de passage profond, qui se porte en avant et va se rendre dans la partie postérieure du lobe du corps calleux. C'est le pli de passage cunéo-limbique ; il est absolument constant; il laisse au-dessus de lui la scissure occipitale et la sépare de la calcarine, dont il forme à ce niveau le bord supérieur. La queue de l'Y appartient donc à la scissure calcarine ; elle en constitue la première portion, et elle se continue directement en arrière avec la seconde portion, qui longe le bord inférieur du cunéus.

Le pli de passage cunéo-limbique, quoique très marqué, est toujours profond chez l'homme, et de la sorte une communication superticielle s'établit à la pointe du cunéus, entre la scissure 
calcarine et l'extrémité inférieure de la scissuręoccipitale interne; la même disposition existe chez les gibbons, mais chez tous les autres primates et chez les lémuriens, le pli cunéo-limbique est superficiel et souvent même très épais, et la scissure occipitale est tout à fait indépendante de la scissure calcarine. En second lieu, la queue de l'Y, c'eśt-à-dire la première portion de la scissure calcarine ne communique jamais avec la fente de Bichat. Elle en est toujours séparée par un pont de substance cérébrale, qui concourt à former le bord de cette fente et qui met l'extrémité inférieure du lobe du corps calleux en continuité avec l'extrémité postérieure de la circonvolution temporale de l'hippocampe ; ce pli, qui passe du lobe du corps calleux (limbique) au lobe temporal, est le pli de passage temporo-limbique. C'est une barrière qui sépare la scissure calcarine de la fente de Bichat. Il est plus ou moins large, plus ou moins épais, mais constant chez l'homme et chez la plupart des primates. Toutefois, chez quelques singes pithéciens et cébiens, il est tellement mince qu'il faut quelque attention pour en apercevoir le rudiment au fond des anfractuosités dilatées. C'est pour cela que Gratiolet, ayant pris le cerveau d'un de ces singes (le cercopithecus sabxus) comme type du cerveau des primates, a cru que la scissure calcarine traversait toute l'épaisseur du bord postérieur de la fente de Bichat, et se continuait, par la rainure de l'hippocampe, jusqu'au crochet de l'hippocampe.

Née dans l'épaisseur du pli de passage temporo-limbique, au dessous du bourrelet du corps calleux, la scissure de l'hippocampe se porte directement en arrière, jusqu'à la bifurcation de l'Y, qui correspond à la pointe du cuneus.

Cette première portion, longue d'environ 2 centimètres, établit une division lobaire. Elle laisse au-dessus d'elle le hord inférieur ou calcarin de l'extrémité postérieure du lobe du corps calleux, et au-dessous d'elle une circonvolution à la fois temporale et occipitale : temporale en avant, où elle forme la circonvolution de l'hippocampe $\mathrm{T}^{5}$; occipitale en arrière, où elle forme la circonvolution laplus interne $0^{5}$ du lobule sous-occipital. On verra plus loin qu'il n'y a aucune ligne de démarcation entre le lobe temporal et le lobe occipital. Le point où la circonvolution $T^{5}$ devient $0^{5}$ est done tout à fait indécis; on peut dire toutefois qu'il 
correspond à peu près au milieu de la première portion de la scissure calcarine.

La seconde portion de la scissure calcarine commence à la bifurcation de l'Y, sous la pointe du cuneus, ou encore sous le pli cunéo-limbique, et se porte de là en arrière, jusqu'à la pointe du lobe occipital, suivant un trajet qui est en moyenne à peu près antéro-postérieur, mais qui n'est jamais rectiligne.

Elle continue d'abord la direction de la première portion, puis se recourbe un peu vers le bas en décrivant une courbe à concavité inférieure, puis elle s'infléchit vers le haut et se termine en formant sur la pointe occipitale une dernière courbe à concavité supérieure. Son extrémité terminale se prolonge ordinairement. de quelques millimètres sur la face convexe dans le petit pli contourné qui est décrit plus loin sous le nom de pôle occipital. Elle est quelquefois bifurquée, mais le plus souvent elle est simple. La forme que nous venons de décrire est la plus fréquente, mais n'est pas constante, la première courbure pouvant être très faible ou même tout à fait nulle.

La seconde portion de la scissure calcarine chemine entre le lobule occipital interne ou cunéus, et le lobule sous-occipital. Séparés par elle dans toute leur longueur, ces deux lobules s'unissent au pôle occipital, aboutissant l'un et l'aụtre au petit pli polaire dans lequel se termine la scissure calcarine.

$5^{\circ}$ Scissure occipitale. La scissure occipitale est une scisșure transversąle qui établit sur la face interne et sur la face externe de l'hémisphère la séparation du lobe occipital et du lobe pariétal. Elle n'existe que chez l'homme, les singes et les lémuriens. Les autres animaux n'ont pas de lobe occipital; chez eux la portion du manteau qui forme le lobe occipital des primates est entièrement fusionnée avec le lobe pariétal.

La portion de cette anfractuosité qui se voit sur la face interne se nomme scissure occipitale interne; celle qui se voit sur la face convexe (ou externe) se nomme scissure occipitale externe. Ces deux portions sont séparées l'une de l'autre par un pli de passage occipito-pariétal (premier pli de passage de Gratioleí) qui correspond à peu près au bord sagittal et qui, chez l'homme, est le plus ordinairement superficiel. On les décrit donc généralement comme formant deux scissures distinctes. Mais chez la 
plupart des singes, et aussi chez quelques hommes, ce pli de passage est profond; la scissure occipitale interne se continue alors sans interruption avec l'externe, et il devient tout à fait évident qu'elles ne forment qu'une seule et même scissure. Il sera toutefois plus commode, dans les descriptions, de les distinguer sous les deux noms que nous venons d'indiquer.

La scissure occipitale interne, presque droite et très profonde, déjà très apparente à travers les membranes, saute pour ainsi dire aux yeux lorsque les membranes sont enlevées. Elle est presque aussi évidente que la scissure de Sylvius. Indiquée en 1780 par Malacarne comme établissant la limite du lobe moyen et du lobe postérieur, figurée en 1786 par Vicq-d'Azyr (1), elle fut décrite avec quelque soin par Burdach en 1822, et depuis lors elle a été mentionnée et plus ou moins décrite par la plupart des auteurs qui ont étudié la face interne de l'hémisphère (2). Quant à la scissure occipitale externe, on n'en aurait jamais soupçonné l'existence si l'on s'était borné à l'étude de l'homme. Lille a été découverte par Gratiolet, qui, éclairé par l'anatomie comparée des primates, a su la retrouver chez l'honme en faisant abstraction des deux plis de passage qui la traversent, la defigurent très diversement, et en rendent la détermination assez dilticile. Aujourd'hui encore, cette détermination constitue le point le plus épineux de l'étude du manteau de l'hémisphère.

Parlons d'abord de la scissure occipitale interne. Elle naît sur le bord supérieur de la calcarine, à l'union des deux portions de cette dernière, et, par conséquent, en arrière de la pointe du cerveau, de là elle remonte en haut et un peu en arrière vers le bord sagittal, auquel elle est à peu près perpendiculaire, d'où est venu le nom de scissure perpendiculaire interne (Gratiolet). Elle dépasse toujours ce bord sagittal, et s'étend sur la face convexe dans une étendue qui varie de 1 à 2 centimètres, et suivant une direction tantôt presque transversale, tantôt un peu oblique

(1) Voir plus haut la note de la page 752.

(2) Il est assez étrange que Cruveilhier, qui a bien décrit la scissure calcarine, n'ait pas vu la scissure occipitale interne, et que Valentin (Névrologie, traduction française, 1843, p. 146) ait dit en parlant de cette dernière scissure, qu'elle existe quelquefois, mais pas toujours. 
en avant. Elle se termine enfin dans le premier pli de passage, ou pli occipital ou pariétal supérieur. Lorsque ce pli est profond, ce qui est assez rare chez l'homme, elle se continue avec la scissure occipitale externe.

A son origine, elle communique superficiellement avec la scissure calcarine, dont elle est séparée profondément par le pli cunéo-limbique. Dans toute la partie de son trajet qui se trouve sur la face interne, elle sépare le cunéus, ou lobule triangulaire, du lobe occipital, du lobule quadrilatère du lobe pariétal; elle émet souvent une incisure antérieure qui pénètre superficiellement dans le lobule quadrilatère du lobe pariétal; elle émet en outre une incisure postérieure qui pénètre superficiellement dans le lobule du cunéus. Ces incisures, lorsqu'elles 'existent, correspondent à un petit pli de passage occipito-pariétal ou cunéo-pariétal interne qui est très profond et souvent rudimentaire. Parvenues sur le bord sagittal, elle envoie une incisure constante, entre le bord supérieur du cunéus et le bord inférieur du pli de passage occipito-pariétal supérieur.

La scissure occipitale interne se voit sur la face convexe de l'hémisphère. Chez les singes, elle est perpendiculaire au bord sagittal, et Gratiolet la nommait pour cela scissure perpendiculaire externe; mais chez l'homme sa direction est souvent plus ou moins oblique; sa position est tout aussi variable que sa direction. Pour faire comprendre la constitution et les variations de cette scissure, il faut dire d'abord que deux gros plis de passage superficiels, l'un supérieur ou sagittal ou premier, l'autre inférieur ou externe ou second, établissent sur la face convexe de l'hémisphère une double continuité entre le lobe occipital et le lobe pariétal. Le premier fait suite à la première circonvolution pariétale, le second à la seconde. Tous deux sont flexueux et décrivent des courbes rapides de la nature des méandres. Voilà ce qui est constant, mais ce qui est variable, c'est la direction de leurs courbures respectives. Le cas le plus ordinaire représenté sur le cerveau schématique est celui où les deux courbures dirigées en sens inverse, s'adossent l'une à l'autre dans leur partie moyenne et où elles forment les deux bords d'une anfractuosité antéro-postérieure qui est la terminaison du grand sillon pariétal. Lorsqu'il en est ainsi, les deux plis de passage adossés 
dans leur partie moyenne, qui est antéro-postérieure, s'écartent brusquement au-dessous ou si l'on veut en arrière de cet adossement, et prennent une direction à peu près transversale, le premier se portant en dedans, le second en dehors; après un trajet d'une longueur variable, tous deux se recourbent de nouveau pour se jeter dans le lobe occipital, le premier sur le bord sagittal de l'hémisphère, le second vers le tiers externe de la face convexe. Entre ces deux insertions occipitales, le bord postérieur (et inférieur) des deux plis de passage s'applique sur le bord antérieur (et supérieur) du lobe occipital dont ils sont séparés par une anfractuosité profonde, à peu près transversale. G'est cette anfractuosité qui constitue la scissure occipitale externe.

Elle se compose donc de deux portions, l'une interne, située derrière le premier pli de passage, l'autre externe, située derrière le second. Ces deux portions se continuent directement l'une avec l'autre; leur limite est indiquée par la scissure pariétale qui vient aboutir à peu près perpendiculairement vers le milieu du bord antérieur ou pariétal de la scissure. Nous indiquerons tout à l'heure les conditions particulières qui peuvent modifier plus ou moins cette disposition dans ses apparences extérieures. Mais nous devons parler d'abord de la scissure occipitale externe et de l'interne.

Pour cela, il faut examiner de plus près le premier pli de passage. Né de l'extrémité postérieure de la première circonvolution pariétale, près du bord sagittal de l'hémisphère, il décrit avant de gagner le bord correspondant du lobe pariétal, deux courbes qui sont deux méandres; l'une est pariétale, l'autre occipitale. Le méandre pariétal s'adosse par sa convexité, qui est dirigée en dehors, avec la partie antérieure du second pli de passage et reçoit dans sa concavité qui est tournée vers le bord sagittal, l'extrémité terminale de la scissure occipitale interne. Le méandre occipital revenant vers le bord sagittal où il se recourbe pour se jeter dans le lobe occipital, embrasse dans sa concavité l'extrémité interne de la scissure occipitale externe. Celle-ci est donc situće derrière l'occipitale interne, et les deux scissures sont séparées par le premier pli de passage, qui forme le bord antérieur de la première et le bord postérieur de la seconde. Par conséquent, lorsque ce pli devient profond, ne fût-ce que dans 
une très petite étendue, la scissure occipitale externe se continue directement avec l'interne. C'est ce qui a lieu chez la plupart des singes, et aussi chez quelques hommes. Il faut alors, pour étudier le premier pli de passđge, écarter les bords de la scissure.

La scissure occipitale externe née dans le méandre occipital du premier pli de passage, commence quelquefois sur le bord sagittal de l'hémisphère, plus souvent à quelques millimètres de ce bord; cela dépend de l'épaisseur du pli et aussi de son degré de flexuosité. De là, elle se porte en dehors suivant une direction plus ou moins transversale et parcourt les deux tiers environ de la largeur de la face convexe, jusqu'à l'insertion occipitale du second pli de passage sur laquelle elle se termine; mais ce dernier pli peut, comme le premier, quoique plus rarement que lui, ètre plus ou moins profond, et alors la scissure occipitale externe se prolonge sur sa base amincie jusqu'au pli courbe, de manière à parcourir presque toute la largeur de la face convexe. Le lobe pariétal se trouve ainsi séparé du lobe occipital presque aussi complètement qu'il l'est chez les singes pithéciens. Enfin, le pli courbe peut devenir profond à ce niveau, et lorsque cette anomalie coïncide avec la précédente, la scissure occipitale externe se trouve en communication avec le premier sillon temporal (appelé par Gratiolet scissure parallèle). L'extrémité postérieure de l'hémisphère paraît alors tout à fait monstrueuse; mais ce n'est qu'une apparence : elle ne diffère de l'état normal que par la position profonde de certains plis d'ailleurs normaux.

Les variétés que nous venons d'indiquer ne sont pas les seules qui puissent modifier la disposition de la scissure occipitale externe. Nous avons dit qu'elle communique vers le milieu de son bord antérieur ou pariétal, avec le sillon pariétal qui sépare la première circonvolution pariétale de la seconde; or, ces deux circonvolutions, à leur extrémité postérieure qui donne naissance respectivement à nos deux plis de passage, sont unies l'une à l'autre par un pli d'anastomose. Celui-ci est ordinairement profond, et le sillon pariétal continue alors son trajet jusqu'à la scissure occipitale; mais il est assez souvent superficiel et forme alors un pont qui sépare la scissure occipitale externe du sillon pariétal. 
D'un autre côté, le bord postérieur de la scissure occipitale, décrit plus haut comme indivis, est quelquefois entaillé par une incisure qui est située sur le prolongement du sillon pariétal. Lorsque cette incisure est courte, elle ne défigure pas sensiblement la scissure occipitale; mais lorsqu'elle est longue, elle pénètre longitudinalement dans le lobe occipital de manière à y séparer profondément quelquefois même jusqu'au voisinage du pôle, les deux circonvolutions ocoipitales $0^{1}$ et $0^{2}$, qui sout ordinairement plus ou moins fusionnées; dans ce cas, le sillon pariétal paraît se prolonger sur le lobe occipital; en même temps, les méandres des deux plis de passage se redressent notablement; ce ne sont plus que des flexuosités plus ou moins profondes, qui parfois ne se produisent pas au même niveau, de sorte qu'il faut beaucoup d'attention pour retrouver, sur le bord externe du premier pli, et sur le bord interne du second, les deux petites anfractuosités qui représentent respectivement les deux parties de la scissure occipitale externe et qui ne sont plus situées exactement l'une vis-à-vis l'autre. Cette anomalie n'est pas rare; elle est très instructive, parce qu'elle sert à démontrer la constitution du lobe occipital comme on le verra plus loin (1), mais elle est très gênante pour les commençants, qui ne connaissent pas encore les caractères polymorphes de la scissure occipitale externe. Le changement qui en résulte paraît excessif; il dépend cependant d'une modification assez légère en soi. La formation de la scissure occipitale externe, avec ses caractères normaux, résulte de l'adossement des deux plis de passage par leur convexité, et de leur séparation brusquement divergente en arrière (et au-dessous) de cet adossement. Or, il suffit que les deux convexités ne soient pas relevées exactement sur le même niveau, pour que la portion convexe de l'une corresponde plus ou moins à la portion concave de l'autre, et pour que les anfractuosités qui pénètrent dans leurs méandres respectifs ne soient plus ni transversales, ni profondes, ni situées sur le prolongement l'une de l'autre.

(1) Ici Broca avait écrit en marge de son manuscrit cette note : Le verra-t-on? Etrange coïncidence, si ce n'est un pressentiment: la partie de son mémoire a laquelle il renvoyait ainsi est précisément celle qui est demeurée inachevée.

(Note de l'Editeur. S. P.) 
J'ai dû décrire avec quelques détails les variétés les plus importantes de la scissure occipitale externe, celles qui la modifient assez pour en rendre la détermination difficile. Je passe sous silence plusieurs autres variétés qui rentrent plus ou moins dans la description générale et qui concernent la direction plus ou moins oblique de la scissure, la longueur absolue ou relative de ses deux portions, la longueur et le degré de flexuosité des plis de pasqage; j'ajoute en terminant que le type qui a servi de base à la description générale et qui est représenté sur le cerveau schématique, est de beaucoup le plus commun, et que les cas réellement difficiles à interpréter sont assez rares.

ART. II. Les lobes et la zone neutre.

La description des scissures nous a fait connaitre les limites des lobes. Nous pourrions passer maintenant à l'étude des circonvolutions qui composent ceux-ci. Toutefois, avant de procéder ì cette analyse, il ne sera pas inutile d'indiquer sommairement la conformation générale des lobes, leur situation et leurs rapports, et de faire connaître la zone neutre.

\section{§1. Les lobes.}

$1^{\circ}$ Lobe du corps calleux. Comme il ne comprend qu'une seule circonvolution, nous n'aurons pas à en donner ici une description qui trouvera mieux sa place dans l'étude spéciale des circonvolutions. Nous nous bornerons donc à dire qu'il se voit sur la face interne, qu'il contourne toute la face convexe du corps calleux depuis son bec jusqu'à son bourrelet ; qu'il est contourné à son tour dans toute sa partie antérieure par la scissure sousfrontale qui le sépare de la face interne du lobe frontal; qu'en arrière il se fusionne plus ou moins avec le lobule quadrilatère du lobe pariétal, qu'enfin son extrémité postérieure s'arrête sur la première portion de la scissure calcarine qui la sépare du lobe occipital et du lobe temporal, à cela près qu'elle se continue avec ce dernier lobe, sous le bourrelet du corps calleux, par le mince pli de passage temporo-limbique.

$2^{\circ}$ Lobe frontal. C'est le plus grand des lobes de l'hémisphère. 
Il forme environ les deux cinquièmes de la surface du manteău. Il occupe sur la face convexe toute la région située en avant de la scissure de Rolando et au-dessus de la scissure de Sylvius, sur la face inférieure toute la région située au-devant de la vallée de Sylvius, et sur la face interne toute la zone comprise entre la scissure sous-frontale et le bord de l'hémisphère.

Il se compose de deux étages, l'un supérieur ou métopique

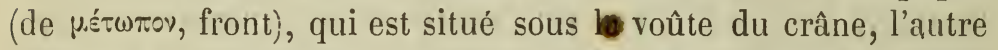
inférieur ou orbitaire, qui repose sur la voûte orbitaire. L'étage inférieur porte le nom de lobule orbitaire. L'étage supérieur, qui forme la plus grande partie du lobe, est la portion qui est située au-dessus du centre ovale de Vieussens.

Les trois circonvolutions longitudinales de cet étage cheminent d'arrière en avant et de haut en bas, passent sur l'extrémité antérieure du centre ovale, se réfléchissent sur elle, passent audessous d'elle et se dirigent d'avant en arrière, de manière à constituer l'étage inférieur. 0 n sait que dans le voisinage de la ligne médiane le centre ovale est formé par la face supérieure du corps calleux ; celle-ci est recouverte par le lobe du corps calleux ; ce n'est donc pas sur le corps calleux lui-même, c'est sur le lobe du corps calleux que se réfléchit la circonvolution frontale la plus externe ; mais les deux autres circonvolutions frontales se réfléchissent directement sur le centre ovale. Toutes les trois se continuent respectivement sur l'extrémité antérieure del'hémisphère avec les trois circonvolutions orbitaires qui n'en sont que les prolongements, et qui vont s'arrêter toutes les trois sur le bord antérieur de la vallée de Sylvius.

Les anfractuosités qui limitent le lobe frontal, le circonscrivent presque entièrement et forment un grand circuit interrompu seulement en trois points.

Nous prendrons d'abord ce circuit sur la face inférieure de l'hémisphère. Il est formé par la vallée de Sylvius, dont le fond constitue l'espace perforé de Vicq-d'Azyr. Il commence sur la ligne médiane, au-devant du chiasma des nerfs optiques et derrière la racine olfactive externe, et se porte de là transversalement en dehors en séparant le bord postérieur de l'étage orbitaire de l'extrémité antérieure du lobe temporal; au niveau du pli falciforme, le circuit, passant sur la face convexe, est formé 
dans une étendue de 4 à 5 centimèlres par la scissure de Sylvius qui sépare le hord inférieur et externe de l'étage métopique du bord supérieur du lobe temporal. La scissure de Rolando forme la seconde partie de notre circuit, en séparant le bord postérieur du lobe frontal du bord antérieur du lobe pariétal. Entre celte scissure et celle de Sylvius existe la première interruption, constituée par le pli de passage fronto-pariétal inférieur. Enfin, la troisième partie du circuit est la scissure sous-frontale qui nous ramène, par la face interne de l'hémisphère, jusqu'à notre point de départ. Gette scissure, dans sa partie postérieure, sépare le lobe frontal du lobe pariétal, lesquels toutefois se continuent l'un avec l'autre, sur le bord sagittal, par le pli de passage frontopariétal supérieur. Dans le reste de son étendue, elle sépare la face interne du lobe frontal du bord convexe du lobe du corps calleux jusqu'à l'origine de ce dernier lobe, au carrefour de l'hémisphère. Les deux plis de passage qui la traversent à son point d'inflexion (pli préovalaire) et à son point de réflexion (pli fronto-limbique), l'interrompentlorsqu'ils sont superficiels, mais ils sont souvent profonds et on ne doit pas confondre ces deux interruptions éventuelles avec celles qui sont constantes et typiques et qui sont seulement au nombre de trois. Nous avons déjà indiqué les deux premières : ce sont les deux plis de passage fronto-pariétaux qui forment à ses deux extrémités la scissure de Rolando. La troisième est constituée par le carrefour de l'hémisphère, petite surface plate située au-devant du bec du corps calleux et de la commissure antérieure, et sur laquelle vient aboutir l'extrémité de la scissure sous-frontale. Entre cette extrémité et le point où nous avons fait commencer notre circuit, existe un petit intervalle de quelques millimètres seulement, correspondant à l'insertion que la face externe de la première circonvolution frontale vient prendre sur le carrefour, en représentant à peu près la moitié inférieure de la hauteur du carrefour. Le lobe frontal se trouve donc entièrement isolé du reste du manteau, si l'on pratique trois petites sections de quelques millimètres chacune, l'une sur le carrefour, de manière à faire communiquer la scissure sous-frontale avec la vallée de Sylvius, et les deux autres sur les deux plis de passage frontopariétaux, de manière à faire commuriquer la scissure de Ro- 
lando avec la sylvienne en bas, avec la sous-frontale en haut.

Tels sont les limites et les rapports du lobe frontal. Il affecte en outre avec le lobe de l'insula, dans la fosse de Sylvius, des rapports qui seront indiqués à l'occasion de ce dernier lobe.

Le lobe frontal présente trois faces : l'une convexe, qui est de beaucoup la plus étendue et qui correspond à la voûte du crâne, l'autre inférieure, qui repose sur la voûte orbitaire et qui forme le lobule orbitaire, l'autre interne, qui s'applique sur la grande faux du cerveau, et qui se décompose en trois lobules correspon. dant aux trois parties de la scissure sous-frontale, savoir : le lobule ovalaire, le lobule métopique et le lobule sus-orbitaire.

$3^{\circ}$ Lobe pariétal. Il apparaît à la foi s sur la face convexe de l'hémisphère et sur sa face interne. Sur la face convexe, il est limité en avant par la scissure de Rolando, qui le sépare du lobe frontal, en arrière par la scissure occipitale externe, qui le sépare du lobe occipital, en bas et en avant par la scissure de Sylvius, qui le sépare du bord supérieur dı lobe temporal ; mais en arrière de la scissure de Sylvius, le bord inféro-externe du lobe pariétal se continue directement avec le lobe temporal par deux gros plis de passage, l'un antérieur, pli rétro-sylvien, qui longe le bord postérieur de la scissure de Sylvius, l'autre postérieur, pli courbe, qui se replie sur le précédent. Rappelons que le lobe pariétal communique avec le lobe frontal aux deux extrémités de la scissure de Rolando, par les deux plis de passage fronto-pariétal supérieur et inférieur, qui sont toujours superficiels à l'état normal. Une troisième communication, qui est constante, mais toujours profonde, estétablie entre ces deux lobes par le pli fronto-pariétal moyen dont il a été question plus haut. En arrière enfin, le lobe pariétal communique avec le lobe occipital par les deux plis de passage pariéto-occipitaux, qui sont presque toujours superficiels chezl'homme. La face interne du lobe pariétal forme, sur la face interne de l'hémisphère, le lobule quadrilatère, limité en avant par la scissure sous-frontale, qui le sépare du lobule ovalaire du lobe frontal; en arrière, par la scissure occipitale externe, qui le sépare du lobule triangulaire (ou cunéus) du lobe occipital; en haut, sur le bord sagittal, le lobule quadrilatère se continue avec la face convexe du lobe pariétal; en bas, il se continue largement avec le lobe du corps calleux; toutefois 
la fusion de ces deux lobes n'est pas complète comme on le ré. pète souvent, et on retrouve toujours entre eux un petit sillon, de forme variable, qui est le vestige de la grande scissure souspariétale des mammifères ordinaires, ce qui n'est plus chez les singes et l'homme qu'un sillon court et peu profond ; c'est le sillon sous-pariétal, sur lequel nous aurons l'occasion de revenir.

$4^{\circ}$ Lobe occipital. C'est le plus petit des lobes superticiels du manteau. Il forme l'extrémité postérieure de l'hémisphère, et apparaît sur les trois faces. Sur la face convexe, il est séparé par la scissure occipitale externe du lobe pariétal, avec lequel il communique par les deux plis de passage pariéto-occipitaux. Sur la face externe, il est séparé du lobule quadrilatère du lobe pariétal par la scissure occipitale interne, et du lobe du corps calleux par la première portion de la scissure calcarine, mais il communique profondément avec ces deux lobes par le pli de passage cunéo-pariétal et par le pli de passage cunéo-limbique. Sur la face inferieure enfin et aussi sur son bord externe, il se continue directement, sans ligne de démarcation, avec les circonvolutions du lobe temporal.

Le lobe occipital se compose de trois lobules correspondant respectivement à ses truis faces: le lobule sus-occipital sur la face convexe, le lobule sous-occipital sur la face inférieure et le lobule oscipital interne ou lobute triangulaire ou cunéus sur la face interue.

Les sillons qui séparent plus ou moins ces trois lobules se terminent en arrière sur une partie indécise qui occupe la pointe du lobe et que nous nommons le pôle occipital.

$5^{\circ}$ Lobe temporal. Ce lobe fait partie de la face convexe de l'hémisplière et de sa face inférieure. Faisant vers le bas une assez forte saillie, il apparâ̂t sur la norma interne de l'hémisphère, mais il n'appartient pas à la face interne.

Son extrémité antérieure, qui se détache sur la face inférieure de l'hémisphère, sous la forme d'une grosse saillie arrondie, est séparée du lobule orbitaire du lobe frontal par la large et profonde vallée de Sylvius.

Son bord supérieur, visible sur la face externe, est limité par la scissure de Sylvius, qui le sépare, en avant du lobe frontal, en arrière du lobe pariétal. 
Son bord interne, qui est aussi son bord inférieur, fait partie du limbe de l'hémisphère, et forme le bord inférieur de la grande fente de Bichat, depuis le point d'émergence de la bandelette optique jusqu'au niveau du point où commence la scissure calcarine. Celle-ci ne pénétrant pas jusqu'à la fente de Bichat, il reste, au-dessous du bourrelet du corps calleux, un mince pli de passage, le pli temporo-limbique, qui fait communiquer le lobe temporal avec le lobe du corps calleux.

La scissure calcarine, la fente de Bichat, la vallée de Sylvius et la scissure de Sylvius constituent les seules limites du lobe temporal, dont l'extrémité postérieure, nommée aussi sa base, se continue sans interruption sur la face convexe avec le lobe pariétal, sur le bord externe et sur la face inférieure avec le lobe occipital. Cette dernière continuité, d'autant plus complète que les circonvolutions s'étendent d'un lobe à l'autre sans changer de direction, constitue une fusion véritable, et l'on conçoit quc beaucoup d'auteurs aient désigné les circonvolutions qui l'établissent sous le nom de circonvolutions temporo-occipitales. Le fait est qu'il est impossible de déterminer, même approximativement, sur ces circonvolutions, la limite de leur portion temporale de leur portion occipitale. Toutefois, si l'on songe que les deux lobes temporal et occipital présentent, dans la série des mammifères, deux évolutions bien différentes(1), on comprendra la nécessité de les considérer comme essentiellement distincts, malgré la continuité de leur base. Il y a, entre ces deux lobes, sur la face inférieure de l'hémisphère, une limite topographique établie par le bord de la tente du cervelet, car le lobe occipital

(1) Le lobe temporal de l'homme et des singes est une ampliation du lobule temporal des autres mammifères; ce lobule est la portion du lobe pariétal sur lequel les deux circonvolutions pariétales du groupe sylvien viennent prendre leur origine en arrière de la scissure de Sylvius. Le lobe pariétal qui forme la plus grande partie de l'hémisphère des mammifères ordinaires, se compose le plus ordinairement de quatre circonvolutions longitudinales, divisées en deux groupes, savoir: deux circonvolutions externes formant le groupe sylvien, et deux internes formant le groupe sagittal. Les deux groupes sont séparés par le grand sillon pariétal primaire, analogue du sillon pariétal des primates. Le lobe temporal des primates procède du groupe sylvien. Leur lobe occipital, au contraire, procède principalement du groupe sagiltal, c'est-à-dire des deux circonvolutions internes qui se prolongent en arrière, au-dessus du cervelet (Revue d'anthropologie, 1878). 
est la portion de l'hémisphère qui entoure la cavité ancyroïde et qui recouvre le cervelet; mais cette limite n'est pas indiquée sur les circonvolutions elles-mêmes.

Le lobe temporal communique en outre: $1^{\circ}$ avec le lobe frontal par le pli falciforme, profondément placé dans l'anfractuosité sylvienne, à l'entrée de la fosse de Sylvius; $2^{\circ}$ avec le lobe du corps calleux par le pli de passage temporo-limbique, sous le bourrelet du corps calleux; $3^{\circ}$ avec le lobe pariétal par le pli rétro-sylvien et par le pli courbe, qui sont superficiels, et par le pli de passage temporo-pariétal profond, qui se voit dans la fosse de Sylvius et qui fait partie du lobe de l'insula.

Toutes les circonvolutions du lobe temporal sont longitudinales, et prennent leur origine, à l'extrémité antérieure du lobe, sur une portion indécise, qui est le pôle temporal.

$6^{\circ}$ Lobe de l'insula. A l'inverse des autres lobes, qui sont apparents à la surface de l'hémisphère, celui-ci est profond. Il est situé dans la fosse de Sylvius, et, pour l'apercevoir, il faut, après avoị enlevé la pie-mère, écarter les bords de la scissure de Sylvius. Il présente, comme la fosse elle-même, la forme d'un triangle rectangle dont le bord inférieur ou temporal constitue l'hypoténuse. Le bord supérieur, horizontal, qu'on aperçoit en relevant l'opercule, correspond en avant au lobe frontal, et en arrière au lobe pariétal; le bord antérieur enfin est vertical et caché sous le cap de la troisième circonvolution du lobe frontal; pour l'apercevoir, il faut rejeter ce cap vers l'avant et relever l'opercule.

Les trois anfractuosités qui limitent le lobe de l'insula portent le nom de rigoles; il y a donc une rigole inférieure ou temporale, une rigole supérieure ou fronto-pariétale et une rigole antérieure ou frontale.

Le lobe se compose de deux portions : l'une antérieure, volumineuse, plissée en éventail, seule décrite par Reil, sous le nom d'insula, et que nous nommerons le lobule de l'insula; l'autre portion, plus étroite, formée par une circonvolution oblique qui s'étend du bord supérieur du lobe temporal au bord inférieur du lobe pariétal et que nous nommons le pli de passage temporoparriétal profond. Ces dispositions du lobe de l'insula doivent être distinguées, car l'anatomie comparée prouve qu'elles procèdent 
de deux plis différents dont le développement relatif est très variable dans la série des mammifères.

Le lobule de l'insula est divisé en plusieurs plis disposés comme un éventail; le sommet de l'éventail, ou pôle de l'insula, est situé dans l'angle antérieur et inférieur de la fosse de Sylvius, immédiatement au-dessus du pli falciforme.

La face profonde du lobe de l'insula repose sur le noyau extraventriculaire du corps strié. La face superficielle est recouverte d'une couche de substance grise qui se continue de toutes parts, à travers les trois rigoles, avec l'écorce des trois lobes environnants. Les connexions de la substance blanche subjacente seront indiquées lorsque nous parlerons des circonvolutions de l'insula.

\section{§2. La zone neutre du manteau.}

Toute la surface du manteau est plissée en circonvolutions, à l'exception d'une étroite zone transversale formée, sur la face inférieure, dans le fond de la vallée deSylvius, par l'espace perforé, et prolongée sur la face interne, où elle constitue le carrefour de l'hémisphère.

Cette zone sans plis pourrait être appelée agyrique, mais, comme elle n'appartient à aucun des lobes de l'hémisphère, je lui donnerai le nom de zone neutre.

Je décrirai successivement les deux parties dont elle se compose.

$1^{\circ}$ Espace perforé. C'est la surface qui est criblée de trous, qui forme le fond de la vallée de Sylvius. Cet espace a été nommé espace perforé antérieur par Vicq-d'Azyr, qui nommait espace perforé postérieur la portion du plancher du troisième ventricule comprise entre les deux pédoncules cérébraux. Le rapprochement qui résultait de cette dénomination commune n'étant nullement justifié, l'espace perforé postérieur a été appelé par Cruveilhier espace interpédonculaire, et dès lors le nom d'espace perforé sans autre épithète ne désigne plus que l'espace perforé antérieur de Vicq-d'Azyr.

Cet espace porte en anatomie comparée le nom d'espace quadrilatère. Il est occupé, chez les mammifères osmatiques dont l'appareil olfactif est complet, par une couche large et épaisse de 
substance grise, doublée d'une couche de substance blanche, le tout formant la racine olfactive grise ou moyenne. Chez les anosmatiques, dont l'appareil olfactif est incomplet ou nul, cette racine disparaît ; à sa place, se produit une dépression transversale, large et profonde, qui est la vallée de Sylvius, et qui, laissant au-devant d'elle le lobule orbitaire du lobe frontal, le sépare de l'extrémité antérieure du lobe temporal. Cette disposition établit entre tous les anosmatiques un caractère commun d'autant plus remarquable que les autres circonvolutions du manteau présentent chez ces animaux des différences très grandes.

Le fond de cette grande dépression, que la pie-mère traverse et au-dessus de laquelle l'arachnoïde passe comme un pont, est formé par une très mince couche de substance grise qui se confond avec la face inférieure du corps strié et qui est criblé de trous pour le passage des veines émanées de ce corps. De là est venu le nom d'espace perforé.

Pour l'étudier, il faut, après avoir divisé l'arachnoïde, écarter les bords de la vallée de Sylvius, en soulevant et attirant en arrière la pointe du lobe temporal. Nous avons déjà décrit dans son ensemble la vallée de Sylvius, mais nous aurons à décrire ici plus complètement l'espace perforé qui forme le fond de cette vallée.

Il a la forme d'un quadrilatère très irrégulier, et présente quatre bords $: 1^{\circ}$ un bord antérieur formé par le lobule orbitaire; $2^{\circ}$ un bord postérieur et externe formé par le lobe temporal ; $3^{\circ}$ un bord postérieur et interne formé par la bandelette optique et le chiasma ; $4^{\circ}$ un bord interne très court, qui fait partie du bord médian de l'hémisphère.

Le bord antérieur ou orbitaire est le plus long de tous; il est transversal et suit toute la largeur du bord postérieur du lobule orbitaire, depuis le bord interne de ce lobule jusqu'à l'entrée de la scissure de Sylvius. Sa limite n'est indiquée que par un simple changement de niveau, l'espace perforé étant un peu plus déprimé que le bord du lobule. Ce bord est longé en outre dans sa partie externe par la racine olfactive externe, et dans sa partie interne par la racine olfactive interne (1). Celle-ci, qui est très

(1) Je me borne à nommer ici les deux racines olfactives blanches, et je passe sous silence la troisième racine dite grise. Les racines olfactives jouent un rôle considérable dans la morphologie cérébrale des mammifères osmatiques; mais 
grêle et très courte, va rejoindre perpendiculairement le bord interne de l'hémisphère, où elle se perd dans la substance grise.

Le bord postérieur et externe, légèrement oblique de dehors en dedans et d'avant en arrière, est formé par le lobe temporal: Il est très profondément caché sous l'extrémité antérieure de ce lobe, dont il parcourt toute la largeur. Il commence en dehors, sur le pli falciforme, qui sépare la vallée de Sylvius de la fosse de Sylvius, et s'étend en dedans jusqu'à l'extrémité antérieure de la ferte de Bichat.

Le bord postérieur et interne, oblique en sens inverse, est formé par le bord antérieur de la bandelette optique et du chiasma. Ce bord est curviligne à convexité antérieure : la bandelette optique, sortant de l'extrémité antérieure de la fente de Bichat, sous le lobule de l'hippocampe, se dirige d'abord en avant et en dedans; puis se recourbe un peu à sa partie antérieure, qui se porte en dedans ou devient enfin transversale, pour se continuer avec le chiasma.

Le bord interne enfin est le plus court de tous. Il est situé sur la ligne médiane et constitué par la partie du bord interne de l'hémisphère comprise entre la racine olfactive interne et le chiasma des nerfs optiques. Ce bord; dont la longueur n'est que de 6 millimètres environ, est une arête mousse formée par la rencontre à angle droit de l'espace perforé qui fait partie de la face inférieure et du carrefour qui fait partie de la face interne, il établit ainsi la continuité des deux portions de la zone neutre. Pour l'apercevoir, il faut soulever et redresser le chiasma, dont le bord adhérent forme une ligne transversale. Cette adhérence est établie par une lame grise dite à tort ou à raison racine grise des nerfs optiques, lame qui, se continuant en arrière avec le plancher du troisième ventricule, se continue en avant avec la couche de substance grise du carrefour et de l'espace perforé.

L'espace perforé, limité par les quatre bords que nous venons d'énumérer, présente naturellement quatre angles, savoir : deux angles internes, un angle postérieur et un angle externe. Les

chez l'homme; comme chez les autres anosmatiques, elles sont rudimentaires, et il n'y a pas lieu de les faire figurer dans la description des circonvolutions. J'ai décrit ces racines en détail dans un mémoire spécial sur les centres olfactifs (Revue d'anthropologie, 1879, p. 385-455, et dans ce volume, p. 383). 
deux angles internes sont à peu près droits; ils se voient sur le bord interne, sur lequel tombent perpendiculairement, en arrière le chiasma, en avant le bord postérieur du lobule orbitaire. Ce dernier bord, fort peu saillant, serait peu distinct s'il n'était longé par la racine olfactive interne. L'angle postérieur, très obtus, correspond au point d'émergence de la bandelette optique. L'angle externe enfin correspond au pli falciforme, qui sépare la vallée de Sylvius de la fosse de Sylvius; il est très aigu, mais il paraît émoussé lorsqu'on soulève le lobe temporal de manière à tendre le pli falciforme.

$2^{\circ}$ Carrefour de l'hémisphère. J'ai donné ce nom à une petite surface plate qui se voit sur la partie inférieure de la face interne de l'hémisphère, au-dessous du genou et du bec du corps calleux (fig. 89, p. 784).

Le carrefour est constitué par une couche indivise de substance grise.

Sa forme est à peu près quadrilatère; son bord postérieur, qui est dirigé de haut en bas et un peu d'avant en arrière, suivant une direction peu éloignée de la verticale, correspond au pilier antérieur de la voûte, dont le sépare en bas la commissure antérieure; son bord supérieur est formé par le bord inférieur du genou du corps calleux; son bord antérieur se continue en haut avec l'origine de la circonvolution du corps calleux et en bas ávec la face interne de la portion sus-orbitaire de la première circonvolution frontale (lobule sus-orbitaire); enfin son bord inférieur se continue sur le bord interne de l'hémisphère avec l'extrémité interne de la vallée de Sylvius, entre la racine grise du chiasma, qui est en arrière, et la racine olfactive interne, qui est en avant.

Les connexions du carrefour sont nombreuses. Le bec du corps calleux se termine en une extrémité effilée qui a été décrite par Vicq-d'Azyr sous le nom de pédoncule du corps calleux, et qui se dirige vers le bas en se plaçant d'abord audevant du pilier antérieur de la voûte. Au moment où le pilier passe derrière la commissure antérieure, le pédoncule du corps calleux s'en sépare, passe au-devant de la commissure, et se perd en partie dans la substance grise du carrefour où l'on peut suivre ses plis jusqu'au bord de l'hémisphère au-devant du 
chiasma; les plus longues dépassent même ce bord et se portant en dehors, dans la vallée de Sylvius, disparaissent dans la substance grise de l'espace perforé. Dans ce trajet, le pédoncule du corps calleux va en s'effilant de plus en plus, en perdant peu à peu ses plis, d'abord dans le carrefour, puis dans la vallée de Sylvius.

Sur le bord inférieur de la face interne de l'hémisphère, la lame grise du carrefour se continue avec celle qui tapisse l'extrémité interne de l'espace perforé, et reçoit en outre en avant l'insertion de la racine olfactive interne, venue de la face inférieure de l'hémisphère. Cette racine se perd dans la couche grise du carrefour. En arrière, sur le même bord, la même couche se continue avec une lame grise qui se détache du bord postéro-supérieur du chiasma des nerfs optiques et de la partie adjacente de la bandelette optique, et qui a reçu le nom de racine grise des nerfs optiques. L'espace compris entre celle-ci et la racine olfactive interne, sur le bord interne de l'hémisphère, varie de 4 à 7 millimètres, c'est la longueur du bord inférieur du carrefour.

En avant enfin, dans toute sa hauteur, qui varie de 15 à 18 millimètres, le carrefour se continue avec les circonvolutions de la face interne de l'hémisphère, par deux prolongements au moins et le plus souvent par trois prolongements qui, en réalité, n'en forment que deux. Le prolongement supérieur est l'origine de la circonvolution du corps calleux; il est séparé de l'autre par la première portion de la scissure sous-frontale; le prolongement inférieur, compris entre cette scissure et le bord inférieur de l'hémisphère, est l'origine ou plutôt la terminaison du lobule sus-orbitaire de la première circonvolution frontale; le lobule est subdivisé par une longue incisure parallèle au bord de l'hémisphère : c'est l'incisure sus-orbitaire. Elle est constante et s'étend ordinairement en arrière jusqu'au carrefour, dont, le bord antérieur se trouve alors subdivisé en trois; quelquefois elle est plus courte, et s'arrête à quelque distance en avant du carrefour.

Le carrefour n'a ni la forme ni la structure des circonvolutions. C'est une surface plate, formée par une couche de substance grise. Il communique avec plusieurs parties sans 
appartenir à aucune d'elles. Il fait donc partie de la zone neutre, comme l'espace perforé, avec lequel il se continue par son bord inférieur sur le bord interne de l'hémisphère.

\section{GHAP. VI. - LES CIRCONVOLUTIONS ET LES SILLONS.}

ARt. I. Circonvolution unique du lobe du corps calleux.

Cette circonvolution, déjà mentionnée par Malacarne, décrite et figurée par Vicq-d'Azyr, appelée par Rolando la circonvolıtion crêtée (processo cristato) at généralement connue sous le nom de circonvolution du corps calleux, forme à elle seule, ainsi que nous l'avons déjà dit, un lobe spécial. - Le nom qui lui convient est celui de lobe du corps calleux; mais ici, dans ce chapitre consacré à l'étude des circonvolutions, nous l'appellerons, pour sacrifier à l'usage, la circonvolution du corps calleux.

Elle appartient exclusivement à la face interne de l'hémisphère. Elle naît, par une extrémité graduellement rétrécie, sur une petite région que nous appelons le carrefour de l'hémisphère, et qui a été décrite plus haut.

La circonvolution du corps calleux, prenant son origine sur la partie antérieure et supérieure du carrefour, au-dessous de la portion réfléchie du corps calleux, se porte de là en avant, puis en haut et en arrière, en suivant toute la convexité du corps calleux jusqu'au bord inférieur du bourrelet, où elle se termine au niveau de la scissure calcarine, en se continuant sur le bord de la fente de Bichat avec la circonvolution de l'hippocampe $\left(\mathrm{T}^{5}\right)$, par le pli de passage temporo-limbique. Son bord concave est séparé du corps calleux par une anfractuosité étroite, linéaire, sans flexuosités, qui est la rainure du corps calleux. Son bord convexe est limité, dans la plus grande partie de son étendue, à partir de son origine, par la scissure sous-frontale, qui le sépare de la face interne du lobe frontal. Cette scissure, ainsi qu'on l'a déjà dit, est traversée au niveau de son point de réflexion qui est situé à peu près au-devant du genou du corps calleux par le pli de passage fronto-limbique, et au niveau de son point d'inflexion qui est situé à peu près au-dessus du 
milieu du corps calleux, par le pli de passage préovalaire. Parvenue sous l'extrémité postérieure du lobule ovalaire, elle remonte vers le bord sagittal et abandonne la circonvolution du corps calleux. De là, jusqu'à la scissure calcarine, le bord convexe de cette circonvolution n'est plus limité par une vraie scissure et se continue largement avec la base du lobule quadrilatère du lobe pariétal.

Quelques auteurs ont même considéré ce lobule comme faisant partie de la circonvolution du corps calleux qui a été appelé d'après cela circonvolution crêtée (processus cristatus), le lobule quadrilatère étant comparé au lobe amplifié qui termine en arrière la crête des gallinacés. Mais il est aisé de reconnaître que le lobule quadrilatère est bien distinct de la circonvolution du corps calleux. Il en est séparé, à défaut d'une scissure véritable, par une petite anfractuosité qui est chez les primates le vestige de la grande et profonde scissure souspariétale des autres mammifères. Toujours courte et peu profonde, et très variable dans son étendue, dans sa forme, dans ses connexions, cette anfractuosité, bien que représentant une séparation lobaire, ne peut, dans l'état rudimentaire où elle se trouve chez les primates, être rangée parmi les scissures; nous l'appellerons donc le sillon sous-pariétal.

Le sillon sous-pariétal est constant. Dans son état le plus ordinaire, il est à peu près parallèle à la rainure du corps calleux; il occupe environ la moitié de la largeur de la base du lobule quadrilatère, et il n'atteint ni la scissure sous-frontale qui est au-devant de son extrémité antérieure, ni la scissure calcarine qui est au-dessous de son extrémité postéro-inférieure; il reste séparé de la sous-frontale par le pli de passage pariétolimbique supérieur, et de la calcarine par le pli de passage pariéto-limbique inférieur. Ces deux plis doivent conserver chez les primates les noms qu'ils portent en anatomie comparée.

L'extrémité antérieure du sillon sous-frontal peut, par exception, se prolonger en avant jusqu'à la scissure sous-frontale; le pli de passage pariéto-limbique supérieur devient alors profond, mais se retrouve toujours au fond du sillon.

Le sillon sous-pariétal, toujours curviligne, est quelquefois plus ou moins sinueux. Sa longueur est de 2 à 3 centimètres. 
Sa concavité est toujours tournée vers le corps calleux; ses deux estrémités, surtout la postérieure, se rapprochent en général de ce corps plus que sa partie moyenne. Il revêt souvent la forme de deux branches qui se séparent à angle obtus et qui entaillent plus ou moins profondément la circonvolution du corps calleux, mais sans atteindre la rainure et sans interrompre par conséquent la continuité de la circonvolution.

L'extrémité postérieure de la circonvolution du corps calleux repose sur la première portion de la scissure calcarine. En écartant les bords de cette scissure et de la scissure occipitale interne qui vient $\mathrm{y}$ aboutir, on aperçoit un pli de passage profond qui, partant du sommet du cunéus et longeant profondément le bord supérieur de la calcarine, va se rendre à la partie profonde de l'extrémité postérieure de la circonvolution du corps calleux. C'est le pli de passage cunéo-limbique, toujours profond chez l'homme, mais superficiel chez tous les singes (à l'exception des gibbons) et séparant alors la scissure calcarine de l'occipitale interne, ainsi qu'on l'a vu plus haut.

La circonvolution du corps calleux commence, sur le carrefour, par une extrémité très étroite, large en moyenne de כ̆ millimètres (min. 3, max. 10); de là, elle va toujours en s'élargissant jusque sous l'extrémité postérieure du lobule ovalaire où elle atteint une largeur moyenne de 16 à 17 millimètres (min. 14, max. 20) (1). Elle va donc en s'élargissant d'avant en arrière jusqu'au point où elle se fusionne avec le lobule quadrilatère. Cette forme de la circonvolution du corps calleux constitue l'un des caractères les plus remarquables du cerveau des primates. Chez les autres mammifères, le lobe du corps calleux atteint son maximum de largeur au-devant du genou du corps calleux et va de là en décroissant d'avant en arrière.

Le bord convexe de la circonvolution du corps calleux toujours sinueux est souvent entaillé par une ou deux incisures ordinairement courtes, émanées de la rainure sous-frontale, máis souvent aussi il est tont à fait indivis. La surface de cette circonvolution est ordinairement assez lisse (ou même tout à fait lisse) et plus simple que celle des autres circonvolutions. On y

(1) Ici Broca avait écrit en marge : "Revoir ces chiffres sur des cerveaux frais. ) S. P. 
remarque toutefois chez quelques individus une incisure longitudinale plus ou moins longue, mais peu profonde.

La circonvolution du corps calleux qui forme, chez les mammifères ordinaires, un lobe volumineux (lobe du corps calleux), est réduite chez les primates à une largeur médiocre, surtout dans sa partie antérieure, mais elle conserve toujours une très grande longueur. Formant la plus grande partie du limbe de l'hémisphère, elle a des connexions avec tous les lobes de l'hémisphère, à l'exception de l'insula; avec le lobe frontal par les deux plis de passage fronto-limbique et préovalaire; avec le lobe pariétal par les deux plis de passage pariéto-limbique; avec le lobe oç̧ipital par le pli de passage cunéo-limbique; avec le lobe temporal, enfin, par le pli de passage temporo-limbique. Elle ne peut donc être rattachée à aucun de ces lobes et doit dès lors être considérée commé formant à elle seule un lobe spécial. Gette conclusion, qui s'impose déjà à l'esprit lorsqu'on décrit le cerveau de l'homme, devient plus évidente encore lorsqu'on étudie, dans la série des mammifères, l'évolution du lobe du corps calleux (1).

ArT. II. Circonvolutions du lobe frontal.

Le lobe frontal comprend quatre circonvolutions, savoir : une circonvolution ascendante ou transverse ou postérieure, $\mathrm{F}^{\circ}$, et trois circonvolutions longitudinales désignées sous les noms de première, seconde et troisième, $\mathrm{F}^{1} \mathrm{~F}^{2} \mathrm{~F}^{3}$.

A. Circonvolution frontale ascendante, $\mathrm{F}^{\circ}$. - Cette circonvolution forme le bord antérieur de la scissure de Rolando, et est par conséquent dirigée comme celle-ci de haut en bas,

(1) Voir moll Mémoire sur le grand lobe limbique, dans la Revue d'anthropologie, 1878, et dans ce volume, p. 259. " Frappé du même fait, voici ce que j'écrivais dès 1875 dans mon article Circonvolutions du Dict.encycl. dessc. méd., t. XVII, p. 370 : “ Il est intéressant de suivre cette circonvolution dans tout son " trajet, dont une partie seulement appartient au lobe frontal interne : on se de" mande alors si l'on ne pourrait pas avec quelque apparence de raison, au lieu " de la fragmenter en divers segments décrits avec chacune des régions qu'elle " traverse, lui conserver une place à part et la considérer comme une grande a commissure, une longue circonvolution de passage, reliant les trois lobes de la " face interne, entre eux d'abord, et en dernier liell au lobe temporal.»S. Pozzı. 
haut en bas, de dedans en dehors et d'arrière en avant. En lui donnant le nom d'ascendante on est convenu de la considérer comme commençant en bas, sur le bord supérieur de la scissure de Sylvius et comme se terminant en haut sur le bord sagittal de l'hémisphère.

On l'appelle encore frontale transverse, parce qu'elle traverse la face convexe de l'hémisphère, au lieu de suivre, comme la plupart des circonvolutions, une direction longitudinale; mais ce nom donne une fausse idée de sa direction qui est très oblique.

Le nom d'ascendante lui-même n'est pas à l'abri de toute objection; il partage avec toutes les dénominations tirées de la direction des parties l'inconvénient de n'être pas applicable à l'anatomie comparée. Le nom de circonvolution prérolandique qui est applicable à tous les cas serait donc préférable; mais dans cet exposé qui concerne exclusivement le cerveau de l'homme, nous nous en tiendrons à la dénomination usuelle.

On décrit ordinairement cette circonvolution comme propre à la face convexe de l'hémisphère ; c'est une erreur ; elle appartient aussi à la face interne où son extrémité élargie, et comme épanouie, forme le lobule ovalaire, désigné sous le nom très défectueux de lobule paracentral.

Nous l'étudierons d'abord sur la face convexe, le long de la scissure de Rolando; elle y présente les mêmes flexuosités que cette scissure, et se compose comme elle de trois portions, savoir : $1^{\circ}$ une portion supérieure et interne comprise entre le bord sagittal de l'hémisphère et le genou supérieur de la scissure, et correspondant à la racine de la première circonvolution frontale; $2^{\circ}$ une portion moyenne, comprise entre les deux genoux de la scissure et correspondant à la racine de la deuxième circonvolution frontale; $3^{\circ}$ une portion inférieure et externe comprise entre le genou inférieur de la scissure et le bord sylvien du lobe frontal.

Il est assez probable qu'il existe entre ces trois portions des différences fonctionnelles, dues à leurs connexions respectives avec les trois circonvolutions frontales longitudinales, sans préjudice d'une fonction motrice qui paraît leur être commune.

La portion inférieure aboutit à la scissure de Sylvius; elle prend 
part à la formation du bord supérieur de cette scissure et de son opercule; elle se continue en arrière, sur ce même bord, avec l'extrémité inférieure de la pariétale ascendante, par le pli de passage fronto-pariétal inférieur, qui est presque toujours entièrement superficiel et qui sépare la scissure de Sylvius de la scissure de Rolando; en avant, et toujours sur le bord sylvien, elle fournit la racine de la troisième circonvolution frontale; audessus de cette racine, le bord antérieur de la portion inférieure que nous étudions est longé par un sillon à peu près parallèle à la scissure de Sylvius. Ce sillon forme la partie inférieure du sillon prérolandique $\left(\mathrm{f}^{\circ}\right)$; il s'étend en bas jusqu'au voisinage de Ja scissure de Sylvius, qu'il peut même rejoindre quelquefois par un prolongement superficiel; en haut, il remonte jusqu'à la racine de la seconde circonvolution frontale.

La portion moyenne, comprise, comme il a été dit plus haut, entre les deux genoux de la scissure de Rolando, donne insertion sur son bord antérieur à la racine de la seconde circonvolution frontale, et reçoit sur son bord postérieur, dans le fond de la scissure, le pli de passage fronto-pariétal moyen, qui est toujour's très profond, et qui est ordinairement simple, mais qui est quelquefois double, c'est-à-dire subdivisé, dans sa partie la plus profonde, en deux plis très rapprochés. Nous donnerons "plus loin, en parlant des circonvolutions pariétales, l'explication de cette subdivision. Sur le bord supérieur de ce pli de passage, existe une petite encoche qui souvent devient assez longue pour constituer une réritable incisure. Celle-ci peut même s'étendre à toute la largeur de la circonvolution ascendante et se mettre en communication avec la portion supérieure du sillon prérolandique. Il semble alors que la circonvolution ascendante soit interrompue, mais elle ne l'est que superficiellement. Ce n'est que dans des cas non seulement très exceptionnels, mais encore anormaux, que l'incisure en question la divise dans toute son épaisseur. Une incisure analogue, mais ordinairement beaucoup moins prononcée, s'observe quelquefois aussi sur le bord inférieur du pli de passage.

La portion supérieure de la frontale ascendante s'étend du genou supérieur de la scissure de Rolando jusqu'au bord sagittal de l'hémisphère. Là elle donne insertion sur son bord antérieur à la 
grosse racine de la première circonvolution frontale; en arrière, elle se prolonge jusque sur l'origine de la scissure de Rolando, qu'elle contourne en se continuant par le pli de passage frontopariétal supérieur avec la pariétale ascendante. On n'a pas oublié qu'à ce niveau, l'extrémité de la scissure de Rolando, recourbée en arrière, présente une direction très oblique, tellement oblique qu'elle est presque parallèle au bord sagittal, dans une étendue moyenne de 1 centimètre (qui va quelquefois jusqu'à 2 centimètres). Dans cette partie de son trajet, le bord antérieur de la scissure devient interne, mais ne cesse pas d'appartenir à la circonvolution frontale ascendante. Celle-ri correspond donc au bord sagittal par une extrémité très élargie, puisque sa largeur se trouve accrue de toute l'étendue du prolongement postérieur de la scissure de Rolando. Cette extrémité est en outre élargie en avant par une sorte de renflement qui correspond à la base d'implantation de la racine de la première circonvolution frontale. Il en résulte que la circonvolution frontale ascendante, dont la largeur moyenne, sur la face convexe de l'hémisphère, n'est que de 12 millimètres environ, occupe sur le bord sagittal une étendue antéro-postérieure de plus de 3 centimètres.

Entre l'insertion de la racine de la première circonvolution frontale, qui se fait sur le bord sagittal, et celle de la racine de la deuxième circonvolution frontale, qui se fait sur la portion moyenne de la frontale ascendante, le bord antérieur de celle-ci est longé par un sillon qui forme la portion supérieure du sillon prérolandique. Ce sillon est à peu près parallèle à la scissure de Rolando ; mais, en approchant du bord sagittal, qu'il n'atteint pas, au lieu de s'infléchir en arrière comme la scissure, il tend plutôt à s'infléchir en avant ; c'est du moins le cas le plus ordinaire. Quelquefois, il est vrai, l'extrémité supérieure du sillon prérolandique est à peu près transversale, et quelquefois aussi elle est légèrement oblique en arrière; mais, même dans ce dernier cas, elle est beaucoup moins oblique que la partie correspondante de la scissure de Rolando, de sorte que l'extrémité supérieure de la circonvolution frontale ascendante est toujours très élargie.

C'est dans cet état d'élargissement que, franchissant le bord sagittal de l'hémisphère, elle passe sur la face interne, où elle 
continue à s'élargir encore et où elle constitue le lobule ovalaire.

Le lobule ovalaire (Pozzi) (1) est une large surface plate, comprise sur la face interne de l'hémisphère, entre le bord sagittal et la troisième portion de la scissure sous-frontale, qui le sépare en arrière du lobule quadrilatère (lobe pariétal) et en bas du lobe du corps calleux. Il est limité en avant par l'incisure préovalaire, qui se dirige du point d'inflexion de la scissure sous-frontale au bord sagittal de l'hémisphère. Cette incisure, peu oblique et quelquefois verticale, communique ordinairement avec la scissure sous-frontale, mais en est assez souvent isolée ; lorsqu'elle est très grande, elle s'étend de la scissure sous-frontale au bord sagittal, qu'elle entaille même un peu, et alors elle sépare complètement le lobule ovalaire de la première circonvolution frontale; mais ordinairement elle est plus courte ou même très courte, et n'établit qu'une séparation incomplète.

La surface du lobule ovalaire présente une ou deux incisures peu profondes, assez simples et ordinairement isolées. Sa longueur varie entre 35 et 55 millimètres, elle est en moyenne de 40 millimètres; sa hauteur, un peu moins variable (de 19 à 26), est en moyenne de 23 millimètres.

Le lobule ovalaire communique en arrière et en haut avec la circonvolution pariétale ascendante par un étroit pli de passage (fronto-pariétal supérieur) qui sépare la scissure de Rolando de la scissure sous-frontale, et qui n'a en moyenne que 6 millimètres d'épaisseur. G'est cette minime connexion qui a fait considérer le lobule ovalaire comme appartenant à la fois à la pariétale ascendante et à la frontale ascendante, mais il suffit de prolonger par la pensée la scissure de Rolando sur le bord sagittal jusqu'à la rencontre de la scissure sous-frontále, pour reconnaître que le lobule ovalaire appartient entièrement à la frontale ascendante, dont il est l'épanouissement, sur la face

(1) C'est en 1875, dans l'article Girconvolutions déjà cité du Dict. encycl. des sc. méd., que je donnai ce nom à la petite région que Betz venait presque en même temps de décrire sous le nom de lobule paracentral. J'ignorais alors les travaux de l'auteur allemand; cette rencontre fortuite dans la distinction d'une particuiarité anatomique jusqu'ici négligée, n'est pas sans intérêt.

S. Pozz: 
interne de l'hémisphère. Ce n'est pas par l'observation, c'est par une vue théorique que ce lobule a été apprécié autrement.

En 1854, Huschke(1), décrivant les deux circonvolutions ascendantes appelées par Foville circonvolutions transversales, leur donna le nom de circonvolutions centrales (antérieure et postérieure), nom motivé, dit-il, à la fois par leur position sur le milieu du cerveau et par leur importance centrale (central Bedeutung); et il débaptisa en même temps la scissure de Rolando pour l'appeler scissure centrale. Ces dénominations furent adoptées par M. Bischoff (2), qui rattacha les deux circonvolutions centrales au lobe pariétal, comme l'avait fait Gratiolet dans l'origine, avant de reconnaître que l'antérieure fait partie du lobe frontal. "Tout ce qui est en avant de ces deux circonvolutions, dit M. Bischoff, appartient au lobe frontal, tout ce qui est en arrière appartient au lobe pariétal et occipital, mais je les comprends dans le lobe pariétal. " Toutefois, il les considérait comme formant un seul système, et sur les belles planches coloriées qui accompagnaient son important mémoire, il leur donna une teinte commune, et distincte de toutes les autres. On fut ainsi conduit à admettre qu'elles formaient dans l'hémisphère un lobule spécial, dont quelques auteurs faisaient même un lobe appelé central. Dès lors, la face interne de ce lobe, celle qui se voit sur la face interne de l'hémisphère, fut désignée par M. Betz sous le nom de lobule paracentral. Ce nom vulgarisé par les travaux de M. Meynert est devenu maintenant usuel en France, où personne n'admet cependant l'existence du lobule ou du lobe central. Si cette dénomination était inoffensive, on pourrait la conserver. Mais elle est la consécration d'une idée erronée qui a eu, en anatomie comparée, des conséquences très fâcheuses. Supposant que le caractère essentiel de la scissure de Rolando et des deux circonvolutions qu'elle sépare était d'être centrales, on a cherché le plus près possible du milieu de l'hémisphère l'anfractuosité qui doit représenter chez les quadrupèdes la scissure de Rolando, et tandis que les uns ont cru la retrouver dans le sillon crucial

(1) Emil Huschke, Schædel, Hirn und Seel, Iéna, 183̆4, in-fol., p. 137.

(2) Bischoff, die Grosshirnwindungen, etc., Munich, 1868, in-4º, p. 39. 
(qui émane de la face interne!), les autres, renonçant à la découvrir, ont déclaré qu'elle n'existait pas; et tous en ont conclu que le type cérébral de l'homme et des primates était essentiellement, radicalement différęnt de celui des autres mammifères. Cette doctrine, contraire à toutes les lois de l'anatomie comparée, ne se serait pas produite si, au lieu de chercher la scissure de Rolando et les circonvolutions rolandiques dans la région centrale où conduisait leur fausse dénomination, on les avait cherchées, sans idées préconçues, en se guidant sur le principe des connexions. On aurait vu ainsi que la scissure de Rolando séparant, sur la face convexe de l'hémisphère, le lobe frontal du lobe pariétal, n'est pas propre aux primates, qu'elle existe aussi, caractérisée par les mêmes connexions, chez les autres mammifères, mais qu'elle est chez eux oblique en sens inverse, et située beaucoup plus en avant, près de la pointe de l'hémisphère, par suite de l'exiguïté du lobe frontal (1).

Les noms de la scissure de Rolando et des deux circonvolutions qui la bordent ne doivent donc pas être empruntés à la position que ces parties occupent, par exception, chez les primates ; et s'il faut cesser de les appeler centrales, il faut cesser aussi d'appeler paracentral le lobule ovalaire qui est situé sur la face interne de l'hémisphère, au-dessous de l'extrémité sagittale de la scissure de Rolando. Les fausses dénominations risquent toujours de faire naître des idées fausses; c'est ce qui est arrivé ici. Le nom du lobule paracentral ne signifiait d'abord qu'une chose : sa position au-dessous de l'extrémité de la scissure " centrale "; mais, comme les deux circonvolutions rolandiques élaient aussi par les mêmes auteurs appelées " centrales", on a été conduit à confondre ensemble toutes ces choses centrales et à admettre que le lobule "paracentral » appartenait à la fois aux deux circonvolutions "centrales ", c'est-à-dire à la frontale ascendante et à la pariétale ascendante, tandis que si l'on avait simplement regardé, on aurait vu qu'il est formé exclusivement par la frontale, la pariétale se bornant à commu-

(1) C'est par le volume excessif du lobe frontal que le cerveau des primates se distingue de celui des autres mammifères. J'ai montré ailleurs que l'anfractuosité décrite par Meynert comme étant l'analogue de la branche ascendante de la scissure de Sylvius, n'est autre que la scissure de Rolando. 
niquer avec lui par un étroit pli de passage. Si nous rappelons, maintenant, que ce nom de lobule paracentral est né de la fausse conception du lobe central, qu'il est lié à une idée théorique contraire à l'anatomie, à l'embryologie et à l'anatomie comparée, on comprendra peut-être qu'il convient d'en laisser l'usage à ceux qui admettent cette idée théorique et de donner la préférence au nom de lobule ovalaire, qui, n'exprimant qu'une forme, ne peut donner lieu à aucune confusion, à aucune discussion.

Les trois racines frontales qui s'insèrent sur le bord antérieur de la circonvolution frontale ascendante donnent naissance aux trois circonvolutions longitudinales désignées sous les noms de première, deuxième et troisième circonvolution frontale.

Toutes les trois cheminent d'abord d'arrière en avant dans l'étage supérieur du lobe frontal, puis se réfléchissent à leur partie antérieure pour aller former l'étage inférieur ou lobule orbitaire de ce lobe. Nous distinguerons donc dans chacune d'elles une portion supérieure et une portion réfléchie; la première, correspondant à la région du front, s'appellera métopique, et la seconde, correspondant à la voûte de l'orbite, s'appellera orbitaire.

B. Première circonvolution frontale, $\mathrm{F}^{2}$. - C'est la plus longue et la plus volumineuse. Elle forme le bord sagittal et elle appartient à la fois à la face interne de l'hémisphère, à sa face convexe et à sa face inférieure ou orbitaire.

1. Face interne. - Étudions d'abord sa face interne, qui est appliquée sur la grande faux. Elle commence en arrière par une extrémité qui est située sous la racine de la circonvolution et qui se continue avec le lobule ovalaire. L'incisure préovalaire n'établit entre ces deux parties qu'une séparation incomplète, et le pli de passage préovalaire qui correspond à cette incisure les fait communiquer l'une et l'autre avec la circonvolution du corps calleux, au niveau du point d'inflexion de la scissure sousfrontale. De là, la face interne de la première circonvolution se porte en avant et un peu en bas jusqu'à la pointe de l'hémisphère, au-dessus de l'arc métopique de la scissure sous-frontale, puis se réfléchit au-devant du point de réflexion de cette 
scissure et se dirige à peu près horizontalement en arrière pour aller se terminer au carrefour de l'hémisphère, après avoir formé toute la partie de la face interne qui est comprise entre le bord inférieur ou orbitaire de cette face et l'arc sous-orbitaire de la scissure sous-frontale.

Au niveau du point de réflexion de la scissure, le pli de pas-

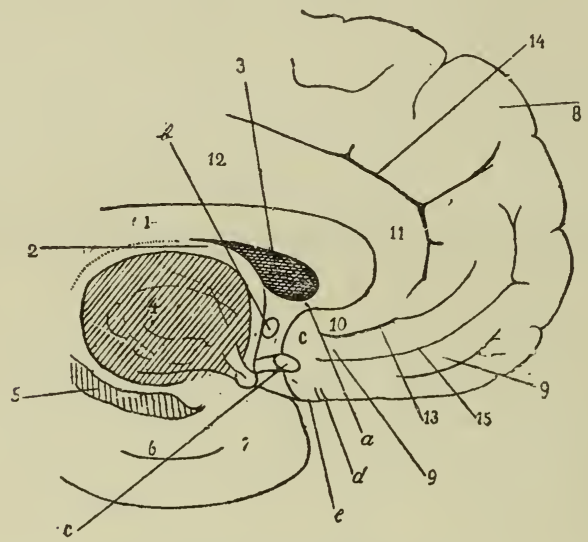

Fig. 89. Carrefour de l'hémisphère '.

1, corps calleux; 2 , voủte à trois piliers; 3 , cloison transparente ; 4 , face interne de la couche optique; 5 , grande fente de Bichat ; 6 , circonvolution de l'hippocampe avec son crochet; 7 , pôle du lobe temporal ; 8 , face interne de l'étage supérieur de la première circonvolution frontale (lobule métopique); 9 , face interne de l'étage inférieur de la même circonvolution (lobule sus-orbitaire); 10, 11, 12, lobe (circonvolution) du corps calleux; 10, son origine ; 13,14, la scissure sous-frontale, interrompue par le pli de passage fronto-limbique; 15, l'incisure sus-orbitaire; $\mathrm{C}$, le carrefour de l'hémisphère; $a$, le bec du corps calleux, terminé en un prolongement filiforme dit pédoncule $d u$ corps calleux; $b$, coupe de la commissure antérieure ; $c$, bandelette optique émergeant entre la couche optique et le lobe temporal, et aboutissant au chiasma qui est coupé; $d$, racine olfactive interne aboutissant au carrefour ; $e$, bord inférieur du carrefour se continuant sur le bord de l'hémisphère entre le chiasma et la racine olfactive interne avec l'extrémité interne de la vallée de Sylvius.

sage fronto-limbique fait communiquer la première circonvolution frontale avec la circonvolution du corps calleux; ordinairement superficiel, mais souvent profond, tantôt large et carré, tantôt assez long et flexueux, ce pli de passage est toujours accompagné d'une incisure plus ou moins longue qui se détache de la scissure sous-frontale, pénètre plus ou moins profondément dans la circonvolution frontale suivant une direction quelquefois horizontale, souvent plus ou moins relevée. C'est l'incisure

(1) Cette figure se rapporte à la description consignée pages 771-773. Elle trouve naturellement sa place ici, avec la description de la face interne de la première circonvolution frontale. 
prélimbique. Cette incisure marque la limite des deux étages de la première circonvolution frontale. L'étage supérieur constitue le lobule métopique; l'étage inférieur constitue le lobule sus-orbitaire.

Le lobule métopique présente plusieurs incisures variables et irrégulières; les unes, plus ou moins longitudinales, sont isolées; d'autres communiquent avec la scissure sous-frontale ; enfin il y en a ordinairement une et souvent deux qui entaillent le bord sagittal et se prolongent plus ou moins sur la face convexe de la première circonvolution frontale.

Le lobule sus-orbitaire est moins variable. Une longue incisure constante, longitudinale, l'incisure sus-orbitaire, le subdivise en deux plis parallèles, qui sont le pli sus-orbitaire supérieur et le pli sus-orbitaire inférieur. Cette incisure existe chez tous les primates qui ont des circonvolutions. Elle se prolonge ordinairement en arrière jusqu'au carrefour; en avant, elle se relève un peu, comme l'arc sus-orbitaire de la scissure frontale, auquel elle est parallèle, et va se terminer un peu au-dessus de la pointe de l'hémisphère. Cette extrémité antérieure se divise souvent en deux branches, dont l'une s'étend vers le bord sagittal, qu'elle atteint quelquefois et qu'elle peut même dépasser. Au-dessous de cette incisure, dont la fixité est remarquable, existe presque toujours chez l'homme et les anthropoïdes une seconde incisure analogue, mais plus courte et limitée ordinairement à la moitié antérieure du lobule; on en voit même quelquefois une troisième, plus courte encore, sur les cerveaux humains très compliqués ou, comme on dit, très riches en circonvolutions (1). Mais la vraie incisure sus-orbitaire, la plus longue, la seule qui soit typique et absolument constante, est la supérieure; les autres peuvent en être distinguées par le nom d'incisures sus-orbitaires accessoires.

$2^{\circ}$ Face convexe. - Occupons-nous maintenant de la face convexe de la première circonvolution frontale. Elle naît de

(1) Gelte expression très usitée, et l'expression opposée de cerveaux pauvres en circonvolutions, datent d'une époque où l'on ne savait pas que le nombre des circonvolutions est le même dans tous les cerveaux. Il faudrait donc dire plutôt cerveaux riches et pauvres en plis secondaires, mais il vaut mieux encore ap. peler ceux-ci cerveaux simples et ceux-là cerveaux compliqués.

T. V. 
l'extrémité supérieure de la frontale ascendante par une grosse racine (première racine frontale), qui, dans la très grande majorité des cas, commençant sur le bord sagittal de l'hémisphère, se porte aussitôt en dehors, s'applique sur le bord antí. rieur de la frontale ascendante, dont elle est séparée par la partie supérieure du sillon prérolandique, et, après un trajst transversal de 2 à 3 centimètres, se recourbe une seconde fois pour se porter en avant. Cette racine est presque toujoul: superficielle; lorsqu'elle est profonde, le sillon prérolandique se prolonge jusqu'au bord sagittal; mais c'est très exceptionnel. Si l'on écarte les bords du sillon prérolandique, on voit qu'il est traversé par un pli profond qui se rend du bord antérieur de la frontale ascendante à la partie externe de la racine et que nous appellerons la racine accessoire de la première frontale. Cette racine accessoire, qui est de la nature des plis d'anastomose, est quelquefois superficielle; on aperçoit alors sur la surface du cerveau deux racines frontales au lieu d'une; il en résulte que le nombre des racines frontales se trouve porté à quatre au lieu de trois et que le sillon prérolandique se trouve divisé en trois portions au lieu de deux. On a cité cette variété, qui n'est pas très rare, comme un exemple de l'instabilité des circonvolutions cérébrales et de leurs connexions; mais, comme la plupart des autres variétés de l'hémisphère, elle n'est que morphologique et non anatomique; elle résulte d'un simple accroissement de volume qui rend superficiel un pli habituellement profond. Ghez quelques sujets, en même temps que la racine accessoire devient superficielle, la racine principale devient profonde; alors la racine paraît unique et implantée à 2 ou 3 centimètres du bord sagittal, au lieu d'être implantée sur ce bord même. D'autres fois enfin, la racine proprement dite et la racine accessoire se confondent en une seule et très grosse racine qui occupe toute la largeur de la base de la première circonvolution et qui se porte directement en avant.

Large de 2 à 3 centimètres environ au niveau de sa racine, Ja première circonvolution frontale chemine d'arrière en avant, le long du bord sagittal, jusqu'à la pointe de l'hémisphère, en conservant à peu près la même largeur, de sorte que le premier sillon frontal $f^{1}$, qui longe son bord externe, est à peu près pa- 
rallèle, abstraction faite de ses sinuosités, au bord sagittal de l'hémisphère. Toutefois, en avant et en bas, en approchant de la pointe, la circonvolution se rétrécit d'une manière plus ou moins rapide, et, sur la pointe même, sa largeur devient presque toujours inférieure à 1 centimètre; elle s'y réduit même quelquefois à 5 millimètres.

Son bord externe, longé par le premier sillon frontal $f$, est sinueux, très irrégulier et surtout très variable. Il semble donc toujours au premier abord échapper à toute description. Toutefois, lorsqu'on étudie comparativement un grand nombre de cerveaux, on voit que ses flexuosités sont soumises à une certaine règle. Résultat de l'excès de longueur de la circonvolution, et d'autant plus fortes que le cerveau est plus compliqué, elles se succèdent de manière à former deux genoux convexes en dehors et séparés par une portion moyenne plus ou moins concave. La largeur de la circonvolution est naturellement un peu augmentée au niveau de ces deux genoux. Le pourtour de ces deux genoux ne peut être apprécié exactement sur les dessins, où la portion antérieure du lobe frontal est vue en raccourci, mais sur le cerveau même ou sur les moules, on voit qu'ils divisent la longueur de la circonvolution, depuis sa base jusqu'à sa pointe, en trois portions à peu près égales. La première portion, comprise entre le genou postérieur et la racine, forme le tiers postérieur de la circonvolution; la seconde est comprise entre les deux genoux, la troisième entre le genou antérieur et la pointe. Le genou postérieur est ordinairement le plus prononcé; c'est aussi le plus important, car il établit la limite antérieure de la première portion de la circonvolution, portion qui paraît posséder des propriétés excito-motrices, comme la frontale ascendante, arec laquelle elle se continue, tandis que la portion movenne et la portion antérieure paraissent n'exercer aucune action directe sur la motilité.

Le bord externe de la première circonvolution frontale communique avec le bord interne de la seconde, à travers le sillon frontal, par un certain nombre de plis d'anastomose, qui quelquefois sont très profonds, de sorte que le sillon est interrompu, mais cela n'est pas commun. Parmi ces plis d'anastomose, on en distingue deux, qui sont généralement plus gros et plus longs, 
et par conséquent plus sinueux que les autres, et qui vont précisément se rendre aux deux genoux ; lorsqu'ils n'arrivent pas à la surface de l'hémisphère, ils en sont du moins très rapprochés, et on les aperçoit aussitôt en écartant légèrement les bords du sillon. Le plus souvent ils sont superficiels l'un et l'autre, et divisent ainsi le sillon en trois portions correspondant respectivement aux trois portions de la circonvolution $\mathrm{F}^{1}$. On trouve assez souvent, au niveau du genou antérieur, deux plis d'anastomose superficiels, au lieu d'un seul.

La surface convexe de cette circonvolution n'est jamais simple. Elle est toujours plus ou moins subdivisée par des incisures dirigées principalement dans le sens de la longueur, mais souvent terminées à leur extrémité postérieure par une branche à peu près transversale (incisure en $\mathrm{T}$ ou en $\mathrm{L}$ ). Dans le type le plus ordinaire, il y a trois incisures longitudinales distinctes, une sur chaque portion ; mais celles des deux premières portions se confondent souvent en une seule (que je n'ai jamais vues se prolonger jusque sur la troisième); alors la circonvolution $\mathrm{F}^{1}$ se trouve subdivisée, dans ses deux tiers postérieurs, en deux plis à peu près parallèles. Lorsqu'en même temps la racine accessoire est superficielle, cette grande incisure longitudinale se prolonge jusqu'à la base de la circonvolution, qui paraît entièrement dédoublée, chacun de ses deux plis longitudinaux s'insérant sur la frontale ascendante par une racine distincte. C'est dans les cas de ce genre qu'on a pu croire que le lobe frontal se composait de quatre circonvolutions longitudinales, et on en a conclu, tantôt que ce nombre était typique, tantôt que le nombre des circonvolutions frontales n'était pas fixe. Mais ce sont des faits exceptionnels, dus à la coïncidence de variétés qu'il faut savoir interpréter.

Parvenue à la pointe du lobe, la circonvolution $\mathrm{F}^{1}$ communique une dernière fois avec $\mathrm{F}^{2}$ par un pli d'anastomose transversal, tantôt large, tantôt étroit, mais toujours superficiel, puis elle se réfléchit brusquement pour passer sur le lobule orbitaire.

$3^{\circ}$ Face inférieure ou portion orbitaire de $\mathrm{F}^{1}$. - Cette partie de la première circonvolution frontale forme la partie la plus interne du lobule orbitaire; on l'appelle encore circonvolution orbitaire; cetle dénomination est comınode, elle est consacrée par l'usage 
et nous l'acceptons. Mais il ne faut pas en conclure que le lobule orbitaire ait des circonvolutions propres; les trois circonvolutions de ce lobule ne sont que le prolongement des étages inférieurs des trois circonvolutions longitudinales du lobe frontal.

La première circonvolution orbitaire $\mathrm{F}^{1}$ (fig. 90 ), droite et rectiligne, est comprise entre le bord interne du lobule et le premier sillon orbitaire, qui la sépare de la deuxième orbitaire, et qui, sous le nom de $f o^{1}$, est la continuation du sillon $f^{1}$ de la face convexe. Dans ce sillon, appelé encore sillon droit ou sillon olfactif, est couchée la bandelette olfactive, nommée à tort nerf olfactif. La première orbitaire s'étend de la pointe du lobe au bord antérieur de l'espace perforé, sur lequel son écorce grise se continue en s'amincissant brusquement. Le premier sillon orbitaire ne se prolonge pas jusqu'à l'espace perforé ; en d'autres termes, il ne s'étend pas jusqu'au bord postérieur du lobule orbitaire; il reste donc, en arrière de son extrémité postérieure, une portion indivise, large de 5 à 8 millimètres, qui établit, sur le bord de l'espace perforé, la continuité de la première circonvolution orbitaire avec la seconde. De même, le premier sillon orbitaire ne s'étend pas en avant jusqu'à la pointe du lobe frontal; il en est séparé par le pli d'anastomose qui a été indiqué plus haut et qui unit les mêmes circonvolutions en séparant $f o^{1}$ du sillon $f^{1}$.

La première circonvolution orbitaire est très étroite dans toute sa longueur ; toutefois elle s'élargit graduellement d'avant en arrière, de sorte que le premier sillon orbitaire suit, d'arrière en avant, une direction légèrement convergente.

Le bord interne de cette circonvolution se continue sur le bord interne du lobule orbitaire avec le lobe sus-orbitaire, déjà décrit sur la face interne. Il n'est jamais entamé par les incisures de ce dernier lobule.

G. Seconde circonvolution frontale, $\mathrm{F}^{2}$ - - La seconde circonvolution frontale, située en dehors de la première, naît de la partie moyenne de la frontale ascendante par la seconde racine frontale, se porte de là en avant jusqu'à l'extrémité antérieure du lobe, et gagne le bord surcilier, sur lequel elle se réfléchit pour passer sur le lobe orbitaire. Nous l'étudierons donc successivement sur la face convexe et sur la face orbitaire de l'hémisphère. 
$1^{\circ}$ Face convexe de la deuxième frontale. - La racine de cette circonvolution naît de la partie moyenne ou seconde portion de la frontale ascendante. Elle est ordinairement volumineuse et superficielle, de manière à interrompre la continuité du sillon prérolandique. Quelquefois cependant elle est profonde; mais on l'aperçoit toujours aisément en écartant les bords dı sillon.

Elle se dirige aussitôt en haut et en dedans, en s'appliquant sur le bord antérieur de la frontale ascendante, dont elle est séparée par le sillon prérolandique ; on n’a pas oublié que la racine de la première circonvolution frontale subit une déviation inverse ; les deux racines se rencontrent donc sur le bord antérieur de la frontale ascendante, en un point qui correspond à peu près au niveau du genou supérieur de la scissure de Rolando. Là elles s'adossent l'une à l'autre, et les deux circonvolutions se recourbent pour se porter en avant, le long du premier sillon frontal, $f^{1}$, qui les sépare ; elles cheminent ainsi, sinueuses l'une et l'autre, jusqu'à l'extrémité antérieure de l'hémisphère, en communiquant entre elles par les plis d'anastomose qui ont été indiqués à l'occasion de la première frontale, et qui marquent également sur la seconde une subdivision en trois portions.

Le bord externe de la deuxième frontale est plus court que son bord interne, et décrit une courbe assez forte qui embrasse dans sa concavité le bord correspondant de la troisième frontale. Le sillon $f^{2}$, qui sépare ces deux bords, est traversé, dans sa partie moyenne, par un pli d'anastomose plus ou moins transversal, qui est presque constamment superficiel, et qui le divise par conséquent en deux portions; la postérieure, comprise entre le pli d'anastomose et le sillon prérolandique, dont elle émane, suit une direction à peu près antéro-postérieure; mais la portion antérieure se recourbe bientôt vers le bas, et gagne le bord surcilier, sur lequel elle se réfléchit pour passer sur la face orbitaire; mais elle est traversée, vers le niveau de ce bord, par un second pli d'anastomose qui est constant, quoiqu'il ne soit pas toujours superficiel.

La face convexe de la deuxième frontale présente d'assez nombreuses incisures, les unes émanées des deux sillons qui la bordent et entament plus ou moins obliquement ses deux bords, les autres isolées et de formes diverses. Ces incisures isolées sont 
MORPhOLOGIE DU CERVEAU DE L'HOMME ET DES PRIMATES. 791

quelquefois assez compliquées; sur la partie antérieure de la circonvolution, elles affectent ordinairement une direction plus ou moins transversale.

La seconde frontale est en général un peu plus large que la première; sa largeur reste à peu près la même dans sa portion postérieure et dans sa portion moyenne; mais, dans sa portion antérieure, au moment d'atteindre le bord surcilier, elle s'élargit d'une manière notable, aux dépens de la première frontale, qui se rétrécit, et souvent aussi aux dépens de la troisième, de sorte qu'elle forme la plus grande partie du bord surcilier de l'hémisphère. Le premier sillon frontal présente à ce niveau une disposition assez variable. Toutefois il aboutit assez généralement ì une incisure à peu près transversale, qui correspond au bord surcilier et qui se porte en dehors, dans l'épaisseur de la seconde frontale, pendant qu'une autre branche, qu'on peut considérer comme représentant le sillon $f^{4}$, se dirige en dedans ver's la pointe du lobe. Cette incisure, que l'on retrouve toujour's a rec un peu d'attention, se prolonge quelquefois en dehors jusqu'au deuxième sillon frontal, et paraît alor's interrompre entièrement la continuité de la face convexe de la deuxième frontale et de sa face orbitaire ; mais ce n'est qu'une apparence, et, en écartant les bords de la partie externe de l'incisure, on roit que la continuité n'est réellement pas interrompue.

Ainsi élargie sur le bord surcilier, la seconde frontale passe immédiatement à la face inférieure du lobe frontal.

$2^{\circ}$ Face inférieure ou portion orbitive de $\mathrm{F}^{2}$. - Cette face quadrilatère, presque aussi étendue en largeur qu'en longueur, constitue plus des quatre cinquièmes de la surface du lobule orbitaire. Elle est comprise entre le premier sillon orbitaire $f o^{1}$, qui forme le bord interne, et le second sillon orbitaire $f o^{2}$, qui est beaucoup plus court et qui forme son bord externe. Son bord antérieur n'est autre que le bord surcilier de l'hémisphère; son bord postérieur enfin est à peu près transversal et limité par une légère dépression qui correspond au bord antérieur de l'espace perforé et de la vallée de Sylvius. Il est longé par la racine olfactive externe.

Sa surface est toujours subdivisée par des incisures assez profondes, qui communiquent les unes arec les autres, mais qui ne 
communiquent pas avec les sillons voisins et qui rentrent par conséquent dans la catégorie des incisures isolées. Si quelquefois l'une d'elles peut se prolonger jusqu'au deuxième sillon orbitaire, on peut toujours reconnaître aisément que ce prolongement est très superficiel, et que l'incisure n'émane pas du sillon.

La disposition de ces incisures, quoique assez variable chez l'homme, se rattache cependant à un type qui a été caractérisé par le nom d'incisure en $\mathrm{H}$ (sillon en $\mathrm{H}$ de Gratiolet) et qui se maintient dans toute la série des primates, avec des modifications relativement légères.

Ramené à l'état où il se trouve invariablement chez les singes d'Amérique, où il se trouve aussi chez la plupart des fœtus humains de six à neuf mois et enfin chez un grand nombre d'hommes adultes, ce type est caractérisé par deux incisures longitudinales, qui représentent les deux branches parallèles d'un $\mathrm{H}$ et par une incisure transversale qui unit leur partie moyenne en formant la troisième branche de l'H (fig. $90, \mathrm{n}^{\circ} 1$ ).

Les deux branches longitudinales $a$ et $b$ sont espacées de manière à diviser la circonvolution, suivant sa largeur, en trois portions à peu près égales. Elles sont quelquefois presque rectilignes, l'externe surtout, mais le plus souvent elles sont un peu courbes et se regardent par leur convexité, de sorte qu'elles sont un peu plus rapprochées à leur partie moyenne qu'à leurs extrémités.

La branche transversale $c$, qui les unit, correspond à cette partie moyenne. Elle est située un peu plus près du bord postérieur du lobule orbitaire que de son bord antérieur. On_sait que la face inférieure de ce lobule repose sur la convexité de la voûte orbitaire. Elle est donc un peu excavée; la branche transversale de l'incisure en $\mathrm{H}$ correspond à la portion la plus excavée.

On me permettra de signaler l'importance de la branche transversale de l'incisure en $\mathrm{H}$ : l'anatomie comparée des osmatiques et des anosmatiques démontre que la partie postérieure des deux premières circonvolutions orbitaires est affectée à l'olfaction, et constitue le centre olfactif antérieur ou orbitaire (1). La limite antérieure de cette portion olfactive du lobule orbitaire n'est pas

(1) Cf. Revue d'anthropologie, Mém. sur les centres olfactifs, 1879, et dans ce volume, page 383. 
indiquée sur la première circonvolution orbitaire, mais elle est marquée chez les primates par la branche transversale de l'H.

Tel est le type de l'incisure en $\mathrm{H}$. Il arrive fréquemment que les deux branches longitudinales décrivent des courbes beaucoup

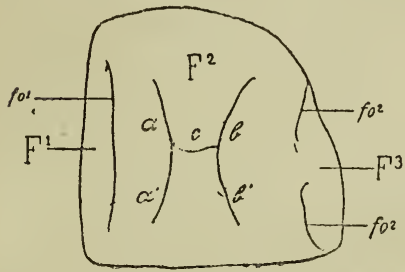

1

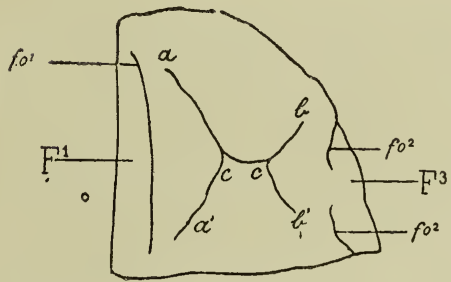

2

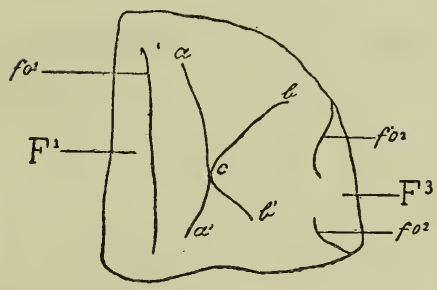

3

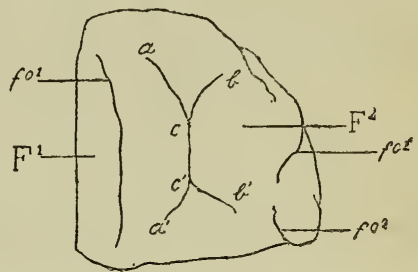

4

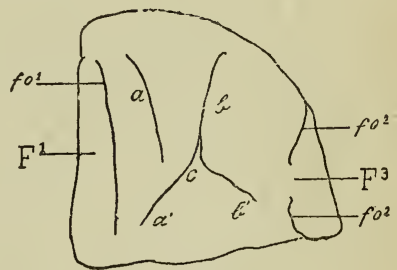

$\ddot{3}$

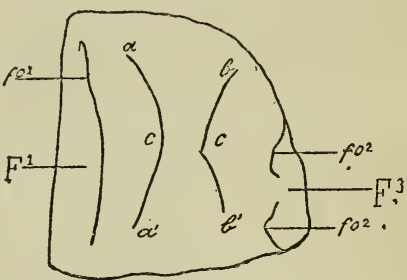

6

Fig. 90. Face inférieure du lobule orbitaire : variétés de l'incisure en $H$ (hémisphère gauche).

$\mathrm{F} o^{1}, \mathrm{~F}_{o^{2}}, \mathrm{~F}_{0^{3}}$ (marquées à tort sur la figure $\mathrm{F}^{1}, \mathrm{~F}^{2}, \mathrm{~F}^{3}$ ) portion orbitaire de la première, de la deuxième et de la troisième circonvolution frontale; $\mathrm{F}_{0}{ }^{\prime}, \mathrm{Fo}^{2}$, premier et second sillon orbito-frontal; $a, a^{\prime}, b, b^{\prime}$, branches longitudinales de l'incisure en $\mathrm{H} ; c$, sa branche trans. versale.

plus fortes, ou même(fig. 90, n²) qu'elles soient disposées sous la forme de deux angles obtus, dont les sommets sont unis par la branche transversale, qui est alors assez longue, de sorte que le système présente la forme d'une incisure transversale en double fourche.

D'autres fois, la branche transversale devient plus ou moins 
oblique, sans cesser pour cela d'aboutir aux deux branches longitudinales. Mais ces modifications sont légères, et le type de l'II, quoique plus ou moins déformé, est toujours parfaitement apparent.

On peut évaluer à 60 pour 100 environ le nombre de cas où les incisures de la deuxième circonvolution sont disposées suivant le type de l'H. Mais, en dehor's de ces cas, il existe plusieurs variétés.

En premier lieu, la branche transversale, dont la longueur peut atteindre jusqu'à 18 et même 20 millimètres, peut devenir très courte, se réduire à 3 ou 4 millimètres, et même se réduire à un point de contact, de sorte que les deux branches longitudinales se touchent par leur convexité, et que l'H devient un $K$ (fig. 90, $\mathrm{n}^{\circ} 3$ ).

Puis, le contact s'étendant de plus en plus, l'ensemble des deux branches longitudinales peut revêtir la forme d'un $x$ italique allongé, et enfin celle d'une incisure longitudinale en double fourche (fig. $90, \mathrm{n}^{\circ} 4$ ). Il semble alors que l'H soit renversé, et que la branche transversale soit devenue longitudinale. C'est une erreur : la branche transversale est nulle; la branche $c c^{\prime}$ est une ligne de contact commune aux deux incisures longitudinales, et celles-ci se séparent l'une de l'autre, en avant et en arrière, comme les deux branches d'une fourche. Cette disposition s'observe assez souvent.

En second lieu, il peut se faire que les deux incisures longitudinales ne conmmuniquent pas entre elles. L'interne (fig. 90, no 5) n'occupe que la partie antérieure du lobule, et s'arrête là où elle est habituellement rejointe par la branche transversale. Mais l'externe $b$, parvenue à ce même niveau, se bifurque en $c$ en deux branches $c b^{\prime}$ et $c a^{\prime}$; la première est évidemment le prolongement de $b$; la seconde, $c a^{\prime}$, doit être considérée comme représentant la branche transversale devenue très oblique.

Enfin il arrive quelquefois que les deux branches longitudinales sont simples l'une et l'autre et disposées comme deux parenthèses retournées (fig. 90, n॰6). Elles ne communiquent pas, et la branche transversale fait défaut. Mais, si l'on examine en $c c$, entre leurs points les plus rapprochés, la surface qui les sépare, on voit qu'elle est déprimée, que l'épaisseur de la couche corticale y est diminuée, et l'on retrouve ainsi la portion de la branche 
transversale effacée. Cette disposition, qui est ordinaire chez les macaques et fréquente chez les anthropoïdes, est assez rare chez l'homme.

Dans l'intervalle assez large qui existe en avant de la branche transversale, entre les deux' incisures longitudinales de l'H, on trouve quelquefois d'autres incisures peu profondes et assez courtes; mais la surface quadrilatère ou triangulaire située en arrière de la branche transversale ne se subdivise jamais.

Je n'ai pas épuisé la liste des variétés de l'incisure en $\mathrm{H}$; mais celles que j’ai décrites conduiront aisément à l'interprétation des autres. Avec un peu d'attention, on retrouvera toujours la trace de la ligne de démarcation établie par la branche transversale de l'H, entre la portion antérieure de la deuxième circonvolution orbitaire et la portion postérieure plus petite, qui paraît affectée à l'olfaction. Cette ligne de démarcation est marquée nettement et de très bonne heure sur le cerveau du fœtus humain, par une incisure transversale qui est située plus près de l'extrémité antérieure du lobule orbitaire qu'elle ne l'est chez l'adulte. Plus tard, lorsque les circonvolutions se développent davantage et commencent à se compliquer, la portion antérieure du lobule orbitaire s'accroît plus que sa portion postérieure, et la refoule plus ou moins irrégulièrement en arrière, en déformant la figure primitive de l'H et en effaçant même quelquefois une partie de cette figure; mais le type fondamental persiste presque toujours dans ce qu'il a d'essentiel, et, s'il est quelquefois plus ou moins masqué, il n'est jamais entièrement dénaturé.

D. Troisième circonvolution frontale, $\mathrm{F}^{3}$. - La troisième circonvolution frontale est très rudimentaire chez les singes ordinaires; elle se développe davantage chez les singes anthropoïdes; mais c'est chez l'homme seulement qu'elle acquiert tout son développement.

(Ici s’arrète le manuscrit de Broca; ce qui va suivre a été rédigé par le docteur S. Pozzi, qui s'est efforcé de se tenir le plus près possible de la pensée du maître, exprimée dans son enseignement oral ou écrit. On a cru devoir observer, dans ce complément, une prudence et une réserve qui feront excuser les omissions.)

Ce développement excessif de la troisième frontale chez l'homme s'explique par l'apparition d'une fonction nouvelle à laquelle elle préside, le 
langage. C'est à Paul Broca que la science est redevable de la démons. tration définitive de ce fait capital; il est donc juste, à l'exemple lles Anglais, de ne plus nommer cette circonvolution autrement que : circonvolution de Broca. D’après ses travaux, l'organe de la parole est $\mathbf{1 3}$ fois sur 14 la troisième circonvolution frontale gauche, 1 fois sur 14 la droite, ainsi que le montre la perte du langage, l'aphémie (mot préférable à celui d'aphasie d'après Broca), à la suite des lésions de cette région du cerveau. Or, coïncidence bien frappante, c'est précisément la même proportion qui se remarque entre droitiers et gauchers dans la race caucasigue.

Cette circonvolution offre un aspect très caractéristique; elle est formée d'un simple pli très gros et très nettement limité qui se recourbe sur luimême et semble presque revenir à son point de départ après avoir fait deux méandres.

Ce pli, comme étranglé et tassé sur lui-même entre son origine et sa terminaison, longe supérieurement la scissure de Sylvius, en formant audessus de l'insula une partie de l'opercule de Burdach, et parvenu audessus de la partie antérieure du lobe temporal se réfléchit sur le lobule orbitaire. On doit donc, comme pour les circonvolutions précédentes, lui distinguer : $1^{\circ}$ une face convexe; 20 une face inférieure ou orbilaire.

$1^{\circ}$ Face convexe de la troisième frontale: On peut la diviser elle-même en trois parties : une première va de son point d'origine à la branche ascendante de la scissure de Sylvius. Une deuxième partie s'étend de la branche ascendante de la scissure sylvienne à sa branche horizontale. Une troisième enfin va de ce dernier point au lobule orbitaire.

La première portion, ou postérieure, nait du pied de la frontale ascendante avec laquelle elle se continue en limitant inférieurement le sillon prérolandique. Elle est marquée d'une profonde incisure à peu près verticale. Un peu avant de se continuer avec la seconde portion, elle reçoit de la seconde circonvolution frontale un pli d'anastomose presque toujours superficiel. Broca croyait pouvoir affirmer que c'était cette portion seule qui était le véritable siège de la faculté du langage articulé.

La seconde portion, ou portion moyenne, est la plus caractéristique cliez l'homme. Transformée régulièrement par une longue et profonde incisure en une sorte de boucle, elle prend en bas la forme d'un petit lobule triangulaire à sommet inférieur, intercepté entre la branche ascendante de la scissure de Sylvius (en arrière) et sa branche horizontale (en avant). Broca a décrit pour la première fois celle disposition dans son Etude sur le cerveau du gorille (1) et il a appelé cap de la troisième circonvolution frontale ce petit lobule particulier. "Son volume et sa forme sont peu variables, dit-il ; toutefois il est plus ou moins aigu selon que les deux branclıes qui le limitent naissent plus ou moins près l'une de l'autre. Ordinairement elles sont séparées à leur origine par une distance de 4 à 5 millimètres seulement; alors le sommet du cap est quelque peu arrondi : quelquefois la distance est plus grande et le sommet s'élargit en proportion ;

(1) Revue d'anthropologie, 1878, p. 20, et dans ce volume, p. 601. 
mais quelquefois aussi cette distance est nulle; les deux branches, quoique divergentes, se confondent à leur origine avant de se séparer, leur ensemble présente la forme d'un $Y$ à courte queue, et le cap se termine en pointe. Celte forme en $Y$ s'observe surtout sur les cerveaux peu riches en circonvolutions.

"Telle est la disposition des deux branches antérieures de la scissure de Sylvius dans le cerveau humain; elle est constante, exception faite d'un petit nombre de cerveaux d'imbéciles et d'idiots, et de quelques cerveaux de microcéphales. Elle est la conséquence du grand développement qu'acquiert chez l'homme la troisième circonvolution (1). "

La troisième portion, ou antérieure, ne mérite pas de description, car elle se réfléchit aussitôt née pour se continuer avec la troisième circonvolution orbitaire qui n'en est que la terminaison, non sans avoir auparavant fourni à la deuxième circonvolution frontale, sur le bord sourcilier, un pli d'anastomose qui est constant, mais non toujours superficiel.

$2^{\circ}$ Face inférieure ou portion orbitaire de $\boldsymbol{F}^{3}$. Sous la forme d'un petit mamelon arrondi et légèrement incisé ou marqué d'une fossette en coup d'ongle, elle constitue la partie la plus externe du lobule orbitaire. Elle est bornée antérieurement par l'extrémité postérieure de $f^{02}$; à la partie postérieure, elle n'est séparée par aucune limite apparente de $\mathrm{F}^{02}$. Elle confine à ce niveau avec la dernière circonvolution temporale : entre elles deux s'étend un repli falciforme.

ART. III. Circonvolutions du lobe pariétal.

Le lobe pariétal, relégué au second plan chez les primates par le magnifique développement du lobe frontal, constitue au-dessous d'eux la presque totalité de ce qu'on pourrait appeler la partie intellectuelle du cerveau. Quoi qu'il en soit, il n'en offre pas moins chez l'homme une richesse tout à fait incomparable à celle qu'il présente même chez les singes supérieurs, et de cetle complexité résulte des différences morphologiques plus apparentes que réelles.

Le lobe pariétal comprend trois circonvolutions : une circonvolution ascendante ou transverse ou antérieure, $\mathrm{P}$, et deux circonvolutions désiguées sous le noun de promière el de seconde $P^{1}$ et $P^{2}$.

A. Circonvolution pariétale ascendante, P. Elle forme le bord postérieur de la scissure de Rolando qu'elle borne parallèlement à la frontale ascendante $\mathbf{F}$ située en avant. De mème qu'à cette dernière on lui a aussi donné le nom de traverse : il vaudrait mieux l'appeler circonvolution postrolandique, si l'usage reçu n'avait pas ici une si grande autorité.

Elle appartient exclusivement à la face externe, car le lobule ovalaire sur lequel elle se termine supérieurement au niveau de la face interne de l'hémisphère doit être considéré (Broca) comme formé par l'épanouisse-

(1) Broca, Loco citato. 
ment de la seule frontale ascendante plutôt que comme une zone neutre et mixte dépendant à la fois de deux circonvolutions marginales de la scissure de Rolando (Pozzi) (1).

$\mathrm{P}$ présente les mêmes flexuosités que $\mathrm{F}$ et reproduit, comme elle, celles de la scissure à laquelle elle sert de limite. On peut aussi lui considérer trois portions, savoir :

$1^{\circ}$ Une portion supérieure et interne comprise entre le bord sagittal de l'hémisphère et le genou supérieur de la scissure; elle correspond aux racines de la première circonvolution pariétale;

$2^{\circ}$ Une portion moyenne, comprise entre les deux genoux de la scissure; elle est longée par le sillon postrolandique;

$3^{\circ}$ Une portion inférieure et externe comprise entre le genou inférieur de la scissure de Rolando et le bord sylvien du lobe pariétal.

Existe-t-il des différences fonctionnelles entre ces trois portions? C'est ce qu'il est absolument impossible de déterminer dans l'état actuel de la science : rappelons seulement que cette circonvolution est comprise pour la plupart des auteurs dans la zone motrice.

La portion inférieure, qui aboutit à la scissure de Sylvius, prend une petite part à la formation du bord supérieur de la scissure et de son opercule. Elle s'unit en avant à la frontale ascendante par le pli de passage fronto-pariétal inférieur, et en arrière fournit la racine antérieure de la seconde circonvolution pariétale.

Lia portion moyenne, comprise entre les deux genoux de la scissure de Rolando, est libre et longée par le sillon postrolandique; ce sillon s'étend en bas jusque vers la scissure de Sylvius avec laquelle un prolongement superficiel peut en apparence le réunir d'une manière anormale; en haut, il est borné par la racine inférieure de $\mathrm{P}^{\mathrm{1}}$. Lorsque celle-ci est très grêle, il peut, en se continuant superficiellement avec une incisure verticale le $\mathrm{P}^{1}$, remonter jusque vers le bord sagittal de l'lémisphère et simuler ajsez bien la scissure de Rolando avec laquelle un œil peu exercé serait. exposé à le confondre.

La portion supérieure de la circonvolution pariétale ascendante va du genou supérieur de la scissure de Rolando jusqu'au bord supérieur de l'hé misphère où elle s'unit avec la frontale ascendante, par un étroit pli de passage (fronto-pariétal supérieur). Elle donne insertion à la double racine de la première circonvolution pariétale, $\mathrm{P}^{\mathbf{1}}$.

Par la description précédente, à peu près calquée sur celle de la frontale ascendante, on voit quel parallélisme exact existe entre ces deux circonvolutions.

Elles n'en sont pas moins profondément séparées, morplıologiquement, et il y a une véritable incohérence d'idées et de mots à les réunir d'une façon factice sous le nom de lobe central.

(1) Article Circonfolutions du Dictionnaire encyclopédique des sciences médicales, 1875. 
Nous intercalons ici un important fragment de Broca, sur ce sujet, trouvé dans ses notes; il nous a paru impossible de ne pas le publier, bien que les opinions qu'il exprime aient déjà été résumées plus haut (Cf. Revue d'anthropologie, 1883, p. 403-405, et dans ce volume, p. 781-783).

"Le prétendu lobe central. - Il ne sera pas inutile de dire quelques mots d'un prétendu lobe central, admis par quelques auteurs allemands (lobe que d'autres appellent seulement lobule central). Il serait formé par la réunion des deux circonvolutions ascendantes (frontale et pariétale) que sépare la longue et profonde scissure de Rolando.

"Abstraction faite du prétendu fait anatomique qu'il exprime, ce nom est bien malheureusement choisi. D'une part, en effet, il y a dans l'hémisphère un autre lobe qui a été appelé central par Gratiolet, et qui l'est réellement : c'est le lobe de l'insula; d'une autre part, les parties qui forment le prétendu lobe central ne sont plus ou moins centrales que chez les primates; chez tous lcs autres mammifères, elles sont situées tout en avant, et on en a méconnu les analogies à cause précisément de cette fausse épithète de centrales qu'on leur a appliquée.

"Toute l'étude du manteau repose sur celle des délimitations établies par les anfractuosités. Ce qui caractérise un lobe, c'cst de ne communiquer avec ses voisins que par des plis de passage, et d'être nettement limité, sinon de toutes parts, du moins dans la plus grande partie de son contour, par des scissures, et ce qui caractérise les scissures, indépendamment de la grandeur et de la profondeur de la plupart d'entre elles, c'est leur fixité anatomique, presque partout très manifeste, dissimulée seulement, sur une partie de la scissure occipitale, par le développement quelque peu variable des plis de passage.

" D’un autre côté, les sillons qui séparent les circonvolutions dans un même lobe se distinguent des scissures par leur extrême régularité, résultant du volıme relatif des circonvolutions voi sines, de leur complication, de leurs anastomoses.

« Examinons à ce double point de vue la constitution du prétendu lobe central.

"Sa circonvolution antérieure est limitée en avant par l'un des sillons les plus variables de l'hémisphère (sillon prérolandique), et se continue presque sans démarcation par trois grosses 
racines avec les circonvolutions longitudinales du lobe frontal. Non moins variable, non moins irrégulier est le sillon postrolandique, qui limite en arrière la circonvolution postérieure, à tel point que les auteurs ne s'accordent pas sur cette démarcation, les uns considérant la "centrale postérieure » comme distincte, les autres comme fusionnée avec la première pariélale. Il en résulte que, si l'on suit le contour du lobe central, on trouve qu'il est variable et vague, interrompu, comme le sont, dans un même lobe, les limites des circonvolutions.

( En second lieu, il existe, entre les deux circonvolutions qui, dit-on, composent ce lobe, une anfractuosité très longue, très profonde, très tixe dans toutes ses parties et ininterrompue: c'est la grande scissure de Rolando, l'une des plus caractéristiques, l'une des plus précoces dans le développement, l'une de celles par conséquent qui établissent des divisions primaires. Les deux circonvolutions, séparées par elle dans toute leur longueur, ne communiquent qu'à leurs extrémités par de minces plis de passage; et on se demande comment on a pu concevoir la pensée de les réunir en un même lobe, lorsqu'il est certain qu'elles sout, d'une part, aussi disjointes que possible, en même temps qu'elles sont, d'une autre part, fusionnées respectivement avec le lobe frontal et avec le lobe pariétal. On aurait ainsi un lobe dont les parties seraient beaucoup plus distinctes l'une de l'autre qu'elles ne le sont des parties voisines, et ce ne serait plus sur l'anatomie, mais sur la fantaisie, que reposerait la distinction des lobes de l'hémisphère.

(Une pareille violation des règles anatomiques ne se concevrait pas si l'on ne tenait compte des circonstances quil'ont amenée.

"Avant les travaux de Gratiolet, ce n'était pas sur le cerveau, mais sur le crâne, qu'on établissait les limites des lobes de l'hémisphère. Le lobe frontal était la portion située sous l'écaille de l'os frontal; le lobe pariétal était la portion recouverte par le pariétal, etc. Gratiolet, le premier, entreprit de tracer ces limites sur l'hémisphère lui-même, c'est-à-dire sur les anfractuosités de sa surface. Mais il n'y parvint pas entièrement du premier coup; en ce qui concerne le lobe frontal, il resta quelque temps encore sous l'influence de ses prédécesseurs, et, comme il lui parut que la circonvolution frontale ascendante était située sous l'os parié- 
tal et non sous l'os frontal, il crut d'abord devoir la rattacher au lobe pariétal. Il la décrivit comme telle, en 1854, dans son célèbre Mémoire sur les Plis cérébraux des Primates, qui donna une impulsion si nouvelle et si féconde à l'étude des circonvolutions cérébrales. Mais, en continuant ses recherches, il ne tarda pas à reconnaître que cette délimitation était fausse, et, en 1857, dans le second volume de l'Anatomie comparée du système nerveux (1), il plaça définitivement sur la scissure de Rolando la limite du lobe frontal (2). Cette nouvelle détermination fut accęptée aussitôt par la plupart des auteurs ; mais quelques-uns avaient déjà pris l'habitude de diviser les lobes suivant la méthode de Gratiolet; et, l'ayant adoptée dans leurs recherches et dans leurs publications, ils y restèrent fidèles, même après que Gratiolet l'eut abandonnée.

"Ils continuèrent donc à comprendre la circonvolution frontale ascendante dans le lobe pariétal, et, dès le moment que les deux circonvolutions ascendantes se trouvaient réunies dans un même lobe, il était assez naturel de les considérer comme formant dans ce lobe une région spéciale; car, étant très semblables l'un à l'autre par leur direction, leur forme et leur simplicité, elles contrastaient d'une manière frappante avec les circonvolutions longitudinales compliquées et polymorphes du reste du lobe. Elles semblaient donc constituer un système à part, et, comme Huschke les avait toutes deux appelées circonvolutions centrales, cette similitude de nom (on sait quelle influence les mots exercent sur les idées) contribua encore à donner le change. On ne pouvait donc faire moins que de constituer dans le lobe pariétal

(1) Le premier volume avait été publié par Leuret en 1838.

(2) Gratiolet, toujours dominé par l'idée que ses prédécesseurs lui avaient inculquée, savoir : que les limites des lobes devaient correspondre aux limites des os, pensa alors qu'il s'était trompé en plaçant la frontale ascendante sous l'os frontal, qu'elle devait être sous l'os pariétal, et que la scissure de Rolando devait se trouver immédiatement au-dessous de la suture coronale; pour s'en assurer, il prit quelques mesures comparatives sur le crâne, le moule intracrânien et cerveau élalé sur la table; mais lorsqu'on a une idée préconçue, on n'est pas bien difficile sur les preuves; il crut donc reconnaître que la scissure de Rolando correspondait à la sulure coronale, erreur énorme, qui fut acceptée avec une facilité élonnante jusqu'au jour où je démontrai par le procédé de fiches que l'extrémité supérieure et postérieure de la scissure de Rolando est située à 4 centimètres au moins, et souvent à plus de 5 centimètres, en arrière de la suture coronale. 
un lobule particulier, le lobule central, formé par la réunion de ces deux circonvolutions. Mais la logique vint à son tour ; il était évident que la centrale postérieure affectait avec les circonvolutions longitudinales du lobe pariétal les mêmes rapports de continuité que la centrale antérieure avec les circonvolutions longitudinales du lobe frontal, que le sillon postrolandique n'était ni moins incomplet ni moins profond, ni moins fixe (ce qui n'est pas beaucoup dire) que le sillon prérolandique, et, puisque celui-ci paraissait suffisant pour établir une division lobaire, il n'y avait vraiment aucune raison pour refuser de donner à celui-là la même importance. - Le lobule central devint donc le lobe central.

"Telle a été la genèse du lobe,central. Il a été formé de deux portions artificiellement détachées des deux lobes adjacents, comme ces Etats neutres que l'on constitue sur les deux côtés d'une frontière naturelle pour séparer deux grands pays.

"Que la scissure de Rolando soit une frontière naturelle, c'est ce que démontre clairement l'embryologie. La scissure de Rolando apparaît vers la fin du cinquième mois, marque déjà la limite du lobe frontal et du lobe pariétal à une époque où la surface de ces deux lobes est encore tout à fait lisse. Les sillons qui les subdivisent n'apparaissent qu'ensuite, sous la forme de dépressions qui, sur le lobe frontal, naissent bien en avant de la scissure. Ces sillons sont longitudinaux; ils s'allongent d'avant en arrière, ou se rapprochent de la scissure de Rolando; la partie postérieure du lobe, celle qui deviendra la frontale ascendante, ne forme pas alors un district particulier ; rien n'indique encore la limite sur laquelle elle s'arrêtera. Mais, plus tard, lorsque les deux sillons longitudinaux ont acquis assez de longueur et de profondeur pour rendre bien distinctes les trois circonvolutions frontales longitudinales, celles-ci s'allongent, deviennent flexueuses dans toute leur étendue, mais surtout au niveau de leur racine, qui décrit, en se repliant, un grand méandre transversal. Le sillon compris dans ce méandre est le sillon prérolandique; il n'est pas complet, il est ordinairement interrompu dans sa partie moyenne par l'insertion de la racine de la seconde circonvolution frontale, mais il indique du moins la limite de la portion du lobe frontal qui s'est subdivisée et de celle qui est restée indivise; c'est cette dernière portion qui constitue la circonvolution fron- 
tale ascendante. Elle fait donc partie intégrante du lobe frontal.

" Le sillon postrolandique se forme chez le fotus, sinon exactement de la même manière, du moins d'une manière tout à fait analogue.

"L'étude de la scissure de Rolando et des sillons des deux lobes adjacents, chez les singes cébiens qui ont peu de circonvolutions, conduit aux mêmes résultats.

" On voit que la conception du lobe central est contraire à tous les faits anatomiques. Les expériences physiologiques et les observations pathologiques ont paru, il est vrai, lui donner quelque appui, en montrant que les deux circonvolutions ascendantes ont une fonction motrice. Certains centres moteurs, qui sont encore à l'étude, y ont été signalés et presque démontrés, mais il est permis de croire que ces centres se rapportent à des mouvements différents, qu'ils correspondent à des parties différentes de l'appareil musculaire. D'autres portions motrices ont été découvertes aussi dans d'autres parties du lobe frontal et du lobe pariétal, et, si l'existence des propriétés motrices devait suffire pour réunir en un même lobe, ou en un même lobule, les deux circonvolutions ascendantes, il faudrait joindre aussi à ce même lobe toute la portion motrice des autres circonvolutions frontales et pariétales. On obtiendrait ainsi un grand lobe moteur qui n'aurait absolument aucune limitc anatomique.

«L'anatomie cérébrale ne relève que d'elle-même, et ne saurait être subordonnée aux doctrines ou hypothèses physiologiques. Sans elle, la physiologie des circonvolutions n'aurait pas même pu naître. On n'a pu concevoir la pensée d'aller à la recherche des localisations cérébrales que lorsqu'on a su, grâce aux progrès de l'anatomie descriptive, distinguer pour soi et désigner pour les autres les parties observées. Les anatomistes ont d'abord démontré qu'il y a, dans la matière de l'hémisphère, en dépit des apparences trompeuses, des divisions et des subdivisions constantes; que ces lobes, ces circonvolutions et ces plis si longtemps qualifiés de "désordonnés " sont dans un ordre parfait; qu'ils sont fixes quant à leur volume et quant à leurs connexions, et que, si l'on y enfonce une épingle en un point quelconque, la position de cette épingle pourra être déterminée exactement de la même manièrc (sauf les synonymies), par tous 
ceux qui ont étudié le cerveau. Cette base anatomique une fois acquise, les physiologistes, jusqu'alors impuissants, ont pu commencer leur's recherches; leur rôle est d'interpréter les faits anatomiques et non de les arranger arbitrairement, en vue de telle ou telle théorie, et celui d'entre eux qui a eu l'honneur, il y a dix-huit ans, de faire le premier pas dans cette voie, a peutêtre le droit d'exprimer ainsi son avis. »

Nous n'avons pas cru nécessuire de poursuivere la description du cerveau schématique au-delà des limites qui nous étaient fixées par la nécessité d'y rattacher cet important fragment.

Dr S. P. 


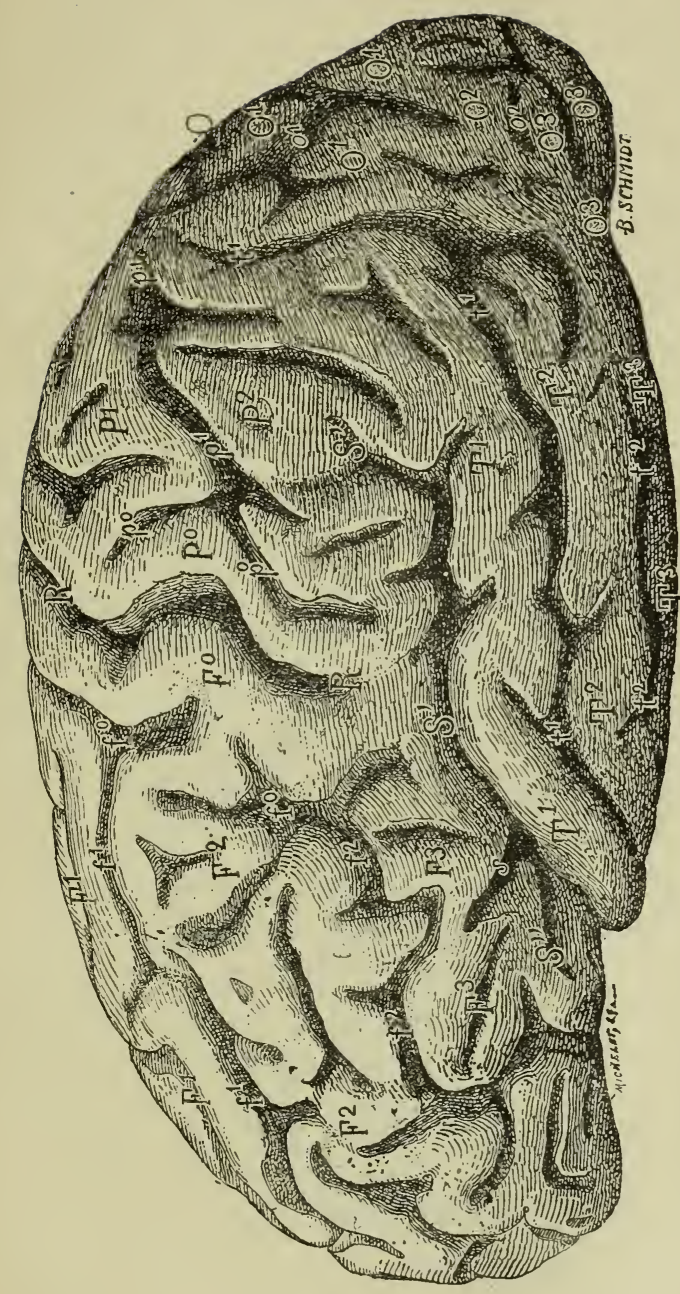

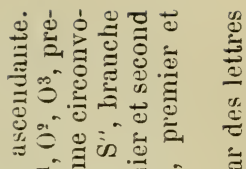

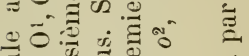

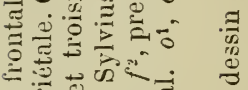

$\approx$ ป

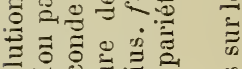

융

हैं

ఏ.

-

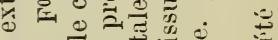

ญ

-

है ठำ

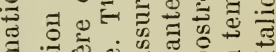

ヨ.ّ.

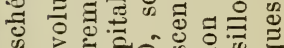

क \.

ฐ

ว. जै

ड

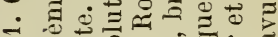

ธ

ن.

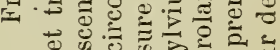

๘

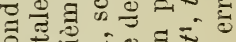

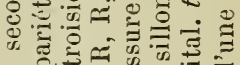

०थ

एँ

ヨี

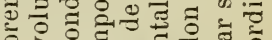

4.

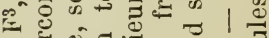

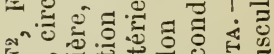

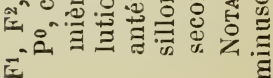





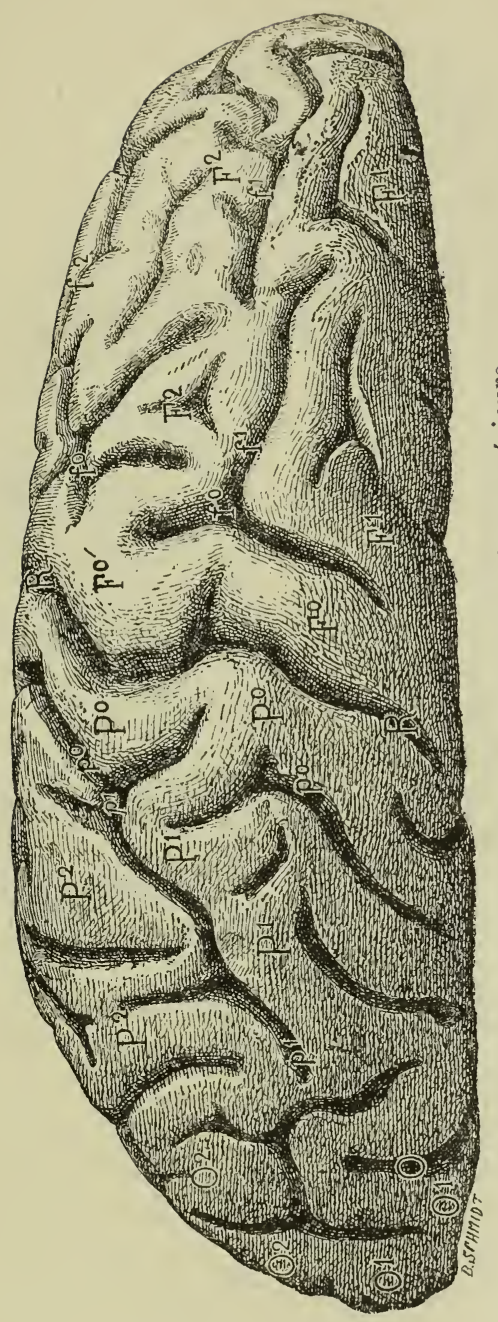

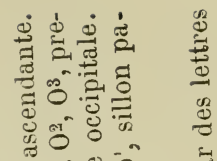

० उँ है है

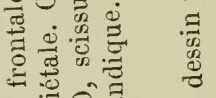

= 0 층

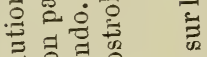

릉. 클

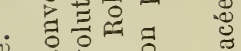

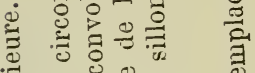

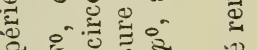

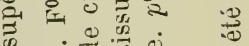

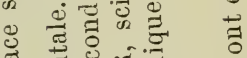

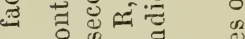

है

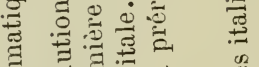

ब्ञ

क्व

ङु

过

๙

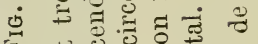

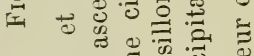

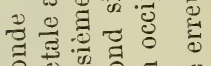

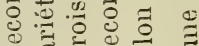

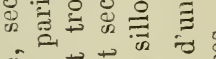

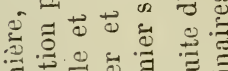

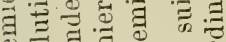

2ㅇํำ

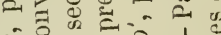

की

ฯ

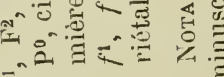

E 


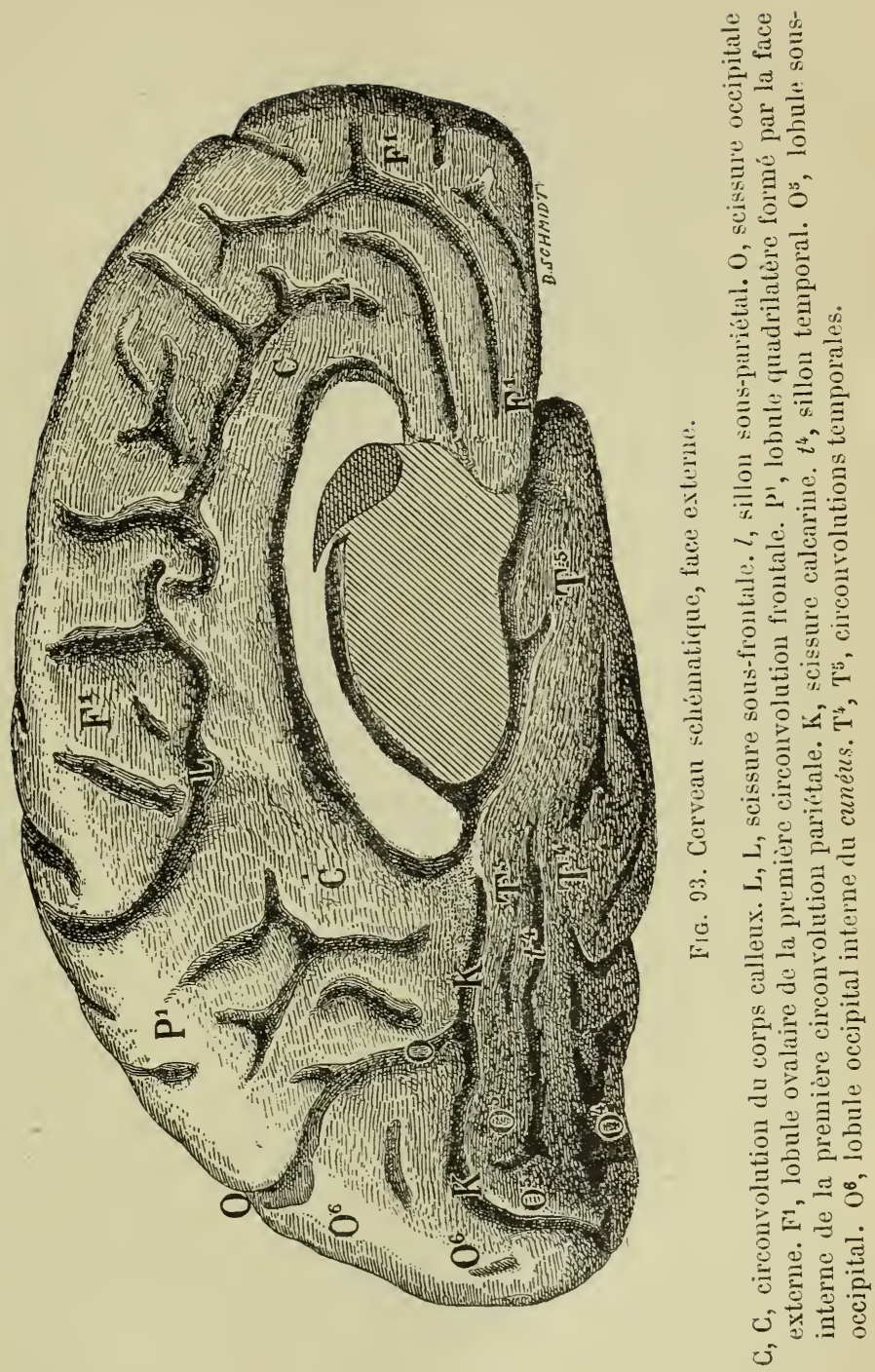



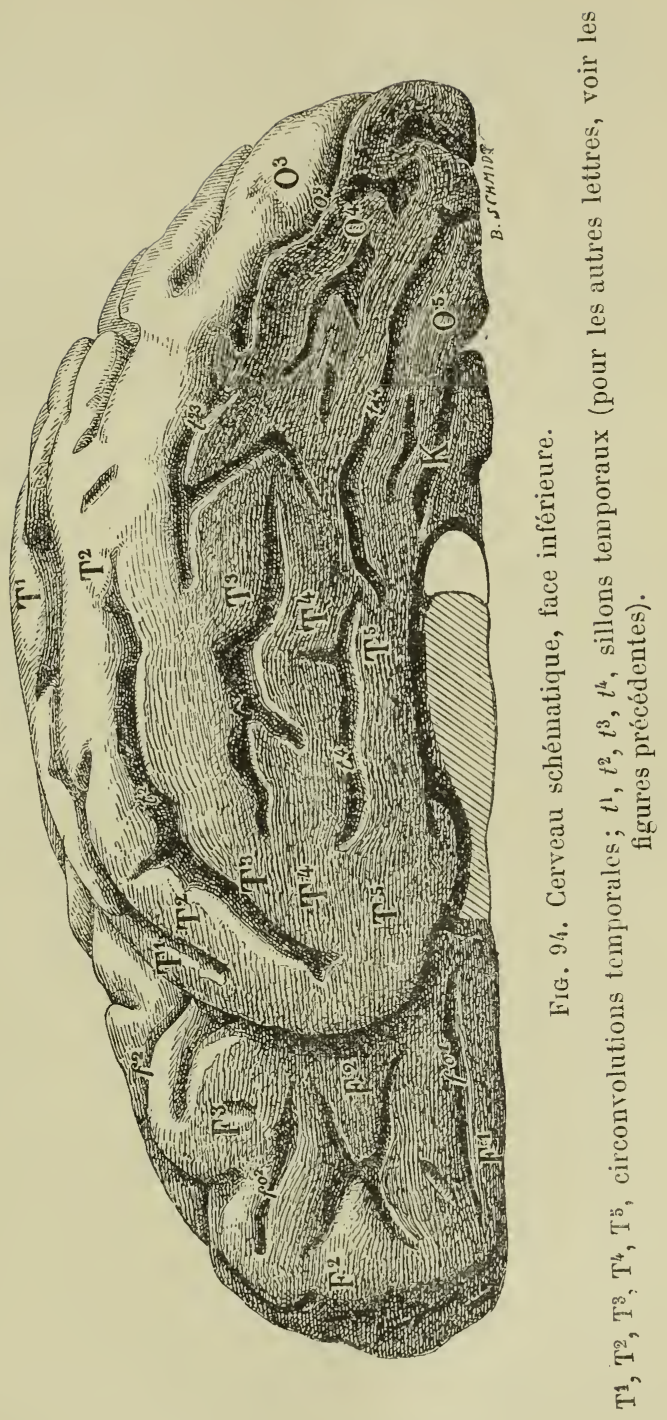





\section{PROCÉdés dE CONSERVATION dES CERVEAUX.}

\section{MOMIFICATION DES GERVEAUX}

(Bulletins de la Sociëté d'anthropologie, 186ว̈, t. VI, p. 26-27.)

M. Broca présente plusieurs hémisphères cérébraux, momifiés depuis quatre ans et durs comme du carton. Ces cerveaux, dont le poids et le volume sont considérablement réduits, ont conservé d'une manière remarquable leur forme et leur modelé, et se prêtent bien mieux que les pièces fraîches à l'étude des circonvolutions. Celles-ci, en effet, se sont un peu séparées les unes des autres, l'œil pénètre aisément jusqu'au fond des sillons, qui sont élargis, et la disposition des plis cérébraux se reconnaît sans aucune hésitation.

Le procédé qui fournit ces résultats pourrait être appliqué à un cerveau entier, mais on n'apercevrait plus les circonvolutions de la face interne des hémisphères. Il vaut donc mieux séparer les deux hémisphères. On les dépouille de leur pie-mère, et on les plonge dans un bain composé de cinq parties d'eau et d'une partie d'acide nitrique. Au bout de deux jours, on double la dose d'acide; deux jours plus tard, on retire les hémisphèes, qui sont déjà assez durs et qui ont perdu environ le quart de leur poids. On les fait égoutter sur des chiffons de linge qu'on renouvelle deux ou trois fois le premier jour. Le lendemain, ils sont déjà assez secs pour ne plus mouiller les doigts; alors on les expose à l'air libre, sur une planche et dans un lieu sec. Il faut avoir soin de les relourner tous les matins, pour qu'ils ne se déforment pas. Par une température de 20 à 25 degrés, les circonvolutions 
les plus superficielles commencent à brunir au bout de cinq ou six jours. A partir de ce moment, on peut se dispenser de retourner les pièces, qui ont déjà une consistance assez ferme. Au bout de douze à quinze jours, les pièces sont bien solides; elles rendent un son sec lorsqu'on les frappe; on peut les laisser tomber sans les briser. Mais il faut les laisser encore à l'air pendant un mois, parce qu'elles continuent à exhaler des vapeurs acides qui altéreraient les linges ou le papier.

Ce procédé de momification est des plus simples; il n'exige presque aucun soin et presque aucune dépense. Les cerveaux ainsi préparés peuvent se transporter dans la poche; on peut les expédier sans emballage; enfin ils se prêtent merveilleusement à l'étude des circonvolutions. M. Broca a donc cru qu'il était utile de le recommander, dans les instructions générales qui vont paraître incessamment, à l'attention des voyageurs et surtout des médecins fixés au milieu des races étrangères. Il a essayé plusieurs autres procédés de momification, mais tous ont l'inconvénient de déformer considérablement le cerveau.

\section{MOMIFICATION DES GERVEAUX}

(Bulletins de la Société d'anthropologie, $2^{\mathrm{e}}$ série, 1866, t. I, F. 440.)

M. le secrétaire général offre à la Société, de la part de 11. Nicolaï, le cerveau momifié d'un Slave de la région de Moscou. La section d'anthropologie de la Suciété des sciences naturelles de cette ville a reçu les Instructions pour l'anthropologie générale, et s'est assimilé plusieurs des méthodes qui y sont indiquées, entre autres le procédé de conservation des cerveaux, dû à M. Broca. Le cerveau offert aujourd'hui par M. Nicolaï et préparé d'après cette méthode est des mieux réussis; il inaugure une collection qui permettra plus tard les recherches les plus instructives sur la morphologie comparée du cerveau des diverses races. 
PROGÉDÉ

$\mathrm{DE}$

\section{CONSERVATION DES CERVEAUX}

(Bulletins de la Société d'anthropologie, 2 e série, 1877, t. XII, p. 191.)

M. Brоса dit que certains cerveaux se prêtent à une longue et parfaite conservation ; certains autres se dógradent très vite. C'est une question d'idiosyncrasie organique.

Quant au procédé de momification par rétraction, il a l'avantage de produire l'écartement des sillons, circonstance qui permet de distinguer ceux qui sont profonds aussi nettement que ceux qui sont superficiels. Or, c'est là un point d'une haute importance dans l'examen des cerveaux complexes comme l'est celui de l'homme en particulier.

\section{CERVEAUX CONSERVÉS PAR LA GALVANOPLASTIE}

(Bulletins de la Société d'anthropologie, 2e série, 1877, t. XII, p. 609.)

M. Broca, en présentant à la Société des cerveaux humains dans un état de conservation parfaite, fait part du progrès sensible accompli, grâce aux travaux de M. Oré, en ce qui concerne l'étude du cerveau, et met sous les yeux de la Société des cerveaux momifiés par le procédé que M. Oré a découvert. Ce procédé réalise un double avaritage : la souplesse et le volume de l'organe sont également conservés.

En outre, M. Oré a trouvé moyen d'appliquer la galvanoplastie au cerveau momifié, condition qui en assure la conscrvation indéfinie. 
SUR LE PROCEDE

$\mathrm{DE}$

\section{CONSERVATION DES GERVEAUX}

DE M. ORÉ

(Bulletins de l'Académie de médecine, $2^{\mathrm{e}}$ série, t. VI, 11 décembre 1877, p. 1299.)

M. Broca : J'ai l'honneur de présenter à l'Académie, de la part de M. Oré, professeur à l'Ecole de médecine de Bordeaux, un certain nombre de cerveaux préparés par de nouveaux procédés.

Les cerveaux conservés dans l'alcool se déforment souvent, sont peu maniables à cause de leur fragilité, et ne se prêtent que très mal à l'étude des circonvolutions, parce qu'il est difficile et quelquefois même impossible d'écarter suffisamment les sillons sans en briser les bords. La momification, au contraire, a l'avantage de dilater les sillons et de les rendre plus apparents qu'ils ne le sont même sur le cerveau frais.

J'ai eu recours, depuis 1861, à un procédé de momification qui pendant longtemps m'a paru excellent, et qui consiste à traiter le cerveau par l'acide nitrique, à doses déterminées, et à l'exposer à l'air au bout d'un temps déterminé. Lorsqu'on se conforme à ce procédé, le cerveau, en se desséchant, ne se fendillepas; il ne se déforme pas; ses diamètres se réduisentde moitié environ, tous les détails des circonvolutions et des sillons se dessinent avec la plus grande netteté, et la pièce, devenue presque aussi dure que du carton, se conserve longtemps sans altération. J'avais même espéré que cette conservation serait définitive, et j'ai pu le croire pendant une dizaine d'années; mais, au bout de 
ce temps, quelques-uns des cerveaux ont commencé à se fendiller, puis à se briser, et, sur vingt-quatre cerveaux préparés en 1861, il n'en reste plus aujourd'hui que huit, qui sont déposés dans le musée de l'Institut anthropologique. D'autres procédés, étudiés dans ces dernières années, ont donné des résultats plus ou moins favorables; mais ils n'ont pas encore subi l'épreuve du temps, et on peut dire que jusqu'ici le problème de la conservation cérébrale n'était pas encore résolu. C'est ce qui fait l'importance des résultats obtenus par M. Oré.

M. Oré a eu recours d'abord à un procédé qui conserve le cerveau sous un volume peu inférieur au volume primitif, et qui lui laisse une certaine souplesse. M. Oré n'indique pas encore la nature de son procédé. D'après le degré de consistance des pièces, et, d'après l'odeur du vernis qui les recouvre, j'ai lieu de croire que ce procédé est assez analogue à celui qui a été imaginé par un interne distingué des hôpitaux, M. Boudet de Pâris; cet anatomiste opère de la manière suivante : la pièce séjourne d'abord dans l'alcool jusqu'à ce que toute l'eau des tissus soit remplacée par le liquide conservateur; on l'expose alors à l'air, en en recouvrant très fréquemment toute la surface d'une couche de vernis Sœhné. Dès qu'une première couche est sèche, on en applique une seconde, puis une troisième, et ainsi de suite pendant plusieurs jours. L'alcool imbibé dans les tissus ne pouvant s'évaporer à travers le vernis, la pièce conserve son volume avec un certain degré de souplesse. M. Boudet de Pâris, qui était mon interne l'année dernière, a préparé ainsi pour notre musée un certain nombre de pièces très remarquables. Il applique son procédé à la conservation des divers viscères, des muscles, des tumeurs, etc.; mais je ne crois pas qu'il l'ait appliqué jusqu'ici à la conservation du cerveau.

Le procédé plus ou moins analogue de $\mathrm{M}$. Oré donne, comme nous pouvons le voir, de très beaux résultats; mais ces résultats seront-ils durables? J'ai lieu de croire que non. Il me parait extrêmement probable que l'imperméabilité du vernis dont il s'est servi ne sera pas absolue, qu'au bout d'un certain nombre de mois ou d'années tout le liquide interstitiel auquel la pièce doit son volume et sa souplesse s'évaporera insensiblement, et que celle-ci, en se desséchant et se ratatinant, se déformera beau- 
coup. Si donc M. Oré s'en était tenu là, il faudrait attendre longtemps encore avant de se prononcer sur la valeur de son procédé.

Mais ce premier résultat une fois acquis, M. Oré en a obtenu un autre beaucoup plus important et entièrement nouveau. Grâce à l'action isolante du vernis qu'il a employé, il a pu préparer la pièce pour la galvanoplastie et faire déposer à sa surface une couche métallique épaisse, très solide, uniforme et indestructible, qui l'entoure complètement et qui en reproduit tous les détails d'une manière merveilleuse. Il a même pu, en opérant successivement sur chaque circonvolution, avec des bains variés, obtenir pour chacune d'elles une coloration particulière due à la nature du sel métallique en solution.

L'application de la galvanoplastie à la préparation et à la conservation du cerveau est entièrement nouvelle. Les tentatives faites jusqu'ici avaient échoué. Notre collègue M. Luys, en particulier, s'en était occupé sans succès. Il s'était servi de pièces enduites de collodion. Mais le collodion, d'une part, forme, dans les anfractuosités de la surface du cerveau, des points irréguliers qui les déforment; d'une autre part, il se rétracte sur cette surface compressible, il se fendille de telle sorte que son action isolante n'est plus complète, et l'expérience échoue.

M. Oré a donc réalisé un progrès important, qui mérite toute l'attention de l'Académie. 


\title{
PRUCLEE
}

POUR

\section{LA CONSERVATION DU GERVEAU}

\author{
AVEC SA FORME, SON VOLUME, SA COULEUR
}

APPLICATION DE LA GALVANOPLASTIE

PAR M. ORÉ

(Bulletins de l'Académie de médecine, 2e série, t. VI, 18 décembre 1877, p.1310.)

M. Broca. Les recherches sur les localisations cérébrales auxquelles les anatomistes, les physiologistes et les cliniciens se livrent depuis plusieurs années m’ont inspiré la pensée de trouver un moyen de conserver le cerveau avec sa forme, sa couleur et son volume. Après avoir essayé, sans résultat satisfaisant, les divers modes de conservation proposés par les expérimentateurs et avoir constaté le degré de ratatinement auquel conduit l'emploi des acides nitrique et chromique, je me suis arrêté au procédé suivant, qui m'a permis de préparer les pièces que M. le professeur Broca a présentées en mon nom à l'Académie.

Description du procédé. - La boîte crânienne une fois ouverte, j'enlève en totalité l'encéphale, que je dépouille aussitôt de ses membranes, de mảnière à mettre complètement à nu la substance cérébrale. Je la plonge alors dans de l'alcool à 90 degrés.

Toutes les douze heures, je la retourne dans le vase qui la renferme, afin que toutes les parties soient également saisies par l'alcool. Quatre jours après, je place dans toutes les scissures des mèches de coton destinées à séparer les circonvolutions, puis je change l'alcool; j'y plonge de nouveau le cerveau. Je renou- 
velle tous les quatre ou cinq jours la même manœuvre pendant vingt, vingt-cinq jours, un mois.

Le cerveau ayant alors acquis une fermeté très grande, je l'enveloppe de trois couches de linges superposées, maintenues en place par des bandes de caoutchouc larges de deux travers de doigt. Je mets habituellement trois bandes en travers, deux dans le sens antéro-postérieur.

La pièce, ainsi disposée, séjourne dans une étuve chauffée à 45 degrés, pendant quinze ou vingt heures au plus. Si l'on ne veut pas que la couleur en soit altérée, il faut la placer à la partie la plus inférieure de l'étuve, à une assez grande distance de la lampe à gaz.

Après quinze ou vingt heures, j'ôte la pièce de l'étuve, j'enlève les bandes de caoutchouc, les compresses de linge, les mèches de coton, et je me trouve alors en présence d'un organe qui a conservé sa forme, sa couleur, presque tout son volume, et dont la consistance est à la fois ferme et élastique.

Ici se place un détail qui a une grande importance. Avant d'envelopper le cerveau dans les compresses de linge, j'introduis dans les ventricules des poires de caoutchouc très simples, très vides, que je remplis d'air. La partie concave est ainsi distendue, et l'affaissement du cerveau est ainsi évité.

Abandonné à l'air au sortir de l'étuve, le volume du cerveau diminuerait rapidement par suite de l'évaporation de l'alcool. Pour éviter cela, j'enduis toute la surface de l'organe, circonvolutions, fentes, scissures, anfractuosités, avec une couche de vernis blanc au caoutchouc. Cette couche, une fois sèche, je la double d'une deuxième, d'une troisième, d'une quatrième, rarement d'une cinquième couche, semblables. $\mathrm{La}$ pièce, ainsi préparée, est complètement finie, je peux l'abandonner à l'air impunément.

T'ont le secret de la préparation consiste donc à faire pénétrer la substance cérébrale, dans toute son épaisseur, par de l'alcool à 90 degrés, qui a, à la fois, la propriété de la durcir et de la conserver, puis à emprisonner l'alcool dans cette substance à l'aide de quatre ou cinq couches de vernis blanc au caoutchouc, qui, s'opposant à l'évaporaiion, permettent de conserver le volume sans en changer la couleur. 
Application de la galvanoplastie. - C'est sur le cerveau ainsi préparé que je fais l'application de la galvanoplastie.

La seule difficulté à vaincre pour que la couche métallique recouvre bien tous les creux de la surface encéphalique réside dans la préparation des surfaces avant de plonger l'organe dans le bain de sulfate de cuivre, la plombagine seule étant infidèle.

On surmontera cette difficulté en procédant ainsi :

Il faut faire fondre de la cire blanche dans de l'essence de térébenthine et y ajouter un peu de plombagine. A l'aide d'un pinceau, on enduit facilement et fidèlement toutes les scissures et fentes les plus profondes ainsi que les circonvolutions.

On fait sécher la pièce. Une fois sèche, on la plombagine en totalité aussi exactement que possible.

G'est alors qu'on la plonge dans un bain métallique en prenant toutes les précautions ordinaires pour la galvanoplastie. Si l'on veut obtenir de belles épreuves, il faut la laisser de quarante-huit à soixante heures sans y toucher.

L'examen qu'on en fait à ce moment révèle-t-il quelques parties non recouvertes par le cuivre ou la plombagine, de nouveau on la replace dans le bain.

Après huit jours, l'organe est alors complètement métallisé. On peut le conserver ainsi indéfiniment:

Si l'on ne veut obtenir, par la galvanoplastie, que la face convexe des hémisphères, rien n'empêche, sur un cerveau entièrement métallisé, de faire deux larges ouvertures à la base, et d'enlever le cerveau lui-même. On a alors un moule inétallique léger d'une espèce de coque en cuivre qui représente très exactement la surface antérieure de l'organe.

A l'aide de bronzes de diverses nuances, on peut distinguer les circonvolutions les unes des autres, ainsi que les divers lobes.

J'ai fait l'application de mon procédé de galvanoplastie au cœur, au rein, à la rate, etc.

J'ai obtenu, pour le cœur, des pièces très remarquables, que j'aurai l'honneur de soumettre prochainement à l'Académie, dans lesquelleż les valvules, les muscles, les cordages des muscles se dessinent avec une netteté parfaite. 


\title{
MOYEN DE CONSERVER LES CERVEAUX
}

\author{
DESTINÉS A EFFEGTUER DE LONGS VOYAGES
}

(Bulletins de la Société d'anthropologie, 3e série, 1879, t. II, p.175-177.)

M. le docteur Breton envoie de Saïgon, par l'intermédiaire de M. Arnaud, qui habite Toulon, un cerveau de Chinoise enfermé dans une boîte de fer-blanc remplie d'alcool et soudée.

Ce cerveau est arrivé dans un tel état de décomposition, qu'il n'a pu être nullement utilisé. Quoique plongé dans l'alcool, il était complètement pourri et répandait une odeur infecte.

Il en a été malheureusement ainsi de tous les cerveaux humains, à l'exception d'un seul, qui nous ont été envoyés des pays lointains. Les cerveaux que M. Berenger-Féraud nous a envoyés du Sénégal ont' de même été perdus. Cela vient de ce que généralement on ne connaît pas les précautions à prendre pour les maintenir en bon état de conservation.

On croit assez généralement qu'îl suffit, pour conserver le cerveau, de le prolonger dans un vase plein d'alcool; mais, d'une part, l'eau que la substance cérébrale renferme en grande abondance affaiblit considérablement l'alcool, dont l'action devient insuffisante; de sorte que, si l'alcool n'est pas renouvelé au bout de quelques jours, la pièce ne se conserve pas.

En second lieu, la pénétration de l'alcool dans les couches profondes est assez lente, et celles-ci sont souvent ramollies sans retour lorsque le liquide conservateur les atteint. Ce qui retarde surtout l'imbibition du liquide, c'est la résistance des membranes extérieures (arachnoïde et pie-mère), dont l'ablation préalable est par conséquent nécessaire.

En troisième lieu, les parties inférieures qui reposent sur le fond du vase sont comprimées par le poids des parties supé- 
rieures à un degré suffisant pour que l'imbibition de l'alcool y soit considérablement retardée. Lorsqu'on examine le cerveau au bout de trois ou quatre jours, on voit que les circonvolutions inférieures ont conservé leur mollesse et leur couleur d'un gris rose, qu'elles ne se sont ni décolorées ni durcies comme le reste de la surface du cerveau. Quelques jours plus tard, elles tombent en putrilage ou s'altèrent à un degré très fâcheux, quoique toutes les circonvolutions de la face convexe soient très bien conservées.

Enfin, un autre inconvénient auquel les cerveaux sont exposés, même lorsqu'on les prépare dans un laboratoire, c'est la déformation qui résulte de leur aplatissement sur le fond du vase. Ils s'affaissent et s'étalent par leur propre poids, puis l'alcool les durcissant dans cet état, il n'est plus possible de leur rendre leur forme naturelle.

Voici donc quelles sont les précautions à prendre pour conserver les cerveaux dans un musée et à plus forte raison pour les faire voyager :

$1^{\circ}$ Le premier jour enlever sinon la totalité, du moins la plus grande partie de la pie-mère des hémisphères.

$2^{\circ}$ Déposer le cerveau sur une couche assez épaisse de ouate, au fond d'un vase d'une contenance de trois litres et verser dans ce vase un litre et demi d'alcool (ou deux litres au moins de tafia).

$3^{\circ}$ Retourner le cerveau tous les deux on trois jours afin d'empêcher d'une part la putréfaction des parties déclives et la déformation de tout l'organe.

$4^{\circ} \mathrm{Au}$ bout de quinze jours environ, lorsque le cerveau paraît partout suffisamment ferme, on renouvelle entièrement le liquide, et le cerveau se conserve définitivement, pourvu qu'il soit toujours entièrement recouvert.

50 Pour expédier le cerveau, on l'entoure entièrement d'une couche de ouate ou d'étoupe, on y joint une petite lame de plomb sur laquelle on a écrit le numéro d'ordre et qu'on fixe sur le paquet avec un lien lâche, puis on introduit le tout dans une petite boîte de fer-blanc, qu'on remplit entièrement d'alcool, et qu'on scelle, après avoir expulsé l'air. 
Toutes ces précautions sont également indispensables. Elles sont indiquées dans les Instructions générales, mais elles sont si peu suivies, qu'il m'a paru utile de les reproduire dans les Bulletins. 


\section{EXTRAGTION}

ET

\section{CONSERVATION DES GERVEAUX}

(Instructions générales pour les recherches anthropologiques à faire sur le vivant, $2^{e}$ édition, 1879, p. 15-23.)

L'extraction et la préparation du cerveau exigent une certaine habileté anatomique et des précautions particulières; mais nous ne saurions trop inviter Messieurs les médecins et naturalistes voyageurs à ne laisser échapper aucune occasion de recueillir cet organe important.

Les variations que présentent dans les diverses races humaines le volume et la forme du cerveau, n'ont pu être appréciées jusqu'ici que d'une manière indirecte; au lieu d'étudier le cerveau lui-même, on a dû se contenter d'étudier la boîte qui le renferme. Si la craniologie joue un si grand rôle dans les recherches anthropologiques, c'est principalement parce que quelquesuns des caractères du cerveau se traduisent extérieurement dans la configuration des os du crâne; mais l'examen direct du cerveau serait bien plus concluant. 0 n peut, en mesurant la capacité interne du crâne, évaluer d'une manière approximative le volume du cerveau; on peut encore, en comparant les dimensions relatives des os du crâne, obtenir quelques données sur le développement relatif des principales régions du cerveau. Cela vaut mieux que rien; mais la substance cérébrale n'occupe pas la totalité de la cavité crânienne; celle-ci renferme en outre des membranes dont l'épaisseur varie, et un liquide dont la quantité varie bien plus encore; en outre, les divers lobes dont se compose chaque hémisphère cérébral sont bien loin de correspondre exactement aux os dont ils portent les noms. L'étude du crâne 
ne peut donc donner qu'une connaissance très insuffisante du cerveau. Enfin, il y a des caractères - et ce sont les plus importants - dont on ne peut constater l'existence qu'en ayant sous les yeux et en comparant attentivement les cerveaux des diverses races. Ce sont ceux qui résultent de l'état des circonvolutions cérébrales, de leur complication, de leur développement relatif. Les cerveaux de races que nous avons pu recueillir à Paris, et que nous conservons précieusement, montrent toute la valeur de ces caractères, dont la détermination, toutefois, ne peut être faite que par des anatomistes spécialement voués à l'étude du cerveau et initiés à la connaissance, toute moderne, des circonvolutions cérébrales. Nous appelons donc tout particulièrement l'attention de Messieurs les voyageurs, et des médecins européens qui résident au milieu des races étrangères, sur la nécessité de recueillir, de conserver et d'envoyer à la Société le plus grand nombre possible de cerveaux des diverses races.

On doit chercher autant que possible à conserver le crâne en même temps que le cerveau; toutefois, il y a des cas où l'on ne peut prendre toute la tête; c'est ce qui a lieu, par exemple, dans les pays plus ou moins civilisés où les autopsies sont permises, mais où les dissections ne le sont pas. Dans ces conditions, on peut du moins conserver la calotte du crâne, détachée à la scie. Cela n'exige aucune préparation. Il sulfit, le jour même de l'autopsie, d'enlever le péricrâne par le grattage, puis de laver la calotte crânienne, de l'essuyer et de l'exposer vingt-quatre heures au grand air : au bout de ce temps elle est sèche et se conserve sans odeur. La calotte du crâne devra, toutes les fois qu'on le pourra, accompagner le cerveau, parce que cet organe se ratatine beaucoup et se déforme toujours un peu dans les liquides conservateurs. La calotte osseuse qui l'accompagne permet de lui restituer par la pensée, et au besoin par le moulage, sa forme et son volume primitifs.

Lorsqu'on pourra disposer d'une tête fraîche, on l'ouvrira le plus tôt possible, parce que dans les pays chauds le cerveau commence déjà à se ramollir au bout de vingt-quatre heures. On procédera de la manière suivante :

On pratiquera d'abord avec une bonne scie la coupe horizontale, bion connue des anatomistes. Cette coupe est difficile, sur- 
tout lorsque la fosse temporale est très déprimée, comme cela a lieu dans beaucoup de races; on doit donc craindre que la scie ne pénètre en certains points dans le cerveau, avant même d'avoir traversé toute l'épaisseur des os dans les points déprimés. C'est pourquoi on doit préférer à la scie à arc la scie à dos et à large lame, dont l'extrémité arronidie et dentelée peut atteindre les points déprimés, sans qu'on soit exposé à pénétrer dans le cerveau avec le reste de l'instrument.

L'épaisseur des os du crâne étant variable et inconnue, on doit procéder à la coupe avec lenteur et circonspection. On trace d'abord une rigole circulaire qui ne dépasse pas la table externe, puis on repasse plusieurs fois dans cette rigole jusqu'à ce qu'on sente en quelques points diminuer la résistance; on arrive ainsi à séparer presque toute la voûte du crâne sans avoịr entamé la dure-mère. On introduit de temps en temps dans la partie antérieure de la coupe, au-dessus des arcades orbitaires, un ciseau mousse large et épais qui sert de levier, et sur lequel on fait de petites pesées en cherchant à faire éclater les ponts osseux qui résistent encore. Lorsque enfin l'écartement obtenu au moyen de ce levier est devenu suffisant, ou passe un crochet de fer sous la partie antérieure de la calotte, et l'on détache celle-ci d'un coup sec, qui produit ordinairement en arrière, vers l'occiput, quelques esquilles insignifiantes.

La dure-mère étant mise à nu, on l'incise sur les deux côtés de la faux, on écarte les lambeaux, on coupe avec des ciseaux l'insertion antérieure de la faux, on renverse celle-ci en arrière en divisant les vaisseaux qu'elle reçoit de la pie-mère; puis on détache le cerveau d'avant en arrière en coupant successivement les nerfs optiques et autres nerfs crâniens; on aperçoit bientôt la tente du cervelet qu'on détache de son insertion antérieure a l'aide d'un bistouri, en rasant le bord postérieur du rocher; enfin, on renverse le cerveau en arrière et on le soutient avec la main gauche pendant que la main droite, armée d'un bistouri assez long, va couper le plus bas possible la moelle épinière dans le canal rachidien.

Le cerveau étant ainsi extrait, on le dépose avec précaution sur une table, où on le laisse pendant quelques moments, afin que la sérosité en excès puisse s'écouler. Lorsqu'il est conve- 
nablement égoutté, on le pèse à un gramme près. Ciette pesée est essentielle, parce que le cerveau perd dans les liquides conservateurs une partie notable et indéterminée de son poids.

Il faut procéder alors à l'ablation de la pie-mère qui recouvre les hémisphères cérébraux. On se sert pour cela de deux pinces et d'une paire de ciseaux avec lesquels on coupe tous les vaisseaux un peu gros qui pénètrent dans l'intérieur du cerveau. On dépouille d'abord la face inférieure des hémisphères, on pénètre avec soin dans la scissure de Sylvius, d'où l'on retire un prolongement considérable de la pie-mère; enfin, lorsqu'on a détaché un lambeau de cette membrane assez grand pour être saisi entre les doigts, on peut abandonner les pinces et dépouiller une à une les circonvolutions de la face inférieure et de la face externe ou convexe des deux hémisphères; quant aux circonvolutions de la face interne ou plane, on ne pourrait les dépouiller entièrement sans les écarter l'une de l'autre, et sans s'exposer à déchirer le corps calleux. Au surplus, il n'est pas nécessaire d'enlever la pie-mère en totalité ; il suffit d'en enlever la plus grande partie pour le but qu'on se propose d'atteindre. Ce but est de faciliter l'imbibition du liquide conservateur dans la substance cérébrale. Si l'on plongeait le cerveau dans l'alcool sans enlever la pie-mère, l'imbibition serait beaucoup plus lente, et il arriverait fréquemment que les couches profondes commenceraient à se ramollir et à se dissocier avant d'avoir subi à un degré suffisant l'action du liquide; la pièce durcirait, mais elle pourrait devenir friable et ne se conserver que pendant quelques mois. Le cervelet, moins volumineux que le cerveau, se laisse plus promptement imbiber jusqu'au centre; on n'a donc pas besoin de toucher à la pie-mère cérébelleuse qui serait, du reste, assez difficile à enlever.

On peut choisir indifféremment, pour conserver le cerveau, l'alcool du commerce ou le tafia. Le cerveau est plongé le plus tôt possible sur un fond de ouate ou d'étoupe, dans un vase contenant au moins un litre et demi d'alcool ou deux litres de tafia; on doit avoir soin de le retourner avec précaution tous les deux ou trois jours, parce que la surface qui repose surle fond du vase ne s'imbibe que très imparfaitement; elle pourrait même se ramollir ou se dissocier si l'on ne retournait pas l'organe. Au 
hout d'environ quinze jours, le cerveau est déjà assez ferme; alors on l'entoure de filasse ou d'étoupe, on y joint une petite lame de plomb sur laquelle on inscrit le numéro d'ordre, et qu'on fixe sur le paquet avec une ficelle, puis on plonge le tout dans un nouveau bain d'alcool ou de tafia. Le premier liquide en effet est mélangé d'une grande quantité d'eau que la substance cérébrale lui a cédée, et il n'est plus assez concentré pour servir à la conservation définitive.

Lorsqu'on a ainsi préparé plusieurs cerveaux, on peut les disposer dans un petit baril où on les emballe avec une quantité suffisante d'étoupes; on remplit ensuite le baril d'alcool ou de tafia, on le ferme hermétiquement et l'on n'a plus à craindre aucun accident. . Toutefois, lorsque le voyage doit durer longtemps, on fera bien de profiter de la première occasion pour expédier les pièces en Europe.

Si les difficultés de la conservation et du transport ne permettaient pas de nous envoyer des cerveaux entiers dans l'alcool, nous recommanderions vivement la préparation suivante, qui a pour but d'obtenir la momification des hémisphères cérébraux.

Après avoir, comme dans le cas précédent, pesé exactement le cerveau avec ses membranes, on coupe les deux pédoncules cérébraux pour enlever le cervelet et la protubérance annulaire (ou pont de Varole), et l'on ne garde ainsi que les deux hémisphères cérébraux. On les sépare l'un de l'autre par une section médiane ongitudinale, puis on les dépouille entièrement de leur piemère, ce qui est très facile, on les pèse séparément à un gramme près, et on les plonge dans un bain composé de six parties d'eau et d'une partie d'acide nitrique du commerce. Au bout de huit ou dix jours, les pièces sont retirées du bain, égouttées et exposées à l'air libre, sur des chiffons de linge qu'on renouvelle plusieurs fois le premier jour. Dès le second jour elles peuvent être placées purement et simplement sur une planche ou sur une assiette, et à partir de ce moment on n'a plus à s'en occuper; la momification s'effectue toute seule, à mesure que le liquide s'évapore. Toutefois, les pièces pourraient se racornir, se fendiller et même tomber en miettes si on les exposait au soleil, ou se ramollir, se déformer et devenir pâteuses si on les plaçait dans un lieı très humide. Lorsqu'on les tient dans un milieu 
bien sec, la préparation réussit presque toujours. La température de 20 à 25 degrés est la plus favorable. Au bout de cinq à six jours, les circonvolutions les plus superficielles commencent à se dessécher et à prendre une teinte roussâtre; au bout de deux semaines les pièces sont déjà assez sèches et assez dures pour qu'on puisse les manier sans aucune précaution pour ce qui les concerne; mais elles continuent à exhaler encore des vapeurs acides qui altéreraient et détruiraient les linges ou papiers dans lesquels on les enfermerait. Il faut donc les laisser à l'air libre pendant quelques semaines de plus.

Les hémisphères cérébraux ainsi momifiés ont une couleur analogue à celle des raisins secs. Ils sont durs comme le carton, et ne craignent ni les chocs ni l'humidité. Ils ont perdu plus des trois quarts de leur poids primitif, et leur volume par conséquent est considérablement réduit; mais leur forme est merveilleusement conservée, ețils se prêtent à l'étude des circonvolutions aussi bien et même mieux ą̀̀certains égards que les cerveaux frais. Nous ne saurions trop recommander l'emploi de ce procédé de momification qui est extrêmement simple et qui n'occasionne niembarras ni dépense (1).

L'étude du cerveau des grands singes anthropoïdes (gorilles, chimpanzés, orangs, gibbons, etc.) est jusqu'ici si peu avancée, et elle est pourtant si intimement liée à celle du cerveau des races humaines, que nous invitons vivement les voyageurs à recueillir, toutes les fois que l'occasion s'en présentera, les cerveaux de ces animaux. On reçoit quelquefois en Europe leurs têtes entières, conservées dans l'alcool, mais le cerveau est presque toujours fort altéré et souvent même tout à fait méconnaissable. Il est donc nécessaire, lorsque l'on conserve une tête de singe, d'extraire le cerveau lorsqu'il est encore frais, et de le préparer isolément comme il vient d'être dit pour les cerveaux humains.

Les personnes qui disposent d'un appareil à injecter pourront éviter la coupe du crâne en se servant du procédé de M. Bischoff, qui consiste à injecter dans le cerveau, à travers l'artère carotide, une solution de chlorure de zinc. Cette opération se fait très aisément sur une tête détronquée; on trouve alors, sur la surface

(1) Bulletins de la Société d'anthropologie, t. VI, 19 janvier 1865. 
de la section, les deux artères vertébrales, que l'on lie, puis les deux carotides. On lie également l'une des carotides, et on pousse l'injection dans l'autre; après quoi, la tête est plongée dans l'alcool, et le cerveau se conserve parfaitement. Si la tête n'était pas détronquée, si l'on voulait envoyer en Europe le corps entier de l'animal, soit dans l'alcool, soit dans le tafia, il faudrait mettre à nu les deux carotides primitives, au moyen de deux incisions longitudinales, introduire une canule dans chacun de ces vaisseaux, et pousser simultanément l'injection, soit avec deux seringues, soit avec une seringue débouchant dans un tube bifurqué; une partie du liquide revient par les deux vertébrales, et se perd dans le corps; mais, le débit des carotides étant supérieur à celui des vertébrales, les vaisseaux du cerveau reçoivent et conservent une quantité de chlorure de zinc parfaitement suffisante.

Si l'on n'avait qu'une seule canule, on ne pourrait injecter que l'une des carotides, après avoir lié l'autre; mais alors il faudrait que la pression fût plus forte, afin que le liquide, débouchant par une seule carotide, n'eût pas le temps de passer en totalité dans les vertébrales.

La solution à injecter se compose de 100 parties d'alcool et de 10 parties de chlorure de zinc.

L'importance hors ligne de l'étude du cerveau explique l'insistance que nous mettons à recommander la préparation et la conservation de cet organe. Un voyageur qui pourrait rapporter seulement cinq ou six cerveaux d'une ou plusieurs races étrangères, rendrait à la science un service inestimable. 



\section{TABLE DES MATIËRES}

\section{SIÈGE DE LA FAGULTÉ DU LANGAGE ARTICULÉ.}

Perte de la Parole, Ramollissement chronique et destruction ParTIElle du lobe antérieur gauche du cerveau.............

ReMARQUES SUR LE SIÈgE DE LA FACULTÉ DU LANGAGE ARTICULÉ SUIVIES D'une observation J'aphéMie.................... Nouvelle observation D'APHÉMIE PROdUtTe PAR UNk: LÉSION DE LA

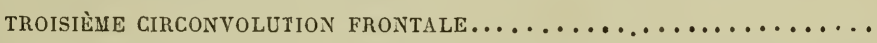
LOCALISATION DES FONCTIONS CÉRÉBRALES, SIÈGF DU LANGAGE ARTICULÉ. Remarques Sur le Siège, Le diagnostic el la Nature de L'APHÉmie... Recherches sur les fonctions cérébrales. . . . . . . . . . . SUR LES MOTS APHÉMIE, APHASIE ET APHRASIE ; LETTRE A I. LE PROFES-

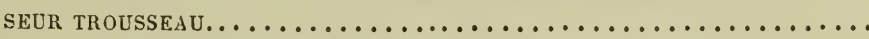

Deux Cas D'Aphémie traumatique produite par LES LÉsions de LA TROISIEME CIRCONVOLUTION FRONTALE GAUCHE, DIAGNOSTIC CHIRURGICAL.

Siège de la faculté du langage articulé. Deux Cas d'aphédie traUMATIQUE PRODUITE PAR DES LÉSIONS DE LA TROISIÈME CIRCONVOLUTION

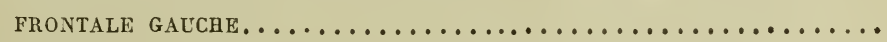

Sur le siège de la faculté du langage articulé. Aphémie traunaTIQUE. LÉSION DE LA TROISIEMEE CIRCONVOLUTION FRONTALE......... SUR L'APHÉMIE . . . . . . . . . . . . . . . . . . . . . .

DU SIÈgE DE LA FACULTÉ DU LANGAGE ARTICULÉ.............. Sur la faculté du LANGa $a$ e ARticulé..................... 101

Discussion SUR UNE OBSERVATION D'APHÉMIti DE M. VOISIN.......... 103 Aphasie traumatique............................ 110

Recherches sur la localisation de la faculté du langage articulé. 113

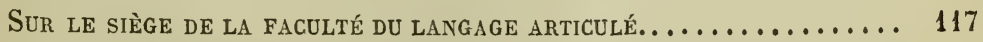
SUR LE POIDS RELATIF DES DFUX HÉMISPMÈRES CÉRÉBRAUX ET DE LEURS LOBES FRONTAUX $\ldots \ldots \ldots \ldots \ldots \ldots \ldots \ldots \ldots \ldots \ldots \ldots \ldots \ldots \ldots \ldots \ldots \ldots$ DE LA DIFFÉRENCE FONGTIONNELLE DES DEUX HÉMISPHÈ ES CÉRÉBRAUX. . 134

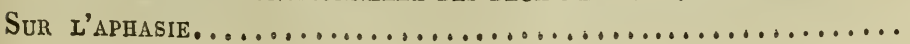




\section{ANATOMIE PATHOLOGIQUE DU CERVEAU}

Perte de substance du cervelet . . . . . . . . . . . . . . .

Sur le volume et la porce du Cerveau a l'État Normal et patioLOGIQUE.

Sur les pétrifications de L'encÉPHALE................

Note POUR SERvir a L'HISTOIRE DU RAMOLLISSEMENT CÉRÉBRAL PROGRESSIF SANS TROUBLES DE L'INTELLIGENCE NI DES FONCTIONS DE

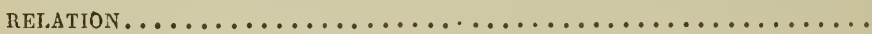

Sur les EMPREintes CÉrébrales a l'État Normal et Pathologique...

LE CRANE DE NÉANDERThal EST-IL PATHologiQue? Discussion sur LE voluME du CERVEAU........................... 178

OSSIFICATION PRÉMATURÉE DES SUTURES.................... 179

Sur l'assassin lemaire et SUR la criminalité............... 180

Sur la déformation toulousaine du CRANE.............. 18 J

SUR UN CAS EXCESSIF DE MICRocépHaLIE (encéphale de 104 grammes). . 202

Déformation congénitale du crane et de la face. Microcéphalie

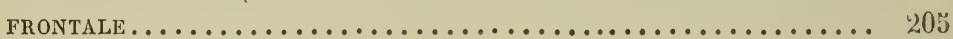

Crane et cerveau d'un homme atteint de la déformation toulousaine. 208

Du poids du Cerveau suivant la RaCe Et LE SEXe (Discussion). . . . . 212

SUR UN CERVEAU hUMAIN INCOMPLÈTEMENT DIVISÉ EN DEUX HÉMISPHÈrES.. 215

Localisations cérébrales. Sur le cerveau d'un fCtromélifn. . . . . 227

Moule d'un cerveau de toulousain .................... 232

LE CERVEAu DE I'ASSASSIN PRÉVOST. . . . . . . . . . . . . . . 234

Localisations CÉrébrales. SUR LE CERVEAU D'UN CUL-DE-JATTE . . . . . 243

\section{ANATOMIE COMPARÉE DU GERVEAU}

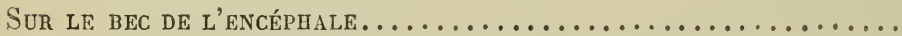

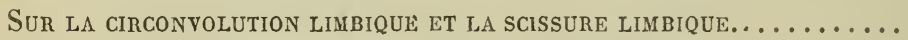

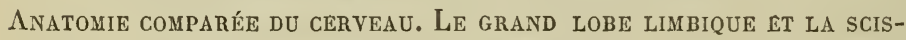
SURE LIMBIQUE DANS LA SÉRIE DES MAMMIFÈrES.............

$\S 1$. La circonvolution limbique du cerveau humain........

$\S 2$. Le lobe olfactif et les sens de l'odorat.............

$\S 3$. Le grand lobe limbique des mammifères décrit d'après le cerveau de la loutre.................... 273

$\S 4$. Cerveaux osmatiques lissencéphales............. 286

$\S$ 5. Cervẹux osmatiques gyrencéphales............. 296

$\S 6$. Cerveaux anosmatiques des mammifères aquatiques.... 332

$\S 7$. Cerveau des primates..................... 343

$\S 8$. Conclusions......................... 379

Localisations cérébrales. ReCuenches sur LES CENTRES OLFACTIFS. . 383 
§ 1. Du rôle de l'anatomie comparée dans l'étude des localisations cérébrales......................... 383

§ 2. L'appareil olfactif des mammifères.............. 387

$\S 3$. Les centres olfactifs chez les anosmatiques.......... 417

$\S 4$. Remarques sur les fonctions respectives des divers centres olfactifs.......................... 443

$\S 5$. Conclusions relatives aux centres olfactifs de l'homme... 460

Anatomie du lobe olfactif $\ldots \ldots \ldots \ldots \ldots \ldots \ldots \ldots \ldots \ldots \ldots \ldots \ldots \ldots$

EtUdes SUR I.E CERVEAU D'UN NÈGRE................. 463

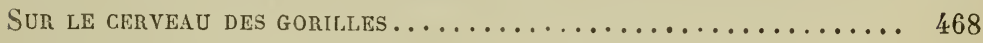

Sur le cerveau des primates..................... 469

Sur le type cÉrÉbral des prinates.................. 469

A propos nu cerveau d'un imbécile.................. 480

\section{MORPHOLOGIE DU GERVEAU DE L'HOMME ET DES PRIMATES}

SUR LA TOPOGRAPHE CRANIO-CÉRÉBRALE OU SUR LES RAPPORTS ANATOmiques du crane et du cerveau................... 481

$\S 1$. Historique de la question. Exposé des procédés.........

§ 2. Application de la topographie cérébrale à la médecine et à l'anthropologie .........................

$\S 3$. De la valeur pratique des procédés.................

$\S 4$. Description du procédé des fiches et de ses diverses appli-

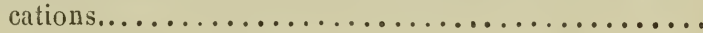

$\S$ ə̈. Sur une application chirurgicale de la topographie cérébrale (Trépanation du crâne). Topographie de l'organe

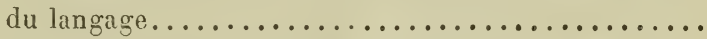

TOPOGRAPHIE CRANIO-CÉRÉbraLe, position relative des diverses parties du crâne et des diverses parties du cerveau (Revue critique).......

Sur LES RaPports des Circonvolutions dU CERVEAU humain aVEC LA SURFACE EXtrême du CRAne ET DE LA TÊTE (Revue critique)....... Sur un cas de lésion arobable du pli courbe (Revue critique).......

LES RAPPORTS TOPOGRAPHIQUES ENTRE LE CRANE ET LE CERVEAU A L'ÉTAT

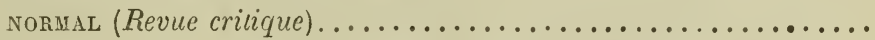

NOTE SUR LA TOPOGRAPHIE CÉRÉbRALE ET SUR QUELQUES POINTS DE L'HIS-

torre des ClRconvolutions..................... \$56

Présentation d'un cerveau de gorille male et adulite......... 567

Sur le cerveau a l'état fetal (Discussion) $\ldots \ldots \ldots \ldots \ldots \ldots \ldots . \quad 372$

SUR la topographie cÉrÉbrale comparéE DE L'HoMme ET DU CYNOCÉ-

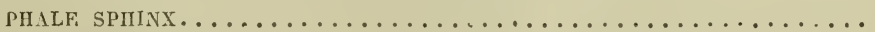

Sur le cerveau du gorille........................

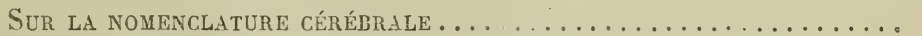

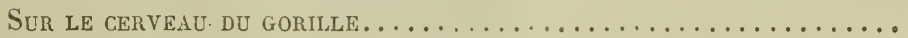

ETUde SUR LE CERVEAU DU GORILLE.... . . . . . . . . . . . . 
Nomenclaturé Gérébrale, dénonination des divisions et subdristoNs

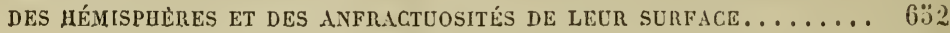

$\S 1$. Nécessité d'une nomenclature fixe............. 6ว̈2

§2. L'hémisphère dans son ensemble.

L'écorce et le manteau. Le corps de l'hémisplière.

Le seuil et le limbe de l'hémisphère......... $60 ̈ 6$

§ 3. Les divisions de l'hémisphère.

Lobes et lobules. Circonvolutions et plis.......... 680

$\S$ 4. Jes anfractuosités de l'hémisphère.

Scissures, sillons et incisures.............. 671

$\S$ 5้. Autres anfractuosités cérébrales............... 689

$\S 6$. De la notation des circonvolutions et des sillons....... 696 Cerveau d'un gorille de neux ans et demi..............

Sur le cerveau du gorille...................... 703

Sur trois chrveaux d'orang.................... 703

DEscription ÉléMentaire des CIRCONVOLUtions CÉrébrales DE L'HOMUE D'APRE'S LE CERVEAU SCHÉMATIOUE. . . . . . . . . . . . . . 707

I. Introduction. Utilité du cerveau schématique......... 707

II. Nomenclature........................... 714

Art. I. L'hémisphère dans son ensemble........... 713

Art. II. Les divisions du manteau de l'hémisphère....... 718

Art. III. Les anfractuosités de l'hémisphère (scissures, anfractuosités, incisures)............ 720

Art. IV. Quelrques mots sur le grand lobe limbique et la scissure limbique................. 723

III. Notation . . . . . . . . . . . . . . . . . . 723

IV. Méthode de description................... 728

V. Lobes et scissures....................... 730

Art. I. Deseription des scissures............... 730

Art. II. Les lobes et la zone neutre.............. 761

$\S$ 1. I.es lobes.................... 761

§. La zone neutre du manteau............ 768

VI. Les circonvolutions et les sillons ............... 773

Art. I. Circonvolution unique du corps calleux........ 773

Art. II. Circonvolutions du lobe frontal........... $7 \% 6$

Art. 111. Circonvolutions du lobe pariétal........... 797

\section{PROGÉDÉ DE CONSERVATION DES CERVEAUX}

Momification des cerveaux...................... 813

Momification des cenveaux ..................... 814

Procédé de conservation des cerveaux............... 815

Cerveaux conservés par la galvanoplastie.............. 810

Sur t.e procédé dF, CONSERVATION DES CERVEAUX DE d. ORÉ........ 816 
Procedé pour la conservation du cerveau, avec sa forae, son volume, sa couleur. Application de lagalvanoplastie par m. oré. 819 Moyen de Conserver les chrveaux destinés a effectuer de longs vortgks............................. 822 Extraction et Conservation des CERVEAUX.............. 825 


\section{TABLE DES FIGURES}

Figures.

1. Pétrification de l'encéphale...................... 16

2. Cerveau de la Toulousaine, face latéralc de l'hémisphère gauche. 189

3. Cerveau de la Toulousaine, face supérieure ............ 189

4. Crâne de la Toulousaine, profil dessiné au stéréographe...... 196

5. Cerveau humain incomplètement divisé en deux hémisphères. . 216

6. Loutre, face inféro-interne de l'hémisphère droit......... 274

7. Loutre, face inférieure du cerveau................ 276

8. Cheval, coupe longitudinale de la partie intérieure de l'hémisphère gauche à 6 millimètres de la ligne médiane...... 279

9. Loutre, face interne de l'hémisphère droit............ 281

10. Loutre, face supérieure du cerveau ................ 283

11. Loutre, face externe de l'hémisphère druit............ 284

12. Marmotte. No I, face inférieure. No II, face interne de l'hémisphère gauche. No III, face externe de l'hémisphère gauche.

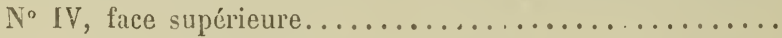

13. Castor. $N^{\circ} I$, face inférieure. $N^{\circ}$ II, face interne de l'hémisphère droit. No III, face externe de l'hémrsphère droit. No IV, face

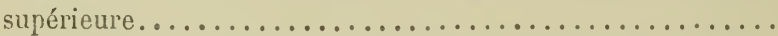

14. Lapin. No I, face inféricure. No II, face interne de l'hémisphère droit. No III, face externe de l'hémisphère droit. No IV, face supérieure............................ 29⿺尢丶

13̆. Cheval, face inféricure du cerveau................. 297

16. Unan. No I, face supérieure du cerveau. No II, face exterue de l'hémisphère droit....................... 304

17. Renard, face externe de l'hémisphère droit........... 307

18. Tapir, face externe de l'hémisphère gauche........... 310

19. Cheval, face externe de l'hémisphère droit............ 311

„20. Chevreuil, face externe de l'hémisphère droit........... 313

21. Porc domestique, face externe de l'hémisphère droit........ 314

22. Porc domestique, face externe de l'hémisphère gauche...... 314

23. Chien de berger, face externe de l'hémisphère gauche...... 316

24. Chien ratier, face externe de l'hémisphère gauche....... 317

23. Tapir, face postéricure et tentoriale de l'hémisphère gauche... 320

26. Chevreuil, face interne de l'hémisphère droit.......... 321

27. Cheval, face interne de l'hémisphère droit........... 323

28. Tapir, face interne de l'hémisphère droit........... 326

29. Renard, face interne de l'hémisphère droit........... 329 
Figures.

30. Chien ratıer, face interne de l'hémisphère droit.... . . . . . . 330

31. Renard, face supérieure du cerveau............... 332

32. Dauphin, face inférieure de l'hémisphère gauche........ . 33/t

33. Phoque, face interne de l'hémisphère droit... . . . . . . . . . 339

34. Phoque, face inférieure de l'hémisphère droit.......... 340

3วั. Lagotriche de Humboldt, face interne de l'hémisphère droit. . . 352

36. Lagotriche de Humboldt, face interne de l'hémisphère droit... 352

37. Cynocéphale papion (Pithécien), face externe de l'hémisphère

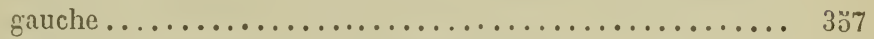

38. Cebus apella, face externe de l'hémisphère droit.......... 363

39. Cebus apella, face externe de l'hémisphère droit.......... 363

40. Cynocéphale papion, face interne de l'hémisphère droit...... 373

41. Chimpanzé, face interne de l'hémisphère droit.......... 377

42. Loutre. Le grand lobe limbique dans son ensemble. Schéma de la face inféro-interne de l'hémisphère droit..........

43. Loutre, face inféricure du cerveau................

44. Mouton, coupe longitudinale verticale pratiquée sur l'hémisphère gauche à 5 inillimètres de la ligne médiane............

45. Cheval, coupe longitudinale de la partie antérieure de l'hémisphère gauche à 6 millimètres de la ligne médiane........

46. Chien, coupe vertico-longitudinale du lobe olfactif, 12 diamètres, ligne demi-schématique.................... 401

47. Chien, coupe horizontale du lobe olfactif, 15 diamètres...... 401

48. Ane, face inférieure de l'hémisphère gauche............ 402

49. Ane, face interne de l'hémisphère gauche........... 403

50 . Chien, grandes cellules multipolaires (motrices) du lobe olfactif, 400 diamètres....................... 412

ว้1. Chien, cellules multipolaires (motrices) des circonvolutions qui bordent le sillon crucial, 400 diamètres..............

52. Homme, coupe de la couche corticale du renflement olfactif, 100 diamètres........................ 414

53. Homme, cellules du renflement olfactif, 400 diamètres..... 41 วั

54. Dauphin, face inférieure de l'hémisphère gauche........ 418

5้. . Cebus apella, face inférieure de l'encéphale............ 42.1

56 . Homme, face inférieure de l'hémisphère gauche........ 422

57. Macaque rhésus, face interne de l'hémisphère droit....... 423

38. Phoque, face inférieure de l'hémisphère droit.......... 426

509. Homme, face inférieure du cerveau d'un homme mort de la varalysie générale des aliénés, demi-grandeur..........

60. Renard, face interne de l'hémisphère droit..............

61. Tapir, face interne de l'hémisphère droit..............

62. Chimpanzé, face interne de l'hémisphère droit...........

63. Phoque, face interne de l'hémisphère droit............

6千. Cynocéphale papion, face interne de l'hémisphère droit.......

65. Crâne de la Toulousaine, profil dessiné au stéréographe...... 
66. Cerveau de la Toulousaine d'après le moule en plâtre, face externe de l'hémisphère gauche...................

67. Cerveau de la Toulousaine d'après le moule en plâtre, face supé-

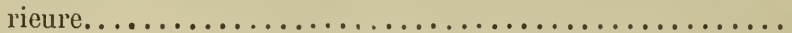

68. Homme. Topographie cérébrale d'un nègre d'Afrique orientale.

69. Homme. Rapport des"circonvolutions avec la surface externe du crâne..................................

70. Gorille mâle et adulte, face supérieure du cerveau..........

71. Homme. Cerveau d'un fœtus humain de quatre mois passés...

72. Homme. Cerveau d'un fœetus mâle de cinq mois et demi à six

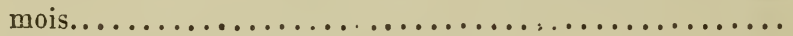

73. Homme. Cerveau d'un fœtus de six nois ...............

74. Homıne. Cerveau d'un fœtus de cent quatre-vingt-un jours....

75. Cynocéphale sphinx. Topographie cérébrale..............

76. Homme (parisien). Topographie cérébrale..................

77. Gorille mâle et adulte, cerveau face supérieure $\left(D^{r}\right.$ Nègre $) . . .$.

78. Gorille femelle adulte; photographie du cerveau altéré (Gra-

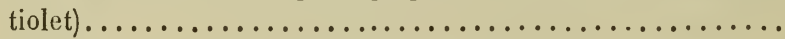

79. Gorille mâle et adulte, hémisphère gauche $\left(D^{r}\right.$ Nègre $) \ldots \ldots \ldots$

80. Gorille mâle et adulte, face inférieure ( $\mathrm{D}^{\mathrm{r}}$ Nègre $) . . \ldots \ldots \ldots$.

81. Homme. La scissure de Sylvius.................. 623

82. Gorille mâle et adulte, face supérieure ( $\mathrm{D}^{\mathrm{r}}$ Nègre $) \ldots \ldots \ldots \ldots .629$

83. Gorille inâle et adulte, face interne ( $\mathrm{D}^{\mathrm{r}}$ Nègrej........... 631

84. Loutre. Schéma de la face inféro-interne de l'hémisphère droit. 724

85. Cheval, face interne de l'hémisphère droit............ 724

86. Homme. Cerveau d'un fœtus humain de cinq mois, face externe. $\quad 736$

87. Figure schématique pour montrer le mode de disposition de la

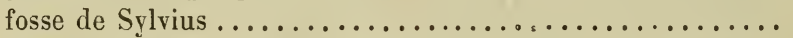

88. Les trois arcs de la scissure sous-frontale (figure schématique).

89. Le carrefour de l'hémisphère.....................

90. Face inférieure du lobule orbitaire : variétés de l'incisure en $H$ (hémisphère gauche)...................... 793

91. Cerveau schématique, face externe............... 805

92. Cerveau schématique, face supérieure.............. 807

93. Cerveau schématique, face interne................ 800

9'. Cerveau schématique, face inférieure............... 811 



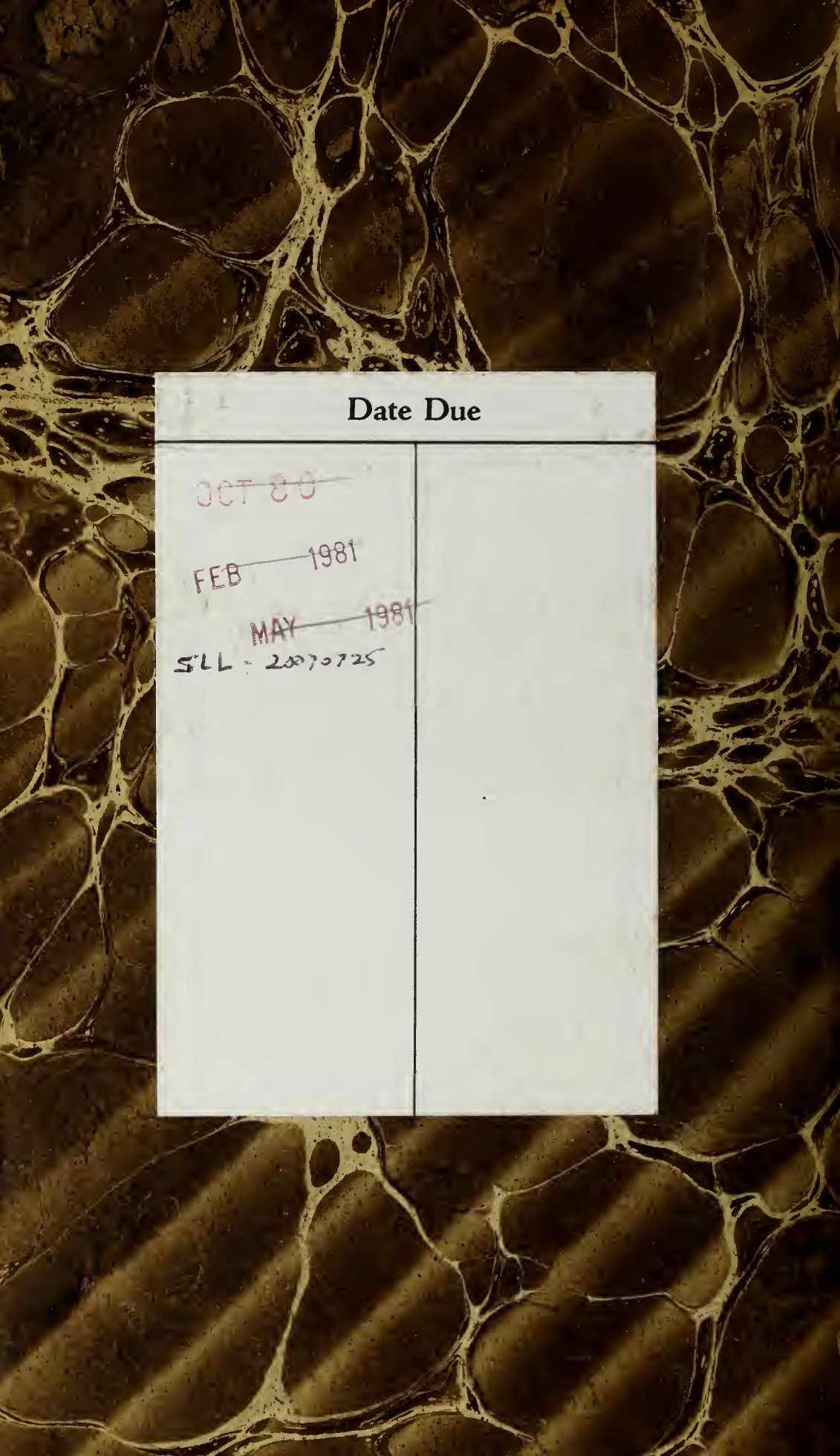




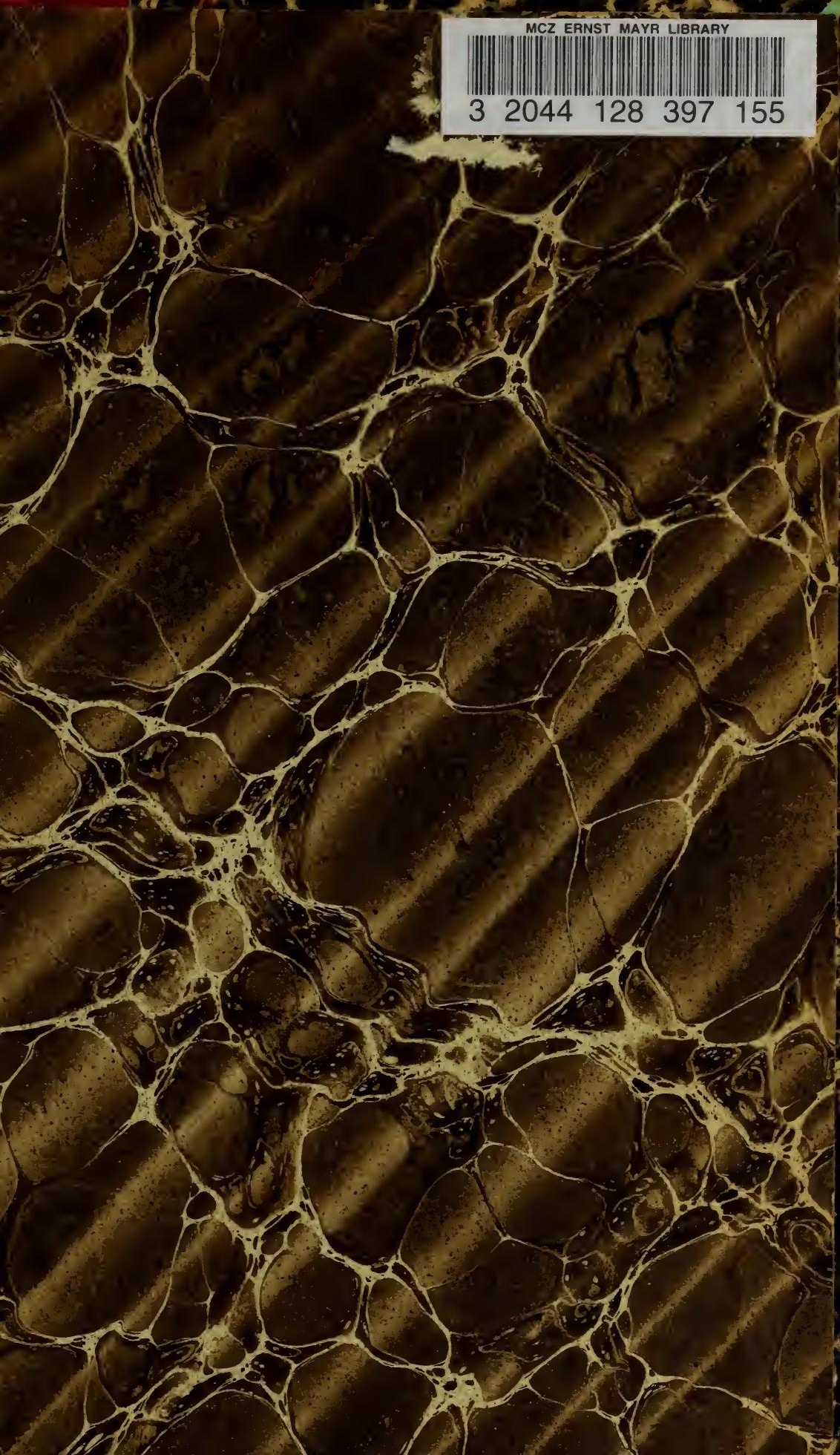


